\title{
Healthy Living: The European Congress of Epidemiology, 2015
}

\author{
Esther Bols $\cdot$ Luc Smits $\cdot$ Matty Weijenberg
}

(C) Springer Science+Business Media Dordrecht 2015

\section{Introduction}

The $8^{\text {th }}$ European Congress of Epidemiology was organised by the Department of Epidemiology of Maastricht University, Maastricht, the Netherlands. The organisation was made possible by the International Epidemiological Association-European Epidemiology Federation (IEA-EEF), the Dutch Epidemiological Society (VvE) and the organisation of the Dutch symposium Onderzoek in Beweging. The conference combined three regular conferences:

- The $8^{\text {th }}$ European Congress of Epidemiology

- The $39^{\text {th }}$ WEON 2015 (the annual conference of the Dutch Epidemiological Society, VvE)

- The $19^{\text {th }}$ 'Onderzoek in Beweging' (the annual symposium for (para)medical disciplines)

Publishing all abstracts presented at the European Congress of Epidemiology 2015 is of great relevance as it is not only a valuable reference work and source of inspiration for our discipline, but may also stimulate further research and collaboration.

The overarching theme of the conference was 'Healthy Living'.

Ongoing demographic and societal changes have a major impact on health and living. Demographic changes are leading to aging societies with increasing prevalence of chronic diseases. Improved diagnosis and treatment options each contribute to longer lives, but also to more years lived with disease and disability. Apart from

This supplement includes the abstracts accepted by the Scientific Committee of the European Congress of Epidemiology, presented in Maastricht, the Netherlands, 25-27 June 2015.

On behalf of the Scientific Committee.

E. Bols $(\varangle) \cdot$ L. Smits

Department of Epidemiology, School for Public Health and Primary Care (CAPHRI), Maastricht University, Maastricht, The Netherlands

e-mail: emj.bols@maastrichtuniversity.nl

\section{Weijenberg}

Department of Epidemiology, GROW—School for Oncology and Developmental Biology, Maastricht University, Maastricht, The Netherlands adding more years to life, there is an increasing need to focus on improving quality of life and thereby adding life to years.

Aging of the population also increases the pressure on the working population. In order to sustain current pension systems, employees have to postpone retirement in most European countries. However, will workers be able to continue to participate as they grow older? Do their skills and health conditions adequately match the demands of the future labor market? Hence, there is an increased need for sustainable employment aimed at fostering the healthy and productive engagement of workers throughout their careers. This requires optimal functioning and participation of workers, even those with one or more chronic health conditions.

Amidst these developments, there is a growing awareness of the seriousness of inequality in health, as these inequalities have a major impact on participation. According to the Dutch National Institute for Public Health and the Environment, RIVM, the life expectancy in the Netherlands for people with a lower education is, on average, 6 years shorter than for people with a higher education (RIVM Forecast Report 2014). When looking at the 'life expectancy in good selfperceived health' this difference is as much as 19 years.

The trends mentioned above give rise to great challenges for epidemiologic research. The first challenge is the shift from merely counting disease occurrence to measuring more complex outcomes such as quality of life, social or work participation, psychosocial factors, functioning in the presence of multimorbidity, or overlapping disease phenotypes leading to new concepts such as the diseasome. Second, there is a growing need to measure increasingly complex exposures that can change over time and for which biomarkers or state-of-the-art devices are promising assessment techniques. The technical potentials are overwhelming, allowing for instance an increasingly comprehensive and simultaneous measurement of multiple biomarkers using omics techniques. Third, studying health across the lifespan requires the consideration of different time-windows with regard to both exposure and outcome and of different trajectories from exposure to outcome. How should we design efficient studies to address these challenges? How should we model the findings? How should we interpret the results in terms of causality? How can we translate the results in order to contribute to public health in 2015 and beyond? Finally, with all these questions accumulating in an increasingly complex research environment with increasing pressures to publish, how do we avoid research waste?

The aforementioned topics were addressed during the Keynote lectures and other scientific sessions of the congress. 


\section{Abstract submission}

The call for abstracts (30 September 2014-9 February 2015) was disseminated online (http://www.healthyliving2015.nl/), announced by direct mail to health and education institutions involved in epidemiology-related research, and issued in several newsletters. During abstract submission, authors were asked to indicate up to three research areas most related to the topic of their abstract (drop-down menu). They could also indicate whether they wanted to be nominated for the Dutch Epidemiological Society (VvE) Student Award and/or VvE Poster Award. Additional information requested included affiliations, preferred mode of presentation, availability as an external reviewer, self-reported fields of expertise and seniority.

\section{Reviewer recruitment and review process}

Reviewers were recruited from three sources: (1) Members of the Department of Epidemiology, Maastricht University $(n=46)$, (2) The pool of professional contacts of members of the Department (invited external reviewers, $n=49$ ) and (3) The pool of volunteers collected via the online submission system $(n=208$ volunteers, 92 of which eventually reviewed abstracts). Each abstract was reviewed by two independent reviewers, preferably one senior (at least 4 years of research experience) and one junior researcher originating from different institutes and (whenever possible) in different countries (other than the main author's country of origin). The online review system was developed by members of the Scientific Committee and the Congress Office for this occasion.

Each reviewer received a unique log into the online review system. Internal reviewers evaluated a maximum of 15 abstracts each and external reviewers a maximum of 10 abstracts. Reviewers were blinded for author names and affiliations. Abstracts were scored on four aspects: (1) Clarity (with respect to abstract, title, hypothesis, method, results (tables/figures), and conclusion), (2) Methodology (appropriateness in view of research question), (3) Importance (are the results, or the methodology, important or relevant for the field of study to which the abstract belongs), and (4) Overall personal impression. Each aspect was rated on a scale of 1 (poor) to 10 (excellent). In case of a difference greater than 3 points between the two reviewers' scores for overall personal impression, a third reviewer was invited. The final score for personal impression was defined as the average value of two evaluations, whereas the median value was used in case of discrepancy. A total of 50 discrepancies were observed ( 5 abstracts $\geq 6$ points difference). In addition to the overall personal impression score, the average score for aspects 1-3 was calculated. Abstracts with at least 5.0 points for both average values (personal impression and clarity/methodology/importance) were accepted for the congress. The top scoring abstracts were selected for an oral presentation.

The call resulted in 790 submitted abstracts. After the review process, the Scientific Committee accepted 81 abstracts for an oral presentation and 661 abstracts for a poster presentation. Forty-two abstracts were rejected and 6 abstracts were withdrawn in the meantime (acceptance rate: $94.6 \%$ ). Table 1 provides an overview of accepted abstracts by research area. Part of the oral presentations in the scientific programme were provided by invited speakers $(n=21)$.

It is of note to mention that several high quality abstracts from Europe and other continents were accepted for oral and poster presentations but eventually had to decline the acceptance of our invitation due to lack of research funds for such activities. It may be worthwhile to try to budget travel awards for some of these participants when organising future conferences.

\section{Scientific programme}

The oral and poster presentations covered a large variety of topics within epidemiology and reflected the interests of the delegates (Table 1). Some of the proposed research areas could not be accommodated in the scientific programme, but another existent coherent session was always found to accommodate all abstracts. The tradition within the Dutch Epidemiological Society is to offer young up-coming scientists the opportunity to present their work, not only during poster sessions, but also during oral presentations in parallel sessions and plenary sessions. Participants were able to discuss these issues in more detail in a smaller more interactive setting with the Keynote speakers during the Meet-the-keynote sessions after every plenary session. In addition, Up-to-date sessions offered interactive lectures on specific methodological and statistical topics. The conference was preceded by a series of pre-conference workshops most of which were sneak-previews of international courses, or courses from master or bachelor programs offered at Maastricht University.

Special attention was paid to the organisation of the poster presentations, with the purpose to provide an additional platform for interaction between delegates and authors. Two poster sessions of 1-h duration were planned on 26 and 27 June between 13.30 and 14.30. The 661 accepted posters were distributed among 56 poster sessions with each a separate theme (e.g. cancer epidemiology or public health). During this session, a "poster moderator", often one of the poster presenters in the session, lead a small group of delegates around the poster session and poster presenters were able to summarize their poster (1-min talk), followed by questions and discussion (4 min) from delegates.

Table 1 Accepted abstracts by primary research area

\begin{tabular}{lr}
\hline Cancer epidemiology & 74 \\
Epidemiologic methods & 65 \\
Epidemiology of cardiovascular disease & 44 \\
Epidemiology of diabetes and obesity & 49 \\
Epidemiology of mental disorders & 27 \\
Epidemiology of musculoskeletal disorders & 23 \\
Epidemiology of reproductive disorders & 8 \\
Ethics and philosophy & 4 \\
Genetic and molecular epidemiology & 21 \\
Health registries & 7 \\
Infectious disease epidemiology & 58 \\
Life-course epidemiology & 23 \\
Nutritional epidemiology & 54 \\
Occupational/environmental epidemiology & 73 \\
Onderzoek in beweging & 27 \\
Other (not specified) & 4 \\
Pharmaco-epidemiology & 15 \\
Public health & 123 \\
Research protocol only & 11 \\
Social epidemiology & 56 \\
Other (specified) & 24 \\
Total & 790 \\
\hline
\end{tabular}




\section{Concluding remark}

It was a great pleasure to welcome so many epidemiologists from all over the world in Maastricht at this years' European Congress of Epidemiology. We were pleased by the large amount of high-quality abstracts, which enabled us to provide an interesting and varying scientific programme. The congress contributed to fruitful collaborations and ongoing discussions aimed at Healthy Living in 2015 and beyond.

\section{Organisation}

\begin{tabular}{|c|c|}
\hline ging voor epidemiclogie & $\begin{array}{l}\text { Vereniging voor Epidemiologie } \\
\text { http://www.epidemiologie.nl/ }\end{array}$ \\
\hline stricht University & $\begin{array}{l}\text { Maastricht University, the Netherlands } \\
\text { http://www.maastrichtuniversity.nl/ }\end{array}$ \\
\hline & $\begin{array}{l}\text { International Epidemiological Association } \\
\text { (IEA)_European Epidemiology } \\
\text { Federation (EEF) }\end{array}$ \\
\hline & $\begin{array}{l}\text { Onderzoek in Beweging } \\
\text { http://www.onderzoekinbeweging.nl/ }\end{array}$ \\
\hline 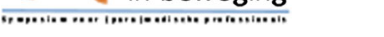 & \\
\hline
\end{tabular}

\section{International Scientific Committee}

Matty P. Weijenberg (chair Scientific Committee), Department of Epidemiology, Maastricht University, the Netherlands;

Esther M.J. Bols, Department of Epidemiology, Maastricht University, the Netherlands;

Rob A. de Bie, Department of Epidemiology, Maastricht University, the Netherlands;

Martien C.J.M. van Dongen, Department of Epidemiology, Maastricht University, the Netherlands;

Maurice P. Zeegers, Department of Complex Genetics, Maastricht University, the Netherlands;

Luc J.M. Smits, Department of Epidemiology, Maastricht University, the Netherlands;

Ilja C.W. Arts, Department of Epidemiology, Maastricht University, the Netherlands;

Bas A.J. Verhage, Department of Epidemiology, Maastricht University, the Netherlands;

Eline van Roekel, Department of Epidemiology, Maastricht University, the Netherlands;

Mariëlle M.H.J. van Os, Department of Epidemiology, Maastricht University, the Netherlands;
Eva Grill, Institute for Medical Informatics, Biometry and Epidemiology (IBE), Ludwig-Maximilians-Universität Munich, Germany;

Adele Seniori Costantini, Cancer Prevention and Research Institute (ISPO), Florence, Italy;

Enkelejda Shkurti, Department of Rehabilitation and Diagnostics Medicine, University of Medicine, Tirana, Albania.

\section{Local Organising Committee}

Martin H. Prins (chair Organising Committee), Department of Epidemiology, Maastricht University, the Netherlands;

Yvonne M.R. Leenders, Department of Epidemiology, Maastricht University, the Netherlands;

Esther M.J. Bols, Department of Epidemiology, Maastricht University, the Netherlands;

Rob A. de Bie, Department of Epidemiology, Maastricht University, the Netherlands;

Matty P. Weijenberg, Department of Epidemiology, Maastricht University, the Netherlands;

Martien C.J.M. van Dongen, Department of Epidemiology, Maastricht University, the Netherlands;

Maurice P. Zeegers (on behalf of the Dutch Epidemiological Society $\&$ the European Epidemiology Federation), Department of Complex Genetics, Maastricht University, the Netherlands;

Harry P.L. van Montfort, Department of Epidemiology, Maastricht University, the Netherlands.

\section{Congress Office}

Klinkhamer Group

Molensingel 476229 PB Maastricht, the Netherlands

$\mathrm{T}+31(0) 43-3627008$

www.klinkhamergroup.com

\section{IEA/EEF representatives}

Maurice P. Zeegers, Department of Complex Genetics, Maastricht University, the Netherlands;

Adele Seniori Costantini, Cancer Prevention and Research Institute (ISPO), Florence, Italy.

\section{Acknowledgements}

Prof. Dr. IJmert Kant for providing input on the text of the introduction.

\section{Reviewers}

The Scientific and Organising Committees would like to thank the following reviewers for having evaluated the abstracts submitted to the congress: 


\begin{tabular}{|c|c|c|c|}
\hline Teun Aalbers & Sarah Dörenkamp & Margreet Lüchtenborg & $\begin{array}{l}\text { Stephane } \\
\text { Sanchez }\end{array}$ \\
\hline $\begin{array}{l}\text { Mehdi Mirzaei } \\
\text { Alavijeh }\end{array}$ & Ghassan El Malki & Nicoline van der Maas & $\begin{array}{l}\text { Mohammed } \\
\text { Sanni Ali }\end{array}$ \\
\hline $\begin{array}{l}\text { Krasimira } \\
\text { Aleksandrova }\end{array}$ & Rachel Elands & $\begin{array}{l}\text { Aristides Machado- } \\
\text { Rodrigues }\end{array}$ & $\begin{array}{l}\text { Zoe Louise } \\
\text { Saynor }\end{array}$ \\
\hline Sandra Alves & Frank Elgar & Joreintje Mackenbach & $\begin{array}{l}\text { Jorgen van der } \\
\text { Schans }\end{array}$ \\
\hline $\begin{array}{l}\text { Ludo van } \\
\text { Amelsvoort }\end{array}$ & Els van der Ende & $\begin{array}{c}\text { Fernanda Penido } \\
\text { Matozinhos }\end{array}$ & Tania Schink \\
\hline $\begin{array}{l}\text { Larissa Fortunato } \\
\text { Araujo }\end{array}$ & Simone Eussen & Amy Matser & $\begin{array}{l}\text { Sabrina } \\
\text { Schlesigner }\end{array}$ \\
\hline $\begin{array}{c}\text { Thiago Machado } \\
\text { Ardenghi }\end{array}$ & Philippine Fassier & Jenab Mazda & $\begin{array}{l}\text { Salome } \\
\text { Scholtens }\end{array}$ \\
\hline Ilja Arts & Mark Ferro & Catherine Mbakwa & Leo Schouten \\
\hline $\begin{array}{l}\text { Leonor Bacelar- } \\
\text { Nicolau }\end{array}$ & Maria Ganczak & Linda Meertens & Holger Schultz \\
\hline Chyi-Huey Bai & $\begin{array}{c}\text { Judith Garcia- } \\
\text { Aymerich }\end{array}$ & Nico van Meeteren & Sarah Seaton \\
\hline Mirjam Bakker & $\begin{array}{r}\text { Genevieve } \\
\text { Gariepy }\end{array}$ & Audrey Merry & $\begin{array}{r}\text { Enkelejda } \\
\text { Shkurti }\end{array}$ \\
\hline Rienke Bannink & $\begin{array}{l}\text { Marleen van } \\
\text { Gelder }\end{array}$ & Ilse Mesters & Judith Sieben \\
\hline Sandhi Barreto & Lode Godderis & Samilly Silva Miranda & Sabine Siesling \\
\hline Carolien Bastiaenen & Fleur Gommans & Monique Mommers & Colinda Simons \\
\hline Manu Batra & Christel van Gool & Farid Najafi & Jet Smit \\
\hline Emmylou Beekman & Eva Grill & Patty Nelemans & Luc Smits \\
\hline Sandra Beijer & Andrej Grjibovski & Roger Nelson & $\begin{array}{c}\text { Joelle Laure } \\
\text { Sobngwi }\end{array}$ \\
\hline $\begin{array}{l}\text { Gabriele Berg- } \\
\text { Beckhoff }\end{array}$ & $\begin{array}{l}\text { Angelique } \\
\text { Grosser }\end{array}$ & Yannick Nielen & Maria Sousa \\
\hline Rob de Bie & Jessica Gubbels & Mark Nieuwenhuijsen & Jacob Spallek \\
\hline $\begin{array}{l}\text { Louise den } \\
\text { Biggelaar }\end{array}$ & Marc Gunter & Masato Nigai & Inge Spronk \\
\hline $\begin{array}{l}\text { Maria del Carmen } \\
\text { Bisi }\end{array}$ & Juliane Hardt & Yeshambel Nigatu & Bart Staal \\
\hline Marieke Blom & Fred Hartgens & Astrid Nooyens & $\begin{array}{l}\text { Sheila Rizzato } \\
\text { Stopa }\end{array}$ \\
\hline Jolanda Boer & $\begin{array}{l}\text { Janneke } \\
\text { Hogervorst }\end{array}$ & Manami Ochi & Dave Stynen \\
\hline Esther Bols & Huub Hoofs & Benson Ogunjimi & $\begin{array}{r}\text { Marie Pierre } \\
\text { Tavolacci }\end{array}$ \\
\hline Bart Bongers & $\begin{array}{l}\text { Thomas } \\
\text { Hoogeboom }\end{array}$ & Olukemi Olugbade & Carel Thijs \\
\hline Gouke Bonsel & Vincent Jaddoe & Sinead O'Neill & $\begin{array}{l}\text { Christophe } \\
\text { Vanroelen }\end{array}$ \\
\hline Hans Bosma & Nicole Jansen & $\begin{array}{l}\text { Charlotte Onland- } \\
\text { Moret }\end{array}$ & Femmie de Vegt \\
\hline Mariska Bot & Kathrin Jobski & Raymond Ostelo & Bas Verhage \\
\hline Martijn Bours & $\begin{array}{l}\text { Samantha } \\
\text { Johnston }\end{array}$ & Raziye Ozdemir & Sita Vermeulen \\
\hline Piet van den Brandt & Ellen Kampman & John Penders & Adri Voogd \\
\hline José Breedveld & $\begin{array}{l}\text { Ijmert van der } \\
\text { Kant }\end{array}$ & $\begin{array}{c}\text { Julia Moreira } \\
\text { Pescarini }\end{array}$ & Frank de Vries \\
\hline Ralph Brinks & Jan Kardaun & Sanne Peters & Martine Vrijheid \\
\hline Hannah Brooke & Thomas Keil & Tobias Pischon & Tanja Vrijkotte \\
\hline Carin de Brouwer & Davood Khalili & Letizia Polito & $\begin{array}{c}\text { Eliseu Alves } \\
\text { Waldman }\end{array}$ \\
\hline Vivian Bruls & $\begin{array}{l}\text { Carolina Klett- } \\
\text { Tammen }\end{array}$ & Koen Pouwels & $\begin{array}{l}\text { Michel } \\
\text { Walthouwer }\end{array}$ \\
\hline Markus A. Busch & Almar Kok & Martin Prins & Petra Wark \\
\hline Lidyane Camelo & Jan Kool & Anita Ravelli & $\begin{array}{l}\text { Matty } \\
\text { Weijenberg }\end{array}$ \\
\hline Géraldine Camilleri & Daniel Korevaar & Nel Reijven & Jannelle van Wel \\
\hline
\end{tabular}

\begin{tabular}{|c|c|c|c|}
\hline Nicola Caranca & Lucian Krille & Teun Remmers & Alet Wijga \\
\hline $\begin{array}{l}\text { Adele Seniori } \\
\text { Costantini }\end{array}$ & Jisca Kuiper & Raoul Reulen & Linda Wijlaars \\
\hline Pieter Dagnelie & Anton Kunst & Stefanie Rewald & Renate Winkels \\
\hline Dorly Deeg & Leanne Kupers & Nathalie Riedel & Hideo Yamazaki \\
\hline Marie Delnord & Jeroen Lakerveld & Shelly Rodrigo & Alfi Yasmina \\
\hline Klodian Dhana & Ton Lenssen & Eline van Roekel & Maurice Zeegers \\
\hline Sonia Pértega Díaz & Frank van Lenthe & Belle de Rooij & Mai Zhi-Ming \\
\hline Boukje van Dijk & Frank van Leth & Iris van Rooij & Iris Zoellner \\
\hline $\begin{array}{c}\text { Martien van } \\
\text { Dongen }\end{array}$ & Hyungryul Lim & Nicole Ruebsamen & \\
\hline
\end{tabular}

\section{Programme overview}

Programme Preconference workshops-Thursday June $25^{\text {th }}$ morning

08.30 Registration desk open-Lobby/Trajectum

09.00-10.30 Preconference workshops (separate registration and fee required)

Molecular Epidemiology of Chronic Diseases-02 Berlin/03 Copenhagen

Ilja Arts, Matty Weijenberg

An introduction to economic evaluations in health care-08 Rome

Carmen Dirksen, Manuela Joore

Systematic reviews-09 Athens

Jos Kleijnen

How to (re)route your research question into the biopsychosocial paradigm: Sneak preview of the trainings trajectory 'Occupational health from a biopsychosocial perspective; an Evidence Based approach'-07 Lisbon

Carin de Brouwer, Yvonne Heerkens, IJmert Kant

The use of social media in epidemiological research

Focus group e-Epidemiology of the Netherlands Society for Epidemiology-01 London

Marleen van Gelder, Anouk Pijpe and Satu Siiskonen

10.30-11.00 Coffee break-Lobby/Trajectum

11.00-12.30 Preconference workshops: continuation

12.30-13.00 Lunch for participants of the preconference workshops-Lobby/Trajectum

Congress Programme-Thursday June $25^{\text {th }}$ afternoon

\begin{tabular}{ll}
\hline 13.30-15.30 & Plenary session 1-Auditorium 1 \\
& Chairs: Matty Weijenberg and \\
& IJmert Kant \\
13.30 & Opening \\
\hline
\end{tabular}




\begin{tabular}{|c|c|}
\hline \multirow[t]{2}{*}{13.45} & $\begin{array}{l}\text { Environmental epidemiology: } \\
\text { beyond epidemiology }\end{array}$ \\
\hline & Paolo Vineis [O 01] \\
\hline \multirow[t]{2}{*}{14.15} & $\begin{array}{l}\text { Work in Life-work and health } \\
\text { from a life course } \\
\text { epidemiological perspective }\end{array}$ \\
\hline & Ute Bültmann [O 02] \\
\hline \multirow[t]{2}{*}{14.45} & $\begin{array}{l}\text { Associations between early body } \\
\text { mass index trajectories and later } \\
\text { metabolic risk in European } \\
\text { children: The IDEFICS Study }\end{array}$ \\
\hline & Claudia Börnhorst [O 03] \\
\hline \multirow[t]{2}{*}{15.00} & $\begin{array}{l}\text { Obstetric mode of delivery and } \\
\text { autism spectrum disorders in } \\
\text { Sweden: A sibling design study }\end{array}$ \\
\hline & Eileen Curran [O 04] \\
\hline \multirow[t]{2}{*}{15.15} & $\begin{array}{l}\text { Performance of variable selection } \\
\text { methods for correlated exposures } \\
\text { and logistic models }\end{array}$ \\
\hline & Viressa Lenters [O 05] \\
\hline 15.30-16.00 & $\begin{array}{c}\text { Coffee break Sponsored by Elevate } \\
\text { Health-Lobby/Trajectum }\end{array}$ \\
\hline
\end{tabular}

\begin{tabular}{|c|c|c|}
\hline \multirow[t]{12}{*}{$16.00-17.00$} & $\begin{array}{l}\text { Parallel activities } \\
\text { Parallel session I (see details in } \\
\text { table) }\end{array}$ & \\
\hline & Epidemiologic methods & Auditorium 1 \\
\hline & Nutritional Epidemiology & 04 Brussels \\
\hline & Social Epidemiology & 05 Paris \\
\hline & $\begin{array}{l}\text { Epidemiology of cardiovascular } \\
\text { disease }\end{array}$ & $\begin{array}{l}02 \text { Berlin/03 } \\
\text { Copenhagen }\end{array}$ \\
\hline & Meet-the-keynote speaker & \\
\hline & Chair: Ilja Arts & \\
\hline & Molecular Epidemiology & 06 Madrid \\
\hline & Paolo Vineis & \\
\hline & Chair: Lenie van Rossem & \\
\hline & Life course epidemiology & 07 Lisbon \\
\hline & Ute Bültmann & \\
\hline 17.30-18.30 & Drink at Town hall & \\
\hline
\end{tabular}

Thursday June $25^{\text {th }}-$ Parallel session I (16.00-17.00)

\begin{tabular}{|c|c|c|c|c|}
\hline Time & $\begin{array}{l}\text { Session 1: Epidemiologic } \\
\text { methods } \\
\text { Chairs: Nicola Orsini \& } \\
\text { Gerhard Zielhuis } \\
\text { Auditorium } 1\end{array}$ & $\begin{array}{l}\text { Session 2: Nutritional } \\
\text { epidemiology } \\
\text { Chairs: Jessica Kiefte-de Jong \& } \\
\text { Franzel van Duinhoven } \\
\text { 04 Brussels }\end{array}$ & $\begin{array}{l}\text { Session 3: Social epidemiology } \\
\text { Chairs: Nynke Smidt \& } \\
\text { Annemarie Koster } \\
\text { 05 Paris }\end{array}$ & $\begin{array}{l}\text { Session 4: Epidemiology of } \\
\text { cardiovascular disease } \\
\text { Chairs: Sabita Soedamah-Muthu } \\
\text { \& Brenda Bongaerts } \\
\text { 02 Berlin/03 Copenhagen }\end{array}$ \\
\hline 16.00 & $\begin{array}{l}\text { Riekie de Vet [O 06] } \\
\text { Specific agreement instead of } \\
\text { Cohen's kappa to quantify } \\
\text { interobserver variation for } \\
>2 \text { raters and }>2 \\
\text { categories }\end{array}$ & $\begin{array}{l}\text { Federica Laguzzi [O } 10] \\
\text { Predictive role of dietary habits in } \\
\text { relation to incident CVD and } \\
\text { all-cause mortality: results } \\
\text { from a Swedish cohort of } \\
\text { 60-year-old }\end{array}$ & $\begin{array}{l}\text { Joost Oude Groeniger [O } 14] \\
\text { The role of material and } \\
\text { behavioral factors measured at } \\
\text { multiple times in adulthood in } \\
\text { explaining socioeconomic } \\
\text { inequalities in mortality }\end{array}$ & $\begin{array}{l}\text { Susanne Tielemans [O 18] } \\
\text { Ten-year blood pressure } \\
\text { trajectories, cardiovascular } \\
\text { mortality and life years lost in } \\
\text { two extinction cohorts }\end{array}$ \\
\hline 16.15 & $\begin{array}{l}\text { Milton Severo [O 07] } \\
\text { Modelling growth across } \\
\text { pediatric age: should } \\
\text { weight be considered as a } \\
\text { smooth function of age or } \\
\text { height and age? }\end{array}$ & $\begin{array}{l}\text { Jaike Praagman [O 11] } \\
\text { The association between saturated } \\
\text { fat and coronary heart disease } \\
\text { depends on the source and type } \\
\text { of fatty acid in EPIC-NL }\end{array}$ & $\begin{array}{l}\text { Sander van Zon [O 15] } \\
\text { Absolute and relative } \\
\text { socioeconomic health } \\
\text { inequalities vary across age } \\
\text { groups: results from the } \\
\text { LifeLines cohort study }\end{array}$ & $\begin{array}{l}\text { Bamba Gaye [O 19] } \\
\text { High depressive symptoms are } \\
\text { inversely associated with ideal } \\
\text { cardiovascular health? The } \\
\text { Paris prospective study III }\end{array}$ \\
\hline 16.30 & $\begin{array}{l}\text { Maarten Burbach [O 08] } \\
\text { Feasibility of using the } \\
\text { "cohort multiple } \\
\text { Randomized Controlled } \\
\text { Trial" design to conduct } \\
\text { the RECTAL BOOST } \\
\text { study }\end{array}$ & $\begin{array}{l}\text { Denes Stefler [O 12] } \\
\text { Mediterranean diet and mortality } \\
\text { in Eastern Europeans: results } \\
\text { from the HAPIEE study }\end{array}$ & $\begin{array}{l}\text { Joanna McHugh [O } 16] \\
\text { An investigation of pathways } \\
\text { from social activity to cognitive } \\
\text { function in older Irish adults }\end{array}$ & $\begin{array}{l}\text { Anneke Damen [O 20] } \\
\text { Prediction models for the risk of } \\
\text { cardiovascular disease in the } \\
\text { general population: a } \\
\text { systematic review }\end{array}$ \\
\hline 16.45 & $\begin{array}{l}\text { Sander van Kuijk [O 09] } \\
\text { Index event bias } \\
\text { compromises the } \\
\text { performance of prediction } \\
\text { models for disease } \\
\text { recurrence: a simulation } \\
\text { study }\end{array}$ & $\begin{array}{l}\text { Matthias Clemens [O 13] } \\
\text { Simulating different assessment } \\
\text { regimes for repeated short-term } \\
\text { dietary measurements to } \\
\text { estimate usual intake } \\
\text { distributions of seasonally } \\
\text { consumed foods }\end{array}$ & $\begin{array}{l}\text { Jisca Kuiper [O 17] } \\
\text { Social relationships and risk of } \\
\text { dementia: a systematic review } \\
\text { and meta-analysis of } \\
\text { longitudinal cohort studies }\end{array}$ & $\begin{array}{l}\text { Nicole Jancovik [O 21] } \\
\text { WHO guidelines for a healthy } \\
\text { diet and mortality from } \\
\text { cardiovascular disease in } \\
\text { European and American } \\
\text { elderly: the CHANCES project }\end{array}$ \\
\hline
\end{tabular}


Programme Friday June $26^{\text {th }}$ morning

08.30-09.00 Registration-Lobby/Trajectum

09.00-10.30 Plenary session II-Auditorium 1

Chairs: Bart Kiemeney and Martin Prins

$09.00 \quad$ Probing causality: can studies of genetic variation have any public health impact?

Nic Timpson [O 22]

09.30 The challenges of health inequalities

Johan Mackenbach [O 23]

$10.00 \quad$ Survival after age 75 : genetic risk is strongly attenuated by lifestyle behaviours

Debora Rizzuto [O 24]

10.15 VvE Publication Award junior researcher [O 25]

10.30-11.00 Coffee break Sponsored by Brightlands-Lobby/Trajectum

11.00-12.30 Parallel activities

Parallel session II (see details in table)

Epidemiologic methods

Occupational and environmental epidemiology

Life-course epidemiology

COREON session: Modalities of consent

Meet-the-keynote speaker (11.00-12.00)

Chair: Sita Vermeulen

Genetic association studies and causality

Nic Timpson

Chair: Hans Bosma

Health inequalities

Johan Mackenbach

Development, Validity, and Backward Compatibility of a new Food Frequency

Questionnaire for The

Netherlands (FFQ-NL1.0)

Chair: Pieter Dagnelie

Boardmeeting EEF
12.30-13.30 Lunch-Lobby/Trajectum

12.30-13.30 Opleidersvergadering Vereniging voor Epidemiologie-Auditorium 1

Programme Friday June $26^{\text {th }}$ afternoon

13.30-14.30 Postersession A-Expo Foyer

14.30-15.30 Parallel session III (see details in table)

Up-to-date: Statistical models for

Auditorium 1

Life-course epidemiology: introduction to the group-based trajectory Model

Up-to-date: Imputation: handling of 04 Brussels missing data

Public Health

05 Paris

Infectious disease epidemiology

02 Berlin/03

Copenhagen

15.30-16.00 Coffee break sponsored by Bruker-Lobby/Trajectum

Auditorium 1

04 Brussels

05 Paris

02 Berlin/03

Copenhagen

06 Madrid

07 Lisbon

18.30

02 Berlin/03

Copenhagen

08 Rome

09 Athens 
Friday June $26^{\text {th }}$-Parallel session II (11.00-12.30)

\begin{tabular}{|c|c|c|c|c|c|}
\hline Time $^{*}$ & $\begin{array}{l}\text { Session 1: Epidemiologic } \\
\text { methods } \\
\text { Chairs: Milton Severo \& } \\
\text { Riekie de Vet } \\
\text { Auditorium } 1\end{array}$ & $\begin{array}{l}\text { Session 2: Occupational } \\
\text { and environmental } \\
\text { epidemiology } \\
\text { Chairs: Adele Seniori } \\
\text { Costantini \& Tim Nawrot } \\
\text { 04 Brussels }\end{array}$ & $\begin{array}{l}\text { Session 3: Life-course } \\
\text { epidemiology } \\
\text { Chairs: Maria Jansen \& } \\
\text { Carel Thijs } \\
\text { 05 Paris }\end{array}$ & $\begin{array}{l}\text { Session 4: COREON } \\
\text { session: Modalities of } \\
\text { consent } \\
\text { Chair: Marjanka Schmidt } \\
02 \text { Berlin/03 Copenhagen }\end{array}$ & $\begin{array}{l}\text { Development, Validity, } \\
\text { and Backward } \\
\text { Compatibility of a new } \\
\text { Food Frequency } \\
\text { Questionnaire for The } \\
\text { Netherlands (FFQ- } \\
\text { NL1.0)** } \\
\text { Chair: Pieter Dagnelie } \\
\text { o8 Rome }\end{array}$ \\
\hline 11.00 & $\begin{array}{l}\text { Sanni Ali [O 26] } \\
\text { Covariate balance } \\
\text { assessment, model } \\
\text { selection and bias in } \\
\text { propensity score } \\
\text { matching: a simulation } \\
\text { study }\end{array}$ & $\begin{array}{l}\text { Sophie Albrecht [O 32] } \\
\text { Women, shift workers, } \\
\text { and public sector } \\
\text { workers report lower } \\
\text { levels of work time } \\
\text { control in a nationally } \\
\text { representative Swedish } \\
\text { sample }\end{array}$ & $\begin{array}{l}\text { Lenie van Rossem [O 38] } \\
\text { Maternal weight gain over } \\
\text { the child's life course, } \\
\text { and child's (over)weight } \\
\text { development and cardio- } \\
\text { metabolic health: the } \\
\text { PIAMA birth cohort }\end{array}$ & $\begin{array}{l}\text { Evert-Ben van Veen [O 44] } \\
\text { Modalities of consent and } \\
\text { their consequences }\end{array}$ & $\begin{array}{l}\text { 11.00 Opening } \\
\text { 11.05 Marga Ocké [E 01] } \\
\text { Introduction on food } \\
\text { frequency } \\
\text { questionnaires and the } \\
\text { BBMRI-FFQ project } \\
\text { 11.30 Simone Eussen [E } \\
\text { 02] }\end{array}$ \\
\hline 11.15 & $\begin{array}{l}\text { Koen Pouwels [O 27] } \\
\text { Comparison of case-only } \\
\text { designs with traditional } \\
\text { study designs: a } \\
\text { systematic review }\end{array}$ & $\begin{array}{l}\text { Monica Ubalde-Lopez [O } \\
\text { 33] } \\
\text { Beyond return to work: } \\
\text { the effect of } \\
\text { multimorbidity on } \\
\text { work functioning after } \\
\text { sick leave due to } \\
\text { common mental } \\
\text { disorders }\end{array}$ & $\begin{array}{l}\text { Dylan Williams [O 39] } \\
\text { 25-hydroxyvitamin D is } \\
\text { not associated with } \\
\text { leukocyte telomere } \\
\text { length in young adults: } \\
\text { findings from the } \\
\text { Northern Finland Birth } \\
\text { Cohort } 1966\end{array}$ & & $\begin{array}{l}\text { Development and } \\
\text { comparison with } \\
\text { existing Dutch FFQs of } \\
\text { the FFQ-NL1.0, a } \\
\text { National Food } \\
\text { Frequency } \\
\text { Questionnaire for The } \\
\text { Netherlands }\end{array}$ \\
\hline 11.30 & Sandra Landwehr [O 28] & Eline Provost [O 34] & Lital Keinan Boker [O 40] & Susanne Rebers [O 45] & 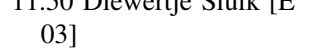 \\
\hline 11.45 & 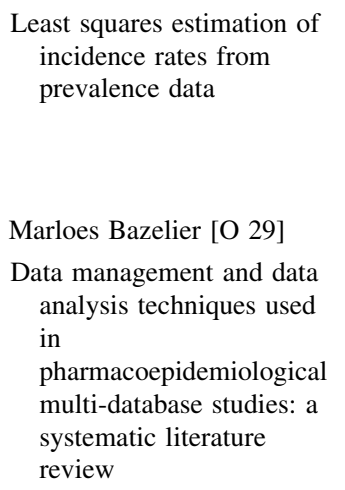 & $\begin{array}{l}\text { Microvascular responses } \\
\text { in association with } \\
\text { recent and chronic } \\
\text { exposure to particulate } \\
\text { air pollution in school } \\
\text { children } \\
\text { Nelly Saenen [O 35] } \\
\text { Prenatal Particulate Air } \\
\text { Pollution And Foetal } \\
\text { Thyroid Function In } \\
\text { Euthyroid Pregnant } \\
\text { Mothers }\end{array}$ & $\begin{array}{l}\text { Klodian Dhana [O 41] } \\
\text { Timing and duration of } \\
\text { overweight in } \\
\text { association with } \\
\text { metabolic health risk } \\
\text { among middle-aged and } \\
\text { elderly: the Rotterdam } \\
\text { Study }\end{array}$ & $\begin{array}{l}\text { A randomized controlled } \\
\text { trial comparing consent } \\
\text { procedures for the use of } \\
\text { residual tissues: } \\
\text { implications for patients, } \\
\text { research, and clinical } \\
\text { practice }\end{array}$ & $\begin{array}{l}\text { Validation of the FFQ- } \\
\text { NL1.0, a National Food } \\
\text { Frequency } \\
\text { Questionnaire for the } \\
\text { Netherlands } \\
12.10 \\
\text { Practical information for } \\
\text { interested users of the } \\
\text { FFQ-NL1.0/Discussion }\end{array}$ \\
\hline 12.00 & $\begin{array}{l}\text { Daniel Korevaar [O 30] } \\
\text { Complete and accurate } \\
\text { reporting of studies of } \\
\text { diagnostic accuracy: } \\
\text { updating the STARD } \\
\text { Statement }\end{array}$ & $\begin{array}{l}\text { Suzan Robroek [O 36] } \\
\text { Health inequalities in } \\
\text { maintaining paid } \\
\text { employment: working } \\
\text { life expectancy in the } \\
\text { Netherlands }\end{array}$ & $\begin{array}{l}\text { Astrid Nooyens [O 42] } \\
\text { Genetic predisposition for } \\
\text { Alzheimer's disease and } \\
\text { cognitive ageing in } \\
\text { healthy persons-The } \\
\text { Doetinchem Cohort } \\
\text { Study }\end{array}$ & $\begin{array}{l}\text { Bart van der Sloot [O 46] } \\
\text { Privacy as societal value }\end{array}$ & \\
\hline 12.15 & $\begin{array}{l}\text { Davood Khalili [O 31] } \\
\text { A systematic approach to } \\
\text { variable selection methods } \\
\text { used in data-mining: a } \\
\text { practice in epidemiology }\end{array}$ & $\begin{array}{l}\text { Jessica Hrudy [O 37] } \\
\text { Neighbourhood } \\
\text { characteristics and } \\
\text { adiposity in preschool } \\
\text { age children: results } \\
\text { from the ABCD study }\end{array}$ & $\begin{array}{l}\text { Monique Mommers [P 122] } \\
\text { Early childhood infections } \\
\text { with rotavirus and } \\
\text { norovirus and the risk of } \\
\text { developing asthma and } \\
\text { atopic manifestations }\end{array}$ & & \\
\hline
\end{tabular}

* Time unless otherwise specified

** Financially supported by BBMRI-NL, a Research Infrastructure financed by the Dutch government (NWO 184.021.007) 
Friday June $26^{\text {th }}-$ Parallel session III (14.30-15.30)

\begin{tabular}{|c|c|c|c|c|}
\hline Time & $\begin{array}{l}\text { Session 1: Up-to-date } \\
\text { Chair: Ludo van } \\
\text { Amelsvoort } \\
\text { Auditorium } 1\end{array}$ & $\begin{array}{l}\text { Session 2: } \\
\text { Up-to-date } \\
\text { Chair: Colinda } \\
\text { Simons } \\
\text { 04 Brussels }\end{array}$ & $\begin{array}{l}\text { Session 3: Public Health } \\
\text { Chairs: Lenie van Rossem } \\
\text { \& Christel van Gool } \\
\text { 05 Paris }\end{array}$ & $\begin{array}{l}\text { Session 4: Infectious disease } \\
\text { epidemiology } \\
\text { Chairs: Monique Mommers } \\
\text { \& Andrej Grjibovski } \\
\text { 02 Berlin/03 Copenhagen }\end{array}$ \\
\hline 14.30 & $\begin{array}{l}\text { Valeria Lima Passos [O 47] } \\
\text { Statistical models for life- } \\
\text { course epidemiology: } \\
\text { introduction to the group- } \\
\text { based trajectory Model }\end{array}$ & $\begin{array}{l}\text { Sander van } \\
\text { Kuijk [O48] } \\
\text { Imputation: } \\
\text { handling of } \\
\text { missing data }\end{array}$ & $\begin{array}{l}\text { Nora Döring [O 49] } \\
\text { The associations between overweight, } \\
\text { weight change and health related quality } \\
\text { of life: Longitudinal data from the } \\
\text { Stockholm Public Health Cohort }\end{array}$ & $\begin{array}{l}\text { Ralf Krumkamp [O 53] } \\
\text { Association between malaria and invasive } \\
\text { non-typhoid Salmonella infection in a } \\
\text { Hospital-Study accounting for } \\
\text { Berkson's Bias }\end{array}$ \\
\hline 14.45 & & & $\begin{array}{l}\text { Michel Walthouwer [O 50] } \\
\text { Differences in effects between a video and } \\
\text { text web-based computer-tailored } \\
\text { obesity prevention intervention: A } \\
\text { randomized controlled trial }\end{array}$ & $\begin{array}{l}\text { Paula Meireles [O } 54] \\
\text { Estimating HIV cases in Portuguese } \\
\text { MSM }\end{array}$ \\
\hline 15.00 & & & $\begin{array}{l}\text { Trudy Voortman [O 51] } \\
\text { Vitamin D status in a multiethnic cohort of } \\
\text { school-age children: Determinants and } \\
\text { associations with obesity and } \\
\text { cardiometabolic health }\end{array}$ & $\begin{array}{l}\text { Mariëtte Lokate [O 55] } \\
\text { Regional outbreak of Serratia marcescens } \\
\text { on neonatal wards }\end{array}$ \\
\hline 15.15 & & & $\begin{array}{l}\text { Linda Pluymen [O 52] } \\
\text { Caesarean section delivery and } \\
\text { cardiovascular markers in childhood and } \\
\text { adolescence }\end{array}$ & $\begin{array}{l}\text { Stephanie Brinkhues [P 487] } \\
\text { Social network characteristics are } \\
\text { associated with self-reported } \\
\text { (symptoms of) infections in the middle } \\
\text { aged and elderly individuals -The } \\
\text { Maastricht Study- }\end{array}$ \\
\hline
\end{tabular}

Friday June $26^{\text {th }}-$ Parallel session IV (16.00-17.00)

\begin{tabular}{|c|c|c|c|c|}
\hline Time & $\begin{array}{l}\text { Session 1: Up-to-date } \\
\text { Chair: Luc Smits } \\
\text { Auditorium } 1\end{array}$ & $\begin{array}{l}\text { Session 2: Up-to-date } \\
\text { Chair: Eline van Roekel } \\
\text { 04 Brussels }\end{array}$ & $\begin{array}{l}\text { Session 3: Cancer } \\
\text { epidemiology } \\
\text { Chairs: Martin Müller \& Piet } \\
\text { van den Brandt } \\
\text { 05 Paris }\end{array}$ & $\begin{array}{l}\text { Session 4: Epidemiology of musculoskeletal } \\
\text { disorders } \\
\text { Chairs: Chris Main \& Emmylou Beekman } \\
02 \text { Berlin/03 Copenhagen }\end{array}$ \\
\hline 16.00 & $\begin{array}{l}\text { Karel Moons [O 57] } \\
\text { Clinical prediction } \\
\text { modelling: where are } \\
\text { we now and where do } \\
\text { we need to go? }\end{array}$ & $\begin{array}{l}\text { Petra Wark [O 58] } \\
\text { mHealth in epidemiological research } \\
\text { and public health interventions: } \\
\text { endless possibilities, yet needs for } \\
\text { validation }\end{array}$ & $\begin{array}{l}\text { Maartje Schellekens [O 59] } \\
\text { Smoking cessation in women } \\
\text { following genetic testing } \\
\text { for a BRCA1/2 mutation } \\
\text { (HOP 2012014) S }\end{array}$ & $\begin{array}{l}\text { Eva Skillgate [O } 63] \\
\text { The influence of a Healthy Lifestyle Behavior on the } \\
\text { prognosis of occasional neck/shoulder/arm pain in } \\
\text { a general population }\end{array}$ \\
\hline 16.15 & & & $\begin{array}{l}\text { Dorien Ripping [O 60] } \\
\text { The benefit of mammographic } \\
\text { screening for women with a } \\
\text { high and low socio- } \\
\text { economic status }\end{array}$ & $\begin{array}{l}\text { Sarah Dörenkamp [O 64] } \\
\text { Patient variables, comorbidities and walking } \\
\text { distances in symptomatic peripheral arterial } \\
\text { disease: findings from a one-year prospective } \\
\text { physiotherapy cohort study }\end{array}$ \\
\hline 16.30 & & & $\begin{array}{l}\text { Elisabeth Kap [O 61] } \\
\text { Genetic variants in T regulatory } \\
\text { cell genes and colorectal } \\
\text { cancer prognosis }\end{array}$ & $\begin{array}{l}\text { Korinna Karampampa [O } 65] \\
\text { Hip fracture trends in Sweden-a study of incidence, } \\
\text { lifetime risk, and survival }\end{array}$ \\
\hline 16.45 & & & $\begin{array}{l}\text { Frederika van Nimwegen } \\
\text { [P 26] } \\
\text { Cardiovascular disease after } \\
\text { Hodgkin lymphoma } \\
\text { treatment: 40-year disease } \\
\text { risk }\end{array}$ & $\begin{array}{l}\text { Annemieke Smid [O 66] } \\
\text { Intermittent claudication affects gait parameters } \\
\quad \text { during treadmill walking } \mathrm{S}\end{array}$ \\
\hline
\end{tabular}

8 S: nominated for the VvE Student Award 
Programme Saturday June $27^{\text {th }}$ morning

08.30-09.00 Registration-Lobby/Trajectum

09.00-10.30 Plenary session III-Auditorium 1

Chairs: Antii Malmivaara and Rob de Bie

09.00 Red, yellow and...blue-low back pain and the psychosocial burden

Chris Main [O 67]

09.30 25 Years of evidence and how it changed management for low back pain

Maurits van Tulder [O 68]

$10.00 \quad$ The role of functional disability assessment in the diagnosis of common mental disorders: a comparison of three different instruments

Elena Olariu [O 69]

10.15 Epidemiological characteristics of Chronic Fatigue Syndrome in an Australian cohort

Samantha Johnston [O 70]

10.30-11.00 Coffee break sponsored by WCRF International-Lobby/Trajectum

11.00-12.30 Parallel activities

Parallel session V (see details in table)

Epidemiology of diabetes and obesity

Genetic and molecular epidemiology

Spinal disorders: Onderzoek in Beweging

Bekken- en heupproblematiek gerelateerd aan rugklachten: Onderzoek in Beweging (Dutch)

Meet-the-keynote speaker (11.00-12.00)

Chair: Martijn Bours

Low back pain and the psychosocial 06 Madrid burden

Chris Main

Chair: Raymond Ostelo

How to handle questionable research practices you come across?

Lex Bouter
07 Lisbon

\subsection{0-13.30 Lunch-Lobby/Trajectum}

12.30-13.30 Algemene ledenvergadering Vereniging voor Epidemiologie-Auditorium 1

Programme Saturday June $27^{\text {th }}$ afternoon

13.30-14.30 Postersession B-Expo Foyer

14.30-15.30 Parallel session VI (see details in table)

Epidemiologic methods

Auditorium 1

Cancer epidemiology

04 Brussels

Low back pain and predictors for

05 Paris return to work: Onderzoek in Beweging

Musculoskeletal disorders: Onderzoek in Beweging

15.30-16.00 Coffee break sponsored by Lifelines-Lobby/Trajectum

16.00-17.00 Plenary session IV-Auditorium 1

Chair: Martien van Dongen

16.00 VvE Poster Award, VvE Student Award and VvE Achievement Award

16.15 What can epidemiologists do to prevent research waste?

Lex Bouter [O 103]

04 Brussels

16.45

Closing
02 Berlin/03

Copenhagen
05 Paris

\section{Berlin/03}

Copenhagen 
Saturday June $27^{\text {th }}$-Parallel session V (11.00-12.30)

\begin{tabular}{|c|c|c|c|c|}
\hline \multirow[t]{4}{*}{ Time } & $\begin{array}{l}\text { Session 1: Epidemiology of } \\
\text { diabetes and obesity } \\
\text { Chairs: Enkelejda Shkurti \& } \\
\text { Dieuwertje Sluik }\end{array}$ & $\begin{array}{l}\text { Session 2: Genetic and molecular } \\
\text { epidemiology } \\
\text { Chairs: Maurice Zeegers \& } \\
\text { Susanne Vijverberg }\end{array}$ & $\begin{array}{l}\text { Session 3: Spinal } \\
\text { disorders } \\
\text { Onderzoek in Beweging } \\
\text { (English) }\end{array}$ & $\begin{array}{l}\text { Session 4: Bekken- en } \\
\text { heupproblematiek } \\
\text { gerelateerd aan } \\
\text { rugklachten }\end{array}$ \\
\hline & \multirow{3}{*}{$\begin{array}{l}\text { Chairs: Enkelejda Shkurti \& } \\
\text { Dieuwertje Sluik } \\
\text { Auditorium } 1\end{array}$} & \multirow{3}{*}{$\begin{array}{l}\text { Chairs: Maurice Zeegers \& } \\
\text { Susanne Vijverberg } \\
\text { 04 Brussels }\end{array}$} & $\begin{array}{l}\text { Chairs: Maurits van Tulder } \\
\text { \& Rob de Bie }\end{array}$ & $\begin{array}{l}\text { Onderzoek in Beweging } \\
\text { (Dutch) }\end{array}$ \\
\hline & & & 05 Paris & $\begin{array}{l}\text { Chairs: Esther Bols \& Ilse } \\
\text { Mesters }\end{array}$ \\
\hline & & & & 02 Berlin/03 Copenhagen \\
\hline \multirow[t]{2}{*}{11.00} & Belle de Rooij [O 71] & Diana van der Plaat [O 77] & Wouter Moojen [O 83] & Marijke Slieker [O 86] \\
\hline & $\begin{array}{l}\text { Sedentary time in 'metabolically } \\
\text { healthy' versus 'metabolically } \\
\text { unhealthy' obese and non- } \\
\text { obese individuals "S }\end{array}$ & $\begin{array}{l}\text { Identification of novel genes } \\
\text { related to airway obstruction in } \\
\text { never-smokers }\end{array}$ & $\begin{array}{l}\text { Scientific evidence in } \\
\text { relation to the daily } \\
\text { practice of patients with } \\
\text { complex spinal disorders }\end{array}$ & $\begin{array}{l}\text { Down Under-Top } \\
\text { Relevant! Het belang van } \\
\text { het signaleren van } \\
\text { bekkenbodemproblemen }\end{array}$ \\
\hline \multirow[t]{2}{*}{11.15} & Tomás Vega [O 72] & Letizia Polito [O 78] & & bij patiënten met lage rug-, \\
\hline & $\begin{array}{l}\text { Diabetes and obesity incidence } \\
\text { in } 5 \text { years follow up of a } \\
\text { Spanish Cohort }\end{array}$ & $\begin{array}{l}\text { Apolipoprotein E } \varepsilon 4 \text { allele } \\
\text { influences serum folate levels in } \\
\text { the elderly with vascular } \\
\text { diseases }\end{array}$ & & \\
\hline \multirow[t]{2}{*}{11.30} & Symen Ligthart [O 73] & Iris van Rooij [O 79] & Wilco Jacobs [O 84] & Rob Langhout [O 87] \\
\hline & $\begin{array}{l}\text { Lifetime risk of prediabetes and } \\
\text { diabetes: a prospective } \\
\text { population-based cohort study }\end{array}$ & $\begin{array}{l}\text { Rare coding variants and the risk } \\
\text { of congenital anorectal } \\
\text { malformations: an exome chip } \\
\text { association study }\end{array}$ & $\begin{array}{l}\text { The science in deciding } \\
\text { (not) to operate }\end{array}$ & $\begin{array}{l}\text { Rugpijn door } \\
\text { heupstoornissen bij } \\
\text { arthrose en sport }\end{array}$ \\
\hline \multirow[t]{2}{*}{11.45} & Simone Eussen [O 74] & Rachel Elands [O 80] & & \\
\hline & $\begin{array}{l}\text { Consumption of dairy foods in } \\
\text { relation to impaired glucose } \\
\text { metabolism and type } 2 \\
\text { diabetes mellitus: The } \\
\text { Maastricht Study }\end{array}$ & $\begin{array}{l}\text { A systematic SNP selection } \\
\text { approach for studying gene- } \\
\text { environment interactions in } \\
\text { cancer epidemiology }\end{array}$ & & \\
\hline \multirow[t]{2}{*}{12.00} & Bahareh Rasouli [O 75] & Dries Martens [O 81] & Paul Willems [O 85] & \multirow{2}{*}{$\begin{array}{l}\text { Annelies Pool-Goudzwaard } \\
\text { [O 88] } \\
\text { Compensatie strategieën bij } \\
\text { lage rug-, bekken- en } \\
\text { heupklachten }\end{array}$} \\
\hline & $\begin{array}{l}\text { Tobacco use and the risk of } \\
\text { latent autoimmune diabetes in } \\
\text { adults (LADA) and type } 2 \\
\text { diabetes: results from the } \\
\text { Swedish ESTRID study }\end{array}$ & $\begin{array}{l}\text { Altered neonatal cord blood } \\
\text { lipidome in association with } \\
\text { exposure to particulate matter in } \\
\text { the early life environment }\end{array}$ & Spinal disorders in children & \\
\hline 12.15 & Alicia Uijl [O 76] & Leanne Küpers [O 82] & & \\
\hline $12.25-12.30$ & $\begin{array}{l}\text { The relative contributions of } \\
\text { visceral fat and liver fat to } \\
\text { insulin resistance and beta-cell } \\
\text { function: The NEO study \& } \mathrm{S}\end{array}$ & $\begin{array}{l}\text { DNA methylation mediates the } \\
\text { effect of maternal smoking } \\
\text { during pregnancy on birth } \\
\text { weight of the offspring }\end{array}$ & & $\begin{array}{c}\text { Onderzoek in Beweging } \\
\text { Poster Awards "OIB }\end{array}$ \\
\hline
\end{tabular}

y S: nominated for the VvE Student Award

Saturday June $27^{\text {th }}$-Parallel session VI (14.30-15.30)

\begin{tabular}{|c|c|c|c|c|}
\hline Time & $\begin{array}{l}\text { Session 1: Epidemiologic methods } \\
\text { Chairs: Luc Smits \& Dorly Deeg } \\
\text { Auditorium } 1\end{array}$ & $\begin{array}{l}\text { Session 2: Cancer epidemiology } \\
\text { Chairs: Janneke Hogervorst \& Adri } \\
\text { Voogd } \\
\text { 04 Brussels }\end{array}$ & $\begin{array}{l}\text { Session 3: Low back } \\
\text { pain and predictors } \\
\text { for return to work } \\
\text { Onderzoek in } \\
\text { Beweging (English) } \\
\text { Chairs: Dave Stynen } \\
\& \\
\text { Ute Bültmann } \\
\text { 05 Paris }\end{array}$ & $\begin{array}{l}\text { Session 4: Musculoskeletal } \\
\text { disorders } \\
\text { Onderzoek in Beweging } \\
\text { (partly in Dutch) } \\
\text { Chairs: Bart Staal \& Carolien } \\
\text { Bastiaenen } \\
02 \text { Berlin/03 Copenhagen }\end{array}$ \\
\hline
\end{tabular}




\subsection{Martin Müller [O 89]}

Development of diagnostic algorithms to estimate prevalence of vestibular disorders in the general population: Results from the Munich Vertigo Study

14.45 Dorly Deeg [O 90]

Increasing life expectancy at older ages despite increasing disease prevalence 1993-2013: explanatory value of life course and period factors

\subsection{Maryse Cnossen [O 91] \\ Adjusting for confounding by indication in observational studies about traumatic brain injury}

15.15 Karel Moons [O 92]

Transparent Reporting of a multivariable prediction model for Individual Prognosis Or Diagnosis: the TRIPOD statement
Simone de Vries [O 62]

Treatment-specific risks of second malignancy and cardiovascular disease in 5-year Hodgkin lymphoma survivors

Kristine Rommens [O 94]

Seasonal variation in the incidence of cutaneous melanoma: link with recent UV exposure. A Belgian population-based study
Annemieke Witteveen [O 95]
Towards personalized follow-up: A conditional prediction model and nomogram for risk of locoregional recurrence in early breast cancer patients
Johanna Wanders [O 96]
Effect of mammographic density on performance of a breast cancer screening program using full-field digital mammography

Allard van der Beek [O 97]

Effectiveness of return to work interventions in employees with low back pain

Antii Malmivaara [O 98]

Real-effectiveness medicine in low back pain
Jeroen van der Velde [O 99]

Meer zitten is gerelateerd aan een lagere loopsnelheid, De Maastricht Studie (Dutch)

Lindy Gommans [O 100]

Gesuperviseerde looptherapie voor patiënten met claudicatio intermittens: mannen doen het beter! (Dutch)

Ester de Jonge [O 101]

Eetpatronen in ouderen en hun relatie tot botdichtheid: de Rotterdam Studie (Dutch)

Deborah Gibson-Smith [O 102]

Trends in anti-osteoporosis drug prescribing after hip fracture in the United Kingdom: 2000-2010 (English)

\section{Postersessions}

\begin{tabular}{|c|c|c|c|}
\hline Poster-numbers & Session A: Friday June $26^{\text {th }}$ & Poster-numbers & Session B: Saturday June $27^{\text {th }}$ \\
\hline P 01-P11 & Cancer epidemiology 1: colorectal cancer & P320-P328 & Cancer epidemiology 3: breast cancer \\
\hline P12-P26 & Cancer epidemiology 2: mixed & P329-P336 & Cancer epidemiology 4: urogenital \\
\hline P27-P36 & Epidemiologic methods 1 & P337-P351 & Cancer epidemiology 5: mixed \\
\hline P37-P46 & Epidemiologic methods 2 & P352-P362 & Epidemiologic methods 3 \\
\hline P47-P58 & Epidemiology of cardiovascular disease 1 & P363-P373 & Epidemiologic methods 4 \\
\hline P59-P69 & Epidemiology of cardiovascular disease 2 & P374-P384 & Epidemiology of cardiovascular disease 3 \\
\hline $\mathrm{P} 70-\mathrm{P} 80$ & Epidemiology of diabetes and obesity 1 & P385-P395 & Epidemiology of cardiovascular disease 4 \\
\hline P81-P91 & Epidemiology of diabetes and obesity 2 & P396-P405 & Epidemiology of diabetes and obesity 3 \\
\hline P92-P104 & Epidemiology of mental disorders 1 & P406-P416 & Epidemiology of diabetes and obesity 4 \\
\hline P105-P115 & Epidemiology of musculoskeletal disorders 1 & P417-P428 & Epidemiology of mental disorders 2 \\
\hline $\mathrm{P} 116-\mathrm{P} 121$ & Health registries 1 & P429-P438 & Epidemiology of musculoskeletal disorders 2 \\
\hline $\mathrm{P} 122-\mathrm{P} 134$ & Infectious disease epidemiology 1 & P439-P447 & Epidemiology of reproductive disorders 1 \\
\hline $\mathrm{P} 135-\mathrm{P} 147$ & Infectious disease epidemiology 2 & P448-P452 & Ethics and philosophy 1 \\
\hline P148-P160 & Life-course epidemiology 1 & P453-P461 & Genetic and molecular epidemiology 1 \\
\hline P161-P170 & Nutritional epidemiology 1 & P462-P474 & Infectious disease epidemiology 3 \\
\hline $\mathrm{P} 171-\mathrm{P} 181$ & Nutritional epidemiology 2 & P475-P488 & Infectious disease epidemiology 4 \\
\hline P182-P189 & $\begin{array}{l}\text { Occupational and environmental epidemiology } 1 \text { : } \\
\text { genetic and molecular epidemiology }\end{array}$ & P489-P498 & Nutritional epidemiology 3 \\
\hline P190-P203 & Occupational and environmental epidemiology 2 & P499-P508 & Nutritional epidemiology 4 \\
\hline P204-P218 & Occupational and environmental epidemiology 3 & P509-P522 & Occupational and environmental epidemiology 4 \\
\hline P219-P225 & Perinatal epidemiology 1 & P523-P536 & Occupational and environmental epidemiology 5 \\
\hline P226-P236 & Pharmaco-epidemiology 1 & P537-P547 & Onderzoek in beweging 1 \\
\hline P237-P247 & Public Health 1: methods & P548-P558 & Onderzoek in beweging 2 \\
\hline
\end{tabular}


Poster-numbers

P248-P258

P259-P273

P274-P288

P289-P297

P298-P308

P309-P319
Session A: Friday June $26^{\text {th }}$

Public Health 2: diabetes and obesity

Public health 3

Public Health 4

Research protocol 1

Social epidemiology 1

Social epidemiology 2
Poster-numbers

P559-P572

P573-P587

P588-P602

P603-P617

P618-P630

P631-P642
Session B: Saturday June $27^{\text {th }}$

Public Health 5: nutrition

Public Health 6

Public health 7

Public Health 8

Social epidemiology 3

Social epidemiology 4 


\section{Abstract \#: 0 01}

\section{Environmental epidemiology: beyond epidemiology}

Paolo Vineis ${ }^{1}$

${ }^{1}$ MRC-PHE Centre for Environment and Health, Imperial College London; Corresponding author's e-mail: p.vineis@imperial.ac.uk

In the study of NCDs epidemiology has faced different combinations of experiment vs observation, single agent vs. complex exposure and strong vs weak associations. Necessary causes have been abandoned very early for NCDs: we know only one necessary cause for cancer, HPV for cervical cancer (with evidence coming also from experiments with the vaccine). Tobacco smoking (a complex exposure) and asbestos (a single agent) are examples of exposures that are definitely causal but not necessary, though they have a very strong association with the risk of disease. In both cases we do not have experiments in humans, but we have good mechanistic evidence. Recent years have seen the appearance of consistent observational evidence for complex exposures or agents that are weakly associated with cancer: e.g. air pollution and second-hand smoke.

Following this historical evolution, etiology is now understood as the interplay of numerous agents/exposures that act along distinct mechanistic pathways, thus creating the opportunity for several steps to be affected (e.g. in the carcinogenic process). This turn seems to imply two key concepts: multiple (weak) exposures contributing to the final outcome; and "remoteness" of some exposures from the target. The simplest case of causation (at one extreme) is a necessary single agent (the Ebola virus), which is strongly associated with and proximal to disease. The same holds true for "magic bullets" in clinical medicine. The other extreme is represented by air pollution, i.e. by a weak association with a mixture (rather than a single agent), possibly remote from disease. Our currently understanding is that these "causes" (like air pollution) belong to genuine casual pathways because we have good mechanistic evidence in addition to being able to satisfy epidemiological criteria of causality like those used by the IARC Monographs. The key concept has now become the ability of such exposures "to perturbate pathways".

The conceptual framework for disease etiology has changed significantly from other points of view in recent decades. First, the concept of non-communicable diseases (NCDs) as a continuum across single disease entities has been developed, with phenotypic overlapping across the traditional clinical categories (cancer, neurological diseases, CVD, metabolic syndrome) and through concepts such as the phenome and diseasome (Loscalzo and Barabasi, 2011). Second, the introduction of a pathogenetic interpretation of "action at a distance", including e.g. the long-term impact of early-life exposures, has revolutionized medical paradigms, since medicine has traditionally perceived causes as events proximal to disease. The combination of these two concepts corresponds to a shift from a bottom-up to a top-down causal framework in public health, i.e. from a reductionist view (e.g. based on key molecules, corresponding to the "magic bullets" of pharmacology) to a holistic view where complex pathways have an effect that is greater than the sum of their components (and also from proximity to remoteness).

See also Wild et al.: Translational Cancer Research: Balancing Prevention and Treatment to Combat Cancer Globally. JNCI J Natl Cancer Inst, 2015, 1-5

\section{Abstract \#: 002}

Work in life-work and health from a life-course epidemiological perspective
Ute Bültmann ${ }^{1}$

${ }^{1}$ University of Groningen, University Medical Center Groningen, Department of Health Sciences, Community and Occupational Medicine, Groningen, The Netherlands; Corresponding author's e-mail: u.bultmann@umcg.nl

Keywords: work, labor market, participation, epidemiology, health, life-course

Good work is fundamental to life-as is good health. Work and health are interdependent, that means work is a determinant of health and health is a determinant of work. In view of changing demographics, work and labor markets, as well social contexts, the key challenges to be addressed in work and health research and practice are (1) to minimize ill-health and its effects on work functioning, (2) to ensure good physical and psychosocial work environments and (3) to accomplish these challenges across the widening socio-economic gradient and across the life course.

Why a life course lens when looking at work and health? The working life is preceded by childhood and adolescence with schooling and training and followed by retirement and older age.

To date, detailed knowledge about the transitions people make, e.g., the transition from school to work, transitions during working life such as changing careers or the transition out of work towards retirement-and the impact of these transitions on trajectories of health, well-being and work functioning is lacking. Hence, to deepen our understanding of the dynamic interplay between work and health, a life-course epidemiological perspective is needed.

If we succeed in adopting a life course perspective and translating our findings into relevant policy and practice measures, we togetherresearchers, health care professionals, employers and policy makerscan make a difference towards "healthy working lives".

\section{Abstract \#: 003}

Associations between early body mass index trajectories and later metabolic risk in European children: the IDEFICS study

Börnhorst $\mathrm{C}^{1}$, Tilling $\mathrm{K}^{2}$, Russo $\mathrm{P}^{3}$, Kourides $\mathrm{Y}^{4}$, Michels $\mathrm{N}^{5}$, Molnár $D^{6}$, Rodríguez $\mathrm{G}^{7,8}$, Moreno $\mathrm{L}^{7}$, Krogh $\mathrm{V}^{9}$, Ben-Shlomo $\mathrm{Y}^{2}$, Ahrens $\mathrm{W}^{1,10}$, Pigeot $\mathrm{I}^{1,10}$

${ }^{1}$ Leibniz Institute for Prevention Research and Epidemiology - BIPS $\mathrm{GmbH}$, Bremen, Germany; ${ }^{2}$ School of Social and Community Medicine, University of Bristol, Bristol, United Kingdom; ${ }^{3}$ Institute of Food Sciences, National Research Council, Avellino, Italy; ${ }^{4}$ Department of Chronic Diseases, National Institute for Health Development, Tallinn, Estonia; ${ }^{5}$ Department of Public Health, Ghent University, Ghent, Belgium; ${ }^{6}$ Department of Pediatrics, University of Pécs, Pécs, Hungary; ${ }^{7}$ GENUD (Growth, Exercise, Nutrition and Development) Research Group, Faculty of Health Sciences, University of Zaragoza, Zaragoza, Spain; ${ }^{8}$ IIS Aragón (Aragon Health Research Institute), Spain; ${ }^{9}$ Department of Preventive and Predictive Medicine, Fondazione IRCCS Istituto Nazionale dei Tumori, Milan, Italy; ${ }^{10}$ Institute of Statistics, Faculty of Mathematics and Computer Science, University of Bremen, Bremen, Germany; Corresponding author's e-mail: boern@bips.uni-bremen.de

Background: Adiposity is a known risk factor for several health outcomes. However, yet it is not clear whether it is mainly the actual weight status or (in addition) the trajectory of adiposity, especially changes throughout early life, that affects health. In this study, a 2-step procedure was applied to investigate the association between body mass index (BMI) trajectories during infancy/childhood and metabolic risk at a later age in order to identify periods of growth related to later metabolic health. 
Methods: In a first step, BMI trajectories of 3301 European children that participated in the multi-centre IDEFICS study were modelled using linear-spline mixed-effects models (step 1). The resulting random coefficients indicating initial subject-specific BMI and rates of change in BMI over time were used as exposure variables in the second step to assess associations between BMI growth and a metabolic risk score as well as with its single components measured in later childhood (mean age at outcome assessment: $8.5 \mathrm{yrs}$ ). The metabolic risk score was derived applying a $\mathrm{z}$-score standardisation based on recently published reference values for young children to combine blood pressure, dyslipidaemia, waist circumference and hyperglycaemia into one continuous variable.

Results: All exposures under investigation, i.e. BMI at birth, rates of BMI change during infancy ( 0 to $<9 \mathrm{mths}$ ), early childhood ( $9 \mathrm{mths}$ to $<6$ yrs) and later childhood ( $\geq 6$ yrs) as well as current BMI z-score were significantly associated with the later metabolic risk score. Associations were strongest for the rate of BMI change in early childhood $(\beta=1.50: p<.0001)$ and for current BMI $\mathrm{z}$-score $(\beta=1.17: \quad p<.0001)$ and least pronounced for BMI at birth $(\beta=0.41: p<.0001)$. Results slightly differed with regard to the single metabolic risk factors.

Conclusion: Rapid growth, especially in the time window of $9 \mathrm{mths}$ to $<6$ yrs, is significantly related to later metabolic risk. Large parts of the associations of early BMI growth may be mediated through its effect on later BMI growth and current BMI.

\section{Abstract \#: 004}

\section{Obstetric mode of delivery and autism spectrum disorders in Sweden: a sibling design study}

Eileen A. Curran ${ }^{1}$, Christina Dalman ${ }^{2}$, Patricia M. Kearney ${ }^{3}$, Louise Kenny $^{1}$, John F. Cryan ${ }^{4}$, Timothy G. Dinan ${ }^{5}$, Ali S. Khashan ${ }^{3}$

${ }^{1}$ The Irish Centre for Fetal and Neonatal Translational Research (INFANT), Department of Obstetrics and Gynaecology, University College Cork, Cork, Ireland; ${ }^{2}$ Division of Public Health Epidemiology, Department of Public Health Sciences, Karolinska Institutet, Stockholm, Sweden; ${ }^{3}$ Department of Epidemiology and Public Health, University College Cork, Cork, Ireland; ${ }^{4}$ Alimentary Pharmabiotic Centre, Department of Anatomy and Neuroscience, University College Cork, Cork, Ireland; ${ }^{5}$ Alimentary Pharmabiotic Centre, Department of Psychiatry, University College Cork, Cork, Ireland; Corresponding author's e-mail: ecurran@ucc.i.e

Objective: As rates of caesarean section (CS) are rising worldwide, it is becoming increasingly important to understand any long-term effects CS may have on child development, such as autism spectrum disorder (ASD). ASD is also on the rise and has been associated with perinatal factors, such as obstetric mode of delivery. Consequently, we investigated the association between obstetric mode of delivery and autism spectrum disorder (ASD) using a large, population-based cohort.

Methods: The study cohort consisted of all singleton live births in Sweden between 1982 and 2010. Perinatal factors were retrieved from the Swedish Medical Birth Register, and ASD diagnoses based on the International Classification of Disease versions 9 and 10 were identified from the National Patient Register. We conducted stratified Cox regression analysis to examine the impact of mode of delivery on ASD, adjusting for perinatal and socio-economic factors. This was followed by sibling design study, which examined association between mode of delivery and ASD among sibling pairs discordant for autism and mode of delivery. The sibling study analysis was performed using conditional logistic regression. Analyses were first partially adjusted for year of birth, then further adjusted for infant gender, maternal age, 5 min Apgar score, maternal and paternal citizenship, small or large for gestational age, birth order, social welfare, family income, and parental psychiatric illness both affective and non-affective. Sibling design analysis was adjusted for all factors with the exception of maternal citizenship, as it was the same for both siblings.

Results: The final cohort included 2,697,315 births, and 28,290 cases of ASD. In adjusted Cox regression analysis, elective $(\mathrm{HR}=1.21$; [95 \% CI: 1.15-1.27]) and emergency (HR $=1.16$; $[95 \% \mathrm{CI}$ : 1.10-1.20]) CS were associated with ASD but assisted vaginal delivery (VD) was not $(\mathrm{HR}=1.03$; [95 \% CI $=0.98-1.07])$. The sibling design study included 13,411 sibling pairs discordant on ASD. In adjusted conditional logistic regression, using siblings as controls, elective CS (OR $=0.89$; [95 \% CI: 0.76-1.04]), emergency CS $(\mathrm{OR}=0.97$; $[95 \% \mathrm{CI}: 0.85-1.11])$ and assisted $\mathrm{VD}(\mathrm{OR}=0.91$; [95 \% CI: 0.82-1.02]) were all not associated with ASD.

Conclusions: This study confirms previous findings that children born by CS are about $20 \%$ more likely to be diagnosed with ASD after adjustment for known confounders. However, the association did not persist when using sibling controls, implying that this relationship is not causal, but may be due to residual confounding by shared familial factors such as genetic predisposition or environmental influences.

\section{Abstract \#: 005}

Performance of variable selection methods for correlated exposures and logistic models

Virissa Lenters $^{1}$, Roel Vermeulen ${ }^{1,2}$, Lützen Portengen ${ }^{1}$

${ }^{1}$ Division of Environmental Epidemiology, Institute for Risk Assessment Sciences, Utrecht University, The Netherlands; ${ }^{2}$ Julius Center for Health Sciences and Primary Care, University Medical Center Utrecht, The Netherlands; Corresponding author's e-mail: V.C.Lenters@uu.nl

Background: To better estimate independent and mutually adjusted effect estimates in epidemiology, there is growing recognition of the need to simultaneously assess multiple exposures. However, there is a paucity of research on the efficacy of statistical strategies for analyzing multicollinear data in relation to binary outcomes. We aimed to compare the performance of variable selection methods for identifying exposure-outcome associations within various data structures typical of occupational and environmental epidemiology analyses.

Methods: We simulated a case-control study with low-dimensional $(p<\mathrm{n})$, multivariate normally distributed data, and varied 5 parameters for a total of 270 simulation scenarios: (1) 10,20 or 50 potential predictors; (2) constant correlation levels of $0.0,0.4$ or 0.8 ; (3) samples sizes of 200,500 or 1000 ; and (4) $0,1,2$ or 5 effective or 'true' predictors; with (5) an effect size $(\beta)$ of $1.2,1.5$ or 2.0. 100 simulations were run per simulation scenario. We compared conventional (univariate, multiple and bidirectional stepwise) logistic regression methods with variable selection methods, readily implementable in $\mathrm{R}$ software, including sparse partial least squares discriminant analysis, and lasso and elastic net penalized regression. We computed the mean square error, sensitivity and false discovery proportion, and also evaluated which simulation parameters most influenced these performance metrics.

Results: Across the 27,000 simulations evaluated per method, the variable selection methods generally yielded improved selection accuracy and less biased effect estimates compared with logistic regression methods, especially for scenarios with higher correlation levels. Penalized methods performed particularly well; however, there was not a single method which consistently outperformed others across all data scenarios, and researchers may need to tailor their 
approach to the structure of the data. To improve statistical inference based on penalized approaches, future refinements might focus on assessing stability of the selection, quantifying uncertainty, and explicitly accounting for multiple testing.

Conclusion: In this extensive simulation study with multicollinear data, we found that several variable selection methods outperformed conventional logistic regression.

\section{Abstract \#: 006}

Specific agreement instead of Cohen's kappa to quantify interobserver variation for $>2$ raters and $>2$ categories

Henrica C.W. de Vet ${ }^{1}$, Rieky Dikmans ${ }^{2}$, Julie M. Fritz ${ }^{3}$, Iris Eekhout ${ }^{1}$

${ }^{1}$ Dept Epidemiology and Biostatistics, EMGO institute for Health and Care Research, VU University medical center, Amsterdam, The Netherlands; ${ }^{2}$ Dept. Reconstructive Surgery, VU University medical center, Amsterdam, The Netherlands; ${ }^{3}$ Division of Physical Therapy, University of Utah, Intermountain Health Care, Salt Lake City, UT, U.S.A.; Corresponding author's e-mail: hcw.devet@vumc.nl

Background: Cohen's kappa is a parameter for reliability, not for agreement. In clinical practice, the questions of interest concern agreement, e.g.: 'What is the probability that a colleague would provide the same answer?'. A recent paper (De Vet et al., 2013) proposed to use the proportion specific agreement for positive or negative outcomes. Now we extend the dichotomous situation (positive or negative) by 2 raters, to $>2$ raters and $>2$ categories, either nominal or ordinal.

Methods: In the dichotomous situation with $>2$ raters the agreement question is: given that one out of $\mathrm{n}$ raters scores positive/negative, what is the probability of a positive/negative score by the other $n-1$ raters. With 4 raters it is possible to present six 2 by 2 tables $(n(n-1) / 2=4 \times 3 / 2=6)$. The proportion specific agreement with 4 raters is based on the summation of the cells of these six 2 by 2 tables. Specific agreement is calculated using the same formula as for the dichotomous situation by 2 raters.

Positive specific agreement Negative specific agreement

$$
P A=\frac{a}{a+(b+c) / 2}, \quad N A=\frac{d}{d+(b+c) / 2}
$$

Ordinal and nominal categories with more than two observers: For ordinal and nominal data with $\mathrm{k}$ categories $(\mathrm{k}>2)$, per pair a $\mathrm{k}$ by $\mathrm{k}$ table is obtained. And these are summated over the number of pairs. Besides the probability of agreement and non-agreement the probability of a score in any of the non-agreement categories can be calculated. For example, in case of an ordinal scale (range 1-5), when one of the raters scores 3 the distribution of all other raters over all 5 scores can be assessed.

Examples: We used data of Dikmans et al. where 4 plastic surgeons scored 50 photo's on breast reconstructions using the 5 point scale ranging from very dissatisfied to very satisfied. For the dichotomous situation we classified the same data as satisfied versus neutral or not satisfied. For the multinominal situation we used the classification of low back pain into three different diagnoses based on the data of Fritz et al. (2006).

Discussion: The proportion specific agreement fits the clinical question better than kappa. It distinguishes between agreement on positive and negative outcomes, with different implications for clinical practice. For the multinominal situation it provides information about which categories are most often confused with each other. The impact of the prevalence on these parameters of agreement will be discussed.

Keywords: Specific agreement, interobserver variation, kappa, reliability.

\section{Abstract \#: 007}

Modelling growth across pediatric age: should weight be considered as a smooth function of age or height and age?

$\underline{\text { Milton Severo }}^{1}$, Joana Araújo ${ }^{1}$, Elisabete Ramos ${ }^{1}$

${ }^{1}$ Department of Clinical Epidemiology, Predictive Medicine and Public Health; Corresponding author's e-mail: milton@med.up.pt

Background: The majority of epidemiologic applications usually model the weight growth in function of age or weight growth in function of age and height (Body Mass Index-BMI). The objective of the study is to compare the use of mixed effects models using fractional polynomials for childhood/adolescent weight growth either as a smooth function of age or as a smooth function of age and height. Methods: We used data from participants measured at 13,17 and 21 years under the EPITeen cohort (Porto, Portugal), and additionally we extracted all measurements of weight and height from child health book records, since birth, We related age and height to natural ln of weight from birth to 21 years of age in 683 girls and boys with 16 measures on average per subject. Mixed effects fractional polynomial was used to predict natural ln of weight, either using age (model 1) or height and age (model 2). The quality of the two models was compared using the Bayesian Information Criterion (BIC), and the Relative Squared Error (RSE), Relative Absolute Error (RAE) and the correlation coefficient between the predictions and observed values. Results: Mixed effects fractional polynomial modelling identified the best-fitting models as being of degree 3 for both models. Model 1 include the powers: age to the power 3 , age to the power 1 and age to the power -3 : while the model of age (model 2 ) include the powers: height to the power 3 , height to the power -3 and height to the power 3 times $\ln$ (height) and age to the power -1 , age to the power -0.5 and age to the power -0.5 times $\ln ($ age $)$. There was significant interaction for sex in both models although the effect was small.

BIC suggested that the model 2 (as function of age and height) was better than the model 1 (as function of age): $\mathrm{BIC}=-27,076.17$ versus $\mathrm{BIC}=-23,643.16$. The RSE, RAE and correlations were $0.28 \%, 4.82 \%$ and 0.9985 for model 2 and $0.35 \%, 5.25 \%$ and 0.9982 for model 1 , respectively.

Differences between the two models were also shown on predicted weight velocity in function of age. According to model 1 , velocity decreases up to 2.9 years, subsequently starts to increase up to 12.4 years and then is followed again by a decrease. When using model 2 , weight velocity presented a fast decrease up to 2.6 years, afterwards it increases up to 11.8 years and then starts to decrease again.

Conclusions: Modelling the effect of ln weight in function of height with a fractional polynomial with one degree, such as the models using BMI, leaves residual confounding of height in the $1 n$ weight. The quality index of the models provided evidence for considering weight as a function of height and age as the best option rather than modelling weight as a function of age, as traditionally is done, although the differences on the predicted values were small.

We acknowledge funding from the Portuguese Foundation for Science and Technology (FCOMP-01-0124-FEDER-015750 and SFRH/ $\mathrm{BD} / 78153 / 2011$ ) 


\section{Abstract \#: 008}

Feasibility of using the 'cohort multiple randomized controlled trial' design to conduct the rectal boost study

J.P.M. Burbach $^{1}$, O. Reerink ${ }^{1}$, M. Intven ${ }^{1}$, J.J.E. Kleijnen ${ }^{1}$, M. Koopman $^{2}$, W.M.U. van Grevenstein ${ }^{3}$, M. van Vulpen ${ }^{1}$, H.M. Verkooijen ${ }^{4}$

${ }^{1}$ Radiotherapy, UMC Utrecht, The Netherlands; ${ }^{2}$ Medical Oncology, UMC Utrecht, The Netherlands; ${ }^{3}$ Surgery, UMC Utrecht, The Netherlands; ${ }^{4}$ Trial office Imaging Division, UMC Utrecht, The Netherlands; Corresponding author's

e-mail: j.p.m.burbach-2@umcutrecht.nl

Rationale: Randomized controlled trials (RCTs) are the gold standard to evaluate effectiveness of new interventions, although RCTs often experience difficulties in recruitment and generalizability.We introduced the 'cohort multiple Randomized Controlled Trial' (cmRCT) to efficiently and simultaneously evaluate multiple oncologic interventions, while maintaining the highest level of evidence.

In the RECTAL BOOST study, response rates of locally advanced rectal cancer (LARC) patients after boost + standard chemoradiation (intervention arm) are compared to those after standard chemoradiation alone (control arm). The boost intervention consists of $5 \times 3 \mathrm{~Gy}$ to the tumor delivered prior to standard chemoradiation $(25 \times 2$ Gy plus capecitabine). This trial is the first clinical and oncological study to be performed according to the cmRCT design. We will evaluate feasibility of the cmRCT design in terms of trial recruitment and boost-offer acceptance.

Methods: A cohort of rectal cancer patients forms the basis of our cmRCT design. These patients consented to prospective data collection (clinical, biobank and quality-of-life), and additionally, were asked to consent to broad randomization for being offered experimental interventions. Within our cohort, patients eligible for boost treatment-LARC $(\mathrm{T} 3+/ 4 \mathrm{NxM} 0$ or TxN2M0) located $\leq 10 \mathrm{~cm}$ from the anus-are included in a sub-cohort. From this sub-cohort, a random sample is offered the boost treatment in addition to standard chemoradiation which may be accepted or refused (intervention arm). Eligible patients who were not randomly selected undergo standard chemoradiation without further notification about the trial (control arm). Data will be analysed according to intention-to-treat.

Results: In the past 4 months 14 eligible patients were eligible. Of these, 13 consented for cohort participation and gave broad consent for randomization. All 13 were randomized: 6 were allocated to the control arm and received chemoradiation, 7 were allocated to the intervention arm where they were offered the boost in addition to chemoradiation. Five accepted and underwent the boost, whereas 2 refused because of personal reasons and reluctance to undergo an MRI-scan.

Conclusion: The cmRCT design shows potential for effective and high trial recruitment (13 of 14 all eligible patients) in a clinical oncologic setting. Five of seven patients accepted their offered intervention and those in the control group adhered to their standard care.

Discussion: If the number of refusing patients increases, this would dilute the treatment effect, thereby demanding advanced statistical solutions (instrumental variable analysis). Updated results will be presented in June 2015. All co-authors approved the abstract. No conflicts of interest.

\section{Abstract \#: 009}

Index event bias compromises the performance of prediction models for disease recurrence: a simulation study
Sander van Kuijk ${ }^{1}$, Simone Sep ${ }^{2}$, Luc Smits ${ }^{3}$

${ }^{1}$ Dept. of Knowledge and Innovation, Regional Public Health Service Zuid Limburg; ${ }^{2}$ Dept. of Internal Medicine, Maastricht University; ${ }^{3}$ Dept. of Epidemiology, Maastricht University; Corresponding author’s e-mail: sander.vankuijk@ggdzl.nl

Background: Index event bias (IEB) can be introduced by restriction to subjects who previously experienced the outcome of interest. Its mechanism has been shown for studies of causal factors of disease recurrence. In this study we evaluate the effect of IEB on the performance of prediction models for disease recurrence.

Methods: We simulated a dataset consisting of 4 mutually unassociated determinants from a multivariate normal distribution, and two dichotomous outcomes (i.e. occurrence and recurrence) from a Bernoulli distribution with probability depending only on the determinants. The prediction model for a first event (the reference model) consisted of two determinants (the other two were assumed unknown and unmeasured), and was estimated using logistic regression. The model for recurrence was estimated on the subset of records that had a first occurrence. Discrimination and calibration measures were computed for both models. All simulations were repeated 10,000 times to obtain stable estimates.

Results: When IEB was present, the model for disease recurrence performed inferior to the reference model. The area under the receiver operating characteristic curve was closer to $50 \%$, and the model fit as quantified by the Hosmer and Lemeshow goodness-of-fit was reduced. The probability of disease recurrence had a strong effect on the relative size of IEB.

Discussion: We have shown by means of stochastic simulation, that index-event bias in the estimates of regression coefficients of predictors for disease recurrence causes a reduction in predictive performance of the model in terms of discrimination and calibration. As of yet, no remedies against IEB have been found, thus identifying scenarios in which it may arise is paramount.

\section{Abstract \#: 010}

Predictive role of dietary habits in relation to incident CVD and all-cause mortality: results from a Swedish cohort of 60-yearold

Federica Laguzzi $^{1}$, Axel C. Carlsson ${ }^{2,3}$, Bruna Gigante ${ }^{1,4}$, Mai-Lis Hellénius ${ }^{5}$, Ulf de Faire ${ }^{1,5}$, Karin Leander ${ }^{1}$

${ }^{1}$ Unit of Cardiovascular Epidemiology, Institute of Environmental Medicine, Karolinska Institutet, Nobels väg 13, Box 210, 17177 Stockholm, Sweden; ${ }^{2}$ Centre for Family and Community Medicine, Department of Neurobiology, Care Sciences and Society, Karolinska Institutet, Huddinge, Sweden; ${ }^{3}$ Department of Medical Sciences, Molecular Epidemiology and Science for Life Laboratory, Uppsala University, Uppsala, Sweden; ${ }^{4}$ Division of Cardiovascular Medicine, Department of Clinical Sciences, Karolinska Institutet, Danderyd Hospital, Stockholm, Sweden; ${ }^{5}$ Cardiology Unit, Department of Medicine, Karolinska Institutet, Stockholm, Sweden; Corresponding author's e-mail: federica.laguzzi@gmail.com

Background and aim: Diet and cardiovascular disease (CVD) are closely related. The role of dietary habits, in particular habits of dietary fat intake, in development of CVD is still uncertain. We aimed to investigate the association between self-reported intake of food items containing different types of dietary fats and incident CVD and all-cause mortality, respectively, in a representative population-based cohort of 60-year-old Swedish men and women. In addition, we studied how fruit and vegetable intake associates with these outcomes. 
Methods: Data were collected between 1997 and 1998 from 4232 randomly selected men $(n=2039)$ and women $(n=2139)$ from Stockholm County. Participants with no previous CVD diagnosis were followed prospectively regarding CVD events and death up to 2012-12-31 using national registers: 359 incident CVD cases and 595 deaths were identified. From nutritional data collected using food frequency questionnaires we created five diet scores reflecting intake of saturated fats in general (1), fats from dairy products (2), fish (3), processed meat (4) and vegetable oils and margarines (5). In addition, binary variables were generated from 16 questions on intake of single dietary items. Cox proportional hazards model was used to estimate hazard ratios (HR) with $95 \%$ confidence interval (CI) for the outcomes associated with the different exposure variables.

Results: None of the diet scores were significantly associated with risk to suffer an incident CVD event or early death in either men or women. Analyses of single dietary item data showed (after adjustments for potential confounding factors related to life style) an increased risk of CVD in women associated with the consumption of $\geq 10 \mathrm{~g} /$ day of spread butter or margarine (vs $<10 \mathrm{~g} /$ day) (HR $=1.49$, $\mathrm{CI}=1.02-2.20)$ and $\geq 2$ times/week consumption of oily potato dishes (vs $<2$ time/week) ( $\mathrm{HR}=2.00, \mathrm{CI}=1.11-3.60)$. In men, none of the 16 single dietary items considered were associated with CVD risk. The consumption of $\geq 10 \mathrm{~g} /$ day of spread butter or margarine was however associated with early death in men (adjusted $\mathrm{HR}=1.57, \mathrm{CI}=1.23-2.02$ ), and so were use of butter (vs margarine), (adjusted HR $=1.28, \mathrm{CI}=1.01-1.62$ ) and intake of eggs $\geq 4$ times/week (vs $<4$ times/week) (adjusted $\mathrm{HR}=1.53, \quad \mathrm{CI}=$ 1.15-2.02). In men, but not in women, a significant inverse association between the daily intake of fruits (vs $<1$ time/day) and early death was observed (adjusted HR $=0.75, \mathrm{CI}=0.60-0.94$ ).

Conclusion: In our study, the quantitative intake of fats from different sources is not predictive of CVD and mortality, whereas intake of a few specific food items shows sex-specific associations with incident CVD and mortality.

\section{Abstract \#: 011}

The association between saturated fat and coronary heart disease depends on the source and type of fatty acid in EPIC-NL

Jaike Praagman $^{1}$, Joline W.J. Beulens ${ }^{1}$, Marjan Alssema ${ }^{2}$, Peter L. Zock $^{2}$, Anne J. Wanders ${ }^{2}$, Yvonne T. van der Schouw ${ }^{1}$

${ }^{1}$ Julius Center for Health Sciences and Primary Care, University Medical Center, Utrecht, the Netherlands; ${ }^{2}$ Unilever Research and Development, Vlaardingen, the Netherlands; Corresponding author's e-mail: j.praagman-2@umcutrecht.nl

Objectives: The association between saturated fatty acid (SFA) intake and coronary heart disease (CHD) risk is debated. We investigated whether intake of SFA was associated with CHD risk, and whether associations depended on (1) the macronutrient that substitutes SFA: (2) the food sources of SFA: and (3) the type of SFA (differing in carbon-chain length).

Methods: We included 35,597 healthy men and women from the EPIC-NL cohort. Baseline (1993-1997) SFA intake was measured using a validated food frequency questionnaire. Multivariable Cox' regression was used to study associations between isoenergetic substitution of SFA with other macronutrients and CHD risk, and between total SFA (per 5 percent of daily energy intake [en\%]), SFA from different food sources (per $1 \mathrm{en} \%$ ), and SFA type (per $0.1 \mathrm{en} \%$ or $1 \mathrm{en} \%$ ) and CHD risk.

Results: During a mean follow-up of 12 years 1807 CHD events occurred. Mean SFA intake was $15( \pm 3)$ en\%, with $41 \%$ from dairy sources. Total SFA intake was associated with a lower CHD risk
(Hazard Ratio (HR) per 5 en\% $=0.83,95 \%$ CI: 0.74-0.93). A higher intake of energy from animal protein, cis-MUFA, PUFA or carbohydrates at the expense of an equal amount of energy from SFA was significantly associated with higher CHD risks (HRs between 1.27 and 1.37 per 5 en\%). No significant associations were observed for substitution of SFA with vegetable protein (HR per 5 en $\%=0.81$, 0.57-1.17). Slightly lower CHD risks were observed for each 1 en\% higher intake of SFA from milk (HR =0.94: 0.90-0.98), cheese $(\mathrm{HR}=0.96: 0.93-0.98)$, and butter $(\mathrm{HR}=0.96$ : 0.93-0.99). Intake of $\mathrm{C} 14: 0$ was associated with a lower CHD risk (HR per 1 en $\%=0.78: 0.65-0.93)$. A small inverse association was also observed for the intake of short- to medium-chain SFA (sum of C4:0 to $\mathrm{C} 10: 0$, HR per $0.1 \mathrm{en} \%=0.970 .95-1.00$ ). For SFA from other food sources (meat, solid and liquid fats, snacks, and cakes) and SFA types no significant associations were observed.

Conclusions: In this general Dutch population a higher total SFA intake was not associated with a higher CHD risk. The lower CHD risk we observed appeared to be driven mainly by SFA from dairy, in particular short-to medium-chain SFAs and C14:0. Our data did not allow complete exclusion of possible residual confounding by transfat, which may explain why we did not observe a lower CHD risk for substitution of SFA with PUFA. Associations need to be established in other populations with larger differences in SFA intake and different dietary patterns, before definitive conclusions are possible.

\section{Abstract \#: O 12}

\section{Mediterranean diet and mortality in eastern Europeans: results} from the HAPIEE study

Denes Stefler ${ }^{1}$, Hynek Pikhart ${ }^{1}$, Ruzena Kubinova ${ }^{2}$, Andrzej Pajak ${ }^{3}$, Sofia Malyutina $^{4}$, Anne Peasey ${ }^{1}$, Martin Bobak ${ }^{1}$

${ }^{1}$ Department of Epidemiology and Public Health, University College London, London, UK; ${ }^{2}$ Centre for Health Monitoring, National Institute of Public Health, Prague, Czech Republic; ${ }^{3}$ Department of Epidemiology and Population Sciences, Jagiellonian University, Krakow, Poland; ${ }^{4}$ Institute of Internal and Preventive Medicine, Siberian Branch of the Russian Academy of Medical Sciences, Novosibirsk, Russia; Corresponding author's e-mail: denes.stefler.10@ucl.ac.uk

Background: Mediterranean-type diet has been found to be related to lower risk of cardiovascular disease (CVD) and other chronic diseases in previous studies, including in non-Mediterranean populations, but its association with health outcomes in Eastern Europe has not been investigated. In this study, we examined the link between Mediterranean diet score (MDS) and total, CVD, coronary heart disease (CHD) and stroke mortality among the participants of the Health Alcohol and Psychosocial factors in Eastern Europe (HAPIEE) prospective cohort study.

Methods: 18,464 adult individuals (age range 45-70 years) from random urban population samples in the Czech Republic, Poland and the Russian Federation were included in the analysis. Dietary data was collected using food frequency questionnaires (FFQ). Participants ' adherence to the Mediterranean diet was estimated using a MDS with nine components proposed by Sofi et al. in 2014. Each component score was based on absolute cut-off values, and the total MDS ranged from 0 to 17 . Data on mortality was ascertained by linkage with death registers, and the association between MDS and mortality was estimated by multivariable adjusted Cox regression models. Missing covariate data was imputed using multiple imputation procedures.

Results: Overall, $22 \%$ of the participants scored more than 10 MDS points (from the maximum of 17). The proportion of high-scorers was 
the largest in Poland and the lowest in the Russian cohort. During the average follow-up time of 7 years, 1234 participants died. Four-point increase in the MDS was found to be inversely associated with mortality from all causes (HR, $95 \%$ CI: 0.90, 0.82-0.99), CVD $(0.80$, $0.67-0.94)$ and stroke $(0.69,0.49-0.96)$ after multivariable adjustment.

Conclusion: Our results suggest that in these Eastern European populations, higher adherence to Mediterranean type diet is associated with lower risk of death from all-causes, CVD and stroke. These findings should be taken into account when dietary or public health recommendations are designed in Eastern European countries. The consistency with results from other population suggests that the MDS is applicable in different settings. The use of MDS with absolute component cut-off values may be also recommended in other population samples.

\section{Abstract \#: 013}

Simulating different assessment regimes for repeated short-term dietary measurements to estimate usual intake distributions of seasonally consumed foods

$\underline{\text { Matthias Clemens }^{1}}{ }^{\text {, Sven Knüppel }}{ }^{1}$, Heiner Boeing ${ }^{1}$

${ }^{1}$ Department of Epidemiology, German Institute of Human Nutrition Potsdam-Rehbruecke, Nuthetal, Germany; Corresponding author's e-mail: Matthias.Clemens@dife.de

Background and aims: Recent statistical advances suggest to combine repeated short-term dietary measurement and non-quantitative information of food frequency questionnaires as the most promising approach to estimate usual dietary intakes. To cover for seasonal variation, most study protocols propose to conduct repeated shortterm dietary assessment on non-consecutive days over a period of 12 months. The aim of this simulation study was to assess if the potential bias of usual food intake estimations derived from repeated dietary measurement within less than 12 month (e.g. 8 weeks) could be attenuated by incorporating indicator variable for season as covariate into the statistical modelling parts.

Methods: The food intake distribution for a hypothetical food item with high seasonal variation was used to simulate individual usual food intakes over a period of 365 days. By random selection of 4 simulation days within different timeframes (8 weeks versus 12 months), distributions of usual food item intake in a study sample of 1000 individuals were repeatedly estimated using no covariate or covariate information on season (bootstrap method, repeats $=100$ ). The derived distributions of estimated usual food item intake were described by the 5th, 25th, 50th, 75th and 95th percentile. Spearman rank order correlation coefficients (r) were determined to test the degree of association between simulated and estimated values of usual food item intake.

Results: The original 5th, 25th, 50th, 75th and 95th percentile of simulated food intake distribution (reference) were 11.20: 17.70: 23.91: 31.26 and $40.26 \mathrm{~g} /$ day: respectively. Estimated percentiles derived from 4 random repeated measurements within 12 months were 14.07: 19.22: 24.31: 28.76: $34.57 \mathrm{~g} /$ day $(\mathrm{r}=0.70$ (95\% CI, $0.66-0.72)$ ). When 4 repeated measurements were assessed within 8 weeks, estimated percentiles amounted to 4.74: 7.04: 9.55: 30.44 : $74.13 \mathrm{~g} /$ day. Spearman correlation coefficient dropped almost about half of its value to 0.39 (95\% CI, 0.32-0.43) but could be adjusted to $0.72(0.71-0.74)$ by including seasonal information into the model.

Conclusion: Epidemiological studies which plan to estimate individual usual food intakes with high numbers of repeated dietary measurements should follow recommendations and conduct repeated short-term dietary assessments on random non-consecutive days over a period of 12 months. If due to feasibility repeated dietary measurements have to be performed within short timeframes estimates of individual consumption probability should be adjusted for seasonal information.

\section{Abstract \#: O 14}

The role of material and behavioral factors measured at multiple times in adulthood in explaining socioeconomic inequalities in mortality

Joost Oude Groeniger ${ }^{1}$, Carlijn B.M. Kamphuis ${ }^{2}$, Johan P. Mackenbach $^{1}$, Frank J. van Lenthe ${ }^{1}$

${ }^{1}$ Department of Public Health, Erasmus MC, Rotterdam, The Netherlands; ${ }^{2}$ Department of Human Geography and Spatial Planning, Utrecht University, Utrecht, The Netherlands; Corresponding author's e-mail: j.oudegroeniger@erasmusmc.nl

Background: Socioeconomic inequalities in mortality are consistently and widely observed. Several explanations have been posed, including higher exposure to unfavourable material and behavioural factors among those in lower socioeconomic groups. Studies examining these explanations usually measure these explanatory factors only at baseline, disregarding possible changes during follow up. We examined the role of behavioral and material factors measured at multiple times in adulthood for the explanation of socioeconomic differences in mortality.

Methods: Data from the Dutch GLOBE-study, a prospective cohort starting in 1991, were linked to mortality register data from Statistics Netherlands up to and including $2013(\mathrm{~N}=5124)$. Socioeconomic position was measured by both highest attained educational level and household occupation at baseline. Potential mediating variables were self-reported and measured in 1991, 1997 and 2004.

The behavioral factors included smoking status, leisure time physical activity, sports participation and body mass index. The material factors included financial difficulties, housing tenure and type of health insurance. Missing data on the behavioural and material factors were imputed by use of multiple imputation. Cox proportional hazard regression models with age as the time scale was used to estimate the hazard ratios and their $95 \%$ confidence intervals for the association between socioeconomic position and mortality. Subsequently, the behavioral and material factors were added to the model as time-dependent covariates. We used inverse probability weighting to account for time-varying confounders. All analyses were carried out separate for men and women.

Results: Both education and occupation were significantly associated with mortality, with the lowest socioeconomic group having higher mortality rates as compared to the highest socioeconomic group. Adding behavioral or material factors to the model reduces part of the increased hazard ratios for those in lower educational and occupational groups. Including all possible risk factors in the same model resulted in the largest attenuation of the hazard ratios.

Conclusion: Our analyses show that measuring both behavioral and material factors several times during follow up impact upon the explanation of socioeconomic inequalities in mortality. Measuring risk factors over the life-course helps to gain better insight into explanations of socioeconomic inequalities in mortality.

\section{Abstract \#: 015}

Absolute and relative socioeconomic health inequalities vary across age groups: results from the lifelines cohort study 
Sander K.R. van Zon ${ }^{1}$, Ute Bültmann ${ }^{1}$, Carlos F. Mendes de Leon ${ }^{2}$, Sijmen A. Reijneveld ${ }^{1}$

${ }^{1}$ Department of Health Sciences Division of Community and Occupational Medicine, University Medical Center Groningen, University of Groningen, Groningen, The Netherlands; ${ }^{2}$ Department of Epidemiology, University of Michigan School of Public Health, Ann Arbor, Michigan, The United States of America; Corresponding author's e-mail: s.k.r.van.zon@umcg.nl

Background: Age affects both measures of socioeconomic position (SEP) and of health outcomes, but in varying degrees. It is not clear whether the effects of age on inequalities differ by SEP indicator, health outcome, and gender. Moreover, it is not clear whether there is a difference between measuring inequalities in absolute or relative terms. The aim is to investigate whether absolute and relative socioeconomic health inequalities differ by indicator of SEP, health outcome, and gender across age groups.

Methods: The study sample was derived from the LifeLines Cohort Study and consisted of 95,432 participants. SEP was measured by educational level and household income, both in eight categories. Physical and mental functioning were measured with the Short Form 36 Health Survey. Age concerned 11 age groups with a 5-year age range. Absolute inequalities were examined by comparing means. Relative inequalities were examined by calculating Gini-coefficients. All analyses were performed for both SEP indicators, and both health outcomes, and stratified for gender.

Results: Absolute socioeconomic health inequalities differed by SEP indicator and health outcome, but not by gender, across age groups. The influence of age was most pronounced in mental functioning by household income. Absolute inequalities were larger in younger compared with older age groups. Relative socioeconomic health inequalities differed by SEP indicator, health outcome, and gender across age groups. The influence of age was most pronounced in mental functioning by educational level. Relative inequalities were small in those aged 25-64 (range Gini-coefficient: 0.04-0.11) and large in those aged $\geq 65$ years (range Gini-coefficient: $0.13-0.20$ ).

Conclusions: Absolute and relative socioeconomic health inequalities differed by SEP indicator, health outcome, and gender across age groups. Researchers should critically consider their SEP indicator, health outcome and age group for research into socioeconomic health inequalities.

\section{Abstract \#: O 16}

\section{An investigation of pathways from social activity to cognitive function in older Irish adults}

Joanna McHugh $^{1}$, Jianjun Tang ${ }^{1}$, Brian Lawlor ${ }^{2}$, Rose Ann Kenny ${ }^{2,3}$, Frank Kee ${ }^{\mathrm{I}}$

${ }^{1}$ Centre for Public Health, Queen's University Belfast; ${ }^{2}$ Institute of Neuroscience, Trinity College Dublin; ${ }^{3}$ Centre for Medical Gerontology, Trinity College Dublin; Corresponding author's e-mail: J.McHugh@qub.ac.uk

Background: It has been suggested that lifestyle impacts cognition via three potential pathways: the stress pathway, the vascular pathway, and the cognitive reserve pathway (Fratiglioni, Paillard-Borg \& Winblad, 2004). We assessed proxies of these three pathways as routes from social activity to cognition among older adults. Education attainment is a common proxy used to assess cognitive reserve, and seated systolic blood pressure was used as a proxy measure of vascular function.

Methods: Data were from the baseline wave of the Irish Longitudinal Study on Ageing (TILDA), a nationally representative cohort of 8500 adults aged over 50. Participants were asked about their frequency of engagement in different categories of social activity, and the Montreal Cognitive Assessment (MoCA) was taken as a proxy of global cognition. Stress was measured using the Perceived Stress Scale (Cohen, Kamarck \& Mermelstein, 1983), vascular health measured using seated systolic blood pressure as a proxy, and education level was taken as a proxy measure of cognitive reserve. Structural equation modelling was used to evaluate each purported pathway as a mediator in the relationship between an overall latent 'social activity' variable and global cognition.

Results: There was some support for the stress hypothesis, as it was found that stress mediated the relationship between social activity and cognition (indirect effect $\beta=-0.046: p<0.005$ : CFI $=0.825$, TLI, 0.794 , RMSEA $=0.056): 17 \%$ of the variance in MoCA scores was accounted for in this model. The cognitive reserve hypothesis was also supported (indirect effect $\beta=-0.4, p<0.001$ : $\mathrm{CFI}=0.83$, TLI $=0.799$, RMSEA $=0.05$ ): $22 \%$ of the variance in MoCA scores was accounted for here. Finally there was also some support for the vascular hypothesis (indirect effect $\beta=-0.021, p<0.001$ : $\mathrm{CFI}=0.825$, TLI $=0.794:$ RMSEA $=0.05$ ): $17 \%$ of the variance in MoCA scores was accounted for. ANOVA was performed to evaluate the goodness of fit across the different models but no significant differences were found.

Conclusions: All three mechanisms may sub-serve the relationship between social activities and cognition in older adults, but the cognitive reserve hypothesis accounted for the greatest amount of variance in MoCA score outcomes. Further longitudinal research is required to compare these three pathways in conjunction with other known causes of cognitive decline and dementia in older adults.

Cohen, S., Kamarck, T. \& Mermelstein, R. (1983). A global measure of perceived stress. Journal of Health \& Social Behavior, 24(4), 385-396. Fratiglioni, L., Paillard-Borg, S. \& Winblad, B. (2004). An active and socially integrated lifestyle in late life might protect against dementia. Lancet Neurology, 3(6), 343-353.

\section{Abstract \#: 017}

Social relationships and risk of dementia: a systematic review and meta-analysis of longitudinal cohort studies

Jisca S. Kuiper ${ }^{1}$, Marij Zuidersma ${ }^{2}$, Richard C. Oude Voshaar ${ }^{2}$, Sytse U. Zuidema $^{3}$, Edwin R. van den Heuvel ${ }^{1,4}$, Ronald P. Stolk ${ }^{1}$, Nynke Smidt ${ }^{1}$

${ }^{1}$ Department of Epidemiology, University of Groningen, University Medical Center Groningen, Groningen, The Netherlands;

${ }^{2}$ Department of Psychiatry, University of Groningen, University Medical Center Groningen, Groningen, The Netherlands;

${ }^{3}$ Department of General Practice, University of Groningen, University Medical Center Groningen, Groningen, The Netherlands;

${ }^{4}$ Department of Mathematics and Computer Science, Eindhoven University of Technology, Eindhoven, The Netherlands; Corresponding author's e-mail: j.s.kuiper@umcg.nl

Background: Poor social relationships are a risk factor for mortality. It is unclear to what extent poor social relationships are also a risk factor for dementia. We conducted a systematic review and metaanalysis to investigate to what extend poor social relationships are associated with the onset of dementia.

Methods: We systematically searched in MEDLINE, Embase, PsycINFO to identify longitudinal cohort studies investigating the association between social relationships factors and dementia in the general population. The methodological quality of all included studies was assessed. Pooled relative risks (RRs) with $95 \%$ confidence intervals (CIs) were calculated using random effects models. 
Results: 19 studies were included in the review, investigating the association between various social relationship factors (social network size ( 8 studies): social participation ( 8 studies): frequency of social contact (9 studies): loneliness (4 studies): satisfaction with social network (4 studies): and other social relationship factors (7 studies)) and dementia. The following social relationship factors were associated with incident dementia: low social participation (RR: 1.41 (95\% CI: 1.13-1.75), less frequent social contact (RR: 1.57 (95\% CI: 1.32-1.85), and loneliness (RR: 1.58 (95\% CI: 1.19-2.09). The results of the association between social network size and dementia were inconsistent. No statistically significant association was found for low satisfaction with social network and the onset of dementia (RR: 1.25 (95\% CI: 0.96-1.62). With regard to the other social relationship factors (mostly composite measures), a statistically significant association between poor social relationships and onset of dementia was found in 5 out of 7 studies.

Conclusion: We conclude that social relationships that represent a lack of social interaction are associated with the onset of dementia. The strength of the association between poor social interaction and the onset of dementia is comparable with other well-established risk factors for dementia, such as low education attainment, physical inactivity and smoking.

\section{Abstract \#: O 18}

Ten-year blood pressure trajectories, cardiovascular mortality and life years lost in two extinction cohorts

$\underline{\text { Susanne M.A.J. Tielemans }}{ }^{1}$, Johanna M. Geleijnse ${ }^{1}$, Alessandro Menotti $^{2}$, Hendriek C. Boshuizen ${ }^{1}$, Sabita S. Soedamah-Muthu ${ }^{1}$, David R. Jacobs $\mathrm{Jr}^{3}$, Henry Blackburn ${ }^{3}$, Daan Kromhout ${ }^{1}$

${ }^{1}$ Division of Human Nutrition, Wageningen University, Wageningen, the Netherlands; ${ }^{2}$ Association for Cardiac Research, Rome, Italy; ${ }^{3}$ Division of Epidemiology and Community Health, School of Public Health, University of Minnesota, Minneapolis, Minnesota, USA; Corresponding author's e-mail: susanne.tielemans@wur.nl

Background: Blood pressure (BP) trajectories derived from measurements repeated over years have low measurement error and may improve cardiovascular disease (CVD) prediction compared to single, average and usual BP (single BP adjusted for regression dilution). We characterized 10-year trajectories of annual systolic BP measurements and examined their association with CVD mortality, all-cause mortality and life years lost in two prospective cohort studies.

Methods: Data from two (nearly) extinct cohorts of middle-aged men, the Minnesota Business and Professional Men Study $(\mathrm{n}=261)$ and the Zutphen Study $(\mathrm{n}=632)$ were used. BP was measured annually during 1947-1957 in Minnesota and 1960-1970 in Zutphen. Systolic BP trajectories were identified by latent mixture modelling (PROC TRAJ in SAS). Cox proportional hazards and linear regression models examined systolic BP trajectories with CVD mortality, all-cause mortality and life years lost. Associations were adjusted for age, serum cholesterol level, smoking habit and diabetes.

Results: Mean initial age was $\sim 50$ years in both cohorts. After 10 years of BP measurements, men were followed until death on average 20 years later. All Minnesota men and $98 \%$ of Zutphen men died. Mean BP at baseline was $124 / 73 \mathrm{mmHg}$ in Minnesota and 141/89 in Zutphen. The best model fit was obtained with a fourtrajectory model. However, because a small fraction of men was assigned to the fourth trajectories, we focused on the comparison of the third trajectories with the first trajectories. In Minnesota, the third systolic BP trajectory was associated with a CVD mortality risk of 3.8 (95\% CI: 2.2-6.6), an all-cause mortality risk of 2.5 (95\% CI:
1.6-3.8) and 8.1 (95 \% CI: 4.4-11.8) life years lost, compared with the first trajectory. In Zutphen, corresponding risks were $2.1(95 \%$ CI: 1.5-2.9) for CVD mortality, 1.5 (95\% CI: 1.2-1.9) for all-cause mortality and the number of life years lost was 3.7 (95 \% CI: 1.8-5.6) years. Compared with the other BP measures (single, average and usual BP), 10-year BP trajectories were the strongest predictors in Minnesota. In Zutphen, average BP was the strongest predictor. Conclusion: We identified 10-year trajectories of systolic BP that were strong predictors of (CVD) mortality and life years lost. In both cohorts, trajectories were stronger predictors than single BP, but the added value compared to the 10-year average depended on the cohort.

\section{Abstract \#: 019}

High depressive symptoms are inversely associated with ideal cardiovascular health? The Paris Prospective Study III

Bamba Gaye $^{1}$, C. Prugger ${ }^{1}$, M.C. Périer ${ }^{1}$, M. Plichart ${ }^{1}$, F. Thomas ${ }^{2}$, C. Guibout $^{1}$, B. Pannier ${ }^{2}$, P. Boutouyrie ${ }^{1}$, X. Jouven ${ }^{3}$, J.P. Empana ${ }^{1}$

${ }^{1}$ INSERM U970, Paris Cardiovascular Research Center (PARCC), Paris Descartes, Sorbonne Paris Cité University, Paris, France; ${ }^{2}$ Preventive and Clinical Investigation Centre, Paris, France; ${ }^{3}$ APHP, Georges Pompidou European Hospital, Pharmacology and Cardiology Departments, Paris, France; Corresponding author's e-mail: m.bamba.gaye@gmail.com

Purpose: The concept of primordial prevention of cardiovascular disease has been re-emphasized recently. To this end, the American Heart Association has proposed the Ideal Cardiovascular Health $(\mathrm{ICVH})$, a simplified 7-items tool to promote cardiovascular health. We hypothesize that High depressive symptoms would be inversely associated with ideal cardiovascular health. We explored this hypothesis using the baseline examination data of 10,157 participants from the Paris Prospective Study III.

Methods: Between 2008 and 2012, 10157 men and women 50-75 years of age were examined in a large health center in Paris (France). The ICVH comprises 4 behavioural (smoking, body mass index, physical activity, and diet) and 3 biological components (total cholesterol blood pressure and blood glucose). Each item is categorized in three levels (poor, intermediate and ideal) and participants with $0-2,3-4$ and 5-7 ideal health components are categorized as having poor, intermediate and ideal cardiovascular health, respectively. High depressive symptoms were defined as a score $\geq 7$ on the 13-item Questionnaire of Depression 2nd version, Abridged or the use of antidepressants. Polytomous logistic regression was performed to examine the relationship between high depressive symptoms and ICVH.

Results: Mean age of the participants was 59.11 (SD 6.3) years and $60.2 \%$ were males. A total of $8.3 \%$ had high depressive symptoms, $5.4 \%$ in men and $12.7 \%$ in women. Poor, intermediate and ideal cardiovascular health was observed in $48.1,44.3$ and $7.6 \%$ of the study participants. After adjusting for age, sex, living alone status and educational level, participants with high depressive symptoms had a $20 \%$ (odds ratio $=0.80: 95 \%$ confidence interval $0.69-0.93$ ) and $30 \%$ (odds ratio $=0.70$ : confidence interval $0.52-0.91$ ) decreased likelihood of having an intermediate and ideal cardiovascular health. Further adjustment for the number of cardiovascular factors at the intermediate level yielded even stronger associations, with respectively a $30 \%$ (odds ratio $=0.70: 95 \%$ confidence interval $0.36-0.74$ ) and $48 \%$ (odds ratio $=0.52$ : confidence interval 0.36-0.74) decreased likelihood of having an intermediate and ideal cardiovascular health. All these associations existed and were statistically significant with the behavioural component of the ICVH only. 
Conclusion: High depressive symptoms were associated with substantially lower odds of being in intermediate and ideal cardiovascular health, specifically regarding the behavioural components.

\section{Abstract \#: O 20 \\ Prediction models for the risk of cardiovascular disease in the general population: a systematic review}

Johanna A.A.G. Damen ${ }^{1}$, Lotty Hooft ${ }^{1}$, Ewoud Schuit ${ }^{2}$, Thomas P.A. Debray $^{1}$, Linda M. Peelen ${ }^{2}$, Gary S. Collins ${ }^{3}$, Ioanna Tzoulaki ${ }^{4}$, Camille M. Lassale ${ }^{4}$, George C.M. Siontis ${ }^{5}$, James A. Black ${ }^{6}$, Pauline Heus ${ }^{1}$, Yvonne T. van der Schouw ${ }^{2}$, Karel G.M. Moons ${ }^{1}$

${ }^{1}$ Dutch Cochrane Centre, Julius Center for Health Sciences and Primary Care, University Medical Center Utrecht, Utrecht, The Netherlands; ${ }^{2}$ Julius Center for Health Sciences and Primary Care, University Medical Center Utrecht, Utrecht, The Netherlands; ${ }^{3}$ Centre for Statistics in Medicine, Nuffield Department of Orthopaedics, Rheumatology and Musculoskeletal Sciences, Botnar Research Centre, University of Oxford, Oxford, UK; ${ }^{4}$ Department of Epidemiology and Biostatistics, Imperial College of Medicine, London, England; ${ }^{5}$ Department of Hygiene and Epidemiology, University of Ioannina School of Medicine, Ioannina, Greece; ${ }^{6} \mathrm{MRC}$ Epidemiology Unit, Cambridge University Biomedical Campus, Cambridge, UK; Corresponding author's e-mail: j.a.a.damen@umcutrecht.nl

Aim: To give an overview of all prognostic models that predict risk of developing cardiovascular disease in the general population, and to describe clinical heterogeneity in predicted outcomes, study populations and included predictors.

Data source: In June 2013 a systematic search was performed in Medline and Embase.

Study selection: Studies were eligible if they described the development, validation or incremental value of a prognostic model predicting cardiovascular disease in the general population.

Data extraction: Separate extraction forms were used for scoring development, validation and incremental value papers and identifying corresponding models. For each model, items such as study design, geographical location, prediction horizon, study population, predicted outcome, predictors in the model, and performance were extracted.

Results: A total of 327 studies were included, consisting of 375 developed prognostic models, 528 external validations and 299 incremental value assessments. Only 79 models (21\%) were externally validated by independent researchers. The Framingham and SCORE prediction models were the most often validated models $(\mathrm{n}=306$ and $\mathrm{n}=57)$. Most models excluded participants diseased at baseline $(n=240)$, and predicted risk of cardiovascular disease or coronary heart disease $(n=104$ and $n=112$ respectively) over a 5or 10-year period $(n=47$ and $n=213)$. Furthermore, most models $(42 \%)$ were not stratified for gender, and included age and/or smoking as a predictor $(>85 \%)$. Other common predictors were systolic blood pressure, diabetes and total cholesterol. The c-statistic was reported for $47 \%$ of the external validations (median 0.744 , range 0.53-0.993). Crucial clinical and methodological information was often missing, and if reported, typically prone to substantial heterogeneity. For example, 70 different age ranges were reported, and over 50 main categories of predictors were identified. In addition, discordant outcome definitions and different composite outcomes were used. Only 83 models were internally validated and for 42 internal validations the c-statistic was reported (median 0.770 , range $0.60-0.872$ ). For 48 models, no prediction horizon was reported and for 66 models the intercepts or baseline hazards were not specified making them unable to use for individual risk predictions.
Conclusion: There is an excess of published prognostic models with a large number of overlapping variables predicting cardiovascular disease in the general population. Despite this overabundance, the usefulness of most models remains unclear due to incomplete presentation of models, a lack of external validations, and heterogeneity in predicted outcomes and study populations.

\section{Abstract \#: 021}

WHO guidelines for a healthy diet and mortality from cardiovascular disease in European and American elderly: the chances project

Nicole Jankovic $^{1,2}$

${ }^{1}$ Division of Human Nutrition, Wageningen University, Wageningen, The Netherlands; ${ }^{2}$ Biometry, and Epidemiology, Institute of Medical Informatics, University Duisburg-Essen, Essen, Germany;

Corresponding author's e-mail: nicole.jankovic@uk-essen.de

Background: Cardiovascular disease (CVD) represents a leading cause of mortality worldwide especially in the elderly. Lowering the number of CVD deaths asks for preventive strategies targeted on the elderly.

Objective: Generate evidence on the association between the World Health Organization (WHO) dietary recommendations and mortality from cardiovascular disease, coronary heart disease (CHD) and stroke in elderly aged 60 years and above.

Design: We analyzed data of ten prospective cohort studies from Europe and the United States (US), comprising a total sample of 281,874 men and women free from chronic diseases at baseline. Components of the Healthy Diet Indicator (HDI) included saturated fatty acids, polyunsaturated fatty acids, mono- and disaccharides, protein, cholesterol, dietary fiber and fruits and vegetables. Cohort specific hazard ratios (HR) adjusted for sex, education, smoking, physical activity, energy and alcohol intake were pooled using a random-effects model.

Results: During 3,322,768 person-years of follow-up, 12,492 people died from CVD. An increase by 10 HDI points (complete adherence to an additional WHO guideline) was on average not associated with CVD mortality (HR: 0.94, 95 \% CI: 0.86-1.03), CHD mortality (HR: $0.99,95 \%$ CI: 0.85-1.14) and stroke mortality (HR: 0.95, $95 \%$ CI: $0.88-1.03$ ). However, after stratification of the data by geographical region, adherence to the HDI was associated with reduced CVD mortality in Southern European cohorts (HR 0.87, $95 \%$ CI: 0.79-0.96) and in the US cohort (HR: 0.85, 95 \% CI: 0.83-0.87).

Conclusion: Overall, greater adherence to the WHO dietary guidelines was not significantly associated with CVD mortality, but the results varied across regions, with clear inverse associations in elderly populations in Southern Europe and the US.

\section{Abstract \#: 023}

\section{The challenge of health inequalities}

Prof. Dr Johan P. Mackenbach ${ }^{1}$

${ }^{1}$ Department of Public Health, Erasmus MC, Rotterdam, Netherlands; Corresponding author's e-mail: j.mackenbach@erasmusmc.nl

Reducing inequalities in health between socioeconomic groups within a country is one of the greatest challenges for public health, even in the highly developed welfare states of Europe. Recognizing this, several countries and the World Health Organization have set quantitative targets for reducing these inequalities. Since these targets 
were set, reports have appeared suggesting that inequalities in mortality have widened instead of narrowed. However, a systematic analysis of progress in reducing health inequalities has not been made. We therefore collected data from 11 European countries to assess whether there has been progress, and if so how much, in reducing relative and absolute inequalities in mortality in the period 1990-2010.

Our findings show that remarkable mortality declines have occurred in lower socioeconomic groups in most European countries. Absolute declines in all-cause mortality were often larger in lower than in higher socioeconomic groups (with absolute inequalities narrowing as a result) among men, but not among women. However, relative declines in mortality were almost always smaller in lower than in higher socioeconomic groups (with relative inequalities widening as a result). Narrowing of absolute inequalities was mainly driven by ischemic heart disease, smoking-related causes (men only), and causes amenable to medical intervention.

Although, as these results show, recent trends in inequalities in mortality in Europe are more encouraging than commonly thought, they also raise important questions, such as whether the observed narrowing of inequalities in mortality can be interpreted as a beneficial effect of explicit policies, e.g. of national programs to reduce health inequalities. Unfortunately, the evidence suggests that that is not the case, and that wherever a narrowing has occurred this has been a side-effect of population-wide behavioural changes and improvements in prevention and treatment of conditions like cardiovascular disease.

Another, more fundamental question is whether in public health we should aim for reducing absolute or relative inequalities in mortality. Quantitative targets are usually ambiguous and do not specify whether relative or absolute inequalities should be reduced. Our findings show that since 1990 no country has achieved a reduction of relative inequalities in mortality, but that in terms of absolute inequalities some countries have even surpassed the $25 \%$ reduction target that the World Health Organization proposed. Should we be satisfied with reducing absolute inequalities in mortality, or keep striving for a reduction of relative inequalities as well?

\section{Abstract \#: 024}

\section{Survival after age 75: genetic risk is strongly attenuated} by lifestyle behaviours

Debora Rizzuto $^{1}$, Lina Keller ${ }^{1,2}$, Nicola Orsini ${ }^{3}$, Caroline Graff ${ }^{2,4}$, Lars Bäckman $^{1,5}$, Rino Bellocco ${ }^{6,7}$, Hui-Xin Wang ${ }^{1}$, Laura Fratiglioni ${ }^{1,4,5}$

${ }^{1}$ Aging Research Center (ARC), Department Neurobiology, Health Care Sciences and Society, Karolinska Institutet and Stockholm University, Stockholm, Sweden; ${ }^{2}$ Alzheimer Disease Research Center, Department Neurobiology, Health Care Sciences and Society, Karolinska Institutet, Stockholm, Sweden; ${ }^{3}$ Unit of Nutritional Epidemiology and Unit of Biostatistics, National Institute of Environmental Medicine, Karolinska Institutet, Stockholm, Sweden; ${ }^{4}$ Department of Geriatric Medicine Karolinska, Karolinska University Hospital, Stockholm, Sweden; ${ }^{5}$ Stockholm Gerontology Research Center, Stockholm, Sweden; ${ }^{6}$ Department of Medical Epidemiology and Biostatistics, Karolinska Institutet, Stockholm, Sweden;

${ }^{7}$ Department of Statistics and Quantitative Methods, University of Milano-Bicocca, Milan, Italy; Corresponding author's e-mail: debora.rizzuto@ki.se
Background: Survival to very old age is the result of a complex combination of genetic, environmental, and biomedical factors. This study explores the association between genes that may be related to human mortality and investigates whether lifestyle behaviours may attenuate genetic risk.

Methods: Data came from the Kungsholmen Project, a communitybased longitudinal study of 1229 individuals aged $\geq 75$ followed for up to 25 years. We examined single nucleotide variations in 14 genes (previously associated with mortality) and relevant risk behaviours (smoking, low or high body mass index, physical inactivity). We evaluated the associations of interest by estimating hazard ratios for mortality with Cox regression and median age at death with Laplace regression.

Results: After controlling for age and sex, we found an increased mortality rate of $20-30 \%$ in people with allelic variation in 3 genes related to cardiovascular diseases and metabolism: APOE $\varepsilon 4$ carriers, APOC1 G carriers, and IDE C carriers. Carrying 1, 2, or 3 of these risk alleles shortened life in a dose-response manner from 1 to 2 years. People with no risk behaviours lived 2 years (difference in median age at death $-2.3,95 \% \mathrm{CI}:-4.0$ to -0.6$)$ longer than people with all 3 risk behaviours. Combining the genetic and environmental factors, we found that individuals with $\geq 1$ risk allele but no risk behaviours had a $50 \%$ lower mortality rate $(\mathrm{HR}=0.50,95 \% \mathrm{CI}$ : $0.38-0.65)$ and 3 years $(3.3,95 \%$ CI: $1.3-5.3)$ longer median survival than individuals with all risk behaviours and $\geq 1$ risk allele.

Conclusions: Our study supports and expands evidence that genetic variations in APOE, APOC1, and IDE are associated with longevity. However, their effects can be strongly attenuated by lifestyle behaviours.

\section{Abstract \#: 025}

Breast cancer risk after salpingo-oophorectomy in healthy bcra1/ 2 mutation carriers: revisiting the evidence for risk reduction

Heemskerk-Gerritsen B.A. ${ }^{1}$, Seynaeve $C^{1}$, van Asperen C.J ${ }^{1}$, Ausems M.G $^{1}$, Collée J.M ${ }^{1}$, van Doorn H.C ${ }^{1}$, Gomez Garcia E.B ${ }^{1}$, Kets C.M ${ }^{1}$, van Leeuwen F.E ${ }^{1}$, Meijers-Heijboer H.E ${ }^{1}$, Mourits M.J' ${ }^{1}$, van Os T.A ${ }^{1}$, Vasen H.F ${ }^{1}$, Verhoef $S^{1}$, Rookus M.A ${ }^{1}$, Hooning M.J ${ }^{1}$, Hereditary Breast and Ovarian Cancer Research Group Netherlands

${ }^{1}$ Affiliations of authors: Department of Medical Oncology (BAMHG, CS, MJH), Department of Clinical Genetics (JMC), and Department of Gynecology (HCvD), Erasmus MC Cancer Institute, Rotterdam, the Netherlands; Department of Clinical Genetics, Leiden University MC, Leiden, the Netherlands (CJvA); Department of Clinical Genetics, University MC Utrecht, Utrecht, the Netherlands (MGEMA); Department of Clinical Genetics, Maastricht University MC, Maastricht, the Netherlands (EBGG); Department of Human Genetics, Radboud University Nijmegen MC, Nijmegen, the Netherlands (CMK); Department of Epidemiology (FEvL, MAR), and Department of Clinical Genetics (SV), Netherlands Cancer Institute, Amsterdam, the Netherlands; Department of Human Genetics, VU University MC, Amsterdam, the Netherlands (HEJMH); Department of Clinical Genetics, Academic Medical Center, Amsterdam, the Netherlands (HEJMJ, TAMvO); Department of Gynecology, University MC Groningen, Groningen, the Netherlands (MJEM); Foundation for Detection of Hereditary Tumours, Leiden, the Netherlands (HFAV); Corresponding author's e-mail: m.hooning@erasmusmc.nl

Published in: J Natl Cancer Inst 2015;107(5). 


\section{Abstract \#: 026}

Covariate balance assessment, model selection and bias in propensity score matching: a simulation study

M Sanni Ali $^{1}$, R.H.H. Groenwold ${ }^{1}$, S.V. Belitser ${ }^{2}$, A.W. Hoes ${ }^{1}$, $\overline{{\text { A. de } \text { Boer }^{2}}^{2} \text { O.H. Klungel }}{ }^{2}$

${ }^{1}$ Julius Center for Health Sciences and Primary Care, University Medical Center Utrecht, Utrecht, The Netherlands; ${ }^{2}$ Division of Pharmacoepidemiology and Clinical Pharmacology, Utrecht Institute for Pharmaceutical Sciences, University of Utrecht, Utrecht, The Netherlands; Corresponding author's e-mail: M.S.Ali@umcutrecht.nl

Background: Covariate balance is a crucial aspect of propensity score (PS) analysis. In constructing the PS model, inclusion of interaction/square terms in addition to the main terms and the use of balance measures have been suggested. However, the impact of assessing balance of different sets of covariates and their interaction/ square terms on bias and precision is not well investigated.

Objective: To assess the impact of assessing balance with respect to different covariates on bias of the estimated treatment effect and PS model selection.

Methods: A simulation study was conducted of binary covariates, treatment and outcome data. In different scenarios, confounding variables (variables related to treatment and outcome), risk factors (variables only related to outcome), instrumental variables (variables only related to treatment but not to the outcome or other covariates, IVs), their interaction and square terms were considered. Treatment effects estimates (risk ratios) were derived after PS matching using Poisson models and covariate balance was assessed using the absolute standardized difference. PS model selection was made based on the balance achieved on different sets of covariates, their interaction and square terms. The types of covariates included in balance assessment was compared with respect to bias and precision of the treatment-outcome relation as well as the PS model selected.

Results: PS model selection based on balance assessed on confounding variables and risk factors provided the least biased treatment effect estimates. Inclusion of interaction and square terms in balance calculation improved the precision of the effect estimate without affecting the bias. Although PS model selection based on balance calculated on all sets of covariates as well as confounding and IVs resulted in similar effect estimate in the absence of unmeasured confounding, inclusion of interaction and square terms in balance calculation increased the bias in the effect estimate (up to $13.6 \%$ bias, for caliper 0.2standard deviation on logit of PS) while reducing the precision. When PS model was selected based on the balance achieved on confounding terms, the PS model containing only confounding terms was often selected followed by the PS model containing confounding terms and risk factors.

Conclusions: In PS model selection based on covariate balance, the choice of covariates, interaction and square terms in balance calculation has substantial impact on bias and precision of the treatment effect. Investigators should consider PS model selection based on the balance achieved on confounding variables, risk factors and important interaction terms among confounders and risk factors.

\section{Abstract \#: 027 \\ Comparison of case-only designs with traditional study designs: a systematic review}

$\underline{\text { Koen B. Pouwels }}{ }^{1}$, Bianca Mulder $^{1}$, Eelko Hak $^{1}$
${ }^{1}$ Unit of PharmacoEpidemiology \& PharmacoEconomics, Department of Pharmacy, University of Groningen; Corresponding author's e-mail: k.b.pouwels@gmail.com

Background: It is increasingly common to compare estimates from case-only designs with those obtained with more traditional designs such as the cohort and case-control studies to gain an indication about the potential influence of unmeasured confounders. We aimed to evaluate the concordance between case-only and case-control or cohort studies in empirical studies and to identify predictors of discrepancies between both types of designs

Methods: The MEDLINE and EMBASE databases were searched through 31 June 2013. Epidemiological studies that used both a caseonly and a case-control or cohort design were identified. Of all included studies, general study characteristics, effect estimates and potential predictors of discrepancies between both type of designs were extracted. Spearman correlation coefficient was used to evaluate the concordance between case-only and cohort or case-control studies. Z-scores were used to assess whether difference in the effect estimates were beyond what would be expected by chance alone. Subsequently, a prediction model was build using multivariable logistic regression to assess whether such discrepancies could be predicted.

Results: The search identified 1367 unique articles, of which 57 articles were included for analysis. In total 526 comparisons were made between case-only and cohort or case-control designs. The correlation coefficient between the treatment effect in case-only and cohort or case-control studies was $0.63(p<1 \mathrm{e}-15)$. In 226 of the 526 comparisons ( $43 \%$ ) the difference between both study designs was beyond what would be expected by chance alone. Backward selection based on the AIC resulted in a final model including the following predictors: intermittent exposure, rare event, acute outcome, length of hazard period, type of case-only design and sample size of the traditional study design. This model had a c-statistic of 0.790 (95\% CI $0.752-0.827$ )

Conclusion: The correlation between effect estimates of case-only and cohort or case-control design is moderate, and discrepancies beyond chance are very common. Such discrepancies could be predicted by failure to meet important assumptions of case-only designs.

\section{Abstract \#: 028}

Least squares estimation of incidence rates from prevalence data $\underline{\text { Sandra Landwehr }}{ }^{1}$, Annika Hoyer $^{2}$, Ralph Brinks ${ }^{2}$

${ }^{1}$ Department of Statistics in Medicine, Faculty of Medicine, Heinrich Heine University Duesseldorf, Germany; ${ }^{2}$ Institute for Biometry and Epidemiology, German Diabetes Center at the Heinrich Heine University Duesseldorf, Germany; Corresponding author's e-mail: sandra.landwehr@ddz.uni-duesseldorf.de

Background: Incidence and prevalence are essential concepts in epidemiology, which are usually estimable via different epidemiological study designs. We show how to estimate the incidence of a chronic disease from cross-sectional data. Recently, Brinks \& Landwehr [Theor Popul Biol 92 (2014), 62-68: Math Med Biol (2015), DOI: 10.1093/imammb/dqu024] presented an approach describing the relation between the prevalence, incidence and mortality in the form of a partial differential equation. We now extend this approach by obtaining the incidence from prevalence data given at two points in time via a least squares approach. We illustrate the quality of this novel method in a simulation study on dementia in Germany.

Methods: We set up a simulation study motivated by the situation of dementia in the German male population. Age-specific incidence rates were provided by Ziegler \& Doblhammer [Gesundheitswesen 71 
(2009), 281-290] and information on mortality rates by the Federal Statistical Office of Germany. When obtaining the prevalence of a disease from given incidence and mortalities data, we speak of a forward problem, whereas the derivation of the incidence from given prevalence and mortalities is called the inverse problem. In the simulation study, we mimic two cross-sections with a time lag of 5 years by solving the forward problem. Having observations of prevalence data at two points in time, we formulate an algorithm to recover the incidence by an iterative least squares optimisation. We compare the reconstructed incidence with the known input data. Moreover, the algorithm is able to obtain standard errors of the age-specific incidence estimates. Two settings are studied: one without sampling uncertainty in the two cross-sections and one with sampling uncertainty using the data from Ziegler \& Doblhammer.

Results: Without sampling uncertainty the algorithm is able to recover the incidence rates in the age range 60-100 with less than $1.5 \%$ relative error. In the setting with sampling uncertainty, we can demonstrate how the error propagates from the prevalence estimates to the incidence estimates.

Conclusion: We suggest a novel approach to calculate incidence rates from prevalence data obtained from two cross-sectional studies. This is an advantage, because cross-sectional studies are less costly-and data collection is less time-consuming - than in cohort studies or registers, which are typically used to assess incidence rates. In a simulation study about dementia in German males, the approach is able to recover the incidence very well.

\section{Abstract \#: 029}

Data management and data analysis techniques used in pharmacoepidemiological multi-database studies: a systematic literature review

Marloes T. Bazelier ${ }^{1}$, Irene Eriksson ${ }^{2}$, Frank de Vries ${ }^{1}$, Marjanka K. Schmidt $^{3}$, Jani Raitanen ${ }^{4}$, Jari Haukka ${ }^{5}$, Jakob Starup-Linde ${ }^{6}$, Marie L. De Bruin ${ }^{1}$, Morten Andersen ${ }^{2}$

${ }^{1}$ Division of Pharmacoepidemiology and Clinical Pharmacology, Utrecht Institute for Pharmaceutical Sciences, Utrecht University, Netherlands; ${ }^{2}$ Centre for Pharmacoepidemiology, Karolinska Institutet, Stockholm, Sweden; ${ }^{3}$ Division of Molecular Pathology, Netherlands Cancer Institute, The Netherlands; ${ }^{4}$ School of Health Sciences, University of Tampere, Finland; ${ }^{5}$ University of Helsinki, Helsinki, Finland; ${ }^{6}$ Department of Endocrinology and Internal Medicine, Aarhus University Hospital, Aarhus, Denmark; Corresponding author's e-mail: m.t.bazelier@uu.nl

Background: Over the past decade, an increasing number of studies have been performed using healthcare databases from multiple countries, regions or healthcare organisations. Using data from multiple databases offers a number of potential advantages such as increased sample size and generalizability. There are various ways to combine data from several independent databases, such as combining aggregate results or combining individual patient data. Our aim was to identify pharmacoepidemiological multi-database studies and to describe data management and data analysis techniques.

Methods: Systematic literature searches were conducted in PubMed and Embase complemented by a manual literature search. We included pharmacoepidemiological multi-database studies published from 2007 onwards that combined data for a pre-planned common analysis or quantitative synthesis. The strategies were based on search terms that included 'databases', 'drugs' and 'epidemiology'/'observational studies' (using MeSH and free text terms). Information was retrieved about study characteristics, methods used for individual- level analyses and meta-analyses, data management and motivations for performing the study.

Regarding the meta-analyses, we defined three different levels of combining data: (1) an aggregate level approach, in which separate analyses are performed on datasets from each database and overall results are collected for meta-analysis, (2) a semi-aggregate level approach, in which stratified datasets with event counts are collected for one common analysis and (3) an individual level approach, in which individual patient data are collected for one common analysis. Results: We found 3083 articles by the systematic searches and an additional 176 by the manual search. After full-text screening of 75 articles, 22 were selected for final inclusion. The number of databases used per study ranged from 2 to 17 (median $=4.0$ ). Most studies used a cohort design $(82 \%)$ instead of a case-control design $(18 \%)$. Logistic regression was most often used for individual-level analyses (41\%), followed by Cox regression $(23 \%)$ and Poisson regression (14\%). As meta-analysis method, a majority of the studies combined individual patient data $(73 \%)$. Six studies performed an aggregate meta-analysis $(27 \%)$, while a semi-aggregate approach was applied in three studies $(14 \%)$. Information on central programming or heterogeneity assessment was missing in approximately half of the publications. Most studies were motivated by power (86\%).

Conclusion: Pharmacoepidemiological multi-database studies, a well-powered strategy to address safety issues, have increased in popularity. To be able to correctly interpret the results of these studies, researchers should systematically report on database management and analysis techniques, including central programming and heterogeneity testing.

\section{Abstract \#: O 30}

Complete and accurate reporting of studies of diagnostic accuracy: updating the stard statement

Daniël A. Korevaar ${ }^{1}$, Lotty Hooft ${ }^{2}$, Jérémie F. Cohen ${ }^{1}$, Patrick M.M. Bossuyt ${ }^{1}$

${ }^{1}$ Department of Clinical Epidemiology, Biostatistics and Bioinformatics, Academic Medical Centre, University of Amsterdam; ${ }^{2}$ Dutch Cochrane Centre, Julius Center for Health Sciences and Primary Care, University Medical Centre Utrecht, University Utrecht; Corresponding author's e-mail: d.a.korevaar@amc.uva.nl

Background: The STARD (STAndards for the Reporting of Diagnostic accuracy studies) statement was published in 2003 to help improve the transparency and completeness of reporting of diagnostic accuracy studies. It includes a checklist of 25 essential items that should be reported to facilitate study identification and assessments of risk of bias and applicability. Since its launch, the checklist has been adopted by more than 200 journals. Evaluations have revealed modest but significant improvements in the reporting quality of diagnostic accuracy studies. In 2013, the STARD steering committee decided that it was time to update the checklist.

Methods: Literature searches were performed to identify potential new items. We evaluated new publications on sources of bias and variation, on methods for design, conduct and analysis. We studied evaluations of adherence to STARD and reporting guidelines in other fields of research. The STARD group was extended to 85 researchers, editors, and journalists and invited to participate in two rounds of web-based surveys. In round one, participants were invited to state whether the 25 existing items should be kept, modified or removed. They were also asked whether potential new items identified during the literature search should be added. In round two, items for which there was no majority response $(>75 \%)$ in the first round were presented again. High-priority issues were discussed and a draft checklist 
was established during a live meeting of the STARD steering committee. After piloting and iterative drafting, the steering committee finalized the checklist during a telephone conference.

Results: Seventy-three (86\%) STARD group members completed the first round of the survey, and $79(93 \%)$ participants the second round. The updated checklist consists of 30 items. Compared to the original checklist, 6 items were added, 2 items were combined, and 3 items were split/extended in two $(\mathrm{n}=2)$ or three new items $(n=1)$. The remaining 17 items were reworded, to make them more comprehensible, or to bring them in line with language used in other major reporting guidelines, such as CONSORT.

Conclusion: The quality of reporting of diagnostic accuracy studies has improved significantly since the launch of the STARD statement in 2003. The update of STARD will be presented in 2015, and is expected to further improve complete and accurate reporting.

\section{Abstract \#: O 31}

A systematic approach to variable selection methods used in datamining: a practice in epidemiology

Farideh Bagherzadeh Khiabani ${ }^{1}$, Azra Ramezankhani ${ }^{1}$, Farzad Hadaegh $^{1}$, Fereidoun Azizi ${ }^{2}$, Davood Khalili ${ }^{3}$

${ }^{1}$ Prevention of Metabolic Disorders Research Center, Research Institute for Endocrine Sciences, Shahid Beheshti University of Medical Sciences, Tehran, Iran; ${ }^{2}$ Endocrine Research Center, Research Institute for Endocrine Sciences, Shahid Beheshti University of Medical Sciences, Tehran, Iran; ${ }^{3}$ Department of Epidemiology, School of Public Health, Shahid Beheshti University of Medical Sciences, Tehran, Iran; Corresponding author's e-mail: dkhalili@endocrine.ac.ir

Background: A serious problem in constructing clinical prediction models is identifying the set of appropriate variables as predictors, hence variable selection techniques have turned into an evident necessity in this domain. The aim of this study is to show the application and importance of data mining variable selection methods in an epidemiological study and recommend them as substitutes for traditional methods.

Methods: A $P$ value based and a number of filter (model-independent) and wrapper (model-dependent) data mining variable selection methods have been implemented to select predictors for a logistic prediction model to predict diabetes occurrence in pre-diabetic females, aged $\geq 20$ years, followed for 10-12 years. Filter methods evaluate the merit of variables without involvement of any models. They take advantage of a criterion which calculates correlation of the variables. On the other hand, wrapper methods observe the performance of a model while evaluating variables. The data was split into 2 disjoint sets named train and test. The sets of appropriate variables were determined using the former. To show the eligibility of the models restricted to these variable subsets, they were tested on the test data set.

Results: We found that the model containing all the variables as predictors had the worst performance (worst AUC and AIC) comparing to all the models constructed. We also found that the best model according to both performance criteria (AUC and AIC) were wrappers. As expected, among wrappers, those with AUC subset evaluation resulted in better AUCs and those with AIC subset evaluation resulted in better AICs. In filter methods, Symmetrical Uncertainty -which is a symmetrical measure of correlation of variables defined on the basis of entropy- gave both the best AUC and AIC.
Conclusion: Our experiment showed that data mining variable selection methods could be applied in epidemiological studies to enhance models' performance.

\section{Abstract \#: 032}

Women, shift workers, and public sector workers report lower levels of work time control in a nationally representative Swedish sample

$\underline{\text { Sophie C. Albrecht }}^{1}$, Göran Kecklund ${ }^{1}$, Philip Tucker ${ }^{1}$, Constanze Leineweber ${ }^{1}$

${ }^{1}$ Stress Research Institute, Stockholm University, Stockholm, Sweden; Corresponding author's e-mail: sophie.albrecht@su.se

Background: Although research on flexible work time arrangements is on the rise, basic differences in the levels of work time control (e.g. control over start and ending times of a work day) between labour sectors, other work-related factors or demographic variables have been neglected. The present study aimed to investigate this matter and additionally analysed the factorial structure of an established measure of work time control.

Methods: The study was based on data from the 2014 data collection $(\mathrm{n}=38,657$, response rate $52 \%$ ) of the Swedish Longitudinal Occupational Survey of Health (SLOSH) which is a follow-up of an approximately representative sample of the Swedish working population. Work time control was measured multi-dimensionally using an established 6-item index. An exploratory and confirmatory factor analysis was performed to assess the factorial structure of the work time control measure. Differences in the levels of work time control were investigated for demographic and work-related factors with Mann-Whitney U and Kruskal-Wallis H tests. Because of the large sample size (valid $n=14,974$ ) focus for interpretation of effects was put on effect sizes instead of significances.

Results: Best model fit was found using 5 items of the work time control measure and a two-factor structure (RMSEA $=.06: 95 \% \mathrm{CI}$ $.04-.09:$ CFI $=.99$ ). The two components were "control of working time' and 'control of time off'. Women were found to perceive lower autonomy than men (Cohen's $d=.30 / .47$ ). Self-employed individuals reported higher control than employed ones $(\mathrm{d}=.23 / .21)$, likewise the private sector reported higher control than the public sector $(\mathrm{d}=.36 / .54)$. Effects of contractual working time (e.g. shift work including nights compared to daytime work, $\mathrm{d}=.41 / .30)$ and actual working hours per week (e.g. 30-39 compared to 40-49 h, $\mathrm{d}=.29 / .24)$ on the perceived work time control levels were found. Working overtime at least once a week resulted in higher reported work time control than working less overtime hours $(\mathrm{d}=.43 / .24)$.

Conclusion: The results point towards important confounding variables when researching work time control. Differences in perceived working time autonomy suggest that some groups (e.g. women, shift workers, the public sector, part-timers) seem to be in greater need of interventions to increase perceived work time control than others.

\section{Abstract \#: 033}

Beyond return to work: the effect of multimorbidity on work functioning after sick leave due to common mental disorders

Monica Ubalde-Lopez ${ }^{1}$, Iris Arends ${ }^{2}$, Josue Almansa ${ }^{2}$, George L Delclos ${ }^{3}$, David Gimeno ${ }^{4}$, Ute Bültmann ${ }^{2}$

${ }^{1}$ CISAL-Center for Research in Occupational Health, Universitat Pompeu Fabra, Barcelona, Spain; ${ }^{2}$ Department of Health Sciences, 
Community and Occupational Medicine, University Medical Center Groningen, University of Groningen, Groningen, the Netherlands; ${ }^{3}$ Southwest Center for Occupational and Environmental Health, Division of Epidemiology, Human Genetics, and Environmental Sciences, The University of Texas Health Science Center at Houston, Texas, USA; ${ }^{4}$ Southwest Center for Occupational and Environmental Health, Division of Epidemiology, Human Genetics, and Environmental Sciences, The University of Texas School of Public Health, San Antonio Campus, Texas, USA; Corresponding author's e-mail: monica.ubalde@upf.edu

Background: Common mental disorders (CMD) are a major concern in working populations due to their high prevalence and as a barrier to remaining healthy and productive at work. Several other morbidities are linked to mental disorders: in general, up to $70 \%$ of CMD patients have at least one co-morbidity. The number and type of chronic health conditions limits the ability to carry out specific work demands and thus the ability to function at work. We aimed to assess the impact of multimorbidity, defined as two or more co-occurring chronic health conditions, on work functioning among workers who had returned to work after sick leave due to CMD.

Methods: Prospective cohort study of 157 workers followed during 1 year after return to work from sick leave due to CMD. Chronic health conditions were measured at baseline. Simple logistic regression analyses were conducted to examine the impact of each condition on self-rated health. Multiple regression models were adjusted for health-related behaviors (alcohol and tobacco consumption, physical activity). A multimorbidity score (MMBS) was computed by counting severity-weighted conditions. Work functioning was measured at baseline and 3, 6 and 12 months follow-up with the Work Role Functioning Questionnaire (WRFQ). Work functioning trajectories (WFTs) were identified using latent class growth analysis which we use to investigate if MMBS predicted WFTs, and whether WRF was affected by MMBS within each trajectory.

Results: A total of $55 \%$ of the workers reported at least one chronic health condition: $33 \%$ had multimorbidity. The most prevalent chronic health conditions were musculoskeletal disorders $(24 \%)$, cardiovascular $(13 \%)$ and skin diseases $(10 \%)$. Four WFTs were identified: WFT1 (11\% of the workers) showed increasing work functioning scores during follow-up, whereas the other WFTs showed stable low [WRT2], medium [WRT3] and high [WFT4] scores $(25,41$ and $23 \%$, respectively). WFTs with lower work functioning scores tended to show higher MMBS. However, MMBS did not predict the membership probability to WFTs. Only within WFT1 levels of work functioning were lower while MMBS increased: one unit of MMBS implied a reduction of 1.5 points on work functioning score.

Conclusions: Multimorbidity has a negative impact on workers' perception of work functioning after return to work from sick leave due to common mental disorders. Return to work programs may benefit from incorporating measures to detect and better manage factors related to decreased work functioning in workers with multimorbidity.

\section{Abstract \#: 034}

Microvascular responses in association with recent and chronic exposure to particulate air pollution in school children

Eline B. Provost ${ }^{1,2}$, Nelly D. Saenen ${ }^{1}$, Michal Kicinski ${ }^{1}$, Tijs Louwies $^{1,2}$, Karen Vrijens ${ }^{1}$, Luc Int Panis ${ }^{2,3}$, Patrick De Boever ${ }^{1,2}$, Tim S. Nawrot ${ }^{1,4}$

${ }^{1}$ Centre for Environmental Sciences (CMK), Hasselt University, Diepenbeek, Belgium; ${ }^{2}$ Environmental Risk and Health, Flemish Institute for Technological Research (VITO), Mol, Belgium; ${ }^{3}$ School for Mobility (IMOB), Hasselt University, Diepenbeek, Belgium; ${ }^{4}$ Department of Public Health \& Primary Care, Leuven University (KU Leuven), Leuven, Belgium; Corresponding author's e-mail: eline.provost@uhasselt.be

Background: Particulate air pollution exposure contributes to cardiovascular morbidity and mortality, with changes in the microcirculation as a potential underlying mechanism. So far, little is known about the effect of exposures during childhood on the microcirculation. It was therefore the aim of this study to investigate the effect of both recent and chronic exposure to particulate air pollution on the microcirculation, as exemplified by retinal vessel calibers, in a panel of healthy children.

Methods: 225 children (49.1\% girls: mean age 9.9 years) were recruited at two primary schools in Flanders, Belgium. Participating children were examined three times at the school over the course of the school year. During the examinations, the fundus of the left and right eye of each participant was photographed with a digital nonmydriatic retinal camera. The caliber of the retinal blood vessels was measured using fundus image analysis software and was summarized as the central retinal arteriolar/venular equivalent (CRAE/CRVE). Recent exposure to PM2.5 was measured at the school prior to the examination. Long-term exposure to particulate air pollution was estimated with a dispersion model and by residential proximity to major roads. Mixed-effect models were used to investigate the association between recent and long-term exposure to particulate air pollution and retinal vessel caliber, while adjusting for gender, age, BMI, blood pressure and heart rate, birth weight, time and day of the examination, education of the mother and passive smoking.

Results: Recent exposure to particulate air pollution was significantly associated with retinal vessel calibers. Each doubling in short-term exposure to PM2.5 was associated with a $0.51 \mu \mathrm{m}$ (95\% CI: $0.17-0.86 \mu \mathrm{m}: p=0.0035$ ) narrowing of the retinal arteriolar caliber (i.e. CRAE), while venular caliber (i.e. CRVE) widened $0.55 \mu \mathrm{m}$ (95\% CI: 0.06-1.04 $\mu \mathrm{m}: p=0.029)$. Children living twice as close to a major road, a surrogate for chronic exposure, had $0.84 \mu \mathrm{m}(95 \%$ CI: -0.0044 to $1.68 \mu \mathrm{m}$ ) narrower arterioles, although this association did not reach statistical significance $(p=0.051)$.

Conclusion: We show that vessel calibers of the retinal microcirculation of healthy children aged 8-12 years respond to recent particulate air pollution exposure. Additionally, children living closer to major roads showed smaller calibers of their arterioles. Since changes in the microcirculation have been associated with cardiovascular disease development, these results suggest that the microcirculation is a pathophysiological target for exposure to particulate air pollution from a young age onwards.

\section{Abstract \#: 035}

Prenatal particulate air pollution and foetal thyroid function in euthyroid pregnant mothers

Bram G. Janssen ${ }^{1}$, Nelly D. Saenen ${ }^{1}$, Narjes Madhloum ${ }^{2}$, Karen Vrijens $^{2}$, Wilfried Gyselaers ${ }^{3,4}$, Joris Penders ${ }^{3,5}$, Harry A. Roels ${ }^{2}$, Tim S. Nawrot ${ }^{2,6}$

${ }^{1}$ Authors equally contributed: Centre for Environmental Sciences, Hasselt University, Diepenbeek 3590, Belgium; ${ }^{2}$ Centre for Environmental Sciences, Hasselt University, Diepenbeek 3590, Belgium; ${ }^{3}$ Department of Physiology, Hasselt University, Diepenbeek 3590, Belgium; ${ }^{4}$ Department of Obstetrics, East-Limburg Hospital, Genk 3600, Belgium; ${ }^{5}$ Laboratory of Clinical Biology, East-Limburg Hospital, Genk 3600, Belgium; ${ }^{6}$ Department of Public Health, Occupational and Environmental Medicine, Leuven University (KU 
Leuven), Leuven 3000, Belgium; Corresponding author's e-mail: nelly.saenen@uhasselt.be

Background: Thyroid hormones are critical for foetal development and growth. Prenatal tobacco smoke exposure is associated with altered maternal and foetal thyroid function. Whether prenatal exposure to particulate matter (PM) may induce similar changes in foetal thyroid function remains an open question.

Methods: Using a chemiluminometric immunoassay, we measured free thyroid hormone concentrations (FT3, FT4) and thyroid stimulating hormone levels (TSH) in cord blood of 556 mother-newborn pairs of the ENVIRONAGE birth cohort. Daily PM2.5 exposure levels were calculated for each participant's home address using a Kriging interpolation model (RIO) in combination with a dispersion model (IFMD). Effects of PM2.5 exposure in utero on thyroid function were studied for various time windows during pregnancy using multiple regression models allowing for covariates and potential confounders including gender, gestational age, maternal age, pregestational BMI, birth weight, smoking, parity, ethnicity, Apgar score, and trimester-specific apparent temperature.

Results: An interquartile range (IQR) increment in PM2.5 exposure, averaged over the entire pregnancy period, was inversely associated with TSH levels in cord blood $(-1.26 \mathrm{mU} / \mathrm{L}, p=0.0008)$ and most pronounced in the first $(-1.37 \mathrm{mU} / \mathrm{L}, p=0.01)$ and third trimester $(-1.26 \mathrm{mU} / \mathrm{L}, p=0.02)$ of pregnancy. The FT4/FT3 ratio was also negatively affected by PM2.5 exposure over the entire pregnancy $(-33.9 \%, p=0.002$, for an IQR increment in PM2.5) and attributed to a raise in FT3 and a reduction in FT4 concentrations. Furthermore, TSH levels in cord blood were lower when mothers stopped or continued smoking during pregnancy $(-1.19 \mathrm{mU} / \mathrm{L}, \quad p=0.02$ and $-1.62 \mathrm{mU} / \mathrm{L}, p=0.006$ respectively) compared to never-smoking mothers.

Conclusion: Exposure to prenatal particulate matter is associated with effects on foetal thyroid function as shown by significant changes in thyroid hormone content of cord blood. These alterations in foetal thyroid function are suggestive of an underlying PM-triggered mechanism with potentially compromising neurodevelopmental consequences.

\section{Abstract \#: 036}

Health inequalities in maintaining paid employment: working life expectancy in the Netherlands

Suzan J.W. Robroek ${ }^{1}$, Marieke Jesse ${ }^{2}$, Coos H. Arts ${ }^{3}$, Ferdy W.J. Otten $^{3}$, Leo Bil ${ }^{2}$, Raymond Brood ${ }^{2}$, Alex Burdorf ${ }^{1}$

${ }^{1}$ Department of Public Health, Erasmus MC, Rotterdam, the Netherlands; ${ }^{2}$ Mercer, Amstelveen, the Netherlands; ${ }^{3}$ Statistics Netherlands, Heerlen, the Netherlands; Corresponding author's e-mail: s.robroek@erasmusmc.nl

Background: With an increasing life expectancy and a rapidly ageing population there is a need to increase labour force participation. The concept of working life expectancy (WLE) expresses the future duration that a person is expected to spend in employment, and can provide insight into the long-term consequences of ill health on labour force participation. This study aims to estimate the WLE stratified by sex and educational level, and describes the working time lost through several routes of exit from paid employment and the influence of ill health in the Dutch population.

Methods: A representative sample of the Dutch population $(\mathrm{N}=15,152)$ was selected to study the relation between ill health and labour force exit using Cox proportional hazards analyses. WLE estimates applied on information of the Dutch population between age 25 and $65(\mathrm{~N}=2,107,802)$ of which Statistics Netherlands ascertained employment status monthly from the period January 2001 to December 2010. Yearly age-, gender-, and education-dependent transition probabilities of states of labour force (non-)participation were calculated. WLE was estimated by using Sullivan life tables and subsequently compared with a Sullivan life table using transition probabilities after full elimination of ill health, using population attributable fractions, to calculate the loss in WLE due to ill health. Results: At age 25, lower educated men 29.6 years in paid employment, and lower educated men women 24.3 years. Men and women with a high educational level have respectively 3.3 and 10.0 years higher WLE at age 25. Most of the working time is lost due to leaving the workforce into no income, unemployment, disability benefits and early retirement. The loss in WLE due to self-perceived poor or moderate health ranged between 20 (men, high education) and 154 weeks (women, low education).

Conclusion: Working life expectancy differs substantially between educational groups. Ill health is an important determinant of working time lost, particularly among workers with a low educational level. Programmes to tackle determinants of ill health and programmes to support those with ill health to remain at work are needed, particularly among workers with a low educational level.

\section{Abstract \#: 037}

Neighbourhood characteristics and adiposity in preschool age children: results from the ABCD Study

\author{
E. Jessica Hrudey ${ }^{1}$, Anton E. Kunst ${ }^{1}$, Karien Stronks ${ }^{1}$, \\ Tanja G.M. Vrijkotte ${ }^{1}$ \\ ${ }^{1}$ Academic Medical Centre; Corresponding author's e-mail: \\ e.j.hrudey@amc.uva.nl
}

Background: Overweight in pre-school aged children is a serious issue worldwide because excessive weight gain early in life is associated with increased risks for overweight and chronic diseases later in life. Unhealthy behaviours that contribute to child adiposity are highly influenced by parental factors, but child adiposity also differs across neighbourhoods suggesting environmental factors may also play a role. Most research investigating the influence of environmental factors on child adiposity has been completed in North America where the neighbourhoods are not comparable to those in Western European settings. Therefore this study aimed to analyse the relationships between neighbourhood characteristics (socioeconomic status (SES), perceived safety, satisfaction with green spaces and perceived physical disorder) and indicators of child adiposity in the Amsterdam region.

Methods: Data on child BMI (measured as a standard deviation score), percent body fat (\%BF) and waist-to-height ratio (WHtR) were obtained from the Amsterdam Born Children and their Development cohort at 5-6 years of age. 3469 children were included in the analyses. Children's environments were defined as their four digit postal code area and neighbourhood data were obtained from the Social Cultural Plan Bureau and the WoON survey. Mixed models, with random intercepts by postal code area to account for neighbourhood clustering effects, were used to analyse the relationships of interest.

Results: In the unadjusted models, increasing neighbourhood SES and perceived safety were inversely associated with BMI (SES: $\beta$ : 
-0.06 : $95 \%$ CI: $-0.1,-0.03$ : safety: $\beta$ : $-0.5: 95 \%$ CI: $-0.7,-0.3)$, \%BF (SES: $\beta$ : -0.6 : $95 \%$ CI: $-0.8,-0.4$ : safety: $\beta$ : -4.5 : $95 \% \mathrm{CI}$ : $-5.9,-3.2)$ and WHtR (SES: $\beta$ : $-0.1: 95 \%$ CI: $-0.2,-0.04$ : safety: $\beta:-1.0: 95 \% \mathrm{CI}:-1.7,-0.3)$, and satisfaction with green spaces was positively associated with \%BF $(\beta: 2.2: 95 \% \mathrm{CI}: 0.4,4.2)$ and WHtR ( $\beta$ : 0.9: $95 \%$ CI: $0.1,1.7)$. However, inclusion of family and individual characteristics eliminated all observed relationships. At the familial and individual level, child adiposity was directly associated with maternal and paternal overweight, overweight in pregnancy, smoking during pregnancy and non-Western ethnicity.

Conclusion: Although child adiposity at age 5-6 years was found to increase as neighbourhood SES and perceived safety decreased, these relationships appear to be a product of family and individual characteristics that are unevenly distributed across neighbourhoods, particularly uneven distributions of non-Western ethnic groups and parents who are overweight. Perhaps at this young age, familial and individual factors play a more important role in influencing child adiposity. Ultimately in the Amsterdam setting, differences in child adiposity cannot be attributed to characteristics of the neighbourhoods themselves.

\section{Abstract \#: 038}

Maternal weight gain over the child's life course, and child's (over)weight development and cardio-metabolic health: the piama birth cohort

Lenie van Rossem ${ }^{1}$, Alet Wijga ${ }^{2}$, Ulrike Gehring ${ }^{3}$, Henriette A. Smit ${ }^{1}$

${ }^{1}$ Julius Center for Health Sciences and Primary Care, UMC Utrecht, Utrecht, the Netherlands; ${ }^{2}$ Center for Prevention and Health Services Research, National Institute of Public Health, Bilthoven, the Netherlands; ${ }^{3}$ Institute for Risk Assessment Sciences (IRAS), Utrecht University, the Netherlands; Corresponding author's e-mail: 1.vanrossem@umcutrecht.nl

Background: Gestational weight gain (GWG) of the mother is suggested to be a risk factor for overweight and cardio-metabolic health in the offspring. It is unknown whether GWG programs the offspring's health or whether GWG reflects a shared familial lifestyle during childhood. We studied the combined effect of GWG and postdelivery maternal weight change as an indicator for shared family lifestyle on child's (over)weight development and cardio-metabolic health.

Methods: We used data of 3367 children participating in the PIAMA study, a birth cohort that started in 1996 in the Netherlands. Parental and child weight and height were parent-reported by questionnaires until 14 years after birth. Measurements of cholesterol, and glycated hemoglobin $(\mathrm{HbA} 1 \mathrm{c})$ were obtained at child's ages of 8,12 , and 16 : blood pressure at child's ages of 12 and 16: and carotid intima media thickness (cIMT) was obtained at child's age 16. GWG was categorized into 'inadequate', 'adequate', and 'excessive', according to the Institute of Medicine (IOM) criteria. Multivariable regression and mixed models were used to study associations between maternal and child BMI changes and cardio-metabolic indicators.

Results: Mothers with excessive GWG had children with a higher BMI z-score and were more likely to be overweight (OR: 1.24, $95 \%$ CI: $1.02,1.51)$ throughout childhood. Children of mothers with a high ( $\geq 1 \mathrm{~kg} /$ year) post-delivery weight gain had a 0.29 (95\% CI: 0.10 , $0.47)$ higher change in BMI $\mathrm{z}$-score between age 1 and 14 than mothers with a low $(<0.5 \mathrm{~kg} /$ year) post-delivery weight gain. Mothers with excessive GWG in combination with a high post-delivery weight gain had children with the highest BMI z-score and overweight risk at age 14 (OR: 2.35, $95 \%$ CI: 1.21, 4.56). There were no differences in child's cholesterol, HbA1c, blood pressure, or cIMT between GWG categories and maternal post-delivery weight gain.

Conclusion: Both GWG and post-delivery weight gain contribute to child's (over)weight development. We conclude that excessive GWG puts children on a BMI track that easily exceeds the limits of a healthy BMI. However, maternal post-delivery weight gain is associated with the further development of childhood (over)weight, which probably reflects underlying shared familial factors, including health behaviors.

\section{Abstract \#: 039}

25-Hydroxyvitamin D is not associated with leukocyte telomere length in young adults: findings from the northern Finland birth cohort 1966

Dylan M Williams $^{1,2}$, Saranya Palaniswamy ${ }^{2}$, Sylvain Sebert ${ }^{2}$, Jessica L. Buxton, Alex I. Blakemore ${ }^{1}$, Elina Hypponen ${ }^{3,4}$, Marjo-Riitta Jarvelin ${ }^{1,2,5}$

${ }^{1}$ Imperial College London, UK; ${ }^{2}$ University of Oulu, Finland;

${ }^{3}$ University of South Australia, Australia; ${ }^{4}$ University College

London, UK; ${ }^{5}$ National Institute for Health and Welfare, Finland;

Corresponding author's e-mail: d.williams@imperial.ac.uk

Epidemiological studies have reported associations of higher vitamin D status, lower adiposity and longer telomere length with lower risk of several chronic diseases and all-cause mortality. However, the relationships between vitamin D status, (measured by circulating 25-hydroxyvitamin D: $25(\mathrm{OH}) \mathrm{D})$, adiposity and telomere length are not well established. We conducted a cross-sectional analysis of associations of $25(\mathrm{OH}) \mathrm{D}$ and body mass index (BMI) with mean relative leukocyte telomere length (LTL) using data on 5096 participants of the Northern Finland Birth Cohort 1966 (NFBC1966) at 31 years of age. $25(\mathrm{OH}) \mathrm{D}$ was not associated with LTL in either basic or confounder/mediator-adjusted models. BMI was inversely associated with LTL after adjustment for potential confounding by age, sex, socioeconomic position, physical activity, diet, smoking, alcohol intake, and use of oral contraceptives (mean difference in LTL per kg/ $\mathrm{m}^{2}$ increase in BMI $\left.=-0.4 \%: 95 \% \mathrm{CI}=-0.6,-0.2\right)$. The BMILTL association was also independent of $25(\mathrm{OH}) \mathrm{D}$, and attenuated slightly, but remained, with adjustment for C-reactive protein, a marker of low-grade inflammation $(-0.3 \%$ : $-0.6,-0.1)$. These findings suggest vitamin D status is unlikely to be an important determinant of LTL, at least by young adulthood. Inflammation may in part mediate any effects that adiposity has on LTL.

\section{Abstract \#: 040}

\section{Chronic health conditions in Jewish holocaust survivors born during World War II}

Lital Keinan Boker $^{1,2}$, Hadas Shasha Lavsky ${ }^{3}$, Sofia Eilat-Zanani ${ }^{3,4}$, Adi Edri-Shur ${ }^{3}$, Shaul M. Shasha ${ }^{5}$

${ }^{1}$ School of Public Health, University of Haifa, Haifa, Israel; ${ }^{2}$ Israel Center for Disease Control, Israel Ministry of Health; ${ }^{3}$ Clalit Health Services, Israel; ${ }^{4}$ Bar Ilan University Faculty of Medicine, Israel; ${ }^{5}$ Faculty of Medicine, The Technion, Haifa, Israel; Corresponding author's e-mail: 1keinan@univ.haifa.ac.il 
Background: Findings of studies addressing outcomes of war-related famine in non-Jewish populations in Europe during Second World War (WWII) confirmed a relationship between prenatal/early life exposure to hunger and adult obesity, impaired glucose metabolism, hypertension, cardiovascular heart disease and the Metabolic Syndrome. Fetal programming was suggested as the explanatory mechanism. Data on physical long-term outcomes in child Holocaust survivors born during WWII are scarce.

Methods: A cross-sectional study on all Jewish Clalit Health Services (CHS)x- North District -members born in 1940-1945 in Europe ('exposed', $\mathrm{N}=653$ ) or in Israel to Europe-born parents ("non-exposed', $\mathrm{N}=433$ ). Data on sociodemographic variables, medical diagnoses, medication procurement, laboratory tests and health services utilization were derived from the CHS computerized database and compared between the groups.

Results: The exposed were significantly more likely than the nonexposed to present with dyslipidemia (81 vs. $72 \%$, respectively), hypertension (67 vs. $53 \%$ ), diabetes mellitus (41 vs. $28 \%$ ), vascular disease (18 vs. $9 \%$ ) and the Metabolic Syndrome (17 vs. $9 \%$ ). The exposed also made lower use of health services but used anti-depressive agents more often compared to the non-exposed. In multivariate analyses adjusted for potential confounders, being born during WWII remained an independent risk marker for hypertension $(\mathrm{OR}=1.52)$, diabetes mellitus $(\mathrm{OR}=1.60)$, vascular disease $(\mathrm{OR}=1.99)$ and the Metabolic Syndrome $(\mathrm{OR}=2.14)$.

Conclusions: The results of this cross-sectional study identify a high risk group for chronic morbidity, and call for higher clinical attention of health care takers in this population. These results also raise a question regarding potential transgenerational effects, impacting the 'second generation'.

\section{Abstract \#: 041}

Timing and duration of overweight in association with metabolic health risk among middle-aged and elderly: the Rotterdam study

Klodian Dhana ${ }^{1}$, Albert Hofman ${ }^{1}$, Maryam Kavousi ${ }^{1}$, Oscar H. Franco ${ }^{1}$

${ }^{1}$ Department of Epidemiology, Erasmus MC, Rotterdam, the Netherlands; Corresponding author's e-mail: k.dhana@erasmusmc.nl

Objective: The impact of timing and duration of overweight in association with metabolic syndrome beyond current BMI status has not been investigated among the middle-aged and elderly.

Methods: This study included 3659 individuals (2099 women and 1560 men: mean age 72 years) from the prospective population-based Rotterdam Study. We used data from the participants attending the third examination of the cohort (visit 3, 1997-1999) to measure the prevalence of metabolic syndrome and then we looked backwards to the first and second examination (visit 1, 1989-1993: visit 2, 1993-1995) for the measures of BMI. Separate logistic regression models were used to examine the associations of metabolic syndrome and each of its components separately with (1) obesity timing [middle-aged overweight (onset $<65$ years), and elderly overweight (onset $\geq 65$ years)] and (2) overweight duration over time (incident overweight, fluctuating overweight, and persistent overweight). The subjects who were never overweight formed the reference category for each analyses.
Results: In multivariable analysis (adjusted for age, smoking, physical activity, education, alcohol use and current BMI levels), middleage overweight women were at higher risk for metabolic syndrome [OR 1.56, $95 \% \mathrm{CI}(1.10-2.22)$ ], diabetes mellitus [OR 1.59, $95 \% \mathrm{CI}$ (1.13-2.23)], hypertriglyceridemia [OR $1.4495 \%$ CI (1.02-2.03)] and low HDL cholesterol [OR 1.59, $95 \%$ CI (1.13-2.24)]. Moreover, women with persistent overweight overtime had a higher risk for developing metabolic syndrome [OR $1.7395 \%$ CI (1.21-2.49)], diabetes mellitus [OR 1.70, $95 \%$ CI (1.05-2.80)], hypertriglyceridemia [OR $1.5095 \% \mathrm{CI}(1.05-2.13)]$ and low HDL cholesterol [OR 1.84, $95 \%$ CI (1.30-2.61)]. Among men, elderly overweight group was associated with the risk of diabetes mellitus [OR 1.87, $95 \%$ CI (1.15-3.07)] and low HDL cholesterol [OR $1.9495 \%$ CI (1.31-2.89)], whereas, the fluctuating overweight was associated with diabetes mellitus [OR 2.85, $95 \%$ CI (1.62-5.01)] and abdominal obesity [OR $4.1095 \% \mathrm{CI}(1.55-12.88)$ ].

Conclusion: The timing and duration of overweight has a significant impact beyond the current levels of BMI on obesity-related comorbidities among middle-age and elderly.

\section{Abstract \#: 042}

Genetic predisposition for Alzheimer's disease and cognitive ageing in healthy persons-the Doetinchem Cohort Study

Astrid C.J. Nooyens $^{1}$, Martin P.J. van Boxtel ${ }^{2}$, W.M. Monique Verschuren

${ }^{1}$ RIVM; ${ }^{2}$ Maastricht University; Corresponding author's e-mail: astrid.nooyens@rivm.nl

Background: Several genetic variations have been associated with Alzheimer's disease, but it is unclear whether these genes are also associated with non-pathological cognitive ageing.

Methods: In the present study, genetic variants of APOE, BIN1 rs744373, CLU rs11136000, ABCA7 rs3764650, CR1 rs3818361, and PICALM rs3851179 are studied in relation to 15-year cognitive decline in 2800 men and women of the Doetinchem Cohort Study, aged 41-74 years at baseline. DNA was extracted from buffy coat samples and genotyped using $\mathrm{KASP}^{\mathrm{TM}}$. Cognitive functioning was assessed up to 4 times: at baseline (1995-2007), at 5, at 10 and at 15 years of follow-up using a cognitive test battery including a verbal learning test, Colour Word Stroop test, Letter Digit Substitution Test, and a fluency test. Based on the results of these tests, a global cognitive score and three cognitive domain scores (memory, speed, and flexibility) were calculated. Associations between genetic variants and (change in) cognitive function with age were analysed using random intercept models, adjusting for age, sex, level of education, and other genetic variants in this study.

Results: Decline in global cognitive function with age was larger in persons with APOE e3/e4 or APOE e4/e4 $(p<0.01$ and $p<0.0001$, respectively) than in persons with APOE e3/e3. These differences were also present for the cognitive domains memory, speed and flexibility ( $p$ 's $=<0.01$ ). On the other hand, persons with APOE e2/ e2 declined less in global cognitive function than persons with APOE $\mathrm{e} 3 / \mathrm{e} 3(p=0.02)$. For the other genetic variants, no significant associations were observed with cognitive decline in our healthy population.

Conclusions: In our relatively young and healthy population, having at least one APOE e4 allele was associated with faster (non-pathological) cognitive decline. 


\section{Abstract \#: 044}

\section{Modalities of consent and their consequences}

Evert-Ben van Veen

${ }^{1}$ L1.M. MedLawconsult; Corresponding author's e-mail: eb.vanveen@medlaw.nl

Informed consent is both in the ethical and privacy debate often seen as the golden standard for the use of patient data in health care. This informed consent obsession is challenged by an important minority of scholars in the context of a learning health care system: a health care system which gives feed back about our current practices in order to improve those. The alternative model is based on fair and save use of patient data under an appropriate governance regime.

Consent is not completely discarded in this model but addressed in a nuanced way. Consent can range from layered consent, to broad consent, to various opt-out mechanisms to no consent at all, as is the case in notifiable communicable diseases. These various modalities will be discussed by the following parameters: their consequences for a learning health care system and epidemiological research in general, consequences for patients and citizens at risk in general, medical confidentiality and "privacy". It is submitted that medical confidentiality is not undermined by 'further use' of patient data for a learning health care system if certain safeguards are met, as referred to earlier. Privacy will prove to be an elusive concept in this discussion and anyhow to be balanced against other societal values.

The feed-back from the health care system about our current practices is sometimes challenged on the ground that it might lead to discrimination and other detrimental aspects for patients or citizens. There is a fundamental flaw in this argument. It presumes the 'status quo', our present arrangements, as being sufficiently balanced already. Insofar as necessary, some examples will be given why this view is obviously wrong.

\section{Abstract \#: 045}

\section{A randomized controlled trial comparing consent procedures for the use of residual tissues: implications for patients, research, and clinical practice}

$\underline{\text { S. Rebers }}^{1}$, Alexander P. Brandenburg ${ }^{1}$, Eric Vermeulen ${ }^{1}$, Neil A. $\overline{\text { Aaronson }}^{1}$, Flora E. van Leeuwen ${ }^{1}$, Marjanka K. Schmidt ${ }^{1,2}$

${ }^{1}$ Psychosocial Research and Epidemiology, Antoni van Leeuwenhoek, Amsterdam, The Netherlands; ${ }^{2}$ Molecular Pathology, Antoni van Leeuwenhoek, Amsterdam, The Netherlands; Corresponding author's e-mail: s.rebers@nki.nl

Background: In the Netherlands, there is continuing discussion of the best consent procedure for the use of residual tissue in medical research. However, there is little evidence about the implications of the different consent procedures for patients, research, and clinical practice.

Methods: In a randomized controlled trial, we compared three consent procedures: opt-out (control group), written informed consent, and opt-out plus, an intermediate procedure in which an opt-out procedure is combined with active information provision to patients. In both the informed consent arm and opt-out plus arm patients were informed verbally by their treating physician or nurse and through a brochure around the time of diagnosis or treatment. Patients and medical staff were asked to complete questionnaires about their experiences with and opinions on the different consent procedures. A subsample of the responding patients were also approached for a semi-structured telephone interview.

Results: Of the 445 and 434 participants in the opt-out and opt-out plus arms, respectively 0 and $0.2 \%$ objected to the use of their tissue. Of the 440 participants in the informed consent arm, $0.2 \%$ actively withheld consent, and $39.1 \%$ did not return the consent form. Of the 641 individuals who completed the questionnaire, 31, 69 and $71 \%$ in the opt-out, opt-out plus, and informed consent arms, respectively, indicated that they were (very) well informed. A majority of respondents indicated in the questionnaire a preference for the informed consent procedure. However, of the 146 interviewees, a majority $(58 \%)$ indicated a preference for an opt-out procedure. Further, most interviewees (67\%) believed that patients should be informed about residual tissue use. Medical staff $(\mathrm{N}=35)$ more often indicated being (very) satisfied about the opt-out plus procedure $(90 \%)$ than the informed consent procedure $(48 \%)(p=0.002)$ or the opt-out procedure $(69 \%)(p=0.039)$. The majority $(66 \%)$ preferred opt-out plus.

Conclusion: Implementing written informed consent would imply that approximately $60 \%$ of residual tissue samples would be available for medical research, as compared to 100 or $99.8 \%$ in an opt-out or opt-out plus situation. Patients are most satisfied with informed consent and opt-out plus. Patients' preferences appear to be subject to change depending on the method of inquiry (questionnaire or interview). Physicians and nurses believe that clinical practice is best served by the opt-out plus procedure. Considering all results together, our findings suggest that the opt-out plus variant is the most optimal consent procedure for residual tissue use in Dutch hospitals.

\section{Abstract \#: 046}

\section{Privacy as societal value}

Bart van der Sloot ${ }^{1,2,3}$

${ }^{1}$ Institute for Information Law, University of Amsterdam;

${ }^{2}$ Amsterdam Platform for Privacy Research; ${ }^{3}$ Scientific Council for Governmental Policy; Corresponding author's e-mail:

b.vandersloot@uva.nl

Keywords: consent, individual rights, Big Data, constitutive, group and societal value.

In legal terms, privacy is currently primarily viewed as an individual right protecting individual interests. Legal instruments grant a natural person rights to control his personal data, to have access to them and to remove them under certain conditions. The right to privacy is generally said to protect individual values such as human dignity, individual autonomy and personal freedom. Current technological developments, known as Big Data, challenge these fundaments. First, many individuals are unaware that their data are gathered and processed by others, who have gathered information via, inter alia, secret surveillances activities, cookies, Google glasses or other means. As they are unaware of this fact, they will not claim their legal rights. But even if they would be aware, in the age of Big Data, data gathering and processing is so omnipresent that it will be undoable for individuals to assess every data processing activity and check whether their personal data are processed, whether this is done according to the applicable legal standards and if this is not the case, to file a court case. Second, 
many of the current data processing activities do not specifically affect individuals. How, for example, has the NSA's data collection affected an ordinary American or European citizen? Likewise, the problem of CTTV camera's on the corner of every street (e.g. London) seems not that this or that specific individual is affected. Rather, the issue is that this affects how citizens in general behave and how the state is able to control its citizens and undermine their freedom. Consequently, there is a general shift from individual to group and societal interests. Re-shifting the focus to protecting general interest might also mean that privacy is no longer protected through the means of individual rights, but for example by group rights, class actions or by private of public privacy organizations. This new approach would partly transpose the interests protected to a higher and more abstract level. It will, for example, frame them in terms of the trust of the citizen in the government (non-abusive of its powers), the protection of weaker groups against discrimination and stigmatization and the protection of the open and free public domain. It will also point to the fact that privacy and confidentiality are the preconditions for the well-functioning of certain sectors, such as the legal domain (secrecy between client and lawyer), journalists (secrecy of sources) and the medical sector (confidentiality between patient and doctor). It is known, for example, that without good privacy protection, patients will not go to a doctor or will not disclose certain aspects of their illness out of fear that this might fall into the wrong hands (whether it be the general public, friend and relatives or the health insurer). Hence, focusing on consent-procedures for the (re-)use of, inter alia, DNA, body material and test results of specific patients is misguided. The protection of privacy should not be the right of an individual patient; it should be a duty of the doctor and the healthcare sector in general.

\section{Abstract \#: E 01 \\ Introduction on food frequency questionnaires and the BBMRI- FFQ project}

Marga C. Ocké ${ }^{1}$, Edith J.M. Feskens ${ }^{2}$, Martien C.J.M. van Dongen ${ }^{3}$, H. Bas Bueno-de-Mesquita ${ }^{1}$ * Pieter van't $\mathrm{Veer}^{2} *$, Pieter C. Dagnelie $^{3 *} ; *$ Joint last author

${ }^{1}$ National Institute for Public Health and the Environment, Bilthoven, The Netherlands; ${ }^{2}$ Division of Human Nutrition, Wageningen University, Wageningen, The Netherlands; ${ }^{3}$ Department of Epidemiology, CAPHRI/CARIM, Maastricht University, Maastricht, The Netherlands

Rationale: Dietary intake is an important risk factor for obesity and various chronic diseases such as cardiovascular disease, diabetes mellitus type 2, and various types of cancer. The relationships between dietary factors and chronic diseases are complex, interact with each other and with other risk factors such as genetic variation. More research is needed to further disentangle the complex dietdisease relationships. Large-scale cohort studies are important type of studies that can contribute to this disentanglement.

Food frequency questionnaires: Food frequency questionnaires are often applied feasible tools in the dietary assessment of participants in cohort studies. They provide insight in the consumption of foods over a longer period of time and allow on-line administration and mechanic processing. Combining food frequency questionnaire data with food composition data provides estimates of nutrient intake. In the development of good quality food frequency questionnaires up-to- date information is needed on the contribution of foods to (variation of) intake of total nutrients of interest for the population of interest. Moreover, experience in optimal ways of designing dietary questionnaires, formulating questions and ways to assess usually consumed portion sizes is essential. Since dietary assessment is never without error, it is crucial to obtain insight in measurement error of newly developed food frequency questionnaires studies.

The BBMRI-FFQ project: The aim of the BBMRI-FFQ project was to develop and validate a generic food frequency questionnaire for use in Dutch cohort studies. The availability of a standardized food frequency questionnaire creates possibilities for future comparison or pooling of results of Dutch cohort studies. A second aim of the BBMRI-FFQ project was to compare the new food frequency questionnaire with Dutch questionnaires previously used in Dutch cohort studies. This comparison allows to explore possibilities for analysing food frequency data for cohort members that are obtained on different points in time with different questionnaires (previous and new).

The project was coordinated by the National Institute for Public Health and the Environment. Project partners were the Department of Epidemiology of Maastricht University, and the Division of Human Nutrition of Wageningen University. The three project partners have ample experience in development and validation of dietary assessment methods.

Keywords: FFQ, food frequency questionnaire, cohort study, standardization, validation, The Netherlands.

Acknowledgement: This research was financially supported by BBMRI-NL, a Research Infrastructure financed by the Dutch government (NWO 184.021.007).

\section{Abstract \#: E 02}

Development, and comparison with existing Dutch FFQs, of the FFQ-nl1.0, a new national food frequency questionnaire for the Netherlands

Simone J.P.M. Eussen ${ }^{1}$, Martien C.J.M. van Dongen ${ }^{1}$, Nicole E.G. Wijckmans-Duysens $^{1}$, Saskia Meijboom ${ }^{2}$, Henny A.M. Brants ${ }^{3}$, Jeanne H. de Vries ${ }^{2}$, H. Bas Bueno de Mesquita ${ }^{3,4,5,6}$, Anouk Geelen ${ }^{2}$, Diewertje Sluik ${ }^{2}$, Edith J.M. Feskens ${ }^{2 *}$, Marga C. Ocke ${ }^{3 *}$, Pieter C. Dagnelie $^{1 *}$;

* Joint last author

${ }^{1}$ Department of Epidemiology, CAPHRI School for Public Health and Primary Care/CARIM School for Cardiovascular Diseases, Maastricht University, Maastricht, The Netherlands; ${ }^{2}$ Division of Human Nutrition, Wageningen University, Wageningen, The Netherlands; ${ }^{3}$ National Institute for Public Health and the Environment, Bilthoven, The Netherlands; ${ }^{4}$ Department of Gastroenterology and Hepatology, University Medical Centre, Utrecht, The Netherlands; ${ }^{5}$ Department of Epidemiology and Biostatistics, The School of Public Health, Imperial College London, London, United Kingdom; ${ }^{6}$ Department of Social and Preventive Medicine, Faculty of Medicine, University of Malaya, Kuala Lumpur, Malaysia; Corresponding author's e-mail:

simone.eussen@maastrichtuniversity.nl

Background: Food frequency questionnaires (FFQs) are an important tool to measure dietary intake in epidemiological studies. The aim of the present study was (1) to develop a standardized national Dutch food frequency questionnaire (FFQ-NL1.0), and (2) to compare its 
content validity with existing Dutch FFQs, varying with regard to study purpose, length and period of development.

Methods: We used the Dutch FFQTOOLTM to develop the FFQNL1.0, by selecting food items that, in the Dutch National Food Consumption Survey (DNFC) 2007-2010, covered at least $80 \%$ of intake and $80 \%$ of variance in intake of energy and 43 nutrients by adults aged 25-69 ys (hereafter called "covered [variance in] nutrient intake"). We compared the number of items and the covered nutrient intake, as well as the covered variance in nutrient intake, of the FFQNL1.0 with The Maastricht Study FFQ (Maastricht-FFQ, version 2.0), the FFQ from the Dutch part of the European Prospective Investigation into Cancer and Nutrition (EPICNL-FFQ), and a FFQ developed by Wageningen University (Wageningen-FFQ).

Preliminary results: The FFQ-NL1.0 comprised 158 food items, as compared to 155 items for the EPICNL-FFQ, 183 items for the Wageningen-FFQ, and 254 items for the Maastricht-FFQ2.0. For the FFQ-NL1.0, the covered intake as compared to the DNFC was $>92 \%$ for energy and all nutrients. The covered variance in nutrient intake varied from 59 to $99 \%$ for energy and macronutrients (including alcohol and water) and from 34 to $93 \%$ for micronutrients. For the other FFQs, the covered intake was $>93 \%$ for energy and all nutrients, and the covered variance in intake was $>75 \%$ for energy and macronutrients; for micronutrients, large variations in covered variance in intake were found (16-96\%), with generally higher scores for longer FFQs and for nutrients for which the concerned FFQ had been developed.

Conclusion: The FFQ-NL1.0 is a relatively short FFQ. The covered intake, based on the DNFC, is $>92 \%$ for all FFQs. Preliminary data on covered variance in intake indicate relatively high values for most FFQs for energy and macronutrients, but lower values for micronutrients with marked variation between micronutrients and FFQs.

Keywords: FFQ, food frequency questionnaire, dietary assessment, standardization, content validity, The Netherlands.

Abstract \#: E 03

Validation of the FFQ-NL1.0, a national food frequency questionnaire for the Netherlands

Diewertje Sluik $^{1}$, Anouk Geelen ${ }^{1}$, Jeanne H. de Vries ${ }^{1}$, Simone J.P.M. Eussen $^{2}$, Henny A.M Brants ${ }^{3}$, Saskia Meijboom ${ }^{1}$, Martien C.J.M. van Dongen $^{2}$, H. Bas Bueno-de-Mesquita ${ }^{3,4,5,6}$, Nicole E.G. WijckmansDuysens ${ }^{2}$, Pieter van't Veer ${ }^{1}$, Pieter C. Dagnelie ${ }^{2 *}$, Marga C. Ocké ${ }^{3}$, Edith J.M. Feskens ${ }^{1} *$; Joint last author

${ }^{1}$ Division of Human Nutrition, Wageningen University, Wageningen, The Netherlands; ${ }^{2}$ Department of Epidemiology, CAPHRI School for Public Health and Primary Care/CARIM School for Cardiovascular Diseases, Maastricht University, Maastricht, The Netherlands;

${ }^{3}$ National Institute for Public Health and the Environment, Bilthoven, The Netherlands; ${ }^{4}$ Department of Gastroenterology and Hepatology, University Medical Centre, Utrecht, The Netherlands; ${ }^{5}$ Department of Epidemiology and Biostatistics, The School of Public Health,

Imperial College London, London, United Kingdom; ${ }^{6}$ Department of Social and Preventive Medicine, Faculty of Medicine, University of Malaya, Kuala Lumpur, Malaysia

Background: Food frequency questionnaires (FFQ) are feasible and cost-effective dietary assessment methods and are therefore widely applied in epidemiology. A standardized national 155-item FFQ was developed for use within Dutch cohort studies, the FFQ-NL1.0. The objective of this study was to validate the FFQ-NL1.0 against repeated measurements of urinary nitrogen and potassium, plasma carotenoids and fatty acids, and multiple telephone- and web-based 24-h recalls.
Methods: The Nutrition Questionnaires plus (NQplus) study is an ongoing longitudinal study within the surroundings of Wageningen, the Netherlands. Between 2011 and 2013, 2048 men and women aged 20-70 years were included. The FFQ-NL1.0 was filled out by a subsample of 149 men and 242 women. Per person, 1-2 urinary and blood samples were available, as well as 1-5 telephone-based and 1-7 web-based 24-h recalls. Validity of the FFQ was studied by estimating dietary intake differences at the group level, correlation coefficients, and attenuation factors. Attenuation factors provide information about the extent to which diet-health associations are affected by measurement error; values closer to one indicate less error.

Results: At group level, the FFQ-NL1.0 underestimated protein intake with $11 \%$ as compared with the urinary biomarker. Compared with multiple 24-h recalls, the FFQ overestimated intake of most nutrients, this was $<10 \%$ for energy and macronutrients. Saturated and trans fatty acids were underestimated by the FFQ, with respectively 5 and $9 \%$. Attenuation factors for both protein and potassium were 0.43 as compared to their recovery biomarkers. Compared with the $24-\mathrm{h}$ recalls, attenuation factors ranged from 0.23 for lutein to 0.76 for alcohol. Spearman correlation coefficients were 0.40 between fish intake and plasma EPA and DHA and 0.43 between fruit and vegetable intake and plasma carotenoids. Reproducibility of the FFQ was good with correlation coefficients ranging from 0.51 for lutein to 0.91 for alcohol.

Conclusion: The overall validity of the FFQ-NL1.0 was acceptable to good. Validity measures were in general better for energy and macronutrients than micronutrients. These findings are in line with the validity studies of other FFQs. This study provides attenuation factors which may be used to correct diet-health associations in future studies.

Keywords: FFQ, food frequency questionnaire, dietary assessment, validity, The Netherlands.

\section{Abstract \#: 049}

The associations between overweight, weight change and health related quality of life: longitudinal data from the Stockholm Public Health Cohort

$\underline{\text { Nora Döring }}^{1}$, Jeroen de Munter ${ }^{1}$, Finn Rasmussen ${ }^{1}$

${ }^{1}$ Public Health Sciences, Karolinska Institutet, Stockholm, Sweden; Corresponding author's e-mail: nora.doering@ki.se

Objective: Cross-sectional studies have shown that people with obesity and overweight report lower health related quality of life (HRQoL).With a clear lack of longitudinal studies, this study aims to investigate the association between 8-year weight change and HRQoL measured by the EQ-5D instrument and to investigate whether the association differs with regard to baseline BMI.

Methods: A population-based survey was conducted among a random sample of 31,182 individuals from Stockholm County aged 18-84 years in 2002 and reassessed in 2010 and supplemented by record linkage with regional and national registers. Poisson regression models with a log link and robust variance were used to estimate the probability of having impairment for each of the EQ-5D dimension. The impact of weight gain on the overall EQ-5D index was examined through linear regression with generalized estimation equation and adjusted standard errors. All analyses were adjusted for socio-demographic and health-related variables and baseline BMI category as effect modifier for the association between weight change and HRQoL.

Results: Individuals with overweight and obesity respectively have 0.014 and 0.039 lower EQ-5D indexes compared to those being 
normal weight. Over the 8-year follow-up, $17.6 \%$ gained moderately ( $\geq 5 \%$ body weight) and $13.9 \%$ gained heavily ( $\geq 10 \%$ body weight) in weight. In the fully adjusted analysis, heavy weight gain was associated with a significantly lower overall EQ-5D index and an increased risk of reporting impairment in all but one EQ-5D dimensions irrespective of baseline BMI category. Weight reduction had no significant preventive effect.

Conclusion: Next to obesity status itself, weight gain leads to impairment in all domains of HRQoL irrespective of BMI category at baseline while 8 year weight loss seems not to have the reversed effect on HRQoL, emphasizing the importance of primary prevention of weight gain.

\section{Abstract \#: $0 \mathbf{5 0}$ \\ Differences in effects between a video and text web-based computer-tailored obesity prevention intervention: a randomized controlled trial \\ $\frac{\text { Michel Walthouwer }}{\text { Vries }^{1}}{ }^{1}$ Anke Oenema ${ }^{1}$, Lilian Lechner ${ }^{2}$, Hein de \\ ${ }^{1}$ Department of Health Promotion, Maastricht University; ${ }^{2}$ Department of Psychology, Open University of the Netherlands; Corresponding author's e-mail: michel.walthouwer@maastrichtuniversity.nl}

Background: Web-based computer-tailored interventions have the potential to improve health behaviors in a cost-effective way. Yet, they often suffer from small effect sizes and high drop-out. It has been hypothesized that the use of videos as delivery format may improve their impact, particularly among people with a low educational level. Hence, the main aim of this study was to evaluate the effects of a video and text version of a web-based computer-tailored obesity prevention intervention in comparison to a waiting list control condition on dietary intake, physical activity level, and BMI among Dutch adults. A related study aim was to examine educational differences in efficacy of the two versions of the intervention.

Methods: A three-armed randomized controlled trial was conducted with a baseline and 6 months follow-up measurement. The intervention was developed using the Intervention Mapping protocol and based on the I-Change Model and self-regulation theories. The intervention consisted of six sessions, which each lasted about $15 \mathrm{~min}$. In the video version, the core tailored information was provided by means of videos. In the text version the same tailored information was provided in text format. The effect analyses were carried out with multiple linear regression analyses and adjusted for confounders.

Results: The baseline questionnaire was completed by 1419 participants and the 6 months follow-up measurement by 1015 participants $(71.53 \%)$. No significant interaction effects of educational level were found on any of the outcome variables. Compared to the control condition, the video version resulted in lower BMI $(\mathrm{B}-0.25, P=.049)$ and lower average daily energy intake from energy-dense food products $(\mathrm{B}-175.58$, $P<.001$ ), while the text version only had an effect on the latter outcome (B $-163.05, P=.001$ ). No effects on physical activity were found.

Conclusion: The video version of the web-based computer-tailored obesity prevention intervention was the most effective intervention. The intervention was further equally effective for people with a lower and higher educational level, regardless of delivery format.

\section{Abstract \#: 051}

Vitamin D status in a multiethnic cohort of school-age children: determinants and associations with obesity and cardiometabolic health

Trudy Voortman ${ }^{1}$, Edith H. van den Hooven ${ }^{1}$, Annemieke C. Heijboer $^{2}$, Albert Hofman ${ }^{1}$, Vincent W.V. Jaddoe ${ }^{1,3}$, Oscar H. Franco ${ }^{1}$

${ }^{1}$ Department of Epidemiology, Erasmus MC, University Medical Center, Rotterdam, the Netherlands; ${ }^{2}$ Department of Clinical Chemistry, VU University Medical Center, Amsterdam, the Netherlands; ${ }^{3}$ Department of Pediatrics, Erasmus MC, University Medical Center, Rotterdam, the Netherlands; Corresponding author's e-mail: trudy.voortman@erasmusmc.nl

Background: Vitamin D deficiency has been associated with obesity and poor cardiometabolic health in adults. However, there is limited information on these associations among young children.

Objectives: Our aims were to describe vitamin D status and its determinants in a large multiethnic cohort of 6-year-old children in the Netherlands: and to examine the associations of vitamin D status with cardiometabolic health.

Methods: We measured serum 25-hydroxyvitamin D (25(OH)D) levels in 4167 children at the age of 6 years, and classified vitamin D status into optimal $(25(\mathrm{OH}) \mathrm{D} \geq 75 \mathrm{nmol} / \mathrm{L})$, sufficient $(50$ to $<75 \mathrm{nmol} / \mathrm{L})$, and deficient $(<50 \mathrm{nmol} / \mathrm{L})$. At the same age, children's body fat percentage (using DXA), blood pressure, blood lipids, and insulin levels were assessed, and age- and sex-specific SD scores were calculated. We used multivariable regression analyses to examine the associations of parental and child sociodemographic and lifestyle variables with vitamin D deficiency: and of vitamin D status with cardiometabolic health outcomes.

Determinants of vitamin D status: Serum 25(OH)D levels ranged from 4 to $211 \mathrm{nmol} / \mathrm{L}$ (median $64 \mathrm{nmol} / \mathrm{L}$ ): $29.8 \%$ of the children were vitamin D deficient: and $33.7 \%$ had optimal vitamin D levels. Prevalence of deficiency was higher in winter $(51.3 \%)$ than in summer $(10.3 \%)$ : and was higher in non-Western children $(54.5 \%)$ compared to those with a Western ethnic background (17.6 \%). Other determinants of vitamin D deficiency in multivariable models included a higher child's age, lower diet quality, more television watching, less playing outside, lower household income, and higher maternal BMI.

Vitamin D status and cardiometabolic health: After adjustment for covariates, vitamin D deficient children had 0.12 SD (95 \% CI 0.03, 0.22) higher insulin levels than children with optimal vitamin D levels. There was a significant interaction between vitamin $\mathrm{D}$ and sex on body fat: vitamin $\mathrm{D}$ deficient girls, but not boys, had a $0.17 \mathrm{SD}$ (95\% CI 0.02, 0.31) higher body fat percentage than those with optimal vitamin D levels. Vitamin D status was not associated with blood pressure or blood lipids.

Conclusions: Suboptimal vitamin D status is common among 6-yearold children in the Netherlands, especially among non-Western children and in winter and spring. Important modifiable factors associated with vitamin D status were overall diet quality, sedentary behavior, and playing outside. Higher vitamin D levels were associated with lower insulin levels, and in girls with lower body fat percentage. Future studies are needed to examine whether prevention of vitamin D deficiency in childhood would improve later health outcomes. 


\section{Abstract \#: 052}

Caesarean section delivery and cardiovascular markers in childhood and adolescence

L.P.M. Pluymen $^{1}$, H.A. Smit ${ }^{1}$, A.H. Wijga ${ }^{2}$, U. Gehring ${ }^{3}$, G.H. Koppelman $^{4}$, J.C. De Jongste ${ }^{5}$, L. Van Rossem ${ }^{1}$

${ }^{1}$ Julius Center for Health Sciences and Primary Care, University Medical Center Utrecht, Utrecht, The Netherlands; ${ }^{2}$ Center for Nutrition, Prevention, and Health Services, National Institute for Public Health and the Environment, Bilthoven, The Netherlands; ${ }^{3}$ Division of Environmental Epidemiology, Institute for Risk Assessment Sciences, Utrecht University, Utrecht, The Netherlands; ${ }^{4}$ Department of Pediatric Pulmonology and Pediatric Allergology, UMCG, GRIAC Research Institute, Beatrix Children's Hospital, University of Groningen, Groningen, the Netherlands; ${ }^{5}$ Department of Pediatrics, Erasmus University Medical Center, Sophia Children's Hospital, Rotterdam, The Netherlands; Corresponding author's e-mail: 1.p.m.pluymen@umcutrecht.nl

Background: Children born after caesarean section (C-section) have a different composition of their gut microbiome and are more often overweight compared to vaginally delivered children. C-section may also increase other cardiovascular markers, possibly through bacterial inflammation of blood vessels. We examined whether children born by $\mathrm{C}$-section had a higher risk of being overweight, had a higher systolic and diastolic blood pressure (SBP, DBP) and thicker carotid intima media (CIMT) during childhood and adolescence compared to vaginally delivered children.

Methods: We used the data from a Dutch population based birth cohort study, the Prevention and Incidence of Asthma and Mite Allergy (PIAMA) Study ( $=3963$ at baseline). We excluded children whose mothers suffered from gestational diabetes. The final study population consisted of 2618 children. Method of delivery was ascertained at 3 months after birth by questionnaire. During medical examinations, length and weight to determine overweight (age 4, 8, 12 and 16 years, $\mathrm{n}$ obs $=5126$ ), blood pressure (age 12 and 16 years, $\mathrm{n}$ obs $=2052$ ) and CIMT (age 16 years, $n=414$ ) were measured. We used multilevel regression analysis for the repeated measurements and linear regression analysis for the CIMT measurement. Analyses were adjusted for maternal pre-pregnancy BMI, maternal smoking- and excessive weight gain during pregnancy, birth weight, gestational age and parental educational level.

Results: Children born by C-section $(\mathrm{n}=230,8.8 \%)$ had a $1.39(95 \%$ CI 1.12, 1.73) higher risk of being overweight and a $1.28 \mathrm{mmHg}(95 \% \mathrm{CI}-0.38,2.95)$ higher SBP during childhood and adolescence compared to vaginally delivered children. DBP was not different in $\mathrm{C}$-section versus vaginally delivered children $(-0.18 \mathrm{mmHg}, 95 \% \mathrm{CI}-1.31,0.96)$. Furthermore, girls born by C-section had a $18.9 \mu \mathrm{m}(95 \%$ CI $0.9,36.9)$ thicker CIM at age 16, while C-section delivered boys had no different CIMT compared to vaginally delivered boys $(-10.7 \mu \mathrm{m}, 95 \%$ CI -34.2 , 12.9).

Conclusion: Compared to vaginally delivered children, children born by $\mathrm{C}$-section had a higher risk of being overweight during childhood and adolescence. Also, C-section delivered girls had thicker CIM at age 16 than vaginally delivered girls. We observed no statistically significant difference in blood pressure, nor in CIMT in 16 year old boys, between the two modes of delivery. Our findings are relevant for public health, as the $\mathrm{C}$-section rate is increasing worldwide.

\section{Abstract \#: 053}

Association between malaria and invasive non-typhoid salmonella infection in a hospital-study-accounting for Berkson's bias

Ralf Krumkamp $^{1}$, Benno Kreuels ${ }^{2}$, Nimako Sarpong ${ }^{3}$, Kennedy Gyau $^{3}$, Geoffrey Foli ${ }^{3}$, Anna Jaeger ${ }^{1}$, Florian Marks ${ }^{4}$, Yaw Adu-Sarkodie ${ }^{5}$, Hajo Zeeb ${ }^{6}$, Jürgen May ${ }^{1}$

${ }^{1}$ Bernhard Nocht Institute for Tropical Medicine (BNITM), Hamburg, Germany; ${ }^{2}$ University Medical Centre Hamburg Eppendorf (UKE), Germany; ${ }^{3}$ Kumasi Centre for Collaborative Research in Tropical Medicine (KCCR), Ghana; ${ }^{4}$ International Vaccine Institute (IVI), Seoul, South Korea; ${ }^{5}$ Kwame Nkrumah University of Science and Technology (KNUST), Kumasi, Ghana; ${ }^{6}$ Leibniz Institute for Prevention Research and Epidemiology (BIPS), Bremen, Germany; Corresponding author's e-mail: krumkamp@bnitm.de

Introduction: There is growing evidence on the association between malaria and invasive non-typhoid Salmonella (iNTS) infection. However, case-control studies conducted within health care facilities also report inverse associations. This may be due to a collider-stratification bias, also called Berkson's bias, which acts if both exposure and outcome are associated with hospital attendance and study participants are selected among attendees only. Berkson already highlighted in 1946, that, to control this bias, cases and controls need the same likelihood to visit the hospital, thus controls should suffer from diseases with case-like symptoms. We established two casecontrol studies with different control selection procedures to show the effect of Berkson's bias and to give a less biased estimate of the malaria-iNTS association.

Methods: Data collected from children $(<15$ years) in two Ghanaian hospitals was used to establish the studies. In both studies children with iNTS were cases: in the first study children without bloodstream infections were controls while in the second, children with a bloodstream infection other than iNTS served as controls. Malaria defined the exposure status. Malaria was diagnosed via microscopy and bloodstream infections via automated blood culture. Age-adjusted odds ratios (OR) were calculated using logistic regression.

Results: Data from 6603 children was eligible for the analyses. iNTS infection was diagnosed in 159 children. 6273 children were bloodculture negative and served as conventional controls. Other febrile bloodstream infections (i.e., control-infection) were detected in 304 children. Using the conventional case-control study an OR of 0.4 (95\% confidence interval [CI]: 0.3-0.7) and in the alternative sampling approach, utilizing a control-disease, an OR of 1.8 (95\% CI: 1.1-3.1) was calculated.

Conclusions: The study highlights how a selection bias may reverse results when an unsuitable control group is used. As the alternative control-selection approach is also prone to biases, it limits the ability to quantify the malaria-iNTS association. However the study adds further evidence on the association between both infections using individual data, which is informative since current research is mainly grounded on ecological study designs.

\section{Abstract \#: 054}

\section{Estimating HIV cases in Portuguese MSM}

Paula Meireles $^{1}$, Raquel Lucas ${ }^{1}$, Ana Martins ${ }^{1}$, Ricardo Fuertes ${ }^{2}$, João Brito $^{2}$, Maria José Campos ${ }^{2}$, Luís Mendão ${ }^{2}$, Henrique Barros ${ }^{1}$ 
${ }^{1}$ EpiUnit, Institute of Public Health, University of Porto, Porto, Portugal; ${ }^{2}$ GAT Portugal, Lisboa, Portugal; Corresponding author's e-mail: paula.meireles@ispup.up.pt

Background: Men who have sex with men (MSM) remain at higher risk of HIV infection. In Portugal after a drug injection driven HIV epidemic, sex between men has gained special relevance as a transmission mode and accounted for $30 \%$ off all HIV diagnosis in 2013. With this study we aimed to estimate HIV incidence among MSM using an on-going urban cohort and calculate the expected yearly number of new HIV cases in the Portuguese MSM population.

Methods: We follow an open cohort of HIV-negative MSM who presented for testing at a community-based voluntary HIV counselling and testing center in Lisbon, Portugal (CheckpointLX). Men testing negative were invited to follow-ups scheduled according to their convenience, ideally every 6 months. Evaluations comprised rapid HIV testing and a structured questionnaire. Incidence rates with $95 \%$ confidence intervals ( $95 \% \mathrm{CI}$ ) were estimated with time at risk defined as the period between recruitment and the most recent followup visit. In MSM who seroconverted, half of the period between the last HIV-negative test and the first HIV-positive test was considered as time at risk. Based on the age specific incidence rate observed in the Lisbon Cohort of MSM we computed the expected number of incident HIV cases for the Portuguese MSM population using data from the 2011 Portuguese Census and data from the 2007 National Health and Sexuality Survey to estimate the proportion of MSM.

Results: Between April 2011 and December 2014, 1155 MSM were followed (1510 person-years) and 41 seroconversions were observed yielding an overall incidence of 2.72 (95\% CI: 2.00-3.69) per 100 person-years: age specific incidence rates were 1.63 (95\% CI: $0.81-3.25)$ for the age group 18-24 yrs.: 3.77 (2.52-5.62) for 25-34 yrs.: 2.26 (95 \% CI: 1.01-5.02) for 35-44 yrs.: 3.36 (95\% CI: $1.08-10.43)$ for $45-55$ yrs. No cases were observed for the ages 55-65. The number of incident cases among Portuguese MSM would be 4199 (95\% CI: 3092-5703) per year, much higher than the observed 450 diagnosis reported in 2012 in Portugal.

Conclusion: Our study showed that MSM epidemic remains very high, namely in the European context and although extrapolation from the Lisbon Cohort of MSM data may result in an overestimation probably a substantial number of infections in Portuguese MSM remain undiagnosed.

\section{Abstract \#: 055}

\section{Regional outbreak of serratia marcescens on neonatal wards}

M. Lokate $^{1}$, K.A. Bergman ${ }^{2}$, K. Zhou ${ }^{1}$, S.B. Debast ${ }^{3}$, M.I. van den Bos ${ }^{3}$, A.W. Friedrich ${ }^{1}$, J.W.A. Rossen ${ }^{1}$

${ }^{1}$ Department of Medical Microbiology, Division of Infection Control, University of Groningen, University Medical Center Groningen, The Netherlands; ${ }^{2}$ Division of Neonatology, Department of Pediatrics, Beatrix Children's Hospital, University Medical Center Groningen, University of Groningen, Groningen, the Netherlands; ${ }^{3}$ Laboratory for Medical Microbiology and Infectious Diseases, Isala, Zwolle, The Netherlands; Corresponding author's e-mail: m.lokate@umcg.nl

Background: Serratia marcescens, a gram-negative bacterium, may cause serious infections in neonates. Infections with Serratia have been associated with high mortality and a high rate of infections of the central nervous system particularly in this patient population. Due to the invasive procedures and long stay on a neonatal ward risk for infections are increased. From August until December 2014, an outbreak with Serratia marcescens (not highly resistant) occurred on a neonatal intensive care unit in a university hospital in the north of the
Netherlands. At the same time, spread of Serratia was seen in two other regional hospitals both in neonates and babies.

Methods: Whole-genome sequencing (WGS) was performed on 48 Serratia marcescens isolates from different hospitals to gain insight in the nosocomial transmission. Transmission routes between and within the hospitals were examined through combining WGS data with epidemiological data.

Results: Analysis of the WGS data revealed that 19 isolates of 15 patients clustered together and that transmission of Serratia occurred not only within but also between the university hospital and two top clinical hospitals. Three patients developed a sepsis caused by the outbreak strain. Also other clinical samples (e.g. positive cultures of pus, sputum and urine) were found to be positive for the outbreak clone. Further transmission in these hospitals was prevented by taking to extra interventions and extensive screening procedures.

Conclusion: As there is an increase in patient transfers between hospitals, the risk of regional outbreaks with (multiresistant) microorganisms will increase. Although Serratia may cause infections in all patient populations, it is mainly a problem in neonates. Hospitals not having this patient population may therefore not pay special attention to the non-resistant Serratia infections or colonizations. This might increase the risk of new introduction if premature babies are transferred to neonatal wards if the care isn't sufficient in the regional pediatric wards. It is therefore important to setup a regional network for efficient infection control in hospitals.

\section{Abstract \#: 058}

mHealth in epidemiological research and public health interventions: endless possibilities, yet needs for validation

Petra A Wark ${ }^{1}$

${ }^{1}$ Global eHealth Unit, Department of Primary Care and Public Health, School of Public Health, Imperial College London, London, UK; Corresponding author's e-mail: p.wark@imperial.ac.uk

mHealth (mobile Health) is a fast growing field. By the end of 2015, over a quarter of the world's population will use a smartphone, with use expected to increase to over a third (2.6 billion users) in 2018; then comprising over half of the mobile phone owners worldwide. In May 2015, 1.5 million apps were already available for Android users and 1.4 million apps for Apple users, with health and fitness apps comprising $2.8 \%$ and medical apps $2.0 \%$. Almost constantly, new apps are being released. Wearable devices are traditionally targeted at the fitness industry, comprising mainly wrist, ankle and leg bands that estimate heart rate and caloric expenditure by making use of embedded accelerometers, heart rate and GPS sensors. Smart watches are now becoming more widely available and being used by a larger group of people, having additional functions such as sleep duration and quality monitoring. Patches with embedded sensors, such as for glucose, are also being released and further developed. Sales of personal health and wellbeing products are expected to be over 1.7 times higher in 2018 than in 2013.

mHealth offers many opportunities for epidemiological researchers as well as for use in public health interventions. Data entry can now take place online by the participant themselves or be collected through objective measurements via wearable devices, devices might be used for detecting early signals or stages of disease, and apps and wearables devices can be used in behavioural change interventions by providing regular feedback to the user. The constant stream of data from such devices is likely to provide us further insight in distribution of risk factors, epidemiology and aetiology of disease and its treatment; provided we ask the right questions and identify appropriate analysis methods. 
However, mHealth technology is still in its infancy, and only few devices have been validated so far. Research methodology to evaluate mHealth interventions and applications is also still in its early stages. The first systematic reviews on mHealth technologies and their efficacy are now appearing in the literature, yet data on effectiveness is still scarce. How people will interact with their devices is also not yet clear, and thereby their health economic impact.

During this session, the state-of-the-art, new developments and needs for validation and evidence-based interventions in this fast moving field will be discussed and put in context.

\section{Abstract \#: 059}

\section{Smoking cessation in women following genetic testing for a BRCA1/2 mutation (HOP 2012014)}

Maartje Schellekens ${ }^{1,2}$, Frederieke van der Baan ${ }^{2}$, Matti Rookus ${ }^{2}$

${ }^{1}$ Wageningen University, Wageningen; ${ }^{2}$ Netherlands Cancer Institute, Amsterdam; Corresponding author's e-mail:

maartjeschellekens@hotmail.com

Background: Little information is available about lifestyle habits of BRCA1/2 mutation carriers and non-carriers following genetic testing. We hypothesize that carriers will be more motivated to change unhealthy lifestyle habits, for example to quit smoking, than noncarriers after BRCA1/2 test disclosure. Aim of this study is to investigate smoking cessation in women who prove to be either a BRCA1/2 mutation carrier or non-carrier following a BRCA1/2 genetic mutation test.

Methods: This study was conducted within the framework of the HEBON study (HEreditary Breast and Ovarian cancer study the Netherlands), an ongoing nationwide cohort study among families tested for a BRCA1/2 mutation. Family members are being invited to participate and complete a questionnaire on lifestyle factors. Women were included in the analysis if they proved to carry or not to carry the pathogenic BRCA1/2 mutation present in their family, had no personal history of cancer, and were current smokers at date of genetic testing.

Results: In total, 639 women were smoking at time of genetic testing. Mean age at time of test was 41.5 years (sd 12.0). Of all women, $32.2 \%$ (206 out of 639) stopped smoking after test disclosure. More BRCA1/2 mutation carriers stopped smoking compared with noncarriers (38.5 vs $28.0 \%$ respectively, $p=0.0053$ ). Also, a Cox regression model using age as time-scale and stratifying for birth cohort showed that smoking cessation following BRCA1/2 genetic testing was significantly different for carriers and non-carriers (HR: 1.34, $95 \%$ CI: $1.01-1.79, p=0.0452$ ).

Conclusion: These results suggest that women's smoking behavior following BRCA $1 / 2$ genetic testing is affected by the BRCA $1 / 2$ test result. However, smoking behavior after genetic testing may also depend on events associated with being a carrier, such as opting for preventive surgery or being diagnosed with cancer. During the coming months we will try to disentangle these reasons for smoking cessation.

\section{Abstract \#: 060}

The benefit of mammographic screening for women with a high and low socio-economic status

$\frac{\text { T.M. Ripping }}{\text { Broeders }^{1,2}}$, A.L.M. Verbeek ${ }^{1}$, D. van der Waal ${ }^{1}$, M.J.M.
${ }^{1}$ Department for Health Evidence, Radboudumc, Nijmegen, The Netherlands; ${ }^{2}$ LRCB, Nijmegen, the Netherlands; Corresponding author's e-mail: dorien.hoeve-ripping@radboudumc.nl

Background: In most population-based mammographic screening programs, age is the only risk factor considered to define the target population. Other risk factors may also affect the balance between the benefits and harms of screening. Social economic status (SES) covers a range of risk factors for breast cancer, such as a mother's age at first birth and the number of children, and is also easily calculated at group level. Previous studies already showed that a high SES is related to higher breast cancer incidence and mortality. The absolute number of breast cancers deaths prevented by mammographic screening might, therefore, be higher in women with a high SES than in women with a low SES. The prerequisite is, however, that the relative effect of mammographic screening is similar for women with a high and a low SES. Currently, it is unknown whether the relative effect of mammographic screening differs by SES. Therefore, the aim of this study is to investigate the effect of mammographic screening in women with a high and a low SES.

Methods: In Nijmegen, the Netherlands, women are invited to participate in biennial mammographic screening since 1974. Postal code is collected at each round and is used to calculate the SES of each woman based on the SES indicator of the Netherlands Institute for Social Research (SCP). The Dutch average was used to classify the SES scores of each woman as either high or low. We designed a casecontrol study to investigate the effect of mammographic screening in women aged 35 and over and calculated the odds ratio (OR) and its $95 \%$ confidence interval $(\mathrm{CI})$.

Results: $9.8 \%$ of the women invited to the mammographic screening program in Nijmegen had a high SES. In women with a high SES, the breast cancer death rate was $61 \%$ lower in screened women than in unscreened women $(\mathrm{OR}=0.39$ : $95 \% \mathrm{CI}=0.18-0.87)$. In women with a low SES, the breast cancer death rate was $25 \%$ lower in screened than in unscreened women $(\mathrm{OR}=0.75: \quad 95 \%$ $\mathrm{CI}=0.60-0.93$ ).

Discussion: Mammographic screening reduces the breast cancer mortality in women with high and low SES. The relative effect of screening is, however, more pronounced in women with high SES. As a consequence, the absolute benefits of mammographic screening is likely to be higher in women with a high SES.

\section{Abstract \#: 061}

Genetic variants in $\mathbf{t}$ regulatory cell genes and colorectal cancer prognosis

Elisabeth J. Kap ${ }^{1}$, Petra Seibold ${ }^{1}$, Akke Botma ${ }^{1}$, Dominique Scherer ${ }^{2}$, Nina Habermann $^{2}$, Yesilda Balavarca ${ }^{2}$, Lina Jansen $^{3}$, Natalia Becker ${ }^{4}$, Hendrik Bläker ${ }^{5}$, Wilfried Roth ${ }^{6}$, Matthias Kloor ${ }^{7}$, Alexis Ulrich ${ }^{8}$, Axel Benner ${ }^{4}$, Cornelia M. Ulrich ${ }^{2}$, Barbara Burwinkel ${ }^{9}$, Michael Hoffmeister $^{3}$, Hermann Brenner ${ }^{3}$, Jenny Chang-Claude ${ }^{1}$

${ }^{1}$ Division of Cancer Epidemiology, German Cancer Research Center (DKFZ), Heidelberg, Germany; ${ }^{2}$ Division of Preventive Oncology, National Center for Tumor Diseases (NCT) and DKFZ, Heidelberg, Germany; ${ }^{3}$ Division of Clinical Epidemiology and Aging Research, DKFZ, Heidelberg, Germany; ${ }^{4}$ Division of Biostatistics, DKFZ, Heidelberg, Germany; ${ }^{5}$ Institute of Pathology, Charite University Medicine, Berlin, Germany; ${ }^{6}$ Department of Pathology, Institute of Pathology, Heidelberg University Hospital, Germany; ${ }^{7}$ Department of Applied Tumor Biology, Institute of Pathology, Heidelberg

University Hospital, Germany; ${ }^{8}$ Department of General, Visceral and Transplantation Surgery, Heidelberg University Hospital, Germany; ${ }^{9}$ Division Molecular Biology of Breast Cancer, Department of 
Gynecology and Obstetrics, Heidelberg University hospital, Germany; Corresponding author's e-mail: 1.kap@dkfz.de

Background: T regulatory cells (Tregs) have an important function in the immune system regulating self-tolerance and immune homeostasis. Through for example cytokine production and cell/cell contact, these cells can suppress the activation, proliferation and function of immune cells. The tumor microenvironment consists of multiple types of immune cells which might influence prognosis. The association of Tregs with colorectal cancer survival seems to differ by microsatellite instability (MSI) status. Furthermore, it has been shown that oxaliplatin treatment increases the immunogenicity of cancer cells and is able to induce an immunogenic cell death. Therefore, we investigated genetic variants in 58 genes involved in the $\mathrm{T}$ regulatory pathway for their association with colorectal cancer survival and possible effect modification by MSI status and treatment with oxaliplatin chemotherapy.

Methods: We included 1749 colorectal cancer patients diagnosed between 1st Jan. 2003 and 31st Dec. 2007 recruited for the DACHS study, a German population-based case-control study, and with longterm follow-up of patients. Genotyping of the 784 SNPs was performed on a customized Illumina GoldenGate assay and the wholegenome Illumina CytoSNP v12.2.1 assay. Single SNP associations with overall survival (OS) were assessed using Cox regression models adjusted for age, sex, TNM stage, BMI, tumor grade and current alcohol intake. MSI was analyzed using a mononucleotide marker panel. Heterogeneity by MSI status and oxaliplatin treatment was determined by using a multiplicative interaction term and evaluated by the log-likelihood ratio test. We adjusted for multiple testing using the FDR by Benjamini \& Hochberg.

Results: Median follow-up time was 5.1 years during which 556 patients $(32 \%)$ deceased. None of the single SNP associations with OS were significant after adjustment for multiple testing. The strongest association was observed for rs3181096 (CD28) with a hazard ratio (HR) of $0.79(0.70-0.90)$ and unadjusted $p$ value 0.0007 . Six further variants associated with OS (unadjusted $p$ value $<0.01$ ) were found, located in TGFBR2, IL2RA and SELP. Additionally, we found six SNPs, which showed a non-significant differential association with OS by MSI status (strongest unadjusted $p$ value 0.0007 ) and nine SNPs which showed marginal heterogeneity by oxaliplatin treatment (strongest unadjusted $p$ value 0.0003 ). As a next step gene level analyses are planned to identify possibly interesting loci.

Conclusion: Our results suggest that genetic variants in the $\mathrm{T}$ regulatory pathway may be prognostic markers in colorectal cancer patient subgroups according to MSI status and oxaliplatin treatment. Confirmation of these findings in larger studies is necessary and will be pursued.

\section{Abstract \#: 062}

Treatment-specific risks of second malignancy and cardiovascular disease in 5-year Hodgkin lymphoma survivors

Simone de Vries ${ }^{1}$, Frederika A. van Nimwegen ${ }^{1}$, Michael Schaapveld $^{1}$, Pieternella J. Lugtenburg ${ }^{2}$, Augustinus D.G. Krol ${ }^{3}$, Judith M. Roesink ${ }^{4}$, John M.M. Raemaekers ${ }^{5}$, Berthe M.P. Aleman ${ }^{6}$, Flora E. van Leeuwen ${ }^{1}$

${ }^{1}$ Department of Epidemiology, The Netherlands Cancer Institute, Amsterdam, The Netherlands; ${ }^{2}$ Department of Hematology, Erasmus University Medical Center, Rotterdam, The Netherlands; ${ }^{3}$ Department of Clinical Oncology, Leiden University Medical Center, Leiden, The Netherlands; ${ }^{4}$ Department of Radiotherapy, University Medical Center Utrecht, Utrecht, The Netherlands; ${ }^{5}$ Department of Hematology, Radboud University Medical Center,
Nijmegen, The Netherlands; ${ }^{6}$ Department of Radiation Oncology, The Netherlands Cancer Institute, Amsterdam, The Netherlands; Corresponding author's e-mail: si.d.vries@nki.nl

Background: Survival following Hodgkin lymphoma (HL) has improved significantly over time due to development of effective radiotherapy (RT) and chemotherapy (CT) regimens. HL survivors are, however, at increased risk to develop long-term treatment-related complications, including second malignant neoplasms (SMNs) and cardiovascular disease (CVD). Both RT and CT contribute to these increased risks. Most research to date has focused on separate risk estimates for these late effects. Therefore, our objective was to assess the combined risk of developing SMN and/or CVD.

Methods: Our multicenter cohort comprised 2480 5-year HL survivors, diagnosed before age 51 and treated between 1965 and 1995 . CVD endpoints (ischemic heart disease [IHD], cardiomyopathy and congestive heart failure [HF] and valvular heart disease [VHD]) were assessed through contacting general practitioners. Data on SMN were derived from linkage with the Netherlands Cancer Registry. Followup was complete until at least October 2013. Standardized incidence ratios (SIRs) were estimated to compare SMN and CVD risk with the general population. Cumulative incidences of SMN and/or CVD were calculated with death as a result of other causes as competing risk, and treatment-specific risks of developing any of these major late complications were quantified using multivariable Cox regression analysis.

Results: After a median follow-up of 22 years, we identified 874 SMNs in $742 \mathrm{HL}$ survivors and 1368 CVDs in $780 \mathrm{HL}$ survivors. 1246 patients developed at least 1 major event, of whom 276 developed both SMN and CVD. HL survivors had a 5.2-fold increased SIR of any SMN (95 \% CI 4.8-5.6), compared to the general population, corresponding to 141 excess cases/10,000 person-years. The SIR of primary IHD or HF was 2.1 (95\% CI 1.1-3.7), resulting in 126 excess cases/10,000 person-years. After 40 years of follow-up, at a median attained age of 60 years, the cumulative incidence of SMN or CVD as a first event was $73.3 \%$ (95\% CI: 70.6-75.7), whereas the cumulative incidence for developing both events was $22.5 \%$ (95\% CI: 19.8-25.3). Both supradiaphragmatic RT (Hazard Ratio [HR]: 2.6, $95 \%$ CI: 2.1-3.1) and anthracycline-containing CT (HR: 1.5, $95 \%$ CI: $1.3-1.7)$ independently increased the risk of SMN and/or CVD. Supradiaphragmatic RT was associated with a strongly increased (HR: 4.4, $95 \%$ CI: 2.6-7.7) risk of developing both SMN and CVD.

Conclusion: HL survivors treated between 1965 and 1995 experience a high disease burden during follow-up, with the cumulative incidence of a SMN and/or a CVD rising to $73 \%$ after 40 years from initial treatment. Supradiaphragmatic RT most strongly increased this risk.

\section{Abstract \#: 063}

The influence of a healthy lifestyle behavior on the prognosis of occasional neck/shoulder/arm pain in a general population

Eva Skillgate $^{1}$, Tony Bohman ${ }^{1}$, Lena W. Holm ${ }^{1}$

${ }^{1}$ Karolinska Institutet; Corresponding author's e-mail:

eva.skillgate@ki.se

Background: A healthy lifestyle behavior (HLB) defined as nonsmoking, physical activity, healthy diet and moderate alcohol use, was a protective prognostic factor, by decreasing the risk of developing long duration troublesome low back pain among women, but not among men with occasional low back pain in a recently published population based cohort study by our research group. The aim of this 
study was to study the influence of HLB on the prognosis of occasional neck/shoulder/arm pain (NSAP) in a general population.

Methods: Stockholm Public Health Cohort is a population-based cohort within the framework of the Stockholm County Council public health surveys. In 2006 a randomly selected and stratified population $(\mathrm{n}=56,634), 18-84$ years of age, received a questionnaire $(61 \%$ responded). The responders received a new questionnaire in 2010 (73\% responded). For this study we included those who at baseline reported occasional NSAP during the past 6 months, defined as having had NSAP, on average, up to a few days per month $(\mathrm{n}=10,133)$. The exposure HLB was categorized as follow: nonHLB had none or one out of the HLB factors: non-smoking, moderate alcohol consumption, recommended consumption of fruit and vegetables and recommended level of physical activity (reference category). HLB2, HLB3 and HLB4 had two, three and four of the HLB factors, respectively. The outcome was defined as at follow-up reporting NSAP at least 2 days a week during the past 6 months that had decreased workability or interfered with other daily activities to some or to a high degree. Binomial regression was used and nine potential confounders were tested. Factors changing the crude rate ratio $\geq 5 \%$ were included the final regression models.

Results: The study population was 10,133 persons of whom information on exposure and outcome was available in 8600 (5053 women and 3547 men). Factors confounding the risk ratio (RR) were sex and socioeconomic status. HLB was protective prognostic factor in all categories compared to a non-HLB: Adjusted RR: (HLB2) 0.74 (95\% CI: 0.64-0.86), (HLB3) 0.63 (95\% CI: 0.53-0.75), (HLB4) 0.61 (96 \% CI: 0.45-0.81). The results were similar when men and women were analyzed separately.

Conclusion: Healthy lifestyle behavior seems to decrease the risk of developing long duration troublesome neck/shoulder/arm pain among men and women with occasional neck/shoulder/arm pain and may be recommended to improve the prognosis. Non-differential misclassification of the exposure and the outcome is a main potential source of bias.

Keywords: Neck pain, life style factors.

\section{Abstract \#: 064}

Patient variables, comorbidities and walking distances in symptomatic peripheral arterial disease: findings from a 1-year prospective physiotherapy cohort study

Sarah Dörenkamp, MSc, PhD Candidate ${ }^{1}$, Ilse Mesters, PhD, Associate Professor ${ }^{1}$, Rob de Bie, PhD, Professor ${ }^{1}$, Joep Teijink, PhD, Professor $^{1,2}$, Gerard van Breukelen, PhD, Professor ${ }^{3}$

${ }^{1}$ Department of Epidemiology and CAPHRI School for Public Health and Primary Care, Faculty of Health, Medicine and Life Sciences, Maastricht University, Maastricht, The Netherlands; ${ }^{2}$ Department of Vascular Surgery, Catharina Hospital, Eindhoven, the Netherlands; ${ }^{3}$ Department of Methodology and Statistics, CAPHRI School for Public Health and Primary Care, Faculty of Health, Medicine and Life Sciences and Faculty of Psychology and Neuroscience, Maastricht University, Maastricht, The Netherlands; Corresponding author's e-mail: sarah.dorenkamp@maastrichtuniversity.nl

Background: Exercise therapy is an appropriate intervention for the management of Intermittent Claudication (IC). Research has shown that the improvement of patients may be influenced by patient-related variables and comorbidity. This study investigated the effects of age, gender, BMI, cigarette smoking, orthopaedic, neurologic, cardiac, pulmonary and internal comorbidity on (improvement in) maximum pain-free walking distance (MPWD) in patients with IC receiving supervised exercise therapy.

Methods: Data was derived from community-based electronic medical records of IC patients $(\mathrm{N}=2295)$. Patients received treatment according to the evidence-based guideline IC of the Royal Dutch Society for Physical Therapy in a community based setting as part of the usual care program. Three-level mixed linear regression analysis was carried out to analyze whether patient-related variables and comorbid conditions were associated with walking capacity after 1,3 , 6 and 12 months.

Results: Being female, increased age and increased BMI were associated with lower MPWD at all-time points $(p<0.001)$. Cardiac comorbidity seemed to be associated with MPWD at a $5 \%$ significance level $(p=0.011)$ at all-time points. MPWD increased steadily over time. And compared to baseline its average was almost $600 \mathrm{~m}$ higher after 1 year. Significant interactions were found for time with age $(p=0.000)$, time with vascular comorbidity $(p=0.023)$ and time with BMI $(p=0.023)$. For vascular comorbidity the reduction in improvement was $85 \mathrm{~m}$ after 12 months. For age the reduction was $8 \mathrm{~m}$ after 12 months, per year increase of age (range 33-93 years). Per unit increase of BMI the decrease in MPWD was $10 \mathrm{~m}$ after 12 month (range $16-44 \mathrm{~kg} / \mathrm{m}^{2}$ ).

Conclusion: Identifying influential co-factors on treatment outcome may allow researchers to recommend treatment adaptations, thereby facilitating optimal care for each patient.

\section{Abstract \#: 065}

\section{Hip fracture trends in Sweden-a study of incidence, lifetime} risk, and survival

Korinna Karampampa $^{1}$, Anders Ahlbom ${ }^{1,2}$, Karl Michaëlsson ${ }^{3}$, Tomas Andersson ${ }^{1,2}$, Sven Drefahl ${ }^{4}$, Karin Modig ${ }^{1}$

${ }^{1}$ Institute of Environmental Medicine, Karolinska Institutet, Stockholm, Sweden; ${ }^{2}$ Centre for Occupational and Environmental Medicine, Stockholm County Council, Stockholm, Sweden; ${ }^{3}$ Uppsala Clinical Research center (UCR) and Department of Surgical Sciences, Uppsala University, Uppsala, Sweden; ${ }^{4}$ Department of Sociology, Demography Unit, Stockholm University, Stockholm, Sweden; Corresponding author's e-mail: korinna.karampampa@ki.se

Background: Hip fracture is a common cause of disability and mortality among the elderly resulting in high healthcare and social costs, as well as impaired quality of life for individuals. Hip fracture incidence has been stable or even declined over the years in Sweden. Still, this condition remains a significant public health problem since Sweden has one of the highest incidences worldwide. Despite its high burden, no lifetime risk or survival trends are denoted in the literature for Sweden. Therefore, the aim of this study was to examine the age-specific incidence and survival trends of hip fractures in Sweden, and relate these to each other and to the change in the lifetime risk.

Methods: The entire Swedish population 60 years-old or above was followed between 1987 and 2010 in the National Patient Register and the Cause of Death Register. The period 1987 through 1994 was used as a wash out period. Annual age-specific hip fracture cumulative incidence was estimated using hospital admissions for hip fractures. Three-month and 1-year survival after the first hip fracture were also estimated. Period life table methodology was used to assess lifetime 
risk of hip fractures occurring from age 60 and above, and the expected mean age of the first hip fracture.

Results: The age-specific hip fracture incidence decreased between 1995 and 2010 in all ages up to 94 years, by on average $1 \%$ per year. The lifetime risk remained almost stable, between 9 and $11 \%$ for men, and between 18 and $20 \%$ for women. The expected mean age of a first hip fracture increased by 2.5 years for men and by 2.2 years for women. No improvements over time were observed for the 3-month survival for men, while for women a $1 \%$ decrease per year was observed. The 1-year survival slightly increased over time for men ( $0.4 \%$ per year) while no improvement was observed for women.

Conclusions: The age-specific hip fracture incidence has decreased over time. Yet the lifetime risk of a hip fracture remained unchanged, or slightly increased, due to the lack of improvement in hip fracture risk among the oldest and the increasing number of old individuals in the population. Overall, no clear improvements in the survival after first hip fracture were observed, especially for women, which may indicate that frailty among these individuals is high.

\section{Abstract \#: 066}

Intermittent claudication affects gait parameters during treadmill walking

A.T. Smid $^{1}$, L.N.M. Gommans ${ }^{1}$, K. Meijer ${ }^{2}$, N. Verhofstad ${ }^{1}$, J.A.W. Teijink $^{1}$

${ }^{1}$ Catharina Hospital Eindhoven, department of vascular sugery;

${ }^{2}$ Maastricht University Medical Centre, department of human movement sciences; Corresponding author's e-mail:

annemieke.smid@gmail.com

Background: Intermittent claudication (IC) is the most common manifestation of peripheral arterial disease (PAD) and presents as exercise induced muscle discomfort and pain of the lower $\operatorname{limb}(\mathrm{s})$, that resolves after a period of rest. Patients with IC demonstrate reduced walking performance, have less isokinetic strength of distal muscles and show lower physical activity levels compared to healthy controls. Supervised exercise therapy (SET) is the first-choice treatment for IC and improves both walking performance and physical function. So far, proper gait analysis during treadmill walking in patients with IC has not been carried out. Therefore, the aim of this study was to investigate gait parameters of patients with IC during treadmill walking.

Methods: Patients with IC and healthy, age-matched, controls walked on a treadmill at a self-selected comfortable pace, while spatio-temporal gait parameters (e.g. step length, swing time and foot settlement) were obtained using the Optogait photoelectric system. This validated system for the assessment of spatio-temporal gait parameters was placed next to the treadmill. For patients, both painfree and painful trials were captured, whereas controls only completed a 'pain-free' session. Parameters were compared between patients and controls as well as within the patient group.

Results: 11 patients and 15 control subjects were examined. Baseline characteristics were similar in both groups. IC patients walked at a slower self-selected pace (IC: $3.5 \mathrm{~km} / \mathrm{h}$ versus controls: $4.3 \mathrm{~km} / \mathrm{h}$, $p=0.031$ ). Besides, step and stride length among patients were significantly shorter in both trials. Step length differences of $9 \mathrm{~cm}$ and $8 \mathrm{~cm}$ were found for respectively, pain-free and painful walking between patients and controls. Within the patient group, total contact time stayed constant $(p=0.832)$ during pain-free and painful walking, whereas evident changes were found in foot settlement. A $1.2 \%$ shorter contact phase $(p=0.006)$ and a $3 \%$ increased foot flat phase $(p=0.022)$ was noted during painful walking. Besides, patients spent $4.4 \%$ more time in double support phase during painful walking, compared to pain-free walking $(p=0.041)$.

Conclusion: Patients with IC appear to have a different walking pattern even before the onset of claudication. They walk slower with shorter steps compared to healthy controls. Besides, foot settlement is disturbed during painful walking as patients with IC tend to drop their foot faster, accompanied with a reduced unwinding of the foot, resulting in an increased overall stance time. Based on these findings, we would suggest physiotherapists to pay attention on feet settlement during SET.

\section{Abstract \#: 069}

The role of functional disability assessment in the diagnosis of common mental disorders: a comparison of three different instruments

Elena Olariu $^{1,2,3}$, Carlos G. Forero ${ }^{2,3}$, Pilar Álvarez ${ }^{4}$, José-Ignacio Castro-Rodríguez $^{1,5}$, Jordi Alonso ${ }^{1,2,3}$

${ }^{1}$ Universitat Pompeu Fabra (UPF), Department of Experimental and Health Sciences; ${ }^{2}$ IMIM-Institut Hospital del Mar d́Investigacions Mèdiques, Health Services Research Unit; ${ }^{3}$ CIBER Epidemiología y Salud Pública (CIBERESP); ${ }^{4}$ Institut de Neuropsiquiatria i Addicions, Hospital Del Mar, Barcelona; ${ }^{5}$ Psychiatry Department, Centro Forum, INAD, Parc de Salut Mar; Corresponding author's e-mail:

eolariu@imim.es

Background: Functional disability is a diagnostic requirement for common mental disorders (e.g. major depressive episode (MDE): generalized anxiety disorder (GAD)). Additionally, changes in functional outcomes can be used to track clinical progress, helping to identify potentially beneficial therapies and facilitating decisionmaking processes. Regarding psychopathology, functional disability assessment is not a straightforward procedure, especially for nonspecialized health care professionals. The objective of this study was to determine the diagnostic value of functional disability assessment in the detection of MDE and GAD. We also studied the responsiveness of three different measures of functional disability.

Methods: Prospective cohort study (baseline, 1-, 3-months assessments) of patients seeking help for incident or aggravated mood or anxiety symptoms from primary, outpatient and inpatient mental health centers. Patients were assessed with the MINI interview for the presence of common mental disorders. Functional disability was assessed with the GAF (Global Assessment of Functioning), a visual analogue scale of functioning (VASF) and WHODAS 2.0 (World Health Organization Disability Assessment Schedule). Hierarchical logistic models were used to determine the diagnostic added value of different methods of assessing functional disability in the detection of MDE and GAD. The diagnostic predictive value of the three measures of functional disability was compared to that of a baseline model that included the number of MDE/GAD symptoms adjusted for age, sex, number of physical disorders. Responsiveness was analyzed based on the evolution of HRSD and HAM-A scores respectively, using Coheńs d as an effect size indicator.

Results: 233 patients were assessed (30.9 \% men: mean age $=49.1$, sd $=14.8) .67 .81 \%$ were active cases, all under psychiatric treatment. In case of GAD, the GAF and VASF improved the diagnostic performance of the number of symptoms ( $p$ value $=0.035, p$ value $<0.0001$ respectively). The VASF model had the highest diagnostic 
accuracy (AUC VASF $=0.905$ vs AUC Baseline Model $=0.858$ ). In case of MDE, the diagnostic prediction of all the measures of functional disability was similar to that of the number of symptoms, with comparable AUCs $\quad(\mathrm{AUCGAF}=0.976: \quad$ AUC $\mathrm{VASF}=0.976$ : AUCWHODAS $=0.971$ vs AUC Baseline Model $=0.976$ ). The GAF was the instrument that, at 3-months, captured the highest change for both GAD and $\mathrm{MDE}(\mathrm{GAD}, \mathrm{dGAF}=0.98$ : MDE, $\mathrm{dGAF}=1.28$ ).

Conclusion: Assessing functional disability improves detection in case of GAD. All measures captured changes in clinical status at 3-months. The use of self-reported measures of functional disability could improve detection of GAD in non-specialized health care levels.

\section{Abstract \#: 070}

\section{Epidemiological characteristics of chronic fatigue syndrome in an Australian cohort}

Samantha C. Johnston ${ }^{1}$, Ekua W. Brenu ${ }^{1}$, Don R. Staines ${ }^{1}$, Sonya M. Marshall-Gradisnik ${ }^{1}$

${ }^{1}$ Griffith Health Institute, Griffith University, Gold Coast, Australia; Corresponding author's e-mail: samantha.johnston3@griffithuni. edu.au

Background: Chronic Fatigue Syndrome (CFS) is characterised by severe, unexplained fatigue accompanied by a myriad of debilitating symptoms. The illness has received increasing public health attention particularly in the United States and United Kingdom as an emerging illness that carries a significant economic burden. No epidemiological investigations have previously been conducted in Australia according to current definitions of CFS. Hence, the aim of this study was to examine sociodemographic and onset characteristics of those reporting CFS symptoms in Australia.

Methods: We present preliminary data on 980 individuals enrolled in a volunteer, university research based registry that were diagnosed with CFS by a primary care physician. These individuals are aged between 18 and 64 years of age, and further case ascertainment involved clinical review of cases according to the Fukuda, and International definitions for CFS. Individuals also completed selfreporting questionnaires regarding their health service utilisation, medications, health related quality of life (HRQoL), as well as a symptom diary.

Results: CFS as characterised by the Fukuda definition was evident in $24.7 \%$ of referred individuals. A further $31.8 \%$ also met the International definition. $27 \%$ were categorised as having alternative physical or psychological diagnoses that explain the fatigue, and $13.5 \%$ had atypical chronic fatigue but did not meet Fukuda or International definitions. The highest frequencies of individuals were female, between the ages of 45 and 54, and more likely to be unemployed. The syndrome was predicated largely by an infectious onset of cases of Epstein-Barr virus (glandular fever), severe influenza, and upper respiratory infection. Significant association was also found with periods of undue stress. More severe onset and increased frequency of symptoms was also associated with poorer prognosis with lower physical and mental HRQoL and disability scores. Further those meeting the International definition reported more severe and frequent symptoms.

Conclusion: The study is identifying a significant cohort of Australians meeting CFS definitions where post-infectious onset and life stressors were strong predictors for their diagnosis. This patient group have a high need for health service provision, utilising primary care, and allied health services, and are at high risk of unemployment. Those meeting the International definition appear to represent a distinct clinical group associated with greater impairment. The integration of these epidemiological characteristics with biological and clinical data contributes further to the understanding of CFS in the Australian population and the potential aetiology and pathomechanism of this illness.

\section{Abstract \#: 071}

Sedentary time in 'metabolically healthy' versus 'metabolically unhealthy' obese and non-obese individuals

Belle H. de Rooij ${ }^{1,2}$, Julianne D. van der Berg ${ }^{1,2}$, Carla J.H. van der Kallen $^{3,4}$, Hans H.C.M. Savelberg ${ }^{5,6}$, Nicolaas C. Schaper ${ }^{3,4}$, Pieter C. Dagnelie $^{2,4,7}$, Miranda T. Schram ${ }^{3,4}$, Ronald R.M.A. Henry ${ }^{3,4}$, Coen D.A. Stehouwer ${ }^{3,4}$, Annemarie Koster ${ }^{1,2}$

${ }^{1}$ Department of Social medicine, Maastricht University, Maastricht, The Netherlands; ${ }^{2}$ CAPHRI School for Public Health and Primary Care, Maastricht University, Maastricht, The Netherlands; ${ }^{3}$ Department of Internal Medicine, Maastricht University Medical Centre (MUMC +); ${ }^{4}$ Cardiovascular Research Institute Maastricht (CARIM), Maastricht University Medical Centre, Maastricht, The Netherlands; ${ }^{5}$ Department of Human Movement Sciences, Maastricht University, Maastricht, The Netherlands; ${ }^{6}$ School for Nutrition and Translational Research in Metabolism (NUTRIM), Maastricht University, Maastricht, The Netherlands; ${ }^{7}$ Department of Epidemiology, Maastricht University, Maastricht, The Netherlands; Corresponding author’s e-mail: b.derooij@maastrichtuniversity.nl

Background: Both obesity and the metabolic syndrome (MetS) are associated with increased risk of cardiovascular diseases and type 2 diabetes. Although both frequently occur together in the same individual, obesity and MetS can also develop independently from each other. The (patho)physiology of "metabolically healthy obese" (i.e. obese without MetS) and "metabolically unhealthy non-obese" phenotypes (i.e. non-obese with MetS) is not fully understood, but sedentary behaviour may play a role. We therefore investigated sedentary behaviour patterns across 4 groups of subjects: I) metabolically healthy obese (MHO), II) metabolically unhealthy obese (MUO), III) metabolically healthy non-obese (MHNO) and IV) metabolically unhealthy non-obese (MUNO).

Methods: Data were available from 2494 men and women, aged 40-75 years who participated in The Maastricht Study from 2010 to 2013. Participants were classified into the 4 groups according to obesity (body mass index $[\mathrm{BMI}] \geq 30 \mathrm{~kg} / \mathrm{m}^{2}$ ) and MetS (Adult Treatment Panel III definition). Physical activity and sedentary behaviour were objectively measured for 7 days with the Activ$\mathrm{PAL}^{\mathrm{TM}}$ physical activity monitor. Sedentary time and step frequency were derived from the ActivPAL data. General Linear Models were used to assess cross-sectional differences in sedentary time across the 4 groups, by Bonferroni-corrected pairwise comparisons.

Results: In our study population, 562 individuals were obese. $19.4 \%$ of the obese individuals and $72.7 \%$ of the non-obese individuals was metabolically healthy. On average, people spent $60.2 \%$ of total waking time sedentary. After adjustment for age, sex, educational level, smoking status and alcohol use, significant differences in sedentary time were found between MHO and MUO (60.3 versus $64.4 \%, P<0.001)$ and between MHNO and MUNO (58.2 versus 
$61.8 \%, P<0.001)$. Additionally, the MUNO were less sedentary then the MUO (61.8 versus $64.4 \%, P<0.001)$. Further adjustments for type 2 diabetes, history of cardiovascular diseases and mean step frequency as a measure for physical activity did not alter the results. Conclusions: Total time spent sedentary was lower in the subgroups without MetS (MHNO and MHO) compared to both subgroups with MetS (MUNO and MUO), independent of level of physical activity. Therefore, sedentary time may partly explain the presence of MetS in obese as well as non-obese individuals.

\section{Abstract \#: 072}

Diabetes and obesity incidence in 5 years follow up of a Spanish cohort

Tomás VEGA ${ }^{1}$, Jose LOZANO $^{1}$, Arancha GARCÍA ${ }^{1}$, Rufino ÁLAMO

${ }^{1}$ Castilla y León Regional Health Ministry; Corresponding author's e-mail: vegaloto@jcyl.es

Background: Diabetes and obesity are considered among the principal threats to the health of the population in the 21 st century. Its relation with the total and specific mortality and morbidity is out of any doubt, as well as the difficulties to prevent and control them in an appropriate way. Cardiovascular diseases are the main consequences of an inadequate glycemic control and the overweight or abdominal obesity. The Cardiovascular Risk Study in Castilla y León (Spain) aims to estimate the cardiovascular risk factors in the regional population and to describe their evolution in a cohort in order to implement effective and cost-benefit control measures.

Methods: In 2004, a medical health exam was carried out in a random sample of 4013 persons from Castilla y León aged 15 and more. Blood pressure, weight, height, waist circumference were measured and the smoking status registered. Fasting plasma glucose (FPG), total cholesterol, LDL-cholesterol, HDL-cholesterol and triglycerides were determined and the global cardiovascular risk calculated. In 2009, the same study was performed in 3000 persons from the cohort. Diabetes was considered when FPG was $\geq 126 \mathrm{mg} / \mathrm{dl}$ or a diagnosis or treatment was registered in the medical record. Overweight and obesity were considered when BMI was $\geq 25$ and 30 respectively. Abdominal obesity (AO) was defined as the waist circumference above $102 \mathrm{~cm}$ in men and $88 \mathrm{~cm}$ in women.

Results: The 5 years cumulated incidence of diabetes was $3.6 \%$. The incidence was higher in men $(4.5 \%)$ than in women $(3.5 \%)$ and in all age groups. The incidence reached a maximum at the 65-69 age group, around $8 \%$. The total prevalence in the cohort population increased from $9.9 \%$ in 2004 to $13.2 \%$ in 2009 . The prevalence of obesity was $22.9 \%$ in 2004 and $26.3 \%$ in 2009 . The 5 years incidence of obesity was $9.1,15.8 \%$ from previous overweight and $1.2 \%$ directly from normal weight. AO appeared in $20.1 \%$ of people with normal waist circumference in 2004. Obesity and AO increased with age and they were more incident in women, particularly the AO from 45 years old on.

Conclusion: Diabetes and obesity are closely related, and involved in severe cardiovascular events. Diabetes diagnosis is often made at a relatively advanced age and metabolic control could take advantage of an early diagnosis. The burden of obesity appears with intensity in middle-aged persons, where changes in life behaviors are still effective to reduce the complications of this health problem.

\section{Abstract \#: 073}

Lifetime risk of prediabetes and diabetes: a prospective population-based cohort study

Symen Ligthart ${ }^{1}$, Thijs T.W. van Herpt ${ }^{1}$, Maarten J.G. Leening ${ }^{1}$, Albert Hofman , Bruno H.Ch. Stricker ${ }^{1}$, Mandy van Hoek ${ }^{2}$, Eric J.G. Sijbrands $^{2}$, Oscar H. Franco ${ }^{1}$, Abbas Dehghan ${ }^{1}$

${ }^{1}$ Department of Epidemiology, Erasmus University Medical Center, Rotterdam, the Netherlands; ${ }^{2}$ Department of Internal Medicine, Erasmus University Medical Center, Rotterdam, the Netherlands; Corresponding author's e-mail: s.ligthart@erasmusmc.nl

Background: Data on the lifetime risk of prediabetes, diabetes and the use of blood glucose lowering medication for the European population have not been published and little is known about the lifetime risk of diabetes in relation to measures of adiposity.

Methods: We calculated lifetime cumulative incidences for prediabetes, diabetes and the use of blood glucose lowering medication adjusted for competing risk of death in 10,050 participants aged 45 years and older of the prospective population-based Rotterdam Study. Incident prediabetes and diabetes diagnosis were defined according to the WHO guidelines. Data on the use of blood glucose lowering medication was derived through linkage with local pharmacies. All analyses were stratified by sex and anthropometric measures.

Results: The mean (SD) age of the population was 65.1 (9.8) years and $56.5 \%$ were women. During a mean follow-up of 7.9 (4.0) years, 828 participants developed diabetes and 1709 deceased. At the age of 45 , the remaining lifetime risk of type 2 diabetes was $31.3 \%$ [95\% CI 29.3-33.3]. Remaining lifetime risk was lower with increasing age and declined to $8.9 \%$ [6.9-10.9] at age 85. At age 45, those with a body mass index $<25 \mathrm{~kg} / \mathrm{m}^{2}$ had a lifetime risk of $18.8 \%$ [15.8-21.7], whilst $56.6 \%$ [46.7-66.6] of those with a body mass index $\geq 35 \mathrm{~kg} / \mathrm{m}^{2}$ developed diabetes. In addition, those aged 45 with a waist circumference $<80 \mathrm{~cm}$ for women and $<94 \mathrm{~cm}$ for men had a lifetime risk of $19.5 \%$ [16.0-23.1], whereas $60.9 \%$ [51.0-70.9] of those with a waist circumference $>108 \mathrm{~cm}$ for women and $>122 \mathrm{~cm}$ for men developed diabetes, respectively. The remaining lifetime risk at the age of 45 for the development of prediabetes was $48.7 \%$ [46.2-51.3]. The lifetime risk for the use of oral blood glucose lowering medication was $28.5 \%$ [25.0-32.0] for those aged 45, whereas $9.1 \%$ [7.8-10.3] became insulin dependent.

Conclusion: Almost one in two individuals aged 45 will develop prediabetes and one in three will develop type 2 diabetes. The vast majority of diabetics will be medication dependent. Our results underscore the importance of lifestyle by showing the effects associated with obesity on lifetime risk of type 2 diabetes.

\section{Abstract \#: 074}

Consumption of dairy foods in relation to impaired glucose metabolism and type $\mathbf{2}$ diabetes mellitus: the Maastricht study

$\underline{\text { Simone J.P.M. Eussen }}^{1,2,3,4}$, Martien C.J.M. van Dongen ${ }^{1,3}$, Nicole Wijckmans $^{1,3}$, Louise den Biggelaar ${ }^{1,2}$ Stefanie J.W.H. Oude Elferink ${ }^{5}$, Cécile M. Singh-Povel ${ }^{5}$, Miranda T. Schram ${ }^{2,6}$, Simone J.S. $\mathrm{Sep}^{2,6}$, Carla J. van der Kallen ${ }^{2,6}$, Annemarie Koster ${ }^{7}$, Nicolaas Schaper $^{2,3,6}$, Ronald M.A. Henry ${ }^{2,6}$, Coen D.A. Stehouwer ${ }^{2,6}$, Pieter C. Dagnelie ${ }^{1,2,3}$ 
${ }^{1}$ Department of Epidemiology, Maastricht University, The Netherlands; ${ }^{2}$ School for Cardiovascular Diseases (CARIM), Maastricht University, The Netherlands; ${ }^{3}$ School for Public Health and Primary Care (CAPHRI), Maastricht University, The Netherlands; ${ }^{4}$ Department of Public health and Primary Health Care, University of Bergen, Norway; ${ }^{5}$ FrieslandCampina, Amersfoort, The Netherlands.; ${ }^{6}$ Department of Internal Medicine, Maastricht University Medical Center + Maastricht, The Netherlands; ${ }^{7}$ Department of Social Medicine, School for Public Health and Primary Care (CAPHRI), Maastricht University, Maastricht, The Netherlands; Corresponding author's e-mail:

simone.eussen@maastrichtuniversity.nl

Background: Observational studies show an inverse association between total dairy intake and diabetes risk. However, there is a lack of information on the relation of specific dairy products with impaired glucose metabolism (IGM, including impaired fasting glucose or impaired glucose tolerance) and type 2 diabetes (T2DM).

Methods: Individuals aged $40-75$ y were recruited into The Maastricht Study. Status of glucose metabolism was assessed by an oral glucose tolerance test and defined according to the WHO 2006 criteria. A 254-food item food frequency questionnaire (FFQ) was filled out, covering 50 specific dairy items that captured differences between full fat, semi-skimmed, and skimmed products, as well as fermented and non-fermented products. Upon FFQ return, participants were informed on their glucose metabolism status. The present analyses include cross-sectional data from the first 3451 participants, who completed the baseline survey between November 2010 and September 2013. Those with previously diagnosed diabetes, defined as self-reported diabetes or the use of glucose lowering medication $(n=883)$, as well as those with implausible energy intake $(n=60)$ were excluded, leaving 2508 individuals for statistical analyses. Logistic regression analyses estimated odds ratios (ORs) with $95 \%$ CI for IGM $(\mathrm{n}=501)$ and newly diagnosed T2DM $(\mathrm{n}=129)$. Analyses were adjusted for age, gender, smoking status, physical activity, BMI, history of CVD, and intakes of energy, fruits, vegetables, meat and fish.

Results: For IGM, fully adjusted analyses revealed inverse associations, with ORs $(95 \% \mathrm{CI})$ comparing the highest with the lowest tertile of intake of $0.67(0.50-0.90)$ for yoghurt, $0.73(0.55-0.96)$ for skimmed products, and $0.74(0.54-0.99)$ for fermented products. These dairy products were not significantly associated with newly diagnosed diabetes. In contrast, full fat products were positively associated with newly diagnosed T2DM (OR $(95 \% \mathrm{CI})=2.01$ (1.16-3.47)), whereas total dairy intake was inversely associated with newly diagnosed T2DM (0.50 (0.24-0.86)).

Conclusions: Individuals with a high consumption of yoghurt, skimmed and fermented products had a lower odds of having IGM, and individuals with a high consumption of total dairy had a lower odds of having newly diagnosed T2DM. High intake of full fat products was not related to IGM, but increased the odds of having newly diagnosed T2DM.

\section{Abstract \#: 075}

\section{Smoking and the risk of latent autoimmune diabetes in adults (LADA): results from the Swedish Estrid Study}

Bahareh Rasouli ${ }^{1}$, Tomas Andersson ${ }^{1,2}$, Per-Ola Carlsson ${ }^{3}$, Mozhgan Dorkhan $^{4}$, Valdemar Grill ${ }^{5,6}$, Mats Martinell ${ }^{7}$, Tiinamaija Tuomi ${ }^{8,9,10}$, Sofia Carlsson ${ }^{1}$, Leif Groop ${ }^{4}$
${ }^{1}$ Epidemiology unit, Institute of Environmental Medicine, Karolinska Institutet, Stockholm, Sweden; ${ }^{2}$ Center for Occupational and Environmental Medicin; ${ }^{3}$ Department of Medical Sciences, Uppsala University, Sweden; ${ }^{4}$ Department of Clinical Sciences in Malmö, Clinical Research Centre, Lund University, Sweden; ${ }^{5}$ NTNU Institute of Cancer Research and Molecular Medicine, Norwegian University of Science and Technology; ${ }^{6}$ Trondheim University Hospital, Trondheim; ${ }^{7}$ Department of Public Health and Caring Sciences, Uppsala University, Sweden; ${ }^{8}$ Department of Medicine, Division of Endocrinology, Helsinki University Central Hospital; ${ }^{9}$ Research Program for Diabetes and obesity, University of Helsinki;

${ }^{10}$ Folkhalsan Research Center, Finland; Corresponding author's e-mail: bahareh.rasouli@ki.se

Aim: Smoking is associated with an increased risk of type 2 diabetes. It has been suggested that smoking may reduce the risk of LADA, possibly through modulating immune response, but few studies have addressed this issue. We aimed to study the impact of smoking on LADA and type 2 diabetes risks.

Methods: Data from a population-based case-control study, including 284 incident cases of LADA (glutamic acid decarboxylase antibodies [GADA] positive) and 888 cases of type 2 diabetes (GADA negative) and 1146 matched controls aged $\geq 35$, was analyzed. Information on history of smoking was collected by validated questionnaires. Using logistic regression we calculated odds ratios and estimated the risk of LADA and T2D in relation to smoking, taking into account duration and amount of cigarettes consumed.

Results: We observed no association between smoking and the risk of LADA, OR was estimated at 1.04, (95\% CI: 0.79-1.37) for current and 1.06, (95 \% CI: 0.63-1.80) for heavy ( $\geq 30$ pack-years) smoking. In contrast, heavy smoking was associated with increased risk of type 2 diabetes: OR was estimated at 1.82 (95\% CI: 1.24-2.68) among those smoking $\geq 30$ pack-years. In patients with LADA, we observed higher levels of HOMA-IR (127 vs. 103: $P=0.0142$ ) and HOMA-B (152 vs. 97: $P=0.003$ ), and lower levels of GADA (100 vs. 138: $P=0.0346)$ among heavy ( $\geq 30$ pack-years) compared to neversmokers.

Conclusion: We could confirm that smoking was associated with an increased risk of type 2 diabetes. In contrast to previous findings, no association was seen between cigarette smoking and the risk of LADA. Our findings are compatible with both negative and beneficial effects of smoking on the pathogenesis of LADA and the lack of association with overall risk may reflect that a potential beneficial effect of smoking on autoimmunity is counterbalanced by increased insulin resistance.

\section{Abstract \#: 076}

The relative contributions of visceral fat and liver fat to insulin resistance and beta-cell function: the NEO study

Alicia Uij $1^{1}$, Karin B. Gast ${ }^{1}$, Eelco J.P. de Koning ${ }^{2}$, Hildo Lamb ${ }^{3}$, Albert de Roos ${ }^{3}$, Frits R. Rosendaal ${ }^{4}$, Martin den Heijer ${ }^{5}$, Renée de Mutsert $^{1}$

${ }^{1}$ Clinical Epidemiology, LUMC, Leiden, Netherlands; ${ }^{2}$ Nephrology and Endocrinology, LUMC, Leiden, Netherlands; ${ }^{3}$ Radiology, LUMC, Leiden, Netherlands; ${ }^{4}$ Clinical Epidemiology and Thrombosis and Hemostasis, LUMC, Leiden, Netherlands; ${ }^{5}$ Clinical Epidemiology and Endocrinology, VU Medical Center, Amsterdam, Netherlands; Corresponding author's e-mail: a.uijl@lumc.nl 
Background: Obesity is associated with insulin resistance (IR) and impaired insulin secretion. It is unclear whether these associations are mainly due to total body fat (TBF), intra-abdominal visceral adipose tissue (VAT), or liver fat. The aim of this study was to investigate the relative contributions of $\mathrm{TBF}$, visceral fat and liver fat to insulin resistance and $\beta$-cell function.

Methods: In this cross-sectional analysis of the Netherlands Epidemiology of Obesity (NEO) study, a population-based cohort of 6671 men and women aged 45-65 years, VAT was assessed by MRI in combination with $1 \mathrm{H}-\mathrm{MR}$ spectroscopy of hepatic triglyceride content (HTGC) in 2076 participants, in addition to TBF with bioelectrical impedance analysis and fasting glucose and insulin concentrations. We calculated the Homeostasis Model Assessment (HOMA) of IR and $\beta$-cell function (B). We performed a linear regression of VAT, HTGC and TBF on HOMA-IR and HOMA-B, adjusted for age, ethnicity, education, alcohol consumption, smoking status, physical activity, dietary energy intake and hormonal therapy, and stratified by sex.

Results: After exclusion of participants with glucose lowering therapy $(\mathrm{n}=88)$, alcohol abuse $(\mathrm{n}=109)$, and missing data $(\mathrm{n}=96)$, 1783 participants $(55 \%$ women) were analysed, with a mean and standard deviation (SD) of age: 55 (6) years, BMI: 26 (4) kg/m², TBF: men $25 \%$ (5), women $36 \%$ (6), VAT: men $111 \mathrm{~cm}^{2}$ (54), women 65 $\mathrm{cm}^{2}$ (41), HTGC: men 6,6\% (7.5), women 4,5\% (7.5). In a joint model including VAT, HTGC and TBF, and confounding factors in men, per SD VAT HOMA-IR was $16.1 \%$ (95 \% CI: 9.9, 22.6) higher, per SD HTGC $15.6 \%$ (9.1, 22.4), and per SD TBF $18.7 \%$ (12.0, 25.7). In women, per SD VAT HOMA-IR was $27.5 \%$ (17.4, 38.5) higher, per SD HTGC $9.7 \%(2.5,17.4)$, and per SD TBF $15.5 \%(5.9,26.1)$. In the multivariate joint model in men, HOMA-B was $5.7 \%(-0.1,11.9)$ higher per SD VAT, $6.6 \%(0.4,13.1)$ per SD HTGC, and $15.1 \%(7.4,23.3)$ per SD TBF. In women, HOMA-B was $13.6 \%(4.8,23.1)$ higher per SD VAT, $2.7 \%(-2.1,7.8)$ per SD HTGC, and $10.1 \%(0.7,20.4)$ per SD TBF.

Conclusion: All markers of total and regional adiposity were associated with insulin resistance and $\beta$-cell function. Joint models showed that VAT was most strongly associated with both HOMA-IR and HOMA-B in women, whereas in men all markers of total and regional adiposity were similarly associated with HOMA-IR and HOMA-B. Visceral fat accumulation may in particular be detrimental in women.

\section{Abstract \#: 077 \\ Identification of novel genes related to airway obstruction in never-smokers}

D.A. van der Plaat ${ }^{1,2}$, K. de Jong ${ }^{1,2}$, J.M. Vonk ${ }^{1,2}$, C.C. van Diemen ${ }^{3}$, C.M. van Duijn ${ }^{4}$, L. Lahousse ${ }^{4,5}$, G.G. Brusselle ${ }^{5,6}$, D.S. Postma ${ }^{2,7}$, H.M. Boezen ${ }^{1,2}$

${ }^{1}$ University of Groningen, University Medical Center Groningen, Department of Epidemiology; ${ }^{2}$ Groningen Research Institute for Asthma and COPD (GRIAC), Groningen, The Netherlands;

${ }^{3}$ University of Groningen, University Medical Center Groningen, Department of Genetics, Groningen, the Netherlands; ${ }^{4}$ Erasmus Medical Center, Department of Epidemiology, Rotterdam, The Netherlands; ${ }^{5}$ Ghent University Hospital, Department of Respiratory Medicine, Ghent, Belgium; ${ }^{6}$ Erasmus Medical Center, Departments of
Epidemiology and Respiratory Medicine, Rotterdam, The Netherlands; ${ }^{7}$ University of Groningen, University Medical Center Groningen, Department of Pulmonary Diseases; Corresponding author's e-mail: d.a.van.der.plaat@umcg.nl

Background: Chronic obstructive pulmonary disease (COPD) is the third leading cause of death in the world and is characterised by impaired lung function and progressive airway obstruction. Cigarette smoking is considered to be the highest risk factor for COPD. Although a striking proportion (25-45\%) of patients with COPD are never-smokers, most genetic susceptibility studies have not focussed on this group exclusively.

Objective: This study aims to identify common genetic variants associated with airway obstruction in never-smokers by performing a genome-wide association (GWA) study on the ratio of forced expiratory volume in $1 \mathrm{~s}$ to forced vital capacity (FEV1/FVC).

Methods: We used linear regression models, adjusted for sex, age and height, to assess the association between 227,981 genotyped single nucleotide polymorphisms (SNPs) and the level of FEV1/ FVC in 5070 never-smokers of the LifeLines study. Results of SNPs with a $p$ value $<1.2 \times 10^{-5}$ were verified in never-smokers of two other cohorts, namely the Vlagtwedde-Vlaardingen study $(n=432)$ and the Rotterdam study I $(n=408)$. Subsequently, the results of the three cohorts were meta-analysed using effect estimates weighted by the inverse of the corresponding standard errors. Results: Seven SNPs were associated with the level of FEV1/FVC in never-smokers of the LifeLines study and selected for verification in the two other cohorts. Although the SNPs did not reach genome-wide significance in the meta-analysis (fixed effects $p$ value $<2.2 \times 10^{-7}$ ) of the three cohorts, we observed similar directions and magnitudes of effects for four SNPs. We identified two novel SNPs, located in introns of AGAP1, which is involved in membrane trafficking and cytoskeleton dynamics, and PACSIN2 which plays a role in vesicle formation and transport. The other two SNPs are located nearby HHIP and in an intron of FAM13A, previously identified in GWA studies on COPD (in smokers).

Discussion: In never-smokers, we found associations of airway obstruction with both novel genetic variants and with genetic variants previously identified in GWA studies on COPD. This could indicate that these genes play a role in the pathogenesis of non-smoking related COPD.

\section{Abstract \#: 078}

\section{Apolipoprotein $\mathrm{E} \& 4$ allele influences serum folate levels} in the elderly with vascular diseases

Letizia Polito $^{1}$, Vincenza Frisardi ${ }^{1}$, Stefano Bellosta ${ }^{1}$, Antonio Guaita ${ }^{1}$

${ }^{1}$ Golgi Cenci Foundation - Abbiategrasso (MI) Italy; Corresponding author's e-mail: 1.polito@golgicenci.it

Background: The role of folic acid (FA) in preventing major cardiovascular outcomes has been reconsidered in light of recent randomized control trials and meta-analyses. However, there is still not a sufficient scientific basis to recommend its supplementation as prevention of cardiovascular diseases. This study aimed to examine whether ApoE genotype might influence serum folate levels in a population with cardiovascular disorders. Furthermore, we explored novel potential gene-environmental interplays. 
Methods: Cross-sectional study of 377 participants aged $72.29 \pm 1.34$ from the InveCe.Ab Study [203 were males $(53.8 \%)$ : $77(20.4 \%)$ were ApoE e4-carriers] with history of major cardiovascular diseases. The association between folate concentration and ApoE genotype was analyzed in the whole group and in those with major cardiac (MCD) or major vascular diseases (MVD) separately. Quantile regression models were constructed to estimate the 25th, 50th, and 75th percentiles of folate concentration distribution conditional on the value of ApoE genotype, gender, folic acid supplementation, serum albumin levels, alcohol and coffee consumption in the study samples. In parallel, we constructed other models taking into account all the aforementioned variables substituting coffee consumption with smoking or raw vegetables consumption. Separate models were run adding the ApoE-alcohol interaction term.

Results: Our data show an association between ApoE\&4 allele and higher serum folate levels in subjects with vascular disorders achieving statistical significance in the MVD group [ $\mathrm{N}=103]$. This association was confirmed also after adjustment for principal confounders, as well as dietary folate supplementation, smoking status, raw vegetable intake, coffee consumption, alcohol drinking, and serum albumin. Noteworthy, this increased folate levels in ApoE $\varepsilon 4$ carriers was observed only in the higher quartile of folate distribution $(p<0.05)$. Taking into account ApoE $\varepsilon 4-$ Alcohol interaction, ApoE $\varepsilon 4$ was associated with higher serum folate levels also in the lower quartiles $(p=0.01)$.

Conclusion: Our study demonstrated a significant association between ApoE e4 and higher serum folate levels in subjects with peripheral vascular diseases. We observed a gene-environmental interplay between ApoE genotype and alcohol consumption. Our results could add a justification to failure of folate supplementation trials on cardiovascular protection, highlightening the importance of knowing the genetic profile of the population at cardiovascular risk. Indeed, if ApoE 84 carriers have a higher risk to develop CVDs without a healthy lifestyle, it could be unnecessary a supplementation with folates. Nonetheless, further analysis, experimental models and longitudinal studies are needed to confirm our suggestions.

\section{Abstract \#: 079}

\section{Rare coding variants and the risk of congenital anorectal malformations: an Exome chip association study}

Charlotte H.W. Wijers ${ }^{1}$, Heiko Reutter ${ }^{2,3}$, Jelle Goeman ${ }^{1}$, Sita H. Vermeulen $^{1,4}$, Tessel Galesloot ${ }^{1}$, Ekkehart Jenetzky ${ }^{5}$, Lambertus A. Kiemeney $^{1}$, Ivo de Blaauw ${ }^{6,7}$, Han G. Brunner ${ }^{4,8}$, Nel Roeleveld ${ }^{1,9}$, I.A.L.M. van Rooij ${ }^{1}$

${ }^{1}$ Department for Health Evidence, Radboud university medical center, Radboud Institute for Health Sciences, Nijmegen, The Netherlands; ${ }^{2}$ Institute of Human Genetics, University of Bonn, Bonn, Germany; ${ }^{3}$ Department of Neonatology, Children's Hospital, University of Bonn, Bonn, Germany; ${ }^{4}$ Department of Human Genetics, Radboud university medical center, Radboud Institute for Molecular Life Sciences, Nijmegen, The Netherlands; ${ }^{5}$ Division of Clinical Epidemiology and Aging Research, German Cancer Research Centre, Heidelberg, Germany; ${ }^{6}$ Department of Surgery-Pediatric surgery, Radboudumc Amalia Children's Hospital, Radboud university medical center, Nijmegen, The Netherlands; ${ }^{7}$ Department of Pediatric Surgery, Sophia's Children's Hospital, Erasmus Medical Centre,
Rotterdam, The Netherlands; ${ }^{8}$ Department of Clinical Genetics, Maastricht University Medical Centre, Maastricht, The Netherlands; ${ }^{9}$ Department of Pediatrics, Radboudumc Amalia Children's Hospital, Radboud university medical center, Nijmegen, The Netherlands; Corresponding author's e-mail: lotte.wijers@ radboudumc.nl

Background: Anorectal malformations (ARM) are rare congenital malformations resulting from disturbed development of the rectum and anus. Knowledge on the genetic etiology of ARM is still scarce. Previous genetic studies on ARM were mainly focused on candidate genes that are involved in embryonic signaling pathways and did not yield any substantial evidence. Hypothesis-generating approaches through genome-wide studies seem valuable to acquire new knowledge.

Methods: We performed genotyping of 598 Caucasian ARM cases and 1931 Caucasian population-based controls using the Illumina Human Exome BeadChip, which contains $\sim 250 \mathrm{~K}$ rare coding variants. Patients were derived from the AGORA (Aetiologic research on Genetic and Occupational/environmental Risk factors for Anomalies in children) data- and biobank in the Netherlands and the German Network for Congenital Uro-REctal malformations (CURENet), while we used controls from the Nijmegen Biomedical Study (NBS). Single variant and gene-based analyses were performed. Statistically significant single variant results after Bonferroni correction $\left(p<1.13 \times 10^{-6}\right)$ were technically validated in patients with targeted sequencing, using the molecular inversion probe (MIP) technique. Additional Caucasian ARM patients from AGORA and CURE-Net and three population-based Caucasian control databases together with Han Chinese patients and controls from the University of Hong Kong were used to replicate the genome-wide findings after validation.

Results: In total, 558 cases, 1886 controls and 241,177 markers passed all quality control steps. These markers included $56 \%$ monomorphic variants, $29 \%$ variants with minor allele frequency $(\mathrm{MAF}) \leq 1 \%$, and $15 \%$ with $\mathrm{MAF}>1 \%$. Variants with MAF $>0.4 \%$ were tested (adjusted by genomic control) and 55 variants reached statistical significance in an allelic model. Only three variants in the CLCN1, LRBA, and ZNF423 genes could be technically validated in the discovery sample. However, replication analyses in Caucasian and Han Chinese cohorts could not confirm these results. The gene-based analyses yielded 86 statistically significant genes, but did not lead to new insights as conditional analyses showed that the gene-based signals were mainly driven by one variant in each gene. These variants were found to be statistically significant or almost statistically significant in the single variant analyses.

Conclusion: We found no evidence for strong associations between ARM and rare coding variants captured by the HumanExome BeadChip. Future studies need large sample sizes to identify common and rare variants with small to moderate effects, while stratifying on phenotypically homogenous groups of ARM patients. Therefore, international collaboration is essential.

\section{Abstract \#: 080}

A systematic SNP selection approach for studying geneenvironment interactions in cancer epidemiology

Rachel J.J. Elands ${ }^{1}$, Mona Riemenschneider ${ }^{2}$, Colinda C. Simons ${ }^{1}$, Leo J. Schouten ${ }^{1}$, Bas A. Verhage ${ }^{1}$, Kristel van Steen $^{3}$, Roger 
Godschalk $^{4}$, Piet A. van den Brandt ${ }^{1}$, Monika Stoll ${ }^{5}$, Matty P. Weijenberg $^{1}$

${ }^{1}$ Department of Epidemiology, GROW School for Oncology and Developmental Biology, Maastricht University, Maastricht, the Netherlands; ${ }^{2}$ Genetic Epidemiology, University of Münster, Münster, Germany; ${ }^{3}$ Systems and Modeling Unit, Montefiore Institute, University of Liege, Liège, Belgium; ${ }^{4}$ Department of Toxicology, NUTRIM-School for Nutrition, Toxicology and Metabolism, Maastricht University, Maastricht, the Netherlands; ${ }^{5}$ Institute of Human Genetics, Genetic Epidemiology, University of Münster, Münster, Germany; Corresponding author's e-mail: rachel.elands@maastrichtuniversity.nl

Background: Genome-wide association studies (GWAS) have employed a number of strategies to identify the best way to incorporate the joint effects of genetic variants and environmental factors into epidemiologic studies of complex diseases. Here, a generalizable systematic approach is described to prioritize genetic variants for complex diseases using all GWAS repository data available. The rationale underlying this prioritization strategy is that GWAS single nucleotide polymorphisms (SNPs) associated with complex diseases tend to cluster in regions of low recombination: this offers the opportunity to select a limited number of SNPs for large-scale studies with exhaustive bio-samples and budget constraints. We have applied this systematic prioritization strategy to select common genetic variants that may mechanistically underpin the association between adult-attained height and post-menopausal breast- and colorectal cancer.

Methods: SNPs were selected from the NHGRI GWAS Catalog and the Johnson \& O'Donnell database with a $p$ value $<10^{-5}$. A clustering algorithm identified genomic regions which included at least one GWAS SNP associated with height and one GWAS SNP associated with either postmenopausal breast- or colorectal cancer risk. In addition, the SNPs in a cluster had to be within 1 megabase pair (Mbp) from each other. SNPs were clustered until no additional SNPs within a sliding window of $1 \mathrm{Mbp}$ could be found. Clusters were prioritized if they contained at least one height-associated SNP and one cancer-associated SNP that were mapped to the same near-by located gene. ConsensuspathDB software was used to identify pathways and overrepresented gene ontology (GO) terms.

Results: Twelve clusters were identified with 44 SNPs annotated to 21 genes. Both height- and cancer-associated SNPs were annotated to the following genes: BMP2, ID4, ZMIZ1, MKL1, PITX1, ESR1, RAD51B, TNS1, TNP1, TET2, FAM46A, DCBLD1, GSDMC. Accounting for all annotated genes in the clusters, the most significant biological pathway was the TGF-beta signaling pathway $(P=$ $3.58 \times 10^{-3}$ ). The most significantly overrepresented biological processes were nucleic acid binding (GO term, $P=4.14 \times 10^{-4}$ ), nucleic acid metabolic process (GO term, $P=7.36 \times 10^{-4}$ ) and gland development (GO term, $P=1.01 \times 10^{-3}$ ).

Conclusions: Our approach revealed genes and pathways pointing to common underlying biological mechanisms which are relevant for both height and cancer. Some of these genes and pathways have previously been proposed in the literature. With this systematic approach, a relatively small number of SNPs were identified which can efficiently be genotyped in large-scale studies in the future to investigate the height-cancer association.

\section{Abstract \#: 081}

Altered neonatal cord blood lipidome in association with exposure to particulate matter in the early life environment

Dries S. Martens ${ }^{1}$, Sandra Gouveia-Figueira ${ }^{2}$, Narjes Madhloum ${ }^{1}$, $\overline{\text { Bram G. Janssen }}^{1}$, Michelle Plusquin ${ }^{1}$, Bertil Forsberg ${ }^{3}$, Malin L. Nording $^{2}$, Tim S. Nawrot ${ }^{1}$

${ }^{1}$ Centre for Environmental Sciences, Hasselt University, Diepenbeek, Belgium; ${ }^{2}$ Department of Chemistry, Umeå University, Umeå, Sweden; ${ }^{3}$ Division of Occupational and Environmental Medicine, Umeå University, Umeå, Sweden; Corresponding author's e-mail: dries.martens@uhasselt.be

Background: The period of in utero life is a critical window programming diseases later in life. We studied determinants of lipidome profiles of oxylipins in early life as potential relevant pathways to later life disease.

Methods: Oxylipins were extracted by SPE (solid phase extraction) from 202 cord blood plasma samples $(750 \mu \mathrm{l})$ from the ENVIRONAGE (ENVIRonmental influence ON AGEing in early life) birth cohort in Belgium. Thirty-seven specific oxylipins reflecting the cyclooxygenase (COX), lipoxygenase (5-LOX and 15-LOX) and cytochrome P450 (CYP) metabolic pathways were quantified by ultraperformance liquid chromatography coupled to electrospray ionization tandem mass spectrometry (UPLC-ESI-MS/MS). Principal component analysis and multiple regression models were applied to associate oxylipin pathways as well as individual metabolites with in utero PM2.5 and PM10 exposure, while adjusting for newborns gender, gestational duration, maternal age, maternal smoking status, maternal BMI, and cord blood total cholesterol and HDL levels.

Results: PM2.5 exposure during pregnancy averaged (25th-75th percentile) $15.8(13.6-17.5) \mu \mathrm{g} / \mathrm{m}^{3}$. Four metabolites combined in a single factor (SF), representing the 5-LOX pathway were positively associated with PM2.5 and PM10 exposures during the entire ( $p=0.04$ and $p=0.05$, respectively) and second trimester of pregnancy $(p=0.01$ and $p=0.02$, respectively). Eleven metabolites combined in a SF representing the 15 -LOX pathway were positively associated with PM2.5 and PM10 exposures during the second trimester ( $p=0.008$ and $p=0.02$, respectively). No associations were found for the COX and CYP pathways.

Conclusions: In utero exposure to particulate matter was associated with the lipoxygenase pathways (5-LOX and 15-LOX) in newborns. These changes at the level of the lipidome indicate an altered inflammatory state of the newborn at birth induced by air pollution.

\section{Abstract \#: 082}

DNA Methylation mediates the effect of maternal smoking during pregnancy on birth weight of the offspring

L.K. Küpers $^{1}$, X. Xu ${ }^{2}$, S.A. Jankipersadsing ${ }_{5}{ }^{3}$, A. Vaez ${ }^{1}$, S. Scholtens ${ }^{4}$, I.M. Nolte ${ }^{1}$, R.C. Richmond ${ }^{5}$, C.L. Relton ${ }^{5}$, J.F. Felix ${ }^{6}$, L. Duijts ${ }^{7}$, J.B. van Meurs ${ }^{8}$, H. Tiemeier ${ }^{9}$, V.W. Jaddoe ${ }^{6}$, X. Wang ${ }^{10}$, E. Corpeleijn1, H. Snieder ${ }^{1}$ 
${ }^{1}$ Department of Epidemiology, University of Groningen, University Medical Center Groningen, Groningen, the Netherlands; ${ }^{2}$ Georgia Regents University, Augusta, Georgia, USA; ${ }^{3}$ Departments of Pulmonology and Genetics, University of Groningen, University Medical Center Groningen, Groningen, the Netherlands; ${ }^{4}$ Department of Epidemiology and LifeLines Cohort Study, University of Groningen, University Medical Center Groningen, Groningen, the Netherlands; ${ }^{5} \mathrm{MRC}$ Integrative Epidemiology Unit, University of Bristol, Bristol, United Kingdom; ${ }^{6}$ Departments of Epidemiology, Pedicatrics and The Generation R Study Group, Erasmus MC, University Medical Center Rotterdam, the Netherlands; ${ }^{7}$ Departments of Epidemiology and Pedicatrics, Erasmus MC, University Medical Center Rotterdam, the Netherlands; ${ }^{8}$ Department of Internal Medicine, Erasmus MC, University Medical Center Rotterdam, the Netherlands; ${ }^{9}$ Department of Epidemiology, Erasmus MC, University Medical Center Rotterdam, the Netherlands; ${ }^{10}$ Georgia Regents University, Augusta, Georgia, USA; Corresponding author's e-mail: 1.k.kupers@umcg.nl

Background: Maternal smoking during pregnancy can explain intrauterine growth restriction and low birth weight (1). We hypothesized that the long-lasting effects of adverse foetal exposures (e.g. smoking) on birth weight and subsequent cardiometabolic risk are, at least partly, caused by DNA methylation (3-5). We examined whether the effect of maternal smoking during pregnancy on birth weight of the offspring was mediated by smoking-induced changes to DNA methylation in cord blood.

Methods: First, we used cord blood of 129 Dutch children exposed to maternal smoking versus 126 unexposed to maternal and paternal smoking ( $53 \%$ male) in the GECKO Drenthe birth cohort. DNA methylation was measured using the Illumina HumanMethylation450 Beadchip. We performed an epigenome-wide association study (EWAS) for the association between maternal smoking and methylation followed by mediation analysis of the top signals (FDR < 0.05). We adjusted both analyses for maternal age, education, pre-pregnancy BMI, offspring's sex, gestational age and white blood cell composition. Secondly, in 175 exposed and 1248 unexposed newborns from two independent birth cohorts, we replicated and meta-analysed results of 8 cytosine-phosphate-guanine (CpG) sites in the GFI1 gene, which showed the most robust mediation. Finally, we performed functional network and enrichment analysis.

Results: We found 35 differentially methylated CpGs (FDR $<0.05$ ) in newborns exposed vs. unexposed to maternal smoking, of which 23 survived Bonferroni correction $\left(P<1 \times 10^{-7}\right)$. These $23 \mathrm{CpGs}$ mapped to eight genes: AHRR, GFI1, MYO1G, CYP1A1, NEUROG1, CNTNAP2, FRMD4A and LRP5. Three of the 8 CpGs in GFI1 partly mediated the effect of maternal smoking on birth weight (Sobel $P<0.05$ ) in meta-analysis of GECKO and the two replication cohorts. Differential methylation of these three GFI1 CpGs explained $12-19 \%$ of the $202 \mathrm{~g}$ lower birth weight in smoking mothers. Functional enrichment analysis pointed towards activation of cellmediated immunity.

Conclusions: Maternal smoking during pregnancy was associated with cord blood methylation differences. We observed a potentially mediating role of methylation in the association between maternal smoking during pregnancy and birth weight of the offspring. Functional network analysis suggested a role in activating the immune system.
1. Aagaard-Tillery KM et al. In utero tobacco exposure is associated with modified effects of maternal factors on fetal growth. Am J Obstet Gynecol 2008: 198: 66 e1-e6.

2. Jaenisch R, Bird A. Epigenetic regulation of gene expression: how the genome integrates intrinsic and environmental signals. Nat Genet 2003: 33 Suppl: 245-254.

3. Godfrey $\mathrm{KM}$ et al. Epigenetic gene promoter methylation at birth is associated with child's later adiposity. Diabetes 2011: 60: 1528-1534.

4. Sebert $\mathrm{S}$ et al. The early programming of metabolic health: is epigenetic setting the missing link? Am J Clin Nutr 2011: 94: 1953S-1958S.

\section{Abstract \#: 085 \\ Spinal disorders in children \\ $\underline{\text { Paul C. Willems, MD, } \mathrm{PhD}^{1}}$ \\ ${ }^{1}$ Orthopedic surgeon, Maastricht University Medical Center, The Netherlands; Corresponding author's e-mail: p.willems@mumc.nl}

Introduction: Although pediatric back pain is assumed to be rare, it has been reported that over $50 \%$ of children have episodes of back pain by 15 years of age. The task of the evaluating surgeon is to identify which children have an underlying musculoskeletal condition. Back pain in young children is worrisome and virtually always caused by a pathologic abnormality. As children reach adolescence, their pain behavior starts resembling the adult population. Plain radiographs are the best screening examination for children with back pain. When suspecting a pathologic bone process, bone scintigraphy or SPECT is recommended. Spinal neoplasms, cord abnormalities, discitis and herniated discs are best evaluated by MRI.

Spondylolysis and spondylolisthesis: Spondylolysis refers to a stress fracture of the pars interarticularis in the lower lumbar spine, most often seen in young athletes. Initial treatment is nonoperative and first involves modifying the patient's level of athletic activity. Spondylolisthesis is a related condition with anterior slippage of the vertebra. Patients often complain of low back pain that may radiate into the legs. Treatment is initially conservative, however, if the magnitude of the slip increases, surgical stabilization may be indicated.

Scheuermann kyphosis: Scheuermann kyphosis is characterized by disc abnormalities and thoracic hyperkyphosis caused by anterior wedging of at least 3 contiguous vertebrae. Management is generally nonoperative. The role of bracing is controversial. In case of severe symptoms, kyphosis with a Cobb angle $>75$ degrees, or significant cosmetic concerns, surgical correction and stabilization can be performed.

Scoliosis: The majority of patients with idiopathic scoliosis do not complain of back pain. There is a coronal as well as rotational deformity present, explaining the rib hump usually observed. Plain radiographs are sufficient for imaging if neurologic abnormalities are absent. Treatment is initially nonoperative but with progression of the deformity bracing or surgical correction and stabilization may be required. Neuromuscular scoliosis is caused by underlying neurologic or muscular deficiencies and commonly featured by large and stiff curves early in life, and pelvic obliquity because of lower extremity contractures and imbalanced spinal deformity. The complexity of the 
deformity and the fragility of the patients makes that management should occur within a multidisciplinary team.

In early onset scoliosis operative treatment is challenging because in children below 10 years of age, spinal fusion will cause long term sequelae such as trunk shortening and thoracic insufficiency. In this patient category, surgery should aim at control of the deformity still allowing spinal growth. Several nonfusion techniques and current progress in research will be highlighted.

\section{Abstract \#: 086}

Down under, top relevant! The importance of pelvic floor knowledge for the physiotherapist

\section{Marijke Slieker-ten Hove}

Corresponding author's e-mail: mcpslieker@gmail.com

Pelvic floor disorders are not always easy to recognize and often a difficult subject to discuss. Symptoms related at a pelvic floor disfunction are f.e. urinary and fecal incontinence, prolapse complaints and urogenital pain. These symptoms are easy to recognize if we ask, but in the treatment of low back pain, hip disorders, stress symptoms or inexplicable complaints can have a relation with pelvic floor disorders. Especially groin pain, but also hamstring complaints can exist in combination with a pelvic floor disorder as the pelvic floor is often compensating. The pelvic floor is functioning in the extension chain does it sound logical that we can not separate the pelvic floor in the hypotheses.

How can I recognize pelvic floor disorders in my patients? Pelvic floor disorders are not for women only during pregnancy or menopause. Pelvic floor disorders definitely exist in men, teenagers and children. We need the competence to recognize the signals and to ask the right questions.

Speaking about pelvic floor is not simple. There still is a taboo and as physiotherapist we never learned to speak with our patients about this. However, it is relevant to recognize all the signals: both the physical as the emotional ones.

How can we spot the signs and what questions do we need to ask? And also important, how do we handle their answers?

Dr. Marijke C.Ph. Slieker-ten Hove is a pelvic physiotherapist since 1992. She was responsable for the Master study Pelvic Physiotherapy, involved in the pelvic floor centre of the ERasmus MC en did her $\mathrm{PhD}$ on pelvic floor function and disfunction in a general female population. She has her own institute for pelvic floor physiotherapy and education, the ProFundum Instituut.

She will take you by the hand in the 'underworld' of the pelvic floor disorders and give answers on the previous mentioned questions.

\section{Abstract \#: 087}

Association between low back pain and hip osteoarthritis in elderly and hip dysfunction in athletes

Rob Langhout MMT PT ${ }^{1}$, Igor Tak MSc MPTS ${ }^{3,4,5}$

${ }^{1}$ Physiotherapy Dukenburg Nijmegen, Aldenhof 7003, 6537 DZ Nijmegen; ${ }^{2}$ Master Musculoskeletal Therapy (SOMT), Amersfoort, The Netherlands; ${ }^{3}$ Dutch Institute for Allied Health Care (NPi), Master of Physical Therapy in Sports, Amersfoort, The Netherlands;
${ }^{4}$ Physiotherapy Utrecht Oost, Sports Rehabilitation and Manual Therapy Unit, Utrecht, The Netherlands; ${ }^{5}$ Academic Medical Centre Amsterdam, Department of Orthopaedics and Sports Traumatology, Amsterdam, The Netherlands; Corresponding author's e-mail: noor.rob@gmail.com

Background: Hip dysfunction has been proposed to relate to low back pain (LBP) because of the anatomical proximity of the hip and lumbopelvic region. In recent years, research findings on the hip-spine syndrome were more clarified by sub-grouping samples based on characteristics such as age and activity demand. To date, findings on longstanding adductor related groin pain (LARGP) in athletes have been inconclusive. Examination of sport specific involvement of neighbouring segments on hip range of motion seems to elucidate the entity of LARGP in athletes.

Methods: Literature of the hip spine syndrome in eldery and athletes was studied. In a cohort study of non-injured football players and players with LARGP, the authors examined sport specific range of motion (SSROM) of both legs with inclinometers in 103 elite, 82 amateur and 57 injured players. Non-injured players were compared with the injured group.

Results: Elderly with hip osteoarthritis demonstrated significant more LBP then those without hip osteoarthritis. In patients with chronic LBP, both surgical and conservative interventions like hip replacement and manually applied hip mobilization, lead to improvements on pain, function and disability. Athletes with a history of LBP who regularly participated in rotation-related sports, displayed significant more asymmetry in ROM hip rotation, earlier timing of lumbopelvic motion and increased lumbopelvic ROM. Non-injured elite football players showed $190( \pm 25)^{\circ}$ and $188( \pm 25)^{\circ}$ of total SSROM for the non-dominant and dominant leg. Non-injured amateurs displayed $188( \pm 25)^{\circ}$ on both sides. Injured players showed significant SSROM deficit with $187( \pm 31)^{\circ}$ on the non-injured side and $135(\mathrm{SD} \pm 29)^{\circ}$ on the injured side.

Conclusion: The hip-spine syndrome seems a relevant clinical entity. Clinical examination and assessment of elderly and athletes with LBP and hip dysfunction is complex, but should be encouraged. Longstanding adductor-related groin pain in athletes is associated with a significant decrease of SSROM of the injured leg.

\section{Abstract \#: 088}

Compensation strategies in low back, pelvic and hip pain patients Dr. A.L.Pool-Goudzwaard ${ }^{1}$

${ }^{1}$ MOVE Institute, Faculty of Behavioral and Human Movement Sciences, VU University, Amsterdam; Corresponding author's e-mail: annelies.pool@gmail.com

Low back pain (LBP) is a worldwide problem with lifetime prevalence rates at 60-70\% in western industrialized countries. Within this LBP group a subgroup of (pregnancy related) pelvic pain (PPP) and/ or SI joint pain exists. Although the prevalence of SI joint pain is $31 \%$, pregnancy related pelvic pain prevalence rates are reported from $37 \%$ (India) to $83 \%$ (Netherlands).

To be able to apply evidence based care, critical appraisal of literature is necessary for the most effective therapy for LBP and/or PPP. However, meta-analysis on LBP and multiple systematic reviews on PPP are not clear to guide the clinician. When e.g. 
education or giving information is advocated, as moderate evidence for positive effect the essence of which information should be shared with the patients is not clear, neither are intensity, frequency and content of exercise therapy. This is the reason why findings of fundamental experimental research are often used as a rationale for treatment.

It is known in LBP patients that motor reflexes are diminished, transmission speed and accuracy of positioning is less and that the brain is altered in response and motor control visible in the smudging of the brain. In addition, literature reports on difference between patients in adaptation, variability and how patients address and compensate for these differences in motor control.

Indeed, in these patterns of compensation and adaptation clinicians see the largest differences.

Compensation strategies involve the hip, spine and pelvis. Overall reported patterns are, increase of tension in superficial large muscles as erector spine, quadratus lumborum, psoas major and rectus abdominous. Furthermore some patients demonstrate rise of intra abdominal pressure during all kind of low load tasks. Some patients can increase their intra abdominal pressure by pushing the belly outwards. Different patterns in activity are also reported in the deep rotators of the hip and the pelvic floor muscles. Sometimes coupled with loss of tone (pushing pelvic floor downwards) or with an increase of tone. Both can cause extra dysfunction in the voluntary and autonomic activation/relaxation of the pelvic floor possibly leading to urinary incontinence, constipation and sexual complaints.

We have to be aware as clinicians that these patterns reported in small cross sectional lab studies and within our clinical expertise are low evidence and therefor cannot be extrapolated to the world population. As example the so-called "instability" present in all LBP patients.

Incorporating science in clinical evidence based practice remains a challenge.

\section{Abstract \#: 089}

Development of diagnostic algorithms to estimate prevalence of vestibular disorders in the general population: results from the Munich vertigo study

Martin Müller $^{1}$, Ralf Strobl ${ }^{1}$, Charlotte Selge ${ }^{2}$, Klaus Jahn ${ }^{2}$, Eva Grill

${ }^{1}$ Institute for Medical Information Processing, Biometrics and Epidemiology and German Center for Vertigo and Balance Disorders, Ludwig-Maximilians-Universität München, Munich, Germany; ${ }^{2}$ Department of Neurology and German Center for Vertigo and Balance Disorders, Ludwig-Maximilians-Universität München, Munich, Germany; Corresponding author's e-mail: martin.mueller@med.uni-muenchen.de

Background: Vestibular disorders are arguably one of the most common complaints seen in primary care and account for a large amount of societal and personal burden. However, information on the prevalence and distribution of specific vestibular disorders is scarce, arguably, because detailed diagnostic work-up is not feasible in population-based studies.

Objectives of this study were (1) to develop a valid diagnostic algorithm for vestibular disorders applicable to population-based studies and to (2) apply this algorithm to a representative sample of the general population to estimate prevalences.
Methods: Algorithms were developed in a sample of 610 patients with verified diagnoses from a specialized tertiary outpatient dizziness clinic using recursive partitioning and choosing the model with the best cross-validated error. Algorithms were then applied to data from a cross-sectional representative telephone survey in Munich, Germany and surrounding counties. Participants were asked for lifetime and one-year prevalence of falls, moderate to severe vertigo and dizziness or balance problems. Those who had vertigo or dizziness or balance problems in the last year were asked for quality and quantity of symptoms, duration of attacks, and potential triggering situations.

Results: A total of 3540 persons, mean age 51.2 years $(54.9 \%$ female) participated in the telephone survey. Lifetime prevalence for moderate to severe vertigo or dizziness or balance problems was $27.5 \%$ (22.2\% in men, $31.8 \%$ in women): 12 -month prevalence was $15.1 \%(11.1 \%$ in men, $18.3 \%$ in women). A total of $252(7.1 \%)$ persons reported rotational vertigo. The best model had a cross-validated error rate of 0.53 . Using this model to identify single vestibular disorders estimated following prevalences: Phobic postural vertigo: $4.7 \%$, benign paroxysmal positioning vertigo: $3.1 \%$, Vestibular migraine: $3.3 \%$, Menierés Disease: $1.7 \%$, bilateral vestibulopathy: $0.9 \%$, downbeat-nystagmus $0.4 \%$.

Conclusion: Findings on the prevalence for moderate to severe vertigo or dizziness or balance problems are mostly in line with literature. The high prevalence of rotational vertigo, a key symptom of vestibular disorders, might indicate that the actual prevalence of vestibular disorders in the general population is higher than previously reported. The algorithms developed in this study might be a useful approach for estimating the prevalence of vestibular disorders in the general population but need further testing and validation.

\section{Abstract \#: 090}

Increasing life expectancy at older ages despite increasing disease prevalence 1993-2013: explanatory value of life course and period factors $\frac{\text { Dorly J.H. Deeg }}{\text { Martijn Huisman }}{ }^{1}$, Henrike Galenkamp-van der Ploeg ${ }^{1}$,

${ }^{1}$ VU University Medical Centre/Dept of Epidemiology and Biostatistics/LASA; Corresponding author's e-mail: djh.deeg@vumc.nl

Background: Western countries have shown continuing declines in mortality, observed at increasingly older ages. The reasons are poorly understood. For the Netherlands, this study examines to what extent the mortality decline at older ages can be explained by improved life course characteristics of subsequent cohorts (higher levels of education) and by period factors such as improved medical care (longer survival with chronic diseases) during the past two decades.

Methods: We studied trends in 4.6-year mortality across six cycles of the nationally representative cohort of the Longitudinal Aging Study Amsterdam for ages 64-84 years. In the pooled sample using Generalised Estimating Equations with a logit link function, we examined the effect of year (1993-2009) on subsequent 4.6-year mortality. We tested the change in the odds ratio of year after adding level of education and chronic diseases, respectively, to a basic model including year, age and gender. 
Results: From 1993 to 2009, the level of education rose (at least some middle education: from 53 to $73 \%$ ), as did the prevalence of chronic diseases (multimorbidity: from 43 to $65 \%$ ). 4.6-year mortality declined from 20 to $13 \%$ ( $p$ (year) $<0.001$ ). Education explained $19 \%$ of the effect of year. By contrast, adding chronic diseases to the basic model increased the effect of year by $25 \%$ : for diabetes this suppressor effect was largest. Thus, if the prevalence of chronic diseases had stayed as low as in 1993, the mortality decline would have been steeper.

Conclusion: The improvement in survival of older people with chronic diseases proved a stronger factor in the explanation of the older-age mortality trend than education. This improvement is likely due to better medical care.

\section{Abstract \#: 091}

Adjusting for confounding by indication in observational studies about traumatic brain injury

M.C. Cnossen $^{1}$, S. Polinder ${ }^{1}$, E.W. Steyerberg ${ }^{1}$, H.F. Lingsma ${ }^{1}$

${ }^{1}$ On behalf of the POCON investigators; Corresponding author's e-mail: m.c.cnossen@erasmusmc.nl

Background: Traumatic brain injury (TBI) is a major public health problem, with 75,000 annual deaths worldwide. Several international large-scale observational studies are currently executed to identify effective treatment interventions for TBI. As observational studies are suspect for confounding by indication, we compared three methods to adjust for confounding by indication, using an example from TBI.

Methods: We used the observational POCON study, including 411 patients with moderate and severe TBI from five Dutch University hospitals between 2008 and 2009. Two interventions were evaluated: ICP monitoring and craniotomy. Outcome was the Glasgow Outcome Scale Extended (GOSE) at 6 months, collapsed into a four-point ordinal scale. As a reference, we estimated unadjusted treatment effects with a proportional odds regression model. Subsequently we used three methods to adjust for potential confounders. First, standard adjustment in a multivariable model. Second, adjustment for the propensity of receiving the treatment, based on relevant baseline- and clinical characteristics. These two methods adjust for observed confounders. Third, we defined treatment on hospital level (percentage of indicated patients treated) and used a random effect model. We included treatment at both hospital and patient level. The hospital level effect can be interpreted as the odds of a more favorable outcome in patients treated in a hospital that is more temped to use ICP monitoring or craniotomy in the treatment of TBI. This approach is expected to adjust for observed and unobserved confounders.

Results: Baseline- and clinical characteristics differed substantially between treated and non-treated patients, to the detriment of those treated. As a result, unadjusted ORs indicated negative effects of treatment on outcome. Also in the multivariate and propensity score adjusted analysis, treatment was associated with less favorable outcome (ICP monitoring: OR (multivariable): 0.73 (95\% CI: 0.46-1.19): OR (propensity): 0.64 (0.38-0.97): Craniotomy: OR (multivariable): 0.98 (0.45-2.41): OR (propensity): 0.50 (0.31-0.81). Treatment varied substantially among hospitals (ICP monitoring: 23-61 \%: craniotomy: $19-42 \%$ ). In random effect analyses, treatment on hospital level was associated with favorable outcome (OR
(ICP) per $10 \%$ more patients treated: $1.12(0.99-1.31)$ : OR (craniotomy): 1.15 (0.94-1.50)).

Conclusion: Strong confounding by indication was present in our example. Multivariable and propensity score adjusted analyses still showed negative estimated treatment effects, indicating residual unobserved confounding. Defining treatment variables on hospital level may provide a less biased estimate of the treatment effects, which should be confirmed in future research.

\section{Abstract \#: 092}

New guideline for the reporting of studies developing, validating, or updating a prediction model: the tripod statement

$\frac{\text { Karel G.M. Moons }}{1}$, Douglas G. Altman ${ }^{2}$, Johannes B. Reitsma ${ }^{1}$, Gary S. Collins ${ }^{2}$

${ }^{1}$ Julius Center for Health Sciences and Primary care, University Medical Center Utrecht, PO Box 85500, 3508 GA Utrecht, The Netherlands; ${ }^{2}$ Centre for Statistics in Medicine, Nuffield Department of Orthopaedics, Rheumatology and Musculoskeletal Sciences, Botnar Research Centre, University of Oxford, Oxford, OX3 7LD, United Kingdom; Corresponding author's e-mail:

k.g.m.moons@umcutrecht.nl

Background and Objective: Patients and care providers are confronted with making numerous decisions based on a probability: a probability that a specific disease or condition is present (diagnostic setting) or a specific event or outcome will occur in the future (prognostic setting). To guide practitioners and patients in these probability estimations, so-called multivariable prediction models are developed. Prediction models convert 2 or more pieces of information, i.e. predictors, from the participant-e.g., an individual's age, gender, symptoms, signs, laboratory and imaging test results-into a diagnostic or prognostic probability. Prediction models are becoming increasingly abundant. In virtually all medical domains, prediction models are being developed, evaluated (validated), extended and implemented. For some specific diseases, there are even an overwhelming number of competing prediction models for the same outcome or target population. It is therefore important that these clinical prediction models and the research done to develop, evaluate or extend these models be transparently reported. However, the overwhelming evidence shows that the quality of reporting of prediction model studies is poor. Only with full and clear reporting of information on all aspects of a prediction model can risk of bias and potential usefulness of prediction models be adequately assessed.

Methods and Results: The Transparent Reporting of a multivariable prediction model for Individual Prognosis Or Diagnosis (TRIPOD) initiative, which has included numerous clinicians, statisticians, epidemiologists and journal editors, has produced a guideline for the reporting of studies developing, validating or updating a prediction model, whether for diagnostic or prognostic purposes. The TRIPOD Statement is a checklist of 22 items, deemed essential for transparent reporting of any prediction model study, and addresses model development, model validation and model extension studies, regardless of the study methods used. The TRIPOD Statement is accompanied by an Explanation and Elaboration article that describes the rationale for the checklist, clarifies the meaning of each item, and discusses why transparent reporting is important, with a view to assessing risk of bias and clinical usefulness of a prediction model. Each item is explained in detail and accompanied by published 
examples of good reporting. The document also provides a valuable reference of issues to consider when designing, conducting, and analyzing prediction model studies.

Conclusions: The endorsement and use this checklist by researchers and medical journal editors will help ensure that medical research findings are complete and accurately reported, understood by readers, and ultimately used by medical practitioners.

\section{Abstract \#: 094}

Seasonal variation in the incidence of cutaneous melanoma: link with recent UV exposure-a belgian population-based study

$\underline{\text { Kristine Rommens }^{1}}$, David Jegou ${ }^{2}$, Hugo De Backer ${ }^{3}$, Joost Weyler ${ }^{1}$

${ }^{1}$ Epidemiology and Community Medicine, University of Antwerp, Edegem, Belgium; ${ }^{2}$ Research department, Belgian Cancer Registry, Brussels, Belgium; ${ }^{3}$ Royal Meteorological Institute of Belgium, Brussels, Belgium; Corresponding author's e-mail:

k.rommens@telenet.be

Background: The aim of this study was to investigate the theory of a short term late promoting effect of UV exposure on the development of cutaneous melanoma as explanation for the summer peak of melanoma incidence. Therefore we studied the seasonal variation of melanoma incidence in relation to recent $\mathrm{UV}$ exposure using direct UV measurements. We wanted to know whether this effect is influenced by gender, age, localization, histologic type or Breslow thickness. We hypothesized a higher effect of recent UV exposure in the first semester since with equal UV intensities, sunburn risk is higher in the first half of the year.

Methods: Data from the Belgian Cancer Registry, for invasive cutaneous melanoma, diagnosed during 2006-2011, were used for analysis. Daily data on UV radiation measurements in Belgium were received from the Royal Meteorological Institute.

Simple and multiple negative binomial regression models were used to investigate the influence of recent UV exposure on melanoma incidence. The sum of the mean UV dose of the 2 months prior to diagnosis (lagsum) was used as proxy for recent UV exposure of the population. To include sunburn risk during the year, a categorical variable 'semester' was created.

Results: The incidence of melanoma in Belgium shows a distinct seasonal variation with peaks in June or July. We found that part of this variation could be explained by the variation in dermatologic activity and therefore used it as an offset in our models. We found a linear relationship with higher melanoma incidence when higher prior UV exposure. The UV exposure had more impact in the first semester (relative risk(RR) per 1000 units change of lagsum: RR sem $1=1.10$, RR sem $2=1.04$ ). The effect of UV exposure was not modified by gender nor age. The interaction between anatomical site and UV exposure was significant $(p<0.001)$ and showed a higher effect on the upper and lower limbs compared to head and neck and trunk $($ RR loc_sem $1=1.13$ and 1.12, RR loc_sem $1=1.07$ and 1.06 respectively).

Conclusion: We found a linear relationship between melanoma incidence and UV dose in the 2 months preceding the diagnosis. Recent UV exposure had more impact in the first semester when sunburn risk is higher. We found a modifying effect by localization with a higher effect on the upper and lower limbs.

\section{Abstract \#: 095}

Towards personalized follow-up: a conditional prediction model and nomogram for risk of locoregional recurrence in early breast cancer patients

Annemieke Witteveen $^{1}{ }^{\text {S Ingrid M.H. Vliegen }}{ }^{2}$, Maarten J. IJzerman ${ }^{1}$, Sabine Siesling ${ }^{3}$

${ }^{1}$ Department of Health Technology and Services Research (HTSR), MIRA institute for Biomedical Technology and Technical Medicine, University of Twente, Enschede, The Netherlands; ${ }^{2}$ Department of Industrial Engineering and Business Information Systems (IEBIS), Center for Healthcare Operations Improvement \& Research, University of Twente, Enschede, The Netherlands; ${ }^{3}$ Department of Registration and Research, Netherlands Comprehensive Cancer Centre Organisation (IKNL), Utrecht, the Netherlands; Corresponding author's e-mail: a.witteveen@utwente.nl

Background: The objective of this study was to develop and validate a conditional logistic regression model for the prediction of locoregional recurrence (LRR) of breast cancer. To make a translation to clinical practice a web based nomogram was made.

Methods: Women first diagnosed with early breast cancer (without distant metastasis or ingrowth in the chest wall or skin) between 2003 and 2006 were selected from the Netherlands Cancer Registry $(\mathrm{N}=37,278)$. In the first five years following primary breast cancer treatment $957(3.0 \%)$ of the selected patients developed a LRR as a first event. Risk factors were determined using logistic regression and the risks were calculated per year, conditional on not being diagnosed with recurrence in the previous year. The presence of interaction and collinearity in the nomogram was assessed, as well as the discrimination by means of the area under the ROC curve and calibration by the Hosmer-Lemeshow goodness-of-fit test in deciles. Bootstrapping was used for internal validation. Data from 43 Dutch hospitals on primary tumours diagnosed between 2007 and 2008 was used for external validation of the performance of the nomogram $(\mathrm{n}=12,318)$.

Results: The final model included the variables grade, size, multifocality, and nodal involvement of the primary tumour, and whether patients were treated with radio-, chemo- or hormone therapy. The modelling group showed an area under the ROC curve of $0.84,0.76,0.70,0.73$ and 0.65 respectively per subsequent year after primary treatment. Model predictions were well calibrated. All effects in the validation group were in the same direction and the estimates in the validation group did not differ significantly from the modelling group. The results were incorporated in a web based nomogram.

Conclusions: This validated nomogram can be used as an instrument to aid clinical decision-making for personalized follow-up and to identify patients with a low or high risk of LRR who might benefit from a less or more intensive follow-up after breast cancer. 


\section{Abstract \#: 096}

Effect of mammographic density on performance of a breast cancer screening program using full-field digital mammography

Johanna O.P. Wanders ${ }^{1}$, Katharina Holland ${ }^{2}$, Wouter B. Veldhuis ${ }^{3}$, Ritse M. Mann ${ }^{2}$, Petra H.M. Peeters ${ }^{1,4}$, Carla H. van Gils ${ }^{1}$, Nico Karssemeijer ${ }^{2}$

${ }^{1}$ Department of Epidemiology, Julius Center for Health Sciences and Primary Care, University Medical Center Utrecht, Utrecht, The Netherlands; ${ }^{2}$ Department of Radiology, Radboud University Medical Center, Nijmegen, The Netherlands; ${ }^{3}$ Department of Radiology, University Medical Center Utrecht, Utrecht, The Netherlands; ${ }^{4}$ MRCPHE Centre for Environment and Health, Department of Epidemiology and Biostatistics, School of Public Health, Imperial College, London, UK; Corresponding author's e-mail:

j.o.p.wanders@umcutrecht.nl

Background: In breast cancer screening programs, film screen mammography (FSM) is increasingly being replaced by full field digital mammography (FFDM). FSM showed reduced screening performance in women with mammographically dense breasts, because dense (fibroglandular) tissue can mask tumors. With FFDM, image contrast can be increased, possibly increasing screening performance. Therefore, we examined to what extent mammographic density still affects screening performance when using FFDM.

Methods: We collected a consecutive series of 69,840 FFDM examinations (2003-2009) from one screening unit of the Dutch biennial screening program (50-75 years). Volumetric mammographic density was automatically assessed with Volpara version 1.5.0 (Matakina, New Zealand). For Dutch FSM screening data, breast density was never routinely reported. We assessed screening performance measures within each of four breast density categories, comparable to the breast density categories defined by the American College of Radiology. For this, we obtained the numbers of recalls (women referred to a hospital for additional examinations), false-positives (women who were recalled, but not diagnosed with breast cancer after additional examinations), and breast cancers detected during screening from the screening registration system. Interval cancers (cancers diagnosed between two screening rounds) were identified through linkage with the Netherlands Cancer Registry. Over incremental breast density categories, screening performance trends were tested with Chi Square linear trend tests.

Results: $19.7 \%$ of the examinations was categorized as density category 1 ('almost entirely fatty'), $43.1 \%$ as category $2,29.4 \%$ as category 3 , and $7.7 \%$ as category 4 ('extremely dense'). In total 413 screen-detected and 150 interval tumors were identified. Recall rates (per 1000 screening examinations) were 15.1, 20.5, 25.0, and $34.9 \%$ in categories $1-4$ respectively ( $p$ trend $<0.001$ ). False-positive rates were higher in women with dense breasts: 11.4, 14.1, 18.3 and 28.6 for categories $1-4$, respectively ( $p$ trend $<0.001$ ). Cancer detection rates were $3.7,6.4,6.6$ and $6.3 \%$ in categories $1-4$, respectively ( $p$ trend $=0.005$ ). Interval cancer rates were higher in higher density categories: $\quad 0.7 \%, \quad 1.9 \%, \quad 3.0$ and $4.5 \%$, respectively ( $p$ trend $<0.001$ ), resulting in a lower sensitivity (proportion of screen-detected tumors among the number of screen-detected and interval tumors together) in the higher density categories: 85.0, 77.6, 69.0 and $58.6 \%$ respectively ( $p$ trend $<0.001$ ). The positive predictive value of screening mammography was 24.5, 31.4, 26.5 and $18.1 \%$ in categories $1-4$, respectively ( $p$ trend $=0.034$ ).

Conclusion: Also when using FFDM for breast cancer screening, program sensitivity is markedly lower in women with dense breasts. Furthermore, high breast density leads to more false-positive screening outcomes.

\section{Abstract \#: 097}

\section{Effectiveness of return to work interventions in employees} with low back pain

Allard van der Beek ${ }^{1,2}$

${ }^{1}$ Department of Public and Occupational Health, EMGO + Institute for Health and Care Research, VU University Medical Center, Amsterdam, The Netherlands; ${ }^{2}$ Body@Work, Research Center Physical Activity, Work and Health, TNO-VU University Medical Center, Amsterdam, The Netherlands; Corresponding author's e-mail: a.vanderbeek@vumc.nl

Low back pain (LBP) remains an enormous burden for employees, employers and society as a whole. LBP is not only associated with personal discomfort and suffering, but also with financial expenses related to health care utilisation (i.e. direct costs) and particularly those related to productivity losses (i.e. indirect costs). Such productivity losses are a result of either being less productive while active at work (i.e. presenteeism), sickness absence (i.e. absenteeism), being work disabled, or even of exit from the labour market by early retirement. Among employees being absent from work due to LBP, there are opportunities to cost-effectively advance return to work (RTW), thereby reducing the risk of long-term sickness absence. The objective is to provide an overview of the scientific evidence as to the effectiveness of RTW interventions among employees absent from work due to LBP.

Several recently published systematic reviews provide evidence of a positive effect in reducing sickness absence due to LBP for various RTW intervention strategies, either by focusing on the worker, such as exercises using a cognitive behavioural approach, or by including the workplace and improving communication between stakeholders using an RTW co-ordinator. However, it is important to realize that the effect sizes in reducing sickness absence duration are overall small, and that cost-effectiveness of more intense intervention strategies needs further research. Nevertheless, even small effect sizes may have a large impact in reducing the societal indirect costs related to LBP. For long-term sickness absence, various RTW interventions have a small, but clinically relevant, effect on reducing sickness absence duration. A positive exception was an integrated care programme in the outpatient setting for patients on sickness absence due to chronic LBP. This integrated care programme showed a huge RTW difference of 94 days between the intervention and control group. The benefit to cost ratio of this programme was: investment of $€ 1$ returned $€ 24.47$. The uptake of this highly cost-effective intervention in managing LBP with chronic sickness absence by clinicians, but also by policy makers and insurers remains challenging. More research regarding how to overcome barriers in various policy specific environments, and with regards to proper financing of RTW management, may help to increase the implementation of cost-effective interventions.

\section{Abstract \#: 098}

\section{Real-effectiveness medicine in low back pain}

\section{Antti Malmivaara, MD, $\mathrm{PhD}$}

${ }^{1}$ Chief Physician, Centre for Health and Social Economics, National Institute for Health and Welfare, Helsinki, Finland; Corresponding author's e-mail: antti.malmivaara@thl.fi

Competence of healthcare personnel is the cornerstone of effective healthcare. In order to guarantee that the treatment is based on the best current evidence, up-to-date scientific evidence from clinical 
guidelines, systematic reviews and randomised controlled trials (RCTs) should constantly be considered and implemented when appropriate. However, this is not enough. We have to obtain continuous data on how low back pain patients are diagnosed and treated at the healthcare units. Documentation of care of patients can be used not only for quality assessment but also for benchmarking with peer units treating similar patients. The novel concept of Benchmarking Controlled Trial (BCT) can be utilized when comparing treatment processes and outcomes between peers. In pursuing best effectiveness for ordinary patients in ordinary settings, the Real-effectiveness medicine (REM) framework suggests that four layers of information and action should be promoted: (1) good medical know-how and skills combined with the patient view, (2) use of up-to-date scientific evidence, (3) continuous documentation of performance in ordinary settings, and (4) benchmarking between health care providers. How this is applied to diagnostic, treatment and rehabilitation of low back pain disorders will be highlighted in the presentation. The REM framework can be used by clinicians, organizations, policy-makers, and researchers.

Keywords: Low back pain, competence, evidence-based medicine, quality, benchmarking, effectiveness, cost-effectiveness, real-effectiveness medicine

\section{Abstract \#: 099}

\section{More sedentary time is associated with slower walking speed: the maastricht study}

Jeroen H.P.M. van der Velde ${ }^{1}$, Hans H.C.M. Savelberg ${ }^{1}$, Nicolaas C. Schaper $^{2}$, Julianne D. van der Berg ${ }^{3}$, Coen D.A. Stehouwer ${ }^{2}$, Paul J.B. Willems ${ }^{1}$, Miranda T. Schram ${ }^{2}$, Simone J.S. Sep ${ }^{2}$, Carla J.H. van der Kallen $^{2}$, Ronald M.A. Henry ${ }^{2}$, Pieter C. Dagnelie ${ }^{4}$, Tineke A.C.M. van Geel $^{5}$, Annemarie Koster ${ }^{3}$

${ }^{1}$ Department of Human Movement Sciences, Maastricht University, Maastricht, the Netherlands; ${ }^{2}$ Department of Medicine, Maastricht University Medical Centre+, Maastricht, the Netherlands; ${ }^{3}$ Department of Social Medicine, Maastricht University, Maastricht, the Netherlands; ${ }^{4}$ Department of Epidemiology, Maastricht University, Maastricht, the Netherlands; ${ }^{5}$ Department of Family Medicine, Maastricht University, Maastricht, the Netherlands; Corresponding author's e-mail: jeroen.vandervelde@maastrichtuniversity.nl

Background: Studies have shown that physical fitness is protective against adverse health effects: in turn, greater moderate to vigorous physical activity (MVPA) has been associated with better physical fitness. People can, however, regularly participate in MVPA and be sedentary for the remainder of the day, which might reduce its beneficial effects. Little is known about the association between sedentary time and physical fitness. Therefore, in this study we examined the association between sedentary time and physical fitness in a relatively large cohort of individuals participating in The Maastricht Study.

Methods: In total 1911 men and women (aged 40-75 years) from The Maastricht Study were selected who reported no physical function limitations. The ActivPAL tri-axial accelerometer, worn on the front right thigh, was used to objectively measure sedentary time and physical activity $24 \mathrm{~h}$ on multiple consecutive days. We calculated percentage of sedentary time per day and mean step frequency from ActivPAL data for all participants who all had at least one valid day ( $>14 \mathrm{~h}$ of monitoring) of ActivPAL wear time. Walking speed from a fast-paced 6-min walk test was used as a measure of physical fitness.
We performed a linear regression analysis with walking speed as dependent variable and mean proportion of sedentary time/day as the independent variable. Adjustments were made for age, sex, educational level, body mass index (BMI), smoking status, alcohol consumption, history of cardiovascular disease, and diabetes status. Additionally, we adjusted for step frequency as a measure of mean physical activity intensity.

Results: We included 993 men and 918 women with an average age of (mean $\pm \mathrm{SD}$ ) $60.4 \pm 8.1$ and $58.0 \pm 8.0$ years, and an average BMI of $27.1 \pm 3.8$ and $25.5 \pm 4.1 \mathrm{~kg} / \mathrm{m}^{2}$ respectively. Average walking speed was $1.66 \pm 0.20 \mathrm{~m} / \mathrm{s}$ and mean proportion of sedentary time per day was $59.3 \pm 10.2 \%$. After adjustment for possible confounders, walking speed was $1.68 \pm 0.46 \mathrm{~m} / \mathrm{s}$ in the quartile with lowest sedentary time and $1.62 \pm 0.41 \mathrm{~m} / \mathrm{s}$ in the quartile with highest sedentary time $(p<0.001)$. Results from linear regression analysis revealed that a $10 \%$ increase in sedentary time was significantly associated with $0.014 \mathrm{~m} / \mathrm{s}$ lower walking speed $(p<0.001)$. Additional adjustment for step frequency did not alter the results.

Conclusion: More sedentary time was associated with slower walking speed, even after adjustment for several confounders including a measure for mean physical activity intensity. These results suggest that, apart from MVPA, sedentary time might be an important determinant for physical fitness and accentuate the potential health risk of sedentariness.

\section{Abstract \#: O 100}

Supervised exercise therapy for intermittent claudication: gender differences following twelve months of follow up

Lindy N.M. Gommans ${ }^{1}$, Marc R.M. Scheltinga ${ }^{2}$, Marc R.H.M. van Sambeek $^{1}$, Angela H.E.M. Maas ${ }^{3}$, Bianca Bendermacher ${ }^{1}$, Joep A.W. Teijink $^{1}$

${ }^{1}$ Dept. vascular surgery, Catharina Hospital, Eindhoven, The Netherlands; ${ }^{2}$ Dept. vascular surgery, Maxima Medical Centre, Veldhoven, The Netherlands; ${ }^{3}$ Dept. cardiology, Radboud University Medical Centre, Nijmegen, The Netherlands; Corresponding author's e-mail: lindygommans@hotmail.com

Background: Peripheral arterial disease (PAD) is a chronic disease of the lower extremities caused by atherosclerosis. Intermittent claudication (IC) is a common manifestation of PAD, and defined as muscle discomfort and pain of the lower $\operatorname{limb}(\mathrm{s})$ elicited by exercise that resolves after a short period of rest. PAD affects more than $12 \%$ of the aging population and has become a global health problem. Prevalence of PAD is equal among men (13.4\%) and women $(15.6 \%)$. But women suffer more and experience greater muscular ischemia during exercise. Supervised exercise therapy (SET) consistently demonstrated beneficial effects on walking performance in patients with IC, and is there for recommended as first choice treatment. So far gender-based analysis of SET in patients with IC have been sparsely conducted and with conflicting results. Paucity of data is in line with a recent scientific statement of the American Heart Association, who released a 'call to action' for more research into gender-based differences of patients with PAD.

Methods: For this purpose we conducted a follow-up analysis on data from a multicentre randomized controlled trial. Patients with IC who were randomized to a SET program were used in the current study. The SET program consisted of exercises provided by trained physiotherapists and based on the guideline of the Royal Dutch Society for Physical Therapy. Standardized treadmill testing, quality of life (Short-Form 36 health survey) and walking (dis)ability (Walking 
Impairment Questionnaire) were measured at baseline and during follow-up at 3, 6, 9 and 12 months.

Results: A total of 113 men and 56 women were available for analysis. Groups were similar in terms of clinical characteristics and baseline walking distances. Women improved from SET, however with significantly shorter maximal walking distances compared to men after 12 months of follow-up (men: 660 meters versus women 565 meters, $P=0.032$ ). Women also reported greater walking disability at several follow-up measures, most particular on walking speed. No differences on quality of life were found between groups, except for a significantly lower mental health for women at baseline $(P=0.031)$.

Conclusion: Women benefit from SET. However, less compared to men after 12 month of follow-up. Besides, women experienced greater walking disability and revealed poorer mental health at baseline. Poor mental health is recently found to be a pronounced risk factor for development of PAV in women. More research is needed on gender-specific outcomes and factors contributing to the greater perceived walking disability in women.

\section{Abstract \#: O 101}

Dietary patterns in an elderly population and their relation with bone mineral density: the Rotterdam study

de Jonge, E.A.L ${ }^{1}$, Rivadeneira, F. ${ }^{2}$, Erler, N.S. ${ }^{3}$, Hofman, A. ${ }^{1}$, Uitterlinden A.G. ${ }^{2}$, Franco, O.H. ${ }^{1}$, Kiefte-deJong, J.C. ${ }^{1,4}$

${ }^{1}$ Department of Epidemiology, Erasmus MC, Rotterdam, The Netherlands; ${ }^{2}$ Department of Internal Medicine, Erasmus MC, Rotterdam, The Netherlands; ${ }^{3}$ Department of Biostatistics, Erasmus MC, Rotterdam, The Netherlands; ${ }^{4}$ Global Public Health, Leiden University College, The Hague, The Netherlands; Corresponding author's e-mail: e.a.l.dejonge@erasmusmc.nl

Background: It is unclear whether dietary patterns influence bone mineral density (BMD) in populations with a relatively high dairy intake. We aimed to identify dietary patterns that are associated with BMD in Dutch elderly subjects.

Methods: Participants were subjects 50 years and over $(\mathrm{n}=5435)$ from The Rotterdam Study, a population-based prospective cohort study. Baseline intake of 28 pre-defined food groups was determined by a validated food frequency questionnaire. Dietary patterns were identified using principal component analysis. Femoral Neck BMD was measured by dual energy X-ray absorptiometry at baseline and at three subsequent visits (1989-2004). The associations between adherence to each pattern and BMD trajectories (all in SDs) were analyzed using mixed models. Analyses were stratified by status of body weight change.

Results: After adjustment for potential confounders, two dietary patterns were significantly associated with higher BMD. A "Traditional" pattern, characterized by high intake of potatoes, meat and fat ( $\beta=0.08: 95 \%$ CI: $0.05,0.11$ in SD BMD per SD pattern-adherence) and a "Mediterranean-like" pattern, characterized by high intake of fruits, vegetables, poultry and fish ( $\beta=0.06$ : $95 \%$ CI: 0.04 , 0.09 ). The "Processed" pattern, characterized by high intake of processed meat and alcohol was associated with lower BMD $(\beta=-0.03$ : $95 \% \mathrm{CI}:-0.06,-0.01)$. The associations of the "Mediterranean-like" and "Processed" pattern were independent of body weight. No effect modification by weight change was observed. Conclusion: In individuals with high dairy intake, a "Mediterraneanlike" and "Traditional" dietary pattern are associated with higher
BMD, whereas a "Processed" dietary pattern is associated with lower BMD.

\section{Abstract \#: O 102}

Trends in anti-osteoporosis drug prescribing after hip fracture in the United Kingdom: 2000-2010

Deborah Gibson-Smith ${ }^{1}$, Corinne Klop ${ }^{1}$, Tjeerd- Pieter van Staa ${ }^{1}$, Petra Elders $^{2}$, Johannes W.J. Bijlsma ${ }^{1}$, Nick Harvey ${ }^{3}$, Paco M.J. Welsing ${ }^{4}$, Hubert Leufkens ${ }^{1}$, F. de Vries ${ }^{1}$

${ }^{1}$ Utrecht Institute for Pharmaceutical Sciences, Utrecht University, Utrecht, The Netherlands; ${ }^{2}$ Department of General Practice and Elderly Care, VU University Medical Centre, Amsterdam, The Netherlands; ${ }^{3}$ MRC Lifecourse Epidemiology Unit, University of Southampton, Southampton General Hospital, Southampton, UK; ${ }^{4}$ Julius Centre for Health Sciences and Primary Care, University Medical Centre Utrecht, Utrecht, The Netherlands; Corresponding author's e-mail: d.gibson-smith@ggzingeest.nl

Background: Osteoporosis is a growing public health issue affecting an estimated 2.4 million people within the United Kingdom. Osteoporosis results in fragility fractures, the most serious of which are hip fractures. Post fracture treatment with anti-osteoporosis drugs is important as a history of hip fracture is associated with a 3.2 increased risk of future fracture. However, despite readily available, effective treatments, pharmacological treatment is sub-optimal. Given the aging population and the increasing number of hip fractures, it is important to know the current trends in primary care prescribing practice for antiosteoporosis drugs. This purpose of this study was to investigate prescribing trends of anti-osteoporosis drugs in treatment naive patients after hip fracture in the United Kingdom between 2000 and 2010.

Method: Data were extracted from the United Kingdom Clinical Practice Research Datalink for patients $\geq 50$ years who had a first hip fracture 2000-2010 $(\mathrm{n}=30,516)$. The proportion of patients who were prescribed anti-osteoporosis drugs (oral bisphosphonates, strontium ranelate, parathyroid hormone, calcitonin and raloxifene) within one year after hip fracture was estimated by Kaplan-Meier life-table analyses. These proportions were stratified according to sex, age groups and drug type. Additionally prescribing trends for calcium, vitamin $\mathrm{D}$ (separately and in combination with anti-osteoporosis drugs) were described.

Results: In the six months before hip fracture, 2974 (9.7\%) patients had received at least one prescription for an anti-osteoporosis drug. After hip fracture an additional $6684(24 \%)$ patients received a prescription for anti-osteoporosis medication. For treatment naive patients, the proportion prescribed any anti-osteoporosis drug after hip fracture increased sixfold from $7.3 \%$ in 2000 to $45 \%$ in 2010 . This increase was more marked in patients $\geq 75$ years. Prevalence and secular increases of anti-osteoporosis therapy were greater in women (10\% in 2000 versus $51 \%$ in 2010) compared to men ( $4 \%$ in 2000 versus $34 \%$ in 2010). Bisphosphonates, and alendronate in particular, were the most frequent form of anti-osteoporosis medication used. The increase in prescribing of anti-osteoporosis drugs was complemented by a similar increase in vitamin $\mathrm{D}$ and calcium provision.

Conclusion: The trends in anti-osteoporosis prescribing reflect drug availability and government guidelines. Although the prescribing of anti-osteoporosis drugs after hip fracture has increased substantially since 2000 , the overall rate is still inadequate, particularly in men. With the continuing increase in the absolute number of hip fractures, further research should be made into the barriers to optimise osteoporosis management in a primary care setting. 


\section{Abstract \#: P 01}

Identification and hierarquization the risk factors for colorectal cancer in Alentejo Litoral: a case control epidemiological study

\section{Sara Letras ${ }^{1}$, Pedro Aguiar ${ }^{2}$, Mário Jorge Santos ${ }^{3}$}

${ }^{1}$ Public Health Unit, Local Health Unit of Alentejo Litoral (ULSLA); ${ }^{2}$ National School of Public Health, Universidade Nova de Lisboa; ${ }^{3}$ Public Health Services, Local Health Unit of Alentejo Litoral (ULSLA); Corresponding author's e-mail: saraletras@gmail.com

Background: Colorectal cancer (CRC) is currently considered a major public health problem internationally and for Portugal. Due to population aging in Alentejo Litoral (AL), the problem get a major magnitude (annual average incidence being around 80 new cases/ 100,000 inhabitants, annual prevalence rate up to 500 cases/100,000, average annual mortality up to 40 deaths $/ 100,000$ ) and that reinforces the importance to identify the main risk factors (RF) for CRC in order to address possible and to maximize effective prevention measures. The main goal of the study it was the identification and its ranking for the main risk factors to CRC.

Methods: It was performed a retrospective analytical epidemiological case-control study, with the Odds ratio (OR) determination. The observation unit it was: to be resident in the $\mathrm{AL}$, and have greater than or equal to 40 years. The study included $90 \mathrm{CRC}$ cases (identified by ROR-Sul) and 201 controls (general medical consultations). Cases and controls were inquired for independent variables included in the study (sociodemographic, family/personal and behavioral history). Descriptive, bivariate and multivariate (logistic regression) statistical analysis (SPPS 20) was performed.

Results: The main results for bivariate analysis (Confidence Interval (CI) $95 \%$ and $\mathrm{p}$ value $<0.10$ ) were: personal history of inflammatory bowel disease $(\mathrm{OR}=9.302)$ : Insulin therapy previous to $\mathrm{CRC}$ $(\mathrm{OR}=6.897)$ : consumption of alcoholic beverages in the past $(\mathrm{OR}=4.853)$ and with a typical frequency greater than 4 times a week $(\mathrm{OR}=3.632)$. The main results for multivariate analysis $(\mathrm{CI}$ $95 \%$ and $p<0.05)$ were: consumption of red meat (adjusted $\mathrm{OR}=6.828$ ), family history for $\mathrm{CRC}$ (adjusted $\mathrm{OR}=6.628$ ) and number of alcoholic drinks (in a typical day) greater than or equal to 3 per day (adjusted OR $=5808$ ).

Conclusion: The main $\mathrm{RF}$ for $\mathrm{CRC}$ in $\mathrm{AL}$ were: red meat consupmtion, family history for CRC and number of alcoholic drinks greater than or equal to 3 per day. The study also concludes that most RF identified for this population are modifiable, can be targeted for interventions of health promotion and disease prevention and establish wich those RF are more cost effective regarding possible health interventions.

\section{Abstract \#: P 02}

Socioeconomic position and incidence of colorectal cancer in the Swedish population

Hannah L. Brooke ${ }^{1}$, Mats Talbäck ${ }^{1}$, Anna Martling ${ }^{2}$, Maria Feychting $^{1}$, Rickard Ljung ${ }^{1}$

${ }^{1}$ Unit of Epidemiology, Institute of Environmental Medicine, Karolinska Institutet, Stockholm, Sweden; ${ }^{2}$ Department of Molecular Medicine and Surgery, Karolinska Institutet, Stockholm, Sweden; Corresponding author's e-mail: hannah.brooke@ki.se

Background: The association between socioeconomic position and incidence of colorectal cancer is unclear. We aimed to clarify this association, in the whole Swedish population. This work may inform policy and prevention strategies designed to reduce health inequalities.

Methods: We conducted a population-based open cohort study using national registry data. We included all individuals, aged $\geq 30$ years, residing in Sweden between 1993 and 2010, without a previous diagnosis of colon or rectal cancer. Socioeconomic position was indicated by (1) highest education level ('Lprimary', 'lower secondary', 'higher secondary', 'lower university' [ $<3$ years], 'higher university' [ $\geq 3$ years]), and (2) personal disposable income (quintiles). The outcome was diagnosis of colon or rectal cancer. We used Poisson regression to estimate incidence rate ratios (IRR) and $95 \%$ confidence intervals $(95 \% \mathrm{CI})$ of colon and rectal cancer, for each exposure. Models were stratified by sex and adjusted for age, year of follow-up, region of residence, and marital status, with mutual adjustment of exposures.

Results: In 100,679,466 person-years of follow-up, 61,793 cases of colon cancer (30,014 men, 31,779 women) and 30,131 cases of rectal cancer (17,379 men, 12,752 women) were diagnosed.

In men and women, IRRs of colon cancer were close to 1.00 for all education levels compared with the least educated, and for all quintiles of personalised disposable income compared with the lowest quintile. However, there was a slightly higher risk of colon cancer in men with 'higher secondary' compared with ' $\leq$ primary' education (IRR [95\% CI]: $1.05[1.02,1.09])$. In women, there was a higher risk of colon cancer in the middle compared with the lowest quintile of personal disposable income (IRR [95 \% CI]: 1.07 [1.03, 1.11]).

Risk of rectal cancer in men and women gradually decreased with increasing education level. Compared with ' $\leq$ primary' education, the IRRs $(95 \% \mathrm{CI})$ of rectal cancer in men with 'lower secondary', 'higher secondary', 'lower university' or 'higher university' education were: 0.99 (0.95, 1.03), $0.94(0.90,0.99), 0.90(0.84,0.96)$, and 0.86 $(0.81,0.92)$, respectively. In women, the corresponding figures were: 0.99 (0.95, 1.04), 0.95 (0.87, 1.04), $0.88(0.82,0.96)$ and 0.87 (0.81, 0.94). Rectal cancer incidence did not differ between quintiles of personal disposable income.

Conclusions: In the Swedish population, incidence of colon cancer was not clearly patterned by socioeconomic position. However, there was lower incidence of rectal cancer in more highly educated groups. To help reduce such health inequalities, further evaluation of potentially preventable mechanisms and health promotion strategies among deprived groups is warranted.

\section{Abstract \#: P 03}

Impact of the choice of life tables on the assessment of socioeconomic inequalities in survival from colorectal cancer

Luis Antunes ${ }^{1}$, Bernard Rachet ${ }^{2}$, Maria José Bento ${ }^{1}$, Denisa Mendonça ${ }^{3}$

${ }^{1}$ North Region Cancer Registry of Portugal, Porto, Portugal; ${ }^{2}$ Cancer Survival Group, London School of Hygiene and Tropical Medicine, London, UK; ${ }^{3}$ EPIUnit - Institute of Public Health, University of Porto, Porto, Portugal; Corresponding author's e-mail: luis.antunes@ipoporto.min-saude.pt

Background: Socioeconomic condition can affect the mortality of a cancer patient from both their cancer and other causes. Assessing socioeconomic inequalities in cancer survival must therefore account for socioeconomic inequalities in background mortality. When the cause of death is unknown (relative survival data setting), net survival is estimated by comparing observed survival with background mortality provided by general population life tables (LTs).

We aimed at evaluating the impact of the choice of LTs in estimating inequalities in colorectal cancer survival by education group. 
Methods: All patients, aged 15-84, diagnosed with a malignant invasive colorectal cancer (ICD-10: C18-C20), in the North Region of Portugal in 2000-2002, were considered for analysis. Education level, the proportion of residents with at least the compulsory level of education in a given census tract, was assigned to each patient based on their area of residence and categorised according to quintiles. Net survival and age-adjusted excess hazards ratios (EHR) were estimated using Pohar-Perme estimator and flexible parametric models, respectively. Three different scenarios were considered for background mortality: no education-specific Portuguese LTs (S1): education-specific LTs considering the same ratios between socioeconomic groups as observed in England (S2): education-specific LTs with a $70 \%$ reduction (relative to S2) in the $\log$ ratio between socioeconomic groups (S3).

Results: A total of 4105 patients (56.3\% male) were analysed. In scenario S1, male patients coming from lower educated areas had comparable 1-year survival to patients from higher educated areas, but a lower 5- and 10-year survival (at 5 years: $\mathrm{EHR}=1.40,95 \% \mathrm{CI}$ 1.07-1.83: at 10 years: $\mathrm{EHR}=1.56,95 \% \mathrm{CI} 1.08-2.25)$. Inequalities in survival decreased substantially in both scenarios S2 (5 years: $\mathrm{EHR}=1.09,95 \%$ CI 0.83-1.43: 10-yrs: $\mathrm{EHR}=1.12,95 \% \mathrm{CI}$ 0.77-1.61) and S3 (5 years: $\mathrm{EHR}=1.30$, $95 \%$ CI $0.99-1.70$ : 10 years: $\mathrm{EHR}=1.41,95 \%$ CI $0.98-2.03$ ).

No significant differences in survival were found in women. Conclusion: No education-specific life tables are available for Portugal. To test the sensitivity of the inequalities found in men to the choice of the LT, we built two sets of education-specific LT in which differences in life expectancy at birth between extreme groups were 7.7 years (S2) and 2.3 years (S3). Cancer survival inequalities observed in $\mathrm{S} 1$ faded out in both scenarios, suggesting that the observed differences are most likely attributable to education inequalities in background mortality, stressing the importance of using the adequate life tables in cancer survival inequalities assessment.

\section{Abstract \#: P 04}

\section{Changes in body weight during and after treatment for colorectal} cancer

Renate M. Winkels ${ }^{1}$, Teunise Snetselaar ${ }^{1}$, Anika Adriaans ${ }^{2}$, Laurence J.C. van Warmerdam ${ }^{3}$, Art Vreugdenhil ${ }^{4}$, Gerrit Slooter ${ }^{4}$, Jan-Willem Straathof ${ }^{4}$, Ellen Kampman ${ }^{1}$, Rianne van Lieshout ${ }^{4}$, Sandra Beijer ${ }^{2}$

${ }^{1}$ Agrotechnology and Food Sciences, Wageningen University, Wageningen, the Netherlands; ${ }^{2}$ Netherlands Cancer Registry, Eindhoven, the Netherlands; ${ }^{3}$ Catharina Hospital, Eindhoven, the Netherlands; ${ }^{4}$ Máxima Medical Centre, Eindhoven, the Netherlands; Corresponding author's e-mail: teunise_s@hotmail.com

Background: Prevalence of overweight and obesity is high among colorectal cancer patients at diagnosis. Literature suggests that body weight may further increase during adjuvant chemotherapy for colorectal cancer. However, so far, weight changes from diagnosis until after treatment have not been studied in this patient group.

Methods: The study population consisted of 485 stage II/III colorectal cancer patients diagnosed between 2007 and 2012 and treated with surgery and adjuvant chemotherapy in one of three selected hospitals in the Netherlands. Eligible patients were selected from the Netherlands Cancer Registry. Data about body weight (at diagnosis, shortly after surgery, shortly after chemotherapy and during followup) and other personal/clinical factors were retrieved from the cancer registry and from medical records.

Results: From diagnosis until shortly after surgery, patients on average lost weight (mean weight loss $-1.9 \mathrm{~kg}$, SD $4.6 \mathrm{~kg}$ ) $(\mathrm{n}=357)$. Body weight increased during chemotherapy with a mean of $2.9 \mathrm{~kg}(\mathrm{SD} 5.8 \mathrm{~kg})(\mathrm{n}=291)$ and continued to increase in the period of follow-up by $2.2 \mathrm{~kg}(\mathrm{SD} 6.6 \mathrm{~kg})(\mathrm{n}=242)$. Overall, from diagnosis until at least 6 months after chemotherapy, there was a mean weight gain of $2.0 \mathrm{~kg}(\mathrm{SD} 6.8 \mathrm{~kg})(\mathrm{n}=283)$. Factors associated with weight gain over this period were a normal BMI (vs patients with a BMI of 25-30), open surgery (vs laparoscopic surgery) and Capecitabine chemotherapy (vs Capecitabine in combination with Oxaliplatin).

Conclusions: Body weight generally decreased from diagnosis until shortly after surgery, while it increased again during and after chemotherapy. At least 6 months after chemotherapy, body weight was higher than at diagnosis. Studies among other patient groupsmostly breast cancer-suggest that these changes may be characterised by unbeneficial changes in body composition, e.g. sarcopenic obesity. Future studies should characterize changes in body weight and composition and the impact on the health and quality of life of colorectal cancer patients.

\section{Abstract \#: P 05}

Interlaboratory variability in grading of dysplasia in a nationwide cohort of colorectal adenomas

Chantal C.H.J. Kuijpers ${ }^{1,2,3}$, Caro E. Sluijter ${ }^{2,4}$, Lucy I.H. Overbeek ${ }^{2}$, Jan H. von der Thüsen ${ }^{5,6}$, Katrien Grünberg ${ }^{6,7}$, Paul J. van Diest ${ }^{1}$, Mehdi Jiwa $^{1,3}$, Iris D. Nagtegaal ${ }^{2,5}$, Stefan M. Willems ${ }^{1,2}$

${ }^{1}$ Department of Pathology, University Medical Centre Utrecht, Utrecht, The Netherlands; ${ }^{2}$ Foundation PALGA, Houten, The Netherlands; ${ }^{3}$ Symbiant Pathology Expert Centre, Alkmaar, The Netherlands; ${ }^{4}$ Department of Pathology, Radboud University Medical Centre, Nijmegen, The Netherlands; ${ }^{5}$ Department of Pathology, Medical Centre Haaglanden, The Hague, The Netherlands; ${ }^{6}$ NVVP (Dutch Society of Pathology), Utrecht, The Netherlands; ${ }^{7}$ Department of Pathology, VU University Medical Centre, Amsterdam, The Netherlands; Corresponding author's e-mail: c.c.h.kuijpers@umcutrecht.nl

Background: Colorectal adenomas are precursor lesions of colorectal adenocarcinoma. One of the risk factors for malignant transformation and future development of a new adenoma or carcinoma is the presence of high-grade dysplasia (HGD). However, this factor is not incorporated in Dutch colonoscopy surveillance guidelines, partly due to high variability in grading dysplasia between pathologists. We aimed to determine, on a nationwide basis, whether histological grading of colorectal adenomas varies in daily practice between Dutch pathology laboratories.

Methods: Using the Dutch Pathology Registry (PALGA), all synoptic pathology reports of colorectal biopsies and polypectomies histologically diagnosed in 2013 as tubular, tubulovillous or villous adenoma were identified. Percentages of low-grade dysplasia (LGD) and HGD were determined for biopsies and polypectomies separately, and clinico-pathological factors associated with HGD were investigated. In a subgroup of 21 Dutch pathology laboratories, each with $\geq 100$ synoptically reported colorectal adenomas, percentages of HGD per laboratory were compared. Univariable and multivariable logistic regression analyses were performed.

Results: Pathology reports of 21,145 colonoscopies of 20,332 patients (57\% males, mean age: 66 year) with $\geq 1$ adenomas were identified. The 32,524 histologically confirmed adenomas included 21,544 adenomas from biopsies and 10,980 adenomas from polypectomies. HGD was diagnosed in $2.6 \%$ and $9.6 \%$ of adenomas from biopsies and polypectomies, respectively. In both subgroups, HGD was significantly associated with advanced age, distal location, 
and villousness. Furthermore HGD was significantly associated with a larger number of biopsies taken, and among polypectomies with larger polyp size and presence of pseudo-invasion. In the subgroup of 21 laboratories, percentages of HGD varied between laboratories from 0 to $11.8 \%$ among 11,742 adenomas from biopsies and from 2.2 to $35.3 \%$ among 5835 adenomas from polypectomies. On multivariable analysis, most laboratories did not seem to differ from each other, except for three laboratories that had a higher frequency of diagnosing HGD on biopsies and polypectomies, and one laboratory that had not diagnosed HGD on biopsies at all.

Conclusion: We observed considerable interlaboratory variation in grading of dysplasia between 21 Dutch laboratories, which can only be partly explained by differences in casemix. Four laboratories $(19 \%)$ were outliers with regard to frequency of diagnosing HGD after multivariable analysis. It is therefore important to find ways to standardize histological grading, and ultimately, if standardization of grading has been achieved, the degree of dysplasia should be incorporated in colonoscopy surveillance guidelines. In future research, we will therefore focus on variation in grading of dysplasia between individual pathologists.

\section{Abstract \#: P 06}

\section{Candidate predictors of health-related quality of life of colorectal} cancer survivors: a systematic review

Martijn J.L. Bours ${ }^{1}$, Roos W.A. van der Linden ${ }^{1}$, Renate M.

\section{Winkels $^{2}$, Fränzel J. van Duijnhoven ${ }^{2}$, Floortje Mols ${ }^{3,4}$, Eline H. van} Roekel $^{1}$, Lonneke V. van de Poll-Franse ${ }^{3,4}$, Ellen Kampman ${ }^{2,5,6}$, Sandra Beijer3, Matty P. Weijenberg ${ }^{1}$

${ }^{1}$ Department of Epidemiology, Maastricht University, GROW School for Oncology and Developmental Biology, Maastricht, the Netherlands; ${ }^{2}$ Division of Human Nutrition, Wageningen University, Wageningen, the Netherlands; ${ }^{3}$ Comprehensive Cancer Center the Netherlands, Netherlands Cancer Registry, Eindhoven, the

Netherlands; ${ }^{4}$ Department of Medical and Clinical Psychology, Tilburg University, Tilburg, the Netherlands; ${ }^{5}$ Department of Health Sciences, VU University Amsterdam, the Netherlands; ${ }^{6}$ Department for Health Evidence, Radboud University Medical Center, Nijmegen, The Netherlands; Corresponding author's e-mail:

m.bours@maastrichtuniversity.nl

Background: Colorectal cancer (CRC) survivors often experience deteriorated health-related quality of life (HRQoL) in both early and late post-treatment phases. Knowledge of predictors of future HRQoL can enable early identification of at-risk individuals and development of tailored strategies for prevention of HRQoL deterioration. A systematic literature review was conducted to identify candidate predictors of HRQoL of CRC survivors.

Methods: Original research articles describing results of multivariable analyses of associations of lifestyle, clinical, sociodemographic, and psychological factors with HRQoL in CRC survivors were searched in PubMed, Embase and Google Scholar, and by citation tracking. Two independent reviewers assessed eligibility and selected articles for inclusion. Strength of evidence for candidate HRQoL predictors was graded based on predefined methodological criteria, i.e., statistical significance, sample size ( $\mathrm{n}<100$ vs. $\mathrm{n} \geq 100$ ), study design (cross-sectional vs. longitudinal), and consistency of findings across studies. The bio-psychosocial framework of the International Classification of Functioning, Disability and Health (ICF) was used to map candidate predictors across the main domains of the ICF framework: health condition, functioning (subdivided in body functions/structures, activities, and participation), and contextual factors (subdivided in environmental and personal factors).
Results: In total, 53 articles published between 1994 and 2014 were included. Evidence for candidate predictors of low HRQoL was most convincing and consistent for the presence of a stoma and more comorbidity (ICF domain: health condition): a higher body mass index and higher levels of fatigue and psychological distress (body functions/structures): lower levels of physical activity (activities): lower socio-economic status and less social support (environmental factors): several psychological and personality variables, such as less optimism and negative cancer threat appraisal (personal factors): and shorter time since diagnosis and lower baseline HRQoL (not covered or defined in ICF). No convincing evidence was found for factors within the participation domain of the ICF. In addition, evidence was inconsistent for factors related to the tumor (health condition), work and marital status (participation), treatment (environmental factors), and gender and age (personal factors) of CRC survivors.

Conclusion: The identified candidate predictors, mapped within the bio-psychosocial ICF framework, will help build comprehensive evidence-based clinical prediction models for estimating future HRQoL in CRC survivors. Such prediction models are useful in clinical practice to identify individual survivors who are at risk of deteriorating HRQoL and thus in need of extra care to prevent HRQoL deterioration.

\section{Abstract \#: P 07}

The association of diagnostic delay and prognosis in colorectal cancer

Sonia Pértega-Díaz ${ }^{1}$, Salvador Pita-Fernández ${ }^{1}$, Luis González-Sáez ${ }^{2}$, Beatriz López-Calviñoo ${ }^{1}$, Teresa Seoane-Pillado ${ }^{1}$, Elena RodríguezCamacho $^{1}$

${ }^{1}$ Clinical Epidemiology and Biostatistics Unit. A Coruña Hospital; ${ }^{2}$ Surgery service. A Coruña Hospital; Corresponding author's e-mail: sonia.pertega.diaz@sergas.es

Background: In symptomatic colorectal cancer (CRC), the association of diagnosis delay with survival is unclear. Some authors have reported an U-shaped association between delay and mortality. The aim is to analyze the relationship between the interval from first symptom to diagnosis (SDI) and survival in colorectal cancer (CRC) patients.

Methods: Observational retrospective cohort study of $n=942$ incident cases of CRC in 1994-2000 ( $n=650$ colon, $n=292$ rectum). SDI was calculated from clinical records as the time from the histologically confirmation of the diagnosis of cancer and the first symptoms noted by the patient and attributed to cancer by the physician.

Colon and rectum tumors were analyzed separately. Survival was compared according to SDI specific quartiles by means of KaplanMeier curves and the log-rank test. Multiple Cox regression was used to estimate 5-year mortality hazard ratios as a function of the diagnosis delay, adjusting for age and gender. SDI was introduced in the models in two ways: as a categorical variable according to SDI quartiles, or as a continuous variable using penalized splines and the specific 50th percentile as reference point.

Results: Median SDI was 3.4 months, significantly higher in rectum than in colon tumors ( 3.7 vs. 3.2 months: $p<0.001$ ). SDI was not significantly associated with stage at diagnosis (Stage I: 3.6 months, Stage II-III: 3.4 months, Stage IV: 3.2 months: $p=0.728$ ). Shorter SDIs corresponded to patients with bowel obstruction (median $=0.9$ months $)$, bowel perforation $(1.2$ months $)$ or intraabdominal abscess (1.3 months), while longer SDIs were observed in patients with mucorrhage (5.2 months) and rectal tenesmus (4.4 months). 
After adjusting for age and gender, in rectum cancers, patients within the first diagnostic interval quartile had significantly lower survival than the rest $(p=0.003)$, while in colon cancer no significant differences were found in prognosis among the four quartile intervals $(p=0.282)$.

The penalized splines regression analysis revealed that, for rectum cancer, 5-year mortality progressively increases for SDIs lower than the median (3.7 months) and significantly decreases as the delay increases until approximately 8 months. In colon cancer, however, no significant relationship was found between SDI and survival.

Conclusion: After CRC symptoms, diagnosis delay seems not to affect survival of patients with tumors located in the colon. For rectum cancers, survival was worst for those patients with lower SDIs.

\section{Abstract \#: P 08}

Associations of total and prolonged sedentary time with healthrelated quality of life in colorectal cancer survivors

Eline H. van Roekel ${ }^{1}$, Elisabeth A.H. Winkler ${ }^{2}$, Martijn J.L. Bours ${ }^{1}$, Brigid M. Lynch $^{3,4,5}$, Paul J.B. Willems ${ }^{6}$, Kenneth Meijer ${ }^{6}$, IJmert Kant $^{7}$, Geerard L. Beets ${ }^{8}$, Silvia Sanduleanu ${ }^{9}$, Genevieve N. Healy $^{2,5,10}$, Matty P. Weijenberg ${ }^{1}$

${ }^{1}$ Department of Epidemiology, GROW School for Oncology and Developmental Biology, Maastricht University, Maastricht, The Netherlands; ${ }^{2}$ The University of Queensland, School of Population Health, Herston, Australia; ${ }^{3}$ Cancer Epidemiology Centre, Cancer Council Victoria, Melbourne, Australia; ${ }^{4}$ Melbourne School of Population and Global Health, Faculty of Medicine, Dentistry and Health Sciences, The University of Melbourne, Melbourne, Australia; ${ }^{5}$ Physical Activity Laboratory, Baker IDI Heart and Diabetes Institute, Melbourne, Australia; ${ }^{6}$ Department of Human Movement Science, NUTRIM School for Nutrition, Toxicology and Metabolism, Maastricht University, Maastricht, The Netherlands; ${ }^{7}$ Department of Epidemiology, CAPHRI School for Public Health and Primary Care, Maastricht University, Maastricht, The Netherlands; ${ }^{8}$ Department of Surgery, GROW School for Oncology and Developmental Biology, Maastricht University Medical Center+, Maastricht, The Netherlands; ${ }^{9}$ Department of Internal Medicine, Division of Gastroenterology and Hepatology, GROW School for Oncology and Developmental Biology, Maastricht University Medical Center+, Maastricht, The Netherlands; ${ }^{10}$ School of Physiotherapy and Exercise Science, Faculty of Health Sciences, Curtin University, Perth, Australia; Corresponding author's e-mail:

eline.vanroekel@maastrichtuniversity.nl

Background: Sedentary behavior (sitting/lying at low energy expenditure while awake) is emerging as an important exposure that may compromise health-related quality of life (HRQoL) of colorectal cancer (CRC) survivors. We examined associations of objectively assessed total and prolonged sedentary time with HRQoL in CRC survivors, 2-10 years post-diagnosis.

Methods: Stage I-III CRC survivors, diagnosed at Maastricht University Medical Center+ (2002-2010), the Netherlands, were included in a cross-sectional study. Sedentary time was measured by the thigh-worn mmoxx 1.0 accelerometer, worn $24 \mathrm{~h} /$ day for seven consecutive days. Linear regression models, adjusting for potential confounders, were used to examine associations of wear-corrected total and prolonged sedentary time (i.e., time accrued in prolonged sedentary bouts $\geq 30 \mathrm{~min}$ ), and usual sedentary bout duration (i.e., $50 \%$ of total sedentary time is accrued in bouts $\geq$ usual bout duration), with HRQoL outcome scores $(n=133-145)$, namely: global health/quality of life; physical; role; and social functioning (EORTC QLQ-C30; 0-100); disability (WHODAS-II; 0-100); fatigue (CIS;
20-140); and, distress (HADS; 0-42). Regression coefficients (ß) are reported, representing the difference in mean HRQoL score per $2 \mathrm{~h} /$ day additional total or prolonged sedentary time, or per $15 \mathrm{~min}$ longer usual sedentary bout duration.

Results: Participants spent 10.2 waking hours/day sedentary (SD, 1.6), and $4.5 \mathrm{~h} /$ day in prolonged sedentary bouts (SD, 2.3) on average. Mean usual sedentary bout duration was $27.3 \mathrm{~min}$ (SD, 16.8). Significantly $(P<0.05)$ lower physical functioning was observed with greater total $(\beta$ : $95 \% \mathrm{CI} ;-7.4:-11.3,-3.4)$ and prolonged sedentary time $(-5.7$ : $-8.7,-2.8)$, and longer usual sedentary bout duration $(-5.7:-8.7,-2.7)$. Significantly higher disability scores were seen with greater total $(5.5: 2.5,8.5)$ and prolonged sedentary time $(4.4: 2.1,6.6)$, and longer usual sedentary bout duration $(4.5: 2.2$, 6.8 ). These sedentary time variables were also associated with significantly higher fatigue scores (8.1: 2.4, 13.8; 7.1: 3.1, 11.1; and 7.2: $3.1,11.2$, respectively). Significantly lower mean global health status/ quality of life and role functioning was observed with greater prolonged sedentary time $(-4.1:-6.9,-1.3$; and $-4.5:-8.5,-0.5$, respectively) and longer usual sedentary bout duration $(-4.5$ : -7.3 , -1.7 ; and -4.9 : $-8.9,-0.8$, respectively). Other associations were non-significant $(P \geq 0.05)$. Most significant associations remained significant following further adjustment for physical activity.

Conclusion: Sedentary time and its accrual in prolonged periods were cross-sectionally associated with poorer HRQoL in CRC survivors. Prospective studies are needed to examine whether sedentary time reduction is a potential target for lifestyle interventions aimed at improving CRC survivors' HRQoL.

\section{Abstract \#: P 09}

\section{Adherence to the WCRF/AICR recommendations in colorectal} cancer survivors: results of the profiles registry

Linde van Lee $^{1}$, Renate Winkels ${ }^{1}$, Martijn Bours ${ }^{2}$, Fränzel van Duijnhoven $^{1}$, Sandra Beijer ${ }^{3}$, Floor Mols ${ }^{3}$, Matty Weijenberg ${ }^{2}$, Meeke Hoedjes ${ }^{4}$, Jeanne de Vries ${ }^{1}$, Anouk Geelen ${ }^{1}$, Ellen Kampman ${ }^{1}$

${ }^{1}$ Division of Human Nutrition, Wageningen University, Wageningen, the Netherlands; ${ }^{2}$ Department of Epidemiology, GROW School for Oncology and Developmental Biology, Maastricht University, Maastricht, the Netherlands; ${ }^{3}$ Comprehensive Cancer Centre the Netherlands, Netherlands Cancer Registry, Eindhoven, the Netherlands; ${ }^{4}$ IHS Institute for Health Sciences, VU University, Amsterdam, the Netherlands; Corresponding author's e-mail: lindevanlee@hotmail.com

Background: Colorectal cancer (CRC) survivors have an elevated risk for recurrence and other chronic diseases and a healthy lifestyle may reduce these risks. Adherence estimates of lifestyle recommendations are lacking in CRC survivors

Objective: To examine the adherence to the World Cancer Research Fund/American Institute for Cancer Research (WCRF/AICR) recommendations and its association with perceived need and beliefs in CRC survivors.

Design: We conducted a cross-sectional study in 1263 CRC survivors diagnosed between 2000 and 2009. All participants filled out a questionnaire on demographics and lifestyle characteristics. A 40-item food frequency questionnaire was used to estimate habitual dietary intake. Information on cancer history and treatment was provided by the Eindhoven cancer registry. We calculated a WCRF/ AICR recommendation adherence score ( $0-8$ points), assigning one point each for eight recommendations. This score was used to calculate prevalence ratios (PR) using Cox proportional hazard statistics adjusting for covariates. 
Results: One survivor completely adhered to all WCRF/AICR recommendations, $12 \%$ of the participants had a score $\geq 6$ points out of the possible 8 points, and $64 \%$ of the participants had a score between 4 and 6 points. Fewest survivors adhered to the recommendations for plant foods, animal foods and sodium. Survivors with a higher adherence score were generally women, older than 65 years, never-smokers, diagnosed less recently, and at early stages. Survivors with a higher adherence score were less likely to have the intention to eat healthier [PR T3 vs. T1 0.69 (95\% CI $0.56-0.86) \quad P$ for trend $=0.001]$. Made dietary changes after cancer diagnosis or received dietary advice was not associated with level of adherence. Conclusions: The present study suggests that CRC survivors could substantially improve their adherence to the WCRF/AICR recommendations, especially for plant foods, animal foods and sodium intake. Survivors with a higher WCRF/AICR score were less likely to have the intention to eat healthier.

\section{Abstract \#: P 10}

\section{Reproductive history and mortality among postmenopausal} colorectal cancer patients

\author{
Mai Kadi ${ }^{1}$, Marc Gunter ${ }^{1}$, Petra Wark ${ }^{2}$, Elio Riboli ${ }^{2}$ \\ ${ }^{1}$ Imperial College London, School of Public Health, Department of \\ Epidemiology and Biostatistics, London, UK; ${ }^{2}$ Global eHealth Unit, \\ Department of Primary Care and Public Health, School of Public \\ Health, Imperial College London, London, UK; Corresponding \\ author's e-mail: mai.kadi09@imperial.ac.uk
}

Background: Circulating oestrogen levels are positively associated with colorectal cancer risk in postmenopausal women. Survival after colorectal cancer diagnosis may also be influenced by sex hormones via similar mechanisms. We investigate the relationship between reproductive factors, which are surrogate measure of lifetime oestrogen exposure, and survival after colorectal cancer diagnosis.

Methods: Study participants included 1556 postmenopausal colorectal cancer patients cases identified from the European Prospective Investigation into Cancer and Nutrition (EPIC). Reproductive factors were assessed at baseline using self-reported questionnaires. During an average of 5.7 years follow up, 527 deaths occurred, 425 of them of colorectal cancer. Cox proportional hazards modelling was used to estimate hazard ratios (HR) and $95 \%$ confidence intervals (CI) reflecting associations between reproductive factors (parity, age at menarche and menopause) and all-cause and colorectal cancerspecific mortality. Models were stratified by age at diagnosis and adjusted for year at diagnosis, tumour stage, tumour grade, smoking and waist circumference.

Result: Compared to women aged 12 years or less at menarche, the participants who had menarche after 15 years of age had a $47 \%$ decreased risk of all-cause and $44 \%$ decreased risk of colorectal cancer-specific mortality (hazard ratio [HR]: 0.53, $95 \%$ confidence interval $[\mathrm{CI}]: 0.34-0.81, \mathrm{P}$ trend: 0.002 and HR: $0.56,95 \% \mathrm{CI}$ : $0.35-0.89$, P trend: 0.002 , respectively). Having ever used oral contraceptive pills (OCs) was associated with $25 \%$ decreased all-cause and $24 \%$ colorectal-cancer specific mortality compared to those who never used them (hazard ratio [HR]: 0.75, $95 \%$ confidence interval [CI]: 0.60-0.94 and HR: 0.76, $95 \%$ CI: 0.60-0.96, respectively).

Conclusion: Our results show that endogenous and endogenous sex hormones may influence prognosis in postmenopausal colorectal cancer patients. If confirmed in other studies, these findings may be further explored in prognostic modelling and influence health monitoring of high-risk groups.

\section{Abstract \#: P 11}

Associations of self-reported lifestyle advice, changes and enjoyment, with health-related quality of life in colorectal cancer survivors

Breedveld-Peters J.J.L. ${ }^{1}$, Bours M.J.L. ${ }^{1}$, van Roekel E.H. ${ }^{1}$, Sanduleanu S. ${ }^{2}$, Beets G.L. ${ }^{3}$, Weijenberg M.P. ${ }^{1}$

${ }^{1}$ Department of Epidemiology, GROW - School for Oncology and Developmental Biology, Maastricht University, Maastricht, The Netherlands; ${ }^{2}$ Department of Internal Medicine, Division of Gastroenterology and Hepatology, GROW - School for Oncology and Developmental Biology, Maastricht University Medical Center+, Maastricht, The Netherlands; ${ }^{3}$ Department of Surgery, GROW School for Oncology and Developmental Biology, Maastricht University Medical Center+, Maastricht, The Netherlands; Corresponding author's e-mail:

jose.breedveld@maastrichtuniversity.nl

Background: There is a paucity of research on lifestyle recommendations for colorectal cancer (CRC) survivors. An important step in this research area is to gain insight into (1) the lifestyle changes that CRC survivors undertake after treatment, (2) the lifestyle advices that they currently receive, and (3) whether survivors enjoy their diet and physical activity (PA) as much as before diagnosis. Furthermore, it is of interest to assess how these factors are associated with healthrelated quality of life (HRQoL) in CRC survivors.

Methods: In the cross-sectional part of the Energy for life after ColoRectal cancer (EnCoRe) study, self-reported dietary advice received, dietary changes, enjoyment of diet, PA advice received, and enjoyment of PA were assessed in 2-10 y post-diagnosis stage I-III CRC survivors $(\mathrm{N}=151)$, who had been treated between 2002 and 2010 at Maastricht University Medical Center+, the Netherlands. Cancer-specific HRQoL was assessed by the EORTC QLQ-C30 (global QoL, physical and social functioning). The EORTC QLQCR29 module was used to measure CRC-specific HRQoL and symptoms. Fatigue was assessed by the Checklist Individual Strength (CIS). Associations of dietary and PA determinants with HRQoL were analyzed by multivariable lineair regression models.

Results: Twenty one percent of CRC survivors $(n=31)$ received dietary advice, $36 \%(\mathrm{n}=54)$ reported dietary changes since diagnose, and $7 \%(\mathrm{n}=11)$ reported less enjoyment of their diet than before diagnosis. PA advice was received by $34 \%(n=52)$ of CRC survivors, and $25 \%(\mathrm{n}=37)$ reported less enjoyment of PA. Dietary advice was only significantly associated with soreness of the anal skin $(p=0.019)$, while dietary change was not significantly associated with any of the HRQoL outcomes. Less dietary enjoyment was significantly related to lower global QoL $(p=0.017)$, lower physical functioning $(p=0.037$, less appetite $(p<0.001)$, more fatigue $(p<0.001)$, and lower body image $(p<0.001)$, bloating $(p=0.012)$, dry mouth $(p=0.007)$, and problems with taste $(p<0.001)$. PA advice was not significantly associated with any of the HRQoL outcomes. Less PA enjoyment was significantly associated with lower global QoL $(p<0.001)$, lower social functioning $(p<0.001)$, more fatigue $(p<0.001)$, and with dry mouth $(p=0.001)$.

Conclusion: A minority of CRC survivors reported to have received dietary and PA advice. No significant associations of self-reported dietary advice, dietary changes and physical activity advice with HRQoL were observed. However, reduced enjoyment of PA and of diet were found to be associated with several HRQoL outcomes and symptoms. Prospective studies are needed to further explore relationships of dietary and physical activity advice, changes, and enjoyment with HRQoL. 


\section{Abstract \#: P 12}

Hypomethylation of the ahrr and F2RL3 genes is associated with lung cancer: genome-wide study in two prospective cohorts

Erica Ponzi ${ }^{1}$

${ }^{1} \mathrm{HuGeF}$ Foundation; Corresponding author's e-mail: erica.ponzi@hugef-torino.org

Background: Epigenetic changes are common events in most, if not all, human malignancies. Among them, DNA methylation is particularly important for stable propagation of gene activity states over cell generations. In particular, genome-wide hypomethylation seems to be an early event in carcinogenesis, and several studies have reported that specific DNA hypomethylation is associated with cancer risk. A decrease in DNA methylation of $\mathrm{CpG}$ sites in the AHRR gene, particularly cg05575921, has also been found as a strong marker of smoking.

Methods: We performed a genome-wide methylation analysis of 132 incident lung cancer cases and 132 controls in the Norwegian Women and Cancer (NOWAC) cohort and then verified our results in the case-control study in a case-control study within the Melbourne Collaborative Cohort Study (MCCS), in which 367 controls were tightly matched to 367 cases on the history of cigarette smoking.

We performed association analysis in both cohorts to detect which probes were significantly associated with lung cancer risk and then considered the impact of smoking cessation on their methylation levels. We applied a mediation analysis to NOWAC data to elucidate whether methylation of these probes was a mediator of the association between smoking and lung cancer.

Results: A CpG site in the AHRR gene (cg05575921) that was previously shown to be strongly hypomethylated in smokers was also the top hit in association with lung cancer risk $\left(p\right.$ value $=3.33 \times 10^{-11}$ in NOWAC). The second hit was CpG cg03636183 in F2RL3, also among the probes previously found to be associated with smoking.

In the MCCS replication cohort we confirmed strong inverse associations with lung cancer risk for both probes $\left(p=1.3 \times 10^{-5}\right.$, for $\operatorname{cg} 0557591$ : and $p=1.5 \times 10^{-4}$ for $\operatorname{cg} 03636183$ ).

Regarding the effect of smoking cessation, we observed in both cases that parallel to a decline in the risk of lung cancer, methylation levels reverted back to the levels of never smokers with years since quitting. Mediation analysis suggested that about $30-35 \%$ of the effect of smoking on lung cancer was explained by methylation of the selected probes.

Conclusions: Our data support the hypothesis that methylation of AHRR and F2RL3 genes is in the pathway between smoking and lung cancer.

\section{Abstract \#: P 13}

Tobacco and alcohol consumption and nasopharyngeal carcinoma (NPC) mortality: a Guangzhou cohort study on 93,672 Chinese males

Jia Huang $\operatorname{Lin}^{1}$, Ching Man $\mathrm{Lo}^{1}$, Zhi Ming Mai ${ }^{1}$, Chao Qiang Jiang ${ }^{2}$, Wei Sen Zhang ${ }^{2}$, Sai Yin $\mathrm{Ho}^{1}$, Tai Hing Lam ${ }^{1}$

${ }^{1}$ School of Public Health, University of Hong Kong, Hong Kong, China; ${ }^{2}$ Guangzhou Occupational Disease Prevention \& Treatment Center, Guangzhou, China; Corresponding author's e-mail: kawongl@hku.hk

Background: Nasopharyngeal carcinoma (NPC) is rare in most of the world, but has high incidence and mortality in North Africa, Southeast Asia, especially in Guangdong, China. Smoking and drinking are well-known risk factors for head and neck cancers. However, for NPC despite over 35 years of research, epidemiologic evidence is conflicting and prospective evidence is scarce. The Guangzhou Occupational Cohort is a large prospective study conducted in an NPC high-risk region. We conducted further analysis to study the effect of smoking and drinking on NPC mortality.

Methods: Worker and drivers in Guangzhou were required to undergo annual medical examinations during 1980s to 1990s. From March 1988 to December 1992, information including demographic characteristics, smoking and drinking, was collected through occupational health examinations in factories or driver examination stations. Vital status and causes of death up to December 1999 were retrieved by two physicians, and verified by an epidemiologist. Subjects who reported having any diseases at baseline and those with missing information on smoking and drinking information were excluded. 93,672 male subjects included in present analysis were divided into 2 sub-cohorts (15,656 workers and 78,016 drivers) and Cox model was used to estimate relative risks (RRs) from hazard ratios.

Results: The mean age (SD) of subjects was 42 (6.6) years, and the mean follow-up was 7.5 years. 35 NPC deaths were observed. Smoking was associated with increased risk of NPC mortality in each of the sub-cohorts, so they were combined for analysis. Compared with never smokers, the crude RR of NPC mortality was 2.5 (95\% CI $1.14-5.60, p=0.02$ ) for daily smokers. RRs after adjusting by age, education and cohort status increased from 1.58 (95 \% CI 0.57-4.39, $p=0.38)$ in smokers of less than 15 cigarettes/day to $2.33(95 \% \mathrm{CI}$ $1.00-5.47, p=0.05$ ) for more than 15 cigarettes/day ( $p$ for trend $=0.05)$. Similar trend was observed for smokers of 1-9 packyears and over 10 pack-years $(p$ for trend $=0.04$ ). For alcohol, in the worker sub-cohort, compared to never drinkers, the crude RR of daily drinkers was 4.56 (95\% CI 1.34-15.60, $p=0.02)$. After adjusting by age, education and smoking status, RR of daily drinkers was 3.67, which was marginally significant (95\% CI 0.95-14.22, $p=0.06)$. In contrast, no significant association between alcohol and NPC mortality was found in the driver sub-cohort.

Conclusions: This is the first large cohort study in a high-risk region which has provided prospective dose-response evidence that smoking can increase the risk of NPC mortality in men. Increased NPC mortality risk for daily drinking was found in the worker sub-cohort, but not in the driver sub-cohort. Further studies are warranted.

\section{Abstract \#: P 15}

Dairy consumption and cancer mortality: a proportional mortality case-control study in Hong Kong

Zhi-Ming Mai ${ }^{1}$, Tai-Hing Lam $^{1}$, Sai-Yin $\mathrm{Ho}^{1}$

${ }^{1}$ School of Public Health, The University of Hong Kong, Hong Kong, P.R. China; Corresponding author's e-mail: maizm@hku.hk

Background: No recommendation for cancer prevention is for dairy product intakes because the evidence is inconclusive and conflicting. In the World Cancer Research Fund/American Institute for Cancer Research (WCRF/AICR) report in 2007 "Food, Nutrition, Physical Activity and the Prevention of Cancer: A Global Perspective", it was stated that there is probable evidence suggesting that milk protects against colorectal cancer, but high consumption of milk and dairy products increase the risk of prostate cancer. Since then, more evidence on the association between dairy consumption and cancer has emerged. Two updated meta-analysis of prospective studies have concluded increased dairy consumption was associated with reduced risk of colorectal cancer, but increased risk of prostate cancer. Consumption of dairy products has greatly increased globally, especially 
in China with rapid economic development. Studies on dairy consumption and cancer among Chinese are scarce, and the association is unclear. The aim of this study is to investigate the association between dairy consumption and cancer mortality among Chinese.

Methods: In the LIMOR (LIfestyle and MORtality) study, a large population-based case-control study including $81 \%$ of all Hong Kong registered deaths in 1998, we used adjusted logistic regression to compare consumption of dairy product (milk (powder), cheese and ice-cream) in decedents aged $\geq 30$ years from malignant neoplasms $(14,726)$ and 13,157 living controls, and 16,379 dead controls (other deaths excluding cancer), adjusting for age (5-year age groups), education, alcohol and smoking.

Results: Using living controls, the adjusted odds ratios (AORs) for all-cancer mortality for dairy product consumption of less than 3 times per month and 1 or more times per week compared with no consumption were 1.14 (95 \% CI: $0.99,1.29)$ and $1.19(1.07,1.33)$ for men, $1.37(1.19,1.58)$ and $1.27(1.12,1.43)$ for women, and 1.21 $(1.10,1.33)$ and $1.20(1.11,1.30)$ for both sexes together. Increased mortality risks were also found using dead controls for less than 3 times per month and 1 or more times per week (AOR: 1.11 (1.01, $1.22)$ and $1.18(1.08,1.28)$ for men, $1.12(0.99,1.91)$ and $1.12(1.02$, $1.24)$ for women, and $1.12(1.04,1.21)$ and $1.16(1.09,1.23)$ for both sexes) compared with no consumption.

Conclusion: Increased dairy product consumption was positively associated with all-cancer mortality among Chinese. Further detailed analyses for different types of dairy products and cause-specific mortality are warranted.

Acknowledgement: Hong Kong Health Services Research Committee (631012) and Hong Kong Council on Smoking and Health.

\section{Abstract \#: P 16 \\ Population mixing and risk of childhood leukaemia in Switzerland}

Judith E. Lupatsch $^{1}$, Christian Kreis ${ }^{1}$, Marcel Zwahlen ${ }^{1}$, Felix Niggli $^{2}$, Roland A. Ammann ${ }^{3}$, Claudia E. Kuehni ${ }^{1}$, Ben D. Spycher ${ }^{1}$

${ }^{1}$ Institute of Social and Preventive Medicine, University of Bern, Switzerland; ${ }^{2}$ University Children's Hospital Zurich, Switzerland; ${ }^{3}$ Department of Pediatrics, University of Bern, Switzerland; Corresponding author's e-mail: judith.lupatsch@ispm.unibe.ch

Background: The aetiology of childhood leukaemia (CL) is still poorly understood. The population mixing hypothesis proposes that CL might be a rare complication to a yet unidentified subclinical infection. Population influxes into rural areas may foster localised epidemics of the postulated infection causing a subsequent increase in the incidence of CL. Previous studies investigating this hypothesis using census data have found mixed results. These studies assessed population mixing during single time periods before or between given censuses. To our knowledge, no study has assessed population mixing continuously over a larger time period to determine whether periods of marked population increase coincide with increased incidence as postulated Kinlen's hypothesis. We aimed to investigate whether incidence of CL was increased in Swiss municipalities in time periods of high population growth and high in-migration.

Methods: We identified incident cases of $\mathrm{CL}<16$ years between 1985 and 2010 though the Swiss Childhood Cancer Registry (SCCR) and obtained population time-series for each municipality for the period 1981-2010 from national statistics. For each municipality and year (1985-2010) we calculated population growth and in-migration during a 5-year period centered on that year as percentage of the 1981 population. To obtain person years at risk, we calculated the proportion of population aged $<16$ years in each municipality at censuses
(1980, 1990, 2000, 2010) and interpolated for the years between accounting for absolute population levels in municipalities in noncensus years. We investigated associations between CL incidence and quintiles of exposure variables using sex and age adjusted Poisson regression models with random effects components for municipalities. We also investigated effect modification by urbanization (urban/ rural) and associations with exposure variables time shifted 1-5 years before diagnosis to allow for possible latent periods.

Results: Our analyses included 1445 incident cases of leukaemia over $39,744,468$ person years at risk. The incidence rate ratio (IRR) comparing the highest with the lowest quintile was 1.31 (95\% CI: 1.07-1.61; global $p=0.074)$ for population growth and 1.11 (95\% CI: $0.92-1.35$; global $p=0.265$ ) for in-migration. There was no evidence of association when accounting for latent periods (global $p>0.357$ for population growth and $p>0.930$ for in-migration) and no evidence of effect modification by urbanization $(p>0.640)$.

Conclusion: Our study is compatible with the population mixing hypothesis but provides little support that the incidence of CL was markedly increased in Swiss municipalities during time periods of high population growth or high in-migration.

\section{Abstract \#: P 17}

Effect of the partner's health and support on cancer patients' GP consultation behaviour

Marianne J. Heins ${ }^{1}$, Petra Hopman ${ }^{1}$, Joke C. Korevaar ${ }^{1}$, Mieke Rijken $^{1}, \mathrm{Ge}$ Donker ${ }^{1}$, Francois Schellevis ${ }^{1,2}$

${ }^{1}$ Netherlands Institute for Health Services Research (NIVEL), Utrecht, The Netherlands; ${ }^{2}$ Department of General Practice/EMGO Institute for Health and Care Research, VU University Medical Centre, Amsterdam; Corresponding author's e-mail: m.heins@nivel.nl

Background: Partners of cancer patients are often an important source of support. However, the diagnosis and taking care may affect their physical and psychological health. They may, therefore, not always be able to provide the support patients need. Patients may then use formal health care as a substitute. In addition, some types of partner support may even adversely affect psychological health of patients and may thus lead to increased health care use. We therefore examined whether the health of partners and the support they provide affects GP consultation behaviour of cancer patients.

Methods: Cancer patients diagnosed at adult age with a cancer type with a 5-year survival rate $>20 \%$ and no distant metastases at diagnosis were sent a questionnaire, as were their partners. Patients' GP consultation behaviour for 'physical', 'emotional', 'practical', 'family-social' and 'religious/spiritual' problems in the past 12 months was assessed. Partner support as perceived by the patient was measured by three scales: active engagement, protective buffering and overprotection.

Results: We included 219 patients and their partners. In the past 12 months, patients (0-19 years post-diagnosis) had most often discussed physical problems with their GP (130 patients, $60 \%)$, followed by emotional problems (61 patients, $28 \%$ ). Other types of problems were less often discussed. Patients were less likely to visit their GP for physical problems when they experienced active engagement from their partner. Patients were less likely to visit their GP for emotional problems when they felt their partner was overprotecting.

Conclusion: We found that partner support affects GP consultation behaviour in cancer patients. GPs and other health care providers should therefore inform partners about beneficial types of partner 
support, especially active engagement. GPs could also inform partners about available support groups and facilities.

\section{Abstract \#: P 18}

Prevalence and correlates of healthy lifestyle behaviors among early cancer survivors in The Netherlands

Iris M. Kanera $^{1}$, Catherine A.W. Bolman ${ }^{1}$, Ilse Mesters ${ }^{2}$, Roy A. Willems ${ }^{1}$, Audrey A.J.M. Beaulen ${ }^{1}$, Lilian Lechner ${ }^{1}$

${ }^{1}$ Faculty of Psychology and Educational Sciences, Open University of the Netherlands, Heerlen, The Netherlands; ${ }^{2}$ CAPHRI School for Public Health and Primary Care, Maastricht University, Maastricht, The Netherlands; Corresponding author's e-mail: iris.kanera@ou.nl

Background: Healthy lifestyle behaviors are associated with positive health outcomes and quality of life in cancer survivors. However, adherence to lifestyle recommendations is low. To improve health enhancing behaviors, insight is needed into the factors that relate to engagement in lifestyle behaviors. This study explored the contribution of distal (socio-demographic, cancer-related, psychological), and proximal factors (attitude, social support, self-efficacy). Abovementioned factors and intention were assessed in relation to five lifestyle behaviors (smoking, physical activity, and alcohol, fruit, and vegetable consumption).

Methods: We conducted a cross-sectional survey among early cancer survivors with various types of cancer. Participants $(\mathrm{N}=255: 70.7 \%$ females: mean age 60.6 years) were recruited from 8 Dutch hospitals. Socio-demographic, cancer-related, psychological, and social cognitive factors, intention, and lifestyle behaviors were assessed with selfreport questionnaires (e.g. EORTC QLQ-C30, HADS, IPAQ). Crosssectional analyses (correlations and regression analyses) were conducted.

Results: The majority ( $>80 \%$ ) of the cancer survivors complied with two to four health recommendations. A small group (11\%) adhered to all five recommendations, while fewer than $7 \%$ adhered to one or no recommendations. Highest adherence to the recommendations was found for engaging in physical activity $(87.4 \%)$, refraining from smoking (82\%), and limiting alcohol consumption (75.4\%). Poor adherence was found for the recommendations relating to fruit $(54.8 \%)$ and vegetable $(27.4 \%)$ intake. The associations between the different behaviors were weak, however, people who drank more alcohol and smoked more were less likely to adhere to the fruit recommendation. Each separate lifestyle behavior was influenced by different factors. Self-efficacy, attitude, and intention were the strongest correlates in all behaviors examined - although with varying strength-while socio-demographic, cancer-related, and psychological factors made much smaller contributions to the explained variance of behaviors. Moreover, adherence to a higher number of recommendations was exclusively significantly correlated to attitude, social support, self-efficacy, and intention.

Conclusions: Adherence to fruit consumption was poor and adherence to vegetable consumption even worse, however, there is room for improvement in all five lifestyle behaviors. Our findings emphasize that all relevant lifestyle behaviors need to be encouraged in cancer survivors, taking into account that each behavior is influenced by a specific set of mainly social cognitive or intention-related factors. These outcomes contributed to the development of a Cancer aftercare intervention, tailored to individual's situations, in particular to social cognitive factors and intentions, and targeting multiple lifestyle behaviors.

\section{Abstract \#: P 19}

\section{Lower gastrointestinal bleeding and risk of gastrointestinal} cancer

Søren Viborg ${ }^{1}$, Kirstine K. Søgaard ${ }^{1}$, Dora K. Farkas ${ }^{1}$, ${ }_{\text {Helene Nørrelund }}{ }^{\text {, Henrik T. Sørensen }}{ }^{1}$

${ }^{1}$ Department of Clinical Epidemiology, Aarhus University Hospital, Denmark; Corresponding author's e-mail: sovi@clin.au.dk

Background: Lower gastrointestinal bleeding is a well-known first symptom of colorectal cancer. However, whether it is also a marker of other types of gastrointestinal cancer in patients with incident bleeding diagnosed in hospital has been sparsely investigated.

Methods: This nationwide cohort study examined the risk of various types of gastrointestinal cancer in patients with lower gastrointestinal bleeding. We used Danish medical registries to identify all patients with a first-time hospital diagnosis of lower gastrointestinal bleeding (1995-2011) and followed them up to 10 years for subsequent gastrointestinal cancer diagnoses. We first calculated absolute risks of cancer, treating death as a competing risk. We then calculated standardized incidence ratios (SIRs) by comparing observed cancer cases with national general population cancer incidence rates.

Results: Among 60,093 patients (49\% men) with lower gastrointestinal bleeding, we observed 2845 gastrointestinal cancers during complete follow-up, corresponding to an overall SIR of $3.91(95 \%$ confidence interval (CI): 3.77-4.06), and a 10-year absolute risk of any gastrointestinal cancer of $5.5 \%$. During the first year of followup, the SIR of any gastrointestinal cancer was 16.1 (95\% CI: 15.4-16.8), mainly due to an excess of colorectal cancers, but all gastrointestinal cancers were diagnosed more frequently than expected. The 1-5 year SIR of any gastrointestinal cancer declined to 1.38 (95 \% CI: 1.26-1.51), and apart from rectal and gall-bladder cancer, the risk of any individual gastrointestinal cancers remained increased in this period. Beyond 5 years of follow-up, the SIR of any gastrointestinal cancer was close to unity: however, the risk of rectal cancer was reduced, whereas the risk of liver and pancreatic cancers persisted 5 years and more after the lower gastrointestinal bleeding. Conclusions: Lower gastrointestinal bleeding is a strong clinical marker of prevalent gastrointestinal cancer, particularly colorectal cancer, but it also predicts an increased risk of gastrointestinal cancer beyond 1 year of follow-up.

\section{Abstract \#: P 21}

Dietary mineral intake and lung cancer risk: the Rotterdam study

Taulant Muka ${ }^{1}$, Bledar Kraja ${ }^{1}$, Rikje Ruiter ${ }^{1}$, Catherine E. de Keyser $^{1}$, Albert Hofman ${ }^{1}$, Oscar H. Franco ${ }^{1}$, Bruno H. Stricker ${ }^{1}$, Jessica C. Kiefte-de Jong ${ }^{1}$

${ }^{1}$ Department of Epidemiology, Erasmus MC, Rotterdam, The Netherlands; Corresponding author's e-mail: t.muka@erasmusmc.nl

Background: Epidemiological studies evaluating the role of dietary mineral intake on lung cancer risk have reported inconsistent results. We investigated whether dietary calcium, copper, iron, magnesium, selenium and zinc intake were associated with lung cancer risk.

Methods: We analyzed data from 5435 participants of the Rotterdam Study, a prospective population-based cohort study among subjects aged 55 years and older. At baseline (1990-1993), diet was measured by a validated food frequency questionnaire. Lung cancer events were 
pathology proven. Hazard ratios (HRs) for energy-adjusted mineral intake were calculated using Cox regression models while adjusting for age, gender, alcohol intake, body mass index, smoking status, physical activity, dietary quality, red meat intake, hormone replacement therapy, type 2 diabetes mellitus, socioeconomic background and family history of cancer.

Results: During a follow-up period of 22 years, we identified 212 incident cases of lung cancer. A higher zinc intake was associated with $43 \%$ reduction in risk for lung cancer (top tertile vs. first tertile: $\mathrm{HR}=0.57: 95 \% \mathrm{CI}=0.35,0.93: P$ for trend $=0.036$ ). Similarly, high intake of iron was associated with reduced risk for lung cancer (top tertile vs. first tertile: $\mathrm{HR}=0.61: 95 \% \mathrm{CI}=0.38,0.96: P$ for trend $=0.033$ ). There was no association between dietary intake of calcium, copper, magnesium and selenium and lung cancer risk.

Conclusion: Our results suggest that dietary zinc and iron intake are associated with reduced risk of lung cancer. No evidence was found for an association between calcium, copper, magnesium and selenium intake and lung cancer risk.

\section{Abstract \#: P 22}

Body mass index and lung cancer survival: results from the ICARE study

Harinakshi Sanikini ${ }^{1}$, Gabrielle Clain ${ }^{1}$, Marie Sanchez ${ }^{1}$, AnneValérie Guizard ${ }^{2}$, Anne-Sophie Woronoff ${ }^{3}$, Brigitte Tretarre ${ }^{4}$, Florence Molinie $^{5}$, Patricia Delafosse ${ }^{6}$, Danièle Luce ${ }^{7}$, $\underline{\text { Isabelle Stücker }}^{1}$

${ }^{1}$ Inserm, Centre for Research in Epidemiology and Population Health (CESP), U1018, Cancer Epidemiology: Gene, Environment Team, F-94807 Villejuif, France; ${ }^{2}$ Registre des Cancers du Calvados, Centre Francois Baclesse, Caen, France; ${ }^{3}$ Registre des Cancers du Doubs, CHU Besançon, Hôpital Jean Minjoz, Besançon, France; ${ }^{4}$ Registre des Cancers de ll'Hérault, ICM Regional Cancer Institute of Montpellier, Montpellier, France; ${ }^{5}$ Registre des Cancers de LoireAtlantique et de Vendée, CHU de Nantes, Nantes, France; ${ }^{6}$ Registre des Cancers de 11'Isère, CH Mutualiste de Grenoble, Grenoble, France; ${ }^{7}$ Santé et Environnement, Inserm UMRS 1085, Pointe-à-Pitre, France ; Corresponding author's e-mail: isabelle.stucker@inserm.fr

Background: Several epidemiological studies have shown that lower body mass index (BMI) is associated with increased risk of lung cancer. However, studies investigating the association between BMI and lung cancer survival are limited. We investigated whether BMI 2 years before interview and BMI at interview is associated with lung cancer survival in the ICARE study (Investigation of occupational and environmental causes of respiratory cancers).

Methods: A population-based case-control study was conducted in ten departments of France between 2001 and 2007. Analyses were based on 2285 lung cancer cases. Data on anthropometric measurements (height, weight 2 years before interview and weight at interview) were collected using standardized questionnaires through face-to-face interviews, and these data were used to calculate BMI 2 years before interview and BMI at interview. BMI $\left(\mathrm{kg} / \mathrm{m}^{2}\right)$, was classified into 4 categories: $(<18.5),(18.5-25),(25-30)$ and $(\geq 30)$. Cox proportional hazard models were used to estimate the hazard ratios (HR) and corresponding $95 \%$ confidence interval (CI). Models were adjusted for age, gender, area of residence, education level and comprehensive smoking index. BMI $\left(18.5-25 \mathrm{~kg} / \mathrm{m}^{2}\right)$ was used as a reference group.

Results: Of the 2285 lung cancer cases that were included in this study, a total of 1988 subjects died. No significant association was observed between BMI 2 years before interview and lung cancer mortality. Adjusted hazard ratios were 0.96 (95 \% CI: 0.73-1.27) for subjects with $\mathrm{BMI}<18.5 \mathrm{~kg} / \mathrm{m}^{2}, 0.98$ (95\% CI: $\left.0.88-1.09\right)$ for subjects with BMI $25-30 \mathrm{~kg} / \mathrm{m}^{2}$ and 1.00 (95\% CI: 0.87-1.14) for subjects with BMI $\geq 30 \mathrm{~kg} / \mathrm{m}^{2}$. For BMI at interview and lung cancer mortality, we found a significant positive association among subjects with $\mathrm{BMI}<18.5 \mathrm{~kg} / \mathrm{m}^{2}$ (HR 1.39: $95 \%$ CI: 1.19-1.64), while a significant inverse association was observed among subjects with BMI $25-30 \mathrm{~kg} / \mathrm{m}^{2}$ (HR 0.78: $95 \%$ CI: 0.70-0.87) and $\mathrm{BMI} \geq$ $30 \mathrm{~kg} / \mathrm{m}^{2}$ (HR 0.82: $95 \%$ CI: 0.69-0.97).

Conclusion: These data suggests that BMI 2 years before interview is not associated with lung cancer mortality. However, lower BMI $\left(<18.5 \mathrm{~kg} / \mathrm{m}^{2}\right)$ at interview is associated with increased lung cancer mortality.

\section{Abstract \#: P 23}

Cancer incidence and survival among children and adolescents in Campinas-SP, BRAZIL

Jane K. O. Friestino $^{1}$, Denisa Mendonça ${ }^{2}$, Pedro Oliveira ${ }^{2}$, Djalma de C. Moreira Filho ${ }^{3}$

${ }^{1}$ CAPES Foundation, Ministry of Education of Brazil, Brasília, Brazil; ${ }^{2}$ Institute of Public Health (ISPUP), University of Porto (UP), Porto, Portugal; ${ }^{3}$ University of Campinas, School of Medical Sciences, Department of Collective Health, Campinas - SP, Brasil; Corresponding author's e-mail: janekos@gmail.com

Background: Childhood cancers (age at diagnosis 0-19 years) comprise a variety of malignancies. Currently in Brazil, neoplasms correspond to the second leading cause of death in children only surpassed by external causes. Campinas-SP, located in the Southwest area in Brazil, has a Population-based Cancer Registry-PBCR operating since 1990. Although, population-based studies concerning childhood cancer incidence and survival are important to evaluate local health services, scarce studies are available for the region. We analyse the incidences and survival time of children (0-14 years) and adolescents (15-19 years) in Campinas-SP-Brazil.

Methods: Data were retrieved of all children and adolescents residing in Campinas, aged 0-19 years, diagnosed between 1996 and 2005. The follow-up censored date was December 31, 2011. Cancers were classified according to the ICCC-3. By design, just the 4 most common groups were studied: Group I-Leukemias: Group II-Lymphomas and reticuloendothelial neoplasm: Group III-Central Nervous System and miscellaneous intracranial and intraspinal neoplasm and Group IX-Soft tissue and other extra osseous sarcomas. Age-standardized incidence rates were calculated using the world standard population. Kaplan-Meier estimation methods, long-rank test and Cox regression were used for survival analysis.

Results: A total of 180 cases were diagnosed: 154-children and 26-adolescents. The most frequent neoplasms were: Acute lymphoblastic (children: $37.7 \%$ : adolescents: $15.4 \%$ ), Acute nonlymphoblastic (children: $13 \%$ : adolescents: $23.1 \%$ ), Astrocytomas (children: $11 \%$ ) and Hodgkin lymphomas (adolescents: $19.2 \%$ ). Pronounced differences in the male/female ratio were observed for Group II. Overall crude incidence rate was 54.2 per million (64.2 in children and 28.2 in adolescents). Overall standardized incidence rates (cases per million) were: 0-19 years, 59.1-Group I-28.8, Group II-11.6, Group III-7.5 and Group IX-4.3. Overall five-year survival was $65.6 \%$ (95\% CI: 57.4-72.9\%) among children and $59.6 \%$ (95\% CI: 40.7-79\%) among adolescents. Significant differences in survival times were found by diagnostic Groups adjusted for age and sex $(p=0.002)$, with lower survival in the Group IX (HRadj $=7.05$-Group-IX vs Group-II) followed by Group III (HRadj $=6.56-$ Group-III vs. Group-II). 
Conclusion: In Campinas-SP, similarly to previous reports from European Registries, the most frequent childhood cancer is Group I, presenting standardized incidence rates lower than those of the majority of European countries but similar to Portugal and Poland. The 5-year survival estimated for adolescents was higher than in children. These findings can be used as baseline indicators of the quality of the healthcare system and as a framework for future improvements and health planning.

\section{Abstract \#: P 24}

Breastfeeding and other early life exposures and the risk of childhood leukemia and lymphoma in Israel: a case control study

Efrat Lev Amitay ${ }^{1}$, Lital Keinan Boker ${ }^{1,2}$

${ }^{1}$ School of Public Health, University of Haifa, Haifa, Israel; ${ }^{2}$ Israel Center for Disease Control, Ministry of Health, Israel; Corresponding author's e-mail: 1keinan@univ.haifa.ac.il

Background: Childhood cancer incidence increases in the western world and although rare, it is a leading cause of mortality. Leukemia and lymphoma comprise together $40 \%$ of all cancers in this age group, but little is known of their etiology. This study aimed to examine the associations of breastfeeding and other early life exposures relating to the 'infectious agent' theory with childhood leukemia and lymphoma.

Methods: A population based case control study was conducted in 2011-2013 comprising of mothers of 190 prevalent (2005-2013) leukemia/lymphoma cases aged 1-19 years at diagnosis and 384 controls. The mothers were interviewed using a computerized structured questionnaire. Association between breastfeeding patterns and childhood leukemia/lymphoma was assessed by multivariate logistic regression models adjusted for potential confounders.

Results: Ever breastfeeding was associated with a $64 \%$ decreased risk for childhood leukemia/lymphoma (OR $=0.36,95 \%$ CI: 0.22 , 0.60). Breastfeeding for 6,12 and $18+$ months, compared to shorter durations, have also been found to be associated with lower risks of childhood leukemia and lymphoma implying a dose-response effect. Other infant exposures associated with cancer risk were iron supplementation (OR $=0.39,95 \%$ CI: $0.26,0.59)$, pet ownership $(\mathrm{OR}=0.50,95 \% \mathrm{CI}: 0.33-0.78)$, paternal smoking $(\mathrm{OR}=1.93$, $95 \% \mathrm{CI}: 1.18,3.15)$ and having older siblings $(\mathrm{OR}=1.18,95 \% \mathrm{CI}$ : $1.05,1.33)$.

Conclusions: Breastfeeding is inversely associated with childhood leukemia and lymphoma risk with a dose-response effect. Pet ownership and iron supplementation are also associated with lower risks.. Paternal smoking and having older siblings are positively associated with cancer risk. These exposures are controllable and modifiable, allowing for public health recommendations.

\section{Abstract \#: P 25}

Regional variations in german head and neck, laryngeal and esophageal cancer mortality rates, and incidence patterns Buttmann-Schweiger, N. ${ }^{1}$, Fragel, B. ${ }^{2}$, Kraywinkel, K. ${ }^{1}$

${ }^{1}$ Robert Koch-Institute, German Centre for Cancer Registry Data; ${ }^{2}$ Sankt Gertrauden-Krankenhaus, Berlin; Corresponding author's e-mail: ButtmannN@rki.de

Background: The draft of a prevention-act has recently passed the German cabinet, and a national prevention strategy is to be implemented. Head and neck cancer (HNC) including laryngeal cancer, and esophageal squamous cell cancer (ESCC) are mainly preventable diseases. Namely tobacco use, alcohol consumption, occupational wood dust exposure, and viral infections are accessible to preventive means. However, it is unclear whether regional variation of HNC\&ESCC incidence and mortality exists in Germany that might call for tailored preventive interventions. We use data from a nationwide mortality reporting system and population based cancer registries to evaluate the geographic variance.

Methods: Nationwide cancer incidence data from 2010/2011 (except for Baden-Württemberg), and mortality data at individual federal state basis, from 1990 onwards were used. HNC and ESCC in 30-79 year olds were identified using ICD-O-3 codes: topography codes C01-06, C09-14, C32, and C15 (SCC morphology codes 805-808). Incidence and mortality rates (ASIR, ASMR) and rate ratios (SIRR/SMRR) were age standardized to the European standard population per 100,000 population and depicted with corresponding $95 \%$ confidence intervals (95\% CI). Average annual percentage changes were calculated on ASMR for all ages and by 10-year age groups.

Results: In total, 24,795 incident HNC and 4943 ESCC were registered in Germany 2010/2011, whereof $78 \%$ occurred in men. Most common HNC subsites were the oropharynx (C01, C05, 09-10, C14), the oral cavity (C00, C02-04, C06) and the larynx. In 2010/2011, significant geographic variance in the HNC and ESCC incidence was found within Germany, predominantly in men: HNC SIRR of 1.3 (95 \% CI 1.2-1.3) and ESCC SIRR of 1.3 (95 \% CI 1.2-1.4) between men in eastern and western federal states, however not uniform across subsites of HNC. Male HNC ASMRs varied from 26.6, to 11.1 on individual federal state basis. Mortality rates from $\mathrm{HNC}$ in men annually decreased by $2.1 \%$ (95 \% CI -2.4 to -1.9$)$ from 1990 to 2011 in Western federal states, and significantly increased in Eastern states. Especially among men in the age groups $<60$ years increased East/West differences in ASMR from oropharyngeal, oral cavity and hypopharyngeal cancer over time.

Discussion: More than 25,000 potentially preventable cancers of the head and neck were registered in 2010/2011. We identified large geographic differences in recent $\mathrm{HNC}$ incidence rates and a continuously growing gap in HNC mortality rates within Germany, particularly among the working-age male population. Inequalities in the HNC burden within Germany will need to be addressed in upcoming cancer prevention strategies.

\section{Abstract \#: P 26}

\section{Cardiovascular disease after Hodgkin lymphoma treatment: 40-year disease risk}

F.A. van Nimwegen ${ }^{1}$, M. Schaapveld ${ }^{1}$, C.P.M. Janus ${ }^{2}$, A.D.G. Krol ${ }^{3}$, E.J. Petersen ${ }^{4}$, J.M.M. Raemaekers ${ }^{5}$, W.E.M. Kok $^{6}$, B.M.P. Aleman ${ }^{7}$, F.E. van Leeuwen ${ }^{1}$

${ }^{1}$ Department of Epidemiology, The Netherlands Cancer Institute, Amsterdam, The Netherlands; ${ }^{2}$ Department of Radiation Oncology, Erasmus MC Cancer Institute, Rotterdam, The Netherlands; ${ }^{3}$ Department of Clinical Oncology, Leiden University Medical Center, Leiden, The Netherlands; ${ }^{4}$ Department of Hematology, University Medical Center Utrecht, Utrecht, The Netherlands; ${ }^{5}$ Department of Hematology, Radboud University Medical Center, Nijmegen, The Netherlands; ${ }^{6}$ Department of Cardiology, Amsterdam Medical Center, Amsterdam, The Netherlands; ${ }^{7}$ Department of Radiation Oncology, The Netherlands Cancer Institute, Amsterdam, The Netherlands; Corresponding author's e-mail: r.v.nimwegen@nki.nl 
Background: Hodgkin lymphoma (HL) survivors are at increased risk of cardiovascular disease (CVD). It is unclear, however, how long the increased risk persists and what the risk factors are for various CVDs. The objective of this study was to examine relative and absolute excess risk up to 40 years since HL treatment, compared to CVD incidence in the general population, and to study treatmentrelated risk factors for different CVDs.

Methods: A retrospective cohort study including 2524 5-year HL survivors, diagnosed age $<51$ (median age 27.3) and treated between 1965 and 1995 was conducted. Data were collected from medical records and GPs. Cardiovascular events [coronary heart disease (CHD), valvular heart disease (VHD), and cardiomyopathy and congestive heart failure (HF)] were graded according to the CTCAEv4.0.

Results: After a median follow-up of 20 years, we identified 1713 cardiovascular events in 797 patients. After $\geq 35$ years, our patients still had a four- to sevenfold increased Standardized Incidence Ratio of $\mathrm{CHD}$ or $\mathrm{HF}$, compared to the general population, corresponding to 887 excess events per 10,000 person-years. Highest relative risks were seen in patients treated before age 25 , but substantial absolute excess risks were also observed for patients treated at older ages. Within the cohort, forty-year cumulative incidence of CVD was $50 \%$ (95\% CI: 47-52\%). Fifty-one percent of patients with CVD developed multiple events. For patients treated before age 25, cumulative incidences at age 60 were 20,31 and $11 \%$ for CHD, VHD and HF as first events, respectively. Mediastinal RT increased the risks of CHD (HR: 2.7, $95 \%$ CI: 2.0-3.7), VHD (HR: 6.6, $95 \%$ CI: 4.0-10.8) and HF (HR: 2.7, $95 \%$ CI: 1.6-4.8) and anthracycline-containing CT increased the risks of VHD (HR: 1.5, $95 \% \mathrm{CI}: 1.1-2.1$ ) and HF (HR: 3.0, $95 \%$ CI: 1.9-4.7) as first events, compared to patients not treated with mediastinal RT or anthracyclines, respectively. Joint effects of mediastinal RT, anthracyclines and smoking appeared to be additive. Conclusions: Throughout life, HL survivors treated at adolescence or adulthood are a high-risk group for various CVDs. Both treating physicians and patients should be aware of the persistently increased risk of CVD throughout life and the results of our study may direct guidelines for follow-up of HL patients.

\section{Abstract \#: P 28 \\ Identification of antithrombotic drugs related to total joint replacement using anonymised free text notes}

Johannes T.H. Nielen ${ }^{1,}$ Bart J.F. van den Bemt ${ }^{2}$, Annelies Boonen ${ }^{3}$, Pieter C. Dagnelie $^{1}$, Pieter Emans ${ }^{4}$, Nicole Veldhorst ${ }^{5}$, Arief Lalmohamed $^{6}$, Tjeerd-Pieter van Staa ${ }^{7}$, Frank de Vries ${ }^{5}$

${ }^{1}$ Department of Epidemiology, Maastricht University, Maastricht, the Netherlands; ${ }^{2}$ Department of Pharmacy, Sint Maartenskliniek, Nijmegen, the Netherlands; ${ }^{3}$ Department of Rheumatology, Maastricht University Medical Center, Maastricht, the Netherlands; ${ }^{4}$ Department of Orthopaedics, Maastricht University Medical Centre, Maastricht, the Netherlands; ${ }^{5}$ Department of Clinical Pharmacy and Toxicology, Maastricht University Medical Centre, Maastricht, the Netherlands; ${ }^{6}$ Department of Clinical Pharmacy, University Medical Center Utrecht, Utrecht, the Netherlands; ${ }^{7}$ Department of Pharmacoepidemiology and Clinical Pharmacology, Utrecht University, Utrecht, the Netherlands; Corresponding author's e-mail: yannick.nielen@mumc.nl

Background: Intensive antithrombotic treatment is recommended to prevent venous thromboembolic events after total joint replacement (TJR) surgery. Antithrombotic drugs are predominantly dispensed at the hospital. Unfortunately, hospital prescription data is often lacking in general practitioner databases, thereby limiting the possibility to use these rich databases to study the longer term effects in real practice. Un-coded anonymised free text from hospital discharge letters may be used to collect additional information on anticoagulant drug use related to TJR. Objective: To design and test a method to extract additional information on anticoagulation therapy in patients undergoing TJR from anonymised free text notes in the Clinical Practice Research Datalink (CPRD).

Methods: Anticoagulant drug use related to total hip (THR) or total knee replacement (TKR) from January 2008 until October 2012 was identified using both anonymised free text and prescription data. Internal validity of our newly designed method was determined by calculating positive predictive values (PPVs) of positive hits for predefined keywords in a random sample anonymised free text notes. To confirm external validity of our method, the proportions of patients using new oral anticoagulants (NOACs) or low molecular weight heparins (LMWHs) were compared with the proportions that have been reported by the UK National Joint Registry. In order to determine the usefulness of our method, TJR patients were then compared with regards to their status of exposure to antithrombotics.

Results: PPVs ranging between 97 and $99 \%$ for NOAC or LMWH exposure related to TJR were obtained with our anonymised free text search method, using manual analysis of the surrounding free text as the gold standard. Aspirin users were identified with PPVs ranging from 91 to $95 \%$. Our anonymised free text algorithm increased detection rates by $57 \%$, yielding a total proportion of $18.5 \%$ of all THR and $18.6 \%$ of all TKR surgeries. Identified users of NOACs and LMWHs were largely similar with regards to age, sex, lifestyle and disease and drug history compared to patients without identified drug use. In contrast, Aspirin users were different as compared to unknown users in particular with regards to a history of ischaemic heart disease. Conclusion: We have developed a useful method to identify additional exposure to NOACs or LMWHs with TJR surgery.

\section{Abstract \#: P 29}

\section{Interaction analysis in the metric of time}

$\underline{\text { Andrea Bellavia }^{1}}$, Matteo Bottai ${ }^{1}$, Nicola Orsini ${ }^{1}$

${ }^{1}$ Unit of Biostatistics and Unit of Nutritional Epidemiology, IMM, Karolinska Institutet, Stockholm, Sweden; Corresponding author's e-mail: andrea.bellavia@ki.se

Interaction is commonly assessed by including a product term between two exposures in the statistical model. Depending on the chosen model, interaction is evaluated as a departure from additivity or multiplicativity, and different papers have emphasized the importance of presenting interaction according to both scales. In survival analysis, however, given the popularity of the Cox proportionalhazard model, evaluation of interaction is usually limited to the multiplicative scale. Measures of additive interaction, such as the relative risk due to interaction (RERI), the attributable proportion due to interaction, and the synergy index, can be calculated using coefficients from a Cox regression, but these are seldom presented in epidemiological studies. Moreover, all measures of interaction in time-to-event analysis, whether additive or multiplicative, are based on the hazard/rate scale, and a constant interaction during the followup period is typically assumed for simplicity. A possible approach for the analysis of time-to-event data is the evaluation of survival percentiles, defined as the time points by which different subpopulations reach the same fraction of events. (1) In this approach the probability of the event of interest is fixed to a specific proportion, and the time variable is evaluated. An interaction analysis in the context of survival percentiles would assess how the impact of one exposure on survival time is affected by another exposure. In addition, this 
approach would make interaction dependent on the proportion of events considered, allowing an evaluation of how interaction is changing during follow-up time. Statistical analysis of interaction could be simply evaluated by including a product-term in a regression model for conditional survival percentiles, such as Laplace regression. (2) Our aim is to introduce the concept of interaction in the metric of survival time, presenting the benefits of focusing on survival percentiles in its evaluation. With the proposed method interaction can be assessed both on the additive or multiplicative scales without assuming constant effects over time.

\section{References}

1. Orsini N, Bottai M, Wolk A. Evaluating Percentiles of Survival. Epidemiology. 2012:23(5).

2. Bottai M, Zhang J. Laplace regression with censored data. Biom J. Aug 2010:52(4):487-503.

\section{Abstract \#: P 30 \\ A Multilevel analysis of the determinants of HIV infection in Mozambique women: evidence from the INSIDA 2009 \\ Sara S. Dias $^{1}$, Francisco Mbofana ${ }^{2}$, Sónia F. Dias ${ }^{3}$, Victor Agadjanian $^{4}$, Maria O. Martins ${ }^{3}$ \\ ${ }^{1}$ Cedoc - Nova Medical School - Faculdade de Ciências Médicas, UIS-ESSLeiria - IPLeiria, Portugal; ${ }^{2}$ Instituto Nacional de Saúde, Moçambique; ${ }^{3}$ Global Health and Tropical Medicine, Instituto de Higiene e Medicina Tropical - UNL, Portugal; ${ }^{4}$ Arizona State University, USA; Corresponding author's e-mail: sara.dias@ipleiria.pt}

Background: Mozambique is one of the ten countries most affected by HIV infection: the national survey on HIV/AIDS prevalence, information and behaviour risks, INSIDA 2009, show that the prevalence among adults aged 15-49 years is $11.5 \%$. Overall, $13.1 \%$ of women age 15-49 are HIV-positive. Numerous factors can be linked to the risk of HIV infection, ranging from individual demographic characteristics to socio-economic status, cultural practice and sexual behaviour risk factors. Moreover existing sociological theories suggest that individual's HIV susceptibility may be affected not only by individual risk factors but also by the contextual factors. In this study, we used INSIDA data to examine individual and contextual factors associated with HIV prevalence in Mozambique women, paying particular attention to possible mechanisms of the association.

Methods: A nationally representative sample of 5527 women aged between 15 and 49 years was analysed. Multilevel logistic regression approach is used to estimate individual and contextual level factors associated with the risk of HIV seropositivity. The key outcome variable is HIV seropositivity while explanatory variables include: background demographic, socio-economic and cultural characteristics including age, urban/rural residence, educational attainment, household socio-economic status and religious affiliation: HIV/AIDS factors, including awareness, stigma and previous testing for HIV: and sexual behaviour factors, including age at first sex, age at first union, union status/type and number of sex partners.

Results: In adjusted models, women living in richer households had increased odds ratio (OR) of being HIV-positive (OR 2.28, 95 \% CI 1.78-2.9). Similar effect is found for women living in female-headed households (OR 1.53, IC $95 \%$ 1.24-1.87). Women with multiple sex partners are more likely to have HIV (OR 1.32, $95 \%$ CI 1.00-1.73), as those less exposed to media (OR 3.49, $95 \%$ CI 1.43-8.58). Stigma is significant only at the contextual level: women living in a region with more stigmatising behaviours had increased odds ratio of being
HIV-positive (OR 1.940, $95 \%$ CI 1.00-3.78). The estimates of intraunit correlations suggest that $23 \%$ of the total unexplained variation in HIV prevalence is attributable to unobserved regional level factors. Conclusion: Contrary to the usual perception that low socioeconomic status individuals tend to be more vulnerable to HIV-infection, we found that both household wealth, and living in female-headed households are positively associated with being HIV-positive. There is a need to take a broader perspective on this issue and consider that wealthier women may have different reasons than poor women for engaging in sexual relationships, but which nonetheless place them at equal risk for HIV. Strategies aimed at reducing HIV-infection in Mozambique needs to be cognizant of the complex social heterogeneity in the individual and regional patterns of HIV-infection.

\section{Abstract \#: P 31}

Appraising of plausible factors, knowledge, attitudes and practice among diabetes patients: hospital-based study in Tabuk city, Saudi Arabia

Radhi A. Alshehri ${ }^{1}$, Abd Allah Al-Temani ${ }^{1}$, Abdulrahman A. Alwakeel $^{1}$, Ahmed A. Eltobgy ${ }^{1}$, Ibrahiem A. Mubarak ${ }^{1}$

${ }^{1}$ Faculty of Medicine, Tabuk University, Saudi Arabia; Corresponding author's e-mail: eltobgy@dr.com

Background: Diabetes Mellitus (DM) is a major global health problem with individual, social and economical consequences. The prevalence of diabetes mellitus in Saudi Arabia is very high $(23.9 \%)$ and ranked sevenith among the top 10 countries around the word. Plausible reasons for the steady increase in the prevalence of DM in many countries may include poor lifestyle, rapid westernization, lack of knowledge and unsatisfactory attitude and practices towards DM among the general population and diabetic patients. Therefore, Knowledge, Attitudes and Practice (KAP) studies are effective in providing baseline data for evaluating intervention programs and improving the service quality. The study aimed to describe the general characteristics of diabetic patients: to determine the degrees of knowledge, attitudes and practice of diabetic patients regarding the pertinent preventive issues: and to examine the interrelated plausible factors of inadequate DM management.

Methods: The clinical epidemiological approach was selected as a suitable investigation strategy in which a cross-sectional analytic research design has been adopted to examine the current diabetic health problem. The systematic stage random sampling technique was applied to recruit a sample size of $5 \%$ diabetic patients (102) from the diabetic center in King Khalid hospital. An interviewing questionnaire was constructed covered the pertinent dependent (knowledge, attitudes and practice) and independent (age, sex, occupation, education, income, marital status and type of diabetes) variables. The questionnaire has been tested and proved valid and reliable during the preparatory research phase. Data have been managed using SPSS v.22.

Results: The mean age of the studied diabetic patients was $37.47 \pm 14.25$ years. The sex distribution was $39.20 \%$ (males) and $59.80 \%$ females. Most of the studied patients $(61.80 \%)$ had inadequate knowledge about DM, while, positive attitudes $(94.90 \%)$ and sound practice $(80.40 \%)$ were satisfactory. Significant plausible factors have been adequately described and analyzed.

Conclusion: Addressing the importance of diabetic knowledge and relevant plausible factors such as poor self-management, lack of motivation, inadequate social support are necessary for sustained life style modification behavior. A patient/self-empowerment approach to diabetes care may enhance the efficiency of DM prevention and control programs. 


\section{Abstract \#: P 32}

The epidemiological analysis of campus injuries and illnesses of high school and elementary school in central Taiwan

Fu-Hui Lee $^{1}$, Li-Feng Lin ${ }^{1}$, Tzu-I Chiu ${ }^{1}$

${ }^{1}$ Central Taiwan University of Science and Technology;

Corresponding author's e-mail: fhlee@ctust.edu.tw

Background: It should always be reported when an injury or illness occurs on campus, regardless of the severity. To describe the epidemiologic patterns of injuries and illnesses on campus is of great importance in injury prevention and health and safety promotion of students. The purpose of this study was to analyze the empirical data of senior and junior high school and elementary school for campus injuries and illnesses in Central Taiwan during one school year period from August 2012 to July 2013.

Methods: By cooperation with the Education Bureau of Taichung City, the data were collected from the Student Health Information Management System. Descriptive method was used in epidemiological analysis of the campus injuries and illnesses data.

Results: The results of this descriptive epidemiology analyses were as follows: It showed that contusion, abrasion, laceration and abdominal pain were the major causes of campus injuries and illnesses in the elementary school, while contusion, abrasion, headache and laceration in the junior high school and dysmenorrhoea, abrasion, dizziness and contusion in senior high school. Boys got contusion, abrasion, laceration and strain more than girls, and girls got headache, dizziness, abdominal pain and stomachache more than boys. Wounds usually occurred on upper limb and lower limb, and then occurred on head and abdomen among all high school and elementary school students. Boys got wounds occurred on limb and face more than girls, and girls usually occurred on head and abdomen. The most common time of campus injuries and illnesses occur were in the morning of a day and in March, May, October and December of a year. The location where most campus accidents and injuries occur was the outside play area and then was classroom.

Conclusion: The findings of the study indicated the health needs of high school and elementary school students. The results of this research shall serve as a reference for the school health administrators and educators to design and implement the school health practice and healthful school environment.

\section{Abstract \#: P 33}

Road traffic injury data collection and analysis in LMICs: an innovative pilot in Bangladesh

A.R.A van der Horst ${ }^{1}$, AKM Fazlur Rahman ${ }^{2}$, M.C.Thierry ${ }^{3}$, J.Vet $^{3}$, K. ul Baset ${ }^{4}$

${ }^{1}$ Traffic Behaviour, TNO, Soesterberg, The Netherlands; ${ }^{2}$ Professor and Head Department of Epidemiology Bangladesh University of Health Sciences Mirpur, Dhaka, Bangladesh; ${ }^{3}$ Safe Crossings, Amsterdam, Netherlands; ${ }^{4}$ Senior Scientist, CIPRB, Dhaka, Bangladesh; Corresponding author's e-mail:

richard.vanderhorst@tno.nl

Background: $92 \%$ of the world's recorded road traffic deaths occur in LMICs. Bangladesh has over 100 road traffic deaths per 10,000 motor vehicles, one of the highest rates in the world. Many crashes occur at 'black spots', concentrated areas with a high road crash burden, and about $70 \%$ of crash fatalities occur in rural areas, often as a result of crashes with high-speed buses.
In 2014, Safe Crossings from the Netherlands and CIPRB from Bangladesh received permission from the government of Bangladesh to implement a traffic-calming program to prevent road crashes. This study is one of the first to consider small-scale measures for traffic calming in rural areas of a LMIC. Part of our study is an innovative approach to record and analyze road traffic injury data. As highlighted by the WHO, UN, and others, official road crash statistics in LMICs are typically incomplete and biased towards (large) motorized traffic. Improved injury data collection is a necessary pre-condition for fighting the road crash epidemic. For practical reasons, we could not use randomized control trials. Thus an innovative approach is needed. Methods: We used a combination of three methods to record and analyse road crash data at three black spots. Firstly, we created our own accident recording system with trained local record-keepers. Secondly, we measured the speed of motorised traffic with a laser gun, backed-up by video measurements. Thirdly, we used a conflict observation study methodology called DOCTOR, developed by the SWOV and TNO in the Netherlands.

Results: Our accident recording system provided complete and accurate road crash statistics in each black spot. On a yearly basis, the three black spots together had 109 accidents with a total of 254 people injured and 11 people killed. The DOCTOR method combined with video analysis enabled us to get a sufficiently large data set in a relatively short period of time. Preliminary analyses showed that buses are involved in $71 \%$ of dangerous road situations, and that pedestrians and cyclists are involved in $34 \%$ of dangerous road situations.

Conclusion: We have three main results. Firstly, it is possible to set up an effective local accident recording system quickly and at relatively low cost. Secondly, the DOCTOR approach with video analysis, combined with speed measurements, is suitable for. Thirdly, it is essential to gain support from the local community.

\section{Abstract \#: P 34}

\section{How large should a pilot study be?}

Wolfgang Viechtbauer ${ }^{1}$, Luc Smits ${ }^{2}$, Daniel Kotz ${ }^{3}$, Luc Budé ${ }^{4}$, Mark Spigt ${ }^{5}$, Rik Crutzen ${ }^{6}$

${ }^{1}$ Department of Psychiatry and Psychology, MHeNS School for Mental Health and Neuroscience, Maastricht University, PO Box 616, 6200 Maastricht, The Netherlands; ${ }^{2}$ Department of Epidemiology, CAPHRI School for Public Health and Primary Care, Maastricht University, PO Box 616, 6200 Maastricht, The Netherlands; ${ }^{3}$ Department of General Practice, CAPHRI School for Public Health and Primary Care, Maastricht University, PO Box 616, 6200 Maastricht, The Netherlands; ${ }^{4}$ Department of Midwifery Education and Studies, Research Centre for Midwifery Science, Zuyd University, PO Box 1256, 6201 BG Maastricht, The Netherlands; ${ }^{5}$ Department of Family Medicine, CAPHRI School for Public Health and Primary Care, Maastricht University, PO Box 616, 6200 Maastricht, The Netherlands; ${ }^{6}$ Department of Health Promotion, CAPHRI School for Public Health and Primary Care, Maastricht University, PO Box 616, 6200 Maastricht, The Netherlands; Corresponding author's e-mail: luc.smits@maastrichtuniversity.nl

Background: One of the goals of a pilot study is to identify unforeseen problems, such as ambiguous in/exclusion criteria or misinterpretations of questionnaire items. Although sample size calculation methods for pilot studies have been proposed, none of them are directed at the goal of problem detection.

This methodological study: In this study, we present a simple formula to calculate the sample size needed to be able to identify, with a chosen level of confidence, problems that may arise with a given 
probability. If a problem exists with $5 \%$ probability in a potential study participant, the problem will almost certainly be identified (with $95 \%$ confidence) in a pilot study including 59 participants.

\section{Abstract \#: P 35 \\ Factors related to rejection of care and behaviours directed towards other in nursing home residents with demetia: a longitudinal study}

Ladislav Volicer, MD, $\mathrm{PhD}^{1}$, Jenny T. Van der Steen, $\mathrm{PhD}^{2}$, Francisca Galindo Garre, $\mathrm{PhD}^{3}$

${ }^{1}$ School of Aging Studies, Florida University of South Florida, Tampa, FL; ${ }^{2}$ Department of General Practice \& Elderly Care Medicine, VU Medical Center, Amsterdam, The Netherlands; ${ }^{3}$ Department of Epidemiology and Biostatistics, VU Medical Center, Amsterdam, The Netherlands; Corresponding author's e-mail: paquigalindo@yahoo.es

Background: The aim of this study is to analyze whether the relationship between the symptoms (lack of understanding and depression) and behaviors directed towards others was mediated by rejection of care in nursing home residents with dementia.

Methods: Relationship of lack of understanding and depression to rejection of care and behaviors directed towards others was explored using four assessments from the Minimum Data Set (MDS) within a period of 15 months on 1101 residents with dementia in Dutch nursing homes. Presence of depressive symptoms was ascertained using a validated MDS scale, and presence of lack of understanding and rejection of care through the individual MDS items. Latent growth mediation models were used to investigate the longitudinal relationship between changes in lack of understanding or depression, changes in rejection of care and physical or verbal behaviors directed towards others.

Results: Changes in lack of understanding predicted changes in rejection of care and there was also a relationship between changes in depression and rejection of care. Changes of behaviors directed towards others were related to changes in lack of understanding and depression. Mediation models suggested that only the relationship of lack of understanding with behaviors directed towards others was mediated by rejection of care.

Conclusion: These results indicate that the lack of understanding and depression are important factors in development of rejection of care and behaviors directed towards others. The relationship between lack of understanding and behaviors directed towards others is mediated by rejection of care. Therefore, improvement in communication between residents and caregivers may prevent or ameliorate these behaviors directed towards others.

\section{Abstract \#: P 36}

Analysis of spatial pattern and distribution of leptospirosis in Mazandaran province using the geographic information system during 2011-2013

$\underline{\text { Roghieh Ramezankhani }}{ }^{1}$, Ali Hosseini ${ }^{2}$, Jalil Shojaee ${ }^{3}$

${ }^{1}$ Ministry of health, Center for communicable disease control; ${ }^{2} \mathrm{PhD}$, Department of Geography and Urban, University of Tehran, Iran; ${ }^{3}$ Faculty of Health, Mazandaran University of Medical Sciences, Sari, Iran; Corresponding author's e-mail: minoo.ramezani@gmail.com

Background: Since the public health and diseases are directly related to the geography of the location, geographic information system
(GIS) can be used in planning and management of public health issues and spread of disease. On the other hand, high capacity of GIS modeling provides opportunities for health policy makers in analyzing the causes of occurrence of diseases. The Most important application of GIS is capability of this system in integrated spatial and statistical analyzing in exploring the diseases. One of the infectious diseases that are related to spatial factors is leptospirosis which is the most common zoonotic diseases in the world. Environmental factors that affect the epidemiology of leptospirosis include heavy rainfall and flooding, high temperatures, exposure to animals, poor sanitation, and poor waste disposal. With global climate change and urbanization, many of these major risk factors are expected to occur with greater frequency and intensity. The aim of this study is pattern analysis and spatial distribution of Leptospirosis in Mazandaran province during 1390-1392 using geographic information system (GIS).

Methods: The location of patients determined by GPS (Global positioning system). Since the district division was not appropriate unit for analyzing, therefore, we used (Modifiable Areal Unit Problem) (MAUP) for correcting the spatial units. However, the spatial resolution requires to creating homogenous region. In these context two subjects is proposed, (1) selection of scale modulation, and (2) the effect of modulation. Scale is related to units of region that we used. Modulation effect is referred to methods that combine the data. A unique code assigned to each grid cell. The location of patients was transferred to the network codes for identifying the location of each point in relation to each cell grid. Spatial analysis was performed after the integration of spatial data based on network coding within each cell.

Results: In our study a cluster distribution of Leptospirosis was observed. Results showed that Sari was main foci and then Ghaemshahr and Amol were the next two foci for disease. Based on our study the disease was more common in men: and the most of Leptospirosis cases were seen in age group 50-59.

Conclusions: Applying of GIS can be useful in determining disease hot spot, spatial analysis in public health, forecasting the spread of disease and controlling the affecting parameters.

\section{Abstract \#: P 37}

Evaluation of non-response bias in a sample of informal caregivers in the lifelines cohort study

Marloes Oldenkamp ${ }^{1}$, Mariët Hagedoorn ${ }^{2}$, Ronald Stolk ${ }^{1}$, Rafael Wittek $^{3}$, Nynke Smidt ${ }^{1}$

${ }^{1}$ Epidemiology, University of Groningen, University Medical Center Groningen, Groningen, the Netherlands; ${ }^{2}$ Health Psychology, University of Groningen, University Medical Center Groningen, Groningen, the Netherlands; ${ }^{3}$ Sociology, University of Groningen, Groningen, the Netherlands; Corresponding author's e-mail: m.oldenkamp@umcg.nl

Background: With current trends such as population ageing and increasing health care costs, caregiving research, specifically focusing on a population of informal caregivers, is increasing in importance. However, little is known about the non-response of informal caregivers in caregiving research. Our aim is to evaluate the non-response bias in a sample of informal caregivers in the LifeLines cohort study. Methods: Within Lifelines, a population based cohort studies of 176.729 participants, informal caregivers aged $\geq 18$ years were identified in the second follow-up questionnaire. All informal caregivers were asked to complete several basic questions about their caregiving situation and experiences, and subsequently all informal caregivers were asked for their consent for participation in the 
additional caregiving questionnaire. Informal caregivers who completed and returned the additional caregiving questionnaire were considered as response informal caregivers. Differences between responding and non-responding informal caregivers were assessed with regard to their socio-demographic characteristics, health status, and caregiving situation and experiences. In addition, multivariable linear regression models, with caregiver burden as main outcome, were estimated separately for responding and all (responding and nonresponding) informal caregivers, and results were compared to assess the presence of non-response bias.

Results: Within Lifelines, 2002 informal caregivers were identified, of which 965 (48\%) responded to the additional informal care questionnaire. Responding informal caregivers were older, more often lower educated, more often caring for their spouse, providing more hours of household and other care tasks (i.e. accompanying with hospital visits), and experienced a higher burden from their caregiving, compared with non-responding informal caregivers. No differences were found for health-related quality of life. Results from the multivariable linear regression models will be presented at the conference.

Conclusion: Informal caregivers who are more involved in their caregiving (i.e. more hours of care, more burden), were somewhat overrepresented in our sample of responding informal caregivers, which might affect the generalizability of our study results. The results of the multivariable linear regression models will provide insights into the extent to which this overrepresentation also lead to non-response bias for relevant study outcomes such as caregiver burden.

\section{Abstract \#: P 38}

An $\mathbf{R}$ algorithm to monitor the start and intensity of influenza seasonal epidemics: the moving epidemic method

$\underline{\text { José E. Lozano }}^{1}$, Tomás Vega ${ }^{1}$

${ }^{1}$ Dirección General de Salud Pública, Consejería de Sanidad, Junta de Castilla y León; Corresponding author's e-mail: lozalojo@jcyl.es

Background: Several methods have been developed to establish epidemic thresholds and intensity levels in the past. It is important those methods to be comparable in order to have a general overview of the state of the influenza epidemic. Since 2012, ECDC and WHOEurope use a standardized method to establish thresholds in Europe: The Moving Epidemic Method (MEM) (Vega \& Lozano). In 2011 the method was implemented as a R language library.

Methods: $\mathrm{R}$ is a system for statistical computation and graphics. It is free software project (GNU) and it is strongly based in the community contributions. The users have different tools to develop their programs (libraries) and share them.

The mem R library was created in 2011 to help country surveillance systems to run the MEM algorithm and calculate thresholds suitable for their data.

Results: The library uses as input Influenza-like illness weekly rates from historical records and produce different results. It has three functions: epimem analyzes the historical epidemics (start, end and shape) and calculates the epidemic threshold, memintensity provides thresholds for intensity levels, memtrend shows indexes for determining the trend of the epidemic and epitiming, which, based on data from a single season, locates the exact timing of that epidemic.

The library creates graphs of a typical influenza epidemic, the start and end and epidemic and intensity thresholds.

Conclusion: The library is currently used by ECDC and WHO-E databases (Tessy and Euroflu) and it is publicly available through the Comprehensive R Archive Network.

\section{Abstract \#: P 39}

How to recruit participants into observational studies? A systematic review

Marleen M.H.J. van Gelder ${ }^{1}$, Richelle Vlenterie ${ }^{2}$, Lucien J.L.P.G. Engelen $^{3}$, Alina Vrieling ${ }^{4}$, Tom H. van de Belt ${ }^{3}$

${ }^{1}$ Department for Health Evidence \& Radboud REshape Innovation Center, Radboud Institute for Health Sciences, Radboud university medical center, Nijmegen, The Netherlands; ${ }^{2}$ Department for Health Evidence, Radboud Institute for Health Sciences, Radboud university medical center, Nijmegen, The Netherlands; ${ }^{3}$ Radboud REshape Innovation Center, Radboud Institute for Health Sciences, Radboud university medical center, Nijmegen, The Netherlands; ${ }^{4}$ Department for Health Evidence, Radboud Institute for Health Sciences, Radboud university medical center, Nijmegen, The Netherlands;

Corresponding author's e-mail: Marleen.vanGelder@radboudumc.nl

Background: Participation in health-related research has declined dramatically in the past decades. Low response rates reduce study power and may produce biased estimates due to non-representativeness between those responding and the target population. Several response-inducing strategies have been proposed and recent metaanalyses have reported on successful and unsuccessful recruitment strategies for randomized clinical trials. However, an up-to-date overview of strategies to increase response to observational studies is lacking.

Methods: We conducted a systematic literature search of PubMed, EMBASE, CINAHL, PsycINFO, and Web of Science from database inception through February 2015 without language restrictions. All studies reporting on strategies directed at study participants to increase participation rates to health-related, observational studies that involve self-reported methods of data collection were included. Two authors independently selected the studies, assessed methodological quality, and extracted data using predefined criteria.

Results: The search strategy identified 11,698 potentially relevant articles, of which 362 were included for full text review. Further results will be presented at the Congress, stratified for method of data collection (paper-based questionnaires, Web-based questionnaires, and interviews), type of strategy (incentive strategies, contact or reminder strategies, and questionnaire strategies), and study population (patients and healthy subjects).

Conclusion: Applying successful response-inducing strategies may increase participation in observational studies in the era of declining response rates. This study identifies these strategies, which epidemiologists may consider to implement when planning an observational study.

\section{Abstract \#: P 40}

\section{Application of net benefit fraction to select cut-off for medical} markers $\frac{\text { Davood Khalili }^{1}}{\text { Fereidoun Azizi }^{2}}$ Farideh Bagherzadeh Khiabani ${ }^{1}$, Farzad Hadaegh ${ }^{1}$,

${ }^{1}$ Prevention of Metabolic Disorders Research Center, Research Institute for Endocrine Sciences, Shahid Beheshti University of Medical Sciences, Tehran, Iran; ${ }^{2}$ Endocrine Research Center,

Research Institute for Endocrine Sciences, Shahid Beheshti

University of Medical Sciences, Tehran, Iran; Corresponding author's e-mail: dkhalili@endocrine.ac.ir

Background: Common cut-off selection methods (e.g. ROC curve analysis and Youden's index) mostly do not consider harms and 
benefits of a decision. Decision-theory-based techniques, which overcome this inefficiency, have not received much attention by epidemiologists since there is usually no clear agreement on these harms and benefits in practice. To us, a modification of such methods which do not need the exact values of harms and benefits would be a valuable achievement for epidemiological studies.

Methods: Net benefit fraction is a true positive rate (sensitivity) penalized by a weight of false positive rate: this weight includes the harm-to-benefit ratio of the decision which equals to odds at the treatment threshold (a probability of disease that a physician/patient decides to start treatment). As a practice, we identified the optimal cutoff point for waist to hip ratio (WHR) in prediction of cardiovascular events in a cohort of 1658 men aged 40-74 years with 10-year follow-up. At each specific treatment threshold, we calculated the NBF for each point of WHR and found the optimal cut-point with the highest NBF. This calculation was repeated for a wide rational range of treatment threshold. All of this was then exported to a plot, as an easy-to-understand summarization, with the range of treatment threshold and/or the corresponding cost ratio as the " $\mathrm{x}$ " axis (bottom), optimal cutoff as the first " $y$ " axis (left), and the best NBF as the second " $y$ " axis (right). In this case, a clinician can pick a cutoff based on his/her own treatment threshold regarding individualized harm and benefit of treatment for a patient. We proved mathematically that other decision-theory-based performance measures, including misclassification cost term (MCT) and generalized Youden's index, are identical to NBF from an optimization point of view. Hence, net benefit fraction as a simple, understandable utility index is suggested in this paper for cutoff selection and developed an $\mathrm{R}$ package.

Results: Our particular application concerned the effect of WHR and its cutoff on CVD among a real-world data. AUC of 0.65 indicates that this marker is promising overall. For a range of threshold probability for treatment $(0,0.5)$ - and equivalently a range of cost ratio $(0,1)$ - the utility of the marker along with its optimal cutoff are shown in the plots.

Conclusion: Our analysis showed that AUC is not enough to show predictive ability of a marker since in spite of a fixed AUC, a marker might be of different value in each specific threshold probabilities for treatment.

\section{Abstract \#: P 42}

\section{Studying the spatial distribution and temporal trends of tuberculosis notifications in India}

Mirjam I. Bakker ${ }^{1}$, Tacilja Ruckert ${ }^{1}$, Sandra Alba ${ }^{1}$, Ente Rood ${ }^{1}$, Masja Straetemans $^{1}$, Stella van Beers ${ }^{1}$

${ }^{1}$ KIT Biomedical Research, Royal Tropical Institute, Amsterdam, The Netherlands; Corresponding author's e-mail: m.bakker@kit.nl

Background: Studying the geographical distribution and temporal trends of tuberculosis (TB) can be very informative to better understand the epidemiology of the disease and to inform control programs towards more targeted interventions, but can routine collected notification data be used for these in-depth studies? We report on an analysis done on data from the National TB Control Program in India (NTC), one of the 22 high-burden TB countries.

Methods: We created a database from publicly available (on-line) data of quarterly case notification data from January 2001 to December 2011 at district level reported as part of the Directly Observed Treatment, Short Course strategy (DOTS) of the national TB program. Population data were obtained from the 2001 and 2011 Indian Census. By combining NTC and population data we were able to derive the following indicators on district level: suspect rate (persons examined for TB per 100,000 population): TB notification rate (total number of TB cases registered per 100,000 population): proportion of notified patients among all suspects: proportion smear positive cases among all TB cases. Spatial data outlining the administrative boundaries of Indian districts were extracted from DIVA-GIS data portal.

Results: The median all forms TB notification rate over all districts in 2011 was 122 per 100,000 population (IQR: 95-149), ranging from a minimum of 26 in the lowest reporting district to 967 in the highest. Mapping TB notification rates showed great heterogeneity between districts, with generally higher notifications than average in the northern districts. Longitudinal time series data from 2001 to 2011 were available for only 5 states (Delhi, Himachal Pradesh, Rajasthan, Tamil Nadu and Kerala). Beyond increases in notification rates due to the expansion of DOTS until 2004, there were no further visible secular trends. However there was evidence of seasonal variation in reporting in the northern regions with a peak in the second quarter. We will also present the results of an analysis entangling TB programmatic factors and socio-environmental factors using public available data including climatic data.

Conclusion: Despite its limitations as changing of reporting requirements over time and inaccessibility of data on patients treated in the private sector, the data made publicly available by the government of India offers the unique possibility to perform in-depth ecological studies combining geo-statistical and time series analyses to better understand the heterogeneity of the TB burden in India and to assess the relative contribution of epidemiological versus programmatic factors towards TB notifications.

\section{Abstract \#: P 43}

Effects of variations in estimated completion time and voucher incentives on questionnaire response

Marleen M.H.J. van Gelder ${ }^{1,2}$, Pauline M. Geuijen ${ }^{1}$, Jeanne H.M. de Vries $^{3}$, Nel Roeleveld ${ }^{1}$

${ }^{1}$ Department for Health Evidence, Radboud Institute for Health Sciences, Radboud university medical center, Nijmegen, The Netherlands; ${ }^{2}$ Radboud REshape Innovation Center, Radboud Institute for Health Sciences, Radboud university medical center, Nijmegen, The Netherlands; ${ }^{3}$ Division of Human Nutrition, Wageningen University, Wageningen, The Netherlands; Corresponding author's e-mail: Marleen.vanGelder@radboudumc.nl

Background: Obtaining a response rate as high as possible is important to reduce selection bias and increase external validity. Some strategies have been found to increase response rates, whereas others have no or ambiguous effects. In this study, we determined whether the estimated completion time and/or a voucher incentive affects questionnaire response.

Methods: Pregnant women participating in the PRegnancy and Infant DEvelopment (PRIDE) Study were asked to fill out a postal food frequency questionnaire after completing the baseline PRIDE Study questionnaire. We employed three strategies: (A) underestimated completion time without incentive, (B) underestimated completion time with an unconditional $€ 5$ voucher, and (C) correctly estimated completion time with the $€ 5$ voucher. Response rates with and without reminder letters and levels of item non-response were compared between the three strategies.

Results: The food frequency questionnaire was sent to 822 women, of which $729(89 \%)$ returned a completed questionnaire. We did not observe differences in completion rates $(p=0.40)$ or the proportion of questionnaires returned without a reminder letter $(p=0.61)$ between the three strategies. Furthermore, the proportion of 
questionnaires with $<5 \%$ item non-response was comparable between the strategies, although it seemed to be somewhat higher in strategy C $(69 \%)$ compared to the strategies with an underestimated completion time $(63 \%)$.

Conclusion: Variations in estimated completion time and a voucher incentive did not affect response to a postal food frequency questionnaire among pregnant women enrolled in a prospective cohort study. However, these strategies might increase response rates in less intrinsically motivated study populations.

\section{Abstract \#: P 44}

Following-up very preterm babies: where can we get the most reliable information: a study at one year of age

Inês Baía ${ }^{1}$, Carina Rodrigues ${ }^{1}$, Raquel Costa ${ }^{1}$, Henrique Barros ${ }^{1}$

${ }^{1}$ EpiUnit, Institute of Public Health, University of Porto, Porto, Portugal; Corresponding author's e-mail: inesbaia@med.up.pt

Background: Epidemiological research has often to rely on selfreported information or on summaries of medical files. The follow up of children poses special challenges because of possible loss of information between health professionals and parents, social representations and valuing of details. Therefore, the aim of this study was to assess the agreement between data obtained using a phone questionnaire answered by mothers and that recorded on medical files to collect information on health outcomes-clinical and developmental—of very preterm infants at 1 year of corrected age.

Methods: Very preterm infants born between June 2011 and May 2012 and discharged alive from the neonatal intensive care units (NICUs) of two regions of Portugal (Northern and Lisbon and Tagus Valley) were invited to participate. Computer-assisted telephone interviews were performed to mothers at one year of corrected age and medical records were abstracted $(n=412)$ : 12 items were evaluated to search for relative validity, that included occurrence of hospitalizations, screening for ophthalmologic and hearing conditions, medical diagnosis, use of nutritional supplements and some infant developmental stages attained. Agreement between sources of information was calculated using intraclass correlation and kappa statistics.

Results: Data on clinical outcomes was successfully obtained either by mothers' report and medical records. However, detailed data on developmental outcomes was lacking on medical records (about $59 \%$ ). Absolute agreements were very high, the lowest being $67.2 \%$ (for respiratory disorders) but reflected the small proportion of positive events. There was substantial agreement for the occurrence of hospitalizations $(\kappa=0.75)$ and moderate agreement for development variables $(\kappa=0.59-0.56)$. For the remaining clinical variables poor to fair agreement $(\kappa=0.03-0.30)$ was found.

Conclusions: These results suggest that medical records and mothers' report should be used for specific purposes and are not interchangeable. As there is no gold standard we need both: medical records seem to be a reliable source of clinical information while mothers' reports seem a more reliable source if developmental data is envisaged.

\section{Abstract \#: P 45}

Validation of subjective and objective outcome measures in patients with nasal obstruction

M.M.H.T. van Egmond ${ }^{1}$, N. van Heerbeek ${ }^{1}$, C.T.M. Hendriks ${ }^{1}$, M.M. Rovers
${ }^{1}$ Otorhinolaryngology, Radboudumc, Nijmegen, Netherlands; ${ }^{2}$ Health Evidence, Radboudumc, Nijmegen, Netherlands; Corresponding author's e-mail: machteld.vanegmond@radboudumc.nl

Background: Septoplasty, i.e., surgical correction of the deviated nasal septum, is the most common ENT operation in adults. Currently the main indication to perform septoplasty is nasal obstruction, a health problem that is a common reason for patients to consult their ENT surgeon. At present, the effectiveness of septoplasty for nasal obstruction in adults with a deviated nasal septum remains uncertain. The effectiveness of septoplasty can be assessed in several ways, for example in terms of health-related quality of life, or in terms of nasal passage. However, most outcome measures are not yet validated for nasal obstruction in patients with a deviated nasal septum. Furthermore, objective measurements do not always correlate with subjective measures to evaluate the effectiveness of septoplasty. The aim of this study is to validate two methods to assess the effectiveness of septoplasty in adults with a deviated nasal septum. Additionally, we will determine to which extent outcomes of subjective and objective measures coincide in this group of patients with a frequently occurring health problem.

Methods: As part of our randomized controlled trial on the effectiveness of septoplasty, measurements of health-related quality of life and nasal passage will prospectively be collected in 100 patients, both before and after septoplasty or non-surgical management (medication or watchful waiting). The measurements of health-related quality of life will be collected using the Glasgow Health Status Inventory (GHSI) and the Glasgow Benefit Inventory (GBI). Objective measurements of nasal patency will be performed using 4-phase rhinomanometry (RhinoLab GmbH, Freiburg im Breisgau, Germany) and peak nasal inspiratory flow (Clement Clarke International, Essex, United Kingdom). Construct validity of the GHSI and GBI questionnaires will be determined by performing factor analysis, itemitem, and item-total correlations. Criterion validity of both subjective as well as objective outcome measures will be assessed by comparing results with outcomes of the Nasal Obstruction Symptom Evaluation (NOSE), which is validated for nasal obstruction in adults undergoin septoplasty. Response sensitivity of the subjective and objective outcome measures will be evaluated by calculating the standardized response mean and the effect size.

Results/Conclusion: Two outcome measures will be validated for nasal obstruction in patients with a deviated nasal septum, focusing on both health-related quality of life as well as nasal passage.

\section{Abstract \#: P 46}

Gain and crossover in life expectancy after elimination of major causes of death in India

Manisha Dubey ${ }^{1}$, F. Ram ${ }^{1}$, Usha Ram $^{2}$

${ }^{1}$ International Institute for Population Science, Mumbai, India;

${ }^{2}$ Centre for Global Health Research, Canada; Corresponding author's e-mail: manikvdlw@gmail.com

Programmatic interventions aims to measure life years gained on reduction of mortality risk and elimination of a few prominent cause of death provide a powerful summary for effectively emphasizing on the need for such intervention. Life expectancy at birth in India has increased from 50 years in 1972 to 66 years in 2008. This rapid and unprecedented increase in human life expectancy was associated with profound changes in prevailing disease patterns, what is known as 'epidemiological transition'. Ideally, a life expectancy curve should monotonically decline with maxima at age zero. However, in India, life expectancies at age one $\left(\mathrm{e}_{0}^{1}\right)$ and five $\left(\mathrm{e}_{0}^{5}\right)$ exceed life expectancy at birth $\left(\mathrm{e}^{0}{ }_{0}\right)$. Probably high mortality during early ages, especially 
during infancy $(<1$ year) and childhood (1-4 years) brings out an anomaly where person at age one or five is likely to live longer than a new-born.

Objectives: The aim of the paper is to quantify gain in $\mathrm{e}_{-} 0^{\wedge} 0, \mathrm{e}_{-} 1^{\wedge} 0$ and e_ $5^{\wedge} 0$ after elimination of a cause of death and its effect on the crossover between life expectancies in India.

Data and Methodology: Using data from Sample Registration System and Million Death Study, overall and disease deleted life tables have been constructed, separately for male and female. The basic principal of the method is based on the fact that death is an inevitable event and hence a person surviving from a specific disease at any age would eventually die due to another disease at a later age. In other words, individual defying the death due to a particular cause " $\mathrm{Ci}$ " at age "Ax" will die due to cause " $\mathrm{Cj}$ ' $\mathrm{i}$ " at age "Ax $+1 \geq \mathrm{x}$ ". The gain in life expectancy after elimination of a cause is calculated as the difference between the life expectancy before and after elimination of cause. The present analysis is carried out for broad categories of causes of death e.g. communicable disease (CD), non-communicable diseases (NCD) and Injuries (I).

Findings: Result shows that in India after eliminating NCD and CD e_ $0^{\wedge} 0$ increased from 62.5 to 64.4 years and 65.5 years, respectively (a gain by nearly $2-3$ years). e_ $1^{\wedge} 0$ has increased by 1.8 years and e_ $5^{\wedge} 0$ by 1.7 years if NCDs are eliminated. While e_ $1^{\wedge} 0$ has declined by 0.8 years and e $5^{\wedge} 0$ by 1.5 years if $C D$ are eliminated. Crossover between e_ $0^{\wedge} 0$ and e_5 $5^{\wedge} 0$ : and e_ $0^{\wedge} 0$ and $\mathrm{e} \_1^{\wedge} 0$ has been noted if risk of dying due to $\mathrm{CD}$ are eliminated.

\section{Abstract \#: P 47}

Common carotid artery echolucency and mortality in elderly men: a prospective cohort study

$\underline{\text { K.A. Groenewegen }^{1}}{ }^{\text {, S.A.E. Peters }}{ }^{1,2}$, M.L. Bots ${ }^{1}$, H.M. den Ruijter ${ }^{1,3}$

${ }^{1}$ Julius Center for Health Sciences and Primary Care, University Medical Center Utrecht; ${ }^{2}$ The George Institute for Global Health, Nuffield Department of Population Health, University of Oxford; ${ }^{3}$ Department of Experimental Cardiology, University Medical Center Utrecht; Corresponding author's e-mail:

k.a.groenewegen@umcutrecht.nl

Background: Carotid intima media echolucency is an ultrasound characteristic associated with cardiovascular risk factors and hypothesized to reflect the contents of the vascular wall. Carotid intima media echolucency has been found to be associated with cardiovascular mortality in elderly men, but these findings need replication.

Methods: We used data from a prospective cohort study conducted in the Netherlands. Men aged 70 and older were asked to participate. The examination included an interview, venous blood draw and ultrasonography of both common carotid arteries. These ultrasound images were used to measure echolucency, quantified as the grey scale median (GSM), of the carotid intima media complex. Low GSM values reflect echolucent (darker) structures, whereas high values reflect echogenic (lighter) structures. Participants were followed up for mortality after four years. Cox proportional hazards models were used to assess the relation between echolucency quantified by GSM (both stratified by tertiles and as a continuous variable) and all-cause and cardiovascular mortality, corrected for potential confounders.

Results: For this study, we used images of 403 men with a median age of 77 years. Median GSM of the far wall of the common carotid artery was 44.8 (SD 8.3). Higher CIMT was associated with a lower GSM ( $(-1.17 /$ SD $(-2.04:-0.31)$ ), suggesting that thicker intimamedia complexes were darker. The highest tertile of GSM (most light) was significantly related to all-cause mortality (HR 2.18 (1.17-4.09)) compared to tertile 1 . For cardiovascular mortality similar associations were observed, the more white the carotid intima-media complex, the higher the risk for cardiovascular mortality (HR respectively $5.00(1.42-17.61)$ and $5.05(1.42-18.00)$, compared to tertile 1).

Conclusion: A high GSM of the carotid intima media complex is associated with a higher risk of all-cause and cardiovascular mortality in elderly men.

\section{Abstract \#: P 48}

Patterns of antiplatelet use in patients with myocardial infarction and subsequent acute coronary syndrome events

Alfi Yasmina $^{1}$, Anthonius de Boer ${ }^{1}$, Patrick C. Souverein ${ }^{1}$, Olaf H. Klungel $^{1}$

${ }^{1}$ Division of Pharmacoepidemiology and Clinical Pharmacology, Department of Pharmaceutical Sciences, Utrecht Institute for Pharmaceutical Science, Faculty of Science, Utrecht University, Utrecht, The Netherlands; Corresponding author's e-mail: A.YasminaNoorsyahdy@uu.nl

Background: Antiplatelet drugs are important for secondary prevention of cardiovascular events after myocardial infarction (MI). The objective of this study was to assess the patterns of antiplatelet drug use in patients who had a MI and to evaluate the impact of subsequent acute coronary syndrome (ACS) events on antiplatelet drug use in the Netherlands.

Methods: A descriptive retrospective cohort study was conducted on 4719 patients in the Utrecht Cardiovascular Pharmacogenetics studies, who had their first MI during 1986-2009. Medication use was assessed through the Dutch PHARMO Record Linkage System (dispensing database linked to the hospital admission registry). Antiplatelet users were classified as continuous users (gap between consecutive prescriptions $\leq 90$ days), discontinued users (gap of $>90$ days or no refills), and restarters (with a new antiplatelet drug episode after earlier discontinuation) and followed for 10 years Antiplatelet drug use in 90 days before and after recurrent consecutive ACS events (MI and unstable angina) following the first MI were also compared.

Results: At 1 year of follow-up, $83.7 \%$ patients continued using antiplatelets, $76.9 \%$ were still on aspirin, and only $36.4 \%$ patients were continuing clopidogrel. Most of the discontinuers restarted antiplatelet drugs later, leading to $74.7 \%$ antiplatelet users, $62.1 \%$ aspirin users and $35.2 \%$ clopidogrel users at 10 years after the index MI. For a subgroup of MI patients who were started dual antiplatelet therapy with aspirin and clopidogrel (DAPT) after hospital discharge in 2002-2009, a total of $28.9 \%$ remained continuous users at 1 year, whereas $24 \%$ of the subjects switched to aspirin or clopidogrel monotherapy. When a recurrent ACS event occurred, antiplatelet use increased by $3.6 \%(p<0.05)$, with the largest increase was observed for clopidogrel $(10.9 \%, p<0.05)$.

Conclusion: A significant proportion of MI patients discontinued antiplatelet drugs at 1 year, although they restarted using antiplatelets again later. Clopidogrel was the most common antiplatelet drug to be discontinued after the index MI.

\section{Abstract \#: P 49}

Prevalence of hypertension and associated factors among residents of ibadan-north local government area of Oyo state, Nigeria 
Mr. Sowemimo, Ibukun O. ${ }^{1}$, Dr. Ajayi Ikeoluwapo O. ${ }^{2}$, Dr. Akpa Onoja $\mathrm{M}^{2}$

${ }^{1}$ Eagle's Heart Foundation; ${ }^{2}$ Department of Epidemiology, Faculty of Public Health, University of Ibadan, Nigeria; Corresponding author's e-mail: ibkprince2000@yahoo.co.uk

Background: Globally, hypertension is a major public health challenge. It is the most important risk factor for cardiovascular diseases. In Nigeria, hypertension is the most frequently diagnosed cardiovascular disorder and its complications constitute a significant proportion of emergency medical admissions in urban hospitals. This study aimed to investigate the prevalence of hypertension and associated factors among the residents of Yemetu community: an urbanslum in Ibadan-North Local Government Area of Oyo State, Nigeria. Methods: A descriptive cross-sectional design was used. The study involved 806 respondents aged from 18 to 90 years from 171 households. They were selected by cluster sampling technique. It was a house-to-house survey. Behavioural risk factors were measured using World Health Organisation (WHO) STEPwise approach to chronic disease risk factor surveillance (STEPS 1 and 2), while physical activities were measured using International Physical Activity Questionnaire (IPAQ). Hypertension was defined as Systolic blood pressure $(\mathrm{SBP}) \geq 140$ and/or Diastolic blood pressure (DBP) $\geq 90 \mathrm{~mm} \mathrm{Hg}$ or currently on anti-hypertensive medication. Data were analysed using descriptive statistics, Chi square and binary logistic regression tests at $p<0.05$.

Results: The overall prevalence of hypertension was $33.1 \%$ (male $36.8 \%$ and female $31.1 \%$ ). The proportion of self reported hypertension was $11.1 \%$, while $5.1 \%$ were currently on anti-hypertensive medication. Prior to the survey, $52.0 \%$ had checked their blood pressure within the past 12 months, $29.4 \%$ had checked more than a year ago, while $18.6 \%$ had never checked. The mean age of the respondents was $38.8 \pm 15.6$ years. The body mass index of the respondents was 5.2, 52.0, 29.5 and $13.3 \%$ for underweight, normal, overweight and obese, respectively. Alcohol and tobacco use were found in 11.5 and $3.2 \%$, respectively. The result of binary logistic regression analysis revealed that hypertension was significantly associated with being in age groups 30-49 years (OR 2.258, $95 \% \mathrm{CI}$ : 1.311-3.884), $\geq 50$ years (OR 7.145, $95 \%$ CI: 3.644-14.011), being overweight or obese (OR 2.281, $95 \%$ CI: 1.022-5.088). Hypertension was inversely associated with being underweight (OR 0.537, 95 \% CI: 0.395-0.832).

Conclusion: This study revealed a high prevalence of hypertension among the inhabitants of Yemetu community, which puts them at risk for cardiovascular disease. These data underscores the need for urgent steps to create awareness and implement interventions for prevention and early detection of hypertension especially among those aged $\geq 30$ years and the overweight or obese.

\section{Abstract \#: P 50 \\ Estradiol, testosterone, and sex hormone binding globulin and cardiovascular health in men and postmenopausal women: the Rotterdam study}

Loes Jaspers $^{1}$, Klodian Dhana ${ }^{1}$, Ester A.L. de Jonge ${ }^{2}$, Taulant Muka ${ }^{1}$, $\bar{C}_{\text {Cindy Meun }}{ }^{3}$, Jessica C. Kiefte-de Jong ${ }^{3,4}$, Joop S.E. Laven ${ }^{3}$, Maryam Kavousi ${ }^{1}$, Oscar H. Franco ${ }^{1}$

${ }^{1}$ Department of Epidemiology, Erasmus University Medical Centre, Rotterdam, The Netherlands; ${ }^{2}$ Department of Epidemiology/Internal Medicine, Erasmus University Medical Centre, Rotterdam, The Netherlands; ${ }^{3}$ Division of Reproductive Medicine, Erasmus University Medical Centre, Rotterdam, The Netherlands; ${ }^{4}$ Leiden
University College, The Hague, The Netherlands; Corresponding author's e-mail: 1.jaspers@erasmusmc.nl

Background: The American Heart Association defined cardiovascular health $(\mathrm{CVH})$ metrics for $\mathrm{CVH}$ promotion and disease reduction. These include ideal levels of 3 health factors: total cholesterol, fasting glucose and blood pressure and 4 health behaviours: physical activity at goal, non-smoking, normal body mass index and a healthy diet. Previous studies have shown that sex hormones can influence individual cardiovascular (risk) factors, but no studies have assessed their role in overall CVH. Therefore, we aimed to assess the association between sex hormones, including estradiol, testosterone, sex hormone binding globulin (SHBG), free androgen index (FAI) and testosterone/estradiol ratio (TER), with CVH in men and postmenopausal women.

Methods: Analyses were conducted within the population-based Rotterdam Study and included 1647 men (68.6 years) and 1564 naturally postmenopausal women (69.6 years) with available measurements of sex hormone levels (estradiol, testosterone and SHBG), from which FAI and TER were additionally calculated. The three health levels $(0=$ poor, $1=$ intermediate and $2=$ ideal health $)$ of the seven $\mathrm{CVH}$ metrics were added up to a total score ranging from 0 to 14 and thereafter categorized into three groups: low (0-7), moderate (8-10) and optimal CVH (11-14). The prevalence of these categories of $\mathrm{CVH}$ were assessed across gender-specific tertiles of each of the five hormone measurements. Thereafter, logistic regression was performed to explore the association between each sex hormone continuously and optimal versus moderate/poor CVH.

Results: Low, moderate and optimal CVH were prevalent in 45.8, 44.9 and $9.3 \%$ of men and in 38.4, 51.2 and $10.4 \%$ of women respectively (no participants had the maximum score of 14).

In men, optimal CVH was most prevalent at moderate levels of estradiol (39.2\%) and TER (36.6) and at high levels of testosterone $(41.2 \%)$ and SHBG $(47.1 \%)$. In women, optimal CVH was most prevalent at low levels of estradiol $(38.9 \%)$, testosterone $(43.8 \%)$ and FAI (47.5\%) and at high levels of SHBG (43.8\%).

Age adjusted logistic regression in men showed that optimal $\mathrm{CVH}$ was significantly associated with higher levels of testosterone (OR $1.062[1.034-1.091], \quad p<0.0001)$ and SHBG (OR 1.018 [1.010-1.025], $p<0.0001)$. In women, optimal CVH was associated with lower estradiol (OR 0.992 [0.986-0.999], $p=0.031$ ) and with higher SHBG levels (OR 1.018 [1.013-1.023], $p<0.0001$ ).

Conclusion: Our study showed an association between $\mathrm{CVH}$ with sex hormones among men and postmenopausal women. A more optimal $\mathrm{CVH}$ profile was associated with higher levels of testosterone and SHBG among men and lower estradiol and higher SHBG levels among women.

\section{Abstract \#: P 51}

Pre-dialysis decline of renal function is a risk factor for survival on dialysis

Chava L Ramspek ${ }^{1}$, Hakan Nacak ${ }^{1}$, Merel van Diepen ${ }^{1}$, Friedo W Dekker ${ }^{1}$

${ }^{1}$ Clinical epidemiology, LUMC, Leiden, the Netherlands; Corresponding author's e-mail: chavaramspek@gmail.com

Background: Chronic Kidney Disease is a worldwide public health problem that often necessitates kidney dialysis. The time period characterized by declining renal function before the start of dialysis is called pre-dialysis. It is unclear if the rate of renal function decline during pre-dialysis is of importance for the patient's prognosis after dialysis initiation. Most studies have focussed on single renal function values instead of looking at a trajectory of renal function decline. The 
few studies that have looked at this decline rate have used estimated glomerular filtration rate (eGFR) to do so. Unfortunately, eGFR gives an overestimation of renal function in patients with low filtration rates. The aim of the present study is to investigate whether the predialysis measured GFR (mGFR) decline rate is associated with mortality on dialysis.

Method: This study included 208 incident dialysis patients from the NECOSAD prospective cohort who had a minimum of two predialysis mGFR measurements (collected retrospectively) and a minimum of 30 days between the first and last measurement. An individual annual decline of mGFR, based on 24-h urine samples, was estimated with linear regression. Patients were divided into two groups based on the rate of renal function decline: fast decliners $\left(>4 \mathrm{~mL} / \mathrm{min} / 1.73 \mathrm{~m}^{2}\right.$ per year, $\left.\mathrm{n}=128\right)$ and slow decliners $(\leq 4 \mathrm{~mL} /$ $\min / 1.73 \mathrm{~m}^{2}$ per year, $\mathrm{n}=80$ ). Cox proportional hazards regression was used to perform multivariable modelling of survival probabilities and to adjust for potential confounders, after the start of dialysis.

Results: After the start of dialysis 82 patients died. The median survival time was 5.3 years. Crude analysis showed a significantly higher risk of death in fast decliners: HR 1.707 (95\% CI: 1.068-2.726). Adjusting for age and gender increased this association: HR 1.951 (95\% CI: 1.219-3.123). Further adjustment for primary kidney disease, cardiovascular disease, diabetes, cancer and ethnicity still gave a significant effect: HR 1.718 (95\% CI: 1.033-2.859). Sensitivity analyses confirmed stability of results.

Conclusions: This study found that a fast mGFR decline during predialysis is a risk factor for survival on dialysis. To the best of our knowledge this is the first study using mGFR to measure renal function decline. The pre-dialysis mGFR slope has importance for the follow up and treatment of dialysis patients, and gives incentive for more frequent GFR measurements in pre-dialysis patients.

\section{Abstract \#: P 52}

Education and cardiovascular risk factors: examining the impact of family factors shared by 215,121 Norwegian siblings

Inger Ariansen $^{1}$, Laust Mortensen ${ }^{2}$, Sidsel Graff-Iversen ${ }^{1}$, Hein Stigum $^{1,3}$, Marte Kjøllesdal ${ }^{3}$, Øyvind Næss ${ }^{1,3}$

${ }^{1}$ Division of Epidemiology, Norwegian Institute of Public Health, Oslo, Norway; ${ }^{2}$ Section of Social Medicine, University of Copenhagen, Copenhagen, Denmark; ${ }^{3}$ Institute of Health and Society, University of Oslo, Oslo, Norway; Corresponding author's e-mail: inger.ariansen@fhi.no

Background: Indicators of childhood socioeconomic circumstances are associated to risk of cardiovascular disease (CVD) in adulthood, indicating that the potential for prevention may start early in life. To assess the impact of factors in early life shared by siblings on educational inequalities in CVD risk factors in adulthood, we investigated the educational gradient between siblings.

Methods: Data on CVD risk factors at age 40-45 years in a cohort from Norwegian health examinations (1974-2003) was linked to national educational data and to generational data from the Norwegian Family Based Life Course study. The study population included 215,121 health survey participants that had a full sibling in one of the health surveys (93,315 sibships). $71 \%$ of participants had one or more siblings with different attained educational level (up to 9 years, 10-11 years, 12 years, 13-16 years, or more than 16 years of education).

Results: A one category disadvantage in educational level was associated with 0.8 (95\% confidence interval $0.8,0.8) \mathrm{mm} \mathrm{Hg}$ higher systolic blood pressure, $0.08(0.07,0.08) \mathrm{mmol} / \mathrm{l}$ higher level of serum total cholesterol, $0.21(0.20,0.22) \mathrm{kg} / \mathrm{m}^{2}$ higher BMI, and 0.05 $(0.05,0.05)$ and $0.03(0.03,0.03)$ percentage points higher predicted risk for 10 -year CVD mortality by the Systematic COronary Risk Evaluation (SCORE) risk prediction score.

When siblings were compared the corresponding educational differences were $0.6(0.5,0.6) \mathrm{mm} \mathrm{Hg}, 0.05(0.05,0.06) \mathrm{mmol} / \mathrm{l}, 0.12$ $(0.10,0.14) \mathrm{kg} / \mathrm{m}^{2}$ and $0.03(0.03,0.03)$ percentage points.

Conclusion: This study indicates that some of the educational inequalities in CVD risk factors are explained by factors shared by siblings.

\section{Abstract \#: P 53}

The modern view of the epidemiology and etiology of intringement cerebral blood circulation in adults

Nazarbek A. Zhumanazarov ${ }^{1}$, Mustafa. I. Omaraliev ${ }^{1}$, Marat. U. Kamyspaev $^{1}$, Anvar. N. Temirbekov ${ }^{1}$, Nurlan. K. Khodzhaev ${ }^{1}$, Samat. S. Doszhanov ${ }^{1}$, Kayrart. M. Tulepbekeov ${ }^{1}$, Dinara. A. Sisenbina ${ }^{1}$, Guljauhar. N. Taskynova ${ }^{1}$

${ }^{1}$ Pathological Anatomy, International kazakh-turkish university af. A.Yassavi, Turkistan, Kazakhstan; Corresponding author's e-mail: j_nazarbek@mail.ru

The prevalence of cerebrovascular disease in the world is very large and is in economically developed countries, about $15 \%$, and mortality from this disease exceeds $30 \%$ and confidently takes $2-3$ place in the structure of total mortality. According to WHO, stroke-a clinical syndrome presented focal neurological and/or cerebral disorders, develops suddenly due to acute infringement cerebrovascular circulation continuing at least $24 \mathrm{~h}$ and ends with the death of the patient in this or earlier. Epidemiological studies are one of the important directions in the study of vascular disease of the brain and provide information about how clinical and applied aspects of the problem.

The purpose of the study: to examine the incidence, mortality, prevalence of risk factors and evaluate ways to predict outcomes of cerebral insult.

Materials and methods: To carry out the study, was chosen southwestern region of the South Kazakhstan region. Cases of insult were recorded for all the inhabitants of the region under study over 25 years.

Results: The results obtained morphological and epidemiological studies have allowed to identify the most important factors in the risk of circulatory system in the first place, hypertension, dyslipidemia, diabetes mellitus, smoking, and the others. At the same time, it was shown that these factors also serve as markers of adverse course of stroke, complications and death. One of the goals of our research is to study the risk factors for cerebral vascular insult, because it is their presence and the combination leads to the development and growth of the incidence of stroke is important in assessing the severity of the clinical outcome of the disease and, determines the tactical actions during prophylaxis. Analysis of rick factors in our study revealed a high incidence of hypertension (96.2\% of cases), ischemic heart disease (40.6\%), myocardial infarction (19.8\%), dyslipidemia (39.7\%), cardiac arrhythmia $(20.4 \%)$, diabetes $(18.8 \%)$ smoking $(30.7 \%)$ in history. Thus, from the results of the data shows that for primary and secondary prevention cerebral vascular insult paramount correction blood pressure as the leading risk factor in the development of to acute infringement cerebrovascular circulation. 


\section{Abstract \#: P 54}

\section{Anthropometric measurements for the secondary prevention} of cardiovascular disease: the Rotterdam study

\section{Adela Brahimaj ${ }^{1}$, Klodian Dhana ${ }^{1}$, Abbas Dehghan ${ }^{1}$, M. Arfan} Ikram $^{1}$, Albert Hofman ${ }^{1}$, Maryam Kavousi ${ }^{1}$, Oscar H. Franco ${ }^{1}$

${ }^{1}$ Department of Epidemiology, Erasmus University Medical Center, Rotterdam, the Netherlands; Corresponding author's e-mail: a.brahimaj@erasmusmc.nl

Background: Anthropometric measures are used in clinical practice mainly to predict the risk of first cardiovascular disease (CVD) events. It is unclear whether they offer the same contribution in secondary prevention. Thus, we evaluated the performance of different anthropometric measures in prediction of subsequent cardiovascular events, in the middle-aged and elderly population.

Methods: The study included 945 participants with history of CVD from the prospective population-based Rotterdam Study. We examined the associations of body mass index (BMI), waist circumference (WC), waist-hip ratio (WHR) and a recently introduced anthropometric measure, a body shape index (ABSI, defined as $\mathrm{WC} /(\mathrm{BMI} /$ 3 *height 1/2)) with subsequent cardiovascular events. Cardiovascular events included prevalent coronary heart disease (CHD), stroke or heart failure. Traditional risk factors included in the study were age, total and high density lipoprotein (HDL) cholesterol, systolic blood pressure, treatment for hypertension, smoking, and diabetes mellitus. Predictive performance of different anthropometric measures was compared using hazard ratios (HR) and c-statistic. We then compared the added predictive value of the strongest anthropometric measure in prediction of CVD above the traditional risk factors using integrated discrimination improvement (IDI) and continuous net reclassification improvement (cNRI).

Results: During 10 years of follow-up there were 250 secondary cardiovascular events, among which $140(56 \%)$ were fatal. In age and gender adjusted models, ABSI (HR (95 \% CIs): $1.18(1.08,1.29))$ and WHR (HR (95\% CIs): $1.18(1.08,1.29))$ were associated with subsequent cardiovascular events. In the multivariate adjusted models, only ABSI (HR (95\% CIs): $1.11(1.08,1.29)$ ) was associated with subsequent cardiovascular events. The models including ABSI had higher c-statistic $(0.640$ (95\% CI) $0.606,0.673)$ ) compared to models including BMI, WC or WHR. When added to the model including traditional risk factors, ABSI provided an improvement in model fit (IDI (95 \% CI): $0.005(0.001,0.009))$ and risk stratification (cNRI $(95 \% \mathrm{CI}): 0.16(0.02,0.31))$ in prediction of subsequent cardiovascular events.

Conclusion: In this population-based study of middle-aged and elderly, among all anthropometric measures, a body shape index (ABSI) was the strongest predictor of subsequent cardiovascular events.

\section{Abstract \#: P 55}

\section{Early and late puberty and differences in cardiometabolic} markers at 16 years

Nina E. Berentzen ${ }^{1,2}$, Henriëtte A. Smit ${ }^{2}$, Lenie van Rossem² ${ }^{2}$ Ulrike Gehring $^{3}$, Alet H. Wijga ${ }^{1}$

${ }^{1}$ Center for Nutrition, Prevention, and Health Services, National Institute for Public Health and the Environment (RIVM); ${ }^{2}$ Julius Center for Health Sciences and Primary Care, University Medical Center Utrecht; ${ }^{3}$ Division of Environmental Epidemiology, Institute for Risk Assessment Sciences (IRAS), Utrecht University; Corresponding author's e-mail: nina.berentzen@rivm.nl
Background: Women with early menarche ( $<12$ years) have increased risks of overweight, hypertension, hypercholesterolemia, and coronary heart disease (CHD). The relation with CHD is likely U-shaped, with both early and late menarche being associated with increased risk. Fewer studies were conducted in men, but results also suggest increased risk with earlier puberty. We examined levels of cardiometabolic markers at 16 years by pubertal status in Dutch adolescents. We hypothesized that adolescents with early and late puberty have unfavourable cardiometabolic markers, compared to those with intermediate puberty.

Methods: We used data of 395 girls and 358 boys participating in the PIAMA birth cohort study, with data on puberty and cardiometabolic markers. Puberty development scale (pds, range 1-4), based on secondary sex characteristics, was self-reported at ages 11, 14 and 16. Based on pds percentile cut-offs, we classified girls as early ( $>$ P75 at age 11 , corresponding with pds $>2.3$, $\mathrm{n}=80)$, late $(<\mathrm{P} 25$ at age 16 , corresponding with pds $\leq 3.2$, $\mathrm{n}=86$ ) or intermediate puberty (all others, $\mathrm{n}=202$ ). Corresponding values for boys were early (pds $>3.4$ at age 14, $\mathrm{n}=92)$, late $(\mathrm{pds}<2.8$ at age $16, \mathrm{n}=80)$, intermediate $(\mathrm{n}=186)$. Additionally, based on age at menarche, we classified girls as early $(\leq 11$ years, $\mathrm{n}=42)$, intermediate $(12-14$ years, $\mathrm{n}=292)$ or late $(\geq 15$ years, $\mathrm{n}=61)$ menarche. Cardiometabolic markers (body mass index (BMI), waist circumference (WC), cholesterol, blood pressure (BP), Hemoglobin A1c) were assessed at age 16. Using multiple regression analyses, we estimated regression coefficients and $95 \%$ confidence intervals (CI) of early and late puberty/menarche for cardiometabolic markers by sex, treating intermediate as reference. We adjusted for age, height (except for BMI), ethnicity, and parental education.

Results: Compared to those with intermediate puberty, girls with early puberty had 0.29 higher BMI z-score $(95 \%$ CI $0.04,0.54)$ and $3.64 \mathrm{mmHg}$ higher systolic BP (95\% CI 1.21, 6.06) at age 16. Girls with late puberty had 0.35 lower BMI z-score $(95 \% \mathrm{CI}-0.59$, -0.10): girls with late menarche had comparably low BMI z-score, and $2.50 \mathrm{mmHg}$ lower diastolic BP (95\% CI -4.53, -0.47). Adjusting for BMI attenuated associations with BP. Boys with late puberty had 0.37 lower BMI z-score $(95 \% \mathrm{CI}-0.64,-0.11)$.

Conclusion: Boys and girls with late puberty had a lower BMI. Girls with early puberty had higher BMI and blood pressure levels. Earlier puberty may be important for cardiometabolic risk already in adolescence, particularly for blood pressure. Contrary to findings in adults, late puberty does not seem to be a risk factor for unfavourable cardiometabolic markers in adolescents.

\section{Abstract \#: P 56}

Genetic predisposition is a major risk factor for atrioventricular septal defects among infants with Down syndrome

Cynthia D.J. Kusters ${ }^{1}$, Mieke S. Kerstjens-Frederikse ${ }^{2}$, Marian K. Bakker $^{3,4}$, Rolf M.F. Berger ${ }^{5}$, Henk Groen ${ }^{1}$, Hermien E.K. de Walle ${ }^{1}$

${ }^{1}$ University of Groningen, University Medical Center Groningen, Department of Genetics, Eurocat Registration Northern Netherlands, Groningen, The Netherlands; ${ }^{2}$ University of Groningen, University Medical Center Groningen, Department of Genetics, Groningen, The Netherlands; ${ }^{3}$ University of Groningen, University Medical Center Groningen, Department of Obstetrics, Groningen, The Netherlands; ${ }^{4}$ University of Groningen, University Medical Center Groningen, Department of Gynaecology, Groningen, The Netherlands;

${ }^{5}$ University of Groningen, University Medical Center Groningen, Department of paediatric cardiology, Groningen, The Netherlands; Corresponding author's e-mail: cynthiakusters@gmail.com 
Objective: $40-55 \%$ of Down syndrome (DS) patients are born with a congenital heart defect (CHD), predominantly, atrioventricular septal defects (AVSD). This study investigates "genetic" and exogenous risk factors for AVSD among children with DS.

Methods: This population-based case-control study used birth defects registry data from 1997 through 2012. Patients with a DS diagnosis and a prenatal ( $\geq 16$ weeks gestation), postnatal or postmortem cardiac screening were selected $(n=274)$. DS patients with an AVSD were classified as cases $(n=80)$ and DS patients without a CHD were classified as controls $(n=194)$. "Genetic" risk factors were defined as risk factors with at least partly a genetic origin, such as gender, ancestry and family history. Information was collected using medical records and parental questionnaires. Univariable and multivariable logistic regression analyses were performed.

Results: Significant risk factors in the univariable logistic regression analyses were: African ancestry (OR 3.03 (95 \% CI: 1.05-8.74)): a positive family history of CHD (OR 3.79 (95 \% CI: 1.03-13.85)): female gender (OR 1.78 (95\% CI: 1.05-3.01)): low parental education (OR 2.57 (95 \% CI: 1.04-6.34)): and body mass index less than 18.5 (OR 10.93 (95 \% CI: 1.19-100.64)). The risk factors for AVSD in the forward selection multivariable regression analysis were: African ancestry (OR 4.22 (95\% CI: 1.16-15.33)): positive CHD family history (OR 5.09 (95\% CI: 1.20-21.62)): and female gender (OR 1.83 (95\% CI: 1.01-3.33)).

Conclusion: This study suggests that the pathomechanism of AVSD among DS patients is primarily a result of a genetic predisposition. Therefore, intervention on exogenous risk factors may only slightly reduce the prevalence of AVSD. Further research using a larger cohort is warranted to determine the exact reasons.

\section{Abstract \#: P 57}

\section{Blood pressure screening in childhood, adolescence and young adulthood only in the presence of obesity or parental history of hypertension?}

Hannelore K. Neuhauser ${ }^{1}$, Carolin Adler ${ }^{1}$, Angelika Schaffrath Rosario ${ }^{1}$

${ }^{1}$ Department of Epidemiology and Health Monitoring, Robert Koch Institute, Berlin, Germany; Corresponding author's e-mail: neuhauserh@rki.de

Background: Guidelines recommend yearly blood pressure (BP) screening in children from age 3 years but more efficient BP screening may be appealing, e.g. BP measurements only in children with obesity or parental history of hypertension. In young adulthood, while women have their BP taken regularly at gynecologist consultations, young men may not seek BP measurement unless they are obese or aware of parental hypertension. Since the aim of screening is that as few as possible get through undetected, this study investigated the sensitivity of selective screening for hi gh BP in children and young adults based on obesity status and on parental history of hypertension.

Methods: The most recent nationally representative German health examination surveys with standardized blood pressure, weight and height measurements were used for analysis: the KiGGS 2003-2006 survey with 14,633 children aged 3-17 years and the DEGS1 2008-2011 study with 1884 young adults aged 18-39 years. The mean of two measurements of resting BP was used. In children, BP percentiles by age and height in non-overweight boys and girls separately were derived using the GAMLSS software, as reported previously. In adults, hypertension was defined as $\mathrm{BP} \geq 140$ / $90 \mathrm{mmHg}$ or intake of antihypertensive medication. History of parental hypertension was available for both surveys (KiGGS1 only telephone follow-up 2009-2012 participants).

Results: $10.4 \%$ of children aged 3-17 years had hypertensive BP ( $\geq$ P95, KiGGS reference). If BP measurements were only taken in obese children (BMI $\geq$ P97, German reference), only $20 \%$ of hypertensive children would be detected (sensitivity $20.2 \%$ (17.4-23.4)) and $80 \%$ would be missed. Sensitivity was lowest in the age group 3-6 years $(8.4 \%)$ and highest in 14- to 17-year-olds $(26.2 \%)$. Parental history of hypertension would also still miss the vast majority of children with hypertensive BP. In 18-39 year old men, BP measurement restricted to the obese would have detected only $39 \%$ of those with hypertension, and restriction to parental history of hypertension only $43 \%$ (women $56 \%$ and $71 \%$ ).

Conclusion: Selective BP screening only in children with obesity or familial history of hypertension cannot be recommended since a relevant proportion of children with hypertensive BP would be missed. Similarly, all young men should have regular BP measurements, including those without obesity or parental history of hypertension.

\section{Abstract \#: P 58}

National economic loss due to non-communicable deaths in India Manisha Dubey $^{1}$

${ }^{1}$ International Institute for Population Science, Mumbai, India; Corresponding author's e-mail: manikvdlw@gmail.com

Economic loss due to ill health and early death are associated not merely with the cost of care but takes a heavy toll in terms of loss of productivity and hence the economy of the nation. From a policy standpoint, the adverse economic outcome resulted from the death of a person is important to perform the cost-benefit analysis of the disease-specific-programs. India is experiencing changes in life style, urbanization, industrialization, socio-economic development, demographic and epidemiological transition, which altogether leads to less prevalence of communicable diseases and growing burden of noncommunicable diseases (NCD) in the country. While early deaths due to any cause impose economic loss to the country, our focus in this study is on NCDs because of their rising burden in India. The central idea which has guided this study is to estimate the economic loss due to early age death by NCD rather the economic costs involve in the treatment of the disease.

The aim of the study is to estimate the economic loss, at national level, resulted from the deaths due to NCD. This study has taken population from Census of India, NCD deaths from Global Burden of Diseases and per capita income (PCI) from Economic Survey of India. Disease deleted life table for NCD and overall life tables are constructed for male and female to estimate the difference in the life expectancy (LE) in the absence of NCD and overall LE. Economic loss is calculated as a product of difference in the life expectancies, number of NCD deaths and PCI. In the absence of NCD, gain in the life expectancy is highest at age zero (two years) and declines with age among male and female. Economic loss in the working age group (15-60) is 6.34 Billion usd for males and 3.14 billion rupees for females at base price of 2010-11. The overall economic loss from NCD is 9.65 Billion usd that is $10.17 \%$ of total expenditure on health sector in year 2010-11 by Government of India.

\section{Abstract \#: P 59}

Metabolically healthy obesity and risk of cardiovascular disease among the elderly: the Rotterdam study 
Klodian Dhana ${ }^{1}$, M. Arfan Ikram ${ }^{1,2}$, Albert Hofman ${ }^{1}$, Maryam Kavousi $^{1}$, Oscar H. Franco ${ }^{1}$

${ }^{1}$ Department of Epidemiology, Erasmus MC, Rotterdam, the Netherlands; ${ }^{2}$ Department of Neurology, Erasmus MC, Rotterdam, the Netherlands; Corresponding author's e-mail:

k.dhana@erasmusmc.nl

Background: Obesity is associated with an increased risk of cardiovascular disease (CVD). Recently, a subgroup of obese individuals called "metabolically healthy obese" (MHO) has been identified which seems to be protected against CVD. No study has addressed whether metabolic syndrome confers a greater risk of CVD compared to obesity among older population. In the present study we investigated whether MHO is associated with CVD among the elderly.

Methods: This study included 5314 individuals (mean age 67 years) from the prospective population-based Rotterdam Study. We categorized our population in groups according to body mass index (BMI) as normal weight, overweight, or obese and according to absence or presence of metabolic syndrome. The risk of CVD was evaluated for each group by using Cox proportional hazard regression analysis.

Results: In our population among obese individuals we identified $25 \%$ MHO subjects. Over the median follow-up of 10.2 years, there were 861 incident cases of CVD. Compared with normal weight subjects both overweight (HR 1.13, $95 \%$ CI $0.97-1.32$ ) and obesity (HR 1.21, $95 \%$ CI 0.99-1.46) were not associated with risk of CVD. Presence of metabolic syndrome increased the risk of incident CVD by $30 \%$ (HR 1.30, $95 \%$ CI 1.14-1.49) regardless of the BMI category. There was not an increased risk for CVD among MHO (HR $1.08,95 \%$ CI $0.76-1.54$ ) individuals when we compared them to metabolically healthy normal weight individuals. Metabolically unhealthy obese subjects were not at increased risk for CVD compare to MHO individuals (HR 1.25, $95 \%$ CI 0.86-1.81).

Conclusion: Our findings suggest that among middle-aged and elderly adults MHO individuals are not at increased risk of CVD, and the presence of the metabolic syndrome confers more risk than being obese alone.

\section{Abstract \#: P 60}

Mortality of metabolic syndrome (METS) in Zanjan and Abhar healthy heart study after eight years of follow up

\section{$\underline{\text { Saeideh Mazloomzadeh }}^{1}$, Fatemeh Karami ${ }^{2}$, Alireza Shoghli ${ }^{3}$}

${ }^{1}$ Zanjan Social Determinants of Health Research Center, Zanjan University of Medical Sciences; ${ }^{2}$ Zanjan University of Medical Sciences; ${ }^{3}$ Social Medicine Department, Medical Faculty, Zanjan University of Medical Sciences; Corresponding author's e-mail: smazloomzadeh@zums.ac.ir

Background: The metabolic syndrome is characterized by the clustering of cardiovascular risk factors including abdominal obesity, elevated blood pressure, hyperglycemia, and dyslipidemia. Several studies about the association between metabolic syndrome and allcause mortality and cardiovascular mortality have been performed in different countries, however, no data on this relationship are available in Iran. The purpose of this study was to determine the association between the Mets and its components with mortality.

Methods: In this retrospective cohort study, we used data from Zanjan and Abhar Healthy Heart Study that was performed on 4000 persons older than 15 years, in 2003. According to NCEP (ATPIII) definition, 1051 of participants who had Mets and 1219 who had no or one of its components were enrolled. Information from 502 subjects with Mets and 523 subjects with no or one of Mets components was collected by telephone call and was analyzed using Cox Proportional hazard models.

Results: The median of follow- up was $104 \pm 10.7$ months. 35 deaths have occurred at this time: including 18 cardiovascular deaths. The hazard ratio of all cause mortality for those who had Mets was 1.75 fold (95\% CI 0.88-3.47) more than controls. The hazard ratio of CVD mortality was 3.66 fold (95\% CI 1.2-11.1) higher in those who had Mets compared to control group which was not significant after adjusting for age and sex. From the metabolic syndrome components, even after adjusting for age and sex, only hypertension predicted a higher risk of all cause and cardiovascular mortality. The risk of mortality has also increased with increasing the number of Mets components.

Conclusion: The findings of this study have indicated a limited utility of Mets for predicting total or CVD mortality compared with cardiovascular risk factors such as hypertension. Higher risks of all cause and cardiovascular mortality for those who had hypertension had implications for conducting early prevention, intervention and therapeutic programs in these patients.

\section{Abstract \#: P 61}

Association between body mass index and health-related quality of life differs by smoking status in high-risk cardiovascular patients

Rebecca Muckelbauer ${ }^{1}$, Ulrike Grittner ${ }^{2}$, Heike Englert ${ }^{3}$, Karl Wegscheider ${ }^{4}$, Heinz Völler ${ }^{5}$, Hugo A. Katus ${ }^{6}$, Stefan N. Willich ${ }^{7}$, Jacqueline Müller-Nordhorn ${ }^{1}$

${ }^{1}$ Berlin School of Public Health, Charité - Universitätsmedizin Berlin; ${ }^{2}$ Biostatistics and Clinical Epidemiology, Charité Universitätsmedizin Berlin; ${ }^{3}$ University of Applied Sciences, Münster; ${ }^{4}$ Department of Medical Biometry and Epidemiology, University of Hamburg; ${ }^{5}$ Rehabilitation Center for Cardiovascular Diseases, Rüdersdorf; ${ }^{6}$ Department of Cardiology, University of Heidelberg; ${ }^{7}$ Institute for Social Medicine, Epidemiology and Health Economics, Charité - Universitätsmedizin Berlin; Corresponding author's e-mail: rebecca.muckelbauer@charite.de

Background: The association of the body mass index (BMI) with mortality as well as with health-related quality of life (HRQoL) seems to be non-linear with an ideal BMI between 25 and 30. We hypothesized that the association between BMI and HRQoL differs between smokers, former smokers, and never smokers in high-risk cardiovascular patients.

Methods: We analyzed cross-sectional baseline data of participants from the ORBITAL (Open Label Primary Care Study: Rosuvastatin Based Compliance Initiatives To Achievements of LDL Goals) study. In this study (ClinicalTrials.gov identifier: NCT00379249) 8108 patients with hypercholesterolemia and an increased cardiovascular risk were randomized. HRQoL was assessed with the Short Form (SF)-12 health status questionnaire. Physicians assessed body weight and height: BMI $\left(\mathrm{kg} / \mathrm{m}^{2}\right)$ was calculated. Smoking status was selfreported. We tested if the smoking status modified the association between SF-12 and BMI by introducing the interaction term. To identify the BMI at which SF-12 summary scores peaked and the direction of the association changed, we plotted a penalized linear b-spline curve. By linear regression, we tested the change of the slope and estimated the coefficients before and after the peak BMI.

Results: We included 7640 participants (mean age: 61 years) with available baseline questionnaires. BMI ranged from 16.0 to $46.0 \mathrm{~kg} /$ $\mathrm{m}^{2}$ (mean: 28.2, standard deviation: 4.2). There was an interaction of smoking status with BMI with regard to physical $(p=0.016)$ but not mental SF-12 summary scores $(p=0.731)$. Thus, we performed 
stratified analyses for physical SF-12. In never smokers, no BMI value at which physical SF-12 peaked was identified: physical SF-12 decreased continuously with increasing BMI over the total BMI range $(\beta=-0.30, p<0.001)$. In smokers and former smokers we identified a BMI value at which physical SF-12 peaked. In smokers, the association between BMI and physical SF-12 changed significantly from $\beta=+0.46$ to $\beta=-0.24(p=0.032)$ at $\mathrm{BMI}=24.1 \mathrm{~kg} / \mathrm{m}^{2}$. Similarly, in former smokers the association changed from $\beta=+0.38$ to $\beta=-0.41$ at $\mathrm{BMI}=25.4 \mathrm{~kg} / \mathrm{m}^{2}(p=0.003)$. A peak BMI was also identified for mental SF-12 summary scores in the total population. The association between BMI and physical SF-12 changed from $\beta=+0.56$ to $\beta=-0.12(p<0.001)$ at $\mathrm{BMI}=23.7$.

Conclusion: Physical HRQoL decreased with increasing BMI but smoking modified the association. In smokers and former smokers, the association between physical HRQoL and BMI was non-linear with a peak in HRQoL at a BMI between 24 and $25 \mathrm{~kg} / \mathrm{m}^{2}$. Mental HRQoL increased with increasing BMI until a BMI of 24 and then decreased slightly.

\section{Abstract \#: P 62}

The prevalence of metabolic syndrome (MES) in Iran: a systematic review

Saeideh Mazloomzadeh ${ }^{1}$, Zahra Rashidi ${ }^{2}$, Nooreddin Moosavinasab ${ }^{2}$

${ }^{1}$ Zanjan Social Determinants of Health Research Center, Zanjan University of Medical Sciences; ${ }^{2}$ Zanjan University of Medical Sciences; Corresponding author's e-mail: smazloomzadeh@zums.ac.ir

Background: Metabolic syndrome is a collection of metabolic disorders which leads to early cardiovascular disease and diabetes type II. Regarding the wide range of its prevalence in Iran, this systematic review and meta-analysis was conducted to determine the overall prevalence of the metabolic syndrome in Iran.

Method: The study was a systematic review and meta-analysis. The Medline, ISI, IranMedex and SID were searched using "metabolic syndrome", "syndromeX", "prevalence" and "Iran" keywords from 2002 to 2012. A total of 223 articles were found in which 14 studies was considered for meta-analysis. Data were analysed using Fixed and Random Modeland Meta Regression in STATA.

Results: The prevalence of MeSfor those who were 20 and older was $23.8 \%$ (95 \% CI 18.99-28.67) and in under 20 was $10.98 \%(95 \% \mathrm{CI}$ $7.75-14.2)$. MeS was more frequent in women $(25.5 \%)$ than in men $(17.16 \%)$ and was increased with increasing age. The most frequent component of MeS was low HDL cholesterol (59.65\%) followed by hypertriglyceridemia $(39.51 \%)$.

Conclusion: Regarding a high prevalence of metabolic syndrome and some of its components such as low HDL and high triglyceride in our country, implementation of prevention programs for changing lifestyle through healthy diet, physical activity and weight control is necessary.

\section{Abstract \#: P 63}

\section{Second hand smoke: a blood pressure determinant in preschool children?}

Maria Cabral $^{1}$, Camila Gonzalez-Beiras ${ }^{1}$, Maria João Fonseca ${ }^{1}$, Ana Cristina Santos ${ }^{1,2}$, Henrique Barros ${ }^{1,2}$

${ }^{1}$ EPIUnit - Institute of Public Health, University of Porto, Porto, Portugal; ${ }^{2}$ Department of Clinical Epidemiology, Predictive Medicine and Public Health, University of Porto Medical School; Corresponding author's e-mail: maria.cabral@ispup.up.pt

Background: Second Hand Smoke exposure (SHSe) during childhood may be associated with permanent damage on blood pressure (BP) control mechanisms. We aimed to examine the association between maternal smoking (currently, during 3rd pregnancy trimester and before pregnancy) and $\mathrm{BP}$ in preschool children using data from a Portuguese birth cohort.

Methods: The sample was composed of 4331 children from Generation XXI, a birth cohort assembled at all public maternities of Porto, Portugal in 2005-2006 and re-evaluated 4 years after birth. At birth, information was collected by face-to-face interviews and additionally abstracted from clinical records. At follow-up, questionnaires were applied, anthropometrics were assessed and children's BP was measured. BP z-scores were calculated, following the recommendations of the American Academy of Pediatrics. Linear and logistic regression models were fitted to estimate the association between SHSe and children's BP levels.

Results: Higher levels of systolic BP were observed among children of current smoking mothers [99.00 (93.00-105.00) vs. 98.00 (92.00-103.00), $p<0.001]$. In the crude analysis, a linear positive association was found between maternal current smoking and children's systolic BP z-score $(\beta=0.11,95 \%$ CI $0.05 ; 0.18)$, whereas no association was observed for diastolic BP z-score $(\beta=0.01,95 \%$ $\mathrm{CI}-0.04 ; 0.06)$. Similar results were found when smoking in the third pregnancy trimester $(\beta=0.09,95 \%$ CI $0.01 ; 0.18)$ and within the three months before pregnancy $(\beta=0.12,95 \%$ CI $0.06 ; 0.18)$ were considered. Maternal education was negatively associated with both systolic $(\beta=-0.02,95 \%$ CI $-0.02 ;-0.01)$ and diastolic $(\beta=-0.01,95 \%$ CI $-0.02 ;-0.00)$ BP z-scores. Conversely, children's body mass index (BMI) was positively associated with systolic $(\beta=0.31,95 \%$ CI $0.28 ; 0.33)$ and diastolic $(\beta=0.20,95 \% \mathrm{CI}$ $0.18 ; 0.22)$ BP z-scores. After adjustment for child's BMI and maternal educational level, preconception maternal smoking remained positively associated with SBP ( $\beta=0.06,95 \%$ CI 0.03 ; 0.12 ). No significant association was found between current maternal smoking $(\mathrm{OR}=1.09$, $95 \% \mathrm{CI}$ 0.93-1.27) and high BP, after adjustment for potential confounders. The same was observed for third pregnancy trimester (OR $=1.10,95 \%$ CI 0.90-1.34). Child's BMI increased the likelihood of having high $\mathrm{BP}(\mathrm{OR}=1.34,95 \%$ CI 1.29-1.40), the opposite being observed for maternal educational level $(\mathrm{OR}=0.98,95 \%$ CI 0.96-0.99). Maternal smoking within the three months before pregnancy remained positively associated with children's high BP (OR = 1.17, $95 \%$ CI 1.00-1.37).

Conclusion: Child's BMI at 4y, maternal educational level, and maternal smoking as early as in the preconception period can impact on children's BP at a very small age.

\section{Abstract \#: P 65}

Maternal thyroid function during 3RD trimester of pregnancy and offspring blood pressure and adiposity at 20 years

Dorte Rytter $^{1}$, Bodil Hammer Bech ${ }^{1}$, Stine Linding Andersen ${ }^{2,3}$, Thorhallur Ingi Halldorsson ${ }^{4,5}$, Tine Brink Henriksen 6 , Peter Laurberg ${ }^{2,3}$, Sjurdur Frodi Olsen ${ }^{4}$

${ }^{1}$ Department of Public Health, Section for Epidemiology, Aarhus University, Aarhus, Denmark; ${ }^{2}$ Department of Endocrinology, Aalborg University Hospital, Denmark; ${ }^{3}$ Department of Clinical Medicine, Aalborg University, Aalborg, Denmark; ${ }^{4}$ Centre for Fetal Programming, Statens Serum Institut, Copenhagen, Denmark; ${ }^{5}$ The Unit for Nutrition Research, Faculty of Food Science and Nutrition, School of Health Sciences, University of Iceland, Reykjavik, Iceland; 
${ }^{6}$ Pediatric Department, Aarhus University Hospital, Aarhus, Denmark; Corresponding author's e-mail: dr@ph.au.dk

Background: Thyroid disease is associated with a number of health problems including cardiovascular disease and obesity. Also maternal thyroid hormones during pregnancy may regulate development of fetal hypothalamic centers governing cardio-vascular function. However, whether maternal thyroid function during pregnancy has the potential to permanently affect offspring adiposity and cardiovascular health is not known.

The objective of the study was to investigate the association between maternal concentrations of TSH and free T4 (fT4) as well as subclinical hypo- and hyperthyroidism in week 30 of gestation and offspring adiposity and blood pressure at 20 years.

Methods: The study was based on the follow up of a Danish birth cohort from 1988 to $89(\mathrm{n}=965)$. A blood sample was drawn from the pregnant women in week 30 of gestation. In 2008-2009 the offspring were followed up with self-reported anthropometrics $(\mathrm{N}=629)$ and a clinical examination including blood pressure measurements $(\mathrm{N}=410)$. Multiple linear regression was used to estimate the association between maternal TSH and fT4 in quintiles as well as subclinical hypo- $(\mathrm{TSH}>3 \mathrm{mIU} / \mathrm{L}$, fT4 within reference levels) and hyperthyroidism (TSH $<0.36 \mathrm{mIU} / \mathrm{L}$, fT4 within reference levels) and offspring BMI, waist circumference and blood pressure adjusting for maternal pre-pregnancy BMI, education, age, parity, smoking and dietary iodine intake during pregnancy as well as offspring sex.

Results: Offspring of subclinical hypothyroid women had higher systolic blood pressure (adjusted dif $=3.6(0.2: 7.0) \mathrm{mmHg}$ ) and a tendency towards higher diastolic blood pressure (adjusted dif $=2.3$ $(-0.2: 4.9) \mathrm{mmHg}$ ) compared to offspring of euthyroid women. Also, blood pressure tended to be higher in offspring of hyperthyroid mothers, but numbers were small. No association was found with offspring BMI and waist circumference.

TSH and fT4 showed inconsistent associations with the outcomes. However, sex specific associations were found between fT4 and offspring systolic blood pressure. Hence whereas a trend towards increased systolic blood pressure with increasing maternal fT4 was observed in girls, an inverse U-shaped association seemed to be present in boys.

Conclusion: Maternal thyroid function during 3rd trimester of pregnancy may affect long term cardiovascular health of the offspring. However, larger studies are needed to replicate the findings also with regard to potential sex specific associations.

\section{Abstract \#: P 67}

Incidence of recurrent CHD and the impact of obesity paradox in a population-based study: Tehran lipid and glucose study

Samaneh Asgari ${ }^{1}$, Davood Khalili ${ }^{1}$, Maryam Barzin ${ }^{2}$, Farhad Hosseinpanah ${ }^{2}$, Farzad Hadaegh ${ }^{1}$, Fereidon Azizi ${ }^{3}$

${ }^{1}$ Prevention of Metabolic Disorders Research Center, Research Institute for Endocrine Sciences (RIES), Shahid Be-heshti University of Medical Sciences, Tehran, Islamic Republic of Iran; ${ }^{2}$ Obesity Research Center, Research Institute for Endocrine Science, Shahid Beheshti University of Medical Science, Tehran, Iran; ${ }^{3}$ Endocrine Research Center, Research Institute for Endocrine Science, Shahid Beheshti University of Medical Science, Tehran, Iran; Corresponding author's e-mail: dkhalili@endocrine.ac.ir

Background: Data on incidence of recurrent coronary heart disease (rCHD) is limited in the Middle East and little is known about the effect of obesity paradox on rCHD in this area.
Methods: Study was conducted in 440 adults, aged $\geq 30$ years, with history of CHD at baseline who attended in first or second examination cycle, first in 1999-2001 and second in 2001-2003, and with at least one year of follow up until March 31, 2010. The incidence of recurrent $\mathrm{CHD}$ was explored. Cox proportional hazard models were used to evaluate the association among various BMI groups and recurrent CHD incidence.

Results: During a median follow-up of 8.44 years, 169 new cases of rCHD occurred (incidence density of rCHD was calculated as 54.53 per 1000 person-year).also the incidence of recurrent CHD was higher in normal BMI compared with overweight individuals and obese category (68.71, 47.56 and 54.46 per 1000 person-year, respectively). Relative to the normal weight group, the age, gender and waist circumference adjusted HRs for recurrent CHD outcomes were protected in both overweight and obese group ( $0.5195 \%$ CI $0.32-0.8$ and 0.52 $95 \%$ CI $0.28-0.99$, respectively). Moreover In the multivariable models with forward stepwise selection approach, obese group lost its significant protection effect $(0.5595 \%$ CI $0.28-1.06)$ despite overweight group (0.48 $95 \%$ CI $0.30-0.80)$.

Conclusion: In this study an apparent protective effect of overweight and obesity on long-term recurrent CHD in patients with history of CHD was shown.

\section{Abstract \#: P 68}

Risk of coronary heart events in symptomatic and asymptomatic individuals: 10 years follow-up in Tehran lipid and glucose study (TLGS)

Mohammad Ali Mansournia ${ }^{1}$, Kourosh Holakouie Naieni ${ }^{1}$, Noushin Fahimfar ${ }^{1}$, Zahra Cheraghi ${ }^{1}$, Amir Almasi-Hashiani ${ }^{1}$, Erfan Ayubi ${ }^{1}$, Fereidoun Azizi ${ }^{2}$, Davood Khalili ${ }^{3}$

${ }^{1}$ Department of Epidemiology and Biostatistics, School of Public Health, Tehran University of Medical Sciences, Tehran, Iran; ${ }^{2}$ Endocrine Research Center, Research Institute for Endocrine Sciences, Shahid Beheshti University of Medical Sciences, Tehran, Iran; ${ }^{3}$ Prevention of Metabolic Disorders Research Center, Research Institute for Endocrine Sciences, Shahid Beheshti University of Medical Sciences, Tehran, Iran; Corresponding author's e-mail: dkhalili@endocrine.ac.ir

Background: There are non-invasive and low-cost methods to assess high risk individuals for CHD such as Rose angina Questionnaire (RQ) and Minnesota ECG coding. Because of difference in the applicability of RQ, we aimed to find the risk of incident CHD based on RQ and ECG in an Iranian population.

Methods: 5431 subjects aged $\geq 30$ years ( 2575 males) were included from the population based cohort study of the Tehran Lipid and Glucose Study. We classified the participants into five categories according to presence of past history of CHD, RQ and ECG status at the baseline i) History-Rose-ECG- (the reference group), ii).

History-Rose-ECG+, iii) History-Rose + ECG-, iv) HistoryRose $+\mathrm{ECG}+$, and v) History + . A Cox regression model was used to detect the role of RQ and ECG independent of other risk factors. Results: During a median follow up of 10.3 years, 562 CHD events (320 males) were observed. Incidence rates (95\% CIs) of new CHD for people with and without history of CHD, were 55.9 (46.7-67) and 9.09 (8.28-9.97) cases per 1000 person-year, respectively. Compared to the reference group, HRs (95\% CIs) were 4.11 (3.27-5.11) for $\mathrm{CHD}+, 2.18$ (1.63-2.90) for History-Rose + ECG-, 1.92(1.47-2.51) for History-Rose-ECG+ and $2.48(1.46-4.20)$ for HistoryRose + ECG + in adjusted model.

Conclusion: High incidence rate of new CHD was detected in subjects with CHD self reported history and must be considered 
precisely. Rose questionnaire showed a good performance in the prediction of CHD events in both gender and can be assumed as a simple and valuable clinical screening tool to detect high risk subjects even in the absence of ECG abnormalities. ECG is also a strong tool to predict CHD events in both genders, especially in asymptomatic patients.

\section{Abstract \#: P 69 \\ Abdominal fat distribution and vascular characteristics in healthy 5 year-old children}

Geertje W. Dalmeijer $^{1}$, Frank L.J. Visseren ${ }^{2}$, Cornelis K. van der Ent $^{3}$, Diederick E. Grobbee ${ }^{1}$, Cuno S.P.M. Uiterwaal ${ }^{1}$

${ }^{1}$ Julius Center for Health Sciences and Primary Care, University Medical Center Utrecht, Utrecht, The Netherlands; ${ }^{2}$ Department of Vascular Medicine, University Medical Center Utrecht, Utrecht, The Netherlands; ${ }^{3}$ Department of Pediatric Pulmonology, Wilhelmina Children's Hospital, University Medical Center Utrecht, Utrecht, The Netherlands; Corresponding author's e-mail:

G.W.Dalmeijer@umcutrecht.nl

Purpose: It is unknown if fat distribution in childhood already affects the young vascular system. Extending on an earlier preliminary study, we assessed the association between body fat distribution and vascular characteristics in healthy 5 year old children.

Methods: In 863 5-year-olds of the Wheezing-Illnesses-Study-Leidsche-Rijn (WHISTLER) birth cohort, abdominal fat distribution was measured in $\mathrm{mm}$ with sonographic measurements. Carotid intimamedia thickness (CIMT) and arterial stiffness were obtained ultrasonographically. Univariable linear regression was performed with vascular characteristics as dependent and measurements of body fat distribution as independent variable. We adjusted for sex, age, birth weight and BMI.

Results: Overall mean CIMT was $387.9 \mu \mathrm{m}$ (SD 41.4) and mean distensibility was $94.9 \mathrm{MPa}-1$ (SD 27.9). Increased intra-abdominal fat was associated to thicker CIMT (linear regression coefficient $1.04 \mu \mathrm{m} / \mathrm{mm}, \quad 95 \%$ CI $0.57: 1.51)$ and lower distensibility (-0.767 MPa-1/mm, $95 \% \mathrm{CI}-1.10:-0.44)$. Subcutaneous fat was not associated with CIMT $(0.72,95 \% \mathrm{CI}-0.20$ : 1.64) and distensibility ( $-0.9995 \% \mathrm{CI}-2.28: 0.30)$.

Conclusion: More intra-abdominal fat is related to thicker and stiffer arteries already in young healthy childhood.

\section{Abstract \#: P 70 \\ Clustering of lifestyle-related behaviours in adolescents: differences between Europe and Brazil}

Tatiana Sadalla Collese ${ }^{1,2}$, Augusto César Ferreira de Moraes ${ }^{1,2}$, Juan Miguel Fernández-Alvira ${ }^{2,3}$, Stefaan De Henauw ${ }^{4}$, Anthony Kafatos ${ }^{5}$, Odysseas Androutsos ${ }^{6}$, Vanesa España ${ }^{7}$, Myriam Galfo ${ }^{8}$, Laurent Beghin ${ }^{9}$, Michael Sjöström ${ }^{10}$, Raquel Pedrero-Chamizo ${ }^{11}$, Luis Moreno Aznar ${ }^{2,12}$, Heráclito Barbosa de Carvalho ${ }^{1}$

${ }^{1}$ School of Medicine, University of São Paulo, YCARE (Youth Child and cArdiovascular Risk and Environmental) Research Group. São Paulo, SP, Brazil; ${ }^{2}$ Faculty of Health Sciences, University of Zaragoza, GENUD (Growth, Exercise, NUtrition and Development; ${ }^{3}$ Epidemiology, Atherothrombosis and Imaging Department, Centro Nacional de Investigaciones Cardiovasculares (CNIC). Madrid, Spain; ${ }^{4}$ Department of Public Health, Ghent University. Ghent, Belgium; ${ }^{5}$ Preventive Medicine \& Nutrition Unit, University of Crete
School of Medicine. Heraklion, Crete, Greece; ${ }^{6}$ Department of Nutrition and Dietetics, School of Health Science and Education, Harokopio University. Athens, Greece; ${ }^{7}$ School of Medicine, University of Granada. Granada, Spain; ${ }^{8}$ Agricultural Research Council, Food and Nutrition Research Centre (CRA-NUT). Rome, Italy; ${ }^{9}$ CIC-1403-Inserm-CH\&U and Inserm U995, Université de Lille. Lille, France; ${ }^{10}$ Department of Biosciences and Nutrition (BioNut), Karolinska Institute. Stockholm, Sweden; ${ }^{11}$ Polytechnic University of Madrid, Health and Human Performance, Madrid/ Spain; ${ }^{12}$ Visiting Professor, School of Medicine of the University of São Paulo - Department of Preventive Medicine, São Paulo/SP, Brazil; Corresponding author's e-mail: tatiana.scollese@gmail.com

Background: Adolescence is a critical period in the development of healthy lifestyle-related behaviors that could prevent obesity. This study aims to describe the clustering of several lifestyle-related behaviours (fruits and vegetables consumption, sugar-sweetened beverages consumption, physical activity, sedentary- and sleeping behaviours) in adolescents, by gender and region (Europe and Brazil). Methods: Adolescents from two cross-sectional studies, one conducted in Europe $(n=1252: 52.72 \%$ females from the HELENA study) and the other in Brazil $(n=682: 54.25 \%$ females from the BRACAH study), were selected by complex sampling, and met all inclusion criteria. Information on fruits and vegetables consumption, sugar-sweetened beverages consumption, physical activity, sedentary behaviour (screen time), and usual sleep duration was assessed by self-reported questionnaires. Gender-specific cluster analysis was performed separately for the HELENA and BRACAH studies, applying a combination of hierarchical and non-hierarchical methods. Results: We identified four clusters for girls and three for boys, which were different between regions: European girls (20.8\% Sedentary: $27.7 \%$ Non-active: $35.7 \%$ Healthy Eating: $15.9 \%$ Active): Brazilian girls $(17.8 \%$ Sedentary: $36.2 \%$ Unhealthy Eating: $31.5 \%$.Healthy Eating: $14.5 \%$ Active): European boys $(23.9 \%$ Sedentary: $33.3 \%$ Unhealthy Eating: $42.7 \%$ Healthy): Brazilian boys (44.7 \% Sedentary: $33.8 \%$ Healthy Eating: $21.5 \%$ Active).

Conclusions: The clustering of lifestyle-related behaviours differed substantially between Brazil and Europe and seem to be genderspecific. However, a cluster mainly characterized by high levels of screen time was identified for both genders and regions. These findings indicate that different health promotion strategies may need to be applied for the prevention of obesity in different regions and these activities may need to be tailor-made for each gender.

\section{Abstract \#: P 71}

Gestational diabetes mellitus and macrosomia in a dutch population: determinants and other adverse pregnancy outcomes

Moniek Looman $^{1}$, Anouk Geelen ${ }^{1}$, Rahul A.K. Samlal ${ }^{2}$, ${ }_{\text {Rik Heijligenberg }}{ }^{3}$, Edith J.M. Feskens ${ }^{1}$

${ }^{1}$ Division of Human Nutrition, Wageningen University, the Netherlands; ${ }^{2}$ Department of Gynaecology and Obstetrics, Hospital Gelderse Vallei Ede, the Netherlands; ${ }^{3}$ Department of Internal Medicine, Hospital Gelderse Vallei Ede, the Netherlands; Corresponding author's e-mail: moniek.looman@wur.nl

Background: Both gestational diabetes mellitus (GDM) and macrosomia (birth weight $>4000 \mathrm{~g}$ ) are common pregnancy complications. Macrosomia is often linked to (undiagnosed) GDM, but it is also common in women with normal glucose tolerance. Numerous studies have investigated the association of either GDM or macrosomia with other adverse pregnancy outcomes. However, few studies have looked at combinations of GDM and macrosomia (i.e. combinations of GDM, impaired glucose tolerance and normal glucose tolerance with 
macrosomia and normal birth weight) and their association with other adverse pregnancy outcomes. Therefore, this study focusses on combinations of GDM and macrosomia in a Dutch population, their main determinants and their associations with other adverse pregnancy outcomes.

Methods: This study was conducted in hospital Gelderse Vallei in Ede, the Netherlands. A universal two step screening approach was used to detect GDM. Seven hundred and fifty women were diagnosed with GDM between 2006 and 2014. Of these, 215 (29\%) gave informed consent to use their medical data for this study. Between 2011 and 2014, 326 women had a positive $50 \mathrm{~g}$ glucose challenge test, but a negative $75 \mathrm{~g}$ oral glucose tolerance test, of which 87 (27\%) gave informed consent to use their data for this study (=impaired glucose tolerance group). Two hundred-five $(23 \%)$ of 882 women who gave birth to a macrosomic child and had normal glucose tolerance between 2011 and 2014 gave informed consent. Of the 3009 women with normal glucose tolerance and who gave birth to a normal weight infant a random sample of $n=950$ was drawn. Of these, 165 $(17 \%)$ gave informed consent. Data on maternal age, ethnicity, education level, body mass index (BMI), parity, history of gestational diabetes, history of macrosomic birth and smoking status was retrieved from medical files. Pregnancy outcomes included mode of delivery, gestational age, birth weight, Apgar score, post-partum haemorrhage, gestational hypertension, preeclampsia, shoulder dystocia and neonatal birth injuries. To compare variables between groups, ANOVA was used for continuous variables and Chi squared tests for categorical variables. Multiple logistic regression was used to adjust for possible confounders including maternal age, BMI, ethnicity, parity and gestational age.

Results will be presented.

\section{Abstract \#: P 72 \\ Revising the International Diabetes Federation diabetes atlas methodology for estimating global and national prevalence of diabetes in adults}

Katherine Ogurtsova ${ }^{1}$, Leonor Guariguata ${ }^{1}$, David Whiting ${ }^{1}$, Nigel Unwin $^{2}$, Clara Weil ${ }^{1}$, Ute Linnenkamp ${ }^{1}$, Joao D. da Rocha Fernandes $^{1}$, Lydia E. Makaroff ${ }^{1}$, Nam H. Cho ${ }^{3}$

${ }^{1}$ International Diabetes Federation; ${ }^{2}$ Chronic Disease Research Centre, University of the West Indies; ${ }^{3}$ Department of Preventive Medicine, Ajou University School of Medicine; Corresponding author's e-mail: Katherine.Ogurtsova@idf.org

Diabetes is a major contributor to the global burden of mortality and morbidity. Global and country-level estimates of diabetes prevalence are needed for coordinated and evidence-based decision making. The International Diabetes Federation (IDF) uses a transparent reproducible methodology to generate global and country level estimates of diabetes prevalence in adults (20-79 years), which are published in the IDF Diabetes Atlas. The described methodology was introduced in the IDF Atlas 5th Edition published in 2011. The IDF estimates are published biannually in the IDF Diabetes Atlas, with updates in alternative years, including in 2014.

A literature search covered studies reporting age-specific prevalence for diabetes and conducted between 1980 and 2014. Sources for country level data include peer-reviewed studies, national health statistics, official reports from international agencies and government, and unpublished data obtained through personal communication. An analytic hierarchy process was used to systematically select studies for inclusion. Studies were characterized and scored according to six criteria.
Logistical regression was used to generate smoothed sex- and agespecific prevalence estimates for adults 20-79 years after data was extracted. The regression used age (as midpoint of each age group) and the quadratic of age as separate independent variables for each sub-group (sex- and urban/rural setting- related) if available. The quadratic term was included to allow for a drop in the prevalence for the oldest age groups. For some sources, where a sample size in a single group was less than 50 , point adjustments were made by combining age groups to reduce variability.

An approach of matching countries by ethnicity, geography, and income group was used to form countries' clusters and then to fill gaps where original data sources were not available within these clusters. Also, country clustering was used for the calculation of median undiagnosed diabetes proportions and median urban/rural ratios in diabetes prevalence, if such values were reported. Data from studies that only reported known diabetes were adjusted to account for undiagnosed diabetes in a cluster. Urban/rural differences in diabetes prevalence and UN urbanization estimates were used to generate detailed distribution of diabetes prevalence. Estimated prevalence ratios were applied to UN population estimates to obtain the absolute number of people with diabetes in each country.

In the 2014 update of the IDF Diabetes Atlas 6th Edition, 173 studies were selected. The estimates revealed a global diabetes prevalence of $8.3 \%$, which translated to 387 million people currently living with diabetes worldwide in 2014.

\section{Abstract \#: P 73}

Impact of sedentary lifestyle on type 2 diabetes prevalence rates: analysis of two health surveys in Chile, $2003 \& 2010$

Marcia Erazo ${ }^{1}$, María Paz Bertoglia ${ }^{1}$, Juan Gormaz ${ }^{2}$, Matías Libuy ${ }^{3}$, Dérgica Sanhueza $^{1}$, Abraham I.J. Gajardo ${ }^{3}$, Roberto Del Águila ${ }^{4}$

${ }^{1}$ Public Health Nutrition Program, School of Public Health, University of Chile, Santiago, Chile; ${ }^{2}$ Molecular and Clinical Pharmacology Program, Bio-Medical Sciences Institute, University of Chile; ${ }^{3}$ School of Medicine, University of Chile; ${ }^{4}$ Pan American Health Organization, Santiago, Chile; Corresponding author's e-mail: merazo@med.uchile.cl

Background: Non-communicable diseases are the leading cause of death worldwide. Type- 2 diabetes (T2DM) represents $4 \%$ of deaths and is the condition that causes more disability. Recently, it was estimated that about 347 million adults have diabetes in the world. The World Health Organization has declared that sedentary lifestyle is among the major risk factors for T2DM, along with unhealthy diet, tobacco smoking and hazardous alcohol consumption. In Chile, T2DM is highly prevalent and most of its risk factors are preventable. We aim to estimate population impact of sedentary lifestyle on T2DM prevalence.

Methods: 8484 subjects with fasting glycaemia levels from two cross-sectional health surveys in 2003 and 2010 (commissioned by the Ministry of Health from Chile) were used ( $n=3191$ in 2003 and $\mathrm{n}=5293$ in 2010). The cross-sectional studies used a stratified, multi-stage random sample of households, with national, regional and rural/urban area representation. The target population was adults aged older than or equal to 15 years. T2DM and sedentary lifestyle prevalence was estimated. Crude and adjusted Odds Ratio to T2DM and its corresponding $95 \%$ confidence interval were estimated through logistic regressions. Attributable fractions were estimated using Kuritz's formula and Population attributable fractions using Levin's formula.

Results: T2DM prevalence in 2003 and 2010 were 4.19 and $9.5 \%$ respectively. Prevalence of sedentary lifestyle was 91.62 and 
$91.66 \%$, respectively. In 2003, the studied risk factor was not significant for T2DM, but in 2010, sedentary lifestyle became significant. During 2010, $64 \%$ of T2DM was explained by sedentary lifestyle. If people become active, a $62.2 \%$ of the T2DM cases would be avoided.

Conclusion: When population impact of sedentary lifestyle is considered, about $54 \%$ of the T2DM cases in Chile could be prevented with cost-effectives strategies focused to prevent sedentary lifestyle. The impact is more important in men than in women (59 vs. $45 \%$, respectively). Its urgent to implement evidence-based polices to decrease T2DM prevalence, its risk factors and consequently, its complications. To attack sedentary lifestyle, the strategies that must be implemented are in the area of education and information campaigns directed to the harms of physical inactivity, increase the hours of physical education in schools and increase the access of safe and adequate locations in the community to motivate people to increase its physical activity levels.

\section{Abstract \#: P 74}

Effectiveness of chronic care models in older patients with type 2 diabetes in Europe: a systematic review and meta analysis

\section{Brenda Bongaerts $^{1}$}

${ }^{1}$ Institute for Biometrics and Epidemiology, German Diabetes Center, Leibniz Center for Diabetes Research at Heinrich Heine University Düsseldorf; Corresponding author's e-mail:

Brenda.Bongaerts@ddz.uni-duesseldorf.de

Background: In the last decade, many interventions have been implemented aiming to improve diabetes care. Yet optimal chronic care is frequently impeded by a fragmented delivery system, poorly designed for the coordinated delivery of chronic care to older people with multi-morbidities. The Chronic Care Model has been developed as an evidence-based approach to improve the quality of chronic care. To date, only one systematic review has described how chronic care management has been applied in diabetes care settings in the US and what the outcomes were of this implementation (1). We performed a systematic review and meta-analysis on the effectiveness of chronic care models for type 2 diabetes, compared to routine diabetes care, in European countries

Methods: Studies were obtained from computerized searches of MEDLINE, EMBASE, CENTRAL and CINAHL, supplemented by hand-searches of reference lists of articles. Randomized controlled trials published from 2000 onwards and which focused on (1) type 2 diabetes, (2) older adult patients, and (3) multifaceted interventions of chronic diabetes care targeting both patient and health care provider were considered for inclusion. Outcome measurements included HbA1c levels, blood lipid concentrations, and blood pressure. Selected trials were analyzed descriptively and meta-analysis was applied for quantitative analysis of the data.

Results: Out of a total 9465 publications, five cluster randomized controlled trials met the inclusion criteria for inclusion in this review. Three trials recruited patients with prevalent type 2 diabetes while the remaining two had studied screen-detected diabetes patients. The studies showed wide-ranging results on $\mathrm{HbA} 1 \mathrm{c}$, cholesterol levels and blood pressure after 12 months of intervention. The overall mean difference in $\mathrm{HbA} 1 \mathrm{c}$ between all intervention and control groups was $0.03 \%$ (95\% confidence interval -0.09 to $0.03 \%$ ). Stratified analyses showed an overall mean HbA1c difference of $0.03 \%(-0.04$ to $0.10 \%$ ) for the patients with prevalent diabetes and a statistically significant mean difference of $-0.20 \%(-0.09$ to $-0.08 \%)$ for patients with screen-detected diabetes. Similar results were obtained for systolic blood pressure and cholesterol.

Conclusions: Effects of multifactorial interventions on $\mathrm{HbAlc}$, cholesterol and blood pressure at 12 months of follow-up seem stronger in screen-detected diabetes patients compared to patients with prevalent diabetes. Yet, the current scientific evidence is low, given the low number of randomized controlled trials.

\section{Abstract \#: P 75}

Body weight gain and risk of colorectal cancer: a systematic review and meta-analysis of observational studies

Sabrina Schlesinger ${ }^{1}$, Wolfgang Lieb ${ }^{1}$, Manja Koch ${ }^{1}$, Veronika $\overline{\text { Fedirko }^{2} \text {, Christina C. Dahm }}{ }^{3}$, Tobias Pischon ${ }^{4}$, Ute Nöthlings ${ }^{5}$, Heiner Boeing ${ }^{6}$, Krasimira Aleksandrova ${ }^{6}$

${ }^{1}$ Institute of Epidemiology, Christian-Albrechts University of Kiel, Kiel, Germany; ${ }^{2}$ Department of Epidemiology, Rollins School of Public Health, and Winship Cancer Institute, Emory University, Atlanta GA, US; ${ }^{3}$ Section for Epidemiology, Department of Public Health, Aarhus University, Aarhus, Denmark; ${ }^{4}$ Molecular Epidemiology Group, Max Delbrueck Center for Molecular Medicine (MDC), Berlin-Buch, Germany; ${ }^{5}$ Department of Nutrition and Food Sciences, Nutritional Epidemiology, University Bonn, Bonn, Germany; ${ }^{6}$ Department of Epidemiology, German Institute of Human Nutrition Potsdam-Rehbrücke, Nuthetal, Germany; Corresponding author's e-mail: sabrina.schlesinger@epi.uni-kiel.de

Background: High body mass index (BMI) measured in middle age or later is an established risk factor for colorectal cancer (CRC), particularly in men. However, whether body weight gain in adulthood is associated with CRC risk is less clear. We performed a systematic review and meta-analysis of observational studies to quantify the association between adult weight gain and risk of CRC, accounting for initial body weight, and taking into consideration potential differences in risk by cancer site, sex and early versus late life period of weight gain.

Methods: The literature search based on MEDLINE (PubMed) databases yielded 12 observational (10 cohort and 2 case-control) studies published until November 2014 with a total of 17,663 incident CRC cases. The summary relative risks (RRs) and $95 \%$ confidence intervals (95\% CIs) were estimated using random effects models. The between-study heterogeneity was evaluated based on I 2 statistics and effect modification was tested by meta-regression.

Results: Overall, high body weight gain was associated with a $21 \%$ higher risk of CRC compared to stable weight [summary RR (95\% CI) 1.21 (1.13-1.29): P for heterogeneity $=0.245: \mathrm{I} 2=15.4 \%]$. In a dose-response meta-analysis, each $5 \mathrm{~kg}$ weight gain was associated with a $4 \%(95 \%$ CI 2-5\%) higher risk of CRC. These findings were independent from initial body weight. The association was present for colon and rectal cancer and in men and women. The strongest association was detected in men for colon cancer [summary RR (95\% CI 1.35 (1.19-1.42)]. No statistically significant differences for risk of CRC have been observed according to early versus late life period of weight gain.

Conclusion: These findings support the evidence that body weight gain in adult life years is associated with a higher risk of CRC, independent of initial body weight. Public health messages should emphasize the need of body weight management already in early adulthood for the prevention of CRC. 


\section{Abstract \#: P 76}

Impact of worsened metabolic syndrome on dementia: a Chinese cohort study

Yen-Chun Fan ${ }^{1}$, Chyi-Huey Bai ${ }^{2}$

${ }^{1}$ School of Public Health, College of Public Health and Nutrition, Taipei Medical University, Taipei City, Taiwan; ${ }^{2}$ School of Public Health, College of Public Health and Nutrition, Taipei Medical University, Taipei City, Taiwan: Department of Public Health; Corresponding author's e-mail: baich@tmu.edu.tw

Background: Many studies paid the attention on the association between metabolic syndrome (MetS) that also expressed as insulin resistance syndrome and dementia. Individuals with dementia increase the public health burden. However, limited data was on the relation between persistent MetS status and the following dementia. The purpose of the study was to examine whether the participants with persistent MetS was predicated with an increased risk of dementia.

Methods: The sample was recruited from Taiwanese Survey on Prevalences of Hypertension, Hyperglycemia, and Hyperlipidemia (TwSHHH). The TwSHHH conducted in 2002 was a nation-wide cross-sectional survey with a subsequent follow-up in 2007. This sample was then linked to National Health Insurance Research Database (NHIRD) that covered almost $99 \%$ residents in Taiwan and was tracked to the end of 2011. The MetS was defined according to National Cholesterol Education Program Adult Treatment Panel III (NCEP ATP III). Criteria for the persistent MetS were MetS existed across two screenings around 5 years. The non-persistent MetS (once for two screenings) was divided into two groups: improved (complied with MetS at first but relieved at the second screening) and worsened (complied without MetS at first then obeyed at the second screening). Dementia events were identified by using the International Classification of Diseases, Ninth Revision, Clinical Modification codes.

Results: A total of 6457 subjects aged from 16 to 94 years were included. During the 60,372 person-years, eighty-nine participants developed dementia during up to the 10-year follow-up period. Subjects with persistent MetS had an increased but nonsignificant risk of dementia during 10-yrs periods after adjusting the confounders (adjusted hazard ratio $(\mathrm{HR})=1.14,95 \%$ confidence interval $(\mathrm{CI}$ $468)$ or improved $(p=0.800)$ MetS did not show the similar risks of dementia.

Conclusion: Worsened MetS within the 5 years among populationbased individuals had an increased risk to develop dementia during the 10-year follow-up. The risk might be higher than those prevalence MetS cases.

\section{Abstract \#: P 77}

\section{Osteocalcin and risk of type 2 diabetes}

Sabine R. Zwakenberg ${ }^{1}$, Caren M. Gundberg ${ }^{2}$, Annemieke M.W. Spijkerman $^{3}$, Daphne L. van der $A^{3}$, Yvonne T. van der Schouw ${ }^{1}$, Joline W.J. Beulens ${ }^{1}$

${ }^{1}$ Julius Center for Health Sciences and Primary Care, University Medical Center Utrecht, the Netherlands; ${ }^{2}$ Department of Orthopaedics, Yale University School of Medicine, New Haven, USA $;{ }^{3}$ Center for Nutrition, Prevention and Health Services, National Institute for Public Health and the Environment, the Netherlands; Corresponding author's e-mail: s.r.zwakenberg-3@umcutrecht.nl

Background: Recent studies indicated a role for the vitamin K-dependent protein osteocalcin in glucose and energy metabolism.
Whether these effects are driven by the carboxylated or uncarboxylated form of osteocalcin is still debated. Therefore this study aims to investigate whether total osteocalcin (tOC), uncarboxylated osteocalcin (ucOC) and percentage of uncarboxylated osteocalcin (\%ucOC) are related to the risk of type 2 diabetes.

Methods: This nested case control study included 1635 participants, 833 incident diabetes cases and 802 non-diabetic control participants, aged 20-70 years from the EPIC-NL cohort. Baseline concentrations of tOC, ucOC and \%ucOC were assessed. Diabetes cases were selfreported and verified by information from general practitioners or pharmacists. The association between the different species of osteocalcin and risk of diabetes was assessed with logistic regression adjusted for diabetes risk factors and nutrient intake.

Results: TOC concentration was not associated with the risk of type 2 diabetes, with an odds ratio (OR) of 0.97 (0.91-1.03). No association between ucOC and \%ucOC and the risk of diabetes was observed either. In sex stratified analyses $(\mathrm{P}$ interaction $=0.07)$, higher \%ucOC tended to be associated with a higher risk of type 2 diabetes in the multivariable adjusted model in women (OR 1.05 for each increment of $5 \%$ ucOC (1.00-1.11), Ptrend $=0.08)$, but not in men (OR 0.96 for each increment of $5 \%$ ucOC (0.88-1.04)).

Conclusions: TOC, ucOC and \%ucOC were not associated with the risk of type 2 diabetes. In women, but not in men, \%ucOC might be associated with a higher risk of type 2 diabetes. Further studies to evaluate the potential role for \%ucOC in type 2 diabetes are warranted.

\section{Abstract \#: P 78}

Insulin-like growth factor-i, insulin-like growth factor-binding protein-3, and risk of type 2 diabetes mellitus in the EPICPotsdam study

Dagmar Drogan ${ }^{1}$, Matthias B. Schulze ${ }^{2}$, Heiner Boeing ${ }^{1}$, Tobias Pischon ${ }^{3}$

${ }^{1}$ Department of Epidemiology, German Institute of Human Nutrition Potsdam-Rehbruecke, Nuthetal, Germany; ${ }^{2}$ Department of Molecular Epidemiology, German Institute of Human Nutrition PotsdamRehbruecke, Nuthetal, Germany; ${ }^{3}$ Molecular Epidemiology Research Group, Max Delbrueck Center for Molecular Medicine (MDC) Berlin-Buch, Germany; Corresponding author's e-mail: tobias.pischon@mdc-berlin.de

Background: Insulin-like growth factor I (IGF-I) and its major binding protein IGFBP3 have been implicated in the aetiology of type 2 diabetes mellitus (T2D). Since their independent influence on T2D risk is less well studied, we aimed to investigate circulating IGF-I, IGFBP-3 and their molar ratio in relation to T2D incidence in a large prospective cohort.

Materials and Methods: Serum concentrations of IGF-I and IGFBP3 were measured in a prospective case-cohort study nested within the European Prospective Investigation into Cancer and Nutrition (EPIC)Potsdam study including a randomly drawn subcohort of 2269 participants and 776 individuals with incident T2D, identified between 1994 and 2005. We used Cox proportional hazards regression adapted for the case-cohort design, with the weighting method described by Prentice and using age as the underlying time variable, to calculate hazard rate ratios (HR), stratified by age at recruitment, and adjusted for sex, BMI, waist circumference, smoking status, educational attainment, physical activity, alcohol consumption from beverages, daily consumption of red meat, whole grain bread, and coffee, and prevalent hypertension, myocardial infarction, and stroke.

Results: Multivariable-adjusted hazard rate ratios in the highest versus lowest quartile of IGF-I, IGFBP-3 and IGF-I:IGFBP-3 were 
$0.91(95 \%$ confidence interval $(\mathrm{CI})$ 0.68, 1.23: $\mathrm{P}$ trend $=0.31), 1.33$ (95 \% CI 1.00, 1.76: P trend $=0.04)$ and 0.77 (95\% CI 0.57, 1.03: P trend $=0.03$ ), respectively. The associations for IGFBP-3 and the IGF-I:IGFBP-3-ratio became marginally stronger after additional adjustment for IGF-I (IGFBP-3, HR $=1.38: 95 \%$ CI 1.04, 1.85: p trend $=0.03$ : IGF-I:IGFBP-3-ratio, HR $=0.63: 95 \%$ CI 0.39, 1.00: $\mathrm{p}$ trend $=0.03$ ).

Conclusions: Our study does not support the hypothesis that total IGF-I levels are associated with risk of T2D in the general study population. In contrast, our data suggest that high IGFBP-3 levels may be related to higher risk of T2D independent of IGF-I levels.

\section{Abstract \#: P 79 \\ Clustering of lifestyle behaviors and abdominal obesity in European and Brazilian adolescents}

$\underline{\text { Elsie C. de O. Forkert }}^{1,2}$, Augusto C.F.de Moraes ${ }^{1,2}$, Heráclito B. Carvalho $^{1}$, Antony Kafatos ${ }^{3}$, Stefaan De Henauw ${ }^{4}$, Magdalena Cuenca-García ${ }^{5}$, Odysseas Androutsos ${ }^{6}$, Laura Censi ${ }^{7}$, Michael Sjöström ${ }^{8}$, Laurent Beghin ${ }^{9}$, Marcela González-Gross ${ }^{10,11}$, Luis A. Moreno ${ }^{2,12}$

${ }^{1}$ Department of Preventive Medicine, School of Medicine of the University of São Paulo, YCARE (Youth/Child and cArdiovascular Risk and Environmental) Research Group, São Paulo/Brazil; ${ }^{2}$ Faculty of Health Sciences of the University of Zaragoza.GENUD (Growth, Exercise, Nutrition and Development) Research Group, Zaragoza/ Spain; ${ }^{3}$ Preventive Medicine and Nutrition Unit, University of Crete School of medicine, Heraklion, Crete/Greece; ${ }^{4}$ Department of Public Health, Ghent University, Ghent/Belgiun; ${ }^{5}$ Department of Medical Physiology, School of Medicine University of Granada, Granada/ Spain; ${ }^{6}$ Department of Nutrition and Dietetics, School of Healthy Science and Education, Harokopio University, Athens/Greece; ${ }^{7}$ Agricultural Research Council, Food and Nutrition Research Centre(CRA-NUT), Rome/Italy; ${ }^{8}$ Department of Biosciences, Unit for Preventive Nutrition, Karolinska Institute, Stockholm/Sweden; ${ }^{9}$ CHRU Lille, Faculté Médecine, Université de Lille/France;

${ }^{10}$ Department of Health and Human Performance, Faculty of Physical Activity and Sport-INEF, Techinical University of Madrid, Madrid/ Spain; ${ }^{11}$ Universität Bonn, Institut für Ernährungs-und ebensmittelwissenschaften-Humanernährung, Rheinische Friedrich Wilhelms, Bonn/Germany; ${ }^{12}$ Department of Preventive Medicine, Visiting Professor, School of Medicine of the University of São Paulo, São Paulo/Brazil; Corresponding author's e-mail: elsie@centerlink.com.br

Background: It is known that obesity, specially abdominal obesity (AO) may be a cardiovascular disease risk factor in children and adolescents. This study aimed to assess the clustering between lifestyle behaviors [weekly consumption of fruits, vegetables and soft drinks, sleep duration, sedentary behaviors (SB) and physical activity (PA)] and, its associations with(AO) indicators in adolescents from two observational studies, HELENA-CSS (Healthy Lifestyle in Europe by Nutrition in Adolescence Cross-Sectional Study) and BRACAH study (Brazilian Cardiovascular Adolescent Health).

Methods: Adolescents were recruited in two cross-sectional studies, one in Europe $(\mathrm{n}=1252$, aged $12.5-17.5 \mathrm{y}$ with $52.7 \%$ girls, HELENA-CSS) and one in Brazil $(\mathrm{n}=682$, aged $14-17.5 \mathrm{y}$ with $54.3 \%$ girls, BRACAH study). Clustering of lifestyle behaviors was determined on the basis of five lifestyle behaviors: PA (time spent performing moderate-and-vigorous PA), SB (time spent using a device such as a computer, television or video game), dietary intake fruits and vegetables consumption and sugar-sweetened beverages (weekly consumption of each food group) and sleep time, which were assessed via self-reported questionnaires. To identify clusters of participants with similar behaviors, clustering analysis with a hierarchical and non-hierarchical combination was applied. After computing the distributions of $\mathrm{z}$-scores of each behavior the clusters were established. Adolescents with complete information on measured waist circumference (WC) and height were included. WC in $\mathrm{cm}$ and the waist to height ratio (WHtR) were evaluated as indicators of AO. To analyze the differences between clusters for the body composition variables, ANOVA- Bonferroni test was used and, in presence of heterogeneous variances, Kruskal-Wallis was applied.

Results: The clusters were different and identified with four and three categories according to the adolescents' gender and the studies that they participated. European girls (sedentary, non-active, healthy eating, active), Brazilian girls (sedentary, un-healthy eating, healthy eating, active), European boys (sedentary, un-healthy eating, healthy) and Brazilian boys (sedentary, healthy eating, active). In European and Brazilian girls there was no significant difference for WC or WHtR between them. On the other hand, the same difference for WC or WHtR was not observed in the cluster of European and Brazilian boys. Although the groups are different according to gender and region, we found no significant association between the groups.

Conclusions: In European and Brazilian adolescents, AO indicators were not associated with any cluster of lifestyle behaviors. Examining younger children would be important to understand, how the clustering of obesogenic behaviors during childhood, will inform the correct timing to interventions.

\section{Abstract \#: P 80}

Markers of the hepatic component of the metabolic syndrome and their association with post-transplantation diabetes mellitus Gerald Klaaseen $^{1}$

${ }^{1}$ Department of Nephrology, University Medical Center Groningen, The Netherlands; Corresponding author's e-mail:

g.klaassen@umcg.nl

Background: Post-transplantation Diabetes Mellitus (PTDM) often occurs in renal transplant recipients (RTR) and negatively impacts long term transplantation outcome. PTDM places RTR at increased risk of infections, graft failure, cardiovascular disease and mortality. Elevations in circulating concentrations of alanine aminotransferase (ALT), aspartate aminotransferase (AST), gamma-glutamyl tansferase (GGT) and alkaline phosphatase (AP) commonly indicate fat deposition in the liver which can lead to decreased hepatic insulin sensitivity and thereby may predict PTDM. We prospectively investigated whether metabolic syndrome and liver enzymes ALT, AST, GGT and AP are associated with PTDM development in RTR. To our knowledge this study is the first to examine the association between liver enzymes and type 2 diabetes development late after renal transplantation taking into account lifestyle factors.

Methods: The primary endpoint of this study was the development of PTDM late after renal transplantation ( $>1$ year). A total of 501 patients with a functioning allograft between 2001 and 2003 and no diabetes at baseline were included. Liver enzymes ALT, AST, GGT and AP where measured at baseline using IFCC method $\left(37^{\circ} \mathrm{C}\right)$. Cases of PTDM were recorded until 2012.

Results: A total of 501 RTR ( $56 \%$ men, mean age $50 \pm 12$ years) were studied. Median [IQR] time at baseline was 6.0 years [2.6-11.5] post-transplant. In cross-sectional analysis, z-scores of ALT, GGT and AP were associated with the metabolic syndrome defined by the NCEP-ATPIII criteria (ORs with $95 \%$ CI are 1.37 (1.08-1.74), 1.28 (1.02-1.60) and $1.46(1.18-1.79)$, respectively). During median [IQR] follow-up time of 9.6 [6.2-10.2] years, 76 subjects (15.2\%) 
developed PTDM. In univariate cox-regression analyses, z-scores of ALT, GGT and AP were significantly associated with PTDM (HR 1.28 per SD, CI 1.12-1.46: HR 1.19 per SD, CI 1.03-1.39 and HR 1.41 per SD, CI 1.17-1.69, respectively), whereas AST was not (HR 1.13 per SD, CI 0.98-1.28). Correction for renal function, immunosuppressive medication, alcohol consumption, smoking and physical activity levels did not materially change these associations. PTDM risk already increased within the normal ranges at serum concentration levels exceeding $22 \mathrm{U} / \mathrm{L}$ for ALT, $35 \mathrm{U} / \mathrm{L}$ for GGT and $80 \mathrm{U} / \mathrm{L}$ for AP (see also figures $1 \mathrm{a}-1 \mathrm{c}$ ).

Conclusions: Liver enzymes ALT, GGT and AP were significantly associated with future PTDM. Notably, even mild elevations in liver enzyme concentrations considered within normal ranges in clinical practice were associated PTDM development.

\section{Abstract \#: P 81}

Correlation among food and mental health in University students in Albania

\section{Shkurti Enkelejda $^{1}$, Shtiza Diamant ${ }^{2}$}

${ }^{1}$ Faculty of Technical Medical Sciences, University of Medicine, Tirana, Albania; ${ }^{2}$ University Hospital Centre: 'Mother Theresa', Tirana, Albania; Corresponding author's e-mail: ledish4@gmail.com

Background/Aim: The present survey appraised, by university and gender, the relationship among dietary behavior, anxiety and depressive indicators in a sample of University students in Albania. Methods: A cross-sectional study was carried out amongst undergraduates signed up across five universities in Albania $(\mathrm{N}=3546)$. Self-administered questionnaires comprised a 10 point food frequency questionnaire, Cohenl's Stress Degree and adjusted Beck Depression Inventory. Sex and university comparisons were undertaken. Multivariable regression analyses were estimated for every outcomesupposed stress and depressive indicators.

Results: The occurrence of using diverse food clusters varied by university and gender, as did depressive indicators and supposed stress. Multivariable regression analyses pointed out that using "unhealthy" foods was considerably completely correlated with supposed stress (simply females) and depressive indicators (equally males and females).In opposition, using "healthy" foods (e.g. fruits, vegetables) was notably negatively related to supposed stress and depressive indicators scores for both genders. There was important negative connection among using sea food and depressive signs between males only. For both genders, using meat/sausage products, dairy products, and cereal merchandises were not related to supposed stress or depressive signs.

Conclusions: The relationship among using "unhealthy" groceries and elevated depressive signs :perceived stress between male and female students in addition to the connections among using "healthy" foods and minor depressive signs and supposed stress between male and female students in Albania recommends that interventions to decrease depressive indicators and stress between students could furthermore result in the utilization of healthier foods and/or vice versa.

Keywords: food, mental health, Albania

\section{Abstract \#: P 82}

Amount of guidance in a combined lifestyle intervention to improve lifestyle of overweight people: the more, the better?
Brenda A.J. Berendsen ${ }^{1}$, Marike R.C. Hendriks ${ }^{1}$, Stef P.J. Kremers ${ }^{2}$, Nicolaas C. Schaper ${ }^{3}$, Hans H.C.M. Savelberg ${ }^{1}$

${ }^{1}$ Human Movement Science, NUTRIM, School for Nutrition and Translational Research in Metabolism, Maastricht University Medical Centre; ${ }^{2}$ Health Promotion, NUTRIM, School for Nutrition and Translational Research in Metabolism, Maastricht University Medical Centre; ${ }^{3}$ Internal Medicine, Maastricht University Medical Centre; Corresponding author's e-mail:

hans.savelberg@maastrichtuniversity.nl

Background: Improving physical activity and dietary behaviour of overweight and obese adults is of increasing interest. We studied a combined lifestyle intervention which is embedded in primary care and is aimed at improving physical activity and dietary behaviour in overweight and obese adults. The one-year intervention comprises guidance by a team of a lifestyle advisor, physical therapist and dietician which can vary in intensity. We assessed the influence of the amount of guidance on improvement in physical activity, sitting time and body composition.

Methods: Every six months, participants $(\mathrm{n}=411)$ in 29 GP practices reported by means of the short version of the International Physical Activity Questionnaire (IPAQ) the amount of moderate to vigorous physical activity (MVPA), walking and sitting. Moreover, we measured BMI at baseline, after the one-year intervention and after two years of follow-up. To indicate amount of guidance, every three months participant reported attendance with lifestyle advisor, physical therapist and dietician. Three-level mixed models were used to study the influence of attendance with lifestyle advisor, physical therapist and dietician on the IPAQ outcomes and BMI, whilst accounting for clustering and repeated measures.

Results: Within the first six months sitting time decreased by $40 \mathrm{~min}$ (10\% of baseline), returning back to baseline values at 1 year. Walking time did not change in the first year. After 1.5 and 2 years, participants increased walking time with 15 and 12 min compared to baseline (respectively 34 and $27 \%$ of baseline). Amount of MVPA did not change. After one year, BMI was decreased with $1.1 \mathrm{~kg} / \mathrm{m}^{2}$, this improvement was still visible after two years. Number of attended meetings with lifestyle advisor, physiotherapist and dietician was not related to changes in physical behaviour. A higher number of lifestyle advisor meetings was associated with a larger decrease in BMI $(\mathrm{r}=-0.35)$.

Conclusion: The BeweegKuur is associated with favourable changes in physical behaviour and BMI. The changes in physical behaviour were independent of the amount of guidance. The change in weight was significantly related to a higher attendance of meetings with the lifestyle advisor, suggesting that intensive guidance by lifestyle advisor is necessary for clinically relevant weight loss.

\section{Abstract \#: P 83}

The Palestinian diabetes complications and control study (PDCCS): prevalence of complications among type 2 diabetes patients in Ramallah Governorate -Palestine

Abdullatif Husseini ${ }^{1}$, Rula Ghandour ${ }^{2}$, Nahed Mikki ${ }^{2}$, Sawsan Imseeh $^{2}$, Lars Jerden ${ }^{3}$, Jan Eriksson ${ }^{3}$, Margareta Norberg ${ }^{3}$

${ }^{1}$ Public health Program, Department of Health Sciences- Qatar University; ${ }^{2}$ Institute of Community and Public Health- Birzeit University; ${ }^{3}$ Umea University; Corresponding author's e-mail: abdullatif@qu.edu.qa

Background: Type 2 diabetes mellitus (DM) is a major public health issue in Palestine in terms of morbidity and mortality. A recent 
STEPS survey conducted in 2010-2011 estimated a DM prevalence rate of $8.5 \%$ among Palestinians in the age group (15-64 years). In 2011, DM was the fourth leading cause of mortality contributing to $8.6 \%$ of total deaths in the West Bank. A recent Markov model analysis estimated a DM prevalence of $20.6 \%$ among patients aged $<25$ years in the year 2020 . The current study aims to estimate the prevalence of selected complications among T2DM patients in Ramallah governorate, West Bank.

Methods: A clinic-based survey was conducted on a representative sample of 517 persons with type 2 DM ( 351 women and 166 men). A proportional to size sample was selected from 11 diabetes clinics operated by the main health care providers in Palestine. The response rate was about $84 \%$. The Diabetes complication index (DCI) was used to assess diabetes complications among patients. Additionally, the study also examined DM control among type 2 patients. Anthropometric measurements: physical examinations of the foot, eye examination: medical laboratory tests including $\mathrm{HbAlc}$, lipid profile, creatinine and microalbumin were performed. Data were gathered during February-June 2012, and analysed using SPSS (version 22.0). Descriptive statistics (means, standard deviations) and bivariate analysis (t-tests and Chi square test) was used. All patients provided verbal informed consent. The study was approved by the ethical committee at the Institute of Community and Public Health (ICPH), Birzeit University.

Results: The mean age $(+\mathrm{SD})$ was $58.1(+9.8)$ years. About $80 \%$ were aged $<50$ years. The mean $(+\mathrm{SD})$ duration of $\mathrm{DM}$ was 9.4 $(+7.5)$ years. More than half of patients reported having the disease for 7 years or more. $15.1 \%$ of patients were current smokers, $62 \%$ had a BMI of $<30$ and only around $20 \%$ achieved good glycemic control $(\mathrm{HbA} 1 \mathrm{c}<7 \%)$. The most prevalent complications were nephropathy (51.6\%), followed by retinopathy (36.7 \%), neuropathy (29.9\%), and CVD $(28.8 \%)$. The mean number of complications among men and among women was 1.83 and 1.30 respectively. $76 \%$ of the participants had at least one complication.

Conclusion: This study indicated that diabetes complications are prevalent among the Palestinian type 2 DM patients in Ramallah governorate. The majority of patients were poorly controlled. The results suggest the need for adopting a prevention approach in DM management to reduce complications and improve disease control.

\section{Abstract \#: P 84}

Validation of web-based self-reported height, weight and BMI among Swedish adolescents

Sandra Ekström $^{1}$, Inger Kull ${ }^{2,3}$, Sara Nilsson ${ }^{4}$, Anna Bergström ${ }^{1}$

${ }^{1}$ Institute of Environmental Medicine, Karolinska Institutet, Stockholm, Sweden; ${ }^{2}$ Department of Pediatrics, Sachs Childrens Hospital, Stockholm, Sweden; ${ }^{3}$ Department of Clinical Science and Education, Karolinska Institutet at Södersjukhuset, Stockholm, Sweden; ${ }^{4}$ Center for Occupational and Environmental Medicine, Stockholm County Council, Stockholm, Sweden; Corresponding author's e-mail: Sandra.Ekstrom@ki.se

Background: Web-collected height and weight are increasingly used in epidemiological studies: however the validity has rarely been evaluated.

Objective: The aim of the study was to validate self-reported height, weight and corresponding BMI among Swedish adolescents, aged approximately 16 years. A secondary aim was to investigate possible prediction factors for validity of self-reported BMI.

Methods: The study included 1698 adolescents from the population based cohort BAMSE. Height and weight were collected through a web-based questionnaire and subsequently measured using standard procedures. Differences between reported and measured height, weight and corresponding BMI were compared by t-tests and agreement was evaluated by Pearson's correlation and Bland-Altman plots. Multivariable linear regression analysis was used to investigate whether lifestyle and demographic factors predicted validity of selfreported BMI.

Results: On average, weight was underestimated by $1.1 \mathrm{~kg}$ and height was overestimated by $0.5 \mathrm{~cm}$, leading to an underestimation of BMI by $0.5 \mathrm{~kg} / \mathrm{m}^{2}$. Correlation coefficients were 0.98 for height, 0.97 for weight and 0.94 for BMI, and highly significant. Females underestimated weight to a higher extent than males, and overweight and obese subjects underestimated weight to a higher extent than normal weight subjects, which resulted in higher underestimation of BMI. Underweight subjects, on the contrary, overestimated weight and correspondingly BMI. Overall, a high proportion of participants were classified into the correct BMI category, however, among overweight and obese subjects, only $60.2 \%$ (139/231) and $46 \%$ (20/44) were correctly classified, respectively. In the multivariable prediction model, only gender and BMI status significantly predicted discrepancy between reported and measured BMI.

Conclusions: Web-collected BMI may be used as a valid, quick and cost-effective alternative to measured BMI among Swedish adolescents. The accuracy of self-reported BMI declines with increasing BMI, and self-reported BMI should not be used to estimate the prevalence of overweight or obesity.

\section{Abstract \#: P 85}

Gender-specific associations between night work, metabolic risk factors and diabetes: baseline results from Brazilian longitudinal study of adult health (ELSA-BRASIL)

Aline Silva-Costa ${ }^{1}$, Lúcia Rotenberg ${ }^{2}$, Aline Araújo Nobre ${ }^{3}$, Maria Inês Schmidt $^{4}$, Claudia Medina Coeli ${ }^{5}$, Dora Chor ${ }^{6}$, Rosane Härter Griep $^{2}$

${ }^{1} \mathrm{Ph} . D$. student - National School of Public Health, Oswaldo Cruz Foundation - ENSP/FIOCRUZ, Brazil; ${ }^{2}$ Researcher on Public Health - Laboratory of Health and Environment Education, Oswaldo Cruz Institute - Fiocruz, Brazil; ${ }^{3}$ Researcher - Scientific Computing Program, Oswaldo Cruz Foundation - Fiocruz, Brazil; ${ }^{4}$ Researcher Postgraduate Studies Program in Epidemiology - School of Medicine, Federal University of Rio Grande do Sul; ${ }^{5}$ Researcher - Institute of Public Health Studies, Federal University of Rio de Janeiro - UFRJ, Brazil; ${ }^{6}$ Researcher on Public Health - Department of Epidemiology, National School of Public Health, Oswaldo Cruz Foundation -

Fiocruz, Brazil; Corresponding author's e-mail: alinecos@ioc.fiocruz.br

Background: Circadian rhythm mismatches resulting from exposure to night work increases the risk of type 2 diabetes. The aim of this study was to test the association between (i) years of exposure to night work and diabetes (DM2)/impaired glucose tolerance (IGT) and (2) work schedule and metabolic risk factors for diabetes among a nondiabetic population using baseline data from ELSA-Brasil.

Methods: ELSA-Brasil, a prospective cohort study, comprises civil servants (35-74 years old at baseline, 2008-2010) sampled from six universities of Brazil. A 12-hour fasting blood sample was drawn for the measurement of serum cholesterol, triacylglycerol, and glucose. Weight, height and waist measurement were collected using standard equipment and techniques. Two analyses were fitted. In the first one (considering the total study population, $\mathrm{N}=14,427$ ), multinomial logistic regression analysis was performed to test the association between years worked at night and the multinomial outcome (normal, DM2 and IGT). The second analysis is based on generalized linear 
models, a gamma regression model with an identity link function to test the association between work schedule and the metabolic risk factors for DM2 (BMI, fasting glucose, total cholesterol, HDL-C, LDL-C, triglycerides, waist circumference, waist-hip ratio) among non-diabetic current workers $(\mathrm{N}=8853)$. For this analysis, participants classified as having diabetes, day workers with previous experience at night work, and retired workers were excluded.

Results: Considering the total study population, $19.6 \%(\mathrm{n}=2825)$ were classified as having DM2 and $20.5 \%$ as having IGT $(\mathrm{n}=2952)$. Among women, the odds ratio derived from multinomial regression for DM2 and IGT were $1.42(95 \% \mathrm{CI}=1.39-1.45)$ and 0.96 (95\% CI $=0.94-0.99)$, respectively, for those exposed to night work for more than 20 years, compared to women who never worked at night, after adjustment for all potential confounders. In men, the odds ratios for DM2 and IGT were 1.06 (95\% CI $=1.04-1.08)$ and $0.99(95 \% \mathrm{CI}=0.98-1.01)$, respectively, for those exposed to night work for more than 20 years, compared to those who never worked at night. In relation to the metabolic risk factors, in women, fasting plasma glucose was the only variable significantly associated with night work after adjustments $(\beta=2.278, p<0.001)$. Among men, the exposure to night work increased in average $0.542 \mathrm{~kg} / \mathrm{m}^{2}$ in BMI $(p=0.032)$ and $1.66 \mathrm{~cm}$ in waist circumference $(p=0.014)$, compared to day workers. For triglycerides, a borderline association was found $(\beta=12.836, p=0.072)$.

Conclusions: Data from this study indicate that working at night is related to metabolic alterations, highlighting a higher chance for DM2 among women. Gender differences both concerning DM2 and metabolic risk factors for DM2 deserve further clarification. Longitudinal studies drawing on the ELSA-Brasil will enable to corroborate or refute these findings.

\section{Abstract \#: P 86}

\section{Cord blood insulin and early life exposure to particulate air pollution}

Narjes Madhloum ${ }^{1}$, Bram G. Janssen ${ }^{1}$, Esmée Bijnens ${ }^{1}$, Wilfried Geyselaers $^{2}$, Joris Penders ${ }^{3,4}$, Michelle Plusquin ${ }^{1}$, Tim Nawrot ${ }^{1,5}$

${ }^{1}$ Centre for Environmental Sciences, Hasselt University, Diepenbeek, Belgium; ${ }^{2}$ Department of Obstetrics, East-Limburg Hospital, Genk, Belgium; ${ }^{3}$ Laboratory of Clinical Biology, East-Limburg Hospital, Genk, Belgium; ${ }^{4}$ Biomedical Research Institute, Hasselt University, Diepenbeek, Belgium; ${ }^{5}$ Department of Public Health, Occupational, Environmental \& Insurance Medicine, Leuven University, Leuven, Belgium; Corresponding author's e-mail:

narjes.madhloum@uhasselt.be

Background and Aim: Cardio-metabolic risk factors including insulin levels are at young age barely perceived as harmful, but over time these risk factors may track and lead to higher risk of metabolic syndrome. Recent studies showed that exposure to air pollution is associated with increase in the risk of insulin resistance in childhood. Further, umbilical cord blood insulin levels are associated with plasma insulin levels later in life. We studied in utero exposure to particulate air pollution and plasma umbilical cord insulin levels.

Methods: In the ENVIRONAGE (ENVIRonmental influence ON AGEing in early life) birth cohort, we investigated in 435 newborns the association between cord plasma insulin levels and exposure to particulate air pollution (PM2.5) in various exposure windows during pregnancy.

Results: Umbilical cord blood samples were obtained from 435 newborns and plasma insulin levels averaged $4.9 \mathrm{mU} / \mathrm{L}(\mathrm{SD}=2.1)$. PM2.5 exposure during pregnancy averaged $16.3(14.5-17.9) \mu \mathrm{g} / \mathrm{m}^{3}$. Cord blood insulin levels correlated negatively with gestational age
( $\beta=-0.35 \mathrm{mU} / \mathrm{L}, p=0.01)$ but positively with newborn's birth weight $(\beta=+0.002 \mathrm{mU} / \mathrm{L}, p<0.0001)$. Multiparous mothers had higher insulin levels compared with primiparous mothers ( $\beta=0.84 \mathrm{mU} / \mathrm{L}, p=0.04$ and $\beta=2.33 \mathrm{mU} / \mathrm{L}, p=0.001 \mathrm{respec}-$ tively). Independent of maternal age, gender, birth weight, parity and maternal education, cord plasma insulin levels increased with $1.22 \mathrm{mU} / \mathrm{L}$ (95\% CI 0.19-2.45, $p=0.02$ ) for each IQR increment of PM2.5 during the last trimester.

Conclusion: Exposure to particulate air pollution late in pregnancy is associated with increases in cord plasma insulin levels. Pollution induced insulin levels in early life might be a risk factor in the development origin of cardio-metabolic disease later in life.

\section{Abstract \#: P 87}

Mind-body practice is associated with body weight status in a general population from the nutrinet-santé study

Géraldine M. Camilleri ${ }^{1}$, Caroline Méjean ${ }^{1}$, France Bellisle ${ }^{1}$, Serge Hercberg ${ }^{1}$, Sandrine Péneau ${ }^{1}$

${ }^{1}$ Université Paris 13, Equipe de Recherche en Epidémiologie Nutritionnelle, Centre de Recherche en Epidémiologie et Statistiques, Inserm (U1153), Inra (U1125), Cnam, COMUE Sorbonne Paris Cité, Bobigny, France; Corresponding author's e-mail:

g.camilleri@eren.smbh.univ-paris13.fr

Background: In industrialized countries characterized by a high prevalence of obesity and chronic stress, mind-body practices such as yoga or meditation may facilitate body weight control. However, virtually no data are available to ascertain whether practicing mindbody techniques is associated with weight status. We aimed to examine the relationship between the practice of mind-body techniques and weight status in a large sample of the adult general population.

Methods: A total of 14,400 men and 49,228 women aged $\geq 18$ y participating in the NutriNet-Santé study were included in this crosssectional analysis. We collected data on mind-body practices as well as self-reported weight and height. The association between the practice of mind-body techniques and weight status was assessed using multiple linear and multinomial logistic regression models adjusted for socio-demographic, lifestyle and dietary factors.

Results: Compared with never users, regular users of mind-body techniques were the least likely to be overweight (OR (95\% CI) 0.74 (0.69-0.79)) or obese (OR (95\% CI) $0.56(0.50-0.62)$ ), followed by occasional and former users (models adjusted for socio-demographic and lifestyle factors). In addition, compared with never users, mindbody users had a lower BMI, particularly in regular users (slope (95\% CI) -0.88 ( -0.99 to -0.76$)$ ) followed by occasional and former users. Further adjustment on dietary quality and total energy intake did not modify the association between mind-body practice and body weight status.

Conclusion: Our data provide novel information about an inverse relationship between mind-body practice and weight status. If causal links were demonstrated in further studies, such practice could be fostered in obesity prevention and treatment.

\section{Abstract \#: P 88}

\section{Fat distribution and mortality: the AGES-Reykjavik study}

Annemarie Koster ${ }^{1}$, Rachel A. Murphy ${ }^{2}$, Gudny Eiriksdottir ${ }^{3}$, Thor Aspelund $^{3,4}$, Sigurdur Sigurdsson ${ }^{3}$, Thomas F. Lang ${ }^{5}$, Vilmundur Gudnason $^{3,4}$, Lenore J. Launer ${ }^{2}$, Tamara B. Harris ${ }^{2}$ 
${ }^{1}$ Department of Social Medicine, CAPHRI School for Public Health and Primary Care, Maastricht University, Maastricht, The

Netherlands; ${ }^{2}$ Laboratory of Epidemiology and Population Sciences, National Institute on Aging, Bethesda, Maryland, USA; ${ }^{3}$ The Icelandic Heart Association, Kopavogur, Iceland; ${ }^{4}$ Faculty of Medicine, University of Iceland, Reykjavik, Iceland; ${ }^{5}$ Department of Radiology and Biomedical Imaging, University of California, San Francisco, USA; Corresponding author's e-mail: a.koster@maastrichtuniversity.nl

Background: The association between BMI and mortality is less pronounced in older adults which is thought to be related to agerelated changes in fat distribution. At present there are very few studies that examined the association between fat distribution, taking into account both fat depots in the abdomen and thigh, and all-cause mortality. This study examined associations of regional fat depots with all-cause mortality over 11 years of follow-up.

Methods: Data were from 2187 men and 2900 women, aged 66-96 years in AGES-Reykjavik Study. Abdominal visceral fat and subcutaneous fat, and thigh intermuscular fat and subcutaneous fat were measured by CT.

Results: During 11 years of follow-up with an average follow-up time of 8 years, 919 men and 882 women died. In men, every standard deviation (SD) increment in thigh intermuscular fat was related to a significantly greater mortality risk (HR:1.17, $95 \%$ CI $1.08-1.26$ ) after adjustment for age, education, smoking, physical activity, alcohol, BMI, type 2 diabetes and coronary heart disease. In women, visceral fat (per SD increment) significantly increased mortality risk (HR:1.13, $95 \%$ CI 1.03-1.25) while abdominal subcutaneous fat (per SD increment) was associated with a lower mortality risk (HR:0.70: $95 \%$ CI 0.61-0.80). Significant interactions with BMI were found in women indicating that visceral fat was a strong predictor of mortality in obese women while abdominal and thigh subcutaneous fat were associated with a lower mortality risk in normal and overweight women.

Conclusion: Fat distribution is associated with mortality over 11 years of follow-up independent of overall fatness. The divergent mortality risks for visceral fat and subcutaneous fat in women suggest complex relationships between overall fatness and mortality.

\section{Abstract \#: P 89}

\section{Quality of life in metabolic diseases, a structural equation modeling application}

Estela Vilhena $^{1,8}$, José Luís Pais Ribeiro ${ }^{2,7}$, Isabel Silva ${ }^{3}$, Luísa Pedro $^{4,7}$, Rute Meneses ${ }^{3}$, Helena Cardoso ${ }^{5,6}$ António Martins da Silva ${ }^{6,9}$, Denisa Mendonça ${ }^{5,8}$

${ }^{1}$ Polytechnic Institute of Cávado and Ave, Barcelos; ${ }^{2}$ FPCE University of Porto; ${ }^{3}$ University of Fernando Pessoa; ${ }^{4}$ ESTeSL Polytechnic Institute of Lisbon; ${ }^{5}$ ICBAS University of Porto; ${ }^{6} \mathrm{HGSA} /$ CHP Hospital Center of Porto; ${ }^{7}$ UIPES Portugal; ${ }^{8}$ EPIUnit, ISPUP University of Porto; ${ }^{9}$ UMIB/ICBAS University of Porto, Portugal; Corresponding author's e-mail: evilhena@ipca.pt

Background: Quality of life (QoL) is a construct composed of a number of factors that contribute to individual's well-being and adjustment to chronic diseases. Metabolic diseases are considered a relevant problem of public health. After the diagnosis, this patients are required to live with the limitations imposed by their conditions.

The aim of this study is to clarify the simultaneous impact of stigma perception, dispositional optimism and positive affect in quality of life of Portuguese metabolic patients and the mediation effect of dispositional optimism.
Methods: This study included a sample of 365 Portuguese metabolic patients approached by their physicians in outpatient departments of main hospitals in Portugal. All patients completed self-report questionnaires to assess socio-demographic, stigma perception, dispositional optimism, positive affect and QoL variables. Structural Equation Modelling (SEM) was used to analyse the hypothesized model, including these factors and a mediation effect of dispositional optimism. Maximum Likelihood (ML) estimation was considered. The Satorra-Bentler Scaled correction to $\chi 2$ test statistic were used. To test the adequacy of the model two other goodness-of-fit indices were used: Comparative Fit Index (CFI) and Root Mean Square Error of Approximation (RMSEA). Analysis were performed using EQS 6.1.

Results: Controlling for socio-demographic and clinical variables, the SEM results showed that the hypothesized model fitted the data reasonably well, $\mathrm{CFI}=0.92, \quad \mathrm{RMSEA}=0.048, \quad S-\chi_{(495)}^{2}=$ 808.1205; $p<0.001$. Patients with a better positive affect and a low perception of stigma had a better QoL in all domains; an optimism attitude contribute simultaneous to a better general well-being and a better physical health. Dispositional optimism exerts a mediator effect between positive affect/stigma perception and QoL and between stigma perception and mental health.

Conclusions: The aim of study was evaluated the simultaneous impact of psychosocial variables in QoL, in a group of metabolic Portuguese patients. SEM models allow us to understand the complexity of the simultaneous relationships between variables. Results suggest that patients with lower perception of stigma, with an optimist attitude, more active and enthusiastic, can facilitate the patient to their new condition of life. These attitudes will contribute to a better quality of life.

\section{Abstract \#: P 90}

Waist circumference and sedentary behaviour assessed by accelerometry in rural youth

Aristides M. Machado-Rodrigues ${ }^{1,2}$, Manuel J. Coelho-e-Silva ${ }^{1}$, Rômulo Fernandes $^{3}$, Luis Ribeiro ${ }^{1}$, Jorge Mota ${ }^{4}$, Robert M. Malina ${ }^{5}$

${ }^{1}$ University of Coimbra, Coimbra; ${ }^{2}$ Escola Superior de Educação IPV, Viseu, Portugal; ${ }^{3}$ Faculty of Science and Technology, São Paulo State University; ${ }^{4}$ University of Porto, Porto, Portugal; ${ }^{5}$ Tarleton State University, Stephenville, Texas, USA; Corresponding author's e-mail: rodriguesari@hotmail.com

Background: Research on relationships between lifestyle behaviours and adiposity in school youth is potentially important for identifying subgroups at risk. This study evaluates the associations among waist circumference (WC) and objective measures of sedentary behaviour (SB) in a sample of rural school adolescents.

Methods: The sample included 254 youth (114 males, 140 females), 13-16 years of age, from rural regions of the Portuguese midlands. Height, weight, and WC were measured. Cardiorespiratory Fitness (CRF) was assessed with the 20-m shuttle-run test. An uniaxial GT1M accelerometer was used to obtain five consecutive days of physically activity (PA) and SB. Multiple linear regression was used to test associations between $\mathrm{WC}$ and $\mathrm{SB}$, adjusted for several potential confounders (age, sex, PA, CRF, parental education).

Results: SB was not significantly associated with the WC, neither in the unadjusted model nor after adjustment for all potential confounders. In the final model, the unique significant predictor of the WC was cardiorespiratory fitness $(\beta=-0.82: 95 \% \mathrm{CI}-1.02$ to $-0.62)$.

Conclusion: WC was not independently associated with SB time in rural school adolescents. Future research is claimed among rural 
adolescents in different geographic contexts to try to clarify recent findings of less studied communities, as well as additional studies to analyze the other side of the energetic equation (i.e. caloric input food habits).

\section{Abstract \#: P 91}

\section{Improving cardiometabolic health by adjusting the obesogenic environment in severe mentally ill residential patients: a randomised controlled trial}

Anne Looijmans ${ }^{1}$, Annemarie Stiekema ${ }^{2}$, Richard Bruggeman ${ }^{3}$, Lisette van der Meer $^{2}$, Ronald P. Stolk ${ }^{1}$, Robert A. Schoevers ${ }^{3}$, Frederike Jörg $^{3,4}$, Eva Corpeleijn ${ }^{1}$

${ }^{1}$ Department of Epidemiology, University of Groningen, University Medical Center Groningen, Groningen, The Netherlands;

${ }^{2}$ Department of Rehabilitation, Lentis Mental Health Care, Zuidlaren, The Netherlands; ${ }^{3}$ University Centre of Psychiatry, University of Groningen, University Medical Center, Groningen, The Netherlands; ${ }^{4}$ Research Department, Friesland Mental Health Services, Leeuwarden, The Netherlands; Corresponding author's e-mail: a.looijmans@umcg.nl

Introduction: Poor cardiometabolic health in severe mentally ill (SMI) patients, mostly schizophrenia, reduces life expectancy with 13-30 years, partly due to unhealthy lifestyle behaviours. The aim of the Effectiveness of Lifestyle Interventions in PSychiatry trial (ELIPS) is to improve cardiometabolic health specifically in residential patients by addressing the obesogenic environment, focusing on diet and physical activity. This is the first large inpatient study which addresses the obesogenic environment. We hypothesized that the intervention stabilised or improved cardiometabolic health in SMI residential patients.

Methods: ELIPS is a multi-site cluster randomised controlled lifestyle trial. All residential and long-term clinical care teams $(\mathrm{N}=29)$ in two Northern Dutch provinces were randomised to the intervention $(\mathrm{N}=15)$ or control $(\mathrm{N}=14)$ arm. In the intervention, lifestyle coaches created a team-tailored lifestyle plan according to pre-set ELIPS goals and strict protocol. In the 3-month implementation phase, lifestyle coaches trained health care teams to create a healthy environment, stimulate health behaviours in patients and achieve their pre-set goals. In the 9 months thereafter, adherence to the lifestyle plan is supervised by a lifestyle coach. Control patients received care as usual. Main outcomes were changes in waist circumference (WC) and body mass index (BMI), measured at baseline, 3 and 12 months. Missing data were imputed and data were analysed using multi-level linear mixed models with adjustment for age, gender, residential or long-term setting, and baseline WC or BMI values, respectively.

Results: Data of 365 intervention and 371 control patients (48 \pm 13 years, $63 \%$ men) were analysed according to the intentionto-treat principle. After full adjustment, results showed no significant treatment effect on waist circumference $(p=.112)$ over time. Mean $\pm \mathrm{SE}$ for changes in WC were $\Delta \mathrm{T} 0-\mathrm{T} 3$ : WC-Intervention $=0.94 \pm 0.54: \Delta \mathrm{T} 0-\mathrm{T} 12:$ WC-Intervention $=0.50 \pm 0.60: \Delta$ T0-T3: $\quad$ WC-control $=0.48 \pm 0.72: \quad \Delta \quad$ T0-T12: $\quad$ WC-control $=1.01 \pm 0.51 \mathrm{~cm}$. Also, no significant treatment effect was found for BMI change $(p=.080): \Delta$ T0-T3: BMI-Intervention $=0.13 \pm 0.13: \Delta$ T0-T12: BMI-Intervention $=0.26 \pm 0.15: \Delta$ T0-T3: $\quad$ BMI-control $=0.19 \pm 0.14: \quad \Delta \quad$ T0-T12: $\quad$ BMI-control $=0.04 \pm 0.14 \mathrm{~kg} / \mathrm{m}^{2}$.

Conclusion: A lifestyle intervention addressing the obesogenic environment through lifestyle coaching for care teams of SMI residential patients had no short-term effects on cardiometabolic health. Against expectations, the control group had a stable BMI instead of an increasing BMI over time. In general, the heterogeneity in the observed changes was large. In the future we will perform per protocol analysis, taking into account non-response in the intervention teams and uncontrolled lifestyle activities in the control teams.

\section{Abstract \#: P 92}

Sports activity and cognitive function among adults in Germany Beate Gaertner ${ }^{1}$, Amanda K. Buttery ${ }^{1,2}$, Steffen Wolfsgruber ${ }^{3,4}$, Jonas Finger $^{1}$, Susanne Krug ${ }^{1}$, Michael Wagner ${ }^{3,4}$, Markus A. Busch ${ }^{1}$

${ }^{1}$ Department of Epidemiology and Health Monitoring, Robert Koch Institute Berlin, Germany; ${ }^{2}$ Health Innovation Network (Academic Health Science Network for South London), London, United Kingdom; ${ }^{3}$ Department of Psychiatry and Psychotherapy, University of Bonn, Germany; ${ }^{4}$ German Center of Neurodegenerative Diseases (DZNE), Bonn, Germany; Corresponding author's e-mail: gaertnerb@rki.de

Background: The positive association between sports and cognitive function in older individuals has been demonstrated in many clinical studies. Less evidence for this association is available for the general population and for adults younger than 65 years. We examined the association between sports activity and cognitive function in a nationwide population-based sample of adults aged 18-79 years in Germany.

Methods: Cross-sectional analysis of data from the mental health module of the German Health Interview and Examination Survey for Adults (DEGS1-MH) $(\mathrm{n}=3667: 51.7 \%$ men: mean age 52.7 years, SD 16.1). Cognitive function was assessed by a comprehensive neuropsychological test battery comprising the digit span backward test (DSBT), the trail making tests (TMT-A/B), the letter digit substitution test (LDST), a verbal fluency test (VFT) and immediate and delayed recall of The Consortium to Establish a Registry for Alzheimer's Disease (CERAD) 10-word list. Subsequent to a Confirmatory Factor Analysis, test scores were z-standardized and combined to create $\mathrm{z}$-scores for executive function (DSBT, TMT-A/ $\mathrm{B}$, LDST, VFT) and memory (immediate and delayed recall). Duration of regular sports activities per week in the last three months was assessed by self-administered questionnaire and categorized as none, $<2 \mathrm{~h} /$ week, and $\geq 2 \mathrm{~h} /$ week. The association between sports activity and executive function and memory $\mathrm{z}$-scores were examined by multivariable linear regression analysis.

Results: No regular sports activity was reported by $30.1 \%,<2 \mathrm{~h} /$ week by $43.7 \%$, and $\geq 2 \mathrm{~h} /$ week by $26.2 \%$ of participants. Higher duration of sports activity per week was significantly associated with higher $\mathrm{z}$-scores for all individual tests, executive function and memory $(p<0.001)$. Participants with no sports activity achieved lower test results than participants who were engaged in any sports. Adjusting for age, sex, education and behavioural risk factors (obesity, current smoking, alcohol consumption risk categories, intake of $<3$ portions of fruit and vegetables per day) regular sports activity was associated with both memory $(<2 \mathrm{~h} /$ week: $\beta=0.08,95 \% \mathrm{CI}$ 0.02-0.14: $\geq 2$ h/week: $\beta=0.07,95 \%$ CI 0.01-0.14: reference: no sports) and executive function $\mathrm{z}$-scores $(<2 \mathrm{~h} /$ week: $\beta=0.12,95 \%$ CI $0.07-0.17: \geq 2 \mathrm{~h} /$ week: $\beta=0.15,95 \%$ CI $0.09-0.21$ ). There was no evidence of interaction between sport activity and age group (18-39, 40-64, 65+ years).

Conclusion: A positive association of sports and cognitive function was found among an adult sample aged 18-79 years in a national health examination survey. The association persisted after adjustment for age, sex, education and behavioural risk factors. Longitudinal analyses to test the causal relationship of sports and cognitive function are required. 


\section{Abstract \#: P 93}

\section{Does the local built environment affect the trajectories} of depression?

\author{
Genevieve Gariepy $^{1}$, Norbert Schmitz ${ }^{2}$ \\ ${ }^{1}$ McGill University; ${ }^{2}$ Douglas Mental Health University Institute; \\ Corresponding author's e-mail: genevieve.gariepy@mail.mcgill.ca
}

Objective: To investigate the effect of the neighbourhood built environment on trajectories of major depression in adults from the general Canadian population.

Research Design and Methods: We used 10 years of data collection (2000/01-2010/11) from the Canadian National Population Health Study $(\mathrm{n}=13,618)$. Major depression episode was identified using the Composite International Diagnostic Interview Short-Form. We assessed the presence of local parks, healthy food stores, health services and cultural services using geospatial data. We used latent class growth modelling (LCGM) to identify different trajectories of major depression in the sample and tested for the effect of time-varying neighbourhood variables on the trajectories over time.

Results: LCGM uncovered three distinct trajectories of major depression over the 10 year study period: low prevalence of depression, moderate prevalence of depression and high persistent prevalence of depression. The presence of local parks and cultural services significantly shifted the trajectory associated with high persistent prevalence of major depression towards lower probability of depression.

Conclusions: For individuals following a trajectory of high persistent prevalence of major depression, living in an area with parks and cultural services was associated with a shift in the trajectory towards lower probability of depression. Future intervention studies are recommended to make policy recommendations.

\section{Abstract \#: P 94}

What compel Indians to attempt suicide? Age, sex \& cause differentials in suicidal deaths in the states of India

\author{
$\underline{\text { Ashish Awasthi }}{ }^{1}$, C.M. Pandey ${ }^{1}$, Uttam Singh ${ }^{1}$, Manisha Dubey ${ }^{2}$ \\ ${ }^{1}$ Dept of Biostatistics \& Health Informatics, Sanjay Gandhi \\ Postgraduate Institute of Medical Sciences, Lucknow, India; \\ ${ }^{2}$ International Institute for Population Science, Mumbai, India; \\ Corresponding author's e-mail: ashishbhuims@gmail.com
}

The World Health Organization (WHO) estimates that nearly 900,000 people worldwide die from suicide every year. The Government of India classifies a death as suicide if it meets the three criteria: it is an unnatural death, the intent to die originated within the person and there is a reason for the person to end his or her life. The reason may have been specified in a suicide note or unspecified. If one of these criterion is met, the death is classified as suicide.

Objective of the present study is to examine the age, sex and cause driving the suicidal tendency in Indian population in last 15 years. Data has been taken from the National Crime Records Bureau and Ministry of Home Affairs, Govt. of India, New Delhi.

There were total 110,596 deaths due to suicide in India in year 1999 which increased by $21.9 \%$ to 134,799 suicidal deaths in year 2013. In year $199959.2 \%$ male committed suicide which increased to $67.2 \%$ in year 2013. In the year, 1999 maximum suicidal tendency was observed in the age group 14-29 (36.8\%) which decreased to $34.2 \%$ of total suicidal deaths in year 2013. Out of total suicidal death in $199918.6 \%$ were from age group 1999 which increased to the $21.1 \%$ in the year 2013. In year maximum $12.56 \%$ suicidal deaths were from the state west Bengal which decreased to $9.68 \%$ in year 2013, however in Maharashtra maximum number of suicidal death of country occurs $12.33 \%$ in 2013 in comparison with the $12.29 \%$ in year 1999. In year $199920.63 \%$ people ended their life due to family problems in comparison with $24.0 \%$ in year 2013 . $19.8 \%$ people committed suicide in year 1999 due to illness in comparison with $19.6 \%$ in year 2013. Out of total suicides, $4.8 \%$ in year 1999 were due to mental illness which increased to the $5.94 \%$ in year 2013. In the year, 1999 cause was unknown in $35.13 \%$ suicide in comparison to the $34.7 \%$ in year 2013. $18.6 \%$ victim of suicide in year 2013 were uneducated, $84.71 \%$ victims of suicide have education up to secondary level in comparison with $90.2 \%$ in year 1999 .

Since the suicide is a widespread phenomenon, it is important to develop to measure for early detection of suicidal tendency.

\section{Abstract \#: P 95}

Impact of parenting style on clinically significant behavior problems after great East Japan earthquake: a follow-up study

\section{Takahiro Miki ${ }^{1}$}

${ }^{1}$ Department of Social Medicine, National Center for Childrenl's Health and Development, Tokyo, Japan; Corresponding author's e-mail: miki-tk@ncchd.go.jp

Background: We have already investigated the impact of exposure to the Great East Japan Earthquake on preschool children, and have found that one in four children still had behavior problems even several years after the disaster. Thereafter, some children have kept having behavioral problems, others have relieved, and the others have gone worse. Their home environment may influence the behavior problems.

Objective: The purpose of this study was to investigate the influence of home environment especially corporal punishment on children's behavior problems after the disaster.

Methods: Participants were children exposed to the 2011 disaster at preschool age. $(\mathrm{n}=157)$. Data was collected from August 2012 to March 2013, and from August 2013 to March 2014 (2 and 3 years after the earthquake), thus participants were aged 5-9 years when assessed. Parenting were assessed by caregivers using Alabama Parenting Questionnaire (APQ), which encompasses parental involvement, positive parenting, poor monitoring/supervision, inconsistent discipline., and corporal punishment in the second year after the disaster. Behavioral problems were assessed by caregivers using the Child Behavior Checklist (CBCL), which encompasses internalizing, externalizing, and total problems in the second and third year after the disaster.

Results: The results show that corporal punishment in the second year after the disaster had negative influence on CBCL total $\mathrm{T}$ score in the third year after the disaster COR: 0.85, $95 \%$ CI 0.16-1.55, $p=0.016$ ), internalizing scale (correlational coefficient (COR): 0.78 , $95 \%$ CI $0.12-1.45, p=0.021$ ), and externalizing scale (correlational coefficient (COR): 0.74, $95 \%$ CI $0.09-1.39, p=0.025)$. All results were adjusted for children's age, sex, number of trauma experiences, maternal education, number of siblings, temporally housing experience, and CBCL each scores in the second year after the disaster.

Discussion: The result suggests that inadequate rearing after a natural disaster had negative impact on the behavioral problems of the affected children. Specifically, corporal punishment had negative influence on children's behavior problems. Other parenting style did not affect children's behavioral problems. Preventive intervention in parents with corporal punishment may reduce behavioral problems. These data will be useful for developing future interventions in child mental health after a natural disaster. 


\section{Abstract \#: P 96}

The association between depressive status and internet addiction in puberty: a community-based cohort study

$\frac{\text { Miri Sato }}{}{ }^{1}$, Kohta Suzuki ${ }^{2}$, Ryoji Shinohara ${ }^{1}$, Sonoko Mizorogi ${ }^{2}$, Zentaro Yamagata ${ }^{2}$

${ }^{1}$ Center for Birth Cohort Studies, University of Yamanashi, Chuo, Japan; ${ }^{2}$ Department of Health Sciences, School of Medicine, University of Yamanashi, Chuo, Japan; Corresponding author's e-mail: miris@yamanashi.ac.jp

Background: Internet addiction (IA) is an emerging health problem in industrialised countries, especially among adolescents. Problematic use of the Internet might have a negative impact on daily life and emotional stability. Several prospective cohort studies have examined risk factors for IA such as internet usage and family factors. Additionally, the results of some studies, particularly cross-sectional studies, have suggested that IA is associated with psychiatric disorders such as depression. The direction of causality and the strength of the connection between IA and depression are unclear. This study aimed to identify the effect of depressive status on IA onset using a community-based cohort study.

Methods: Study participants were 1045 students in the 5th, 6th and 7th grades (age 10-12 years) who participated in an on-going community-based birth cohort study in a rural area of Japan. At baseline and after 1 year (Year 1), the presence of depressive symptoms was assessed using the Birleson Depression Self-Rating Scale for Children, and the hours of internet usage were noted. One year later (Year 2), IA was assessed using Young Internet Addiction Test (IAT). Participants were identified as average internet users, or users with moderate or severe addiction. In the analyses, we excluded children who used the internet for $>2 \mathrm{~h}$ per day at baseline or at Year 1, because these children might already have IA. We examined the relationships between IA at Year 2 and depressive status at baseline and at Year 1 separately to examine the effect of exposure duration. Adjusting for age and sex, multiple logistic regression analyses were conducted to examine the associations between depressive status and IA.

Results: The number of participants at baseline and Year 1 were 858 and 804 , respectively. Depressive symptoms were found in $5.0 \%$ and $6.1 \%$ of the participants at baseline and Year 1, respectively. At Year $2,8 \%$ were defined as having some degree of addiction based on IAT results. The follow-up rate was $87 \%$. Depressive symptoms at baseline and Year 1 were significantly associated with IA (odds ratio [OR], 2.3: $95 \%$ confidence interval [CI], 1.1-4.7 and OR, 3.5: $95 \%$ CI 1.6-7.6, respectively).

Conclusion: Depressive symptoms were associated with later onset of IA and the impact might be changed by exposure duration. There are some potential factors which affect the association between longer exposure duration and IA. In future studies with this cohort, we will examine the effect of IA on depression.

\section{Abstract \#: P 97}

Low socioeconomic status increases the adverse effect of negative life events on anxiety and depression during pregnancy

Verbeek, T. ${ }^{1}$, Bockting, C.L.H. ${ }^{2}$, Beijers, C. ${ }^{3}$, Meijer, J.L. ${ }^{\text {, , van Pampus, M.G. }}{ }^{4}$, Burger, H. ${ }^{5}$

${ }^{1}$ Department of Epidemiology, University of Groningen, University Medical Center Groningen, Groningen, The Netherlands;

${ }^{2}$ Department of Clinical and Health Psychology, Utrecht University, Utrecht, The Netherlands; ${ }^{3}$ Interdisciplinary Center Psychopathology and Emotion Regulation, University of Groningen, University Medical Center Groningen, Groningen, The Netherlands; ${ }^{4}$ Department of Obstetrics and Gynecology, University of Groningen, University Medical Center Groningen, Groningen, The Netherlands; ${ }^{5}$ Department of General Practice, University of Groningen, University Medical Center Groningen, Groningen, The Netherlands; Corresponding author's e-mail: t.verbeek@umcg.nl

Background: Prevention, identification, and treatment of maternal psychopathology may be favorable for both mother and child. Both low socioeconomic status (SES) and negative life events are risk factors for symptoms of anxiety and depression during pregnancy. It is unknown whether the adverse effect of negative life events is modified by SES. Objective. To investigate the relationships between symptoms of anxiety and depression in the first trimester of pregnancy and negative life events, and how they are modified by SES. Methods: The population based Pregnancy, Anxiety and Depression (PAD) study is a prospective cohort study in Dutch obstetric care. We performed a cross-sectional analysis using the baseline data on symptoms of anxiety and depression in pregnant women, SES (educational level of mother and partner, work status of mother and partner and family income), and the number of maternal negative life events, categorized by period in life. The association of the number of negative life events with anxiety and depression, as well as effect modification by SES was tested using linear regression analyses.

Results: We included 4272 participants. The number of negative life events and low SES were associated with symptoms of anxiety and depression during pregnancy (effect size per life event in Z-scores for depression $0.083-0.117, \quad p<0.001$ and anxiety $0.077-0.123$, $p<0.001)$. Additionally, we found that the aspects of SES: low maternal educational level, maternal unemployment, and low family income may increase the adverse effect of negative life events $(p<0.001-0.016)$

Conclusion: Low SES increases the adverse impact of negative life events. In an early screening for anxiety and depression during pregnancy, the number of negative life events and, more important, the above-mentioned aspects of SES should play a key role. Identification and subsequently early treatment of antenatal psychopathology may ultimately prevent psychosocial problems in the offspring.

\section{Abstract \#: P 98}

Urban residential greenness and depressive symptoms: results from the Heinz Nixdorf recall study

Ester Orban $^{1}$, Kelsey McDonald ${ }^{1}$, Nico Dragano ${ }^{2}$, Karl-Heinz Jöckel $^{1}$, Susanne Moebus ${ }^{1}$

${ }^{1}$ Institute of Medical Informatics, Biometry and Epidemiology, University Hospital Essen, University of Duisburg-Essen, Essen, Germany; ${ }^{2}$ Institute of Medical Sociology, University Hospital Düsseldorf, Heinrich Heine University Düsseldorf, Düsseldorf, Germany; Corresponding author's e-mail: ester.orban@uk-essen.de

Background: Evidence suggests access to green spaces, contact with nature and outdoor physical activity may be beneficial to mental health: however, only limited evidence exists from population-based data. Therefore, our aim was to investigate the association between urban residential greenness and depressive symptoms using crosssectional data from a German population-based study.

Methods: We analyzed baseline data of 4374 participants from the Heinz Nixdorf Recall study, aged 45-75 years and residing in the metropolitan Ruhr area in western Germany. Level of greenness or green vegetation within a 100-meter buffer of each participant's residence was defined using the Normalized Difference Vegetation 
Index (NDVI), which was calculated from satellite imagery using a geographic information system. Greenness exposure was dichotomized, defining low greenness as the lowest third of the NDVI value range within the $100 \mathrm{~m}$ buffer. Depressive symptoms were assessed by the CES-D 15-item short form. Participants who reported antidepressant medication use and/or had a CES-D score $\geq 17$ were classified as having depressive symptoms. Logistic regression was used to estimate odds ratios (OR) and their $95 \%$-confidence intervals (95\%-CI), adjusting for age, sex, socioeconomic status, neighborhood socioeconomic status, distance to nearest major road, comorbidities, physical activity, BMI and smoking.

Results: Overall, $10.8 \%$ of the participants had low greenness within $100 \mathrm{~m}$ of their homes. The overall prevalence of depressive symptoms was $18.4 \%$ in women and $8.6 \%$ in men. In participants with high and low greenness we found a prevalence of $13.2 \%$ and $16.6 \%$, respectively. The adjusted OR of depressive symptoms was 1.18 (95\%-CI 0.88-1.58: crude OR: $1.31,1.01-1.70)$ comparing those with low greenness to those with high greenness. Sex-stratified analyses suggest differences by gender (adjusted OR women: 1.30, 0.92-1.83: adjusted OR men: $0.90,0.52-1.57$ ).

Conclusion: The observed results hint at an association between greenness and depressive symptoms, which is in line with previous research, but did not reach statistical significance after adjustment. Studies examining change in residential greenness and corresponding changes in depressive symptoms, as well as individuals' interaction with the greenness (e.g. use of parks), may provide important insight into this relationship. Gender-specific effects deserve further attention, keeping in mind that measurement of depressive symptoms might be gender-insensitive. As greenness may only be of relevance considering mild to moderate depressiveness, future analyses should also examine severity of depressive symptoms in this context.

\section{Abstract \#: P 99}

\section{Serum proteomic profiling of major depressive disorder}

Mariska Bot ${ }^{1}$, Man K. Chan ${ }^{2}$, Rick Jansen ${ }^{1}$, Femke Lamers ${ }^{1}$, Nicole Vogelzangs $^{1}$, Johann Steiner ${ }^{3}$, F. Markus Leweke ${ }^{4}$, Matthias Rothermundt $^{5}$, Jason Cooper ${ }^{2}$, Sabine Bahn ${ }^{2}$,

Brenda W.J.H. Penninx ${ }^{1}$

${ }^{1}$ Department of Psychiatry, VU University Medical Center, and GGZ inGeest, Amsterdam, The Netherlands; ${ }^{2}$ Department of Chemical Engineering and Biotechnology, Institute of Biotechnology, University of Cambridge, Cambridge, UK; ${ }^{3}$ Department of Psychiatry, University of Magdeburg, Germany; ${ }^{4}$ Department of Psychiatry and Psychotherapy, Central Institute of Mental Health, Medical Faculty Mannheim, Heidelberg University, Mannheim, Germany; ${ }^{5}$ University of Münster, Germany and Evangelisches Klinikum Niederrhein, Oberhausen, Germany; Corresponding author's e-mail: m.bot@ggzingeest.nl

Background: Major depressive disorder (MDD) is a burdensome and multi-factorial psychiatric disorder. Several biological mechanisms have been related to MDD, but a detailed understanding of the pathophysiology of MDD is currently lacking. This study aims to gain insight into contributing mechanisms by identifying serum proteins related to MDD in a large psychiatric cohort study.

Methods: An exploratory molecular profiling study was performed in 1589 participants of the Netherlands Study of Depression and Anxiety (NESDA), comprising 687 individuals with current MDD (cMDD), 482 individuals with remitted MDD (rMDD), and 420 controls. A total of 171 proteins involved in various hormonal, immunological, and metabolic pathways were detected in serum on a multi-analyte profiling platform. We studied the relationship between MDD status and the levels of these 171 proteins using linear regression analyses, which were adjusted for potential confounders. Furthermore, pooled analyses of two independent validation cohorts (totalling 78 MDD cases and 156 controls) was carried out to validate our top markers. In this exploratory study, $p$ values $<0.05$ were considered statistical significant.

Results: Twenty-eight analytes differed significantly $(p<0.05$ : false discovery rate q-value range $0.09-0.30$ ) between cMDD cases and controls, while 10 partly overlapping markers differed significantly between rMDD cases and controls. Antidepressant medication use and comorbid anxiety status did not substantially impact on these findings. Sixteen of the cMDD-related markers had been assayed in the pooled validation cohorts. Of these, 7 markers were associated with MDD in the validation cohorts. The analytes prominently associated with cMDD related to diverse cell communication and signal transduction processes (pancreatic polypeptide, macrophage migration inhibitory factor, ENRAGE, interleukin-1 receptor antagonist, tenascin-C), immune response (growth-regulated alpha protein), and protein metabolism (von Willebrand factor).

Conclusions: Several proteins were implicated in depression. Changes in protein levels were more prominent in cMDD, suggesting that molecular alterations in serum are associated with acute depression symptomatology. These findings could improve our understanding of the molecular mechanisms related to depression.

\section{Abstract \#: P 100}

Serum proteomic profiles of depressive subtypes in a depression cohort study

Femke Lamers ${ }^{1}$, Mariska Bot ${ }^{1}$, Rick Jansen ${ }^{1}$, Man K Chan ${ }^{2}$, Jason Cooper $^{2}$, Sabine Bahn ${ }^{2}$, Brenda W.J.H. Penninx ${ }^{1}$

${ }^{1}$ Department of Psychiatry and EMGO Institute for Health and Care research, VU University Medical Center, Amsterdam, the Netherlands; ${ }^{2}$ Department of Chemical Engineering and Biotechnology, Institute of Biotechnology, University of Cambridge, Cambridge, UK; Corresponding author's e-mail:

f.lamers@ggzingeest.nl

Background: The pathophysiological mechanisms of depression are not yet fully understood. Depression is however a highly heterogeneous disorder, and an increasing body of research implicates biological and genetic differences between subtypes of depression that are more homogeneous with respect to symptom presentation. New proteomic techniques are now available that allow for the simultaneous quantitative measurement of a wide array of proteins. This proteomic approach may provide a more complete representation of pathophysiological processes potentially involved in subtypes of depression. The aim of the current study was to evaluate differences in serum protein profiles between persons with atypical and melancholic depressive subtypes, and to compare these profiles with serum protein levels of healthy controls.

Methods: We used baseline data from the Netherlands Study of Depression and Anxiety (NESDA), an ongoing longitudinal cohort study. For the current study we included 414 controls, 231 persons with a melancholic depressive subtype and 128 persons with an atypical depressive subtype for whom proteomic data was available. Subtypes were previously established using data-driven analysis. One hundred and seventy-one serum proteins were detected on a multianalyte profiling platform and analyzed with linear regression models. To account for multiple testing, a false discovery rate (FDR) was calculated (q-value).

Results: We found 24 markers to be differentially expressed between the three groups. We observed differences in analytes between the 
atypical and melancholic depressive subtypes $(9$ analytes, $\mathrm{q}<0.05)$ and between atypical depression and controls ( 23 analytes, $\mathrm{q}<0.05$ ). Eight of the 9 markers differing between the atypical and melancholic subtype overlapped with significant markers from the comparison atypical subtype vs controls (mesothelin, leptin, insulin-like growth factor binding protein 1, insulin-like growth factor binding protein 2 , fatty acid binding protein-adipocyte, insulin, complement $\mathrm{C} 3$, and beta-2-microglobulin). No markers were significantly different between the melancholic depressive subtype and controls. The 24 identified markers were mainly involved in cell communication and signal transduction, protein metabolism, immune response, and metabolism and energy pathways.

Conclusion: This study shows how the application of a proteomic approach can help to increase our understanding of pathophysiological mechanisms of depressive subtypes. The results of this study support the notion of atypical depression as a syndrome characterized by metabolic disturbances and inflammation, underline the importance and relevance of subtypes of depression in biological and genetic research, and may be relevant as well for the treatment of depression.

\section{Abstract \#: P 101}

Do sleep disturbances modify the effect of psychosocial work characteristics on future depression? A study of time-varying causal effect modification

Linda L. Magnusson Hanson $^{1}$, Paraskevi Peristera ${ }^{1}$, Holendro Singh Chungkham $^{2}$, Hugo Westerlund ${ }^{1}$

${ }^{1}$ Stress Research Institute, Stockholm University, Stockholm, Sweden; ${ }^{2}$ Indian Statistical Institute, Tezpur India; Corresponding author's e-mail: linda.hanson@su.se

Background: Stress in combination with poor restitution may lead to more long-term health problems including depression, and sleep is one of most important component of restitution. However, it is unknown whether sleep disturbances can moderate the relationship between work stressors, such as high work demands, low control and low support at work, and depression. The present study sought to investigate this.

Methods: The study sample was derived from the SLOSH (Swedish Longitudinal Occupational Survey of Health) study, a longitudinal cohort survey with repeated measures every second year on an originally representative sample of the Swedish working population. We used data on high work demands, low decision authority and low social support (exposures), sleep disturbances (putative moderator) and a number of time invariant and time-varying covariates from 5 time points (1537 respondents working all time points). The outcome variable was depression severity measured at time point 5 . We primarily applied a structural nested mean model (SNMM) and twostage parametric regression-with-residual-estimation for the parameters of the SNMM to assess the moderated time varying causal effects of interest in a conditional mean model given time-varying exposures, putative moderator and covariates. For comparability we also tested multiplicative and additive interaction using regression models.

Results: While more proximal (time point 4) high demands predicted depression severity (time point 5), there was no indication that sleep disturbances moderated this association when adjusting for education, income, civil status, sex and age. Low decision authority on the other hand did not predict depression severity in the adjusted model. Both proximal and distal (time point 2) low social support at work, however, predicted later depression severity, and disturbed sleep at time point 3 moderated the association between low social support at time point $4(p<0.05)$ and depression. A tendency to an additive interaction between social support and sleep disturbances was also found when using prospective regression analysis.

Conclusion: Disturbed sleep seemed to moderate the prospective relationship between low social support at work and depression severity to some extent, but overall the results indicate that disturbed sleep is not a strong moderator of the relationship between work stressors, such as demands, decision authority and support, and subsequent depression. A major strength is that the putative moderator is measured prior to the exposure and the main results are unbiased by past exposure, but future studies with even more proximal measurements than two years apart may be needed for definite conclusions.

\section{Abstract \#: P 102}

The Rhineland study: a novel platform for epidemiologic research into Alzheimer disease and related disorders

Monique M.B. Breteler ${ }^{1}$

${ }^{1}$ German Center for Neurodegenerative Diseases, Population Health Science, Bonn, Germany; Corresponding author's e-mail: monique.breteler@dzne.de

Background: The insights from epidemiologic research over the last decades into the causes of neurodegenerative diseases also delineate the need for a new prospective cohort to study these further: First, neurodegenerative diseases typically develop over decades rather then years until they manifest late in life. Second, most age-related neurodegenerative diseases occur as the resultant of varying combinations of protective, restorative, and detrimental factors. This asks for additional measurements and approaches to study the etiology of neurodegenerative diseases beyond a focus on determinants of occurrence of disease, including a focus on normal physiology, brain plasticity and repair capacity. Finally, tremendous technological progress has been made in the last decades, particularly in the areas of imaging and -omics and we need to include those more advanced measurements, in larger numbers, and in the potentially more relevant age-groups in our epidemiologic studies.

Methods: We here present the Rhineland Study, a new prospective cohort study that is primarily designed to investigate causes and biomarkers for neurodegenerative and neuropsychiatric diseases and to investigate determinants of normal and pathological brain structure and function over the adult life course. It is a single center community-based study that will recruit up to 30,000 people aged 30 years or over at baseline. All participants will undergo a $7 \mathrm{~h}$ core protocol that includes brain imaging on 3 dedicated 3T MRI scanners, detailed cognitive, neurologic and sensory assessments, cardiovascular measurements, assessment of physical activity and physical fitness, and extensive collection of biomaterials (including plasma, serum, DNA, RNA, cells, urine, stool). Full re-examinations will take place every 3-4 years. The study is planned to run for decades. The study design and setting allows for additional examinations in subsets of the cohorts.

Results: First pilot studies are ongoing.

\section{Abstract \#: P 103}

Major depressive disorder, antidepressant use and subsequent 2-year weight change patterns in The Netherlands study of depression and anxiety 
Deborah J. Gibson-Smith ${ }^{1}$, Mariska Bot ${ }^{1}$, Yuri Milaneschi ${ }^{1}$, Jos Twisk ${ }^{2}$, Marjolein Visser ${ }^{3}$, Ingeborg Brouwer ${ }^{3}$, Brenda W.J.H. Penninx ${ }^{1}$

${ }^{1}$ Department of Psychiatry/EMGO Institute for health and care research, VU University Medical Center, and GGZ inGeest Amsterdam, The Netherlands; ${ }^{2}$ Department of Epidemiology and Biostatistics, EMGO Institute for Health and Care Research, VU University Medical Center Amsterdam, The Netherlands; ${ }^{3}$ Department of Health Sciences, Faculty of Earth and Life Sciences and EMGO Institute for Health and Care Research, VU University, Amsterdam, the Netherlands; Corresponding author's e-mail: d.gibson-smith@ggzingeest.nl

Background: Major depressive disorder (MDD) and obesity are two major causes of disability adjusted life years (DALYs) worldwide. Given the huge impact these two disorders have on society, understanding the causes of depression and obesity is of importance. Depression and obesity have been (bi-directionally) linked, however little is known about subsequent weight changes following major depressive disorder (MDD). This study compared 2-year weight changes between patients with clinically diagnosed current MDD (cMDD), patients with remitted MDD (rMDD) and healthy controls. Additionally, we examined the relationship between antidepressant medication use and 2-year weight change and compared general demographic and health characteristics and the depressive symptom profiles between depressed weight gainers and losers.

Method: Data from 2542 adults aged 18-65y was sourced from the Netherlands Study of Depression and Anxiety. Data was collected at baseline and after 2, 4, and 6 years (9/2004-04/2013). Depression status was established with the Composite International Diagnostic Interview. Subsequent, measured, 2-year weight changes were categorised as weight loss ( $>5 \%$ loss), weight stable (within $5 \%$ weight loss or gain) and weight gain ( $>5 \%$ gain). The association of depression status with subsequent 2 -year weight change, using controls and weight stable as reference category, were studied by combining all repeated measurements in a mixed multinomial logistic regression model.

Results: Using 5390 observations, cMDD was significantly associated with both weight gain and weight loss over a 2-year period after adjustment for covariates (odds ratio (OR) 1.96, $95 \%$ confidence interval (CI) $1.64-2.35, p<0.001$; OR $1.6095 \%$ CI $1.03-1.63$, $p=0.045$ respectively). rMDD was not related to weight changes. Antidepressant use was associated with weight gain, however, when MDD status was included in the model, only cMDD remained associated with weight gain and loss. Compared to cMDD patients who lost weight, weight gainers had lower initial weight, were younger, had more comorbid anxiety disorders, and reported poorer quality of mood and reduced appetite as depressive symptoms.

Conclusions: Compared to controls, cMDD participants have greater odds of either gaining or losing weight over a 2-year period, regardless of antidepressant use. This provides empirical evidence for the heterogenic nature of MDD and suggests that that it is the underlying depressive disorder that is responsible for any weight gain, rather than antidepressant medication use. This is important considering fear of weight gain is a major reason for drug treatment noncompliance in depressed patients and may contribute to a hesitancy to start with antidepressant treatment.

\author{
Abstract \#: P 104 \\ Perceived barriers to accessing mental health services \\ among ethnic minorities: a qualitative study in Southeast \\ England
}

A. Memon ${ }^{1}$, K. Taylor ${ }^{1}$, L. Mohebati ${ }^{1}$, V. Collins ${ }^{1}$, M. Campbell ${ }^{2}$, A. Porter ${ }^{2}$, A. Dale ${ }^{3}$, E. Hope ${ }^{3}$, P. Koroma ${ }^{3}$, D. Ndebele ${ }^{3}$, R. O. de Visser, M. Cooper

${ }^{1}$ Brighton and Sussex Medical School, UK; ${ }^{2}$ NHS Brighton and Hove, UK; ${ }^{3}$ BME Community Partnership, Brighton and Hove, UK; Corresponding author's e-mail: a.memon@bsms.ac.uk

Background: In most European countries, there are significant disparities in the understanding of mental health conditions and access to mental health services among ethnic minority groups. In general, people from ethnic minorities are less likely to contact their general practitioner about mental health issues, and have negative perceptions or experiences of mental health services. This often leads to disengagement from the services and poor clinical and social outcomes. It is unclear whether these disparities represent variation in mental health needs, or result from personal/environmental factors and/or relationships between service users and healthcare providers. This qualitative study sought to identify perceived barriers to accessing mental health services among individuals from ethnic minorities in Southeast England to inform the development of effective and culturally acceptable services.

Methods: Twenty-six adults from ethnic minorities were recruited by community development workers to participate in two focus groups. Discussions were facilitated by researchers trained in cross-cultural communication and the qualitative methodology. Thematic analysis was conducted to identify key emerging themes.

Results: Participants highlighted several personal/environmental factors that influenced their access to mental health services. These included: positive impact of social networks (family/friends): inability to recognise and accept mental health problems: reluctance to discuss psychological distress (especially men): cultural identity: and negative impact of prejudice, discrimination and stigmatisation of mental illness. The relationship between service users and healthcare providers was also perceived to affect access, especially: long waiting times for initial assessment: language barriers and poor communication between service users and providers, inadequate recognition/ response to mental health needs: the imbalance of power and authority between service users and providers: cultural naivety and insensitivity towards the needs of service users: and lack of awareness of different mental health services among service users and providers. Conclusion: Members of ethnic minorities require greater mental health literacy and practical support to raise awareness of mental health issues, and provided with appropriate information about the different services and pathways to access these services. Healthcare providers need to be supported in developing effective communication strategies to deliver individually tailored and culturally sensitive care. The engagement of ethnic minorities in the development and delivery of culturally appropriate mental health services could also facilitate better understanding of mental health conditions and improved utilisation of mental health services.

\section{Abstract \#: P 105}

Long-term effects on physical activity and body weight after participation in a randomized weight loss trial in postmenopausal women

Martijn de Roon $^{1,2}$, Evelyn Monninkhof ${ }^{1,2}$

${ }^{1}$ Department of Epidemiology, Julius Center for Health Sciences; ${ }^{2}$ Primary Care, University Medical Center Utrecht, Utrecht, the Netherlands; Corresponding author's e-mail: martijnderoon@live.nl

Purpose: The aim of this study was to determine body weight and physical activity 12 months after participation in a randomized 
16-week weight loss program in postmenopausal women. Furthermore we aimed to investigate whether the effects are different for weight loss induced by diet or exercise.

Methods: Twelve months after participation in a randomized weight loss intervention study, women were contacted again for measurement of bodyweight and physical activity (using both the ActiGraph ${ }^{\circledR}$ accelerometer and the Physical Activity Scale for the Elderly (PASE) questionnaire). In the intervention study, 195 postmenopausal inactive women had been randomized into an exercise $(\mathrm{N}=98)$ and a diet group $(\mathrm{n}=97)$. During 16 weeks, subjects received an exercise or diet intervention aiming at a 5-6 kg reduction in bodyweight, which was obtained in both groups.

Results: At 12 months, both the exercise $(-4.3 \mathrm{~kg}$ ) and diet group $(-3.4 \mathrm{~kg})$ showed a reduced bodyweight compared to start of the intervention study (baseline) but had slightly regained bodyweight compared to end of the intervention study $(+1.3 \mathrm{~kg}$ and $+1.5 \mathrm{~kg}$, respectively). Both the exercise ( +39 points) and diet group $(+14$ points) scored higher (i.e., more active) on the PASE questionnaire at 12 months compared to baseline. The ActiGraph ${ }^{\circledR}$ showed that the exercise group had less sedentary $(-1.5 \% 95 \%$ CI $-2.73:-0.26)$ and more moderate to vigorous activity $(+0.7 \% 95 \% \mathrm{CI} 0.17: 1.31)$ compared to baseline. The exercise group also showed significantly less sedentary time and more moderate and vigorous activity when compared to diet.

Conclusion: This study shows highly sustained weight loss and increased levels of physical activity 12 months after completing a 16 -week exercise program in overweight postmenopausal women with an inactive lifestyle. However, although the exercise group became more physically active compared with diet, maintenance of weight loss did not differ between the two groups.

\section{Abstract \#: P 106}

The influence of weather conditions on outdoor physical activity among older people with and without osteoarthritis

Erik Timmermans ${ }^{1}$, Suzan van der Pas ${ }^{1}$, Laura Schaap ${ }^{2}$, Dorly Deeg ${ }^{1}$

${ }^{1}$ Department of Epidemiology and Biostatistics, VU University Medical Center; ${ }^{2}$ Department of Health Sciences, VU University Medical Center; Corresponding author's e-mail:

ej.timmermans@vumc.nl

Older people with osteoarthritis (OA) often report that their disease symptoms, including joint pain and stiffness, are exacerbated by weather conditions. This study addresses the question whether outdoor physical activity (PA) in older people with OA is more strongly influenced by weather conditions than in those without OA. Baseline data from the population-based European Project on OSteoArthritis (EPOSA) were used. American College of Rheumatology classification criteria were used to diagnose OA in older people (65-85 years). Outdoor PA in minutes per day was assessed using the LASA Physical Activity Questionnaire. Daily average values for temperature, precipitation, atmospheric pressure, relative humidity and wind speed were obtained from local weather stations. The study included 2687 participants. Their mean age was $74.0(\mathrm{SD}=5.0)$ and 802 $(30.5 \%)$ participants had OA in knee, hand and/or hip. Participants with OA spent fewer minutes in outdoor PA than those without OA $($ Median $=42.9, \mathrm{IQR}=20.0-85.7$ versus Median $=50.7, \mathrm{IQR}=$ 21.4-98.6). In the full sample, temperature $(\mathrm{B}=1.51: p<0.001)$ and relative humidity $(\mathrm{B}=-1.31: p<0.001)$ were independently associated with total outdoor PA. The association of temperature with outdoor PA was stronger in participants without $\mathrm{OA}(\mathrm{B}=1.94$ : $p<0.001)$ than in those with $\mathrm{OA}(\mathrm{B}=0.58: p=0.35)$. In particular, the influence of temperature on outdoor walking was stronger in participants without OA $(\mathrm{B}=0.69: p=0.02)$ compared to their counterparts with $\mathrm{OA}(\mathrm{B}=-0.32: p=0.43)$. The influence of weather conditions on outdoor PA was not greater in older people with OA than in those without OA. In fact, the influence of temperature on total outdoor PA and walking was greater in those without OA. Regardless of OA, researchers and clinicians should take weather conditions into account when designing and interpreting the results of interventions aimed at increasing PA in older populations.

\section{Abstract \#: P 107}

Towards a preventive strategy for cans: the role of help seeking behavior

Bruls V.E.J. Msc, Dr Jansen N.W.H., Prof. Dr. De Bie R.A., Dr Bastiaenen C.H.G., Prof. dr. Kant Ij; Corresponding author's e-mail: vivian.bruls@maastrichtuniversity.nl

Introduction: Non-traumatic complaints of the arm, neck and/or shoulder (CANS) are highly prevalent in the working and student population. Symptoms are usually mild at first, but may worsen over time to a level at which they become chronic and disabling, representing a major cause for temporary and sometimes long-term sickness absence from work or study. Once on sick leave, individuals may encounter difficulties to return to work or to resume study activities. Effectiveness of treatments, intended to reduce the duration of sick leave once complaints have become chronic and disabling, is limited. It is hypothesized that a more effective strategy would be to intervene at an earlier stage, before absenteeism and disability occur. Therefore, a preventive approach for CANS should be considered aimed at individuals with beginning and mild symptoms, while still at work. However, precisely those individuals with a mild level of complaints are unlikely to display any help seeking behavior and subsequently might refuse participation in a prevention program comprising screening and subsequent early intervention. To develop an effective early preventive strategy, insight in the determinants of help seeking behavior within the target population is fundamental.

The objectives of this study were (1) to examine whether the perceived severity of the complaint is associated with help seeking behavior in employees and students identified with CANS (2) to determine if factors related to functioning and participation, workenvironment and demographics influence the association between the perceived severity of the complaint and help seeking behavior. Outcomes of this study might result in the identification of certain subgroups which will enable tailored prevention strategies, in order to optimize screenings uptake and treatment adherence.

Methods: A cross-sectional survey was conducted among employees and students of two universities in the Netherlands. Approximately 5600 employees and 30,000 students received a survey questionnaire by email. In total, 1724 employees $(30.8 \%)$ and 3327 students $(11.1 \%)$ responded. The questionnaire included questions to assess (1) the experience of CANS (2) perceived severity (3) help seeking behavior and (4) demographics.

A subpopulation of the survey, consisting of employees and students with self-reported CANS, received additional questionnaires to examine the impact of (1) functioning and participation (2) workenvironment and social support on help seeking behavior.

Results: Preliminary results show that perceived severity of CANS and impact of CANS on individual functioning indeed are related to help seeking behavior.

Detailed results, including for subgroups will be available in June 2015. 


\section{Abstract \#: P 108}

\section{Hip fractures among people 67 years and older attending} the Emergency Department at Landspitalinn 2008-2012

Sigrún Sunna Skúladóttir ${ }^{1}$, Elísabet Guðmundsdóttir ${ }^{2}$, Helga Rósa Másdóttir ${ }^{1}$, Ingibjörg Sigurpórsdóttir ${ }^{1}$, Hlíf Guðmundsdóttir ${ }^{3}$, Lovísa Jónsdóttir $^{1}$, Pórdís K Porsteinsdóttir ${ }^{1,4}$

${ }^{1}$ Emergency Department, Landspitali University Hospital, Reykjavik, Iceland; ${ }^{2}$ Department of Economy, Landspitali University Hospital, Reykjavik, Iceland; ${ }^{3}$ Department of Geriatrics, Landspitali University Hospital, Reykjavik, Iceland; ${ }^{4}$ Faculty of Nursing University of Iceland; Corresponding author's e-mail: sigrskul@landspitali.is

Background: Hip fracture is a serious injury. Epidemiological studies have revealed that people over 50 years of age account for over $90 \%$ of hip fractures and the risk is $2-3$ times higher for women than men. Patients with hip fractures need fast and good services to avoid complications, and surgery is almost always required.

Aim: To increase knowledge on the epidemiology of elderly patients with hip fractures in Iceland, with the aim to improve care at the Emergency Department (ED) and promote preventive measures.

Method: Data was obtained retrospectively from the clinical data warehouse at Landspítali University Hospital on all patients older than 67 years admitted to the ED during 2008-2012 with collum, pertrochanteric and subtrochanteric fractures. Data was analyzed descriptively and by regression analysis to find predictors for fractures, arrival time, waiting time in the Emergency Department and for surgery as well as mortality.

Results: During the study period a total of 1053 patients were admitted to the ED with hip fractures: the oldest was 107 years old. The prevalence rate was 3.9 men and 6.9 women per 1000 inhabitants at the same age. Number of yearly visits ranged from 193 to 222, with significantly more fractures during winter than summer season. Widowed had a higher prevalence than those married or single. Men waited longer for surgery than women, 21.5 vs $18.9 \mathrm{~h}(p<0.05)$. Elderly living in rural areas waited significantly longer than elderly from the urban area. Mortality within 12 months was $21 \%$ among women and $36 \%$ among men $(p<0.05)$.

Conclusions: The outcome for elderly people suffering from a hip fracture can be severe. The high proportion of hip fractures among elderly people in Iceland implies the need for preventive measures among groups at risk.

\section{Abstract \#: P 109}

\section{Osteoarthritis in Germany: current burden of disease} and prognosis to 2050

Thomas Petzold $^{1}$, Elisabeth Haase ${ }^{2}$, Fritz Uwe Niethard ${ }^{3}$, Jochen Schmitt ${ }^{2}$

${ }^{1}$ Center for Evidence-Based Healthcare, Department of Quality and Medical Risk Management, University Hospital Carl Gustav Carus Dresden, Dresden, Germany; ${ }^{2}$ Center for Evidence-Based Healthcare, University Hospital Carl Gustav Carus Dresden, Dresden, Germany; ${ }^{3}$ German Society for Orthopaedics and Traumatology, Germany; Corresponding author's e-mail: thomas.petzold@uniklinikum-dresden.de

Background: Osteoarthritis is among the most common chronic diseases in industrialized countries and constitutes substantial disease burden. Currently, approximately $20 \%$ of the population suffer from osteoarthritis of the knee, hip, and/or shoulder. We aimed to investigate the current burden due to osteoarthritis for the German healthcare system and estimate the future demand due to medical care of patients with osteoarthritis until 2050.

Methods: We utilized complementary data on the population prognosis from the German federal bureau of statistics, healthcare utilization data of the German Federal Statistical Office including data from the statistical offices of the federal states (FDZ) and data of the scientific institute of the AOK, a large German statutory health insurance (WidO). We included all inpatient cases of the FDZ from 2005 to 2012 and all outpatient cases of the WidO from 2007 to 2012. Based on 2012, we predicted the number of inpatient and outpatient cases with osteoarthritis in Germany until 2050 assuming stable prevalences and healthcare utilization behavior. Sensitivity analyses were performed to assess the robustness of the estimations from variations in prevalence, demographic developments, and healthcare utilization behaviour.

Results: In 2012, a total of 7,736,651 cases were treated for osteoarthritis of shoulder, knee or hip in Germany. The projected burden of disease of osteoarthritis is predicted to increase from 2012 to 2040 and then to decline slightly until 2050. The burden of osteoarthritis is currently twice as high in women compared to men. In the age group of 65 years and older, the annual number of cases will increase by more than 2 million from 4,956,702 in 2012 up to $7,188,886$ in 2040 .

Conclusion: For the medical treatment of the predicted number of cases more service providers are required until 2040. Medical-technical progress that may influence the prevalence and/or prognosis of osteoarthritis, potential relocation of services to other sectors and between disciplines, socio-cultural and health policy changes and modifications of our social system were not considered in our model and may significantly impact the future healthcare demand. A continuous monitoring of disease burden is required to ensure that the medical demand for the care of osteoarthritis can be adequately met.

\section{Abstract \#: P 110}

The influence of weather conditions on joint pain in older people with osteoarthritis from six European countries

$\underline{\text { Erik Timmermans }}{ }^{1}$, Laura Schaap ${ }^{2}$, Suzan van der Pas ${ }^{1}$, Dorly Deeg ${ }^{1}$

${ }^{1}$ Department of Epidemiology and Biostatistics, VU University Medical Center; ${ }^{2}$ Department of Health Sciences, VU University Amsterdam; Corresponding author's e-mail:

ej.timmermans@vumc.nl

This study examined whether daily weather conditions, ambient weather conditions (average weather conditions over a 3-day period before a pain report) and changes in weather conditions influence joint pain in older people with osteoarthritis (OA) in six European countries. Data from the population-based European Project on OSteoArthritis (EPOSA) were used. American College of Rheumatology classification criteria were used to diagnose OA in older people (65-85 years). After the baseline-interview, at six months, and after the 12-18 months follow-up interview, joint pain was assessed using two-week pain calendars. Daily values for temperature, precipitation, atmospheric pressure, relative humidity and wind speed were obtained from local weather stations. Multilevel regression modelling was used to examine the associations between the weather parameters and joint pain, adjusted for sex, age, country, depression and medication use. The study included 810 participants with OA in knee, hand and/or hip. Their mean age was 73.6 years $(S D=5.5)$. After adjustment, there were significant associations of joint pain with daily average humidity $(\mathrm{B}=0.004: p<0.01)$ and ambient humidity ( $\mathrm{B}=0.004: p=0.01)$, but not with other daily or ambient weather parameters. Effect sizes were 0.063 and 0.054 for daily average 
humidity and ambient humidity respectively. A significant ambient humidity by ambient temperature interaction effect on joint pain was found. The effect of ambient humidity on pain was stronger in relatively cold weather conditions. Changes in weather parameters between two consecutive days were not significantly associated with reported joint pain. Humidity is associated with joint pain severity in older people with OA. The influence of ambient humidity on joint pain is stronger when the temperature is low. Knowledge about the relationship between joint pain in OA and weather may help physicians and patients to better understand and manage fluctuations in pain.

\section{Abstract \#: P 111}

Healthy lifestyle behavior reduces the risk of long-duration troublesome neck/shoulder/arm pain in women: results from the Stockholm public health cohort

$\underline{\text { Lena W Holm }}^{1,2}$, Tony Bohman ${ }^{1,3}$, Eva Skillgate ${ }^{1,4}$

${ }^{1}$ Institute of Environmental Medicine, Musculoskeletal \& Sports Injury Epidemiology Center, Karolinska Institutet, Stockholm, Sweden; ${ }^{2}$ Institute of Health Policy, Management and Evaluation, University of Toronto, Toronto, Ontario, Canada; ${ }^{3}$ Department of Neurobiology, Care Sciences and Society, Karolinska Institutet, Stockholm, Sweden; ${ }^{4}$ Scandinavian College of Naprapathic Manual Medicine, Stockholm, Sweden; Corresponding author's e-mail: Lena.Holm@ki.se

Background: Healthy lifestyle behavior (HLB) is known to prevent several health problems. In a recent study it was found that HLB was a protective prognostic factor in women with occasional low-back pain. No effect was seen in men. If HLB also is protective in a population without pain at baseline, has to date not been studied. Our aim was to assess the association between HLB in population with no neck/shoulder/arm pain and subsequent long-duration troublesome neck pain (NSAP).

Methods: Stockholm Public Health Cohort is a population-based cohort within the framework of the Stockholm County Council public health surveys. In 2006 a randomly selected and stratified population $(\mathrm{n}=56,634)$, age $18-84$, received a questionnaire, (61\% responded). The responders received a new questionnaire in 2010, (73\% responded). For this study we included those who at baseline reported no NSAP during the past six months The exposure categories were as follow: non-HLB had none or one out of the HLB factors: nonsmoking, moderate alcohol consumption, recommended consumption of fruit and vegetables and recommended level of physical activity (reference category). HLB2, HLB3 and HLB4 had two, three and four of the HLB factors, respectively. The outcome was defined as at follow-up reporting NSAP at least two days a week during the past six months that had decreased workability or interfered with other daily activities to some or to a high degree. Binomial regression was used and we tested for nine potential confounders in men and women separately. Factors changing the rate ratio $\geq 5 \%$ were included the final regression models.

Results: The study population was 9304 persons of whom information on exposure and outcome was available in 7760:(3503 women and 4257 men). Factors confounding the risk ratio (RR) in women was socioeconomic status and in men socioeconomic status, age and economic stress: In women HLB was protective for developing NSAP in all categories of HLB compared to a non-HLB: Adjusted RR: (HLB2) 0.74 (95 \% CI 0.52-1.09), (HLB3) 0.52 (95 \% CI 0.34-0.79), (HLB4) 0.53 (96 \%CI 0.29-0.95). Similar pattern was not seen in men. In contrary, the highest category HLB4 seemed to be a risk factor for NSAP in men (1.64: (95\% CI 0.95-3.91).
Conclusion: In a population free of neck pain, women, but not men, who have a healthy life style behavior, seem to have a lower risk of developing long-duration troublesome NSAP. Non-differential misclassification of the exposure and the outcome is a main potential source of bias.

\section{Abstract \#: P 112}

A self-management programme for employees with complaints of the arm, neck, or shoulder: short and middle term effects

Nathan Hutting ${ }^{1}$, J. Bart Staal ${ }^{2}$, Josephine A. Engels ${ }^{1}$, Yvonne F. Heerkens $^{1}$, Sarah I. Detaille ${ }^{1}$, Maria W.G. Nijhuis-van der Sanden ${ }^{2}$

${ }^{1}$ HAN University of Applied Sciences, Nijmegen, The Netherlands; ${ }^{2}$ Radboud university medical center, Nijmegen, The Netherlands; Corresponding author's e-mail: nathan.hutting@han.nl

Background: Complaints of the arm, neck, or shoulder (CANS) have a multifactorial origin and can cause decreased work productivity, sickness absence, and job loss. There is a need for intervention programs for people with CANS. Self-management is an approach used in chronic disease care to improve self-efficacy and behaviour to facilitate participants to make informed choices and carry them out, so that employees with CANS have the ability to manage the symptoms, healthcare utilization, physical and psychosocial consequences, and lifestyle changes inherent to living with CANS.

Purpose: To get insight in the short and middle long term effectiveness of a self-management programme (including ehealth) and compare it to usual care among employees with chronic non-specific CANS (lasting $>3$ months).

Methods: Participants were randomized to either the intervention group (IG) or control group (CG). The IG participated in a selfmanagement programme consisting of six group sessions and an ehealth module. The CG is allowed to use all usual care available. The self-reported disability of arm, shoulder, and hand (DASH) was the main outcome measure. Other outcome measures were pain in the previous week (NPRS), self-efficacy at work, and presenteeism (SPS6). Data were collected at baseline (T0), and at 3 months (T1), 6 months (T2), and 12 months (T3). Outcomes at T0, T1 and T2 are available and were evaluated using between group analysis of covariance.

Results: In total 123 participants were randomized of which 115 filled in the baseline questionnaire (IG: 64, CG: 51 participants). Questionnaires at $\mathrm{T} 1$ at $\mathrm{T} 2$ were filled in by 103 (IG: 59, CG: 44 participants) and 92 participants (IG: 54, CG: 38) participants, respectively. Mean age was 46.9 (SD 12.8 years). No significant differences $(p>0.05)$ between the IG and the CG were found in any of the outcome measures. Between group effects for disability were -1.98 (95\% CI -5.06 to 1.6$)$ at $\mathrm{T} 1$ and -2.9 (95\% CI -6.80 to $1.04)$ at $\mathrm{T} 2$, for pain -0.27 (95\% CI -0.97 to 0.53$)$ at $\mathrm{T} 1$ and -0.22 (95\% CI -97 to 0.53$)$ at $\mathrm{T} 2$, for self-efficacy at work $4.30(95 \% \mathrm{CI}$ -0.99 to 9.48$)$ at $\mathrm{T} 1$ and $1.43(95 \% \mathrm{CI}-4.97$ to 7.83$)$ at $\mathrm{T} 2$, and for presenteeism -0.74 (95\% CI 1.25-4.80) at $\mathrm{T} 1$ and -0.14 (95\% CI -1.05 to 1.20$)$ at $\mathrm{T} 2$.

Conclusions: A self-management programme for employees with CANS seems not to be effective on disability, pain, self-efficacy at work, and presenteeism compared to usual care in short (3 months) and middle long term (6 months). In general, both the IG as well as the CG showed improvements, with slightly better, non-significant, improvements in the IG. Implications Implementation of the programme is not recommended at this moment. The results of the long term evaluation (T3: 12 months) must be awaited before drawing firm conclusions. The data used in this abstract will be presented on a 
poster presentation on the WCPT congress and in a peer-reviewed article in 2015.

\section{Abstract \#: P 113}

Prognostic factors of complaints of arm, neck and/or shoulder: a systematic review of prognostic cohort studies

Bruls, V.E.J. Msc., Dr. Bastiaenen C.H.G., Prof. Dr. Bie de R.A.;

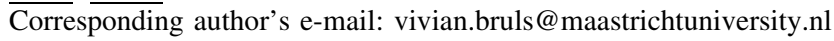

Complaints of the arm, neck or shoulder (CANS) are common musculoskeletal disorders. To gain insight in prognostic factors of CANS that are associated with recovery, we conducted a systematic review. We included longitudinal prognostic cohort studies which investigated associations between prognostic factors and recovery in terms of symptoms, disability or sickness absence. Twenty-six papers reporting on 20 cohorts were included following a search of electronic databases (Pubmed, Embase, Cinahl and Psychinfo). The risk of bias (ROB) was independently assessed by two reviewers using the Quality In Prognosis Studies (QUIPS tool). Sixteen studies were assessed as having 'low' ROB, and ten studies were assessed as having 'high' ROB. Because of heterogeneity in included studies, pooling was not possible. In the qualitative analysis, the number of studies that evaluated a factor, the ROB of each cohort, and consistency of available evidence were taken into account when summarizing the evidence. We examined whether follow-up duration altered the association of prognostic factors with recovery. The results of our best-evidence synthesis showed that for short term follow-up ( $<6$ months) longer duration of complaints, higher symptom severity, more functional limitations, the use of specific coping styles, and accident as 'patients opinion regarding cause' were negatively associated with recovery. For long term follow-up, we found that longer duration of complaints at presentation had an unfavorable prognostic value for recovery. Our evidence synthesis revealed strong evidence for no prognostic impact of many factors which are suggested to be associated with recovery according to the primary studies.

\section{Abstract \#: P 114}

Experiences of participants in a self-management program for employees with complaints of the arm, neck or shoulder (CANS)

Nathan Hutting ${ }^{1}$, Sarah I. Detaille ${ }^{1}$, Yvonne F. Heerkens ${ }^{1}$, Josephine A. Engels ${ }^{1}$, J. Bart Staal ${ }^{2}$, Maria W.G. Nijhuis-van der Sanden ${ }^{2}$

${ }^{1}$ HAN University of Applied Sciences, Nijmegen, The Netherlands; ${ }^{2}$ Radboud university medical center, Nijmegen, The Netherlands; Corresponding author's e-mail: nathan.hutting@han.nl

Background: Complaints of the arm, neck, or shoulder (CANS) have a multifactorial origin including physical characteristics, psychosocial characteristics, personal factors, and environmental factors. CANS is persistent and can cause decreased work productivity, sickness absence, and, job loss. Following the intervention mapping protocol, a self-management program, including an ehealth module, was developed. Together with a randomized controlled trial (RCT), designed and planned to evaluate the quantitative effects of the intervention on the outcome measures, a process evaluation was planned to investigate whether the developed self-management program and the program topics fitted the needs of employees with CANS.

Methods: The first groups of consecutive participants of the intervention group participating in the RCT were interviewed shortly after their last group session. Of the first six groups, 31 of the 32 participants were willing to participate. Three of them did not complete the self-management sessions. All semi-structured interviews were guided by an interview guide and audio-recorded. The interview guide focused on the participants' reasons to participate, expectations, benefits, future expectations, and experiences with the action plans, group sessions and ehealth module. Data were analysed using theoretical thematic analysis in Atlas.ti by two authors, and the emerging themes were discussed.

Results: Participants liked the diversity of the program. They benefitted from the interaction with others and mentioned the social support of the group was valuable. Especially the section with the exercises in the ehealth module was experienced as positive. Participants obtained more insight in their complaints and experienced an increased awareness, which contributed to the acceptance of and coping with the complaints. Almost all participants said that they indeed changed their behaviour. Some of the participants had expected a more physical approach or would like to see more physical activity in the sessions. The lack of a follow-up session was also point of criticism, as many participants indicated that some kind of followup would be valuable for them. Half of the participants did not use the ehealth module extensively, mostly, because they had limited time or did not think that it was interesting for them.

Conclusions: Although there were some points of criticism, participants were generally satisfied with the program. The intervention fitted the needs of employees with CANS. In almost all participants a behavioral change was facilitated, although some felt that continuing their changed behaviour would be a challenge. The results of the RCT should indicate whether the program leads to adequate behavioral change on the long term. The data used in this abstract will be presented on the WCPT congress and a peer-reviewed article in 2015 and are partly presented at the international pain congress in october 2014.

\section{Abstract \#: P 115}

\section{Chronic pain}

Anna Wouters ${ }^{1}$, Janke Oosterhaven ${ }^{1}$

${ }^{1}$ Department of Lifestyle and health, University of Applied Sciences Utrecht, Utrecht, The Netherlands; Corresponding author's e-mail: annawouters@live.nl

Aim: Chronic pain is a problem with which $18 \%$ of the adult Dutch population struggle with. Research shows that it has a big influence on people and, in turn, society. Psychosocial factors like illness perceptions and coping play an important part in developing and living with chronic pain.

Objective: This case study is part of the qualitative research PREPGO (Pain Revalidation for Patients with low Health Literacy). PREPGO research aims to show whether there is a way to predict when patients will not respond to the chronic pain treatment and how they may prevent this from occurring.

Purpose: "What are the illness perceptions and what is the coping of a patient with chronic pain on the waiting list for a mulDesign:

Data collection: A chronic pain patient on the waiting list of a chronic pain treatment in rehabilitation center "De Hrecorded on audio and video. Data-analysis and data processing: A transcript is made of the audio recording and analyzed afterwards (open, axial and selective). MAXQDA is used for analyzing the transcript. Results: The case is a 42-year-old woman with chronic pain. The results are obtained with the main labels 'illness perception' and 'coping' and described based on the Common Sense Model (CSM). Illness perceptions are divided in Obstacle, Thoughts, Emotion and Identity. 
Coping is divided into Identity, Coping, Family, Work and Spare time.

Discussion: The illness perceptions of the case show that there is not a complete understanding of the pain. Research shows that when the complaints are not understood there is a high possibility of poor treatment results. For coping it looks like the case performs too many activities and she does not realize this could prolong her pain. In previous situations with pain, doing more had a positive effect for the case.

Conclusion: The illness perceptions of the case are suggesting that the case experiences a lot of obstacles because of chronic pain. She cannot live the life she did previously, this has left her disappointed and angry. The case describes herself as someone who is persistent. The coping of the case is to always continue. It appears that incorrect illness perceptions have sustained an incorrect coping.

\section{Abstract \#: P 116}

\section{Netherlands cancer registry: from population based registry towards patient and tumor registries}

$\underline{\text { Sabine Siesling }}^{1,2}$, Otto Visser ${ }^{1}$, Janneke Verloop ${ }^{1}$, Aiko de Raaf ${ }^{1}$, Heidi van Doorne-Nagtegaal ${ }^{1}$, Valery Lemmens ${ }^{1,3}$, Peter Huijgens ${ }^{1}$

${ }^{1}$ Netherlands Comprehensive Cancer Organisation; ${ }^{2}$ University of Twente; ${ }^{3}$ Erasmus University Rotterdam; Corresponding author's e-mail: s.siesling@iknl.nl

Background: The Netherlands Cancer Registry (NCR) is a population based registry and includes all newly diagnosed cancer cases in the Netherlands. The main sources of notification are the national automated pathology database (PALGA) and the hospital discharge registries. Specially trained registrars retrieve data directly from the patient files. To answer the increased need for information and research on quality of care, guideline implementation and outcomes research (i.e. disease free survival, costs, quality of life) of the total patient pathway, the NCR requires supplementary data.

Methods: The current item set of the NCR is re-evaluated with clinical and health economic experts for all tumour groups. The complete health care pathway (including diagnostics, treatment, etc. for primary and recurrent disease) is taken into account including the existing guidelines and gaps in knowledge.

Results: More than 10 tumour expert groups are working on the item sets. For 5 tumour types the registration system is being adapted in 2015 and new registration manuals are being developed. Agreements with the hospitals on data collection and regular feedback are made. Expansion to all tumour types will be initiated later.

Conclusion: Evaluation of clinical guidelines, heath technology assessment studies, economic evaluations will be possible more in detail and over the complete patient pathway (diagnosis, cure and care) with the new NCR. Support of clinical audits by regular feedback to clinicians will improve the quality of care. The NCR will be more useful and have more value then ever.

\section{Abstract \#: P 117}

\section{Analysis on the changing trend of causes of death between 1980 and 2013 in Turkey} $\frac{\text { Raziye Ozdemir }}{\text { Unal }^{4}}$, Gonul Dinc Horasan ${ }^{2}$, Kaan Sozmen ${ }^{3}$, Belgin

${ }^{1}$ Karabuk University, School of Health, Department of Occupational Health and Safety, Karabuk, Turkey; ${ }^{2}$ Celal Bayar University, Faculty of Medicine, Department of Biostatistics, Manisa, Turkey; ${ }^{3}$ Katip Celebi University, Faculty of Medicine, Department of Public Health, Izmir, Turkey; ${ }^{4}$ Dokuz Eylul University, Faculty of Medicine,

Department of Public Health, Izmir, Turkey; Corresponding author's e-mail: ozdemirr75@hotmail.com

Background: The analyses of causes of death (CoDs) data are very important to define public health policy priorities and to evaluate health programs.

Objective: The aim of this study was to examine age group and sex specific trends of CoDs in Turkey between 1980 and 2013.

Methods: Data on the number of deaths by year, sex, age group and CoDs were obtained from the Turkish Statistical Institute. The CoDs were classified as Group I: Communicable, maternal, perinatal and nutritional conditions, Group II: Non-communicable diseases, Group III: Injuries. Unknown or ill-defined CoDs which signs, symptoms and ill-defined conditions and senility were distributed to the causes within Group I and Group II: ill-defined cardiovascular CoDs distributed to within three groups proportionately. Age Standardized Mortality Rates (ASMRs) per 100,000 were calculated using European Standard Population 1976. Changes in mortality rates per 100 were calculated using the formula (the rate of last year of period - the rate of first year of period)/the rate of first year of period. Sex and age specific data were analyzed using the Joinpoint Software to examine trends and significant changes in trends of mortality rates. Results: Crude death rates per 100,000 for Group I, Group II and Group III in both sexes were 159.5, 133.7 and 11.0 in 1980: 128.5, 284.3 and 16.2 in 1990: 60.3, 319.1 and 12.6 in 2000: 24.5, 291.9, 20.7 in 2013. ASMRs for Group I, Group II and Group III were 142.2, 342.0, 18.4 in 1980: 127.6, 649.4, 21.1 in 1990: 68.3, 652.8, 16.1 in 2000: $28.9,387.6,23.1$ in 2013. The trends of rates were similar in both sexes. Deaths due to Group III were approximately two fold higher in male than in female. While deaths due to communicable diseases declined, non-communicable diseases and injuries increased over 33 years period. Change in ASMRs per 100 was found -79.7, 13.3 and 25.2 for Group I, Group II and Group III respectively between 1980 and 2013. The mortality rates of Group I for almost all age groups particularly under five years of age decreased significantly. Deaths due to Group III showed a dramatic increase in both sexes and age groups in 1999 when the big Marmara earthquake occurred.

Conclusion: This study clearly shows that Turkey is a country undergoing epidemiological transition. An analysis such as ours can identify details of epidemiological change, and hence provide evidence on which risk factors should be priorities for policy intervention.

\section{Abstract \#: P 118}

Vecas: a Dutch project to obtain valid data from electronic patient records for midwifery research

Hennie Wijnen $^{1}$, Bert Zeegers ${ }^{1}$

${ }^{1}$ Department of Midwifery Research, Maastricht; Corresponding author's e-mail: h.wijnen@av-m.nl

Evidence based medicine in Dutch midwifery practice is mainly based on research outside their domain and the availability of evidence is therefore frequently suboptimal. The domain of Dutch midwifery is the care of normality of the reproductive process. National and international research in obstetrics are mainly performed in mixed low and high risk or solely high risk populations which limits generalizability of evidence to the population of pregnant women under care of Dutch midwives. The Dutch obstetric care system is internationally well known for its philosophy of low- 
intervention and its high rate of home-births. The Dutch population is therefore ideal for research of the normality of pregnancy and childbirth. Dutch midwives register prenatal, natal and postnatal data of their care in different Electronic Patient Record (EPR) systems. Data are registered of every consultation which includes anamnestic information, physical examinations, ultrasounds, laboratory tests, data of decisions for referral, perinatal outcomes, etc. Data of the physiological course of the reproductive process are therefore theoretically available for research. Challenges to utilize these data for research include to understand and enhance the validity of the data and to extract and combine data from different EPR systems. To embrace these challenges the project Midwifery Caseregistration System (Verloskundig Casusregistratie Systeem $=\mathrm{VeCaS}$ ) was initiated. $\mathrm{VeCaS}$ realized a cooperation of 25 primary care midwifery practices throughout the Netherlands, creating a cumulative extraction of approximately 9000 cases annually. Six consensus meetings attended by at least one midwife of each midwifery practices resulted in a Consensus Manual, now used by all participating midwives to improve data validity. During the project software was developed to extract data from two EPR systems including a system for continuous recoding of data into a final general dataset. From 2012 onwards midwives started obtaining informed consent from pregnant women resulting in continues cumulation of research data. Biannual midwifery practices are visited by a researcher to talk through a summary of their own registered data. This gives feedback to their midwifery practices and to their quality of registration again to enhance data validity. $\mathrm{VeCaS}$ is committing itself to become a useful tool for research in the normality of pregnancy and childbirth and to contribute to evidence based medicine. The dataset of $\mathrm{VeCaS}$ is growing annually, and $\mathrm{VeCaS}$ hopes to grow in national and international partnerships in performing joined research.

\section{Abstract \#: P 119}

\section{Measuring disability in patients with cervical dystonia by applying the international classification of functioning and health}

Joke De Pauw $^{1}$, Kevin Van der Velden ${ }^{1}$, Patrick Cras ${ }^{2}$, Rudy Mercelis $^{2}$, Steven Truijen ${ }^{1}$, Willem De Hertogh ${ }^{1}$

${ }^{1}$ Department of Rehabilitation Sciences and Physiotherapy, University of Antwerp, Antwerp, Belgium; ${ }^{2}$ Faculty of Medicine and Health Sciences, University of Antwerp, University Hospital Antwerp, Born Bunge Institute Antwerp, Belgium; Corresponding author's e-mail: joke.depauw@uantwerpen.be

Background: Idiopathic Cervical Dystonia is a movement disorder, a focal dystonia, in which one or more muscles of the neck contract involuntary. This results in an abnormal head posture or involuntary movements of the neck and head. The impact of Cervical Dystonia on quality of life is well documented and numerous disease specific rating scales are available to measure its severity. It is however unclear how severe disability is perceived by patients with Cervical Dystonia and to what extent a patients' functioning is limited. Therefore, the aim is to describe the health status and severity of disability in Cervical Dystonia, using the International Classification of Functioning (ICF) as a neutral framework, proposed by the World Health Organization in 2001.

Methods: The Toronto Western Spasmodic Rating Scale (TWSTRS) and Cervical Impact Profile (CDIP-58) were administered to a sample of 30 patients with Cervical Dystonia. By linking the individual answers to the ICF model, the frequency and severity of reported impairments and restrictions was estimated using a count based method. This to calculate to what extent and how severe impairments and restrictions were present.

Results: Patients most frequently reported impairments linked to 'neuromusculoskeletal and movement-related functions' and 'mental functions', representing the characteristics of $\mathrm{CD}$ and the mental burden which is often accompanied. Most restrictions in activities were related to 'interpersonal interactions and relations', 'major life areas' and 'community, social and civic life'. All patients reported limitations in walking and more than $80 \%$ of the patients reported limitations of engaging recreational activities, socializing with other individuals or enjoying arts and culture. $60-86.7 \%$ of the patients reported problems in carrying out daily routine, personal hygiene or eating. Of the reported limitations in activities or participation, onethird can be labeled as severe disability. Implying that one-third of the activities can no longer be executed due to $\mathrm{CD}$, or that $\mathrm{CD}$ has a severe negative impact on the ability to execute functional routines or engage interpersonal relationships. No information on contextual factors is recorded in the TWSTRS and CDIP-58.

Conclusion: Notwithstanding that $\mathrm{CD}$ is a focal dystonia, impairments and restrictions on multiple levels of a patients' functioning in life appear to be highly prevalent. Since contextual factors are not incorporated in the TWSTRS and CDIP-58, these need to be evaluated separately.

\section{Abstract \#: P 120}

Development of the chariot research register for the prevention of Alzheimer's disease

Lefkos Middleton $^{1}$, Darina Bassil ${ }^{1}$, Mark Larsen ${ }^{2}$, Lisa Curry ${ }^{1}$, Nikolaos Mastellos ${ }^{2}$, Sonia Marquardt ${ }^{1}$, Catherine Robb ${ }^{1}$, Josip Car ${ }^{2}$

${ }^{1}$ Neuroepidemiology and Ageing Research Unit, School of Public Health, Imperial College London, London, United Kingdom; ${ }^{2}$ Global eHealth Unit, School of Public Health, Imperial College London, London, United Kingdom; Corresponding author's e-mail:

1.middleton@imperial.ac.uk

There is growing interest in defining and refining public health recommendations based on studies aiming at dementia prevention. Furthermore, as the target population for interventional trials for disease modifying drugs are shifting towards at risk pre-symptomatic individuals: detecting cognitively healthy individuals who are at high risk for developing dementia is now essential in observational and interventional research studies. Similar to other research studies, dementia studies in cognitively healthy individuals strongly rely on a high participation rate of a largely representative sample. Access to the population of interest poses a challenging issue, as dementia studies usually include participants recruited through secondary and tertiary services. The United Kingdom primary care system permits the recognition of a prodromal population preceding to the emerging of dementia symptoms and specialist referrals. We present the CHARIOT register, a primary care-based recruitment register developed for research into the prevention of dementia. West and central London general practice participant lists were identified and included into the study. Healthy participants, without a diagnosis of dementia, and between the age of 60 and 85 were invited to join the register via posted letters. Upon consent to join the register, minimal demographic and contact information was extracted for these individuals for the purpose of contacting them for future studies looking into the prevention of dementia.

123 surgeries participated in the register, recruiting a total of 24,509 participants - a response rate of $22.3 \%$. The age, gender and ethnicity profiles of participants closely match that of the overall eligible population. Higher response rates tended to be associated 
with larger practices $(\mathrm{r}=0.34)$, practices with a larger older population $(r=0.27)$, less socioeconomically disadvantaged practices $(\mathrm{r}=0.68)$, and practices with a higher proportion of White patients $(\mathrm{r}=0.82)$.

Findings from the Chariot register demonstrate a high interest rate and motivation for participation in research targeting the prevention of dementia. Furthermore, the results have been shown to be comparable to other registers reported in the literature, whilst providing a simple, feasible and low cost approach.

\section{Abstract \#: P 121 \\ Development of the Australian registry for chronic fatigue syndrome: applying new methods of e-research and mixed design in epidemiology \\ $\underline{\text { Samantha C. Johnston }}^{1}$ Ekua W. Brenu ${ }^{1}$, Don R Staines ${ }^{1}$, Sonya M. Marshall-Gradisnik ${ }^{1}$ \\ ${ }^{1}$ Griffith Health Institute, Griffith University, Gold Coast, Australia; Corresponding author's e-mail: \\ samantha.johnston3@griffithuni.edu.au}

Background: Chronic Fatigue Syndrome (CFS) is a highly debilitating illness of unknown cause. International prevalence studies, particularly in the United States and United Kingdom, have demonstrated that CFS has a significant public health and economic burden. Very little evidence is available on the impact of this illness in Australia despite its increasing attention in primary health care. The Australian Research Registry for CFS (ARRC) serves as the first database recording evidence on the impact of CFS at the patient, as well as public health level in Australia. As an often misdiagnosed condition, CFS is contrary to most chronic illness or disease in that the most clinically severe cases are underreported. Hence, traditional methods of extracting data from general practice databases may miss severely bedridden and housebound cases. Further, CFS may be relapsing in nature as patients can experience prolonged periods of remission and periods of severe exhaustion.

Methods: Participants are referred to the registry from participating general practices, university research programs and community based support networks across Australia. The registry has looked into innovations in e-research to enrol and collate data from participants. This includes the development of mobile device applications for patients to record and update their health service use, symptoms, medications and therapies, and nutrition longitudinally and further developing this towards biometric measurements. In addition to quantitative surveys, the registry collects supplementary data from structured interviews and clinical observations of those that attend a specialised clinic and blood analysis of those that donate to an ongoing immunological investigation on potential biomarkers for CFS.

Results: Since August 2012, 980 potential cases of CFS have enrolled in the registry. This has provided the opportunity to examine the relationship between epidemiological, clinical, and biological data. Accordingly, several papers on the registry's preliminary findings have been published on emerging patterns found in this patient group. This includes the identification of a more severely impaired clinical group, identification of further immunological anomalies, and environmental versus non-environmental triggers for the illness.

Conclusion: As a university based research registry, the ARRC operates at a smaller scale to government public health based registries: however its integrated data has been a key approach to determining epidemiological characteristics, as well as the potential aetiology, pathomechanism and management of this illness through a mixed design approach.

\section{Abstract \#: P 122}

Early childhood infections with rotavirus and norovirus and the risk of developing asthma and atopic manifestations

Dianne de Korte-de Boer ${ }^{1}$, Monique Mommers ${ }^{1}$, Melanie Ogunyena ${ }^{1}$, John Penders ${ }^{2}$, Johan Reimerink ${ }^{3}$, Foekje Stelma ${ }^{4}$, Marion Koopmans ${ }^{3,5}$, Carel Thijs ${ }^{1}$

${ }^{1}$ Dept. of Epidemiology, School for Public Health and Primary Care (Caphri), Maastricht University, Maastricht, the Netherlands; ${ }^{2}$ Dept. of Medical Microbiology, School for Public Health and Primary Care (Caphri) and School for Nutrition and Translational Research in Medicine (NUTRIM), University Hospital Maastricht, Maastricht, the Netherlands; ${ }^{3}$ The Laboratory for Infectious Diseases and Screening, National Institute of Public Health and the Environment (RIVM), Bilthoven, The Netherlands; ${ }^{4}$ Radboud University Medical Centre (UMC), Department of Medical Microbiology, Nijmegen,

The Netherlands; ${ }^{5}$ Department of Viroscience, Erasmus Medical Centre, Rotterdam, the Netherlands; Corresponding author's e-mail: monique.mommers@maastrichtuniversity.nl

Background: According to the 'hygiene hypothesis' early viral infections protect children against asthma and atopic manifestations. In previous work we investigated gastrointestinal infections in the KOALA cohort and found an unexpected positive association between rotavirus and wheeze. We now present results of longer follow-up, and including asthma as outcome measure.

Aim: To investigate whether infection with gastrointestinal viruses during infancy is associated with the development of childhood asthma and related atopic manifestations.

Methods: In 591 children from the KOALA Birth Cohort Study, IgG serology for rotavirus and norovirus (GGII.4 and GGI.1) was determined at age 1 year and gastrointestinal symptoms were assessed by repeated questionnaires until age 1 year. Associations with childhood asthma, wheeze and eczema between 2 and 9 years of age, and allergic sensitisation (specific IgE against eight common allergens) at ages 2 and 6-7 years were analysed using multivariable logistic regression and Generalized Estimating Equations. Multivariable models were adjusted for sex, recruitment group, parental history of atopy, season of birth, mode and place of delivery, breastfeeding duration, daycare, and for rotavirus, norovirus GGII.4 and/or norovirus GGI.1, respectively.

Results: Children seropositive for rotavirus had an increased risk for wheeze until age 9 years (adjusted odds ratio 1.63: $95 \%$ CI 1.08-2.48), with the highest risk observed in 2-year-old (4.49: 2.33-8.64) and 4-year-old (3.02: 1.66-5.50) children with reported gastrointestinal symptoms. After the age of 5 years, the association with wheeze was no longer statistically significant. Rotavirus seropositivity was not associated with asthma, allergic sensitisation, or eczema.

Norovirus GGII.4 seropositivity at age 1 year was associated with a decreased asthma risk until age 9 years $(0.35$ : $0.16-0.77)$ and norovirus GGI.1 seropositivity was associated with decreased risk of allergic sensitisation at the age of 6-7 years (0.50: $0.25-1.00)$, but not at age 2 years (1.17: 0.64-2.15).

Conclusion: Early life rotavirus and norovirus infections are associated with wheeze and asthma development, respectively, but in opposite directions. Symptomatic rotavirus infection might reveal an underlying general susceptibility to wheeze or may be a causal factor for wheeze if viremia occurs. Norovirus infection might protect against allergic sensitisation and asthma development. 


\section{Abstract \#: P 123}

\section{Are people with HIV/AIDS (PLWHAS) taking adequate} precautions/self care practices - a real cause for concern

\section{Dr Sandhya Ghodke ${ }^{1,2}$, Dr R.N. Hiremath ${ }^{2}$}

${ }^{1} \mathrm{MO} ;{ }^{2} \mathrm{Govt}$; Corresponding author's e-mail:

sandhyaghodke@yahoo.com

Introduction: In the absence of a complete cure for HIV/AIDS, proper maintenance of health and self-care is essential for people living with HIV/AIDs (PLWHAs) for not only prolonging the life but also quality implied in it. This can be achieved only when PLWHAs have appropriate knowledge regarding self-care.

Materials and Methods: A cross-sectional Hospital based survey was carried out in tertiary care centre and all the HIV positive persons who were not on ART and who consented were included in the study. Results: The mean age was 35.55 years +7.44 years. $58.8 \%$ were educated up-to class $\mathrm{X}$ while $35.3 \%$ were intermediate and $3.9 \%$ were graduate. When asked about basic parameters of self-care, majority $99 \%(101)$ felt that good diet is very much essential in order to prevent progression of the disease while others felt that good hygiene sanitation $(44,43.1 \%)$, yoga and meditation $(43,42.2 \%)$, good compliance to treatment $(43,42.2 \%)$ and follow up $(2.2 \%)$ are essential in order to be able to lead healthful life. $79.4 \%$ were having satisfactory knowledge about kind of diet to be taken. Similarly 61.8 , $45.1,28.4,19.6,11.8$, and $11.8 \%$ were found to have satisfactory knowledge regarding self-care to be taken during fever, pain/headache, diarrhea, chronic cough, skin problems and nausea/vomiting respectively. Only $37.3 \%$ were aware of precautions to be taken while preparing food. As per predetermined knowledge score using all studied parameters to assess the overall level of knowledge regarding self-care, it was seen that only $22.5 \%$ had satisfactory knowledge regarding self-care. $11.6 \%$ of the respondents with HIV positivity of $<24$ months had satisfactory knowledge about self-care than compared to $30.5 \%$ of the respondents with $\geq 24$ months duration. The relation between duration of HIV positivity and Knowledge about self-care was statistically significant $(p$ value $=0.02)$

Conclusion: Very low percentage of respondents had satisfactory knowledge regarding self-care which is again a matter of concern.

\section{Abstract \#: P 124}

\section{IL28B polymorphism in a cohort of $\mathrm{HCV}$ infected patients} in Rome and its association with HCV genotypes and liver fibrosis

Nosotti Lorenzo ${ }^{1}$, Petrelli Alessio ${ }^{1}$, Genovese Domenico ${ }^{2}$, Argentini Claudio $^{2}$, Vella Stefano ${ }^{2}$, Rossi Alessandra ${ }^{1}$, Miglioresi Lucia ${ }^{1}$, Costanzo Gianfranco ${ }^{1}$, Fortino Antonio ${ }^{1}$, Chessa Luchino ${ }^{3}$, Mirisola Concetta ${ }^{1}$

${ }^{1}$ National Institute for Health, Migration and Poverty (NIHMP); ${ }^{2}$ National Institute for Health (NIH); ${ }^{3}$ University of Cagliari; Corresponding author's e-mail: nosotti@inmp.it

Background: Different factors associated with hepatitis $\mathrm{C}$ virus $(\mathrm{HCV})$ or with the host may predict the outcome of chronic patients under treatment. Virus genotype, viral load, gender, age, BMI, fibrosis staging and ethnic group have been used for years in order to identify patients to be treated. Recently, attention was given to another factor, single nucleotide polymorphism (SNPs) in the interleukin 28B gene (IL28B). IL28B CC was associated with virus spontaneous clearance and with sustained virological response (SVR) to antiviral therapy. Aims of the study are: 1)to assess the prevalence of IL28B genotypes and the distribution of HCV genotypes in a cohort of immigrant and Italian patients: 2) to investigate the prevalence of IL28B favourable genotype (CC) in genotypes HCV 1 and HCV4: 3) to verify the role of IL28B polymorphism as a predictor of SVR: 4)to study the relationship between the severity of liver fibrosis and the IL28B polymorphism.

Methods: 175 patients with chronic HCV infection were observed between 2013 and 2014. For each patient, demographical, virological, biochemical, and genetic characteristics were investigated. Liver fibrosis staging was assessed by transient elastometry.

Results: The mean age of the patients $(71.7 \%$ males, $28.3 \%$ females) is 44 years: $68.6 \%$ of individuals were from Europe, $20.6 \%$ from Africa, $5.7 \%$ from America and $5.1 \%$ from Asia. HCV genotypes are distributed as follows: HCV4 in $55.2 \%$ of patients, $\mathrm{HCV} 1$ in $30.7 \%$, HCV3 in $12.3 \%$, HCV2 in $1.8 \%$. IL28B polymorphism prevalence is the following: $\mathrm{CC}$ in $24.7 \%$ of patients, CT in $58.8 \%$, TT in $16.5 \%$. IL28B genotypes frequencies do not appear to be associated with ethnic origin. HCV1 and HCV4 genotypes were observed in 140 patients. They had a similar prevalence of genotype IL28B CC (53.1\% and $57.8 \%$ respectively). In 140 patients with HCV1 and HCV4, IL28B CC increases the chances of SVR: in the same patients, we cannot observe a significant statistical association between IL28B CC and liver fibrosis.

Conclusions: In our cohort, the HCV 4 genotype is the most frequently observed, with a higher prevalence compared to that described in literature in Italy (55.2 versus $10 \%)$. IL28B plymorphism does not show significant differences among ethnic groups and in HCV1 and HCV4 genotypes. As described in literature, IL28B CC genotype is confirmed as predictor of SVR in both Caucasians and Africans. In patients with HCV1 and HCV4 no statistically significant association was observed between IL28B polymorphism and liver fibrosis.

\section{Abstract \#: P 125}

Mapping leprosy morbidity in municipalities of an endemic area in Brazil, 2009-2011

Leila Posenato Garcia ${ }^{1}$, Gabriela Drummond Marques da Silva ${ }^{2}$, Lúcia Rolim Santana de Freitas $^{2}$

${ }^{1}$ Instituto de Pesquisa Econômica Aplicada; ${ }^{2}$ Universidade de Brasília; Corresponding author's e-mail: gabidrum@yahoo.com.br

Background: Leprosy is an important public health problem in Brazil, despite the economic growth and the improvement in access to health services. The disease is heterogeneously distributed across the country, affecting mainly the poorest regions and populations. This study aimed to estimate and to map the leprosy incidence rate in municipalities of an endemic area in Brazil.

Methods: This is a echological study with data from the National Notifiable Diseases Information System (SINAN) regarding the 2708 municipalities located in the North, Northeast and Midwest regions of Brazil, in 2009-2011. In order to reduce the random fluctuation of the estimates, we used a spatial hierarchical Bayesian model, estimated through Integrated Nested Laplace Approximation (INLA). Model adjustment was obtained with the addition of covariates related to the municipalities' socioeconomic characteristics and to the performance of the epidemiological surveillance.

Results: In 2009-2011, 85,592 cases of leprosy were reported in 2271 municipalities ( $84 \%$ of the municipalities in the study area). Before the adjustment, the minimum annual incidence rate was zero and the maximum was 330.5 cases per 100,000 inhabitants (in a municipality with 3197 inhabitants). The final model included as covariates the percentage of household contacts investigated and the poverty rate, in 
addition to the spatial and non-spatial random effects. The adjusted correlation with leprosy incidence rate was positive for the percentage of household contacts investigated and the percentage of urban population, although it is negative for the poverty rate. After adjustment, 84 municipalities, mainly located at the states of Tocantins $(n=27)$, Goiás $(n=16)$ and Mato Grosso $(n=15)$, have become hyperendemic (incidence rate $>40$ cases per 100,000 inhabitants). Also, 58 municipalities were no longer considered hyperendemic, most of them located in the Northeast region of the country $(n=41)$.

Conclusion: The incidence rate estimation using a Bayesian model resulted in a clearer spatial pattern, highlighting the higher morbidity in the states of Mato Grosso, Tocantins and Rondônia, located upcountry. The results at the municipality level are useful to indicate areas with very higher incidence rates after data modeling, where active search for leprosy cases should be prioritized. The response variable's negative correlation with poverty rate and its positive correlation with the percentage of household contacts investigated provide evidence of underreporting of cases.

\section{Abstract \#: P 126}

Comparison of the effects of online and pen-and-paper participation on study responses in a large panel in Lower Saxony, Germany

Nicole Rübsamen ${ }^{1}$, André Karch ${ }^{1}$, Beate Zoch $^{1}$, Rafael T. Mikolajczyk ${ }^{1}$

${ }^{1}$ Helmholtz Centre for Infection Research, Braunschweig, Germany; Corresponding author's e-mail: Nicole.Ruebsamen@helmholtz-hzi.de

Background: The widespread use of the Internet and the familiarity with it allow researchers to apply web-based questionnaires in surveys or even longitudinal panels. However, there is little information whether participants in web-based studies differ from participants in studies using pen-and-paper questionnaires with respect to their sociodemographic characteristics and to their responses in the questionnaires.

Methods: We invited a random sample of 27,000 adults aged 15-69 years from four districts of the federal state of Lower Saxony, Germany to participate in the longitudinal panel "Hygiene and Behaviour Infectious Diseases Study" (HaBIDS). Participants could choose to participate either by filling in pen-and-paper or web-based questionnaires. We compared the two groups of participants with respect to their socio-demographic characteristics. In addition, we compared responses between the groups based on exemplary items (frequency of upper respiratory infections in the past 12 months and prevention of infections by washing hands) using direct standardization (population of Lower Saxony according to age and sex as standard population).

Results: A total of 2380 persons participated in the study. Participants in the online group $(n=1444)$ were younger than in the pen-andpaper group $(\mathrm{n}=936)$ and were more likely to have an academic degree. In the online group, $25.5 \%$ of the participants stated to have had more than two upper respiratory infections in the past 12 months while $32.6 \%$ in the pen-and-paper group did so. Standardization changed these percentages to $25.0 \%$ and $36.0 \%$, respectively. With respect to hand washing as a prevention measure against upper respiratory infections, $91.4 \%$ in the online group and $93.4 \%$ in the penand-paper group stated to wash hands several times a day. After standardization, the percentages were $88.6 \%$ in the online group and $89.8 \%$ in the pen-and-paper group.

Conclusion: The characteristics of the participants in the HaBIDS study varied dependent on survey mode. The frequency of upper respiratory infections between both groups differed, too. As age standardization did not change the observed difference in frequency, it is likely that participants who chose online participation are different from those choosing pen-and-paper with respect to other factors influencing the risk of getting infected. However, this finding might not be a universal tendency for other outcomes.

\section{Abstract \#: P 127}

Systematic literature review on the incubation period and period of infectiousness/shedding of child infectious diseases

Mariken J. Tijhuis ${ }^{1}$, Rui $\mathrm{Cai}^{1}$, Marije Vonk Noordegraaf-Schouten ${ }^{1}$, Ida Czumbel ${ }^{2}$

${ }^{1}$ Pallas health research and consultancy, Rotterdam, the Netherlands; ${ }^{2}$ European Centre for Disease Prevention and Co; Corresponding author's e-mail: tijhuis@pallashrc.com

Background: Currently, there is no common EU-approach to the control of communicable diseases in school and other child care settings, e.g. by exclusion policies. To support the development of such an approach based on the best available relevant scientific information, we performed a systematic literature review for the European Centre for Disease Prevention and Control on three key parameters: 1. incubation period: 2. period of infectiousness and/or the duration of shedding: and 3. exclusion period and its effectiveness, for 30 of the most common childhood infections or those with a particular concern.

Methods: We performed a search (search date: August 11th 2014) in PubMed using terms for A. the selected infections: B. children aged 1 month to 18 years: C. incubation period: D. period of infectiousness or shedding: E. setting-specific exclusion period: F. studies investigating humans. No language or time restrictions were applied. The search identified approximately 3100 references, which were first screened for relevance by title and abstract: those selected were further screened full text for eligibility with respect to age, study design, confounding or modifying factors and outcome, and for basic quality issues. In addition, full text articles were extensively screened for relevant references. Besides the information from scientific journal articles, we also searched websites of key institutions and relevant handbooks for the key parameters.

Results: In total, 500 articles were screened full text and 75 articles with relevant information were included in the review. In addition, information was included from 2 websites (CDC, WHO), one handbook (AAP Red Book) and an evidence base from 2001 on the same topic. In general, the review showed: 1 . an unexpected paucity in the reporting of literature sources for the key parameters in all investigated data sources: 2. an unexpected scarcity in literature fitting the eligibility criteria for the key parameters: 3 . diversity in study characteristics, such as population age, (a)symptomatology, treatment, vaccination, diagnostic tools, viral load, study design and (reporting of) key definitions. To broaden the information base, the search terms may be expanded in PubMed, the inclusion criteria may be expanded for some parameters and design types, e.g. to lower evidence designs as case series and modelling studies, and by searching a second database.

Conclusion: This review may serve as a building block for the development of a document on exclusion and other strategies to control the spread of infectious diseases in day care and school settings. 


\section{Abstract \#: P 128}

\section{Application of predictive models in early detection of bacterial} meningitis in Iran: comparison of three selected algorithms

Atiehsadat Mirkhani ${ }^{1}$, Jamal Mirzaie ${ }^{2}$, Mahmoud Nabavi ${ }^{3}$, Mohammad Tavakkoli ${ }^{4}$, Omid Pournik ${ }^{5}$

${ }^{1}$ Faculty of Biomedical Engineering, Amirkabir University of Technology, Tehran, Iran; ${ }^{2}$ Hepatitis Research Center, Department of Internal Medicine, Faculty of Medicine, Birjand University of Medical Sciences, Birjand, Iran,; ${ }^{3}$ Imam Hossein hospital, Shaheed Beheshti medical university, Tehran, Iran; ${ }^{4}$ Department of Global Health and Population, Harvard School of Public Health, Boston, US; ${ }^{5}$ Medical Informatics Department, Faculty of Medicine, Iran University of Medical Sciences, Tehran, Iran; Corresponding author's e-mail: mirkhania461@yahoo.com

Bacterial meningitis is a dangerous infectious disease epidemics of which can influence an entire community. Using data mining and machine learning techniques to create predictive models could facilitate early detection of outbreaks and prevent bacterial meningitis epidemics. The object of this study is to screen bacterial meningitis from non-bacterial meningitis in early phases. This study was conducted using the standard data mining and knowledge discovery methodology Cross Industry Standard Process for Data Mining CRISP-DM on the national meningitis registry of the Iranian Center for Disease Control and Prevention (ICDCP), including 7475 cases of meningitis in 2009 and 2010. The data was partitioned into the training class $(70 \%)$ to make the model and the test class $(30 \%)$ to evaluate the model. The modeling was performed using probabilistic neural network (PNN), self-organizing tree algorithm (SOTA) and naïve Bayes algorithms. Finally, the models were compared to each other in terms of their sensitivity accuracy. The PNN was shown to be the best predictor with a sensitivity of $99.9 \%$ and an accuracy of $93.7 \%$ while naïve Bayesian ranked second with $99.7 \%$ sensitivity and $93.1 \%$ accuracy, SOTA turned out to be the weakest with $94.7 \%$ sensitivity and $88.8 \%$ accuracy. The comparative study of multiple prediction models for early detection of bacterial meningitis using a national-level dataset yielded sensitivities higher than $90 \%$. As a conclusion, the purpose of this study was to develop the predictive models that can screen bacterial meningitis from non-bacterial meningitis in early phases with maximum sensitivity and accuracy to support public health decision making in shortest time. As a result, it is understood that the model developed by PNN algorithm is the most suitable model for screening.

\section{Abstract \#: P 129}

\section{What is the optimal time to retest STI clinic patients} with a urogenital chlamydia infection?

Jannie J. van der Helm ${ }^{1}$, Rik H. Koekenbier ${ }^{1}$, Martijn S. van

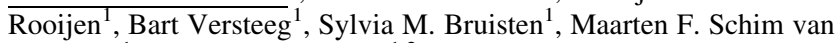
der Loeff ${ }^{1}$, Henry J.C. de Vries ${ }^{1,2}$

${ }^{1}$ Public Health Service Amsterdam, Amsterdam, the Netherlands;

${ }^{2}$ Academic Medical Center, Amsterdam, the Netherlands;

Corresponding author's e-mail: jvdhelm@ggd.amsterdam.nl

Background: Chlamydia trachomatis $(\mathrm{Ct})$ is a common and often recurring sexually transmitted infection. Retesting can be an effective strategy to prevent onward transmission and late sequellae. However, the recommendation of when to retest varies widely by current guidelines. Therefore, we assessed the optimal time to retest following treatment of urogenital $\mathrm{Ct}$.
Methods: Included in the study were heterosexual clients of the Amsterdam STI outpatient clinic who were positive for urogenital Ct by a nucleic acid amplification test (NAAT). After diagnosis, treatment and counselling, they were offered retesting for Ct. Participants were randomly assigned for retesting after 2,4 or 6 months. Participants could choose between two retest options: collect a sample (urine for men, vaginal swab for women) at home or return to the clinic, in both cases using the same type of self-collection kit. Participation and $\mathrm{Ct}$ positivity rates at follow-up of individuals randomized to the 3 retest intervals were calculated. An intention-to-treat analysis was performed using follow-up data until 8 months after the recruitment visit.

Results: Between May 2012 and March 2013, 2257 individuals were included ( $45 \%$ were male, median age 23 years [IQR 21-26]). The participation rate of retesting was significantly higher in the 2-months-group $(65.8 \%)$ compared to the $4-(50.5 \%)$ and 6-monthsgroup ( $40.9 \%, p<0.001)$ while the positivity rate was comparable between the three groups $(9 \%, p=0.859)$. Retesting more than 1 week before the scheduled retest moment occurred significantly more often in the 6-month group (20\%) compared to the 2-monthsgroup $(5 \%, p<0.001)$, however the positivity rate was significantly higher in the 2 -months group ( $36 \%$ vs $21 \%, p=0.026$ ). The intention-to-treat analysis showed that the return rate within 8 months after the initial visit (regardless of timing) was significantly higher in the 2-months-group (76.7\%) compared to the 6-months-group $(63.8 \%, p<0.001)$ : however the positivity rates were comparable $(p=0.998)$.

Conclusion: We recommend to offer retesting to STI clinic patients not later than 4 months following an initial diagnosis of urogenital $\mathrm{Ct}$. The participation rate was higher when earlier retesting was offered and positivity rates were comparable. Moreover, $20 \%$ of individuals scheduled in the 6 month group re-tested $>1$ week before their scheduled retest moment, indicating that a 6 month interval is too long.

\section{Abstract \#: P 130}

Analysis of the epidemiological situation of leprosy in an endemic area in Brazil: spatial distribution, 2001-2012

\section{Lucia R. S. Freitas $^{1}$, Leila P. Garcia ${ }^{2}$, Elisabeth C. Duarte ${ }^{3}$}

${ }^{1}$ Programa de Pós-Graduação em Medicina Tropical, Universidade de Brasília - UnB, Brasília - DF, Brazil; ${ }^{2}$ Instituto de Pesquisa Econômica Aplicada - Ipea, Brasília - DF, BraZil; ${ }^{3}$ Faculdade de Medicina, Universidade de Brasília - UnB, Brasília - DF, Brazil; Corresponding author's e-mail: lucia.rolim@gmail.com

Background: In Brazil, the spatial distribution of leprosy is heterogeneous: the most socioeconomically developed states in the Southern region achieved the goal of elimination of leprosy as a public health problem. However, areas with high transmission remain in the Northern, Center-Western and Northeastern regions of the country. This study objective is to describe the spatial distribution of leprosy in Brazilian municipalities with high risk of transmission in the period 2001-2012.

Methods: This is an ecological study with data from the Brazilian Notifiable Diseases Information System (SINAN). Were included 692 municipalities located at states of Mato Grosso, Tocantins, Rondônia, Pará and Maranhão. The following leprosy indicators were calculated per 100,000 inhabitants: incidence rate, incidence rate in under 15 years-old, and the rate of new cases with grade 2 disabilities. The spatial scan statistic was used to detect significant clusters $(p \leq 0.05)$ within the study area, in the periods 2001-2003 and 2010-2012. The 
clusters were identified using purely spatial analysis with a bandwidth of up to $100 \mathrm{~km}$.

Results: In the period 2001-2012, 176,929 cases of leprosy were reported in the study area, equivalent to $34.6 \%$ of all cases in Brazil. In the period 2001-2003, the spatial scan statistic identified 44 significant clusters for leprosy incidence rate. Of these, 30 are located in the states of Pará (12) and Mato Grosso (18). In the period 2010-2012, the scan spatial statistics identified 42 significant clusters for leprosy incidence rate. Of these, 28 are located in the states of Pará (11) and Mato Grosso (17). In the period 2001-2003, 20 significant clusters were identified for the incidence rate of leprosy in under 15 years-old, 7 in the state of Pará. In the period 2010-2012, the scan spatial statistics identified 14 significant clusters for the incidence rate in under 15 years-old. For the rate of new cases with grade-2 disabilities, the scan spatial statistics identified 19 significant clusters in the period 2001-2003, 7 of them located in the state of Pará, and 14 significant clusters in the period 2010-2012, with homogeneous distribution between the five Brazilian states belonging to the studied aggregate.

Conclusion: Despite the reduction in the detection of leprosy cases, there is a need to step up disease control actions, especially in the identified clusters, in order to achieve the goal of eliminating the disease as a public health problem in Brazil.

\section{Abstract \#: P 131}

Determining the most likely source of infection: an application to Neisseria gonorrhoeae infection among men who have sex with men

\section{Amy Matser ${ }^{1}$, Maarten Schim van der Loeff ${ }^{1}$, Ronald Geskus ${ }^{1}$}

${ }^{1}$ Dep. of Infectious Diseases, GGD Amsterdam; Corresponding author's e-mail: amatser@ggd.amsterdam.nl

Introduction: For public health purposes it is useful to know from which person and where (e.g. at home, in clubs or in public transport) most infections are acquired. To inform directed prevention measures, we developed a method to estimate from whom an infection was acquired when there are multiple possible sources. We estimated the infection risk of Neisseria gonorrhoeae (NG) among men who have sex with men (MSM) per meeting location.

Methods: In 2008-2009, we collected information from 2983 MSM attending the Sexually Transmitted Infections clinic of Amsterdam. For $\leq 4$ partners per participant (8028 in total), meeting location, partner, and partnership characteristics were asked. Meeting location was categorised into 16 categorizes: 6 locations in Amsterdam, 5 websites, the Netherlands, abroad, and steady and casual long-lasting partners with non-relevant meeting locations, and other. We used logistic regression to relate infection risk per partnership to participant and partner characteristics and meeting location. In the likelihood we accounted for partially unobserved transmission information per partnership. We predicted the probability of a partner having NG. Partnerships were assumed to be the source based on the relative size of the probabilities. Each source was linked to a meeting location. We used a Bayesian method. The statistical properties of the method were assessed by a simulation study.

Results: Rectal NG was diagnosed in 157 MSM who reported data on 422 possible sources, urethral NG in 126 reporting 285 relevant partners, and pharyngeal NG in 162 reporting 451 relevant partners. We estimated that most infections were acquired from long-lasting steady partners (21.5:95\% 18.0-25.2\%), but MSM had the highest infection risk at a street in Amsterdam where gay venues are located (4.2\%: $95 \%$ CI 2.4-5.9\%). In the simulation study, the distribution of infections and the probabilities of infection per meeting location were close, but not always similar to the theoretical values: there was a small bias.

Conclusions: The presented method estimates the source of infection when there are multiple possible sources and enables the summation over all kinds of epidemiological characteristics (here meeting locations) that are relevant for prevention. The presented method can be used for all types of infections, including STIs and airborne infections.

\section{Abstract \#: P 132}

Trends of the main epidemiological indicators of leprosy in an endemic area in Brazil, 2001-2012

$\underline{\text { Lucia R. S. Freitas }}^{1}$, Leila P. Garcia ${ }^{2}$, Elisabeth C. Duarte ${ }^{3}$

${ }^{1}$ Programa de Pós-Graduação em Medicina Tropical, Universidade de Brasília - UnB, Brasília - DF, Brazil; ${ }^{2}$ Instituto de Pesquisa

Econômica Aplicada - Ipea, Brasília - DF, Brazil; ${ }^{3}$ Faculdade de Medicina, Universidade de Brasília - UnB, Brasília - DF, Brazil; Corresponding author's e-mail: lucia.rolim@gmail.com

Background: In Brazil, leprosy incidence has declined in recent years. However, the incidence of cases with visible deformities showed to be stable over time. This study objective was to investigate the trends of the main indicators of leprosy in Brazilian municipalities with high risk of transmission in the period 2001-2012.

Methods: This is an ecological time-series study with data from the National Notifiable Diseases Information System (SINAN). We included all 692 Brazilian municipalities located in the states of Mato Grosso, Tocantins, Rondônia, Pará and Maranhão. The following leprosy indicators were calculated per 100,000 inhabitants: incidence rate, incidence rate in under 15 years-old, and rate of new cases with grade-2 disabilities. Maps were generated to show the percentage change in the incidence of leprosy between the periods 2001-2003 and 2010-2012. Joinpoint log-linear regression was applied to identify yearly trends. For the rate of new cases with grade- 2 disabilities, in 2007, the Arima model was used to value imputation.

Results: In the period 2001-2012, 176,929 cases of leprosy were reported in the study area, equivalent to $34.6 \%$ of all cases of Brazil, distributed in only $10 \%$ of the population. Regarding incidence rate, in 2001-2003, $404(58.4 \%)$ municipalities in the study area were considered hyperendemic (incidence rate $\geq 40$ per 100,000). Of these municipalities, comparing both periods, $63(9.1 \%)$ endemic municipalities showed an over $20 \%$ increase in the incidence rate. Regarding the incidence rate in under 15, in 2001-2003, $362(52.3 \%)$ municipalities were considered hyperendemic (incidence $\geq 10$ per 100,000). Of these municipalities, comparing both periods, $58(8.4 \%)$ of those showed an over $20 \%$ increase. In the aggregate of municipalities, there was a reduction of leprosy incidence rate of 89.10 new cases in 2001 to 56.98 new cases per 100,000 inhabitants in 2012 . This rate decreased significantly in the period 2003-2012 (APC: $-6.2 \%, 95 \% \mathrm{CI}-7.2$ to $-5.2 \%)$. Among under 15 , the rate also showed a significant reduction (APC: $-5.6 \%$ : $95 \% \mathrm{CI}-7.2$ to $-4.1 \%$ ) in the period 2003-2012. However, the rate of new cases with grade- 2 disabilities was stable in the twelve years period (APC: $-1.1 \%$ : $95 \% \mathrm{CI}-3.5$ to $-1.3 \%$ ).

Conclusion: Despite the reduction in leprosy incidence rate, there is a need to improve control strategies to identify cases with grade-2 disabilities, especially in hyperendemic municipalities of the aggregate, with the objective of prevent deformities and reduce the burden of disease. 


\section{Abstract \#: P 133}

\section{Maternal and paternal indoor or outdoor smoking and the risk} of asthma in their children

Akahiro Tabuchi ${ }^{1}$, Takeo Fujiwara ${ }^{2}$, Tomio Nakayama ${ }^{1}$, Isao Miyashiro ${ }^{1}$, Hideaki Tsukuma ${ }^{1}$, Koken Ozaki ${ }^{3}$, Naoki Kondo ${ }^{4}$

${ }^{1}$ Center for Cancer Control and Statistics, Osaka Medical Center for Cancer and Cardiovascular Diseases, 3-3, Nakamichi 1-chome, Higashinari-ku, Osaka 537-8511, Japan; ${ }^{2}$ Department of Social Medicine, National Research Institute for Child Health and Development, 2-10-1, Okura, Setagaya-ku, Tokyo 157-8535, Japan; ${ }^{3}$ Graduate School of Business Sciences, University of Tsukuba, 3-291, Otsuka, Bunkyo-ku, Tokyo 112-0012, Japan; ${ }^{4}$ School of Public Health, The University of Tokyo, 7-3-1, Hongo, Bunkyo-ku, Tokyo 113-0033, Japan; Corresponding author's e-mail: tabuchitak@gmail.com

Background: Little is known about the differential impact of combinations of parental smoking behavior (indoor or outdoor smoking, or not smoking) on preventing childhood asthma. Our objective was to examine the association between parental smoking behavior and children's asthma.

Methods: A nationally representative population-based birth cohort of 40,580 babies, aged 0.5 years in 2001 (response rate, $87.8 \%$ ), was studied to estimate adjusted odds ratios of combinations of maternal and paternal indoor or outdoor smoking at home for physician visits and hospitalization for childhood asthma up to 8-years-old, and population attributable fractions.

Results: Odds of hospitalization for asthma among children whose father alone smokes indoors at home did not largely increase (up to $20 \%$ ). However, if the mother also smokes indoors at home, the odds strongly increased. After adjusting for demographic, perinatal and socioeconomic factors, the increase in odds for children whose father and mother both smoke indoors compared to children with nonsmoking parents was $54 \%$ (95\% confidence interval 21-96\%), $43 \%(8-90 \%)$ and $72 \%(22-143 \%)$ for children aged $0.5<-2.5$, $2.5<-4.5$ and $4.5<-8$ years-old, respectively. the odds ratios of smoking outdoors did not largely differ from those indoors. our estimation population attributable fractions revealed that if all parents in japan quit smoking, hospitalization children for asthma could be reduced by $8.3 \%(2.2-14.3 \%), \quad 9.3 \% \quad(0.9-17.6 \%) \quad 18.2 \%$ (7.7-28.8\%),

Conclusions: parental indoor at home increased exacerbated children's asthma. home, whether it is or outdoor, may increase risks attacks their children.

\section{Abstract \#: P 134}

The effects of lutein on respiratory health across the life course: a systematic review

Debora, Melo van Lent ${ }^{1}$, Elisabeth T.M., Leermakers ${ }^{1}$, Sirwan K. L., $\overline{\text { Darweesh }}{ }^{1}$, Eduardo M., Moreira ${ }^{1}$, Myrte J., Tielemans ${ }^{1}$, Taulant, Muka $^{1}$, Anna, Vitezova ${ }^{1}$, Rajiv, Chowdhury ${ }^{2}$, Wichor M., Bramer ${ }^{3}$, Janine F., Felix ${ }^{1}$, Guy G., Brusselle ${ }^{4}$, Jessica C., Kiefte ${ }^{1}$

${ }^{1}$ Department of Epidemiology, Erasmus MC, Rotterdam, the Netherlands; ${ }^{2}$ Department of Public Health \& Primary Care, Cardiovascular Epidemiology Unit, University of Cambridge, Cambridge, United Kingdom; ${ }^{3}$ Medical Library, Erasmus MC, University Medical Center Rotterdam, the Netherlands; ${ }^{4}$ Department of Respiratory Medicine, Erasmus MC Rotterdam, the Netherlands; Corresponding author's e-mail: d.melovanlent@erasmusmc.nl
Background: Lutein, a fat-soluble carotenoid present in green leafy vegetables and eggs, has strong antioxidant properties and could therefore be important for respiratory health.

Methods: We systematically reviewed literature for articles that evaluated associations of lutein (dietary intake, supplements or blood levels) with respiratory outcomes, published in Medline, Embase, Cochrane Central, PubMed, Web of Science and Google Scholar, up to August 2014.

Results: We identified 13 studies (one trial, five longitudinal and seven cross-sectional studies). In children: two studies reported on bronchopulmonary dysplasia (BPD) and four on asthma. In adults: one reported on asthma, five on respiratory function and one on respiratory mortality.

For BPD, the longitudinal observational study found no difference in plasma lutein levels between neonates who developed BPD and controls. The intervention study found no significant effect of lutein/ zeaxanthin supplementation in neonates $(140 \mathrm{mcg}$ lutein/ $0.6 \mathrm{mcg}$ zeaxanthin daily for 36 weeks) on lowering risk of BPD (OR 0.43 (95 $\%$ CI $0.15: 1.17$ ) versus placebo), but this study was not sufficiently powered.

For asthma, one longitudinal and three cross-sectional studies in children found lower lutein intake or blood levels in asthma patients as compared to controls, but this association disappeared after adjustment for sociodemographic and lifestyle variables.

In adults, a case-control study also reported lower lutein blood levels in asthma cases versus controls, but no adjustment for potential confounders was made.

For respiratory function (FEV1, FVC and FEV1/FVC-ratio), three out of five observational studies, all in adults, found small, but significant, beneficial effects of lutein. One cross-sectional study in patients with chronic airflow limitation found both intake and levels of lutein to be positively associated with increases in FEV1, FVC and FEV1/FVC-ratio. One longitudinal study in adults from a general population reported a beneficial effect of lutein intake on FVC only. The third study again found a beneficial effect of lutein blood levels on slower declines of FVC only. No association was found in the other two studies.

For respiratory mortality, one longitudinal study in elderly showed that higher lutein blood levels were associated with a decreased risk (HR 0.77 (95 \% CI 0.60: 0.99), per SD increase in lutein level).

Conclusion: The published literature suggests possible beneficial effects of lutein on adult respiratory health, especially for respiratory function. However, literature is scarce, especially on respiratory function in children, and most studies are observational. Further large scale prospective cohort studies and intervention studies are needed to clarify the role of lutein in respiratory health.

\section{Abstract \#: P 135}

\section{Performance of health services on tuberculosis care in the municipality of North-Eastern Brazil}

Ewerton William Gomes Brito ${ }^{1}$, Severina Alice Da Costa Uchoa ${ }^{1}$, Zenewton André Da Silva Gama ${ }^{1}$, Ardigleusa Alves Coêlho ${ }^{1}$, José Vilton Costa ${ }^{1}$, Pétala Tuani Cândido De Oliveira Salvador ${ }^{1}$, Kisna Yasmin Andrade Alves ${ }^{1}$, Douglas Ferreira Enedino Albino ${ }^{2}$, Ricardo Alexandre Arcêncio $^{3}$, Inês Fronteira ${ }^{4}$

${ }^{1}$ Universidade Federal do Rio Grande do Norte; ${ }^{2}$ Secretaria municipal de Saúde de Natal; ${ }^{3}$ Universidade de São Paulo; ${ }^{4}$ Universidade de Nova Lisboa; Corresponding author's e-mail: ewerton_brito@hotmail.com

In Brazil and in other priority countries in the control of tuberculosis, there is little access to evaluations of structures and processes that 
guide the good practice in primary care. The aim of this study is to assess, from the perspective of health professionals, the performance of health services in the care of tuberculosis patients in the municipality of north-eastern Brazil. A cross-sectional study was conducted in Natal/Rio Grande do Norte/Brazil, between August 2011 and February 2012. 240 questionnaires with Family Health professionals (doctors, nurses, assistant nurses and community health agents were applied. We analyzed the structure and process components, using descriptive statistics, significance, box-plot and Pareto chart statistics. As for the structure, involvement in tuberculosis care of $100 \%$ of the nurses, doctors and community health agents and $73.9 \%$ of assistant nurses is found. $96.6 \%$ of nurses, $84.1 \%$ of physicians, $13.6 \%$ of the assistant nurses and $38.7 \%$ of community health agents participated in training. The daily record access of the Directly Observed Treatment (DOT) account was $63.7 \%$, the book of record $69.9 \%$ : the availability of pots for sputum test $78.2 \%$ : liaison with other levels of care, $83.4 \%$ : difficulty supporting DOT, $65.2 \%$. For processes, information about the disease has reached $99.7 \%$ : supply of DOT in health units, $51.5 \%$ : educational activities routinely, only $31.5 \%$. The greatest variability was in professional qualification with amplitude from 17.4 to 89.4 and interquartile range 44.8-66.2. It was a statistically significant $(p<0.05)$ for involvement of professionals in attendance, professional qualification and access to recording instruments and DOT. Although the systematic incorporation of shares of tuberculosis care in patients to the routine of the work of health teams represent a breakthrough in performance of health services, some weaknesses can hamper the sustainability of the actions of tuberculosis in Primary Health Care, such as the low professional qualification, difficulties for the realization of the DOT, and the lack of standardization in the distribution of inputs and the use of recording instruments for epidemiological and operational diagnosis of tuberculosis.

\section{Abstract \#: P 136}

\section{A simple method to quantify outbreak risk in highly vaccinated populations with application to Belgian serology on mumps and measles}

$\underline{\text { Steven Abrams }}^{1}$, Philippe Beutels ${ }^{2}$, Niel Hens ${ }^{1,2}$

${ }^{1}$ Interuniversity Institute for Biostatistics and statistical Bioinformatics, Hasselt University, Diepenbeek, Belgium; ${ }^{2}$ Centre for Health Economics Research and Modelling Infectious Diseases, Centre for the Evaluation of Vaccination, Vaccine \& Infectious Disease Institute (WHO Collaborating Centre), University of Antwerp, Antwerp, Belgium; Corresponding author's e-mail: steven.abrams@uhasselt.be

Background: The incidence of measles and mumps has declined dramatically in many European countries since the introduction of the trivalent measles-mumps-rubella (MMR) vaccine. Recently, however, large outbreaks of mumps and measles have been reported in highly vaccinated (European) populations, e.g. a large mumps outbreak occurred in Flanders, Belgium in 2012 and a measles outbreak is currently ongoing in the US. This can be explained, at least partly, by waning of vaccine induced immunity and primary vaccine failure. We present a simple method to identify regions of high outbreak potential based on Belgian serological current status data and available vaccination coverage information.

Methods: Age-dependent susceptibility to mumps and measles infection in the Belgian municipalities in 2012 is estimated using a generalized additive model fitted to the Belgian serology from 2006, while accounting for available vaccination coverage information, primary vaccine failure and waning of vaccine induced immunity.
The age-dependent susceptibility profile leads to local estimates for the effective reproduction number $\mathrm{R}$ reflecting the epidemic potential for both diseases. More specifically, if $\mathrm{R}$ exceeds the threshold value of one, an infection can invade the population after being introduced whereas values smaller than one will lead to eradication.

Results: Based on our methodology, using spatial serological survey data, MMR vaccination coverage data and data on social contacts, we find effective reproduction numbers significantly higher than 1 for both mumps as well as measles in Belgium. This indicates that upon re-introductions, outbreaks are likely to occur. The predicted average effective reproduction number increases over a 30 year time span from about 1.4 to 2.4 , and 1.3 to 2.2 for mumps and measles, respectively. The expected relative incidence is highest in infants under one year of age, in adolescents and young adults. Our predictions coincide well with the observed incidence during the 2012 mumps outbreak in Flanders.

Conclusion: Despite long-standing 2-dose measles-mumps-rubella (MMR) vaccination with moderate to high vaccination coverage in the Belgian regions, the study reveals that large mumps and measles outbreaks are likely to occur in the future when no appropriate intervention measures are undertaken. Gradually increasing susceptibility for adolescents and young adults, together with their active social life, provide avenues for mumps and measles to resurge in large outbreaks upon re-introduction in Belgium. Furthermore, infants form an important vulnerable group during future outbreaks. However, additional vaccination campaigns proof valuable in decreasing susceptibility in the Belgian population, thereby reducing the risk of large-scale outbreaks.

\section{Abstract \#: P 137}

\section{Comparison of rapid and venous HIV testing strategies among vulnerable populations}

Uccella Ilaria $^{1}$, Cammilli Marina ${ }^{1}$, Silvia De Carolis ${ }^{1}$, Alessio Petrelli ${ }^{1}$, Maria Fenicia Vescio ${ }^{2}$, Patrizio Pezzotti ${ }^{2}$

${ }^{1}$ National Institute for Health, Migration and Poverty (INMP/ NIHMP), Rome, Italy; ${ }^{2}$ Istituto Superiore di Sanité (ISS), Rome, Italy; Corresponding author's e-mail: uccella@inmp.it

Background: Despite ongoing prevention and educational efforts, the incidence of new HIV infections in 2012 in Italy is estimated 5.0/ 100,000 among Italians, and 22.3/100,000 among foreigners. Many new infections, more than half in AIDS stage, are diagnosed in persons unaware their serostatus.

The objective of the study was to compare rapid and venous HIV testing strategies among vulnerable populations.

Methods: The differences between the use of venous HIV testingroutinely provided to migrant populations at the INMP/NIHMP - and the Rapid Finger HIV Antibody Test (4 generation test-Alere)-offered with counseling in the framework of a project aimed at promoting access to HIV testing and disseminating knowledge about STIs-were analyzed. When rapid test results were positive, a confirmatory venous test was required. From 2010 to 2014, 5676 HIV tests were performed and 5202 patients were tested.

Results: 4676 venous "V" and 1000 rapid "R" were performed ( 1 test/person in both cases). " $\mathrm{R}$ " test was used in $55.2 \%$ of cases vs $16.8 \%$ of the "V" test. The number of vulnerable populations as irregular migrants (43 vs $14 \%: p<0.001$ ), and homeless (15 vs $0.9 \%: p<0.001$ ) was significantly higher in the group " $\mathrm{R}$ " than in the "V" group, respectively. None missed the test result in the " $R$ " group vs 5 in the "V" group $(11.1 \%)$. "V" test was indeterminate in 18 cases $(0.4 \%)$ and " $\mathrm{R}$ " in $9(0.9 \%)$. "V" test results were available after 10-14 days and " $\mathrm{R}$ " results in $20 \mathrm{~min}$. Furthermore, rapid 
HIV test was also offered in closing hours of the laboratory. HIV prevalence was similar in both groups $(1.3 \%$ in "V" vs $1.5 \%$ in " $\mathrm{R}$ "). The median CD4 cell count at diagnosis was remarkably different (347 cell/mmc in "R" vs 108 cell/mmc in "V"). Out of HIV positive patients in the "V" group (11\%), 5/44 had almost one AIDS related disease vs $1 / 10$ in "R" patients (10\%). HIV patients "in care" of the "V" group were 89 vs $100 \%$ of the "R" group.

Conclusion: Advantages of HIV rapid test were: reduction of barriers to testing for vulnerable populations (higher feasibility in females): increase in the number of people aware of their serostatus: reduction in the number of "failure to return": possibility to reduce the number of "late presenters" and to increase referral to appropriate care and prevention services.

\section{Abstract \#: P 138}

Evaluation of the efficacy and safety of bitherapy on Moroccan patients treated from hepatitis $\mathrm{C}$

\section{Ghassan El Malki ${ }^{1}$}

${ }^{1}$ Research Team of Pharmacoepidemiology \& Pharmacoeconomics. Laboratory of Pharmacology - Toxicology, Faculty of Medicine and Pharmacy, Rabat, Morocco; Corresponding author's e-mail: gh.elmalki@gmail.com

Background: In Morocco studies estimate a prevalence of $\mathrm{HCV}$ to $3.5 \%$ for the whole population, and $5 \%$ for the age group between 45 and 75 years. According to these results, the number of patients with HCV in Morocco reached 300,000 and only 15,000 patients treated.

Objectives: The aim of this work is to find out the efficacy and safety of treatment with interferon alfa- $2 \mathrm{a}$ and ribavirin in Moroccan patients suffering from hepatitis $\mathrm{C}$ and identify factors associated with treatment response.

Methods: This is a retrospective study conducted between February and August 2012 in the regions of Casablanca, El Jadida, Mohammedia, Khouribgua, Beni Mellal, Safi: concerning clinical, paraclinical and therapeutic data collected from patients suffering from hepatitis $\mathrm{C}$ and treated over a period of 24 weeks (genotype $2,3,4$ ) or 48 weeks (genotype 1 ).

Results: At the end of the study, 143 patients were followed up. The average age was 58.1 years. The sex ratio M/F was 0.90 . Genotype 1 was observed in 63 patients $(43.4 \%)$, genotype 2 in 59 patients $(41.3 \%)$. The positive response was observed in 73 patients $(51.4 \%)$, relapse in 42 patients $(29.4 \%)$ and negative response in 28 patients $(19.5 \%) .14$ patients stopped their treatment $(9.8 \%)$. The main side effects were asthenia $(88.8 \%)$, metabolic and liver complications: the hepatocellular carcinoma, cirrhosis, graft cases, leukopenia, acute anemia $(4.9 \%)$. In multivariate analysis, the associated factor with healing was discontinuation of treatment $(p=0.005$ OR $=12.2$ $95 \%$ CI [-60.705 2.482]. Predictive Values of a Positive Response at week 12: $83.01 \%$ for genotype 1 and $95 \%$ for genotype 2. Predictive Values of Negative Response at week 12: $59.25 \%$ for genotype 1 and $33.33 \%$ for genotype 2 .

Conclusions: Discontinuations constitute a significant risk to the success of the treatment, improving treatment outcomes of hepatitis $\mathrm{C}$ depends on the continuity of the treatment itself, compliance with protocols and patient education.

\section{Abstract \#: P 139}

\section{Nutritional status of people living with HIV/aids in an urban community}

$\underline{\text { Dr RN Hiremath }}^{1}$, Dr Shailaja Patil ${ }^{2}$

${ }^{1}$ Student; Prof, Dept of Community Med; Corresponding author's e-mail: drshekar80@gmail.com

Background: Poor nutritional knowledge and dietary practices common among the most affected households significantly contribute to the worsening of HIV status. Addressing gaps in nutrition among people living with HIV and AIDS (PLWHA) is essential because nutrition plays a vital role in the care and management of HIV and AIDS as it is intrinsically linked to immune function.

\section{Objectives}

(1) To assess the knowledge regarding nutrition aspects and care to be taken to stay healthy

(2) To assess the nutritional status of PLWHA on ART (3) To assess the association of BMI with related factor.

Methods: A cross-sectional study, with a sample of 208 PLWHA on ART was conducted by means of predesigned and pretested questionnaire. Systematic random sampling technique was used to collect the sample.

Results: Mean age of the study population was $38.5+9.6$ years and mean duration since HIV positivity was 40.19 months, $48 \%$ were males, $52 \%$ females, $70 \%$ were employed out of which $23.6 \%$ were drivers. $16.3 \%$ were illiterate, $33.7 \%$ belonged to rural areas and $28.8 \%$ were undernourished while $20 \%$ were overweight and $46.2 \%$ were anemic. $74 \%$ had satisfactory knowledge regarding the general ways to stay healthy while only $29.8 \%$ were having satisfactory knowledge regarding nutrition aspects.CD4 count and Hemoglobin levels increased significantly with ART. A significantly higher BMI was seen in individuals who were employed and those with longer duration of ART. The better nourished respondents showed significant increase in CD4 count compared to under-nourished after starting ART ( $p$ value $<0.05$ ).

Conclusion: There exists a gap in PLWHAs knowledge regarding nutritional aspects. The study also shows that good nutrition has good influence on CD4 count. Therefore there is felt need for Nutritional interventions like introduction of protein rich food supplementation and educational material in the form of small nutritional booklets which can be given to each individual and the importance of nutrition can be emphasized during each follow-up visit.

\section{Abstract \#: P 140}

Assessment of treatment outcome and determinant factor among re-treatment tuberculosis patients, eastern Ethiopia: retrospective cohort study

Yonatan Moges Mesfin $^{1}$, Kelemu Tilahun Kibiret ${ }^{2}$

${ }^{1}$ Haramaya University; ${ }^{2}$ Welega university; Corresponding author's e-mail: mogesyoni@gmail.com

Introduction: Tuberculosis is the leading causes of mortality among infectious diseases worldwide. The management of previously treated tuberculosis patients (retreatment cases) is a challenge for National Tuberculosis Programmes in resource-limited settings such as Ethiopia. Aims of this study was to determine the treatment outcomes of the different categories of retreatment cases and factors influencing these outcomes in Eastern Ethiopia.

Methods: A retrospective cohort study was conducted from 2002 to 2006 in selected health facilities in Dire Dawa. Study included all TB cases registered as 'relapse', 'failure' or 'default' between September 2002-March 2006 in Dilchora Referal Hospital and from September 2004-March 2006 in Legehare Health Center. Data were sourced from TB health unit registers from the two health institutions using a structured data form. 
Result: A total of 1667 TB patients were treated from September 2002-March 2006 in Dilchora Referal Hospital and in Legehare Health Center. Of these: 103 of the patients were recorded as TB retreatment cases. The retreatment rate in the follow up period was 6.24. Among the 103 retreatment cases, $52(50.5 \%)$ had a treatment outcome registered in the health unit register: $22(21.4 \%)$ cases were transferred out and $29(28.2 \%)$ had no record of outcome in the register. Of the 52 category II TB treated patients $29(55.7 \%)$ were cured, $14(26.9 \%)$ died, $5(9.6 \%)$ retreatment failure cases and 4 (7.7\%) developed multidrug resistance tuberculosis (MDR-TB). Cure rate was maximum $(40.0 \%)$ and $(27.4 \%)$ in the patients belonging to the default and relapse category respectively.

Conclusion: The analysis has shown that relapse cases were the most common retreatment category and treatment success rates for relapse cases were low $(55 \%)$. Persistent monitoring of treatment outcomes of relapse, failure and default cases is crucial for improving TB control.

\section{Abstract \#: P 141}

Knowledge of youngsters about sexually transmitted infections: influence of organizational family culture

Manuela Ferreira $^{1}$, Ana Marques ${ }^{2}$, João Duarte ${ }^{1}$, Joana Andrade ${ }^{3}$

${ }^{1}$ Polytechnic Institute of Viseu - Health School - CI\&DETS; ${ }^{2}$ Health School of Viseu; ${ }^{3}$ Hospital Tondela Viseu Center; Corresponding author's e-mail: mmcferreira@gmail.com

Background: STIs are now considered a major public health problem because of its magnitude, the difficulty of identifying the correspondent symptoms and, above all, for being great facilitators of HIV transmission. A large percentage of adolescents do not know any form of infection or treatment of STIs, which shows us that despite the dissemination of information, it has not reached effectively the population.

Objectives: Analyze the influence of sociodemographic, academical, sexual and organizational family culture variables on teens' knowledge about sexual transmitted infections.

Method: Quantitative, descriptive, correlational and cross study with a non-probabilistic sample consisting of 809 adolescents attending vocational schools from the central region. The instrument for data collection: Sociodemographic Questionnaire, Knowledge Scale on Sexually Transmitted Infections and Organizational Family Culture Inventory.

Results: Adolescents aged between 14 and 23 years, 343 girls and 466 boys. Mostly single $(96.9 \%)$, Portuguese $(84.5 \%)$, caucasian $(83.2 \%)$ and catholic $(91.4 \%)$. The age when they started sexual intercourse varied between 12 and 20 years old among girls and between 10 and 21 among boys. $63.0 \%$ have already started having sexual relations. Young foreigners $(\mathrm{OM}=423.72)$, living with their families $(\mathrm{OM}=252,956.5), 18$ or older $($ average $=12.62)$, male (average $=13.19)$, married $(\mathrm{OM}=406.29)$, catholic (397.07), studying in schools of Beira Baixa $(\mathrm{OM}=423.20)$, of 12 th grade (average $=12.81)$, attending health courses $(\mathrm{OM}=486.08)$ and whose parents have higher education have more knowledge about sexually transmitted infections. The gender, the grade, the course they attend, feeling pressure towards starting sexual intercourse, being in love with the person with who they first had sexual relations, the organizational culture in the family where they belong, can influence their knowledge about STIs ( $p \geq 0.000$ and $p \leq 0.003$ ).

Conclusion: In addition to the factors that enhance a sexual risk behavior at any age, there are specific risk factors during adolescence. Sexual maturity, the need to experiment, impulsivity, and the organizational culture of the family where the teenagers are inserted are variables to consider in sex education programs in schools.

\section{Abstract \#: P 142}

Risk perceptions during the 2014 Ebola virus disease epidemic: results of a german survey in lower saxony

Nicole Rübsamen $^{1}$, Stefanie Castell ${ }^{1}$, Johannes Horn ${ }^{1}$, André Karch ${ }^{1}$, Jördis J. Ott ${ }^{1}$, Heike Raupach-Rosin ${ }^{1}$, Beate Zoch $^{1}$, Gérard Krause ${ }^{1}$, Rafael T. Mikolajczyk ${ }^{1}$

${ }^{1}$ Helmholtz Centre for Infection Research, Braunschweig, Germany; Corresponding author's e-mail: Nicole.Ruebsamen@helmholtz-hzi.de

Background: Several Ebola virus disease (EVD) outbreaks have occurred during the last decades, but none has affected European countries like the 2014 EVD epidemic in West Africa. To date, no information is available on risk perceptions regarding EVD in European populations. We aimed to address this gap by means of a survey on risk perceptions and behaviour related to EVD in Germany. Methods: We developed a questionnaire that was sent to the participants of a longitudinal online panel addressing hygiene and preventive behaviour regarding infections (HaBIDS) in four districts of the federal state of Lower Saxony, Germany. This panel consisted of 1376 adults aged 15-69 years who were sampled randomly from the population registries in the four districts. The questionnaire covered risk perceptions, knowledge about transmission routes of EVD, media use, personal reactions to the outbreak, attitudes towards measures to prevent the spread of EVD to Europe, willingness to volunteer for aid missions, and attitudes towards vaccination against EVD.

Results: A total of 974 participants completed the questionnaire. Twenty-nine percent expressed fear of EVD. Only four percent of all participants stated the transmission routes of EVD correctly. Seventyfour percent answered that airborne transmission or transmission by asymptomatic patients was possible. If a patient was flown in from Africa for treatment in a close-by hospital, the majority of participants (87\%) would adopt additional preventive behaviours. Of these, $16 \%$ would avoid using public transportation, $75 \%$ would increase their hygiene behaviour (e.g., washing hands more often), and $30 \%$ would not visit friends admitted to the same hospital. Among the measures to prevent the spread of EVD, providing information on EVD for all travellers coming from affected areas and advice in case of developing signs and symptoms was the measure supported by most of the participants $(97 \%)$. Other measures were supported by fewer participants, e.g. a ban on return transport of Germans getting infected during aid missions in West Africa $(9 \%)$ or mandatory quarantine for all returning volunteers $(38 \%)$.

Conclusion: Although the majority of participants was not scared of EVD at the time the study was conducted, misperceptions regarding transmission routes were common and could trigger irrational behavioural changes. Prevention measures which are likely to negatively impact on willingness to participate in aid missions would also be supported by a fraction of the participants. Thus, risk perception in countries not yet affected could influence control of the outbreak in the affected countries.

\section{Abstract \#: P 143}

Living conditions and tuberculosis: an analysis in endemic area under intense pressure from changes in urban space 
Alexandre San Pedro ${ }^{1}$, Gerusa Gibson ${ }^{1}$, Luciano Medeiros de Toledo $^{1}$, Paulo Chagastelles Sabroza ${ }^{1}$, Rosely Magalhães de Oliveira ${ }^{1}$

${ }^{1}$ National School of Public Health, Oswaldo Cruz FoundationFIOCRUZ; Corresponding author's e-mail:

alexsan9578@hotmail.com

Background: the precarious living conditions associated with occurrence of tuberculosis underscore a situation of socioeconomic inequity in which worsening socioeconomic inequalities figure prominently, particularly in areas under intense pressure from changes in urban space.

Objective: to analyse the relationship between occurrence of tuberculosis and socioeconomic and tuberculosis control operating indicators, as well as to discuss the influence of variations in those indicators on the distribution of tuberculosis from 2000 to 2011.

Method: An ecological study whose units of analysis were the districts of municipality of Itaboraí, Rio de Janeiro State, an area hosting the installation of the largest petrochemical complex of South America. The data was incorporated into a negative binomial regression model.

Results: From 2000 to 2011, there was a direct association between tuberculosis incidence and the poverty density, homes connected to the sewage collection network, income distribution inequality and household crowding.

Conclusion: The findings suggest that the economic development observed in the study area has been accompanied by worsening socioeconomic inequalities, resulting in differentiated forms of exposure to, and illness from, tuberculosis connected with the organisation of urban space. Overall, the presence of a population in a situation of greater socioeconomic vulnerability to tuberculosis, disconnected from policies designed to eliminate social inequities, restricts resolution of the problem to tuberculosis control programmes.

\section{Abstract \#: P 144}

Eosinophilia and parasitic infections identified in foreigners: data from the national institute for health, migration and poverty (INMP/NIHMP), Rome, Italy

Ilaria Uccella $^{1}$, Marina Cammilli ${ }^{1}$, Laura Pecoraro ${ }^{1}$

${ }^{1}$ National Institute for Health, Migration and Poverty (INMP/

NIHMP), Rome, Italy; Corresponding author's e-mail:

uccella@inmp.it

Background: The European GeoSentinel surveillance data on travelrelated diseases reported that prevalent diagnoses in migrants are latent tuberculosis (43\%) and eosinophilia (15\%). Regional variations are observed for specific diagnoses such as parasitic infections. In Italy, national data of travel-related diseases in newly arrived people are not recorded.

Objective of the study was to evaluate the prevalence of eosinophilia and parasitic infections among foreigners

Methods: A retrospective analysis of eosinophilia and parasitic infections diagnosis in foreigners attending the INMP/NIHMP from January 2010 to December 2014 was conducted. In the framework of a screening for newly arrived people including white blood cell count (WBC), Mantoux test, hepatitis, syphilis and HIV test, parasitic infections were investigated. Eosinophilia was defined as almost 450 cell $/ \mathrm{mmc}$.

Results: During the study period, 41,090 foreigners were visited. Out of them, $5.560(8.8 \%)$ were newly arrived. Eosinophilia was diagnosed in 477 cases $(1.2 \%)$, mostly in males $(82.2 \%), 90(18.8 \%)$ under $18,63 \%$ with median age $18-34$ years. 194 (40.7\%) were asylum seekers and undocumented migrants $(55.3 \%)$. They came from Africa $(66.8 \%)$, Asia (19.7\%) and South America $(5.5 \%)$. Main origin countries were Nigeria (16.1\%), Bangladesh $(11.2 \%)$, Senegal $(7.7 \%)$, Gambia $(6.8 \%)$ and Mali $(4.8 \%)$. In 117 cases (24.5\%), eosinophilia resulted associated to a parasitic infection: Anchilostostomiasis (23\%), Blastocystis hominis (21.4\%), Amebiasis, (15.4\%) Schistosomiasis (12\%), Strongylodiasis $(8.5 \%)$, Giardiasis $(6.8 \%)$, Trichuriasis $(3.4 \%)$, other Helminthic infections $(5.1 \%)$, Trichinosis $(1.7 \%)$, E. histolytica $(1.7 \%)$, Enterobiasis $(0.8 \%)$. Parasitic infections were $394(0.9 \%)$. Out of them, 96 $(24.4 \%)$ with eosinophilia. Prevalence in asylum seekers (5560) was $1.5 \%$. Newly arrived people resulted the migrant group with the highest rate of parasitic infections $(21.3 \%)$. Main diseases were: Blastocystis hominis (26.6 \%), Amebiasis (21.8\%), Anchilostostomiasis $(15.2 \%)$, Schistosomiasis $(7.8 \%)$, Giardiasis $(6.8 \%)$, Strongylodiasis $(4.3 \%)$, Enterobiasis $(3.5 \%)$, Trichuriasis $(3.1 \%)$, other Helminthic infections $(2.6 \%)$, Trichinosis $(2.6 \%)$. Few cases of other infections: E. histolytica (2\%), Hookworm (1.3\%), Filariasis $(1 \%)$, Echinococcosis (1\%), Ascariasis $(0.8 \%)$, Malaria $(0.8 \%)$, Tripanosomiasis $(0.25 \%)$, cutaneous Leishmaniosis $(0.25 \%)$.

Conclusions: Prevalence of eosinophilia resulted higher in newly arrived people, including asylum seekers (mostly Africans), than in other foreign groups. This blood alteration seems poorly associated with parasitic infections (mostly non-pathogenic), and other causes must be investigated. To increase the probability of new diagnosis, we propose to include parasitic infections in screening for migrants from high prevalence countries at their first access to health services, independently from the time of arrival and the presence of eosinophilia in the WBC.

\section{Abstract \#: P 145}

\section{Survival time of patients coinfected with HIV and hepatitis C in Brazil}

Silvano Barbosa de Oliveira ${ }^{1}$, Leila Denise Alves Ferreira Amorim ${ }^{2}$

${ }^{1}$ Ministry of Health; ${ }^{2}$ University Federal of Bahia; Corresponding author's e-mail: silvano.oliveira@aids.gov.br

Introduction: In Brazil, the estimate for those coinfected with HIV/ $\mathrm{HCV}$ varies from 5 to $20 \%$. Coinfected individuals present a higher chance of progression to diseases related to the liver, including cirrhosis, hepatocellular carcinoma, and hepatic coma, than those infected with only HCV. Liver diseases have been considered one of the emerging causes in relation to deaths from AIDS. This study aims to estimate survival time and associated factors among those coinfected with HIV/HCV in Brazil.

Materials and methods: 3355 records of patients coinfected with $\mathrm{HIV} / \mathrm{HCV}$ were considered, identified by the probabilistic linkage of the databases of the period from 2002 to 2012 . The survival time of the coinfected patients, according to epidemiological variables, was estimated using the Kaplan-Meier method and the risk factors through the Cox regression model. Survival time was defined as the difference between the date of diagnosis of co-infection and the date of death.

Results: Of the overall, $72.8 \%$ are of the male sex, $86.7 \%$ are between 25 and 49 years of age, $32.0 \%$ are black or mixed, $78.5 \%$ were in treatment for HIV and $42.2 \%$ are from the exposure category of injectable drug users (IDU). Individuals aged 50 years or more have a lower median survival time (43 months) and a 2.5-timeshigher risk of death compared to those under 25 years. Black and mixed also have a lower median time compared to the other races and an $21 \%$ higher risk of death. Individuals who are not in treatment for 
HIV have a risk of death three times higher than those who are in treatment.

Conclusion: Vulnerability, difficulty in access to the health care services, lack of awareness of adherence to treatment and prevention for HIV/AIDS and viral hepatitis are some of the factors which may explain the higher risk of death and the lower survival time among individuals over 50 years of age and among black and mixed.

The history of active treatment for HIV/AIDS among patients coinfected with HIV/HCV shows that there is a better response to the survival time when these patients have already started treatment, proving the positive impact of ART on the survival of coinfected patients as well.

\section{Abstract \#: P 146}

Effect of farming environment on childhood asthma incidence in Canada: results from the national longitudinal survey of children and youth

\section{Marc Parsons ${ }^{1}$, Ambikaipakan Senthilselvan ${ }^{1}$, Jeremy Beach ${ }^{2}$}

${ }^{1}$ School of Public Health, University of Alberta, Edmonton, Canada; ${ }^{2}$ Division of Preventive Medicine, Faculty of Medicine and Dentistry, University of Alberta, Edmonton, Canada; Corresponding author's e-mail: maparson@ualberta.ca

Background: Asthma is the most prevalent chronic disease among children globally. This disease costs national healthcare systems significant sums and contributes to school absenteeism among young students. Some studies have suggested that living in a farming environment is protective against asthma. The hygiene hypothesis is often cited as the underlying mechanism for this effect. In this cohort study, children living in farming and non-farming environments were followed from childhood to adolescence and the association between farming environment and asthma incidence was investigated.

Methods: After excluding children with asthma, wheeze, and missing data on living environment (non-rural, rural residential, and rural farming) at the baseline in 1994-1995 (Cycle 1), over 10,900 children who participated in the National Longitudinal Survey of Children and Youth conducted by the Statistics Canada were considered in this study. These children were followed up biennially until either the end of the study in 2008-2009 (Cycle 8), they reached 25 years of age, or they were lost to follow-up. These health and demographic data were collected from interviewer-administered questionnaires which were answered by the person most knowledgeable of the children less than 16 years old or by children themselves if they were of ages 16 years or greater. Replicate sample weights were used to adjust for the survey design effect. Cox proportional hazards regression was used to estimate hazard ratios for the exposures of interest and possible confounders.

Results: Living in a rural farming environment rather than a non-rural one was found to have a strong protective effect against asthma incidence when adjusting for potential confounders $(\mathrm{HR}=0.58$ : $95 \%$ CI: $[0.43,0.80])$ while living in a rural residential environment had a weaker effect $(\mathrm{HR}=0.79: 95 \% \mathrm{CI}$ : $[0.62,1.02])$. Other factors found to be associated with asthma include number of older siblings, child ever-allergy, family asthma history, and dwelling needing repairs.

Conclusion: This population-based longitudinal study suggests that living in a farming environment reduces childhood risk of developing asthma. The results from this study add to the evidence supporting the hygiene hypothesis as a mechanism for reducing asthma development in children.

\section{Abstract \#: P 147}

The influence of nonprescription and prescription medications on the gut microbiome in community volunteers

Mary A.M. Rogers ${ }^{1}$, Alyxandria M. Schubert ${ }^{1}$, Patrick D. Schloss ${ }^{1}$, Vincent B. Young , David M. Aronoff ${ }^{2}$

${ }^{1}$ University of Michigan; ${ }^{2}$ Vanderbilt University; Corresponding author's e-mail: maryroge@umich.edu

Background: Approximately $70 \%$ of Americans take over-thecounter (OTC) medications for headaches and more than half use OTC drugs to treat colds and flu, heartburn, and muscle/joint pain. Prescription drug use is common as well but the gastrointestinal effects of drug combinations are not fully known. In particular, the composition of the gut microbiome for individuals using different types of medications has not yet been described. We hypothesized that the gut microbiome is responsive to the entirety of medications used at a given time and that the microbiome of those not taking any medications would differ from those who do.

Methods: Participants were 155 adult community residents in Michigan, USA. A complete assessment of OTC and prescription drugs taken within the past 30 days was ascertained. Subsequently, each participant submitted a stool sample for bacterial DNA analyses. PowerSoil $^{\circledR}$-htp 96 Well Soil DNA Isolation Kits were utilized to extract total bacterial DNA. The V35 region of the 16S rRNA gene was amplified and sequenced, followed by curation using mother software. Phylogenetic analysis was performed with operational taxonomic unit (OTU) clustering. Differences in the relative abundance of bacteria were ranked between medication classes. Logit models were generated to discriminate bacteria present by medication class and area under the curve (AUC) was calculated for receiver operator characteristic curves. Two-limit tobit regression was used, with standard error adjustment for within-person clustering, to evaluate overall changes in bacterial relative abundance.

Results: Bacteria of genus Prevotella, Barnesiella, Alistipes and Dialister tended to be more frequent in individuals not taking any medication. Organisms of genus Prevotella, Bacteroides, Barnesiella and family Ruminococaceae discriminated aspirin users versus no medication $(\mathrm{AUC}=0.96: 95 \%$ CI $0.84,1.00$ ). The AUC for ibuprofen was 0.98 (95\% CI: $0.93,1.00)$ with microbiome data, while the AUC for nonsteroidal anti-inflammatory drugs (NSAIDs) was 0.86 (95\% CI: 0.78, 0.94) when compared to no medication. Bacteria of genus Bacteroides and Erysipelotrichaceae discriminated NSAIDs with proton pump inhibitors versus NSAIDs (AUC $=0.96$ : $95 \%$ CI: $0.87,1.00)$. The microbiome also varied by whether the person took laxatives and/or antidepressants with NSAIDs. The greatest decrease in relative abundance was seen in those who took both antibiotics and laxatives (coefficient $-2.53, p<0.001$ ). Antibiotics with NSAIDs and antidepressants was also strongly related to a relative reduction in gut bacteria (coefficient -2.27 , $p<0.001)$ when compared to no medication.

Conclusion: Bacteria in the gastrointestinal tract reflect the types and combinations of medications that people ingest.

\section{Abstract \#: P 149}

\section{Self-care behaviors in hypertension patients}

Fazel Zinat Motlagh ${ }^{1}$, Reza Chaman ${ }^{1}$, Ahmad Ali Eslami ${ }^{2}$, Sayed Rashid Ghafari ${ }^{1}$

${ }^{1}$ Social Determinants of Health Research Center, Yasuj University of Medical Sciences, Yasuj, Iran; ${ }^{2}$ Department of Health Education and 
Promotion, School of Health, Isfahan University of Medical Sciences, Isfahan, Iran; Corresponding author's e-mail: motlaghf@yahoo.com

Background: Assessment of individuals' hypertension self-care behavior may provide important information to clinicians and practitioners working to control high blood pressure. The objective of this study was to investigate the self-care behaviors among hypertensive patients.

Methods: This cross-sectional study was conducted on a 1836 patients of female and male patients diagnosed with hypertension in urban and rural health centers in the province kohgiluyeh va boyerahmad in south of Iran, during 2014. Were randomly selected to participate voluntarily in the study. Self-care activities were measured using the H-SCALE (Hypertension Self-Care Activity Level Effects). Data were analyzed by SPSS-20 statistical tests.

Results: Respondents were average age of 63 (range 30-92), $36.1 \%$ of participants reported adhering to medication recommendations levels. Physical activity levels (27.9\%, respectively). Following practices related to weight management was less frequent $(39.2 \%)$ and adherence to low-salt diet recommendations was also low (12\%). $86.7 \%$ were nonsmokers and $100 \%$ abstained from alcohol.

Conclusions: Based on our result, it seems that designing and implementation of educational programs to increase hypertension self-care behavior.

\section{Abstract \#: P 150}

\section{Association of childhood abuse history and dementia: results from JAGES study}

Takeo Fujiwara $^{1}$, Yukako Tani ${ }^{2}$, Toyo Ashida $^{2}$, Naoki Kondo ${ }^{2}$, Katsunori Kondo ${ }^{3}$

${ }^{1}$ Department of Social Medicine, National Research Institute for Child Health and Development, Tokyo, Japan; ${ }^{2}$ Department of Health and Social behavior, School of Public Health, The University of Tokyo, Tokyo, Japan; ${ }^{3}$ Center for Preventive Medical Sciences, Graduate School of Medicine, Chiba University, Chiba, Japan; Corresponding author's e-mail: fujiwara-tk@ncchd.go.jp

Background: Childhood abuse history is associated with the onset of adult disease, such as cardiovascular disease or mental disorder. However, few studies investigate the association between childhood abuse history and dementia, which is the purpose of this study.

Method: JAGES study 2013, a population-based study for elderly aged 65 years old or older without nursing care, with valid response on childhood abuse history was used $(\mathrm{N}=24,822)$. Dementia was defined as under-treatment or having subsequent complication on dementia, including Alzheimer disease, inquired via questionnaire. Childhood abuse history was assessed on whether having experience on physical abuse, psychological abuse, or psychological neglect up to 15 years old. Multivariate logistic regression was performed to investigate the association between childhood abuse history and dementia, adjusted for age, sex, education, equivalent income longest job, and marital status, and further existence of domestic violence in childhood was used as instrument variable.

Results: Dementia under treatment was found for 143 cases $(0.59$ [95\% confidence interval (CI): 0.50-0.69] \%). In crude model, physical and psychological abuse was significantly, and psychological neglect was marginally, associated with dementia. In multivariable model adjusted for covariates, elderly with any time of childhood abuse history was significantly associated with dementia (odds ratio [OR]: $1.75,95 \%$ CI: 1.19-2.57). The significant association remains in the instrumental variable model.
Conclusion: Among elderly without nursing care, childhood abuse history was associated with dementia. Further longitudinal study using more valid outcome on dementia is needed.

\section{Abstract \#: P 151}

Adverse socioeconomic trajectories and higher cardiovascular risk predict worse performance on cognitive function tests in adults (Elsa-Brasil)

Larissa Fortunato Araujo ${ }^{1}$, Luana Giatti ${ }^{2}$, Valéria Maria Azeredo Passos $^{1}$, Isabela M. Benseñor ${ }^{3}$, Rodrigo Citton Padilha dos Reis ${ }^{1}$, Mohammad Arfan Ikram ${ }^{4}$, Henning Tiemeier ${ }^{4}$, Sandhi Maria Barreto ${ }^{1}$

${ }^{1}$ Universidade Federal de Minas Gerais; ${ }^{2}$ Universidade Federal de Ouro Preto; ${ }^{3}$ Universidade de São Paulo; ${ }^{4}$ University Medical Center Rotterdam; Corresponding author's e-mail:

fortunatolarissa@yahoo.com.br

Background: The onset of cognitive decline in old age is the result of a long-term pathological process that evident starts around at the age of 45 years.

Methods: We examined the effects of early nutritional and social conditions, cardiovascular disease risk and also the cumulative disadvantage from childhood through adulthood on the cognitive performance among 10,773 civil servants, aged 35-64 years, workers of public universities and research institutes, in six Brazilian states. Results: After mutual adjustments for all exposures, the lower maternal educational attainment, smaller trunk lengths and higher cardiovascular disease risk assessed by the Framingham Risk Score were associated with worse performance in general cognitive factor and also with specific domains task, by the learning, recall and word recognition tests, semantic and phonemic verbal fluency tests, and the trail-making test version $\mathrm{B}$.

Conclusions: Life course disadvantages seem to operate through biological and social mechanisms representing active cognitive reserve. Prevention of dementia should start early in life and continue through life span as seen with many other chronic diseases.

\section{Abstract \#: P 152}

\section{Contribution of chronic diseases to mild and severe disability} burden in Belgium

Renata T. C. Yokota ${ }^{1,2}$, Stefaan Demarest ${ }^{1}$, Johan Van der Heyden ${ }^{1}$, Jean Tafforeau ${ }^{1}$, Willma J. Nusselder ${ }^{3}$, Patrick Deboosere ${ }^{2}$, Herman Van Oyen ${ }^{1}$

${ }^{1}$ Department of Public Health and Surveillance, Scientific Institute of Public Health, Brussels; ${ }^{2}$ Department of Social Research, Interface Demography, Vrije Universiteit Brussel; ${ }^{3}$ Department of Public Health, Erasmus MC; Corresponding author's e-mail: renata.yokota@wiv-isp.be

Background: Population aging accompanied by increased longevity with disability has raised international concern, due to its costs to the health care systems. Chronic diseases are the main causes of physical disability and their simultaneously occurrence in the population can have different impact in the disablement process, resulting in different severity levels. In this study, the contribution of chronic diseases to mild and severe disability prevalence burden in Belgium was assessed.

Methods: Data on 21 chronic diseases and disability from 35,799 individuals aged 15 years or older who participated in the 1997, 2001, 2004, or 2008 Belgian Health Interview Surveys were analysed. 
Disability was defined based on six activities of daily living (ADL) and/or mobility limitations. Individuals who reported some difficulties in at least one of the ADLs or declared that they were unable to walk without stopping more than $200 \mathrm{~m}$ were considered mildly disabled: severe disability was defined as the inability to perform at least one ADL alone or being unable to walk without stopping after only few steps. The attribution method was used to assess the contribution of chronic diseases to the disability prevalence, using multiple additive hazard models to each disability outcome, separately for men and women.

Results: Different chronic diseases were identified as major contributors to the burden of disability, according to severity level and gender: back pain and arthritis were the most important contributors to the mild disability burden in men and women, while chronic respiratory diseases (CRD) and arthritis contributed most to the severe disability burden in men and women, respectively. This contribution also differed by age: for mild disability, depression and CRD were important contributors among young individuals, while heart attack had a large contribution for older individuals. For severe disability, neurological diseases and stroke presented a large contribution in young and elderly individuals, respectively.

Conclusions: Our results show that the main contributors to the disability burden vary according to the disability severity level. Therefore, this distinction can assist policymakers in the definition of strategies to tackle disability, involving prevention, rehabilitation programs, support services, and training for disabled individuals.

\section{Abstract \#: P 153}

Perinatal health and all-cause mortality: A sibling analysis using the Uppsala birth cohort study

$\underline{\text { Sol P. Juárez }}^{1,2}$, Anna Goodman ${ }^{1,3}$, Bianca DeStavola ${ }^{3}$, Ilona Koupil ${ }^{1}$

${ }^{1}$ Centre for Health Equity Studies (CHESS), Stockholm University/ Karolinska Institute; ${ }^{2}$ Division of Environmental and Occupational Medicine, Lund University; ${ }^{3}$ London School of Hygiene and Tropical Medicine (LSHTM); Corresponding author's e-mail:

sol.juarez@chess.su.se

Background: In this paper we study the association between perinatal health measures and all-cause mortality by age-specific intervals, exploring the contribution of maternal socioeconomic characteristics and the presence of maternal-level confounding.

Methods: Our study is based on the Uppsala Birth Cohort Study (UBCoS), which comprises all births at the Uppsala University Hospital between 1915 and 1929. We restricted analyses to 12,564 singletons. Birthweight was classified into low weight $(<3000)$, normal weight (3000-3999) and macrosomia (>4000). Gestational age was categorized into preterm ( $<37$ gestational weeks), term (between 37 and 41 weeks) and post-term ( $>42$ weeks). Birthweight standardized by sex and gestational age was categorized into "small-forgestational age" (below the 10th percentile), "adequate-birthweightfor gestational age (between 10th and 90th percentile) and "large-forgestational age" (above the 90th percentile). We used Cox regression models to estimate age-varying hazard ratios (HR) of overall mortality and their $95 \%$ confidence intervals for the following bands: $<1$, $1-4,5-24,25-44,45-59,60-64,65-69$ and $>75$ years of age. We conducted between and within-family survival analyses to assess maternal-level confounding.

Results: Being low birthweight and small-for-gestational age was associated with higher rates of mortality up to 44 years (HRs between 1.2 and 3.1), while being preterm up to 4 years (HR: 3.1). We did not find evidence that observable early-life socioeconomic disadvantage explains or modifies the association between perinatal health and mortality. The associations with birthweight and gestational age were confirmed in the sibling analysis analyses, indicating that any residual maternal confounding is limited. There was a small indication of negative confounding of low birthweight on infant mortality, leading to underestimation.

Conclusions: This study provides evidence that light, small, and preterm newborns have a higher rate of mortality. These associations varied by age and measure under scrutiny. Our findings support the message that policies oriented towards improving population health should invest in improving birth outcomes and hence, in maternal health.

Keywords: mortality, life-course, birth weight, fetal development, gestational age, Sweden.

\section{Abstract \#: P 154}

Maternal pre-pregnancy obesity and child neuropsychological development at 4 years

Maribel Casas $^{1}$, Joan Forns ${ }^{1,2}$, David Martínez ${ }^{1}$, Martine Vrijheid ${ }^{1}$, on behalf of the INMA Project

${ }^{1}$ Centre for Research in Environmental Epidemiology (CREAL), Universitat Pompeu Fabra (UPF), CIBER Epidemiología y Salud Pública (CIBERESP), Barcelona, Spain; ${ }^{2}$ Department of Genes and Environment, Division of Epidemiology, Norwegian Institute of Public Health, Oslo, Norway; Corresponding author's e-mail: mcasas@creal.cat

Background: Maternal pre-pregnancy obesity may be associated with impaired infant neuropsychological development: however, it is unclear if reported associations are due to intrauterine mechanisms or reflect residual confounding by genetic, postnatal, socioeconomic, and familial factors. We previously reported in INMA and RHEA birth cohorts that children of obese mothers had lower cognitive development scores at 1-2 years than those of normal weight mothers. In this new analysis we assessed whether maternal pre-pregnancy obesity affected infant's neuropsychological development at the age of 4 years in 1746 mother-child pairs from the INMA birth cohort study.

Methods: Measured maternal height and reported pre-pregnancy weight at the first prenatal visit were used to calculate maternal body mass index (BMI). At 4 years, cognitive and motor development was assessed using McCarthy Scales of Children's Abilities. Attentiondeficit hyperactivity disorder (ADHD) symptoms was assessed using the Criteria of Diagnostic and Statistical Manual of Mental Disorders, Fourth Edition (ADHD-DSM-IV) form and autism spectrum disorder (ASD) using the Childhood Asperger Syndrome Test (CAST). Paternal BMI reported by the mother at the first prenatal visit was used as negative control exposure. Underweight mothers and fathers were excluded from the analysis.

Results: The percentage of obese mothers and fathers was 8 and $12 \%$, respectively. Children of obese mothers had lower cognitive and motor development scores than those of normal weight mothers in the unadjusted models. Adjustment for socioeconomic and other variables resulted in coefficients closer to the null, and adjusting for paternal BMI had only very small effects. After full adjustment only the verbal subscale remained statistically significantly reduced $(\beta=-3.1: 95 \% \mathrm{CI}=-5.7,-0.5)$. Both maternal and paternal overweight and obesity were associated with an increased risk of ADHD symptoms and ASD.

Conclusion: Maternal pre-pregnancy obesity seems to be associated with reduced verbal development scores at 4 years. The reported associations with ADHD and ASD could be attributed to unmeasured familial confounding. 


\section{Abstract \#: P 155}

Total and central adiposity throughout adolescence: direct and indirect effects on systolic blood pressure at 21 years of age $\underline{\text { Joana Araújo }^{1}}{ }$, Milton Severo ${ }^{1}$, Henrique Barros ${ }^{1}$, Elisabete Ramos ${ }^{1}$ ${ }^{1}$ EPIUnit - Institute of Public Health, University of Porto, Porto, Portugal: Department of Clinical; Corresponding author's e-mail: jfaraujo@med.up.pt

Background: We aimed to study the role of total and central adiposity on systolic blood pressure (SBP) in adulthood, measuring the direct and the indirect effects of the adiposity tracking during adolescence.

Methods: We analysed data from a population-based cohort (EPITeen study) that recruited 13-year-old adolescents enrolled at public and private schools of Porto, Portugal, in 2003/2004 school year (77.5\% participation). For this analysis we included 1105 adolescents evaluated at 13, 17 and 21 years of age. We used body mass index (BMI) as a measure of total adiposity and waist-to-weight ratio as a measure of central adiposity independent of total weight, calculated as [waist circumference/sqrt(weight)]. For each study wave, sex-, age, and height-specific z-scores of the sample were calculated for SBP. Path analysis was used to evaluate the relationships between adiposity (BMI and waist-to-weight ratio) and SBP. Final path analysis included the tracking of adiposity and of SBP, the cross-sectional associations between adiposity and SBP at 13, 17 and 21 years, and the longitudinal associations. Models were stratified by sex and results are presented as correlations (r: $p$ value).

Results: The total effect of BMI at $13 \mathrm{y}$ on SBP at $21 \mathrm{y}$ was $\mathrm{r}=0.189$ $(p<0.001)$ for females and $\mathrm{r}=0.178(p<0.001)$ for males. Path analysis showed moderate positive correlations for the cross-sectional associations of BMI with SBP at 13, 17 and 21 years of age, in both sexes. On the contrary, inverse correlations were found for the longitudinal associations, significant throughout adolescence in girls ( $\mathrm{r}=-0.192: p=0.010$ for BMI at $13 \mathrm{y}$ with SBP at $17 \mathrm{y}:$ and $\mathrm{r}=-0.139: p=0.037$ for BMI at $17 \mathrm{y}$ with SBP at $21 \mathrm{y}$ ), and between 17 and 21 years in boys $(\mathrm{r}=-0.190: p=0.006)$. There was a significant tracking of BMI between 13 and 21 years of age, which also contributes to the positive association with SBP at 21 years.

For central adiposity, we obtained significant moderate correlations for the tracking of waist-to-weight ratio between 13 and 21 years in both sexes, but in general non-significant correlations between central adiposity and SBP were found, both in the crosssectional and longitudinal associations.

Conclusion: Our results from a path analysis showed a global positive association between BMI across adolescence and SBP in early adulthood. The results from waist-to-weight ratio did not support the existence of this effect for central adiposity suggesting that for these ages the role of total adiposity may be more relevant than the effect of body fat distribution.

We acknowledge funding from the Portuguese Foundation for Science and Technology (FCOMP-01-0124-FEDER-015750 and SFRH/BD/78153/2011).

\section{Abstract \#: P 156}

Sex differences in violence profile among youth victims treated in public emergency departments in Brazil

Alice C. M. das Neves ${ }^{1}$, Leila P. Garcia ${ }^{2}$

${ }^{1}$ Postgraduate Programs in Public Health. University of Brasilia. Brasília. Brazil; ${ }^{2}$ Institute for Applied Economic Research. Brasilia. Brazil; Corresponding author's e-mail: acnmedeiros@gmail.com
Background: In Brazil, the Youth Act was enacted in 2013, seeking to develop intersectoral policies and programs for youth. Violence is the main cause of youth mortality in Brazil. This study aims to describe the gender differences in the profile of youth victims of violence treated in Public Emergency Departments, in 2011.

Methods: This is a descriptive study conducted with data from the Brazilian Surveillance System for Violence and Accidents (VIVA), a multicenter survey conducted by the Ministry of Health in 71 public emergency departments, located in 24 State capitals and the Federal District. Data were collected from the victims treated in randomly selected 12-h shifts during 30 consecutive days, in September 2011. Data analysis was conducted taking into account the complex sampling design.

Results: 1894, 1422 male (75.1\%) and 472 female (24.9\%) victims aged between 15 and 29 years were included in this study. The male: female ratio was 3.0. Male aggressors prevailed overall, and were involved in 83.1 and $69.7 \%$ of violence cases among male and female victims, respectively. Among male victims, $55.4 \%$ of the violence episodes took place at streets or public places, while among female $43.6 \%$ took place at the household. The perpetrator's relation to the victim was quite different according to sex. The perpetrator was a stranger in 49.7 and $26.8 \%$ of cases among male and female victims, respectively, while was a partner or ex-partner in 3.9 and $31.5 \%$ of the cases among male and female victims, respectively. There were also important differences regarding the type of injury. The prevailing mechanisms of assault were sharp objects $(28.9 \%)$ and firearms $(23.0 \%)$ among men, and physical force $(58.7 \%)$ and sharp objects $(21.6 \%)$ among women. Men have suffered more frequently cuts or lacerations $(59.9 \%)$ and multiple injuries $(10.5 \%)$, than women (48.7 and $6.5 \%$, respectively). Less severe injuries, such as bruises and sprains were more frequent among women $(29.5 \%)$ than men $(15.5 \%)$. The greater severity of injuries among men was consistent with the lower proportion of discharge and higher proportion of deaths in the first $24 \mathrm{~h}$ amongst them (59.5 and $2.1 \%$ ) compared to women (76.4 and $0.2 \%)$.

Conclusion: The violence profile among youth victims treated in Public Emergency Departments was substantially different according to the victims' sex. Among males, injuries with greater severity prevailed, mostly resulting from violence perpetrated by strangers or legal agents. Among females, less severe injuries prevailed, often in circumstances consistent with domestic violence.

\section{Abstract \#: P 157}

\section{Daytime sleep duration and the development of childhood overweight: the koala birth cohort study}

Renee Bolijn ${ }^{1}$, Jessica S. Gubbels ${ }^{1}$, Ester F.C. Sleddens ${ }^{1}$, Stef P.J. Kremers ${ }^{1}$, Carel Thijs ${ }^{2}$

${ }^{1}$ Maastricht University, Department of Health Promotion, NUTRIM School for Nutrition, Toxicology and Metabolism, PO Box 616, 6200 MD Maastricht, The Netherlands; ${ }^{2}$ Maastricht University, Department of Epidemiology, CAPHRI School for Public Health and Primary Care, PO Box 616, 6200 MD Maastricht, The Netherlands; Corresponding author's e-mail:

renee.bolijn@alumni.maastrichtuniversity.nl

Background: Reduced nighttime sleep duration has been recognized as an independent risk factor for childhood overweight, but the association between daytime sleeping and overweight is unknown. The aim of this study is to examine the association between daytime sleep duration and childhood overweight development, independently from nighttime sleeping, in a longitudinal setting. 
Methods: Data from the KOALA Birth Cohort Study on daytime and nighttime sleep duration at 2 years $(\mathrm{N}=2322)$, and Body Mass Index (BMI) around 2, 5, 6, 7, 8, and 9 years were used. To examine the independent, cross-sectional association between daytime and nighttime sleeping and BMI z-scores and overweight at age 2, linear and logistic regression analysis were performed, correcting for potential confounding by covariates. General Estimating Equations (GEE) regression analyses were performed to examine the longitudinal associations between sleep duration and BMI and overweight at ages $5,6,7,8$, and 9 years.

Results: Nighttime sleep duration was significantly associated with lower BMI z-scores (beta $=-0.082,95 \% \mathrm{CI}=-0.120$ : -0.044 , $P=0.00003)$ and lower risk of overweight $(\mathrm{OR}=0.86,95 \%$ $\mathrm{CI}=0.76-0.97, P=0.017)$ at follow up. Daytime sleep duration was not significantly associated with follow up BMI (beta $=-0.011$, $95 \% \mathrm{CI}=-0.065: 0.043, P=0.70)$ or overweight $(\mathrm{OR}=1.10$, $95 \% \mathrm{CI}=0.91-1.33, P=0.31)$. When adjusted for baseline BMI $\mathrm{z}$-score at age 2 , the result for nighttime sleep was attenuated but remained significant (beta $=-0.054,95 \% \mathrm{CI}=-0.088:-0.020$, $P=0.002$ ), and interactions with age of BMI measurement were non-significant, both indicating that the effect of nighttime sleep duration was lasting beyond the contemporaneous association at age 2. The $95 \%$ confidence intervals for daytime sleep exclude the effect size found for nighttime sleep, which shows that it is highly unlikely that daytime sleep would have an effect size similar to nighttime sleep on BMI development into childhood.

Conclusion: This study was, to our knowledge, the first study examining daytime sleep, in addition to nighttime sleep, as a potential independent risk factor for longitudinal childhood overweight development. The results confirm that daytime sleep duration is not associated with BMI growth or overweight development independently from night time sleep. We conclude that daytime sleep is less relevant for childhood overweight prevention.

\section{Abstract \#: P 158}

'Grey spaces' as an early life determinant for respiratory health. Presentation of a scientific project

Christina Tischer $^{1}$, Jordi Sunyer ${ }^{2}$, Josep M Anto ${ }^{2}$

${ }^{1}$ Helmholtz Zentrum München, Institute of Epidemiology I, Neuherberg, Germany; ${ }^{2}$ CREAL - Center for Research in

Environmental Epidemiology: CIBER Epidemiología y Salud Pública (CIBERESP): Hospital del Mar Medical Research Institute (IMIM): University Pompeu Fabra, Barcelona, Spain; Corresponding author's e-mail: christina.tischer@helmholtz-muenchen.de

Background: Early infancy is considered as periods of both great opportunity and considerable risk regarding environmental hazards and later respiratory health status, however, most studies only focused on a single exposure type. The impact of possible interrelationships between outdoor, indoor and socio-economic determinants, especially focusing on urban environments ('Grey Spaces') on later respiratory health impairment is understudied. There is further a need to respond and to react to the current demographic transition towards urbanization and to possibly identify also beneficial urban living environments.

Study objective: This European study aims to investigate longitudinally the association between early exposure to 'Grey Spaces' and various indices of respiratory health (lung function, asthma (symptoms) and atopy) in 11 ongoing European birth cohorts of different geographical regions with particular emphasis on determining beneficial urban conditions.
Methods: The project duration is 2 years. This study will be based on a comprehensive, already available database generated within the "MeDALL: Mechanisms of the Development of ALLergy" project, using a longitudinal study design until early adulthood. A concept of 'Grey Spaces' will be established by integrating data from multiple exposures and by defining patterns of co-existing exposures, and their determinants, using advanced multivariate statistical tools.

Expected scientific output: The project provides a direct contribution to the prevention and treatment of lung diseases by simultaneously addressing the rising increase in urban population and the global burden of respiratory diseases, especially in children. In addition, the holistic concept of 'Grey Spaces' aims to identify not only harmful but also beneficial environmental determinants in urban areas which will inform policymakers and urban planners for better and more innovative European preventive strategies in relation to the burden of respiratory diseases.

\section{Abstract \#: P 159}

The association of air pollution with birth weight and gestational age, evidence from Hong Kong's "children of 1997" birth cohort Jian V Huang ${ }^{1}$, Gabriel M Leung ${ }^{1}$, $\underline{\text { C Mary Schooling }}{ }^{1}$

${ }^{1}$ University of Hong Kong; Corresponding author's e-mail: cms1@hku.hk

Air pollution is a potentially modifiable driver of birth weight (BW) and gestational age (GA). Previous studies, mainly from Western settings have found inconsistent associations of air pollutants with BW and GA, which are open to residual confounding by socio-economic position as both air pollution and BW tend to be socially patterned. In the contrasting developed non-Western setting of Hong Kong, with high levels of air pollution, but little social patterning of $\mathrm{BW}$ or GA, we assessed the association of PM10, $\mathrm{SO}_{2}, \mathrm{NO}$ and $\mathrm{NO}_{2}$ exposure (obtained from air quality monitoring) with BW and GA in a large population-representative birth cohort "Children of 1997" using partial least square regression to account for the collinearity between air pollutants. PM10 and NO (per standard deviation higher) were associated with BW lower by $75.2 \mathrm{~g}$ ( $95 \%$ confidence interval 61.1-90.0) and $46.5 \mathrm{~g}(33.1-61.5)$ respectively, while $\mathrm{SO}_{2}$ and $\mathrm{NO}_{2}$ was associated with BW higher by $94.4 \mathrm{~g}$ (77.4-112.2) and $13.3 \mathrm{~g}$ (0.9-25.0) respectively, adjusted for household income, mother's migration status and parental education: these estimates were substantially attenuated by further adjustment for GA. Similarly adjusted, PM10 and NO were associated with GA shorter by 3.2 days (2.8-3.6) and 1.6 days (1.3-2.1) respectively, while $\mathrm{SO}_{2}$ and $\mathrm{NO}_{2}$ were associated with GA longer by 3.8 days (3.4-4.3) and 0.7 days (0.4-1.0) respectively: these estimates were little changed by adjustment for BW. Our results are similar in magnitude to the effect of maternal secondhand smoking or maternal asthma on BW. Our mixed findings are similar to those reported previously, and suggest a complex effect of air pollution during a critical period. Mechanisms by which different air pollutants affect health have not yet been fully confirmed in experimental studies and may depend on the constituents of PM10 and interactions between air pollutants possibly acting via oxidative stress and inflammation, which need further investigation.

\section{Abstract \#: P 160}

Different subjective health indicators and all-cause mortality: The rotterdam study 
Ayesha Sajjad $^{1}$, Rosanne L. Freak-Poli ${ }^{1,2}$, Albert Hofman ${ }^{1}$, M. Arfan Ikram $^{1}$, Henning Tiemeier ${ }^{1}$

${ }^{1}$ Department of Epidemiology, Erasmus MC, Rotterdam, The Netherlands; ${ }^{2}$ Department of Epidemiology and Preventive Medicine, Monash University, Melbourne, Australia; Corresponding author's e-mail: a.sajjad@erasmusmc.nl

Background: Different subjective indicators of health and well-being have been related to mortality but their independent relation in prediction of mortality remains unknown. These subjective health measures cover perceived health in a range of domains from self-rated physical functioning, quality of life and mental health. In this study, we assessed the association of basic and instrumental activities of daily living, quality of life, positive and negative affect with all-cause mortality.

Methods: We investigated the independent relation between several subjective health indicators with all-cause mortality in 5586 individuals (mean age: 64.3 years $(\mathrm{SD}=6.7)$ ) using Cox proportional hazard models.

Results: During a follow-up of 51,531 person years, 1624 participants died of all-cause mortality. All subjective health indicators were associated with mortality after adjusting for age, gender, education level, MMSE, joint problems, prevalent stroke, prevalent MI, prevalent diabetes mellitus, prevalent COPD, prevalent heart failure, systolic blood pressure, diastolic blood pressure, total cholesterol, HDL cholesterol, BMI, smoking, and alcohol consumption. Once mutual adjustments for other subjective health measures were made, only basic activities of daily living (HR 1.15: $95 \%$ CI: 1.08-1.22) and instrumental activities of daily living (HR 1.20: $95 \% \mathrm{CI}$ : $1.13-1.27)$ remained independently associated with mortality, while the associations of other subjective health indicators with mortality were strongly attenuated. Additional analyses stratified by presence of prevalent chronic comorbidities showed that instrumental activities of daily living (HR 1.31: $95 \%$ CI: 1.21-1.42) but also quality of life (HR 0.91: $95 \%$ CI: 0.84-0.99) were independently associated with mortality in persons with prevalent chronic diseases.

Conclusions: These findings indicate that self-rated physical functioning assessed by basic and instrumental activities of daily living is the best predictor of mortality in older individuals. The prediction of mortality by quality of life depends on prevalent diseases. In contrast, positive and negative affect do not predict mortality if self-rated physical functioning is accounted for.

\section{Abstract \#: P 161}

\section{Nut consumption and cardiovascular risk in older Chinese: the Guangzhou Biobank cohort study}

Yangbo Sun BMed ${ }^{1}$, Chaoqiang Jiang $\mathrm{MD}^{2}$, Kar Keung Cheng $\mathrm{MD}^{3}$, Weisen Zhang $\mathrm{MD}^{2}$, Gabriel M Leung MD ${ }^{1}$, Tai Hing Lam MD ${ }^{1}, \mathrm{C}$ Mary Schooling, $\mathrm{PhD}{ }^{1,4}$

${ }^{1}$ School of Public Health, Li Ka Shing Faculty of Medicine, The University of Hong Kong, SAR, China; ${ }^{2}$ Guangzhou Number 12 Hospital, Guangzhou, China; ${ }^{3}$ Department of Public Health and Epidemiology, University of Birmingham; ${ }^{4}$ School of Public Health, CUNY, New York, USA; Corresponding author's e-mail: violasun@connect.hku.hk

Background: In Western contexts nut consumption is associated with better health. We examined the associations of nut consumption with cardiovascular disease risk in the non-Western setting of Southern China.

Methods: In the Guangzhou Biobank Cohort Study we used multivariable linear regression to examine the adjusted associations of baseline nut consumption (none $(\mathrm{n}=6688),<3$ portions/week $(\mathrm{n}=2596)$ and $\geq 3$ portions/week $(\mathrm{n}=2444))$ with follow-up assessment of Framingham cardiovascular disease score (excluding smoking) and its components in older Chinese ( $\geq 50$ years) (follow-up rate $57.8 \%$ ).

Results: Nut consumption was unclearly associated with Framingham score $(<3$ portions/week compared to none: $0.13,95 \%$ confidence interval (CI) 0.01 to $0.26: \geq 3$ portions/week: $0.02,95 \% \mathrm{CI}-0.11$ to $0.15)$, systolic blood pressure $(<3$ portions/week: $-0.55 \mathrm{mmHg}$, $95 \% \mathrm{CI}-1.78$ to $0.68: \geq 3$ portions/week: $-0.66 \mathrm{mmHg}, 95 \% \mathrm{CI}$ -1.94 to 0.62$)$, diastolic blood pressure ( $<3$ portions/week: $-0.64 \mathrm{mmHg}, \quad 95 \% \mathrm{CI}-1.36$ to $0.09: \geq 3$ portions/week: $-0.69 \mathrm{mmHg}, 95 \% \mathrm{CI}-1.44$ to 0.07 ), HDL-cholesterol ( $<3$ portions/week: $-0.02 \mathrm{mmol} / \mathrm{L}, 95 \% \mathrm{CI}-0.03$ to $-0.004: \geq 3$ portions/ week: $-0.01 \mathrm{mmol} / \mathrm{L}, 95 \% \mathrm{CI}-0.02$ to 0.005$)$, LDL-cholesterol $(<3$ portions/week: $0.01 \mathrm{mmol} / \mathrm{L}, 95 \% \mathrm{CI}-0.02$ to $0.04: \geq 3$ portions/ week: $-0.01 \mathrm{mmol} / \mathrm{L}, 95 \% \mathrm{CI}-0.05$ to 0.02$)$ or fasting glucose $(<3$ portions/week: $0.03 \mathrm{mmol} / \mathrm{L}, 95 \% \mathrm{CI}-0.02$ to $0.09: \geq 3$ portions/ week: $0.04 \mathrm{mmol} / \mathrm{L}, 95 \% \mathrm{CI}-0.02$ to 0.09 ), adjusted for baseline values, energy intake, age, sex, phase of recruitment, socio-economic position, lifestyle and baseline health status.

Conclusion: This large study from a non-western setting suggests observations concerning the benefits of nut consumption may be contextually specific.

\section{Abstract \#: P 162}

The usefulness of adjusting food portion size to estimate dietary intake from a food frequency questionnaire among pre-school children

$\underline{\text { Sofia Vilela }}{ }^{1}$, Milton Severo $^{2}$, Teresa Moreira ${ }^{1}$, Elisabete Ramos ${ }^{2}$, Carla Lopes $^{2}$

${ }^{1}$ EPIUnit - Institute of Public Health, University of Porto, Porto, Portugal; ${ }^{2}$ EPIUnit - Institute of Public Health, University of Porto, Porto, Portugal: Department of Clinical Epidemiology, Predictive Medicine and Public Health of University of Porto Medical School, Porto, Portugal; Corresponding author's e-mail: sofia.vilela@ispup.up.pt

Background: Measuring accurately dietary intake is considered as one of the greatest challenge for epidemiological purposes. The aim of this study was to test two different methods to quantify foods and nutrients from a qualitative food frequency questionnaire (FFQ) in 4-year-old children.

Methods: Participants are from a population-based birth cohort assembled in Porto, Portugal during 2005/2006 (Generation 21, $\mathrm{n}=8647$ ). During the follow-up at 4 years of age dietary intake was assessed by a qualitative 35 -item-FFQ, applied to main caregivers and by food diaries using 3 non-consecutive days. The present analysis comprises data from 2373 children with data on FFQ and complete $3 \mathrm{~d}$ food diaries. Detailed instructions were given to parents on how to fill food diaries, including how to use household measures and standard units to quantify food portions. To estimate daily consumption from FFQ, the nine frequency responses were transformed into daily frequencies for each food item and multiplied by a portion. This portion was estimated through two methods: (a) standard method, using a standard mean portion and (b) z-score method, adjustment of FFQ with the reported mean portion from $3 \mathrm{~d}$ food diaries, applying the formula $\mathrm{Z}=[(\mathrm{FFQ}-\mathrm{MeanFFQ}) /(\mathrm{SDFFQ})]+$ (SDDiary + MeanDiary)]. The total energy intake and nutrients obtained from the two quantity estimation methods were compared with the $3 \mathrm{~d}$ food diaries, using Pearson's correlation and intra-class correlation coefficient (ICC). 
Results: Comparing with $3 \mathrm{~d}$ food diaries, the standard method seemed to overestimate the food and nutrient intake when compared with the z-score method. The lower ICC obtained was 0.059 (95\% CI $-0.007: 0.123$ ) for 'chocolate' in the standard method while the same item using the z-score method had a ICC of 0.142 (95\% CI 0.103:0.180). The higher ICC was found in the z-score method for 'vegetable soup' $[(\mathrm{ICC}=0.536,95 \%$ CI $0.508: 0.564)]$ comparing to a ICC of 0.373 (95\% CI $-0.013: 0.027$ ) using the standard method. The mean of total energy intake obtained through the food diaries and $\mathrm{z}$-score method was similar $(1628 \mathrm{kcal})$ against $2285 \mathrm{kcal}$ in the standard method. While the Pearson's correlation is similar across methods, the ICC increased significantly in the majority of nutrients evaluated through the $\mathrm{z}$-score method, comparing to the standard method.

Conclusion: The study supports that adjustment in the portion size when converting a qualitative FFQ to nutrients by using a z-score method, seems to increase the accuracy of dietary data in young children.

\section{Abstract \#: P 163}

\section{Energy intake in a population of pregnant women}

Amina Boufars ${ }^{1}$, Hakim Belghiti ${ }^{1}$, Hassania Guerinech ${ }^{1}$, El arbi Bouaiti ${ }^{1}$, Rachid Razine ${ }^{2}$, Mustapha Mrabet $^{1}$

${ }^{1}$ Health service and community medicine; ${ }^{2}$ Department of public health; Corresponding author's e-mail: boufarsamina@gmail.com

Background: During pregnancy, a balanced diet is essential for normal fetal growth and to avoid deficiencies in pregnant woman. The aim of our study is to determine the nutritional status of pregnant women.

Methods: this is a descriptive study of a sample of pregnant women admitted in 2009 to the nutrition unit of the military hospital of instruction Mohammed V in Rabat. The recruitment was carried out by simple random sampling with the list of pregnant women in the same service. Were included women at 12 weeks gestation, with no associated pathology and having a single pregnancy. For each woman a standardized food questionnaire with 24-h recall was filled to study the energy inputs and $5 \mathrm{ml}$ of blood was carried out to study the contribution of macronutrients (carbohydrates, fats and proteins), and elements (calcium, zinc and sodium) and vitamins (vitamin C, vitamin E, vitamin B1 and folic acid).

Objectives: of the study were clearly explained to women while respecting confidentiality and anonymity.

Results: were included in our study 50 pregnant women whose average age was $27 \pm 6$ years. Food survey revealed daily energy intake $(2116.8 \pm 98 \mathrm{kcal})$ lower than the WHO recommendations $(2250 \pm 50 \mathrm{kcal})$, it is the same for fat intake (28\% of total energy intake AET) while carbohydrate intake (56\% of AET) and protein (16\% of AET) are slightly higher. Mean intakes observed for trace elements (calcium $673.5 \pm 60 \mathrm{mg}$, zinc $4.9 \pm 0.4 \mathrm{mg}$ and sodium $2511 \pm 140 \mathrm{mg}$ ) and vitamins (vitamin C $68 \pm 6 \mathrm{mg}$, vitamin E $3 \pm 0.4 \mathrm{mg}$, vitamin B1 $0.9 \pm 0.06 \mathrm{mg}$ and folic acid 239,17 $\mu \mathrm{g}$ ) are lower than average nutritional needs.

Conclusion: Our study shows that the status of pregnant women studied is unbalanced. Food choices, nutrition education, information and proper monitoring are interesting during pregnancy.

\section{Abstract \#: P 164}

The association between vitamin $K$ and the metabolic syndrome: a ten year follow-up study

Veerle Dam $^{1}$, Geertje W. Dalmeijer ${ }^{1}$, Cees Vermeer ${ }^{2}$, Nadja E. Drummen $^{2}$, Marjo H. Knapen ${ }^{2}$, Yvonne T. van der Schouw ${ }^{1}$, Joline W. J. Beulens ${ }^{1}$

${ }^{1}$ Julius Center for Health Sciences and Primary Care, University Medical Center Utrecht, Utrecht, The Netherlands; ${ }^{2}$ VitaK, Maastricht University; Corresponding author's e-mail: v.dam@umcutrecht.nl

Background: The Metabolic Syndrome (MetS) is a cluster of metabolic abnormalities and is associated with increased risk of diabetes and cardiovascular diseases. Phylloquinone, menaquinones and vitamin K status are associated with several components of MetS, but the association with MetS has hardly been studied to date.

Methods: This study comprised of two cohorts, one of 402 women and one of 400 men (40-80 years). At follow-up 625 participants were still alive and willing to participate. Data were analyzed both cross-sectionally and longitudinally with Poisson and linear regression adjusted for multiple confounders. Baseline phylloquinone/menaquinone intakes were measured with a validated food frequency questionnaire and vitamin $\mathrm{K}$ status with serum desphosphouncarborxylated matrix-Gla protein level.

Results: At baseline 285 (35.6\%) participants had MetS and 172 (35.7\%) at follow-up. Cross-sectionally, high menaquinone intakes were associated $\left(P_{\text {trend }}=0.03\right)$ with a lower prevalence of MetS with a prevalence ratio (PR) of 0.70 (95\% CI: 0.51:0.96) for the highest versus the lowest tertile. At follow-up, the highest tertiles of menaquinones intake ( $\mathrm{PR}=0.62,95 \% \mathrm{CI}$ : 0.40:0.95) and vitamin $\mathrm{K}$ status $\quad(\mathrm{PR}=0.61, \quad 95 \% \quad \mathrm{CI}: \quad 0.40: 0.92) \quad$ were associated $\left(P_{\text {trend }}=0.02\right)$ with a lower occurrence of MetS. These associations were mainly driven by relations with lower triglyceride concentrations for menaquinones and lower waist circumference for vitamin $\mathrm{K}$ status. Phylloquinone intake was not associated with MetS prevalence.

Conclusion: This study shows that a high intake of menaquinones and high vitamin $\mathrm{K}$ status are associated with a lower occurence of MetS.

\section{Abstract \#: P 166}

Associations between dietary intake of methyl donor nutrients in early childhood and body composition at school age

Kim V.E. Braun $^{1}$, Trudy Voortman ${ }^{1,2}$, Jessica C. Kiefte-de Jong ${ }^{1}$, Vincent W.V. Jaddoe ${ }^{1,2,3}$, Albert Hofman ${ }^{1}$, Oscar H. Franco ${ }^{1}$, Edith H. van den Hooven ${ }^{1}$

Epidemiology, Erasmus Medical Center, Rotterdam, Netherlands; ${ }^{2}$ The Generation R Study Group; ${ }^{3}$ Department of Peadiatrics; Corresponding author's e-mail: k.braun@erasmusmc.nl

Background: Deficiency of methyl donor nutrients may lead to dysregulation of DNA methylation, which might generate a disturbed energy and lipid metabolism. We aimed to explore whether intake of methyl donor nutrients in early childhood is associated with body composition at the age of 6 years. 
Methods: This study was performed in 2922 children participating in the Generation R Study, a population-based prospective cohort study. Dietary intakes of vitamin B6, B12, folate, folic acid, and methionine were assessed at a median age of 12.9 months using a validated food frequency questionnaire and were adjusted for total energy intake using the residual method. At the age of 6 years height and weight were measured and BMI was calculated. Body fat was measured using dual-energy X-ray absorptiometry (DXA) and body fat percentage and android:gynoid ratio were calculated.

Analyses: Multivariable linear regression analyses were used to analyse associations between methyl donor nutrient intake and body composition. Nutrient intakes were analysed both as continuous variables and categorized into tertiles. The models were adjusted for maternal age, BMI, education, parity, folic acid supplement use, smoking and alcohol consumption during pregnancy: for household income: and for child's ethnicity, birth weight Z-score, breastfeeding during infancy, intake of infant formula, total energy intake, diet quality score, screen time, and participation in sports.

Results: In models adjusted for all confounders, children with folic acid intake in the highest tertile had a 0.16 SD lower weight $(95 \%$ CI $-0.31,-0.02)$ and a 0.14 SD lower BMI (95\% CI -0.26 , -0.01 ) compared to those in the lowest tertile. Children with vitamin B12 intake in the highest tertile had a 0.13 SD higher android:gynoid ratio (95\% CI $0.00,0.25)$ compared to those in the lowest tertile. A higher intake of methionine was associated with a higher BMI, body fat percentage, and android:gynoid ratio. No associations were observed between vitamin B6 or folate intake and body composition outcomes.

Conclusions: In this population of children, higher folic acid intake was associated with a lower body weight and BMI. In contrast, higher vitamin B12 intake was associated with a higher android:gynoid ratio and a higher methionine intake was associated with a higher BMI, body fat percentage and android:gynoid ratio. Future studies should investigate if intake of methyl donor nutrients in early childhood affects health later in life.

\section{Abstract \#: P 167}

Influence of food insecurity on cognitive ability among adults from six middle-income countries

\section{Ashish Awasthi ${ }^{1}, \mathrm{CM}$ Pandey ${ }^{1}$, Manishsa Dubey ${ }^{2}, \mathrm{Uttam}_{\mathrm{Singh}}{ }^{1}$}

${ }^{1}$ Dept of Biostatistics and Health Informatics, Sanjay Gandhi Postgraduate Institute of Medical Sciences, Lucknow, India; ${ }^{2}$ International Institute for Populations Sciences, Mumbai, India; Corresponding author's e-mail: ashishbhuims@gmail.com

United States Department of Agriculture (USDA) defines Food insecurity is defined as limited or uncertain availability of nutritionally adequate and safe foods or limited or uncertain ability to acquire acceptable foods in socially acceptable ways. Food insecurity is associated with nutrient inadequacy and a variety of unfavorable health outcomes. However, little is known about whether food security is associated with lower cognitive function in the elderly population.

Objective of the present study is to investigate prevalence of food security and effect of this on cognition in adult population of six countries. Data for the present study is borrowed from World Health Organization Study on Global Ageing and adult Health (WHOSAGE) wave-I. Food insecurity was recoded as ever or never having experienced hunger or limiting one's food consumption. Seven cognitive performance tests were used to create a summary variable of cognitive function for each participant. Analysis of Covariance was used to test the relation of cognition with different variables.
Total 45,531 individual of age more than 18 were included in the analysis. Food insecurity was prevalent in the $15.80 \%$ individuals. China has lowest prevalence $(1.22 \%)$ of food insecurity and Ghana has highest (43.54\%) food insecurity among participants of study, India was on third place $(16.90 \%)$ in terms of food insecurity. Average Cognitive score was 43.56 with SD of 11.30, Russian participants have highest cognitive score $46.83 \pm 12.79$ and Indian participants have lowest cognitive score $39.89 \pm 9.25$. Average cognition score among participants who faced food insecurity was low $(40.46 \pm 10.69)$ in comparison of participants who have not suffered food insecurity $(44.13 \pm 11.13)$. ANCOVA reveals that food insecurity is predictor of cognitive ability of individual when controlled with age, sex, place of residence and country of participants.

These results suggest that food insecurity is a related with the lower cognition among adult participants of developing countries.

\section{Abstract \#: P 168}

Bidirectional association between parental child-feeding practices and weight status at 4 and 7 years

Lisa G. Afonso ${ }^{1}$, Carla Lopes ${ }^{2}$, Milton Severo ${ }^{2}$, Susana Santos ${ }^{1}$, Helena Real $^{1}$, Henrique Barros ${ }^{2}$, Andreia Oliveira ${ }^{2}$

${ }^{1}$ EPIUnit, Institute of Public Health, University of Porto, Porto, Portugal; ${ }^{2}$ Department of Clinical Epidemiology, Predictive Medicine and Public Health, University of Porto Medical School, Porto, Portugal and 1EPIUnit, Institute of Public Health, University of Porto, Porto, Portugal; Corresponding author's e-mail: lisa.afonso@ispup.up.pt

Background: Parental child-feeding practices may influence eating behaviors and weight status of children but may also be responsive to their child's weight. Previous studies hampers inferences about the direction of those associations. We aimed to examine the bidirectional associations between parental child-feeding practices (including overt and covert control) and child's weight status at 4 and 7 years of age. Design: Generation XXI is a population-based birth cohort assembled during 2005-2006 at all public maternity units of Porto, Portugal. This analyses include singleton children with data on parental childfeeding practices and weight status at 4 and 7 years old $(n=3708)$. Feeding practices were assessed through a self-administered questionnaire, already adapted and validated to Portuguese pre-school children, that combines the Child Feeding Questionnaire and the Overt/Covert Control scale. Body mass index (BMI) z-scores for sex and age were computed based on the World Health Organization Growth References. Linear regression models were run to estimate the bidirectional association between BMI and each practice. Crosslagged analyses were performed to compare the directions of those associations.

Results: Pressure to eat and overt control at 4 years old showed a significant and negative association with BMI z-score at 7 years old ( $\beta=-0.05495 \% \mathrm{CI}-0.079:-0.030$ and $\beta=-0.04995 \% \mathrm{CI}$ -0.088 : -0.010 respectively). For pressure to eat, a bidirectional association was found, but the strongest association was from BMI $\mathrm{z}$-score (at $4 \mathrm{y}$ ) to the practice (at $7 \mathrm{y}$ ), as confirmed by the Likelihood-Ratio test $(\beta \quad$ standardized $=-0.17 \quad$ vs. $\beta$ standardized $=-0.04$ : Likelihood-Ratio Test: $p<0.001$ ). A higher BMI z-score at 4 years old was significantly associated with higher levels of restriction and covert control at 7 years old, while a lower BMI z-score at 4 years old was significantly associated with higher levels of pressure to eat at 7 years old (restriction: $\beta=0.05695 \% \mathrm{CI}$ 0.033: 0.079: covert control: $\beta=0.06095 \%$ CI 0.037: 0.083 : pressure to eat: $\beta=-0.17395 \% \mathrm{CI}-0.201:-0.145)$. Monitoring showed no association with BMI. 
Conclusion: Parents tend to exert restriction and covert control in response to their child́s BMI, while overt control seems to influence later BMI. Pressure to eat showed a bidirectional association, although BMI influence in the practice was stronger than the reverse.

\section{Abstract \#: P 169 \\ Concordance of different methods of assessment of nutritional status in patients included on the waiting list for liver transplantation}

Beatriz López-Calviño ${ }^{1}$, María Teresa García-Rodríguez ${ }^{2}$, Salvador Pita-Fernández ${ }^{1}$, Sonia Pértega-Díaz ${ }^{1}$,

Teresa Seoane-Pillado ${ }^{1}$, María del Carmen Piñón-Villar ${ }^{2}$

${ }^{1}$ Clinical Epidemiology and Biostatistics Research Group, Instituto de Investigación Biomédica de A Coruña (INIBIC), Complexo Hospitalario Universitario de A Coruña (CHUAC), SERGAS, Universidade da Coruña, A Coruña, Spain; ${ }^{2}$ Department of Digestive Diseases, Complexo Hospitalario Universitario de A Coruña (CHUAC), SERGAS, Universidade da Coruña, A Coruña, Spain; Corresponding author's e-mail: Beatriz.Lopez.Calvino@sergas.es

Background: Malnutrition is associated with progressive liver failure, the most malnourished patients have a worse prognosis for the illness. There isn't consensus among the most effective methods for assessing the nutritional status of these patients, as the disease itself affects the values obtained.

Aim: To study the concordance between different valuation methods to determine the nutritional status

Methods:

Design: Observational study.

Setting: A Coruña Hospital(northwest Spain).

Period: 2010-2014.

Inclusion criteria: Patients $\geq 18$ years in transplant waiting list.

Exclusion criteria: Patients with impaired.

Sample size: $\mathrm{n}=110$ (precision $= \pm 9.4 \%$; security $=95 \%$ ).

Measurements: Demographic characteristics, body mass index(BMI), etiologies, decompensation, Child-Pugh and MELD scales, System of Nutritional Control(CONUT), Nutritional Risk Index(NRI), Prognostic Nutritional Index(PNI-O), SENPE criteria, Subjective Global Assessment Test(SGA).

Analysis: Descriptive, concordance(Kappa index). Diagnostic validity:Area Under Curves(AUC), Youden index, sensitivity, specificity. Ethical-legal aspects: Approved by Clinical Research Ethical Committee of Galicia (2010/081).

Results: The mean age was $56.9 \pm 8.2$ years, $72.7 \%$ men and $58.2 \%$ with IMC $\geq 25 \mathrm{~kg} / \mathrm{m}^{2}$. The score of liver dysfunction according to MELD scale of $14.9 \pm 6.0$ and Child-Pugh score of $8.4 \pm 2.1$, being the most frequent degree of liver dysfunction as rated by the Child-Pugh was B $(48.6 \%)$. The most common etiologies were alcoholic cirrhosis $(52.7 \%)$, viral $(30.9 \%)$ and liver tumor (30.9\%). Decompensation most frequently presented were ascites $(79.1 \%)$, encephalopathy $(69.9 \%)$ and portal hypertension $(68.2 \%)$. The percentage of malnutrition at the time of inclusion in liver traplante list varies according to the method of valuation: $90.9 \%$ according to the CONUT, $89.1 \%$ Nutritional Risk Index (NRI), $87.3 \%$ PNI-O, 64.6 \% SENPE criteria, $50.9 \%$ Subjective Global Assessment Test (SGA). There isn't good agreement between nutritional methods, being the PNI-O which gets better agreement, with an overall kappa of $\mathrm{K}=0.364 ; p<0.001$. Considering the PNI-O as a reference criterion to assess the nutritional status in these patients. It was observed a very good agreement with NRI $(\mathrm{K}=0.826$; $p<0.001)$; moderate with SENPE criteria $(\mathrm{K}=0.420 ; p<0.001)$ and CONUT $(\mathrm{K}=0.441 ; p<0.001)$ and poor with SGA
$(\mathrm{K}=0.042 ; p=0.519)$. The diagnostic validity of NRI and CONUT is studied to identify the state of malnutrition being the reference criterion the PNI-O. Observed that the best predictor would be the NRI with AUC $=0.987$ and identified as optimal cutoff 98.39 with Youden Index $=0.958$ (sensitivity: $96.8 \%$; specificity: $92.9 \%$ ). The CONUT obtain an AUC $=0.847$ and identified as optimal cutoff 3.50 with Youden.

Index $=0.536$ (sensitivity:75.0\%;specificity:78.6\%).

Conclusion: Patients included in liver transplant list show a high percentage of malnutrition.

Methods to identify the state of malnutrition in these patients would be Nutritional Prognostic Index(PNI-O), Nutritional Risk Index(NRI) and CONUT.

\section{Abstract \#: P 170}

Intuitive eating is inversely associated with body weight status in a general population from the nutrinet-santé study

Géraldine M Camilleri ${ }^{1}$, Caroline Méjean ${ }^{1}$, France Bellisle ${ }^{1}$, ${ }_{\text {Valentina A Andreeva }}{ }^{1}$, Emmanuelle Kesse-Guyot ${ }^{1}$, Serge Hercberg ${ }^{1}$, Sandrine Péneau ${ }^{1}$

${ }^{1}$ Université Paris 13, Equipe de Recherche en Epidémiologie Nutritionnelle, Centre de Recherche en Epidémiologie et Statistiques, Inserm (U1153), Inra (U1125), Cnam, COMUE Sorbonne Paris Cité, Bobigny, France; Corresponding author's e-mail:

g.camilleri@eren.smbh.univ-paris13.fr

Background: In a social context where thinness is perceived as an ideal, weight loss programs based on energy restriction are becoming more and more common despite their limited effect on the long-term and associated psychological and physiological adverse effects. Conversely, intuitive eating is an adaptive dietary behavior that emphasizes eating in response to physiological hunger and satiety cues, together with low preoccupation toward food. It has been proposed as an alternative to weight loss programs that would promote a healthier food-mind-body connection. We therefore aimed at studying the relationship between intuitive eating and weight status in a large sample of adult general population.

Methods: A total of 11,774 men and 40,389 women aged $\geq 18$ years participating in the NutriNet-Santé cohort were included in this crosssectional analysis. Self-reported weight and height were collected as well as intuitive eating levels using the validated French version of the Intuitive Eating Scale-2 (IES-2). The association between intuitive eating, its subscales (categorized into quartiles) and overweight/ obesity was assessed using multinomial logistic regression models adjusted for socio-demographic and lifestyle factors.

Results: Median IES scores were moderately greater in men than in women (3.6 vs. $3.3, P<0.0001)$. A higher IES score was strongly associated with lower overweight/obesity in both men and women. The strongest associations were observed in women [quartile 4 vs. 1 for both overweight (excluding obesity) (OR (95\% CI): 0.19 (0.17, $0.20)$ ) and obesity (OR: $0.09(0.08,0.10))]$. Associations in men were as follow [quartile 4 vs. 1 for both overweight (excluding obesity) (OR: $0.43(0.38,0.48))$ and obesity (OR: $0.14(0.11,0.18))]$. All IES subscales were inversely associated with weight status: in particular Eating for Physical Rather than Emotional Reasons subscale, followed by Reliance on Hunger and Satiety Cues and Unconditional Permission to Eat subscales.

Conclusion: Greater intuitive eating is associated with a lower prevalence of overweight and obesity which supports the importance of eating in response to hunger and satiety signals. These data bear out the relevance to promote such behavior for obesity prevention and treatment. 


\section{Abstract \#: P 172}

Influence of household food insecurity on the nutritional status of under-five children in urban and rural areas of Ondo state

Dr. Francis Adegoke Akanbiemu (MBBS, MPH, FWACP) Consultant Community \& Public Health Physician ${ }^{1}$, Dr. Akinola Ayoola Fatiregun (MBBS, MSc: Epid \& Med Stat., FWACP) Consultant Community \& Public Health Physician ${ }^{2}$, Dr.Adeniyi Francis Fagbamigbe $(\mathrm{PhD})^{3}$

${ }^{1}$ Ondo State Hospitals Management Board, Ministry of Health, Akure, Ondo State, Nigeria; ${ }^{2}$ Ondo State Coordinator, World Health Organization, Akure, Ondo State, Nigerian; ${ }^{3}$ Department of Epidemiology and Biostatistics, Faculty of Public Health, College of Medicine, University of Ibadan, Ibadan, Nigeria; Corresponding author’s e-mail: adegokeakans@yahoo.co.uk

Introduction: Food and other related factors have been reported to influence the occurrence of malnutrition in under-five children. However, study confirming this association in Ondo state is scarce. This study assessed the relationship between microbial, Socio-economic and household food insecurity factors on the nutritional status of under-five children in Ondo State, Nigeria.

Methodology: This study was a comparative cross-sectional study conducted in rural and urban Areas of Ondo State between July and August 2012. A four-stage sampling technique was used to select 826 primary care givers and their under-five children. Anthropometric measurements and laboratory assessments were used to measure nutritional status and biological parameters of the under-five children, while household food insecurity was assessed using an adapted United States Department of Agriculture Household Food Insecurity Access Scale. Data was analysed using descriptive statistics, Chi square test to determine association between outcome and explanatory variables, and t-test to compare outcomes in rural and urban settings and predictors of the nutritional status were determined by binary logistic regression with statistical significance set at $5 \%$.

Results: The mean age of the children was $20.5 \pm 13.9$ months. The predictor of stunting in the rural areas was food insecure households (95 \% CI: 1.112-6.196: $p=0.028$ ), while in urban areas, they were household heads lack of formal education (95\% CI: 0.5-1.0: $p=0.048$ ), food insecure households (95 \% CI: 3.5-15.0: $p<0.001$ ) and lower wealth quintiles (95\% CI: 8.0-44.5: $p<0.001$ ). In the rural areas, being in food insecure households (95\% CI: 1.2-8.9: $p=0.020$ ) and lower wealth quintiles (95\% CI: 0.4-0.8: $p<0.001$ ) were the predictors of under-weight, while in urban areas, food insecure households (95\% CI: 1.1-6.6: $p=0.032$ ) and lower wealth quintiles (95\% CI: 0.3-0.7: $p<0.001$ ) predicts underweight. The proportion of children with intestinal parasite were higher in urban area $(39.9 \%)$, compared with $12.7 \%$ in the rural area $(p<0.001)$. The most predominant parasitic infestation was Ascaris lumbricoides. The rural area has the highest prevalence of moderate to severe anaemia $(23.5 \%)$ compared to urban area $(1.0 \%)$. Policies that will improve the socio-economic status of its citizens by increasing universal access to education and reduce unemployment were suggested.

\section{Abstract \#: P 173}

Motives for dishes choice during home-meal preparation: results from a large sample of the nutrinet-santé study

Pauline Ducrot ${ }^{1}$, Caroline Méjean ${ }^{1}$, Benjamin Alles ${ }^{1}$, $\overline{\text { Philippine Fassier }}^{1}$, Serge Hercberg ${ }^{1}$, Sandrine Péneau ${ }^{1}$

${ }^{1}$ Université Paris 13, Equipe de Recherche en Epidémiologie

Nutritionnelle, Centre de Recherche en Epidémiologie et Statistiques,
Inserm (U1153), Inra (U1125), Cnam, COMUE Sorbonne Paris Cité, F-93017 Bobigny, France; Corresponding author's e-mail: p.ducrot@eren.smbh.univ-paris13.fr

Background: Although cooking practices have greatly changed over time, there are few data available on contemporary dishes choices during meal preparation. Understanding dishes choice motives seems particularly important since some practices such as ready-meal consumption have been associated with lower quality diet and with a greater risk of overweight whereas benefits have been attributed to foods cooked at home. We therefore aimed at describing individual motives when choosing the dishes that will be prepared at home during weekdays and weekend.

Methods: The importance of 27 criteria associated with the choice of dishes to be prepared at home was assessed in 53,025 participants of the NutriNet-Santé study. Dimensions of dishes choice motives were investigated using exploratory factor analysis. Mean ratings of motives during weekdays and weekend were compared using Student t-test. Socio-demographic profiles of individuals giving importance to constraints were described and compared to others by using Student's tests, Chi square or non-parametric Wilcoxon's rank-sum tests.

Results: Five dimensions of dishes choice motives emerged from the exploratory factor analysis: healthy diet (explained variance: $48.3 \%$ ), constraints $(19.0 \%)$, pleasure $(12.1 \%)$, specific diets $(11.0 \%)$ and organization $(9.6 \%)$. The healthy diet factor was the most important in weekdays (mean rating: 3.93) and weekends (3.90). Pleasure (3.61) had higher score than constraints $(3.54)$ in weekend $(p<0.0001)$ while the opposite was observed during weekdays (3.42 vs 3.77 , respectively) $(p<0.0001)$. Organization was more important during weekdays $(2.89)$ than weekend $(2.75)(p<0.0001)$ and specific diets was no significantly different during both contexts. People who considered constraints as important during weekdays were more often women, younger, had lower income, were more likely to have medium cooking skills, enjoyed cooking less and spent less time in meal preparation (all $p<0.0001$ ).

Conclusion: This study highlighted the factors involved in the choice of dishes to be prepared at home during weekdays and weekends, as well as characteristics of individuals who perceive constraints as important. These data allow a better identification of levers to promote home dishes preparation, which can have implication for public health.

\section{Abstract \#: P 174}

Effectiveness of front-of-pack nutrition labels in french adults: results from the nutrinet-santé cohort study

Pauline Ducrot $^{1}$, Caroline Méjean ${ }^{1}$, Chantal Julia ${ }^{1}$, Emmanuelle Kesse-Guyot $^{1}$, Mathilde Touvier ${ }^{1}$, Léopold Fezeu ${ }^{1}$, Serge Hercberg ${ }^{1}$, Sandrine Péneau ${ }^{1}$

${ }^{1}$ Université Paris 13, Equipe de Recherche en Epidémiologie Nutritionnelle, Centre de Recherche en Epidémiologie et Statistiques, Inserm (U1153), Inra (U1125), Cnam, COMUE Sorbonne Paris Cité, F-93017 Bobigny, France; Corresponding author's e-mail: p.ducrot@eren.smbh.univ-paris13.fr

Background: Front-of-pack (FOP) nutrition labels have been shown to be efficient tools to increase consumer awareness regarding product healthiness. To date, no consensus has emerged on the most appropriate FOP nutrition label to help consumers in making informed choices. We aimed to compare in a large sample of adults, effectiveness of three types of labels currently in use: nutrient-specific, graded and simple summary systems.

Methods: The FOP labels' effectiveness was assessed by measuring the labels' acceptability and understanding in 13,578 participants of 
the NutriNet-Santé cohort, representative of the adult French population. Participants were exposed to five conditions, including four FOP formats: Guidelines Daily Amount (GDA), Multiple Traffic Lights (MTL), 5-Color Nutrition Label (5-CNL), Green Tick and a "no label" condition. Five different product categories were tested: prepared fish dishes, pizzas, dairy products, breakfast cereals, and appetizers. Acceptability was evaluated by several indicators: attractiveness, liking and perceived cognitive workload. Objective understanding was assessed by the percentage of correct answers when ranking three products according to their nutritional quality. Labels' effectiveness was assessed among various socio-demographic subgroups. Differences in labels' effectiveness were compared with Chi square tests.

Results: The 5-CNL label was viewed as the easiest format to identify and as the one requiring the lowest amount of effort and time to understand. GDA was considered as the least easy to identify and to understand, despite being the most attractive and liked label. All FOP labels were found to be effective in ranking products according to their nutritional quality compared with the "no label" situation, although they showed differing levels of effectiveness $(p<0.0001)$. Globally, the 5-CNL performed best, followed by MTL, GDA and Tick formats. Similar findings were observed across socio-demographic characteristics.

Conclusion: The graded 5-CNL label was easy to identify, simple and rapid to understand: it performed well when comparing the products' nutritional quality. Therefore, it is likely to present advantages in real shopping situations where choices are usually made quickly.

\section{Abstract \#: P 175}

\section{Relationship between serum vitamin $d$ and longitudinal development of BMI and waist-HIP ratio: repeated measures analysis extending over three decades}

Susanna Lehtinen-Jacks ${ }^{1}$, Monica Leu ${ }^{2}$, Monica Hunsberger ${ }^{2}$, Henrik Zetterberg $^{3}$, Lauren Lissner ${ }^{2}$

${ }^{1}$ School of Health Sciences, University of Tampere, Tampere, Finland: Department of Public Health and Community Medicine, Institute of Medicine, Sahlgrenska Academy, University of Gothenburg, Gothenburg, Sweden; ${ }^{2}$ Department of Public Health and Community Medicine, Institute of Medicine, Sahlgrenska Academy, University of Gothenburg, Gothenburg, Sweden; ${ }^{3}$ Department of Psychiatry and Neurochemistry, Institute of Neuroscience a Physiology, Sahlgrenska Academy, University of Gothenburg, Gothenburg, Sweden: UCL Institute of Neurology, Queen Square, London, United Kingdom; Corresponding author's e-mail: susanna.lehtinen-jacks@uta.fi

Background: Serum vitamin D (25(OH)D) levels are associated with adiposity: however, the direction of the association remains unclear. We assessed the relationship between $25(\mathrm{OH}) \mathrm{D}$ and longitudinal development of body mass index (BMI) and waist-hip ratio (WHR) in middle-aged women followed for as many as 35 years.

Methods: The Population Study of Women in Gothenburg started in $1968 / 1969$ as a health study of 1462 randomly selected women aged 38-60 years residing in Gothenburg, Sweden. Four follow-up examinations were made between $1974 / 75$ and 2000/03. Levels of $25(\mathrm{OH}) \mathrm{D}$ were analyzed in serum samples drawn at baseline in 1227 $(84 \%)$ women. The $25(\mathrm{OH}) \mathrm{D}$ values were dichotomized into the lowest quartile $(51.45 \mathrm{nmol} / \mathrm{L})$ versus the upper three quartiles combined. BMI, used as an indicator for overall adiposity, and WHR for central adiposity, were measured at each study visit. We used linear regression and repeated measures models (SAS PROC
MIXED), respectively, to model the baseline cross-sectional and the longitudinal associations between $25(\mathrm{OH}) \mathrm{D}$ and BMI or WHR. We built the repeated measures models (1) including all study visits in the longitudinal analysis: (2) including only the follow-up visits and adjusting for the baseline difference in BMI or WHR. Age at baseline, country of origin, education, socioeconomic position, smoking, menopause, leisure time physical activity, and season of $25(\mathrm{OH}) \mathrm{D}$ measurement were taken into account in the models.

Results: At baseline, mean BMI was $25.2 \mathrm{~kg} / \mathrm{m}^{2}$ in women with low $25(\mathrm{OH}) \mathrm{D}$ and $23.8 \mathrm{~kg} / \mathrm{m}^{2}$ in the other women $\left(\mathrm{X}^{2}\right.$ test for the difference, $p<0.001)$. The respective figures for WHR were 0.75 and $0.74(p<0.001)$. Adjusted for potential confounders, low $25(\mathrm{OH}) \mathrm{D}$ was associated with higher BMI at baseline $(\beta=1.45,95 \% \mathrm{CI}$ $0.97: 1.92)$ and in the longitudinal analyses including all visits ( $\beta=1.47,95 \%$ CI 0.99:1.94). When we analyzed only the follow-up visit and adjusted for baseline BMI, the difference was negligible $(\beta=0.01$, $95 \%$ CI $-0.27: 0.24)$. Similarly, low 25(OH)D was associated with higher WHR at baseline $(\beta=0.012,95 \% \mathrm{CI}$ 0.006:0.019) and longitudinally when all study visits were included ( $\beta=0.011,95 \%$ CI $0.005: 0.018$ ), but not when the baseline difference in WHR was taken into account in the repeated measures model ( $\beta=-0.001,95 \% \mathrm{CI}-0.007: 0.005)$.

Conclusion: The associations observed between baseline 25(OH)D level and the longitudinal development of BMI and WHR were explained by the baseline difference in the respective adiposity measures. Serum $25(\mathrm{OH}) \mathrm{D}$ level in middle-aged women predicted long-lasting differences in BMI and WHR.

Conflict of Interest: None Disclosed.

Funding: Research relating to this abstract was funded by the FORTE Research Center EpiLife, the Swedish Research Council, and the International Guest Researcher Program at the Institute of Medicine, the Sahlgrenska Academy, University of Gothenburg.

\section{Abstract \#: P 176}

Effects of B-vitamin supplementation on depressive symptoms and health-related quality of life in older adults with elevated homocysteine levels

Elisa J. de Koning ${ }^{1}$, Nikita L. van der Zwaluw ${ }^{2}$, Janneke P. van Wijngaarden $^{2}$, Evelien Sohl, ${ }^{1}$ Elske M. Brouwer-Brolsma ${ }^{2}$, Harm W.J. van Marwijk ${ }^{3}$, Anke W. Enneman ${ }^{4}$, Karin M.A. Swart ${ }^{1}$, Suzanne C. van Dijk ${ }^{5}$, Annelies C. Ham ${ }^{5}$, Nathalie van der Velde ${ }^{5}$, André G. Uitterlinde $^{5}$, Brenda. J.W.H. Penninx ${ }^{6}$, Petra J.M. Elders ${ }^{7}$, Paul Lips ${ }^{8}$, Rosalie A.M. Donukshe-Rutten ${ }^{2}$, Natasja M. van Schoor ${ }^{1}$, Lisette C.P.G.M. de Groot ${ }^{2}$

${ }^{1}$ Dpt. of Epidemiology and Biostatistics/EMGO Institute for Health and Care Research, VU University Medical Centre Amsterdam, The Netherlands; ${ }^{2}$ Division of Human Nutrition, Wageningen University, Wageningen, The Netherlands; ${ }^{3}$ Dpt. of General Practice and Elderly Care Medicine/EMGO Institute for Health and Care Research, VU University Medical Centre Amsterdam, The Netherlands; ${ }^{4}$ Dpt. of Internal Medicine, Erasmus University Medical Centre, Rotterdam, The Netherlands; ${ }^{5}$ Dpt. of Internal Medicine, Erasmus University Medical Centre, Rotterdam, The Netherlands; ${ }^{6}$ Dpt. of Psychiatry/ EMGO Institute for Health and Care Research, VU Univerisity Medical Centre, Amsterdam, The Netherlands; ${ }^{7}$ Dpt. of General Practice and Elderly Care Medicine, VU University Medical Centre Amsterdam, The Netherlands; ${ }^{8}$ Dpt. of Internal Medicine,

Endocrinology section/EMGO Institute for Health and Care Research, VU University Medical Centre Amsterdam, The Netherlands; Corresponding author's e-mail: ej.dekoning@vumc.nl 
Background: Elevated plasma homocysteine (Hcy) levels are common in older persons and are a possible risk factor for depressive symptoms and reduced health-related quality of life (HR-QoL). Lowering Hcy levels by supplementing vitamin B12 and folic acid may reduce depressive symptoms and improve HR-QoL in older adults.

Methods: Data from the B-vitamins for the PRevention Of Osteoporotic Fractures (B-PROOF) study were used: a randomized, doubleblind, placebo-controlled trial. Depressive symptoms and HR-QoL were predefined secondary outcome measures of the B-PROOF study. Participants $(\mathrm{N}=2919, \geq 65$ years $)$ with elevated Hcy levels $(12-50 \mu \mathrm{mol} / \mathrm{L})$ were randomized to either $500 \mu \mathrm{g}$ vitamin B12 and $400 \mu \mathrm{g}$ folic acid daily or placebo for two years. Both tablets contained $15 \mu \mathrm{g}$ vitamin D3. Depressive symptoms were measured with the Geriatric Depression Scale-15 (GDS-15). HR-QoL was assessed with the Short Form-12 (SF-12, mental and physical components) and EuroQol-5 Dimensions (EQ-5D, Index score and Visual Analogue Scale). Cross-sectional baseline associations were examined using Cox regression with fixed time points. Two-year change scores of depressive symptoms and HR-QoL were tested for differences between treatment groups with Analysis of Covariance, adjusted for age, sex, study location, and baseline Hcy levels.

Results: Seven percent $(\mathrm{N}=200)$ of participants had a GDS-15 score of $\geq 5$ at baseline, indicating clinically relevant depressive symptoms. At baseline, no significant cross-sectional associations between Hcy and depressive symptoms or HR-QoL were observed in the fully adjusted models. Longitudinally, Hcy concentrations decreased more in the intervention group, but two-year change scores of the GDS-15 and three of the four HR-QoL measures did not differ between groups. However, the placebo group slightly declined on the EQ-5D Index score, whereas the intervention group remained stable over time (mean change -0.02 vs. $0.00, p=0.004$ ).

Conclusion: Baseline cross-sectional analyses and two-year supplementation with vitamin B12 and folic acid in older adults with elevated Hcy levels showed that lowering Hcy concentrations did not reduce depressive symptoms and had a limited positive effect on HRQoL.

\section{Abstract \#: P 177}

\section{Adherence to the WCRF/AICR dietary recommendations for cancer prevention and risk of cancer in elderly, the chances consortium}

\section{Nicole Jankovic $^{1}$}

${ }^{1}$ Division of Human Nutrition, Wageningen University, Wageningen, The Netherlands and Biometry, and Epidemiology, Institute of Medical Informatics, University Duisburg-Essen, Essen, Germany; Corresponding author's e-mail: nicole.jankovic@uk-essen.de

Background: In 2007, the World Cancer Research Fund/American Institute for Cancer Research (WCRF/AICR) formulated specific dietary recommendations for cancer prevention. We examined the association between the adherence to these recommendations and cancer risk in elderly from Europe and the US.

Methods: Meta-analysis of individual participant data including 362,114 participants ( $>60$ years old: $43 \%$ women), from seven prospective cohort studies, who were free from cancer at enrolment. The WCRF/AICR diet score was constructed based on the WCRF/ AICR recommendations regarding: (1) energy-dense foods and sugary drinks, (2) plant foods, (3) red and processed meat, and (4) alcoholic drinks. Cox proportional hazards regression was used to examine the association between the diet score and cancer risks. Adjusted, cohort-specific hazard ratios (HR) were pooled using random-effects meta-analysis. Risk Advancement Periods (RAP) were calculated to quantify the time period by which the risk of cancer was postponed among those adhering to the recommendations. Results: After a median follow-up of 11-15 years across cohorts, 69,708 cancer cases were identified. One point increase in the WCRF/ AICR diet score (range 0 (no adherence) to 4 (complete adherence)) was statistically significantly associated with a lower risk of total (HR: 0.94, $95 \%$ CI: 0.92-0.97), and cancer at specific anatomical sites of colorectal (HR: 0.84, $95 \%$ CI: 0.80-0.89), and prostate (HR: 0.94, $95 \%$ CI: 0.92-0.97), but not breast or lung. The adherence to an additional component of the WCRF/AICR diet score significantly postponed the incidence of any cancer site by -1.6 (95\% CI: -4.09 to -2.16 ) years.

Conclusion: Adherence to the WCRF/AICR dietary recommendations is associated with lower risk of cancer, later in life.

\section{Abstract \#: P 178}

\section{Socioeconomic status associations with body mass index in adolescents of Brazil and Europe: the mediation effect of energy balance-related behaviors}

Marcus Vinícius Nascimento-Ferreira, PhD Student ${ }^{1}$, Tara RendoUrteaga, PhD ${ }^{1,2}$, Augusto César F. de Moraes, PhD ${ }^{1,2}$, David Jimenez-Pavón, $\mathrm{PhD}^{2,3}$, Anthony Kafatos, $\mathrm{PhD}^{4}$, Stefaan De Henauw, $\mathrm{PhD}^{5}$, Odysseas Androutsos, $\mathrm{PhD}^{6}$, Romana Roccaldo, $\mathrm{PhD}^{7}$, Frederic Gottrand ${ }^{1}$

${ }^{1}$ Youth/Child and cArdiovascular Risk and Environmental (YCARE) Research Group, School of Medicine, University of São Paulo, São Paulo, Brazil; ${ }^{2}$ Growth, Exercise, NUtrition and Development (GENUD) Research Group, School of Health Science (EUCS), University of Zaragoza, Zaragoza, Spain; ${ }^{3}$ GALENO Research Group, Department of Physical Education, University of Cadiz, Puerto Real, Spain; ${ }^{4}$ University of Crete, School of Medicine, Greece; ${ }^{5}$ Department of Nutrition and Dietetics, School of Health Science and Education, Harokopio University, Athens, Greece; ${ }^{6}$ Agricultural Research Council, Food and Nutrition Research Centre (CRA-NUT), Rome, Italy/Centre d'Investigation Clinique, CIC-9301-InsermCH\&U, Lille, France; ${ }^{7}$ Department of Biosciences, Unit for Preventive Nutrition, Karolinska Institutet, Stockhol, Sweden/ImFine Research Group. Department of Health and Human Performance. Technical University of Madrid, Madrid, Spain; Corresponding author's e-mail: marcus_nascimento_@hotmail.com

Background: Energy balance-related behaviors (EBRBs) are associated with overweight and obesity. Among adolescents, patterns of EBRBs may be different between countries and socioeconomic groups. Our objective was to assess the association between socioeconomic status (SES) and body mass index (BMI) and to examine the possible mediation effect of physical activity (PA), sedentary behavior (SB) and sleep time in these associations among Brazilian and European adolescents.

Methods: Data were obtained from adolescents (aged 12-19 years) from two cross-sectional studies, one conducted in ten European cities (HELENA study: $\mathrm{n}=3308,52.2 \%$ females) and the other in Brazil (BRACAH study: $\mathrm{n}=991,54.5 \%$ females). The outcome of this study was BMI. The predictor variable was family SES and the possible mediating variables were: PA (active when they accumulated at least $60 \mathrm{~min} /$ day of moderate-to-vigorous PA), SB (total time habitually spent in front of the television, the computer and/or playing video games) and sleep time (average hours sleep per day). The confounding variables were: gender, age and country (only for Europe). To qualify as a mediator, the presumed mediators (PA, SB or sleep) had to be associated with the predictor variable (SES, path a) 
and with the outcome (BMI, path b). The association between SES and BMI was analyzed by linear regression. To assess whether these associations were mediated by the EBRBs, the product of coefficients test proposed by MacKinnon (path $\mathrm{a}^{*}$ path $\mathrm{b}$, indirect effect or mediated effect) and direct effect (c', no mediated effect calculated after the inclusion of the mediators in the model) was performed. Bootstrap corrected confidence intervals (CI) were used for indirect and direct effect. The proportion of total effect mediated $(a * b /(a * b$ $\left.+c^{\prime}\right)$ ) was calculated.

Results: We found significant association between SES and BMI ( $\beta$ : -0.344 : $95 \%$ CI: -0.530 to 0.158 ) in both studies. However, no mediation effects were found between this association neither in Brazilian adolescents nor in European boys. For European girls, only SB was identified as a mediator, where: path a was $\beta$ : $-0.114(95 \%$ CI: -0.173 to -0.056 ) and path b was $\beta$ : 0.244 (95 CI \%: 0.022 to $0.467)$. Moreover, the indirect and direct effects were statistically significant for this association $(\beta$ : $-0.114: 95 \mathrm{CI} \%:-0.173$ to -0.056 and $\beta$ : -0.474 : $95 \mathrm{CI} \%$ : -0.747 to -0.201 , respectively). Thus, the proportion of total effect mediated by SB was $6 \%$.

Conclusion: There are significant associations between SES and BMI in Brazilian and European adolescents. However, only for European girls, the association was partially mediated by sedentary behavior.

\section{Abstract \#: P 179}

\section{The relationship between vitamin $K$ and peripheral arterial} disease and congestive heart failure

Linda E.T. Vissers ${ }^{1}$, Geertje W. Dalmeijer ${ }^{1}$, Jolanda M.A. Boer ${ }^{2}$, W. M. Monique Verschuren ${ }^{1}$, Yvonne T. van der Schouw ${ }^{1}$, Joline W.J. Beulens ${ }^{1}$

${ }^{1}$ Julius center; ${ }^{2}$ RIVM; Corresponding author's e-mail: l.e.t.vissers@students.uu.nl

Background: Dietary intake of vitamin K1 (phylloquinone) and vitamin $\mathrm{K} 2$ (menaquinones) is thought to decrease cardiovascular disease risk by reducing vascular calcification. Although studies so far have focused mainly on coronary heart disease and stroke, a recent study suggested a relationship between vitamin $\mathrm{K}$ status and peripheral arterial disease (PAD) and congestive heart failure (CHF). The relationship of vitamin $\mathrm{K}$ intake with PAD or CHF has not been investigated to date.

Methods: We investigated the association between intake of phylloquinone and menaquinones with $\mathrm{PAD}$ and $\mathrm{CHF}$ in a prospective cohort with 36,629 participants. Occurrence of PAD and CHF was obtained by linkage to national hospital discharge registries. Baseline intake of phylloquinone and menaquinones were estimated using a validated 178-item food-frequency questionnaire. Multivariate cox regression adjusted for cardiovascular risk factors, lifestyle and dietary factors was used to estimate the associations of intake of phylloquinone and menaquinones with PAD and CHF.

Results: During a follow-up of $12.1 \pm 2.1$ years, 489 incident cases of $\mathrm{PAD}$ and 243 cases of $\mathrm{CHF}$ were documented. Menaquinones intake was associated with a reduced risk of PAD (hazard ratio (HR) 0.72 , $95 \%$ CI [0.52-0.96]) for the highest versus lowest quartile: p-tren$\mathrm{d}=0.08$ )but was not associated with CHF (HR 0.96, $95 \% \mathrm{CI}$ $[0.70-1.33])$ for the highest versus lowest tertile. Phylloquinone intake was not associated with risk of PAD HRQ4 versus Q1 1.18, $95 \% \mathrm{CI}$ [0.92-1.53]) or CHF (HRT3 versu T1 0.96, $95 \%$ CI [0.70-1.33]).

Conclusion: High intake of menaquinones, but not phylloquinone, was associated with a reduced risk of PAD. Neither menaquinones nor phylloquinone were associated with risk of $\mathrm{CHF}$.

\section{Abstract \#: P 180}

\section{Dietary patterns during pregnancy and gestational weight gain:} the generation $\mathbf{R}$ study

Myrte J. Tielemans ${ }^{1}$, Nicole S. Erler ${ }^{2}$, Elisabeth T.M. Leermakers ${ }^{1}$, Marion van den Broek ${ }^{1}$, Vincent W.V. Jaddoe ${ }^{3}$, Eric A.P. Steegers ${ }^{4}$, Oscar H. Franco ${ }^{1}$, Jessica C. Kiefte-de Jong ${ }^{1}$

${ }^{1}$ Department of Epidemiology, Erasmus MC University Medical Center, Rotterdam the Netherlands; ${ }^{2}$ Department of Statistics, Erasmus MC University Medical Center, Rotterdam the Netherlands; ${ }^{3}$ Department of Paediatrics, Erasmus MC University Medical Center, Rotterdam the Netherlands; ${ }^{4}$ Department of Obstetrics and gynaecology, Erasmus MC University Medical Center, Rotterdam the Netherlands; Corresponding author's e-mail: m.tielemans@erasmusmc.nl

Background: Abnormal weight gain during pregnancy (i.e. too little or too much) may increase risk of adverse pregnancy outcomes. Nutrition during pregnancy may influence gestational weight gain (GWG), and research has mainly focussed on effects of macronutrients, however little is known about overall diet during pregnancy in relation to GWG.

Objective: To examine whether different dietary patterns (guidelinebased or data-driven) are associated with gestational weight gain in Dutch pregnant women participating in a population-based cohort study.

Methods: We included 2591 Dutch pregnant women with a normal pre-pregnancy BMI who participated in the Generation $\mathrm{R}$ cohort study, a population-based study in Rotterdam, the Netherlands (enrolment 2002-2006). Diet was assessed in early pregnancy by a 293-item food frequency questionnaire. We used Principal Component Analysis to identify three data-driven dietary patterns, namely a 'Vegetable, oil and fish', a 'Nuts, high fibre cereals and soy', and a 'Margarine, sugar and snacks' dietary pattern. Together, they explained $25.8 \%$ of the variance in maternal dietary intake. An adapted version of the Dutch Healthy Diet index, reflecting adherence to the Dutch dietary recommendations, served as a guideline-based dietary pattern. Women visited the research centre during each trimester of pregnancy to have their weight measured. Total GWG and trimester specific GWG were calculated using the measured weights and a self-reported pre-pregnancy weight. The dietary patterns were evaluated in standard deviation adherence scores (SDS). Multivariable linear regression was performed with adjustment for sociodemographic and lifestyle factors.

Results: Higher adherence to the 'Vegetable, oil and fish' dietary pattern was associated with higher total GWG $(0.19 \mathrm{~kg}$ (95\% CI 0.02: 0.37) per SDS), and trimester-specific GWG in first trimester (0.21 kg (95\% CI 0.08: 0.34) per SDS), but not with GWG later in pregnancy. The 'Nuts, high fibre cereals and soy' dietary pattern was associated with a lower GWG in first trimester $(-0.14 \mathrm{~kg}(95 \% \mathrm{CI}$ -0.27 : -0.01$)$ per SDS), but not later in pregnancy or with total GWG. Higher adherence to the 'Margarine, sugar and snacks' dietary pattern was associated with a lower total GWG $(-0.39 \mathrm{~kg}(95 \%$ CI -0.75 : -0.04$)$ per SDS), but not with trimester-specific GWG. The Dutch Healthy Diet index was not associated with total GWG or with trimester-specific GWG.

Conclusion: Our results suggest that dietary patterns can be related to GWG, mainly in first trimester. However, we found no association between adherence to the guideline-based dietary pattern and GWG, which implies that further research is needed to improve dietary guidelines during pregnancy. 


\section{Abstract \#: P 181}

\section{The influence of socio-economic determinants on changes} in dietary quality in elderly people

Josje D. Schoufour $^{1}$, Samuel P. T. Nunn ${ }^{1}$, Ester A. L. de Jonge ${ }^{1}$, Jessica C. Kiefte-de Jong ${ }^{1}$, Albert Hofman ${ }^{1}$, Oscar H. Franco

${ }^{1}$ Department of Epidemiology, Erasmus MC, University Medical Center, Rotterdam, The Netherlands; Corresponding author's e-mail: j.schoufour@erasmusmc.nl

Background: Dietary quality is an important contributing factor to healthy aging. Dietary behavior develops during childhood and remains relatively stable throughout adulthood. Nevertheless, with increasing age, dietary patterns seem to change which could lead to poor dietary quality or even malnutrition. Dietary quality is largely influenced by socio-economic determinants, such as, income, social class, education and social support. Far less is known about the influences of socio-economic determinants on changes over time in dietary quality in elderly. Therefore the aim of our study is to evaluate which socio-economic determinants are associated with a changes in dietary quality in old age.

Methods: This study will be performed within the framework of The Rotterdam Study. This is a prospective population-based cohort study comprising subjects aged over 55 years living in Ommoord, a district in Rotterdam in the Netherlands. Participants were included in 1990 and were examined every 3-4 years. Social determinants included education level, occupation, employment status, income, living situation, marital status, recent life events (e.g. death of a loved one), and level of social support. A dietary quality score was determined at baseline and repeated after 20 years of follow-up, using the Dutch Healthy Diet Index. This index includes eight components reflecting the Dutch Guidelines for a healthy diet (vegetables, fruit, fiber, fish, saturated fat, trans fat, sodium, and alcohol). In total, 1441 participants were included in the analysis. Linear regression models were used to test the univariate associations between social determinants and changes in dietary quality score. Variables with $p<0.1$ were subsequently included in a regression model to test their independent effects. All analyses were stratified by gender.

Results: We found that in females, any life event in the 5 years before baseline was significantly associated with a lower dietary quality. Furthermore, education is associated with dietary quality at baseline in men, and income associated with dietary quality at baseline in women. Comprehensive and final results will be discussed at the congress.

Conclusion: Our preliminary results show that dietary quality is influenced by socio-economic determinants.

\section{Abstract \#: P 182}

\section{Genetic associations for hypospadias: review of current} knowledge and replication

Loes F.M. van der Zanden ${ }^{1}$, Nathasja van Leeuwen ${ }^{1}$, Remco R. Makkinje ${ }^{2,3,4}$, Wout F.J. Feitz ${ }^{5}$, Nel Roeleveld ${ }^{6}$, Iris A.L.M. van Rooij ${ }^{1}$

${ }^{1}$ Department for Health Evidence, Radboud university medical center, Radboud Institute for Health Sciences, Nijmegen, The Netherlands; ${ }^{2}$ Department of Human Genetics, Radboud university medical center, Radboud Institute for Health Sciences, Nijmegen, The Netherlands; ${ }^{3}$ Department of Psychiatry, Radboud university medical center, Nijmegen, The Netherlands; ${ }^{4}$ Donders Institute for Brain, Cognition and Behaviour, Radboud University, Nijmegen, The Netherlands; ${ }^{5}$ Department of Urology-Pediatric urology, Radboudumc Amalia
Children's Hospital, Radboud university medical center, Nijmegen, The Netherlands; ${ }^{6}$ Department for Health Evidence, Radboud university medical center, Radboud Institute for Health Sciences, Nijmegen, The Netherlands and Department of Pediatrics, Radboudumc Amalia Children's Hospital, Radboud university medical center, Nijmegen, The Netherlands; Corresponding author's e-mail: Loes.vanderZanden@radboudumc.nl

Background: Hypospadias is a common congenital malformation of the male external genitalia with a multifactorial etiology. More and more research is performed to find polymorphisms associated with hypospadias, but replication of results is often lacking. The aim of this study was to identify all genetic associations reported for hypospadias and to replicate the most promising ones.

Methods: We thoroughly searched the literature to identify all genetic association studies on hypospadias and selected seven single nucleotide polymorphisms (SNPs) for replication based on association present, minor allele frequency (MAF), and previous replication. We used Taqman assays to genotype 816 Caucasian nonsyndromic hypospadias patients and 668 population-based controls derived from the AGORA (Aetiologic research on Genetic and Occupational/environmental Risk factors for Anomalies in children) data- and biobank of the Radboudumc.

Results: When excluding studies using microarrays performed after our SNP selection, 36 polymorphisms in diacylglyceraol kinase, kappa (DGKK) and 67 polymorphisms in 16 other genes were studied for their association with hypospadias. We already examined DGKK successfully in our cohort and this gene was also associated with hypospadias in several other Caucasian populations, but much less strong in a Chinese. In the other genes, 52 polymorphisms were examined only once, of which 12 showed an association. Among these 12 , seven had a MAF $<2 \%$ in Caucasians and one was associated with severe hypospadias only. The remaining four SNPs were selected for this study. Of the 15 polymorphisms that were examined more than once, three were repeats, two were deletions, three were consistently shown not to be associated with hypospadias, and four were already genotyped in our cohort in a previous replication study. The remaining three were selected for this study. As a result, we tried to replicate previously found associations between hypospadias and SNPs in hydroxysteroid (17-beta) dehydrogenase 3 (HSD17B3), estrogen receptor 1 (ESR1), ESR2, mastermind-like domain containing 1 (MAMLD1) (2 SNPs), and activating transcription factor 3 (ATF3) (2 SNPs). None of these SNPs was associated with hypospadias in our study population, with the possible exception of rs944050 in ESR2 (OR $=1.5,95 \%$ CI: $1.0-2.3)$. This association was in the same direction as in a Swedish study and in the opposite direction compared to a Japanese study.

Conclusions: Summarizing all currently reported genetic association studies on hypospadias shows that risk polymorphisms differ between populations. This stresses the importance of studying generalizability of genetic association results and points towards the need for geneenvironment interaction analyses.

\section{Abstract \#: P 183}

Associations between long-term exposure to air pollution and perturbations in the transcriptome

N.S. Mostafavi ${ }^{1}$, J.J. Vlaanderen ${ }^{1}$, L. Portengen ${ }^{1}$, M. Chadeau-Hyam ${ }^{2}$, R. Beelen ${ }^{1}$, L. Modig ${ }^{3}$, D. Palli ${ }^{4}$ I. Bergdahl $^{3}$, P. Vineis ${ }^{2}$, G. Hoek ${ }^{1}$, S.A. Kyrtopoulos ${ }^{5}$, R.C.H. Vermeulen ${ }^{1}$

${ }^{1}$ Division of Environmental Epidemiology, Institute for Risk Assessment Sciences, Utrecht University, 3584 CM, Utrecht, the Netherlands; ${ }^{2}$ Medical Research Council-Health Protection Agency 
Centre for Environment and Health, Department of Epidemiology and Biostatistics, Imperial College London, London, United Kingdom; ${ }^{3}$ Department of Public Health and Clinical Medicine, Occupational and Environmental Medicine, University of Umeå, 901 87,Umeå, Sweden; ${ }^{4}$ Molecular and Nutritional Epidemiology Unit, Cancer Prevention and Research Institute (ISPO), Florence, Italy; ${ }^{5}$ National Hellenic Research Foundation, Institute of Biology, Pharmaceutical Chemistry and Biotechnology, Athens, Greece; Corresponding author's e-mail: N.S.MostafaviMontazeri@uu.nl

Epidemiologic studies have consistently shown associations between long-term exposure to ambient air pollution and chronic health effects such as cardiovascular disease, non-malignant respiratory disease, and lung cancer. We hypothesized that early indications of biological perturbations due to ambient air pollution could be detected in genome-wide gene expression profiles from peripheral blood mononuclear cells (PBMC) in healthy individuals.

We assessed genome-wide gene expression in PBMC samples from study participants of two prospective cohorts from Italy $(n=95)$ and Sweden $(n=455)$. We estimated long-term air pollution (NOx) exposure levels for our study participants using exposure models developed within the European Study of Cohorts for Air Pollution Effects (ESCAPE).

We used three complementary statistical approaches: univariate analysis (coupled with a $5 \%$ false discovery rate (FDR) control to account for multiple testing), elastic net regression, and Graphical Unit Evolutionary Stochastic Search (GUESS: a recent Bayesian variable selection approach), to identify for which genes expression level was altered by long-term exposure to NOx.

There was little overlap between the distribution of NOx exposure levels in the Italian and the Swedish cohort (median: 5th-95th percentile: 94.2 : $7.8-124.6$ and 6.7 : $4.8-19.5 \mu \mathrm{g} / \mathrm{m}^{3}$, respectively). We therefore conducted our primary analyses in the two cohorts separately.

Using the univariate approach we identified one (unannotated) transcript in the Italian cohort and no transcripts in the Swedish cohort that were significantly associated at an FDR of $5 \%$. While elastic net regression provided consistent results, GUESS provided less indication for an association with this probe in the Italian data.

Our analyses were complicated by the fact that on the one hand, exposure levels in our largest cohort ( $83 \%$ of the available data) were low, and on the other hand the Italian cohort was likely too small to detect subtle changes in gene expression. In summary, our study does not provide strong indications of transcriptomic changes related to air pollution within the exposure range studied.

\section{Abstract \#: P 184}

Fetal growth restriction and exposure to particulate air pollution in the early-life environment of twins

Esmée Bijnens $^{1}$, Tim S Nawrot ${ }^{1}$, Marij Gielen ${ }^{2}$, Ellen Winckelmans ${ }^{1}$, Catherine Derom $^{3}$, Robert Vlietinck ${ }^{3}$, Maurice P Zeegers ${ }^{2}$

${ }^{1}$ Centre for Environmental Sciences, Hasselt University, Belgium; ${ }^{2}$ Department of Complex Genetics, NUTRIM School for Nutrition, Maastricht University, The Netherlands; ${ }^{3}$ Centre of Human Genetics, University Hospitals Leuven, Belgium; Corresponding author's e-mail: esmee.bijnens@uhasselt.be

Background: Several studies in singletons have shown that maternal exposure to ambient air pollutants is associated with restricted fetal growth. About half of twins have low birth weight compared with $6 \%$ in singletons and twin pregnancies may be more susceptible to external drivers. However, to the best of our knowledge, no studies have investigated the association between maternal air pollution exposure and fetal growth in twins.

Methods: We studied the association between in utero exposure to air pollution and fetal growth among in 3347 twins (2002-2013) of the East Flanders Prospective Twin Survey. Maternal air pollution exposure was estimated using a spatial temporal interpolation method. Mixed models accounting for newborn's sex, birth order, parity, gestational age (linear and quadratic), birth year (linear and quadratic), season of birth, zygosity and chorionicity, maternal age, median income neighbourhood and insertion of the umbilical cord were used to study the association between fetal growth and maternal residential exposure to PM10, and $\mathrm{NO} 2$ over various time windows during pregnancy.

Results: Birth weight averaged (SD) 2397 g (542 g). PM10 exposure during pregnancy averaged (25th-75th percentile) $30.8 \mu \mathrm{g} / \mathrm{m}^{3}$ $\left(27.5-33.8 \mu \mathrm{g} / \mathrm{m}^{3}\right)$ and $24.6 \mu \mathrm{g} / \mathrm{m}^{3}\left(20.0-28.6 \mu \mathrm{g} / \mathrm{m}^{3}\right)$ for NO2. Birth weight decreased by $29.75 \mathrm{~g}$ ( $95 \% \mathrm{CI}:-55.4 \mathrm{~g}$ to $-4.1 \mathrm{~g}: p=0.02$ ) for each $10 \mu \mathrm{g} / \mathrm{m}^{3}$ increase in $\mathrm{NO} 2$ concentration during pregnancy. The corresponding odds ratio for small for gestational age was 1.26 (95\% CI: $1.00-1.58: p=0.05$ ) for NO2. A $10 \mu \mathrm{g} / \mathrm{m}^{3}$ increase in PM10 exposure during the third trimester was associated with an odds ratio of 1.30 (95\% CI: $1.04-1.62: p=0.02$ ).

Conclusion: Twin pregnancies are vulnerable to the effects of in utero exposure to particulate air pollution and fetal growth restriction. An achievement of a $10 \mu \mathrm{g} / \mathrm{m}^{3}$ reduction is highly relevant for the protection of susceptible population groups such as twins in their early life.

\section{Abstract \#: P 185}

Altered placental Mirna expression in association with in utero exposure to particulate air pollution

Maria, Tsamou $^{1}$, Karen, Vrijens ${ }^{1}$, Narjes, Madhloum ${ }^{1}$, Wilfried, Gyselaers ${ }^{2}$, Tim, S, Nawrot ${ }^{3}$

${ }^{1}$ Center for Environmental Sciences, Hasselt University, Diepenbeek, Belgium; ${ }^{2}$ Department of Obstetrics, East-Limburg Hospital, Genk, Belgium; ${ }^{3}$ Department of Obstetrics, East-Limburg Hospital, Genk, Belgium: Department of Public Health, Occupational, Environmental and Insurance Medicine, Leuven University (KU Leuven), Leuven, Belgium; Corresponding author's e-mail: maria.tsamou@uhasselt.be

Background: Particulate matter exposure during in utero life is associated with adverse health outcomes later in life. Epidemiological studies have linked air pollution's adverse effects to alterations in gene expression profiles, which can be regulated by epigenetic mechanisms. MicroRNAs (miRNAs) have been implicated in diverse biological processes. We investigate the potential influence of air pollution exposure in early life on miRNA expression in human placental tissue.

Methods: Within the framework of the ENVIRONAGE birth cohort, the expression of four miRNAs (miR-16, miR-21, miR-146a and miR-222) was analyzed by qRT-PCR in placental tissue from 211 mother-newborn pairs. Relative miRNA expression levels in human placental tissue were associated with air pollution data estimated for the home address of the pregnant women using a Kriging interpolation and dispersion model. Multiple regression models were used to study placental miRNA expression and in utero exposure to particulate matter (PM2.5) over various time windows during pregnancy. In silico analysis was performed to predict genes and pathways targeted by the studied miRNAs.

Results: All four measured placental miRNAs were associated with air pollution exposure in early life. For each $5 \mu / \mathrm{m}^{3}$ increase in PM2.5 exposure, the expression of miR-16 and miR-21 was reduced by 
$39.1 \%(p=0.0006)$ and $29.2 \%(p=0.0463)$ during the 2 nd trimester, respectively. The expressed miR-146a and miR-222 were significantly decreased by various exposure windows during pregnancy including exposure during 2nd trimester for miR-146a, and 2nd and 3rd trimester exposure with miR-222. The effects were independent of mother's age, pre-gestational BMI, smoking status, parity and educational status, and newborn's gender, gestational age and birth weight, and temperature. Pathway analysis based on in silico predicted miRNA targets revealed immune responses as the core pathways targeted by the studied miRNAs.

Conclusions: Environmental exposure to particulate air pollution in early life was associated with altered expression of miRNAs-16, -21, $-146 \mathrm{a}$ and -222 in placental tissue at birth. These miRNA might be relevant targets for PM induced effects in fetal programming.

\section{Abstract \#: P 186}

\section{The impact of ambient air pollution on the human blood exposome}

Jelle Vlaanderen $^{1}$, N.A. Janssen ${ }^{2}$, G. Hoek ${ }^{1}$, D.K. Barupal ${ }^{3,}$ A. Scalbert $^{4}$, F.R. Cassee ${ }^{2}$, I. Gosens ${ }^{1}$, M. Strak ${ }^{1}$, M. Steenhof ${ }^{1}$, B. Brunekreef $^{1}$, R.C.H. Vermeulen ${ }^{1}$

${ }^{1}$ Division of Environmental Epidemiology, Institute for Risk Assessment Sciences, Utrecht University, The Netherlands; ${ }^{2}$ National Institute for Public Health and the Environment (RIVM), Bilthoven, The Netherlands; ${ }^{3}$ International Agency for Research on Cancer, Lyon, France; ${ }^{4}$ National Institute for Public Health and the Environment (RIVM), Bilthoven, The Netherlands: Division of Environmental Epidemiology, Institute for Risk Assessment Sciences, Utrecht University, The Netherlands; Corresponding author's e-mail: j.j.vlaanderen@uu.nl

Epidemiological studies have consistently shown associations between exposure to air pollution and (acute) cardiovascular and respiratory disease, and lung cancer. Early biological perturbations due to exposure to ambient air pollution might be reflected in the composition of the set of compounds present in blood originating from internal metabolic pathways and external exposures (the blood exposome).

In a semi-experimental study design we exposed 31 healthy adult volunteers at four different locations to varying levels of air pollution. Volunteers were exposed for $5 \mathrm{~h}$, exercising intermittently, 3-7 times at different sites. On site, we measured a range of air pollution markers including particulate matter (PM) mass and number concentration, and gaseous pollutants (ozone, nitrogen oxides). We collected blood from the participants before exposure (t0) and $2(\mathrm{t} 1)$ and $18 \mathrm{~h}$ (t2) after exposure. We employed untargeted metabolite profiling using an ultra-high pressure liquid chromatography time of flight mass spectrometry to assess levels of up to 2700 compounds in the blood samples. We conducted univariate analysis to assess which metabolite concentrations measured at $\mathrm{t} 1$ and $\mathrm{t} 2$ were significantly associated with air pollution markers. The analyses were adjusted for the metabolite levels measured at $\mathrm{t} 0$ in addition to a set of likely confounding factors. The false discovery rate (FDR) was controlled at $5 \%$.

We observed a strong signal for one compound that was significantly associated to PM2.5 $(p=8.59 \mathrm{e}-06)$ and PM10 $(p=4.32 \mathrm{e}-05)$ at $\mathrm{t} 1$, but not at $\mathrm{t} 2$. A sensitivity analyses demonstrated that this association was not sensitive to sequentially leaving out data from one of the study locations.

Relaxing our criterion for statistical significance to an FDR of $20 \%$, we identified one additional compound that was associated with PM $\neg 2.5$, but not with PM10. In addition, we identified one more compound that was highly correlated $(\mathrm{r}>0.8)$ with our most significant metabolite. No significant associations were observed between the other air pollution markers and any of detected compounds. We are currently in the process of chemical identification of the significantly associated compounds using MS/MS spectra and spectral library searches.

Our study provides some indication for an association between particulate components of ambient air pollution and a subtle acute perturbation of the blood exposome $2 \mathrm{~h}$ after exposure. Significantly associated perturbations observed $2 \mathrm{~h}$ after exposure were no longer observed $18 \mathrm{~h}$ after exposure, suggesting a rather acute increase and wearing off of the effect of PM on the human blood exposome. Strengths of our study are the semi-experimental design (standardizing nutritional intake and physical activity), repeated collection of blood samples, and use of a high resolution mass spectrometer for metabolite profiling.

\section{Abstract \#: P 187}

Iron metabolism gene, lead exposure, and homocysteine level: a longitudinal gene-environment interaction study

$\underline{\text { Koung-Nam Kim }}^{1}$, Yun-Chul Hong ${ }^{1}$

${ }^{1}$ Department of Preventive Medicine, Seoul National University College of Medicine, Seoul, Republic of Korea; Corresponding author's e-mail: kkn002@snu.ac.kr

Background: Homocysteine is an independent risk factor for cardiovascular and neurological disease. Evidences supporting the relationship between lead and homocysteine have been accumulating, while most prior studies were conducted in cross-sectional design and have not focused on interaction with genetic polymorphism.

Methods: From a community-based prospective cohort, 386 participants aged 41-71 with available blood $\mathrm{Pb}$ and homocysteine measurement were included in the present study. $\mathrm{Pb}$ was measured between 2001 and 2003, and homocysteine in 2007. Interaction of $\mathrm{Pb}$ with 47 genotyped SNPs in 5 genes (TF, HFE, CBS, BHMT, and MTR) was assessed via $2 \mathrm{df}$ joint and $1 \mathrm{df}$ interaction test. Further, in the secondary analyses, 58 imputed SNPs in TF and MTHFR were also assessed.

Results: Blood $\mathrm{Pb}$ concentration was positively associated with homocysteine level ( $p$ trend $=0.0276$ ) in adjusted linear model. Six SNPs in TF and MTR were screened through 2 df joint test, and 3 SNPs (rs2715632, rs2715631, and rs2715627) in TF were selected to show significant interaction ( $p$ value $<0.0083$ ). Seven SNPs in MTHFR had significant main effect on homocysteine ( $p$ value $<0.05$ ), despite no significant interaction with $\mathrm{Pb}$.

Conclusions: Significant association between blood $\mathrm{Pb}$ and homocysteine 4-6 years later was found, and 3 SNPs in TF locus were demonstrated to modify the association. The current results not only support the causal relationship between $\mathrm{Pb}$ and homocysteine but also implicated the direction of the future mechanistic study of $\mathrm{Pb}-\mathrm{ho}-$ mocysteine association.

\section{Abstract \#: P 188}

Children's blood pressure in association with mitochondrial DNA content

Nelly D. Saenen ${ }^{1}$, Martien Peusens ${ }^{1}$, Eline B. Provost ${ }^{1}$, Michal Kicinski $^{1}$, Nicky Pieters ${ }^{1}$, Karen Vrijens ${ }^{1}$, Tim S. Nawrot ${ }^{2}$ 
${ }^{1}$ Centre for Environmental Sciences, Hasselt University, Diepenbeek 3590, Belgium; ${ }^{2}$ Centre for Environmental Sciences, Hasselt University, Diepenbeek 3590, Belgium: Department of Public Health and Primary care, Leuven University (KU Leuven), Leuven 3000, Belgium; Corresponding author's e-mail: nelly.saenen@uhasselt.be

Background: Mitochondrial DNA content is a determinant of the mitochondrial function. Mitochondrial changes have been linked with ageing. Childhood blood pressure is an important predictor of hypertension and cardiovascular disease later in life. We investigated the association of mitochondrial DNA content (mtDNAcn) on blood pressure in primary schoolchildren.

Methods: Within the COGNition and Air pollution in Children (COGNAC) study, 331 children, aged 9-12 years, were recruited from three different primary schools (Tienen, Zonhoven, Kiewit) in Flanders, Belgium. Each child was studied on three different examination days. Blood pressure was measured, on each visit 5 times consecutively, using a full automatic upper-arm blood-pressure monitor with a special cuff size for children. Relative mtDNAcn was measured from buccal DNA using a quantitative polymerase chain reaction. We regressed the association between blood pressure and mtDNAcn, while accounting for child's gender, age, education of the mother (middle-high), heart rate, length, weight, playing on computer (hours/week) and watching television (hours/week).

Results: 331 children (50.8\% girls) had a mean age (SD) of 10.2 (1.3) years and their BMI averaged $17.3(2.9) \mathrm{kg} / \mathrm{m}^{2}$. A reduction in buccal mtDNA content with $50 \%$ was associated with a higher systolic blood pressure $(+0.87 \mathrm{mmHg}, 95 \%$ confidence interval [CI]: $0.04, \quad 1.70 \mathrm{mmHg}: \quad p=0.04)$ and diastolic blood pressure $(+1.12 \mathrm{mmHg}, 95 \%$ CI: 0.11, $2.13 \mathrm{mmHg}: p=0.03)$.

Conclusion: MtDNA content has been implicated in age-related diseases. We found an inverse association between blood pressure and buccal mtDNA content in children, illustrating the significance of early life changes in mtDNA content. The prospective value of mtDNA content in blood pressure, tracking from childhood to adulthood, should be elucidated in the future.

\section{Abstract \#: P 189}

Lower placental telomere length may be attributed to maternal residential traffic exposure: a twin study

Esmée Bijnens $^{1}$, Maurice P Zeegers ${ }^{2}$, Marij Gielen ${ }^{2}$, Michal ${\text { Kicinski }{ }^{1} \text {, Geja J Hageman }}^{3}$, Daniëlle Pachen ${ }^{3}$,

Catherine Derom ${ }^{4}$, Robert Vlietinck ${ }^{4}$, Tim S Nawrot ${ }^{1}$

${ }^{1}$ Centre for Environmental Sciences, Hasselt University, Belgium; ${ }^{2}$ Department of Complex Genetics, NUTRIM School of Nutrition and Translational Research in Metabolism, Maastricht University, The Netherlands; ${ }^{3}$ Department of Toxicology, NUTRIM School of Nutrition and Translational Research in Metabolism, Maastricht University, The Netherlands; ${ }^{4}$ Centre of Human Genetics, University Hospitals Leuven, Belgium; Corresponding author's e-mail: esmee.bijnens@uhasselt.be

Background: High variation in telomere length between individuals is already present before birth and is as wide among newborns as in adults. Environmental exposures likely have an impact on this observation, but remain largely unidentified. We hypothesize that placental telomere length in twins is associated with residential traffic exposure, an important environmental source of free radicals that might accelerate aging. Next, we intend to unravel the nature-nurture contribution to placental telomere length by estimating the heritability of placental telomere length.

Methods: We measured telomere length in placental tissue of 211 twins in the East Flanders Prospective Twin Survey. Maternal traffic exposure was determined using Geographic information system. Additionally, we estimated the relative importance of genetic and environmental sources of variance.

Results: In this twin study, variation in telomere length in placental tissue was mainly determined by the common environment. Maternal residential proximity to a major road was associated with placental telomere length: a doubling in the distance to the nearest major road was associated with a $5.32 \%(95 \% \mathrm{CI}: 1.90-8.86 \%: p=0.003)$ longer placental telomere length at birth. In addition, an interquartile increase $(22 \%)$ in maternal residential surrounding greenness $(5 \mathrm{~km}$ buffer) was associated with an increase of $3.62 \%$ (95\% CI: 0.20 to $7.15 \%: p=0.04$ ) in placental telomere length.

Conclusion: In conclusion, we showed that maternal residential proximity to traffic and lower residential surrounding greenness is associated with shorter placental telomere length at birth. This may explain a significant proportion of air pollution-related adverse health outcomes starting from early life, since shortened telomeres accelerate the progression of many diseases.

\section{Abstract \#: P 190}

What is the role of supportive work and caregiving environments for a successful combination of informal care and paid work?

Marloes Oldenkamp ${ }^{1}$, Mariët Hagedoorn ${ }^{2}$, Ronald Stolk ${ }^{1}$, Rafael Wittek $^{3}$, Ute Bültmann ${ }^{4}$, Nynke Smidt ${ }^{1}$

${ }^{1}$ Epidemiology, University of Groningen, University Medical Center Groningen, Groningen, the Netherlands; ${ }^{2}$ Health Psychology, Health Sciences, University of Groningen, University Medical Center Groningen, Groningen, the Netherlands; ${ }^{3}$ Sociology, University of Groningen, Groningen, the Netherlands; ${ }^{4}$ Community and Occupational Medicine, Health Sciences, University of Groningen, University Medical Center Groningen, Groningen, the Netherlands; Corresponding author's e-mail: m.oldenkamp@umcg.nl

Background: Currently, in the Netherlands more than $70 \%$ of all informal caregivers aged 65 years or younger combines informal care with paid work. This percentage is expected to increase in the future, due to the ageing population. Studies investigating the consequences of combining informal care and paid work for informal caregiver's health and work performance found inconsistent results. This suggests that for some informal caregivers the combination may be beneficial, while for others it may be harmful to combine informal care and paid work. We studied the role of support at work, support at home, and support from official organizations for the ability to combine informal care and paid work. In addition, we studied to what extent such supportive environments might buffer health problems due to the informal caregiving.

Methods: Within LifeLines, a population-based cohort study including 176.729 participants, a subcohort of informal caregivers with paid work aged $\geq 18$ years $(\mathrm{N}=651: 77 \%$ female, mean age 50 (SD 7.9) years) was formed. All informal caregivers filled out a questionnaire about their caregiving situation (i.e. hours and tasks of care, support), their caregiving experiences (Caregiver Reaction Assessment, including health problems due to caregiving), and their work situation (i.e. work status, hours, support, work accommodations, psychosocial work environment (Copenhagen Psychosocial Questionnaire)). Multivariable linear regression models will be used to analyse the role of support at work, at home, and from official organizations for the ability to combine informal care and paid work, and health problems due to caregiving. Furthermore, interactions between the caregiving intensity (i.e. hours and types of informal care tasks) and levels of support will be included.

Results: Results will be presented at the conference. 
Conclusion: Results will provide insight into the situations in which informal care and paid work can be successfully combined without health problems, and how the support in the work environment and caregiving environment may contribute to this. This is useful information for the development of effective policies and caregiver support interventions.

\section{Abstract \#: P 191 \\ Cohort profile: the Spanish working life social security (WORKss) cohort study}

María-Andrée López Gómez ${ }^{1}$, Xavier Durán ${ }^{1}$, Elena Zaballa ${ }^{1}$, Albert Sanchez-Niubo ${ }^{1}$, George L Delclos ${ }^{2}$, Fernando G Benavides ${ }^{1}$

${ }^{1}$ Center for Research in Occupational Health (CiSAL), Department of Experimental and Health Sciences, Pompeu Fabra University, Barcelona, Spain; ${ }^{2}$ Division of Epidemiology, Human Genetics and Environmental Sciences, The University of Texas School of Public Health (UT), Houston, USA; Corresponding author's e-mail: andree.gomez01@estudiant.upf.edu

Cohort profile: The Spanish WORKing life Social Security (WORKss) Cohort Study, The global economy is changing the labor market and the social protection systems, especially the Welfare States in the European countries. The effect of both changes on health needs to be monitored in view of an aging population and the resulting increase in the number of people with chronic health conditions. The Spanish WORKing life Social Security (WORKss) Cohort Study provides unique longitudinal data to study the impact of labor trajectories and employment conditions on health, in terms of sickness absence, permanent disability and death. In 2004, the cohort initiated with 1022,779 Social Security affiliates who had a registered labor trajectory from 1981 or later. The WORKss cohort is updated annually and includes demographic characteristics, chronological data about employment history, retirement, permanent disability and death. Researchers interested in working with the WORKss cohort study are welcome to contact the Center for Research in Occupational Health (CiSAL) at Pompeu Fabra Univesity at salutlaboral@upf.edu.

\section{Abstract \#: P 192}

Asthma prevalence in children living in rural and non-rural environments- influence of indoor air pollution in nurseries and primary schools

Sousa S.I.V. ${ }^{1}$, Branco P.T.B.S. ${ }^{1}$, Nunes R.A.O. ${ }^{1}$, Alvim-Ferraz M.C.M. ${ }^{1}$, Martins F.G. ${ }^{1}$

${ }^{1}$ LEPABE - Laboratory for Process Engineering, Environment, Biotechnology and Energy, Faculty of Engineering, University of Porto; Corresponding author's e-mail: sofia.sousa@fe.up.pt

Background: Association between exposure to indoor air pollution (IAP) and childhood asthma has been demonstrated in several studies performed across the world. Evidence is suggesting that some air pollutants may contribute to both exacerbation and development of asthma, but some uncertainty remains concerning the specific causative role of IAP.

Objectives: This study aimed to associate exposure to IAP with the prevalence of asthma in children living at rural and non-rural environments.

Methods: The study was based on data collected in Northern Portugal from 7 non-rural nurseries and 5 primary schools influenced by traffic emissions in Porto district and from 7 rural nurseries and 3 primary schools in Bragança district. The indoor concentrations of $\mathrm{CO} 2, \mathrm{CO}$, $\mathrm{O} 3, \mathrm{NO} 2$, total volatile organic compounds and formaldehyde were continuously measured using a Haz-Scanner IEMS monitor (measurements were taken each minute and hourly means were calculated), and those of particulate matter (PM1, PM2.5, PM10 and TSP) were continuously measured using a TSI DustTrak DRX 8534 particle monitor. Samplings were performed from February 2013 to November 2014. The studied population comprised 1369 children at non-rural sites and 700 at rural sites, aged 1-13 years, enrolled in the nurseries and schools of the municipalities (coincident with the locations of the monitoring sites). Data concerning asthma prevalence were collected through questionnaires similar to those used in ISAAC.

Results: Both gaseous and PM concentrations were found higher at non-rural sites than at rural sites. A total of 2069 questionnaires were distributed in the nurseries and schools, from which $55 \%$ were answered by the children parents. Around $50 \%$ of the studied children presented at least one of the respiratory symptoms investigated (wheeze, dyspnoea and cough) in the absence of upper respiratory infections. The prevalence of wheezing for lifetime period and in the past year were 30 and $13 \%$, respectively. According to the answers to the questionnaires, asthmatic children were identified when wheezing and dyspnoea were simultaneously mentioned in the absence of upper respiratory infections, or when self-reported. Therefore, the lifetime prevalence of asthmatic symptoms at non-rural sites was $13 \%$ and at rural sites was $7 \%$.

Conclusions: The higher pollutant concentrations and prevalence of childhood asthma observed in non-rural sites than at rural ones suggested that IAP might influence the development of childhood asthma. A complete study of asthma risk factors considering the medical examination of the studied population is currently on.

\section{Abstract \#: P 193}

Second-hand smoke exposure during pregnancy intensifies the harmful effect of prenatal cadmium exposure on birth weight

Surabhi Shah-Kulkarni ${ }^{1}$, Byung-Mi Kim ${ }^{1}$, Eunjeong Kim ${ }^{1}$, Yun-Chul Hong $^{2}$, Hyesook Park ${ }^{1}$, Yangho $\mathrm{Kim}^{3}$, Mina $\mathrm{Ha}^{1}$, Eun-Hee $\mathrm{Ha}^{1}$

${ }^{1}$ Department of Preventive Medicine, Ewha Medical Research Center, School of Medicine, Ewha Womans University, Seoul, Republic of Korea; ${ }^{2}$ Institute of Environmental Medicine, Medical Research Center, Seoul National University, Seoul, Republic of Korea;

${ }^{3}$ Department of Occupational and Environmental Medicine, Ulsan University Hospital, University of Ulsan College of Medicine, Ulsan, Republic of Korea; Corresponding author's e-mail: surabhi.3007@gmail.com

Background: Cadmium is a persistent environment pollutant which has entered our food chain. The main exposure sources of cadmium are smoking, due to high cadmium content in tobacco leaves and food resulting from cadmium contaminated soil. Studies on the effect of prenatal cadmium exposure on the development of child are very scare and the results are inconsistent. Thus, in this study we explore the effect of secondhand smoke exposure of pregnant women on the action of prenatal cadmium exposure on infant and child's growth. Methods: The study participants comprises of 1751 pregnant women and their offspring's recruited from 2006 and 2010 in a prospective birth cohort study: Mother and children's environment (MOCEH) study. Maternal socio-demographic, environmental factors and prenatal secondhand smoke exposure were obtained by questionnaire during pregnancy. Mother's blood at $<20$ weeks and at time of delivery and cord blood was collected to measure the pre-natal cadmium exposure. Length and weight of children were measured at 
birth, 6, 12 and 24 months of age using standard protocols. Age specific z-scores were calculated for length and weight of children. Generalized linear model was constructed and analysis by prenatal secondhand smoke exposure was conducted to estimate the effects of prenatal cadmium exposure on children's growth at each time point using SAS (version 9.3).

Results: The geometric mean cadmium blood levels of all participating mothers were 1.41 and $1.50 \mu \mathrm{g} / \mathrm{L}$ at early and late pregnancy respectively. In the second-hand smoke exposure group of mothers during pregnancy, blood cadmium levels in late pregnancy was found to be significantly associated in reduction in the birth weight $\mathrm{z}$-scores ( $\beta=-0.29,95 \% \mathrm{CI}-0.56$ to -0.02$)$ while in the no secondhand smoke exposure group the association was not significant ( $\beta=-0.21,95 \% \mathrm{CI}-0.49$ to 0.08$)$. However, cadmium exposure during entire gestation affects growth in children: where intensified effect was seen in secondhand smoke exposure group during late pregnancy.

Conclusions: Thus, our results show that adverse effect of prenatal cadmium exposure on birth weight is intensified due to secondhand smoke exposure during pregnancy. This shows that accurate measures to reduce the secondhand smoke exposure in vulnerable population like pregnant mothers is essential.

\section{Abstract \#: P 194}

\section{Cross-cultural adaptation to Brazilian Portuguese of the effort-} reward scale in domestic work

Ilmeire Ramos Rosembach de Vasconcellos ${ }^{1}$, Rosane Härter Griep ${ }^{2}$, Luciana Portela ${ }^{1}$, Márcia Guimaraes de Mello Alves $^{3}$, Lucia Rotenberg $^{2}$

${ }^{1}$ National School of Public Health. Oswaldo Cruz Foundation. Rio de Janeiro, RJ, Brazil; ${ }^{2}$ Oswaldo Cruz Institute, Oswaldo Cruz Foundation. Rio de Janeiro, Brazil; ${ }^{3}$ Institute of Community Health. Federal Fluminense University. Niteroi, RJ, Brazil; Corresponding author's e-mail: rohgriep@gmail.com

Objective: To present the steps of cross-cultural adaptation of the scale of the "effort-reward imbalance model to household and family work" in a Brazilian population.

Method: We conducted translation, back translation and initial psychometric evaluation. The component 'effort' was measured by eight items referring to demanding aspects of work environments of mothers by emphasizing quantitative workload. The component Reward is measured by 11 items, divided into four subscales: iintrinsic value of family and household work, ii- societal esteem, iiirecognition from the spouse/partner, and iv- affection from the chil$\mathrm{d}(\mathrm{ren})$. Overcommitment (personal component of the model) was measured by four items. Each item has four categories ranging from (1) 'totally disagree' to (4) 'totally agree'. The scale was included in a multidimensional questionnaire of a sectional study in a hospital in the city of Rio de Janeiro and applied among 1045 female nursing professionals. A subsample of 222 participants completed the questionnaire for the second time with an interval of 7-15 days (test-retest study). Data were collected between October 2012 and May 2013. The internal consistency of the scale was assessed using Cronbach's Alpha coefficient and test-retest reliability was assessed using the quadratic weighted Kappa index and the Kappa adjusted for prevalence.

Results: Prevalence-adjusted reliability (ka) varied from 0.80 to 0.83 for over commitment, 0.78 to 0.90 for effort and 0.76 to 0.93 for reward subscale. In the majority of dimensions, the values of minimum and maximum score, mean, standard deviation and Cronbach's alpha in the test and retest were very similar. Only in the social esteem subdimension (reward) there was a small variation in the standard deviation (2.24 in the test and 3.36 in retest) and Cronbach's alpha coefficient ( 0.38 in the test and 0.59 in the retest).

Conclusion: The Brazilian version of the scale had adequate levels of reliability in terms of temporal stability. This suggests the suitability of the scale for use in populations with similar characteristics to the study.

\section{Abstract \#: P 195}

(Un)Healthy in the city: respiratory, metabolic and mental health associated with urbanicity

Wilma L. Zijlema ${ }^{1}$, Bart Klijs ${ }^{1}$, Ronald P. Stolk ${ }^{1}$, Judith G.M. Rosmalen ${ }^{2}$

${ }^{1}$ Department of Epidemiology, University of Groningen, University Medical Center Groningen, Groningen, The Netherlands;

${ }^{2}$ Department of Psychiatry, University of Groningen, University Medical Center Groningen, Groningen, The Netherlands; Corresponding author's e-mail: w.l.zijlema@umcg.nl

Background: Research suggests that there are urban-rural health differences, but studies often focus on one particular health outcome, use self-report outcomes and do not take into account neighborhood socioeconomic status. Living in urban areas can be associated with both positive and negative effects on health. We therefore investigated four different objectively measured health outcomes in relation to urbanicity, while taking into account individual and neighborhood socioeconomic status.

Methods: Data from 74,733 participants, with $42 \%$ males and mean age of 43.8 years (standard deviation 11.6 years), were available from the LifeLines cohort study: a population based sample from the North of the Netherlands. Urbanicity was expressed as address density categorized into five levels ranging from rural to urban. Health outcomes were assessed by means of spirometry, anthropometry, collection of fasting blood samples, and a psychiatric interview.

Results: Multilevel models adjusted for age, sex, neighborhood and individual socioeconomic status, smoking and physical activity showed that, compared to living in rural neighborhoods, living in a semi-urban neighborhood was associated with decreased lung function $(\beta-1.17,95 \%$ confidence interval $(\mathrm{CI})-1.61:-0.74)$, and increased odds for depression (OR 1.54, $95 \%$ CI 1.29:1.85). Living in urban neighborhoods was associated with increased odds for anxiety (OR 1.47, $95 \%$ CI 1.26:1.72), but with decreased odds for metabolic syndrome (OR 0.80, $95 \%$ CI 0.70:0.92), when compared to rural neighborhoods.

Conclusions: Living in urban neighborhoods was associated with decreased lung function and increased odds of MDD and GAD, compared to rural neighborhoods, while for metabolic syndrome this was the other way around. Whether living in urban areas is less healthy than in rural areas, depends on the health outcome that is assessed.

\section{Abstract \#: P 196}

Inverse association between the distance from semipalatinsk nuclear weapons tests site and the prevalence of selected cardiovascular diseases

Akbayan Markabayeva ${ }^{1}$, Aiman Kerimkulova ${ }^{1}$, Andrej M Grjibovski $^{2,3}$, Zhanat T Moldagalieva ${ }^{4}$, Viktor R.Veber ${ }^{5}$, Zoya Ensegenova $^{6}$, Ainur S Ospanova ${ }^{1}$ 
${ }^{1}$ State Medical University, Semey, Kazakhstan; ${ }^{2}$ Norwegian Institute of Public Health, Oslo, Norway; ${ }^{3}$ Northern State Medical University, Arkhangelsk, Russia; ${ }^{4}$ Semey Diagnostic Centre, Semey, Kazakhstan; ${ }^{5}$ Novgorod State University, Novgorod, Russia; ${ }^{6}$ Kazakh National Medical University, Almaty, Kazakhstan; Corresponding author's e-mail: andrej.grjibovski@gmail.com

Background: Several studies have reported elevated risks for cardiovascular diseases among Hiroshima and Nagasaki survivors. Semipalatinsk was the main site for testing Soviet nuclear weapons in 1949-1991, but little information on health of the population living close to the test site is available. We studied associations between the prevalence of selected cardiovascular diseases and proximity to the nuclear test site in Semipalatinsk region, Kazakhstan.

Methods: Altogether, 24707 adults (69.7\% women) aged 18-102 years participated in a cross-sectional study. Participants were classified in four groups by proximity of their place of permanent residence to the test site. Data on a history of myocardial infarction (MI), hypertension, valvular disorders, and angina pectoris were collected. Associations between proximity of the place of permanent residence to the test site and the outcomes were studied using multiple logistic regression with adjustment for age, gender, ethnicity, smoking and drinking habits.

Results: The prevalence of a history of myocardial infarction, hypertension, valvular disorders, and angina pectoris, were 1.8, 29.8, 1.1 , and $8.5 \%$, respectively. An inverse gradient between the proximity to the test site and hypertension was observed. Those living closest to the site had the lowest odds of hypertension ( $\mathrm{OR}=0.66$, $95 \%$ CI: 0.54-0.80) compared to those who lived on a territory with the lowest risk of radioactive pollution. The groups who were classified as the second and the third closest to the test site by their place of residence had OR $=0.70,95 \%$ CI: $0.61-0.81$ and $0.79,95 \%$ CI: $0.69-0.91$, respectively. No significant associations between the proximity to the nuclear test site and other outcomes were found. Age, gender, ethnic background, smoking and alcohol were all associated with all studied outcomes.

Conclusions: We observed an inverse association between the prevalence of hypertension and the proximity to the Semipalatinsk nuclear weapons test site among the population permanently residing in the Semipalatinsk region. Given that the study was performed 20 years after the test site was closed, the results may be partly explained by a healthy survivor effect or competing mortality from cancers. Other potential explanations will be discussed.

\section{Abstract \#: P 197}

Are blood cadmium levels associated with depressive symptoms in elderly population?

\section{Changwoo Han ${ }^{1}$, Yun-Chul Hong ${ }^{1}$}

${ }^{1}$ Seoul National University College of Medicine, Seoul, Korea; Corresponding author's e-mail: cwohan@snu.ac.kr

Background: Neurotoxic agent cadmium $(\mathrm{Cd})$ and its association with elderly depression and the involvement of cognitive dysfunction were poorly evaluated before. Therefore, by investigating the relationship between blood cadmium levels and the score of a depression screening test, we aimed to investigate the impact of cadmium exposure on elderly depression. By performing mediation analysis, we also tried to explore whether cognitive dysfunction is involved in the relationship.

Methods: From 2008 to 2010, a total 560 elderly subjects residing in Seoul, Korea were evaluated. Demographic data and life style information were obtained from a systemized questionnaire and blood samples were collected and stored for analysis. Subjects underwent the Korean Version of Short Form Generic Depression Scale test (SGDS-K) and the Functional Activity Questionnaire (FAQ) for screening depression and cognitive dysfunction. Associations were estimated using logistic regression models adjusted for potential confounders and mediation analysis was done with Sobel test.

Results: Blood cadmium levels were significantly related to risk of depressive symptoms. Compared to lowest quartile blood cadmium group $(\mathrm{Cd}<0.85 \mu \mathrm{g} / \mathrm{L})$, the highest quartile group $(1.53 \mu \mathrm{g} / \mathrm{L} \leq \mathrm{Cd})$ showed increased risk for depressive symptoms. (Odds Ratio of highest quartile: $3.39,95 \%$ CI: 1.19 to 9.67$)$ By performing mediation analysis, we found that the score of FAQ mediated the relationship between log transformed blood cadmium level and depressive symptom score. (Percent mediated: $24.4 \%$, p value for Sobel test: 0.01).

Conclusion: Our findings suggest that exposure to cadmium is associated with depressive symptoms in elderly population, and the relationship is partially mediated by cognitive dysfunction.

\section{Abstract \#: P 198}

Association of cardiovascular diseases emergency admissions with short term peaks of air pollution and traffic intensity at home address

Angelo G Solimini ${ }^{1}$, Matteo Renzi ${ }^{2}$

${ }^{1}$ Department of Public Health and Infectious Diseases, Sapienza University, Roma, Italy; ${ }^{2}$ Department of Epidemiology, Rome E Health Authority, Rome, Italy; Corresponding author's e-mail: angelo.solimini@uniroma1.it

Background: The morbidity caused from exposure to air pollution is higher for cardiovascular diseases (CVD) than for other conditions. It is unclear if chronic exposure to traffic interacts with short term peaks of pollutants in determining acute CVD outcomes.

Methods: For the city of Rome, we collected 8 years time series data of daily emergency admissions for CVD codes in a large hospital and hourly monitoring data of air pollution (PM10, PM2.5 and NO2). Cross-sectional modeled traffic volume at peak hour within $50 \mathrm{~m}$ from patient home addresses was obtained for different years. Poisson time series regression was run using traffic $\mathrm{x}$ pollutant interaction terms for CVDs at different lags. All models were adjusted for sex, age, long and seasonal terms, temperature, relative humidity, day of the week, influenza.

Results: We found significant short term effects at lag 0-1 of PM10 and NO2 on total CVD admissions $(\mathrm{N}=17998)$. Prolonged effect (lag 0-5) was significant for PM10 and retarded effect (lag 2-5) was significant for $\mathrm{NO} 2$ only. The interaction term traffic volume $\mathrm{x}$ pollutant was non significant for any pollutant at all lags tested.

Conclusion: Our data support the hypothesis that short term exposure to air pollution increases the risk of acute CVD outcomes shortly after air pollution peaks. However, patients residing at home addresses with different traffic intensities had similar risks in all models tested.

\section{Abstract \#: P 199}

\section{Lingering effect of exposure to air pollution during pregnancy on weight of children}

Sanghyuk Bae ${ }^{1}$, Young Ah Lee ${ }^{2}$, Youn-hee $\mathrm{Lim}^{3}$, Choong Ho Shin ${ }^{2}$, Yun-Chul Hong ${ }^{3}$

${ }^{1}$ Public Health Medical Service, Seoul National University Hospital, Seoul, Korea; ${ }^{2}$ Department of Pediatrics, Seoul National University 
Children's Hospital, Seoul, Korea; ${ }^{3}$ Environmental Health Center, College of Medicine, Seoul National University, Seoul, Korea; Corresponding author's e-mail: sanghyukbae@snu.ac.kr

Background: Exposure to air pollution during pregnancy was associated with adverse birth outcomes, such as low birth weight. We hypothesized that the exposure during the pregnancy may have lingering effects even in the childhood.

Aim: The aim of the present study was to analyze the associations of the mean concentrations of air pollutants during pregnancy and childhood with the weight of the child.

Methods: A cohort of 13,278 pregnant women was followed-up until the birth of the child from Aug. 2008 to Jul. 2011. The mothers' addresses were matched to the nearby monitoring stations, and mean concentrations of air pollutants (PM10, SO2, NO2, CO and O3) during the pregnancy were calculated. We randomly selected and measured the weight of 301 children at the age of 2 and 351 children at the age of 4 . We analyzed the associations of the ambient concentration of pollutants during the pregnancy with birth weight and weight of the children at age 2 and 4 using linear regression models. The associations between mean pollutants after the birth and weight were also analyzed.

Results: Exposures to $\mathrm{O} 3$ during the entire pregnancy and $\mathrm{CO}$ during the first trimester were significantly and negatively associated with birth weight $(\mathrm{g})$ after adjusting potential confounders. The regression coefficients of $\mathrm{CO}$ and $\mathrm{O} 3$ for $10 \mathrm{ppb}$ increase were -1.897 $(\mathrm{SE}=0.944, \quad P=0.0450) \quad$ and $\quad-104.350 \quad(\mathrm{SE}=51.766$, $P=0.0444)$, respectively. The weight $(\mathrm{kg})$ at age 2 was still significantly and negatively associated with $\mathrm{O} 3$. $\mathrm{CO}$ was significantly and negatively associated with weight at age 4 , but not O3. At age 4 , mean $\mathrm{CO}$ concentration during the first 3 years was negatively associated with weight.

Conclusion: We observed that the exposure to air pollution during pregnancy was associated not only with birth weight but also with weight in early childhood.

\section{Abstract \#: P 201}

\section{The effect of extreme cold temperatures on the risk of death in two major cities of Portugal}

Liliana Antunes ${ }^{1}$, Susana Silva ${ }^{2}$, Jorge Marques ${ }^{1}$, Baltazar Nunes ${ }^{2}$, Sílvia Antunes ${ }^{1}$

${ }^{1}$ Division of Climate and Climate Change, Portuguese Sea and Atmosphere Institute, Lisbon, Portugal; ${ }^{2}$ Department of Epidemiology, National Health Institute Dr. Ricardo Jorge, Lisbon, Portugal; Corresponding author's e-mail: liliana.antunes@ipma.pt

Background: Periods of extreme cold have been shown to be associated with an increase risk of death during the winter, in particular with cardiovascular and respiratory deaths. Despite the temperate climate, Portugal presents one of the highest excess winter deaths in Europe. The objective of the present work was to estimate the daily effect of minimum temperature in the principal cities of Portugal aiming the development of a real-time warning system.

Methods: Poisson regression models combined with distributed lag non-linear models were applied to assess the exposure-response relationship and lag patterns of the association between minimum temperature and all cause and both respiratory and circulatory system diseases mortality from 1992 to 2012, for the period of November to March, in two different Portuguese districts (Lisbon and Oporto). Models were adjusted for the confounding effect of influenza by including as a covariate the influenza-like illness incidence rate and adjusted for over dispersion and population size. Models based on different assumptions for the delay effects of exposure were applied in order to assess goodness of fit while assuming a linear exposureresponse relationship along minimum temperature.

Results: The best fitting model by Akaike's criterion for quasi Poisson models(QAIC) described delay effects by a polynomial of degree 4 for all groups. As expected, the minimum temperature effect was higher for respiratory and circulatory system diseases mortality than for all cause mortality. The overall Relative Risk (RR) for respiratory and circulatory mortality was 2.34 [IC 95: 2.13-2.57] in Lisbon and 1.98 [IC 95: 1.79-2.20] in Oporto. For all cause mortality, the overall effect of minimum temperatures was 1.83 (CI $95 \%$ [1.72-1.95]) in Lisbon and 1.67 (CI $95 \%$ [1.57-1.79]) in Oporto. Maximum RR was found for -2 and $0{ }^{\circ} \mathrm{C}$ minimum temperatures in Oporto and Lisbon, respectively. Overall, cold effect persists from 1 to a maximum of 26-28 days followed by a harvesting effect from lag 29-31 days. The maximum risk was estimated after 6 days for both causes in Lisbon, and after 6 and 7 days respectively for all causes and respiratory and circulatory system disease mortality in Oporto.

Conclusion: The effect of minimum temperatures on mortality is significant for several consecutive days in Lisbon and Oporto and higher in respiratory and circulatory system disease mortality in Lisbon.

The results found in this work were also observed in other European cities (PHEWE project), namely the stronger and more persistence through time association of minimum apparent temperature with respiratory system disease mortality.

Acknowledgment: This work was financially supported by national funds from FCT-FRIESA PROJECT (EXPL/DTP-SAP/1373/2013).

\section{Abstract \#: P 202}

Occupational exposure to beryllium and mortality from lung cancer and beryllium disease

$\underline{\text { Paolo Boffetta }}^{1}$, Tiffany A. Fordyce ${ }^{2}$, Jack S. Mandel ${ }^{2}$

${ }^{1}$ Ichan School of Medicine at Mount Sinai; ${ }^{2}$ Exponent Inc.; Corresponding author's e-mail: paolo.boffetta@gmail.com

We aimed at studying mortality from lung cancer and beryllium disease (BD, comprising acute and chronic disease) among workers exposed to soluble and insoluble beryllium compounds.

We conducted a study of 16,115 workers ( $84 \%$ males) employed between 1925 and 2009 at 16 US beryllium manufacturing plants and distribution centers. Sixty percent of the workers were exposed to soluble beryllium and $36 \%$ to insoluble beryllium. Follow-up was through 2011: $0.6 \%$ of workers were lost to follow-up, and cause of death was missing for $7.6 \%$ of deceased workers: SMRs were calculated using national rates.

The SMR for all causes was 1.00 (95\% CI 0.98-1.02, 7868 observed deaths), that for lung cancer was 1.01 (95\% CI 0.93-1.09, 666 deaths) and that for 'other non-malignant respiratory diseases' (ONMRD), a category comprising BD, was $1.29(95 \%$ CI 1.15-1.43). The SMR for lung cancer was 1.07 (95 \% CI 0.98-1.18) among workers exposed to soluble beryllium and 0.88 (95\% CI 0.74-1.03) among those exposed to insoluble beryllium ( $\mathrm{p}$ for heterogeneity [p-het] 0.04). Corresponding SMRs for ONMRD were 1.33 (95\% CI 1.16-1.52) and 1.10 (95\% CI 0.85-1.40: p-het 0.2). The SMR for lung cancer was 1.16 (95\% CI 1.06-1.28) among workers hired before 1955 and 0.82 (95\% CI 0.72-0.93) among workers hired in 1955 or later (p-het $<0.001)$. Corresponding SMRs for ONMRD were 1.28 (95\% CI 1.11-1.47) and 1.30 (95\% CI 1.07-1.56) (p-het 0.9). The increased SMR for lung cancer among workers hired before 1955 was restricted to those exposed to soluble beryllium (SMR 1.24, 95 \% CI 1.12-1.38). 
This is the largest mortality study ever conducted among beryllium workers. It detected no increased mortality from lung cancer overall, and among workers exposed to insoluble beryllium or hired in 1955 or later. An increased mortality was observed among workers exposed to soluble beryllium and hired before 1955. Mortality from ONMRD, including BD, did not differ according to beryllium form or date of hire.

\section{Abstract \#: P 203}

\section{Cattle mortality as a sentinel for the effects of air pollution} on human health

$\underline{\text { Bianca Cox }}^{1}$, Jaco Vangronsveld ${ }^{1}$, Tim S. Nawrot ${ }^{1}$

${ }^{1}$ Centre for Environmental Sciences, Hasselt University, Diepenbeek, Belgium; Corresponding author's e-mail: bianca.cox@uhasselt.be

Background: Air pollution has been found to be a risk factor for human mortality. The use of animal sentinels as models for epidemiologic studies of human diseases and environmental exposures might improve causal inference. Therefore, we examined the role of ozone (O3), particulate matter (PM10) and nitrogen dioxide (NO2) on the risk of mortality among dairy cows.

Methods: Data on cattle mortality were linked with air pollution concentrations at the level of the municipality. We used a casecrossover design on 87,108 dairy cow deaths from 2006 to 2009, across the warm (April-September) and the cold (October-March) period of the year. We applied distributed lag nonlinear models (DLNM) to assess the potential nonlinear and delayed effects of air pollution on cow mortality.

Results: Across the warm period, we observed significant effects of each of the pollutants on cattle mortality. An IQR $(29.9 \mu \mathrm{g} / \mathrm{m} 3)$ increase in average $\mathrm{O} 3$ of the day and the preceding day (lag0-1) was associated with an increase in dairy cow deaths of $3.92 \%$ (95\% CI: 1.08: 6.84). The estimated increase in mortality for an IQR $(14.5 \mu \mathrm{g} /$ $\mathrm{m} 3$ ) increase in PM10 (lag0-1) was $2.99 \%$ (95 \% CI: 0.50: 5.55) and the corresponding estimate for an IQR $(10.6 \mu \mathrm{g} / \mathrm{m} 3)$ increase in NO2 (lag0-1) was $6.47 \%$ (95\% CI: 3.57: 9.45). We did not find evidence for air pollution induced mortality during the cold period of the year. Conclusion: Our study in cattle adds to the existing human findings and further improves its causality. We observed a substantial higher risk for short-term effects of air pollution on cattle mortality compared with epidemiological derived risks in human populations. Cow mortality may be a sensitive indicator of air pollution hazards and provide an early warning system for public health intervention.

\section{Abstract \#: P 204}

The combined effects of job insecurity and environmental stress on self-rated health: a german national Representative cohort study

\section{Natalie Riedel ${ }^{1}$, Adrian Loerbroks ${ }^{2}$, Peter Angerer ${ }^{2}, \mathrm{Jian}_{\mathrm{Li}^{2}}{ }^{2}$}

${ }^{1}$ University of Bremen, Faculty of Human and Health Sciences, Institute of Public Health and Nursing Research, Department of Social Epidemiology; ${ }^{2}$ Heinrich-Heine-University of Düsseldorf, Medical Faculty, Institute of Occupational and Social Medicine; Corresponding author's e-mail: nriedel@uni-bremen.de

Objective: With the labour market growing more and more unpredictable, job insecurity has been spreading as a chronic stressor affecting employees' health. Meanwhile, economic restructuring involves an increase in transportation which is a major cause for chronic exposure to environmental pollution in residential areas. Therefore, we assume that work-related and environmental stress may accumulate and have a joint impact on people's health. Yet, research linking concepts of work stress and environmental stress in one study is sparse. Hence, we examined the combined effects of job insecurity and environmental stress on health in Germany.

Methods: Using the national representative data from the German Socio-Economic Panel (G-SOEP) Study in 2009 and 2011, we included 6544 employed persons who were free from poor self-rated health (being reported as not so good or bad) in 2009. At baseline in 2009 , high job insecurity (JI) was defined by respondents' ratings of the probability $(\geq 50 \%)$ of losing their job within the next 2 years. Respondents also rated the extent to be affected by noise or air pollution and this exposure was dichotomised as high environmental stress (ES, being perceived as just bearable, strong or very strong) versus low. Information on JI and ES was combined in one composite variable with four exposure categories (with low JI and low ES as reference group). The associations of JI and ES in 2009 with poor self-rated health in 2011 was quantified by risk ratios (RRs) with $95 \%$ confidence intervals (CIs) using Poisson regression modelling, adjusting for socio-demographic factors, employment contract, and health-related behaviours.

Results: After a 2-year follow-up, 571 (9\%) participants rated their health as poor. As compared to the reference group (both low IJ and ES), participants with high JI and low ES, and with low JI and high ES had similar risks of poor self-rated health $(\mathrm{RR}=1.42,95 \%$ $\mathrm{CIs}=1.15-1.74$, and $\mathrm{RR}=1.49,95 \% \mathrm{CI}=1.22-1.83$, respectively), while the combined exposure of both high JI and high ES was associated with a further excess risk of poor self-rated health $(\mathrm{RR}=1.92,95 \% \mathrm{CI}=1.47-2.51)$.

Conclusions: Our study shows that both job insecurity and environmental stress can have independent adverse impacts on employees' self-rated health. Further, their simultaneous exposure is likely to exert an additive effect.

\section{Abstract \#: P 205}

\section{Heat disorders: complex disease, simple solution}

Chika U. Obi ${ }^{1}$, Oludoyinmola O. Ojifinni ${ }^{1}$, Obioma C. Uchendu ${ }^{1}$

${ }^{1}$ Community Medicine Department, University College Hospital, Ibadan, Nigeria; Corresponding author's e-mail: oludoyinmola@gmail.com

Background: Heat disorders resulting from exposure to high temperature environments are preventable using simple interventions. The experience of heat disorders among workers close to a gas flare was assessed.

Methods: A descriptive cross-sectional study was performed among a group of workers at a gas plant using interviewer-administered, structured questionnaire to collect information on the workers' sociodemographic characteristics and experience of symptoms of heat disorders. Physical examinations were also carried out and data retrieved from the clinic records on symptoms that required medical care.

Results: A total of 64 workers were evaluated with mean age $37.0 \pm 7.7$ years. The mean ambient temperature overall was $35.9 \pm 5.5{ }^{\circ} \mathrm{C}$. The temperature varied from $46.18 \pm 6.7{ }^{\circ} \mathrm{C}$ in the area closest to the flare to $32.28 \pm 3.3{ }^{\circ} \mathrm{C}$ about $500 \mathrm{~m}$ away from the flare. The self-reported symptoms of mild heat stress were skin rashes $(93.8 \%)$, muscle cramps $(79.2 \%)$ and dry mouth $(60.4 \%)$. Selfreported symptoms of moderate to severe heat stress were similar to the outpatient clinic records and included irrational behaviour 47 $(88.7 \%)$, restlessness $42(79.2 \%)$ fainting spells $53(100 \%)$ and 
vomiting $51(83.6 \%)$. Physical examination revealed an increase in the number who had abnormal systolic blood pressure (SBP) from 51.6 to $54.7 \%$ most of the workers reported experiencing more heat $(95.3 \%)$, sweating more $(90.6 \%)$ and being more thirsty $(93.8 \%)$ at work than in their residence. More than two-thirds $(81.3 \%)$ drink above three litres of water in a day while $57(89.1 \%)$ drink water most while at work especially during the day shift $(60.9 \%)$. The preferred season was the wet/rainy season for $53(82.8 \%)$ due to the coolness of the weather $(49 / 76.6 \%)$.

Conclusion: The control of morbidity secondary to heat exposure involves simple measures such drinking water that is fortified with electrolytes. Long term prevention through harvesting rather than flaring of natural gas is highly important in occupational settings.

\section{Abstract \#: P 206}

Repeated measure of oxidative stress biomarkers in residents after the Hebei spirit oil spill

Jung Ah Kim ${ }^{1}$, Myungsook Park ${ }^{1}$, Eun Kyoung Yang ${ }^{1}$, Young-Hyun $\mathrm{Choi}^{1}$, Mina $\mathrm{Ha}^{2}$, Hae-Kwan Cheong ${ }^{3}$

${ }^{1}$ Taean Environmental Health Center, Korea; ${ }^{2}$ Dankook University College of Medicine, Korea; ${ }^{3}$ Sungkyuonkwan University School of Medicine, Korea; Corresponding author's e-mail: jungah6365@gmail.com

Background: The Hebei Spirit oil tanker spilled 12,547 kl of crude oil in December 2007 in South Korea. Repeated measure of oxidative stress biomarker were conducted approximately 1.5, 3, 4 and 7 years after the accident among residents who live in exposed areas to assess the possible long-term persistence and recovery associated with the exposure to spilled crude oil.

Methods: Urinary 8-hydroxy-2'-deoxyguanosine (8-OHdG) and urinary malondialdehyde (MDA) levels were measured as indicators of oxidative DNA damage and lipid peroxidation, respectively. The each survey contained 65 participants whose samples were repeatedly analysed in 2009, 2010, 2012 and 2014.

Results: The geometric mean of urinary $8-\mathrm{OHdG}$ and MDA levels, $1.5,3,4$ and 7 years after the oil spill were $5.6,5.8,6.7$ and $5.3 \mu \mathrm{g} / \mathrm{g}$ creatinine and $4.0,2.5,2.3$ and $2.1 \mu \mathrm{mol} / \mathrm{g}$ creatinine, respectively. The urinary 8 -OHdG levels had not decreased as time passed among residents in affected area by crude oil.

Conclusions: Although time passed, the urinary 8-OHdG levels were not recovered during the follow-up period. The mid- and long-term adverse health effects due to the oil spill exposure are a concern in inhabitants.

\section{Abstract \#: P 207}

\section{Databased observational surveillance of occupational non-hodgkin lymphoma}

$\underline{\text { Delphine Rieutort }}^{1}$, Régis de Gaudemaris ${ }^{1,2}$, Dominique J. Bicout ${ }^{1,3}$

${ }^{1}$ UJF-Grenoble 1/CNRS/TIMC-IMAG UMR 5525 (EPSP team -

Environment and Health Prediction of Populations), Grenoble, F-38041, France; ${ }^{2}$ Occupational and Environmental Diseases Centre, Grenoble Teaching Hospital (CHU Grenoble), Grenoble, France; ${ }^{3}$ Biomathematics and Epidemiology EPSP-TIMC, VetAgro Sup, Veterinary Campus of Lyon, Marcy l'Etoile, France; Corresponding author's e-mail: delphine.rieutort@imag.fr

Background: The lymphoma is a rare cancer of lymphatic system (3\% of all cancers) and non-Hodgkin lymphoma (NHL) represents
$90 \%$ of all lymphomas. In most developed countries, NHL incidence was found increasing from 1970s until the end of the 20th century and starting to level off in the last few years. Causes of the increase and associated risk factors remain largely unknown, because immunological factor and behavioral factors, such as tobacco or alcohol, cannot explain this entirely. In this context, hypotheses on exposures such as solvents or agricultural products have been suggested, but the very widespread multi-exposure at the workplace prevents to conclude from epidemiological studies.

Objectives: Use the observational database of the RNV3P network (French National Network of Surveillance and Prevention of Occupational Diseases) to investigate the relationship structure of NHL with occupational (multi)exposures and draw the spectrum of NHL's occupational (multi)exposures.

Methods: We employed the newly developed Observational Surveillance method, based on the Occupational Exposome approach, for an optimal use of the RNV3P database. The method involves structuring the data in terms of an occupational exposome that is a relational network of significant occupational exposures associated with the disease of interest. It allows the identification of significant associations (exposures and/or sectors related to the disease) and the study of temporal evolution of the exposure spectrum.

Results: In 2011, RNV3P network recruited about 205 OHP of NHL ( $86.8 \%$ of men). They were recorded with 142 distinct hazards founded in 43 different occupational sectors, mostly manufacturing industries. Risk of NHL seemed to be more related to occupational exposure to organic solvents and thinners, such as benzene or trichloroethylene, agricultural products and exposure to ionizing radiations, with an important report since 2001. Observational Surveillance method allowed highlighting the importance of multiexposure related to NHL with nine particular associations of 2 exposures, four with 3 associated hazards and one with 4 associated hazards. Moreover, 13 were highlighted as emerging, such as polycyclic aromatic hydrocarbons or the association organophosphates pesticides/herbicides. Sectors that seemed to be most at risk were agriculture, chemical industry and construction.

Conclusion: Observational Surveillance of NHL from the RNV3P has allowed to highlight new occupational (multi)exposures, in addition to those already known, and to study the dynamic structure of occupational exposures and NHL relationship in specific workplace. It would be interesting to conduct further epidemiological investigations of these associations. This approach is not specific to occupational databases and can be applied to other databases.

\section{Abstract \#: P 208}

Environmental dioxin exposure and risk of breast cancer: the geo3n pilot study in the rhône-alpes region, France

Aurélie Danjou $^{1,2}$, Thomas Coudon ${ }^{1,2}$, Laure Dossus ${ }^{3}$, Elodie Faure ${ }^{1}$, Guillaume Harel $^{1}$, Françoise Clavel-Chapelon ${ }^{3}$, Béatrice Fervers ${ }^{1,2}$,

${ }^{1}$ Unité Cancer et Environnement, Centre Léon Bérard, Lyon France; ${ }^{2}$ Laboratoire Santé, Individu, Société EAM 4128, Université Claude Bernard Lyon 1, Lyon, France; ${ }^{3}$ Inserm, CESP, UMR-S U1018, Equipe 9 : Mode de vie, gènes et santé: épidémiologie intégrée transgénérationnelle, Université Paris-Sud; Corresponding author's e-mail: aurelie.danjou@lyon.unicancer.fr

Background: Dioxins are environmental pollutants emitted from many sources including industries with combustion activity such as waste incinerators, metal and cement industries. Known to be endocrine disrupting chemicals, dioxins are suspected to increase breast cancer (BC) risk: Tetrachlorodibenzo-p-dioxin (TCDD) has been classified as Group 1 carcinogen by the International Agency for 
Research on Cancer. The GEO3 $\mathrm{N}$ project aims to investigate the hypothesis of an association between environmental dioxin exposure and $\mathrm{BC}$ risk in a case-control study $(5500 \mathrm{BC}$ cases and 5500 controls) nested within the French E3N prospective cohort ("Etude Epidémiologique auprès de femmes de l'Education Nationale", involving 98,995 women aged 45-60 in 1990, www.e3n.fr).

Methods: A first stage, involving $525 \mathrm{BC}$ cases and 953 controls (matched on age, place of residence, menopausal status and date of inclusion in E3N), was conducted in the Rhône-Alpes region to develop and optimize the Geographic Information System (GIS) to assess environmental dioxin exposure from 1990 to 2008. Dioxin emitting sources were identified through national and regional databases and their emission level was estimated via emission factors from the United National Environment Program (UNEP) Toolkit ( www.unep.org/tnt-unep/toolkit/). We used the ArcGIS software to locate dioxin emitting sources and participant residential history between 1990 and 2008. Environmental dioxin exposure will be assessed using three different surrogate exposures (within $3 \mathrm{~km}$ around the subject address, presence/absence of a source, distance to the nearest source and number of years of residence close to the source). We will use conditional logistic regression to estimate BC risk associated with environmental dioxin exposure adjusting for individual $\mathrm{BC}$ risk factors (reproductive factors, alcohol, body mass index...).

Results: Results on the association between BC risk and dioxin surrogate exposures will be presented and discussed at the European Congress of Epidemiology 2015.

Conclusion: This first stage of the GEO3 $\mathrm{N}$ project will allow developing the GIS based method to estimate the association between environmental dioxin exposure and $\mathrm{BC}$ risk in a large prospective cohort. In a second stage, a more complex dioxin exposure score will combine the distance to the sources, the emission levels, the exposure duration, as well as meteorological and topographical data and some technical characteristics of the sources such as stack height. Future analyses will also include both dietary and environmental dioxin exposures.

\section{Abstract \#: P 209}

The ageing shift worker: a prospective cohort study on need for recovery, disability and retirement intentions

Fleur Gommans ${ }^{1}$, Nicole Jansen ${ }^{1}$, Dave Stynen ${ }^{1}$, Andries de Grip ${ }^{2}$, IJmert Kant

${ }^{1}$ Department of Epidemiology, CAPHRI School for Public Health and Primary Care, Faculty of Health, Medicine and Life Sciences, Maastricht University, Maastricht, The Netherlands; ${ }^{2}$ Research Centre for Education and the Labour Market (ROA), Maastricht University, Maastricht, The Netherlands; Corresponding author's e-mail: fleur.gommans@maastrichtuniversity.nl

Background: This study investigates whether different shift work schedules, compared to day work, are associated with differences in employees' need for recovery (NFR), future disability and retirement intentions across the work career. Also, the association between exposure duration to shift work and NFR is investigated as well as the possible presence of a healthy worker effect. All analyses distinguish between workers employed in the industry and the healthcare sector as well as between two age categories: $35-44$ and 45-60 years.

Methods: Data from the prospective Maastricht Cohort Study was used. A subsample of industry ( $\mathrm{n}=1877$, all men) three-shift, fiveshift and day workers and a subsample of $(\mathrm{n}=818,624$ women and 194 men) irregular shift and day workers in healthcare were investigated for a follow-up period of 32 months. GEE and Cox regression analyses were performed to investigate NFR. Future disability was investigated using Cox regression and retirement intentions using logistic regression analysis. Exposure duration was investigated with Poisson regression analysis and the healthy worker effect with time-lag GEE analysis.

Results: In the 35-44 age category, three-shift and five-shift workers had significantly higher NFR scores as compared to day workers. In the 45-60 age category, only three-shift workers had a higher NFR compared to day workers. Irregular shift work was associated with higher NFR in the 45-60 category. Three- and five-shift industry workers were at risk for future disability and irregular shift work was a risk factor for disability among irregular shift workers aged 45-60. No significant Results: were found regarding retirement intentions. This study shows that shift workers with a higher NFR had a higher risk of altering to a daywork job, compared to shift workers with lower NFR scores, implying a possible healthy worker effect.

Conclusion: Shift work was generally associated with higher levels of NFR and a higher risk of disability. Different types of shift work schedules are differently associated with these outcomes, and associations varied across the work career and economic sectors. Moreover, the findings in this study might be an underestimation of the true results since secondary selection processes seem to have taken place.

The implementation of shift work (type) should carefully be considered, and the health and performance of shift workers should be monitored throughout the work career. Different shift work types exist and shift work schedules allow for optimisation, indicating that preventive measures for adverse outcomes should be tailored for different types of shift work across the work career.

\section{Abstract \#: P 210}

Analysing the relationship between temperature and west midlands ambulance data

$\underline{\text { Paul Fisher }}^{1}$

${ }^{1}$ University of Birmingham; Corresponding author's e-mail: p.a.fisher@bham.ac.uk

Background: Although there has been some research on the impact of extreme weather on the number of ambulance call-out incidents, especially heat waves, there has been very little research on the impact of cold weather on ambulance call-outs and response times. In the UK, there is a target response rate of $75 \%$ of life threatening incidents (Category A) that must be responded to within $8 \mathrm{~min}$.

Methods: This research compares daily air temperature data with ambulance call-out data for Birmingham over a 5-year period (2009-2014).

Results: A significant relationship between extreme weather and increased ambulance call-out and response times can clearly be shown. Both hot and cold weather have a negative impact on response times. In December 2010 (the coldest December for more than 100 years), the response rate fell below $50 \%$ for 3 days in a row (18 December-20 December 2010) with a mean response time of $15 \mathrm{~min}$. For every reduction of air temperature by $1{ }^{\circ} \mathrm{C}$ there was a reduction of $1.3 \%$ in performance.

Conclusion: Improved weather forecasting and the take up of adaptation measures, such as the use of winter tyres, are suggested for consideration as management tools to improve ambulance response resilience during extreme weather. Also it is suggested that ambulance response times could be used as part of the syndromic surveillance system at Public Health England (PHE). 


\section{Abstract \#: P 211}

\section{Calux-teq levels of the Hebei oil spill on the residents of Taean,} Korea

\author{
Myung S. Park ${ }^{1}$ \\ ${ }^{1}$ Taean Environmental Health Center, Taean, Republic of Korea; \\ Corresponding author's e-mail: pms1816@gmail.com
}

Background: The oil spill from the Heibei Spirit oil tank in December 2007 contaminated the Yellow Coast of South Korea. Crude oil has high level of Dioxin. We evaluated the CALUX-TEQ levels of residents who lived near oil spill area.

Methods: TEQ levels were measured using DRE-CALUX bioassays. Stable DRE-Luc-Hepa1c1c7 cells were seeded in 96 well plates at a density of $5 \times 103$ cells and treated high or low exposure serum samples for $24 \mathrm{~h}$, lysed, and assayed for luciferase activity by Luciferase assay system (Promega, Madison, WI). Adding luciferin as a substrate and measuring the emitted bioluminescence by a luminometer (Berthold Detection System, Oak Ridge, TN, USA). Luciferase activity was normalized to protein content.

Results: TEQ levels were significantly higher in high-exposure area serum samples compared to controls $(151.7 \pm 4.962$ vs. $120.6 \pm 5.157 \mathrm{pg}-\mathrm{TEQ} / \mathrm{L}, P<0.0001)$. TEQ levels in high exposure area serum samples did not differ according to age.

Conclusions: This study suggested that exposure an oil spill is a risk factor for higher CALUX-TEQ level.

\section{Abstract \#: P 213}

Older workers' fatigue, work enjoyment and retirement intentions: the longitudinal impact of work-related and personal resources

\section{Dave Stynen ${ }^{1}$, Nicole Jansen ${ }^{1}$, IJmert Kant ${ }^{1}$}

${ }^{1}$ Department of Epidemiology, CAPHRI School for Public Health and Primary Care, Faculty of Health, Medicine and Life Sciences, Maastricht University, Maastricht, The Netherlands; Corresponding author's e-mail: dave.stynen@maastrichtuniversity.nl

Background: Due to the greying of the workforce, an important striving throughout the European Union is to enhance the work participation of older ( $\geq 45$ years) workers. To date, much emphasis is put on accommodating work in such a way that older workers are protected from demanding working conditions rather than to stimulating working conditions or resources that foster active ageing. In this longitudinal study a resource perspective is adopted on a key concept of the retirement decision process: retirement intentions. More specifically, this study investigates the impact of a broad set of work-related (skill discretion, decision authority, co-worker and supervisor social support, developmental and accommodative support) and personal resources (mastery, general health and educational level) on older workers' retirement intentions. Furthermore, guided by theoretical models like the Job Demands-Resources model this study tries to advance our understanding on the various ways resources may impact retirement intentions. Thereby this study aims to investigate (1) the pathways that may explain the relationships between resources and early retirement intentions: fatigue and work enjoyment: and (2) the buffering role of resources in the relationship of psychological job demands and these work-related outcomes.

Methods: Data from the prospective Maastricht Cohort Study was used. A subsample of full time day workers $(\mathrm{N}=2080)$ was followed for 4 years (October 2008-October 2012). Cross-sectional and longitudinal associations were investigated among older ( $\geq 45$ years) and younger workers by means of linear, logistic and cox regression analyses and adjusted for potential confounders.

Results: Longitudinal results indicate that among older workers, decision authority related negatively to prolonged fatigue $(\mathrm{HR}=0.97,95 \% \mathrm{CI}, 0.94-1.00)$ and that mastery and general health related negatively to prolonged fatigue $(\mathrm{HR}=0.87,95 \% \mathrm{CI}$, 0.82-0.93: $\mathrm{HR}=0.42,95 \% \mathrm{CI}, 0.30-0.59$, respectively) and work disenjoyment $(\mathrm{HR}=0.90,95 \% \mathrm{CI}, 0.81-0.99: \mathrm{HR}=0.47,95 \%$ CI, $0.27-0.80$, respectively). A low educational degree was positively related to prolonged fatigue $(\mathrm{HR}=2.16,95 \% \mathrm{CI}, 1.24-3.77)$. In turn, prolonged fatigue is positively associated with older workers' retirement intentions ( $\mathrm{HR}=2.03,95 \% \mathrm{CI}, 1.32-3.14)$. Finally, there was little evidence for effect modification by resources in the relationship between psychological job demands and the outcomes. Whereas personal resources played a less prominent role among younger workers, both decision authority $(\mathrm{HR}=0.89,95 \% \mathrm{CI}$, 0.81-0.99) and skill discretion ( $\mathrm{HR}=0.85,95 \% \mathrm{CI}, 0.76-0.96)$ related negatively to work disenjoyment among these workers.

Conclusion: Resources are of value to workers to prevent fatigue and work disenjoyment. For the retention of older workers it is important to consider the potential beneficial influence of personal resources as well as work-related resources, like more decision authority.

\section{Abstract \#: P 214}

Chronotype, social jetlag, and anthropometric measures

Mei Yong ${ }^{1}$, Christina Germann ${ }^{1}$, Stefan Lang $^{1}$, Christoph Oberlinner ${ }^{1}$

${ }^{1}$ Occupational Medicine and Health Protection, BASF SE;

Corresponding author's e-mail: mei.yong@basf.com

Background: The individual phase of entrainment (chronotype) of the circadian system regulates physiology and behavior so that peaks and troughs occur earlier or later within the $24 \mathrm{~h}$ day. A misalignment between the individual biological time, which can be assessed by chronotype, and social time, as determined by work schedules, results in a so-called social jetlag. Both chronotype and social jetlag may potentially influence metabolism and possibly promote development of obesity. The present study investigates the impact of chronotype, sleep duration, and social jetlag on anthropometric measures of fat accumulation and abdominal fat distribution, within an industrial setting.

Methods: Between 2011 and 2013, 2474 employees participated in a health check offered by an occupational health promotion program and filled out the Munich ChronoType Questionnaire, adapted to the $4 \times 12 \mathrm{~h}$ shift schedule. We computed sleep duration on work and free days, chronotype (based on mid-sleep timing on work days and corrected for sleep debt accumulated during the work week) and social jetlag (difference between mid-sleep on work and free days). Body height, weight, and waist circumference were measured during the physical examination, and body mass index (BMI) and waist-toheight-ratio (WHR) were then derived und used as anthropometric measures. We used linear regression models to examine the impact of chronotype, sleep duration, and social jetlag on BMI and WHR after adjusting for potential confounders such as age, gender, smoking behavior, and job level.

Results: The median BMI and WHR of the present study population was 25.5 (inter-quartile range: $23.1-28.4$ ) and $52.0 \%$ (inter-quartile range: $47.9-56.5 \%$ ), respectively. Participants reported an average daily sleep duration of $7.35 \mathrm{~h}$ (SD: 1.2), had an average chronotype of 3:08 am (SD: $1 \mathrm{~h}$ ). Average social jetlag corresponded to $1.96 \mathrm{~h}$ (SD: 2.05). Increasing social jetlag was associated with an increasing BMI, while longer sleep duration was inversely associated with BMI. With 
respect to WHR, social jetlag showed positive impact, while estimate of sleep duration didn't reach the statistical significance.

Conclusion: In line with a growing body of literature, our results show that circadian misalignment, but not chrono-type per se, may be related with fat metabolism. Our results suggest that sleep duration might have impact on fat accumulation rather than abdominal fat distribution.

\section{Abstract \#: P 215}

\section{A conceptual framework to describe and measure shift work in epidemiological studies}

Van de Langenberg, Daniella ${ }^{1}$, Vlaanderen, Jelle ${ }^{1}$, Rodenburg, Wendy $^{2}$, Rookus, Matti ${ }^{3}$, Vermeulen, Roel ${ }^{1}$

${ }^{1}$ IRAS, Institute for Risk Assessment Sciences, Utrecht University; ${ }^{2}$ RIVM, National Institute for Public Health and the Environment; ${ }^{3}$ NKI, Netherlands Cancer Institute; Corresponding author's e-mail: D.vandeLangenberg@uu.nl

Background: Shift work is a multidimensional exposure and disrupts both lifestyle and circadian rhythms. Identifying which dimensions contribute most to shift work induced adverse health effects is important both from an etiological and a public health perspective. We developed a conceptual framework that describes the multidimensionality of exposure to shift work in order to optimally utilize future epidemiological studies through implementation of improved exposure metrics. Furthermore, this framework can be used to develop risk analyses through the use of Directed Acyclic Graphs. Finally, we also identified measurement methods that can optimally assess each of the identified exposure dimensions.

Methods: We conducted a literature review of epidemiological and animal studies to identify exposure dimensions and associated biological pathways. We structured the exposure dimensions and biological pathways to provide a clear overview of overlap and interactions. Measurement methods were primarily identified from existing epidemiological studies or studies focused on exposure assessment.

Results: The conceptual framework describes the wide range of (biological, social and behavioral) aspects and includes the following shift-work dimensions: disturbed social pattern, behavioral changes including diet and physical activity, disturbed sleep, nutrition at night, light at night, and sun exposure. We have identified a suite of tools which are capable of measuring these dimensions including traditional epidemiological results (e.g. questionnaires), objective exposure measurement devices (e.g. actimetry and light sensors), and biological markers (circadian disruption).

Conclusions: Accurately measuring shift-work aspects in future research, using our conceptual framework, will result in innovative new research directions and might lead to promising clues for interventions. We used the conceptual framework to design a molecular epidemiology study among nurses which aims to identify the most relevant exposure dimensions of night-shift work using detailed questionnaires and objective monitors. In addition we aim to identify biomarkers for both acute and chronic circadian disruption associated to these specific night-shift work dimensions. Linking accurate measurements of specific night-shift work dimensions with biomarkers reflecting circadian disruption might provide insights into which dimensions are most harmful to human health.

\section{Abstract \#: P 217}

Development of an instrument to evaluate the effectiveness of a re-employment programme in a non-experimental setting

M. Schuring PhD, H.F. Lingsma PhD, Prof. A. Burdorf

Corresponding author's e-mail: m.schuring@erasmusmc.nl

Background: There is a need for more evidence on effective strategies to promote entering paid employment. However, in this area randomized controlled trials are often not feasible. New evaluation strategies are needed based on observational studies. A new approach was used by use of a quality indicator which presents the ratio of observed number of persons entering paid employment over expected number of persons entering paid employment. In the current study a prognostic model with validation is developed to estimate the expected number of persons entering paid employment.

Methods: Longitudinal data from the Statistics Netherlands with a representative sample of Dutch non-employed persons (age 18-64 years) receiving unemployment- or social security benefits, was used to develop a prognostic model for entering paid employment within 1 year. Factors that predicted entering paid employment were selected in the multivariate logistic regression model with the backward selection procedure. A prediction rule was made using the regression coefficients to predict entering paid employment based on a person's characteristics. The discriminatory power of the prognostic model was assessed in the dataset in which the model was developed (development AUC) as well as in other datasets (validated AUCs). External validity was investigated in three longitudinal datasets with (1) 225 unemployed persons living in one of the largest cities in the Netherland, (2) 750 older unemployed persons (age 45-64), and (3) 7279 non-employed persons receiving unemployment or social security benefits.

Results: Sociodemographic characteristics (age, sex, education), household situation (marital status, children in the household), perceived health and type of benefit (unemployment or social security benefit) were included in the prognostic model. The development AUC was 0.80 , and the validated AUCs ranged from 0.60 to 0.75 . The calibration plot showed that the expected probabilities agreed well with the observed frequencies in the dataset in which the model was developed as well as in the three external datasets.

Conclusion: The effectiveness of a re-employment programme can be evaluated by comparing the observed number of persons that entered paid employment with the expected number of persons that entered paid employment, taking into account the characteristics of the participants. Using prognostic models to investigate the effectiveness of re-employment programmes is a promising strategy when randomized controlled trials are not feasible.

\section{Abstract \#: P 218}

Effectiveness of co-oximetry and minimum advice for smoking cessation in kidney transplant recipients

Salvador Pita-Fernández ${ }^{1}$, Francisco Valdés-Cañedo ${ }^{2}$, Rocío Seijo-Bestilleiro ${ }^{1}$, Teresa Seoane-Pillado ${ }^{1}$, Beatriz López-Calviño ${ }^{1}$, Sonia Pértega-Díaz ${ }^{1}$

${ }^{1}$ Clinical Epidemiology and Biostatistics Unit, A Coruña Hospital;

${ }^{2}$ Nephrology service, A Coruña Hospital; Corresponding author's e-mail: sonia.pertega.diaz@sergas.es 
Background: In kidney transplant patients, smoking is an important factor associated with graft loss and mortality. It is important to promote smoking cessation, as many adverse events after transplantation may be associated with smoking in these patients.

The aim of this study is to determine the efficacy of the measurement of exhaled carbon monoxide (CO) plus brief advice, in comparison with brief advice alone, for smoking cessation in kidney transplant recipients.

Methods: Design: Randomized, controlled trial.

Setting: A Coruña Hospital (northwest Spain).

Period: 2012-2014.

Inclusion criteria: Smoking kidney transplant recipients, with informed consent.

Exclusion criteria: Smokers in the preparation stage of change, patients with terminal illness/mental disability. Randomization: To control (brief advice for smoking cessation) or intervention (brief advice + exhaled $\mathrm{CO}$ measurement) group.

Measurements: Sociodemographic characteristics, cardiovascular risk factors. Self-reported smoking habit, Fagerstrom Test for Nicotine Dependence, motivation to stop smoking (Ritchmond Test).

Outcomes: The effectiveness will be evaluated at 3, 6, 9 and 12 months by: (a) Smoking cessation rate, (b) Tobacco consumption, (c) Motivation to quit smoking, (d) Nicotine dependence.

Analysis: Descriptive, Mann-Whitney and Chi squared tests. Relative risk (RR), relative risk reduction (RRR), absolute risk reduction (RAR) and number needed to treat (NNT).

Results: Out of the $\mathrm{n}=1006$ patients contacted, 498 (49.5\%) were smokers before transplantation and $127(12.6 \%)$ at the time of conducting the study $(12.4 \pm 8.6$ cigarettes/day). The mean score of Richmond questionnaire was $6.2 \pm 2.4$ and $1.9 \pm 2.2$ for the Fagerstrom test.

Of all smoking patients, $\mathrm{n}=125$ agreed to participate in the study (60 allocated to the intervention and 65 to the control group). No differences were found between groups regarding demographic characteristics, time since transplant, and basal nicotine dependence or motivation to quit smoking. results at 12 months of follow-up are presented. Smoking cessation rate, confirmed by urine cotinine test, was significantly higher in the intervention group (46.6 vs. $25.9 \%$ : $p=0.018)(\mathrm{RR}=0.72: 95 \% \mathrm{CI}: 0.54-0.95)(\mathrm{NNT}=5: 95 \% \mathrm{CI}:$ 3-26). Self-report smoking cessation rate was also higher in the intervention group, although with no significant differences (46.6 vs. $32.3 \%, p=0.109$ ).

A decrease in tobacco consumption was found in both groups ( -6.7 vs. -6.1 cigarettes/day: $p=0.852$ ). No differences were found in the motivation to quit smoking or in the level of dependency.

Conclusions: An intervention based in $\mathrm{CO}$ measurement, combined with minimum advice for smoking cessation, contributes to decrease smoking prevalence and cigarette consumption in kidney transplant recipients.

\section{Abstract \#: P 219}

Are congenital urinary tract and genital anomalies related to folic acid?

Fenneke Blom $^{1}$, Jorieke E.H. Bergman ${ }^{2}$, Cynthia Kusters ${ }^{2}$, Rien J.M. Nijman ${ }^{3}$, Mireille N.M. van Poppel ${ }^{4,5}$, Martina C. Cornel ${ }^{1}$, Hermien E.K. de Walle ${ }^{2}$

${ }^{1}$ VU University Medical Center, Department of Clinical Genetics and EMGO Institute for Health and Care Research, Community Genetics Section, Amsterdam, The Netherlands; ${ }^{2}$ University of Groningen, University Medical Center Groningen, Eurocat Registration Northern Netherlands, Department of Genetics, Groningen, The Netherlands; ${ }^{3}$ University of Groningen, University Medical Center Groningen,
Department of Urology, Groningen, The Netherlands; ${ }^{4}$ VU University Medical Center, Department of Public and Occupational Health and EMGO Institute for Health and Care Research, Amsterdam, The Netherlands; ${ }^{5}$ Institute for Sport Science, Karl-Franzens University, Graz; Corresponding author's e-mail: f.blom@vumc.nl

Background: The literature on the association between congenital urinary tract and genital anomalies (CUGA) and maternal use of folic acid (FA) is inconclusive.

Objective: We investigated possible associations of CUGA with periconceptional FA supplementation and use of FA-antagonists. We also tested whether CUGA are more likely to co-occur with FArelated congenital anomalies than with other congenital anomalies.

Design, setting and participants: Case-control study using data from EUROCAT Northern Netherlands, a population-based birth defects registry. Case definitions were based on ICD codes. The control group consisted of other congenital anomalies not related to FA.

Outcome measurements and statistical analysis: Using logistic regression, we analysed associations between CUGA and (1) FA supplementation, (2) FA-antagonists use, and (3) the co-occurrence with FA-related congenital anomalies.

Results and limitations: We included 868 CUGA cases and 3088 controls born 1997-2012. We found no associations between overall CUGA and use of FA or FA-antagonists, although FA was associated with a lower prevalence of cystic kidney disease (adjusted OR 0.58, $95 \%$ CI $0.33-1.00$ ). Users of FA-antagonists also had a higher risk for cystic kidney (OR 2.38, $95 \%$ CI 1.01-5.59). Infants with multiple congenital anomalies (MCA) with at least one CUGA and no known aetiology were less frequently affected by other FA-related congenital anomalies than MCA cases without CUGA (OR 0.30, $95 \%$ CI 0.19-0.46).

Conclusions: Our results suggest there is no association between FA supplementation and the occurrence of CUGA. We observed no increased occurrence of CUGA with FA-related congenital anomalies, this hypothesis needs further investigation.

\section{Abstract \#: P 220}

Effects of fetal perfluorinated compounds (PFCs) on thyroid hormones and birth outcome: results from the retrospective cohort study of EWHA birth \& growth

Byung-Mi Kim ${ }^{1}$, Hye sook Park ${ }^{1}$, Young lim $\mathrm{Kho}^{2}$, Eun Ae P ark ${ }^{3}$, Young Ju Kim ${ }^{4}$, Eun Jin Kwon ${ }^{1}$, Eun-Kyo Park ${ }^{1}$, Eunjeong Kim ${ }^{1}$, Eun Hee $\mathrm{Ha}^{1}$

${ }^{1}$ Department of Preventive Medicine, School of Medicine, Ewha Medical Research Center, EwhaWomans University; ${ }^{2}$ Department of Health, Environment \& Safety, Eul ji University, Seongnam, South Korea; ${ }^{3}$ Department of Pediatrics, School of Medicine, Ewha; Corresponding author's e-mail: kbm80@hanmail.net

Background: and aims: Perfluorinated chemicals (PFCs) have been widely used in a variety of products worldwide for years. Previous studies suggested that serum PFCs may influence on reproductive development and thyroid function, but the results remain unclear. Therefore, we aimed to investigate the relationship between concentrations of PFCs in serum and fetal thyroid hormone concentrations or birth outcome.

Methods: We collected 151 pregnant women and newborns through a retrospective cohort study of Ewha Birth \& Growth between 2006 and 2010 in Ewha Woman's University. Information on birth weight was obtained from medical records. Cord serum samples were analyzed for perfluorooctanoic acid (PFOA), perfluorodecanoic acid (PFDA), perfluorotridecanoic acid (PFTrDA), perfluorododecanoate 
(PFDoDA) and perfluorooctane sulfonate (PFOS) by high-performance liquid chromatograph coupled with a Triple Quad LC-MS/MS system. Total concentrations of thyroxin (T4), triiodothyronin (T3) and thyroid stimulating hormone (TSH) in blood serum were also quantified. We assessed the association between the cord serum PFCs levels and thyroid hormones or birth outcome by multiple linear regression analysis.

Results: PFOS, PFDA and PFDoDA showed a significantly negative association with birth weight after adjusting for major covariates (PFOS : adjusted $\beta=-0.08, p=0.03$, PFDA : adjusted $\beta=-0.14$, $p=0.05$, PFDoDA: adjusted $\beta=-0.17, p=0.01$ ). Fetal PFOS and PFDA were a significantly positive association with fetal total T3 concentrations. However, Most of the relationships between PFCs and T4 or TSH were no longer statistically significant.

Conclusion: Serum concentrations of fetal PFDA and PFOS are associated with total T3 or birth weight. More studies are warranted to clarify the causal relationship between PFCs and thyroid function or birth weight. Also, since thyroid hormones are crucial in the early development of the fetus, its clinical implication should be evaluated.

\section{Abstract \#: P 221}

Prediction models for length of stay in the neonatal unit: a systematic review

$\underline{\text { Sarah E. Seaton }}{ }^{1}$, Lisa Barker ${ }^{1}$, Elizabeth S. Draper ${ }^{1}$, Keith Abrams ${ }^{1}$, Bradley N. Manktelow ${ }^{1}$

${ }^{1}$ University of Leicester; Corresponding author's e-mail: sarah.seaton@le.ac.uk

Background: In the UK, 1 in 10 babies will require specialist neonatal care. The care required will depend on the needs of the baby but can last from a few hours to months. The ability to predict length of stay (LOS) is useful for resource planning, decision making and parent counselling. This review investigates all proposed prediction models for LOS, and identifies which factors may be considered clinically important for predicting LOS.

Methods: MEDLINE, Embase and Scopus were systematically searched for papers from 1994 to 2013 for research which investigated neonatal LOS. Exclusion criteria included: conference proceedings: review articles: clinical trials and specific disease areas (e.g. E-coli outbreaks). Data was extracted related to: study characteristics: primary outcome: clinical predictors and quality of the study. Clinical factors used to predict LOS were grouped into categories of: inherent factors: antenatal and maternal factors: conditions of the baby and organisational factors.

Results: Eight papers were identified that investigated multivariable prediction of LOS. Statistical methods used to predict LOS included: linear regression: logistic regression and competing risks. The eight studies presented a total of 35 factors considered predictive of LOS. Factors were selected using prior clinical knowledge in at least four studies.

All studies used inherent factors in their prediction, with the most popular being birthweight, gestational age and sex. These factors are simple to measure and present at birth, allowing for quick prediction of LOS. However, it was acknowledged that predicting LOS may need to use more information than just early occurring factors. Six studies attempted to do this by using the clinical condition of the baby to improve the LOS prediction. A wide range of clinical factors were used including early occurring conditions (e.g. reason for admission to the neonatal unit) and late occurring (e.g. occurrence of retinopathy of prematurity). Whilst important to account for clinical condition, there was little consensus on what that factor should be. However, irrespective of condition, in most instances, the main determinant of LOS is hypothesised to be prematurity.

Conclusion: Prediction of neonatal LOS is a complex issue, and there is little consensus on the most important clinical factors to use. However, it is likely that in many cases, predicting using only inherent factors will possibly suffice. Estimates of LOS could be updated as clinical conditions develop, however it is hypothesised that in most cases, LOS is determined by inherent factors.

\section{Abstract \#: P 222}

Decrease in perinatal mortality after introduction of prenatal screening in Monchegorsk (North-West Russia): birth registries study

Vitaly A. Postoev $^{1,2}$, Andrej M. Grjibovski ${ }^{2,3,4}$, Evert Nieboer ${ }^{5}$, Jon Øyvind Odland ${ }^{1}$

${ }^{1}$ Department of Community Medicine, UiT- The Arctic University of Norway, Troms $\varnothing$, Norway; ${ }^{2}$ International School of Public Health, Northern State Medical University, Arkhangelsk, Russia;

${ }^{3}$ Department of International Public Health, Norwegian Institute of Public Health, Oslo, Norway; ${ }^{4}$ Department of Preventive Medicine, International Kazakh-Turkish University, Turkestan, Kazakhstan; ${ }^{5}$ Department of Biochemistry and Biomedical Sciences, McMaster University, Hamilton, ON, Canada; Corresponding author's e-mail: vpo000@post.uit.no

Introduction: Prenatal diagnostics allow the detection of more than $50 \%$ a birth defects prior to delivery. It was established in Russia in 2000 as a routine method of screening for birth defects (BD). The aim of our study was to assess changes in perinatal mortality after perinatal screening was implemented in the city of Monchegorsk, Murmansk County, North-West Russia.

Methods: The Murmansk County Birth Registry and the Kola Birth Registry were the primary sources of information, and include 30,448 pregnancy outcomes in Monchegorsk during 1973-2011. The data from these registries were supplemented with information about pregnancy terminations for the period 2000-2007.

Results: The perinatal mortality among newborns with any kind of malformation decreased from 107.7 per 1000 newborns with BD $(95 \% \mathrm{CI}=86.3-129.1)$ in $1973-2000$ to $21.2(95 \% \mathrm{CI}=4.3-38.1)$ in 2001-2011, whereas that for all newborns and those without BD decreased two-fold. When including pregnancy termination data related to birth defects during 2000-2007, the perinatal mortality among all newborns became $17.7 / 1000(95 \% \mathrm{I}=14.7-22.0)$ instead of 12.4/1000 (95\% CI $=9.2-15.6)$. Logistic regression analysis indicated that mothers who had undergone at least one ultrasound examination during pregnancy $(n=9883)$ had a lower chance of having a newborn die during the perinatal period [adjusted $\mathrm{OR}=0.49$ (95\% CI: 0.27-0.89)]

Conclusion: Improved detection of severe malformations with subsequent termination is likely to have been the main contributor to the decrease in perinatal mortality in Murmansk County, Russia.

\section{Abstract \#: P 223}

Variations in very preterm births rates in Europe: Can valid comparisons be made using routine data?

\author{
Marie Delnord $^{1}$, Ashna D. Mohangoo ${ }^{2}$, Jennifer Zeitlin ${ }^{1}$ \\ ${ }^{1}$ Inserm UMR 1153, Obstetrical, Perinatal and Pediatric \\ Epidemiology Research Team (Epopé), Center for Epidemiology and
}


Statistics Sorbonne Paris Cité, Paris Descartes University; ${ }^{2}$ TNO Netherlands Organisation for Applied Scientific Research; Corresponding author's e-mail: marie.delnord@inserm.fr

Background: Very preterm infants ( $<32$ weeks gestational age (GA)) face high risks of mortality, neonatal morbidity and long term cognitive and motor impairments. Preterm birth rates vary greatly in Europe, but less is known about variations in very preterm birth. It is unclear if routine data can be used to make valid international comparisons. We investigated very preterm birth (VPTB) rates in Europe and assessed the impact of periviable births and stillbirths on country rates and rankings.

Methods: Using routine aggregate data from 2010 collected by the Euro-Peristat project from 32 European countries/regions covering $4,450,135$ births, we computed extremely ( $<28$ weeks GA) and very $(<32$ weeks GA) preterm birth rates. We studied the impact of including stillbirths as well as births at $22-23$ weeks GA on these rates.

Results: VPTB rates ranged between 8.8 and 19.8 per 1000 total births with a median of 12.9 (IQR 11.5-14.3) and between 6.5 and 14.1 per 1000 live births with a median of 10.3 (IQR 8.2-13.0). Removing births at 22-23 weeks GA reduced the median to 11.7 per 1000 total births (IQR 10.5-13.0) and to 10.0 per 1000 live births (IQR 9.0-11.3), with reductions of $20 \%$ in some countries. Stillbirths represented between 2 and $62 \%$ of births 24-27 weeks GA (median: $21 \%$ ) and between 3 and $26 \%$ of births $28-31$ weeks GA (median: $8 \%$ ). For births at 28-31 weeks GA, rankings were almost identical with and without stillbirths (rho $=0.94, p=0.00$ ): this association was also strong at 24-27 weeks GA (rho $=0.80, p=0.00)$ and weaker for births at 22-27 weeks GA (rho $=0.56, p=0.00)$.

Conclusion: VPTB birth rates vary substantially across Europe, but differences in the proportion of births at 22-23 weeks GA and of stillbirths appear to strongly influence these variations. Sensitivity analyses removing stillbirths and periviable births can be used to flag countries where registration practices and thresholds for recording live and stillbirths require further investigation.

\section{Abstract \#: P 224}

Linking databases on perinatal health: a review of the literature and current practices in Europe

Marie Delnord $^{1}$, Katarzyna Szamotulska ${ }^{2}$, Ashna Mohangoo ${ }^{3}$, Mika Gissler $^{4}$, Carmen Barona ${ }^{5}$, Sylvie Berrut ${ }^{6}$, Rachael Wood ${ }^{7}$, Nirupa Dattani ${ }^{8}$, Luule Sakkeus ${ }^{9}$, Irisa Zile ${ }^{10}$, Jennifer Zeitlin ${ }^{1}$

${ }^{1}$ Inserm UMR 1153, Obstetrical, Perinatal and Pediatric

Epidemiology Research Team (Epopé), Center for Epidemiology and Statistics Sorbonne Paris Cité, Paris Descartes University;

${ }^{2}$ Department of Epidemiology, National Research Institute of Mother and Child, Warsaw, Poland; ${ }^{3}$ Department Child Health, TNO

Netherlands Organization for Applied Scientific Research, Leiden, the Netherlands; ${ }^{4}$ Department of Information, THL National Institute for Health and Welfare, Helsinki, Finland \& NHV Nordic School of Public Health, Gothenburg, Sweden; ${ }^{5}$ General Directorate of Public Health. Generalitat Valenciana; ${ }^{6}$ Section Health, Swiss Federal Statistical Office; ${ }^{7}$ Information Services Division, NHS National Services Scotland; ${ }^{8}$ Maternal and Child Health and Care Research Centre, City University; ${ }^{9}$ Estonian Institute for Population Studies, Tallinn University; ${ }^{10}$ Centre for Disease Prevention and Control, Riga, Latvia; Corresponding author's e-mail: marie.delnord@inserm.fr

Background: International comparisons of perinatal health are complicated by the heterogeneity of data sources on pregnancy, maternal and neonatal outcomes. Record linkage by harmonizing data systems, enhances coverage, increases the information available about each birth and has been shown to improve the validity and quality of routine data. Linkage of data systems enables countries to develop their capacity for high quality perinatal health monitoring. We sought to assess the extent to which routine sources are linked for perinatal health research and reporting.

Methods: We conducted a systematic review of the literature by searching PubMed for studies on perinatal health citing linkage of routine databases or of a cohort study to a routine database from 2001 to 2011. We also surveyed European health monitoring professionals participating in the Euro-Peristat project about use of linkage for national perinatal health monitoring.

Results: 536 studies fit our inclusion criteria. Nearly half were from the US, Sweden and the UK: a further 24 countries contributed at least one publication. Most studies linked combinations of vital statistics data, hospital records, medical birth registers and cohort studies. Other sources were specific registers (cancer $(\mathrm{N}=61)$, congenital anomaly (53) and ART (19)) as well as census (63), occupational (43), insurance (23) prescription (20), and educational (10) databases. Eighteen of 29 (62\%) European countries reported using at least one linked dataset in 2010 to derive Euro-Peristat perinatal indicators and 5 used 2 or more.

Conclusion: Studies using linkage are concentrated in a few countries and routine linkages are not systematic practice in Europe. Linkage of routine data systems is a readily available option for improving the quality and completeness of perinatal health indicators. Linkage techniques have proven especially useful for public health scientists to answer complex research questions, conduct original life-course analyses, confidential enquiries, provide subgroup estimates of maternal or infant mortality, and to study trends in perinatal health indicators. Broader adoption of linkage could yield substantial gains for research and surveillance of perinatal health nationally and internationally.

\section{Abstract \#: P 225}

\section{Advanced paternal age and stillbirth rate}

Stine K. Urhoj ${ }^{1}$, Per K. Andersen ${ }^{2}$, Laust H. Mortensen ${ }^{1}$, George D. Smith ${ }^{3}$, Anne-Marie N. Andersen ${ }^{1}$

${ }^{1}$ Department of Public Health, University of Copenhagen, Copenhagen, Denmark; ${ }^{2}$ Department of Biostatistics, University of Copenhagen, Copenhagen, Denmark; ${ }^{3}$ School of Social and Community Medicine, University of Bristol, Bristol, United Kingdom; Corresponding author's e-mail: stur@sund.ku.dk

Background: Advanced paternal age has been associated with a variety of rare conditions and with diseases of great public health impact. An increased number of de novo point mutations in sperm with increasing age has been suggested as a mechanism, which would likely also affect fetal viability. Our aim was to investigate the association between advanced paternal age and stillbirth rate while adjusting for maternal age.

Methods: A population-based register-linkage study of all pregnancies in Denmark from 1994 to 2010 carried to a gestational age of at least 22 completed weeks was undertaken. Taking into account competing risks from other pregnancy outcomes (life birth, termination of pregnancy, hydatidiform mole), we estimated the relative rate of stillbirth ( $\geq 22$ weeks), and subgroups of stillbirths, according to paternal age by hazard ratios (HR) with $95 \%$ confidence intervals (CI), using a robust standard error estimator. Gestational age was used as underlying time. In Denmark, the paternity registration is more complete for live births $(98 \%)$ than for stillbirths $(83 \%)$, and for other pregnancy outcomes no such information is directly available. 
Aiming at a non-biased identification of fathers according to the pregnancy outcome, we applied a step-wise procedure for identification of paternity of all pregnancies. Paternal age was modeled categorically in six categories and by use of restricted cubic splines. We adjusted the analysis for maternal age modeled in 1-year categories, year of pregnancy outcome, and parental educational levels. Results: From a total of 1,125,864 pregnancies, the father could be identified from health registers in $98 \%$ (primarily live births). Based on our procedure, paternity was established in $84 \%$, a figure rising to $87 \%$ after restriction to parents aged $25+$ years $(n=911,700)$. A total of 4414 stillbirths were registered. Compared with fathers aged 30-34 years, the adjusted HRs of stillbirth among fathers in other age groups were as follows: 25-29 years, 1.03 (95 \% CI: 0.95-1.12): 35-39 years, 1.16 (95 \% CI: 1.06-1.26): 40-44 years, 1.40 (95\% CI: 1.24-1.57): 45-49 years, 1.17 (95\% CI: 0.94-1.46): and 50+ years, 1.42 (95 \% CI: 1.03-1.96). These estimates attenuated slightly when further adjusted for parental educational levels. Likewise, higher HRs with higher paternal age were found for fathers older than the reference group (32 years) when paternal age was modeled using restricted cubic splines. Analyses of preterm ( $\geq 22$ and $<37$ weeks), non-preterm ( $\geq 37$ weeks), early ( $\geq 22$ and $<28$ weeks) and late ( $\geq 28$ weeks) stillbirths separately showed similar results (All results: are preliminary).

Conclusions: The relative rate of stillbirth increased slightly with higher paternal age, but advanced paternal age plays a minor role in the etiology of stillbirth.

\section{Abstract \#: P 226 \\ Uptake and characteristics of zoledronic acid and denosumab patients and physicians in Ontario, Canada: impact of drug formulary access}

Andrea M. Burden ${ }^{1,2}$, Mina Tadrous ${ }^{1}$, Andrew Calzavara ${ }^{3}$, Yannick J. Nielen $^{2}$, Frank de Vries ${ }^{2,4}$, Suzanne M. Cadarette ${ }^{1,3}$

${ }^{1}$ Leslie Dan Faculty of Pharmacy, University of Toronto, Toronto, Canada; ${ }^{2}$ Department of Epidemiology, Maastricht University, Maastricht, Netherlands; ${ }^{3}$ Institute for Clinical Evaluative Sciences, Toronto, Canada; ${ }^{4}$ Department of Clinical Pharmacy and Toxicology, Maastricht University Medical Centre+, Maastricht, Netherlands; Corresponding author's e-mail: andrea.burden@gmail.com

Purpose: We sought to describe the use of zoledronic acid and denosumab by physicians and patients over time, and examine the impact of a 2012 provincial formulary modification that removed prescribing restrictions for zoledronic acid.

Methods: We identified users of zoledronic acid and denosumab using Ontario pharmacy claims data. The number of new patients and physicians were plotted and examined over time. Interrupted timeseries analysis examined the impact of a formulary modification to zoledronic acid. Descriptive characteristics of patients and prescribers were summarized pre- and post-formulary change for zoledronic acid and overall for denosumab.

Results: We identified 1463 zoledronic acid users treated by 627 physicians and 16,736 denosumab users treated by 2904 physicians. In the first two months on the market, we identified a rapid uptake of denosumab ( $>450$ new physicians and $>1200$ new patients) in contrast to zoledronic acid ( $<10$ new physicians and $<10$ new patients). Zoledronic acid use increased significantly in the two months postformulary change with $>80$ new physicians and $>200$ new patients. Prior to the formulary change, more zoledronic acid users had a history of osteoporosis therapy (41 vs. $26 \%$ ) or bone density testing (30 vs. $10 \%$ ). Compared to zoledronic users initiating after the formulary change, more denosumab users had prior osteoporosis therapy (55 vs. $26 \%$ ), yet fewer had a gastrointestinal diagnosis (6 vs. $11 \%)$.

Conclusion: We identified a rapid uptake of denosumab in only 15 months of observation. A provincial formulary modification removing administrative burden on physicians prescribing zoledronic acid resulted in an increase in utilization and impacted patient characteristics.

\section{Abstract \#: P 227}

Riboflavin, vitamin B12 and homocysteine status in children and their associations with dietary intake of B-vitamins from different food groups

Ellen G.H.M. van den Heuvel $^{1}$, George Moschonis ${ }^{2,3}$, Renske Dekkers ${ }^{1}$, Christina Mavrogianni ${ }^{2}$, Eva Grammatikaki ${ }^{2}$, Yannis Manios ${ }^{2}$

${ }^{1}$ Department of Nutritional Sciences, FrieslandCampina, Amersfoort, The Netherlands; ${ }^{2}$ Department of Nutrition and Dietetics, Harokopio University of Athens, Kallithea, Athens, Greece; ${ }^{3}$ EnviNHealth S.A., Athens, Greece; Corresponding author's e-mail:

ellen.vandenheuvel@frieslandcampina.com

Background: Dairy products contribute highly to dietary riboflavin and vitamin B12 intake in Vitamin B6 can be found in a wide variety of animal and plant foods. Regarding folate, its main dietary sources are dark green leafy vegetables, fruits, nuts, beans, peas and grains. The aim of the present study was to examine the associations between dietary intakes of certain B-vitamins from different food groups with plasma levels of riboflavin, methylmalonic acid (MMA) and total homocysteine (THcy) in children from Greece.

Methods: A representative subsample of 600 9-13 year-old children participating in the Healthy Growth Study was examined. Dietary intake of vitamins B2, B12, B6 and folate derived from different food groups and plasma levels of riboflavin, MMA and THcy were assessed. Multivariate linear and non-linear regression analyses were performed to examine the associations between dietary intake and status indices, controlling for potential confounders.

Results: The prevalence of plasma riboflavin levels below the normal range threshold was $22.8 \%$. A positive linear association was observed between vitamin B2 derived from milk and plasma riboflavin levels $(P=0.004)$. Furthermore, nonlinear associations were observed between vitamin B12, folate and vitamin B6 derived from milk and plasma THcy levels $(P=0.003, P=0.021$ and $P<0.001$ respectively): and between folate and vitamin B6 derived from cereals and plasma THcy levels $(P=0.034$ and $P=0.031$ respectively).

Conclusion: A considerably high prevalence of poor plasma riboflavin status was observed in children. Milk-derived vitamins B2 and B12 were positively associated with plasma riboflavin and negatively with plasma THcy levels. Additionally, folate and vitamin B6 derived from milk and cereals were also negatively associated with plasma THcy levels. Although a possible favorable effect of milk and cereals on plasma riboflavin and THcy levels could be implied, further bioavailability and clinical studies are needed to investigate the exact causal associations.

\section{Abstract \#: P 228}

The effect of platelet storage time on clinical outcomes in patients receiving platelet transfusions: a systematic review and metaanalyses 
Camila Caram-Deelder ${ }^{1}$, Justin Jacobse ${ }^{1}$, Rutger A. Middelburg ${ }^{1}$, Johanna G. van der Bom ${ }^{1}$

${ }^{1}$ Center for Clinical Transfusion Research, Sanquin Research/ Department of Clinical Epidemiology, Leiden University Medical Center, Leiden LUMC- Leiden, The Netherlands; Corresponding author's e-mail: C.Caram-Deelder@lumc.nl

Background: Platelets, or thrombocytes, are essential for haemostasis. Platelet transfusion can be indicated for patients with thrombocytopenia. Platelets, as all blood products, have a complex and controlled production process before being stored to ultimately get transfused. Optimal storage time before transfusion is a delicate balance between avoiding waist and product quality. Many papers have been published relating storage time of blood products and clinical outcomes. However, divergent results and often questioned methodology of such papers hamper inferences and consensus about the effect of storage in platelet products. Therefore the objective of this systematic review and meta-analysis is to assess the effect of platelet storage time on clinical outcomes.

Methods: Relevant published studies and meeting abstracts were identified using MEDLINE (PubMed), EMBASE, Cochrane, CINAHL, Academic Search Premier, ScienceDirect and Web of Science databases until April 2014. No restriction of language or year of publication was used. Two reviewers systematically reviewed titles and abstracts to select studies that address platelets storage time and clinical outcomes and scored the quality of the selected papers using structured forms. Further steps to be performed in the following months: reviewers will (i) extracted relevant data of the selected papers and (ii) perform a meta-analysis using the software STATA version 12.1 .

Results: The search retrieved 2795 papers and meeting abstracts of which $607(22 \%)$ did not provide an abstract. From the 2188 analysed papers and meeting abstracts 84 met eligibility criteria and 59 had relevant data to be included in the meta-analyse. Main reasons to exclude studies were: not being about platelet transfusion product (47\%) and not presenting a clinical outcome (32\%). The Kappa statistic for agreement between reviewers was 0.703 (95\% confidence interval: $0.620-0.785$ ) for the selection of studies, which was considered good according to Fleiss criteria. Principal clinical outcomes observed were mortality: platelet recovery, survival and increment: corrected count increment $(\mathrm{CCI})$ : adverse reactions (including TRALI, fever and sepsis): and bleeding.

Conclusion: A meta-analyses will be performed to investigate the relation between storage time of platelet transfusion products and different clinical outcomes. Sensitivity analysis will be performed per type of platelet transfusion product (e.g.: apheresis or buffy coat derived) to determine if the effect of storage time on clinical outcomes is different for a specific type of platelet product. Finally, in addition to the composite outcome, the possible effect of platelet storage time on specific clinical outcomes will be investigated by individual analysis.

\section{Abstract \#: P 229}

Treatment patterns and outcomes of patients diagnosed with ovarian cancer in the Netherlands: a registry study

$\underline{\text { E. Houben }}^{1}$, H.G.M. van Haalen ${ }^{2}$, W. Sparreboom ${ }^{2}$, J.A. Overbeek ${ }^{1}$, N. Ezendam ${ }^{3}$, J.M.A. Pijnenborg ${ }^{4}$, M.P.P. van Herk-Sukel ${ }^{1}$

${ }^{1}$ PHARMO Institute for Drug Outcomes Research, Utrecht, Netherlands; ${ }^{2}$ AstraZeneca, Zoetermeer, Netherlands;

${ }^{3}$ Comprehensive Cancer Centre the Netherlands, Eindhoven, Netherlands; ${ }^{4}$ Tweesteden Ziekenhuis, Tilburg, Netherlands; Corresponding author's e-mail: eline.houben@pharmo.nl
Background: In the Netherlands, around 1300 women are diagnosed with ovarian cancer (OC) each year. Of these patients $70 \%$ is diagnosed with advanced disease resulting in a 5-year survival rate of only $35 \%$. Currently, surgery followed by chemotherapy is the backbone of OC treatment. Little information is available on the patterns of chemotherapy administered for the treatment of OC and the resulting survival outcomes. The objective for this study was to describe current chemotherapy patterns for $\mathrm{OC}$ in the Netherlands and to evaluate survival outcomes following subsequent lines of chemotherapy.

Methods: Data from the Eindhoven Cancer Registry (ECR), including data on all newly diagnosed cancer patients, was linked to the PHARMO Database Network (PHARMO) including, among other things, information on in- and out-patient drug use. Patients diagnosed with primary OC between January 2000 and December 2010 were selected. First and subsequent chemotherapy regimens were defined as the start of a different (combination of) chemotherapeutic agent(s) or a gap $>42$ days between two treatment cycles. Median survival after first and second line chemotherapy was determined and stratified by type of chemotherapy.

Results: From the ECR-PHARMO cohort, 261 OC patients with detailed chemotherapy data available were selected, who had a mean age of 63 (standard deviation \pm 12 ) years. Of those, $95 \%$ received chemotherapy as initial treatment. Pathological tumor stage was recorded for $77 \%$ of patients, of whom 11,55 and $17 \%$ of patients had disease stage II, III, and IV, respectively. In first line chemotherapy, $76 \%$ of patients received platinum/taxane doublet chemotherapy. Of the 161 patients receiving second line chemotherapy, platinum-containing chemotherapy was received by $63 \%$ of patients (101 of whom $13(13 \%)$ received platinum monotherapy). In third line chemotherapy this was $51 \%$ (53 patients). At least eight lines of chemotherapy were identified in 12 cases. Median survival after first line chemotherapy was 32 months and after second line chemotherapy this remained 14 months. Patients treated with platinum based chemotherapy showed median survival of 34 months compared to 10 months for patients treated with nonplatinum based chemotherapy in first line chemotherapy. For second line chemotherapy this was 23 and 11 months, respectively.

Conclusion: This study provides detailed information on the type of chemotherapy regimens administered to OC patients at initial diagnosis and during subsequent lines of chemotherapy as well as the survival following these lines of chemotherapy.

\section{Abstract \#: P 230}

Drug utilization among 10-year-old children and 15-year-old adolescents from the German GINIplus and LISAplus birth cohorts

\section{Salvatore Italia $^{1}$, Joachim Heinrich ${ }^{2}$, Silke B. Wolfenstetter ${ }^{2}$}

${ }^{1}$ Department of International Health, School for Public Health and Primary Care (CAPHRI), Faculty of Health, Medicine and Life Sciences, Maastricht University, Maastricht, The Netherlands; ${ }^{2}$ Helmholtz Zentrum München, German Research Center for Environmental Health, Institute of Epidemiology I, Neuherberg, Germany; Corresponding author's e-mail: salvatore.italia@maastrichtuniversity.nl

Background: According to the available data from various European countries, the prevalence of drug utilization may be very high among adolescents, but longitudinal studies on pediatric drug use are rare. The objective of this study was to compare drug utilization among 10-year-old children from two large German birth cohorts (GINIplus and LISAplus, 10-year follow-up) with the results from the 15-year follow-up of the same birth cohorts. 
Methods: Data on pediatric drug utilization (use within the last 4 weeks) were collected with a self-administered questionnaire for 10-year-old children $(\mathrm{n}=3642)$ and 15-year-old adolescents $(n=4677)$ from a pooled sample of two German birth cohorts (GINIplus and LISAplus). The drugs were grouped into various therapeutic categories (e.g. conventional drugs with chemically defined active pharmaceutical ingredients, homeopathic drugs, herbal drugs, etc.) and were classified according to the anatomical therapeutic chemical classification system (ATC). The results of both age groups were compared with regard to prevalence of drug utilization and distribution of ATC codes.

Results: In summary, the prevalence of overall drug use was similar in children $(42.3 \%)$ and adolescents $(41.1 \%)$. Within the observation period of 4 weeks, 10-year-old children used slightly fewer conventional drugs than 15-year-old adolescents (30.3 vs. $33.9 \%$ ). The 4-week prevalence of homeopathy use fell substantially from $14.3 \%$ (children) to $8.1 \%$ (adolescents), and adolescents used fewer herbal drugs as well (5.9 vs. $8.9 \%$ ). The proportion of drugs available without prescription was $75 \%$ (children) and $71 \%$ (adolescents). 10 -year-old children used far more drugs from the ATC groups R03 (Drugs for obstructive airway diseases) and R05 (cough and cold preparations), but fewer drugs from the ATC groups N02 (analgesics) and R06 (antihistamines). Compared with children, adolescents used more than twice (adolescent girls more than thrice) antiinflammatory drugs (ATC code M01, mainly ibuprofen).

Conclusion: The drop in homeopathy and herbal drug use may in part be explained by the fact that they are normally not reimbursed by statutory health insurance for children older than 12 years. The substantial increase in antiinflammatory drug use among adolescents should be considered by decision makers in their further decisionmaking process, also with respect to possible (further) liberalizations of drugs sales.

\section{Abstract \#: P 231 \\ Meta-analysis of observational studies on the effect of incretin treatment on fracture risk}

Johanna H.M. Driessen ${ }^{1}$, Hein van Onzenoort ${ }^{2}$, Ronald M.A. Henry ${ }^{3}$, Peter Vestergaard $^{4}$, Frank de Vries ${ }^{1}$

${ }^{1}$ Division of Pharmacoepidemiology and Clinical Pharmacology, Utrecht Institute of Pharmaceutical Sciences, Utrecht, Netherlands; ${ }^{2}$ Department of Pharmacy, Radboud University Nijmegen Medical Centre, Nijmegen, The Netherlands; ${ }^{3}$ Department of Medicine, Maastricht University Medical Centre+, The Netherlands; ${ }^{4}$ Clinical Institute, Aalborg University, Aalborg, Denmark; Corresponding author's e-mail: annemariek.driessen@mumc.nl

Introduction: In Europe, approximately 60 million patients suffer from Type 2 Diabetes Mellitus (T2DM). T2DM patients are at increased risk of fracture. Incretin agents are used to treat T2DM and have been marketed since 2007. It includes two classes: glucagonlike-peptide 1 receptor agonists (GLP1-RAs) and dipeptidyl peptidase-4 inhibitors (DPP4-Is). A $40 \%$ reduced risk of fracture with use of DPP4-Is has been reported in a meta-analysis of randomised controlled trials. However, the individual studies had a small sample size and a limited duration of follow-up. Currently, there is no data available from electronic healthcare databases. Objective of this study was to evaluate the association between incretin agents and risk of fracture.

Methods: We used data from the UK Clinical Practice Research Datalink (CPRD), the world's largest primary care database which is representative for the total UK population $(2007-2012, \mathrm{n}=13$ million) and from the full country of Denmark (2007-2011, $\mathrm{n}=5.5$ million). We used a cohort design and Cox regression analysis with CPRD data and a case-control study with conditional logistical regression in Denmark (which comprised all patients with a first fracture matched to controls). We compared current incretin use with non-use. A meta-analysis extracted hazard- (HRs) and odds ratios and their corresponding $95 \%$ confidence intervals (CIs) using generic inverse variance methods assuming a random effects model.

Results: There was no reduced fracture risk with use of incretin agents in both countries (adj. pooled risk ratio DPP4-I 1.01: $95 \% \mathrm{CI}$ 0.92-1.12, adj. pooled risk ratio GLP1-ra 1.03: $95 \%$ CI 0.87-1.22). A higher cumulative dose (sitagliptin equivalents, CPRD data) did not further decrease risk of fracture yielding adj. HRs of 1.07: $95 \% \mathrm{CI}$ 0.90-1.27 (0-18.2 mg) adj. HR 0.84: $95 \%$ CI $0.67-1.06$ (18.3-36.5 mg) adj. HR 1.05: $95 \%$ CI 0.81-1.37 (36.6-54.7 mg), adj. HR 0.97: $95 \%$ CI 0.78-1.20 (>54.7 mg). Results were similar for GLP-1RAs and in Denmark.

Discussion: Use of incretin agents was not associated with a decreased fracture risk in both countries, and higher cumulative dosages did not result in an inverse association. Our results do not support the conduct of further clinical research to study beneficial effects of incretin agents on fracture risk.

\section{Abstract \#: P 232}

\section{Adherence to statin therapy and the hazard of cardiovascular} mortality in the Netherlands

Maarten J. Bijlsma ${ }^{1}$, Stijn Vansteelandt ${ }^{2}$, Fanny Janssen ${ }^{3}$, Eelko Hak ${ }^{1}$ ${ }^{1}$ University of Groningen, Department of Pharmacy; ${ }^{2}$ University of Ghent, Department of Applied Mathematics, Computer Science and Statistics; ${ }^{3}$ University of Groningen, Population Research Centre; Corresponding author's e-mail: maarten.bijlsma@rug.nl

Background: The biological efficacy of statin therapy has been demonstrated in various clinical trials. However, end users may differ from trial participants in relevant ways. Therefore, observational studies are needed to assess clinical effectiveness. Our objective was to assess the clinical effectiveness of adherence to statin therapy in reducing cardiovascular mortality in the Netherlands.

Methods: Individual-level mortality information from Statistics Netherlands was linked to pharmacy dispensing data came from the representative database IADB.nl. We used extended Cox models with adherence to statin therapy as the primary exposure and time to cardiovascular mortality as the primary outcome. We adjusted for age, sex, birth cohort, socio-economic status, diabetic status, and the utilization of various cardiovascular drugs. Covariates were allowed to vary over time. We achieved population-averaged effect estimates through implementation of the parametric G-formula. We also performed a subset analysis by calendar period corresponding to periods of particular cardiovascular prescribing guidelines, and a subset analysis by dispensing background to assess the influence of healthy adherer bias.

Results: The conditional estimate was that being fully adherent to statins reduced the hazard of cardiovascular mortality by about $47 \%$ (HR: 0.53: $95 \%$ CI: 0.46-0.61), compared to being fully non-adherent to statins. The population averaged estimate was of similar magnitude. In addition, we found evidence that estimates of clinical effect approached estimates of trials just after the introduction of statins in the population, but became potentially more confounded in later calendar years.

Conclusion: The study provides evidence of the clinical effectiveness of statins, though the final estimates may still be affected by healthy adherer bias. 


\section{Abstract \#: P 233}

Use of thiazolidinediones and the risk of elective hip or knee replacement: a population based case-control study

Johannes T.H. Nielen ${ }^{1}$, Bart van den Bemt ${ }^{2}$, Arief Lalmohamed ${ }^{3}$, Anthonius de Boer $^{4}$, Annelies Boonen ${ }^{5}$, Pieter C. Dagnelie ${ }^{1}$, Pieter Emans ${ }^{6}$, Frank de Vries ${ }^{7}$

${ }^{1}$ Department of Epidemiology, Maastricht University, Maastricht, the Netherlands; ${ }^{2}$ Department of Pharmacy, Sint Maartenskliniek, Nijmegen, the Netherlands; ${ }^{3}$ Department of Clinical Pharmacy, University Medical Center Utrecht, Utrecht, the Netherlands; ${ }^{4}$ Division of Pharmacoepidemiology \& Clinical Pharmacology, Utrecht University, Utrecht, the Netherlands; ${ }^{5}$ Department of Rheumatology, Maastricht University Medical Center, Maastricht, the Netherlands; ${ }^{6}$ Department of Orthopaedics, Maastricht University Medical Center, Maastricht, the Netherlands; ${ }^{7}$ Department of Clinical Pharmacy and Toxicology, Mastricht University Medical Center, Maastricht, the Netherlands; Corresponding author's e-mail: yannick.nielen@mumc.nl

Background: Currently no disease modifying drugs exist for the treatment of osteoarthritis (OA). Previous in vivo studies suggest glitazons may have anti-arthritic properties. These effects have not been investigated in humans before. Objective: To determine the risk of total joint replacement (TJR) in patients using thiazolidinediones (TZDs) compared to those not using TZDs.

Methods: A population based case-control study was performed using the Clinical Practice Research Datalink (CPRD). Cases $(\mathrm{n}=94,609)$ were defined as patients $>18$ years of age who had undergone TJR between 2000 and 2012. Controls were matched by age, gender and general practitioner (GP) practice. Conditional logistic regression was used to estimate the risk of total knee (TKR) and total hip replacement (THR) associated with use of TZDs. We additionally evaluated risk of TJR in current TZD users compared to DM patients using other ADs. In order to determine a dose effect relationship, we also stratified TZD users by total number of prescriptions prior to surgery.

Results: There is no difference in risk of TKR (OR $=1.11(95 \%$ $\mathrm{CI}=0.95-1.29)) \quad$ or $\mathrm{THR} \quad(\mathrm{OR}=0.87 \quad(95 \% \mathrm{CI}=0.74-1.02))$ between TZD users and patients not using TZDs. Furthermore, there is no difference in risk of TKR $(\mathrm{OR}=1.03(95 \% \mathrm{CI}=0.88-1.22)$ ) and THR (OR $=0.90(95 \% \mathrm{CI}=0.75-1.08)$ ) when TZD users are compared to other AD users. Finally, we did not find a dose response effect with increasing number of prescriptions. Conclusion: Despite promising results from in vivo studies, this study did not find any evidence for an anti-arthritic effect of TZDs.

\section{Abstract \#: P 234}

Proton pump inhibitor use is associated with an increased risk of hypomagnesemia in the general population

Brenda C.T. Kieboom ${ }^{1,2,3}$, Jessica C Kiefte-de Jong ${ }^{1,4}$, Mark Eijgelsheim $^{1,2}$, Oscar H. Franco ${ }^{1}$, Ernst J. Kuipers ${ }^{2}$, Albert Hofman ${ }^{1}$, Robert Zietse $^{2}$, Bruno H. Stricker ${ }^{1,2,3}$, Ewout J. Hoorn ${ }^{2}$

${ }^{1}$ Department of Epidemiology, Erasmus Medical Center, Rotterdam, The Netherlands; ${ }^{2}$ Department of Internal Medicine, Erasmus Medical Center, Rotterdam, The Netherlands; ${ }^{3}$ Inspectorate for Health Care, Utrecht, The Netherlands; ${ }^{4}$ Leiden University College, The Hague, The Netherlands; Corresponding author's e-mail: b.kieboom@erasmusmc.nl
Background: Proton pump inhibitor (PPI) use has been associated with hypomagnesemia in case reports and hospital-based cohorts studies, which could have been influenced by selection bias. Our aim was to determine whether PPI use is associated with hypomagnesemia in the general population and whether this association is also found in users of H2-receptor antagonists.

Methods: Within the Rotterdam Study, a prospective populationbased cohort, we investigated the association between PPI-use and hypomagnesemia (defined as serum magnesium $\leq 0.72 \mathrm{mmol} / \mathrm{L}$ ) in 9818 participants, aged 45 and over. All analysis were adjusted for age, gender, body composition, kidney function, diabetes mellitus, and the use of thiazide or loop diuretics. The same analysis were performed in H2-receptor antagonist users.

Results: Serum magnesium was $0.012 \mathrm{mmol} / \mathrm{L}$ lower in PPI users ( $\mathrm{n}=724,95 \%$ confidence interval $[\mathrm{CI}]-0.016$ to -0.007$)$ compared to non-users. PPI-use increased the risk of hypomagnesemia (odds ratio [OR] 1.73, $95 \%$ CI 1.06-2.83). Effect modification was found between the use of PPIs and loop diuretics $(\mathrm{n}=45, P$ for interaction 0.03), which further increased the risk of hypomagnesemia (OR 6.19, $95 \%$ CI 1.59-24.07). The increased risk of hypomagnesemia was only seen after prolonged use (range 182-2618 days, OR 2.99 , $95 \%$ CI 1.74-5.16). Users of H2-receptor antagonists $(\mathrm{n}=250)$ also had lower serum magnesium levels $(-0.09 \mathrm{mmol} / \mathrm{L}$, $95 \% \mathrm{CI}-0.016$ to -0.001$)$ and an increased risk of hypomagnesemia (OR 2.07, $95 \%$ CI 1.12-3.81), but we found no effect modification with loop diuretics.

Conclusions: The use of PPIs is associated with hypomagnesemia in the general population. Prolonged use and concomitant use of loop diuretics increases the risk of hypomagnesemia. Similar but weaker associations were found in users of $\mathrm{H} 2$-receptor antagonists, except for effect modification by loop diuretics. Health care professionals should be aware of the risk of hypomagnesemia and should consider measuring serum magnesium levels for patients on prolonged treatment or those who combine PPIs with diuretics.

\section{Abstract \#: P 235}

Low osteoporosis treatment initiation rate in 445 women after distal forearm or proximal humerus fracture

Marie Viprey $^{1,2}$, Pascal Caillet ${ }^{3,4}$, Guillaume Canat ${ }^{1}$, Roland Chapurlat $^{1,5}$, Susan Jaglal ${ }^{6}$, Anne-Marie Schott ${ }^{1,2,3}$

${ }^{1}$ Pôle Information Médicale, Evaluation, Recherche, Hospices Civils de Lyon, Lyon, France; ${ }^{2}$ Université de Lyon, RECIF, Lyon, France; ${ }^{3}$ Service de Rhumatologie \& de Pathologie Osseuse, Immunologie Clinique Cancérologie Osseuse, Inserm U INSERM 1033 Pavillon F, Lyon, France; ${ }^{4}$ Service d'Hygiène, d'Evaluation Médicale et de Santé Publique, CHU Amiens, Amiens, France \& Service de Rhumatologie; ${ }^{5}$ de Pathologie Osseuse, Immunologie Clinique Cancérologie Osseuse, Inserm U INSERM 1033 Pavillon F, Lyon, France; ${ }^{6}$ Toronto Rehabilitation Institute-UHN Chair, Department of Physical Therapy, University of Toronto, Toronto, Canada; Corresponding author's e-mail: marie.viprey@chu-lyon.fr

Background: Treatment initiation rates following fragility fractures have often been reported to be low and in recent years numerous programs have been implemented worldwide to increase them. This study aimed at describing osteoporosis (OP) treatment initiation in a representative sample of women who were hospitalized for a distal forearm fracture (DFF) or proximal humerus fracture (PHF) in 2009-2011 in France.

Methods: A retrospective cohort study was carried out using a nationwide representative sample of 600,000 individuals, extracted from the French National Insurance Healthcare System database. All 
women aged 50 years and older who were hospitalized for a first DFF or PHF between 2009 and 2011 were included. An initial OP prescription was defined as a reimbursement for bisphosphonates, hormonal replacement therapy, raloxifene, strontium ranelate, calcium, vitamin $\mathrm{D}$, or teriparatide, with no prior reimbursement for one of these drugs during the year preceding the index date. The primary outcome was the OP treatment initiation rate. Secondary outcomes were the mean time elapsed between fracture and OP treatment initiation and characteristics of patients and prescribers who initiated OP treatment. Data were controlled, validated and analysed centrally using SAS ${ }^{\circledR}$ Enterprise Guide Software V.4.3 (SAS Institute Inc., Cary, NC, USA).

Results: From 2009 to 2011, 729 women were hospitalized for a DFF or a PHF and $284(39 \%)$ were on OP treatment at the time of the fracture occurrence. Among the 445 women who had no prevalent OP treatment, 173 women $(39 \%)$ were prescribed an OP treatment in the year following the fracture. One hundred thirty one patients $(76 \%)$ initiated vitamin D and/or calcium only and 42 patients (24\%) initiated pharmacological OP treatments including bisphosphonates $(12 \%)$, strontium ranelate $(8 \%)$, hormonal replacement therapy $(2 \%)$, or raloxifene $(2 \%)$. Median time elapsed between fracture and OP treatment initiation was 96 days, with an interquartile range between 49 and 184 days. General practitioners prescribed $75 \%$ of initial OP treatments.

Conclusion: Despite the guidelines published in 2006 and the numerous initiatives to promote post-fracture OP treatment, OP treatment initiation rate in women who were hospitalized for a first fragility fracture remained low in 2009-2011 in France.

\section{Abstract \#: P 236 \\ Medication-induced microscopic colitis: Do recency and duration of use matter? \\ Bas P.M. Verhaegh $^{1}$, Frank de Vries ${ }^{2}$, Ad A.M. Masclee ${ }^{1}$, Ali Keshavarzian $^{2}$, Anton de Boer ${ }^{2}$, Patrick Souverein ${ }^{2}$, Daisy M.A.E. Jonkers ${ }^{1}$, Marieke J. Pierik ${ }^{1}$ \\ ${ }^{1}$ Division of Gastroenterology-Hepatology, Maastricht University Medical Center+; ${ }^{2}$ Dept. of Pharmacoepidemiology, Utrecht Institute of Pharmaceutical Sciences; Corresponding author's e-mail: b.verhaegh@maastrichtuniversity.nl}

Background: Microscopic colitis (MC) is a colonic disorder characterised by chronic watery diarrhoea. There is increasing evidence that exposure to non-steroidal anti-inflammatory drugs (NSAIDs), proton pump inhibitors (PPIs), selective serotonin reuptake inhibitors (SSRIs), and statins is associated with an increased risk of MC. The attributive risk of recent or longer use and the effect of higher dosages has never been investigated. However, this would give more insight in the possible mechanism of medication-induced MC.

Methods: A case-control study was conducted using the Clinical Practice Research Datalink. Cases were diagnosed with microscopic, lymphocytic (LC) or collagenous (CC) colitis between 1992-2013 and linked to 5 randomly selected controls without MC, matched by age, gender, and practice. The index date of the case defined that of the controls. Prescriptions within 60 days prior to index date were excluded to take into account a latency (lag) time. Exposure status was classified as current (61-90 days), recent (91-150 days), and past use ( $>150$ days) according to the time since most recent prescription prior to index date. In current users, duration of continuous use and average daily dose were assessed. Conditional logistic regression was applied to quantify the strength of the associations and to correct for confounders.
Results: We identified 1.211 cases (394 CC, 292 LC, 525 undefined $\mathrm{MC}$ ) and 6.041 controls. Current use of NSAIDs (OR 1.79, $95 \% \mathrm{CI}$ 1.36-2.36), PPIs (OR 3.93, $95 \%$ CI 2.25-4.74), and SSRIs (OR 2.27, $95 \%$ CI 1.79-2.89) was associated with an increased risk of MC compared to never and past use. Especially a 4-12 month continuous use of NSAIDs, PPIs, and SSRIs increased the risk of MC. Exposure to more than 1.25 standardised daily dosages was associated with an elevated risk of MC in PPI (OR 6.90, $95 \%$ CI 3.82-12.49) and SSRI users (OR 4.15, $95 \%$ CI 2.47-6.97). Analysis per MC subtype showed a positive association between current use of NSAIDs (OR $2.28,95 \%$ CI 1.46-3.54) and PPIs (OR 6.15, $95 \%$ CI 4.41-8.58) in CC and current use of PPIs (OR 2.40, $95 \%$ CI 1.60-3.59) and SSRIs (OR 2.65, $95 \%$ CI 1.69-4.15) in LC. Statin use was not associated with MC. A sensitivity analysis with a lag time of 90 days showed conclusion consistent with the primary analysis.

Conclusion: Use of NSAIDs, PPIs, and SSRIs is associated with an increased risk of MC. Especially in current users with continuous exposure duration of 4 to 12 months, drug exposure as cause for MC should be considered.

\section{Abstract \#: P 237}

Exploring health and health behaviours in students with cognitive function disorders: from methodological issues to a study protocol $\underline{\text { Sentenac Mariane }}^{1}$, Godeau Emmanuelle ${ }^{1,2}$

${ }^{1}$ Inserm, UMR 1027, Université de Toulouse III Paul Sabatier, Toulouse, France; ${ }^{2}$ Service médical du rectorat de Toulouse, France; Corresponding author's e-mail: marianesentenac@yahoo.fr

Background: The principle of inclusive education has been increasingly recognised over recent decades by most countries. Although the number of children with a disability or a chronic condition in mainstream settings has considerably increased, few studies have explored the life and well-being at school of such children. Large-scale epidemiologic studies, such as the Health Behaviour in School-aged Children (HBSC) survey, based on self- administrated questionnaires are commonly used to collect respondent's point of view in general school-population, but are particularly cognitive demanding for respondents.

The objective of this project was to implement an adequate methodology to collect data on well-being, health, health behaviours and their determinants among students with mild or moderate intellectual disability (MMID) that could be compared to data collected among others students through the HBSC protocol.

Methods: A multi-steps approach has been adopted. First, a literature review has been conducted to identify methodological issues in collecting self-reported data among young people with MMID and to identify solutions and alternatives to standard conditions to facilitate their comprehension, retrieval, judgment and response. In a second stage, a panel of experts was consulted and group sessions with students schooled in special classes organized to finalize a study protocol adapted from the HBSC standardized protocol. And last, the final material has been administrated to a national representative sample of students with MMID as part of the 2014 HBSC France survey.

Results: The review literature provided a clear mapping with an important literature discussing methodological difficulties and sparser publications on alternatives to mainstream standardised conditions. The reliability and validity of the self-reported data were discussed, as well as ways to improve question and response options understanding. Numbers of components of the HBSC standardized protocol, including the questionnaire design and content, were identified as problematic for students with cognitive disorders after this review and therefore modified. Then data on eating habits, risk behaviours, 
school, health, bullying behaviours, family, social network and sports have been collected from 701 students with MMID aged between 11 and 20. Preliminary results will be presented.

Conclusion: These data should lead to improve our knowledge on health, health behaviours, well-being and determinants of youth with MMID and provide relevant information to inform health policy and health education actors broader about the quality of life of this population, and further to act upon the results.

\section{Abstract \#: P 238}

Screening policies in health impact assessment: easier decision making through cluster analysis

$\underline{\text { Leonor Bacelar-Nicolau }}^{1,2,3}$, José Pereira Miguel ${ }^{1,2}$, Gilbert Saporta ${ }^{4}$

${ }^{1}$ Institute of Preventive Medicine, Faculty of Medicine, Universidade de Lisboa, Lisboa, Portugal; ${ }^{2}$ ISAMB-Institute of Environmental

Health, Faculty of Medicine, Universidade de Lisboa, Lisboa, Portugal; ${ }^{3}$ DataScience Cons., Oeiras, Portugal; ${ }^{4}$ Laboratoire CEDRIC, Conservatoire National des Arts et Métiers, Paris, France; Corresponding author's e-mail: 1nicolau@medicina.ulisboa.pt

Background: Screening policies to identify candidates for applying Health Impact Assessment (HIA) is generally a qualitative process. Our aim is to show how the use of multivariate exploratory statistical methods such as cluster analysis may help the screening step of HIA in getting a shortlist of policies as HIA candidates quickly and reliably.

Methods: A panel of 7 international renowned public health experts from Nova University in 2011 rated 76 policies proposed by the Technical Group planning the Portuguese hospital reform on a 10 -point scale (1-Very low to 10 -Very high) regarding Potential Impact, Ease of implementation and Implementation costs. Hierarchical cluster analysis is used to identify groups of similar policies regarding these 3 dimensions and prioritize those more pertinent of being considered for HIA according to chosen priority criteria, given different scenarios.

Results: The analysis identified 7 groups of policies and showed that Ease of implementation was not as relevant to differentiate these groups as were Potential impact and Implementation costs: 3 groups present higher mean levels of potential impact (one with also higher mean levels of implementation costs, another with intermediate costs and a last one with lower costs): 2 groups present intermediate mean levels of potential impact (one with higher mean costs and another with lower ones): 2 groups present lower mean levels of potential impact (one with higher mean costs and another with lower ones). The groups of policies were then re-ranked according to different priority criteria, but Group 1 lead all possible scenarios being the group of most obvious candidates for HIA.

Conclusion: Although multivariate statistical methodologies may be complex, decision-makers may thus obtain more evidence-based knowledge and not necessarily in a harder way to grasp. Applying multivariate statistical methods such as hierarchical cluster analysis as early as the screening step of HIA may be very helpful towards obtaining evidence based, reliable, participatory, flexible and more efficient ways of selecting policies for HIA.

\author{
Abstract \#: P 239 \\ Evaluation of blood biomarkers stability during transport \\ in multicenter studies
}

R. Assali Serruya ${ }^{1}$, A.C. Ferreira de Moraes $^{1}$, L.A. Moreno ${ }^{2}$, G.A. Berg $^{3}$, T. Rendo-Urteaga ${ }^{1}$, M.V. Nascimento-Ferreira ${ }^{1}$, T. Sadalla Collese $^{1}$, H. Barbosa de Carvalho ${ }^{1}$

${ }^{1}$ Youth/Child and cArdiovascular Risk and Environmental (YCARE) Research Group, School of Medicine, University of São Paulo, São Paulo; ${ }^{2}$ Growth, Exercise, NUtrition and Development (GENUD) Research Group, School of Health Science (EUCS), University of Zaragoza; ${ }^{3}$ Laboratory of Lipids and Lipoproteins - Department of Clinical Biochemistry of the School of Pharmacy and Biochemistry of University of Buenos Aires; Corresponding author's e-mail: raphaelserruya91@yahoo.com.br

Background: Blood biomarkers are used in analysis on several epidemiologic studies. On multicenter studies, that need to transport biological samples, standardization and quality assurance of the analysis are especially important. Thus, the aim of this study was to systematically review the literature about analysis standardization and blood biomarkers stability during transport in multicenter studies.

Methods: Five databases (BioMed Central, PubMed, SciELO, Scopus, Web of Science) were used on the research, using Boolean operators to combine five commands groups: 1- Transportation related expressions 2- Quality assurance related expressions 3- Blood related expressions 4- Multicenter related expressions 5- Standardization related expressions. Studies in English, Portuguese and Spanish were considered and the last search was run on 12 December 2014. Relevant studies were selected by the following sequence: 1Titles review, 2- Abstracts review and 3- Full-text articles read. The coefficient of variation (CV\%) of each biomarker was evaluated.

Results: The literature search yielded 63 potentially relevant articles, of which 4 articles were eligible according to the inclusion criteria established for this review. One article did not quote the coefficients of variation values, just described the logistical and methodological aspects of transportation (multicenter in Brazil). Two articles evaluated the effect of transportation on triglycerides, their CV were from 0.13 (multicenter in India) to 0.55 . (multicenter in 8 European countries). Some others blood biomarkers were evaluated in one study, with a CV range of -0.25 to $0.05 \%$ (multicenter in 10 European cities).

Conclusion: Following standards protocols for pre analytical phase, transportation and blood analysis reduces potential damages to blood samples and guarantees more reliable results. Nevertheless, there is a lack of studies that describe blood biomarkers stability during transportation in multicenter studies.

\section{Abstract \#: P 240}

Social support directly and indirectly associated with physical activity in women from socioeconomically disadvantaged neighbourhoods: a longitudinal mediation analysis

Anouk Middelweerd ${ }^{1}$, Saskia J te Velde ${ }^{1}$, Gavin Abbott ${ }^{2}$, Anna Timperio $^{2}$, Johannes Brug ${ }^{1}$, Kylie Ball ${ }^{2}$

${ }^{1}$ VU University Medical Centre/EMGO Institute for Health and Care Research; ${ }^{2}$ Deakin University, Centre for Physical Activity and Nutrition Research, School of Exercise and Nutrition Sciences, Faculty of Health, MELBOURNE, Australia; Corresponding author's e-mail: a.middelweerd@vumc.nl

Purpose: Levels of physical activity (PA) decrease when transitioning from adolescence into young adulthood. Theoretical models, such as the social-cognitive theory and the socio-ecological model, and evidence suggest that social support and personal factors (self-efficacy, outcome expectations, enjoyment) are associated with PA. However, little is known about what mediates the association between 
social factors and PA. Furthermore, the majority of this research is cross-sectional: longitudinal studies are needed. Therefore the aim of the present study was to explore whether associations between social support from family and social support from friends, and leisure-time PA (LTPA) were mediated by personal factors (i.e. enjoyment of PA, PA outcome expectations, self-efficacy for PA) using cross-sectional and longitudinal data obtained from young Australian women (aged 18-30 years) living in disadvantaged neighbourhoods.

Methods: Survey data was collected as part of the READI study in 2007-2008 (T1, $\mathrm{N}=1197), \quad 2010-2011 \quad(\mathrm{~T} 2, \mathrm{~N}=427)$ and 2012-2013 (T3, N = 337). A series of single-mediator models were tested using baseline (T1) data only. These single-mediator models were repeated using longitudinal data from all three time points (based on residual change scores for changes between two measurements).

Results: Cross-sectional analyses found that associations between social support from family and social support from friends, and LTPA, were mediated by all personal factors. Social support was both directly and indirectly associated with LTPA, with each personal factor explaining between 79.7 and $97.6 \%$ of the associations. In the longitudinal analyses, enjoyment and self-efficacy were significant mediators of the association between social support from friends and LTPA, explaining 56 and $34.1 \%$ of the relationship, respectively.

Conclusion: The current longitudinal analyses found that self-efficacy for, and enjoyment of, PA partly explained the relationship between social support from friends and LTPA in young Australian women living in disadvantaged neighbourhoods. Thus, these results suggest that a supportive network of friends can facilitate LTPA through increases in women's self-efficacy and enjoyment of PA. Interventions to increase LTPA targeting young adult women may benefit from strategies to enhance social support from friends.

\section{Abstract \#: P 241}

The context dependency of mental health questionnaires within the public health: a cross-sectional study between two administration settings

\section{H. Hoofs $^{1}$, N.W.H. Jansen ${ }^{1}$, D.C.L. Mohren ${ }^{2}$, M.W.J. Jansen ${ }^{2,3}$,} I.J. Kant

${ }^{1}$ Department of Epidemiology, CAPHRI School for Public Health and Primary Care, Faculty of Health, Medicine and Life Sciences, Maastricht University, Maastricht, The Netherlands; ${ }^{2}$ Academic Collaborative Centre for Public Health Limburg, Public Health Service Southern Limburg, Geleen, The Netherlands; ${ }^{3}$ Department of Health Services Research, CAPHRI School for Public Health and Primary Care, Faculty of Health, Medicine and Life Sciences, Maastricht University, Maastricht, The Netherlands; Corresponding author's e-mail: huub.hoofs@maastrichtuniversity.nl

Background: Within the public health, mental health problems are often assessed using questionnaires. Applications of these questionnaires within the preventive child healthcare $(\mathrm{PCH})$ can roughly be divided into two settings, the "individual" and the "collective" setting. The individual setting focuses on the early detection of mental health problems. Questionnaires are applied to facilitate screening by the healthcare professional. The collective setting, in contrast, is characterised by the aggregation of information across a population. In such a setting instruments are used to characterize the overall mental health of populations, for health policy-making. Assessment in the individual setting is confidential for clinical applications, while assessment in the collective setting can be anonymous. Due to these administration differences it remains unclear whether results and conclusions of both settings actually can be used interchangeably. To illustrate this, the present study investigates whether a cross-use of scores between settings is possible for the Strength and Difficulties Questionnaire (SDQ) which is developed to screen for psychopathology in children and adolescents.

Methods: Two independent samples were retrieved (mean age $=14.07$ years), one from an individual setting ( $\mathrm{PCH}$ : $\mathrm{N}=6,594$ ) and one from a collective setting (youth monitor: $\mathrm{N}=4,613$ ). The SDQ was administered in the second year of secondary school in both settings. Samples come from the same sociogeographic population in the Netherlands.

Results: Confirmatory factor analysis showed that the SDQ was measurement invariant/equivalent across settings and gender. On average, children in the individual setting scored lower on total difficulties $\left(\eta^{2}=.04\right)$ compared to those in the collective setting. This was also reflected in the cut-off points for caseness, defined by the 90th percentiles, which were lower in the individual setting. Using cut-off points from the collective in the individual setting therefore resulted in a smaller number of cases, $\sim 3 \%$, while $\sim 10 \%$ is expected.

Conclusion: The SDQ has the same connotation across the individual and collective setting. The observed structural differences regarding the mean scores, however, undermine the validity of the cross-use of absolute SDQ-scores between these settings. Applying cut-off scores from a collective setting in an individual setting could, therefore, result in invalid conclusions and unintentional misuse of the instrument. This study shows that cut-off scores of the SDQ should be retrieved from the setting in which they are applied. These findings, therefore, advocate thorough investigation of the validity of cross-use also for other instruments measuring mental health within public health.

\section{Abstract \#: P 242}

Structural invariance of quality of life in neurological and metabolic diseases a multigroup analysis

Estela Vilhena $^{1,2}$, José Luís Pais Ribeiro $^{3}$, Isabel Silva ${ }^{4}$, Luísa Pedro ${ }^{5}$, Rute Meneses $^{4}$, Helena Cardoso ${ }^{6,7}$, António Martins da Silva ${ }^{7,8}$, Denisa Mendonça ${ }^{2,6}$

${ }^{1}$ Polytechnic Institute of Cávado and Ave, Barcelos; ${ }^{2}$ EPIUnit, ISPUP University of Porto, Porto; ${ }^{3}$ FPCE University of Porto: UIPES Portugal; ${ }^{4}$ University of Fernando Pessoa; ${ }^{5}$ ESTeSL Polytechnic Institute of Lisbon; ${ }^{6}$ ICBAS University of Porto; ${ }^{7} \mathrm{HGSA} / \mathrm{CHP}$ Hospital Center of Porto; ${ }^{8}$ UMIB/ICBAS University of Porto, Portugal; Corresponding author's e-mail: evilhena@ipca.pt

Background: Quality of life (QoL) has become an important concept for health care. It is a construct composed of a number of factors that contribute to individual's well-being and adjustment to chronic diseases. Neurological and metabolic disease patients are required to live with the limitations imposed by their condition and their QOL is considered a relevant problem of public health in modern societies.

The structure of QoL is represented by three factors: general wellbeing, physical and mental health. But is this structure the same for diseases with different characteristics?

The aim of the present study was to test the structural invariance of QoL across Neurological and Metabolic Diseases.

Methods: This study included a sample of 365 Portuguese metabolic and 199 neurological patients approached by their physicians in outpatient departments of the main hospitals in Portugal. All completed a self-report questionnaires to assess QoL variables.

In order to test the invariance of QoL structure between disease groups, MultiGroup Factor Analysis was applied using Structural Equation Model (SEM), with equality constraints. 
To test the adequacy of the model two goodness-of-fit indices were used: Comparative Fit Index (CFI) and Root Mean Square Error of Approximation (RMSEA). Analysis were performed using EQS 6.1.

Results: We started the analysis by performing a Confirmatory Factor Analysis of each measure in each of the two samples, by testing the factorial structure of QoL, including all the observed and latent variable. The results indicated a good fit of models: $\chi_{16}^{2}=63.0139$, $p<0.001, \mathrm{CFI}=0.97$, RMSEA $=0.08: \chi_{16}^{2}=46.6181, p<0.001$, $\mathrm{CFI}=0.98$ and RMSEA $=0.074$ for the group of neurological and metabolic patients, respectively.

Multigroup results, suggest that the models fit the data well, revealing a structural invariance of QoL among the neurological and metabolic groups (measurement model $\chi_{37}^{2}=98.3414, p<0.001$, $\mathrm{CFI}=0.972$, RMSEA $=0.055$ : structural model $\chi_{40}^{2}=106.3413$, $p<0.001, \mathrm{CFI}=0.97$, RMSEA $=0.055)$.

Conclusions: The principal goal of the study was to evaluate the structural invariance of QoL across different disease groups with different characteristics: neurological and metabolic.

Using SEM, MultiGroup Factor Analysis results suggest there is a structural invariance among the groups and the structure of QoL is well represented by subjective well-being, physical and mental health.

\section{Abstract \#: P 243}

The use of kernel density estimation to examine associations between destination intensity, walking and physical activity

$\underline{\text { Tania King }}^{1}$, Lukar Thornton ${ }^{2}$, Rebecca Bentley ${ }^{1}$, Anne Kavanagh ${ }^{1}$

${ }^{1}$ Centre for Health Equity, Melbourne School of Population Health, University of Melbourne, Melbourne, Australia; ${ }^{2}$ Centre for Physical Activity and Nutrition Research, School of Exercise and Nutrition Sciences, Deakin University, Burwood, Victoria, Australia; Corresponding author's e-mail: tking@unimelb.edu.au

Background: Local destinations have previously been shown to be associated with higher levels of both physical activity and walking, but little is known about how the distribution of destinations is related to activity. Kernel density estimation is a spatial analysis technique that accounts for the location of features relative to each other. Using kernel density estimation, this study sought to investigate whether individuals who live near destinations (shops and service facilities) that are more intensely distributed rather than dispersed: 1) have higher odds of being sufficiently active: 2 ) engage in more frequent walking for transport and recreation.

Methods: The sample consisted of 2349 residents of 50 urban areas in metropolitan Melbourne, Australia. Destinations within these areas were geocoded and kernel density estimates of destination intensity were created using kernels of $400 \mathrm{~m}$ (meters), $800 \mathrm{~m}$ and $1200 \mathrm{~m}$. Using multilevel logistic regression, the association between destination intensity (classified in quintiles Q1(least)-Q5(most)) and likelihood of: (1) being sufficiently active (compared to insufficiently active): (2) walking $\geq 4 /$ week (compared to walking less), was estimated in models that were adjusted for potential confounders.

Results: For all kernel distances, there was a significantly greater likelihood of walking $\geq 4$ /week, among respondents living in areas of greatest destination intensity compared to areas with least destination intensity: 400 m (Q4 OR $1.4195 \%$ CI 1.02-1.96: Q5 OR $1.4995 \%$ CI 1.06-2.09), $800 \mathrm{~m}$ (Q4 OR 1.55, $95 \%$ CI 1.09-2.21: Q5, OR 1.71, $95 \%$ CI 1.18-2.48) and $1200 \mathrm{~m}$ (Q4, OR 1.7, $95 \%$ CI 1.18-2.45: Q5, OR $1.8695 \%$ CI 1.28-2.71). There was also evidence of associations between destination intensity and sufficient physical activity, however these associations were markedly attenuated when walking was included in the models.
Conclusions: This study, conducted within urban Melbourne, found that those who lived in areas of greater destination intensity walked more frequently, and showed higher odds of being sufficiently physically active - an effect that was largely explained by levels of walking. The results suggest that increasing the intensity of destinations in areas where they are more dispersed, and or planning neighbourhoods that have greater destination intensity will increase residents' likelihood of being sufficiently active for health.

\section{Abstract \#: P 244}

Determinants of health seeking behavior in Pakistan: a complex health survey design

Dr. Shafquat Rozi ${ }^{1}$, Dr. Sadia Mahmud ${ }^{2}$, Gillian Lancaster ${ }^{3}$

${ }^{1}$ Department of Community Health Sciences, Aga Khan University, Karachi, Pakistan; ${ }^{2}$ Visiting Faculty, Department of Medicine, Aga Khan University, Karachi, Pakistan; ${ }^{3}$ Department of Mathematics and Statistics, Lancaster University, Lancashire United Kingdom; Corresponding author's e-mail: shafquat.rozi@aku.edu

Background: The health care delivery system in Pakistan is complex in nature and limited resources are available for the health care sector. Hence it is important to understand the health seeking behavior of the population and trend of health services utilization in Pakistan. To investigate the determinants of health seeking behavior in Pakistan we suggest a multilevel pseudo maximum likelihood (MPML) approach to estimates model parameters for the complex survey design.

Method: The sampling strategy of the National Health Survey was stratified two stage cluster sampling. Overall 18,315 subjects were interviewed. This is three level data with PSUs at the third level, household at the second level and persons at the first level. Health care utilization was considered as a binary outcome.

Results: We found age, gender, marital status, household ownership of durable goods, urban/rural status, community development index, and province as significant predictors of health care utilization $(p$ value $<0.05)$. We also found two significant interactions: between gender and marital status ( $p$-value $<0.005)$, and between the community development index and urban/rural status ( $p$-value $<0.045$ ).

The variances of the random intercepts are estimated as 0.135 for PSU level and 0.224 for $\mathrm{HH}$. The results are significantly different from zero ( $p$-value $<0.05)$ and indicate considerable heterogeneity in health care utilization with respect to HHs and PSUs.

Conclusion: Our study results reveal the inequalities between socioeconomic groups and between urban and rural residents of Pakistan especially in terms of health care utilization. There is also a need to consider gender sensitive programs. Policy makers in Pakistan frequently make decisions without data. This study builds the case for Pakistan that data can safely be used for decision makings. It also gives support to health advocates who use data to promote health reform. This study gives advocates a stronger position in relation to decision makers in the government, as they marshal data to promote their policies for reform.

\section{Abstract \#: P 245}

The use of linked health and mortality data to inform injury prevention strategies

Ruth Baker $^{1}$, Laila J Tata ${ }^{2}$, Elizabeth Orton ${ }^{1}$, Denise Kendrick ${ }^{1}$

${ }^{1}$ Division of Primary Care, University of Nottingham, Nottingham, United Kingdom; ${ }^{2}$ Division of Epidemiology and Public Health, 
University of Nottingham, Nottingham, United Kingdom; Corresponding author's e-mail: mcxrb12@nottingham.ac.uk

Background: Within the United Kingdom national guidance on preventing injuries in children and young people recommends a strategic approach to injury prevention, targeting interventions to those at greatest risk. Despite this, understanding patterns of injury remains a challenge due to a lack of national injury surveillance data. Through recent linkage of a large primary care research database to secondary care and mortality data, we describe the epidemiology of three common injury types, poisonings, fractures and burns.

Methods: We conducted an open cohort study of 2,147,853 0-24 year olds living in England who had linked primary care, secondary care and mortality data from the Clinical Practice Research Datalink, Hospital Episode Statistics and Office for National Statistics, respectively, during the period 1997-2012. Incidence rates, per 10,000 person-years (PY) with $95 \%$ confidence intervals (95\% CI) were estimated by age, sex and socioeconomic status. Mechanisms of injury (e.g. falls) were identified for injuries leading to hospitalisation or death.

Results: Among the cohort we identified 42,985 poisoning, 185,517 fracture, and 36,719 burn events, giving overall incidence rates of 41.9/10,000 PY (95\% CI 41.4, 42.4), 180.8 (95 \% CI 179.8, 181.7) and 35.8 (95\% CI 35.4, 36.1), respectively.

Different patterns of injury incidence were seen according to age and injury type, with incidence peaking at $2(75.2 / 10,000 \mathrm{PY})$ and again at 18 years old $(74.7 / 10,000 \mathrm{PY})$ for poisonings: compared to 13 years old for fractures (306.5/10,000 PY) and 2 years old for burns $(117.8 / 10,000 \mathrm{PY})$. Large differences in rates were seen by sex for poisonings and fractures $(p<0.0001)$. For each injury type, those from more deprived socioeconomic groups had higher injury rates: although the strength of gradient varied by injury type and age. The strongest association was seen for poisonings, with those in the most deprived areas having a two-fold higher incidence than those in the least deprived areas (incidence rate ratio $2.24,95 \%$ CI 2.17, 2.31). Mechanisms of injury also varied by age: for example, proportions of fractures due to transport incidents and animate mechanical forces increased with age, as did the proportion of poisonings due to intentional self-harm.

Conclusion: Linkage of primary care data to other data sources allows detailed description of injury epidemiology. Differing patterns according to age and injury type reflect differences in underlying mechanism and intent, highlighting the importance of developing tailored preventative interventions across the life course. Inequalities in injury occurrences support the targeting of preventative interventions to households in the most deprived areas.

\section{Abstract \#: P 246}

Tuberculosis notification in private sector physicians: an application of the structural equation model and the theory of planned behavior

\section{Ayat Ahmadi $^{1}$, Reza Majdzadeh ${ }^{2}$}

${ }^{1}$ Department of Epidemiology and Biostatistics, School of Public Health, Tehran University of Medical Sciences; ${ }^{2}$ Knowledge Utilization Research Center, Tehran University of Medical Sciences; Corresponding author's e-mail: ayat1049@gmail.com

Background: A small proportion of physicians adhere to disease notification, particularly in private sector. Private physicians are generally not aware of what they need to report and where they should report to. The aim of this study was to estimate the effect of such knowledge deficiency and the other behavioural determinants on TB notification behaviour.
Methods: A cross sectional population-based study was conducted. A total sample of 503 physicians was selected from private sector physicians in Tehran, Iran's capital. The questionnaire was designed based on the Theory of Planned Behavior (TPB), which included the history of TB notification as Behavior and knowledge of TB notification as Perceived Behavioral Controls (PBC). For measuring Attitude and Subjective Norm(SN) constructs, appropriate indicators were measured, also.

Statistical analysis: Structural Equation Modeling(SEM) was applied to estimate different parameters of the TPB model by using the maximum likelihood estimation. The detection of multivariate outliers was done by Mahalanobis D2. After appropriate transformations the multivariate normality was met (Mardi's test $=1.2$ ). AMOS edition 20 was used for data analysis.

Results: The response rate of the study was $89.3 \%(n=449)$. The standardized effect's coefficient of the PBC on Behavior was 0.118 $(p=.027)$, while it was $-0.123(p=.024)$ for Behavioral Intention. The standardized effect of Intention on Behavior was 0.107 $(p=.021)$.The effect of Attitude and $\mathrm{SN}$ on Intention were 0.525 $(p<.001)$ and $0.146(p=.076)$ respectively. The PBC had a significant correlation with $\mathrm{SN}(\mathrm{r}=0.210, p=.035)$, but it was not significantly correlated with Attitude $(\mathrm{r}=-0.02, p=0.75)$. The value of the GOF Chi square was $29.34(\mathrm{df}=20, p=.081)$ for the established model. The other important GOF indexes values were as follow: $\quad$ AGFI $=.968, \quad$ GFI $=.986, \quad$ TLI $=.959, \quad$ CFI $=.977$, RMSEA $=.032$ and HOELTER $=479$.

Discussion: The perceived knowledge deficiency about TB notification, as an impediment for the physician's self efficacy for notification, had an adverse effect on their Behavior. On the other hand, there was a positive relation between the perceived knowledge deficiency and Intention. This relation may indicate that physicians, who have behavioral intention for the TB notification, have perceived their knowledge deficiency as a barrier for notification, while the others have not perceived so. However, SN about the TB notification and Attitude toward TB notification have enhanced Behavioral Intention significantly.

Conclusion: For private sector physicians, who have a positive intention to notification, giving practical information about the TB notification could increase their adherence to the TB notification. For the others, interventions to enhance attitude and subjective norms toward the TB notification may have more priority.

\section{Abstract \#: P 247}

A new approach to estimating weight change and its reference intervals during the first 96 hours of life

Maria-João Fonseca $^{1,2}$, Milton Severo ${ }^{1,2}$, Ana-Cristina Santos ${ }^{1,2}$

${ }^{1}$ EPIUnit - Institute of Public Health, University of Porto, Porto, Portugal; ${ }^{2}$ Department of Clinical Epidemiology, Predictive Medicine and Public Health, University of Porto Medical School, Porto,

Portugal; Corresponding author's e-mail: mjfonseca@med.up.pt

Background: After birth, newborns lose weight, but expert opinions disagree about what constitutes a normal newborn weight loss. Recently, the question "At what weight-loss percentage is it medically necessary to formula supplement a breastfeeding infant?" was classified as the most important in the research agenda for newborn care. Since newborn weight is the basis for multiple clinical decisions, knowing the pattern of newborn weight change in the population is of major clinical utility. Therefore, we aimed to estimate weight change and its reference intervals during the first $96 \mathrm{~h}$ of life and the time of weight nadir, among full-term singletons, applying a novel approach. 
Methods: This study involved 1288 full-term singletons, from the Generation XXI birth cohort. Recruitment occurred between 2005 and 2006 in all five public units providing obstetrical and neonatal care in Porto, Portugal. Birth weight was abstracted from clinical records and the subsequent newborn weight was obtained by trained examiners. The second weight measurement was performed during the hospital stay and the time of measurement varied between 6.3 and $96 \mathrm{~h}$ of life. Longitudinal mixed effects models were tested to estimate the weight according to newborn's age in hours. Weight change was expressed as weight difference (birth weight minus weight) and as weight ratio (weight divided by birth weight). The age-related reference intervals were estimated using absolute residuals. Curves for newborn's mean weight change expressed as weight ratio and its $95 \%$ confidence interval and percentiles 3, 10, 90 and 97 were constructed.

Results: The chosen model was weight $(\mathrm{t}) \sim 3241.442+(-9.378) \times$ $\mathrm{t}+0.119 \times \mathrm{t} 2+0.000 \times \mathrm{t} 3+\mathrm{b} 0 \mathrm{i}+\mathrm{b} 1 \mathrm{i} \times \mathrm{t}$, where $\mathrm{t}$ represented the newborn's age in hours and bi represented the random coefficients. The estimated mean birth weight was $3241 \mathrm{~g}$ and, after birth, newborns began to lose weight. We estimated that at $6,12,24,36$, and $48 \mathrm{~h}$ of life the mean weight ratio and 10th-90th percentiles were 0.978 (0.968-0.988), 0.968 (0.953-0.983), 0.951 (0.928-0.974), $0.939(0.909-0.969)$, and $0.933(0.898-0.969)$ respectively. The curve inflection point (nadir) was achieved at $52.3 \mathrm{~h}$ of life, corresponding to a weight ratio of $0.933(0.896-0.970)$ and a loss of $218 \mathrm{~g}$. After $52.3 \mathrm{~h}$ of life, newborns started to gain weight.

Conclusion: For the first time, newborn weight change was described as a continuous variable, along with reference intervals, and the exact time of weight nadir was estimated. Using the weight change curves, clinicians can rapidly observe if a newborn is experiencing excessive weight loss and initiate primary interventions.

\section{Abstract \#: P 248}

Cardiometabolic risk profiles in pre-diabetes and diabetes defined by fasting plasma glucose and HbA1c levels in middle-aged adults

$\underline{\text { Seán R. Millar }}{ }^{1}$, Ivan J. Perry ${ }^{1}$, Catherine M. Phillips ${ }^{1}$

${ }^{1}$ University College Cork; Corresponding author's e-mail: s.millar@ucc.i.e

Background: Glycated haemoglobin A1c (HbA1c) measurement is recommended as an alternative to fasting plasma glucose (FPG) for the diagnosis of pre-diabetes and type 2 diabetes. However, evidence suggests discordance between HbA1c and FPG. The aim of this study is to investigate which test more accurately identifies individuals at increased cardiometabolic risk by examining diabetes risk feature and biomarker relationships with both criteria.

Methods: This was a cross-sectional study involving a random sample of 2047 men and women aged 50-69 years. Binary and multinomial logistic regression were used to explore risk feature associations with pre-diabetes [either HbA1c levels 5.7-6.4\% (39-46 $\mathrm{mmol} / \mathrm{mol}$ ) or impaired FPG levels 5.6-6.9 mmol/1] and type 2 diabetes [either $\mathrm{HbAlc}>6.5 \%(>48 \mathrm{mmol} / \mathrm{mol})$ or $\mathrm{FPG}>$ $7.0 \mathrm{mmol} / \mathrm{l}]$. Receiver operating characteristic curve analysis was used to compare the ability of $\mathrm{HbA} 1 \mathrm{c}$ to discriminate pre-diabetes or diabetes defined by FPG.

Results: A higher percentage of pre-diabetic individuals with diabetes-related phenotypes were identified by FPG compared to HbAlc. Cardiometabolic profiles for subjects with type 2 diabetes were broadly similar according to diagnosis by either assay. Odds ratios of having three or more metabolic syndrome features were noticeably increased in pre-diabetic participants classified by both tests (OR: 4.0,
$95 \%$ CI: 2.8-5.8) compared to subjects diagnosed by either HbA1c (OR: 1.4, $95 \%$ CI: $1.2-1.8$ ) or FPG (OR: 3.0, $95 \%$ CI: 1.7-5.1) individually.

Conclusions: In middle-aged Caucasian-Europeans, HbA1c alone is a poor indicator of diabetes risk compared to FPG. However, combined use of HbA1c and FPG may be of additional benefit for identifying individuals at greatest risk of future diabetes development.

\section{Abstract \#: P 249}

The impact of maternal obesity on mother and neonatal health: study in Idrissi Hospital of Kenitra, Morocco

\section{HSAINI Asmae $^{1}$}

${ }^{1}$ Professor Youssef ABOUSSALEH, laboratory nutrition and food, University of ibn tofail kenitra; Corresponding author's e-mail: Asmaehsaini9@gmail.com

Introduction: Obese during pregnancy increases the risk of complications.

Our objective is to evaluate the deleterious effects of maternal obesity on obstetrical complications.

Methods: Historical cohort study including 300 patients delivered in our maternity of kenitra. Intra uterine and fetal loss before 22 weeks were excluded. Women we categorized by the body Mass index. Less than 25, between 25 and 30 , and more than 30.Maternal data, obstetrical, labor and its complications were studied.

Results: $51 \%$ of patients were overweight and $13.7 \%$ were obese. Obstetrical pathologies (hypertension and fetal macrosomie) and mode of delivery were more significantly frequent in obese patient $(p<0.01)$, we noted twice more caesarean sections during labor in obese patients: obese and normal patients representing respectively $9.75 \%: 4.45 \%$ caesarean.

Conclusion: Our study confirms the obesity is the cause of major obstetric complications in pregnant. Pregnancy in obese women must be considered to be ?at risk?, regardless of any complications of obesity. It is thus important to help obese women become more aware of the importance of a balanced diet for themselves and their children. The presence of an obese adult in the household quadruples the risk of obesity in children.

\section{Abstract \#: P 250}

Time trends in the prevalence of obesity and diabetes comorbidities in Belo Horizonte, 2006-2012

Larissa Loures Mendes ${ }^{1}$, Mayara Santos Mendes ${ }^{2}$, Fernanda Penido Matozinhos $^{2}$, Crizian Saar Gomes ${ }^{2}$, Mariana Santos FelisbinoMendes $^{2}$, Gustavo Velasquez-Melendez ${ }^{2}$

${ }^{1}$ Universidade Federal de Juiz de Fora, Juiz de Fora, MG, Brazil;

${ }^{2}$ Universidade Federal de Minas Gerais, Department of Maternal and Child Nursing and Public Health, Belo Horizonte, MG, Brazil; Corresponding author's e-mail: nandapenido@hotmail.com

Background: Obesity and diabetes are public health problems that affect great part of population. Studies show that these factors tend to occur simultaneously and their combination represents an overall increased risk for cardiovascular disease. The aim of this study was to analyze time trends in the prevalence of obesity and diabetes comorbidities in the population of Belo Horizonte, between the years 2006 and 2012.

Methods: This is a historical series developed using the data from Surveillance System for Risk and Protective Factors for Chronic 
Diseases by Telephone Survey (VIGITEL), from 2006 to 2012. The sample of this study included adults aged 18 or more years old. Obesity was defined from body mass index (BMI) self-reported by the participants, $>=30 \mathrm{~kg} / \mathrm{m}^{2}$, according to the recommendation of the World Health Organization. The diagnosis of diabetes was also selfreported by the subject: and comorbidity was characterized by the simultaneous presence of obesity and diabetes. The prevalence of obesity and diabetes and their combination were described for the total population by gender and by the relative frequency. Linear regression (beta coefficient) was used to analyze trends from 2006 to 2012. For the statistical analysis, we used the STATA statistical package, version 12.0. For all analyses, a $5 \%$ significance level was defined.

Results: There was an increase in the prevalence of obesity and diabetes, as well as their combination during the study period. The prevalence of obesity increased from $8.9 \%$ (2006) to $14.2 \%$ (2012) $(P=0.001)$ and diabetes increased from $4.3 \%$ (2006) to $6.6 \%$ (2012) $(P=0.016)$. The prevalence increased from $0.9 \%$ (2006) to $1.8 \%(2012)(P=0.020)$. It was found that there was a significant increasing trend for both conditions for each sex, except for diabetes men.

Conclusion: The results showed that the prevalence of obesity and diabetes alone or combined are on the rise in the population of Belo Horizonte. The continuity of the surveillance system can be helpful to understand and stimulate control policies of the weight gain in the population of Brazilian urban centers.

\section{Abstract \#: P 251}

\section{Hospitalizations in adults due to hypertension and diabetes} and the brazil's family health strategy

Camila Nascimento Monteiro ${ }^{1}$, Bárbara Laisa Alves Moura ${ }^{1}$, Eduardo Luiz Andrade Mota ${ }^{2}$, Moisés Goldbaum ${ }^{1}$, Márcia Furquim de Almeida ${ }^{1}$

${ }^{1}$ University of São Paulo; ${ }^{2}$ Federal University of Bahia; Corresponding author's e-mail: c.nascimentomonteiro@gmail.com

Background: Hypertension and diabetes mellitus are a major cause of morbidity among adults in Brazil. Such diseases are public health priorities and individuals affected rely on the national governmental primary health care program called Family Health Strategy to control its effects. The aim was to analyze hospitalization tendencies in diabetic and hypertensive adults and the program coverage (FHSC) in the city of São Paulo, Brazil.

Methods: Secondary data of diabetes mellitus and hypertension hospitalizations registered between 2001 and 2012 among adults of São Paulo comprised an ecological study. Data sources were Hospital Information System, Primary Care Information System of Ministry of Health and the Brazilian Institute of Geography and Statistics. Historical series by descriptive analysis, exponential and linear regression were constructed.

Results: The FHSC average proportion was 17.7 and increased $29.3 \%$ per year $\left(\mathrm{R}^{2}=0.86\right)$. The diabetes mellitus and hypertension hospitalization average rates per 100.000 inhabitants were respectively 55.6 and 74.4. Hospitalizations' rates decreased annually by $1.5 \%\left(\mathrm{R}^{2}=0.47\right)$ for diabetes mellitus and by $1.0 \%\left(\mathrm{R}^{2}=0.80\right)$ for hypertension. For each one point increase in FHSC there was an average decrease of $15.7 \%$ in diabetes mellitus hospitalization rate $\left(\mathrm{R}^{2}=0.37\right)$ and an average decrease of $1.6 \%$ in hypertension hospitalization rate $\left(\mathrm{R}^{2}=0.24\right)$.

Conclusions: The trends over time revealed much wanted changes in the rates of hospital admission for diabetes mellitus and hypertension. The coverage growth of Brazil's Family Health Strategy may have contributed to this outcome. As those health problems are of high priority for primary health care, results suggested the benefits of Family Health Strategy in controlling preventable complications of diabetes mellitus and hypertension and in reducing unnecessary hospitalization by these illnesses.

\section{Abstract \#: P 252}

\section{Brazilian immunization program for adults with diabetes:} population-based health surveys in São paulo, brazil

Camila Nascimento Monteiro ${ }^{1}$, Sheila Rizzato Stopa ${ }^{2}$, Reinaldo José Gianini $^{1}$, Marilisa Berti de Azevedo Barros ${ }^{3}$, Chester Luiz Galvão Cesar $^{2}$, Moisés Goldbaum ${ }^{1}$

${ }^{1}$ Department of Preventive Medicine, Faculty of Medicine, University of São Paulo, São Paulo, Brazil; ${ }^{2}$ Faculty of Public Health, University of São Paulo, São Paulo, Brazil; ${ }^{3}$ Faculty of Medicine, University of Campinas, Campinas, Brazil; Corresponding author's e-mail: c.nascimentomonteiro@gmail.com

Background: Immunization program is one of the most successful experiences in Brazilian health system. Immunization against influenza and pneumonia among adults with diabetes promotes health in this population. The aim is to analyze private and public health services use to immunization against influenza and pneumonia in adults with diabetes self-reported in São Paulo, Brazil.

Methods: Data from two cross-sectional population-based health surveys (ISA-Capital 2003 and ISA-Capital 2008), carried out by home interviews. Data analysis was carried out by comparison of prevalences at two different periods of time and their $95 \%$ confidence intervals. A combined dataset was performed and the difference between the periods was estimated by Chi squared tests (Rao-Scott) and logistic regression considering the complex sample design of the surveys.

Results: Regarding to immunization against pneumonia, immunization proportion in elderly was 9.4 times (CI $95 \%$ : 2.1-41.5) higher than in adults, also adjusted as described earlier. Health services use for immunization against influenza and pneumonia was predominantly public. In 2003-2008 period, more than $90 \%$ of adults used public health services for immunization, despite of it was not found a significant difference in the period. Regarding immunization against influenza, a significant difference $(p<0.001)$ was found in the period (adults: $15.5 \%$ versus elderly: $71.0 \%$ ). Regarding to immunization against pneumonia, in 2003 immunized adults percentage was 4.5 versus $29.3 \%$ in elderly $(p=0.015)$ and in 2008, adults 1.7 versus $24.3 \%$ in elderly $(p<0.001)$. Immunization proportion in elderly was 4.1 times (CI95 \%: 2.4-41.5) higher than in adults, adjusted by sex, schooling and period.

Conclusions: Major immunization proportion in elderly may be explained by immunization campaigns to this population. Since 2003, public health service achieves large immunization coverage. Brazilian Immunization Program to vulnerable population, wich includes diabetics adults, is reference to other countries regarding to immunization coverage through public health system.

\section{Abstract \#: P 253}

The combined effects of obesity, abdominal obesity and major depression/anxiety disorders on health-related quality of life: the lifelines cohort study

Yeshambel T. Nigatu ${ }^{1}$, Sijmen A. Reijneveld ${ }^{1}$, Peter de Jonge ${ }^{2}$, Elisabeth van Rossum $^{3}$, Ute Bültmann ${ }^{1}$ 
${ }^{1}$ Department of Health Sciences, University Medical Center

Groningen, University of Groningen, The Netherlands;

${ }^{2}$ Interdisciplinary Center Psychopathology and Emotion Regulation, Department of Psychiatry, University Medical Center Groningen, University of Groningen, Groningen, the Netherlands; ${ }^{3}$ Department of Internal Medicine, Erasmus Medical Center, Rotterdam, the Netherlands; Corresponding author's e-mail: y.t.nigatu@umcg.nl

Background: Obesity, major depressive disorder (MDD) and anxiety have reached epidemic proportions globally. Obesity and MDD/ anxiety disorders often co-occur and are bidirectionally inter-related. To date, little is known about the potential interaction effects between obesity and MDD/anxiety on poor health-related quality of life (HRQoL). The aim of this study was to examine the combined effect of obesity and MDD/anxiety disorders on HR-QoL in a Dutch population.

Methods: Cross-sectional data was collected on $\mathrm{N}=90,015$ participants from LifeLines, an ongoing, large population-based cohort study. Body weight was categorized using body mass index (BMI in $\left.\mathrm{kg} / \mathrm{m}^{2}\right)$ as normal weight $(<25)$, overweight $(25-29.9)$, mild obesity (30-34.9) and moderate/severe obesity ( $\geq 35)$, abdominal obesity was defined as waist circumference of $\geq 102$ and $\geq 88 \mathrm{~cm}$ for males and females, respectively. Mental disorders, i.e. MDD and anxiety, were diagnosed with the Mini-International Neuropsychiatric Interview. HR-QoL was assessed using the 36-item Short Form Health survey, from which the physical and mental component summary scores (PCS, MCS) were computed. Multiple linear regression analysis technique was used, adjusted for potential confounders.

Results: The combined effect of obesity and MDD/anxiety on the reduction of PCS- and MCS-scores was larger than that of the sum of the separate effects of obesity and MDD/anxiety: the regression coefficients of the interaction effects on an additive scale $(95 \%$ confidence interval) were: $-0.81(-1.01:-0.62)$ and $-0.32(-0.53$ : -0.11 ), respectively. Mean PCS-and MCS-scores were 4.45 and 5.52 points lower in obese individuals with MDD/anxiety compared to those without MDD/anxiety, respectively. The poorest HR-QoL was observed in obese individuals with MDD/anxiety.

Conclusions: The co-occurrence of obesity and MDD/anxiety disorders substantially decreases the HR-QoL of people. Thus, optimal management of MDD/anxiety disorders and reduction of excess weight may be important routes to improve their HR-QoL.

\section{Abstract \#: P 254}

Assessing cardiometabolic risk in middle-aged adults using body mass index and waist-height ratio: Are two indices better than one?

Seán R. Millar ${ }^{1}$, Ivan J. Perry ${ }^{1}$, Catherine M. Phillips ${ }^{1}$

${ }^{1}$ University College Cork; Corresponding author's e-mail: s.millar@ucc.i.e

Background: A novel obesity classification method has been proposed utilising body mass index (BMI) and waist-height ratio (WHtR) together. However, the utility of this approach is unclear. In this study we compare the metabolic profiles in subjects defined as overweight or obese by both measures. We examine a range of metabolic risk features, pro-inflammatory cytokines, acute-phase response proteins, coagulation factors and white blood cell counts to determine whether a combination of both indices more accurately identifies subjects at increased cardiometabolic risk.

Methods: This was a cross-sectional study involving a random sample of 2047 men and women aged 50-69 years. Metabolic and anthropometric profiles were assessed in study participants. Independent $t$ or Mann-Whitney $U$ tests were used to compare lipid, blood pressure, glycaemic and inflammatory biomarker levels between BMI and WHtR tertiles. Multinomial logistic regression was performed to determine cardiometabolic feature and biomarker risk factor associations for BMI and WHtR groupings.

Results: In both overweight and obese groups, the combination of BMI and WHtR tertiles identified consistent metabolic variable differences relative to those characterised on the basis of one index. Similarly, odds ratios for cardiometabolic risk factors were noticeably increased in subjects classified as overweight or obese by both indices when compared to study participants categorised by either BMI or WHtR individually. In a fully adjusted model, only subjects within the highest percentile for both indices displayed a significant and positive association with pre-diabetes (OR: 3.4, $95 \%$ CI: 2.0-6.0, $P<.001)$.

Conclusions: Risk stratification using a composite index may provide a more accurate method for identifying high and low-risk subjects.

\section{Abstract \#: P 255}

Optimal central obesity measurement site for assessing cardiometabolic and type 2 diabetes risk in middle-aged adults

$\underline{\text { Seán R. Millar }}{ }^{1}$, Ivan J. Perry ${ }^{1}$, Jan Van den Broeck ${ }^{2}$, Catherine M. Phillips ${ }^{1}$

${ }^{1}$ University College Cork; ${ }^{2}$ University of Bergen; Corresponding author's e-mail: s.millar@ucc.i.e.

Background: Despite recommendations that central obesity assessment should be employed as a marker of cardiometabolic health, no consensus exists regarding measurement protocol. This study examined a range of anthropometric variables and their relationships with cardiometabolic features and type 2 diabetes in order to ascertain whether measurement site influences discriminatory accuracy. In particular, we compared waist circumference (WC) measured at two sites: (1) below the lowest rib (WC rib) and (2) between the lowest rib and iliac crest (WC midway), which has been recommended by the World Health Organisation and International Diabetes Federation.

Methods: This was a cross-sectional study involving a random sample of 2002 men and women aged 50-69 years. Metabolic profiles and WC, hip circumference, pelvic width and body mass index (BMI) were determined in study participants. Logistic regression and area under the receiver operating characteristic curve analyses were used to evaluate obesity measurement associations and discrimination of metabolic risk phenotypes and type 2 diabetes.

Results: WC Rib measures displayed the strongest associations with non-optimal lipid profiles, high blood pressure, insulin resistance, impaired fasting glucose, a clustering of metabolic risk features and type 2 diabetes, in both genders. Rib-derived indices improved discrimination of type 2 diabetes by 3-7 \% compared to BMI and 2-6 \% compared to WC midway (in men) and 5-7 \% compared to BMI and 4-6\% compared to WC midway (in women).

Conclusions: WC rib is easier to assess and our data suggest that it is a better method for determining obesity-related cardiometabolic risk than WC midway. The clinical utility of rib-derived indices as potentially more accurate predictors of type 2 diabetes requires further investigation.

\section{Abstract \#: P 256}

Health-related quality of life in relation to obesity grade, type 2 diabetes, metabolic syndrome and inflammation 
Sandra N. Slagter ${ }^{1}$, Jana V. van Vliet-Ostaptchouk ${ }^{1}$, André P. van Beek $^{1}$, Joost C. Keers ${ }^{2}$, Helen L. Lutgers ${ }^{1}$, Melanie M. van der Klauw $^{1}$, Bruce H.R. Wolffenbuttel ${ }^{1}$

${ }^{1}$ Department of Endocrinology, University of Groningen, University Medical Center Groningen, Groningen, The Netherlands; ${ }^{2}$ Van Swieten Research Institute, Martini Hospital, Groningen, The Netherlands; Corresponding author's e-mail: s.n.slagter@umcg.nl

Background: Health-related quality of life (HR-QoL) may be compromised in obese individuals, depending on the presence of other complications. We assessed whether HR-QoL in obese individuals was associated with grade of obesity, the presence of type 2 diabetes (T2D) or metabolic syndrome (MetS), and the level of inflammation. Methods: From the Dutch population-based LifeLines Cohort Study we included 13,686 obese individuals (body mass index (BMI) $\geq 30 \mathrm{~kg} / \mathrm{m}^{2}$ ), aged $18-80$ years. Diabetic individuals were those with known T2D or a fasting blood glucose $>7.0 \mathrm{mmol} / \mathrm{L}$ at screening. MetS was defined according to the NCEP ATP III criteria (2005). Level of inflammation was measured by testing for high-sensitivity $\mathrm{C}$-reactive protein (hs-CRP). HR-QoL was measured in eight health domains using the RAND 36-Item Health Survey. We calculated the percentage of obese individuals with poor HR-QoL, i.e. those scoring below the domain and sex specific cut-off value derived from the normal weight population. The risk of having a poor HR-QoL domain score, was assessed using logistic regression, stratified by sex and adjusted for age and a wide range of morbidities.

Results: Higher BMI and the additional presence of T2D was associated with a lower HR-QoL, particularly in the domains of physical functioning and general health $(P$ values $<0.0001)$. Obese individuals with MetS, especially women (all $P$ values $<0.01$ ), rated their quality of life as lower than that of obese individuals without MetS. We observed a linear trend between inflammation degree and the percentage of obese individuals who had poor scores in the HR-QoL domains $(P$ values $<0.01)$. This was most pronounced in women and in individuals with hs-CRP levels above $3 \mathrm{mg} / \mathrm{L}$. Physical functioning and general health were again mostly reduced in individuals who had MetS and in those with a higher degree of inflammation $(P$ values $<0.01$ ).

Conclusions: Obesity grade, T2D, MetS and inflammation were associated with poor outcomes in HR-QoL domains, mainly in those related to physical health. The impact varied per HR-QoL domain and was stronger in women than in men. Mental well-being was less affected.

\section{Abstract \#: P 257}

Public health surveillance of diabetes control within the context of health care reform in the United States

Carlos J. Crespo ${ }^{1}$, Ellen Smit $^{2}$

${ }^{1}$ Portland State University; ${ }^{2}$ Oregon State University; Corresponding author's e-mail: ccrespo@pdx.edu

Background: The State of Oregon, USA, is working to transform the health care delivery system to improve population health, provide better medical care and lower costs. To accomplish these goals surveillance mechanisms to assess a variety of metrics have been established to monitor progress across several health indicators including diabetes control.

Methods: The Oregon Health Authority tracks 17 local Coordinated Care Organizations (CCO) to determine if CCOs are effectively and adequately improving care, making quality care accessible, eliminating health disparities, and controlling costs for the populations that they serve. The primary method to achieve this goal is through the implementation of Patient Centered Primary Care Homes (PCPCH) within CCOs.

Results: There has been an increased of $55 \%$ since 2011 in the enrollment of Oregonians in PCPCH. Our most recent findings (June, 2014) show that there has been an increase in primary care cost and a reduction in emergency room visits. The rate of adult patients (ages 18 years and older) with diabetes who had a hospital stay because of a short-term problem from their disease was 192.9 in 2011, and 174.9 in June 2014. Rates are reported per 100,000 member years. The percentage of adult patients (ages 18-75 yrs) with diabetes who received at least one A1c blood sugar measurements showed continual improvement since 2011. All CCOs improved from $78.5 \%$ in 2013 to $82.7 \%$ in June 2014. HbA1c testing has approached the benchmark $(87 \%)$ across all racial and ethnic groups. Newly enrolled members use emergency rooms less frequently and have fewer avoidable emergency room visits than persons who had been enrolled in the Oregon Health Plan prior to health reform. Lastly, financial data indicates $\mathrm{CCO}$ are continuing to hold down costs.

Conclusion: Our findings provide data on population based indicators of diabetes control within the context of health reform in the United States and in the state of Oregon. The results are part of the establishing metrics to assess quality of care among persons with diabetes based on the most recent available data. It is also the first report to include data on some key measures for the more than 380,000 additional Oregonians who have enrolled in the Oregon Health Plan since the Affordable Care Act (ACA) took effect January 1, 2014. Oregon is staying within the budget that meets its commitment to the federal government to reduce the growth in spending by two percentage points per member, per year.

\section{Abstract \#: P 258}

Communicational and critical health literacy impact on self-care behaviors in Iranian patients with type 2 diabetes

Mahnoush Reisi ${ }^{1}$, Homamodin Javadzade ${ }^{1}$, Gholamreza Sharifirad ${ }^{2}$, Firouzeh Mostafavi ${ }^{3}$, Behzad Mahaki ${ }^{4}$

${ }^{1}$ Department of Health Education and Health Promotion, Faculty of Health, Bushehr University of Medical Sciences, Bushehr, Iran;

${ }^{2}$ Department of Health Education and Health Promotion, Faculty of Health, Qom University of Medical Sciences, Qom, Iran;

${ }^{3}$ Department of Health Education and Health Promotion, Faculty of Health, Isfahan University of Medical Sciences, Isfahan, Iran. of;

${ }^{4}$ Department of Bio-statistics, Faculty of Health, Isfahan University of Medical Sciences, Isfahan, Iran; Corresponding author's e-mail: reisi_mr@yahoo.com

Introduction: Diabetes is a chronic disease that may cause some acute and debilitating complications like cardiovascular diseases, blindness and renal failure. Inadequate health literacy is a serious obstacle in diabetes management and it can affect patient's participation in self-care. The present study is conducted to investigate health literacy of patients with type 2 diabetes in functional, communicative and critical domains and to determine the relationship between these domains and self-care behaviors.

Materials and Methods: We conducted a cross-sectional descriptive correlational design on a convenience sample of 187 type 2 diabetes patients referred to Hazrat Ali diabetes clinic in Isfahan, Iran. Health literacy and self-care behavior in patients were assessed by standard questionnaires of FCCHL and DMSES, respectively. The collected data was analyzed using Analysis of variance, Independent t-test, Pearson correlation coefficient and linear regression tests and by means of SPSS vr.16 software. 
Findings: The mean age of studied patients was $57.40 \pm 11.07$ years. The average health literacy and self-care behaviors score of participants was $2.28 \pm 0.57$ and $3.84 \pm 1.56$ respectively. While there was a statistically significant association between total health literacy, communicative and critical health literacy and self-care behaviors, there was no statistically significant association between functional health literacy and self-care behavior. Communicative health literacy was related to self-care behaviors of diet adherence, performing physical activity, blood sugar monitoring and feet care. Critical health literacy in patients was related to physical activity, blood sugar monitoring, feet-care and medication intake. In the analysis, no statistically significant association between communicative health literacy and medication intake and also between critical health literacy and diet adherence was observed. From various domains of health literacy, communicative health literacy was identified as a better predictor of self-care behavior in diabetic patients.

Conclusion: Since in diabetic patients, critical and communicative health literacy was related to self-care behaviors, paying attention to the skills which are related to these domains of health literacy in healthcare system and also providing information according to patients' health literacy level, looks to be necessary. Because functional health literacy in patients that it is more related to reading and writing skills, had no relationship with self-care behaviors, so it is not recommended to merely rely on people's educational level to communicate and provide training for them.

\section{Abstract \#: P 259}

\section{Exploration of oral health-related quality of life among Indian} patient population

\section{Manu Batra ${ }^{1}$, Rishabh Kumar Rana ${ }^{2}$}

${ }^{1}$ Department of Public Health Dentistry, Surendera Dental College and Research Institute, SRI Ganganagar, India; ${ }^{2}$ WHO Country Office Surveillance Medical Officer NPSP Unit Begusarai, India;

Corresponding author's e-mail: drmanubatra@aol.com

Objective: The objective of the study is to establish to what extent the patients' complaints affect their quality of life and to determine if there was a possible association between gender, education, harmful habits and their oral health quality of life.

Materials and Methods: Study was carried out on 1054 subjects and the examination of subjects and recording of questionnaires were carried out by four researchers. Two patient-centred outcome measures, the 14 item Oral Health Impact Profile (OHIP-14) and the 16 item Oral Health related Quality of Life (OHQoL-UK) measure were used in this study.

Results: Highest OHIP-14 scores and lowest OHQoL-UK scores were in patients who came with trauma. OHIP-14 scores were lowest and OHQoL-UK scores were highest in subjects who had orthodontic or aesthetic complaint. It was seen that there was statistically significant difference in OHQoL-UK $(p<0.001)$ and OHIP-14 $(p<0.001)$ scores of subjects according to complaints $(p<0.01)$.

Conclusion: OHQoL is associated with the oral complaints of patients. Furthermore, OHQoL may not only be associated with the oral health status of patients, but factors such as gender, education and harmful habits may also play a role.

\section{Abstract \#: P 260}

Renal transplant: early rejection and association with pre-discharge creatinine level
Sonam Bedi $^{1}$, C.M. Pandey ${ }^{1}$, R.K. Sharma ${ }^{2}$, Uttam Singh ${ }^{1}$

${ }^{1}$ Department of Biostatistics \& Health Informatics, Sanjay Gandhi Postgraduate Institute of Medical Sciences, Lucknow, India;

${ }^{2}$ Department of Nephrology, Sanjay Gandhi Postgraduate Institute of Medical Sciences, Lucknow, India; Corresponding author's e-mail: sonam.bedi09@gmail.com

Background: Rise in end stage renal disease (ESRD) is a major concern to developing nations including India. Poor renal dysfunction reflected by elevated serum creatinine remains a strong risk factor and highly correlated with graft failure. Present analysis was carried to assess early renal allograft dysfunction, its pattern and the extent of additional risk with elevated base line creatinine level along with sensitivity of creatinine level to predict rejection.

Methods: All the 323 transplant cases during December 2009 to April 2013 at SGPGI, Lucknow in State Uttar Pradesh were considered and followed for a total of 90 days. The diagnosis of allograft rejection was based on graft biopsy findings. The parameters recorded were age, gender, blood group of both recipient and donor, HLA matching, pre-discharge serum creatinine level of recipient, immunosuppressant and vascular access. Chi square was used to establish strength of association. Cox regression analysis was carried to find out the possible predictors of early renal allograft rejection. Survival of allograft on the basis of different categories of predictors was obtained by Kaplan Meir method. Statistical significance was checked at $p=0.05$.

Results: The base line creatinine level of $32.2 \%$ cases was below $1.10 \mathrm{mg} / \mathrm{dl}$ while of $11.5 \%$ greater or equal to $1.50 \mathrm{mg} / \mathrm{dl}$. Normal graft function was achieved in $58.2 \%$ cases without any complication in immediate post-operative period. Allograft dysfunction rate was about 5 times $(\mathrm{OR}=5.40, \mathrm{CI}=2.12-13.74)$ higher in those with base line creatinine level $\geq 1.5 \mathrm{mg} / \mathrm{dl}$ than those below $1.5 \mathrm{mg} / \mathrm{dl}$. Patients treated with Cyclosporine-Mycophenolate mofetil-Prednisolone combination of immunosuppressant are $3(\mathrm{OR}=3.36$, $\mathrm{CI}=1.39-8.13)$ times more prone to have early allograft rejection than those treated with Tacrolimus-Mycophenolate mofetil-Prednisolone. A sharp increase in allograft dysfunction noticed in those with creatinine $\geq 1.5$ till 50th day and remains constant thereafter. The sensitivity to predict graft dysfunction was reasonably good as area under the curve was 0.69 .

Conclusion: Transplant cases with elevated base line creatinine require frequent follow up for interventions in need.

\section{Abstract \#: P 261}

\section{Self-perception of oral health and impact on quality of life} among the elderly

$\underline{\text { Tatiana R. de C. Mello }}{ }^{1}$, Ivan Junho Bolivio ${ }^{1}$

${ }^{1}$ University of Mogi das Cruzes, School of Dentistry, Mogi das Cruzes, Brazil; Corresponding author's e-mail: tatianar@umc.br

Background: Given the demographic changes initiated in the last century, the importance of ensuring the elderly not only a longer life expectancy, but also a good quality of life has grown significantly. The objective of this study was to describe the impact of oral health conditions in the elderly.

Methods: Fifty individuals aged 60 years or more took part in the study. We used the GOHAI questionnaire, composed of 12 closed questions about dental problems that affect physical and functional roles, psychological, pain and discomfort of the elderly. For social factors, we included questions related to socioeconomic status. For the epidemiological survey of the teeth conditions, the criteria recommended by WHO was used. 
Results: The average for CPO-D was $24.84(\mathrm{SD}=5.71)$. Clinical examination showed that all participants had a history of injury to oral health with tooth loss. The average GOHAI was $29.74(\mathrm{SD}=4.33)$, which was low, showed a high impact of oral health on quality of life for elders.

Conclusion: Women and people over 70 years and edentulous had the lowest scores, showing that these groups consider high the negative impact of their oral health on their quality of life. The epidemiological profile of this population and the impact of this profile in the quality of life allows the planning of promotion, protection and recovery of health.

\section{Abstract \#: P 262}

\section{Accident with exposure to biological material in Bahia, brazil \\ Paula Muniz do Amaral ${ }^{1}$, Tânia Maria de Araújo ${ }^{1}$, Fernanda de Oliveira Souza ${ }^{1}$ \\ ${ }^{1}$ Núcleo de Epidemiologia/Universidade Estadual de Feira de Santana - Bahia/Brasil; Corresponding author's e-mail: aluapmuniz@hotmail.com}

With the purpose to improve the quality and expand the coverage of registration of accidents at work, the Unified Health System (SUS) has incorporated to the Notification System of Grievance Information (SINAN) 11 diseases and disorders related to work. Among these contained accidents involving exposure to biological material.

Objective: to analyze reports of accidents with biological material in Bahia, Brazil.

Methodology: descriptive study, with Notification System of Grievance Information of Occupational Health Reference Center (CEREST) data. Whereas the registry in SINAN of injuries related to work, as the Ministry of Health Order n 777 of 28 April 2004, was implemented in Bahia since 2007, all accidents with biological material present in the SINAN, from 2007 to 2014, were included.

Results: Bahia has 14 regional CEREST's, where were recorded 10,570 accidents with biological material. During this period there was a significant increase in the reporting of this condition. In 2007, 221 cases of accidents with biological material were reported, and in 2014, 1813 cases were reported. Of these, 8268 (78.22\%) were females, $2301(21.76 \%)$ males and $1(0.009 \%)$ ignored. Regarding the type of exposure, 7649 (72.36\%) were percutaneous type, 995 $(9.41 \%)$ mucocutaneous and $149(1.40 \%)$ another exhibition. Regarding the situation of the skin, $3819(36.13 \%)$ had intact skin and $469(4.43 \%)$ not intact skin. Regarding the circumstance of the accident, $1004(9.5 \%)$ were intravenous medication administration, $956(9.04 \%)$ inappropriate disposal on the ground, $825(7.80 \%)$ improper disposal in the trash, $847(8.01 \%)$ surgical procedure and $765(7.23 \%)$ handling perforating/cutting box.

Conclusion: The data suggests an increase in the reporting of accidents with biological material. However, despite the progress, it is estimated that this number is much higher. Previous studies in Brazil indicated that absence of notification does not necessarily indicate absence of cases, but underreporting. It is noteworthy that since 2002, was established by SUS the National Network of Integral Care for Workers' Health (RENAST) and has a network of information and health practices organized to perform assistance, surveillance and health promotion actions. Despite the effort established, it is still necessary to stimulate and educate health professionals to commit themselves to the important role of the notification. The results of this study represent important epidemiological assessment task, toward the improvement of the information system as well as the promotion, prevention, control and rehabilitation of workers' health.

\section{Abstract \#: P 263}

Components of the life expectancy divergence across developed countries

Sergey Timonin $^{1}$, Vladimir M. Shkolnikov ${ }^{2,3}$, Domantas Jasilionis ${ }^{2}$, $\overrightarrow{\text { Pavel Grigoriev }}^{2}$

${ }^{1}$ National Research University Higher School of Economics;

${ }^{2}$ Max Planck Institute for Demographic Research, Germany;

${ }^{3}$ New Economic School, Russia; Corresponding author's

e-mail: stimonin@hse.ru

Although the worldwide lengthening of the human lifetime is continuously progressing, the country-specific trends are rather diverse. Mortality has been decreasing in most of the developed countries since the end of 1970s, however several countries have experienced slow-downs and even reversals. This phenomenon has led to a rise of the inter-country disparity in the length of life.

Using high quality data on mortality up to age 95+ from the Human Mortality Database, we examine this disparity across 36 developed countries that are divided into three groups of high income (HI), Central and Eastern European (CEE), and the former Soviet Union (FSU) countries.

The average population-weighted standard deviation (a measure of a life expectancy disparity) has increased by almost three times for men (from 2.0 to 5.6 years) and by about two times for women (from 1.0 to 3.6 years) in 1970-2010. Its value was constantly rising till the mid-2000s (maximum was reached in 2005 for men and in 2004 for women). After the period of growing divergence, standard deviation value slightly decreased that might indicate the starting transition from divergence to the convergence process, mainly induced by the recent health improvements in FSU countries.

We use the stepwise replacement algorithm to decompose the changes in the life expectancy and its variance according to age categories. The decomposition results document meaningful changes in importance of mid-life and old-age mortality components across time and groups of countries.

Finally we decompose changes in the population-weighted life expectancy variance into between- and within-group partitions. The outcomes reveal a steep rise of the between-group part of the variance due to the growing geo-political polarization in mortality.

The inter-country mortality disparities continue to be a major health challenge even among the developed countries. Understanding determinants of the convergence-divergence processes, factors and conditions leading to success or failure in fight against death is an important research priority.

\section{Abstract \#: P 264}

Immunogenicity of three- versus two-dose HPV immunization schedules in preadolescent girls and young women: a systematic review and meta-analysis

Robine Donken $^{1,2}$, Mirjam J. Knol ${ }^{1}$, Johannes A. Bogaards ${ }^{1}$, Fiona R.M. van der Klis ${ }^{1}$, C.J.L.M. Meijer ${ }^{2}$, Hester E. de Melker ${ }^{1}$

${ }^{1}$ Centre for Infectious Disease Control, RIVM; ${ }^{2}$ Department of Pathology, VUmc; Corresponding author's e-mail: robine.donken@rivm.nl

Background: Human Papillomavirus (HPV) is the necessary cause of cervical cancer. Primary prevention is possible by vaccination. Initially HPV vaccination was licensed in a three dose schedule. For young girls approval was based on immunobridging studies, where the immunogenicity was compared to immunogenicity in adult 
females, in which clinical efficacy against (pre)cancerous lesions was proven. The European Medicines Agency (EMA) recently approved two-dose immunization schedules for bivalent (HPV 16/18) and quadrivalent (HPV 6/11/16/18) human papillomavirus (HPV) vaccines in nine to fourteen and thirteen year-old-girls, respectively. Registration was based on trials comparing immunogenicity of threedose schedules in young women 15-26 years versus two-dose schedules in girls 9-14 years. Decision-making on HPV vaccination on the basis of immunological correlates is complicated because the correlate of protection is unknown, therefore it is uncertain what the value of antibody titers or concentrations is and what level is considered to be protective. We evaluate comparability of antibody levels between and within age groups and discuss potential implications for monitoring the effectiveness of HPV vaccination.

Methods: A systematic literature search was performed for studies comparing immunogenicity of three- versus two-dose schedules of HPV vaccination. We compared geometric mean concentrations (GMCs) of vaccine-type antibodies between different dosing schedules across different age groups. Meta-analysis was used to estimate pooled GMC ratios (bivalent vaccine) of three- versus two-dose schedules within girls.

Results: For both vaccines, two-dose immunization of girls yielded non-inferior GMCs relative to a three-dose schedule in young women up to respectively 36 and 48 months follow-up. Pooled GMC ratios showed the two-dose in girls becoming inferior to the three-dose schedule in girls for HPV 16 at approximately 2 years after the first dose. For the quadrivalent vaccine, inferior antibody responses comparing the three-dose with the two-dose schedule in girls were shown from 18 months follow-up onwards.

Conclusion: Two-dose immunization of girls has non-inferior immunogenicity compared to a three-dose schedule among young women. However, non-inferior immunogenicity of two- versus threedose schedules within girls has not been shown. Implementation of two-dose HPV vaccination needs to be monitored closely.

\section{Abstract \#: P 265}

A change of the life style among inhabitants of Koper region and Slovenia in the period 2004-2012

\author{
Marina Sucic Vukovic ${ }^{1}$ \\ ${ }^{1}$ Nationa Institute of Public Health; Corresponding author's e-mail: \\ marina.sucic-vukovic@ijz.si
}

Background: Many researches about the connection between life style and health in different countries were performed.

In the years 2001, 2004, 2008 and 2012 we were included in performing of national researches "The life style connected to the health among inhabitants of Slovenia"

Our objective is to present the results of analyses of acquired data in the field of tobacco and alcohol use, nutrition and physical exercise habits among inhabitants of Koper region and Slovenia as well the change of them in the period between 2004, 2008 and 2012.

The life style is established as an important determinant of health. We wanted to establish a change of the life style among our inhabitants in a period of 8 years and to connect it with health indicators. Methods: The researches were performed on the representative sample of adult inhabitants of Slovenia according to single regions in the years 2004, 2008 and 2012. We collected the data with the questionnaires sent by post. We processed data with computer programs SPSS, Microsoft Excel and Microsoft Word.

Results: We analysed and established statistical significant differences into collected dates about the spread of tobacco and alcohol use, nutrition and physical exercise habits among inhabitants of Koper region in these years.

We established in year 2012 compared to year 2004 and 2008 many favourable changes in the field of smoking, physical exercise habits and nutrition habits and less favourable results on the field of alcohol use among our inhabitants.

Conclusions: The results of analyses will be used to estimate our performed health promotion programs and as a background for our further planed activities.

\section{Abstract \#: P 266}

Prevalence of smoking and risk factors among physical education and sports school students at Ahi Evran University, Kirsehir, Turkey

Ulken T. Babaoglu ${ }^{1}$, Sibel Cevizci ${ }^{2}$, Serhat Ozdenk ${ }^{3}$

${ }^{1}$ College of Health, Ahi Evran University, Kirsehir, Turkey; ${ }^{2}$ Department of Public Health, Onsekiz Mart Medical University, Canakkale, Turkey; ${ }^{3}$ Colleges of Physical Education and Sports, Ahi Evran University, Kirsehir, Turkey; Corresponding author's e-mail: utbabaoglu@ahievran.edu.tr

Background: The aim of this study was to investigate smoking prevalence and related risk factors among students at the Physical Education and Sports School of Ahi Evran University in Kirsehir, Turkey.

Methods: A cross-sectional study was conducted on total of 1082 students continuing their education at the school from February and May 2014. A multiple-item questionnaire was applied to students using the "answering-under-supervision" technique during lessons. Statistical analyses were made by using SPSS version 17.0 software package.

Results: A total of 675 responders were female $(62.4 \%)$ and 407 responders were male $(37.6 \%)$ out of 1082 participants. Of the 1082 students, $256(23.7 \%)$ were current smokers and the average age for beginning smoking was $16.61 \pm 2.72$. The participants had mostly graduated from public $(96.0 \%)$ school, while $4.0 \%$ had graduated from private high schools. In this study, we found that the smoking prevalence associated with some variables such as sex, classroom grade, age, place of residence, cigarette or tobacco use in the living place and knowledge status of students about their teacher's smoking habits $(p \leq 0.05)$.

Conclusion: Our study results revealed that smoking prevalence was high among the students, who engaged in sports actively. These results also provide that sports school students who will be a role model presenting physical activity as a sports people for increasing stop smoking and healthy living behaviors in public should be trained to improve their knowledge and awareness about health risks of smoking.

\section{Abstract \#: P 267}

Total income of the neighbourhood and obesity among adults: a multilevel analysis of an urban Brazilian context

Fernanda Penido Matozinhos ${ }^{1}$, Larissa Loures Mendes ${ }^{2}$, Crizian Saar Gomes $^{1}$, Milene Cristine Pessoa ${ }^{3}$, Gustavo Velasquez-Melendez ${ }^{1}$

${ }^{1}$ Universidade Federal de Minas Gerais, Department of Maternal and Child Nursing and Public Health, Belo Horizonte, MG, Brazil;

${ }^{2}$ Universidade Federal de Juiz de Fora, Juiz de Fora, MG, Brazil; 
${ }^{3}$ Universidade Federal de Viçosa, Viçosa, MG, Brazil; Corresponding author's e-mail: nandapenido@ hotmail.com

Background: The prevalence of obesity is constantly increasing worldwide and the environmental factors affect the risk of it. It has been recognized that the socioeconomic status of the neighbourhood can influence the health of individuals and affect the prevalence of obesity through environmental factors, such as access to food stores, healthy restaurants, sports facilities, and parks and the price and availability of healthy foods. The objective of this study is to estimate the association of the total income of the neighbourhood and individual factors with obesity in adults in a Brazilian urban context.

Methods: A cross-sectional study was conducted using the Surveillance System for Risk and Protective Factors for Chronic Diseases by Telephone Survey (VIGITEL) from 2008 to 2010. The body mass index (BMI) was calculated from the participants' self-reported weight and height. Obesity was defined as a BMI $\geq 30 \mathrm{~kg} / \mathrm{m}^{2}$. The area covered by the basic health units was defined as a neighbourhood unit. The total income of the neighbourhood was used to characterise the social environment. In addition, individual variables were considered. A multilevel logistic regression was performed. Estimates of the odds ratio (OR) and $95 \%$ confidence interval $(95 \% \mathrm{CI})$ were used.

Results: This study included 5273 individuals, with an average age of 43.67 years $(\mathrm{SD}=16.65)$. A total of $43.51 \%$ of participants were male, and $40.24 \%$ had 9-11 years of education. The prevalence of obesity was $12.13 \%$ (95\% CI: $11.20-13.12$ ). It was observed that individuals living in neighborhoods with more total income $(\mathrm{OR}=0.96,95 \%$ CI: 0.94-0.98) had decreases odds of being obesity. This association remained statistically significant after controlled by age, sex, education and consumption of meat with visible fat. Conclusion: Obesity is considered a significant public health problem. This study contributes to a better understanding of the complex interaction between environmental and individual determinants of obesity and may aid in the development of effective interventions, such as the expansion of obesity control programs and health promotion strategies.

\section{Abstract \#: P 268}

Impact of the post-2008 economic crisis on harmful drinking in the Dutch working-age population

Moniek C.M. de Goeij ${ }^{1,2}$, Bregje van der Wouden $^{1,2}$, Jan-Willem Bruggink $^{3}$, Ferdy Otten ${ }^{3}$, Anton E. Kunst ${ }^{1,2}$

${ }^{1}$ Department of Public Health, Amsterdam Medical Center (AMC); ${ }^{2}$ University of Amsterdam, Amsterdam, The Netherlands;

${ }^{3}$ Department of Statistical Analysis, Division of Social and Spatial

Statistics, Statistics Netherlands, Heerlen, The Netherlands;

Corresponding author's e-mail: m.c.degoeij@amc.uva.nl

Background: Studies on the impact of economic crises on alcohol consumption have yielded ambiguous results. Therefore, we studied changes in trends in harmful drinking among the Dutch working-age male and female population after the post-2008 economic crisis has started. We also assessed whether these trend changes differed across age categories and socioeconomic groups.

Methods: We used repeated cross-sectional data from the Dutch Health Interview Survey conducted by Statistics Netherlands. Representative respondent samples were independently drawn each month between January 2004 and December 2013. Our study sample consisted of 43,487 working-age (25-65 years) individuals. For men and women, binge drinking was defined as drinking $\geq 6$ glasses of alcohol on one day at least once a week. Hazardous drinking was defined as consuming $\geq 14$ glasses/week for women and $\geq 21$ glasses/ week for men. Socioeconomic groups were defined by three separate variables: work status (employed, unemployed, and not part of the labor force), disposable household income (divided in tertiles), and educational level (low, middle, and high). Segmented logistic regression was used to model the trend changes among the Dutch population after the crisis has started (October 1, 2008).

Results: An initial trend of decreasing prevalence of hazardous drinking among men and women slowed down after the economic crisis has started. None of these trend changes varied by age or socioeconomic group. Regarding binge drinking, the prior upward trend among men aged 25-34 changed to a downward trend after the economic crisis has started, while in men aged 35-54 this was the other way around. No differences were found across socioeconomic groups.

Conclusion: During the post-2008 economic crisis, the decline in hazardous drinking slowed down among men and women, while binge drinking started to increase among men aged 35-54. These results suggest that the economic crisis had an upward effect on chronic and episodic drinking, especially among men aged 35-54.

\section{Abstract \#: P 269}

Correlation between neighborhood walkability, residential density, land-use mix with moderate physical activity and sedentary time: an exploratory study in Porto

Roseanne Autran $^{1}$, Hugo Teixeira ${ }^{2,3}$, Ana Isabel Ribeiro ${ }^{2,3,4}$, Maria de Fátima de Pina $^{2,3,5}$, Jan Dygryn ${ }^{6}$, Jorge Mota ${ }^{1}$, Maria Paula Santos ${ }^{1}$

${ }^{1}$ Centro de Investigação em Atividade Física, Saúde e LazerFaculdade de Desporto da Universidade do Porto, Portugal; ${ }^{2} \mathrm{i} 3 \mathrm{~S}-$ Instituto de Investigação e Inovação em Saúde, Universidade do Porto, Portugal; ${ }^{3}$ INEB - Instituto de Engenharia Biomédica,

Universidade do Porto, Portugal; ${ }^{4}$ ISPUP - Instituto de Saúde Pública da Universidade do Porto, Portugal; ${ }^{5}$ ICICT/FIOCRUZ - Instituto de Comunicação e Informação Científica e Tecnológica em Saúde, Fundação Oswaldo Cruz -, Rio de Janeiro, Brazil; ${ }^{6}$ Institut of Active Lifestyle, Faculty of Physical Culture, Palacky University, Olomouc, Czech Republic; Corresponding author's e-mail:

roseautran@gmail.com

Background: Research has shown that characteristics of the place of residence, such as neighborhood walkability (a function of street connectivity, residential density and land use mix) are associated with physical activity (PA), and sedentary time (ST). However, there are still gaps in the literature about which environmental variable is more strongly associated with PA and SB. The aim of this study is to explore whether there is an association between walkability index (as well as each of the variables that compose the index) and neighborhood SES, with moderate PA and ST in adolescents living in Porto, Portugal.

Methods: This cross-sectional study included 153 adolescents (56.2\% girls), aged 14-18 years-old attending in 3 public schools in Porto municipality. Walkability index (WI) was calculated using Geographic Information System (GIS) based on: residential density, street connectivity and land use mix, and was dichotomized for analysis using deciles (1st to 5th corresponds low walkability and 6th to 10th corresponds high walkability area). Using GIS, circular buffers of 500 meters around participants residences were generated. Then, WI, land-use mix, street connectivity and residential density were calculated for each buffer and attributed to each participant. PA was measured with accelerometers (Actigraph GT1 M) considering minimum of 4 consecutive days ( 1 weekend day) and $8 \mathrm{~h}$ per day. Evenson (2008)1 cut-points were used to classify moderate PA and ST. Pearson bivariate correlations analysis was performed. 
Results: The mean and standard deviation of moderate PA (min/day) in adolescents was $(31.59 \pm 12.55)$ and ST (min/day) (486.44 \pm 70.87 ). $50.2 \%$ of adolescents lived in a low walkability area. There was no significant correlation between walkability index $(\mathrm{r}=0.10, p=0.20)$, street connectivity $(\mathrm{r}=-0.11, p=0.89)$, land-use mix $(\mathrm{r}=0.06, p=0.45)$ with moderate PA and ST $(p>0.05)$. However, we found a positive correlation between household density and moderate PA $(\mathrm{r}=0.16, p=0.04)$.

Conclusion: Our results suggest that household density might affect moderate physical activity.

Reference: Evenson KR, Catellier DJ, Gill K, Ondrak KS, McMurray RG. Calibration of two objective measures of physical activity for children. Journal of sports sciences. 2008 Dec:26(14):1557-65. PubMed PMID: 18949660.

Acknowledgement: CAPES Foundation, Ministry of Education of Brazil.

\section{Abstract \#: P 270}

\section{The periodontal disease as a risk factor for acute myocardial} infarction

Samilly S. Miranda ${ }^{1}$, Julita M.F. Coelho ${ }^{1}$, Isaac S. Gomes-Filho ${ }^{1}$, Monalisa N. Pereira $^{2}$, Simone S. da Cruz ${ }^{1}$, Johelle de S. Passos ${ }^{1}$,

${ }^{1}$ Health, UEFS, Feira de Santana, Brazil; ${ }^{2}$ Major public hospital in São Paulo; Corresponding author's e-mail: samillymiranda@gmail.com

Background: Researches currently exist on the relationship between periodontal disease and systemic conditions, since persistent bacterial aggression in the oral cavity may overtake the oral tissues, triggering serious repercussions throughout the entire body. Aim: Evaluate the contribution of Chronic Periodontitis in Acute Myocardial Infarction events.

Methods: Was performed case-control study among patients seen in the emergency room of two hospitals in Salvador, Bahia, September 2008 to April 2009, were evaluated in a paired comparison. The estimated sample size was 414 individuals, the case group was composed of those patients with acute myocardial infarction, and the control group of those with no history of acute myocardial infarction. This study design was submitted to and approved by the Research Ethics Committee of the State University of Feira de Santana, Bahia (Protocol $n^{\circ}$ 025/2004). Participants underwent a complete periodontal examination by a previously trained dentist as well as a questionnaire constructed for research.

Results: Through brute association of data analysis, it was found that among individuals with Periodontal Disease (PD) the chance for Acute Myocardial Infarction (AIM)was higher than among those without PD (OR bruta $=1.73$ : IC $95 \%$ [1.10- 2.72], $p=0.016)$. After adjusting for confounding variables (smoking, level of education, occupation, diabetes and HDL-cholesterol), PD showed an independent effect for AMI (OR adjusted $=1.92$ : IC $95 \%$ [1.13-3.23]), $p=0.015$ ).

Conclusion: It can be concluded that there is a statistically significant association between the occurrence of DP and AMI and these results can help to support prevention and control measures for the two abovementioned diseases.

\section{Abstract \#: P 271}

Donor-recipient age relation: an impact on early renal allograft rejection
Sonam Bedi ${ }^{1}$, C.M. Pandey ${ }^{1}$, R.K. Sharma ${ }^{2}$, Uttam Singh ${ }^{1}$

${ }^{1}$ Department of Biostatistics \& Health Informatics, Sanjay Gandhi Postgraduate Institute of Medical Sciences; ${ }^{2}$ Department of Nephrology, Sanjay Gandhi Postgraduate Institute of Medical Sciences; Corresponding author's e-mail: sonam.bedi09@gmail.com

Background: The early allograft rejection is a major issue. Elevated creatinine has been found to contribute significantly for early allograft rejection. Many studies have shown the impact of donor-recipient age difference on long and short-term graft and patient survivals: no study was found to substantiate the role of recipient-donor age on early allograft survival. Present analysis is an attempt to assess the role of recipient-donor age on early allograft survival.

Methods: A total, 451 kidney transplant recipients on regular follow up at a tertiary care centre, during December 2009 to November 2013 were studied. Characteristics like age, gender, blood group and HLA matching of donors and recipients, vascular access, post-transplant serum creatinine at the time of discharge were categorized. The early rejection of allograft was considered based on biopsy report. Donor and recipient ages were divided into 2 subgroups: Similar groups: I (donor $<50$ and recipient $<50$ ) and (donor $>=50$ and recipient $>=50$ ) and dissimilar groups: II (donor $<50$ and recipient $>=50$ ), and (donor $>=50$ and recipient $<50$ ). Characteristics between rejected (cases) and non-rejected (controls) were compared and significance was checked at $p=5 \%$.

Results: $86 \%$ recipients were younger i.e. age below 50 years whereas $62.2 \%$ donors were younger. Creatinine below $1.5 \mathrm{mg} / \mathrm{dl}$ was of $90.9 \%$ recipients. Allograft rejection was seen in $9.3 \%$ cases. None of the characteristics except creatinine and similar-dissimilar age groups of recipient-donors combined together were found to be associated with early allograft rejection $(p<0.000)$. Similar age groups of recipients and donors are 4 times at higher risk of early allograft rejection than the dissimilar groups $(\mathrm{OR}=4.21$ : 1.14-15.53): while recipients with serum creatinine $>=1.5 \mathrm{mg} / \mathrm{dl}$ are more prone to rejection than with creatinine $<1.50$ ( $\mathrm{OR}=32.27$ : 9.74-106.87).

Conclusion: Donor-recipient age relation signifies the role to early renal allograft rejection: a large scale analysis is further required to validate the findings. This will support in decision making to choose a donor for better survival of transplanted allograft.

\section{Abstract \#: P 272}

Post-nuclear disaster evacuation and survival amongst elderly people in Fukushima: a comparative analysis between evacuees and non-evacuees

Shuhei Nomura $^{1}$, Marta Blangiardo ${ }^{2}$, Masaharu Tsubokura $^{3}$, Yoshitaka Nishikawa ${ }^{4}$, Stuart Gilmour ${ }^{5}$, Masahiro Kami ${ }^{3}$, Susan Hodgson ${ }^{2}$

${ }^{1}$ Department of Epidemiology and Biostatistics, School of Public Health, Imperial College London, Norfolk Place, London W2 1PG, United Kingdom; ${ }^{2}$ MRC-PHE Centre for Environment and Health, School of Public Health, Imperial College London, Norfolk Place, London W2 1PG, United Kingdom; ${ }^{3}$ Division of Social Communication System for Advanced Clinical Research, the Institute of Medical Science, University of Tokyo, 4-6-1 Shirokanedai, Minato-ku, Tokyo 108-8639, Japan; ${ }^{4}$ Soma Central Hospital, 3-5-18 Okinouchi, Soma, Fukushima 976-0016, Japan; ${ }^{5}$ Department of Global Health Policy, Graduate School of Medicine, University of Tokyo, 7-3-1 Hongo, Bunkyo-ku, Tokyo 113-0033, Japan; Corresponding author's e-mail: s.nomura13@imperial.ac.uk 
Background and aims: Although the evacuation is an important response to a major disaster, little is investigated about evacuationrelated mortality risks. We conducted a retrospective cohort survival study of elderly evacuees including comparative analyses with nonevacuees, after the Japan's Fukushima Dai-ichi nuclear incident on 11th March 2011.

Methods: A total of 1216 residents from seven nursing homes located $20-40 \mathrm{~km}$ from the nuclear plant who were admitted in the 5 years before the incident participated in this study. Demographic and clinical characteristics were obtained from medical records, and evacuation histories were tracked until mid 2013. To assess the relationships between evacuation and mortality after the incident, the pre- and post-disaster survival probability and relative mortality incidence, as well as hazard ratios in Cox hazard regression were employed.

Results: There was a large variation in post-incident mortality risks across the five evacuated facilities ranging from 0.77 to 2.88 . Evacuation (initial and subsequent) was associated with 1.69 times higher mortality (95\% CI: 1.14-2.51) than non-evacuation after adjusting for covariates, while experience of the disasters did not have a significant influence on mortality. Particularly, initial evacuation from the original facility had substantial impact on mortality with hazard ratio of 3.37 (95\% CI: 1.66-6.81) against non-evacuation, though subsequent evacuations had no significant mortality risk. In addition, no statistically significant influence of evacuation distance on mortality was observed.

Conclusion: The findings of this study indicate that evacuation may not be the best life-saving strategy for elderly people. Following the Fukushima nuclear incident, evacuations of some facilities were inevitable due to staff deficiencies and/or other resource shortages, but at other sites sheltering in situ might have minimized health risk. Moreover, facility-specific disaster response strategies, such as on-site relief and care, may have a strong influence on survival of elderly people. In a mass displacement disaster, careful planning and coordination with other nursing homes, evacuation sites and government disaster agencies is of paramount importance to reduce the mortality.

\section{Abstract \#: P 274}

\section{Association between oral microflora and oral health} and socio-demographic variables among adolescents

Nélio Veiga ${ }^{1,2,3}$, Carlos Pereira ${ }^{2}$, Carlos Resende ${ }^{4}$, Odete Amaral $^{2}$, Manuela Ferreira $^{2}$, Claudia Chaves ${ }^{2}$, Paula Nelas ${ }^{2}$, João Duarte ${ }^{2}$, José Carlos Machado $^{4}$, Luis Cirnes ${ }^{4}$, Paula Ferreira ${ }^{3}$, Ilidio J Correia ${ }^{5}$

${ }^{1}$ Health Sciences Department - Portuguese Catholic University; ${ }^{2}$ CI\&DETS - Polytechnic Institute of Viseu; ${ }^{3}$ Beira Interior University: CIEPQPF, Chemical Engineering Department University of Coimbra; ${ }^{4}$ Institute of Molecular Pathology and Immunology of the University of Porto; ${ }^{5} \mathrm{Health}$ Sciences Research Centre - Health Sciences Faculty, Beira Interior University; Corresponding author's e-mail: nelioveiga@gmail.com

Background: The oral cavity is inhabited by hundreds of bacterial species that play vital roles in maintaining oral health or in shifting to a diseased state such as dental caries. The aim of the present study was therefore to investigate the prevalence of salivary Streptococcus mutans, Lactobacillus and Aggregatibacter actinomycetemcomitans and associated risk of development of dental caries, and cross the data with socio-demographic aspects among a sample of Portuguese adolescents.

Methods: A epidemiological cross-sectional study was designed including a final sample of 447 adolescents aged between 12 and 19 years old, attending a public school in Sátão, Portugal. A self- administered questionnaire about socio-demographic variables and oral health behaviours was filled out by the adolescents in the classroom. Clinical examination of oral health status was carried out and saliva collection was accomplished by the passive drool method. The identification of the different types of bacterial strains was accomplished using the Polymerase Chain Reaction technique.

Results: The prevalence of Streptoccoccus mutans in the sample studied was $80.8 \%$, Lactobacillus spp. $99.5 \%$ and Aggregatibacter actinomycetemcomitans only $15.2 \%$. The presence of Streptococcus mutans was associated with gender (male $=76.1 \%$ vs female $=83.6 \%, p=0.04)$ and dental pain in the presence of severe dental caries $(77.3 \%$ vs $87.8 \%, p=0.006)$. The presence of Aggregatibacter actinomycetemcomitans was associated with age $(<15 \mathrm{yrs}=12.3 \%$ vs $\geq 15 \mathrm{yrs}=20.3 \%, p=0.03)$ and residence area (rural $=18.2 \%$ vs urban $=11.0 \%, p=0.04$ ), and may be related with a higher risk of periodontal disease development in adulthood.

Conclusions: Aggregatibacter actinomycetemcomitans infection was found to be associated with socio-demographic variables, suggesting that, if not clinically well identified and treated, may cause serious oral diseases during adulthood. Adolescents who suffered one or more episodes of dental pain due to dental caries had a higher incidence of Streptococcus mutans. The presence of oral microflora is clearly one of the main etiological factors for dental caries and periodontal diseases development, but cannot be considered in an isolated manner. The imbalances in the resident microflora may be the ultimate mechanism of dental caries development. Oral diseases can appear in the presence of changes of the oral bacterial communities' structure and that may be related with the shift from health to disease.

\section{Abstract \#: P 275}

Physical activity is inversely associated with deficit accumulation in older adults: results from the KORA-age study

Ralf Strobl ${ }^{1,2}$, Anna-Janina Stephan ${ }^{1}$, Martin Müller ${ }^{1,2}$, Rolf Holle ${ }^{3}$, Barbara Thorand ${ }^{4}$, Birgit Linkohr ${ }^{4}$, Annette Peters ${ }^{4}$, Eva Grill ${ }^{1,2}$

${ }^{1}$ Institute for Medical Information Processing, Biometrics and Epidemiology, Ludwig-Maximilians-Universität München, Munich, Germany; ${ }^{2}$ German Center for Vertigo and Balance Disorders, Ludwig-Maximilians-Universität München, Munich, Germany; ${ }^{3}$ Institute of Health Economics and Health Care Management, Helmholtz Zentrum München, Neuherberg, Germany; ${ }^{4}$ Institute of Epidemiology II, Helmholtz Zentrum München, Neuherberg, Germany; Corresponding author's e-mail: ralf.strobl@med.unimuenchen.de

Background: Aging may be associated with successive accumulation of physiological deficits. Consistent with this idea of increasing loss of physiological resilience, a deficit accumulation index can be constructed that sums up information on deficits including all kinds of signs, symptoms, health conditions and impairments. One of the factors that may play an important role in preventing or delaying deficit accumulation is physical activity (PA). The effect of exercise/ leisure time PA on health of older persons is well investigated: the effect of household PA is less clear. The objective of this study was to examine the association of leisure time and household physical activity with deficit accumulation (DA).

Methods: Data originates from the baseline assessment of the Cooperative Health Research in the Region of Augsburg (KORA)Age study from Southern Germany conducted in 2008/09. The DA index was constructed from 33 potential health- and age-related deficits which covered a broad range of health domains. The DA index ranges from 0 (no deficits) to 1 (all potential deficits present). 
Leisure and household PA was measured with the Physical Activity Scale for the Elderly (PASE). The association of DA and PA was assessed through negative binomial regression analysis.

Results: We analyzed 960 participants (mean age 76 years, $49.0 \%$ female) with a DA index ranging from 0 to 0.64 . Controlling for potential confounders, both leisure and household physical activity showed a significant negative association with deficit accumulation. Point estimates indicated a $16 \%$ (CI: $2-27 \%$ ) to $35 \%$ (CI: 24-45\%) deficit difference for higher versus lower physical activity levels. Differences were higher for household than for leisure physical activity.

Conclusion: Physical activity as a whole and specifically household physical activity is inversely associated with deficit accumulation and might be a promising modifiable protective factor with regard to deficit accumulation. Further research efforts are needed to investigate the temporal sequence of this relationship and to examine whether PA is causally related to DA.

\section{Abstract \#: P 276}

Health investments using European structural founds: the Portuguese experience

$\underline{\text { Andreia Olhero }}^{1,2}$, Sandra Alves ${ }^{1,2,3}$, Fátima Pina ${ }^{2,3,4}$

${ }^{1}$ INEB-Instituto de Engenharia Biomédica, Universidade do Porto, Portugal; ${ }^{2} \mathrm{i} 3 \mathrm{~S}$ - Instituto de Investigação e Inovação em Saúde, Universidade do Porto, Portugal; ${ }^{3}$ Escola Superior de Tecnologia da Saúde do Porto, Instituto Politécnico do Porto (ESTSP/IPP), Vila Nova de Gaia, Portugal; ${ }^{4}$ ICICT/FIOCRUZ - Instituto de Comunicação e Informação Científica e Tecnológica/Fundação Oswaldo Cruz, Rio de Janeiro, Brazil; Corresponding author's e-mail: andreiaolhero@gmail.com

Background: Investment in health is necessary guarantee health care to all. Structural funds have been used in European Union (EU) to support the economic development, minimizing countries and regional disparities. Since 1989 Portugal benefits from regular community financial support (structural funds). The aim of this work is to characterize EU health investments in Portugal (1989-2013).

Methods: Data of health investments supported by structural funds in Portugal were analyzed considering the different investment phases: 1989 to 1993-Quadro Comunitário de Apoio -(QCA) I: 1994 to 1999_QCA II: 2000 to 2006-QCA III: 2007 to 2013-Quadro de Referência Estratégico Nacional-QREN). Data was provided from Financing Institute for the Regional Development and QREN Observatory. For each project we analyzed: executor entity: total investment: reimbursement percentage: approved investment: coverage area of the project. For each municipality the total investment was calculated summing, weighted by the population, the investments from different projects in which the municipality was beneficiated. We also calculated the per capita investment by dividing the total investment by the total population.

Results: In the study period 2550 projects were identified. The absolute investment is 2,095,005,034.51€, where $1,464,599,784.39 €$ $(69.9 \%)$ was supported by structural funds. The remaining $630,405,250.13 €$ were provided from national counterpart. Overall investment per capita was lower in QCAI (mean 9.89, SD 27.1) and higher in QCAIII (mean 121.32, SD 116.4). The last communitarian framework decreased to a value similar to QCAII (in average each municipality received about 50€). QREN had the higher reimbursement percentage $(75 \%)$ and QCAI the lower $(67 \%)$.Considering the projects influence areas $69 \%$ of the funds had a regional impact, $26 \%$ had a local impact and $5 \%$ a national impact. Hospitals executed $33 \%$ of the total investment (considering all programs), followed by the central and regional health authorities (31 and $19 \%$, respectively).

Conclusion: Investment in health increased until QCAIII (1989-2006), during this period reorganization of health policies in Portugal occurred. The investment decrease in QREN may reflect the need to invest in other areas than health. The higher reimbursement percentage in the QREN indicates a lower capacity of executors to provide national counterpart, a consequence of the financial crisis. More than half of the funds were applied at regional level and about a third directly in hospitals potentiating a reduction of regional disparities.

\section{Abstract \#: P 277}

Association between arthritis and physical mobility among Brazilian older adults: findings from the national household sample survey (PNAD)

Clarissa de Matos Nascimento ${ }^{1}$, Juliana Vaz de Melo Mambrini ${ }^{1}$, ${\text { Cesar de } \text { Oliveira }^{2} \text {, Sergio Viana Peixoto }}^{1}$

${ }^{1}$ Centre of Public Health and Ageing Studies. René Rachou Research Centre/CPqRR/FIOCRUZ, Belo Horizonte, Brazil; ${ }^{2}$ Department of Epidemiology and Public Health, University College London/UCL, London, England, United Kingdom; Corresponding author's e-mail: clarissamn@hotmail.com

Background: Few studies have examined the association between arthritis and disability over time among older adults. The aim of this study was to investigate the magnitude of this association among Brazilian older adults over 10 years.

Methods: The data came from the Brazilian National Household Sample Survey (PNAD) of 1998, 2003 and 2008. Information on sociodemographic status, health status (including self-reported arthritis), use of health services and physical mobility were obtained by interview in the residence of participant. 28,943 participants aged 60 years and older were investigated in 1998, 35,042 in 2003 and 41,269 in 2008 . The dependent variable was the physical mobility index (PMI), constructed based on Model Item Response Theory (IRT) using the following items: "normally, because of a health problem, do you have difficulty in": 1 . Running, lifting weight, doing sports or doing heavy work?: 2. Pushing a table or doing a home repair?: 3. Going up a steep hill or stairs?: 4. Stooping or kneeling?: 5. Walking about 100 meters? The score from IRT was categorized into 3 groups. The first group was composed of elderly who had no difficulty in any of the activities considered (namely "no difficulty"). The other two groups were defined based on the median score, excluding the individuals in the first group (namely "moderate difficulty" and "greater difficulty"). The analysis was performed using the multinomial logistic regression, considering the no difficulty group as a reference.

Results: The prevalence of arthritis decreased over the decade studied, being $37.5 \%$ (95\% confidence interval 36.6 to $38.3 \%$ ) in 1998 , $27.3 \%$ (95\% CI 26.6 to $27.9 \%$ ) in 2003 and $24.2 \%$ (95\% CI 23.6 to $24.8 \%$ ) in 2008 . Physical mobility was significantly associated with arthritis, even after adjusting for confounders, but the magnitude of this association increased from 1998 (group 2-OR: 2.1: $95 \% \mathrm{CI}$ : 1.9-2.3 and group 3-OR: 3.5: $95 \%$ CI: 3.3-3.9) to 2008 (group 2OR: 2.5: $95 \%$ CI: 2.3-2.7 and group 3-OR: 4.5: $95 \%$ CI: 4.1-5.0). Conclusion: Despite the decrease in prevalence of arthritis over 10 years, the strength of its association with mobility increased over time. Therefore, it is necessary to control this chronic condition in order to reduce its impact on physical mobility among older adults. 


\section{Abstract \#: P 278}

Prediction of marijuana use among Iranian early youth: an application of integrative model of behavioral prediction

$\underline{\text { Mehdi Mirzaei Alavijeh }}{ }^{1}$, Farzad Jalilian ${ }^{2}$, Behzad Karami Matin ${ }^{2}$

${ }^{1}$ Department of Health Education \& Promotion, Faculty of Medical Sciences, Tarbiat Modares University, Tehran, Iran; ${ }^{2}$ Department of Public Health, School of Health, Kermanshah University of Medical Sciences, Kermanshah, Iran; Corresponding author's e-mail: m.mirzaeialavijeh@modares.ac.ir

Background: Marijuana is the most widely used illicit drug worldwide, especially among adolescents and young adults, which can cause numerous complications. The aim of this study was to determine the pattern, motivation use, and factors related to marijuana use among Iranian youths based on the integrative model of behavioral prediction.

Methods: A cross-sectional study was conducted among 174 youths marijuana user in Kermanshah County and Isfahan County, during summer 2014 which was selected with the convenience sampling for participation in this study. A self-reporting questionnaire was applied for collecting data. Data were analyzed by SPSS version 21 using bivariate correlations and linear regression statistical tests at $95 \%$ significant level.

Results: The mean marijuana use of respondents was 4.60 times at during week (95\% CI: 4.06, 5.15). Linear regression statistical showed, the structures of integrative model of behavioral prediction accounted for $36 \%$ of the variation in the outcome measure of the marijuana use at during week $(\mathrm{R} 2=36 \%$ and $P<0.001)$ : and among them attitude, marijuana refuse, and subjective normswere a stronger predictors.

Conclusion: Comprehensive health education and prevention programs need to emphasize on cognitive factors that predict youth's health-related behaviors. Based on our findings it seems, designing educational and behavioral intervention for reduce positive belief about marijuana, marijuana self-efficacy refuse promotion, and reduce subjective norms encourage marijuana use has effective potential to protect youths marijuana use.

\section{Abstract \#: P 279}

Tracking of antenatal mothers and neonates in rural and remote area by mobile e-health model: an innovative approach

Dr. Abhay Mudey ${ }^{1}$, Dr. Ramchandra Goyal ${ }^{1}$, Dr. Gargi DangreMudey $^{1}$, Dr. Meenakshi Khapre ${ }^{1}$

${ }^{1}$ Jawaharlal Nehru Medical College, Sawangi, Wardha MS; Corresponding author's e-mail: abhaymudey@hotmail.com

Background: India is a vast country with 1.4 billion populations consisting of 29 states and 6 union territories with limited national health insurance policy coverage.

India is "making progress" in declining maternal mortality, but not "on track" to meeting its goal under the U.N. Millennium Development Goals. There is great deal of disparity in quality and access to health care between rural and urban.

As India has the good network of mobile connectivity, low usage cost and high acceptability and availability of mobiles in community, it could be used for providing basic health services to rural and remote people.

With aim to study effectiveness of mobile phones in improving maternal and neonatal health and seeks to innovate measures for ensuring access to skilled attendance at delivery through an intervention called 'Mobile-e-health' this study was conducted with an initial focus on antenatal care and neonate.

Methods: This is an experimental non-randomized study comparing the effectiveness of mobile e-health in maternal and neonatal health with control group, receiving routine care. Mothers registered for ANC between Jan-Jun 2010 willing to use mobile phone and motivated to complete follow up visit till 28 days postpartum period and neonates born to mothers recruited for study. Total 178 and 206 mothers were included in interventional and control group respectively.

Results: There is significantly improvement (12\%) increase in $\geq 4$ ANC visits and $(9 \%)$ increase in early registration before 12 weeks in interventional group. Most of high risk cases i.e. hypertension, diabetes were detected at least 4-7 weeks earlier than control group. In interventional group significant increase in mean weight and hemoglobin was observed in third trimester.

Institutional delivery has increased and prevalence of LBW babies had decreased by $8 \% .92 \%$ of neonates in interventional group was immunized with BCG and OPV within 7 days compared to $73 \%$ in control group.

Conclusions: Thus overall the intervention was found to be effective and acceptable. Result can be generalized to rural India where there is good network connectivity and mothers are willing to use the mobile.

It is not only due to communication system, that is mobile technology but also motivation, monitoring and supervision had played role in improving the maternal and neonatal health services.

\section{Abstract \#: P 280}

Prevalence and correlates of sitting in the $28 \mathrm{EU}$ member states: a Eurobarometer study

Anne Loyen $^{1}$, Hidde van der Ploeg ${ }^{2}$, Jeroen Lakerveld ${ }^{1}$, Adrian Bauman ${ }^{3}$, Johannes Brug ${ }^{1}$

${ }^{1}$ Department of Epidemiology and Biostatistics, EMGO Institute for Health and Care Research, Amsterdam, the Netherlands; ${ }^{2}$ Department of Public and Occupational Health, EMGO Institute for Health and Care Research, Amsterdam, the Netherlands; ${ }^{3}$ Sydney Medical School, University of Sydney, Sydney, Australia; Corresponding author's e-mail: a.loyen@vumc.nl

Background: Sedentary behaviour is increasingly recognized as an important health risk, but comparable sitting data across European countries are scarce. This study explores the prevalence and correlates of self-reported sitting in the 28 European Union (EU) member states. Methods: 919 Europeans (approximately 1000 per EU member state) were interviewed face-to-face. Sitting time on a usual day was assessed using the IPAQ question and dichotomised into sitting lessand more than $7.5 \mathrm{~h}$ per day. Uni- and multi-variate odds of sitting more than $7.5 \mathrm{~h}$ per day were assessed by country, gender, age, marital status, age when stopped full-time education, occupation, type of community, number of children in the household, television-, computer-, and car ownership, internet use frequency, difficulties paying bills and life satisfaction using logistic regression.

Results: 18.5 percent of European adults reported sitting $7.5 \mathrm{~h}$ or more per day, ranging from 32.1 percent in the Netherlands to 8.9 percent in Spain. The odds of sitting more than $7.5 \mathrm{~h}$ per day were highest in the Netherlands (OR 1.74 (95 \% CI 1.42-2.14) and Denmark (1.71 (1.38-2.10) and lowest in Portugal (0.44 (0.33-0.57) and Italy $(0.42(0.32-0.55)$ in the multivariate model. In addition, the multivariate model showed that women, people living in rural areas, people with three or more children and car owners had significant lower odds of sitting, while several occupation categories (self-employed, manager, white collar, unemployed, retired and student), daily 
internet use frequency and being fairly, not very or not at all satisfied with life showed significant higher odds.

Conclusion: There is great variation in the prevalence of sedentary behaviour in Europe, both across countries and sociodemographic groups. These results provide a starting point for additional research and intervention development, especially aimed at high-sitting populations.

\section{Abstract \#: P 281}

Number of restaurants and obesity among Brazilian adults: a multilevel analysis

Larissa Loures Mendes ${ }^{1}$, Fernanda Penido Matozinhos ${ }^{2}$, Crizian Saar Gomes $^{2}$, Milene Cristine Pessoa ${ }^{3}$, Gustavo Velasquez-Melendez ${ }^{2}$

${ }^{1}$ Universidade Federal de Juiz de Fora, Juiz de Fora, MG, Brazil; ${ }^{2}$ Universidade Federal de Minas Gerais, Department of Maternal and Child Nursing and Public Health, Belo Horizonte, MG, Brazil; ${ }^{3}$ Universidade Federal de Viçosa, Viçosa, MG, Brazil; Corresponding author's e-mail: nandapenido@hotmail.com

Background: Obesity is a global problem of epidemic proportions and has significant health consequences, especially in the development and progression of many chronic diseases. The objective of this study is to estimate the association of the number of restaurants and individual factors with obesity in adults in a Brazilian urban context. Methods: A cross-sectional study was conducted including adults, aged 18 years and older, who were participants in the Surveillance System for Risk and Protective Factors for Chronic Diseases by Telephone Survey (VIGITEL) from 2008 to 2010 in the city of Belo Horizonte, Minas Gerais. Obesity was defined as body mass index (BMI) $>=30 \mathrm{~kg} / \mathrm{m}^{2}$. Individual-level data included sociodemographic and lifestyle habits (eating patterns and physical activity). Each participant's residence geographic coordinates were obtained on their zip code. Georeferenced data were used for identification the number of restaurants of the coverage areas (CAs) of the Basic Health Units (BHUs). The CABHUs were used as neighbourhood units. The STATA statistical package (svy function) was used for the statistical analyses. A fixed-effects multilevel logistic regression with random intercept was applied. Estimates of the odds ratio (OR) and $95 \%$ confidence interval $(95 \% \mathrm{CI})$ were used. For all analyses, a $5 \%$ significance level was defined.

Results: This study included 5273 individuals, with an average age of 43.67 years $(\mathrm{SD}=16.65)$. A total of $43.51 \%$ of participants were male, and $40.24 \%$ had 9-11 years of education. The prevalence of obesity was $12.13 \%$ (95\% CI: 11.20-13.12). For every increase in the number of restaurants $(\mathrm{OR}=0.97,95 \% \mathrm{CI}: 0.96-0.99)$, the likelihood of obesity significantly decreases. This association remained statistically significant after controlled by age, sex, education and consumption of meat with visible fat.

Conclusion: The results showed that the food environment of the neighbourhood may influence the prevalence of obesity among adults. These data corroborate previous studies of urban settings in developed countries. Thus, addressing the obesity problem will require the development of effective intervention strategies and the expansion of programs that address aspects of the physical environment.

\section{Abstract \#: P 282}

\section{Oral and gastric helicobacter pylori: effects and associations}

Nélio Veiga $^{1,2,3}$, Carlos Pereira ${ }^{2}$, Carlos Resende $^{4}$, Odete Amaral $^{2}$, Manuela Ferreira $^{2}$, Claudia Chaves ${ }^{2}$, Paula Nelas ${ }^{2}$, João Duarte ${ }^{2}$, José Carlos Machado ${ }^{4}$, Luis Cirnes ${ }^{4}$, Paula Ferreira ${ }^{3}$, Ilidio J Correia ${ }^{5}$

${ }^{1}$ Health Sciences Department - Portuguese Catholic University; ${ }^{2}$ CI\&DETS - Polytechnic Institute of Viseu; ${ }^{3}$ Beira Interior University: CIEPQPF - Chemistry Engeneering Department, University of Coimbra; ${ }^{4}$ Institute of Molecular Pathology and Immunology of the University of Porto; ${ }^{5}$ Health Sciences Research Centre - Health Sciences Faculty, Beira Interior University; Corresponding author's e-mail: nelioveiga@gmail.com

Background: This study consisted in the comparison of the prevalence of Helicobacter pylori (H. pylori) present in the stomach and in saliva in a cohort of Portuguese adolescents and determining the association between $\mathrm{H}$. pylori infection with socio-demographic variables and prevalence of dental caries.

Methods: A cross-sectional study was designed including a sampling cohort of 447 adolescents aged 12-19 years old, attending a public school in Sátão, Portugal. A questionnaire about socio-demographic variables and oral health behaviors was applied. Gastric H. pylori infection was determined using the urease breath test (UBT). Saliva collection was obtained and DNA was extracted by Polymerase Chain Reaction (PCR) in order to detect the presence of oral H. pylori.

Results: The prevalence of gastric H. pylori detected by UBT was $35.9 \%$. Within the adolescents with a gastric UBT positive, only $1.9 \%$ were positive for oral $\mathrm{H}$. pylori. The presence of gastric $\mathrm{H}$. pylori was found to be associated with age $(>15$ years, Odds ratio $(\mathrm{OR})=1.64, \quad 95 \% \quad \mathrm{CI}=1.08-2.52$ ), residence area (urban, $\mathrm{OR}=1.48,95 \% \mathrm{CI}=1.03-2.29)$ and parentś professional situation (unemployed, $\mathrm{OR}=1.22,95 \% \mathrm{CI}=1.02-1.23$ ). Among those with detected dental caries during the intra-oral observation, $37.4 \%$ were positive for gastric H. pylori and $40.2 \%$ negative for the same bacterial strain $(p=0.3)$.

Conclusions: The prevalence of oral H. pylori is an infrequent event, even among individuals with a positive UBT, revealing that the oral cavity cannot be considered a reservoir for infection of $\mathrm{H}$. pylori. Gastric H. pylori infection was found to be associated with sociodemographic variables such as age, residence area and socioeconomic status.

\section{Abstract \#: P 283}

\section{Inequalities in maternal and child health in Latin America and the Caribbean: an unfinished agenda}

María C. Restrepo-Méndez ${ }^{1}$, Giovanny V.A. França ${ }^{1}$, Fernando Wehrmeister $^{1}$, Aluisio J.D. Barros ${ }^{1}$, Cesar G. Victora ${ }^{1}$

${ }^{1}$ International Center for Equity in Health, Federal University of Pelotas, Pelotas, Brazil; Corresponding author's e-mail: mcrestrepo@equidade.org

Background: We assessed socioeconomic inequalities in reproductive, maternal, newborn and child health (RMNCH) and time trends in inequalities in Latin America and The Caribbean (LAC) region. 
Methods: We used data from Demographic and Health Surveys and Multiple Indicator Cluster Surveys. Thirteen countries were selected because they had at least one survey available at the time of analyses, including information on wealth. Our outcome indicators included coverage of 12 preventive and curative interventions along the RMNCH continuum of care. We also assessed under-five mortality rate and stunting in children under-five years of age. These indicators were disaggregated by quintiles of household wealth. We also calculated two summary measures of inequalities: slope index of inequality and concentration index.

Results: When the most recent surveys from each country were analyzed, their average values on intervention coverage showed monotonic patterns over wealth quintiles, with coverage lowest among the poorest quintile and increasing for each quintile. Important inequalities persist for family planning satisfied, antenatal care and skilled birth attendant (SBA). SBA was the most inequitable intervention as it was virtually universal in the wealthiest quintile, but available to only about $60 \%$ of women in the poorest quintile. Some interventions, such as vaccines, were quite equitable at high coverage levels for all quintiles. Others also seem to be relatively equitable (insecticide treated nets, vitamin $\mathrm{A}$, oral rehydration therapy and pneumonia careseeking) but coverage was low. Early initiation of breastfeeding was more common among the poor than among the rich. Except for Guyana, under-five mortality was highest in the poorest quintile. Similar patterns were observed for stunting prevalence. Guatemala, Bolivia, Peru, Haiti and Nicaragua showed large inequalities in both impact indicators. Brazil has almost eliminated inequalities in stunting, and Suriname, Colombia and the Dominican Republic showed small absolute inequalities compared to other countries. In five countries with four surveys over time, we studied trends in coverage, mortality and stunting. In general, all five countries tended to increase intervention coverage among the poor and to reduce absolute inequalities in coverage over time. Progress was also observed for mortality and stunting, but these inequalities varied from country to country.

Conclusion: LAC is regarded as a region with substantial progress in terms of RMNCH and nutrition. However, in most countries there are still subgroups of the population-particularly the poorest $20 \%$ of mothers and children - that are lagging behind, and that will require special attention if further gains are to be made.

\section{Abstract \#: P 284}

\section{Applying intervention mapping as a framework for developing an intervention aiming to prevent maternal distress among Dutch pregnant women}

Evelien B.M. van Limbeek $^{1}$, Yvonne Fontein-Kuijpers ${ }^{1}$, Marianne Nieuwenhuijze $^{1}$, Marlein Ausems ${ }^{1}$

${ }^{1}$ Department of Midwifery Science, Zuyd University, Maastricht, The Netherlands; Corresponding author's e-mail: e.vanlimbeek@av-m.nl

Background: Maternal distress (MD) is expressed as one of the major concerns for perinatal health. It encompasses different constructs such as anxiety, depression, and stress and can manifest during pregnancy, either caused by pregnancy or non-pregnancy related reasons. It varies in severity from women with low MD experiencing stress, worry and concern to women with high MD that feel unhappy, anxious and depressed. MD can have extensive adverse consequences for women and their families, including obstetric complications, severe maternal mental health problems and affects child neurobehavioral and cognitive development. In 2012 the prevalence of MD among Dutch pregnant women with uncomplicated pregnancies was $39.1 \%$. Since barely any effective interventions are available midwifery care is assisted with the development of evidence-based interventions that focus on screening and support of pregnant women experiencing maternal distress.

Methods: An intervention for the prevention of MD among pregnant women in midwife-led care was systematically developed by use of the Intervention Mapping (IM) approach. IM is a stepwise approach for theory and evidence-based program development, implementation and evaluation. It guides program planners in making balanced decisions by combining theoretical evidence with practical information. A planning group of stakeholders (e.g. pregnant women, midwives, psychologists) was involved in the six steps of IM: needs assessment, program objectives, behavioral change methods and applications, intervention design, implementation and evaluation. The presentation will describe the content of every step accompanied by a description of how that step was executed in the development of the 'Wazzup Mama' intervention and how existing and newly acquired evidence was used to underpin the development.

Results: The 'Wazzup Mama' intervention consists of one part for pregnant women aimed at prevention or reduction of MD using a web-based computer-tailored program. The program part for midwives aims to provide a format for the implementation and coordination of care for MD and to support self-disclosure and selfmanagement and consists of a toolkit for practice, consisting of several components such as a guideline including a clinical pathway for consultation and referral and a regional health care map focusing at psychological and emotional wellbeing.

Conclusion: IM was found to be a useful tool for systematically developing an intervention for preventing maternal distress, with stakeholder involvement and may lead to more effective interventions and lesser implementation problems. Moreover, it also gives insight in the current theoretical and empirical knowledge regarding maternal distress.

\section{Abstract \#: P 285}

Impact evaluation of interventions aiming to promote healthy living in youths in canada: a critical review of published studies

Philippe De Wals $^{1}$, Judith Lapierre ${ }^{2}$, Nathalie Dumas ${ }^{3}$, Maude Dionne $^{3}$, Jasmine Prevost ${ }^{3}$, Michael Gandonou ${ }^{3}$

${ }^{1}$ Departement of Social and Preventive Medicine, Laval University, Quebec City, Canada; ${ }^{2}$ Faculty of Nursing, Laval University, Quebec City, Canada; ${ }^{3}$ Heart and Lung Institute Research Centre, Quebec City, Canada; Corresponding author's e-mail: philippe.dewals@criucpq.ulaval.ca

Background: In Canada, numerous interventions have been implemented in recent years to promote healthy lifestyles and counter the rise in obesity and related chronic diseases. A majority of interventions are targeting youths and are involving considerable human and financial resources. A systematic and rigorous valuation of their effects is important for public health authorities and the research community.

Objectives: To identify and characterize published studies involving measurement of effects at individual level.

Methods: A systematic search in seven electronic databases was performed in peer-reviewed journals in 2000-2013, using inclusion criteria: (i) population-based intervention focusing on primary prevention, (ii) targeting individuals less than 18 years of age (iv), with measures of effects at individual level, (v) including changes in psychological, behavioral, physiological or biometric parameters. Interventions were classified according to 16 characteristics pertaining to the nature of the intervention, and 22 pertaining to the nature of the evaluation. 
Results: Out of 28,044 citations screened, 22 were retained. Three additional studies were found following selective search by authors, and 3 others following a review of references, for a total of 28 studies pertaining to 22 interventions. Most interventions (19/22) were implemented in a school setting. Two were of policy nature, and the others consisted of a promotion program or activity. The target was nutrition in 10 , physical activity in 5 , and these two behaviors in 7 cases.

There was an increasing trend in publication frequency: 1.0 publication per year in 2000-2004, 2.3 in 2005-2008 and 3.8 in 2009-2013. All but one studies followed the classical cause-effect paradigm, and one was based on a logic 'hierarchy of effects' model. One study was experimental, 8 were cluster-randomized trials and 19 quasi-experimentations with concurrent, historic or internal controls. Most evaluations were short-term, and relying on self-reported indicators. Overall, documented impact results were small.

Interpretation: For valid evaluations, conditions have to be met: clear and realistic objectives, early planning of the evaluation, collaboration of all stakeholders with an experienced academic team, allocation of necessary resources, sound design and validated measurement tools, as well as an assessment of the level of implementation of the intervention. Two major obstacles are remaining: the magnitude of expected effects is usually low, requiring large sample sizes, and the presence of multiple interventions complicating the assessment of causality. More emphasis should be given to progress measurement at societal level integrating the combined effect of concomitant interventions, using population-surveys and administrative databases.

\section{Abstract \#: P 286}

\section{Impact of mandatory seat restraining on child deaths in car accidents in Brazil}

Leila Posenato Garcia ${ }^{1}$, Lucia Rolim Santana de Freitas ${ }^{2}$, Elisabeth Carmen Duarte ${ }^{2}$

${ }^{1}$ Institute of Applied Economic Research; ${ }^{2}$ University of Brasilia; Corresponding author's e-mail: leilapgarcia@gmail.com

Background: In Brazil, on September 1st, 2010, the use of restraining devices became mandatory for transportation of children in Objective: To assess the impact of mandatory child restraint on the occurrence of deaths of children under 10-years-old caused by car accidents in Brazil.

Methods: A time-series study with before-and-after design was conducted, considering the period between September 1st, 2005 and August 31st, 2012. Information on deaths was obtained from the National Mortality Information System. Mortality ratios were estimated per 1,000,000 vehicles. The monthly numbers of licensed vehicles in the Country were obtained from the National Department of Transportation. The Box-Jenkins method was used to adjust the time series model. The Seasonal Autoregressive Integrated Moving Average (SARIMA) was used to model the monthly mortality ratios. The intervention analysis was performed using the Box and Tiao method, comparing the behavior of the time series before and after the intervention.

Results: During the study period, there were registered 1845 deaths of children under ten-years-old in car accidents in Brazil. Within the 5 years before the mandatory restraining the average annual number of deaths was 267.8, while within the 2 years after it was 253.0 $(-5.5 \%)$. The average annual death ratios per $1,000,000$ vehicles before and after the enactment of the law were, respectively, 0.73 and 0.54. One-third of deaths $(33.5 \%)$ were concentrated during the school vacation period (December, January and July). The SARIMA parameter estimate for the montlhy mortality ratios was fitted $(p=0.0015)$.

Conclusion: There was a statistically significant reduction in the mortality of children under 10-years-old in car accidents in Brazil, 2 years after the enactment of the "Child Car Seat Lawâ".

\section{Abstract \#: P 287}

Incidence and costs of bicycle-related traumatic brain injuries in the Netherlands

Annemieke C. Scholten ${ }^{1}$, Suzanne Polinder ${ }^{1}$, Martien J.M. Panneman $^{2}$, Ed. F. van Beek ${ }^{1}$, Juanita A. Haagsma ${ }^{1}$

${ }^{1}$ Department of Public Health, Erasmus University Medical Centre, Rotterdam, The Netherlands; ${ }^{2}$ Research Department, Consumer and Safety Institute, Amsterdam, The Netherlands; Corresponding author's e-mail: a.scholten@erasmusmc.nl

Background: Cycling is a popular form of transport and recreation worldwide, and especially in the Netherlands. Despite the high levels of road safety, in the Netherlands approximately 350,000 injuries occur due to cycling each year. The main cause of death and serious disability in bicycle accidents is traumatic brain injury (TBI). The aim of this population-based study was to assess the incidence and costs of bicycle-related TBI across various age groups, and in comparison to all bicycle-related injuries, to identify main risk groups for the development of preventive strategies.

Methods: Data from the National Injury Surveillance System and National Medical Registration were used for all patients with bicyclerelated injuries and TBI who visited a Dutch emergency department (ED) between 1998 and 2012. Demographics and national, weighted estimates of injury mechanism, injury severity and costs were analysed per age group. Direct healthcare costs and indirect costs were determined using the incidence-based Dutch Burden of Injury Model. Results: Between 1998 and 2012, the incidence rate of ED treatments due to bicycle-related TBI strongly increased with $54 \%$, to 43 per 100,000 persons in 2012. However, the incidence rate of all bicyclerelated injuries remained stable, from 444 in 1998 to 456 per 100,000 in 2012. The incidence rate of hospital admissions increased in both TBI $(92 \%)$ and all injuries from cycling $(71 \%)$. Highest increase in incidence rate of both ED treatments and hospital admissions was seen in adults aged 55+. Children and adolescents (aged 0-24) had highest incidence rate of ED treatments due to bicycle-related injuries. Total costs of bicycle-related TBI were $€ 74.5$ million annually. Although bicycle-related TBI accounted for $9 \%$ of the incidence rate of all ED treatments due to cycling, it accounted for $20 \%$ of the total costs due to all bicycle-related injuries ( $€ 410.7$ million). Men in the working population (age 15-64) had highest indirect costs following injuries from cycling, including TBI. Older cyclists (aged 55+) were identified as main risk group for TBI, as they had highest ED attendance, injury severity, admission to hospital or intensive care unit, and costs.

Conclusion: The incidence rate of ED treatments due to cycling are high and often involve TBI, imposing a high burden on individuals and society. Older cyclists aged $55+$ were identified as main risk group for TBI to be targeted in preventive strategies, due to their high risk for (serious) injuries and ever-increasing share of ED visits and hospital admissions. 
Abstract \#: P 288

Maternal education and the use of health care services and medication in coeliac children: a population-based birth cohort study

Cristina Canova ${ }^{1}$, Gisella Pietter ${ }^{1}$, Lorenzo Simonato ${ }^{1}$, $\overline{R e n z o}_{\text {Zanotti }}{ }^{1}$, Loris Zanier ${ }^{2}$, Jonas F. Ludvigsson ${ }^{3}$

${ }^{1}$ Department Molecular Medicine, University of Padova, Italy; ${ }^{2}$ Epidemiological Service, Health Directorate, Friuli Venezia-Giulia Region, Udine, Italy; ${ }^{3}$ Department of Medical Epidemiology and Biostatistics, Karolinska Institutet, Stockholm, Sweden; Corresponding author's e-mail: cristina.canova@unipd.it

Background: Celiac disease (CD) has been linked to several diseases as well as to impaired quality of life, particularly in adults. Little is however known about patients' use of healthcare services and medication and its association with socio-economic factors. We used a population-based cohort design to estimate healthcare use in $\mathrm{CD}$ children compared to matched references, and its variation across maternal educational level.

Methods: We examined health data in children born and registered in the regional Medical Birth Register of Northeast Italy between 1989 and 2012. CD incident cases were identified through pathology reports, hospital discharges and exemption from prescription charges for clinical tests. Each coeliac child was matched with five references through the Medical Birth Register by sex and year of birth. We retrieved data on first hospital discharges by cause of disease according to ICD9-CM classification and drug prescriptions according to first level of Anatomical Therapeutic Chemical (ATC) Classification after date of diagnosis (index date in references). Follow-up started 30 days after the index date and ended at the date of death, emigration, or date of first hospitalization or drug prescription. Cox regression models were used to estimate the Hazard Ratios (HRs) for health care use in patients with CD compared to reference individuals within the same stratum. Analyses were also stratified by maternal education (up to middle school: secondary school: university level).

Results: We identified 1294 children with CD (920 (71\%) histopathologically confirmed) and 6470 references. CD children were at increased risk of hospital admission for any cause (HR: 2.25: $95 \%$ CI: 2.01-2.50) and for all major groups of causes except complications of pregnancy and puerperium, diseases of skin, muscoloskeletal tissue, and injuries and poisoning. A significant increase of prescriptions was also detected in CD children for all drug categories except dermatologicals $(\mathrm{ATC}=\mathrm{D})$ and genito-urinary medications $(\mathrm{ATC}=\mathrm{G})$. For most diseases and drug categories, the risks were stronger in the first year after $\mathrm{CD}$ diagnosis but remained significant also afterwards. Risks of hospitalization and consumption of systemic hormonal $($ ATC $=\mathrm{H})$, anti-infectives $($ ATC $=\mathrm{J})$, nervous system $($ ATC $=N)$, antiparasitic, insecticides and repellents $(\mathrm{ATC}=\mathrm{P})$ and respiratory $(\mathrm{ATC}=\mathrm{R})$ medicines were not observed among $\mathrm{CD}$ children born to graduated mother but only to less educated mothers.

Conclusion: Children with diagnosed CD seem to be at increased risk of hospitalization and medication use compared to the general child population, also 1 year after diagnosis: however, for most outcomes, risks were only observed in children born to less educated mothers. A poorer educational and socio-economic condition may adversely affect health of celiac children, possibly because of a poorer adherence to gluten-free diet.
Abstract \#: P 289

Custom made outcome assessment in the randomized evaluation of secondary prevention by outpatient nurse specialists 2 trial (response 2)

Sangeeta Lachman $^{1}$, Madelon Minneboo ${ }^{1}$, Marjolein Snaterse ${ }^{2}$, Harald T. Jorstad ${ }^{1}$, Gerben ter Riet ${ }^{2}$, Wilma J. Scholte Op Reimer ${ }^{2}$, Matthijs Boekholdt ${ }^{1}$, Ron J.G. Peters ${ }^{1}$

${ }^{1}$ Department of Cardiology, Academic Medical Center, Amsterdam, The Netherlands; ${ }^{2}$ Amsterdam University of Applied Sciences, School of Health Professions, The Netherlands; Corresponding author's e-mail: s.lachman@amc.uva.nl

Background: Patients with coronary artery disease (CAD) are at high risk of recurrent events. Therefore, current guidelines also recommend lifestyle targets. The Randomized Evaluation of Secondary Prevention by Outpatient Nurse SpEcialists (RESPONSE) trial in 754 patients demonstrated that nurse-led outpatient clinics improve drug treatment for cardiovascular risk factors. Yet, lifestyle-related risk factors including smoking, overweight and physical inactivity, were common and remained largely unchanged at follow-up in the majority of the patients $(66 \%)$. Objectives: The aim of the current study is to evaluate the impact of three community-based lifestyle interventions in patients who have been hospitalized for CAD.

Methods: A multicenter randomized trial to test the efficacy of referral of patients $(n=1000)$ and their partners to a comprehensive lifestyle program consisting of up to three widely available commercial lifestyle interventions, on top of usual care, compared to usual care alone. The community- based programs are aimed at smoking cessation (Luchtsignaal ${ }^{\circledR}$ ), weight loss (Weight Watchers ${ }^{\circledR}$ ) and improving physical fitness (Philips Directlife ${ }^{\circledR}$ ).

Expected results: The current study started in April 2013 and is still ongoing. The study results are expected by the fall of 2016. Outcome variables at 12 months are abstinence of smoking as confirmed by a urinary cotinine test, reduction in body mass index ( $\geq 5 \%$ weight reduction), and improvement of physical activity as measured by a 6-minute walk test (increase of $>10 \%$ in walking distance). The primary outcome will be assessed at patient level. The following 7 patient categories are distinguishable according to lifestyle risk factors 1)smoking only, 2)increased BMI only, 3)physical inactivity only, 4)smoking plus increased BMI, 5)smoking plus physical inactivity, 6)increased BMI plus physical inactivity and 7) all the three risk factors. Treatment success is defined per patient as achieving the target for at least one of the three lifestyle-related risk factors, without deterioration in any of the other. In case of non-smoking status an increase of $\leq 2.5 \%$ of BMI or BMI $\leq 25 \mathrm{~kg} / \mathrm{m}^{2}$ is considered as success. And in case of improved walking distance an increase of $\leq 2.5 \%$ of BMI or BMI $\leq 25 \mathrm{~kg} / \mathrm{m}^{2}$ is considered as success as well. A relative increase of at least $30 \%$ in treatment success in the proportion of patients who achieve at least one improvement in lifestyle-related risk factors, compared to usual care alone, is considered clinically relevant.

\section{Abstract \#: P 290}

The physical exercise following esophageal cancer treatment (perfect) study: rationale and study design

Jonna K. van Vulpen ${ }^{1}$, Peter D. Siersema ${ }^{2}$, Richard van Hillegersberg $^{3}$, Petra H.M. Peeters ${ }^{1}$, Anne M. May ${ }^{1}$ 
${ }^{1}$ Julius Center for Health Sciences and Primary Care, University Medical Center Utrecht, Utrecht, The Netherlands; ${ }^{2}$ Department of Gastroenterology and Hepatology, University Medical Center Utrecht, Utrecht, The Netherlands; ${ }^{3}$ Department of Surgery, University Medical Center Utrecht, Utrecht, The Netherlands; Corresponding author's e-mail: j.k.vanvulpen-2@umcutrecht.nl

Background: For several years esophageal cancer has been one of the malignancies with the fastest rising incidence rates in the Western world. Esophageal cancer surgery is an extensive procedure with high risk of post-operative complications and persisting morbidity. These patients are often fatigued and have limited health-related quality of life and physical functioning in the short- and long-term. Therefore, interventions that potentially improve physical functioning and health-related quality of life are needed. Exercise training interventions have shown to be effective in patients with various other types of cancer. Evidence for patients with esophageal cancer is lacking. Therefore, we designed the Physical ExeRcise Following Esophageal Cancer Treatment (PERFECT) Study to investigate effects of a 12-week combined supervised endurance and resistance training program on health-related quality of life (primary outcome) and physical fitness, fatigue, functional wellbeing and symptoms, anxiety and depression, diet, sleep quality, body composition, work ability and blood markers (secondary outcomes). In addition, we will explore the effect of the exercise program on disease recurrence and survival. Patients will be included shortly after curatively intended esophageal cancer surgery.

Methods: PERFECT is a multicenter randomized controlled trial in 150 patients with esophageal cancer who have undergone surgery with curative intent. Recruitment will start in February 2015. Patients will be randomized to either the exercise intervention $(n=75)$ or to usual care $(n=75)$. Health-related quality of life will be assessed at baseline, directly after the intervention (12-weeks post-baseline) and 24-weeks post-baseline by means of the EORTC QLQ-C30 questionnaire. Physical fitness and muscle strength will be assessed at baseline and 12-weeks post-baseline using maximal cardiopulmonary exercise testing for cardiorespiratory fitness and a microFET ${ }^{\circledR}$ hand held dynamometer, respectively. Also blood samples will be taken. Effectiveness of the exercise program will be determined by analyzing changes between groups from baseline to 12 and 24 weeks post-baseline using mixed linear regression models. Physical fitness and blood outcomes will be analyzed using ANCOVA.

Clinical implications: Positive outcome of the exercise program may lead to implementation in usual care for esophageal cancer patients after surgery and improve long-term quality of life in these patients.

\section{Abstract \#: P 291}

\section{Survival study in kidney transplant patients: cancer,} cardiovascular events and peripheral arterial disease

Teresa Seoane-Pillado ${ }^{1}$, Salvador Pita-Fernandez ${ }^{1}$, Francisco ValdesCañedo $^{2}$, Sonia Pertega-Diaz ${ }^{1}$, Rocio Seijo-Bestilleiro ${ }^{1}$, Beatriz Lopez-Calviño ${ }^{1}$, Constantino Fernandez-Rivera ${ }^{2}$

${ }^{1}$ Clinical Epidemiology and Biostatistics Unit. Instituto de Investigación Biomédica de A Coruña (INIBIC), Complexo Hospitalario Universitario de A Coruña (CHUAC), SERGAS, Universidade da Coruña; ${ }^{2}$ Department of Nephrology. Instituto de Investigación Biomédica de A Coruña (INIBIC), Complexo Hospitalario Universitario de A Coruña (CHUAC), SERGAS, Universidade da Coruña; Corresponding author's e-mail: maria.teresa.seoane.pillado@sergas.es

To determine, in kidney transplant recipients:
- Patient and renal graft survival and associated factors

- Event-free survival (cancer and cardiovascular events)

- Prevalence of peripheral arterial disease and associated cardiovascular risk factors.

The study will include all the kidney transplants performed at the Nephrology Department of A Coruña Hospital (Spain) during 1981-2013. An observational prospective follow-up study with a retrospective component. 2594 kidney transplants were performed, corresponding to 2281 patients. The study has received written approval from the region's Ethics Committee for Clinical Research.

Patients who had received transplants are identified through the hospital's transplant registry. Information includes donor and recipient characteristics, patient and graft survival and cancer incidence after transplantation. The follow-up period for each patient starts on the day of transplantation and continues until death or last reported contact.

The sample size will make it possible to detect as significant, in a Cox regression model, a relative risk $\geq 1.3$ associated with a prevalence of exposure to a risk factor of $50 \%$ and a censored data percentage of $80 \%$ (Security: $95 \%$ : Statistical power: $80 \%$ ).

Incident cancer is considered as new cases which have anatomopathological confirmation (Classification of Diseases-9th revision). Post-transplant cardiovascular events are defined as the presence of myocardial infarction, invasive coronary artery therapy, cerebral vascular events, new-onset angina, congestive heart failure, rhythm disturbances and peripheral vascular disease. The presence of peripheral arterial disease will be determined using ankle brachial index measured using Doppler techniques.

Competing risk survival analysis methods will be applied to estimate the cumulative incidence of developing events. The regression model of Fine and Gray will be used for multiple regression analyses. Nomograms to estimate the prognosis after kidney transplantation will be developed, predictive performance will be evaluated (discrimination and calibration values). A bootstrap resampling approach with 1000 replications will be used to reduce overfit bias. Discrimination will be quantified with the concordance C index. The nomogram will be then calibrated graphically. A Loess curve will be fit to the data and compared with an ideal fit. Additionally, the Brier score will be calculated to measure the predictive accuracy.

Generalized Additive Models (GAM) will be explored to identify factors associated to the incidence of events and the prevalence of peripheral arterial disease. Agreement between clinical symptoms and peripheral arterial disease will be analyzed with the Bland-Altman method. Validity indexes will be calculated. ROC curve will be calculated for assessing the performance of the diagnostic test.

\section{Abstract \#: P 292}

\section{Different insulin types and regimens for pregnant women} with pre-existing diabetes

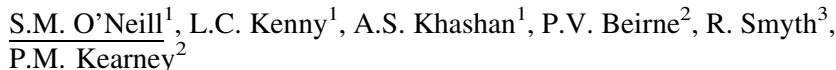

${ }^{1}$ INFANT, Cork, Ireland; ${ }^{2} \mathrm{UCC}$, Cork, Ireland; ${ }^{3} \mathrm{MU}$, Manchester, UK; Corresponding author's e-mail: sinead.oneill@ucc.i.e

Background: Diabetes Mellitus (DM) is on the rise globally and the number of adults with diabetes has more than doubled over nearly three decades driven by both population growth and ageing. Recent figures from Canada report that in $19960.7 \%$ of pregnant women had pre-existing DM, which doubled to $1.5 \%$ of pregnant women with pre-existing DM in 2010. Similar increasing trends of DM in pregnant women have been reported in the United States and the United 
Kingdom. Furthermore, DM is the most common pre-existing medical condition complicating pregnancy and the outcome for diabetic pregnancy remains poor despite improvements in care. Pre-existing DM complicates approximately $1.3 \%$ of pregnancies, with one third being type $1 \mathrm{DM}$ and two thirds type $2 \mathrm{DM}$.

Methods: To conduct a full Cochrane review.

Objectives: To assess the effects of different insulin types and regimens in pregnant women with pre-existing diabetes.

Types of studies: Randomised controlled trials irrespective of language or publication status.

Types of participants: Women with a singleton pregnancy who have pre-existing diabetes and randomised to receive different insulin types and/or regimens.

Types of interventions: We will include randomised controlled trials making any of the following comparisons:

1) Comparisons between similar insulin regimens with different insulin types used within the regimens

2) Comparisons between different insulin regimens with similar insulin types used within the regimens

3) Comparisons between different insulin regimens and different insulin types used within the regimens.

Outcomes: Infant-Macrosomia (birth weight greater than $4000 \mathrm{~g}$ and birth weight greater than $4500 \mathrm{~g})$ : Maternal-(Glycaemic control (HbA1C, blood glucose control).

Several systematic searches will be conducted and the include RCTs will be assessed for risk of bias and heterogeneity. Results will be pooled where applicable to perform a meta-analysis.

Conclusion: Diabetes is on the increase and women with pregnancies complicated by type 1 and $2 \mathrm{DM}$ are at an increased risk of serious perinatal outcomes including stillbirth, perinatal mortality and congenital malformations. The management of diabetes in pregnancy is complex and includes the controlling glucose levels using careful combinations of diet, exercise and insulin or other anti-diabetogenic drugs (Ballas 2012: Balaji 2011). Whilst there have been advances in the different insulin types and regimens available which have crossed into the field of obstetrics, further research is needed to address the safety and efficacy of these new drugs on the market to improve compliance and glycaemic control especially during pregnancy.

\section{Abstract \#: P 293}

\section{Impact of green/blue spaces on specific morbidity and cause- specific mortality in Belgium: the GRESP-HEALTH project protocol (2015-2019)}

Lidia Casas ${ }^{1}$, Isabelle Thomas ${ }^{2}$, Tim Nawrot ${ }^{3}$, Catherine Bouland ${ }^{4}$, Patrick Deboosere ${ }^{5}$, An Van Nieuwenhuyse, Benoit Nemery ${ }^{1}$

${ }^{1}$ Centre Environment and Health, Department of Public Health and Primary Care, KU Leuven, Leuven, Belgium; ${ }^{2}$ Ecole de géographie, Faculté des sciences, Université Catholique de Louvain, Louvain-laNeuve, Belgium; ${ }^{3}$ Centre for Environmental Sciences, Hasselt University, Hasselt, Belgium; ${ }^{4}$ Ecole de Santé Publique, Centre de Recherches en Santé, Environnementale et Santé au Travail Université Libre de Bruxelles, Brussels, Belgium; ${ }^{5}$ Interface Demography, Department of Sociology, Vrije Universiteit Brussel, Brussels, Belgium; ${ }^{6}$ Unit Health and Environment, Scientific Institute of Public Health, Brussels, Belgium; Corresponding author's e-mail: lidia.casasruiz@med.kuleuven.be

Living in green/blue areas is associated with better health. This may be due to low air/noise pollution, opportunities for physical activity, facilitation of social contacts, and/or promotion of recovery from fatigue and stress. Yet, socio-economic (SE) factors also explain inequalities in both health and access to green/blue spaces. The GRESP-HEALTH (Impact of green/blue spaces on specific morbidity and cause-specific mortality in Belgium) project intends to evaluate the associations between living in/close to a green or a blue area on morbidity and mortality in Belgium, at different stages of life (infancy to elderly). It assesses all-cause and cause-specific mortality, specific morbidities and perceived health and takes into account environmental pollutants and SE factors at different levels of spatial aggregation.

The project includes all individuals registered in the official Belgian censuses of 1991 and/or 2001. Three main levels of observation will be studied: individual, statistical sector and group of statistical sectors having similar characteristics in green/blue areas and air pollution. This allows to follow individual and ecological designs. Mortality will be based on the National Mortality Database (a linkage between cause-specific mortality and SE factors using the 1991 and 2001 censuses and cause-specific mortality for 2000-2010). Perceived health information is available from the 2001 census. Specific morbidity (2000-2010) will be derived from the IMA-AIM ("Intermutualistisch Agentschap-Agence Inter-Mutualiste") database, which contains reimbursement data of individual prescriptions. For green/blue spaces, the surface, shape, accessibility and type will be calculated for each statistical sector. Residential area-specific exposure to air pollutants will be obtained from satellite images (CORINE) and traffic noise databases are used whenever possible. Material deprivation, educational attainment, and professional status will be indicators of SE status (SES). The analyses will be conducted separately in different age specific populations (infants, children and adults). We will use multilevel models for clustered data within geographical areas (i.e. individuals, households, nested scales, statistical sectors/groups of statistical sectors and municipalities). When possible, the models will be controlled for SES, perceived environment, air/noise pollution, and type of area (urban, sub-urban, rural, etc.). Interactions of green/blue spaces with air pollution and SE factors will be evaluated and stratified analyses in areas with similar SE and environmental characteristics will be performed. Moreover, more specific population groups (elderly, gender, employment status) will be considered.

The GRESP-HEALTH project will improve the scientific knowledge about the hitherto uncertain associations between living close to green/blue spaces and health.

\section{Abstract \#: P 294}

Design of heterogenic donors: a follow-up of donor insight on genetic determinants of declining versus stable hemoglobin levels

Tiffany C. Timmer ${ }^{1}$, Katja van den Hurk ${ }^{1}$, Kazem Nasserinejad ${ }^{2}$, Wim L.A.M. de Kort ${ }^{1}$

${ }^{1}$ Department of Donor Studies, Sanquin Research, Amsterdam, the Netherlands; ${ }^{2}$ Department of Biostatistics, Erasmus MC, Rotterdam, the Netherlands; Corresponding author's e-mail: t.timmer@sanquin.nl

Introduction: Frequent whole blood donations may carry the risk of iron depletion and subsequent hemoglobin $(\mathrm{Hb})$ level decrease. There are large differences in $\mathrm{Hb}$ level and $\mathrm{Hb}$ level recovery between donors, resulting in different $\mathrm{Hb}$ trajectories. Some donors show stable $\mathrm{Hb}$ levels over time, while others show a decline. Several factors including sex, age, and season are known to influence $\mathrm{Hb}$ levels. Still a good explanation for the different $\mathrm{Hb}$ trajectories between donors is lacking. Genetic factors may play a role in the synthesis of $\mathrm{Hb}$. It has never been studied whether these genetic factors are also associated with recovery from blood donation and $\mathrm{Hb}$ 
trajectories in donors during their donor career. Therefore, the aim of this project is to investigate the association between genetic factors and declining versus stable $\mathrm{Hb}$ levels in blood donors. Furthermore, health effects of iron deficiency in blood donors will be examined.

Methods: study population

A total of 3000 Dutch whole blood donors who previously participated in Donor InSight (DIS)-I and/or -II will be included in DIS-III. We will select two groups of 1333 donors with declining and stable hemoglobin levels based on latent class growth analyses. Another randomly selected group of 1334 donors will be added to this cohort, in order to enable analyses and prediction modeling on a representative sample of the donor population. Capillary $\mathrm{Hb}$ is measured as part of routine donor health assessments and these data will be extracted from the donor database.

Methods: measurements

When the donor visits the blood bank, blood samples will be collected from the sampling pouch, or -in case of deferral-from venipuncture, in order to perform a full blood count, analyze markers of iron metabolism and isolate DNA. DNA will be sequenced using the UK Biobank Axiom ${ }^{\circledR}$ Array (Affymetrix, CA, USA). Potential health effects of iron deficiency and background data will be gathered using questionnaires, including the Short Form (SF)-12 Health Survey, the International Physical Activity Questionnaire, a shortened Food Frequency Questionnaire for dietary iron intake, and questions on cognitive functioning, menstruation patterns, smoking and alcohol use.

Projections: Data collection will be completed towards 2016. Increased knowledge on genetic factors distinguishing donors with different $\mathrm{Hb}$ trajectories after repeated blood donations will help selecting donor subpopulations and tailor their donation intervals in order to prevent iron depletion and anemia.

\section{Abstract \#: P 295}

2B active: outpatient rehabilitation for adolescents with chronic pain

C. Dekker, $\mathrm{MSc}^{1}$, M.E.J.B. Goossens, $\mathrm{PhD}^{1,2}$, C.H.G. Bastiaenen, $\mathrm{PhD}^{3}$, J.A.M.C.F. Verbunt, MD, $\mathrm{PhD}^{1,4}$

${ }^{1}$ Department of Rehabilitation Medicine, CAPHRI, Maastricht University; ${ }^{2}$ Department of Clinical Psychological Sciences, EPP, Maastricht University; ${ }^{3}$ Department of Epidemiology, CAPHRI, Maastricht University; ${ }^{4}$ Maastricht University Medical Center (MUMC+), Maastricht and Adelante Centre of Expertise in Rehabilitation and Audiology, Hoensbroek; Corresponding author's e-mail: carolien.dekker@maastrichtuniversity.nl

Chronic musculoskeletal pain (CMP) in adolescents is a common problem. Living with CMP not only impacts on the adolescent's functioning and well-being, but also has negative consequences for the family and society. According to the Fear Avoidance Model [5] of CMP, fear of movement and pain catastrophizing play an important role in the occurrence and maintenance of chronic pain complaints and functional disability. The intervention, a multimodal rehabilitation program (MRP), aims at decreasing functional disability by reducing fear of movement and pain catastrophizing. The aim of this study is to evaluate the effectiveness of a multimodal rehabilitation program in reducing functional disability (measured with the Functional Disability Inventory) for adolescents with CMP by comparing to care as usual (CAU). There is a focus on the long term results, at 12 months after start of treatment.

The design is a multicenter randomized controlled trial, characterized as a pragmatic intervention study, since the goal is to study the effectiveness of a set of treatment modules. Because of the pragmatic approach, results are directly applicable in practice. Participants are allocated (ratio 1:1) to MRP or care as usual (CAU). The randomization method minimization was chosen to balance sex, age and treatment center in this small trial. Randomization and treatment allocation are blinded. Treatment duration varies between 7 and 16 weeks. Self- reported measurements are at baseline and at 2, 4, 10 and 12 months after start of the treatment. In total, 124 adolescents and their parents will be recruited (Sample size is based on a $25 \%$ difference in group mean on the primary outcome (FDI), with alpha of $5 \%$, power of $80 \%$ and expected $15 \%$ loss to follow up, taking repeated measurements into account. Adolescents between 12 and 21 years with a certain degree of fear, a certain level of functional disability and an indication for outpatient rehabilitation treatment are eligible. Treatment teams of four Dutch rehabilitation centers were trained to provide the MRP in this study. Intention to treat analysis will be performed for all outcome variables. Intervention: MRP is a multidisciplinary outpatient individual rehabilitation program. MRP consists of 3 treatment modules, based on graded exposure therapy. A combination of modules is chosen, depending on the needs of the patient. Control: CAU consists of the care currently provided in Dutch rehabilitation centres, based on a consensus approach for treatment of adolescents with chronic fatigue and pain.

Clinicaltrials.gov ID: NCT02181725, METC NL47323.068.13/ METC13-3-062.

\section{Abstract \#: P 296}

\section{Active2Gether: innovative and smart coaching strategies} to promote physical activity: a research protocol

Anouk Middelweerd $^{1}$, Saskia J. te Velde ${ }^{1}$, Michel C.A. Klein ${ }^{2}$, Aart T. van Halteren ${ }^{3}$, Johannes Brug ${ }^{1}$

${ }^{1}$ VU University Medical Centre/EMGO Institute for Health and Care Research; ${ }^{2}$ Vrije Universiteit Amsterdam; ${ }^{3}$ Vrije Universiteit Amsterdam/Philips Research; Corresponding author's e-mail: a.middelweerd@vumc.nl

Background: Because $67.5 \%$ of Dutch adults is not sufficiently physically active, effective innovative and smart physical activity (PA) promotion is warranted. The ubiquitous nature of smartphones offer new possibilities in PA promotion. Because of their built-in sensors and their ability to connect with the internet or third-party sensors, smartphones offer the possibility to monitor the behavior continuously, provide personalized, real-time and context specific feedback. Active2Gether (A2G) makes use of these possibilities and uniquely combines behavior change techniques with a model-based reasoning system in order to provide context specific coaching messages via smartphones to empower young adults to be and remain more physically active.

Intervention: $A 2 \mathrm{G}$ is an app-based intervention that focuses on PA. The overall aim is to increase levels of weekly moderate-vigorous PA (MVPA). It surpasses existing apps as the coaching messages are theory- and literature- based and tailored to psychological and social concepts (e.g. outcome expectations, self-efficacy, social support) as well as to the physical and social context. The messages are based on proven behavior change techniques (e.g., self-monitoring, goal-setting, social-comparison) and are framed in an autonomy-supportive style. Furthermore, social network techniques will be used to influence beliefs and subsequent behavior. The intervention will start with an intake and an initial monitoring phase followed by 6 months of coaching.

Methods: A 3-arm randomized trial with a baseline and three followup assessments will be carried out with approximately 150 young adults (18-30 years) in April 2015. PA and determinants of PA will 
be measured for two purposes: for the evaluation of the intervention and to serve as input for the personalized coaching messages. Actigraph accelerometers will measure weekly PA at baseline and at 3and 6-months during and at 6 month after the intervention. Additionally, PA will continuously be assessed with a Fitbit One activity tracker. An extensive survey at baseline and the follow-ups will assess demographic characteristics and determinants of PA, while these determinants will repeatedly be measured during the intervention by means of short versions of existing questionnaires.

Analysis: An outcome and a process evaluation will be conducted to estimate the effect of the A2G intervention on levels of weekly MVPA and to evaluate the underlying processes and mechanisms with mixed models and latent class growth analysis to explain the (lack of) effect.

This research is supported by Philips and Technology Foundation STW, Nationaal Initiatief Hersenen en Cognitie NIHC under the Partnership programme Healthy Lifestyle Solutions.

\section{Abstract \#: P 297}

Using reduced set of genetic markers with iterative pruning principal component analysis (IPPCA) to retrieve sub patients

Kridsadakorn Chaichoompu $^{1}$, Ramouna Fouladi ${ }^{2}$, Isabelle Cleynen ${ }^{1}$, Kristel Van Steen ${ }^{1}$

${ }^{1}$ Montefiore Institute, University of Liege, Belgium; ${ }^{2}$ Department of Clinical and Experimental Medicine, University of Leuven, Belgium; Corresponding author's e-mail:

kridsadakorn.chaichoompu@ulg.ac.be

Background: Single Nucleotide Polymorphisms (SNPs) are commonly used to capture variations between populations. Often genomewide SNP data are pruned based on linkage disequilibrium patterns or small subsets of SNPs are selected (e.g. PCA-correlated SNPs [1]) to reproduce the genomic structure of the complete data set. In addition, iterative pruning principal component analysis (ipPCA) [2] has been shown to be a powerful tool to cluster subpopulations based on SNP profiles [3].

Methods: To identify and differentiate between sub patients using reduced sets of genetic markers for these purposes can become challenging. Here, we do genome-wide association study to obtain reduced set of disease markers by PLINK. We then use ipPCA to identify and characterize sub patients in an unsupervised way. This approach are applied to real-life data consisting of $\sim 19,000$ Crohn's disease patients from the International IBD Genetics Consortium [4]. Results: Preliminary results indicate that ipPCA applied to the reduced SNP data give additional information about subgroups of patients. Moreover, we found that missing genotype affected clustering results. We did experiments to figure out the optimal percentage of missingness to prune out the missing genotype during preprocessing step.

Conclusion: In this work, we propose to use small subsets of SNPs with the ipPCA machinery to retrieve sub patients. Despite the complexities that are associated with genome-wide association study, added value can be obtained.

\section{References:}

1. Paschou, P., et al., PCA-correlated SNPs for structure identification in worldwide human populations. PLoS Genet, 2007. 3(9): p. $1672-86$.

2. Intarapanich, A., et al., Iterative pruning PCA improves resolution of highly structured populations. BMC Bioinformatics, 2009. 10: p. 382.
3. Limpiti, T., et al., Study of large and highly stratified population datasets by combining iterative pruning principal component analysis and structure. BMC Bioinformatics, 2011. 12: p. 255.

4. Maus, B., et al., Molecular reclassification of Crohn's disease: a cautionary note on population stratification. PLoS One, 2013. 8(10): p. e77720.

\section{Abstract \#: P 298}

Analysis of mortality in Brazil according to race/color skin and gender: What says the mortality information system?

Kelly Albuquerque de Oliveira ${ }^{1}$, Jenny Rose Smolen ${ }^{1}$, Edna Maria de Araújo ${ }^{1}$, Tânia Maria de Araújo ${ }^{1}$

${ }^{1}$ UEFS; Corresponding author's e-mail: kellyalbuquerque84@gmail.com

Background: Data on a population's mortality can serve as an indicator of the general health situation of that population. Although Brazilian researchers are beginning to recognize the importance of race in relation to health, no recent studies have evaluated mortality data by race. The objective of this study is to analyze mortality in Brazil according to race and gender.

Method: This descriptive study evaluates mortality from 2008-2012, using data from the Ministry of Health's Mortality Information System, as well as data from the Information System on Live Births. The measures of mortality used in this study are proportional mortality by age, leading causes of death, general mortality by age, and infant mortality. This study's analysis shows that the mortality patterns in Brazil are different according to race.

Results: Whites die at an older age, while the black population die at an active age. The causes of death also differ by gender, with men dying more of external causes while women die of chronic disease. This is true of all races, though external causes affect black men more than white men. The indigenous population has a mortality pattern completely distinct from the rest of Brazil, one indicative of a less developed society.

Conclusion: This study shows that racial disparities still exist in Brazil-the policies put in place to reduce and eliminate these disparities should use this mortality data to guide interventions.

\section{Abstract \#: P 300}

Prevalence and socio-demographic characteristics associated with methamphetamine use among male adolescents in the west of Iran

Seyyed Nasrollah Hosseini ${ }^{1}$, Farzad Jalilian ${ }^{2}$, Mehdi Mirzaei Alavijeh $^{3}$, Behzad Karami Matin ${ }^{2}$, Parvin Nokhasi ${ }^{2}$, Mohamad Reza Amoei ${ }^{4}$

${ }^{1}$ Ministry of Health and Medical Education, Tehran, Iran; ${ }^{2}$ Department of Public Health, School of Health, Kermanshah University of Medical Sciences, Kermanshah, Iran; ${ }^{3}$ Department of Health Education \& Promotion, Faculty of Medical Sciences, Tarbiat Modares University, Tehran, Iran; ${ }^{4}$ Applied Research Bureau of the Police of Kermanshah, Kermanshah, Iran; Corresponding author's e-mail: hoseyniseyyed@yahoo.com

Background: Methamphetamine use is the one of the common illicit drug use in worldwide, especially among adolescents, which can cause numerous complications. The main objective of this study was 
determined the prevalence and socio-demographic characteristics associated with methamphetamine use among Iranian male adolescents.

Methods: This cross sectional study was carried out on 163 male adolescents in Kermanshah County the west of Iran, were randomly selected to participate voluntarily in the study. A self-report written questionnaire was applied to collect data. Data were analyzed by SPSS-20 using logistic regression statistical tests.

Results: The mean age of respondents was 16.94 years [SD: 1.24], ranged from 15 to 19 years. Almost $10.8 \%$ of the participants had history of last one methamphetamine use in lifetime. The initiation age for drug use was 12.The best predictor for methamphetamine use was drug suggestion by friends with odds ratio estimate of 10.55 [95\% CI: 3.41, 32.59].

Conclusions: Prevalence of methamphetamine use among participate can be warning to health policy makers in Iran: and should be the focus of special attention. Based on our result, it seems that designing and implementation of educational programs to increase assertiveness skills and drug refuse skills may be usefulness of the results in order to prevent of methamphetamine use.

\section{Abstract \#: P 301}

\section{Equity in health care services: the challenges in Brazil}

Camila Nascimento Monteiro $^{1}$, Mariëlle A. Beenackers ${ }^{2}$, Moisés Goldbaum $^{1}$, Marilisa Berti de Azevedo Barros ${ }^{3}$, Reinaldo José Gianini $^{1}$, Chester Luiz Galvão Cesar ${ }^{4}$, Johan P. Mackenbach ${ }^{2}$

${ }^{1}$ Department of Preventive Medicine, Faculty of Medicine, University of São Paulo, São Paulo, Brazil; ${ }^{2}$ Department of Public Health, Erasmus University Medical Centre, Rotterdam, the Netherlands; ${ }^{3}$ Faculty of Medicine, University of Campinas, Campinas, Brazil; ${ }^{4}$ Faculty of Public Health, University of São Paulo, São Paulo, Brazil; Corresponding author's e-mail: c.nascimentomonteiro@gmail.com

Background: Socioeconomic inequalities are a big problem in the Americas. Brazil, and in particular São Paulo, is experiencing considerable inequalities in access to, and use of, the health care service. The aim of this study is to understand how socioeconomic indicators are associated with such access and use in São Paulo, Brazil.

Method: Data were obtained from two household health surveys from São Paulo (ISA-Capital 2003 and ISA-Capital 2008). We used logistic regression to analyze the associations between socioeconomic indicators and access to and use of health care services.

Results: The study showed almost universal access to health care services (94.91\% in 2003 and $94.98 \%$ in 2008). In 2003 and 2008 there were some socioeconomic differences in health care seeking and resolution of the health problem after seeking care. The use of public sector health services was higher in lower income groups, those with lower education and non-Caucasians in 2003 and 2008. Resolution of the health problem was reported more often by people of high socioeconomic position and those with private health insurance than by people who used the public sector.

Conclusions: This study shows that access to health care services is high in São Paulo: this increase may have been influenced by private health plans and public strategies such as the Family Health Program. However, inequalities in the use of health care services were observed and the results indicate problems in the quality of health care services that seem to disfavor the lower socioeconomic groups who more often use public services. The Brazilian Health System faces the challenge of decreasing the socioeconomic disparities between individuals. Deeper knowledge about the use of, access to health services, together with a better understanding of inequalities, is important to inform health policies and to provide better understanding of the potential barriers to reducing inequalities in the use of health services.

\section{Abstract \#: P 302}

Socioeconomic inequalities in dental services use: the case of the smiling Brazil program

Camila Nascimento Monteiro ${ }^{1}$, Mariëlle A. Beenackers ${ }^{2}$, Moisés Goldbaum $^{1}$, Marilisa Berti de Azevedo Barros ${ }^{3}$, Reinaldo José Gianini $^{1}$, Chester Luiz Galvão Cesar ${ }^{4}$, Johan P. Mackenbach ${ }^{2}$

${ }^{1}$ Department of Preventive Medicine, Faculty of Medicine, University of São Paulo, São Paulo, Brazil; ${ }^{2}$ Department of Public Health,

Erasmus University Medical Centre, Rotterdam, the Netherlands; ${ }^{3}$ Faculty of Medicine, University of Campinas, Campinas, Brazil; ${ }^{4}$ Faculty of Public Health, University of São Paulo, São Paulo, Brazil; Corresponding author's e-mail: c.nascimentomonteiro@gmail.com

Background: Access to, and use of, dental services in Brazil have been improving since 2003 due to the increase of private health plans and public strategies which aim to increase access, such as the Smiling Brazil program. However, we do not yet know if inequalities in dental services use persist after the improvement in access. The aims of this study are to analyze socioeconomic differences in dental services use in 2003 and in 2008 in São Paulo and to examine changes in these associations after the implementation of the Smiling Brazil program in 2005.

Method: We used data from two cross-sectional population-based household health surveys (ISA-Capital 2003 and ISA-Capital 2008) which investigated living and health conditions, health status and use of health care services. We used logistic regression to analyze the associations between socioeconomic factors and health status and use of dental services. Additionally, we studied trends from 2003 to 2008 regarding socioeconomic differences in dental services.

Results: Overall dental services use increased between 2003 and 2008 and was at both time points more common in those who had higher income, higher education, better housing conditions, private health plan and were Caucasian. The main reasons of not seeking dental care were not having teeth and financial difficulty, both more common in lower socioeconomic groups.

Conclusions: The Brazilian national health strategy 'Smiling Brazil' seems to have contributed slightly to the increase in dental services use, but has not influenced socioeconomic inequalities. The Brazilian Health System faces the challenge of decreasing the socioeconomic disparities between individuals. Acquiring deeper knowledge about inequalities in dental service use will contribute to understanding the potential barriers to reducing these inequalities.

\section{Abstract \#: P 303}

\section{History of violence in childhood and vulnerabilities of women}

Bianka Sousa Martins Silva $^{1}$, Maura Maria Magalhães ${ }^{1}$, Tânai Maria Araújo ${ }^{1}$

${ }^{1}$ Universidade Estadual de Feira de Santana; Corresponding author's e-mail: biankabio@bol.com.br

The present study aimed to identify risk behaviors in women who have witnessed family violence during their childhood and were victims of violence in adulthood and profile of these women according to sociodemographic and economic conditions. This is a cross-sectional study conducted with 2819 women over the age of 
15 years living in the city of Feira de Santana-BA. Data were collected during home visits with use of household and individual record form. Bivariate and the Chi square test analyzes were performed considering $95 \% \mathrm{CI}$ and $p \leq 0.05$ for statistically significant association.

The results indicated a prevalence $(12.14 \%)$ history of childhood violence among women victimized in adulthood. It was observed that women who have never been to school (15.08\%), non-white $(12.61 \%)$ and had an income of up to 1 minimum wage $(14.17 \%)$ had a higher incidence of physical violence in childhood. The women drinkers had 1.43 times higher prevalence of experiencing violence in childhood and, in relation to smoking, this prevalence increased to 1.56. Despite the complexity of violence, it is necessary to get her out of invisibility and initiating various measures, such as preparing the care of victims of violence and the deployment of a service to protect the victims, because many are silent for fear of reprisals by their abusers.

\section{Abstract \#: P 304}

The impact of smoke-free legislation on educational differences in birth outcomes

\section{Britt McKinnon $^{1}$, Nathalie Auger ${ }^{2}$, Jay S. Kaufman ${ }^{3}$}

${ }^{1}$ Institute for Health and Social Policy, McGill University, Montreal, Canada; ${ }^{2}$ Institut National de Santé Publique du Québec, Montreal, Canada; ${ }^{3}$ Department of Epidemiology, Biostatistics and Occupational Health, McGill University, Montreal, Canada; Corresponding author's e-mail: britt.mckinnon@mcgill.ca

Background: Emerging evidence suggests smoke-free legislation may have positive effects on birth outcomes. Given that smoking and exposure to second hand smoke during pregnancy vary with socioeconomic position, it is possible the effects of this legislation may be greater in some socioeconomic groups. For this study, we evaluate the impact of the 2006 ban on smoking in public places in the Canadian province of Quebec on educational differences in preterm birth, small-for-gestational age birth, and birth weight.

Methods: We analyzed data on singleton births in Quebec between 2003 and 2009. Logistic regression was used to model the association of smoke-free legislation with preterm birth ( $<37$ weeks), moderate/ extreme preterm birth ( $<34$ weeks), small-for-gestational-age birth, and birth weight, adjusting for secular trends before and after the legislation. Interaction terms were included to assess differential effects by level of maternal education.

Results: We estimated the smoke-free legislation was associated with average reductions of 0.31 preterm births (95\% CI: $0.01,0.60), 0.23$ moderate/extreme preterm births (95\% CI: 0.9, 3.7), and 0.59 smallfor-gestational age births (95\% CI: 2.6, 9.3) per 100 live births, and a 17.1-g increase in mean birth weight (95\% CI: 10.7, 23.6). The legislation was consistent with meaningful effects on birth outcomes across categories of maternal education, with no statistical evidence of differential effects of the legislation by maternal education for any of the outcomes.

Conclusion: Smoke-free legislation in Quebec was associated with reductions in preterm and small-for-gestational-age births, and an increase in birth weight. There was no compelling evidence the legislation impacted educational gradients in birth outcomes.

\section{Abstract \#: P 305}

Perceived stress in Kazakhstani adults: prevalence and association with metabolic syndrome

Mira Zhunissova ${ }^{1}$, Karlygash Sadykova ${ }^{1}$, Kamshat Madenbay ${ }^{1}$, Gulnaz Nuskabaeva $^{1}$, Zhanar Shalkharova ${ }^{2}$, Zhanat Shalkharova ${ }^{1}$, Andrej M. Grjibovski ${ }^{3,4}$

${ }^{1}$ International Kazakh - Turkish University, Turkestan, Kazakhstan; ${ }^{2}$ Kazakh Medical University of Continuing Education, Almaty, Kazakhstan; ${ }^{3}$ Northern State Medical University, Arkhangelsk, Russia; ${ }^{4}$ Norwegian Institute of Public Health, Oslo, Norway; Corresponding author's e-mail: mira.zhunissova@gmail.com

Background: Stress has been reported to be associated with several components of the metabolic syndrome (MetS). However, the results are not consistent across populations warranting further research in other settings. We failed to identify studies on stress and MetS in former Soviet Republics of Central Asia, which are still undergoing economic and social transition. The aim of this study is to assess perceived stress among adult urban population in Southern Kazakhstan and to study its association with MetS and its components.

Methods: Altogether, 938 adults aged 18-74 years were enrolled in a cross-sectional study in Turkestan, Southern Kazakhstan. All subjects completed an anonymous questionnaire and underwent a physical examination. Stress levels were assessed using a 10-items Perceived Stress Scale (PSS-10). Scores 31-40 indicate intense stress, 21-30moderate stress, 11-20-light stress and 10 and below-absent stress. Blood tests were taken and analyzed in the International KazakhTurkish University laboratory using calibrated equipment. MetS was defined using IDF criteria (2005). Association between stress and metabolic syndrome and its components was studied using multiple logistic regression with adjustment for age, gender, education and marital status. The analyses were repeated using multiple linear regression with components of MetS as continuous dependent variables. PSS-10 scores were used as the main continuous independent variable in the models.

Results: Mean PSS scores were 18.5 (95 \% CI: 18.0-19.0) among women and 17.4 (95\% CI: 16.7-18.2) among men, $p<0.001$. The prevalence of intense, moderate and light stress was $1.8 \%, 37.1$ and $49.2 \%$ in women. The corresponding data for men were $0.9,39.5$ and $54.5 \%$, respectively. No significant association was found between PSS scores and either MetS (OR $=0.99,95 \%$ CI: $0.96-1.02)$ or any of its components: blood glucose $(\mathrm{OR}=1.01,95 \% \mathrm{CI}$ : 0.98-1.04), abdominal obesity ( $\mathrm{OR}=1.01,95 \% \mathrm{CI}: 0.98-1.05)$, triglycerides $(\mathrm{OR}=1.01,95 \%$ CI: $0.98-1.04)$, high density lipoproteins $(\mathrm{OR}=1.01,95 \%$ CI: 0.97-1.05), blood pressure $(\mathrm{OR}=0.98,95 \%$ CI: 0.95-1.01). Linear regression models yielded similar results.

Conclusions: The mean PSS scores observed in this study are considerably higher than normative values reported from European countries. No association between perceives stress and either MetS or any of its components was found in the Kazakhstani setting. Cultural, behavioral and lifestyle factors behind our findings will be discussed.

\section{Abstract \#: P 307}

Cost of maternal isolation for child-rearing: a large populationbased study in Japan 
Yukiko H. Honda $^{1,2}$, Takeo F. Fujiwara ${ }^{2,3}$

${ }^{1}$ Global Health and Socio-Epideiology, Kyoto University School of Public Health, Kyoto, Japan; ${ }^{2}$ Social Medicine, National Research Institute for Child Health and Development, Tokyo, Japan; ${ }^{3}$ Public Health and Occupational Medicine, Mie University Graduate School of Medicine, Tsu, Japan; Corresponding author's e-mail: fujiwara-tk@ncchd.go.jp

Background: Social capital is beneficial for both maternal and child health, but few study investigated the amount of social capital as monetary value. Comparing child-rearing cost between mothers with and without social network can be a good marker to estimate it. The aim of this study was to assess the association between maternal isolation and child-rearing cost.

Methods: We used the Data from a population-based birth cohort study in Japan, the Longitudinal Survey of Babies in the 21st Century $(n=40,643)$. Maternal isolation was defined mothers who distressed on child-rearing but not having someone to consult with. Cost of child-rearing per month at 6 month of age was self-reported as continuous variable. The association between maternal isolation and child-rearing cost was assessed by using multiple regression adjusted for covariates (child birth outcomes, maternal and paternal socioeconomic status, and urban/rural differences).

Results: Isolated mothers were found for $258(0.6 \%)$. In crude model, isolated mothers more likely pay 6980 (95\% confidence interval [CI]: $3168-10,793)$ JPY than non-isolated mothers, which is similar in multivariable model (coefficient: 4573 (95\% CI: 857-8289) JPY) and model of multiple imputation for covariates (coefficient: 5214 (95\% CI: 1483-8946)).

Conclusion: Isolated mothers spend 5,214 JPY (equivalent to 50 USD) per month higher amount of child-rearing cost than non-isolated mothers, which can be considered the cost of social capital among mothers. Provision of social network and support for isolated mothers might be helpful to save child-rearing cost for isolated mothers.

\section{Abstract \#: P 308}

The impacts of early paternal involvement of childcare on later child behavioral problems

Manami Ochi $^{1,2}$, Takeo Fujiwara ${ }^{1,2}$

${ }^{1}$ National Research Institute for Child Health and Development; ${ }^{2} \mathrm{Mie}$ University Graduate School of Medicine; Corresponding author's e-mail: mon.ami728@gmail.com

Background: Several studies in Western countries have reported that adverse effect of maternal employment during the first year of life on children's cognitive scores and more behavior problems at later ages. However, few studies investigate the impact of paternal spending hours with children on later behavioral outcomes.

Objective: To examine the impact of paternal working hours and spending time with their children aged $0.5-1.5$ years old on later behavioral developments at age 5.5 years old.

Methods: We used the data of "Longitudinal Survey of Babies in 21st Century", which was a Japanese population-based longitudinal data. Sample were born in 2001 limited to singleton without low birth weight $(\mathrm{N}=31,819)$. Responses to six survey questions about ageappropriate behaviors at age 5.5 years were used as indicators of behavioral development: "Listening without fidgeting", "Focusing on one task", "Being patient", "Expressing emotions appropriately", "Getting along with others in a group setting", and "Keeping promises". The impact of the paternal and maternal working hours at 6 months after birth and spending time with the child at 1.5 years on the behavioral problems at 5.5 years were investigated using multivariate logistic regression.

Results: Children whose fathers who did not work or worked less than $20 \mathrm{~h}$ in a week at age 0.5 years showed significantly poorer behavioral developments at their age 5.5 years. The associations between the length of the hours with their children in weekdays and the later behavioral development outcomes were not consistent, however, children whose father spending time with the child over $6 \mathrm{~h}$ in weekend less likely to show child behavioral problems, except for "Focusing on one task", than children whose father spending with the child less than $4 \mathrm{~h}$ in weekends.

Conclusion: A high degree of parental involvement in holidays with young children at age 1.5 years old, might have protective effects on behavioral developments at age 5.5 years old.

\section{Abstract \#: P 309}

Status of disability in India: trends, prospects and disability deprivation index of the districts in India

Ashish Awasthi $^{1}$, C.M. Pandey ${ }^{1}$, Uttam Singh ${ }^{1}$

${ }^{1}$ Sanjay Gandhi Postgraduate Institute of Medical Sciences, Lucknow, India; Corresponding author's e-mail: ashishbhuims@gmail.com

Context: With the dawn of the civilization, disability exists in various dimensions human life. World Health Organization (WHO) defines disability as an umbrella term, covering impairments, activity limitations, and participation restrictions. Disability is thus not limited to health problems. It is a complex phenomenon, reflecting the interaction between features of a human body and features of the society in which they live. Globally approximate 1 billion people have some form of disability and approximate $20 \%$ have significant functioning disabilities.

Objectives: Present study aims to estimate the level, trend and prospects of disability in the districts of India and further to construct disability deprivation index.

Material \& methods: Data for the present study has been borrowed from the Census of India $2001 \& 2011$. There were 640 districts in Census 2011 in place of 593 districts in year 2001. It is assumed that population of new districts in year 2011 follow same distribution of population characteristics of parent district in 2001. Disability index was calculated at district level and state level indexing is done using disability deprivation index. Population for year 2021 is projected using exponential growth rate method. Disability deprivation index was calculated using child labor, adult unemployment, illiteracy in disabled population and ratio of beggars in disabled population.

Results: The study reveals that disability in India has increased from $2.10 \%$ in 2001 to $2.21 \%$ in 2011. Maximum disability $4.51 \%$ was observed in Hyderabad district and minimum disability percentage $0.76 \%$ was observed in Daman district in 2011. 66 districts were in first quartile of the disability index in year 2011 in comparison of 96 districts in year 2001. According to disability deprivation index Maharashtra was best performing state and Assam was worst performing state in India in 2011 whereas Chandigarh was best performing state and Nagaland was worst performing state in India in 2001. There were 49.04 million new cases of disability in India during 2001-11 out of which 15. 19 million confirmed cases of non-congenital disability. By 2021 , the disability percent will raise to 32.81 million $(2.3 \%)$.

Conclusions: There is a rise in the disabled population in India which needs special attention. The situation of the disabled working status is gloomy. The majorities of the disabled people are non-working and need effective rehabilitation measures which would facilitate employment. 


\section{Abstract \#: P 310}

Association between social participation and influenza infection: a cross sectional study in Japanese older people

Yugo Shobugawa $^{1}$, Takeo Fujiwara ${ }^{2}$, Reiko Saito ${ }^{1}$, Katsunori Kondo ${ }^{3}$

${ }^{1}$ Niigata University Graduate School of Medical and Dental Sciences; ${ }^{2}$ National Research Institute for Child Health and Development;

${ }^{3}$ Chiba University Graduate School of Medicine; Corresponding author's e-mail: yshobugawa@gmail.com

Background: Influenza infection can cause severe disease especially in older people. In high aging countries as like Japan, influenza outbreak has potentially large impact. Influenza vaccination is only an option to prevent severe disease besides prophylactic administration of neuraminidase inhibitor. The World Health Organization highly recommends for older people to receive annual vaccination of influenza. However, even in developed country, part of older people cannot have chance to be vaccinated, further cannot be diagnosed as influenza when they have fever due to poor medical access. In this study, we tried to elucidate what is obstacle to see a doctor and be diagnosed as influenza.

Method: People aged 65 or older who were without the need of nursing care were enrolled from 30 municipalities in Japan. The data used were collected by a mail survey in 2013 on influenza infection, influenza vaccination, social participation, age, sex, household income and other socioeconomic characteristics. We explored the factors which can affect influenza infection using logistic regression models. This study was conducted as JAGES (Japan Gerontological Evaluation Study) 2013, and we used a part of the data.

Results: In total, 26,461 older people were enrolled. Of all, 15,356 $(58.0 \%)$ were vaccinated, and $993(3.8 \%)$ were diagnosed as influenza infection. For the model investigating factors on influenza infection, people participating in two or more groups $[\mathrm{OR}=1.24$ (95 \% CI: 1.02-1.51)] compared with no participating were more diagnosed as influenza after adjusting sex, age, educational attainment, income, and influenza vaccination.

Discussion: In Japan, influenza infection can be diagnosed by commercial rapid antigen test kit in outpatient settings. However, older people who can see a doctor when they get sick are limited. Sociable persons who participate social group have rich social network and can obtain health and medical information. And, such sociable persons can go to see a doctor when they have fever because of better medical access based on their rich social network.

Conclusion: This study showed more influenza infected cases were reported in vaccinated and sociable person. The result indicates that older people who have rich social network can reach medical resource easily.

\section{Abstract \#: P 311}

\section{Association of social capital and functional disability among elderly living in community: a population-based study \\ Cristina Franco Gontijo ${ }^{1}$, Juliana Vaz de Melo Mambrini ${ }^{1}$, Tatiana Chama Borges Luz ${ }^{1}$, Antônio Ignácio de Loyola Filho ${ }^{1}$ \\ ${ }^{1} \mathrm{CPqRR} /$ Fiocruz, Brazil; Corresponding author's e-mail: cfgontijo@yahoo.com.br}

Functional disability is defined as difficulty or inability to perform certain activities of daily life, typically linked to survival (Basic Activities of Daily Living or BADL) and social life (Instrumental Activities of Daily Living or IADL) and it is frequent in the elderly. In addition to sociodemographic and health factors, the disabling process may also be influenced by the relationship between individual and social context characteristics. The present study aimed to estimate the prevalence of disability and its association with social capital among 2271 older residents of the Belo Horizonte Metropolitan Area-BHMA. Approximately one third of participants $(33.1 \%)$ presented himself unable to at least one IADL and/or BADL and the prevalence of functional disability for BADL/IADL was $18.6 \%$ and just for IADL disability was $14.5 \%$. The dependent older for IADL/ BADL had higher odds of poor social capital, but only the element of neighborhood perception of attachment proved to be independently associated with functional disability $(\mathrm{OR}=1.80 ; \quad 95 \%$ $\mathrm{CI}=1.12-2.88)$. Our results show the importance of social capital in research on the functional disability determinants and it indicate the need for other public policies are implemented, in the social and environmental areas, as the needs of the older require measures beyond those inherent to the health sector.

\section{Abstract \#: P 312}

Lifestyle-related factors for mediator between change in socioeconomic status due to disaster and poor subjective health

Masato Nagai ${ }^{1,2}$,Tetsuya Ohira ${ }^{1,2}$, Hideto Takahashi ${ }^{1}$, Zhang Wen ${ }^{2}$, Hironori Nakano $^{1,2}$, Masaharu Maeda ${ }^{1,2}$, Seiji Yasumura ${ }^{1,2}$, Masafumi Abe ${ }^{1}$

${ }^{1}$ Radiation Medical Science Center for the Fukushima Health Management Survey, Fukushima Medical University, Fukushima, Japan; ${ }^{2}$ Department of Epidemiology, School of Medicine, Fukushima Medical University, Fukushima, Japan; Corresponding author's e-mail: m-nagai@med.tohoku.ac.jp

Background: Change in socioeconomic status (SES) due to disaster associates with poor subjective health. However, it is unavoidable in disaster. Therefore, we examined the lifestyle-related factors for mediator which attenuate the impact of SES change due to disaster on poor subjective health.

Methods: We delivered a self-administered questionnaire of Mental Health and Lifestyle Survey in Fukushima Health Management Survey to all residents aged $\geq 16$ years living in the evacuation zones due to radiation accident in January 2012. For the current analysis, 24,634 men and 30,521 women were analyzed. The participants who changed living status, became unemployed, changed jobs, or decreased income after disaster classified as change in SES. Poor subjective health was defined as participants who responded "poor" or "very poor". Compared with participants who did not change in SES, the risk ratios (RRs) and $95 \%$ confidence intervals (CIs) for poor subjective health in participants who changed in SES were calculated by Poisson regression with robust error variance adjusted for confounders (age, history of diseases, mental illness, activities of daily living, education, and evacuation place) in men and women (RR1). We further adjusted lifestyle-related factors which is smoking, drinking, satisfaction of sleep, participation in recreation and community activities, and exercise habit as intermediate variables (RR2). The percentage excess risks mediated by lifestyle-related factors was calculated as follows: (RR1-RR2)/(RR1 - 1)) × $100 \%$.

Results: After adjusted lifestyle-related factors, RRs (95\% CIs) in men and women who changed in SES decreased from 1.68 $(1.58-1.79)$ and $1.56(1.48-1.64)$ to $1.39(1.31-1.48)$ and 1.31 (1.25-1.38), respectively. Controlling for lifestyle-related factors yielded 43.0 and $44.1 \%$ attenuation in the impact of SES change on poor subjective health in men and women, respectively. RR2s (95\% CIs) in men and women were 5.28 (4.83-5.78) and 5.34 (4.87-5.85) in complaint about satisfaction of sleep (reference: content), 1.77 $(1.55-2.02)$ and $1.80(1.58-2.05)$ in no participation in recreation and 
community activities (reference: often), and 1.21 (1.12-1.31) and $1.18(1.10-1.28)$ in exercise habit $\leq 1$ time/week (reference: $\geq 5$ times/week), respectively. RR2 (95\% CIs) was 1.11 (1.03-1.20) in current smoker among women (reference: never smoker), while it did not show statistically significant association in smoking among men and drinking among men and women.

Conclusion: Smoking (only women), satisfaction of sleep, participation in recreation and community activities, and exercise habit mediated the impact of SES change due to disaster on poor subjective health. Even though change in SES is unavoidable in disaster, improvement of lifestyle-related factors might prevent from poor subjective health.

\section{Abstract \#: P 313}

Awareness of HIV transmission, attitudes and practices amongst secondary students in an urban metropolis in Ibadan, south west Nigeria 2012

$\frac{\text { A. Aderinoye }^{1}}{\text { A. Olayinka }^{1}}$, O. T. Olugbade ${ }^{1}$, I. Ojedokun $^{2}$, O. Ajumobi $^{3}$,

${ }^{1}$ Department of Social Work, Faculty of Education, University of Ibadan, Oyo state, Nigeria; ${ }^{2}$ Nigeria Field Epidemiology Laboratory Training Program, Abuja, Nigeria; ${ }^{3}$ National Malaria Elimination Program, Federal Ministry of Health, Abuja, Nigeria; Corresponding author's e-mail: aderinoyeadegboyega@gmail.com

Background: The World Health Organization estimates 2.6 million new cases of Human Immunodeficiency Virus (HIV) Infections worldwide annually, with nearly $370,000(14 \%)$ occurring in persons under 15 years. Nigeria has the 2 nd highest number of new infections world-wide with $3.7 \%$ of the population living with HIV. Lack of information about sexual health and HIV, poor awareness of transmission and unprotected intercourse amongst sexually active adolescents, are major drivers of HIV infections, and constitute barriers to ending the pandemic in at risk groups. We assessed the awareness of HIV, and Sexually Transmitted Infections (STIs), and sexual practices amongst secondary school students in this vulnerable age group.

Methods: We conducted a descriptive cross sectional survey, amongst adolescent secondary school students in Ibadan, Oyo state, Nigeria. We selected 270 respondents using a multistage sampling technique. Information on socio demographic characteristics: knowledge about transmission of HIV, STIs and sexual practices was collected using a self-administered questionnaire. Multivariate analysis was done using Chi square $\left(\chi^{2}\right)$.

Results: Mean age of respondents was $14.5 \pm 1.5$ years. Of 270 students, $102(38 \%)$ were male, $224(83 \%)$ affirmed that sex should be delayed till completion of studies to prevent teenage pregnancy, however $26(10 \%)$ of the students were sexually active, with all, having unprotected intercourse. A total $124(45.9 \%)$ believe that unprotected sex and 120 (44\%) are aware STIs increases the risk of HIV/AIDS and unplanned teenage pregnancy, with awareness being associated with female gender $\left(\chi^{2}=8.8: p=0.013\right)$ and being a senior secondary student $\left(\chi^{2}=53.758: p=0.0001\right)$.

Conclusion: Sexual health education with improved access to information on safe sexual behavior through youth-oriented public awareness campaigns should be incorporated into the secondary school curriculum in Oyo state.

\section{Abstract \#: P 314}

Racial differences on oral health-related quality of life in adolescents - a multilevel analysis

Thiago M. Ardenghi ${ }^{1}$, Bruno Emmanuelli ${ }^{1}$, Fernanda Tomazoni ${ }^{1}$, Simone Tuchtenhagen ${ }^{1}$, Bernardo A. Agostini ${ }^{1}$

${ }^{1}$ Department of Stomatology, Federal University of Santa Maria, Santa Maria, Brazil; Corresponding author's e-mail: thiardenghi@hotmail.com

Background: Ethnic/racial inequalities has been recognized as an important predictor for disparities in oral health outcomes. However, there is scarce information on the influence of racial dissipativities on self-perceived oral health and quality of life of children and adolescents.

Objective: The aim of this study was to assess the influence of racial/ ethnics on Oral Health-Related Quality of Life (OHRQoL) of Brazilian adolescents.

Methods: This study followed a cross-sectional design, with a multistage random sample of 1134 12-year-old schoolchildren, representative of Santa Maria, a southern city in Brazil. Participants completed the Brazilian version of the Child Perceptions Questionnaire (CPQ11-14), their parents or guardians answered questions on socioeconomic status, and a dental examination provided information on the prevalence of caries, dental trauma and occlusion. The survey adopted a classification of the ethnic groups according to the criteria established by the Brazilian Institute of Geography and Statistics. The assessment of associations used multilevel Poisson regression models. Results: Higher impacts on OHRQOL were observed for adolescents from minority racial/ethnic group (non-white children). The mean CPQ 11-14 scores were 1.08 higher for non-white children than for their counterparts. Social Well-being and Emotional Limitation were the most affected domains, being the non-white children presented a mean score 1.19 and 1.14, respectively, higher than white ones (RR 1.19: $95 \%$ CI 1.07-1.33 and RR 1.14: $95 \%$ CI 1.04-1.24). This association remained significant, even after adjusting for individual and contextual-level covariates.

Conclusion: Oral health-related quality of life disparities persist in adolescents from minority racial/ethnic group: reducing health inequalities may demand dental programs and policies targeting minority racial/ethnic group.

\section{Abstract \#: P 315}

Using ecometrics to explore the relation between social capital and self-rated health, BMI and obesity-related behaviours: the European spotlight project

$\underline{\text { J.D. Mackenbach, On behalf of the SPOTLIGHT consortium }}{ }^{1}$

${ }^{1}$ Department of Epidemiology \& Biostatistics, EMGO Institute for Health and Care Research, VU Medical Center Amsterdam; Corresponding author's e-mail: j.mackenbach@vumc.nl

Background: Where we live matters for our health. Neighbourhood resources may explain variations in health behaviour and subsequent health outcomes. Understanding how contextual factors such as the neighbourhood physical or social environment affect behaviours and health may help to improve living conditions. Social capital has been proposed as an explanation for neighbourhood effects on health. 
However, evidence for this relation is not unambiguous. This may partly be due to the operationalization of social capital as contextual (rather than an individual) factor. By decomposing the social capital variables into individual and contextual variation, ecometrics allows for analysis with a social capital variable at the neighbourhood level, independent of the variation that can be attributed to individual response patterns. The objectives of this study were to apply an ecometric approach to assess the association between neighbourhood aspects of social capital and self-rated health, body mass index (BMI) and obesity-related behaviours across Europe.

Methods: Survey data were analysed from 6038 adult participants sampled within 60 neighbourhoods (heterogeneous in socioeconomic status and housing density) in five European countries. Factor analysis was used to identify constructs underlying the 13 social capital item responses. Ecometric measures of aspects of social capital were compared to aggregated neighbourhood sumscores of the same aspects and associated with self-rated health, BMI and obesity-related behaviours.

Results: Two factors labelled social cohesion (SC, range 5-25) and social network (SN, range 4-20) were identified. SC was highest in the Netherlands $($ mean $=15.4)$ and lowest in Hungary $($ mean $=13.5)$, while BMI was lowest in France $($ mean $=24.7)$ and highest in Hungary (mean $=26.3$ ). Preliminary analyses showed that individuals in the highest quartile of SC and SN (aggregate neighbourhood sumscores) had better self-rated health (B for SC $\mathrm{Q} 4=3.75$ (1.65: 5.86), B for SN Q4 = $2.66(0.54: 4.79)$, lower BMI (B for SC Q4 $=-0.86(-1.44:-0.28)$, B for SN Q4 $=-0.65$ $(-1.20:-0.10)$ after adjustment for covariates, but also less walking than individuals in the lowest quartile. Using quartiles of the ecometric measures of SC and SN generally generated similar associations (for example B for SN Q4 on general health $=2.64$ (0.28: 5.00)

Discussion: This is the first study to provide evidence for an association between SC and SN and BMI in a European context. Aspects of social capital and BMI differed between European countries. The results confirm those of earlier research by demonstrating an association between social capital and general health. Associations with obesity-related behaviours were less clear and need further study.

\section{Abstract \#: P 316}

\section{Hazardous alcohol consumption and its socio-demographic correlates among Russian adults: the Arkhangelsk-2012 study}

Olga Kotilevskaya ${ }^{1}$, Alexander Kudryavtsev ${ }^{1,2}$, Andrej M. Grjibovski $^{1,3}$

${ }^{1}$ International School of Public Health, Northern State Medical University, Arkhangelsk, Russia; ${ }^{2}$ University of Troms $\varnothing$, Norway; ${ }^{3}$ Norwegian Institute of Public Health, Oslo, Norway; Corresponding author's e-mail: andrej.grjibovski@gmail.com

Background: Hazardous alcohol consumption is a considerable public health problem with a substantial contribution to high mortality from external and cardiovascular causes in Russia. However, the prevalence of alcohol consumption varies substantially between the regions. The aim of this study is to study the prevalence of hazardous alcohol consumption and its socio-demographic correlates in an urban setting in Northwest Russia.

Methods: A cross-sectional, population-based study was conducted in 2012-2013 in Arkhangelsk, Northwest Russia. Participants were invited using a systematic sample from service coverage lists at municipal policlinics. Altogether, 448 individuals aged $18+(24 \%$ men and $76 \%$ women) participated in the study. Alcohol consumption was assessed using the AUDIT questionnaire. Hazardous alcohol consumption was defined as the AUDIT scores of 4 for women and 5 for men. Data on age, gender, occupation and marital status were also collected.

Results: The prevalence of hazardous alcohol consumption was 42.7 (95 \% CI: 32.7-52.8) among men and $19.6 \%$ (95 \% CI: 14.9-24.4) among women. In multivariable analysis, men $(\mathrm{OR}=2.9,95 \% \mathrm{CI}$ : 1.6-5.3), cohabiting partners $(\mathrm{OR}=4.7,95 \% \mathrm{CI}: 1.3-13.7)$, bluecollar workers $(\mathrm{OR}=2.1,95 \% \mathrm{CI}$ : $1.1-4.1)$ were more likely while responders in the age-group $60+$ years were less likely $(\mathrm{OR}=0.2$, $95 \%$ CI: 0.1-0.6) to report hazardous alcohol drinking. No interaction between the variables was observed.

Conclusions: A high proportion of both men and women reported hazardous alcohol consumption. Male gender, blue collar occupation and surprisingly cohabiting family status were associated with the outcome while older age was associated with lower odds for hazardous drinking. Further studies are needed on the validity of alcohol reporting and its effects on mortality and morbidity in Russia.

\section{Abstract \#: P 317}

\section{Perceived classism in the Netherlands: psychometric properties} of the perceived classism scale

Audrey M.W. Simons ${ }^{1}$, Danielle A.I. Groffen ${ }^{1}$, Annemarie Koster ${ }^{1}$, Hans Bosma

${ }^{1}$ Maastricht University; Corresponding author's e-mail: audrey.simons@maastrichtuniversity.nl

Background: High expectations of being successful in life and strong beliefs in personal responsibility might have a downside of increasing classism in Western countries. People who end up at the bottom of the socioeconomic hierarchy might feel ashamed, blamed, and looked down upon by others. Studies into perceived classism are mostly conducted in less egalitarian countries (e.g. US and UK). However, less is known about perceived classism in more egalitarian countries. In the comparatively egalitarian Netherlands, we studied both the psychometric properties of the Perceived Classism Scale (PCS), a questionnaire measuring perceived classism, and the prevalence of perceived classism.

Methods: In February 2013, the PCS was completed by 2096 participant of the Dutch Longitudinal Internet Studies for Social Sciences (LISS) $(78.9 \%$ : mean age $=50.79$ years, SD 17.24: $45.9 \%$ men). The original 8-item 'Perceived Stigmatisation Scale' (divided into two subscales: internalised and experienced stigma) was translated to Dutch and slightly adapted in order to more broadly cover socioeconomic grouping (e.g. "there have been times when I have felt ashamed because of my financial situation, educational level or occupation"). The reliability of the PCS was assessed by factor analyses and Cronbach $\alpha$ 's. To look at the construct validity, one-way analyses of variance and spearman's rho correlations were used to assess the association of perceived classism with socioeconomic status, mental and physical health characteristics, and felt inferiority characteristics (social inadequacy, shame, and self-esteem).

Results: The factor analysis of the 8 items generally confirmed the original factor structure, although 2 negatively-worded items tended to load on a separate factor. $62.5 \%$ of the variance was explained and both the 6- and the 8-item PCS showed good internal consistency (respectively, $\alpha=0.85$ and 0.75 ). The lowest income and occupations groups had the highest scores on the PCS. Higher scores on the PCS were further significantly associated with worse scores on physical and mental health variables, and with adverse scores on the inferiority measures. On average, $9.4 \%$ felt ashamed because of their class and $19.8 \%$ of the sample experienced some kind of classism. 
Conclusion: This study showed that the Perceived Classism Scale is a valid and reliable instrument to measure classism in a Dutch sample, and that classism also exists in a quite egalitarian country, such as the Netherlands. However, based on our results, we recommend both some changes in the PCS and further studies into the role of classism e.g. in explaining socioeconomic health inequalities.

\section{Abstract \#: P 318}

\section{The Italian network of metropolitan longitudinal studies} for health monitoring of immigrants and vulnerable groups: study introduction and cohorts description

Nicola Caranci ${ }^{1}$, Barbara Pacelli ${ }^{1}$, Nicolás Zengarini ${ }^{2}$, Teresa Spadea $^{2}$, Laura Grisotto ${ }^{3}$, Gianna Terni ${ }^{3}$, Serena Broccoli ${ }^{4,5}$, Paola Ballotari ${ }^{4,5}$, Paolo Giorgi Rossi ${ }^{4,5}$, Laura Cacciani ${ }^{6}$, Anna Maria Bargagli $^{6}$, Cristina Canova ${ }^{7}$, Laura Cestari ${ }^{7}$, Gianfranco Costanzo ${ }^{8}$, Alessio Petrelli ${ }^{8}$, and INMP working group*

${ }^{1}$ Regional Agency for Health and Social Care, Emilia-Romagna Region, Bologna, Italy; ${ }^{2}$ Epidemiology Unit, ASL TO3 Piedmont Region, Grugliasco (TO), Italy; ${ }^{3}$ Department of Statistics, University of Florence, Florence, Italy; ${ }^{4}$ Epidemiology Unit, Azienda Unità Sanitaria Locale, Reggio Emilia, Italy; ${ }^{5}$ IRCCS Arcispedale Santa Maria Nuova, Reggio Emilia, Italy; ${ }^{6}$ Department of Epidemiology, Lazio Regional Health Service, Rome, Italy; ${ }^{7}$ Department of Molecular Medicine University of Padova, Padova, Italy; ${ }^{8}$ National Institute for Health, Migration and Poverty (INMP), Rome, Italy;

* Alessio Petrelli, Gianfranco Costanzo, Concetta Mirisola, Teresa Spadea, Nicolás Zengarini, Luisa Mondo, Paolo Carnà, Giuseppe Costa, Nicola Caranci, Barbara Pacelli, Serena Broccoli, Laura Bonvicini, Paola Ballotari, Paolo Giorgi Rossi, Cristina Canova, Laura Cestari, Lorenzo Simonato, Laura Grisotto, Gianna Terni, Annibale Biggeri, Laura Cacciani, Anna Maria Bargagli, Nera Agabiti, Marina Davoli; Corresponding author's e-mail: ncaranci@ regione.emilia-romagna.it

Background: Italy has recently faced a rise in immigration flows. The evaluation of immigrant health status is complex, because of issues related to their identification in the health and population data, dynamics of migration patterns, and heterogeneity of immigrant populations. Due to the lack of longitudinal data on immigrants, previous studies conducted in Italy, mainly cross-sectional, saw only part of the phenomenon. In order to overcome these limitations, a study on immigrants health based on Census cohorts of residents has been set up by the Italian network of metropolitan longitudinal studies (MLSs). The objectives of this presentation are to describe the participating studies, with a first step of closed cohort design, and to estimate the mortality risks associated with immigrant status, which is the first condition among socio-demographic vulnerabilities having been analysed.

Methods: The MLSs network to date includes the cohorts from the cities of Rome, Turin, Venice, Reggio Emilia and Florence. Archives from the registry office, population census, cause of death registry and hospital discharge have been integrated. The closed cohort were defined by residents on 21/10/2001 followed up until 31/12/2012 (2010 Florence). Immigrants are defined as residents with citizenship of a High Migration Pressure Country. Incidence Rate Ratios (IRR) and $95 \%$ confidence intervals were estimated by means of Poisson model. Analysis were restricted to people aged 1-64 years and adjusted for age, gender and calendar year.

Results: The study population includes 3,646,774 members; the percentage of immigrants was $2.5 \%$ (ranging from $1.9 \%$ in Venice to $4.2 \%$ in Reggio Emilia). The number of deaths was 49,253 overall and 11,226, 1917, 1649, 27,797, 6227 respectively in Turin, Florence, Reggio Emilia, Rome, and Venice. Compared to Italians, immigrants had lower mortality in all cities, with the following IRRs $(95 \% \mathrm{CI})$ : Turin 0.73 (0.64-0.84), Florence 0.53 (0.28-0.98), Reggio Emilia 0.75 (0.55-1.03), Rome 0.62 (0.57-0.69), Venice 0.61 (0.42-0.90). IRRs were similar between males and females in Turin and Rome, while indications of heterogeneities were observed in the other cities (with lower risks for males in Florence and higher in Reggio Emilia and Venice).

Conclusion: The findings of this study are consistent with scientific evidences showing that immigrants living in Italy have lower mortality than native populations. These results can be partly explained by self-selection of people who migrate (healthy migrant effect) and by the return to the country of origin of severely ill people (salmon effect). Risks by gender seemed heterogeneous across cities. Moreover the immigrants benefit decreases in cities with higher migration prevalence in 2001, which probably have more foreigners with long stay in Italy. Other information gaps are to be closed; accounting for new entries in the cohorts will allow to enroll many immigrants arrived in Italy after 2001.

\section{Abstract \#: P 319}

Cigarette smoking and non-medical use of new type of drugs associated with family, school, and psychosocial factors among Chinese adolescents

\section{Ciyong $\mathrm{Lu}^{1}$, Lan Guo ${ }^{1}$, Wei-Hong Zhang ${ }^{2}$}

${ }^{1}$ Department of Medical Statistics and Epidemiology, School of Public Health, Sun Yat-sen University, Guangzhou 510080, People's Republic of China; ${ }^{2}$ Biostatistics and Clinical Research Centre, School of Public Health, Université Libre de Bruxelles (ULB), Belgium; Corresponding author's e-mail: 260004774@qq.com

Objective: To investigate the prevalence of cigarette smoking and non-medical use of new type of drugs among Chinese adolescents, to explore their common predictors and connections among family, school, and psychosocial domains.

Method: A national cross-sectional study was involved in a total of 105,752 students in grades 7 th-12th sampled from schools in eighteen cities of China. An anonymous self-administered questionnaire was used to survey main variables for interest included family factors, school factors, and psychosocial adjustment. Multilevel logistic regression analyses were used to explore potentially influential factors.

Results: Of the 105,752 participants, 48,906 were boys: a total of $49.1 \%$ were from grade 7 th to grade 9 th. The mean onset age of smoking is 13.16 (SD 4.84) years old. The adjusted prevalence of heavy smoking, problem drug use, and drug use disorder was $2.12 \%$, 0.52 and $0.24 \%$, respectively: there was a uniformly higher prevalence of mild use than use-related problems or disorders. Boys were 4.78 times more likely to smoke heavily than girls, and the mean onset age of boys is earlier than girls. Having 1-2 siblings was the protective predictor for heavy smoking: being a school bully or victim were both risk predictors for using most substances. It is rather remarkable that mostly negative family, school and psychosocial factors were associated with cigarette smoking and drug use.

Conclusion: This study indicates that cigarette smoking and new type of drugs use among Chinese adolescents is a major public health problem nationwide, and a well-established surveillance is needed in China. 


\section{Abstract \#: P 320}

Serum 25 hydroxyvitamin D and total mortality among women diagnosed with breast cancer

Johanna Eyrun Torfadottir ${ }^{1,2}$, Unnur A. Valdimarsdóttir ${ }^{1,3}$, Thor Aspelund $^{1,4}$, Laufey Trygg vadóttir ${ }^{5}$, Thorhallur I. Halldorsson ${ }^{2}$, Tamara B. Harris ${ }^{6}$, Mary Frances Cotch ${ }^{7}$, Meir J. Stampfer ${ }^{3,8,9}$, Lorelei A. Mucci ${ }^{3,8,9}$, Edward L. Giovannucci ${ }^{3,8,9}$, Vilmundur Gudnason $^{4,10}$, Laufey Steingrimsdottir ${ }^{2}$

${ }^{1}$ Centre of Public Health Sciences, Faculty of Medicine, University of Iceland; ${ }^{2}$ Unit for Nutrition Research, Faculty for Food Science and Nutrition, University of Iceland, Reykjavik; ${ }^{3}$ Department of Epidemiology, Harvard School of Public Health, Boston; ${ }^{4}$ The Icelandic Heart Association, Kopavogur; ${ }^{5}$ The Icelandic Cancer Registry, Reykjavik \& Faculty of Medicine, University of Iceland; ${ }^{6}$ Laboratory of Epidemiology, Demography, and Biometry, Intramural Research Program, National Institute on Aging, Bethesda; ${ }^{7}$ Division of Epidemiology and Clinical Applications, National Eye Institute, National Institutes of Health, Bethesda; ${ }^{8}$ Channing Division of Network Medicine, Department of Medicine, Brigham; ${ }^{9}$ Women's Hospital and Harvard Medical School, Boston; ${ }^{10}$ Faculty of Medicine, University of Iceland; Corresponding author's e-mail: jet@hi.is

Background: Limited data are available on the association of vitamin $\mathrm{D}$ status with total mortality among women later diagnosed with breast cancer (BC). Our aim was to examine whether prediagnostic levels of vitamin $\mathrm{D}$ measured in serum from older women who develop BC, was associated with all-cause mortality.

Methods: Participants in this analysis were 91 women, aged 66-97 years, who were diagnosed with breast cancer after entry to the AGES-Reykjavik cohort and had 25-hydroxy-vitamin-D (25-OHD) measured at study entry (2002-2006). The women were followed for all-cause mortality through 2013. Cox proportional hazard regression models were used to assess the association between 25-OHD and allcause mortality. Analysis were adjusted for age at entry, age at BC diagnosis, smoking status, body mass index and physical activity.

Results: Mean age (SD) at diagnosis was 80.7 (6.1) years and mean serum 25-OHD levels among the women at study entry (on average 4.6 years earlier) was $51.4(24.1) \mathrm{nmol} / \mathrm{L}$. A total of $34(37 \%)$ women died during follow-up. There was statistically significantly lower all-cause mortality (hazard ratio $(\mathrm{HR})=0.32,95 \%$ confidence interval (CI): 0.11-0.92) among women in the top tertile of 25-OHD $(\geq 59.4 \mathrm{nmol} / \mathrm{L})$ and the mid-tertile $(39.4-59.3 \mathrm{nmol} / \mathrm{L})(\mathrm{HR}=0.72$ : 95\% CI: 0.32-1.66) compared with those in the bottom tertile $(\leq 39.3 \mathrm{nmol} / \mathrm{L})$. Further adjusting for receptor- and lymph node status did not alter the results significantly.

Conclusion: Although based on small numbers, our data suggest that higher prediagnostic serum 25-OHD levels may be associated with improved survival among women diagnosed with breast cancer late in life.

\section{Abstract \#: P 321}

The additional value of mammography to MRI screening in BRCA1 and BRCA2 mutation carriers: an individual patient data meta-analysis

Xuan-Anh Phi ${ }^{1}$, Sepideh Saadatmaand ${ }^{2}$, Nehmat Houssami ${ }^{3}$, Madeleine M.A. Tilanus-Linthorst ${ }^{2}$, Edwin R. Van den Heuvel ${ }^{1}$, Geertruida H. De Bock ${ }^{1}$

${ }^{1}$ Department of Epidemiology - University Medical Center Groningen - Groningen - The Netherlands; ${ }^{2}$ Department of Surgery - Erasmus

University Medical Center Rotterdam - Rotterdam - The Netherlands;
${ }^{3}$ School of Public Health, Sydney Medical School, Univers; Corresponding author's e-mail: x.a.phi@umcg.nl

Introduction: Women with BRCA1/2 mutation are recommended with annual breast screen by both MRI and mammography. Due to low sensitivity of mammography both in women at young age and older than 50, there is discussion whether women with BRCA1/2 mutations can be screened with only MRI and if mammography is needed, at which age it should start. This study investigates the evidence on the contribution of mammography to MRI screening in women with a BRCA1/2 mutations at different ages.

Methods: We conducted an Individual Patient Data meta-analysis by pooling patient data from six high-risk screening trials. Women with a BRCA1/2 mutation, having at least one screening by both MRI and mammography were included. Sensitivity of the combination of two tests and of MRI alone were estimated and compared in groups of women according to their BRCA mutation and age using generalized linear mixed model. The number of screens needed for mammography to contribute one breast cancer was also calculated.

Results: In women with a BRCA1 mutation, adding mammography to MRI increased the screening sensitivity by $9.3 \%$ in women younger than $40(P>0.05)$ and in older women the sensitivity did not change statistically significant. In women with a BRCA2 mutation, adding mammography to MRI increased screening sensitivity by $34.5 \%$ in women age $\leq 40(P>0.05)$, by $5-10 \%$ in women age above age $40(P>0.05)$. Further 641 screens are needed for mammography to detect one breast cancer not detected by MRI in women with BRCA1 mutation which is four times higher than that for women with BRCA2 mutation.

Conclusion: The additional screening sensitivity from mammography above that from MRI is limited in BRCA1 mutation carriers, whereas it seems relevant in BRCA2 mutation carriers especially those aged $\leq 40$ years. Our work highlights that a differential screening schedule by BRCA status is worth considering.

\section{Abstract \#: P 322}

The order of radiotherapy and chemotherapy in early breast cancer and its effect on outcome

S.Siesling ${ }^{4,5}$, E.H. Gort ${ }^{1}$, S.G. Elias ${ }^{1,2}$, P.H.M. Elkhuizen ${ }^{1}$, S. Siesling ${ }^{2}$, V. Ho ${ }^{3}$, O. Visser ${ }^{4}$ and S.C. Linn ${ }^{1}$

${ }^{1}$ Antoni van Leeuwenhoek Hospital/Netherlands Cancer Institute; ${ }^{2}$ Julius Center for Health Sciences and Primary Care, University Medical Center Utrecht, the Netherlands; ${ }^{3}$ Comprehensive Cancer Center Netherlands, Utrecht, the Netherlands; ${ }^{4}$ Netherlands Comprehensive Cancer Organisation (IKNL), Dept Registration and Research, Utrecht, the Netherlands; ${ }^{5}$ University of Twente MIRA Institute for Biomedical Technology and Technical Medicine, Enschede, the Netherlands; Corresponding author's e-mail: s.siesling@iknl.nl

Background \& introduction: Early breast cancer patients with unfavorable prognostic factors are often treated with both chemotherapy (CT) and radiotherapy (RT). Whereas a delay in start of RT or CT may result in increased risk of local and systemic recurrence respectively, the optimal sequence of $\mathrm{RT}$ and $\mathrm{CT}$ is subject for debate. The effect of RT and CT sequence on overall-survival (OS) and recurrence-free survival (RFS) was studied using a population-based cancer registry.

Materials \& methods: Data from all primary breast cancer patients diagnosed in the Netherlands from 1999 to 2008 were obtained from the Netherlands Cancer Registry. Cox and competing risk regression was used to compare OS and RFS in two treatment groups (RT first or 
CT first) while adjusting for important prognostic variables. Sequencing was arbitrary and not according to national guidelines, Results: Between 1999 and 2008 22,045 primary breast cancer patients that received both $\mathrm{CT}$ and RT were registered by the Netherlands Cancer Registry. Of these patients 9977 (45 \%) received RT first and 12,068 (55\%) received CT first. During a total of 112,872 patient years of follow-up (median 57 months), 3075 patients died. Multivariate analysis with adjustments for age, stage, number of positive lymph nodes, histological grade and administration of hormone therapy, showed no overall survival difference for CT first compared to RT first (hazard ratio (HR) 0.97 ; $95 \%$ CI 0.90-1.05; $P=0.46)$. Additional adjustment for hormone receptor status, HER2 status and tumor diameter available for a subgroup of 9951 patients confirmed these results (HR 0.96; $95 \%$ CI $0.79-1.16$; $P=0.66$ ). In 6582 patients data on recurrent disease was specifically documented in the registry of whom 1259 patients experienced one or more recurrences during a total of 30,762 patient years of follow-up (median 61 months). No significant recurrence-free survival benefit was seen for either treatment sequence (HR 0.97; $95 \%$ CI $0.86-1.10$; $P=0.64)$.

Conclusions: The sequence of radio- and chemotherapy as practiced in routine clinical care did not affect overall-survival or recurrencefree survival in a large analysis of prospectively collected cancer registry data of primary breast cancer patients after a median followup of approximately 5 years.

\section{Abstract \#: P 323}

\section{Survival analysis of breast cancer in patient living rabat/morocco (2005-2008)}

Nada Bennani Mechita ${ }^{1}$, Mohammed Adnane Tazi ${ }^{2}$,

Abdelouahed Er-raki ${ }^{3}$, Noureddine Benjaafar ${ }^{3}$, Asma Saadi ${ }^{1}$, Mustapha Mrabet ${ }^{1}$, Rachid Razine ${ }^{1}$

${ }^{1}$ Department of Public Health, Faculty of Medicine and Pharmacy, University Mohammed V Rabat: Morocco; ${ }^{2}$ Direction of Epidemiology and Disease Control, Cancer Registry of Rabat, Morocco; ${ }^{3}$ Cancer Registry Rabat, Morocco; Corresponding author's e-mail: nadaben7@gmail.com

Background: Breast cancer is a major public health problem worldwide, it is the second most common cancer in terms of frequency in the world affecting 1.7 million cases in 2012, representing $11.9 \%$ of total cancers. Breast cancer is the most common cause of death in women worldwide (522,000 deaths). The aim of this work is to estimate the overall survival rate for breast cancer in patients living in the district of Rabat and diagnosed for breast cancer between 2005 and 2008.

Materials and methods: This is a prognostic study in all patients diagnosed for breast cancer from 2005 to 2008 , living in the district of Rabat and recorded in the Register of Rabat cancers (population register). The variables collected were: age, date of diagnosis, histological type, tumor stage and type of treatment. Data on survival status of the patients were collected at the level of patient's care units and from the registry of deaths in the city of Rabat. The date of inclusion in the study was considered as the date of histological diagnosis of cancer. The point date was set at 07/01/14. Estimation of survival was performed by the Kaplan-Meier method, and the comparison between the different classes of a variable has been performed by the log rank test. The study of factors associated with survival was performed by the Cox model. The significance level was set at 0.05 . Data analysis was performed by SPSS software version 13.0

Results: During the study period 619 cases of breast cancer were collected. The mean age was $51.0 \pm 11.2$ years. The upper outer quadrant was the predominant site of the tumor $(41.7 \%: \mathrm{n}=129)$ and the histological type most frequently observed was invasive ductal carcinoma ( $84.3 \%: \mathrm{n}=519)$. The cancer was stage $\mathrm{I}$ in 83 patients $(15.3 \%)$ and stage II in 262 patients (48.3\%). Only 40 patients $(7.4 \%)$ were stage IV. The surgery was performed in 556 patients $(90.0 \%)$, radiation therapy in 455 patients $(73.5 \%)$, chemotherapy in 484 patients $(78.2 \%)$ and hormonal therapy in 306 patients $(49.4 \%)$. The percentage of death was $19.1 \%(n=118)$. Overall survival at one year was $96.9,87.9 \%$ at 3 years and $76.9 \%$ at 5 years. In univariate analysis, survival was statistically associated with age $(p<0.001)$, the size of the tumor $(p<0.001)$, the class of lymph nodes $(p<0.001)$, the presence of metastases $(p<0.001)$ and the stage of the tumor $(p<0.001)$. The 5 -year survival was better in the age group $40-54$ years $(80.3 \%)$, for tumors classified T1 $(90.8 \%)$, in the absence of lymphadenopathy $(88.7 \%)$, in the absence of metastases $(82.3 \%)$ and for stage I $(90.3 \%)$. In multivariate analysis, survival of breast cancer was statistically associated with age $(p<0.001) \quad(40-54$ years: $\mathrm{RR}=0.55 \quad[0.30-0.99]$, 55-69 years: $R R=0.68[0.36-1.29]$, more than 70 years: $R R 2.63$ [1.28-5.40] Reference Class $>40$ years), the size of the tumor $(p=0.017) . \quad(\mathrm{T} 2: \quad \mathrm{RR}=2.41 \quad[0.84-6.88] \quad \mathrm{T} 3: \quad \mathrm{RR}=3.66$ [1.14-11.84] T4: $\mathrm{RR}=5.79$ [1.80-18.64]. reference class $\mathrm{T} 1)$ and the metastases $(p=0.038)(\mathrm{M}+: \mathrm{RR}=5.47$ [1.09-27.31]).

Conclusion: The size of the tumor and metastases influence survival in cancer, hence the need to strengthen screening to improve the prognosis of this cancer.

\section{Abstract \#: P 324}

Population-wide effects of an organised mammography screening program in north-western Germany - a register-based time trend study

Hans-Werner Hense $^{1}$, Jan Heidrich ${ }^{2}$, Hiltraud Kajüter ${ }^{2}$, Ina Wellmann $^{1}$, Oliver Heidinger ${ }^{2}$

${ }^{1}$ University Münster, Institute of Epidemiology and Social Medicine, Münster, Germany; ${ }^{2}$ Epidemiological Cancer Register NRW, Münster, Germany; Corresponding author's e-mail: hense@unimuenster.de

Background: The population-wide systematic, quality-controlled mammography screening program (MSP) in Germany invites all resident women aged 50-69 years (target population) bi-annually for screening examinations that are free of charge. We investigated changes in the epidemiology of breast cancer in the target population of north-western Germany observable in the wake of this program.

Methods: Invasive (ICD-10: C50) and in situ (D 05) breast cancer incidence rates were recorded in the Epidemiological Cancer Registry of North-Rhine Westphalia (EKR NRW): since 1994, this register covered the District Münster (RB-MS, 2.7 million population) and, since 2006, the encompassing Province of Westphalia-Lippe (W-L: 8.6 million population). Completeness of registration is high $(>90 \%)$. The MSP started in October 2005 in RB-MS and was subsequently implemented in all of W-L (completed by 2009). We examined changes in the numbers of incident breast cancers and shifts in their stage distributions in the entire target population. Three-yearsaveraged incidence rates of advanced stages (UICC stages II+) were calculated and assessed by join-point analyses, after imputing missing tumor stage data. Interval cancers (IC) for the initial screening round (up to end of 2008) were identified in MSP participants during the 24 months after an inconspicuous screening mammography. We used data from the RB-MS to describe the period 1994-2005 and from W-L for the ensuing period 2006-2012. 
Results: Among the more than 1 million women of the target population in W-L, MSP participation was at about $55 \%$. The incidence of invasive breast cancer rose initially after MSP start and showed still elevated levels in 2012. DCIS rates remained clearly elevated up to 2012. Thus, in 2012, $14 \%$ of all incident cases in the entire target population were DCIS (compared to $7 \%$ in 2002-2004) while the proportion of cancers $>20 \mathrm{~mm}$ had declined to $33 \%$ (42\%, resp.) After a short rise during the initial MSP phase, the incidence rates of advanced stages (UICC II+) declined significantly and consistently after 2007 (annual percentage change $-6 \%$ ). IC rates for the first screening round (follow-up until end 2010) were 2.45 per 1000 women negatively screened and accounted for about 1 in 5 cancer cases among MSP screenees.

Conclusions: The MSP has markedly changed the epidemiology of breast cancer in the target population of this large study region. The role of overdiagnosis and the impact on breast cancer mortality are presently unclear and require further concurrent epidemiological evaluation of the on-going program.

\section{Abstract \#: P 325}

\section{Insulin treatment and breast cancer risk: a systematic review} of in vitro, animal and epidemiological evidence

Heleen K. Bronsveld ${ }^{1}$, Bas ter Braak ${ }^{2}$, Øystein Karlstad ${ }^{3}$, Peter Vestergaard $^{4}$, Jakob Starup-Linde ${ }^{5}$, Marloes T. Bazelier ${ }^{6}$, Marie L. de Bruin $^{6}$, Anthonius de Boer ${ }^{6}$, Christine L. E. Siezen ${ }^{7}$, Bob van de Water $^{8}$, Jan Willem van der Laan ${ }^{8}$, Marjanka K. Schmidt ${ }^{9}$

${ }^{1}$ Division of Molecular Pathology, The Netherlands Cancer Institute, Amsterdam, Netherlands; ${ }^{2}$ Division of Toxicology, Leiden Academic Centre for Drug Research, Leiden University, Leiden, the Netherlands; ${ }^{3}$ Department of Pharmacoepidemiology, Norwegian Institute of Public Health, Oslo, Norway; ${ }^{4}$ Departments of Clinical Medicine and Endocrinology, Aalborg University, Aalborg, Denmark; ${ }^{5}$ Department of Endocrinology and Internal Medicine (MEA), Aarhus University Hospital THG, Aarhus, Denmark; ${ }^{6}$ Division of Pharmacoepidemiology \& Clinical Pharmacology, Utrecht University, Utrecht, Netherlands; ${ }^{7}$ Medicines Evaluation Board (MEB), Utrecht, The Netherlands; ${ }^{8}$ Division of Toxicology, Leiden Academic Centre for Drug Research, Leiden University, Leiden, the Netherlands; ${ }^{9}$ Division of Molecular Pathology, The Netherlands Cancer Institute, Amsterdam, Netherlands; Corresponding author's e-mail: h.bronsveld@nki.nl

Background: In 2009 the concern has been raised that insulin analogues, especially insulin glargine, might increase risk of (breast) cancer. Many in vitro and epidemiological, and some animal studies have been performed, but there is still no clarity on this issue. This systematic review, therefore, investigates the association between insulin and insulin analogue treatment and breast cancer development, and plausible mechanisms, based on in vitro, animal and epidemiological evidence.

Methods: A systematic literature search was performed on breast cell-line, animal and human studies using the key words 'insulin analogue' and 'breast neoplasia' in MEDLINE at PubMed, EMBASE and ISI Web of Science databases. A quantitative and qualitative review was performed on the epidemiological data and a complete overview was composed for in vitro and animal studies. Protein and gene expression was analysed for the cell lines most frequently used in the included in vitro studies.

Results: 16 in vitro, 5 animal, 2 in vivo human and 29 epidemiological papers were included. Insulin AspB10 showed mitogenic properties in in vitro and animal studies. Glargine was the only clinically available insulin analogue for which an increased proliferative potential was found in breast cancer cell lines. However, the pooled analysis of 13 epidemiological studies did not show evidence for an association between insulin glargine treatment and increased breast cancer risk $(\mathrm{HR}=1.04,95 \% \mathrm{CI}=0.91-1.17$, $p=0.49)$ versus no glargine in patients with diabetes mellitus. It has to be taken into account that animal data was limited, epidemiological studies were underpowered and suffered from methodological limitations.

Conclusion: There is no compelling evidence that any clinically available insulin analogue increases breast cancer risk. Overall, the data suggests that insulin treatment is not involved in breast tumour initiation, but might induce breast tumour progression by up regulating mitogenic signalling pathways.

\section{Abstract \#: P 326}

Cholesterol and breast cancer risk: a systematic review and metaanalysis of prospective studies

Philippine Fassier ${ }^{1}$, Paule Latino-Martel ${ }^{1}$, Mathilde His ${ }^{1}$, Teresa Norat $^{2}$, Doris SM Chan ${ }^{2}$, Jacques Blacher ${ }^{3}$, Serge Hercberg ${ }^{1}$, Pilar Galan ${ }^{1}$, Nathalie Druesne-Pecollo ${ }^{1}$, Mathilde Touvier ${ }^{1}$

${ }^{1}$ Sorbonne Paris Cité Epidemiology and Biostatistics Research Center, Nutritional Epidemiology Research Team (EREN), Inserm U1153, Inra U1125, Cnam, University Paris 13, University Paris 5, University Paris 7, F-93017, Bobigny, France; ${ }^{2}$ Department of Epidemiology and Public Health, Imperial College, London, UK; ${ }^{3}$ Diagnosis and Therapeutic Center, Hôtel-Dieu Hospital, F-75004 Paris, France; Corresponding author's e-mail:

p.fassier@uren.smbh.univ-paris13.fr

Background: Our objective was to conduct the first systematic review and meta-analysis of prospective studies on total cholesterol (T-C), high density lipoprotein cholesterol (HDL-C), and low density lipoprotein cholesterol (LDL-C) and breast cancer risk.

Methods: Relevant studies were identified in PubMed (up to January 2014). Inclusion criteria were original peer-reviewed publications with a prospective design. Random effects models were used to estimate summary hazard ratios (HRs) and $95 \%$ confidence intervals. Distinction was made between studies that did or did not exclude cancer cases diagnosed during the first years of follow-up, thereby eliminating potential preclinical bias.

Results: Overall, summary associations with breast cancer risk were $\mathrm{HR}=0.97(0.94-1.00)($ dose-response per $1 \mathrm{mmol} / \mathrm{L}$ increment, 13 studies) for T-C and $\mathrm{HR}=0.86(0.69-1.09)$ (dose-response per $1 \mathrm{mmol} / \mathrm{L}$ increment, 6 studies) for HDL-C, with high heterogeneity (I2 $=67$ and $47 \%$, respectively). When focusing on studies that eliminated preclinical bias, T-C (dose-response $\mathrm{HR}=0.94$ (0.89-0.99), 7 studies, I2 $=78 \% /$ highest versus lowest $\mathrm{HR}=0.82$ (0.66, 1.02), 9 studies, I $2=81 \%$ ) and HDL-C (dose-response $\mathrm{HR}=0.81(0.65-1.02), 5$ studies, $\mathrm{I} 2=30 \% /$ highest versus lowest $\mathrm{HR}=0.82(0.69-0.98), 5$ studies, I $2=0 \%)$ were inversely associated with breast cancer risk. There was no association between LDL$\mathrm{C}$ and breast cancer risk (4 studies).

Conclusions: These meta-analyses support the evidence of a modest but statistically significant inverse association between $\mathrm{T}-\mathrm{C}$, and more specifically HDL-C and breast cancer risk, consistent with mechanistic plausibility from experimental studies. Further large prospective studies that adequately control for preclinical bias are needed.

If confirmed, these results suggest that controlling cholesterol level and its fractions may contribute to breast cancer prevention. 


\section{Abstract \#: P 327}

Towards personalized screening: cumulative risk of breast cancer screening outcomes in women with and without first-degree relatives with breast cancer

\section{$\underline{\text { T.M. Ripping }}^{1}$, A.L.M. Verbeek ${ }^{1}$, R.A. Hubbard ${ }^{2}$, M.J.M. Broeders ${ }^{3,4}$}

${ }^{1}$ Department for Health Evidence, Radboudumc, Nijmegen, The Netherlands; ${ }^{2}$ Department of Biostatistics, University of Pennsylvania, Philadelphia, PA, USA; ${ }^{3}$ Department for Health Evidence, Radboudumc, Nijmegen, The Netherlands; ${ }^{4}$ LRCB, Nijmegen, The Netherlands; Corresponding author's e-mail: dorien.hoeve-ripping@radboudumc.nl

Background: In virtually all population-based mammographic screening programs, the target population for screening is defined by age alone. Other characteristics, such as family history of breast cancer, may also influence the benefits and harms of screening. Until now, it is unknown to what extent a family history of breast cancer influences the balance between these benefits and harms. Therefore, the aim of this study is to calculate the cumulative risk of primary screening outcomes-screen-detected breast cancer, interval cancer, and false-positive results-in women with and without a first-degree relative with a history of breast cancer. Our study population includes women from the start of screening at age 50 and invited to participate until age 74 .

Methods: The Nijmegen Screening Program holds data on screening attendance, recall, screen-detected breast cancer, interval cancer and false positive results for each woman living in Nijmegen, the Netherlands. We used a discrete time survival model to calculate the cumulative probability of each major screening outcome.

Results: Women with a family history of breast cancer have slightly higher risks for all screening outcomes, both benefits and harms. For women who are screened biennially from 50 to 74 , the cumulative risk screen-detected breast cancer, interval cancer and a false positive result are 9.2, 4.4 and $10.8 \%$, respectively, for women with a family history and 6.3, 2.7 and $7.5 \%$, respectively, for women without a family history.

Discussion: Women who participate in screening and have at least one first-degree relative with a history of breast cancer are more likely to have a screen-detected breast cancer, interval cancer and falsepositive result than women without a family history. As a consequence, harms of screening as well as the cancer detection rate are higher for women with a family history than for women without a family history. We need, however, to investigate whether observed increases in the screen-detected cancer rate translate into decreases in breast cancer mortality for women with a family history before we can compare the benefit-harm balance.

\section{Abstract \#: P 328}

Low-grade inflammation and oxidative stress and risk of invasive post-menopausal breast cancer - a nested case-control study

Joana A. Dias ${ }^{1}$

${ }^{1}$ Lund University; Corresponding author's e-mail: joana.alves_dias@med.lu.se

Background: The established risk factors for breast cancer (such as early menarche, late menopause, no children, no breastfeeding, old age at the time of first born) are associated with a lifetime exposure to estrogens. Lifestyle choices like alcohol and hormone use (i.e. contraceptives or menopausal hormone therapy, MHT) and height are documented to increase the risk for post-menopausal breast cancer.
Obesity may contribute to breast cancer risk, through several mechanisms involving inflammatory pathways or deregulated metabolism. Inflammation promotes the increase of free radicals and stimulates oxidative stress, which could influence DNA-methylation. Although it is accepted that cancer promotes inflammation, it is less well established role of inflammation in tumor-genesis.

Objectives: The aim was to examine if low-grade inflammation is associated with post-menopausal breast cancer in women from the Malmö Diet and Cancer cohort (MDC) and the potential influence of obesity on this association.

Methods: The association between inflammation and breast cancer was examined prospectively in a nested case-control study in the MDC. Out of 8513 women cancer-free at baseline (1991-1996) and $\geq 55$ years old, 459 were diagnosed with invasive breast cancer during follow-up (until December 31st, 2010). Plasma samples were available and laboratory analysis was successful in 446 cases, and in 910 controls ( 2 controls per case, matched on age and date of measurement).

We analyzed several inflammation markers: oxidized-LDL, IL-1 $\beta$, IL-6, IL-8, TNF- $\alpha$, and white blood cells, lymphocyte and neutrophil counts. We considered potential confounders: BMI, waist-to-hip ratio, MHT, parity, smoking status, alcohol intake, physical activity, and education. We calculated the odds ratios $(\mathrm{OR})$ and $95 \%$ confidence intervals (CI) for breast cancer comparing the higher tertiles to the lowest using multivariate unconditional logistic regression.

Results: Participants in the higher tertiles of ox-LDL had lower breast cancer risk compared to the first tertile (OR: 0.71: 95 \% CI: 0.52-0.97 and OR: 0.69: $95 \%$ CI: $0.50-0.96$ for 2 nd and 3rd tertiles, respectively), when adjusting for matching factors and confounders ( $p$ trend $=0.03$ ). The opposite was seen for IL-1 $\beta$ : those in the highest category had higher risk, OR: 1.70: $95 \%$ CI: 1.19-2.43 ( $p$ trend $=0.009)$. Being overweight carried increased risk, OR: 1.32 : $95 \%$ CI: $1.03-1.70$ when compared to normal weight. However, when adding obesity variables to the models as confounders, no change in estimates was observed.

Conclusion: Our results do not suggest that inflammation increases the risk of post-menopausal breast cancer. Although obesity was associated with breast cancer, and with some biomarkers, associations were not affected.

\section{Abstract \#: P 329}

\section{Bladder tumors infiltrating the muscle (BTIM)} in the octogenarian in SFAX, TUNISIA

Asma S. Saadi ${ }^{1}$, Nada B. M. Bennani Mechita ${ }^{1}$, Mohamed Nabil M. Mhiri $^{2}$, Mustapha M. Mradet ${ }^{3}$, Rachid R. Razine ${ }^{1}$

${ }^{1}$ Department of Public Health, Faculty of Medicine and Pharmacy, Rabat, MOROCCO; ${ }^{2}$ Department of Urology, University Hospital Habib Bourguiba, Sfax, TUNISIA; ${ }^{3}$ Department of Environmental Health, Military Hospital of Instruction Mohamed V, Rabat, MOROCCO; Corresponding author's e-mail: asmasaadi372@gmail.com

Background: A muscle invasive bladder tumor is a malignant tumor characterized by the invasion of the bladder muscle by urothelial cells. Bladder cancer typically occurs in the elderly between 50 and 70 years, but with the improved life expectancy, the incidence of bladder tumors in old age is becoming higher with octogenarian patients. The objectives of this study were: to draw the epidemiological profile of patients with BTIM among all octogenarians, study the evolution and prognosis of these tumors in these patients. Methods: A retrospective descriptive study from 1982 to 2010 performed at the Department of Urology at the University Hospital 
Habib Bourguiba in Sfax, Tunisia: on 22 cases of BTIM in patients aged 80 and over, and this among 52 cases of bladder tumors in the octogenarian. Moreover, 1100 cases of bladder tumors all ages were collected in this service. Data were collected from hospital records on pre established fact sheets about: biographical data of the patients (age, sex, geographic origin) and data on hospitalization (consultation period, length of stay, medical and surgical history, comorbid conditions, circumstances of discovery, clinical, radiological and histological, therapeutic alternatives, evolution and prognosis.

Results: The mean age was $84.2 \pm 3.2$ years. The sex ratio M/F was 1.2. All patients had varied severity of defects. Cardiovascular comorbidity factors were present in $100 \%$ of patients, the discovery was fortuitous in 7 patients $(31.8 \%)$, hematuria was the most important reason for revelation (100\% of cases). Our patients were classified according to the score of the American Association of Anesthesiology ASA I-II: 7 patients (31.8\%) and ASA III-IV: 15 patients $(68.18 \%)$. The conventional urothelial carcinoma was the most common 12 patients $(54.5 \%)$, there was metastasis in a patient operated and in 09 patients followed by radiation therapy, therapy abstention has involved 10 patients in relation to their high comorbidity, a radical cystectomy with cystoplasty was performed in 4 patients, radiation therapy was performed in 8 patients. Early postoperative course was good in $100 \%$ of cases and perioperative mortality was zero. Late postoperative course was marked by the appearance of nocturnal urinary leakage in 10 patients or erectile impotence in 05 patients.

Conclusion: The chronological age should not be against the surgical treatment of BTIM, it would be rather physiological age modified by comorbidities and locoregional extension assessment of tumor affecting the prognosis of BTIM in elderly patients 80 years and older.

\section{Abstract \#: P 331}

\section{Aftercare for prostate cancer survivors: relevant information for general practitioners in international guidelines}

\author{
Inge Spronk ${ }^{1}$, Francois G. Schellevis ${ }^{1}$, Joke C. Korevaar ${ }^{1}$
}

${ }^{1}$ IVEL, Netherlands Institute for Health Services Research, Utrecht, The Netherlands; Corresponding author's e-mail: i.spronk@nivel.nl

Background: Due to improved treatment results and an aging population, the number of cancer survivors requiring aftercare is rising. This places extra burden on hospital care. Therefore there are increasing calls for greater involvement of General Practitioners (GPs) in the aftercare of cancer, so also for prostate cancer survivors. To date, there is little information for GPs on the most optimal way to provide this aftercare. The aim of this overview is to identify relevant topics from existing guidelines to be synthesized into a guideline for GPs on aftercare of prostate cancer survivors.

Methods: As part of CanCon (EU Joint Action Cancer Control), an inventory of international guidelines was undertaken. Internet and literature was searched and a survey was sent to European Union Member States to reveal national and regional guidelines on aftercare for curatively treated prostate cancer survivors. Relevant information and recommendations for GPs were extracted and summarized in topics.

Results: So far, thirteen guidelines from nine countries were included: three were specific on aftercare and two were specifically made for GPs. Eight relevant topics were identified. Most attention was paid to the detection of cancer recurrence. All guidelines highlighted the importance of PSA testing. Wide agreement exists about the frequency of PSA testing, namely at least every six months in the first 3 years after treatment and thereafter (semi-)annually. The majority of guidelines discussed digital rectal examination (DRE): however there is disagreement about its value. Few guidelines reported information on treatment of potential complications, and only one mentioned health promotion for survivors.

Conclusion: This inventory gives GPs a comprehensive view of evidence based guidance and opinion based advices on aftercare for prostate cancer survivors. Guidelines yielded several topics on aftercare. The main subject in all guidelines is the detection of cancer recurrence, including the topics PSA testing, DRE and signs of recurrence. There is overall agreement on the value of PSA testing, but not on the use of DRE. Other important topics to include in the guideline for GPs on aftercare are: potential complications, treatment of complications, health promotion, shared care among caregivers and patient information.

\section{Abstract \#: P 332}

Survival analysis in cervix cancer in cancers registry of rabat, morocco from 2005 to 2008

Asma S. Saadi ${ }^{1}$, Mohamed Adnane T. Tazi ${ }^{2}$, Abdelwahed E. Er-raki ${ }^{3}$,

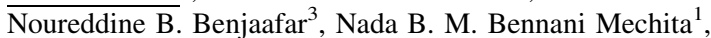
Mustapha M. Mrabet ${ }^{4}$, Rachid R. Razine ${ }^{1}$

${ }^{1}$ Department of Public Health, Faculty of Medicine and Pharmacy, Rabat, Morocco; ${ }^{2}$ Department of Epidemiology and Disease Control, Ministry of Health, Rabat, Morocco; ${ }^{3}$ Cancers Registry, Rabat, Morocco; ${ }^{4}$ Environmental Health Department, Military Hospital of Instruction Mohamed V, Rabat, Morocco; Corresponding author's e-mail: asmasaadi372@gmail.com

Background: In the world, cervix cancer is the second female cancer in terms of incidence with 528,000 cases each year, and the fourth leading cause of cancer death in women with 266,000 deaths in 2012. In Morocco, it represents a public health problem: it is the second most common cancer in women after breast cancer, with around 2000 new cases each year. The main objective of this study is to estimate overall survival in cervical cancer patients living in Rabat.

Methods: This is a prognostic study of all cases of cancer of the cervix registered in the cancers registry of Rabat (population registry) between 2005 and 2008. The main collected variables were: age, date of diagnosis, histological type, stage at diagnosis, and treatment type. Data on survival status of the patients was collected from health units (public and private) and prefectural deaths registry of Rabat. The chosen date of inclusion in the study was the date of histological diagnosis of cancer and the time point was set at July 1st 2014. The survival estimation was performed by the Kaplan-Meier method. The study of prognostic factors was performed using the log-rank test for comparison between different groups of the same variable and the Cox model for multivariate analysis. The significance level was set at 0.05. Data analysis was performed using SPSS software (version 13.0).

Results: A total of 191 cases of cervical cancer were collected at Rabat between 2005 and 2008. All cases of cancer were confirmed histologically. One hundred and fifty cases $(93.8 \%)$ were found following symptoms, the mean age was $55.2 \pm 12.4$ years, one hundred and thirty patients $(69.1 \%)$ were aged between 35 and 64 years. Squamous cell carcinoma was found in 164 patients $(85.9 \%)$. Stage I was the most frequent 171 patients $(89.5 \%)$. One hundred and sixty four patients $(85.9 \%)$ received radiotherapy. The total number of registered deaths was $62(32 \%)$. Overall survival was $85.3 \%$ at 1 year, $64.9 \%$ at 3 years and $50 \%$ at 5 years. Survival was better in patients aged between 35 and 64 years with a 5-year survival of $60.7 \%$, whereas it was $19 \%$ among in those under age 35 and $35.9 \%$ in those over 65 years $(p=0.001)$. The survival was better in 
patients with stage I relatively to the other stages $(p<0.001)$. The 5-year survival was 77.3, 55.3, 37.7 and $13.2 \%$ for stage I, II, II and IV. In multivariate analysis, the variables "age classes" and "stage" were significant. The age group below 35 years being the reference, the relative risk was $0.52\{0.11-0.79\}$ for the age group 35-64 years and $1.11\{0.24-1.74\}$ for over 65 years $(p<0.001)$. For stage, the relative risk was $2.65\{1.06-6.60\}$ for stage II, 2.03-12.63 5.06\{\} for stage III and $10.453 .65\{-29.88\}$ for stage IV, stage I being the reference class $(p=0.005)$.

Conclusion: The age and stage are cancer prognosis factors of cervix cancer. The prognosis is better when the cancer is diagnosed at an early stage where the interest of a mass screening program.

\section{Abstract \#: P 333}

\section{Modifiable risk factors of bladder cancer: a quantitative review of meta-analyses}

Abdulmohsen Alzalabani ${ }^{1}$, Anke Wesselius ${ }^{2}$, Kelly F. J. Stewart ${ }^{2}$, Maurice P. Zeegers ${ }^{2}$

${ }^{1}$ Taibah University, Family and Community Medicine Department, College of Medicine, P.O. Box 42317, Madinah 41541, Saudi Arabia; ${ }^{2}$ University of Maastricht, Department of Complex Genetics, School for Nutrition, Toxicology and Metabolism (NUTRIM), P. O. Box 616, 6200 MS Maastricht, The Netherlands; Corresponding author's e-mail: k.stewart@maastrichtuniversity.nl

Background: Each year, 430,000 people are estimated to be diagnosed with bladder cancer, of which about $77 \%$ are male. For men, it ranks 7 th of all cancers. Several genetic and environmental risk factors have been proposed, including smoking, occupational exposure and several dietary factors. Due to the relapsing nature of the disease, primary prevention is paramount. Therefore, we investigated the modifiable risk factors associated with primary bladder cancer, based on results from meta-analyses.

Methods: PubMed was systematically searched for meta-analyses (MAs) and pooled analysis on modifiable risk factors of primary bladder cancer, published between 1995 and 2015. When multiple MAs were identified, a meta-meta-analysis (MMA) was performed in which the results were combined into a single estimate. When combining was not appropriate, the most comprehensive MA was selected based on the number of included studies and the number of events in each MA.

Results: Of 1496 articles identified, 63 articles were selected for inclusion based on title and abstract. Of these, 10 were suitable for MMA on consumption of vegetables and coffee, smoking, and workers of the petroleum or chemical industry. Significant protective associations were found for higher levels of selenium (serum/toenail: $\mathrm{RE}=0.61: 95 \% \mathrm{CI}: 0.42-0.87$ ) and vitamin $\mathrm{D}$ (serum: $\mathrm{RE}=0.75$ : $95 \%$ CI: $0.65-0.87)$, higher intake of fruit $(\mathrm{RE}=0.77: 95 \% \mathrm{CI}$ : 0.69-0.87) and vegetables ( $\mathrm{RE}=0.83$ : $95 \% \mathrm{CI}: 0.75-0.92$ ), citrus fruit $(\mathrm{RE}=0.85: 95 \% \mathrm{CI}: 0.76-0.94)$ and cruciferous vegetables $(\mathrm{RE}=0.84: 95 \% \mathrm{CI}: 0.77-0.91)$, and higher physical activity levels $(\mathrm{RE}=0.86: 95 \% \mathrm{CI}: 0.77-0.95)$. Current $(\mathrm{RE}=3.67: 95 \% \mathrm{CI}$ : 3.31-4.03) or former $(\mathrm{RE}=2.17: 95 \% \mathrm{CI}: 2.00-2.34)$ cigarette smoking, pipe $(\mathrm{RE}=1.9$ : $95 \% \mathrm{CI}: 1.2-3.1)$ or cigar $(\mathrm{RE}=2.3$ : $95 \% \mathrm{CI}: 1.6-3.5)$ only smoking, beta-carotene $(\mathrm{RE}=1.52: 95 \% \mathrm{CI}$ : 1.03-2.24) or antioxidant ( $\mathrm{RE}=1.52: 95 \% \mathrm{CI}: 1.06-2.17)$ supplements, coffee consumption of $>5$ cups/day $(\mathrm{RE}=1.36: 95 \% \mathrm{CI}$ : 1.17-1.54), higher processed meat consumption ( $\mathrm{RE}=1.22: 95 \%$ CI: 1.04-1.43), and exposure to water disinfection by-products $(\mathrm{RE}=1.31: 95 \% \mathrm{CI}: 1.16-1.49)$ and chlorinated drinking water $(\mathrm{RE}=1.2: 95 \% \mathrm{CI}: 1.1-1.4)$ significantly increased the risk. Finally, occupational factors that are associated with increased risk include working as a painter $(\mathrm{RE}=1.14: 95 \% \mathrm{CI}$ : 1.06-1.22), hairdresser $(\mathrm{RE}=1.35: 95 \% \mathrm{CI}: 1.13-1.61)$, motor vehicle driver $(\mathrm{RE}=1.11$ : $95 \% \mathrm{CI}: 1.04-1.18)$, petroleum industry worker $(\mathrm{RE}=1.41: 95 \%$ CI: $1.20-1.61)$, dry cleaning worker $(\mathrm{RE}=1.47: 95 \%$ CI: 1.16-1.85), and occupational exposure to inorganic led compounds $(\mathrm{RE}=1.41: 95 \% \mathrm{CI}: 1.16-1.71)$.

Conclusion: While smoking remains one of the key risk factors in the development of bladder cancer, this study shows that also several diet-related and occupational factors prove to be very relevant. We recommend that preventative strategies focus on these risk factors in order capture the greatest potential of disease risk reduction.

\section{Abstract \#: P 334}

Urothelial cell carcinoma incidence, lifetime smoking behaviour and exposure to environmental tobacco smoke: a meta-analysis

Frits H.M. van Osch ${ }^{1,2}$, Sylvia H.J. Jochems ${ }^{1,2}$, Frederik-Jan van Schooten $^{3}$, Richard T. Bryan ${ }^{2}$, Maurice P.A. Zeegers ${ }^{1}$

${ }^{1}$ Department of Complex Genetics, NUTRIM School for Nutrition and Translational Research in Metabolism, Maastricht University, Maastricht, The Netherlands; ${ }^{2}$ School of Cancer Sciences, University of Birmingham, Birmingham, United Kingdom; ${ }^{3}$ Department of Toxicology, NUTRIM School for Nutrition and Translational Research in Metabolism, Maastricht University; Corresponding author's e-mail: f.vanosch@maastrichtuniversity.nl

Background: With approximately $20 \%$ of the Western population currently smoking, cigarette smoking remains a common and essential risk factor for urothelial cell carcinoma (UCC). UCC is characterized as a transitional cell carcinoma mainly occurring in the bladder, ureter or renal pelvis. Incidence rates of UCC vary by duration and intensity of smoking. Recent evidence suggests that smoking cessation might decrease risk of developing UCC and that it is probable that exposure to environmental tobacco smoke (ETS) slightly increases UCC risk. However, pooled estimates on the effects of smoking cessation and exposure to ETS are lacking. This metaanalysis sets out to update and further enhance the meta-analysis on smoking and UCC risk published by Zeegers et al. in 2000 .

Methods: PubMed and EMBASE were used to search for epidemiologic studies, either case-control or cohort studies, on cigarette smoking and UCC incidence. Studies were excluded if they were not involving humans. The search included the (Mesh) search terms "urinary bladder neoplasms", "incidence", "risk", "smoking" and "epidemiologic studies" in different combinations and resulted in a total count of 2,112 articles. Studies which did not present effect measures or other useable data on smoking status and duplicate publications within the same patient population were excluded. After independent, full-text evaluation of risk of bias by two reviewers (FvO and SJ) using the Newcaste-Ottowa scale, 93 studies were included in the meta-analysis. Data is currently being extracted from these articles. Random effects models will be applied and heterogeneity tests will include I2 and Cochran's Q tests. A cumulative meta-analysis will be performed for smoking status, comparing current and former smokers to never smokers, and UCC risk. Doseresponse meta-analyses will be carried out to investigate how smoking intensity (by 10 cigarettes per day) and smoking cessation (by 10 years prior to diagnosis) influence risk of UCC. The effects of duration of smoking and exposure to ETS will be investigated using a classical meta-analysis approach. Subgroup analyses include comparing results between gender, adjusted and unadjusted effect measures and between case-control studies and cohort studies.

Results: Since data is currently being extracted from all 93 included studies, results cannot be presented yet. However, pooled UCC risk 
estimates regarding smoking behaviour and exposure to ETS, based on a total of approximately 60,000 cases of UCC, can be presented at the Healthy Living Congress 2015.

\section{Abstract \#: P 335}

\section{Lifestyle factors and bladder cancer prognosis: a systematic} review

Ellen Westhoff ${ }^{1}$, Ellen Kampman ${ }^{1,2}$, J. Alfred Witjes ${ }^{3}$, Antoine van der Heijden $^{3}$, Lambertus A. Kiemeney ${ }^{4}$, Alina Vrieling ${ }^{4}$

${ }^{1}$ Radboud Institute for Health Sciences, Radboud University Medical Center, Nijmegen, The Netherlands; ${ }^{2}$ Division of Human Nutrition, Wageningen University, Wageningen, The Netherlands; ${ }^{3}$ Department of Urology, Radboud University Medical Center, Nijmegen, The Netherlands; ${ }^{4}$ Health Sciences, Radboud university medical center, Nijmegen, The Netherlands; Corresponding author's e-mail: ellen.westhoff@radboudumc.nl

Background: Urologists are frequently confronted with questions by urinary bladder cancer (UBC) patients how they can influence their prognosis, for example by making lifestyle changes. Lifestyle factors have been linked to risk and prognosis of many cancers and may also influence UBC prognosis. However, to our knowledge no systematic review of studies on the association of body mass index (BMI), diet and other lifestyle factors, with prognosis of UBC patients has been performed thus far. Such a systematic review only exists for smoking. The aim of this systematic review is to qualitatively summarize the evidence on dietary and lifestyle factors and to identify next steps in research.

Methods: We performed a systematic search of the literature up to September 2014 using PubMed. Relevant articles reporting on epidemiologic and intervention studies in UBC survivors with respect to BMI, diet, dietary supplements or physical activity in relation to recurrence, progression, CSM or all-cause mortality (ACM) were included.

Results: Twenty-six articles were included. Two out of eight observational studies found a higher BMI associated with increased risk of recurrence, progression, CSM, or ACM. However, one study found a decreased risk and five studies reported no association. Four out of five observational studies on diet and five out of 13 intervention studies with dietary supplements found a potential protective effect on recurrence or mortality for single foods and supplements, such as broccoli, a megadose of multiple vitamins and Lactobacillus casei preparation. Studies on physical activity were not available.

Conclusion: Approximately half of the studies found an association between dietary and lifestyle factors and UBC prognosis. However, results were conflicting, most studies conducted to date have methodological shortcomings and many aspects have not been studied at all. Prospective studies with multiple measurements, long follow up and investigating multiple dietary and lifestyle factors in a homogenous population need to be undertaken in order to develop evidencebased guidelines with regard to diet and lifestyle for bladder cancer patients.

\section{Abstract \#: P 337}

Risk of cancer incidence before the age of fifteen after exposure to computed tomography: a german cohort study

$\frac{\text { Lucian Krille }^{1,2}}{\text { Maria Blettner }}{ }^{2}$, Steffen Dreger ${ }^{3}$, Roman Pokora ${ }^{2}$, Hajo Zeeb ${ }^{3,4}$,
${ }^{1}$ International Agency for Research on Cancer, Lyon, France; ${ }^{2}$ University Medical Center Mainz, Mainz, Germany; ${ }^{3}$ Leibniz Institute for Prevention Research and Epidemiology - BIPS, Bremen, Germany; ${ }^{4}$ Health Sciences Bremen, University of Bremen, Bermen, Germany; Corresponding author's e-mail: lbk.sub2015@gmail.com

Methods: The aim of this cohort study was to assess the risk of developing cancer, specifically leukaemia, tumours of the central nervous system and lymphoma, before the age of 15 years in children previously exposed to computed tomography (CT) in Germany. Data for children with at least one CT between 1980 and 2010 were abstracted from 20 hospitals. Cancer cases occurring between 1980 and 2010 were identified by stochastic linkage with the German Childhood Cancer Registry (GCCR). For all cases and a sample of non-cases, radiology reports were reviewed to assess the underlying medical conditions at time of the CT. Cases were only included if diagnosis occurred at least 2 years after the first CT and no signs of cancer were recorded in the radiology reports. Standardised incidence ratios (SIR) using incidence rates from the general population were estimated.

Results: The cohort included information on 71,073 CT examinations in 44,584 children contributing 161,407 person-years at risk with 46 cases initially identified through linkage with the GCCR. Seven cases had to be excluded due to signs possibly suggestive of cancer at the time of first CT. Overall, more cancer cases were observed $(\mathrm{O})$ than expected (E), but this was mainly driven by unexpected and possibly biased results for lymphomas. For leukaemia the SIR $(\operatorname{SIR}=\mathrm{O} / \mathrm{E})$ was 1.72 (95\% CI: $0.89-3.01, \mathrm{O}=12)$ and for CNS tumours the SIR was 1.35 (95\% CI: $0.54-2.78, \mathrm{O}=7$ ).

Conclusion: Despite careful examination of the medical information, confounding by indication or reverse causation cannot be ruled out completely and may explain parts of the excess. Furthermore the CT exposure may have been underestimated as only data from the participating clinics were available. This should be taken into account when interpreting risk estimates.

\section{Abstract \#: P 338}

Differences at transcriptome level reflect genetic susceptibility to environmental carcinogens

\author{
Almudena Espín-Pérez ${ }^{1}$ \\ ${ }^{1}$ Toxicogenomics, Maastricht University; Corresponding author's \\ e-mail: a.espin@maastrichtuniversity.nl
}

Background: Cancer risk has been associated with exposure to a wide range of environmental pollutants. In order to identify subjects with an increased susceptibility to chemical induced carcinogenesis, it is important to assess the impact of genetic background on responses to environmental carcinogens. We studied the impact of the genotype on the relationship between exposure and gene expression, identifying exposure-dependently co-regulated genes and genetic pathways.

Methods: We used data from 134 adults of Flemish Environment and Health Survey. The population was selected based on an integration score calculated per subject (index of internal exposure), which comprises the arithmetic mean of the Z-Scores for cadmium, lead, polychlorinated biphenyls, $\mathrm{p}, \mathrm{p}^{\prime}$-dichlorodiphenyldichloroethylene, hexachlorobenzene and 1-OH-pyrene. To assess the impact of the genotype on the relationship between exposure and gene expression we used mixed models, a type of regression models capable of correcting for both confounders and laboratory variables within the same analysis. We ran mixed models to correlate data on gene expression with exposure, genotype, age, sex and biomarkers of cancer risk. Then, we studied cancer development-related pathways using ConsensusPathDB. 
Results: We found generic and carcinogenesis-related pathways deregulated in both sexes but males presented a bigger influence at transcriptome level than females. Spliceosome and RNA transportrelated pathways are at the top of significant pathways among females, while NOD1/2 signaling, $\mathrm{TNF} \alpha-$ and apoptosis-related pathways are most significantly modulated among males. After filtering based on a ratio average/standard deviation for each gene, we highlighted NOTCH1, CBR1, ITGB3, ITGA4, ADI1, HES1, NCOA2 and SMARCA2 in view of their direct link with cancer development. Two of these, NOTCH1 and ITGB3, are also known as genes that are responsive to $\mathrm{PCBs}$ and cadmium chloride exposure in rodents and to lead in human, respectively.

Conclusions: Genotype, exposure and particularly the interaction between these two variables showed an impact on gene expression levels. Significant gender differences have been observed and specific pathways appear to be related to cancerous processes. Subjects carrying a high number of risk alleles are more responsive to exposure with respect to the induced expression of some of these cancer-related genes. The observed correlations are statistically significant but the regression coefficients are low, making it difficult to interpret the biological consequences of these findings in terms of cancer risk for those individuals carrying a higher number of risk alleles and exposed to higher levels of environmental exposures.

\section{Abstract \#: P 339}

Probabilistic and deterministic record linkage: application in the population-based cancer registry of São-Paulo (PBCR-SP), Brazil

Stela Verzinhasse Peres ${ }^{1}$, Maria do Rosário Dias de Oliveira Latorre ${ }^{1}$, Fernanda Alessandra Silva Michels ${ }^{1}$, Mônica Laporte ${ }^{2}$, Claudia Medina Coeli ${ }^{3}$, Márcia Furquim de Almeida ${ }^{1}$

${ }^{1}$ Department of Epidemiology - School of Public Health (USP), São Paulo, Brazil; ${ }^{2}$ SEADE Foundation, São Paulo, Brazil; ${ }^{3}$ Institute of Public Health Studies (IESC/UFRJ), Rio de Janeiro, Brazil; Corresponding author's e-mail: svperes@usp.br

Background: The aim of this study was to assess the performance of the probabilistic and deterministic record linkage between PBCR-SP for incident cancer cases between São Paulo death database and drugs/medical procedures database.

Methods: In this study we used the database of the RCBP-SP composed of 343,306 incident cancer cases in the Municipality of São Paulo registered in the period between 1997 and 2005 with ages raging from under one to 106 years, from both sexes. To complete the database of the RCBP-SP three databases were used, namely: PROAIM, APAC-SIA/SUS and FSeade. Crude incidence (CIR), mortality rates (CMR) were calculated and overall survival analysis was performed using the Kaplan-Meier technique and for the comparison between curves, the log rank test was employed.

Results: After record linkage, it was verified a gain of $101.5 \%$ for the variable address, $31.5 \%$ for death date and $80.0 \%$ for the date of latest information. As for the variable motheŕs name, in the database of the RCBP-SP before record linkage, this information represented only $0.5 \%$, having been completed, in general, in 76,332 registries. The overall survival analysis showed that before the record linkage there was an underestimation of the probability of being alive for all periods assessed. In general, for the truncated survival at 7 years, the probability of being alive at the first year of follow up before record linkage was lower when compared to the probability of being alive at the first year of follow up after record linkage $(48.8 \% \times 61.1 \%$ : $p<0.001$ ).
Conclusion: Probabilistic and deterministic record linkage were effective to complete/improve information from the database of the RCBP-SP. Moreover, the CIR had a gain of de $3.4 \%$. As for the CMR, there was a gain of $25.8 \%$. After using the record linkage technique, it was verified that values for overall survival were underestimated for both sexes, all age groups, and cancer sites.

\section{Abstract \#: P 340}

Trends in major cancer mortality in Korea, 1983-2012: join point analysis

Daroh $\mathrm{Lim}^{1}$, Mina $\mathrm{Ha}^{2}$, Inmyung Song ${ }^{3}$

${ }^{1}$ Center for Health Industry Information and Statistics, Korea Health Industry Development Institute; ${ }^{2}$ Department of Preventive Medicine, Dankook University College of Medicine; ${ }^{3}$ Department of Pharmaceutical Industry, Korea Health Industry Development; Corresponding author's e-mail: minaha@dku.edu

Background: Cancer is the leading cause of death in Korea. This study aims to examine changes in temporal trends in major cancer mortality rates.

Materials and methods: Mortality data for 1993-2012 were obtained from the Korean Statistics Information Service (KOSIS) database and age-standardized to the 2000 Korean population. Joinpoint analysis was used to identify significant changes in trends over time. The annual percentage rate change (APC) was computed for each segment of the trends.

Results: The age-standardized mortality rates (ASR) for all cancer sites combined decreased by 9.1 and $1.1 \%$ in men and women, respectively, from 1983 to 2012. ASRs from esophagus, stomach, liver and cervical cancer decreased substantially, whereas ASRs from cancer for all other sites increased markedly. ASRs for all cancer sites combined increased until 1994 and thereafter decreased significantly in both genders except for the period 1998-2002 (APC: $-5.5 \%$ for men $[p<.05]$ and $0.07 \%$ for women). asrs esophagus, stomach liver cancer increased until the early 1990s thereafter declined, leading to significant decreases $[p<0.05]$ esophagus (apc: $-1.85 \%$ men $-3.82 \%$ women), $-4.06-4.07-1.55-0.56 \%$ women) over 3 decades. from cervical declined significantly consistently $-3.13 \%$ ). in contrast, colorectal $4.254 .01 \%$ pancreatic $2.043 .51 \%$ lung 1.37 $2.2 \%$ decades although they leveled off or started decrease at various time points. most increasing trend was observed non-Hodgkin's lymphoma $3.555 .29 \%$ women: number of join points =l'0).।' greatest asr increase seen prostate $6.28 \%$ ).

Conclusion: While mortality decreased recent Korea, challenges still remain many types, especially, pancreatic, breast lymphoma. Surveillance trends can lend valuable insight as prevention control cancer.

\section{Abstract \#: P 341}

Psychosocial factors as predictors of quality of life in cancer Portuguese patients: a structural equation modeling application

Estela Vilhena ${ }^{1}$, José Luís Pais Ribeiro ${ }^{2}$, Isabel Silva ${ }^{3}$, Luísa Pedro ${ }^{4}$, Rute Meneses $^{3}$, Helena Cardoso ${ }^{5}$, António Martins da Silva ${ }^{6}$, Denisa Mendonça $^{5}$

${ }^{1}$ Polytechnic Institute of Cávado and Ave, Barcelos: EPIUnit, ISPUP University of Porto, Porto; ${ }^{2}$ FPCE University of Porto: UIPES Portugal; ${ }^{3}$ University of Fernando Pessoa; ${ }^{4}$ ESTeSL Polytechnic Institute of Lisbon; ${ }^{5}$ ICBAS University of Porto: HGSA/CHP 
Hospital Center of Porto; ${ }^{6} \mathrm{HGSA} / \mathrm{CHP}$ Hospital Center of Porto: UMIB/ICBAS University of Porto, Portugal; Corresponding author's e-mail: evilhena@ipca.pt

Background: Living with a chronic disease is a demanding experience that may affect multiple aspects of an individual's life. In general, cancer patients are responsible for the management of a wide range of psychosocial factors which contribute to their quality of life (QoL). QoL has become an important concept for health care and to evaluate QoL in cancer patients is an increasingly important issue. The aim of this study is to clarify the role of dispositional optimism, treatment adherence and social support in quality of life of Portuguese cancer patients, controlling for socio demographic variables. Is was hypothesized that these psychosocial factors are simultaneous relevant predictors of quality of life domains (general well-being and mental health).

Methods: This study included a sample of 210 Portuguese cancer patients approached by their physicians in outpatient departments of the main hospitals in Portugal. All patients completed self-report questionnaires to assess socio-demographic and clinical, psychosocial and QoL variables.

Structural Equation Modelling (SEM) were used to test the quality of the hypothesized model using Maximum Likelihood (ML) estimation. The Satorra-Bentler Scaled correction to $\chi^{2}$ test statistic were used. Standard errors were corrected to adjust for the extent of nonnormality. To test the adequacy of the model two other goodness-offit indices were used: Comparative Fit Index (CFI) and Root Mean Square Error of Approximation (RMSEA). Analysis were performed using EQS 6.1.

Results: The mean age of patients was 48.8 years $(\mathrm{SD}=10.1)$ and $67.1 \%$ were female. Mean education level was 9.4 years $(\mathrm{SD}=4.8)$ and mean severity of disease perception was $6.4(\mathrm{SD}=2.9)$.

The SEM results showed that the hypothesized model fitted the data reasonably well, CFI $=0.80$, RMSEA $=0.06,[S-\chi]_{(348)}^{2}=$ $616.422 p<0.001$ (test sensible to sample size). All factors had a simultaneous independent and positive impact on general well-being, physical and mental health. Patients with higher optimism, a better treatment adherence and a better social support had a better QoL in all domains.

Conclusions: The principal goal of this study was to clarify the simultaneous impact of psychosocial variables on QoLto analyse. The use of SEM allows us to understand the complexity of the simultaneous relationships between these variables. Results revealed that an attitude more optimistic, a better treatment adherence and more social support contribute to a better general well-being, a better physical health and a better mental health, which in turn will affect positively their quality of life.

\section{Abstract \#: P 342}

\section{The role of dietary factors on survival in common cancers with a high survival rate: a systematic literature review}

Sylvia H.J. Jochems ${ }^{1,2}$, Frits H.M. van Osch ${ }^{1,2}$, Frederik-Jan van Schooten $^{3}$, K.K. Cheng ${ }^{4}$, Maurice P.A. Zeegers ${ }^{2}$

${ }^{1}$ School of Cancer Sciences, University of Birmingham, Birmingham, United Kingdom; ${ }^{2}$ Department of Complex Genetics, NUTRIM School for Nutrition and Translational Research in Metabolism, Maastricht University, Maastricht, the Netherlands; ${ }^{3}$ Department of Toxicology, NUTRIM School for Nutrition and Translational Research in Metabolism, Maastricht University, Maastricht, The Netherlands; ${ }^{4}$ Department of Public Health, Epidemiology and Biostatistics, University of Birmingham, Birmingham, United
Kingdom; Corresponding author's e-mail:

s.jochems@maastrichtuniversity.nl

Background: As more people are surviving cancer, there is an increased need for evidence-based recommendations on maintaining quality of life and long-term survival. Survival rates have improved for almost all cancers and vary greatly between cancer sites ranging from a 10-year survival of $98 \%$ for testicular cancer to a 10 -year survival of $1 \%$ for pancreatic cancer. An important consideration is that many cancer survivors are motivated to make lifestyle changes to reduce their risk of recurrence. Hence, research should focus on identifying modifiable risk factors among individuals. There is evidence that cancer survivors could benefit from a healthy diet to improve their survivorship from cancer. However, current literature linking diet to cancer prognosis is inconclusive and limited at best. Therefore, the objective of this systematic literature review is to provide a summary of the published literature with regard to dietary factors in preventing recurrent or progressive cancer among cancer survivors.

Methods: For this systematic literature review, ten of the most common cancer sites with a 10-year survival of $50 \%$ or more (excluding malignant melanoma) were selected. These include cancer of the testis, breast, prostate, uterus, larynx, cervix, bowel and bladder and multiple myeloma and (non-) Hodgkin's lymphoma. The literature search was performed using the PubMed/Medline and EMBASE database for published studies to explore the association between diet and cancer recurrence or progression before January 2015. The search strategy included keywords relating to cancer prognosis and mortality, dietary patterns and macro- and micronutrients. Considered endpoints were total and cancer-specific mortality, cancer-specific recurrence or progression and second primary cancers. Literature on both randomised controlled trials and epidemiologic studies were reviewed.

Results: Results have not yet been obtained but will have at the time of the congress. Associations between dietary patterns and nutrients will be reviewed separately for each type of cancer. The main purpose of this review is to provide current patients and health professionals with scientifically based evidence on the interplay between dietary factors and cancer survival.

\section{Abstract \#: P 343}

\section{A descriptive analysis of gastric cancer in Alaska}

Fabrice E. Evengue $^{1}$, Nancy A. Nix ${ }^{1}$, Virginia A. Miller ${ }^{1}$, David O'Brien ${ }^{2}$

${ }^{1}$ University of Alaska Anchorage; ${ }^{2}$ Alaska Department of Health and Social Services-Division of Chronic Disease Prevention and Health Promotion; Corresponding author's e-mail: feevengue@alaska.edu

Gastric cancer or stomach cancer represents a major public health problem in the contiguous United States and in Alaska. In fact, stomach cancer is the fourth most common malignancy and the second most common cause of cancer-related deaths throughout the world. A retrospective study of gastric cancer cases from 1996 to 2011 was undertaken and data were extracted from the Alaska Cancer Registry where cases are consistently recorded and centralized. An analysis of data was done using the National Cancer Institute's SEER* Statistical program software (version 8.1.5). The goal of the project was to provide a detailed epidemiologic descriptive analysis of gastric cancer to better inform health professionals, the public and to provide additional resources for future research. Results showed that gastric cancer incidence rates in Alaska are significantly higher than the rest of the nation. Alaska Natives and American Indians in Alaska have the highest rate of gastric cancer than all races combined. 
The males are twice at risk of gastric cancer than females. The Alaska Native male and Asian/Pacific Islander male incidence rates are much higher than males from other races. In addition, Southeast Alaska Natives' incidence rates are higher than rates for non-Southeast Alaska Natives. Based on findings, the study recommended promoting primary prevention strategies by an amelioration and improvement of lifestyle through exclusion of causal factors: promoting secondary prevention measures through early screening, detection and treatment of premalignant lesions of high risk-groups: promoting daily consumption of fresh fruits, vegetables and supplement vitamins: and improvement of treatment by well-designed clinical trials, coupled with molecular characterization of tumors.

\section{Abstract \#: P 344}

\section{Utilization of inpatient treatment as an estimator for disease} burden due to sarcoma in Germany

$\frac{\text { Thomas Petzold }^{1}}{\text { Jochen Schmitt }^{3}}$, Markus Schuler ${ }^{2}$, Freya Trautmann ${ }^{3}$,

${ }^{1}$ Center for Evidence-Based Healthcare, Department of Quality and Medical Risk Management, University Hospital Carl Gustav Carus Dresden, Dresden, Germany; ${ }^{2}$ Department of Internal Medicine I, University Hospital Carl Gustav Carus Dresden, Dresden, German; ${ }^{3}$ Center for Evidence-Based Healthcare, University Hospital Carl Gustav Carus Dresden, Dresden, German; Corresponding author's e-mail: thomas.petzold@uniklinikum-dresden.de

Background: Sarcomas are a heterogeneous disease entity and comprise approximately $1 \%$ of all malignant neoplasms in adults. Currently, the burden of sarcoma in Germany is unclear as a centralized and comprehensive documentation is missing. German cancer registries do not allow a straight forward assessment of the epidemiology of sarcoma as cancers are classified mainly by their primary tissue of origin and not their histopathology. We aimed to estimate the utilization of inpatient treatment due to sarcoma in Germany as a marker of disease burden.

Methods: We analyzed healthcare utilization data from the research data centers of the German Federal Statistical Office and the statistical offices of the federal states (FDZ) to estimate the degree of healthcare utilization due to sarcoma in different age groups. The FDZ data were documented annual at patient case level from 2005 to 2012 and cover the complete inpatient-treatment data from Germany (ca. 18 Mio cases/year). By means of descriptive statistics we assessed reasons of admission and discharge, as well as regional differences in healthcare utilization.

Results: On average, a total of 25,338 sarcoma inpatient cases (range 24,224-25,909) were treated per year in Germany. These are $0.1 \%$ of all inpatient cases in Germany. Approximately half of the cases $(54.2 \%: \mathrm{n}=13,745$ (range 12,359-14,200)) were aged 50 years and older. The most common reason for admission was referral from an ambulatory physician (79.7 \%: $\mathrm{n}=19,625$ (range 18,979-20,655)). Stationary emergency admission was the second most common reason (19.5\%: $\mathrm{n}=4649$ (range 4332-5056)). There were significant differences in healthcare utilization patterns due to sarcoma between the federal states of Germany. In each federal state 1589 inpatients (range 291-5582) were treated on average per year.

Conclusion: The analysis of healthcare utilization data appears useful as a first step to estimate the burden of sarcoma in Germany. However, patient-level analyses are required to estimate disease prevalence, incidence, and survival, as well as treatment effects and outcomes. Therefore, a prospective clinical registry should be established to investigate the burden, course of disease, treatment benefits and harms of sarcoma patients and to address the patients' perspective by collecting patient-reported outcomes and patient preferences.

\section{Abstract \#: P 345}

Mediating biomarkers in the relation of coffee and tea intake with hepatocellular carcinoma risk in the epic study

Krasimira Aleksandrova ${ }^{1}$, Christina Bamia ${ }^{2,3}$, Dagmar Drogan ${ }^{1}$, Pagona Lagiou $^{2,4,5}$, Antonia Trichopoulou ${ }^{2,3}$, Mazda Jenab ${ }^{6}$, Veronika Fedirko ${ }^{7,8}$, H. Bas Bueno-de-Mesquita ${ }^{9,10,11}$, Marc J. Gunter $^{11}$, Rudolf Kaaks ${ }^{12}$, Tobias Pischon ${ }^{13}$, Elio Riboli ${ }^{7}$, Heiner Boeing $^{1}$, Dimitrios Trichopo ${ }^{3,4,5}$

${ }^{1}$ Department of Epidemiology, German Institute of Human Nutrition Potsdam-Rehbrücke, Nuthetal, Germany; ${ }^{2}$ WHO Collaborating Center for Food and Nutrition Policies, Department of Hygiene, Epidemiology and Medical Statistics, University of Athens Medical School, Athens, Greece; ${ }^{3}$ Hellenic Health Foundation, Athens, Greece; ${ }^{4}$ Department of Epidemiology, Harvard School of Public Health, Boston, USA; ${ }^{5}$ Bureau of Epidemiologic Research, Academy of Athens, Athens, Greece; ${ }^{6}$ International Agency for Research on Cancer (IARC-WHO), Lyon, France; ${ }^{7}$ Department of Epidemiology, Rollins School of Public Health, Emory University, Atlanta GA, US; ${ }^{8}$ Winship Cancer Institute, Emory University, Atlanta, GA, US; ${ }^{9}$ National Institute for Public Health and the Environment (RIVM), Bilthoven, the Netherlands; ${ }^{10}$ Department of Gastroenterology and Hepatology, University Medical Center, Utrecht, The Netherlands;

${ }^{11}$ Division of Epidemiology, Public Health and Primary Care, Imperial College, London, United Kingdom; ${ }^{12}$ Division of Cancer Epidemiology, German Cancer Research Centre, Heidelberg, Germany; ${ }^{13}$ Molecular Epidemiology Group, Max Delbrueck Center for Molecular Medicine (MDC), Berlin-Buch, Germany;

Corresponding author's e-mail: krasimira.aleksandrova@dife.de

Background: Higher coffee and tea intakes have been purportedly related to a lower risk of hepatocellular carcinoma (HCC). However, whether this association may be accounted for by specific biological mechanisms remains unclear. We, therefore, evaluated mediating effects of inflammatory, metabolic, liver injury, and iron metabolism biomarkers on the association between coffee and tea intakes with HCC risk taking simultaneously into account established HCC risk factors.

Methods: In a prospective nested case-control study among 125 incident HCC cases matched to 250 controls, we estimated relative risks (RRs) and $95 \%$ confidence intervals (CIs) for the association between coffee and tea intake and HCC risk using conditional logistic regression. Mediating effects of 21 biomarkers were evaluated based on changes in the regression coefficients and corresponding CIs of models with and without adjustment for biomarkers individually and in combinations.

Results: In multivariable-adjusted model, compared to participants with coffee and tea intakes of less than 4 cups per day, those with more than 4 cups per day had $75 \%$ (95\%CI: 0.11-0.62) and $65 \%$ (95 \% CI: 0.10-0.95) lower HCC risk, respectively. These associations were not modified by non-alcoholic fatty liver disease, hepatitis infection, smoking or alcohol consumption. A statistically significant attenuation of the association of coffee with HCC risk was observed for interleukin-6, glutamate dehydrogenase, aminotransferases and bilirubin which in combination attenuated the multivariable-adjusted $\beta$ coefficients by $72 \%$ (95 \% CI: 7-239\%). The association between tea intake and HCC risk was mostly accounted for by interleukin- 6 and glutamate dehydrogenase attenuating the association by $42 \%$ (95 \% CI: 7-239\%). 
Conclusions: These data suggest that the beneficial effects of coffee and tea on biomarkers of inflammation and hepatocellular injury may represent a plausible biological pathway explaining its inverse relation with HCC risk. Having the difficulties in conducting long-term randomized trials to test these relations, our findings may provide important insight into the current knowledge for the prevention of $\mathrm{HCC}-$ one of the most lethal tumors around the world.

\section{Abstract \#: P 346}

Regional variation in cancer survival and the impact of stage, socioeconomic status, comorbidity and treatment

Katrine Damgaard Skyrud ${ }^{1}$, Yngvar Nilssen ${ }^{1}$, Morten Tanberg Eriksen $^{2}$, Freddie Bray ${ }^{3}$, Bjørn Møller ${ }^{1}$

${ }^{1}$ Cancer Registry of Norway; ${ }^{2}$ Oslo University Hospital; ${ }^{3}$ International Agency for Research on Cancer; Corresponding author's e-mail: katrine.damgaard@kreftregisteret.no

Background: Universal health systems aim to provide equal health care to all citizens, irrespective of socioeconomic status and place of residence. Despite, disparities in the quality of care are known to exist, it is yet to investigate how prognostic factors and regional variations are associated.

Methods: Data from the Cancer Registry of Norway were used to identify 258675 patients diagnosed with cancer between 1.1.2002 and 31.12.2011. They were followed up until June 30, 2013. The six most common cancer sites and all sites combined were investigated. The prognostic factors evaluated were stage at diagnosis, socioeconomic status (SES), a modified Charlson Comorbidity Index (CCI) and treatment. The effect of adjusting for prognostic factors on regional variation was investigated. To adjust for the prognostic factors, flexible parametric models were used to examine regional variations in relative survival. The median difference in relative excess risk (RER) of death between regions was calculated to quantify variations explained by the individual prognostic factors.

Results: All of the prognostic factors were highly associated with both survival and region. The RER of death for patients diagnosed with prostate and lung cancer having a CCI of 3 or more was 9.5 and 1.7 , respectively compared to those not admitted to a hospital. Statistically significant regional variations in an unadjusted analysis of RER of death were found for three out of six cancer sites and for all sites combined. The median differences in RER became smaller when adjusting for tumour stage for all sites combined and prostate cancer. Including SES, comorbidity and treatment had small effect on the differences in RER of death for all the cancer sites.

Conclusions: Stage, SES, and comorbidity differed across the health service regions, but they did not explain the regional differences observed in survival. An exception was smaller regional variation among patients diagnosed with prostate cancer, when controlling for stage. Thus, there still exist unexplained variations between the regions which may be due to inequality of care delivered by the health services.

\section{Abstract \#: P 347 \\ Overweight duration in the elderly and cancer risk: a study of cohorts in Europe}

Melina Arnold ${ }^{1}$, Heinz Freisling ${ }^{2}$, Rachael Stolzenberg-Solomon ${ }^{3}$, Frank Kee ${ }^{4}$, Mark O’Doherty ${ }^{4}$, José Ordonez Mena ${ }^{5}$, Yikyung Park ${ }^{3}$, Tom Wilsgaard ${ }^{6}$, Freddie Bray ${ }^{1}$, Mazda Jenab ${ }^{7}$, Isabelle

Soerjomataram ${ }^{1}$
${ }^{1}$ Section of Cancer Surveillance, International Agency for Research on Cancer, Lyon, France; ${ }^{2}$ Section of Nutrition and Metabolism, Dietary Exposure Assessment Group, International Agency for Research on Cancer, Lyon, France; ${ }^{3}$ Nutritional Epidemiology Branch, National Cancer Institute (NCI/DCEG), Bethesda, USA;

${ }^{4}$ Centre for Public Health, School of Medicine, Dentistry and Biomedical Sciences, Queen's University Belfast, UK; ${ }^{5}$ Division of Clinical Epidemiology and Aging Research, German Cancer Research Center, Heidelberg, Germany; ${ }^{6}$ Department of Community Medicine, The Arctic University of Norway, Troms $\varnothing$, Norway; ${ }^{7}$ Section of Nutrition and Metabolism, Nutritional Epidemiology Group, International Agency for Research on Cancer, Lyon, France; Corresponding author's e-mail: arnoldm@fellows.iarc.fr

Background: Globally, nearly half a million cancer cases were associated with overweight and obesity in 2012. Almost 200,000 of those cases occurred in Europe, corresponding to $5.8 \%$ of all cancer cases in the European regions in that year. The relationship between overweight and the risk of cancer has previously been quantified but was often limited to single measurements of body mass index (BMI) at one point in life. Recent studies have suggested that the risk of cancer related to overweight and obesity compounds with time. In this study, we aimed to estimate the burden of cancer related to overweight duration in the elderly.

Methods: We included study participants from seven European cohort studies with at least two BMI measurements, without a history of cancer and complete information smoking status and physical activity at baseline $(\mathrm{n}=38,563)$. BMI trajectories were estimated across ages using a quadratic growth model with random intercept and random slope. Using this model, overweight $(\mathrm{BMI} \geq 25)$ duration and weighted cumulative overweight years (OWY, taking into account the degree of overweight over time) were calculated. We then applied cox proportional hazard models to determine the cancer risk associated with overweight duration and calculated the fraction of cancer cases attributable to overweight at any age during follow-up. Results: A longer duration of overweight was significantly associated with the incidence of obesity-related cancer (overall HR per 10-yr increment: 1.68: $95 \%$ CI: 1.48-1.91), breast (HR: 1.72: $95 \%$ CI: 1.39-2.14) and colorectal cancer (HR: 1.62: $95 \%$ CI: 1.34-1.95). Risks associated with a longer overweight duration and intensity were higher in men (overall HR per 100 units of OWY: $1.51: 95 \% \mathrm{CI}$ : 1.35-1.68) than in women (HR: 1.32: $95 \% \mathrm{CI}: 1.23-1.42$ ) and were attenuated by smoking (overall HR per 100 units of OWY: 1.50: $95 \%$ CI: $1.38-1.65$ in never smokers vs. $1.24: 95 \%$ CI: $1.02-1.52$ in current smokers). The fraction cancer cases explained by overweight was $16 \%$ in all study participants and $24 \%$ in never smokers for all obesity-related cancers combined.

Conclusion: Overweight duration and intensity are positively associated with cancer risk and modified by smoking status. These findings provide further insights into the role of overweight duration in the aetiology of cancer, needed for the development of effective cancer prevention strategies.

\section{Abstract \#: P 348}

\section{Cancer risk following hospital admissions for pneumonia}

TienYu Owen Yang ${ }^{1}$, Benjamin J. Cairns ${ }^{1}$, Gillian K. Reeves ${ }^{1}$, Jane Green $^{1}$, Valerie Beral ${ }^{1}$

${ }^{1}$ Nuffield Department of Population Health, University of Oxford; Corresponding author's e-mail: owen.yang@ceu.ox.ac.uk

Background: Some investigators have reported excess risks of lung cancer in the short-term and also in the long-term following an episode of pneumonia. The risks of other cancers following pneumonia 
are less studied. We report here on the short-term and long-term incidence of 18 site-specific cancers following hospital admission for pneumonia in a large cohort of women for whom information of risk factors for cancer, such as smoking, body mass index, and alcohol consumption, were recorded.

Methods: In the prospective Million Women Study, 158,501 incident cancers were identified among 1.3 million women during an average of 13 years of follow-up. Using a nested case-control design we selected up to 30 controls for each of the 18 most common types of cancer (with 1000 or more incident cases), matched by year of birth, year of recruitment, region, and socioeconomic status, and followed up to the date when the cancer case was diagnosed. Hospital admissions for pneumonia were identified through linkage to routinely collected National Health Service hospital admission data. Conditioned logistic regression was used to estimate relative risks for each of the 18 cancers $0-1,2-4$, and $5+$ years after hospital admissions for pneumonia.

Results: For 8 of the 18 cancers investigated excess risks were found in the 2 years after hospital admissions for pneumonia (after adjustment for year of birth, year of recruitment, region, and socioeconomic status, adult height, body mass index, smoking status, and alcohol consumption: $p<0.05$ after correction for multiple comparisons). All cancer risks declined substantially more than 2 years after hospital admission for pneumonia, although the risks of oropharyngeal cancer, lung cancer, and myeloma remained significantly elevated 5 or more years after an admission for pneumonia. Risks estimates for oropharyngeal cancer and lung cancer were sensitive to adjustment for smoking, and the association observed even 5 years after hospital admission for pneumonia may well be due to residual confounding. The risk of myeloma was not sensitive to adjustment by smoking or other factors, and may reflect a sub-clinical disease even five or more years before clinical diagnosis.

Conclusion: Most of the observed excess risk of cancer after an episode of pneumonia may be due to the existence of sub-clinical cancer or due to residual confounding by smoking.

Funding: The Million Women Study is funded by Cancer Research UK and Medical Research Council.

\section{Abstract \#: P 349}

Baseline analysis of primary care requesting patterns for immunoglobulins (IG) in the south-west of Ireland: a cross sectional study

$\underline{\text { Sharon L. Cadogan }}{ }^{1}$, John P. Browne ${ }^{1}$, Colin P. Bradley ${ }^{2}$, Mary R. Cahill ${ }^{3}$

${ }^{1}$ Department of Epidemiology and Public Health, University College Cork, Ireland; ${ }^{2}$ Department of General Practice, University College Cork, Ireland; ${ }^{3}$ Department of Haematology, Cork University Hospital, Cork, Ireland; Corresponding author's e-mail: scadogan@ucc.i.e

Background: Each year, approximately 76 million laboratory tests are analysed in Ireland. General practitioners (GPs) are responsible for up to $50 \%$ of these requests, yet no studies to date have examined their requesting patterns. The aim of this descriptive research was to identify the initial rate of Immunoglobulin (Ig) requested by GPs in South-West region (Cork-Kerry) of Ireland in 2013, analysing the abnormal test results. A secondary objective was to assess the variation in requesting patterns between GPs and within practices.

Methods: Cross-sectional analysis of routine laboratory data on Ig requests by 489 GPs using the laboratories in the Cork-Kerry region. Data were extracted for 2013 using the hospital's Cognos impromptu system to interrogate APEX laboratory system. Data were exported, cleaned and statistical analysis including frequencies and cross tabs were performed using Stata v12.

Results: In 2013, the CUH analysed over 19,000 GP-requested Ig tests (IgA, IgG and $\operatorname{IgM}$ ) for approximately 6000 primary care patients. Of the IgA tests, $84 \%$ were normal, $4 \%$ low abnormal and $12 \%$ high abnormal. Of the IgG tests, $92 \%$ were normal, $2 \%$ low abnormal and $6 \%$ high abnormal. Of the IgM tests, $80 \%$ were normal, $12 \%$ low abnormal and $8 \%$ high abnormal. Requesting volumes per GP ranged from 1 to 1,588 tests/year. The highest requesting practice accounted for over $25 \%$ of requests (2076 tests between 11 GPs in one practice), with one GP requesting over $75 \%$ (1588) of these tests.

Conclusions: Variation exists in requests patterns for Igs both at practice level and a GP level. Results show a variable requesting pattern unlikely to be related to level of probable underlying pathology. This presents an opportunity to develop an intervention to improve laboratory testing in the baseline requesting patterns.

\section{Abstract \#: P 350}

\section{Space-time clustering of childhood cancers in Switzerland} Christian Kreis $^{1}$, Michael Grotzer ${ }^{2}$, Heinz Hengartner ${ }^{3}$, Ben Spycher ${ }^{1}$

${ }^{1}$ Institute of Social and Preventive Medicine, University of Bern, Switzerland; ${ }^{2}$ University Children's Hospital Zurich, Zurich, Switzerland; ${ }^{3}$ Children's Hospital Eastern Switzerland, St. Gallen, Switzerland; Corresponding author's e-mail: ben.spycher@ispm.unibe.ch

Background: The aetiology of childhood cancer remains largely unknown. If specific infections are involved, incident cases may cluster in space and time. Results from previous studies on spacetime clustering of childhood cancers are mixed, except for childhood leukaemia for which a majority of studies were positive. However, failure to control for regional population shifts may have biased the results of these studies. Moreover, few studies used precise geocodes of residential locations or investigated space-time clustering both at birth and at diagnosis, which could provide insights into the timing of potentially causative infections.

Methods: In a nationwide study, we investigated the presence of space-time clustering of cancers among children aged $<16$ years in Switzerland between 1985 and 2010. Geo-coded residential locations of incident cases were available at diagnosis and at birth from the Swiss Childhood Cancer Registry. The Knox test was computed for a range of distance lags $(0.5,1,2,5,10 \mathrm{~km})$ and temporal lags $(0.5,1$, $1.5,2$ years) and Baker's Max method (Baker, J Appl Stat 1996:23:543-554) was used to correct for multiple testing. In order to adjust for uneven regional population shifts, $P$-values were obtained by Monte Carlo simulation using weighted random sampling of residential locations of the general child population available from national censuses in 1990, 2000 and 2010 (Kulldorff \& Hjalmars, Biometrics 1999:55:544-552). Tests were performed separately for leukaemia, acute lymphoblastic leukaemia (ALL), lymphomas, tumours of the central nervous system, neuroblastomas and soft tissues sarcomas.

Results: We included 3779 cases of childhood cancer (1493 cases of leukaemia). For childhood leukaemia, we found evidence of spacetime clustering at the time of birth, i.e. using residence at birth and date of birth $(P$ value adjusted for multiple testing $=0.029)$. The evidence was strongest for distances of $<1 \mathrm{~km}$ and time lags of $<2$ years $(P<0.001)$. Results were similar for ALL. We found no evidence of space-time clustering for leukaemia at time of diagnosis or for other cancer types. 
Conclusion: Our study provides further evidence that childhood leukaemia tends to cluster in space and time. In particular, it suggests that the observed clustering is a real phenomenon and not a spurious finding caused by uneven regional population growth. The excess of close case pairs observed at the time of birth but not at diagnosis could indicate that an infection in utero or in the first years of life is involved in these cases.

\section{Abstract \#: P 351}

Modeling cancer risk associated to inhalation exposure to polycyclic aromatic hydrocarbons in industries

Pascal Petit $^{1}$, Dominique J. Bicout ${ }^{1,2}$, Renaud Persoons ${ }^{1,3}$, Anne Maître $^{1,3}$

${ }^{1}$ UJF-Grenoble 1/CNRS/TIMC-IMAG UMR 5525 (EPSP team Environment and Health Prediction of Populations), Grenoble, F-38041, France; ${ }^{2}$ Biomathematics and Epidemiology EPSP-TIMC, VetAgro Sup, Veterinary Campus of Lyon, Marcy l'Etoile, France; ${ }^{3}$ Occupational and Environmental Toxicology Unit, Biochemistry Toxicology and Pharmacology Department, Biology and Pathology Institute, Grenoble teaching Hospital, Grenoble, France; Corresponding author's e-mail: pascal.petit@imag.fr

Background: About 2 million workers are exposed to Polycyclic Aromatic Hydrocarbons (PAHs) mixtures in many industrial sectors (silicon, coke production, road paving ...) in France. Some of these pollutants are among the strongest known carcinogens. Due to a wide diversity of mixtures composition, the mixtures toxicity is variable and dependent on the emission sources. In addition to lung, skin and bladder cancers, PAHs are also responsible for immune and reproduction disorders and are ranked as second priority pollutants, just after asbestos, in France. Due to a lack of occupational exposure data and precise chemical characterization, the estimation of the cancers risk associated to exposures to PAHs' mixtures is poorly known and into mixture synergies of PAHs is rarely taken into account in risk assessments.

The aims of this study were to assess the risk of cancers for workers exposed to PAHs mixtures in the industries and identify occupational activities at risk.

Materials and Methods: In a previous work, an approach was developed to identify and group occupational activities (from French companies) sharing similar PAHs exposure functions-using the Exporisq-HAP database-and accurately characterize PAHs mixtures. Occupational activities-exposure function matrices were constructed and the groups' exposure functions were modeled in terms of correlated multivariate lognormal distributions. Using the modeled exposure functions, probabilistic cancer risks of the previously identified groups (40 occupational activities) were estimated using a classical approach with 7 indicators-e.g.: hazard ratios, excess and incremental lifetime cancer risk (ELCRTI and ILCR)and using a model we developed that took into account mixtures synergy.

Results: Result showed cancer risk distributions for the two approaches. For instance, two ILCR distributions had a 95th percentile above the acceptable risk level of 10-6 indicating an unacceptable potential cancer risk for activities from Silicon, Aluminum and Cathodes production. Results from both approaches were compared. Regardless of the indicators, two types of responses were observed: easy decision (above or under the limit) or difficult decision (at the frontier limit). Each group was not homogeneously classified depending on the indicator chosen. We created a score taking into account all indicators decisions to decide whether or not there was a potential cancer risk and rank activities according to this risk.

Conclusion: The developed approach-combination of characterization and construction of PAH exposure functions and modeling of cancer risk-is very promising as allowing a finer risk assessment of cancers.

\section{Abstract \#: P 352}

Joint spatial modeling of gender-specific cancer data at subregional level

Verena Jürgens $^{1}$, Joachim Kieschke ${ }^{2}$, Antje Timmer ${ }^{1}$

${ }^{1}$ Department of Health Services Research, Division of Epidemiology and Biometry, University of Oldenburg, Oldenburg, Germany; ${ }^{2}$ Epidemiological Cancer Registry of Lower Saxony, Oldenburg, Germany; Corresponding author's e-mail: verena.juergens@unioldenburg.de

Background: In 2011, breast and prostate cancer were the most common cancers diagnosed in females and males, respectively. Among others, reproductive factors have been reported to increase breast cancer risk, while in general, risk factors for prostate cancer risk are less well studied. However, similarities between the two diseases and common risk factors, such as family history and genetic mutations, have been reported. Potential determinants of the spatial and temporal distribution for both cancer sites are implementation of and access to screening. We hypothesize that a common spatial pattern exists for age group-specific male prostate cancer and female breast cancer risk. A joint spatial modeling approach was employed to analyze the geographical association between the diseases.

Methods: Age-specific female breast and male prostate cancer data at subregional level during 2008-2012 were provided by the Epidemiological Cancer Registry Lower Saxony. Incidence data were indirectly age-standardized based on the overall population of Lower Saxony as a reference. A Bayesian spatial Poisson regression model, so-called shared component model, was used to jointly model breast and prostate cancer incidence data. Model formulation considered a disease-specific unstructured and spatially structured random effect as well as one component allowing to account for a common risk between the two diseases. A conditional autoregressive prior was assigned to the spatial random terms.

Results: The relative risk ratio, which was estimated to be lower than 1 , indicated greater contribution of the shared component to breast cancer incidence (in comparison to prostate cancer). Furthermore, disease-specific and shared variability were higher for breast cancer. Overall, the spatial pattern shared by both diseases was rather minor, however, results indicated that a considerable amount of variation for breast cancer was explained by the shared component.

Conclusion: A refined study of those areas showing a common risk distribution, illustrated by disease maps, should be considered to explore its determinants. Future work might address joint modeling at a larger geographical scale, such as the country level. Moreover, model formulation might include information on hospital density or screening services/accessibility.

\section{Abstract \#: P 353}

Meta-analysis for the comparison of two tests for the screening of type 2 diabetes using information from multiple cutpoints

Annika Hoyer ${ }^{1}$, Wolfgang Rathmann ${ }^{1}$, Oliver Kuss ${ }^{1}$ 
${ }^{1}$ German Diabetes Center, Institute for Biometry and Epidemiology, Duesseldorf, Germany; Corresponding author's e-mail: annika.hoyer@ddz.uni-duesseldorf.de

Background: Most meta-analyses of diagnostic tests report more than one cutpoint for each test. Selecting only one cutpoint per study for meta-analysis, which is current practice, however, leads to a loss of information. Therefore it is necessary to account for multiple cutpoints. We propose a novel statistical model that explicitly enables us to use complete information of a diagnostic test. As an example we have compared the diagnostic utility of fasting plasma glucose (FPG) and $\mathrm{HbA} 1 \mathrm{c}$ for diabetes (reference: oral glucose tolerance test).

Methods: We analyzed 37 studies in total which have been collected in two systematic reviews. We assume binomial distributions for the true positives and true negatives for the two tests and fit a quadrivariate generalized linear mixed model using the logit link with the penalized quasi-likelihood (PQL) method to estimate differences of sensitivity and specificity between two tests. Thereby we treat the study-specific cutpoints as a covariate in a regression model. Differences of sensitivity and specificity then can be estimated as simple linear combinations of model parameters.

Results: We estimated the differences of sensitivity and specificity between the HbA1c and FPG at different cutpoints, these cutpoint ranging from 5.8 to $6.8 \mathrm{mmol} / \mathrm{l}$ on the FPG-scale. We always observed a higher sensitivity using FPG and mostly a higher specificity favoring $\mathrm{HbA} 1 \mathrm{c}$ independent of cutpoint. The estimated difference in sensitivities is about $20 \%$ (95\%-CI: [5\%,35\%]) for the lowest cutpoint and about $6 \%$ (95\%-CI: $[-10 \%, 22 \%])$ for the highest cutpoint. The estimated differences of specificity range from $13 \%$ (95\%-CI: [4\%, $23 \%]$ ) for the lowest cutpoint to $0 \%$ (95\%-CI: [-1\%, $1 \%])$ for the highest cutpoint. For a clinically sensible cutpoint of $6.5 \%$ on the $\mathrm{HbA} 1 \mathrm{c}$-scale and $6.7 \mathrm{mmol} / \mathrm{l}$ on the FPG-scale, we find a difference in sensitivity of $7 \%$ (95\%-CI: [-9\%, $23 \%])$, favoring FPG and a difference in specificity of $0 \%$ (95\%-CI: $[-1 \%, 1 \%])$.

Conclusion: With our new approach and using the complete information from the single studies, we have shown that there is a difference in the diagnostic accuracy of HbA1c and FPG which depends on the chosen cutpoint.

\section{Abstract \#: P 354}

Review of illness-death models in the epidemiology of noncommunicable diseases (NCDS)

\section{Ralph Brinks ${ }^{1}$}

${ }^{1}$ German Diabetes Center, Institute for Biometry and Epidemiology, Duesseldorf, Germany; Corresponding author's e-mail:

ralph.brinks@ddz.uni-duesseldorf.de

Introduction: State models have a long history in the field of infectious disease epidemiology - at least going back to the work of Kermack \& McKendrick in 1927. In the early 1950ies Fix and Neyman applied state models to NCDs [1], which lead to a variety of interesting theoretical results with practical implications reviewed in [2]. Currently, the most prominent application of state models in epidemiology is the Global Burden of Disease Study [3].

Methods and results: Beside a brief history of a simple state model, the illness-death model (IDM) [4], theoretical extensions to the IDM are presented. It is shown how basic epidemiological measures such as incidence rate of an NCD and mortality in a population determine the prevalence of an NCD. These basic measures determine other important outcomes, e.g. the age at onset of an NCD, disease-free survival, excess costs, the years of life lost attributable to a NCD. Furthermore, the effect of a possible intervention on these outcomes can be predicted by using IDMs. This helps to guide decision making in the health sciences [5]. The methods presented in this work are illustrated by examples from dementia and diabetes [6-8].

Conclusions: Illness-death models gain insights how basic epidemiological measures, like the incidence of an NCD, are related to relevant health related outcomes, e.g. lost lifetime or cost of illness. They may make important contributions for extending the methodological toolbox used in current epidemiology, which has been advocated for recently $[9,10]$.

\section{References}

[1] Fix E, Neyman J (1951) A simple stochastic model of recovery, relapse, death and loss of patients. Hum Bio, 205-241.

[2] Keiding N (1991) Age-specific incidence and prevalence: a statistical perspective. J Royal Stat Soc A, 371-412.

[3] Murray CJ, Lopez AD (2013). Measuring the global burden of disease. New Engl J Med 369(5), 448-457.

[4] Kalbfleisch JD, Prentice RL (2002) The statistical analysis of failure time data. John Wiley \& Sons.

[5] Waldeyer R, Brinks R, Rathmann W, Giani G, Icks A (2013) Projection of the burden of Type 2 diabetes mellitus in Germany: a demographic modelling approach to estimate the direct medical excess costs from 2010 to 2040. Diab Med 30(8), 999-1008.

[6] Brinks R, Tamayo T, Kowall B, Rathmann W (2012) Prevalence of type 2 diabetes in Germany in 2040: estimates from an epidemiological model. Eur J Epi 27(10), 791-797.

[7] Brinks R, Landwehr S, Waldeyer R (2013) Age of onset in chronic diseases: new method and application to dementia in Germany. Pop Health Metrics 11(1), 6.

[8] Brinks R, Landwehr S (2014). Age-and time-dependent model of the prevalence of non-communicable diseases and application to dementia in Germany. Theor Popul Bio 92, 62-68.

[9] Chubb MC, Jacobsen KH (2010) Mathematical modeling and the epidemiological research process. Eur J Epi 25(1), 13-19.

[10] Ughade S (2013) Statistical modeling in epidemiologic research: Some basic concepts. Clinl Epi Global Health 1(1), $32-36$.

\section{Abstract \#: P 355}

Comparing sewage-based epidemiology with epidemiological research on drug use in the general population: preliminary data

Janelle H.P. van Wel ${ }^{1}$, Juliet Kinyua ${ }^{2}$, Alexander van Nuijs ${ }^{2}$, Adrian Covaci ${ }^{2}$, Guido Van Hal ${ }^{1}$

${ }^{1}$ Dep. Epidemiology and Social Medicine, University of Antwerp, Antwerp, Belgium; ${ }^{2}$ Dep. Pharmaceutical Sciences, University of Antwerp, Antwerp, Belgium; Corresponding author's e-mail: janelle.vanwel@uantwerpen.be

Drug use is quite prevalent in the European Union. For example, over $21 \%$ of all adults (15-64 years of age) indicate having ever used cannabis. To estimate the number of drug users, population surveys are the most used technique. However, these self-report measures suffer from report biases. Therefore, policy makers and scientists wanting to make use of this information are at a disadvantage because the data might be biased. One possible way to circumvent this issue is to add more objective measures of drug use to the current methods. Sewage-based epidemiology is such a measure. Hereby, samples of wastewater are collected and analyzed for the presence of drugs and their metabolites. Since this is a relatively new technique, no studies 
have been performed to compare the results of sewage-based epidemiology to more conventional epidemiological techniques. This study aimed at combining these two measures to provide a validation for sewage-based epidemiology.

During a 12-week period (Autumn 2014), a website was opened on which inhabitants of a selected community (a town of $<30.000$ inhabitants) were asked to indicate their drug use in the past week. At the same time, wastewater samples were taken from the wastewater treatment plant (WWTP) collecting from the community. This way, comparisons could be drawn between what inhabitants of the city reported themselves about their drug use and the drug concentrations present in the water. The wastewater samples were analyzed using a validated method based on solid phase extraction (SPE) and liquid chromatography coupled to tandem mass spectroscopy (LC-MS/MS).

Answers on the questionnaires (on average $\mathrm{N}=263$ every week) showed that apart from alcohol and tobacco (60.8 and $17.5 \%$ users on average per week respectively), cannabis was the most used drug each week among the persons answering the questionnaire $(1.3 \%$ on average per week). Chemical analyses showed that cocaine (and metabolites), amphetamine, ecstasy and cannabis metabolite(s) were present in the wastewater.

Preliminary data show that it is difficult to directly compare wastewater analysis with survey data on illicit drug use, mostly because of the low response rate of the latter (1\%), possibly due to the sensitive nature of the topic. Future research on combining the two approaches should focus on either a more general approach, such as in national population surveys, or take place in a more focused setting, such as festivals, where a higher degree of drug use can be expected.

\section{Abstract \#: P 356 \\ Generalizability, validity, and efficiency of the cohort multiple randomized controlled trial design}

Joanne M. van der Velden ${ }^{1}$, H.M. (Lenny) Verkooijen ${ }^{1,2}$, Danny A. Young Afat ${ }^{1,2}$, J.P.M. (Maarten) Burbach ${ }^{1}$, Marco van Vulpen ${ }^{1}$, Carla H. van Gils ${ }^{2}$, Anne M. May ${ }^{2}$, Rolf H.H. Groenwold ${ }^{2}$

${ }^{1}$ Imaging Division, University Medical Center Utrecht, Utrecht, The Netherlands; ${ }^{2}$ Julius Center for Health Sciences and Primary Care, University Medical Center, Utrecht; Corresponding author's e-mail: j.m.vandervelden@umcutrecht.nl

Rationale and objective: The "cohort multiple Randomized Controlled Trial" (cmRCT) is proposed as an alternative to Randomized Controlled Trials (RCT). In a cmRCT study, participants with a certain condition are recruited in a prospective cohort and all receive care-as-usual. Subject characteristics are captured at baseline, and clinical and patient-reported outcomes are measured at fixed time intervals. When new interventions are ready for formal evaluation, eligible participants within the cohort are identified. From this subcohort, some participants will be randomly selected and invited to undergo the new intervention. Participants who were not randomly selected, do not receive an invitation and continue receiving care-asusual. Outcomes of the selected participants offered the new intervention will be compared to the outcomes of the participants who were not randomly selected. We aimed to review the cmRCT design, with a focus on potential methodological issues.

Results: Generalizability of trial results depend on patient trialspecific selection criteria and knowledge of underlying biological mechanisms: cmRCTs and classic pragmatic RCTs do not differ substantially. Naturally, the results of a cmRCT will only be generalizable to those settings with similar care as usual.
Validity: In a cmRCT, the timing of randomization relative to informed consent procedure differs from a classic RCT. Furthermore, only participants randomly selected to the experimental treatment group are provided with information about their allocation. As a result, only participants in the intervention arm can refuse the intervention. Since they will be followed-up and included in the analysis (intention to treat (ITT)), baseline comparability between treatment arms is maintained. Treatment effects in a cmRCT will therefore be underestimated by ITT analysis, if the treatment is effective. However, since participants in the control group are very unlikely to receive the experimental treatment this underestimation may be less pronounced as compared to a classic RCT.

Efficiency: In a cmRCT, there is unbalanced compliance: participants in the control group will most likely be $100 \%$ compliant, while compliance in the experimental group is $<100 \%$. Non-compliance in the intervention arm can be accounted for by instrumental variable (IV) analysis. Irrespective of the analysis, effect estimates tend to be more precise in a cmRCT, compared to a classic RCT with noncompliance in both treatment arms.

Conclusion: Results from RCTs conducted according to the cmRCT design are at least as generalizable and valid as results obtained in classic pragmatic RCTs, and may be even more efficient.

\section{Abstract \#: P 357}

To estimate the prevalence of disability among adults in Brazil. This study used secondary data from the national household survey

Keitty R. C. Andrade ${ }^{1}$, Ana Claudia Morais Godoy Fugueiredo ${ }^{1}$, Marcus T. Silva ${ }^{2}$, Tais F. Galvão ${ }^{2}$, Fabiana A. F. da Mata ${ }^{1}$, Priscilla Perez da Silva Pereira ${ }^{1}$, Mauricio G. Pereira ${ }^{1}$

${ }^{1}$ Faculty of Medicine, University of Brasilia, Brasilia, Distrito Federal, Brazil; ${ }^{2}$ Faculty of Medicine, University of Amazonas, Manaus, Amazonas, Brazil; Corresponding author's e-mail: keittyregina@hotmail.com

Background: To estimate the prevalence of disability among adults in Brazil and its associated factors.

Methods: This study used secondary data from the National Household Survey-PNAD 2008 (health supplementary edition). The dependent variable was disability among adults aged 18-65 years measured by the difficulty to walk one hundred meters. Odds ratios (OR) and their raw confidence intervals $(95 \% \mathrm{CI})$ were calculated and adjusted for study variables by ordinal logistic regression following a hierarchical model. In all calculations we considered the PNAD sample weights.

Results: We included 18,745 subjects, $74 \%$ of which constituted women. More than a third of adults reported having functional disability. The failure was significantly higher among men $(\mathrm{OR}=1.17$, $95 \%$ CI: $1.09-1.27)$, persons aged $35-49$ years $(\mathrm{OR}=1.30,95 \%$ CI: $1.17-1.45)$ and $50-65$ years $(\mathrm{OR}=1.38,95 \% \mathrm{CI}: 1.24-1.54)$ : individuals without occupation ( $\mathrm{OR}=2.21,95 \% \mathrm{CI}: 1.65-2.96)$ : adults who reported having cardiovascular disease ( $\mathrm{OR}=1.13,95 \%$ CI: $1.03-1.24)$, diabetes (OR $=1.16,95 \%$ CI: 1.05-1.29), hypertension (OR $=1.10,95 \% \mathrm{CI}: 1.02-1.18)$, and arthritis/rheumatism $(\mathrm{OR}=1.24$, $95 \% \mathrm{CI}: 1.15-1.34)$ : and participants who were hospitalized in the last twelve months $(\mathrm{OR}=2.35,95 \%$ CI 1.73-3.2). Conclusions: Functional disability is common among Brazilian adults. Hospitalization is most strongly associated factor, followed by occupation and chronic diseases. Sex, age, education and income are also associated. The results indicate specific actions to address the main factors associated with disability targets, and contribute to the 
projection of interventions for improving well-being and promoting quality of life of Brazilian adults.

\section{Abstract \#: P 358}

Compliance to oral bisphosphonate therapy and fracture risk: influence of exposure misclassification in pharmacy claims data

Andrea M. Burden ${ }^{1,2}$, Andrea Gruneir ${ }^{3}$, J. Michael Paterson ${ }^{3,4}$, Yannick J. Nielen $^{2}$, Frank de Vries ${ }^{2,5}$, Suzanne M. Cadarette ${ }^{1,4}$

${ }^{1}$ Leslie Dan Faculty of Pharmacy, University of Toronto, Toronto, Canada; ${ }^{2}$ Department of Epidemiology, Maastricht University, Maastricht, Netherlands; ${ }^{3}$ Institute of Health Policy, Management and Evaluation, University of Toronto, Toronto, Canada; ${ }^{4}$ Institute for Clinical Evaluative Sciences, Toronto, Canada; ${ }^{5}$ Department of Clinical Pharmacy and Toxicology, Maastricht University Medical Centre+, Maastricht, Netherlands; Corresponding author's e-mail: andrea.burden@gmail.com

Background: Pharmacy claims data are commonly used to estimate drug exposure. We previously identified misclassification in days supply values that underestimated medication compliance, particularly in long-term care (LTC). In this study we examined the impact of oral bisphosphonate exposure misclassification in days supply values on estimates of drug effectiveness in reducing hip fractures. Methods: We used Ontario administrative claims data to identify new users of oral bisphosphonates aged 66 or more years between 2001 and 2011. Medication compliance was quantified by the proportion of days covered (PDC) and categorized into groups according to a 365-day ascertainment period. PDC was calculated using observed and cleaned days supply values. Hip fracture rates within 365 days after the ascertainment period were calculated using Cox proportional hazard models, adjusted for behavioural and fracture risk factors. Low compliance (PDC $<20 \%$ ) was the referent. Analyses were completed overall and separately for patients in community and LTC settings.

Results: The rate of hip fracture was higher in LTC (2.4/100 patientyears) than in the community (1.0/100 patient-years). Overall, cleaning days supply values increased the estimated benefit of high compliance $(\mathrm{PDC} \geq 80 \%)$ on fracture prevention (HRobserved $=0.74,95 \% \quad \mathrm{CI}=0.66-0.83: \quad$ HRcleaned $=0.65,95 \%$ $\mathrm{CI}=0.57-0.74)$. Risk estimates were similar among communitydwelling patients (HRobserved $=0.68,95 \% \quad \mathrm{CI}=0.60-0.77$ : HRcleaned $=0.65,95 \% \mathrm{CI}=0.56-0.75)$, yet differed substantially in LTC before data cleaning (HRobserved $=0.96, \quad 95 \%$ $\mathrm{CI}=0.73-1.26:$ HRcleaned $=0.64,95 \% \mathrm{CI}=0.46-0.91)$.

Conclusion: Misclassified days supply values can bias estimates of drug effectiveness. Larger effects on risk estimates were noted in LTC, where fracture risk is highest. Overall, little change was noted in the community setting, where most studies are completed. Thus, results caution researchers to examine days supply accuracy in pharmacy claims data when estimating the relationship between drug exposure and health outcomes, particularly when including LTC settings.

\section{Abstract \#: P 359}

What are the most important targets for the management of progression in CKD?

Marieke M.H.C van Rijn ${ }^{1}$, Jan A.J.G. van den Brand ${ }^{1}$, Arjan D. Van Zuilen $^{2}$, Peter J. Blankestijn ${ }^{2}$, Jack F. Wetzels ${ }^{1}$
${ }^{1}$ Nephrology, RadboudUMC, Nijmegen, The Netherlands;

${ }^{2}$ University Medical Centre Utrecht, the Netherlands; Corresponding author's e-mail: mariekevanrijn@live.nl

Background: KDIGO guidelines for management of CKD recommend interventions on blood pressure, proteinuria, glycemic control, salt intake, lifestyle, anemia and calcium-phosphorus metabolism. We previously showed that close adherence to these guidelines reduced the risk of progression in CKD (Peeters, JASN, 2014). However, simultaneously addressing all aspects of CKD management can be challenging for doctor and patient. The purpose of this study was to identify the most important treatment factors, so that interventions may be prioritized.

Methods: The MASTERPLAN study was a RCT performed in 9 Dutch hospitals. The intervention consisted of 14 targets based on guidelines. In total 788 patients with eGFR between 20 and $70 \mathrm{ml} /$ min per $1.73 \mathrm{~m}^{2}$ were included. The outcome was a composite of death, end stage renal disease, and $50 \%$ increase in serum creatinine. The total effect of the MASTERPLAN can be split up in a direct and indirect effect. The indirect effect is defined as the proportion of the total effect explained by a change in treatment target at one year follow-up. It can be determined with mediation analysis (Imai, APSR, 2011).

Results: We were able to explain $57 \%$ of the risk reduction in the intervention arm through a reduction in blood pressure, proteinuria and LDL-cholesterol. However, a substantial proportion of risk reduction due to nurse practitioner aided care remained unexplained.

\begin{tabular}{llllll}
\hline & TE & $95 \%$ CI & ID & DE & $\%$ mediated \\
\hline $\begin{array}{l}\text { Log protein } \\
\quad \text { creatinine ratio }\end{array}$ & -227 & -468 to 17 & -55 & -171 & 24 \\
LDL cholesterol & -264 & -481 to -50 & -34 & -231 & 13 \\
Blood pressure & -210 & -425 to 4 & -31 & -181 & 20 \\
$\begin{array}{l}\text { Log parathyroid } \\
\quad \text { hormone }\end{array}$ & -266 & -489 to -47 & -1 & -207 & 0 \\
Total & & & & & \\
\hline
\end{tabular}

The estimates for total effect (TE), indirect effect (IE) and direct effect (DE) can be interpreted as the decreased number of patients with a composite endpoint per 10,000 patients a year.

Conclusions: The reduced risk due to the MASTERPLAN intervention was mediated through lower protein creatinine ratio, LDL cholesterol and blood pressure, which may thus be priorities for a more focused intervention.

\section{Abstract \#: P 360}

On the distinction between average number of events per person-time and geometric rate

Matteo Bottai ${ }^{1}$, Nicola Orsini ${ }^{1}$

${ }^{1}$ Unit of Biostatistics, Institute of Environmental Medicine, Karolinska Institutet; Corresponding author's e-mail: nicola.orsini@ki.se

Measures of occurrence of events over time have long been studied and widely utilized in many areas of modern epidemiology. The incidence rate, defined as number of events over person-time, is one 
of the most popular among them. This measure, however, is often misunderstood.

We aim to describe a different type of rate, the geometric rate. The geometric rate represents the probability of an event per unit time, whereas the traditional incidence rate represents the average number of events per unit time. We show that the two types of rates measure different quantities and should not be used interchangeably. Which of the two is the more appropriate depends on the type of event being investigated.

We define the geometric rate and describe its properties, its connection to the Kaplan-Meier estimator, and its dissimilarities with the traditional person-time rate. The geometric rate is then applied to analyze survival in a large prospective cohort. Too frequently the traditional incidence rate is reported when the geometric rate would be preferable.

\section{Abstract \#: P 361}

New insight into type 2 diabetes in the light of data mining: results from TLGS prospective study

Azra Ramezankhani ${ }^{1}$, Esmaeil Hadavandi ${ }^{2}$, Omid Pournik ${ }^{3}$, Jamal Shahrabi $^{2}$, Fereidoun Azizi ${ }^{4}$, Farzad Hadaegh ${ }^{1}$

${ }^{1}$ Prevention of Metabolic Disorders Research Center, Research Institute for Endocrine Sciences, Shahid Beheshti University of Medical Sciences, Tehran, Iran; ${ }^{2}$ Industrial Engineering Department, Amirkabir University of Technology, Tehran, Iran; ${ }^{3}$ Department of Community Medicine, School of Medicine, Iran University of Medical Sciences, Tehran, Iran; ${ }^{4}$ Endocrine Research Center, Research Institute for Endocrine Sciences, Shahid Beheshti University of Medical Sciences, Tehran, Iran; Corresponding author's e-mail: ma.ramezankhani@gmail.com

Background: The aim of this study was to develop the prediction models for type 2 diabetes in adult men and women using the most common classification methods and gain further insight into the factors contributing to incidence of diabetes.

Methods: Data on 6647 (2885 men) participants of Tehran Lipid and Glucose Study aged $>20$ years, without diabetes at the baseline that completed a 9 year follow-up were used to develop a series of risk prediction models by 3 types of Decision Trees (the Classification and Regression tree $(\mathrm{C} \& \mathrm{R})$, Quick Unbiased Efficient Statistical Tree (QUEST), and C5.0). The most important predictor variables were selected by two filter approach (Genetic Algorithm and Best First) from the demographic characteristics, smoking status, medical and drug history and laboratory measures. For handling the imbalanced problem in datasets we applied SMOTE (Synthetic Minority over sampling Technique). We compared the classification efficiency of decision trees to logistic regression using sensitivity and G-Mean.

Results: During follow-up, 729 individuals (302 men) developed diabetes. The QUEST had the highest sensitivity and G-mean among all models for both men and women. Sensitivity was $78 \%$ for both men and women in models that included 2-h post-challenge plasma glucose (2-h PCPG). For models without 2-h PCPG, the sensitivity was $68 \%$ and $73 \%$ for men and women respectively.

Sensitivity analysis showed that the most important variable in the models is fasting plasma glucose (FPG): the next three were 2-h PCPG, waist-to-height ratio (WHtR) and mean arterial blood pressure (MAP). The QUEST produced a set of rules, which each rule separate the study samples into several subgroups. The rules for women showed that if the WHtR is $<0.52$, diabetes will not occur at least with probability $74 \%$ independent of the FPG and 2 h-PCPG levels. The rules for men showed that, if FPG level is $<88 \mathrm{mg} / \mathrm{dl}$, diabetes does not occur in $86 \%$ of the time. Therefore, in men, FPG level had more predictive effect compared to women. Results showed that the age $>43$ years and a MAP $>92 \mathrm{mmHg}$ is a risk factor for men who have FPG level greater than $95 \mathrm{mg} / \mathrm{dl}$. In women, a MAP $>97 \mathrm{mmHg}$ is a risk factor when $\mathrm{WHtR}$ is $>0.66$.

\section{Abstract \#: P 362}

Including unpublished results in a systematic review of diagnostic accuracy studies: impact on summary estimates?

Daniël A. Korevaar ${ }^{1}$, Guus A. Westerhof ${ }^{2}$, Junfeng Wang ${ }^{1}$, Jérémie F. Cohen ${ }^{1}$, René Spijker ${ }^{3}$, Peter J. Sterk ${ }^{2}$, Elisabeth H. Bel ${ }^{2}$, Patrick M.M. Bossuyt ${ }^{1}$

${ }^{1}$ Department of Clinical Epidemiology, Biostatistics and Bioinformatics, Academic Medical Centre, University of Amsterdam; ${ }^{2}$ Department of Respiratory Medicine, Academic Medical Centre, University of Amsterdam; ${ }^{3}$ Medical Library, Academic Medical Centre, University of Amsterdam; Corresponding author's e-mail: d.a.korevaar@amc.uva.nl

Background: Many research results remain unpublished. Favorable results have higher chances of getting published than unfavorable results. This could introduce bias in approaches to aggregate data. We performed a systematic review of the diagnostic accuracy of minimally invasive markers for the detection of airway eosinophilia in asthmatic patients, and aimed to include unpublished results.

Methods: We searched MEDLINE, Embase and PubMed for studies evaluating the diagnostic accuracy of Fraction of Exhaled Nitric Oxide (FeNO), blood eosinophils or total Immunoglobulin E (IgE), against a reference standard of induced sputum, bronchoalveolar lavage and/or endobronchial biopsy in adults with (suspected) asthma (inception-August 2014). Unpublished results were obtained by contacting authors of studies that did not report on diagnostic accuracy, but seemed to have data from which estimates could be calculated. Such studies were selected if they had included at least 50 patients, and had performed at least one index test and one reference standard, as defined above. Random effects meta-analysis was used to produce summary estimates.

Results: Analysing 2,919 search results, we found 12 studies reporting on the accuracy of FeNO, blood eosinophils and/or IgE. We considered these as published results. We contacted the authors of 2 of these studies, and obtained additional diagnostic accuracy data of markers that had also been assessed, but for which no accuracy results were reported. We considered these as unpublished. Authors of another 14 articles were contacted because they could have data from which accuracy estimates could be calculated. Seven shared the requested data. We also included a conference poster. These data were all considered as unpublished. Overall, we included 20 studies. Adding unpublished data led to a $28 \%$ increase in sample size for FeNO and blood eosinophils, and $103 \%$ for IgE. The summary area under the receiver operator curve for FeNO was 0.75 (0.72-0.78: 9 studies: 2263 patients) based on published data, versus 0.73 (0.68-0.78: 7 studies: 627 patients) for unpublished data (overall $0.74: 0.72-0.77$ ). For blood eosinophils, these numbers were 0.77 (0.71-0.83: 7 studies: 1592 patients) versus 0.77 (0.71-0.83: 5 studies: 446 patients) (overall 0.77: 0.72-0.81). For IgE, these were 0.64 (0.57-0.70: 2 studies: 300 patients) versus 0.61 (0.54-0.67: 4 studies: 310 patients) (overall 0.62: 0.58-0.67).

Conclusion: Many authors were willing to share unpublished results. Adding these data led to a considerable increase in precision, but barely affected summary estimates of accuracy. Unfortunately, FeNO, blood eosinophils and IgE have moderate accuracy in detecting airway eosinophilia. 


\section{Abstract \#: P 363}

\section{A comparison of one-stage vs. two-stage dose-response meta-} analysis

\author{
Alessio Crippa $^{1}$, Nicola Orsini ${ }^{1}$ \\ ${ }^{1}$ Institute of Environmental Medicine, Karolinska Institutet, \\ Stockholm, Sweden; Corresponding author's e-mail: \\ alessio.crippa@ki.se
}

The objective of a dose-response meta-analysis is to summarize the available scientific evidence in order to characterize the shape of the association between a quantitative exposure and a health outcome. The standard method is a two-stage approach. First the dose-response trends are estimated separately for each study included in the meta-analysis. The study-specific coefficient estimates together with the corresponding (co)variances are then used as primary data for meta-analysis.

One-stage approach has never been fully described and applied. It involves pooling study-specific data before investigating the doseresponse association. Trend coefficients and between-studies heterogeneity are estimated simultaneously using multivariable random effects models. The main advantage of this procedure is to use of the whole amount of information available. It would also allow the proper use of established measures to compare alternative models such as information criteria.

To the best of our knowledge, no formal comparison between the two approaches has been conducted. Our aim is to investigate analogies and dissimilarities between the techniques based on either theoretical or real examples. Features to be compared include model definition, estimation methods hypothesis testing, prediction, and comparison of model fit. The one- and two-stage approaches will be implemented in a novel package in the statistical software R.

\section{Abstract \#: P 364}

\section{Reporting weaknesses in journal abstracts of diagnostic accuracy} studies

Daniël A. Korevaar ${ }^{1}$, Jérémie F. Cohen ${ }^{1}$, Lotty Hooft ${ }^{2}$, Patrick M.M. Bossuyt ${ }^{1}$

${ }^{1}$ Department of Clinical Epidemiology, Biostatistics and Bioinformatics, Academic Medical Centre, University of Amsterdam; ${ }^{2}$ Dutch Cochrane Centre, Julius Center for Health Sciences and Primary Care, University Medical Centre Utrecht, University Utrecht; Corresponding author's e-mail: d.a.korevaar@amc.uva.nl

Background: Informative journal abstracts are crucial for the identification and initial appraisal of studies. We evaluated the informativeness of abstracts of diagnostic accuracy studies.

Methods: We searched PubMed for reports of studies that had evaluated the diagnostic accuracy of one or more tests against a clinical reference standard, published in twelve higher-impact journals in 2012. Two authors independently evaluated the information contained in included abstracts using 21 items. These items were selected based on published guidance for adequate reporting and for study quality assessment. The items focused on study identification, rationale, objectives, methods for recruitment and testing, participant baseline characteristics, missing data, test results and reproducibility, estimates of diagnostic accuracy, and discussion of study findings, implications and limitations.

Results: We included 103 abstracts. Crucial information on study population, setting, patient sampling, and blinding, as well as confidence intervals around accuracy estimates were reported in less than half of the abstracts. Only research aims, study design, information on the index test, the number of participants, estimates of diagnostic accuracy, and a discussion of the diagnostic accuracy of the index test under evaluation were reported in more than $75 \%$ of the abstracts. The mean number of reported items per abstract was 10.1 out of 21 (SD 2.2: range 6-15). The mean number of reported items was significantly lower for multiple-gate (case-control) studies compared to single-gate (cohort) studies $(9.0$ vs. $10.6(p=0.001)$ ), in reports in specialty journals compared to those in general journals (9.6 vs. 12.2 $(p<0.001))$, and for studies with sample sizes and word counts below the median compared to those above (9.4 vs. $10.8(p=0.001)$, and 9.5 vs. $10.6(p=0.008)$, respectively). No significant differences were found between studies evaluating imaging tests, laboratory tests and other types of tests (10.6 vs. 9.6 vs. $10.1(p=0.13))$.

Conclusion: Many abstracts of diagnostic accuracy study reports in higher-impact journals fail to report essential elements, such as descriptions of the study population, or do not refer to imprecision. Developing and implementing guidelines for such abstracts could help the transparency and completeness of reporting.

\section{Abstract \#: P 365}

Increased homicide mortality in men of 15-19 years old in the municipality of Maceio, Alagoas, Brazil: an intervention proposal

Alice C. M. das Neves ${ }^{1}$, Ana Lucía G. Barroso ${ }^{2}$, Bruno Z. De Oliveira $^{3}$, Célia M. C. Aly ${ }^{4}$, Cintia C. G. Da Silva ${ }^{5}$, Henrique de B. M. Beltrão ${ }^{6}$, María Belén J. Sanabria ${ }^{7}$, Maria M. U. Gutierrez ${ }^{8}$

${ }^{1}$ Postgraduate Programs in Public Health. University of Brasilia. Brasília. Brazil; ${ }^{2}$ Epidemiologist. Department of Epidemiology, Ministry of Health of Mexico. DF, Mexico; ${ }^{3}$ Nutritionist. Ministry of Health of Brazil. Brasilia. Brazil; ${ }^{4}$ Psychologist. Ministry of Health of Brazil. Brasilia. Brazil; ${ }^{5}$ Bachelor in Public Health. Ministry of Health of Brazil. Brasilia. Brazil; ${ }^{6}$ Biomedical. Ministry of Health of Brazil. Brasilia. Brazil; ${ }^{7}$ Nurse. Ministry of Health and Social Protection of Colombia. Bogotá, Colombia; ${ }^{8}$ Doctor. Department of Public Health. University of Brasília. Brasilia. Brazil; Corresponding author's e-mail: acnmedeiros@gmail.com

Background: In Brazil, the Youth Act was enacted in 2013, seeking to develop intersectoral policies and programs for youth. Violence is the main cause of youth mortality in Brazil. The city of Maceio (capital of Alagoas) is the most violent of Brazil and is ranked 5th in the world, according to the Citizen Council for Public Safety and Criminal Justice. This study aims to propose an intervention to reduce mortality from homicide among young people 15-19 years of age in the city of Maceio, Alagoas, Brazil.

Methods: In this project the Multilevel Model of Social Determinants of Health was selected. Own calculations were performed to reverse the determinants that were behaving as protective factors to allow calculation of the population attributable risk in order to prioritize the key determinants affecting the population under 20 years of age. By reviewing literature and by using the nominal group technique priority determinants were identified and prioritized: education or schooling: lack of social support: access to illicit drugs: poverty: unemployment and delinquent behavior or police record.

Results: A decision process with four strategies was conducted: intersectoral actions focused on equity: implementation of an integral center for culture, education and sport: incentives to complete and attend school, and, the strengthening of promotion health activities. To determine the highest score of the strategies, the nominal group technique and a combination of the Hanlon/CENDES technique was used. The strategy selected focuses on the National Policy for Health Promotion and represents the one that will allow us to influence a 
combination of several of the intersectoral factors. Actions, targets and indicators of structure, process, outcome and impact to evaluate the actions were defined. The main goal of the intervention was established as the reduction of homicide mortality in men of 15-19 years old living in Maceio/Alagoas, in $20 \%$ within 5 years, from 2015 to 2020 . To assess the impact and effectiveness of the strategy in the population two strategies were developed: a health situation room to monitor the number of cases of interpersonal violence and murders and a strategy to evaluate the proposed intervention by a quasi-experimental study type design "before and after with a comparison group".

Conclusion: In Maceio/Alagoas, health promotion strategies need to be strengthened in order to reduce violent youth mortality, as well as, the implementation of violence surveillance and effective use of data for decision making and impact evaluation of interventions.

\section{Abstract \#: P 366}

\section{Assessment of premature deaths in Istanbul using geographic information system}

Ulken Tunga Babaoglu ${ }^{1}$, Ahmet Topuzoğ lu ${ }^{2}$, Halim Issever ${ }^{3}$, Bilge Hapçıoglu ${ }^{3}$, Huseyin Turoglu ${ }^{4}$

${ }^{1}$ Ahi Evran University, College of Health, Kirsehir, Turkey; ${ }^{2}$ Marmara University, Faculty of Medicine, Department of Public Health, Istanbul, Turkey; ${ }^{3}$ Istanbul University, Faculty of Medicine, Department of Public Health, Istanbul, Turkey; ${ }^{4}$ Istanbul University, Faculty of Letters, Department of Geography, Istanbul, Turkey; Corresponding author's e-mail: ulkentunga@yahoo.com

Background: Analysing mortality rates and reasons in Turkey's urban allocations using geographic information systems (GIS) is a new research area. While planning health services, knowing the reasons and mortality rates according to age and residental area is a big source for health planners and for people who give health service. The aim of this research is to analyse the deaths under 5 year age in big cities depending on the socio economical range by using GIS. Also it will be possible to determine geographical risky places.

Methods: In this ecological research, under 5 year deaths that occurred between 2005 and 2009 were analyzed. Turkish statistical institute (Turk Stat) population and age specified death data's: Istanbul Metropolitan municipality (IMM) cemetery directorate death records and Ministry of Finance land unit prices (in order to determine the social-economical level of the districts) have been used as data resources. $\mathrm{Z}$ value of each year's death, depending on the districts age ranges and average of all years $Z$ value has been calculated and shown on the map.

Results: While under- 5 mortality rates was 19.37 per thousand in 2005, it was 14.31 per thousand in 2009. Deaths under 5 year old especially took place in Istanbul European side (in districts where the land value is low).

Conclusions: Priority should be given to health promotion programs especially in areas with low socioeconomic level where premature death rates are observed more common.

\section{Abstract \#: P 367}

\section{A lots quality assurance sampling (LQAS) strategy for antimicrobial resistance surveillance informing empirical treatment guidelines}

Frank van Leth ${ }^{1}$, Casper den Heijer ${ }^{2,3}$, Ellen Stobberingh ${ }^{2}$, Ge Donker ${ }^{4}$, Constance Schultsz ${ }^{1,5}$
${ }^{1}$ Department of Global Health, Academic Medical Center, University of Amsterdam, Amsterdam Institute for Global Health and Development, Amsterdam, The Netherlands; ${ }^{2}$ Department of Medical Microbiology, Maastricht University, School of Public Health and Primary Care, Maastricht, The Netherlands; ${ }^{3}$ Department of Sexual Health, Infectious Diseases and Environmental Health, Public Health Service South Limburg, Geleen, The Netherlands; ${ }^{4}$ Netherlands Institute for Health Service Research (NIVEL), Utrecht, The Netherlands; ${ }^{5}$ Department of Medical Microbiology, Academic Medical Center, University of Amsterdam, Amsterdam, The Netherlands; Corresponding author's e-mail: f.vanleth@aighd.org

Background: Antimicrobial resistance (AMR) is a major threat to global health. AMR-surveillance is typically based on laboratory isolates only. Community-based AMR surveillance in research settings is expensive, time-consuming and needs large sample sizes, precluding timely and locally relevant estimates. The absence of upto-date community-based AMR estimates jeopardizes empirical treatment for common infections. A Lots Quality Assurance Sampling (LQAS) strategy assesses whether AMR is above or below certain boundaries rather than estimates the prevalence of AMR, thereby using markedly smaller sample sizes than conventional surveillance. We assessed the performance of LQAS-based AMR surveillance for Escherichia coli causing urinary tract infections (UTI) in patients attending general practitioners (GP) in The Netherlands.

Methods: Data on AMR for 8 antibiotics for UTI were prospectively obtained from 42 GP practices (Sentinel Practices of NIVEL Primary Care Database). AMR boundaries for "low" and "high" were defined with corresponding LQAS sample size and "decision rule" (minimum number resistant isolates needed for classification "high"). AMR boundaries (sample size: decision rule) were 2-10\% (76:4), 5-20\% (45:5), 10-30 \% (42:7), 15-40\% (34:9), and 20-50 \% (30:10). The sample sizes were drawn 100 times with replacement from all available data. Mean proportion LQAS samples denoting "high AMR" was compared with AMR prevalence in the full data set. LQAS classification was adequate when misclassification did not exceed pre-set allowable misclassification (5 and $10 \%$ for "low" and "high" AMR, respectively).

Results: LQAS classification depended on the relationship between point estimate with $95 \%$ confidence interval (CI) of AMR prevalence, and set boundaries. Being good when point estimate and CI were fully below or above boundaries, being moderate when point estimate was between lower and upper boundary but CI crossed one boundary, and being poor when point estimate and CI were fully between boundaries.

AMR against all antibiotics was adequately classified with the following boundaries (AMR point prevalence): $2-10 \%$ [nitrofurantoin $(0.1 \%)$, augmentin $(0.5 \%$ ], 5-20\% [norfloxacin $(2.4 \%)$, ciprofloxacin $(2.4 \%)$, gentamicin $(2.5 \%)$ ], 10-30\% [trimethoprim $(7.5 \%)$ ], and 20-50\% [cotrimoxazole (7.5\%), amoxicillin (33\%]. Conclusion: LQAS-based AMR surveillance can adequately capture background AMR prevalence, making it a valuable tool in providing timely and locally relevant information for empirical treatment guidelines. Deciding on clinically relevant AMR boundaries is crucia for good performance of the strategy. Boundary setting can be done through expert opinion, use of laboratory surveillance, and/or from a posterior distribution derived in a Bayesian analysis of surveillance data where available.

\section{Abstract \#: P 368}

Determinants for baseline participation rates in large populationbased biobank studies: a systematic review 
Sander K.R. van Zon ${ }^{1}$, Salome Scholtens ${ }^{2}$, Sijmen A. Reijneveld ${ }^{1}$, Nynke Smidt $^{3}$, Ute Bültmann ${ }^{1}$

${ }^{1}$ Department of Health Sciences Division of Community and Occupational Medicine, University Medical Center Groningen, University of Groningen, Groningen, The Netherlands; ${ }^{2}$ LifeLines Cohort Study and Biobank; ${ }^{3}$ Department of Epidemiology, University Medical Center Groningen, University of Groningen, Groningen, The Netherlands; Corresponding author's e-mail: s.k.r.van.zon@umcg.nl

Background: Insight into baseline participation rates in large population-based biobank studies and into their determinants is crucial for the design and planning of future biobank studies and provides an indication of the internal validity of studies. Therefore, we conducted a systematic review of baseline participation rates in large longitudinal population-based biobank studies and their determinants.

Methods: All studies registered within the Public Population Project in Genomics and Society (P3G) and Biobanking and Biomolecular Resources Research Infrastructure (BBMRI) catalogues were screened for potentially eligible large ( $\mathrm{n}>10,000$ participants), longitudinal population-based biobank studies. Subsequently, the full text (design) papers of the biobank studies were retrieved. Participation rates were considered high if they were $\geq 75 \%$.

Results: Twenty-five population-based biobank studies, with participation rates varying from 5 to $96 \%$, were included in this review. High participation rates were most often found in studies that used a face-to-face recruitment method (4/4 studies: $100 \%$ ), visited participants for an interview or physical examination (3/4 studies: $75 \%$ ), collaborated with a trusted research organization or governmental body (5/8 studies: $63 \%$ ), were conducted in Asia (5/6 studies: $83 \%$ ), started recruiting participants between 1990 and 1999 (6/11 studies: $55 \%$ ) or included 50,000-100,000 participants (4/7 studies: $57 \%$ ). Conclusions: Participation rates vary widely between populationbased biobank studies. Modifiable design and recruitment factors like face-to-face recruitment, visiting participants for an interview or physical examination and collaboration with a trusted research organization or governmental body were associated with high participation rates. These insights may help future population-based biobank studies to increase participation rates and thereby increasing the validity of their findings.

\section{Abstract \#: P 369}

Surveilling the incidence of non-communicable diseases (NCDS) using low resources

\section{Ralph Brinks ${ }^{1}$}

${ }^{1}$ German Diabetes Center, Institute for Biometry and Epidemiology, Duesseldorf, Germany; Corresponding author's e-mail: ralph.brinks@ddz.uni-duesseldorf.de

Introduction: In 2010 more than $60 \%$ of all global deaths were caused by NCDs [1]. Surveillance as demanded by the Moscow Declaration [2] and the United Nations' General Assembly [3] is an important step to encounter the growing global burden. Considering the prevalence of NCDs is not sufficient as the prevalence may increase if survival of the diseased persons increases. Thus, the Moscow Declaration requests surveilling the incidence of NCDs.

Typically trends in incidence are examined by disease registers or a series of subsequent cohort studies. In countries with low resources both is difficult to obtain. Compared to registers and a number of cohort studies, a series of cross-sectional requires less effort.

In [4] we described a method how two cross-sectional studies combined with information about the mortality can be used to estimate the incidence of a chronic disease. Here we take this approach further and show how a series of cross-sections can examine possible trends in incidence.

Methods: Based on data from the Danish Diabetes Register [5] we simulate a sequence of cross-sectional studies describing the agespecific prevalence of diabetes in the years 1995-2004. We assume that the general mortality in the population and the hazard ratio of the mortality between diseased and non-diseased subjects are known.

Then, we estimate the age-specific incidence rates by repetitively applying the algorithm of [4] to the simulated prevalence data. Finally, the incidence rate used for the simulation ("true incidence") and the estimated incidence are compared in terms of the absolute relative error (ARE).

Results: The median ARE (calculated over all age groups and all years 1995-2004) is 0.8 per mille. The maximum ARE is $1.47 \%$, which indicates a very accurate estimation. The trend from the simulated data is clearly detectable.

Discussion: The proposed method provides accurate estimates of the trend in the age-specific incidence. The accuracy is likely to be sufficient for surveillance of chronic diseases without running expensive disease registers or cohort studies. Our approach just requires a series of cross-sectional studies, the mortality rate of the general population (typically available from official vital statistics) and a series of case-control studies [6].

The method provides a means of detecting trends in the incidence of a chronic disease with relatively low resources. Hence, the algorithm is advantageous for use in developing countries or other settings where resources are sparse.

\section{References:}

1) Lozano et al., Lancet 2012

2) Moscow Declaration, April 2011

3) UN Resolution 66/2

4) doi: 10.1093/imammb/dqu024

5) Carstensen et al., Diabetologia 2008

6) Pearce, International Journal of Epidemiology 1993

\section{Abstract \#: P 370}

\section{Drug survival studies- principles, purposes and pitfalls}

J.M.P.A. van den Reek ${ }^{1}$, W. Kietvit ${ }^{2}$, R. Gniadecki ${ }^{3}$, J.J. Goeman ${ }^{2}$, J. Zweegers $^{1}$, P.C.M. van de Kerkhof ${ }^{1}$, M.M.B. Seyger ${ }^{1}$, M.G.J. de Jong $^{1}$

${ }^{1}$ Department of Dermatology, Radboudumc, Nijmegen, The Netherlands; ${ }^{2}$ Department for Health Evidence, Radboudumc, Nijmegen, The Netherlands; ${ }^{3}$ Department of Dermatology, Bispebjerg Hospital, Bispebjerg, Denmark; Corresponding author's e-mail: juul.vandenreek@radboudumc.nl

With rising health-care costs and a growth of pharmaceutical options, health professionals are continuously looking for better methods to evaluate treatments. The method of 'drug survival' is a comprehensive outcome covering effectiveness, safety, and patients' and doctors' preferences. Drug survival is the time patients remain on a specific drug. It is typically research that can be done in daily clinical practice and it is investigated using the technique of Kaplan-Meier survival analysis, in which the 'event' refers to the actual discontinuation of a drug. Identifying drugs with long drug survival rates as well as ways to prolong drug survival is important. It is particularly suitable for chronic diseases that require long-term treatment, such as biologic drugs in rheumatology and dermatology. Frequently used synonyms for drug survival are 'drug retention' and 'drug longevity'.

Drug survival studies have been used and analyzed for various purposes: head-to-head comparisons between different drugs can be 
used to identify the drug with the longest survival rates. These comparisons are important in clinical decision making. Furthermore, subanalyses split for different reasons of discontinuation can be used to deepen insight in reasons for short drug survival. Knowledge on the reason for discontinuation is extremely important for the clinician. Another important purpose is to identify useful predictors for long drug survival, using Cox-regression analysis. In clinical practice, these predictors can be used to select those patients that will benefit most from a certain drug. Also, studying and comparing drug survival of different (disease) groups may provide insight whether or not we can exchange knowledge between those groups. Finally, drug survival rates can be combined with quality-of life-measures. As we put effort in prolonging drug survival-rates, we think it is also of importance to know whether being 'on drug' is compatible with acceptable qualityof-life outcomes.

As methodological designs and patient-selection criteria differ among published drug survival studies, these studies cannot always simply be compared. Moreover, it must be kept in mind that drug survival is influenced by behavioral factors and secular trends, such as the physicians' preferences and the changing availability of therapeutic alternatives.

This paper provides an overview of the methodology, purposes and limitations of drug survival. In order to make future drug survival studies more comparable and of high quality, we formulated 7 suggestions to harmonize outcomes. For indexing and to prevent false interpretations of the concept, we argue that the number of synonyms used should be reduced and propose the worldwide adoption of the term 'drug survival'.

\section{Abstract \#: P 371}

Cortisol validation study: one awakening salivary cortisol sample is reliable in pregnant women

\section{$\underline{\text { Richelle Vlenterie }}^{1}$, Nel Roeleveld ${ }^{1}$, Marleen M.H.J. van Gelder ${ }^{1}$}

${ }^{1}$ Radboud Institute for Health Science, Radboud university medical center, Nijmegen, The Netherlands; Corresponding author's e-mail: richelle.vlenterie@radboudumc.nl

Background: Maternal depression, anxiety, and stress during pregnancy have been associated with adverse effects on maternal as well as fetal health and may increase the risk of severe adverse pregnancy outcomes. Elevated cortisol levels are associated with these mood, anxiety, and stress disorders and can be measured in saliva. Several studies have already been conducted to measure cortisol levels in pregnant women using multiple measurements on consecutive days, which may lead to participation burden and high costs. Objective: The aim of this validation study was to examine whether one awakening salivary cortisol measurement will suffice to classify pregnant women as having normal or elevated cortisol levels compared to collecting awakening salivary cortisol measurements on three consecutive working days.

Methods: Salivary cortisol samples used for this validation study were collected in a sub-cohort of the PRegnancy and Infant DEvelopment (PRIDE) Study. Women were asked to collect three saliva samples on consecutive working days within 10 min after waking up. The Intraclass correlation coefficient (ICC) and Cohen's kappa coefficient were calculated for the cortisol samples measured on day one and the average of the cortisol samples measured on three consecutive days. Sub-analyses within stratified factors as time of sampling, employment and other maternal characteristics were done for both the continuous and categorical data to further examine the reliability of the cortisol measurements.
Results: The total study population consisted of 199 women. The overall ICC between day one and the average of awakening cortisol levels on the three consecutive days was 0.79 (95\% CI 0.73-0.84). The kappa coefficient for agreement between the two measurements was 0.74 (95\% CI 0.63-0.85).

Conclusion: One awakening salivary cortisol sample provides a reliable measurement for classifying pregnant women as having normal or elevated cortisol levels in epidemiologic studies.

\section{Abstract \#: P 372}

Application of the self-controlled case series design in pharmacoepidemiological studies: a cautionary note

Md. Jamal Uddin ${ }^{1}$, Rolf H.H. Groenwold ${ }^{2}$, M. Sanni Ali ${ }^{2}$, Gianmario Candore $^{3}$, Mark C.H. de Groot ${ }^{2,4}$, Patrick C. Souverein ${ }^{2,4}$, Yolanda Alvarez $^{3}$, Svetlana V. Belitser ${ }^{2,4}$, Arno W. Hoes ${ }^{2}$, Anthonius de Boer $^{2,4}$, Kit C.B. Roes ${ }^{2}$, Olaf H. Klungel ${ }^{2,4}$, Helga Gardarsdottir ${ }^{4,5}$

${ }^{1}$ Epidemiology and Biostatistics Group Department of Statistics Shahjalal University of Science and Technology Sylhet 3114, Bangladesh; ${ }^{2}$ Julius Center for Health Sciences and Primary Care, University Medical Center Utrecht, Utrecht, the Netherlands; ${ }^{3}$ European Medicines Agency, EMA, London, United Kingdom; ${ }^{4}$ Division of Pharmacoepidemiology and Clinical Pharmacology, University of Utrecht, Netherlands; ${ }^{5}$ Department of Clinical Pharmacy, Division of Laboratory and Pharmacy, University Medical Center Utrecht, Utrecht, Netherlands; Corresponding author's e-mail: M.S.Ali@umcutrecht.nl

Background: The self-controlled case-series (SCCS) design has been applied to control for time-fixed (un)measured confounding in pharmacoepidemiological studies. Although previous studies acknowledged that violations of the key SCCS assumptions lead to biased exposure effects, little is known about the impact of the violations in empirical studies. We aimed to evaluate the impact of various levels of violation of assumptions of the SCCS design and different definitions of observation/risk periods in a study of antidepressants use and risk of hip/femur fracture (HF).

Methods: Information on adults with a hip/femur fracture (HF) who used antidepressants at any time during the observation period 2001-2009 was extracted from the UK THIN (6632 cases) and the Dutch Mondriaan (136 cases) databases. The incidence rate ratio (IRR) using this design was defined as the rate of events during exposed periods and during all other observed periods. The IRR of HF was estimated using conditional Poisson regression.

Results: The IRRs appeared extremely biased when all subjects were censored at their first/last HF or when the analysis was restricted to subjects experiencing hip fracture after initiating antidepressant use. For example in THIN, IRRs for $>365$ days of exposure were 1.26 [1.13-1.42] when complete follow-up was considered and 40.1 [32.2-49.9] when censoring was at the first event. However, results were consistent when including subjects who were exposed at the start of follow-up and for different risk period definitions.

Conclusion: The SCCS design is sensitive to violations of the assumptions and yields apparently biased estimates when a significant number of subjects is censored at the event or when the analysis is restricted subjects who experienced hip fracture after initiating antidepressants. The performance of this design may differ across studies and across databases. Therefore, in each SCCS study, correct specification of the SCCS design should be carefully assessed and reported. 


\section{Abstract \#: P 373}

Checklist for data extraction and critical appraisal for systematic reviews of prediction modelling studies: the charms checklist

Karel G.M. Moons ${ }^{1}$, Joris A.H. de Groot ${ }^{1}$, Walter Bouwmeester ${ }^{1}$, $\overline{\text { Yvonne Vergouwe }}^{1}$, Susan Mallett ${ }^{2}$, Douglas G. Altman ${ }^{3}$, Johannes B. Reitsma ${ }^{1}$, Gary S. Collins ${ }^{3}$

${ }^{1}$ Julius Center for Health Sciences and Primary Care, UMC Utrecht, Utrecht, The Netherlands; ${ }^{2}$ Department of Primary Care Health Sciences, New Radcliffe House, University of Oxford; ${ }^{3}$ Centre for Statistics in Medicine, University of Oxford, Botnar Research Centre, Windmill Road, Oxford OX3 7LD, UK; Corresponding author's e-mail: k.g.m.moons@umcutrecht.nl

Background: Prediction models, both diagnostic and prognostic, are becoming increasingly abundant in the medical literature, and they are increasingly being appraised and recommended for formal risk assessment in clinical guidelines. Diagnostic prediction models aim to calculate the probability that an individual has a certain disorder in those suspected of that disorder. Prognostic prediction models estimate the probability of the future occurrence of a particular outcome or event in individuals at risk of such event. Frequently, there are numerous models for the same target population or same outcome. For example, there are over 150 models predicting cardiovascular risk in the general population, over 100 models predicting outcome after brain trauma, over 60 models for breast cancer prognosis, and over 45 models predicting prevalent and incident type 2 diabetes. Healthcare professionals and guideline developers are often in doubt which of all these models to use or appraise in which situation. Hence, as applies to therapeutic interventions, systematic reviews of published prediction modelling studies are increasingly required to identify and critically appraise existing evidence. Yet, in the domain of prediction modelling studies no specific guidance exists to help framing a welldefined review question, nor to determine which details to extract and critically appraise from primary prediction modelling studies. Existing guidance for synthesizing studies of prognostic factors does not address studies of multivariable prediction models. Instead, current reviews of prediction model studies have all created their own checklist with variable inclusion of key details.

Methods and results: Therefore, after examining existing reporting guidelines, quality assessment tools, and key methodological publications, we identified 7 items important for framing a clear systematic review question (diagnostic vs. prognostic models: intended scope of the review: type of prediction modelling studies: target population: outcome to be predicted: time span of the prediction: intended moment of using the model). We also recommend 11 domains to critically appraise the included primary studies (source of data: participants: outcome: predictors: sample size: missing data: model development: model performance: model evaluation: results: interpretation). Both were combined into the $\mathrm{CHecklist}$ for data extraction and critical Appraisal for systematic Reviews of prediction Modelling Studies: the CHARMS Checklist.

Conclusions: The checklist is designed to help form a focused review question for and appraisal of all types of primary prediction modelling studies. This includes model development, model validation and incremental value papers, regardless the method (regression, neural network, genetic programming or vector machine learning methods) used to develop the model.

\section{Abstract \#: P 374}

Retinal vessel diameter and the risk of mortality in the general population: the rotterdam study
U. Mutlu ${ }^{1}$, M.K. Ikram $^{2}$, F.J. Wolters ${ }^{3}$, A. Hofman ${ }^{4}$, C.C. Klaver ${ }^{1}$, M.A. Ikram ${ }^{4}$

${ }^{1}$ Department of Epidemiology, Department of Ophthalmology, Erasmus Medical Center, Rotterdam, The Netherlands; ${ }^{2}$ Singapore Eye Research Institute, Singapore National Eye Center, Singapore, Singapore; ${ }^{3}$ Department of Epidemiology, Department of Neurology, Erasmus Medical Center, Rotterdam, The Netherlands; ${ }^{4}$ Department of Epidemiology, Erasmus Medical Center, Rotterdam, The Netherlands; Corresponding author's e-mail: u.mutlu@erasmusmc.nl

Background: The retinal vasculature is an acknowledged marker of systemic vascular disease. Both arteriolar narrowing and venular dilatation have been associated with (sub)clinical cardiovascular disease and short-term cardiovascular mortality. Yet, it remains uncertain whether arterial and venular abnormalities reflect the same disease process, and whether they differentiate in long-term prognosis.

We aimed to determine the association between midlife measurements of arteriolar and venular diameters and cause-specific mortality in the general population.

Methods: From 1990 to 1993, arteriolar and venular diameters were measured on digitized images with a semi-automated system, as part of the ongoing population-based Rotterdam study. Follow-up for mortality was complete up till 2 July 2014. We determined the association between retinal vessel diameter (arteriolar and venular) and mortality, using the Cox hazard model, adjusting for age and sex, arteriolar or venular diameter and cardiovascular risk factors. Finally, we assessed the very long-term associations by excluding the first 10 years of follow-up as immortal person time.

Results: Out of 5674 participants (mean age 68.0 years, $59 \%$ women), 3675 (64.8\%) died during a mean follow-up of 14.9 years. Venular diameters were associated with an increased risk of all-cause mortality (adjusted HR [95\% CI] per standard deviation increase: 1.07 [1.03-1.12]), in particular for those below 70 years of age ( $p$ interaction $<0.05$ ). Associations were similar for cardiovascular and non-cardiovascular death. In contrast, arteriolar diameters tended to be associated with cardiovascular (aHR 1.06 [0.99-1.16]), but not with non-cardiovascular mortality (aHR 1.03 [0.98-1.09]). Excluding the first 10 years of follow-up strengthened the association between both arteriolar and venular diameters and cardiovascular mortality (aHR 1.14 [1.08-1.20] and 1.14 [1.02-1.28], respectively), but not for non-cardiovascular mortality.

Conclusions: Retinal venular diameters are associated with long-term cardiovascular and non-cardiovascular mortality, whereas arteriolar diameters are associated with cardiovascular mortality only. The role of venular and arteriolar changes in disease etiology and prognosis warrants further investigation.

\section{Abstract \#: P 375}

\section{Increased liver cancer risk among patients with pulmonary} tuberculosis

Tsui-Ming Chang ${ }^{1}$, Fung-Chang Sung ${ }^{1}$

${ }^{1}$ Department of Public Health, China Medical University; Corresponding author's e-mail: tmchang@ctust.edu.tw

Objectives: Patients with tuberculosis $(\mathrm{Tb})$ are at higher risk for other complications. Limited study has examined whether Tb patients are at higher risk of Liver cancer.

This study investigated this association using insurance data. Methods: From the Taiwan National Health Insurance reimbursement data, we established a Tb cohort of 6856 patients newly diagnosed with tuberculosis from 1998 to 2009, and a non-Tb cohort 
of 27,424 persons free of $\mathrm{Tb}$ with sex, age and diagnosis year frequency matched. Incidence of liver cancer was estimated for both cohorts until 2010. Association with comorbidity of hepatitis infections were considered as well.

Results: The incidence rate was $58 \%$ higher in the Tb cohort than in the non-Tb cohort (1.94 vs. 1.23 per 10,000 person-years). The incidence was higher for men and the elderly, although the agespecific $\mathrm{Tb}$ to non- $\mathrm{Tb}$ incidence rate ratio showed a higher relative risk for younger patients. The Nest case-control analysis for the TB cohort at the end of follow-up time showed that the estimated risk of liver cancer was much greater for Tb patients with comorbidity of hepatitis $\mathrm{B}$ infection $(\mathrm{OR}=9.55,95 \%$ confidence interval $(\mathrm{CI})=4.78-19.0)$ and hepatitis $\mathrm{C}$ infection $(\mathrm{OR}=8.72,95 \% \mathrm{CI}$ 4.14-18.3).

Conclusion: Patients with tuberculosis are at an elevated risk of liver cancer, particularly for men and those with comorbidity of hepatitis.

\section{Abstract \#: P 376}

Peripheral proportion of regulatory t-cells and the risk of myocardial infarction and stroke: findings from the epic-heidelberg case-cohort study

$\underline{\text { Sebastian Barth }}{ }^{1}$, Tilman Kühn ${ }^{1}$, Sven Olek ${ }^{2}$, Rudolf Kaaks ${ }^{1}$

${ }^{1}$ Division of Cancer Epidemiology, German Cancer Research Center (DKFZ), Heidelberg, Germany; ${ }^{2}$ Ivana Türbachova Labor für Epigenetik, Epiontis GmbH, Berlin, Germany; Corresponding author's e-mail: s.barth@dkfz-heidelberg.de

Background: Regulatory T-cells (Tregs) are critical in maintaining immune homeostasis and tolerance, which has been found to be involved in experimental atherosclerosis. The present study aimed to evaluate whether low circulating Treg proportions relate to increased cardiovascular disease (CVD) risk in humans.

Methods: A nested case-cohort study was conducted comprising 1183 diabetes-free participants of the European Prospective Investigation into Cancer and Nutrition (EPIC)-Heidelberg cohort. Primary endpoints were occurrence of a first myocardial infarction $(n=276)$ or ischemic stroke $(n=151)$. Treg and total T-lymphocyte (tTL) numbers were measured by qPCR-based DNA methylation analyses of two T-cell genes (FOXP3, and CD3g respectively) in peripheral blood leukocyte samples. Prentice-weighted Cox proportional hazard models were used to estimate hazard ratios for associations between Tregs, expressed as percentage of tTLs, and CVD risks.

Results: After multivariable adjustment, low Treg proportions were not associated with either the risk of MI (lowest vs. highest sexspecific quartile: Hazard Ratio: 0.72, $95 \%$ Confidence Interval: 0.46-1.13: $p$ trend $=0.54$ ) or stroke (HR: $0.90,95 \%$ CI: 0.51-1.59: $p$ trend $=0.77$ ).

Conclusions: In contrast to experimental data, our findings do not support a protective role of Tregs in CVD development.

\section{Abstract \#: P 377}

\section{A comparison of the prevalence and clustering of cardiovascular} risk factors in the Netherlands and China

Xin Wang ${ }^{1}$, Junqi Niu ${ }^{2}$, Michiel Bots ${ }^{1}$, Fang Yang ${ }^{2}$, Arno Hoes ${ }^{1}$, $\underline{\text { Ilonca Vaartjes }}{ }^{1}$

${ }^{1}$ Julius Center for Health Sciences and Primary Care, UMC Utrecht, the Netherlands; ${ }^{2} 1$ st Hospital of Jilin University, Changchun, China; Corresponding author's e-mail: c.h.vaartjes@umcutrecht.nl
Aims: To compare the prevalence and clustering of major cardiovascular risk factors among adults in the Netherlands and China. Methods: 6542 adults were recruited from 2001 to 2006 for the Utrecht Health Project (UHP), an ongoing cohort study among inhabitants of a newly developing area near Utrecht, the Netherlands. 37,141 employees who received health screening in Changchun City, China were enrolled from 2003 to 2010. 3850 residents from Dehui, another city from Northeast China were enrolled in 2007.

Results: In each age group, Dutch population had higher levels of systolic blood pressure and total cholesterol for both men and women compared to Chinese population. In the younger age group (18-44 years old), Dutch men and Chinese men had similar body mass index (BMI) level. Age-standardized prevalence of current smoking was much higher in Chinese men compared to Dutch men $(p<0.05)$, while excessive alcohol intake was more prevalent in Dutch women compared to Chinese women $(p<0.05)$. Age-standardized prevalence of hypercholesterolemia was higher in Dutch than in Chinese $(p<0.05)$. For both Dutch and Chinese adults, older participants and men were more likely to have clustering of cardiovascular risk factors. The clustering patterns of risk factors were different between Dutch and Chinese. For example, the smokingheavy drinking cluster was most common in Chinese young men, while smoking and hypercholesterolemia commonly went together in Dutch young population.

Conclusions: Cardiovascular risk profiles and clustering patterns are different between Caucasians and Asians and seem to differ between men and women. This calls for race and sex specific targeted prevention programs.

\section{Abstract \#: P 379}

Trends in case-fatality of myocardial infarction and ischemic stroke, by social class in Sweden 1987-2007

Ninoa Malki M.Sc, Alexander Ploner PhD, Professor Ilona Koupil, Sara Hägg PhD, Sanna Tiikkaja PhD, Professor Paul Lambert, Professor Pär Sparén

Corresponding author's e-mail: ninoa.malki@ki.se

Background: Improvements in medical care and better case-ascertainment have led to a decrease in case-fatality rate of cardiovascular diseases. The question is if these improvements have been equally distributed across different socioeconomic position (SEP) groups. In this study we will address this question by analyzing both short and long term case-fatality rates in myocardial infarction (MI) and ischemic stroke (IS) over time by different SEP in Sweden.

Method: All Swedish residents born between 1932 and 1960 were included. Study period was from 1987 to 2010: individual follow-up for each subject started at date of first disease event and continued until either death, censoring at the cut-point of interest, or end of study. Individuals who developed MI or IS were selected from the Hospital inpatient register and the Cause of death register. Individuals who died before reaching the hospital or at the disease event day were analyzed separately using logistic regression. Individuals who survived the first day were analyzed using time to event analysis for periods of 28 days, 90 days and 365 days. Flexible parametric models were used to estimate the mortality rates per 100,000 person years as well as mortality rate ratios comparing different SEP groups. Both civil status (single: widow/divorce: cohabiting/married) and birth country were considered as potential confounders, but only civil status was retained in the final analysis.

Results: In total 142,223 MI cases and 77,652 IS cases where identified. We found large socioeconomic inequalities in mortality rate in immediate death and 1 year case-fatality for both MI and IS among 
men and women. For both MI and IS, mortality rates in immediate death and 1 year case-fatality decreased over time to the same extent for all SEP groups among men and women. Sex differences in mortality rates in immediate death were less pronounced in IS compared to MI.

Conclusion: The large socioeconomic inequality in mortality rate for both MI and IS, persisted over the study period.

\section{Abstract \#: P 380}

\section{Association of interleukin 8 levels with incident cardiovascular disease and all-cause mortality. Results from a Swedish cohort of 60-year-olds}

Ilais Moreno Velásquez ${ }^{1}$, Karin Leander ${ }^{1}$, Anita Berglund ${ }^{1}$, Ashwini Gajulapuri $^{1}$, Ulf de Faire ${ }^{1}$, Bruna Gigante ${ }^{1}$

${ }^{1}$ Unit of Cardiovascular Epidemiology, Institute of Environmental Medicine, Karolinska Institutet, Stockholm, Sweden; Corresponding author's e-mail: ilais.moreno.velasquez@ki.se

Background: Interleukin 8 (IL8) has pro-inflammatory and pro-angiogenic properties. Population-based observational studies analyzing the association between IL8 and atherosclerosis related cardiovascular diseases (CVD) and all-cause mortality are scarce and have yielded conflicting findings.

Aims: To study the association between IL8 serum levels and incident CVD in the cohort of 60 years old men and women from Stockholm using a nested case-control design. A secondary aim was to explore the association of IL8 with all-cause mortality.

Methods: Every third man and woman living in Stockholm County born between 1 July 1937 and 30 June 1938 was randomly selected from the register of the total population in Sweden and invited to participate in a health screening for CVD from August 1997 to March 1999. Of 5460 subjects invited to participate, 4232 (2039 men and 2193 women) $(78 \%)$ agreed to be enrolled. We conducted a nested case-control study to investigate the associations between IL8 and CVD. For each incident CVD $(n=491)$, two controls were randomly selected from the cohort (with the exception of 1 male participant to whom only 1 control was eligible) $(\mathrm{n}=981)$ matched by sex and time at risk ( \pm 60 days). IL8 serum levels were measured by Mesoscale and divided into quartiles (Q). Conditional logistic regression was used to estimate odds ratio $(\mathrm{OR})$ with $95 \% \mathrm{CI}$ for the association of IL8 with CVD. ORs were adjusted for hypertension, diabetes, hypercholesterolemia, body mass index and smoking. Unconditional logistic model was applied for the association of IL8 with all-cause mortality, adjusted for hypertension, diabetes, obesity, and smoking.

Results: Compared to the lowest IL8 Q, exposure to the highest Q was not associated with incident CVD in all, with an adjusted OR of 0.9: (95\% CI 0.7-1.3), women (OR 0.7: $95 \%$ CI 0.4-1.4), and in men (OR 1.1: $95 \%$ CI 0.7-1.6). Elevated levels of serum IL8 ( $>50$ th) were associated with increased all-cause mortality in all, with an adjusted OR of 1.6: (95\% CI 1.2-2.2). Men with IL8 above the median had $70 \%$ higher all-cause mortality, as compared to IL8 < 50th (OR 1.7: $95 \%$ CI 1.1-2.5). No association was observed in women.

Conclusion: Elevated levels of serum IL8 were not associated with an increased risk of incident CVE, but with increased risk of all-cause mortality. Our findings add evidence for a link between IL8 and total mortality in the elderly population. Molecular epidemiologic studies aiming at disentangle IL8's role in atherogenesis and tumorigenesis are warranted.

\section{Abstract \#: P 382}

Survival with favorable neurologic outcome after out-of-hospital cardiac arrest in persons $\mathbf{Z 7 0}$ years

$\underline{\text { M.T. Blom }}^{1}$, S.G. Beesems ${ }^{1}$, R.W. Koster ${ }^{1}$, H.L. Tan ${ }^{1}$

${ }^{1}$ Department of Cardiology, Academic Medical Center, Amsterdam, The Netherlands; Corresponding author's e-mail: m.t.blom@amc.uva.nl

Introduction: Advanced age is associated with low survival after outof-hospital cardiac arrest (OHCA). We aimed to establish survival rate and neurological outcome at hospital discharge after OHCA in older patients in The Netherlands. Second, we aimed to evaluate whether pre-OHCA factors are associated with favorable outcome in older patients.

Methods: Using data from an ongoing prospective registry of all cardiopulmonary resuscitation attempts after OHCA in the province of North Holland (The Netherlands), we established survival rates in patients aged $\geq 70$ in whom resuscitation after OHCA with nontraumatic etiology was attempted in 2009-2011 $(\mathrm{n}=851)$. PreOHCA factors (age, gender, residing in long-term care institution, Charlson Comorbidity Index [CCI] score) and resuscitation parameters were regressed with neurologically intact survival at hospital discharge as primary outcome.

Results: We found a $12 \%$ survival to discharge rate after OHCA in patients aged $\geq 70$ (25\% among patients with shockable initial rhythm). Among those patients who survived until hospital discharge, $96 \%$ survived with favorable neurologic outcome. Of the pre-OHCA factors only age was significantly associated with outcome (age OR $0.94,95 \%$ CI $0.90-0.97, p=0.003$ ). A CCI score $\geq 4$ was not statistically significantly associated with survival (7 vs. $12 \%$, OR 0.53 , $95 \% \mathrm{CI}(0.25-1.13), p=0.10)$. When adjusted for resuscitation parameters, none of the studied pre-OHCA factors remained statistically significant.

Conclusion: In The Netherlands, the survival rate in older patients is $12 \%$ : the greater majority of older patients $(96 \%)$ survived with favorable neurologic outcome. Resuscitation-related factors are more important than the pre-OHCA factors including comorbidity score when predicting outcome in older patients.

\section{Abstract \#: P 383}

Negative interaction between chronic kidney disease and metabolic syndrome for coronary heart disease incidence in non-obese individuals: risk factors paradox

Mohammad Hossein Panahi ${ }^{1}$, Farzad Hadaegh ${ }^{2}$, Parvin Yavari ${ }^{3}$, Sara Kazempour-Ardebili ${ }^{4}$, Yadollah Mehrabi ${ }^{2}$, Fereidoun Azizi ${ }^{5}$, Davood Khalili $^{4}$

${ }^{1}$ Department of Epidemiology, School of Public Health, Shahid Beheshti University of Medical Sciences, Tehran, Iran; ${ }^{2}$ Prevention of Metabolic Disorders Research Center, Research Institute for Endocrine Sciences, Shahid Beheshti University of Medical Sciences, Tehran, Iran; ${ }^{3}$ Department of Community Medicine, School of medicine, Shahid Beheshti University of Medical Sciences, Tehran, Iran; ${ }^{4}$ Prevention of Metabolic Disorders Research Center, Research Institute for Endocrine Sciences, Shahid Beheshti University of Medical Sciences, Tehran, Iran; ${ }^{5}$ Endocrine Research Center, Research Institute for Endocrine Sciences, Shahid Beheshti University of Medical Sciences, Tehran, Iran; Corresponding author's e-mail: dkhalili@endocrine.ac.ir 
Background: Some studies have shown that chronic kidney disease $(\mathrm{CKD})$ is an independent risk factor for cardiovascular diseases while others have not. We aimed to investigate the effect of CKD and its joint effect with Metabolic Syndrome (MetS) on coronary heart disease (CHD) in different body mass index (BMI) categories.

Methods: 2823 men and 3684 women aged $>=30$ years, and free of cardiovascular disease and cancer at baseline (1999-2001), followed for 10 years. They were classified to four CKD/MetS subgroups: CKD-/MetS-, CKD+/MetS-, CKD-/MetS+, CKD+/MetS+. Multivariable adjusted hazard ratio (HR) of CHD was estimated for each subgroup compared to CKD-/MetS - group by gender and BMI levels below and above $27 \mathrm{~kg} / \mathrm{m}^{2}$. The interaction term of $\mathrm{CKD} \times$ MetS and also CKD $\times$ MetS-components were assessed in Cox proportional hazard models as well.

Results: CKD, without MetS, showed a significant effect on CHD only in subjects with BMI $<27$ (HR $=2.06$, CI $95 \%: 1.28-3.31$ in men and 2.56, 1.04-6.31, in women): however the joint effect of CKD and MetS decreased to one third of their multiplicative effect in this subgroup, all indicating a negative interaction between CKD, MetS and Obesity. The same interaction was observed between CKD with hypertension in both genders and with Type 2 Diabetes in men as well.

Conclusions: Our results showed that CKD is an independent risk factor for CHD only in non-obese individuals: however its risk was wiped out when joined to MetS. Following the concept of "obesity paradox" the term of "risk factors paradox" also needs more attention to explain these findings.

\section{Abstract \#: P 385}

\section{Are twins at increased risk of congenital heart disease?}

Kate E. Best ${ }^{1}$, Judith Rankin ${ }^{1}$

${ }^{1}$ Insitute of Health \& Society, Newcastle University, UK; Corresponding author's e-mail: kate.best@ncl.ac.uk

Background: Congenital anomalies are abnormalities that are present before birth. Research consistently suggests that compared to singletons, twins are at increased risk of congenital anomalies. The risk of congenital anomalies amongst twins that share a placenta, known as monochorionic (MC) twins, reportedly exceeds that of twins that do not share a placenta, dichorionic (DC) twins. However, the risk of congenital heart disease (CHD), amongst twins is less well researched, particularly in relation to chronicity. The aim of this study was to examine the relative risk (RR) of CHD in MC and DC twins compared to singletons, according to CHD severity.

Methods: Twins and singletons with CHD notified to the Northern Congenital Abnormality Survey (NorCAS) between 1998 and 2010 were included. Information on chronicity was obtained from the Northern Survey of Twins and Multiple Pregnancy. Prevalence was calculated as the number of cases occurring in live births, fetal deaths ( $\geq 20$ weeks) and terminations of pregnancy for fetal anomaly, per 10,000 live and stillbirths. The risk of CHD in twins compared to singletons was estimated using Poisson regression.

Results: Excluding cases with extra-cardiac anomalies, there were 3078 cases notified to NorCAS: 140 twins and 2938 singletons (prevalence $=117.9,95 \% \mathrm{CI}: 99.3-139.0$ and $73.5,95 \% \mathrm{CI}$ : 70.9-76.2 per 10,000 total births, respectively). Twins were at $50 \%$ significant increased risk of CHD compared to singletons $(\mathrm{RR}=1.5$, $95 \%$ CI: $1.3-1.8)$. Eighty-nine twins were DC (63.6\%, prevalence $=103.4,95 \%$ CI: 83.1-127.1 per 10,000 total births) and 41 $(29.3 \%$, prevalence $=177.0,95 \%$ CI: 127.3-239.3) were MC. DC and MC twins were at 30 and $130 \%$ significant increased risk of CHD compared to singletons $(\mathrm{RR}=1.3,95 \% \mathrm{CI}: 1.1-1.6$ and
$\mathrm{RR}=2.3,95 \% \mathrm{CI}: 1.7-3.1$, respectively). $\mathrm{DC}$ twins were at $40 \%$ $(\mathrm{RR}=1.4,95 \% \mathrm{CI}: 0.5-3.8), 60 \%(\mathrm{RR}=1.6,95 \% \mathrm{CI}: 1.1-2.4)$ and $30 \%(\mathrm{RR}=1.3,95 \% \mathrm{CI}: 1.0-1.7)$ increased risk of severe, moderate and mild CHD respectively, although this did not reach statistical significance for severe CHD $(p=0.526)$. MC twins were at $290 \%(\mathrm{RR}=3.9,95 \% \mathrm{CI}: 1.2-12.3)$ significant increased risk of severe $\mathrm{CHD}$ and $160 \%(\mathrm{RR}=2.6,95 \% \mathrm{CI}: 1.8-3.8)$ significant increased risk of mild CHD. There was no significant effect amongst cases of moderate CHD (RR = 1.2, $95 \% \mathrm{CI}: 0.5-2.9)$.

Conclusion: Compared to singletons, twins are at increased risk of $\mathrm{CHD}$, the risk being substantially higher amongst MC twins. The risk of severe CHD in MC twins compared to singletons was particularly high. This information is important for health professionals when counselling women with a twin pregnancy.

\section{Abstract \#: P 386}

Classification and decision trees in prediction of adverse cardiac events in patients with chronic heart failure

Aleksander J. Owczarek $^{1}$

${ }^{1}$ Division of Statistics, SPLMS in Sosnowiec, Medical University of Silesia, Katowice, Poland; Corresponding author's e-mail: aowczarek@sum.edu.pl

Heart failure (HF), despite substantial progress in the prevention and modern methods of treatment is a growing problem in terms of health and socio-economic background. It is the most common and most costly cause of hospitalization among cardiovascular diseases, and has a high percentage of mortality. Therefore it is crucial to develop a useful prediction model which enables a rational and economical use of therapeutic methods by estimating the risk of death or adverse cardiac events in patients with HF. In the 10-year follow-up clinical data of 630 consecutive patients with chronic heart failure were analyzed. The endpoints included: 1) death from all causes, 2) the occurrence of MACE (cardiac death, re-hospitalization due to the worsening of heart failure). In the observation period which lasted 41 months on average, the percentage of deaths from all causes was $16.4 \%$ and adverse cardiac events $37.6 \%$, in the analyzed group of patients with chronic heart failure. The most useful decision tree to predict mortality from all causes proved to be a CART tree with the Gini index and double penalty for a false-negative misclassification than false-positive one. The risk factors in the model included: the concentration of NT-pro BNP and uric acid, left ventricular end systolic dimension, the average pulmonary arterial pressure and prothrombin time. The most useful decision tree to predict MACE proved to be a CART tree with the Gini index and double penalty for a false-negative misclassification. The risk factors in the model included: the concentration of NT-pro BNP, uric acid and LDL cholesterol, left ventricular end systolic volume and prothrombin time.

\section{Abstract \#: P 387}

Mediation effects of body composition on the association of early puberty and apolipoproteins $a-i$ and $b$ in adolescents: Helena study

Tara Rendo-Urteaga ${ }^{1,2}$, Marcus Vinicius Nascimento-Ferreira ${ }^{1}$, Augusto César Ferreira de Moraes ${ }^{1,2}$, Francisco Leonardo Torres Leal $^{3}$, Odysseas Androutsos ${ }^{4}$, Chantal Gilbert ${ }^{5}$, Michael Sjöström ${ }^{6}$, Anthony Kafatos ${ }^{7}$, María Plada ${ }^{7}$, Kurt Widhalm ${ }^{8}$, Frederic Gottrand ${ }^{9}$, Catherine Leclercq ${ }^{10}$, Ascensión Marcos ${ }^{11}$, Marcela González- 
Gross $^{12,13}$, Stefaan De Henauw ${ }^{14}$, Marika Ferrari ${ }^{15}$, Manuel Castillo $^{16}$, Jonatan R. Ruiz ${ }^{6,16}$, Francisco B. Ortega ${ }^{16}$, Dénes Molnár $^{17}$, Peter Stehle ${ }^{18}$, Jean Dallongeville ${ }^{19}$, Heráclito B. Carvalho $^{1}$, Luis A. Moreno ${ }^{2,20}$, on behalf of the HELENA study group*

${ }^{1}$ Youth/Child and cArdiovascular Risk and Environmental (YCARE) Research Group, School of Medicine, University of São Paulo, São Paulo, Brazil; ${ }^{2}$ Growth, Exercise, NUtrition and Development (GENUD) Research Group, School of Health Science (EUCS), University of Zaragoza, Zaragoza, Spain; ${ }^{3}$ Department of Biophysics and Physiology, Health Science Center, Federal University of Piauí, Piauí, Brazil; ${ }^{4}$ Department of Nutrition and Dietetics, School of Health Science and Education, Harokopio University, Athens, Greece; ${ }^{5}$ Campden BRI (United Kingdom); ${ }^{6}$ Unit for Preventive Nutrition, Department of Biosciences and Nutrition, Karolinska Institutet, Huddinge, Sweden; ${ }^{7}$ Department of Social Medicine, School of Medicine, Preventive Medicine and Nutrition Clinic, University of Crete, Crete, Greece; ${ }^{8}$ Department of Pediatrics, Private Medical University, Salzburg, Austria; ${ }^{9}$ INSERM U995, University Lille Nord de France, Lille, France; ${ }^{10}$ Nutrition Assessment \& Nutrient Requirements Group Nutrition Division (ESN) Food and Agricultural Organization of the United Nations Viale Terme di Caracalla, Rome, Italy; ${ }^{11}$ Immunonutrition Research Group,

Department of Metabolism and Nutrition, Instituto del Frío, Institute of Food Science and Technology and Nutrition (ICTAN), Spanish National Research Council (CSIC), Madrid, Spain; ${ }^{12}$ ImFINE Research Group, Department of Health and Human Performance, Faculty of Physical Activity and Sport-INEF, Technical University of Madrid, Madrid, Spain; ${ }^{13}$ Institut für Ernährungs- und

Lebensmittelwissenschaften - Ernährungphysiologie, Rheinische Friedrich Wilhelms Universität, Bonn, Germany; ${ }^{14}$ Department of Public Health, Ghent University, Ghent, Belgium; University College Ghent Vesalius, Ghent, Belgium; ${ }^{15}$ Agricultural Research Council, Research Centre for Food and Nutrition (CRA-NUT), Rome, Italy; ${ }^{16}$ PROFITH "PROmoting FITness and Health Through Physical Activity" Research Group, Department of Physical Education and Sports, Faculty of Sport Sciences, University of Granada, Granada, Spain ${ }^{17}$ Department of Pediatrics, Medical Faculty, University of Pécs, Pécs, Hungary; ${ }^{18}$ Institut für Ernährungs- und

Lebensmittelwissenschaften - Ernährungphysiologie. Rheinische Friedrich Wilhelms Universität (Germany); ${ }^{19}$ Institut Pasteur de Lille (France); ${ }^{20}$ Visiting Professor, School of Medicine of the University of São Paulo - Department of Preventive Medicine, São Paulo, Brazil; Corresponding author's e-mail: trendo@usp.br

Keywords: Mediated effects; Early Puberty; Apolipoproteins; Youth

Background: Early puberty (EP) is defined as the appearance of signs of puberty at an early age. Obesity leads to the appearance of these signs in girls, although there are controversial results for boys. Moreover, obesity is associated with an increased cardiovascular risk in adolescents. It has been showed that apolipoproteins A-I (apoAI) and $\mathrm{B}(\mathrm{apoB})$ have been shown to be good predictors of atherosclerotic diseases. There is scarce evidence about the role of obesity in the association between EP and apolipoproteins in adolescents. Thus, the aim of this study was to assess the possible mediation role of body composition (waist circumference [WC] and body mass index [BMI]) in the association between EP and apolipoproteins in adolescents.

Methods: A sample of 984 adolescents (12.5-17.5 years; $52.2 \%$ female) from the HELENA cross-sectional study (Healthy Lifestyle in Europe by Nutrition in Adolescence) was included. Sexual maturation was determined based on the stages of development proposed by Tanner. We consider that a young age at a sexual maturation stage indicates precocious sexual maturation. To qualify as a mediator, the presumed mediators (WC and BMI) have to be associated with the principal exposure (EP) (path a) and the outcome variables (apoAI or
apoB) (path b). To assess whether these associations were mediated by body composition (WC and BMI), the product of coefficients test proposed by MacKinnon $\left(a^{*} b\right.$; indirect or mediated effect) was performed. Total associations between EP and apolipoproteins were estimated by regression models without the mediators (path c). The mediated proportions were calculated as the indirect effect divided by the total effect $(\mathrm{a} * \mathrm{~b} / \mathrm{c})$. Bootstrap corrected confidence intervals $(\mathrm{CI})$ were used for indirect and direct effects. All regression models were adjusted for potential confounders (age, physical activity and socioeconomic factors).

Results: The results showed that WC and BMI were mediators on the association between EP and apoAI or ApoB in boys. However, in these multiple mediations, the indirect effects were not statistically significant. In girls, only WC was found to be a mediator in the association between EP and apoAI or apoB. The indirect effect was statistically significant on the relation of EP and apoAI $(\square=-0.01$; CI: -0.03 to -0.001$)$ or apoB $(\square=0.01$; CI: $0.0004-0.02)$. Moreover, WC had a suppressive effect on the relationship between EP and apoAI $(-30 \%)$ and apoB $(-19 \%)$ in girls.

Conclusion: The associations between EP and apolipoproteins A-I and $\mathrm{B}$ were partially mediated by WC in girls.

\section{Abstract \#: P 388}

Differences in myocardial mass and left ventricular remodeling between Kazakhstani and Russian hypertension patients

Aiman S. Kerimkulova ${ }^{1}$, Akbayan M. Markabayeva ${ }^{1}$, Andrej M. Grjibovski ${ }^{2,3}$, Viktor R. Veber ${ }^{4}$, Lyudmila M. Pivina ${ }^{1}$, Zoya Ensegenova $^{5}$, Ainur S. Ospanova ${ }^{1}$

${ }^{1}$ State Medical University, Semey, Kazakhstan; ${ }^{2}$ Norwegian Institute of Public Health, Oslo, Norway; ${ }^{3}$ Northern State Medical, University, Arkhangelsk, Russia; ${ }^{4}$ Novgorod State University, Novgorod, Russia; ${ }^{5}$ Kazakh National Medical University, Almaty, Kazakhstan; Corresponding author's e-mail: andrej.grjibovski@gmail.com

Introduction: Kazakhstan has one of the highest mortality from cardiovascular diseases in the world while Russia has the highest. Autopsy studies are becoming more popular in the field of cardiovascular epidemiology, but no studies have been published from Kazakhstan. We studied the differences in myocardial mass and left ventricle wall thickness between ethnic Kazakhs and ethnic Russians who had hypertension.

Methods: All patients with confirmed diagnosis of arterial hypertension who were autopsied Semey, Kazakhstan, in 1999-2012 comprised the study base. Data on ethnic background (Kazakh or Russian), age at death, gender, myocardial mass and left ventricle wall thickness were obtained from autopsy protocols at the Municipal Bureau of Forensic Medicine. Continuous data are presented as means (M) and standard deviations (SD). Categorical data were compared using Chi squared tests. Differences in myocardial mass and left ventricle wall thickness between Kazakhs and Russians were studied by multiple linear regression.

Results: Altogether, there were 365 ethnic Russians and 169 ethnic Kazakhs among the autopsied patients. Men comprised $53.2 \%$ among Russians and $71.8 \%$ among Kazakhs $(p<0.001)$. Myocardial mass was greater among Russians for both men $(\mathrm{M}=582.3 \mathrm{~g}$, $\mathrm{SD}=142.0$ vs. $\mathrm{M}=536.7 \mathrm{~g}, \mathrm{SD}=134.5, p=0.007)$ and women $(\mathrm{M}=543.0 \mathrm{~g}, \quad \mathrm{SD}=136.1 \quad$ vs. $\quad \mathrm{M}=478.2 \mathrm{~g}, \quad \mathrm{SD}=158.8$, $p=0.009)$. Left ventricle wall was thicker in both Russian men $(\mathrm{M}=1.73 \mathrm{~cm}, \quad \mathrm{SD}=0.52 \quad$ vs. $\mathrm{M}=1.68 \mathrm{~cm}, \quad \mathrm{SD}=0.53$, $p=0.021)$, and women $(\mathrm{M}=1.68 \mathrm{~cm}, \quad \mathrm{SD}=0.54$ vs. $\mathrm{M}=$ $1.38 \mathrm{~cm}, \mathrm{SD}=0.49, p=0.001)$ compared to Kazakhs in bivariate analysis. After adjustment for gender and age, the differences 
between Russians and Kazakhs were 44.9 g (95 \% CI: 18.6-77.1) for myocardial mass and $0.20 \mathrm{~cm}(95 \% \mathrm{CI}: 0.09-0.31)$ for left ventricle wall thickness.

Conclusions: Ethnic Russians have greater myocardial mass and left ventricular wall thickness than ethnic Kazakhs even after adjustment for age and gender suggesting ethnic variations in remodeling patterns among hypertension patients. Limitations of the study and potential explanatory factors will be discussed.

\section{Abstract \#: P 389}

\section{CVRCALC: a cardiovascular risk calculator}

Teresa Seoane-Pillado ${ }^{1}$, Miguel Rodriguez-Muiños ${ }^{2}$, Salvador Pita-Fernandez ${ }^{1}$, Xurxo Hervada-Vidal ${ }^{2}$, Sonia Pertega-Diaz ${ }^{1}$, Beatriz Lopez-Calviño ${ }^{1}$

${ }^{1}$ Clinical Epidemiology and Biostatistics Unit. Instituto de Investigación Biomédica de A Coruña (INIBIC), Complexo Hospitalario Universitario de A Coruña (CHUAC), SERGAS, Universidade da Coruña; ${ }^{2}$ Dirección General de Innovación y Gestión de la Salud Pública, Xunta de Galicia; Corresponding author's e-mail: maria.teresa.seoane.pillado@sergas.es

Background: There is great interest in developing cardiovascular risk prediction models that will allow us to take action at the level of primary prevention. Software tools are available which allow us to automatically calculate this risk according to any established model. This type of program (commonly referred to as the I'cardiovascular risk calculatorsl') are usually based on risk prediction tables presenting estimates of the risk of cardiovascular disease.

But problems arise when we want to do an assessment of a set of individuals previously collected in a database. Current tools only allow us to perform these calculations manually by entering the requested information for each of the individual cases to assess.

Methods: CVRCALC is a program developed in $\mathrm{R}$ which allows us to estimate the cardiovascular risk of a database of individuals applying algorithms from different models. The project is hosted in $\mathrm{R}$-Forge (http://r-forge.r-project.org/projects/cvrcalc/) and is available under the GNU/GPL license.

There are two ways to install the CVRCALC package in R: 1. Download the file http://r-forge.r-project.org/R/?group_id=1731 and proceed to the installation from a ZIP archive. 2. -Run the following command from the R command console:>install.packages( $($ 'cvrcalcl', repos=l'http://R-Forge.R-project.orgl').

Results: In R, load CVRCALC: require (cvrcalc) and call your graphical environment: cvrcalcgui(). From the main window, we must supply the program, through an Excel file, with the variables: sex, age, cholesterol, hdl, tas, tad, smoking, diabetes and hypertrophy for each individual that have been previously collected. In addition, we must select the model that we want to apply: Framigham classic, Framingham-Wilson, Regicor, Dorica, High Risk Score or Low Risk Score. The result will appear as a new column in the Excel file supplied, with the estimation of the cardiovascular risk for each of the individuals on the database.

Conclusions: CVRCALC is a cardiovascular risk calculator that allows to load a database, select the model you wish to apply and which allows us to calculate, in one step, the cardiovascular risk for each of the records in the database and store the results.

\section{Abstract \#: P 392}

Prediction models for intracranial haemorrhage or major bleeding in patients on antiplatelet therapy: a systematic review of the literature

$\underline{\text { Nina A. Hilkens }}{ }^{1}$, Jacoba P. Greving ${ }^{1}$, Ale Algra ${ }^{1,2}$

${ }^{1}$ Julius Center for Health Sciences and Primary Care, University Medical Center Utrecht, The Netherlands; ${ }^{2}$ Department of Neurology and Neurosurgery Brain Center Rudolf Magnus, University Medical Center Utrecht, The Netherlands; Corresponding author's e-mail: n.a.hilkens-3@umcutrecht.nl

Background and purpose: Antiplatelet therapy is widely used in secondary prevention after TIA or ischaemic stroke. Bleeding is the main adverse effect of antiplatelet therapy and is potentially lifethreatening. Identification of patients at increased bleeding risk may target antiplatelet therapy. This study sought to identify existing prediction models that predict the risk of intracranial haemorrhage or major bleeding in patients on antiplatelet therapy.

Methods: We systematically searched PubMed and Embase for existing prediction models up to December 2014. Methodological quality of the included studies was assessed with the CHARMS checklist. We investigated model performance and predictors used in the included models.

Results: Five prediction models were identified, of which two were developed in patients with previous cerebral ischaemia. One study assessed intracerebral haemorrhage, all others studied major bleeding. None of the studies met criteria of good quality, three were of moderate quality, one of low quality, one was only published as an abstract and provided insufficient information for proper quality assessment. External validation was performed in three studies with an area under the curve (AUC) ranging from 0.64 to 0.72 . Frequently used predictors were age, hypertension, renal failure and use of anticoagulants.

Conclusion: A limited number of prediction models are available that predict intracranial haemorrhage or major bleeding in patients with a previous stroke. Methodological quality of the studies varied, but was generally low. In order to reliably predict the risk of bleeding in patients on antiplatelet therapy, development of a prediction model according to current methodological standards is needed.

\section{Abstract \#: P 393}

Effect of potassium intake on heart rate: a meta-analysis of randomized controlled trials

Lieke Gijsbers $^{1}$, Famke J.M. Mölenberg ${ }^{2}$, Johanna M. Geleijnse ${ }^{1}$

${ }^{1}$ Top Institute Food and Nutrition, Wageningen, The Netherlands: Division of Human Nutrition, Wageningen University, Wageningen, The Netherlands; ${ }^{2}$ Division of Human Nutrition, Wageningen University, Wageningen, The Netherlands; Corresponding author's e-mail: lieke.gijsbers@wur.nl

Background: Potassium intake is known to reduce blood pressure (BP). The effect of increased potassium intake on heart rate (HR), which is closely related to BP, has not yet been established. We aimed 
to determine this effect in a meta-analysis of randomized controlled trials.

Methods: Randomized, placebo-controlled, trials with a minimum duration of 2 weeks, investigating the effect of increased potassium intake on HR in adult populations were searched via PubMed (1966October 2014). In addition, reference lists from previous meta-analyses on potassium intake and BP was hand-searched. Two reviewers independently extracted the data. Random-effects meta-analysis was performed to calculate the pooled estimate (mean difference with $95 \%$ confidence interval). Subgroup analyses and meta-regression were also conducted.

Results: The search yielded 22 eligible trials (including 1065 participants), of which 21 reported on office HR and 5 on 24-h ambulatory HR. No effect of increased potassium intake on office HR ( $0.23 \mathrm{bpm}, 95 \% \mathrm{CI}:-0.42$ to 0.87$)$ was observed. Increased potassium intake tended to increase $24-\mathrm{h}$ HR by $0.99 \mathrm{bpm}$ (95\% CI: $-0.73: 2.71$ ), but this effect was no longer present after the exclusion of one outlier. Subgroup analyses indicated that increased potassium intake induced small rises in office $\mathrm{HR}(\geq 0.7 \mathrm{bpm})$ in populations with high body weight $(>73 \mathrm{~kg})$, low initial HR ( $\leq 69 \mathrm{bpm})$ and SBP $(\leq 145 \mathrm{mmHg})$, and in studies with a long duration ( $>4$ weeks). Metaregression suggested a positive linear trend between dose of potassium and effect on office HR: each $1 \mathrm{~g}$ increase of potassium nonsignificantly increased HR by $0.38 \mathrm{bpm}(95 \% \mathrm{CI}:-0.56$ to 1.32$)$.

Conclusions: This meta-analysis of randomized, placebo-controlled, trials showed no overall effect of increased potassium intake on HR. In specific subgroups of the population potassium-induced increases of $0.7 \mathrm{bpm}$ in office HR were observed. Whether this small increase in HR has consequences for public health needs to be further investigated.

\section{Abstract \#: P 394}

\section{Profile of valve disease bearers in a reference hospital in Brazil}

Aline Magalhaes Bessa Andrade $^{1}$, Maíra Costa Ferreira ${ }^{1}$, Cláudia Fernanda Trindade Silva ${ }^{2}$, Fernanda de Oliveira Souza ${ }^{3}$, Layla Baptista Cavalcante Wright ${ }^{3}$

${ }^{1}$ Universidade Federal da Bahia; ${ }^{2}$ Hospital Ana Neri; ${ }^{3}$ Universidade Estadual de Feira de Santana; Corresponding author's e-mail: linebmagalhaes@hotmail.com

Introduction: According to government information, for each of 100 hospital admissions for cardiovascular disease during the period from January of 2012 to January of 2013, 37 had as etiology the cardiac valve disease.

Methodology: A quantitive and transversal research, with 39 patients with aortic valve disease admitted in the cardiac unit of a public hospital reference in heart disease in the state of Bahia/Brazil. The selection of the sample was done through a non probabilistic method with the patients admitted in the hospital with a medical diagnosis of aortic insufficiency or estenosis, with or without co-morbidities, of both genders, during the months of October-January 2013. A questionnaire with 51 questions about their social-economical, epidemiological and clinical profile was given to those participating. The absolute and relative frequencies were calculated and the medium value and standard shunting line from the IBM SPSS Software Statistics version 19.0.

Results: Among the 39 patients interviewed, $51.3 \%$ were female and $48.7 \%$ were male: average age was $42.3 \pm 13.7$ years. The majority of the individuals $(46.1 \%$ ) had low education (incomplete elementary school), $64.1 \%$ had income lower than the minimum wage and systemic arterial hypertension. Only $2.5 \%$ reported current tobacco use and $43.59 \%$ said to be ex-smokers for a medium of
$16 \pm 10$ years. $5.1 \%$ of the interviewed already had an encephalic vascular accident with a medium time of $1.6 \pm 0.5$ years and $28.2 \%$ had already gone through heart surgery before. As to the kind of aortic valve disease, the majority of those interviewed had aortic insufficiency, having as the most common etiology the rheumatic heart disease. It was observed the co-existence of other heart valve lesions in $36(93.3 \%)$ of the 39 patients of the sample. Among these, $33.3 \%$ had mitral insufficiency associated with aortic valve disease. As to the ventricular ejection fraction (FEVE), $18.9 \%$ of the patients had already developed IC had FEVE $<50 \%$, while $81.1 \%$ had IC with FEVE preserved. However, $73 \%$ of those interviewed were hemodynamicly compensated.

Conclusions: those with aortic valve disease which collaborated in the research had low education and low income, characteristics that may bring repercussions in the intra-hospital and home management. Thus, it is peremptory that health professionals elaborate strategies which will make an easier understanding for the patients, considering their social context and living habits.

\section{Abstract \#: P 395}

Psychosocial stress at work and blood pressure in ELSA-Brasil: methodological aspects of the operationalization of exposure and outcome variables

Leidjaira J. Lopes ${ }^{1}$, Enirtes C. P. Melo ${ }^{1}$, Marilia S. Carvalho ${ }^{2}$, Dora Chor ${ }^{1}$, Jose G. Mill ${ }^{3}$, Rosane H. Griep ${ }^{4}$

${ }^{1}$ National School of Public Health Sergio Arouca, Oswaldo Cruz Foundation, Rio de Janeiro, Brazil; ${ }^{2}$ Scientific Computing Program, Oswaldo Cruz Foundation, Rio de Janeiro, Brazil; ${ }^{3}$ Health Sciences Center, Federal University of Espírito Santo, Vitória, Brazil; ${ }^{4}$ Laboratory of Environment and Health Education. Oswaldo Cruz Institute, Oswaldo Cruz Foundation, Rio de Janeiro, Brazil; Corresponding author's e-mail: leidjaira_lopes@hotmail.com

Background: Work stress, resulting from the combination of high demands and low control in the workplace, is an important cardiovascular risk factor. The rise in blood pressure levels has been indicated as a possible mediator in this relationship, but to explore the association between work stress and blood pressure can be a complex task, especially because of the difficulties related to the definition of exposure and outcome variables. This study aims to discuss methodological issues related to the operationalization of exposure and outcome variables, taking as an example the association between psychosocial work stress and blood pressure levels.

Methods: We evaluated 12,096 active participants at the baseline (2008-2010) of the Longitudinal Study of Adult Health (ELSABrasil), a multicenter cohort of civil servants between 35 and 74 years old. Work stress was measured by the Brazilian version of the Swedish questionnaire of social demand-control-support, and assessed according to the formulation proposed by Karasek, in which the combination of high/low demand and high/low control form the quadrants 'low strain', 'active job', 'passive job' and 'high strain'. Due to the homogeneity of the studied group regarding demand and control scores, we adopted the procedure that deletes the segment belonging to the second tertile of both dimensions. The subjects were grouped into high stress (high strain and passive) and low stress (low strain and active) at work. Blood pressure was taken as the average of the last two out of three measurements performed by standard techniques, defining the hypertension condition (systolic and/or diastolic pressure $\geq 140 / 90 \mathrm{mmHg}$, and/or use of antihypertensives). Density charts present three groups: normotensive, medicated hypertensive and unmedicated hypertensive. The relationship between occupational stress and hypertension was also tested by logistic regression. 
Results: We observed a distance between the curves of high stress and low stress at work among hypertensive subjects. However, distributions of blood pressure according to work stress categories were similar when we considered the total population. The use of antihypertensive drugs appears to attenuate the effects of work stress, especially among younger individuals. The results were less consistent for the diastolic pressure: no gender differences were found. The work stressed subjects showed a significant increase of $35 \%$ in the odds of hypertension.

Conclusion: The relationship between work stress and blood pressure is not homogeneous in the population, and the process of operationalization of exposure and outcome variables strongly influences the results in the assessment of the effects of the event of interest.

\section{Abstract \#: P 397}

Weight change and subsequent physical and mental health functioning: a follow-up study among ageing employees

Anna Svärd ${ }^{1}$, Jouni Lahti ${ }^{1}$, Eira Roos ${ }^{1}$, Ossi Rahkonen ${ }^{1}$, Eero Lahelma $^{1}$, Tea Lallukka ${ }^{1,2}$

${ }^{1}$ Department of Public Health, Faculty of Medicine, University of Helsinki, Helsinki, Finland; ${ }^{2}$ Finnish Institute of Occupational Health, Helsinki, Finland; Corresponding author's e-mail: anna.svard@helsinki.fi

Background: Weight gain is associated with diseases, work disability and mortality. Longitudinal studies suggest an association between weight change and poor subsequent physical health functioning, whereas the association with mental health functioning is poorly understood. This study aimed to examine whether weight change among normal weight, overweight and obese female and male employees is associated with poor subsequent physical and mental health functioning. A further aim was to examine whether key covariates affect the associations.

Methods: The Helsinki Health Study baseline surveys were conducted in 2000-2002 among 40-60-year-old employees of the City of Helsinki, Finland $(n=8960)$. The first follow-up was conducted in 2007 and the second in 2012 (70\% responded to both follow-up surveys). The participants were categorized according to their baseline body mass index (BMI) and their weight change (BMI change $\geq 5 \%$ ) between the first two surveys. Physical and mental health functioning were measured with the Short Form 36 Health Survey (SF-36), the lowest quartile representing those with poor functioning. Logistic regression analysis was used to examine the associations between weight change and subsequent physical and mental health functioning until 2012. Covariates were age, sociodemographic factors, health behaviours, diagnosed diseases, strenuousness of work, employment status and prior functioning.

Results: Weight gain between baseline and first follow-up was common among women $(37 \%)$ and men $(27 \%)$. Among female normal weight weight-gainers $(\mathrm{OR}=1.81: 95 \% \mathrm{CI}=1.46-2.25)$, overweight weight-gainers $(\mathrm{OR}=3.11$ : $\mathrm{CI}=2.45-3.94)$ and obese weight-gainers $(\mathrm{OR}=5.62$ : $\mathrm{CI}=4.12-7.66)$ a strong association with poor physical health functioning was found. A strong association was also found among overweight and obese weight-maintainers. Covariates did not explain the associations. Among obese $(\mathrm{OR}=4.86: \quad \mathrm{CI}=2.49-9.50) \quad$ and $\quad$ overweight $\quad(\mathrm{OR}=1.94$ : $\mathrm{CI}=1.18-3.20)$ male weight-gainers and obese weight-maintainers $(\mathrm{OR}=3.44: \quad \mathrm{CI}=1.94-6.09)$, an association with poor physical health functioning was found. After adjustments, the association remained for obese, but not for overweight men. Among women, but not men, weight gain and maintenance of high weight was weakly associated with poor mental health functioning (obese weight-gainers:
$\mathrm{OR}=1.41: \mathrm{CI}=1.02-1.96)$. However, the associations disappeared after adjustments. Weight loss among women was associated with both poor physical and mental health functioning, whereas weight loss among men was associated with poor physical but not mental health functioning.

Conclusion: Weight-gaining normal weight and overweight employees might have a higher risk for poor physical health functioning than weight-maintainers. Preventing weight gain likely helps maintaining good physical health functioning and work ability.

\section{Abstract \#: P 398}

Extraction of risk patterns for type 2 diabetes using association rule mining

Azra Ramezankhani ${ }^{1}$, Omid Pournik ${ }^{2}$, Jamal Shahrabi ${ }^{3}$, Davood Khalili $^{1}$, Fereidoun Azizi ${ }^{4}$, Farzad Hadaegh ${ }^{1}$

${ }^{1}$ Prevention of Metabolic Disorders Research Center, Research Institute for Endocrine Sciences, Shahid Beheshti University of Medical Sciences, Tehran, Iran; ${ }^{2}$ Department of Community Medicine, School of Medicine, Iran University of Medical Sciences, Tehran, Iran; ${ }^{3}$ Industrial Engineering Department, Amirkabir University of Technology, Tehran, Iran; ${ }^{4}$ Endocrine Research Center, Research Institute for Endocrine Sciences, Shahid Beheshti

University of Medical Sciences, Tehran, Iran; Corresponding author's e-mail: ma.ramezankhani@gmail.com

Background: The aim of this study was to identify risk patterns for type 2 diabetes incidence using Association Rule Mining.

Methods: For a 6647 population without diabetes, aged $\geq 20$ years, followed for 10-12 years, a set of risk patterns for diabetes occurrence was identified. Study variables included demographic and anthropometric characteristics, smoking status, medical and drug history and laboratory measures. Association rules were generated in two steps. First, a set of frequent item sets or patterns were generated. Second, these patterns were used for generation of association rules. Support and confidence were used for evaluation of statistical significance and strength of the rules respectively. For the current study, we used the Apriori algorithm, the best-known and standard approach, for discovering association rules in a cohort dataset.

Results: The results showed for women, impaired fasting glucose (IFG) and impaired glucose tolerance (IGT) in combination with BMI $\geq 30$, Family history of diabetes, wrist circumference $>16.5 \mathrm{~cm}$ and waist to height $\geq 0.5$ can increase the risk for development of diabetes. For men a combination of IGT, IFG, length of stay $>40$ years in the city, central obesity, cholesterol to HDL ratio $\geq 5.3$, low physical activity, chronic kidney disease (CKD) and wrist circumference $>18.5 \mathrm{~cm}$ was identified as risk patterns for diabetes occurrence.

Conclusion: we showed the usefulness of Association Rule Mining for extracting risk patterns from a large prospective database.

\section{Abstract \#: P 399}

Glucose and the risk of hypertension in first-degree relatives of patients with type 2 diabetes

$\underline{\text { Mohsen Janghorbani }}{ }^{1}$

${ }^{1}$ Isfahan University of Medical Sciences; Corresponding author's e-mail: janghorbani@hlth.mui.ac.ir

Objective: To test the hypothesis that plasma glucose levels is associated with the incidence of hypertension (HT) in non-diabetic 
and non-hypertensive first degree relatives (FDR) of people with type 2 diabetes (T2D).

Methods: A total of 1089 FDR without diabetes and/or HT of consecutive patients with T2D 30-70 years old were examined and followed for a mean (SD) of 6.9 (1.7) years for HT incidence. At baseline and through follow-up, participants underwent a standard 75-g 2-h oral glucose tolerance test. HT was defined according to the criteria of the JNC7. We used Cox proportional hazard models to estimate hazard ratio (HR) for incident $\mathrm{HT}$ and plotted a receiver operating characteristic curve to assess discrimination.

Results: The plasma glucose levels at baseline were associated with incidence of HT, independently of age, gender, obesity and high cholesterol, high-density lipoprotein cholesterol, low-density lipoprotein cholesterol, triglycerides, education and systolic blood pressure. Those with impaired glucose tolerance were $54 \%$ (HR 1.54: $95 \%$ CI $1.33,1.77)$ more likely to develop HT than those with normal glucose tolerance. Those with impaired fasting glucose were also $23 \%$ (HR 1.23: $95 \%$ CI 1.01, 1.50) more likely to develop HT. Conclusions: High plasma glucose levels were consistently associated with incident HT.

\section{Abstract \#: P 400 \\ Prevalence and incidence of diagnosed type 1 diabetes in Germany 2009-2010: analysis of claims data from 65 million persons}

Ralph Brinks ${ }^{1}$, Joachim Rosenbauer ${ }^{1}$, Annika Hoyer ${ }^{1}$, Teresa Tamayo $^{1}$, Oliver Kuss ${ }^{1}$, Wolfgang Rathmann ${ }^{1}$

${ }^{1}$ German Diabetes Center, Institute for Biometry and Epidemiology, Duesseldorf, Germany; Corresponding author's e-mail: ralph.brinks@ddz.uni-duesseldorf.de

Background: There are only few population-based data on the ageand sex-specific prevalence and incidence of type 1 diabetes (T1D) in Germany. The statutory health insurance (SHI) in Germany covers more than $85 \%$ of the population. Recently, the complete claims data from 2009 to 2010 were made available for research purposes.

Materials and methods: All claims data from 2009 to 2010 (outpatient, inpatient, statutory sick pay) from persons of the SHI with consistent data on age, sex and period of coverage were analysed for ICD-10 diagnoses. A person was considered to have type T1D if at least one E10 diagnosis and no E11, E12, or E13 diagnoses were present. Overall and age- and sex-specific prevalences are estimated for both years.

Age-specific incidence of diagnosed T1D can be estimated from two cross-sections if the age-specific relative mortality (RM) of persons with T1D compared to persons without T1D is known [1]. We used three scenarios about RM for the German population to assess the ageand sex-specific incidence. In the first scenario, RM is taken from the Scottish Diabetes Register [2], in the second and third scenario RM is $20 \%$ lower and $20 \%$ higher than in Scotland, respectively.

Results: In 2009 and 2010, 65.64 and 64.93 million persons met the inclusion criteria ( 46.5 and $46.6 \%$ males). For the age range from 1 to 55 years, the data provide consistent prevalence estimates. In higher ages, the claims data yield questionable results. From 2009 to 2010, the overall prevalence of diagnosed T1D in the age range 1-55 rose only slightly from 216 to 217 per 100,000 in men, and from 147 to 148 per 100,000 in women.

The age-specific incidence rate peaks at about 10 years of age at 21 and 16 per 100,000 per year in the male and female population, respectively. After the peak, the incidence rate decreases linearly with age at rate of 0.47 and 0.44 per 100,000 person-years per year. The scenarios about RM have very little impact on these results.

Conclusions: The data provides consistent estimates for the prevalence of diagnosed T1D in the age range from 1 to 55 years. For higher age groups the diagnoses in the claims data seem to have low validity. For the first time, we were able to estimate the incidence of T1D in the age range $20-55$.

\section{References:}

[1] Brinks R, Landwehr S. doi 10.1093/imammb/dqu024, 2015

[2] Livingstone SJ, Levin D, Looker $\mathrm{HC}$ et al. doi 10.1001/jama.2014.16425, 2015

\section{Abstract \#: P 401}

Prevalence of the metabolic syndrome and its components in southern Kazakhstan: a cross-sectional study using three international criteria

Kamshat Madenbay ${ }^{1}$, Mira Zhunissova ${ }^{1}$, Karlygash Sadykova ${ }^{1}$, Assel Sadykova $^{1}$, Zhanar Shalkharova ${ }^{2}$, Zhanat Shalkharova $^{1}$, Andrej M. Grjibovski ${ }^{3,4}$

${ }^{1}$ International Kazakh - Turkish University, Turkestan, Kazakhstan; ${ }^{2}$ Kazakh Medical University of Continuing Education, Almaty, Kazakhstan; ${ }^{3}$ Norwegian Institute of Public Health, Oslo, Norway; ${ }^{4}$ Northern State Medical University, Arkhangelsk, Russia; Corresponding author's e-mail: kamshat.madenbay@gmail.com

Introduction: The metabolic syndrome (MetS) is a cluster of risk factors associated with cardiovascular (CVD) morbidity and mortality. Kazakhstan has one of the highest CVD mortality in the European WHO Region, particularly among men. However, the prevalence of MetS in Kazakhstan remains unknown. The aim of this study is to assess the prevalence of MetS and its components in an urban Kazakhstani setting using different international criteria to ensure comparability with international data.

Methods: Altogether, 938 adults aged 18-74 years were enrolled in a cross-sectional study in Turkestan, Southern Kazakhstan. All subjects completed an anonymous questionnaire and underwent a physical examination. Blood tests were taken and analyzed in the International Kazakh-Turkish University laboratory using calibrated equipment. MetS was defined using ATP-III (2001), AHA/NHLBT (2005) and IDF (2005) criteria.

Results: Among women, the prevalence of MetS was $15.8 \%(95 \% \mathrm{CI}$ : 13.2-18.9), $23.1 \%$ (95\% CI 20.0-26.6) and $20.2 \%$ (95\% CI: 17.2-23.5) for the ATP-III, AHA/NHLBT and IDF criteria, respectively. The corresponding data for men were $16.0 \%(95 \% \mathrm{CI}$ : 11.8-21.4), $29.3 \%$ (95\% CI: $23.8-35.6$ ) and $25.8 \%$ (95\% CI: 20.5-31.9). In women, the prevalence of MetS defined using IDF criteria progressively increased from $1.1 \%$ in the age group of $18-29$ years to $49.0 \%$ in the age group of $60+$ years. Among men, the prevalence for the same age-groups was 6.1 and $34.9 \%$, respectively. In the full sample, abdominal obesity was the most prevalent component of MetS using IDF criteria among women $(74.5 \%)$ followed elevated blood pressure $(29.7 \%)$ and high blood glucose $(26.5 \%)$ while for men the three most common components were abdominal obesity (70.2\%), elevated blood pressure (51.6\%) and triglycerides (22.2\%).

Conclusions: The prevalence of MetS in a Southern Kazakhstani urban setting is comparable to many other settings in the European WHO Region. However, the distribution of components of MetS is different and varies across genders. Our results suggest that MetS may contribute to high CVD mortality in Kazakhstan, particularly among men. The reasons behind the observed pattern of the prevalence of 
MetS and its components of will be discussed. Further research on the factors leading to development of MetS in a Kazakhstani population and its association with high CVD mortality is needed.

\section{Abstract \#: P 402}

Perceptions of obesity among (former) centrally obese adults with and without additional cardiometabolic risk factors

Rimke C. Vos ${ }^{1}$, Corine den Engelsen ${ }^{1}$, Mieke Rijken ${ }^{2}$, Guy E.H.M. Rutten ${ }^{1}$

${ }^{1}$ Julius Center for Health Sciences and Primary Care, University Medical Center Utrecht, the Netherlands; ${ }^{2}$ NIVEL, Netherlands Institute for Health Services Research, Utrecht, the Netherlands; Corresponding author's e-mail: r.c.vos-3@umcutrecht.nl

Background: People's perceptions of illness are important determinants of their health behaviour. Knowledge of perceptions regarding obesity, might lead to more effective interventions, by challenging these perceptions through lifestyle modification programs. Several studies have evaluated causal attributions with regard to obesity, but other domains of illness perceptions, such as the perceived consequences of obesity and their perceived controllability, have not yet been studied. Therefore, the aim of the current study was to assessed if centrally obese adults with and without an elevated cardiometabolic risk and with or without weight loss after screening for metabolic syndrome differ in their perceptions regarding the causes, consequences and time course of their obesity, their control beliefs and concerns.

Methods: Three samples from a longitudinal study were selected based on their baseline and 3 years follow-up profile: people with central obesity and metabolic syndrome at both occasions ("persistent cardiometabolic-risk group', $\mathrm{n}=80$ ), people with central obesity but without the metabolic syndrome at both occasions ('persistent obese group', $n=63$ ), and formerly obese people ('improved cardiometabolic-risk group', $\mathrm{n}=49$ ). Illness perceptions were assessed by an adapted version of the Brief Illness Perception Questionnaire (BIPQ).

Results: Mean BIPQ-scores did not differ significantly between those with persistent obesity and those with a persistent cardiometabolic risk after adjusting for age and gender. However, those who improved their cardiometabolic risk perceived their obesity as shorter (timelines: $p$ value $<0.001$ ) and felt more personal control over their weight $(p$ value $<0.05)$

Conclusion: Obese adults who improved their cardiometabolic risk profile felt more personal control and rightly considered their obesity of shorter duration. Controlled trials should demonstrate whether discussing 'personal control' could be favorable in lifestyle interventions.

\section{Abstract \#: P 403}

Objectively measured sedentary behaviour patterns according to diabetes status- the Maastricht Study

Julianne D. van der Berg ${ }^{1}$, Coen D.A. Stehouwer ${ }^{2}$, Hans Bosma ${ }^{1}$, Jeroen H.P.M. van der Velde ${ }^{3,4,5}$, Paul J.B. Willems ${ }^{3}$, Hans H.C.M. Savelberg ${ }^{3}$, Miranda T. Schram ${ }^{2}$, Simone J.S. Sep ${ }^{2}$, Carla J.H. van der Kallen $^{2}$, Ronald M.A. Henry ${ }^{2}$, Pieter C. Dagnelie ${ }^{2,6,7}$, Nicolaas C. Schaper ${ }^{2,5,6}$, Annemarie Koster ${ }^{1,6}$

${ }^{1}$ Department of Social Medicine, Maastricht University \& CAPHRI School for Public Health and Primary Care, Maastricht University;
${ }^{2}$ Cardiovascular Research Institute Maastricht (CARIM), Maastricht University \& Department of Internal Medicine, Maastricht University Medical Centre (MUMC+); ${ }^{3}$ Department of Human Movement Sciences, Maastricht University; ${ }^{4}$ School for Nutrition and Translational Research in Metabolism (NUTRIM), Maastricht University; ${ }^{5}$ Division of Endocrinology, Department of Internal Medicine, MUMC $+;{ }^{6}$ CAPHRI School for Public Health and Primary Care, Maastricht University; ${ }^{7}$ Department of Epidemiology, Maastricht University: Maastricht, The Netherlands; Corresponding author's e-mail: j.vanderberg@maastrichtuniversity.nl

Introduction: Studies have shown that physical activity (PA) plays a role in the development and management of type 2 diabetes mellitus (T2DM). Next to the role of PA, there has been growing interest in the role of sedentary behaviour. However, large-scale studies with objectively measured sedentary behaviour in a population with T2DM are scarce. Therefore, we examined differences in objectively assessed sedentary behaviour patterns between participants with T2DM, with prediabetes (impaired glucose metabolism) and without T2DM. Methods: We included 2529 participants (aged 40-76 years) of The Maastricht Study who wore an activPAL accelerometer $24 \mathrm{~h} /$ day for seven consecutive days on the front right thigh. We calculated percentage sedentary time (ST) of waking time, mean number of breaks in ST per day ( $\geq 1$ min of ST followed by $\geq 1$ min activity), and mean step frequency per day (measure for PA). To determine glucose metabolism status, all participants underwent an oral glucose tolerance test. Associations between glucose metabolism status and percentage ST were examined using linear regression analysis. Results: 726 participants $(28.7 \%)$ had T2DM, 391 (15.5\%) had prediabetes, and $1412(55.8 \%)$ had no T2DM. Participants provided on average 6.4 valid days (defined as $\geq 14 \mathrm{~h}$ of monitoring), and $91 \%$ wore the device six or more days. Participants with T2DM spent on average (mean $\pm \mathrm{SD}$ ) $10.2 \pm 1.8 \mathrm{~h}$ per day sedentary, as compared to on average $9.5 \pm 1.8$ in participants with prediabetes and $9.1 \pm 1.6$ in participants without T2DM $(P<0.001)$. Mean number of sedentary breaks per day was $35.8 \pm 9.3$ among participants with T2DM, and $37.4 \pm 8.8$ and $38.0 \pm 8.5$ among participants with prediabetes and participants without T2DM $(P<0.001)$. After adjustment for demographic factors (sex, age, level of education), health factors (smoking status, body mass index, mobility limitation, history of cardiovascular diseases and chronic obstructive pulmonary disease), and mean step frequency, participants with T2DM had on average $22.5 \mathrm{~min}$ more ST per day $(95 \% \mathrm{CI}=16.8-28.2$ : $P<0.001)$ compared to those without T2DM.

Conclusion: Having T2DM was significantly associated with on average 22.5 sedentary minutes more per day. The results suggest that next to PA, sedentary behaviour could be an important determinant of the development and management of T2DM.

\section{Abstract \#: P 404}

Self-reported hypoglycaemia and mortality in type 2 diabetes patients treated with insulin in the diabetes care system WestFriesland, The Netherlands

Simone P. Rauh ${ }^{1}$, Femke Rutters ${ }^{1}$, Giel Nijpels ${ }^{2}$, Amber A.W.A. van der Heijden $^{2}$, Iris Walraven ${ }^{1}$, Petra J. Elders ${ }^{2}$, Martijn W. Heymans ${ }^{1}$, Jacqueline M. Dekker ${ }^{1}$

${ }^{1}$ EMGO Institute for Health and Care Research, Department of Epidemiology and Biostatistics, VU University Medical Centre, Amsterdam, The Netherlands; ${ }^{2}$ EMGO Institute for Health and Care Research, Department of General Practice, VU University Medical Centre, Amsterdam, The Netherlands; Corresponding author's e-mail: s.rauh@vumc.nl 
Background: Hypoglycaemia is a major side effect of insulin treatment in patients with type 2 diabetes. Previous studies suggested that objectively measured hypoglycaemic events are associated with mortality in patients with type 2 diabetes. However, for patients and clinicians it is also relevant to know the associated mortality risk of self-reported hypoglycaemia. Self-reported hypoglycaemia might better reflect hypoglycaemic sensations experienced in everyday life. Up until now, little is known about self-reported hypoglycaemia and the associated risk on mortality in the general type 2 diabetes population in usual care. Our aim was to study the association between self-reported mild and severe hypoglycaemia and all-cause mortality in type 2 diabetes patients treated with insulin in usual care.

Methods: Demographics, clinical characteristics and mortality data were obtained from 1667 type 2 diabetes patients treated with insulin in the Diabetes Care System West-Friesland, the Netherlands. Hypoglycaemia was self-reported. Mild hypoglycaemia was defined as hypoglycaemic events not requiring help from others, while severe hypoglycaemia was defined as hypoglycaemic events requiring help from others. Logistic regression analysis was performed to analyse the association between hypoglycaemia and mortality.

Results: During a median follow-up of 1.9 years, 804 patients (48 \%) reported no hypoglycaemia, $744(45 \%)$ reported only mild hypoglycaemia and 119 (7\%) reported severe hypoglycaemia. During follow-up, 98 patients $(5.9 \%$ ) died. Reporting only mild hypoglycaemia was significantly associated with lower mortality risk during follow-up (OR: $0.48,95 \%$ CI $0.29-0.81$ ), while reporting severe hypoglycaemia was not significantly associated with mortality (OR: 0.77, $95 \%$ CI: 0.33-1.80), compared to reporting no hypoglycaemia. These results were adjusted for age, sex, diabetes duration, haemoglobin A1c (HbA1c) levels, hypertension, smoking, sulfonylurea use, and micro-/macro-vascular complications.

Conclusions: We observed that insulin-treated type 2 diabetes patients reporting mild hypoglycaemia had a lower mortality risk during a median follow-up of 2 years compared to patients reporting no hypoglycaemia. If this finding is confirmed in other studies, it may be reassuring for patients and their physicians to know that experiencing hypoglycaemic sensations not requiring medical help is associated with reduced short-term mortality risk.

Support: This work was supported by funding from Novo Nordisk A/S.

\section{Abstract \#: P 405}

The association of pre-schoolers' fussy eating with body composition at 6 years of age in a prospective birth cohort

Lisanne M. de Barse $^{1}$, Henning Tiemeier ${ }^{1}$, Elisabeth T.M. Leermakers ${ }^{1}$, Trudy Voortman ${ }^{1}$, Lisa R. Edelson ${ }^{2}$, Albert Hofman ${ }^{1}$, Vincent W.V. Jaddoe ${ }^{1}$, Oscar H. Franco ${ }^{1}$, Pauline W. Jansen ${ }^{1}$

${ }^{1}$ Department of Epidemiology, Erasmus-MC University Medical Center, Rotterdam, the Netherlands; ${ }^{2}$ Consumer and Sensory Science, Nestlé Research Center, Lausanne, Switzerland; Corresponding author's e-mail: 1.debarse @erasmusmc.nl

Background and objective: Children's fussy eating behavior has been related to both underweight and overweight in cross-sectional studies, but the direction of these associations and the relations with other measures of body compositions remain unclear. We aimed to examine whether fussy eating at age 4 years is longitudinally related to body mass index (BMI), body fat percentage (BF\%), fat mass index (FMI) and fat free mass index (FFMI) at 6 years of age.

Design: This study was embedded in Generation R, a populationbased, prospective cohort study from fetal life onwards.
Materials and methods: In 4191 children, data was available on exposure and at least one of the outcomes. The Child Eating Behaviour Questionnaire (CEBQ) was administered at age 4 . We used a fussy eating profile, based on a latent profile analysis with five CEBQsubscales. This eating profile is characterized by high scores on food avoidant scales and low scores on food approach scales. At age 6, height and weight were measured at our research center. Body fat mass, bone mass, and lean mass were measured by dual-energy-X-ray absorptiometry scans. We used age- and sex-specific standard deviation (SD) scores for all outcomes. Regression analyses were performed, adjusting for potential confounders. We additionally adjusted for baseline BMI at age 4 .

Results: A fussy eating profile was related to a lower BMI (B $=-0.36,95 \%$ CI: $-0.47:-0.26)$, explained by both a lower FMI (B $=-0.22,95 \% \mathrm{CI}:-0.32:-0.11)$ and FFMI $(\mathrm{B}=-0.41$, $95 \% \mathrm{CI}:-0.53$ : -0.29 ). When adjusting for baseline BMI at 4 years of age, a fussy eating profile at age 4 predicted a 0.12 lower BMI-SD at age 6 (95\% CI: $-0.20:-0.05)$. This was mainly due to a lower FFMI (B $=-0.19,95 \% \mathrm{CI}:-0.30:-0.09)$. Moreover, fussy eaters even had a higher risk of becoming underweight than non-fussy eaters $(\mathrm{OR}=2.41,95 \%$ CI: 1.43: 4.05).

Conclusions: Our findings suggest that young fussy eaters are at risk of having a lower fat free mass and of becoming underweight in a 2-year period.

\section{Abstract \#: P 406}

Association between obesity, abdominal obesity and estimated risk for coronary heart disease in asymptomatic Brazilian adults

Regina Célia Vilanova Campelo ${ }^{1}$, Marcus Vinicius Nascimento Ferreira $^{2}$, Germano da Paz Oliveira ${ }^{3}$, Maurício Batista Paes Landim ${ }^{1}$, $\underline{\text { Francisco Leonardo Torres-Leal }}{ }^{1}$

${ }^{1}$ Department of Biophysics and Physiology, Health Science Center, Federal University of Piauí, Piauí, Brazil; ${ }^{2}$ Youth/Child and cARdiovascular Risk and Environmental (YCARE) Research Group, Research Group, School University of São Paulo, São Paulo, Brazil; ${ }^{3}$ Hospital of the Federal University of Piauí, Piauí, Brazil; Corresponding author's e-mail: torresleal@ufpi.edu.br

Background: Studies have shown that obesity causes or accelerates the development of cardiovascular diseases, being recognized as global epidemic and important public health problem. For the control of this pathology is necessary to know the cardiovascular risk and identify factors associated with that risk. The Framingham Risk Score (FRS) is used in the assessment of cardiovascular risk, considered a reliable score, simple and low cost, it is a way to detect the onset of the disease and establish primary prevention strategies and possibly reduce future costs in asymptomatic patients, so it fits that Brazil is a developing country. The objectives of our study were to assess the estimated risk for coronary heart disease (CHD) and to examine your association with obesity and abdominal obesity in asymptomatic Brazilian adults.

Methods: We conducted a cross-sectional study with asymptomatic patients $(\mathrm{n}=201)$, with stratified by gender ( $\%$ men and women), between 20 and 60 years of age, in Teresina (Piauí, Brazil), selected by probability sampling simple random. Were included in the study patients without previous diagnosis of atherosclerotic disease and that didn't make use of continuous medication. The estimate of risk for coronary artery disease (outcome) was evaluated by the FRS exposure factors analyzed were: overweight and obesity (body mass index, BMI), and abdominal obesity (waist circumference, WC). Confounding variables were sex, age and physical activity ( $\geq 60 \mathrm{~min} /$ day). Associations were examined by multilevel linear 
regression, with confidence interval of $95 \%$. The analyses were adjusted for confounding variables.

Results: The prevalence of estimated risk for CHD was $7.0 \%$ in asymptomatic Brazilian adults. In women's, obesity $(\beta$ : 1.89 : CI95 \%: 0.09-3.06) and abdominal obesity ( $\beta$ : 2.18: $95 \% \mathrm{CI}$ : 0.09-2.82) showed association with estimated risk for CHD, in adjusted analyses. We not found association between exposition factors and outcome in men's.

Conclusion: The prevalence of estimated risk for coronary heart disease (CHD) is low in asymptomatic Brazilian adults. However, the risk of CHD rises in women's with obesity and abdominal obesity.

\section{Abstract \#: P 407 \\ Epicatechin intake and markers of glucose and insulin metabolism: the Zutphen elderly study}

Morgan O. Obura, $\mathrm{BSc}^{1}$, James Dower, MSc, Daan Kromhout, $\mathrm{PhD}^{1}$, Peter C.H. Hollman, $\mathrm{PhD}^{1}$, Marianne Geleijnse, $\mathrm{PhD}^{1}$,

Sabita Soedamah-Muthu, $\mathrm{PhD}^{1}$, Janette de Goede, $\mathrm{PhD}^{1}$

${ }^{1}$ Division of Human Nutrition, Wageningen University, Wageningen, The Netherlands; Corresponding author's e-mail:

Janette.degoede@wur.nl

Background: Consumption of flavonoid-rich foods like cocoa and tea is associated with a reduced risk of type 2 diabetes and improved glycemic control. Cocoa and tea are both important dietary sources of the flavonoid epicatechin. Our objective therefore was to examine whether dietary epicatechin intake is associated with markers of glucose and insulin metabolism.

Methods: We conducted a prospective study in 437 men from the Zutphen Elderly Study aged 65-84 years at baseline (1985), free of diabetes and cancer. Dietary epicatechin and other nutrients were assessed at baseline using a validated cross-check dietary history method. Glucose and insulin areas under the curve (AUC) were determined in response to an oral glucose tolerance test (OGTT) performed in 1990. Homeostasis model assessment of insulin resistance (HOMA-IR) as well as Matsuda index were calculated from fasting glucose (mmol/l) and insulin (mU/l) levels. Glycated hemoglobin $(\mathrm{HbA} 1 \mathrm{c})$ was measured in 1993. Adjusted means and $95 \%$ confidence intervals $(95 \% \mathrm{CI})$ across quintiles of epicatechin intake and trend analysis were calculated using multivariable linear regression. Models were adjusted for age, BMI, socioeconomic status, family history of diabetes, lifestyle (smoking, physical activity and alcohol consumption), dietary factors (total energy and dietary fiber) and cardiovascular disease.

Results: The median intake of epicatechin in the highest quintile (Q5) was $26.6 \mathrm{mg} /$ day compared to $5.5 \mathrm{mg}$ /day in the lowest quintile (Q1). Tea contributed $50 \%$ of epicatechin intake, followed by apples $(29 \%)$, cocoa $(8 \%)$ and red wine $(2 \%)$. Epicatechin intake was not associated with any of the markers of glucose and insulin metabolism ( $p$ trends $>0.05$ for all). The adjusted mean $(95 \% \mathrm{CI})$ for HbA1c $(\%)$ was $4.9(4.7-5.1)$ in $\mathrm{Q} 1$ and $4.7(4.5-4.9)$ in Q5 $(p$ trend $=0.32)$. The adjusted means (95\% CI) for Q1 and Q5 were 90.5 (81.4-100.6)and 93.4 (83.6-104.4) ( $p$ trend $=0.52$ ) for the Matsuda index, 891 $(842-942)$ and $882(832-935)(p$ trend $=0.94)$ for glucoseAUC (mmol/L min), 4777 (4323-5279) and 4995 (4498.8-5547.4) ( $p$ trend $=0.86)$ for insulinAUC $(\mathrm{mU} / \mathrm{L} \mathrm{min}$ ) and $2.3(2.0-2.5)$ and $2.1(1.9-2.4)$ ( $p$ trend $=0.29)$ for HOMA-IR. There was no interaction with age, family history of diabetes, smoking status and BMI. Excluding men with cardiovascular disease $(n=57)$ did not alter the associations.

Conclusions: In Dutch elderly non-diabetic men, epicatechin intake was not associated with markers of glucose and insulin metabolism.

\section{Abstract \#: P 408}

Severity of diabetes mellitus and risk of total hip or knee replacement: a population based case-control study

Johannes T.H. Nielen ${ }^{1}$, Bart van den Bemt ${ }^{2}$, Arief Lalmohamed ${ }^{3}$, Anthonius de Boer ${ }^{4}$, Annelies Boonen ${ }^{5}$, Pieter C. Dagnelie ${ }^{1}$, Pieter Emans ${ }^{6}$, Frank de Vries

${ }^{1}$ Department of Epidemiology, Maastricht University, Maastricht, the Netherlands; ${ }^{2}$ Department of Pharmacy, Sint Maartenskliniek, Nijmegen, the Netherlands; ${ }^{3}$ Department of Clinical Pharmacy, University Medical Center Utrecht, Utrecht, the Netherlands; ${ }^{4}$ Department of Pharmacoepidemiology and Clinical Pharmacology, Utrecht University, Utrecht, the Netherlands; ${ }^{5}$ Department of Rheumatology, Maastricht University Medical Centre, Maastricht, the Netherlands; ${ }^{6}$ Department of Orthopaedics, Maastricht University Medical Centre, Maastricht, the Netherlands; ${ }^{7}$ Department of Clinical Pharmacy and Toxicology, Maastricht University Medical Centre, Maastricht, the Netherlands; Corresponding author's e-mail: yannick.nielen@mumc.nl

Background: Previous research indicates that diabetes mellitus (DM) might be an independent risk factor for osteoarthritis (OA). Increased risk of $\mathrm{OA}$ has been found in non-weight-bearing joints eliminating the possibility of BMI associated mechanically induced cartilage damage. Aims: To evaluate the risk of hip or knee replacement, as a proxy for severe, in patients with DM. We additionally evaluated the risk of total joint replacement (TJR) with various proxies for increased DM severity.

Methods: We performed a population based case-control study using the Clinical Practice Research Datalink (CPRD). Cases $(n=94,609)$ were defined as patients $>18$ years who had undergone TJR between 2000 and 2012. Controls were matched by age, gender and general practitioner (GP) practice. Conditional logistic regression was used to estimate the risk of total knee (TKR) and total hip replacement (THR) surgery associated with use of antidiabetic drugs (ADs). We additionally stratified current $\mathrm{AD}$ users by proxies for OA severity.

Results: Current AD use was significantly associated with a lower risk of TKR $[\mathrm{OR}=0.86(95 \% \mathrm{CI}=0.78-0.94)]$ and $\mathrm{THR}$ $[\mathrm{OR}=0.90(95 \% \mathrm{CI}=0.82-0.99)]$ compared to patients not using ADs. Moreover, risk of TKR and THR was decreased with increasing HbA1c.

Conclusions: Contrary to previous research, our study suggests that DM patients are less likely to suffer from severe OA as compared to non-users. Moreover, risk of severe OA necessitating TJR decreases with increasing DM severity, based on HbAlc values. This is possibly due to dissimilarities in methodology, a decrease in eligibility for surgery, or variability of OA phenotypes.

\section{Abstract \#: P 409}

Individuals with type $\mathbf{2}$ diabetes mellitus are at an increased risk of gout but this is not due to diabetes

Caroline M.P.G.van Durme ${ }^{1}$, José M.A. Wijnands ${ }^{1}$, Johanna H.M. Driessen $^{2}$, Annelies Boonen ${ }^{1}$, Corinne Klop ${ }^{3}$, Bert Leufkens ${ }^{3}$, Coen D.A. Stehouwer ${ }^{1}$, Frank de Vries ${ }^{2}$, Cyrus Cooper ${ }^{4}$

${ }^{1}$ Department of Internal Medicine, Division of Rheumatology, Maastricht University Medical Centre+, Maastricht, the Netherlands; ${ }^{2}$ Department of Clinical Pharmacy and Toxicology, Maastricht University Medical Centre+, Maastricht, the Netherlands; ${ }^{3}$ Utrecht Institute of Pharmaceutical Sciences, Utrecht University, Utrecht, the Netherlands; ${ }^{4}$ MRC Lifecourse Epidemiology Unit, University of 
Southampton, Southampton General Hospital, Southampton, United Kingdom; Corresponding author's e-mail: carolinevd@gmail.com

Objective: The objective of this study was to understand the role of diabetes itself and its comorbidities within the association between type 2 diabetes mellitus (T2DM) and gout.

Methods: We conducted a retrospective cohort study using the UK Clinical Practice Research Datalink (CPRD) GOLD. Patients with T2DM were defined as individuals on a non-insulin antidiabetic drug (NIAD) between 2004 and 2012, and matched to one control based on age, sex, and general practice. We estimated gout risk in NIAD users using Cox regression analysis and performed subgroup analyses within these individuals to further explore the role of HbA1c levels. All analyses were stratified for sex.

Results: Compared with controls, NIAD users had an increased risk of gout (HR 1.48: $95 \%$ CI 1.41-1.54). This association was stronger in women (HR 2.23: $95 \%$ CI 2.07-2.41) as compared with men (HR 1.19: $95 \%$ CI 1.13-1.26). However, after adjustments for BMI, eGFR, hypertension, renal transplantation, and the use of diuretics, statins, low dose aspirin, ciclosporin, and tacrolimus, the risk disappeared in women (HR 1.01: $95 \%$ CI 0.92-1.11) and reversed in men (HR 0.61: $95 \%$ CI 0.58-0.66) ( $p$ for interaction <.001). When stratifying gout risk according to hbalc in male and female niad users, we found an inverse association between incident men only ( $p$ for interaction $\leq 0.01$ ). Further adjustment gave similar results.

Conclusion: Individuals with $\mathrm{t} 2 \mathrm{dm}$ are at increased of compared population controls. However, this is not due diabetes itself, which actually associated patients a decreased men, but the comorbid conditions these individuals. CVD JW participated equally work.

\section{Abstract \#: P 410}

General and central obesity measurement associations with markers of chronic inflammation and type 2 diabetes

$\underline{\text { Seán R. Millar }}{ }^{1}$, Ivan J. Perry ${ }^{1}$, Catherine M. Phillips ${ }^{1}$

${ }^{1}$ University College Cork; Corresponding author's e-mail: s.millar@ucc.i.e

Background: Inflammation has been suggested a possible mechanism linking adiposity with type 2 diabetes. Central obesity indicated by waist circumference (WC) measurement is thought to be a greater risk factor for chronic inflammation compared to general obesity characterised by body mass index (BMI). However, evidence for this association is still equivocal. In this study we compare biomarker relationships with BMI and WC measures and type 2 diabetes. We examine a range of pro-inflammatory cytokines, acute-phase response proteins, coagulation factors, white blood cell counts and a combination of these markers to determine which measurement is more strongly associated with diabetes-related inflammation.

Methods: This was a cross-sectional study involving a random sample of 2002 men and women aged 50-69 years. Correlation and logistic regression analyses were used to explore general and central obesity measurement relationships with non-optimal biomarker levels, biomarker combinations and type 2 diabetes.

Results: When compared with BMI, WC was more strongly related to a majority of inflammatory markers, adverse biomarker clustering and type 2 diabetes. In multivariable analysis, only WC remained significantly associated with type 2 diabetes (OR: 2.96, $95 \%$ CI: 1.93-4.55) after adjusting for BMI (OR: 0.73, $95 \%$ CI: 0.49-1.10) four or more markers (OR: 4.67, $95 \% \mathrm{CI}$ : 2.64-8.27) and other potential confounders.

Conclusions: These data suggest that central obesity is a greater risk factor for type 2 diabetes and associated chronic inflammation than BMI. However, our results also demonstrate that the relationship between obesity and diabetes-related inflammation cannot be completely accounted for by surrogate adiposity measures.

\section{Abstract \#: P 411}

Differences in health behaviours and BMI according to neighbourhood socio-economic status and residential area density among adults across Europe

Jeroen Lakerveld $^{1}$ on behalf of the SPOTLIGHT consortium

${ }^{1}$ Department of Epidemiology and Biostatistics, EMGO Institute for Health and Care Research, VU University Medical Center, Amsterdam, the Netherlands; Corresponding author's e-mail: j.lakerveld@vumc.nl

Background: People from lower socio-economic status (SES) and those living in neighbourhoods with high residential area density (RAD) have been found to be less likely to engage in health behaviors and more likely to be overweight and obese. However, it is unclear if such differences are present in different countries in Europe that vary according to neighbourhood SES and RAD. This study describes a sampling approach for defining categories of urban neighbourhoods and explores how health behaviours and BMI differs across neighbourhood types across and within Belgium, France, Hungary, the Netherlands and the United Kingdom.

Methods: In this cross-sectional, observational study, 60 urban neighbourhoods were selected (12 per country), representing a variety in SES and RAD. Self-report online and paper questionnaires was used to assess physical activity, sedentary behaviours, dietary habits and BMI. Multivariate analyses were conducted to study differences in these behaviors and self report BMI according to neighbourhood SES and RAD across and within countries.

Results: 6038 adults (mean age 52 years, $56 \%$ female) reported a median of $52.9 \mathrm{~min} /$ day leisure time physical activity and $22.9 \mathrm{~min} /$ day on active transport. Almost $9 \mathrm{~h} /$ day was spent on sedentary activities. Median weekly intake was 7.0 for pieces of fruit, 7.0 for vegetables, 0.5 for fish, 2.0 for sugary drinks and 3.0 for sweets. Across all countries, residents from low SES neighbourhoods ate less fruit, vegetables and fish (most notable in Hungary), drank more sugary drinks (France), and had a higher BMI (especially in Hungary and the UK). SES differences in sedentary behaviours were only observed in France (low SES sat 84.3 min/day more (CI -114.4 to -54.2 ). Low RAD residents were less physically active during leisure and for transport (except for Belgium). They ate less often vegetables and fish in the UK, and ate more sweets in the Netherlands. BMI differences by RAD were inconsistent across countries.

Conclusions: The sampling approach of the survey revealed that both country and neighbourhood type are important correlates of adults' health behaviours and BMI. Further studies should focus on how and why.

\section{Abstract \#: P 412}

Colonization of the gut with the archaeon methanobrevibacter smithii is associated with childhood weight development

Catherine Akwi Mbakwa ${ }^{1,2}$, John Penders ${ }^{1}$, Paul H. Savelkoul ${ }^{1}$, Carel

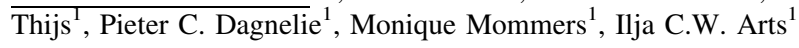

${ }^{1}$ Maastricht University; ${ }^{2}$ TIFN; Corresponding author's e-mail: catherine.akwimbakwa@maastrichtuniversity.nl

Background: Recent studies have indicated that, next to the many bacterial species, indigenous archaea play a crucial role in the 
metabolic capacity of the gut microbiota. However, few of these studies have been conducted in humans. Moreover, to date no study has investigated intestinal archaea with respect to weight gain over time in children.

Aim: Prospectively investigate whether the presence and abundance of archaea in faeces of 472 children at school age are associated with weight development from 6 to 10 years of age.

Methods: The present study was conducted within the prospective KOALA Birth Cohort study. Faecal samples of 472 children were analysed by qPCR to quantify archaea (M. smithii and M. stadtmanae). Parents were sent repeated questionnaires to collect anthropometric data and information on potential confounders. Main outcomes were overweight (BMI $\geq 85$ th percentile), and age- and sexstandardized BMI z-scores, weight $\mathrm{z}$-scores and height $\mathrm{z}$-scores at ages (mean $\pm \mathrm{SD}$ ) of $6.2 \pm 0.5, \quad 6.8 \pm 0.5, \quad 7.8 \pm 0.5$, and $8.8 \pm 0.5$ years. Generalised estimating equations (GEE) for repeated measures was used for the statistical analysis while controlling for the following confounders: maternal educational level, weight gained during pregnancy, place and mode of delivery, birth weight, household size, antibiotic use, physical activity, sex, and dietary intake of fibre, fat, carbohydrates and total energy.

Results: The presence and high abundance of M. smithii were associated with an increased risk of overweight (adjusted odds ratio $(\mathrm{OR})=2.69: 95 \%$ CI $0.96-7.54:$ OR $=3.27: 95 \%$ CI $1.0-9.83$, respectively) from 6 to 10 years of age. Moreover, the presence of $M$. smithii was also associated with higher weight $\mathrm{z}$-scores (adjusted beta 0.18: $95 \%$ CI 0.01-0.36), but not with height z-scores. We found a statistically significant interaction $(P=0.008)$ between $M$. smithii and age for BMI z-scores, implying that the strength of this association increased over time.

Conclusion: The presence/higher abundance of $M$. smithii in the gut of children is associated with higher weight $\mathrm{z}$-scores, higher BMI $\mathrm{z}$-scores and overweight.

\section{Abstract \#: P 413}

\section{Screen-based behaviours of adolescents in Bangladesh}

Asad K. ${ }^{1}$, Nicola W. Burton ${ }^{2}$

${ }^{1}$ School of Health \& Rehabilitation Sciences, The University of Queensland; ${ }^{2}$ School of Human Movements \& Nutrition Sciences; Corresponding author's e-mail: a.khan2@uq.edu.au

Background: Screen-based behaviours (i.e., television/DVD, video games, computer use, face-book/twitter) of adolescents have been adversely associated with body composition, cardiorespiratory and musculoskeletal fitness, wellbeing, and academic performance. Understanding the magnitude and patterns of these behaviours may be the first step to design targeted interventions, but as yet, no studies in Bangladesh have examined adolescents' screen-based behaviours. The purpose of this study was therefore to describe the prevalence and correlates of screen time among adolescents in Bangladesh.

Methods: A self-administered written survey was conducted among students from eight secondary schools of Dhaka city, Bangladesh. A total of 758 students ( 366 boys, 392 girls: mean age 14.3 years, SD 1.14) from grades 7 to 10 completed a modified version of the Adolescent Sedentary Activity Questionnaire, which was used to collect information on screen time on past week, separating weekdays and weekends. Total screen time was dichotomized as $\leq 2 \mathrm{~h}$ (low) or $>2 \mathrm{~h}$ (high) per day, reflecting a widely used screen time recommendation. Parents completed a separate questionnaire to provide house-hold/family level data.

Results: On average, Bangladeshi adolescents reported $4.47 \mathrm{~h}$ $(\mathrm{SD}=3.10)$ of screen time daily with a higher average screen time among boys $(4.78 \mathrm{~h} /$ day) than girls $(4.19 \mathrm{~h} /$ day $)$. About $79 \%$ of the adolescents reported more than $2 \mathrm{~h}$ /day of screen time, with more participants having high screen time on weekends $(89 \%)$ than weekdays $(75 \%)$. About $83 \%$ of the boys reported high screen time compared to $76 \%$ of the girls. The main contributors to screen time included watching television and use of social media with about $3 \mathrm{~h} /$ weekend day each. Multilevel modelling revealed that high screen time was associated with travel to school by car, high family income, having carbonated soft-drink $3+$ times per week, lack of vegetable/ fresh fruit on daily basis and not having a play field in the school.

Conclusion: Screen time in excess of the recommended guidelines was highly prevalent among Bangladeshi adolescents. This study identifies some characteristics of adolescents who need special attention to reduce their screen time. Longitudinal research is needed to examine temporal trends in screen-based behaviours and their correlates in Bangladeshi adolescents, which can facilitate developing multi-level strategies targeting individual behaviours, families and settings.

\section{Abstract \#: P 414}

Pain experience and perception in the obese subject: a systematic review

B. Torensma MSc, $\mathrm{PhD}^{1}$, A. Dahan PhD, MD, $\operatorname{prof}^{2}$, I. Thomassen $\mathrm{PhD}, \mathrm{MD}^{3}$, B. In 't Veld, $\mathrm{MD}, \mathrm{PhD}^{1}$

${ }^{1}$ Dutch Obesity Clinic the Hague; ${ }^{2}$ LUMC, Leiden Aneshtsiology;

${ }^{3}$ Kennemergasthuis, Haarlem; Corresponding author's e-mail: info@barttorensma.nl

Background: Pain is an integral part of life and plays an important protective function. Pain perception has been shown to differ between subjects with different gender, race and culture. In addition, it has been suggested that obesity influences pain perception and that obesity can be a risk factor for increased pain threshold. The aim of this systematic review was to examine the perception of pain and pain thresholds in obese subjects compared to non-obese subjects and to explore demographic variables of influence to pain sensitivity and pain perception in obese subjects.

Methods: The electronic databases Cochrane Central Register of Controlled Trials (CENTRAL), PubMed and EMBASE were searched using combinations of terms for obese, pain measurement, visual analog scale, quantitative sensory testing and pain perception. Excluded were studies without comparison as well as cross-sectional studies, case series, and case reports. The search was conducted without restrictions on language or date of publication.

Results: A total of 1818 studies were identified: seven studies fulfilled the inclusion criteria. Two studies showed a lower pain threshold and four studies a higher pain threshold in obese subjects compared to non-obese subjects. Two studies showed no difference in pain threshold before and after substantial body weight loss due to surgery. The body weight loss and gender could not be identified as factors for higher pain threshold in obese subjects.

In view of the heterogeneity of the studies, the variability of the subjects of study and differences in methodological quality, a metaanalysis could not be performed.

Conclusion: There is a tendency headed for a higher pain threshold in obese subjects. Neither substantial body weight loss nor gender, were factors explaining this increased threshold. Therefore, other variables are of influence to the higher pain and sensitivity threshold in obese subjects. Future methodological well-conducted randomized controlled trials in larger groups of subjects, exploring various demographic variables that assumed the influence to pain, are 
necessary to investigate pain threshold and pain perception in obese subjects.

\section{Abstract \#: P 415 \\ Association between glycemic status and current depressive symptoms-results from the German health interview and examination survey for adults}

Beate Weikert ${ }^{1}$, Christin Heidemann ${ }^{1}$, Nina Rieckmann ${ }^{2}$, Rebecca Paprott $^{1}$, Ulrike E. Maske ${ }^{1}$, Christa Scheidt-Nave ${ }^{1}$, Markus A. Busch ${ }^{1}$

${ }^{1}$ Department of Epidemiology and Health Monitoring, Robert Koch Institute, General-Pape-Str. 62-66, 12101 Berlin, Germany; ${ }^{2}$ Berlin School of Public Health, Charité - University Medicine Berlin, Seestr. 73, 13347 Berlin, Germany; Corresponding author's e-mail: buschm@rki.de

Background: We examined the association between glycemic status and current depressive symptoms in a representative sample of the general adult population in Germany.

Methods: Data for this cross-sectional study came from the nationwide, population-based German Health Interview and Examination Survey for Adults (DEGS1) $(\mathrm{n}=6385$ adults aged $18-79$ years with complete data for all variables). Glycemic status was classified into three categories: known diabetes (self-reported diagnosis or current intake of antidiabetic medication), prediabetes or unknown diabetes $(\mathrm{HbAlc} \geq 5.7 \%[\geq 39 \mathrm{mmol} / \mathrm{mol}])$ and normoglycemia (HbAlc $<5.7 \% \quad[<39 \mathrm{mmol} / \mathrm{mol}])$. Current depressive symptoms were defined as a score of $\geq 10$ on the nine item depression subscale of the Patient Health Questionnaire (PHQ-9). Associations between glycemic status and current depressive symptoms were analyzed by multiple logistic and linear regression models adjusting for age, sex, socio-economic status, partnership, obesity (BMI $\geq 30 \mathrm{~kg} / \mathrm{m}^{2}$ ), sports activity, current smoking and alcohol consumption (Audit-C). In sensitivity analyses we further adjusted for comorbidities and cardiovascular risk factors, which are discussed as intermediate factors in the literature.

Results: Prevalence of current depressive symptoms was $7.7 \%$ for those with normoglycemia, $6.2 \%$ for those with prediabetes or unknown diabetes and $11.8 \%$ for those with known diabetes. In models adjusted for socio-demographic and lifestyle factors, known diabetes was associated with higher odds of current depressive symptoms [odds ratio (OR) 1.55: $95 \%$ CI 1.00-2.40] and higher mean PHQ-9 score ( $\beta$ coefficient 0.71: 0.24-1.19) compared with normoglycemia, while no significant associations were found between prediabetes or undiagnosed diabetes and current depressive symptoms (OR 0.91: 0.64-1.28) and PHQ-9 score (-0.01: $-0.28,0.26)$. Further adjustment for cardiovascular risk factors or comorbidities attenuated the association between known diabetes and depression outcomes. The associations were similar among persons with and without antidiabetic medication.

Conclusions: Known diabetes, but not prediabetes or undiagnosed diabetes was associated with current depressive symptoms and severity of depressive symptoms in this representative sample of the general adult population in Germany. This supports the hypothesis that the awareness of diabetes diagnosis and its consequences (diabetes management and comorbidities) may have a stronger impact on the development of depressive symptoms than glycemic metabolism itself.

\section{Abstract \#: P 416}

Serum gamma-glutamyltransferase within normal range, total body fat and regional body fat distribution: the Rotterdam study

Taulant Muka ${ }^{1}$, Fernando Rivadeneira ${ }^{2}$, Harry L.A. Janssen ${ }^{3}$, Sarwa Darwish Murad ${ }^{3}$, Bledar Kraja ${ }^{1}$, Albert Hofman ${ }^{1}$, Jessica Christina Kiefte-de Jong ${ }^{1}$, Oscar H. Franco ${ }^{1}$

${ }^{1}$ Department of Epidemiology, Erasmus MC, Rotterdam, the Netherlands; ${ }^{2}$ Department of Internal Medicine, Erasmus MC, Rotterdam, the Netherlands; ${ }^{3}$ Department of Hepatology, Erasmus MC, Rotterdam, the Netherlands; Corresponding author's e-mail: t.muka@erasmusmc.nl

Background: Little data is available on serum gamma-glutamyltransferase (GGT) within normal range and body fat distribution in healthy individuals. We examined whether GGT within normal range is prospectively associated with total body fat (TF) and regional body fat distribution.

Methods: This study was embedded in the Rotterdam Study, a prospective follow-up study among subjects 55 years and older $(\mathrm{n}=1715)$. GGT was measured between 1997 and 1999 by enzymatic photometry method. TF, android fat (AF), gynoid fat (GF) and android/gynoid ratio (A/G ratio) was assessed using Dual-energy X-ray absorptiometry during follow-up visit (2002-2004). Regression coefficients and $95 \%$ Confidence Intervals were calculated using multivariable linear regression models adjusting for age, gender, education level, income level, smoking status, alcohol intake, physical activity, body mass index Dutch Healthy Diet-index, cardiometabolic diseases, serum total cholesterol and medication use.

Results: Mean value of GGT of the study population was $21.64 \mathrm{U} / \mathrm{L}$ (ranging from 6 to $48 \mathrm{U} / \mathrm{L}$ ). There was no association between GGT and TF. After adjustments for potential confounders, increased GGT was associated with higher AF (top tertile relative to the lowest: $\beta=0.35: 95 \%$ CI: $0.19,0.52$ ), lower GF (top tertile relative to the lowest: $\beta=-0.48$ : $95 \% \mathrm{CI}:-0.69,-0.27$ ) and higher AF/GF ratio (top tertile relative to the lowest: $\beta=0.04$ : $95 \% \mathrm{CI}$ : $0.03,0.06$ ). These associations were independent of $\mathrm{C}$-reactive protein, insulin resistance and non-alcoholic fatty liver disease.

Conclusions: This study suggests that an increase in GGT concentrations within its physiological normal range is a sensitive and early biomarker of unfavorable body fat distribution. As measurement of serum GGT is reliable, easy and inexpensive, its assessment may have clinical utility in identifying individuals at high risk of metabolic disturbances later in life that could benefit from effective preventive interventions.

\section{Abstract \#: P 417}

Association of attention-deficit/hyperactivity disorder and atopic diseases: a case-control study

Jurjen van der Schans ${ }^{1}$, Janine C. Pleiter ${ }^{1}$, Tjalling W. de Vries ${ }^{2}$, Catharina C.M. Schuiling-Veninga ${ }^{1}$, Jens H.J. Bos ${ }^{1,3}$,

Pieter J. Hoekstra ${ }^{4}$, Eelko Hak ${ }^{1}$

${ }^{1}$ Department of Pharmacy, Pharmacoepidemiology \& Pharmacoeconomics, University Groningen, The Netherlands; ${ }^{2}$ Medical Centre Leeuwarden, The Netherlands; ${ }^{3}$ IADB.nl, The Netherlands; ${ }^{4}$ University of Groningen, University Medical Centre 
Groningen, Department of Psychiatry, The Netherlands; Corresponding author's e-mail: j.van.der.schans@rug.nl

Background: Data on the association between attention-deficit/hyperactivity disorder (ADHD) and atopic diseases have been inconclusive. We assessed whether children using ADHD medication are more likely to receive drug treatment for asthma, allergic rhinitis, and/or eczema than children not using ADHD medication.

Methods: We conducted a retrospective nested case-control study among children between 6 and 12 years of age using the IADB.nl prescription database. Cases were defined as children with at least two prescriptions of methylphenidate within 12 months. Cases were matched to four controls on age, sex, and area code. We further assessed the exposure of parental use of medication for ADHD and atopic diseases as a predicting parameter on receiving ADHD medication in childhood. Conditional logistic regression analysis was applied to obtain odds ratios and corresponding $95 \%$ confidence intervals $(\mathrm{CI})$.

Results: We identified 4257 children, drug-treated for ADHD, and 17,028 controls. Drug treatment for asthma, allergic rhinitis, and eczema were more common in cases than controls with odds ratios of 1.4 (95\% CI 1.3-1.6), 1.4 (95\% CI 1.1-1.8) and 1.3 (95\% CI 1.1-1.5), respectively. Association of parental use of medication for atopy on receiving ADHD medication in the offspring (OR: 1.1: $95 \%$ CI 1.0-1.2) was higher in cases compared to controls, but lower than the association between medication for atopy and ADHD in the child (OR: 1.4: $95 \%$ CI 1.2-1.5) $(p=0.006)$.

Conclusion: This study provides further evidence on the hypothesis that ADHD is associated with atopic diseases. Our results suggest that the association may have a genetic component.

\section{Abstract \#: P 418}

Body dissatisfaction and behaviors indicative to the development of eating disorders in adolescents

Franca C. ${ }^{1}$, Colares V. ${ }^{1}$, Silva T. ${ }^{1}$, Valensa P. ${ }^{1}$, Coelho, M.E. ${ }^{1}$

${ }^{1}$ University of Pernambuco, Camaragibe, Brazil; Corresponding author's e-mail: carolina.franca@upe.br

Background: Body image may influence health behaviours of adolescents showing a negative impact on their growth and development. Objective: To investigate the association between body dissatisfaction and behaviours indicative to the development of eating disorders in Brazilian adolescent students.

Methods: A cross-sectional study was conducted on a representative random sample of 1059 adolescents (aged 15-19 years) from public schools in the city Olinda-PE, Brazil. Data were collected through validated version of the Youth Risk Behavior Survey. To evaluate the association between categorical variables we used the Chi square test and to assess the strength of association in categorical variables was obtained crosses the value of the odds ratio (OR). In order to check the influence of the dependent variable or response in the variable "Body dissatisfaction" we adjusted a multivariate logistic regression model with the variables that were significant $<20 \%(p<0.20)$ in the bivariate study. The margin of error used was $5 \%$ and the intervals were obtained with $95.0 \%$ confidence interval (CI) and the software used for the statistical calculations was the SPSS (Statistical Package for Social Sciences), version 21.

Results: Among the respondents $53.4 \%$ were female and $72.7 \%$ were 16-to-19-year-old. The prevalence of body dissatisfaction was $58.5 \%$ and there was no significant association between body dissatisfaction and sex. Crude analysis revealed that the body dissatisfaction showed higher prevalence among: those who reported some initiative to change body weight OR: 2.42 (95\% CI:
1.82-3.22): those who practiced some physical activity to lose weight OR: 1.31 (95\% CI:1.01-1.69): those who had undergone change of eating habits OR: 1.68 (95 \% CI: 1.27-2.21) in the last month: among those who underwent total fast for $24 \mathrm{~h}$ OR: 1.97 (95\% CI: 1.23-3.15) and those who made use of laxatives or vomiting OR: 2.62 (95\% CI: 1.06-6.53). Logistic regression adjustment revealed that only the initiative to change body weight in the last month remained associated with body dissatisfaction $\mathrm{OR}=2.23 \quad(95 \% \quad \mathrm{CI}$ : 1.64-3.03).

Conclusion: The present results suggest that adolescents dissatisfied with their body image have more chance to engage in behavior indicative development of eating disorders.

\section{Abstract \#: P 419}

Prevalence and associated factors of depression in the population of Feira de Santana, Bahia

Thaís Marins S. Cerqueira ${ }^{1}$, Tânia Maria de Araújo ${ }^{1}$, Kiona Bernardes $^{1}$, Fernanda de O. Souza ${ }^{1}$, Morgana S. Mascarenhas ${ }^{1}$, Iracema Lua $^{1}$, Layla B. Cavalcante Wright ${ }^{1}$

${ }^{1}$ Programa de Pós Graduação em Saúde Coletiva - Núcleo e Epidemiologia (Nepi) - Universidade Estadual de feira de Santana, Feira de Santana, Brazil; Corresponding author's e-mail:

laylabc@hotmail.com

Depression is a subject of great importance in public health, given its rising character, which may lead it among the first diseases with high morbidity in the population in 2030, according to the UN.

One in every 20 persons is committed by an episode of moderate or severe depression during life. In every 50 persons diagnosed, one needs to be hospitalized. Over more $15 \%$ of severe cases commit suicide.

The high morbidity of individuals with mental illness has gained visibility for health researchers, since a person with mental illness has twice as much complaints of physical health when compared to those without mental illness.

In Brazil, studies based on population to evaluate the prevalence of depression in non specific populations are rare. Thus, there is still a gap in literature on the incidence of depression in population groups.

This study therefore, has as an objective to describe the factors associated to depression in the urban population of Feira de Santana, Bahia, Brazil.

It is an epidemiological study with transversal bias with a sample of the urban population of Feira de Santana, Bahia. We used a questionnaire (Patient Health Questionnaire-PHQ) conc erning the evaluation of the outcome of depression. To evaluate the degree of depression, they were grouped in five categories according to the score presented: 0-4 with no depression: 5-9 light depression: 10-14 moderate to severe depression: over 20 , severe depression. The data was processed by SPSS and OpenEpi. A bivariated analysis was done in absolute and relative frequency and as a statistical significance criterion: it considered the confidence interval (IC) of $95 \%$. This study followed the 466/2012 resolution.

Analyzing the prevalence of depression in the population of Feira de Santana we found 1259 (35.4\%) persons presented depression varying from light to severe, analyzed by the signs and symptoms present in the PHQ-9, where above score 5 the individual already presents the illness. The profile of the population of Feira de Santana that have depression in 2007, are characterized as persons of female gender $(80.1 \%)$, under the age of 39 years old $(57 \%)$, dark skin $(52.8 \%)$, low education level $(94.1 \%)$ and single $(51.3 \%)$.

These results consonance with literature show us that it is necessary to look at this population in risk, and leads us to think to 
elaborate and to develop actions towards this population. They may be psychosocial interventions, medical care and educational actions, in order to control the symptoms present, prevent future depressive syndromes and more so, the suicidal act, very common in the depressive.

Keywords: Depression, Prevalence, Epidemiology, Mental health.

\section{Abstract \#: P 420 \\ Psychiatric co-morbidity in a population of alcoholic immigrants in Rome \\ Nosotti Lorenzo $^{1}$, Morgillo Teresa ${ }^{1}$, Petrelli Alessio ${ }^{1}$, Mandolini Donatella' $^{1}$, Maggio Daria ${ }^{1}$, Costanzo Gianfranco ${ }^{1}$, Sarchiapone Marco $^{1}$, Fortino Antonio ${ }^{1}$, Mirisola Concetta ${ }^{1}$ \\ ${ }^{1}$ National Institute for Health, Migration and Poverty (NIHMP); Corresponding author's e-mail: nosotti@inmp.it}

Background: Alcoholism constitute a serious social and health problem. Alcohol consumption is the cause of $3.8 \%$ of world's mortality. In Europe alcoholics are assessed to be about 23 million (with a prevalence of $6.2 \%$ ). Accurate epidemiological data about alcohol dependence in immigrant populations are not available in Europe and Italy.

Alcoholics show high rates (70-95\%) of co-morbidity with other psychiatric disorders. Existence of associated psychiatric disorders in alcoholism implies a worse prognosis in the disease evolution. Management of alcohol dependence can be more difficult because these patients have more physical, psychological, familial and social problems than alcoholics without co-morbidity.

Methods: Since March 2013-June 2014, 1225 immigrants attending the Outpatient Clinic of National Institute for Health, Migration and Poverty (NIHMP) were visited.

Alcohol dependence was detected by physicians and psychologists according to a multidisciplinary approach.

Diagnostic tools used for assessing alcoholism were the AUDIT (Alcohol Use Disorders Inventory Test) questionnaire and the SCID I questionnaire.

Association with other psychiatric disorders was assessed through clinical interviews and administration of SCID I and SCID II questionnaires. In particular, the SCID I questionnaire is aimed at assessing mood disorders, anxiety disorders, psychotic disorders, adjustment disorders. The SCID II questionnaire, instead, assesses personality disorders.

Results: Out of the total 1225 immigrants enrolled in the study, 81 alcoholics were identified, with a prevalence of $6.6 \%$. Patients' origin was distributed as follows: $58 \%$ of the sample was from Europe, $20 \%$ from Africa, $12 \%$ from America, $10 \%$ from Asia.

Gender distribution showed a clear prevalence of men $(87.7 \%$ men): mean age was 44.8 years. The percentage of patients with a dual diagnosis, namely alcoholism and a psychiatric disorder, was $92 \%$. Psychiatric disorders most frequently associated with alcoholism were depressive disorders $(54.1 \%)$, personality disorders $(11 \%)$, anxiety disorders (10.8\%), adjustment disorders $(9.5 \%)$, psychotic disorders $(9.5 \%)$.

Conclusions: The low percentage $(11 \%)$ of personality disorders and the high percentage $(54.1 \%)$ of depressive disorders are remarkable in the considered sample (in literature the prevalence of personality disorders in alcoholics is about $30 \%$ and the prevalence of depressive disorders ranges from 22 to $33 \%$ ).

It is important to outline how doubtful is the temporal and causal relationship between alcohol and psychiatric disorders. In fact, it is often difficult to decide which is the primary diagnosis, namely if alcoholism is the cause or a consequence of the psychiatric disorder.

\section{Abstract \#: P 421}

Mood and substance use disorder among emerging adults with chronic health conditions

Mark A. Ferro ${ }^{1}$

${ }^{1}$ Department of Psychiatry and Behavioural Neurosciences, McMaster University, Hamilton, Canada; Corresponding author's e-mail: ferroma@mcmaster.ca

Background: It is well-established that chronic physical health conditions and mental disorder are highly comorbid and that the diagnosis of a chronic health condition early in life can have a detrimental impact on mental health later in adulthood. However, data on the prevalence of mental and substance use disorder among emerging adults with and without chronic health conditions is lacking. Using population data, we examined the prevalence and trends of mental and substance use disorder in a representative sample of emerging adults with and without chronic health conditions and determined the extent to which individuals with chronic health conditions are at risk for such disorders compared to healthy controls.

Methods: Data were obtained from the Canadian Community Health Survey-Mental Health, a large epidemiological study of Canadians. Respondents for this analysis were $15-25$ years of age $(n=3101)$ and self-reported whether they had a chronic health condition. The World Health Organization Composite International Diagnostic Interview 3.0 was used to assess the presence of lifetime and 12-month mental and substance use disorder. The disorders included: major depressive episode, suicidal behaviour, bipolar disorder, generalized anxiety disorder, and alcohol, cannabis, and other drug abuse/ dependence. Sex-specific prevalence across age categories (15-17, $18-21,22-25$ years) were estimated for participants with and without a chronic health condition. The Cochran-Armitage trend test was used to examine differences in the distribution of disorder across age groups. Logistic regression was used to quantify the association between having a chronic health condition and disorder, controlling for sociodemographic factors.

Results: The prevalence of any lifetime mental or substance use disorder for males and females with chronic health conditions was 37.7 and $38.8 \%$, respectively. For controls it was 25.7 and $17.6 \%$ $(p<0.001)$. Twelve-month prevalence was 25.6 and $17.6 \%$ for males and females with chronic health conditions, respectively. For controls it was 14.9 and $9.3 \%(p<0.001)$. Trends of increasing prevalence were found for most mental and substance use disorders. Controlling for sociodemographic factors, having a chronic health condition was associated with mental or substance used disorder in males, odd ratio: $1.76(1.07,2.91)$ and females, odds ratio: $3.20(2.23$, 4.60).

Conclusion: Associations were found between having a chronic health condition and mental and substance use disorder among emerging adults. Reducing or preventing physical and mental multimorbidity early in life should be a public health priority that could lead to reductions in health service utilization and costs across the life-course.

\section{Abstract \#: P 422}

Depression and health related quality of life in patients on antiretroviral therapy in a rural district in North West Cameroon

Serra Lem Asangbeh ${ }^{1}$, Joëlle Laure Sobngwi ${ }^{2}$, Gabriel Loni Ekali ${ }^{3}$, Christian Eyoum ${ }^{4}$, Philippe Msellati ${ }^{5}$ 
${ }^{1}$ Recherche Sante et Developpement, Yaounde, Cameroon; ${ }^{2}$ Catholic University of Central Africa, Yaounde, Cameroon; ${ }^{3}$ National AIDS Control Committee, Yaounde, Cameroon; ${ }^{4}$ Faculty of Medicine and Public health, University of Douala, Cameroon; ${ }^{5}$ UMI 233 IRD, Institut de Recherche et Developpement, Montpellier, France; Corresponding author's e-mail: sobngwijoelle@gmail.com

Background: Depression in people living with HIV/AIDS (PLWHA) increases risky HIV transmission behaviour, disease progression to AIDS, negatively affects drug adherence and is a risk for the development of drug-resistant strains. Studies suggest that depression may also be associated with poor quality of life (QOL). This study sought to assess the relationship between depression and health-related QOL (HRQOL) and to identify psychosocial, clinical and socio-economic determinants of depression in a rural setting in Cameroon.

Methods: A cross sectional study was carried out from September 2013 to November 2013 in the Mbengwi district hospital of the North West region. Included were all patients on ART at least 21 years old who gave informed consent. Using structured pre-tested questionnaires, depression (PHQ-9), HRQOL (WHOQOL-BREF), stigma, social support, clinical and demographic characteristics of patients on ART were measured. Proportions and means were used to describe participants' characteristics while the association between QOL and depression was assessed with ANOVA. To identify predictors of depression, a logistic regression stepwise model was used.

Results: Of the 202 recruited patients, $58(28.7 \%)$ had a positive depression screen. Mean QOL scores for all domains but the environmental domain were significantly and inversely correlated $(p<.05)$ with depression implying that patients depressive symptoms had poorer HRQOL. The independent predictors of were found to be monthly income $<20,000 \mathrm{fcfa}$ (around 40 US \$) (aor = "2.48:" $95 \%$ $\mathrm{ci}=$ "1.06-5.82)" cd4 count $<200 \mathrm{cls} />\mu \mathrm{l} \quad(\mathrm{AOR}=4.26: 95 \%$ $\mathrm{CI}=1.31-11.96)$ and presence of AIDS symptoms (AOR $=2.58$ : $95 \% \mathrm{CI}=1.26-5.29)$. There was no significant correlation between stigma, social support, duration on ART, marital status, gender and depression.

Conclusion: Early diagnosis and treatment of depressed patients should be incorporated into intervention programs to improve patient outcomes, QOL and success of health promotion activities. More research is needed to investigate the impact of antidepressant therapy in PLWHA on the evolution of treatment and QOL.

Keywords: depression, quality of life, HIV/AIDS, ART, CD4 count, income.

\section{Abstract \#: P 423}

\section{Serum n-3 polyunsaturated fatty acids are inversely associated with longitudinal changes in depressive symptoms during pregnancy}

Thatiana J. P. Pinto ${ }^{1}$, Dayana R. Farias ${ }^{1}$, Ana Amélia F. Vilela ${ }^{1}$, Jaqueline Lepsch ${ }^{1}$, Juliana S. Vaz ${ }^{2}$, Geraldo M. Cunha ${ }^{3}$, Gilberto $\mathrm{Kac}^{1}$

${ }^{1}$ Nutritional Epidemiology Observatory, Department of Social and Applied Nutrition, Institute of Nutrition Josué de Castro, Federal University of Rio de Janeiro, RJ, Brazil; ${ }^{2}$ Faculty of Nutrition, Federal University of Pelotas, Rio Grande do Sul, Brazil; ${ }^{3}$ Department of Epidemiology, National School of Public Health, Oswaldo Cruz Foundation, RJ, Brazil; Corresponding author's e-mail: thatiana.pinto@gmail.com

Background: There is no consensus regarding the association between serum fatty acids (FA) concentration and depression during pregnancy. Objective: To evaluate the association between $n-3$ and n-6 polyunsaturated fatty acids (PUFAs) concentration and the occurrence of depressive symptoms throughout pregnancy.

Methods: A prospective cohort of 208 Brazilian women was followed at 5-13th, 20-26th and 30-36th weeks of gestation. The presence of depressive symptoms during pregnancy was evaluated using the Edinburgh Postnatal Depression Scale (EPDS) $\geq 11$. The serum n-3 and n-6 FA were assayed using fast gas-liquid chromatography and were expressed as absolute concentrations $(\mu \mathrm{g} / \mathrm{ml})$. The analyses were performed using logistic model with random intercept, adjusted for confounders.

Results: The prevalence of depressive symptoms was high in all gestational trimesters $\quad(1 \mathrm{st}=33.6 \%$ : $2 \mathrm{nd}=20.4 \% \quad$ and 3rd $=17.1 \%)$ and $44.2 \%$ of the women presented depressive symptoms at some point during pregnancy. The women presented a $5 \%$ decrease in the odds of having depressive symptoms for each one-unit increase in the gestational age. Women with depressive symptoms at baseline presented higher mean of pre-pregnancy BMI $\left[25.4(\mathrm{SD}=5.4)\right.$ vs. $\left.24.2(\mathrm{SD}=4.5) \mathrm{kg} / \mathrm{m}^{2}, \quad p=0.040\right]$ and monthly per-capita family income [334.6 $(\mathrm{SD}=190.4)$ vs. 279.2 ( $\mathrm{SD}=194.1)$ dollars, $p=0.027$ ], and a higher frequency of previous history of depression $(65.2$ vs. $35.1 \%, p<0.001)$, single marital status ( 31.4 vs. $15.2 \%, p=0.006)$, inter-partum interval $<48$ months ( 25.8 vs. $11.2 \%, p=0.008)$ and unplanned pregnancy (38.6 vs. $13.8 \%, p<0.001$ ), compared to those without the symptoms. Higher concentrations of eicosapentaenoic acid (EPA: OR $=0.927: 95 \%$ CI: 0.859-0.999), docosahexaenoic acid (DHA: OR $=0.964: 95 \%$ CI: 0.934-0.995), n-3 docosapentaenoic acid (DPA: OR $=0.876$ : $95 \%$ CI: $0.774-0.991)$ and total $n-3(\mathrm{OR}=0.976: 95 \% \mathrm{CI}$ : 0.957-0.995) were associated with a lower odds of depressive symptoms for a particular woman during pregnancy, while higher total n-6/total n-3 ratio represented a greater odds of depressive symptoms $(\mathrm{OR}=1.399: 95 \% \mathrm{CI}: 1.084-1.805)$. We found no significant associations between $\mathrm{n}-6$ fatty acids and depressive symptoms.

Conclusion: Higher serum concentrations of DHA, EPA and n-3 DPA and a lower n-6/n-3 ratio were associated with lower odds of depressive symptoms throughout pregnancy.

\section{Abstract \#: P 424}

Vitamin K status is not associated with cognitive decline in middle aged adults

E.G.H.M. van den $\mathrm{Heuvel}^{1,2}$, N.M. van $\mathrm{Schoor}^{1}$, C. Vermeer ${ }^{3}$, R.M.L. Zwijsen $^{2}$, M. den Heijer ${ }^{1,4}$, H.C. Comijs ${ }^{5}$

${ }^{1}$ Department of Epidemiology and Biostatistics, EMGO Institute for Health and Care Research, VU University Medical Center,

Amsterdam, The Netherlands; ${ }^{2}$ Department of Nutritional Sciences, FrieslandCampina, Amersfoort, The Netherlands; ${ }^{3}$ VitaK BV,

Maastricht University, BioPartner Center, Maastricht, The

Netherlands; ${ }^{4}$ Department of Internal Medicine and Endocrinology, VU University Medical Center, Amsterdam, The Netherlands; ${ }^{5} \mathrm{GGZ}$ in Geest/Department of Psychiatry, EMGO Institute for Health and Care Research, VU University Medical Center, Amsterdam, The Netherlands; Corresponding author's e-mail:

ellen.vandenheuvel@frieslandcampina.com

Background: Cognitive functioning declines during life, however, the rate of cognitive decline differs considerably among persons. Previous studies have focused on modifiable factors that influence the rate of decline, such as nutrition, yet little is known about a possible effect of suboptimal vitamin $\mathrm{K}$ levels on cognitive decline. The aim of this study was to examine the association between dephospho- 
uncarboxylated matrix Gla protein (dp-ucMGP), an indicator of vitamin $\mathrm{K}$ status, and cognitive decline, and the modifying role of 25(OH)D.

Methods: Data were collected during 6 years follow-up as part of the Longitudinal Aging Study Amsterdam ( $\mathrm{N}=599$, age 55-65 years) and analyzed using generalized estimating equations (GEE). Two cognitive measures were included in our analyses, information processing speed and general cognitive functioning. Information processing speed is hypothesized to be especially affected by vitamin $\mathrm{K}$-induced changes in signaling events via its impact on brain sphingolipid metabolism within the brain. General cognitive functioning was measured with a composite Z-score by combining three domains of cognition: information processing speed, episodic memory, and fluid intelligence.

Results: No significant associations was found between dp-ucMGP and decline in general cognitive functioning. Vitamin D modified the association between dp-ucMGP and speed of information processing $(p<0.05)$. In the group with a $25(\mathrm{OH}) \mathrm{D}$ concentration $>50 \mathrm{nmol} / \mathrm{l}$, the highest tertile of dp-ucMGP ( $>406 \mathrm{pmol} / \mathrm{l})$, which corresponds to lower vitamin $\mathrm{K}$ levels, was associated with 1.5 higher score on information processing speed ( $p=0.023$ ) as compared to the lowest tertile of dp-ucMGP.

Conclusion: In contrast to our hypothesis, a suboptimal vitamin $\mathrm{K}$ was not associated with cognitive decline in middle-aged adults.

\section{Abstract \#: P 425 \\ Exploring predictors of change in behavioral problems over a 1-year period in preterm born preschoolers}

\section{R. Schappin $^{1}{ }^{1}$ L. Wijnroks ${ }^{2}$, M.M.A.T. Uniken Venema ${ }^{1}$,} M.J. Jongmans ${ }^{2}$

${ }^{1}$ Department of Medical Psychology and Social Work, Wilhelmina Children's Hospital, UMC Utrecht, Utrecht, The Netherlands; ${ }^{2}$ Department of Child, Family and Education Studies, Faculty of Social and Behavioral Sciences, Utrecht University, Utrecht, The Netherlands; Corresponding author's e-mail: r.schappin@umcutrecht.nl

Background: Although predictors of the prevalence of behavioral problems in preterm-born children have been frequently studied, predictors of behavioral change in these children remain unknown. Therefore, in this study we explore predictors of short-term changes in problem behavior in preterm-born preschoolers, an age period characterized by rapid behavioral change.

Methods: Two- to 5-year-old children born with a gestational age $<32$ weeks and/or birth weight $<1500 \mathrm{~g}$ were eligible, because of their high risk for behavioral problems. Following screening, 59 children with a t-score $\geq 60$ on the Child Behavior Checklist (CBCL) were included in the study. Linear mixed modeling was used to investigate predictors of change in behavioral problems over a 1-year period.

Results: A higher level of parenting stress and parent perceived child vulnerability, and a lower educational level of the mother at baseline significantly predicted an increase in externalizing behavior problems. No significant predictors of changes in internalizing problems were found. Mean levels of internalizing behavior remained stable during the study period, whilst externalizing behavior decreased.

Conclusion: Parenting stress and parent perceived child vulnerability were the only modifiable predictors of an increase in externalizing behavior. We propose that stress and worries may directly influence parents' reports on behavioral measures, because due to stress they may be bothered by behavior that they would otherwise perceive as normal. Therefore, future interventions for parents of preterm-born children should primarily address parenting stress and parental perceptions of the child.

\section{Abstract \#: P 426}

Renal dysfunction and risk for cognitive impairment: a systematic review and meta-analysis

Kay Deckers $^{1}$, Ileana Camerino ${ }^{1}$, Martin van Boxtel ${ }^{1}$, Frans Verhey ${ }^{1}$, Kate Irving $^{2}$, Carol Brayne ${ }^{3}$, Miia Kivipelto ${ }^{4}$, John M. Starr ${ }^{5}$, Kristine Yaffe $^{6}$, Sebastian Köhler ${ }^{1}$

${ }^{1}$ Department of Psychiatry \& Neuropsychology I School for Mental Health and Neuroscience, Maastricht University; ${ }^{2}$ School of Nursing and Human Sciences, Dublin City University, Dublin, Ireland; ${ }^{3}$ Department of Public Health and Primary Care, University of Cambridge, Cambridge, UK; ${ }^{4}$ Alzheimer Disease Research Center, Karolinska Institute, Stockholm, Sweden; ${ }^{5}$ Centre for Cognitive Ageing and Cognitive Epidemiology, University of Edinburgh, UK; ${ }^{6}$ Departments of Psychiatry, Neurology, Epidemiology and

Biostatistics, School of Medicine, University of California, San Francisco, USA; Corresponding author's e-mail:

kay.deckers@maastrichtuniversity.nl

Background: Renal dysfunction has been associated with cognitive impairment and may be a risk factor for dementia. Therefore, we aimed to summarize the evidence on the association between several markers of renal dysfunction and risk for cognitive impairment or dementia in a systematic review and meta-analysis.

Methods: We searched Medline, Embase and Cochrane Database of Systematic Reviews for all publications until 24th July 2013.The search strategy included: (1) predictor-related terms (e.g. renal, kidney, creatinine, albuminuria): (2) outcome-related terms (e.g. dementia, cognitive decline): and (3) limitations (e.g. only studies in humans). All publications included if they fulfilled the inclusion criteria (prospective design, $\geq 100$ participants, aged $\geq 45$ years, $\geq 1$ year follow-up). Assessment of study quality and extraction of data was conducted by two independent assessors. We conducted meta-analyses using random-effects models.

Results: The literature search yielded 5294 abstracts, of which 39 fulfilled the inclusion criteria after full-text scrutiny. Markers of renal dysfunction identified were: estimated glomerular filtration rate (eGFR), proteinuria or albuminuria, Cystatin C (CysC), and elevated serum creatinine level. Meta-analyses of 14 population-based studies representing 54,085 individuals showed increased risk of cognitive impairment or dementia in those with any marker of renal dysfunction $(\mathrm{OR}=1.65,95 \%$ CI 1.33-2.03, $p<0.001)$. Results were similar for the outcomes of cognitive impairment $(\mathrm{OR}=1.68,95 \% \mathrm{CI}$ $1.35-2.09, p<0.001)$ and dementia $(\mathrm{OR}=1.64,95 \%$ CI $0.92-2.92$, $p=0.094)$. The risk was increased in individuals with eGFR level $<60 \mathrm{ml} / \mathrm{min} / 1.73 \mathrm{~m}^{2}(\mathrm{OR}=3.67,95 \%$ CI 1.73-7.79, $p=0.001)$ and albuminuria $(\mathrm{OR}=1.58,95 \% \mathrm{CI} 1.23-2.02, p<0.001)$, and was highest for CysC level $>1.25 \mathrm{mg} / \mathrm{L}(\mathrm{OR}=1.82$, $95 \% \mathrm{CI}$ 1.39-2.39, $p<0.001)$.

Conclusion: Renal dysfunction is associated with an increased risk of cognitive impairment and dementia, but there is substantial heterogeneity among studies. Future research into underlying pathways is needed to assess whether the association is causal or due to shared risk factors. 


\section{Abstract \#: P 427}

Association between hand grip strength and depressive symptoms in Europeans aged 50 years and older

$\underline{\text { Markus A. Busch }^{1}}$, Bruno Neuner ${ }^{2}$, Amanda K. Buttery ${ }^{3}$

${ }^{1}$ Dept. of Epidemiology and Health Monitoring, Robert Koch Institute, Berlin, Germany; ${ }^{2}$ Dept. of Anesthesiology and Intensive Care Medicine, Charité - Universitaetsmedizin Berlin, Campus Charité Mitte and Campus Virchow-Klinikum, Berlin, Germany; ${ }^{3}$ Dept. of Epidemiology and Health Monitoring, Robert Koch Institute, Berlin, Germany; Corresponding author's e-mail: buschm@rki.de

Background: Reduced hand grip strength is associated with various adverse health outcomes in older adults, but little is known about the association of grip strength with mental health. We investigated the reciprocal relationships between hand grip strength and depressive symptoms in a longitudinal analysis.

Methods: We analysed data from 8248 women and 7252 men who participated in wave 1 (2004-2005) and wave 2 (2006-2007) of the Survey of Health, Ageing and Retirement in Europe (SHARE), a longitudinal study of non-institutionalized people aged $\geq 50$ years in 11 countries. We used mixed-effects regression models to examine bidirectional longitudinal relationships between grip strength (measured in $\mathrm{kg}$ with a handheld dynamometer) and current depressive symptoms (defined as a score of 4 or more on the EURO-D scale) at wave 1 and 2 .

Results: At baseline (wave 1) $53.2 \%$ were female: mean age was 63.7 years: mean maximum grip strength was $44.3 \mathrm{~kg}$ in men and $27.1 \mathrm{~kg}$ in women and depressive symptoms were $14.3 \%$ in men and $29.5 \%$ in women. Higher grip strength at baseline was associated with lower odds of depressive symptoms at follow-up (wave 2) in women (HR for highest vs. lowest quartile, 0.60: $95 \%$ CI, 0.51-0.72) and men (HR 0.51: $95 \%$ CI, 0.51-0.84), adjusting for baseline depressive symptoms, socio-demographics, body mass, height and health-related behaviours. The association remained largely unchanged after additional adjustment for chronic diseases, cognitive function, change in grip strength or when participants with baseline depressive symptoms were excluded. Depressive symptoms at baseline were associated with lower grip strength at follow-up in men ( $\beta$, -0.7 : $95 \% \mathrm{CI},-1.2$ to -0.2$)$ but not in women $(\beta,-0.3$ : $95 \% \mathrm{CI}$, -0.7 to 0.1 ), adjusting for baseline grip strength, sociodemographics, body mass and height, and health-related behaviours. After additional adjustment for chronic diseases and cognitive function effect sizes were attenuated for both men and women and were no longer significant.

Conclusions: Hand grip strength is inversely associated with depressive symptoms in women and men 50 years or older in Europe. The effect of grip strength on depressive symptoms is stronger than the reciprocal effect.

\section{Abstract \#: P 428}

\section{Depressive symptoms are associated with gait domains in community-dwelling population}

Ana Maksimovic MD MSc, V.J.A. Verlinden MSc, J.N. van der Geest, A. Hofman MD PhD, H. Tiemeier MD PhD, M.A. Ikram MD $\mathrm{PhD}$

Corresponding author's e-mail: a.maksimovic@erasmusmc.nl

Background: Depressive symptoms are highly prevalent in elderly persons and lead to severe co-morbidity, including impaired physical functioning. However, it remains unclear to what extent this impairment is objectively quantifiable or is merely subjective and related to worse quality of life, physical functioning and gait impairment. Impairment in gait can lead to falls and mortality. We investigated the association between depressive symptoms with objective gait assessment and assessed the influence of subjectively assessed physical functioning on this association and specific gait domains in elderly.

Methods: In 3050 participants from the Rotterdam Study (aged 45 and older, $54 \%$ women) we assessed depressive symptoms using the CES-D questionnaire. Gait was assessed using an electronic walkway and obtained variables were summarized into seven gait domains (Rhythm, Variability, Phases, Pace, Tandem, Turning and Base of Support). Global gait was obtained by summarizing all seven gait domains and dividing the result by 7 . Linear regression analyses were performed to determine the association between depressive symptoms and gait. We investigated the association between depressive symptoms and gait in three different models: in the first model we adjusted for age, sex, height and weight, in the second model we additionally adjusted for various cardiovascular risk factors and education and in the third model models we investigated the same association adjusted for subjective assessment of physical functioning measured by the Dutch version of Stanford Health Assessment Questionnaire.

Results: A higher CES-D score was associated with worse Global gait $[\beta$ per SD with $95 \%$ CI: $-0.07(-0.11:-0.04)]$. This association was driven by the following domains: variability $[\beta$ per SD with $95 \%$ CI: $-0.06(-0.10:-0.03)$ ], pace $[\beta$ per SD with $95 \%$ CI: $-0.05(-$ 0.08:-0.02)] and Turning [ $\beta$ per SD with $95 \%$ CI: $-0.04(-0.08$ : $-0.01)$ ] after adjustment for cardiovascular risk factors and education. The association remained in Global gait, Variability and Pace after adjustment for subjective assessment of physical functioning.

Conclusion: In our population -based study we found that depressive symptoms in persons of the age 45 and older are associated with impairment on objectively measured poor gait, especially in the domains of Variability and Pace. More importantly, these association was independent from subjectively assessed physical functioning.

\section{Abstract \#: P 429}

Adherence to physiotherapy among patients with low back pain $\underline{\text { Md. Monoarul Haque }}{ }^{1}$, Monirul Haqe ${ }^{1}$, Md. Shafiullah Pradhania ${ }^{1}$

${ }^{1}$ DPRC Specialized Hospital \& Research Center; Corresponding author's e-mail: monoarmunna@yahoo.com

Background: Low back pain is well documented to be an extremely common health problem and a leading cause of activity limitation and absenteeism in work throughout much of the world however, its burden is often considered trivial.

Methods: An observational analytical study with cross-sectional design was conducted to assess adherence to physiotherapy among patient with low back pain in six teaching hospitals advanced centers with specialized physiotherapy setup in Dhaka, Bangladesh. A total of 370 samples were taken using non probability convenient sampling technique. A semi-structured questionnaire and visual analogue pain scale was used as research tool.

Results: Mean \pm SD age of respondents was $41.05 \pm 11.96$ years. Low back pain in female and male was 57 and $43 \%$. The study also revealed that proportion of adherent to physiotherapy was 81.9 and $18.1 \%$. Busy with other work, financial problem and long distance/transportation problem were the reasons of non-adherent. The outcome of physiotherapy treatment was found that $83.5 \%$ had improved and $16.5 \%$ had not improved. Strong statistical significant association was found among occupation and income with adherent to 
physiotherapy $(p<0.05)$. Statistically significant relationship was found among treatment cost affordability, duration of suffering, initial pain status and adherence to physiotherapy among patient with low back pain with adherence to physiotherapy $(p<0.05)$. Multivariate logistic regression model showed some predictors as associated with adherent to physiotherapy, among them affordability of treatment, pain status during admission, pain status during discharge, progress of treatment, behavior of physiotherapist, income, occupation and education were strongly associated with adherent to physiotherapy among the target population.

Conclusion: Adherent to physiotherapy was necessary to relive the pain among the patient with low back pain.

\section{Abstract \#: P 431}

\section{Etiological determinants of pregnancy-related pelvic girdle pain}

\section{A.H.H. Merry $^{1}$, C.H.G. Bastiaenen ${ }^{1}$}

${ }^{1}$ Department of Epidemiology, CAPHRI School for Public Health and Primary Care, Maastricht University, Maastricht, the Netherlands; Corresponding author's e-mail: audrey.merry@maastrichtuniversity.nl

Background: Pelvic girdle pain has a high prevalence during pregnancy. In some of these women, the pain symptoms even persist after pregnancy. The aim of this study was to investigate which etiological factors are associated with an increased risk of pregnancy-related pelvic girdle pain (PPGP) during pregnancy in a prospective cohort study.

Methods: The cohort consisted of 7526 pregnant women, living in the south-eastern part of the Netherlands, who were recruited at 10-14 weeks of pregnancy by both midwives and gynaecologists between November 2000 and November 2002. Participants completed self-administered questionnaires at baseline, at 30 weeks of pregnancy, and at 2 weeks, 6 months and 1 year after delivery. Through these questionnaires, information was gathered about physical complaints, limitations in activities, restriction in participation, work situation, demographics, lifestyle factors, pregnancy-related factors and psychosocial factors. Incidence rate ratios and $95 \%$ confidence intervals for the etiological determinants were estimated using Cox proportional hazards models. By using survival analyses, it was possible to take into account the time of onset of PPGP during pregnancy.

Results: During pregnancy, $84.3 \%$ of the pregnant women reported that they experienced pain in the lower back, the buttocks, the symphyses, the groins or radiation into the legs during pregnancy. In multivariable analyses, a history of low back pain (LBP) or PPGP, younger maternal age, higher body mass index, smoking before and during pregnancy, and multiparity were associated with the onset of pregnancy-related pelvic girdle pain. In addition, performing sports during pregnancy was associated with a lower risk of developing PPGP. No associations were found for level of education, twin pregnancy, and number of hours of physically strenuous work, after adjustment for other determinants.

Conclusion: This study shows that a history of LBP/PPGP, younger maternal age, higher body mass index, smoking before and during pregnancy, multiparity, and performing sports during pregnancy are associated with the occurrence of PPGP during pregnancy. In addition, it should be investigated whether these factors are also associated with the occurrence of persistent PPGP after pregnancy in a large prospective cohort study. Using this knowledge, it may be possible to predict which women are at highest risk of developing PPGP during and after pregnancy.

\section{Abstract \#: P 432}

Sagittal standing posture in school-aged girls and boys: from the same classification to different clinical meanings $\underline{\text { Fábio A. Araújo }}{ }^{1}$, A. Rita Gaio $^{2}$, Nuno Alegrete ${ }^{3}$, Raquel Lucas ${ }^{1}$ ${ }^{1}$ EPIUnit - Institute of Public Health, University of Porto, Porto, Portugal; ${ }^{2}$ Department of Mathematics, Science Faculty, University of Porto, Porto, Portugal; ${ }^{3}$ Department of Surgery, University of Porto Medical School, Porto, Portugal; Corresponding author's e-mail: fabio.araujo@ispup.up.pt

Background: Non-neutral sagittal posture is an important cause of musculoskeletal pain in adolescence and adulthood. However, little is known about the timing in life when postural patterns differentiate into neutral and non-neutral variants. Similarly, it is unknown if nonneutral patterns may cause symptoms since early ages. Our hypothesis is that empirically-obtained patterns in children are consistent with those observed in adults in terms of morphology and clinical manifestation.

Methods: Children were consecutively recruited during the 7-yearold evaluation of the population-based birth cohort Generation XXI (2012/2013). Sagittal posture was assessed through lateral photographs during habitual standing position and by retro-reflective markers paced on predefined body landmarks. Twelve spino-pelvic parameters were computed. Postural patterns were defined from trunk, lumbar and sway angles by clusters analysis, separately for girls and boys. The final cluster solution was based on the following sequential steps: (1) hierarchical analysis by Ward's method: (2) K-means algorithm using the centers obtained in (1): (3) interpretation of the obtained clusters: (4) criterion validity through postural parameters not used in the cluster algorithm, age, and anthropometric measures. The four sequential steps were performed in two validation samples (50\% of children) and further repeated to the whole sample. Back pain was evaluated using the Lubeck Pain-Screening Questionnaire applied to parents.

Results: 1147 girls and 1266 boys were evaluated (respectively, 79.7 and $81.2 \%$ of eligible children). Cluster analysis identified 4 patterns within each subpopulation, with a "neutral" sagittal posture and 3 other non-neutral types: "sway", "flat" and "hyperlordotic". However, for the same pattern, girls and boys differed regarding the three considered angles (highest difference of $6.18^{\circ}$ in lumbar angle in the "sway" pattern, $p<0.001)$. Girls showed higher odds for a "flat" $(\mathrm{OR}=1.38,95 \% \mathrm{CI}: 1.10-1.74)$ or a "hyperlordotic" $(\mathrm{OR}=1.29$, $95 \%$ CI: 1.01-1.64) than for a "neutral" pattern. Postural patterns differed according to the three considered angles $(p<0.001)$, all other cervico-thoraco-lumbo-pelvic alignment parameters $(p \leq 0.006$ in girls and $p \leq 0.009$ in boys), age $(p<0.001)$ and body mass index $(p<0.001)$. Among boys, the pattern characterized by accentuated curves ("hyperlordotic") was associated with back pain (OR $=3.00$, $95 \%$ CI: 1.13-9.41), independently of age and anthropometrics.

Conclusion: Clinically meaningful sagittal postural patterns were identified in school-aged children, and they resemble those previously described among adolescents and adults, providing the basis for a lifecourse hypothesis of posture differentiation. Classification was similar between genders, but the underlying sagittal organization and clinical meaning diverge, suggesting gender-specific mechanical frameworks for paediatric back pain development.

\section{Abstract \#: P 433}

IS vigorous-intensity physical activity required for improving bone mass in adolescence? Findings from a Brazilian birth cohort 
Renata Moraes Bielemann ${ }^{1}$, Virgílio Viana Ramires ${ }^{1}$, Bernardo Lessa Horta $^{1}$, Ana Maria Baptista Menezes ${ }^{1}$, Helen Gonçalves ${ }^{1}$, Maria Cecília Formoso Assunção ${ }^{1}$, Pedro Curi Hallal ${ }^{1}$

${ }^{1}$ Post-Graduate Program in Epidemiology - Federal University of Pelotas; Corresponding author's e-mail: renatabielemann@hotmail.com

Background: Beyond mechanical stress related mainly to impact on bones promoted by physical activity, physiological aspects related to the intensity of physical activity could be important in the bone mass accrual. The aim of this study was to evaluate longitudinal and independent associations of moderate- and vigorous-intensity physical activity with bone mineral density (BMD) in adolescents.

Methods: Moderate- and vigorous-intensity commuting and leisuretime physical activity were assessed at 11,15 and 18 years of age by self-report. Time spent in moderate- and vigorous-intensity physical activity was determined using metabolic equivalents. Lumbar spine and femoral neck BMD were measured by DXA at 18 years. A peak strain score (a proxy of impact) was also generated at 11 and 15 years of age based on ground reaction forces of different physical activities. Statistical analyses evaluated the independent effects of moderateand vigorous-intensity physical activity on BMD, after adjusting for possible confounders and the peak strain score.

Results: DXA scans were conducted for 3947 and 3960 individuals for lumbar spine and femoral neck BMD, respectively. Time spent in moderate-intensity physical activity was not associated with BMD in neither sex, after adjustment for the peak strain score. Vigorous-intensity physical activity in all periods was linearly and positively related to both lumbar spine and femoral neck BMD in boys. Girls who achieved $75+\mathrm{min} /$ week of vigorous-intensity physical activity at least in two follow up visits showed higher BMD at 18 years of age. Boys who reached $75+\mathrm{min} /$ week of vigorous-intensity physical activity in all follow-ups had on average $0.117 \mathrm{~g} / \mathrm{cm}^{2}$ higher femoral neck BMD than boys who did not achieve this threshold-reducing in around $120 \%$ their risk for hip fractures in the future.

Conclusions: Vigorous-intensity physical activity is required to increase BMD during adolescence. Girls had less benefit from vigorous physical activity than boys. Physical activity promotion in adolescence is essential for the development of bone health.

\section{Abstract \#: P 434}

\section{Socioeconomic position as determinant of widespread pain} syndrome in young adults: a study from the epiteen cohort

Dimitrovova, Klara $^{1}$, Nkata, Hamida ${ }^{1}$, Lucas, Raquel ${ }^{2}$, Samorinha, Catarina $^{2}$, Lourenço, Sara ${ }^{2}$, Barros, Henrique ${ }^{3}$

${ }^{1}$ University of Porto and Nova University of Lisbon; ${ }^{2}$ EPIUnit Institute of Public Health of the University of Porto; ${ }^{3}$ Department of Clinical Epidemiology, Predictive Medicine and Public Health University of Porto Medical School and EPIUnit Institute of Public Health of the University of Porto; Corresponding author's e-mail: k.dimitrovova@gmail.com

Background: Regional and widespread pain are more frequent in more deprived socioeconomic groups. Despite that, little is known about the timing in the life course when such disparities begin.

Our aim was to estimate the associations between socioeconomic position (SEP) indicators and the severity of widespread pain syndrome features in a population-based sample of young adults.

Methods: We studied 1764 21-year-old participants of the EPITeen study in Porto, Portugal. As outcome variables we used the Widespread Pain Index (WPI), the Symptom Severity Score (SSS) and the Polysymptomatic Distress scale (PDS) - the latter as a summary measure of the former two. As explanatory variables we used complete years of education, weighted household income and subjective social class (self-assessment of individuals in one of three categories based on their perceived position in the socioeconomic hierarchy).

Logistic regressions (sex adjusted OR and $95 \%$ confidence intervals $(95 \% \mathrm{CI})$ ) were computed to estimate the strength of the associations of education, income and subjective social class with WPI, SSS and PDS. WPI, SSS and PDS were dichotomized with the median being the cutoff.

Results: Overall, we did not find socioeconomic gradients for WPI, SSS or PDS. Regarding the association between education and PDS, adjusted OR were $1.143,95 \% \mathrm{CI}=0.802-1.629, p=0.461$ for secondary education and $\mathrm{OR}=1.170,95 \% \mathrm{CI}=0.937-1.460$, $p=0.166$ for post-secondary education, as compared with people with the 3rd cycle. As for income and PDS, adjusted OR were $\mathrm{OR}=1.100,95 \% \mathrm{CI}=0.861-1.406, p=0.446$ for the middle income class and $\mathrm{OR}=0.924,95 \% \mathrm{CI}=0.713-1.197, p=0.550$ for the high income class, as compared with the lower income class. Concordantly subjective social class was also not significantly associated with PDS: adjusted OR were $\mathrm{OR}=0.759, \quad 95 \%$ $\mathrm{CI}=0.506-1.139, p=0.183$ for the "medium low" social class and $\mathrm{OR}=0.709,95 \% \mathrm{CI}=0.467-1.076, p=0.106$ for the "medium high and high" social class, as compared with the "low" social class. Conclusion: We did not find significant associations between widespread pain syndrome features and SEP in these young adults. Socioeconomic disparities in symptoms might only be detectable in later years. Also, the overall high educational level of these individuals may attenuate these differences.

\section{Abstract \#: P 435}

Reliability of the Dutch translation of the kujala patellofemoral score questionnaire

Ummels P.E.J. ${ }^{1,2,3}$, Dr. Lenssen A.F. ${ }^{1,4}$, Barendrecht M. ${ }^{3}$, Prof. Dr. Beurskens A.J.H.M ${ }^{5,6}$

${ }^{1}$ Zuyd University, Faculty of Health, Department of Physiotherapy, Heerlen, The Netherlands; ${ }^{2}$ Paramedic Centre Sittard Zuid, Sittard, The Netherlands; ${ }^{3}$ Avans Plus Improving Professionals, Breda, The Netherlands; ${ }^{4}$ Maastricht University Medical Center, Maastricht, The Netherlands; ${ }^{5}$ Zuyd University, Faculty of Health, Research Centre Autonomy and Participation of patients with a chronic illness, Heerlen, The Netherlands; ${ }^{6}$ Maastricht University, Caphri School, Maastricht, The Netherlands; Corresponding author's e-mail: rob.ummels@zuyd.nl

Objective: There are no Dutch-language disease-specific questionnaires for patients with patellofemoral pain syndrome (PFPS) available that could help Dutch physiotherapists to assess and monitor these symptoms and functional limitations. The aim of this study was to translate the original disease-specific Kujala Patellofemoral Score (KPS) into Dutch and evaluate its reliability.

Methods: The questionnaire was translated from English into Dutch in accordance with internationally recommended guidelines. Reliability was determined in 50 stable subjects with an interval of 1 week. The patient inclusion criteria were age between 14 and 60 years, and knowledge of the Dutch language. The presence of at least three of the following symptoms: pain while taking the stairs, pain when squatting, pain when running, pain when cycling, pain when sitting with knees flexed for a prolonged period, grinding of the patella and a positive clinical patella test. The internal consistency, test-retest reliability, measurement error, and Limits of Agreement (LoA) were calculated. 
Results: Internal consistency was 0.78 for the first assessment and 0.80 for the second assessment. The intraclass correlation coefficient (ICCagreement) between the first and second assessments was 0.98. The mean difference between the first and second measurements was 0.64 and SD 5.51. The standard error measurement (SEM) was 3.9 and smallest detectable change (SDC) 11. The Bland and Altman plot shows that the Limits of agreement (LoA) are -10.37 and 11.65 . Conclusions: The results of the present study indicated that the testretest reliability translated Dutch version of the KPS questionnaire is equivalent of test-retest original English-language version and has good internal consistency. Trial registration NTR $($ TC $=3258)$.

\section{Abstract \#: P 436 \\ Predictors of poor functional outcome of an episode of distal upper limb pain: the arm pain trial}

Daniel Whibley ${ }^{1}$, Kathryn R. Martin ${ }^{1}$, Karina Lovell ${ }^{2}$, Gary J. Macfarlane $^{1}$, Keith T. Palmer ${ }^{3}$, David Coggon ${ }^{3}$, Karen Walker-Bone ${ }^{3}$, Kim Burton ${ }^{4}$, Peter J. Heine ${ }^{5}$, Candy McCabe ${ }^{6}$, Paul McNamee ${ }^{7}$, Alex McConnachie ${ }^{8}$, Gareth T. Jones ${ }^{1}$

${ }^{1}$ Musculoskeletal Research Collaboration (Epidemiology Group), University of Aberdeen; ${ }^{2}$ School of Nursing, Midwifery and Social Work, University of Manchester; ${ }^{3}$ MRC Lifecourse Epidemiology Unit, University of Southampton; ${ }^{4}$ Centre for Health and Social Care Research, University of Huddersfield; ${ }^{5}$ Warwick Clinical Trials Unit, University of Warwick; ${ }^{6}$ The Royal United Hospital NHS Foundation Trust, Bath: University of West of England, Bristol; ${ }^{7}$ Health Economics Research Unit, University of Aberdeen; ${ }^{8}$ Robertson Centre for Biostatistics, University of Glasgow; Corresponding author's e-mail: daniel.whibley@abdn.ac.uk

Background: Approximately $15 \%$ of the UK population consult their GP for arm pain annually, almost half reporting pain in the distal region. Resultant work incapacity presents a substantial economic burden. Psychosocial factors have been identified as important prognostic markers in other musculoskeletal pain syndromes. Identification of psychosocial factors that predict functional impairment associated with distal upper limb pain may inform management strategies, public and occupational health initiatives. This study aimed to identify factors that predict poor functional outcome 6 months after presentation to physiotherapy services with distal upper limb pain.

Methods: This analysis used data from a randomised controlled trial investigating management of distal arm pain (ISRCTN79085082). Participants were recruited after referral to physiotherapy and randomised to: advice to rest the arm during the usual waiting period (6-7 weeks), advice to remain active, or immediate physiotherapy. The primary outcome was persistent disability at 6 months, measured using the modified Disabilities of the Arm, Shoulder and Hand questionnaire (range 0-11). Outcome was dichotomised, with poor outcome defined as any disability (1-11). Information on a range of putative prognostic factors was collected using a self-completed questionnaire. Forward stepwise multivariable logistic regression (inclusion $p<0.1$, exclusion $p>0.15$ ) identified factors that independently predicted poor functional outcome.

Results: Of 431 participants (55\% female), $63 \%$ experienced functional limitation 6 months post-randomisation. After adjustment for sex and baseline pain and disability, 21 factors predicted outcome at significance level $p<0.2$ and were offered to the stepwise regression model. Independent predictors of functional limitation at 6 months included being female (OR 2.16, $95 \%$ CI 1.03-4.52), presenting with moderate $(3.48,1.01-11.92)$ or severe pain $(5.78$, 1.63-20.48) compared to mild, holding the belief that physical activity might be harmful for arm pain $(1.81,0.72-4.55)$, scoring in the middle or upper third on the Modified Somatic Perception Questionnaire (2.54, 1.11-5.83 and 5.34, 1.88-15.16 respectively), and undertaking non-computer-based tasks at work involving repetitive wrist or finger movement for $>4 \mathrm{~h} /$ day $(2.47,1.20-5.09)$.

Conclusion: Nearly two-thirds of participants reported disability 6 months after presentation. Two psychological factors predicted poor outcome: negative beliefs about activity and features of somatisation. Patients with distal upper limb pain and such predictors of poor outcome may benefit from psychological interventions in addition to the original referral for physiotherapy. These results may help to inform stratified models of care where those at higher risk of persistent disability are identified early and treated accordingly.

\section{Abstract \#: P 437}

Comorbidities force physiotherapists to deviate from guidelines: a vignette study

Sarah Dörenkamp MSc PhD Candidate ${ }^{1}$, Ilse Mesters PhD Associate Professor $^{1}$, Joep Teijink MD PhD Professor ${ }^{2,3}$, Rob de Bie PhD Professor $^{1}$

${ }^{1}$ Department of Epidemiology and CAPHRI School for Public Health and Primary Care, Faculty of Health, Medicine and Life Sciences, Maastricht University, Maastricht, The Netherlands; ${ }^{2}$ Department of Vascular Surgery, Catharina Hospital, Eindhoven, the Netherlands and Department of Epidemiology; ${ }^{3}$ CAPHRI School for Public Health and Primary Care, Maastricht University, Maastricht, The Netherlands; Corresponding author's e-mail: sarah.dorenkamp@maastrichtuniversity.nl

Background: How patients with comorbidities are treated in daily practice is unknown. Understanding the decision making process of physical therapists (PTs) and exploring the treatment of comorbid patients in daily practice might be a first step to unravel the complexity of treating multi-diseased patients. The aim of this Vignette study was to assess whether PTs make reasoned adaptations to evidence based treatment recommendations when comorbidity influences the initial treatment of the index disease.

Methods: To study the influence of comorbidities on treatment recommendations, three vignettes were created based on authentic Intermittent Claudication (IC) patient data. In the first vignette a single-diseased IC patient was described, in the second comorbidity Chronic Obstructive Pulmonary Disease (COPD) was added and in the third vignette additionally Knee Osteoarthritis was added. Therapists were instructed to describe three detailed treatment plans and their decision rationale. A random selection of 100 Dutch Claudication Network members (ClaudicatioNet) was invited to participate in this qualitative study: expecting a $50 \%$ response.

Results: Response rate was $61 \%$. Thirty percent of the physical therapists did not adjust treatment despite comorbidity. Another $30 \%$ partly adapted the treatment plan when comorbidity was added to the vignette. The presence of comorbidity induced $40 \%$ of the therapists to abandon guideline recommendations and to create an individualised treatment plan based on the health needs of the vignette patient. Conclusion: This study showed, for better or for worse, that the majority of PTs makes adaptations to evidence-based recommendations when comorbidity is present, but not leading to uniformity. Exactly the same patient was treated in various ways by different PTs. 


\section{Abstract \#: P 438}

\section{Association between psychosocial factors, physical activity and sedentary behaviors: sex differences in adolescents from Rio de Janeiro-BRAZIL}

\section{$\underline{\text { Viviane S. Straatmann }}^{1}$, Aldair J. Oliveira ${ }^{2}$, Claudia S. Lopes ${ }^{1}$}

${ }^{1}$ University of Rio de Janeiro State, Social Medicine Institute/ Epidemiology, Rio de Janeiro- Brazil; ${ }^{2}$ Rural Federal University of Rio de Janeiro, Physical Education Departament, Rio de JaneiroBrazil; Corresponding author's e-mail: vica_s@hotmail.com

Purpose: Psychosocial factors including social support, violence, well-being and psychological distress, are able to influence health behaviors. The purpose of this study was to evaluate the association between psychosocial factors, estimated by body bullying and psychological well-being, physical activity and sedentary behavior among adolescents.

Methods: This is a cohort study called "Longitudinal Study of Adolescent Nutritional Assessment (ELANA)" conducted among elementary school students from two public and four private schools in Rio de Janeiro in the years 2010, 2011, 2012 and 2013. For the present study we analyzed the baseline data (2010) from 809 adolescents (435 male and 374 female) aged between 9 and 15 years. Psychosocial factors were evaluated by "The Child-Adolescent Teasing Scale" (CATS) questionnaire, used to assess body bullying: psychological well-being was assessed by the psychological domain of the questionnaire "KIDSCREEN 27". Both scales/domains were consolidated and categorized into tertiles. Physical activity (PA) was evaluated by the "International Questionnaire of physical activity (IPAQ)". Those who practiced $60 \mathrm{~min}$ or more per day of moderate to vigorous PA were classified as active. Sedentary behavior was evaluated with objective questions about daily time in front of the screen (television, video games and computed). Those with screen time of $4 \mathrm{~h}$ or more per day were classified as sedentary. Logistic regression was used to analyze the associations estimating the odds ratio (OR) and confidence intervals (CI $95 \%$ ), stratified by sex.

Results: The mean age of male and female adolescents was 11.4 years (standard deviation $= \pm 1.2$ years) and 11.2 (standard deviation $= \pm 1.1$ years), respectively. The statistic models did not provide significant $(p<0.05)$ results, except among female adolescents exposed to bad psychological well-being $(\mathrm{OR}=1.5: 1.5-3.2 \mathrm{CI}$ $95 \%$ ), which were more likely to take sedentary behavior compared with those in the higher level of psychological states.

Conclusions: There was an association between bad psychological well-being and sedentary behavior only among female adolescents. Therefore, there is a sex difference in the association between psychological well-being and sedentary behaviors, assuming different coping strategies across sexes.

\section{Abstract \#: P 439}

\section{Epidemiology of congenital malformations of the CNS Turkestan region}

Nazarbek A. Zhumanazarov ${ }^{1,2}$, Marat U. Kamysbayev ${ }^{1}$, Mustapha I. Omaraliyev $^{1}$, Anvar N. Temirbekov ${ }^{1}$, Nurlan K. Khodjayev ${ }^{1}$, Samat S. Doszhanov ${ }^{1}$, Kayrat M. Tolepbekov ${ }^{1}$, Guljaukhar N. Taskynova ${ }^{1}$, Dinara A. Sisenbina ${ }^{1}$

${ }^{1}$ Pathological Anatomy, International kazakh-turkish university af. A.Yassavi, Turkistan, Kazakhstan; ${ }^{2}$ International kazakh-turkish university af. A.Yassavi, Turkistan, Kazakhstan; Corresponding author's e-mail: j_nazarbek@mail.ru
According to WHO, $20 \%$ of infant morbidity and disability, as well as $15-20 \%$ of child deaths caused by malformations, such as defects of the central nervous system are the second place, behind congenital malformations of the circulatory system. The CM CNS account for about $25 \%$ of all birth defects and cause of neurological symptoms.

The purpose of work: Identify pathological and epidemiological features of congenital malformations of the central nervous system in fetuses and dead children.

Materials and methods based on CCP medical institution I'RPC no. 3"-Turkestan was studied 130 protocol sectional study of deaths from various causes fetuses and children with morphologically confirmed CNS malformations.

Results of study: Protocols sectional study of 130 fetuses and children who died during the period 2009-2014, of which 95 were selected protocol $(12.2 \pm 1.3 \%$ of the total) with morphologically confirmed congenital malformations (the $\mathrm{CM}$ ) of the central central nervous system (CNS). Age of the fetus and children ranged from 22 weeks gestation to 3 years. Ultrasound study in CCP RPC no. 3 I-trimester it possible to detect such gross malformations of the central nervous system, as anencephaly, traumatic brain and spinal hernia. An ultrasound conducted in II-trimester, identified anencephaly, congenital hydrocephalus, spinal hernia. In the III-trimester pregnancies were detected the CM CNS congenital hydrocephalus and combined forms the CM CNS. Of 52 women consented to termination of pregnancy gave 37 people $(75.8 \pm 3.9 \%)$. Fifteen women $(35.3 \pm 4.5 \%)$ kept the pregnancy, but in all cases the pregnancy ended in stillbirth.

Epidemiology the CM CNS in Turkestan in general affect ethnic, genetic, medical and organizational, biological, sanitary and environmental factors.

The results obtained morphological and epidemiological studies can be used in the planning of the necessary volume of medical care for children with congenital malformations (calculation of the number of children in need of inpatient and outpatient treatment, surgical correction and rehabilitation). The presented data on the frequency and structure defects of the central nervous system are necessary for the prenatal diagnosis of doctors at work with families at risk for the CM: to develop tactics of pregnancy and childbirth in the detection of fetal neural tube defects. For information about the course and frequency of neurological syndromes in children with congenital malformations of the central nervous system allow clinicians (neurologists, pediatricians, neonatologists) to determine the methods dynamics of the treatment observation of such patients.

\section{Abstract \#: P 440}

Interaction between MTHFR $677 \mathrm{C}>\mathrm{T}$ and periconceptional folic acid supplementation in the risk of hypospadias

Elisabeth M.J. Dokter ${ }^{1,2}$, Iris A.L.M. van Rooij ${ }^{1}$, Charlotte H.W. Wijers $^{1}$, Johanne M. Groothuismink ${ }^{1}$, Jan Jaap van der Biezen ${ }^{2}$, Wout F.J. Feitz ${ }^{3}$, Nel Roeleveld ${ }^{1,4}$, Loes F.M. van der Zanden ${ }^{1}$

${ }^{1}$ Radboud Institute for Health Sciences, Department for Health Evidence, Radboud University Medical Center, Nijmegen; ${ }^{2}$ Department of Plastic Surgery and Hand Surgery, Medical Centre Leeuwarden, Leeuwarden; ${ }^{3}$ Department of Urology, Paediatric Urology, Radboudumc Amalia Children's Hospital, Nijmegen; ${ }^{4}$ Department of Paediatrics, Radboudumc Amalia Children's Hospital, Nijmegen; Corresponding author's e-mail: elsemieke.dokter@radboudumc.nl

Background: Hypospadias is a congenital malformation of the urethra in which the meatus is located on the ventral side of the penis. Both environmental factors and genetic predisposition are believed to 
play a role in the pathogenesis. Folate is important for rapid cell division and gene expression and is therefore essential for cellular growth and differentiation during embryogenesis. Folate levels are reduced due to the C677T polymorphism in the methylenetetrahydrofolate reductase (MTHFR) gene, as this polymorphism reduces MTHFR enzyme activity. This polymorphism is involved in the etiology of several congenital malformations, but it was never studied in relation to hypospadias. Maternal periconceptional use of folic acid supplements may compensate for reduced folate levels and thereby play a role in the prevention of congenital malformations. Although this has been confirmed for some congenital malformations, such as neural tube defects, the results for hypospadias are inconsistent. Therefore, we investigated independent associations and interactions of maternal periconceptional use of folic acid supplements and maternal and infant MTHFR C677T polymorphisms with the risk of hypospadias.

Methods: A case-control study was conducted among 914 hypospadias cases and 711 Dutch population-based controls from the AGORA data- and bio-bank (Aetiologic research into Genetic and Occupational/environmental Risk factors for Anomalies in children). Cases and controls were all born between 1990 and 2012. Information about folic acid use was derived from questionnaires filled out by the mother, and DNA samples from mother and child were used to determine the MTHFR C677T polymorphism using the Taqman assay. In the analyses, we assumed a dominant effect of the polymorphism.

Results: Preliminary univariable analysis showed a small protective effect of maternal periconceptional use of folic acid supplements on the risk of hypospadias [odds ratio $(\mathrm{OR})=0.8,95 \%$ confidence interval (95\% CI): 0.6-1.0]. No associations were found for the infant or maternal MTHFR C677T polymorphism (both OR $=1.1$ : $95 \%$ CI: 0.9-1.4). However, lack of folic acid supplement use in combination with carrying the MTHFR C677T polymorphism increased the risk of hypospadias (infant polymorphism: $\mathrm{OR}=1.5$ : $95 \%$ CI: $1.1-2.1$, maternal polymorphism: $\mathrm{OR}=1.5,95 \% \mathrm{CI}$ : 1.1-2.2).

Conclusion: This first study investigating interactions between maternal periconceptional use of folic acid supplements and infant and maternal MTHFR C677T polymorphisms in the aetiology of hypospadias showed an increased risk of hypospadias when no folic acid supplements were used and infants or mothers carried the MTHFR C677T polymorphism.

\section{Abstract \#: P 441}

Maternal depression and use of antidepressant during pregnancy and the risk of adverse pregnancy outcomes: an IPD metaanalysis

$\underline{\text { Richelle Vlenterie }}^{1}$, Marleen M.H.J. van Gelder ${ }^{1}$, Monica PopPurceleanu $^{2}$, Nel Roeleveld ${ }^{1}$

${ }^{1}$ Radboud Institute for Health Science, Radboud University Medical Center, Nijmegen, The Netherlands; ${ }^{2}$ Department of Psychiatry, Radboud University Medical Center, Nijmegen, The Netherlands; Corresponding author's e-mail: richelle.vlenterie@ radboudumc.nl

Background: Recent systematic reviews and meta-analyses examining the adverse effects of maternal depression and the use of antidepressants during pregnancy show inconclusive results. Therefore, it is still difficult for prospective parents, prenatal care provides, general practitioners, and psychiatrists to make evidence-based decisions on treatment possibilities.

Objective: To perform an individual patient data (IPD) meta-analysis on the association between non-pharmacologically managed maternal depression and antidepressant use during pregnancy and the occurrence of miscarriages, preterm birth, low birth weight, small for gestational age, poor Apgar scores, and major birth defects.

Methods: A systematic literature search was conducted using PubMed, Embase, and PsycINFO until November 2013. For eligible publications $(n=176)$, the methodological quality was assessed with the Newcastle-Ottawa Scale. Both selection and extraction of papers and the methodological quality assessment were done independently by two reviewers. We invited all authors of the included studies to share their original data, which include individual patient characteristics. After assessment of validity and completeness, the databases will be merged and analyzed in early 2015 .

Results and conclusion: Combined odds ratios adjusted for confounding will be calculated for data of high methodological quality for each of the pregnancy outcomes. This IPD meta-analysis will overcome the inconclusive results of other systematic reviews and meta-analyses and will provide evidence-based information on the potential adverse effects of maternal depression and the use of antidepressants during pregnancy on pregnancy outcomes.

\section{Abstract \#: P 442}

Maternal bereavement during pregnancy and preterm delivery risk: the importance of gestational age and of the precursor of preterm birth

Krisztina D. László ${ }^{1}$, Jiong Li $^{2}$, Jørn Olsen ${ }^{3}$, Mogens Vestergaard ${ }^{4}$, Tobias Svensson $^{1}$, Carsten $\mathrm{Obel}^{5}$, Sven Cnattingius ${ }^{1}$

${ }^{1}$ Clinical Epidemiology Unit, Department of Medicine, Karolinska University Hospital and Karolinska Institute, Stockholm, Sweden;

${ }^{2}$ Section for Epidemiology, Department of Public Health, Aarhus University, Aarhus, Denmark; ${ }^{3}$ School of Public Health, University of California, Los Angeles, California; ${ }^{4}$ Research Unit for General Practice, Department of Public Health, University of Aarhus, Aarhus, Denmark and Section for General Practice, Department of Public Health, University of Aarhus, Aarhus, Denmark; ${ }^{5}$ Research Unit for General Practice, Department of Public Health, University of Aarhus, Aarhus, Denmark: Section for General Practice, Department of Public Health, University of Aarhus, Aarhus, Denmark and Research Program for Mental Child Health, Department of Public Health, University of Aarhus and Institute of Handicap and Communication, The Central Region, Aarhus, Denmark; Corresponding author's e-mail: krisztina.laszlo@ki.se

Background: Maternal stress during pregnancy may increase risk of preterm delivery, but possible mechanisms remain unknown. We investigated maternal loss of a close relative and risks of very and moderately preterm delivery ( $<32$ and $32-36$ weeks, respectively) and spontaneous and medically indicated preterm delivery.

Methods: We studied live singleton births in Denmark during 1978-2008 and in Sweden during 1973-2006 $(\mathrm{n}=4,940,764)$. We retrieved information on death of family members and on maternal characteristics through nationwide registry linkages.

Results: The death of a close family member the year before or during pregnancy was associated with an $8 \%$ increased risk of preterm (<37 weeks) delivery $[95 \%$ confidence intervals (CI): 1.05-1.11]. The corresponding hazard ratios (HR) and $95 \% \mathrm{CI}$ were $1.26(1.15-1.38)$ for death of a child, 1.31 (1.03-1.66) for death of a partner, and 1.07 (1.04-1.10) for death of a parent: loss of a sibling was not associated with the outcome. Death of an older child and death of the partner (during pregnancy) was more related to risk of very preterm [HR (95\% CI): $1.70(1.37-2.13)$ and 2.07 (1.15-3.74), respectively] than moderately preterm delivery [HR $(95 \% \mathrm{CI}): 1.21$ (1.09-1.33) and $1.22(0.94-1.58)$, respectively]. Death of an older 
child was associated with an increased risk of both spontaneous [HR (95\% CI): 1.45 (1.13-1.84)] and medically indicated preterm delivery [HR (95\% CI): $1.61(1.12-2.30)]$.

Conclusions: Severe stress during pregnancy may foremost increase risk of very preterm, but also risk of moderately preterm delivery. Possible mechanisms include both spontaneous and medically indicated preterm birth.

\section{Abstract \#: P 443 \\ Predictors of gestational weight gain \\ Marlein Ausems ${ }^{1}$, Astrid Merkx ${ }^{1}$ \\ ${ }^{1}$ Research Centre for Midwifery Science Maastricht, Zuyd University; Corresponding author's e-mail: marlein.ausems@av-m.nl}

Background: Gestational weight gain (GWG) is associated with mothers' and babies' health. Too much GWG is associated with overweight and obesity in the long term, both in mothers and their offspring. Furthermore, too much GWG is associated with pregnancy related pathology including gestational diabetes and pregnancy-induced hypertension. On the other hand, too little GWG is associated with prematurity and small for gestational age babies. Recommendations for healthy GWG are described in the GWG-guidelines of the Institute of Medicine (IOM-guidelines) and depend on the Body Mass Index before pregnancy (prepregnancy BMI). Studies on GWG mostly included specific subgroups like obese women or women with higher risk for gestational diabetes. This study focusses on the GWG distribution of Dutch healthy pregnant women in their third trimester according to the IOM guidelines, and on predictors (measured at the beginning of the pregnancy) of (un)healthy GWG.

Methods: A longitudinal study design was used. At the beginning of their pregnancy, 215 healthy pregnant women completed a digital baseline questionnaire, 144 of them completed the follow-up questionnaire in the third trimester of their pregnancy. Both questionnaires included questions related to demographic variables, prepregnancy BMI, gestational weight gain, lifestyle behaviors (diet and physical activity) and their determinants. Descriptive and multinomial regression analyses are used, contrasting women gaining below with within and above with within IOM guidelines to distinguish relevant baseline predictors.

Results: Preliminary results show that almost halve of the women gained weight above the IOM guidelines. Halve of the women with a normal prepregnancy BMI did not gain within IOM guidelines. Further analyses will point at factors that predict (un)healthy weight gain, for instance prepregnancy BMI, baseline diet and physical activity, the change in diet and physical activity during the pregnancy course, and demographics such as parity, maternal age, ethnicity, education, income.

Conclusion: This study will improve our understanding of the distribution of healthy women gaining weight below, within, or above the IOM guidelines and will bring us to predictors of (un)healthy GWG. The study will help us developing programs aiming firstly at reaching a healthy GWG and secondly better health for mothers and their offspring.

\author{
Abstract \#: P 444 \\ Pregnancy-induced hypertensive disorders and use \\ of antihypertensive drugs following the 2008 economic collapse \\ in Iceland
}

Védís H. Eiríksdóttir ${ }^{1}$, Unnur A. Valdimarsdóttir ${ }^{1,2}$, Tinna L. Ásgeirsdóttir ${ }^{3}$, Sigrún H. Lund ${ }^{1}$, Arna Hauksdóttir ${ }^{1}$, Helga Zoega ${ }^{1}$

${ }^{1}$ Centre of Public Health Sciences, Faculty of Medicine, University of Iceland, Reykjavik, Iceland; ${ }^{2}$ Department of Epidemiology, Harvard School of Public Health, Boston, Massachusetts, USA; ${ }^{3}$ Faculty of Economics, University of Iceland, Reykjavik, Iceland; Corresponding author's e-mail: vedis.helga@gmail.com

Background: Data are scarce on the potential influence of macroeconomic recessions on maternal diseases during pregnancy. Previous research indicates that stress may play a role in the development of hypertensive disorders of pregnancy. We aimed to assess a potential change in the prevalence of pregnancy-induced hypertensive disorders, including gestational hypertension and preeclampsia, and use of antihypertensive drugs during the first years of the major national economic recession in Iceland, which started abruptly in October 2008.

Methods: Women whose pregnancies resulted in live singleton births in Iceland in 2005-2012 constituted the study population $(\mathrm{N}=35,211)$. Data on pregnancy-induced hypertensive disorders were obtained from the nationwide Medical Birth Registry and use of antihypertensive drugs during pregnancy, including $\beta$-blockers and calcium channel blockers, from the Icelandic Medicines Registry. With pre-collapse period as reference, we used logistic regression analysis to assess annual change in hypertensive disorders of pregnancy and use of antihypertensives during the first four consecutive years after the economic collapse, adjusting for demographic and pregnancy characteristics as well as aggregate economic indicators. Results: Compared with the pre-collapse years, we observed an increase in gestational hypertension in the first year following the economic collapse (2.4 vs. $3.9 \%$ : adjusted odds ratio [aOR] 1.47: $95 \%$ confidence interval [95\% CI] 1.13-1.91) but not in the subsequent 3 years. The observed association attenuated when adjusting for aggregate unemployment rate (aOR 1.04: $95 \%$ CI 0.74-1.47). Similarly, there was an increase in prescription fills of $\beta$-blockers in the first year following the collapse (1.9 vs. $3.1 \%$ : aOR 1.43: $95 \%$ CI 1.07-1.90), which attenuated when adjusting for aggregate unemployment rate (aOR 1.05: $95 \%$ CI 0.72-1.54). No changes were observed for preeclampsia or use of calcium channel blockers.

Conclusion: The results suggest an increase in gestational hypertension and use of $\beta$-blockers among pregnant women in Iceland in the first year following the national economic collapse. The severity of the aggregate economic climate at that time with a gradual yet slow recovery during the following years is a likely explanation for the observed pattern.

\section{Abstract \#: P 445}

Obstetric mode of delivery and risk of neonatal or infant death: a register-based cohort

S.M. O'Neill ${ }^{1}$, E. Agerbo $^{2}$, A.S. Khashan ${ }^{1}$, P.M. Kearney ${ }^{3}$, T.B. Henriksen $^{4}$, P.B. Mortensen ${ }^{2}$, R.A. Greene ${ }^{5}$, L.C. Kenny ${ }^{1}$

${ }^{1}$ INFANT, Cork, Ireland; ${ }^{2}$ NCRR, Aarhus, Denmark; ${ }^{3}$ UCC, Cork, Ireland; ${ }^{4} \mathrm{AU}$, Aarhus, Denmark; ${ }^{5} \mathrm{NPEC}$, Cork, Ireland; Corresponding author's e-mail: sinead.oneill@ucc.i.e

Background: Caesarean section rates have increased globally with rates of over $50 \%$ reported in some countries. In women with one previous Caesarean section choosing the optimum mode of delivery in the next birth remains controversial. Is a trial of labour (TOL) or an elective repeat Caesarean section (ERCS) safer?

Methods: A population-based cohort study using the Danish National Registers was conducted. Women with two deliveries between 1982 
and 2010 were identified using the Medical Birth Register $(\mathrm{n}=550,273)$. Exclusion criteria included multiple births (twins or more), non-cephalic presentation and gestation outside 37-43 weeks. Multiple logistic regression models adjusting for key social and obstetric variables were generated using SAS software.

Results: Neonatal death ( $<28$ days) and infant death ( $<365$ days) in women with a first Caesarean section and subsequent TOL or an ERCS were compared to women with a first vaginal delivery and subsequent TOL (reference group) or an elective CS. Compared to the reference group, women with a first CS who had an ERCS had a reduced odds of neonatal death (odds ratio $0.61,95 \%$ CI $0.50,0.98$ ). No significantly increased odds of neonatal death were found in women with a first CS and subsequent TOL (OR 1.01, $95 \%$ CI 0.82 , $1.45)$. Women with a first vaginal delivery and subsequent $\mathrm{CS}$ had a $45 \%$ increased odds of neonatal death $(1.45,95 \%$ CI 1.05, 2.01). No increased odds of infant death was found in women with a first CS and subsequent ERCS or TOL. However, women with a first vaginal delivery and subsequent CS had a $63 \%$ increased odds of infant death(OR 1.63, $95 \%$ CI 1.31, 2.10).

Conclusions: This, one of the largest studies to date comparing neonatal and infant death in women with a first CS and subsequent TOL or ERCS found no increased odds in the prior CS group. Women with a first vaginal delivery and subsequent CS had a significantly increased odds, which could be due to unknown confounders and underlying medical conditions. More analyses using detailed confounder information is on-going.

\section{Abstract \#: P 446}

The role of previous miscarriages and single nucleotide polymorphisms in developmental genes in the etiology of anorectal malformations

Romy van de Putte ${ }^{1}$, Charlotte H.W. Wijers ${ }^{1}$, Ivo de Blaauw ${ }^{2,3}$, Carlo L.M. Marcelis ${ }^{4}$, Cornelius E.J. Sloots ${ }^{3}$, Paul M.A. Broens ${ }^{5}$, Nel Roeleveld $^{1,6}$, Loes F.M. van der Zanden ${ }^{1}$, Iris A.L.M. van Rooij ${ }^{1}$

${ }^{1}$ Department for Health Evidence, Radboud university medical center, Radboud Institute for Health Sciences, Nijmegen, The Netherlands; ${ }^{2}$ Department of Surgery-Pediatric surgery, Radboudumc Amalia Children's Hospital, Radboud university medical center, Nijmegen, The Netherlands and; ${ }^{3}$ Department of Pediatric Surgery, Sophia's Children's Hospital, Erasmus Medical Centre, Rotterdam, The Netherlands; ${ }^{4}$ Department of Human Genetics, Radboud university medical center, Radboud Institute for Molecular Life Sciences, Nijmegen, The Netherlands; ${ }^{5}$ Department of Surgery, Division of Pediatric Surgery, University Medical Centre Groningen, Groningen, The Netherlands; ${ }^{6}$ Department of Pediatrics, Radboudumc Amalia Children's Hospital, Radboud university medical center, Nijmegen, The Netherlands; Corresponding author's e-mail:

iris.vanrooij@radboudumc.nl

Background: Anorectal malformations (ARM) are one of the most frequently occurring birth defects of the gastrointestinal tract. In the majority of patients, multiple congenital malformations are present. The etiology still remains unknown, but is likely to be heterogeneous with both environmental and genetic factors playing a role. Having miscarriages before the index child is born seems to be a risk factor for several congenital malformations and genetic factors are often the cause of early miscarriages. The aims of this study were to test the association of ARM with previous miscarriages and two known polymorphisms in the developmental genes BMP4 and GLI2, as well as the GxG and GxE interactions.

Methods: A case-control study was performed in a Caucasian population of 431 nonsyndromic ARM cases and 661 population-based controls using parental questionnaires and DNA samples of the children. Patients and controls were derived from the AGORA (Aetiologic research on Genetic and Occupational/environmental Risk factors for Anomalies in children) data- and bio-bank of the Radboud university medical center in Nijmegen, The Netherlands. Maternal questionnaire information was used for information about miscarriages, defined as having a fetal loss before 20 weeks of pregnancy. Genotyping was performed using Kompetitive Alleles Specific PCR assays.

Results: Among the control mothers, $19 \%$ reported a miscarriage before the index child was born, versus $25 \%$ of the case mothers, resulting in an elevated OR of 1.4 (95\% CI 1.1-2.0). The non-synonymous SNP rs17563 in BMP4 was not associated with ARM, but we did find associations between ARM and the non-synonymous SNP rs3738880 in GLI2, especially in ARM patients with multiple congenital malformations (homozygous GG genotype: $\mathrm{OR}=2.1$ : $95 \%$ CI: 1.2-3.7). A fourfold increased risk was shown when homozygous variant genotypes of both genes were present $(\mathrm{OR}=4.1: 95 \% \mathrm{CI}$ : 1.0-17.8). The combination of previous miscarriages and infant genotypes of BMP4 and GLI2 did not show a clear gene-environment interaction.

Conclusion: We showed independent associations between ARM and miscarriages and between the variant in GLI2 and ARM in patients with multiple congenital malformations, which is consistent with a multifactorial etiology. In addition, a gene-gene interaction was demonstrated between BMP4 and GLI2, both downstream genes in the Sonic Hedgehog ( $\mathrm{SHH})$ pathway, which is involved in several important developmental processes.

\section{Abstract \#: P 447}

Incidence of congenital malformations of the circulatory system in semey (former Semipalatinsk), Kazakhstan, in 2009-2013

Madina R. Madiyeva ${ }^{1}$, Tamara Kh. Rymbaeva ${ }^{1}$, Aizhan Abylgazinova $^{1}$, Andrej M. Grjibovski ${ }^{2}$

${ }^{1}$ Semey State Medical University, Semey, Kazakhstan; ${ }^{2}$ Department of International Public Health, Norwegian Institute of Public Health, Osl; Corresponding author's e-mail: andrej.grjibovski@gmail.com

Background: Congenital malformations including malformations of the circulatory system are among the main causes of infant mortality and childhood morbidity representing a considerable public health problem. Elevated incidence of birth defects has been reported from settings exposed to ionizing radiation. More than 450 nuclear tests were performed at the Semipalatinsk test site during Soviet time, but the official data on health of the population were unreliable before breakup of the USSR. Even 20 years after the test site was closed, the data are still scarce and their validity has been questioned. The aim of this study was to assess the incidence of congenital malformations of the circulatory system in a city of Semey (former Semipalatinsk).

Methods: Data on all newly diagnosed congenital malformations of the circulatory system (ICD-10 codes: Q20-Q28) registered among children aged 0-18 years in a city of Semey in 2009-2013 were obtained from the regional medical authorities. Data on population of children $0-18$ years (for overall incidence of birth defects) and newborns (for calculation of perinatal incidence of birth defects) was obtained from the regional statistical office. The incidence of congenital malformations of the circulatory system is presented by year with $95 \%$ confidence intervals (CI).

Results: Altogether, there were 1091 registered birth defects during the study period including 336 (30.8\%) defects of circulatory system. The overall incidence of congenital malformations of the circulatory system was 0.89 (95 \% CI 0.65: 1.13) in 2009, 1.33 (95\% CI 1.05: 
$1.61)$ in $2010,1.01(95 \%$ CI $0.77,1.13)$ in $2011,1.02$ (95\% CI 0.76 , 1.13 ) in 2012 and 0.69 (95\% CI 0.51: 0.88) in 2013. In the structure of congenital malformations of the circulatory system the most common defects were ventricular septal defect (Q21.0), tetralogy of Fallot (Q21.3) followed by atrial septal defect (Q21.1). The incidence of perinatally diagnosed malformations of the circulatory system was 0.18 per 1000 in 2009, 0.22 in 2010, 0.19 in 2011, 0.17 in 2012 and 0.20 in 2013.

Conclusions: The incidence of congenital malformations of the circulatory system in Semey is similar to other regions of Kazakhstan. Comparisons with European countries will be presented. Less than one-third of defects of circulatory system are diagnosed perinatally. There is a serious need for effective prenatal detection of severe malformations with their subsequent elimination, which has a potential to reduce perinatal mortality in the study area.

\section{Abstract \#: P 448}

Physician burnout and power issues in physician-patient relationship: the case of Russia

\section{Elena Tarasenko}

${ }^{1}$ National Research University Higher School of Economics: Corresponding author's e-mail: etarasenko@hse.ru

Now Russia reforms its health care sector. The structure of public health system is changing rapidly: we can see trends towards strengthening primary care provision and competition stimulation among health care providers, beds number' reduction in hospitals, managed care, there is less time for doctor-patient contacts, an increase in paperwork and so on. Additional demanding characteristics of physicians' professional work-such as time urgency, a continuous need to develop new skills and practice routines-are making physicians a high-risk group for developing burnout. Additionally Semashko health model' paternalism approach towards patients is sometimes thought them as if they were maladaptive children so some physicians may feel unable to respect patients.

Qualitative research (September 2014-February 2015):

35 Moscow physicians (50\% works in private hospitals, $50 \%$ works in public healthcare organizations) were interviewed (deep interviews) for qualitative phenomenological study exploring their experiences of burnout syndrome.

Interviews were fully analyzed within an interpretive phenomenological framework.

Results of the discussion "Why we need to respect patients?" in professional physicians community in FACEBOOK (Russian segment) on October 2014.

Main findings:

Physicians are faced with different chaotic and unpredictable situations in their everyday job practice and they need to manage burnout syndrome.

- The majority of respondents think that burnout syndrome is a "natural" result of their job practice. Burnout self-management requires effort.

- Burnout is an individual experience that is specific to the job context.

- There are no any working instruments and organizational practices for burnout syndrome management in public hospitals (grass-roots approach).

- There are special organizational practices and rules for burnout syndrome prevention in private hospitals (grass-roots approach). In this case we can see hiding true, negative attitudes towards patients and job in order to conform to professional or organisational norms-i.e. conforming to display rules.
- Physicians who are experiencing burnout can have a negative impact on their colleagues.

- Physicians professional community sometimes very loyal for burnout expressions and consequences (when physicians are cynical or critical at work).

- There are no any special coach support agencies for burnout management.

Conclusion: Interventions aiming at reducing job-related stress can be effective approaches to prevent burnout among physicians. Solutions should be multidisciplinary and combine preventive measureschanges to the job environment and management systems, successful strategies for physicians empowerment-with special courses to manage burnout, including stress-management techniques that teach medical practitioners how to cope better with stressful situations.

\section{Abstract \#: P 449}

\section{Netherlands research integrity network: the first 7 months}

Fenneke Blom $^{1}$, Lex M. Bouter ${ }^{1}$

${ }^{1}$ VU University Medical Center, Department of Epidemiology and Biostatistics; Corresponding author's e-mail: f.blom@vumc.nl

Recently several serious violations of research integrity have come to light in the Netherlands, which placed Research Integrity (RI) high on the public and academic agenda. More systematic and explicit attention is clearly needed, especially with respect to prevention. Although many institutions are committed to improve the research culture and to offer better Responsible Conduct of Research (RCR) education, there is little contact between the actors in the field. The newly launched Netherlands Research Integrity Network (NRIN) aims to facilitate collaboration, exchange and mutual learning. Other elements of NRIN's mission include information supply: training and education for different audiences: development and exchange of teaching materials and best practises: enabling of consultation and intervision: and providing solicited and unsolicited advice to decision makers.

Participants include RI counsellors: RI committee chairs and members: RCR educators: and researchers and policymakers in the field of RI. Relevant and important topics urging NRIN attention will be identified through interviews with stakeholders and policy document analysis. Among these topics are differences in regulations and litigation: re-use of previous published materials: acceptability of anonymous complaints: and new national and international insights and innovations. Seminars, workshops, think tanks, public debates and other events will be organised in close co-production with active participants. Working on RI requires intrinsic motivation rather than top-down policy alone. Therefore we aim to identify early adopters and start working with, and learning from them.

We will present our experiences and lessons learned from the first 7 months of the Netherlands Research Integrity Network.

\section{Abstract \#: P 450}

\section{Privacy protection in cohort studies. The lifelines solution}

Salome Scholtens ${ }^{1}$, Marie-Jose Bonthuis ${ }^{1}$, Jan-Lucas van der Ploeg ${ }^{2}$, Aafje Dotinga $^{1}$, Morris A. Swertz ${ }^{3}$, Ronald P. Stolk ${ }^{1}$

${ }^{1}$ LifeLines Cohort Study, Groningen, the Netherlands; ${ }^{2}$ University Medical Center Groningen, Groningen, the Netherlands; ${ }^{3}$ Genomic Coordination Center, University Medical Center Groningen, 
Groningen, the Netherlands; Corresponding author's e-mail: s.scholtens@umcg.nl

Background LifeLines is a prospective population-based cohort study and biobank that will follow 167,229 individuals for at least 30 years in the northern part of the Netherlands in a three generation design. High quality data, by means of physical examinations, questionnaires and analysis of biomaterial, are collected to push forward research on l'Healthy Ageing'. Also, linkage is being established with medical registries, national registry data and environmental exposures. Privacy protection of participants is essential, because the dataset contains sensitive information. The aim of LifeLines is to be a resource for the national and international scientific community for research on Healthy Ageing. The data and biomaterial are available for researchers worldwide. In combination with the intention of LifeLines to maximally protect the privacy of the participants, this aim is challenging and asks for novel solutions in ICT and data access procedures. Methods When the data are processed for data release, the dataset is stripped from all personal details to prevent (in-)direct identification (pseudonimized) and is moved to a new database in a separate geographic location. Even after this procedure re-identification is possible, in particular after linkage with other data sources. We consider the level of possible re-identification in each dataset by assessing the level of uniqueness of subjects in each dataset using k-anonimity analysis. Based on the outcomes of this analysis, the aggregation level of the variables that contribute to the re-identification are adjusted. Each researcher gets access to a tailor-made dataset with only those data that are specifically needed to answer the research question. The data are made available for researchers by means of a private virtual environment: the LifeLines workspace. Results the adjusted aggregation level are implemented in each data release. A survey is being conducted among researchers to investigate the impact of this aggregation on scientific findings. Conclusion LifeLines has developed an innovative ICT and data management solution based on adjustment of the aggregation level of data provided to researchers. This can be implemented in other biobank and cohort studies.

\section{Abstract \#: P 451}

\section{The cohort multiple randomized controlled trial design:} introducing staged-informed consent to avoid pre-randomization

Young-Afat D.A. ${ }^{1}$, Verkooijen H.M. ${ }^{2}$, Van Gils C.H. ${ }^{1}$, Elias S.G. ${ }^{1}$, Van der Velden J.M. ${ }^{3}$, Burbach J.P.M. ${ }^{3}$, Van Delden J.J.M. ${ }^{4}$, Relton C. ${ }^{5}$, Van Vulpen M. ${ }^{3}$, Van der Graaf R. ${ }^{4}$

${ }^{1}$ Department of Clinical Epidemiology, Julius Center for Health Sciences and Primary Care, University Medical Center Utrecht, Utrecht, Netherlands; ${ }^{2}$ Imaging Division, University Medical Center Utrecht, Utrecht, Netherlands; ${ }^{3}$ Department of Radiation Oncology, University Medical Center Utrecht, The Netherlands; ${ }^{4}$ Department of Medical Humanities, Julius Center for Health Sciences and Primary Care, University Medical Center Utrecht, Utrecht, Netherlands; ${ }^{5}$ School of Health and Related Research (Public Health section), University of Sheffield, Sheffield, United Kingdom; Corresponding author's e-mail: d.a.youngafat@umcutrecht.nl

The "cohort multiple Randomized Controlled Trial" (cmRCT) is a new design for pragmatic trials, which embeds multiple trials within a cohort. The cmRCT is an attractive alternative to conventional RCTs in fields where recruitment is difficult, multiple interventions have to be tested, where experimental interventions are highly preferred by physicians and patients and the risk of disappointment bias, crossover and contamination is considerable. In order to prevent most of these unwanted effects, the cmRCT design provides information on randomization to the intervention group/arm only, and only after they have been randomized (i.e. pre-randomization). To some this is not ethically acceptable, especially when applied in a hospital setting.

We propose a staged-informed consent procedure that leads to the avoidance of pre-randomization. In the first stage, at entry into the cohort, all potential participants are asked for their informed consent to participate in a cohort study and broad consent to be either randomly selected to receive an experimental intervention or to serve as control without further notice during participation in the cohort. In a second stage, at the initiation of an RCT within the cohort, informed consent to receive the experimental intervention is then only sought in those randomly selected for the intervention arm. At the third stage, after an RCT has been completed, all cohort participants receive aggregate disclosure of the results of the RCTs performed within the cohort (if they opt-into receive this information).

We applied the staged-informed consent model at our hospital in three ongoing cmRCT cohorts and found that it leads to promising recruitment rates. In these ongoing $\mathrm{cmRCT}$ cohorts including patients with rectal cancer (PICNIC, $\mathrm{n}=255$ ), bone metastases (PRESENT, $\mathrm{n}=370$ ) and breast cancer (UMBRELLA, $\mathrm{n}=515$ ), $85 \%$ of all rectal cancer patients who were asked to participate in the cohort were enrolled, $92 \%$ in the bone metastases cohort and $90 \%$ in the breast cancer cohort. Broad consent to be randomized was given by $87 \%$ of all cohort participants in PICNIC (rectal cancer), $84 \%$ in PRESENT (bone metastases) and $89 \%$ in UMBRELLA (breast cancer). These numbers show that asking broad consent for randomization works well in a clinical setting, especially since recruitment of patients into studies is known to be difficult in oncologic populations.

The staged-informed consent procedure avoids pre-randomization in cmRCT and aims to keep participants actively engaged in the research process.

\section{Abstract \#: P 452}

An efficient model for ethical review of epidemiological research: outside the scope of the medical research involving human subjects act

Peggy Manders ${ }^{1}$, Frans M. van $\mathrm{Agt}^{2}$, Evert-Ben van Veen ${ }^{3}$, Piet J. $\overline{\text { Slootweg }}^{4}$, Gerhard A. Zielhuis ${ }^{1}$

${ }^{1}$ Radboud Biobank, Radboudumc, Nijmegen; ${ }^{2}$ Research Ethics Committe, Radboudumc, Nijmegen; ${ }^{3}$ Med Law consult, the Hague; ${ }^{4}$ Dept. of Pathology, Radboudumc, Nijmegen; Corresponding author's e-mail: peggy.manders@radboudumc.nl

Only scientific research within the scope of the Dutch Medical Research Involving Human Subjects Act (WMO) is being reviewed by an accredited research ethics committee (REC). However, that is a minority of all scientific research in healthcare. Most epidemiological studies do not fit the criteria of the WMO.

The idea is to develop a system for all (epidemiological) studies currently not (sufficiently and formally) reviewed. Basically, there are four types of research that do not fit the WMO: (1) studies with personal data obtained in the context of healthcare, (2) studies with residual tissue, (3) biobanks and registries, both the installation of these research infrastructures as well as their use for specific studies, and (4) the feasibility of multicentre biobank initiatives, already approved at the coordinating centre, at the participating centre.

The Radboud university medical center, Nijmegen, developed a model with a 'REC-light' to deal with the formal assessment on these four types of situations in a way that warrants both quality and efficiency. REC-light is a research ethics committee authorized by the Board of Radboudumc with multidisciplinary senior expertise to review all non-experimental studies. With a standard 'fit-for-purpose' 
procedure, standard internet forms, automated procedures for standard situations and frequent meetings, the model should enhance research quality and is practical, quick, easy to handle and efficient.

The next step is to harmonize the procedure for multicenter studies in such a way that a review carried out by one REC-light is accepted by all participating centers. In the end these initiatives will serve quality of epidemiological studies, transparency and public trust in the discipline and prevent research waste.

\section{Abstract \#: P 453}

Environmental programming of respiratory allergy in childhood: the applicability of saliva

Sabine A.S. Langie ${ }^{1}$, Katarzyna Szarc vel $\mathrm{Szic}^{2}$, Ellen Van Der Plas ${ }^{1}$, Ken Declerck $^{2}$, Matthieu Moisse ${ }^{3}$, Gudrun Koppen ${ }^{1}$, Anne Schepers ${ }^{4}$, Guy Van Camp ${ }^{4}$, Diether Lambrechts ${ }^{3}$, Greet Schoeters ${ }^{1,2}$, Wim Vanden Berghe $^{2}$, Patrick de Boever ${ }^{1,5}$

${ }^{1}$ Environmental Risk and Health unit, Flemish Institute of Technological Research (VITO), Mol, Belgium; ${ }^{2}$ Department of Biomedical Sciences, University of Antwerp, Wilrijk, Belgium; ${ }^{3} \mathrm{VIB}$ Vesalius Research Center, KU Leuven, Campus Gasthuisberg, Leuven, Belgium; ${ }^{4}$ Laboratory of Cancer Research and Clinical Oncology, Center for Medical Genetics, University of Antwerp, Edegem, Belgium; ${ }^{5}$ Centre for Environmental Sciences, Hasselt University, Agoralaan gebouw D, Diepenbeek, Belgium; Corresponding author's e-mail: sabine.langie@ vito.be

Background: Environmental exposures during fetal and early life may contribute to complex disease later in life such as allergy. The underlying mechanisms are still undiscovered, but changes in the epigenetic regulation of gene expression (such as DNA methylation) is a prominent candidate mechanism. Longitudinal birth cohorts are instrumental to study the relation between early-life environmental factors and the development of complex diseases, but investigations are hampered since blood sampling in children is kept minimal for practical and ethical reasons. Saliva is suggested as an alternative DNA source because the samples can be obtained in a decentralized manner with less constraints. It is of interest to know how saliva DNA methylation patterns compare with the blood patterns, with the latter being considered as a reflection of systemic effects.

Methods: We hypothesize that prenatal exposures (environmental pollutants, mother's lifestyle) can alter fetal DNA methylation patterns, and thereby predispose the child to develop respiratory allergy (RA) later in life. Furthermore, we hypothesized that differential methylation regions (DMR) can be detected in saliva and blood of 11 years old RA cases $(n=20)$ when compared to healthy controls $(n=20)$. R-based software was used for data normalization and Comb-p tool to identify DMR. DNA methylation analyses were performed via Illumina Methylation 450K BeadChips.

Results: The methylation status between blood and saliva was comparable with about $11 \%$ of the probes having a differential methylation pattern (Padj $<.05$ and $\left.\left|\hat{\mathrm{I}}^{2}\right|>0.1\right)$, indicating that saliva is a suitable matrix for DNA methylation studies. Comparing allergy cases versus controls revealed 75 DMR in blood from 11 year olds. An overlap with DMR detected in cord blood and saliva revealed 2 DMR in common. These 2 plus 4 other DMR were selected for further biological and technical validation with bisulfite pyrosequencing in 60 additional cases of the same birth cohort as well as in an independent cohort $(\mathrm{N}=79)$.

Conclusion: This project will provide new levels of insight in the molecular mechanisms through which prenatal and early life environmental factors predispose children to RA. The use of saliva will simplify the assessment of the impact of environmental exposures on
DNA methylation patterns in human biomonitoring studies, especially for children where blood collection is often cumbersome. The ultimate goal of the project is to contribute to the development of prevention strategies, particularly in children, thereby reducing the family and societal burden associated with RA.

\section{Abstract \#: P 454}

\section{The perfluoroalkyl compounds on birth weight by GSTT1, GSTM1 and CYP1A1 polymorphisms}

/Eun Hee Ha/Eun Jin Kwon/Joon Soo Shin/Byung Mi Kim/Hyesook Park/Young lim Kho/Eun Ae Park/Young Ju Kim/

${ }^{1}$ Department of Preventive Medicine, School of Medicine, Ewha Womans University, Seoul, South Korea; /Department of Preventive Medicine, School of Medicine, Ewha Womans University, Seoul, South Korea; /Chicago Medical School, Rosalind Franklin University; Corresponding author's e-mail: eunheeha@ewha.ac.kr

Background: The aim of this study to investigated whether the association between prenatal exposure to perfluorooctanoic acid (PFOA), perfluorodecanoic acid (PFDA), perfluorotridecanoic acid (PFTrDA), and perfluorooctane sulfonate (PFOS) and birth weight differs by GSTT1, GSTM1, and CYP1A1 polymorphisms.

Methods: Our study investigated 255 pregnant women and their infants obtained from a retrospective cohort of Ewha Birth and Growth between 2006 and 2010. Information on birth outcomes were obtained from medical charts. Cord blood was analyzed for PFOA, PFDA, PFTrDA, and PFOS by high-performance liquid chromatography coupled with a triple quadrupole mass spectrometry (LC-MS/ MS). The glutathione S-transferase T1 (GSTT1), glutathione S-transferase M1 (GSTM1) null, and cytochrome P4501A1 (CYP1A1) Ile462Val polymorphisms were examined using PCR and PCR-RFLP methods. Multiple linear regression was assessed the association between the cord blood PFCs levels and birth weight according to GSTT1, GSTM1 and CYP1A1 polymorphisms.

Results: PFOS levels in cord serum showed a significantly negative association with birth weight (adjusted $\beta=-43.96, P=0.05$ ). For mothers with GSTT1 null type, PFDA levels were marginally significant associated with lower birth weight (adjusted $\beta=-161.62 \mathrm{~g}$, $P=0.06$ ). The birth weight of infants whose mothers had the CYP1A1 Val variants significantly decreased with PFOS exposure (adjusted $\beta=-129.21 \mathrm{~g}, P=0.04$ ).

Conclusion: This study suggests that GSTT1, GSTM1, and CYP1A1 polymorphisms may contribute to the inverse association between prenatal exposure to PFCs and birth weight.

\section{Abstract \#: P 455}

Study of the genetic polymorphism in the spoligotype Haarlem family using 4 loci MIRU-VNTR: MIRU04, MIRU40, ETRA and ETRC

\section{$\underline{\text { Ndamen Njabo Arlette }}^{1}$}

\section{${ }^{1}$ Assam Assam Jean Paul; Corresponding author's e-mail:} arletteange@yahoo.fr

Tuberculosis is a disease caused by mycobacteria of the Mycobacterium tuberculosis complex. It is the major cause of suffering and death in the world, with almost $95 \%$ of deaths occurring in low- and middle-income countries. The advent of molecular biology has seen new technologies that have revolutionized the search fields, prevention and control of tuberculosis. New molecular typing techniques 
have thus been developed including the MIRU-VNTR technique that has been the subject of this study.

The overall objective of this research was to study the genetic polymorphism in the Haarlem family by MIRU-VNTR typing technique using four loci: MIRU04, MIRU40, ETRA and ETRC. For this study, 33 isolates were selected at random from a Mycobacterium tuberculosis strain bank belonging to the Haarlem family previously characterized by spoligotyping. After amplification of each isolate, the PCR products were revealed by agarose gel electrophoresis at $3 \%$, the size of the amplicons was determined and the number of allele was assigned.

The results reveal allelic diversity of -0.03 for loci MIRU 04 , ETRA and ETRC and allelic diversity of 0.48 for the locus MIRU 40: two types of MIRU-VNTR profiles 2233 and 2333 in the proportion 48.5 and $51.5 \%$ respectively, and finally a discriminating power of 0.52 .

In conclusion, it appears that in the context of our study, the genetic diversity of the Haarlem family, although low, is indeed existing and needs to be investigated on a larger sample.

\section{Abstract \#: P 456}

Tuberculosis transmission between natives and immigrants in middle-income countries: the illustrative case of the city of São Paulo

Julia M. Pescarini $^{1}$, Vera Simonsen ${ }^{2}$, Rosângela S. Oliveira ${ }^{2}$, Sumie M Figueiredo $^{3}$, Regina R.G. Lemos ${ }^{3}$, Cirene Silva ${ }^{3}$, Naomi K. Komatsu $^{3}$, Lucilaine Ferrazoli ${ }^{2}$, Eliseu A. Waldman ${ }^{1}$

${ }^{1}$ Epidemiology Department, School of Public Health, University of São Paulo, São Paulo, Brazil; ${ }^{2}$ Instituto Adolfo Lutz, São Paulo, Brazil; ${ }^{3}$ São Paulo Municipal Department of Health, São Paulo, Brazil; Corresponding author's e-mail: juliapescarini@usp.br

Background: Like any other large city in middle-income countries, São Paulo has received a growing number of immigrants from countries with a high incidence of tuberculosis (TB), which has created a potentially favorable condition for a reversion of the TB declining tendency. This issue has been little investigated, thus a study to evaluate genotypic clustering of Mycobacterium tuberculosis (MTB) among Bolivian and Brazilian individuals (mixed clusters) and the potential TB cross-transmission between these two population groups was conducted.

Methods: A cross-sectional study was developed involving individuals with pulmonary TB residing in the city's districts with high proportions of cases among Latin American immigrants. We selected all cases confirmed by sputum culture regardless of their nationality. In Sao Paulo, TB diagnosis is confirmed by culture in individuals with risk factors for multi resistant TB (MR-TB) and socially vulnerable populations including immigrants. MTB isolates were tested for antimicrobial resistance by MGIT-960 and typed by RFLP-IS6110 analysis. We obtained sociodemographic, clinical and epidemiological information from the Sao Paulo Tuberculosis Control Program and laboratory information from Instituto Adolfo Lutz.

Results: The Human Development Index (HDI) of Sao Paulo is 0.805 , ranging from 0.432 to 0.799 in the 30 districts selected. We studied 330 individuals, of which $72.7 \%$ were male, $16.3 \%$ South American immigrants, $70.9 \%$ new TB cases, $27.9 \%$ co-infected with HIV-TB, $17.0 \%$ alcoholics, $20.9 \%$ drug addicts, and $65.8 \%$ on directly observed treatment. A total of $221 \mathrm{M}$. tuberculosis isolates were typed $(66.7 \%)$ and identified clusters consisting of 2-14 individuals. Similar profiles in individuals of the same nationality (simple clusters) were identified in $34.9 \%$ of Latin American immigrants and $39.9 \%$ in Brazilians. Mixed clusters were identified in 18.6 and $3.9 \%$ of Latin American immigrants and Brazilians, respectively. Conclusions: The proportion of mixed clusters found in our study is not negligible suggesting potential cross-transmission and socialization among immigrant and native populations. These data contrast with those reported in developed countries and stress out the need for different interventions consisting of not only specific measuresscreening for active and latent TB and expansion of DOTS coverage in socially vulnerable populations-but also intersectoral policies aimed at improved living conditions and income generation and health equity promotion in large cities of middle-income countries.

\section{Abstract \#: P 457}

Placental DNA methylation as a proxy for fetal neurodevelopment in association with in utero particulate air pollution

Nelly D. Saenen ${ }^{1}$, Bram G. Janssen ${ }^{1}$, Karen Vrijens ${ }^{1}$, Wim Vanden Berghe $^{2}$, Patrick De Boever ${ }^{1,3}$, Tim S. Nawrot ${ }^{1,4}$

${ }^{1}$ Centre for Environmental Sciences, Hasselt University, Diepenbeek 3590, Belgium; ${ }^{2}$ Department of Biomedical Sciences, PPES

Epigenetic Signaling Lab, University of Antwerp, Wilrijk 2610,

Belgium; ${ }^{3}$ Unit Environmental Risk \& Health, Flemish Institute for Technological Research, Mol 2400, Belgium; ${ }^{4}$ Department of Public Health \& Primary care, Leuven University (KU Leuven), Leuven 3000, Belgium; Corresponding author's e-mail: nelly.saenen@uhasselt.be

Background: The placenta contributes to fetal neurodevelopmental processes by transmitting adaptive responses to the maternal environment. Exposure to particulate air pollution during pregnancy affects fetal development due to a suboptimal intra-uterine environment. Epigenetic mechanisms, including promoter DNA methylation, could play an essential role in these developmental changes during early life. Within the ENVIRONAGE birth cohort, we investigated whether in utero exposure to fine particle air pollution in different periods of prenatal life is associated with differences in placental DNA methylation of neurodevelopmental genes at birth.

Methods: DNA methylation of the promoter region of the BrainDerived Neurotrophic Factor (BDNF) and Leptin (LEP) genes was measured in 391 placental tissue samples, randomly selected from the ENVIRONAGE birth cohort, using highly quantitative bisulfite-PCR pyrosequencing. Daily PM2.5 exposure levels were calculated for each participant's home address using a Kriging interpolation model (RIO) in combination with a dispersion model (IFMD).

Results: We fitted mixed-effect models to evaluate the associations between PM2.5 exposure and BDNF and LEP methylation. Independent of newborns gender, maternal age, maternal education, maternal smoking status, gestational age, $\mathrm{CpG}$ site and season, placental BDNF methylation was $0.36 \%$ higher (95\% confidence interval [CI]: $0.06,0.66 \%, p=0.02$ ) for an interquartile range (IQR) increment in PM2.5 during the second trimester of pregnancy. Independent of aforementioned covariates, we also found a $-1.2 \%$ lower placental LEP methylation associated with second trimester PM2.5 exposure (95\% CI: $-3.9,-0.5 \%: p=0.01$ ).

Conclusions: Placental promoter methylation of BDNF and LEP, two genes implicated in normal neurodevelopmental trajectories, are influenced by in utero exposure to PM2.5. Future studies should elucidate the significance of the PM2.5 impact on placental promoter methylation of the BDNF and LEP genes with respect to neurodevelopment later in life. 


\section{Abstract \#: P 458}

Energy restriction, genetic variants in the insulin-like growth factor pathway and colorectal cancer risk in The Netherlands cohort study

Colinda C.J.M. Simons ${ }^{1}$, Leo J. Schouten ${ }^{1}$, Roger Godschalk ${ }^{2}$, Manon van Engeland $^{3}$, Piet A. van den Brandt ${ }^{1}$, Frederik-Jan van Schooten ${ }^{2}$, Matty P. Weijenberg ${ }^{1}$

${ }^{1}$ Department of Epidemiology, GROW - School for Oncology and Developmental Biology, Maastricht University, Maastricht, the Netherlands; ${ }^{2}$ Department of Toxicology, NUTRIM - School for Nutrition and Toxicology, Maastricht University, Maastricht, the Netherlands; ${ }^{3}$ Department of Pathology, GROW - School for Oncology and Developmental Biology, Maastricht University Medical Center, Maastricht, the Netherlands; Corresponding author's e-mail: colinda.simons@maastrichtuniversity.nl

Background: Early life nutritional status may affect later life colorectal (CRC) cancer risk through effects on insulin-like growth factors. Using unique data from the Netherlands Cohort Study (NLCS, $n=120,852$ ), we investigated joint effects of early life energy restriction with genetic variants in the insulin-like growth factor (IGF) pathway on CRC risk.

Methods: The Netherlands Cohort Study was initiated in 1986. NLCS participants were 12-28 years old at the time of the Dutch Hunger winter (1944-1945). In particular living in a Western city during the Hunger Winter indicated severe energy restriction for $\sim 5$ months. The place of residence during this period and two other proxy variables for energy restriction were available from the baseline questionnaire. Participants also provided toenail clippings. Using a case-cohort approach and 16.3 years follow-up, toenail DNA was available for genotyping from 3768 subcohort members and 2580 CRC cases. Genotyping included 18 single nucleotide polymorphisms (SNPs) in eight genes in the IGF pathway, for which unfavorable alleles (potentially increasing CRC risk as based on literature) were aggregated into a genetic sum score. We estimated joint effects of early life energy restriction with the genetic sum score and an IGF1 19-CA repeat polymorphism in relation to sex- and subsite-specific CRC risks using Cox regression. To evaluate gene-environment $(\mathrm{GxE})$ interactions, the coding of the energy restriction variables was reversed, because exposure to energy restriction in combination with a low number of unfavorable alleles was hypothesized to be potentially beneficial.

Results: In the presence of an accumulation of unfavorable alleles, no exposure as compared to exposure to the Dutch Hunger Winter yielded increasingly higher CRC risks, especially colon cancer risks, in men (joint effect: $\mathrm{HR}=2.04,95 \% \mathrm{CI}$ : $1.39,3.00$ ). This risk pattern was less clear in women and when using the place of residence during the War Years (1940-1944) or the employment status of an individual's father during the Economic Depression (1932-1940) as proxy variables for energy restriction. The IGF1 19-CA repeat polymorphism halved CRC risk in women, irrespective of energy restriction. Tests for multiplicative and additive GxE interactions were not significant.

Conclusion: The risk pattern in these unique observational data indicates that the extent to which exposure to early life energy restriction influences CRC risk in later life in men might depend on genetic variation in the IGF pathway. However, GxE interactions were not significant.

\section{Abstract \#: P 459}

Birth weight and ambient air pollution: the role of mitochondrial DNA content

Diana B.P. Clemente ${ }^{1,2}$, Maribel Casas ${ }^{2}$, Jordi Sunyer ${ }^{2}$, Martine Vrijheid $^{2}$, Tim Nawrot ${ }^{1}$

${ }^{1}$ Centre for Environmental Sciences (CMK), Hasselt University, Diepenbeek, Belgium; ${ }^{2}$ Centre for research in environmental epidemiology (CREAL), Barcelona, Spain; Corresponding author's e-mail: diana.clementebatalhapardal@uhasselt.be

Background: Mitochondria, the energy producers of the cells, are particularly sensitive to environmental toxicants due to their lack of repair capacity. Fetus adapt their mitochondrial structure and metabolism when the supply of nutrients and oxygen is limited. Changes in mitochondrial DNA (mtDNA) content, may represent a biological relevant endpoint on the mechanisms underlying the association between air pollution and fetal growth restriction. In this study we investigated whether placental mtDNA content is a possible mediator of the association between prenatal NO2 exposure and birth weight. Methods: We used data from two independent European cohorts: INMA ( $\mathrm{n}=376$ : Spain) and ENVIRONAGE $(\mathrm{n}=550$ : Belgium). Relative placental mtDNA content was determined as the ratio of two mitochondrial genes (MT-ND1 and MTF3212/R3319) to two control genes (RPLP0 and ACTB). Effect estimated for individual cohorts and the pooled dataset were calculated using multivariate linear regression or mixed models. We also stratified by cohort and performed a mediation analysis.

Results: Each $10 \mu \mathrm{g} / \mathrm{m} 3$ increment in prenatal NO2 exposure during the entire pregnancy was associated with a relative decrease in placental mtDNA content of $5 \%$ [95\% confidence interval (CI): -9.3 , $-0.3 \%: \mathrm{n}=926]$. Placental mtDNA content was significantly and positively associated with birth weight (140 g: $95 \%$ CI: 43, $237 \mathrm{~g}$ : $\mathrm{n}=926$ ). For each $10 \mu \mathrm{g} / \mathrm{m}^{3}$ higher prenatal NO2 exposure birth weight decreased significantly with $48 \mathrm{~g}$ (95\% CI: $-87,-9 \mathrm{~g}$ : $\mathrm{n}=926$ ), particularly in INMA. Only in the INMA cohort, we found significant mediating effects of placental mtDNA content on the association between prenatal $\mathrm{NO}_{2}$ exposure and birth weight (proportion of mediation was $10 \%$ ).

Conclusion: Our results suggest that mtDNA content can be one of the potential mediators of the association between prenatal air pollution exposure and birth weight.

\section{Abstract \#: P 460}

Salivary extracellular fraction of miRNA candidates in association with recent exposure to ultrafine particles in school children

Annette Vriens ${ }^{1}$, Tim S. Nawrot ${ }^{1}$, Nelly Saenen ${ }^{1}$, Michal Kicinski ${ }^{1}$, $\overline{\text { Eline Provost }}^{1}$, Karen Vrijens ${ }^{1}$, Patrick De Boever ${ }^{2}$, Michelle Plusquin $^{3}$

${ }^{1}$ Centre for Environmental Sciences, Hasselt University, Diepenbeek, Belgium; ${ }^{2}$ Environmental Risk and Health, Flemish Institute for Technological Research (VITO), Mol, Belgium; ${ }^{3} \mathrm{MRC} / \mathrm{PHE}$ Centre for Environment and Health, School of Public Health, Imperial College, London, UK; Corresponding author's e-mail: annette.vriens@uhasselt.be 
Background: Intercellular communication via extracellular vesicles (EV) is influenced by external triggers such as growth factors, hypoxia, etc. which results in specific packaging of miRNAs into EVs. The transfer of these specific miRNAs can alter the phenotype of recipient cells and contributes to pathophysiological processes. Environmental stimuli, such as air pollution, may induce changes in cellular miRNA expression patterns. Studies in adults found evidence for alterations in miR-222 and miR-146a in response to particulate matter (PM) exposure on a cellular level. miR-222 functions in cell cycle and vascular biology, whereas miR-146a is important in inflammation. Recently, in an occupational exposed population, PM was shown to affect the miRNA content in EVs. We investigate the association of miRNA content in salivary EVs and acute changes in small particulates in 8-12 years old children.

Methods: Saliva was collected and stabilized for RNA preservation from 90 children at two different time points, circa 11 weeks apart. EVs in saliva were obtained by means of differential centrifugation and ultracentrifugation. The expression levels of miR-222 and miR$146 \mathrm{a}$ were profiled by qPCR. We regressed on the EV miRNA expression and recent exposure to ultrafine and fine particulates measured at the school site (i.e. class room and playground) by use of mixed models, while accounting for gender, age, BMI, exposure to second-hand smoke, maternal education, hours of television per week, time of the day and day of the week.

Results: Recent exposure to UFP was associated with an increase in miR-222 expression in EV in saliva. An IQR increase in indoor UFP concentration is associated with an $24.96 \%$ increase (95\% CI: $7.15-42.77 \%)$ in EV miR-222 levels $(p=0.0091)$. Similarly, an IQR increase in outdoor UFP concentration is associated with a $30.87 \%$ increase (95\% CI: 11.69-50.05\%) in miR-222 expression in salivary $\mathrm{EV}(p=0.0022)$. No associations between EV miR-146a content and UFP exposure was found. On the other hand, recent exposure to larger particles (PM2.5) was not found to induce changes in miR-222 or miR-146a expression in salivary vesicles.

Conclusion: Children attending school on days with higher ambient concentrations of ultrafine particulates (diameter smaller than $300 \mathrm{~nm}$ ) had higher expression in saliva of miRNA-222, but not miR$146 \mathrm{a}$, in extracellular vesicles. This rapid response was independent of exposure to larger particulates, suggesting that UFP exposure is particularly relevant in the process of rapid adaptations of EV miRNA's. This implies that UFP exposure can influence cell-cell communication.

\section{Abstract \#: P 461 \\ Epigenome-wide DNA methylation profiles of exposure to ambient air pollution}

Michelle Plusquin $^{1}$, Florence Guida ${ }^{1}$, Gianluca Campanella ${ }^{1}$, Roel Vermeulen $^{1,2,3}$, Marc Chadeau-Hyam ${ }^{1,2}$, Paolo Vineis ${ }^{1,4}$, On behalf of the EPIC-Italy and EPIC-NL consortium

${ }^{1}$ MRC-PHE Centre for Environment and Health, Department of Epidemiology and Biostatistics, School of Public Health, Imperial College London, Norfolk Place, London W2 1PG, UK; ${ }^{2}$ Institute for Risk Assessment Sciences, Utrecht University, Utrecht, The Netherlands; ${ }^{3}$ Julius Center for Health Sciences and Primary Care, University Medical Center Utrecht, Utrecht, The Netherlands; ${ }^{4} \mathrm{HuGeF}$, Human Genetics Foundation, Torino, Italy; Corresponding author's e-mail: m.plusquin@imperial.ac.uk

Background: Outdoor air pollution has been associated with increased mortality and morbidity of cardiovascular, respiratory diseases and cancers. It is a complex mixture of toxicants with several pathways of action that are not fully understood yet. There is evidence that exposure to particulate matter induces changes in DNA methylation. However results from untargeted genome wide approaches are currently lacking.

Methods: In this study, we performed epigenome-wide association studies (EpWAS) nested in a prospective healthy cohort with participants from Italy $(n=457)$ and the Netherlands $(n=167)$, by means of the Illumina 450K Methylation assay. Long-term air pollution exposure estimates of PM2.5, PM10, PM2.5abs, NOx and $\mathrm{NO}_{2}$ were calculated using exposure models developed within the European Study of Cohorts for Air Pollution Effects (ESCAPE). Beta regression was conducted using a confounder sets (age, gender, smoking, chip and position on chip being a cancer case later in life) and controlling for a family wise error of $5 \%$.

Results: Exposure to $\mathrm{NOx}$ and $\mathrm{NO}_{2}$ was inversely associated with somatic global methylation, calculated by taking the average of the methylation of the somatic probes. In locus-by-locus comparative analyses, one $\mathrm{CpG}$ site (c16orf63) showed significant hypomethylation in association with $\mathrm{NOx}$ and $\mathrm{NO} 2$. On the other hand the methylation of $12 \mathrm{CpG}$ sites was significantly associated with exposure to PM2.5, PM10, PM2.5abs. We observed a hypomethylation of the tumor suppressor gene MGMT (O-6-Methylguanine-DNA Methyltransferase) with PM as well as with NOx.

Conclusion: Our result suggests that NOx exposure is associated with a global hypomethylation of the genome. At the gene-specific level we can identify several CpG sites whose methylation is modified by air pollution. They can contribute to further understand the biological processes that are induced by air pollution exposure.

This research was supported by a Marie Curie Intra European Fellowship within the 7th European Community Framework Programme (62885).

\section{Abstract \#: P 462}

Prevalence of $\boldsymbol{H}$. pylori infection in a population of Italians and immigrants in Rome and rate of response to therapy

Nosotti Lorenzo $^{1}$, Petrelli Alessio ${ }^{1}$, Caruso Anna ${ }^{1}$, Rossi Alessandra ${ }^{1}$, Costanzo Gianfranco $^{1}$, Fortino Antonio ${ }^{1}$, Mirisola Concetta ${ }^{1}$

${ }^{1}$ National Institute for Health, Migration and Poverty (NIHMP); Corresponding author's e-mail: nosotti@inmp.it

Background: It is estimated that Helicobacter Pylori (HP) infection affects approximately half of the world's population, causing diseases such as gastritis, peptic ulcer and gastric neoplasms.

Although its impact in developed countries has been reduced over the last decades, the prevalence of the disease remains high, equal to $10-20 \%$ in individuals under the age of 50 and to $40-50 \%$ in individuals over the age of 50 .

However in developing countries, the prevalence of the infection is higher compared to developed countries (reaching up to $90 \%$ in some African and Asian countries).

Aims of the study are: (1) to assess the prevalence of HP infection in a population of Italians and immigrants with chronic dyspepsia, (2) to evaluate the rate of responders to first line therapy.

Methods: Between January 2014 and November 2014, 366 patients with dyspeptic symptoms attending the outpatient clinic of NIHMP were visited.

Among these, 311 were submitted to urea breath test (UBT) for the diagnosis of HP infection.

The distribution by gender demonstrates a slight prevalence of males $(51.4 \%)$ with a mean age of 43.1 years. The distribution of patients, related to the geographical areas of origin, was the following: Europe $50.2 \%$ (of which Italy was $23.5 \%$ ), Africa $29.3 \%$, Asia $10 \%$, South America $10.6 \%$. 
HP positive patients after UBT were submitted to first line therapy.

First line therapy was differentiated on the basis of the country of origin: individuals from countries with a high percentage $(>20 \%)$ of antibiotic resistance to clarithromycin (Europe, Asia) were treated with sequential therapy, whereas individuals from countries with a low percentage $(<20 \%)$ of antibiotic resistance to clarithromycin (Africa, America) were treated with standard triple therapy with clarithromycin.

Results: Among the 311 patients submitted to UBT, 188 (67.6\%) were HP positive, and among these 163 began a treatment cycle.

Among the 101 patients that were examined post-therapy, the overall response rate was $75.2 \%$, of which $80.6 \%$ concerned sequential therapy and $78.3 \%$ standard triple therapy.

Conclusions: The prevalence of HP infection in the cohort studied is high $(67.6 \%)$ : this confirms that HP infection represents one of the main causes of chronic dyspepsia.

The low response rate to sequential and standard triple therapy highlights the need to individuate more efficient therapy schemes in order to eradicate HP infection and to go beyond the bacterial resistances to antibiotic therapy, that appeared in geographical areas where they have never been before.

\section{Abstract \#: P 463}

\section{Poliomyelitis in the population health of Pakistan}

\section{Faheem Ahmed $^{1}$}

${ }^{1}$ Partnership in Global Health Society; Corresponding author's e-mail: fah_khan@yahoo.com

The government of Pakistan is committed to erdicate the poliomylitis in partnership with the World Health Organization (WHO), International Monetary Fund (IMF) and the World Bank. This paper outlines poliomyelitis situation in Pakistan and examines the current public health prevention and control of poliomyelitis in order to protect global community. We comments to meet the challenges of preventive service provision, achievement of millennium development goals and polio end game strategic plan, Pakistan needs to improve health status of its society by priority setting partnership, health promotion, vaccination, health system, ownership, honesty, altruitism, primary health care etc. in providing health care services.

\section{Abstract \#: P 464}

\section{Surveillance of infectious diseases in the south of Germany 2014}

Iris Zoellner $^{1}$, Fee Koehler ${ }^{1}$, Dorothee Lohr ${ }^{1}$, Corinna Langhans ${ }^{1}$, Elisabeth Aichinger $^{1}$

${ }^{1}$ Baden-Wuerttemberg State Health Office, Stuttgart, Nordbahnhofstr. 135, D-70191 Stuttgart, Germany; Corresponding author's e-mail: Iris.Zoellner@rps.bwl.de

Introduction: The amount of data which has to be analysed for the surveillance of notifiable infectious diseases is substantial and specific graphs can be a help when a quick overview is needed. The aim of the graphical summary of aggregated data in weekly reports is the early detection of outbreaks.

Methods: Since the numbers of notified cases per week are not necessarily normally distributed, a method for the analysis and visualisation of infectious disease data was developed. The weekly updated graphs are easy to read and interpret since they allow a direct visual comparison of current data with the data observed in the five preceding years. The method can be applied to all notifiable infectious disease categories and is independent of the distribution of notifications. Many notifiable infections shows different seasonal trends which can be shown in the graphs. The visualisation of data includes the range of notified cases per week observed in preceding years and current data.

Results: This method will be shown for several notifiable infectious diseases: salmonellosis, giardiasis, listeriosis, cryptosporidiosis, hepatitis B, and Hanta virus infections in Baden-Wuerttemberg 2014 and compared to results in outbreak situations. The STEC outbreak detected in 2011 in the Southwest of Germany was part of a nationwide epidemic caused by contaminated sprouts. Although most cases of the epidemic were observed in the North of Germany, where the sprouts were produced, the outbreak also reached the Southwest due to travelling activities and (at least) one restaurant, where the sprouts from the North were served for diner.

Conclusion: Surveillance graphs have proved successful in practice as an approach to detect disease clustering in time. The method presented here is used in weekly reports for Baden-Wuerttemberg in the surveillance of about twenty categories of notifiable diseases.

\section{Abstract \#: P 465}

\section{Epidemiology of HIV infection in a city of Semey (former} Semipalatinsk) in 2009-2014

Saule Maukayeva ${ }^{1}$, Daria Shabdarbaeva ${ }^{1}$, Bakhyt Alimbekova ${ }^{1}$, Nurlan Saykenov $^{1}$, Saulesh Apbasova ${ }^{1}$, Akmaral Zhakipova ${ }^{1}$, Ludmila Pivina ${ }^{1}$, Zhanna Mussabekova ${ }^{1}$, Andrej M. Grjibovski ${ }^{2,3}$

${ }^{1}$ Semey State Medical University, Semey, Kazakhstan; ${ }^{2}$ Norwegian Institute of Public Health, Oslo, Norway; ${ }^{3}$ Northern State Medical University, Arkhangelsk, Russia; Corresponding author's e-mail: andrej.grjibovski@gmail.com

Background: Nearly 78 million people have been infected HIV and about 39 million people have died of HIV since the beginning of the epidemic worldwide. The burden of HIV greatly varies between countries and regions However, the information about HIV infection in Kazakhstan is relatively scarce and often limited to Alma-Ata and Astana warranting studies in other parts of the country. The aim of this study was to summarize the information about HIV infection in the region.

Methods: This is a descriptive records-based study conducted in a city of Semey (former Semipalatinsk, population 314,000 in 2013), East Kazakhstan. Data were obtained from the anonymized records at the municipal HIV Center from 2009 to 2014.

Results: Altogether there were 288 new cases of HIV in Semey since the first case was registered in 1997 and 32 of them died. The incidence of HIV slightly increased from 8.6 per 100,000 in 2009 to 9.0 per 100,000 in 2014. The prevalence of HIV was 50.3 per 100,000 in 2014. More than a half of cases were aged 20-29 years (51.7\%) and $30-39$ years $(37.0 \%)$ in 2009 while in 2014 the corresponding proportions were 44.0 and $28.0 \%$. Heterosexual transmission was the most common (55\%) way of transmission in 2014 followed by parenteral $(31 \%)$ while in 2009 parenteral transmission dominated $(77.8 \%)$ with heterosexual transmission being the second most common $(18.5 \%)$. Men comprised $88.9 \%$ of all new cases in 2009 and $48.0 \%$ in 2014 . The proportion of unemployed among incident cases decreased from $77.8 \%$ in 2009 to $41.0 \%$ in 2014. Altogether, four infants were infected from 1997 to 2014 (vertical transmission$75 \%$, parenteral-25\%.

Conclusions: Although the data suggest that Semey is the setting with a low prevalence of HIV it may soon face a rapid HIV spreading among general population unless the appropriate preventive strategies are applied in the near future. The pattern of HIV infection in Semey 
is changing from being the most common among young male intravenous drug users to women with heterosexual way of transmission. More detailed data on the epidemiology of HIV in the region and the reasons behind the data will be discussed.

\section{Abstract \#: P 466 \\ Voluntary counseling and HIV testing (VCCT) aimed at preventing mother to child HIV transmission}

$\underline{\text { Biljana Kocic }}^{1}$, Branislav Petrovic ${ }^{1}$

${ }^{1}$ Faculty of Medicine University of Nis, Public Health Institute Nis; Corresponding author's e-mail: biljaizzz@yahoo.com

Background: VCCT has been accepted in developed and in majority of developing countries, and became part of a standard package of prevention strategies. People, who are offered personalized VCCT intervention are likely to reduce their practice of risky sexual and other HIV-related behaviours, and prevent HIV infection. At the end of 2004, voluntary confidential counseling and testing service (VCCT) for HIV was established in Nis, in the Public Health Institute (PHI). A great effort was made to promote this service and to disseminate information to the community in order to facilitate utilization of the service. Four thousand and five hundred clients were voluntary, free of cost, counseled and tested at PHI. The aim of the paper was to explore the prevalence of pregnant women among clients attending VCCT center in Nis from 2004 to 2014, and to monitor the trend and outcome of their testing aimed at preventing MTCT.

Methods: The Register of the clients was used as a study matherial. Results: In the observed period, 1187 pregnant women, during the first 3 months of pregnancy and for the first time, were counseled and tested for HIV. In 2004, eight of them were tested. Majority of them were tested in 2005 (667) and 2006 (504), due to collaboration with their gynecologists and the promotion of opt out testing of pregnant women as a part of Global fund project which took part in Serbia. Implementation of this project, without other capacities and financing available (for tests and etc.), with also resistance towards this intervention by some health providers reported, resulted in decrease of testing in years to follow (from 2007 to 2011 eight pregnant women were tested, and none from 2012 to 2014). Within the observed period, one HIV infected pregnant woman was registered and received antiretroviral therapy for PMTCT and gave birth to a healthy, HIV-free child. In 2014, one HIV infected child was registered due to MTCT.

Conclusion: Despite great value of HIV testing of pregnant women and availability of HIV treatment since 2000 , the rate of testing is negligible. It is important to increase collaboration with other health providers, especially gynecologists, in order to increase coverage of HIV tested pregnant women and prevent MTCT.

\section{Abstract \#: P 467}

\section{Diphtheria antibody seroprevalence among adults in Baden-} Wuerttemberg 2010/2011

Iris K. Zoellner $^{1}$, Christiane Wagner-Wiening ${ }^{1}$, Silke Fischer ${ }^{1}$, Doris Reick $^{1}$

${ }^{1}$ Epidemiology, Baden-Wuerttemberg State Health Office, Stuttgart, Germany; Corresponding author's e-mail: Iris.Zoellner@rps.bwl.de

Introduction: Recent data on diphtheria antibody seroprevalence among adults in Germany are rare. A laboratoy study with blood samples from patients of medical doctors from 1991 to 1993 revealed a negative relationship of seropositivity to age. The following questions should be answered by our study among adults in BadenWuerttemberg which is located in the Southwest of Germany: Is there a decrease in diphtheria antibody prevalence with age? How many adults received a vaccination against diphtheria during the last 10 years before investigation?

Methods: A purpose of our study was to estimate the diphtheria antibody seroprevalence among civil servants in Baden-Wuerttemberg who took part in a study conducted by ten local health offices and the Baden-Wuerttemberg State Health Office in 2010/2011. The study partici-pants were asked to complete a self-administered questionnaire and to donate a blood sample. Among other laboratory tests, diphtheria antibody seroprevalence was determined by the laboratory of the State Health Office in Stuttgart.

Questionnaire data and laboratory results were analysed using the statistical software package of SAS Institute, Cary, version 9.3.

Results: The following health offices in Baden-Wuerttemberg took part in the investigation: Aalen, Boeblingen, Konstanz, Kuenzelsau, Loerrach, Ludwigsburg, Offenburg, Ravensburg, Rastatt, and Waldshut-Tiengen. In total, 1396 study participants completed the question-naire and 1356 blood samples could be serologically analysed and evaluated. The follow-ing seroprevalences of diphtheria antibodies were found: $89-94 \%$ in the age group younger than 30 years, $88-97 \%$ in the age group $30-39$ years, $75-86 \%$ in 40-49 year-olds, 58-72\% in 50-59 year-olds, and 50-65\% among study participants above 60 years of age, respectively. When asked for diphtheria vaccination within the last 10 years before the in-vestigation $71 \%$ of the age group younger than 30 years reported vaccination ( $18 \%$ did not know if they received vaccination against diphtheria in the last decade), $61 \%$ of the age group 30-39 years (19\% did not know), $59 \%$ of the age group 40-49 years (13\% did not know), $50 \%$ of the age group 50-59 years (13\% did not know), and $47 \%$ of the age group above 60 years of age (12\% did not know), respectively.

Conclusion: The seroprevalence of diphtheria antibodies among study participants decreased substan-tially with increasing age. This could be relevant for public health even if diphtheria cases are rare in Germany. Since the investigation was restricted to civil servants the results may not be representative for the whole population of BadenWuerttemberg. On the other hand, they give a first insight into the current distribution of the immune status among adults in the Southwest of Germany.

\section{Abstract \#: P 468}

Prevalence of healthcare-associated infections in four pediatric hospitals in northwest Russia in 2014

Ekaterina Krieger $^{1}$, Olga Samodova ${ }^{1}$, Daria Shabdarbayeva ${ }^{2}$, Saule Maukayeva ${ }^{2}$, Andrej M. Grjibovski ${ }^{2,3}$

${ }^{1}$ Northern State Medical University, Arkhangelsk, Russia; ${ }^{2}$ Semey State Medical University, Semey, Kazakhstan; ${ }^{3}$ Norwegian Institute of Public Health, Oslo, Norsway; Corresponding author's e-mail: andrej.grjibovski@gmail.com

Background: Hospital-acquired infections (HAI) represent a significant public health problem reducing the quality of hospital care and increasing the cost of treatment. Surveillance of healthcare-associated infections (HAI) in Russia has been considered to suffer from underreporting and use of questionable criteria complicating international comparisons and warranting development and implementation of alternative surveillance measures such as regular point-prevalence surveys to improve hospital infection control. The 
aim of this study was to assess the prevalence of HAI in 4 pediatric hospitals in Northwest Russia in 2014.

Methods: One-day point-prevalence surveys were conducted in four pediatric hospitals in March and in December 2014 in cities of Arkhangelsk (population 350,000) and Severodvinsk (population 187,000). A CDC definition of HAI was applied to ensure international comparability of the findings. All patients staying in the hospital for more than $48 \mathrm{~h}$ at 9 a.m. on the day of the survey were included. The data are presented with $95 \%$ confidence intervals calculated using Wilson's method.

Results: Altogether, 1134 inpatients were involved. The highest prevalence of HAI was observed in the pediatric department of the Arkhangelsk regional hospital where $8.3 \%$ (95\% CI: 2.3-25.8) patients had HAI in February and $13.9 \%$ (95 \% CI: 6.1-28.7) in December while the lowest prevalence was observed in Severodvinsk municipal pediatric hospital where $4.1 \%$ (95\% CI: 2.2-7.7) and $0.7 \%$ (95\% CI: 0.3-2.2) were infected in March and in November 2014, respectively. The prevalence of HAI in the pediatric unit of Arkhangelsk municipal hospital was $8.6 \%$ (95\% CI: 8.0-22.4) in February and $7.1 \%$ (95 \% CI: 2.5-19.0) in December 2014. In the regional pediatric hospital the prevalence was $6.5 \%(95 \% \mathrm{CI}$ : 4.2-10.1) in February and $6.7 \%$ (95\% CI: 4.5-9.9) in December. The most common HAI recorded in pediatric hospitals were upper respiratory tract infections (45\%), followed by viral gastroenteritis $(22 \%)$ and urinary tract infections (22\%).

Conclusions: The overall prevalence of HAI in the two Northwestern Russian cities is comparable with the findings from other European settings. Moreover, we observed seasonal variation in HAI and identified the most common types of HAI. Further research is needed to identify risk factors for HAI in Russia. Our results should be interpreted with caution because of considerable differences between hospitals in the country. Development and implementation of universal surveillance system of HAI and use of international definitions of HAI in Russia is also warranted.

\section{Abstract \#: P 469}

\section{Genetic drift of influenza A viruses in a subtropical city Hong Kong, 2013-2014}

Pei-Hua Cao ${ }^{1}$, Lin Yang ${ }^{2}$, Kwok-Hung Chan ${ }^{3}$, Leo Lit-Man Poon ${ }^{1}$, King-Pan Chan ${ }^{1}$, Chit-Ming Wong ${ }^{1}$

${ }^{1}$ School of Public Health, The University of Hong Kong; ${ }^{2}$ School of Nursing, The Hong Kong Polytechnic University; ${ }^{3}$ Department of Microbiology, The University of Hong Kong; Corresponding author's e-mail: cphcc@hku.hk

Background: Influenza is an infectious disease that causes significant morbidity and mortality and shows distinct seasonal patterns under different climates. In subtropical and tropical regions such as Hong Kong, influenza shows less clear seasonal patterns, with multiple peaks observed throughout the year. Antigenic variation of influenza viruses has been proposed to play an important role in regulating influenza seasonality.

Methods: We conducted a comprehensive study to understand the characterization of the antigenicity and explore the temporal trend of antigenic changes of influenza $A$ virus (both $\mathrm{A} / \mathrm{H} 1 \mathrm{~N} 1$ and $\mathrm{A} / \mathrm{H} 3 \mathrm{~N} 2$ subtypes) in Hong Kong from January 2013 to May 2014. Clinical samples from 1038 patients with confirmed $\mathrm{A} / \mathrm{H} 1 \mathrm{~N} 1$ and $\mathrm{A} / \mathrm{H} 3 \mathrm{~N} 2$ influenza viruses were collected from two public hospitals in Hong Kong during the study period.

Results: 290 specimens were randomly selected for a hemagglutinin amino acids (HA) sequence analysis. Based on sequence analysis, all influenza viruses for both $\mathrm{A} / \mathrm{H} 1 \mathrm{~N} 1$ and $\mathrm{A} / \mathrm{H} 3 \mathrm{~N} 2$ subtypes were characterized by at least 5 fixed amino acid mutations for A/H1N1: D114, N138S, K180Q, I251V, and A273T, and at least 3 fixed amino acid mutations for A/H3N2: Y110H, T144A, and R158Q, with respect to WHO recommended vaccine composition strains A/California/07/ 2009(for A/H1N1) and A/Victoria/361/2011 (for A/H3N2) in 2013-2014. We further randomly selected 111 specimens (58 $\mathrm{A} / \mathrm{H} 1 \mathrm{~N} 1$ and $53 \mathrm{~A} / \mathrm{H} 3 \mathrm{~N} 2$ ) for hemagglutinin-inhibition (HI) test. All the $\mathrm{HI}$ titer results for both $\mathrm{A} / \mathrm{H} 1 \mathrm{~N} 1$ and $\mathrm{A} / \mathrm{H} 3 \mathrm{~N} 2$ were over 320 , which indicated that there were no significant differences between our samples and the reference WHO strains. We compared the HI titer results among different age groups $(<18,18-65$, and $>65$ years $)$, and no significant differences were found. (A/H1N1: $P=0.643$ : A/H3N2: $P=0.844$ ).

Conclusion: The substitutions observed in sequence analysis suggested that there were antigenic drifts of the influenza A (both $\mathrm{A} / \mathrm{H} 1 \mathrm{~N} 1$ and $\mathrm{A} / \mathrm{H} 3 \mathrm{~N} 2$ subtypes) viruses. Our study may contribute to develop the knowledge of the evolution of influenza A virus circulated in Hong Kong.

\section{Abstract \#: P 470}

Prevalence and associated factors of single anorectal chlamydia/gonorrhoea in women and men who have sex with men reporting anal sex/symptoms

Geneviève A.F.S. van Liere, ${ }^{1,2}$, Martijn S. van Rooijen ${ }^{3}$, Christian J.P.A. Hoebe ${ }^{1,2}$, Titia Heijman ${ }^{4}$, Henry J.C. de Vries ${ }^{5,6,7,8}$ Nicole H.T.M. Dukers-Muijrers ${ }^{1,2}$

${ }^{1}$ Department of Sexual Health, Infectious Diseases and Environmental Health, Public Health Service South Limburg, Geleen; ${ }^{2}$ Department of Medical Microbiology, School of Public Health and Primary Care (CAPHRI), Maastricht University Medical Center (MUMC+), The Netherlands; ${ }^{3}$ Public Health Laboratory, Department of Research, STI Outpatient Clinic Public Health Service of Amsterdam (GGD Amsterdam), Amsterdam, The Netherlands; ${ }^{4}$ Department of Research, STI Outpatient Clinic Public Health Service of Amsterdam (GGD Amsterdam), Amsterdam, The Netherlands; ${ }^{5}$ STI Outpatient Clinic Public Health Service of Amsterdam (GGD Amsterdam), Amsterdam; ${ }^{6}$ Department of Dermatology, Academic Medical Center (AMC), University of Amsterdam, Amsterdam; ${ }^{7}$ Centre for Infection and Immunology Amsterdam (CINIMA), Amsterdam; ${ }^{8}$ Centre for Infectious Disease Control, National Institute of Public Health and the Environment, Bilthoven, The Netherlands; Corresponding author's e-mail: genevieve.vanliere@ggdzl.nl

Background: Anorectal chlamydia infections are common in both men who have sex with men (MSM) and women. Undetected infections could lead to further spread into the population and development of sequelae within individuals. Anorectal chlamydia infections can be single infections or concurrent anorectal/urogenital infections. Concurrent infections can be coincidentally treated. Insight in associations between single anorectal infections and demographic and behavioural factors can be useful to determine high prevalence groups to guide anorectal testing.

Methods: At the outpatient STI clinics of Amsterdam and South Limburg from January 2011-December 2012, all MSM $(\mathrm{n}=9549)$ and women $(\mathrm{n}=11,113)>16$ years who were tested for chlamydia $(\mathrm{Ct})$ and/or gonorrhoea $(\mathrm{Ng})$ at both urogenital and anorectal sites were included $(n=20,662$ unique individuals). Data included a standardised medical and sexual history, including demographic information, self-reported symptoms and sexual behaviour in the past 6 months. Associated factors for single infections were assessed using univariable and multivariable logistic regression. 
Results: In MSM, anorectal Ct/Ng prevalence was $9.8 \%$ (693/7094)/ $4.2 \%(397 / 9534)$, in women this was $9.5 \%(439 / 4597) / 0.9 \%$ (96/ 10,972) $(P=0.86 / P<0.001)$ respectively. Among positives, single anorectal $\mathrm{Ct} / \mathrm{Ng}$ infections were found in $85.9 \%(595 / 693) / 85.6 \%$ (340/397), in women this was $22.1 \%(97 / 439) / 20.8 \%(20 / 96)$ respectively. In MSM, not being sex worker (OR 0.4, CI 0.2-1.0), exclusively having sex with men (OR 3.4, CI 1.7-6.8) and not having urogenital symptoms (OR 0.2, CI 0.2-0.4) were independent factors for a single anorectal $\mathrm{Ct}$ infection. For women these were older age ( $\geq 28$ years) (OR 1.8, CI 1.0-3.2) and non-Western nationality (OR 2.1 , CI 1.1-3.9). Independent factors for a single anorectal $\mathrm{Ng}$ infection in MSM were warned by an (ex)partner (OR 2.9, CI 1.1-7.5), oropharyngeal $\mathrm{Ng}$ infection (OR 2.4, CI 1.0-5.3) and not having urogenital symptoms (OR 0.02 , CI $0.01-0.04$ ). In women no factors were independently associated with a single anorectal $\mathrm{Ng}$ infection.

Conclusion: Prevalence of anorectal Ct was substantial with $10 \%$ in both MSM and women. The majority of MSM had single anorectal $\mathrm{Ct} / \mathrm{Ng}$ infections, while in women the majority had concurrent urogenital infections. Given the diversity of factors and lack of strong indicators for single infections, this study emphasizes the need for anorectal testing in both MSM and women who report receptive anal sex and/or anal symptoms.

\section{Abstract \#: P 471 \\ Pandemrix vaccination or infection with pandemic influenza A(H1N1)PDM09 and risk of Guillain-Barré syndrome: a nationwide population-based cohort study}

Sara Ghaderi $^{1}$, Nina Gunnes ${ }^{2}$, Inger Johanne Bakken ${ }^{2}$, Per Magnus ${ }^{3}$, $\overline{\text { Lill Trogstad }}^{4}$, Siri Eldevik Håberg ${ }^{5}$

${ }^{1}$ Department of Health Registries, Division of Epidemiology, Norwegian Institute of Public Health, Bergen, Norway; ${ }^{2}$ Department of Genes and Environment, Division of Epidemiology, Norwegian Institute of Public Health, Oslo, Norway; ${ }^{3}$ Institute management and staff, Norwegian Institute of Public Health, Oslo, Norway;

${ }^{4}$ Department of Vaccines, Division of Infectious Disease Control, Norwegian Institute of Public Health, Oslo, Norway; ${ }^{5}$ Institute management and staff, Norwegian Institute of Public Health, Oslo, Norway; Corresponding author's e-mail: Sara.Ghaderi@fhi.no

Introduction: Guillain-Barré syndrome (GBS) is a rare but serious disease in which the immune system attacks nerve cells causing muscle weakness, paralysis, and in some cases death. GBS may be triggered by respiratory infections such as influenza. Concerns regarding development of GBS after influenza vaccination were first raised during the influenza epidemic in 1976-1977 in the United States. Studies of vaccinations and GBS show contradictory results, and only few studies have considered the role of influenza infection. The aim of the current study was to explore the association between exposure to influenza A(H1N1)pdm09 infection or Pandemrix vaccination and occurrence of GBS during the pandemic season.

Methods: The total Norwegian population in 2009 was included as our study cohort. Information regarding vaccinations, influenza infections, and GBS diagnoses were obtained from national health registries: the Norwegian Immunisation Registry (SYSVAK), the Directorate of Health for reimbursement data (KUHR), the Norwegian Surveillance System for Communicable Diseases (MSIS), and the Norwegian Patient Register (NPR). Self-controlled case series (SCCS) and Cox regression analyses with time-varying covariates were used to estimate the risk of GBS in 42-day periods following influenza $\mathrm{A}(\mathrm{H} 1 \mathrm{~N} 1)$ pdm09 infection or vaccination with Pandemrix.
Results: At the onset of the influenza pandemic in 2009, there were $4,832,211$ individuals residing in Norway. An influenza diagnosis was recorded for $3 \%$ of the population while vaccination coverage in the total population was $39 \%$. There were 410 new GBS cases registered after October 1, 2009 of which 46 new cases occurred during the peak period of the influenza pandemic. The incidence rate ratio (IRR) of GBS during the pandemic peak as estimated by SCCS was significantly elevated when compared with the other periods [IRR: $1.46,95 \%$ confidence interval (CI): 1.08-1.98]. The adjusted hazard ratio (HR) of GBS after an influenza diagnosis was 4.89 (95\% CI: 1.17-20.36) and the HR after vaccination was 1.11 (95\% CI: $0.51-2.43)$.

Conclusion: Our results indicated that there was a significantly increased risk of GBS during the pandemic season and after pandemic influenza infection. Infection with pandemic influenza was associated with an approximately fivefold increased risk of GBS. Vaccination with Pandemrix, on the other hand, was not associated with an increased risk of GBS.

\section{Abstract \#: P 472}

\section{Impact of 2014/2015 influenza season in Alentejo region of Portugal}

Eleonora Paixão ${ }^{1}$, Paula Valente ${ }^{2}$, Ana Mendes ${ }^{1}$, Sandra Guerra ${ }^{1}$, Anabela David $^{1}$, José Marques Robalo ${ }^{3}$

${ }^{1}$ Alentejo Regional Administration of Health, Technical support unit for the areas of planning, investments and statistics; ${ }^{2}$ Alentejo Regional Administration of Health, Department of Public Health; ${ }^{3}$ Alentejo Regional Administration of Health, Directive Council; Corresponding author's e-mail: eleonora.paixao@arsalentejo.minsaude.pt

Background: Influenza activity in Europe started week 50/2014 with subtype $\mathrm{A}(\mathrm{H} 3 \mathrm{~N} 2)$ viruses as dominant. In Portugal was reported medium intensity of influenza activity in week 1/2015 to European Centre for Disease Prevention and Control, with B as dominant subtype.

The main purpose of study is early estimation of impact 2014/2015 influenza season in excess mortality, consultations in primary healthcare, hospital admissions and emergency episodes in Alentejo.

Methods: Mortality data were obtained from daily mortality surveillance system (VDM). The number of consultations by flu syndrome (code R80 in International Classification of Primary Care) and vaccines administrated was obtained from SIARS Informatics System. The number of hospital admissions and emergency episodes were obtained from local hospitals registered in ALERT ADW and SONHO informatics systems.

The period studied, observed period (O), was week 1-5 2014/2015 season.

The expected number $(\mathrm{E})$ of deaths was obtained by taking the sum of variables from an equivalent set of reference with same days week, month from season 2013/2014.

Rate ratios ( $R R$ ), $R R=O / E$, comparing observed versus reference period, were calculated for number of total deaths, consultations, hospital admissions and emergency episodes per week, and $95 \%$ confidence intervals (CI) used methodology described by Hoshiko et al. (2009).

Results: During these season was administrated, at least, $80 \%$ of total (76.768) influenza vaccines purchased (92\% was individuals with 65 or more years).

In week 3/2015 was registered in Alentejo the peak of excess deaths, corresponding 118 deaths with a $\mathrm{RR}=1.61$ (95\% CI: 1.19-1.40). 
During the period of study was estimated an excess of: 2274 consultations by R80 in primary health care, 189 hospital admissions and 766 emergency episodes in priority colors of Manchester Triage System.

Particularly, week 5/2015 registered an $\mathrm{RR}=2.95$ (95\% CI: 2.65-3.30) in consultations by R80 and week 4/2015 registered a $\mathrm{RR}=1.359$ (95\% CI: $1.25-1.48)$ in emergency pediatric episodes. Conclusions: These results are considered preliminary, because in Alentejo the peak of influenza activity may not have been reached. Week 5/2015 has showed incidence rate of 255.5/100.000 inhabitants.

The excess mortality can be overestimated, because the VDM have included municipalities in Alentejo that aren't in influence area of Alentejo Regional Administration of Health. Otherwise, consultations by flu in primary health care, can be under estimated, because depends of registries made by general practitioners.

Surveillance in primary and secondary healthcare services should continue, be enhanced or implemented to facilitate early public health risk assessment, prepare health services as well management and treatment severe cases.

\section{Abstract \#: P 473 \\ Impact of meningococcal $\mathrm{C}$ conjugate vaccine in children under 5 years of age: São Paulo (SP), Brazil}

Mônica Tilli Reis Pessoa Conde ${ }^{1}$, Rachel Maria Borelli Paradella Fernades $^{2}$, Rosa Naria Dias Nakazaki ${ }^{2}$, Maria Ligia Bacciotte Ramos Nerger $^{2}$, Ines Suarez Romano ${ }^{2}$, Wilma Tiemi Morimoto ${ }^{2}$, Silvia Silva de Oliveira ${ }^{2}$, Claudio Sacchi ${ }^{3}$, Ana Paula Lemos ${ }^{3}$, Eliseu Alves Waldman $^{1}$

${ }^{1}$ Faculdade de Saúde Pública da Universidade de São Paulo, Brazil; ${ }^{2}$ Centro de Controle de Doenças/Secretaria da Saúde do Município de São Paulo; ${ }^{3}$ Instituto Adolfo Lutz; Corresponding author's e-mail: eawaldma@usp.br

Background: São Paulo is the largest Brazilian State capital and one of the most affected by meningococcal disease (MD), with high incidence, especially in the children under 5 years old. Since 2010 , the meningococcal $\mathrm{C}$ conjugate vaccine $(\mathrm{MCCV})$ was introduced in the routine immunization schedule for children under 2 years. Vaccination coverage has reached about $90 \%$ in São Paulo. This study aimed to assess the impact of MCCV on incidence and mortality rates in children under 5 years in the city of Sao Paulo, 2 years after its introduction.

Methods: A descriptive study comprising MD cases in children under five living in the city of São Paulo from 2008 to 2012. We estimated population prevented fractions (FPP) (1-rate ratio) to assess the impact of MCCV on incidence and mortality rates from MD of all serogroups and of serogroup $\mathrm{C}$ in children under five. The estimate was made by comparing the rates of incidence and mortality from 2012 to 2010.

Results: There were 945 confirmed cases of MD in children under five living in São Paulo during the study period. About $70 \%$ of the cases were serogroup C. In this age group the incidence and mortality rates were 31.9 and 6.3 per 100,000 /year in 2010 and 14.3 and 1.6 in 2012 , respectively. Comparing the rates of incidence and mortality in 2012 with the of 2010, the impact of MCCV on the incidence of MD of all serogroups, measured by FPP, was $55.1 \%$ in children under five: and 63.6, 69.8, and $72.3 \%$ in those under one, aged 1 year and aged 2 years. The impact of MCCV on the incidence of MD of serogroup C was $72.0 \%$ in children under five, and $84.2 \%$ in those under 2 years old. The impact of MCCV on mortality rates from DM of all serogroups was $74.9 \%$ in children under five: and 88.2, 92.9, and $76.6 \%$ in those under one, aged one and aged two. The decline in incidence and mortality rates was not statistically significant in children aged three and 4 years, belonging to unvaccinated birth cohorts. Conclusions: The MCCV schedule showed a high impact in children for whom vaccination is recommended. There was a more significant impact on mortality rates, which suggests that this vaccine does not just protect against the disease but also prevents the most severe forms of MD. However, this last result should further studies to be confirmed.

\section{Abstract \#: P 474}

\section{HBV infection prevalence and vaccination in an immigrant} population in Rome

Nosotti Lorenzo $^{1}$, Petrelli Alessio ${ }^{1}$, Rossi Alessandra ${ }^{1}$, Miglioresi Lucia $^{1}$, D’Arca Teresa ${ }^{1}$, Costanzo Gianfranco ${ }^{1}$, Fortino Antonio ${ }^{1}$, Pecoraro Laura ${ }^{1}$, Mirisola Concetta ${ }^{1}$

${ }^{1}$ National Institute for Health, Migration and Poverty (NIHMP); Corresponding author's e-mail: nosotti@inmp.it

Background: HBV chronically infects about 400 million people, in some geographical areas reaching and exceeding an $8 \%$ prevalence. Italy is placed in a HBV low prevalence range (about $1 \%$ ) among native population. Still lack of information is observed about the diffusion of HBV infection in immigrant populations. Aim of the study is to improve immigrant's access to screening, diagnosis and treatment.

Methods: Between March 2013 and June 2014 a screening for HBV infection was proposed to 516 immigrants coming from different high and intermediate endemic areas. 449 of them accepted the blood test.

Vaccination against Hepatitis B was offered to the 209 patients negative for all markers of $\mathrm{HBV}$ infection.

Results: HBsAg positivity prevalence in patients accessing the Outpatient Clinic of NIHMP was $7.7 \%$, (41 \% of them coming from East Europe, $39 \%$ from Africa, $20 \%$ from Asia). $67.3 \%$ of patients were males, mean age was $39.5 \pm 11$.

Among the 449 patients submitted to serological screening, 35 were positive for HBV infection (HBsAg positive), while 414 patients were negative for HBV infection (163 patients with immune response generated by previous infection, 42 with immune response generated by vaccination and 209 negative for all markers). The prevalence of HBV infection in the cohort studied is $7.7 \%(35 / 449)$,

Among the 209 patients who received the vaccination against HBV infection, $68(32.5 \%)$ refused the vaccination, while 141 $(67.4 \%)$ began the vaccination cycle.

In addition, the screening showed the low percentage $(9.3 \%)$ of patients that had been vaccinated against HBV (42/449), due to a lack of health education and of vaccination campaigns in their countries of origin.

Conclusions: In the cohort of patients studied, HBV infection prevalence resulted higher than in the general Italian population ( 7.7 versus $1 \%$ ). The reason for this difference can be explained by considering immigrant's places of origin, which are often countries with high endemic HBV infection. We emphasize that, besides offering the screening test, also the vaccination option was offered to the immigrants resulting HBV sero-negative: in particular it is crucial to extend vaccination to cohabiting people and sexual partners of HBsAg positive subjects.

It is also worth noting how, thanks to the effectiveness of counseling and the free administration of the vaccine, a high percentage $(67.4 \%)$ of individuals who had not been immunised began the vaccination cycle. 


\section{Abstract \#: P 475}

Factors associated with the use of community health workers in the management of uncomplicated malaria among children in rural Cameroon

\section{Fidèle Bemadoum Mbezo ${ }^{1}$, Benjamin Alexandre Nkoum ${ }^{2}$}

${ }^{1}$ Public health professionnal; ${ }^{2}$ Director of School of Health Sciences/ Catholic University of Central Africa; Corresponding author's e-mail: mbezofidele@yahoo.fr

Background: More than 10 million children under five die every year from easily preventable diseases and disorders for which there are treatments, such as malaria (UNICEF, 2001). This disease is the major public health problem in children under five, especially in subSaharan Africa, with major consequences on the economic and social development. Community care to train and supervise community health workers (CHWs) to treat children against this scourge in the most affected countries is the key strategy recommended by WHO and UNICEF, to contribute to a sustainable reduction of mortality infant (OMS/UNICEF, 2012). This study examines factors associated with the utilization of CHWs by caregivers less than 5 years in the management of malaria in rural Cameroon.

Method: We conducted a cross-sectional study in the Sa'a health district which is located in a region classified as highly endemic malaria area. Households with children under 5 years were selected for this study. Households were selected in the villages of the health district proportionate to size using probability sampling. A semistructured questionnaire was administered to caregivers in selected households. Data were entered into Epiinfo statistical software, and analyzed using SPSS Statistics 18.0, SPAD 5.5 and STATA 11.

Results: Of the 222 individuals who responded to the questionnaire, $49.1 \%$ used the services of CHWs. The results of this study suggest that caregivers who have more than 10 children in their care, were 28 times more likely to use the services of CHWs compared to those who have one or two children $(\mathrm{OR}=28.08, \mathrm{CI}=20.23-352.42)$. Compared to the poorest, those poor $(\mathrm{OR}=0.049, \mathrm{CI}=0.007-0.344)$ and those rich $(\mathrm{OR}=0.386, \mathrm{CI}=0.187-0.795)$, respectively, 0.049 and 0.386 times less likely to use care of CHWs in case of malaria in children under 5 . When the child had fever, caregivers are 7 times more likely to seek advice and/or treatment in a health care provider, compared to those who do not seek $(\mathrm{OR}=6.82, \mathrm{CI}=1.98-23.51)$. Compared to a caregivers whose child has not had uncomplicated malaria in the past 6 months, one whose child was a victim almost 2 times more likely to visit a CHW (OR $=1.731, p=0.003)$. Among the reasons for using the services of CHWs raised by the caregivers, the main two are free medication $(98.2 \%)$ and free care for children $(92.7 \%)$.

Conclusion: CHWs are the primary caregivers in the treatment of uncomplicated malaria in children under 5 years. However, there is a still trust issue with the CHWs. This is as well as organizing awareness sessions on the presence and the need to consult the CHW if child's illness: minimize stock outs of drugs and motivate CHWs are some recommendations at the end of this study.

\section{Abstract \#: P 476}

Antimicrobial resistance in nasal isolates of Staphylococcus aureus: A cause of concern in rural tertiary care center of Central India

Dr. Gargi Dangre-Mudey ${ }^{1}$, Dr. Abhay Mudey ${ }^{1}$, Dr. Neelima Tankhiwale
${ }^{1}$ Jawaharlal Nehru Medical College, Sawangi, Wardha MS; Corresponding author's e-mail: gargimudey@ hotmail.com

Background: Staphylococcus aureus is a common pathogen responsible for community as well as hospital-associated infections. The infections caused by Staphylococcus aureus have clinical range from minor skin infections to severe life threatening infections.

[1] Within the hospital colonized health care workers and medical students act as a reservoir for the spread of Staphylococcus aureus to uncolonized susceptible patients. The anterior nares have been shown to be the main reservoir of Staphylococcus aureus in both children and adults. The Staphylococcus aureus is transmitted to nares by contaminated hands and from surfaces where it can survive for months.

[2] Treatment of infection caused by Staphylococcus aureus has become more problematic since the development of Methicillin resistant Staphylococcus aureus (MRSA). MRSA strain are resistant to all $\beta$-lactam antibiotics and the treatment options are limited. MRSA strains also show multidrug resistance.

[3] For MRSA infections MLS B (macrolid, lincosamide, streptogramin) family of antimicrobials were used frequently. But widespread use of MLS B family of antimicrobials has led to the emergence of resistance to these.

[4] Topical mupirocin is an effective Staphylococcus aureus nasal decolonization agent however: mupirocin resistance (High level and low level) is observed by many researchers.

[5] In view of the above facts \& observations it was felt necessary to study the Prevalence of nasal carriage of Staphylococcus aureus and its antimicrobial resistance pattern in health care workers and medical students.

Methods: Nasal swabs of 200 medical students (Final MBBS) Group I and 192 Health care workers (Nursing Staff) Group II were collected aseptically and cultured using standard microbiological methods. Antibiotic susceptibility was done by disc diffusion method as per CLSI guidelines.

Results: Prevalence of Nasal carriage was 16 and $14.58 \%$ in group I and II respectively. MRSA carriage was more $(2.08 \%)$ in group II as compared to group I $(1.5 \%)$. Inducible clindamycin resistance and Constitutive clindamycin resistance were 3.5 and $4 \%$ in group I which was very high in comparison to group II $(0.5$ and $0 \%)$. No isolate was high level mupirocin resistant. Low level resistance was seen in $0.5 \%$ among group $\mathrm{I}$.

Conclusion: To reduce the prevalence and antimicrobial resistance, emphasis should be given on continuous surveillance and following hospital infection control practices. Antibiotic policy should be strictly followed and antibiotic abuse discouraged.

\section{Abstract \#: P 477}

Platelets and infections, storagetime and storage medium: Does it matter?

A.L.Kreuger ${ }^{1}$, R.A. Middelburg ${ }^{1}$, J-.L. H. Kerkhoffs ${ }^{2}$, J.G. van der Bom $^{1}$

${ }^{1}$ Clinical epidemiology, LUMC, Leiden, Netherlands: Clinical transfusion research, Sanquin, Leiden, Netherlands; ${ }^{2}$ Hagaziekenhuis, den Haag, Netherlands: Clinical transfusion research, Sanquin, Leiden,Netherlands; Corresponding author's e-mail: a.l.kreuger@lumc.nl

Background: Extended storage of platelet products causes soluble immune modulators to accumulate. It also increases the ability to detect bacteria, by increasing the time to culture. Storage in platelet 
additive solution (PAS) reduces the biofilm capacity of bacteria, possibly leading to earlier detection of contamination. Plasma contains proteins which could influence bacterial growth. The aim of this study is to determine the effect of storage time and storage medium of platelet products on bacterial infections in transfusion recipients.

Methods: Since 2002, adverse transfusion reactions are reported to TRIP (Transfusion Reactions In Patients). The storage medium of products leading to transfusion-transmitted bacterial infections was compared with the medium of products involved in all reported transfusion reactions.

In a cohort study in 2 hospitals, 1 using PAS, 1 using plasmastored platelets, all platelet transfusions between 2006 and 2013 were included. Cases were haematological patients who received a platelet transfusion and had a positive blood culture the next day. The observed storagetime was calculated in cases as the number of products in 3 age categories (young $\leq 2$ days, middle 3-4 days, old $\geq 5$ days). As a control, the expected storagetime was determined based on all transfusions given in that hospital, stratified by day of the week and rhesus D. For each age category and storage medium an observed-expected ratio was calculated. This estimates the rate ratio for a positive blood culture after transfusion per age category, using both other age categories as reference.

Results: Fourteen cases of transfusion-transmitted bacterial infections were reported. In $8(57.1 \%)$ a PAS-stored product was involved and in $6(42.9 \%)$ a plasma product. In all reported reactions, $20.5 \%$ of platelet products were stored in PAS. (RR $5.0095 \%$ CI 1.73: 14.46). 376 positive blood cultures after transfusion of plasma-stored platelets and 116 after transfusion of PAS-stored platelets were included. The rate ratio for plasma-stored platelets was for young products 1.08 (95\% CI 0.96: 1.22), for middle 0.90 (95\% CI 0.82-0.99) and for old products 1.12 (95\% CI 0.90: 1.35). For PAS-stored platelets, the rate ratios were 0.97 , (95\% CI 0.81: 1.12) for young, 0.92 (95\% CI 0.73 : 1.12) for middle and 1.93 (95\% CI 0.79: 3.07) for old platelets.

Conclusion: We observed an association of storage medium and transfusion-transmitted bacterial infections, with an increased incidence after exposure to PAS-platelets. Storage time of plasma-stored platelets appears not to affect the incidence of bacterial infections in haematology patients, but in PAS-stored platelets the risk of infections may increase using older platelets.

\section{Abstract \#: P 478}

Differences in the impact of meningococcal $C$ conjugate vaccine in children under 5 years of age in Brazilian capital cities

Eliana T. Masuda ${ }^{1}$, Ana P. Sato ${ }^{1}$, Eliseu A. Waldman ${ }^{1}$

${ }^{1}$ Department of Epidemiology, School of Public Health, University of São Paulo, São Paulo, Brazil; Corresponding author's e-mail: elianamasuda@usp.br

Background: Meningococcal disease (MD) has high incidence rates in Brazil especially among children under 5 years of age. Endemic rates of MD vary among Brazilian capital cities and according to population size and sociodemographic, economic and health indicators. The meningococcal $\mathrm{C}$ conjugate vaccine (MCCV) was introduced in 2010 for children under 2 years of age. In this study, we estimated the impact of MCCV in Brazilian capital cities 3 years after its introduction and examined differences in the vaccine impact according to sociodemographic, economic and health indicators.

Methods: An ecological study comprising MD cases in children under 5 years of age living in 26 state capital cities and the Federal District in Brazil between 2009 and 2013. The Human Development Index (HDI) of Brazilian capital cities ranged between 0.721 and 0.847 . The data source was the database of Health Ministry. We estimated population prevented fractions (PPF) (1-rate ratio) to assess the impact of MCCV by macroregion (North, Northeast, Centralwest, Southeast, South) over a 3-year-period (from 2009 when the vaccine was introduced to 2013). To examine sociodemographic and economic indicators that can potentially influence the impact of MCCV we used Pearson's correlation coefficient at $5 \%$ significance level.

Results: There were 4152 cases of MD in children under five, of which 1618 were living in state capital cities. Serogroup data were available for $43.8 \%$ of the cases, and of these $63.7 \%$ were serogroup C. The average MCCV coverage was $84.8 \%$ in 2013, ranging from 68.8 to $96.3 \%$ among the cities. Three years after the introduction of MCCV the overall PPF in Brazil was $47.6 \%$ (95 \% CI: 41.8-52.8 \%) and it varied by macroregion as follows: $79.7 \%$ in the Central-west (95\% CI: $56.7-90.5 \%$ ): $60.4 \%$ in the Northeast (95\% CI: 35.6-75.6\%): $59.0 \%$ in the South (95\% CI: 7.4-81.8\%): and $54.8 \%$ in the Southeast (95\% CI: 44.6-63.1\%). We found no vaccine impact in the capital cities of the North region. PPF showed a positive correlation with local HDI $(\mathrm{r}=0.421, p=0.029)$ and a negative correlation with average number of people per household $(\mathrm{r}=-0.406, p=0.036)$.

Conclusions: Because of a low proportion of cases with serogroup information available, the vaccine impact was likely underestimated as we estimated PPF for total cases of MD. Nevertheless, there was a significant reduction of MD in children under five after the introduction of MCCV in Brazilian capital cities with wide variations by macroregion. The uneven impact of the vaccine can be explained by social disparities and differences in vaccine coverage despite high rates.

\section{Abstract \#: P 479}

STIs prevalence among vulnerable populations at the national institute for health, migration and poverty (INMP) in Rome (2010-2014)

Valeska Padovese ${ }^{1}$, Marina Cammilli ${ }^{1}$, Raffaella Fazio ${ }^{1}$, Gennaro Franco $^{1}$, Maria Carla Valenzano ${ }^{1}$, Ilaria Uccella ${ }^{1}$

${ }^{1}$ National Institute for Health, Migration and Poverty (INMP), Rome, Italy; Corresponding author's e-mail: uccella@inmp.it

Background: In Italy, few data are available about STIs prevalence in migrants, since most infections are not subjected to mandatory notification and because of access barriers to health services for this population group.

Methods: A single-center retrospective observational study was conducted on patients with STIs attended the National Institute for Health, Migration and Poverty (INMP) in Rome, from January 2010 to December 2014.

Results: During the 5 year period, a total of 4733 STIs were diagnosed, including 54 HIV new infections $(1.1 \%)$ over a total of 3980 patients $(53.5 \%$ females). Of them, $48 \%$ were in the age range 18-34 years. Foreigners were $72 \%$. Most of them came from African countries $(34 \%)$ and Romania $(18.1 \%)$. The $51.2 \%$ of migrants were irregulars, the median age was 32 years, resulting younger than Italians (median age 46 years). $\mathrm{HBV}$ and $\mathrm{HCV}$ infections were diagnosed in 836 migrants $(17.6 \%)$, syphilis in 266 (5.6\% most of them latent infections), genital warts in $254(5.3 \%)$. Only 69 cases of genital herpes were recorded $(1.4 \%)$. The new HIV positive diagnosis in migrants were $54(1 \%)$, in some cases associated with other STIs. Few diagnoses of Chlamydia $(0.5 \%)$ and Gonococcal infection $(0.1 \%)$ were made. Non-specific vaginitis were recorded in 1692 migrants $(41.4 \%)$ whereas non-specific urethritis in $131(2.8 \%)$. In 
the study period, $5676 \mathrm{HIV}$ antibody tests were performed: only 10 patients having an STI refused the test.

Conclusion: Migrants subgroups resulted to have an higher risk for hepatitis B and C, latent syphilis and HIV infection compared to Italians. Although conducted on a small population, this study provides a basis for targeting prevention and control strategies on our high-risk patients, and the need of paying special attention to gender and cultural issues.

\section{Abstract \#: P 480}

Testing times ahead: a systematic review of interventions for improving primary care laboratory use

$\underline{\text { Sharon L. Cadogan }}^{1}$, John P. Browne ${ }^{1}$,Colin P. Bradley ${ }^{2}$, Mary R. Cahill ${ }^{3}$

${ }^{1}$ Department of Epidemiology and Public Health, University College Cork, Ireland; ${ }^{2}$ Department of General Practice, University College Cork, Ireland; ${ }^{3}$ Department of Haematology, Cork University Hospital, Cork, Ireland; Corresponding author's e-mail: scadogan@ucc.i.e

Background: Laboratory testing is an integral part of day-to-day primary care practice underlying approximately $70 \%$ of diagnoses and treatment decisions. Research suggests that a large proportion of requests are avoidable. The aim of this systematic review was to comprehensively search the literature for studies evaluating the effectiveness of interventions for improving general practitioners (GPs) laboratory use.

Methods: PubMed, Cochrane Library, Embase and SCOPUS (from inception to 09/06/14) were searched using the relevant search terms. Systematic reviews, randomised controlled trials, controlled clinical trials, controlled before and after studies and interrupted time series analysis of interventions objectively (volume of tests) assessing Primary Care requesting patterns were considered for inclusion. Studies were only considered if participants were primary care physicians. Quality and risk of bias was assessed using a modified version of EPOC Data Collection Checklist and Quality Criteria.

Results: In total, 6166 titles and abstracts were reviewed, followed by the review of 87 full texts. 11 studies met the eligibility criteria and were included in the review.Due to heterogeneity, results could not be meta-analysed. All, but one intervention significantly reduced the volume of test requests. The reduction of test requests resulting from the interventions varied from 5 to $60 \%$. Feedback and education based interventions proved most effective for changing GP requesting behaviour. However, the complex health systems of included studies indicate further qualitative research is required prior to developing an intervention.

Implications: Each type of intervention (education, feedback, cost displays and guidelines) may improve laboratory use among GPs. However, quality of studies and heterogeneity between results indicate further research is needed.

\section{Abstract \#: P 481}

Safety profile of vaccines using an e-health system, Araraquara, Brazil

Ana Paula S. Sato $^{1}$, Eliseu A. Waldman ${ }^{1}$, Vinicius L. de R. Ferreira ${ }^{1}$, Laura C. Rodrigues $^{2}$, Mariana B. Barros ${ }^{1}$, Márcia de C. Tauil ${ }^{1}$, Walter M. Figueiredo ${ }^{3}$, Ângela A. Costa ${ }^{3}$, Marta Inenami $^{3}$, Edmar Martineli $^{4}$, Maria de Lourdes R. Lirani ${ }^{4}$
${ }^{1}$ School of Public Health, University of São Paulo, São Paulo, Brazil; ${ }^{2}$ London School of Hygiene and Tropical Medicine, University of London, London, UK; ${ }^{3}$ Special Health Center of Araraquara, University of São Paulo, Araraquara, Brazil; ${ }^{4}$ Center of Information Technology of São Carlos, University of São Paulo, São Carlos, Brazil; Corresponding author's e-mail: sah@usp.br

Background: As vaccination programs achieve their goals to control diseases, concerns shift to vaccine safety, with increased awareness of adverse events following vaccination (AEFV). E-health vaccine system is an innovative tool for more accurate monitoring of vaccination actions including vaccine's safety profile.

Objective: To describe AEFV from 2000 to 2013 in the city of Araraquara, SP, Brazil.

Methods: Araraquara is a middle-sized city, where an e-health vaccine system (Juarez System) has been used since 1987. The AEFV surveillance is passive. This is a time series study conducted with data on children up to 2 years old enrolled in Juarez System. We also collected data from AEFV reports available in the health services. Variables were: age, date of notification, vaccine type, symptoms and hospitalization. We estimated rates using the number of AEFVs as numerator and the number of administered doses of any vaccine and when appropriate for specific vaccines as denominator. We also estimated the number of administered doses per AEFV. We used Prais-Winsten regression for the time series analysis. (Project funded by FAPESP: 2014/05183-9)

Results: We found 1438 AEFV reports, of which $60 \%$ were confirmed. The rate of confirmed AEFV was 11.4/10,000 doses, without a trend in this 14 years period $(p=0.474)$. We found $566 \mathrm{AEFV}$ reports with detailed information. Among detailed reports, $70 \%$ occurred in children under 12 months old. Local reactions rate was $5.5 / 10,000$ doses of any vaccine (1:1825 doses). Fever rate was $4.5 /$ 10,000 doses of any vaccine (1:2218 doses). Convulsion rate was $0.3 /$ 10,000 doses of any vaccine (1:30,516 doses). About $3 \%$ of children with AEFV were hospitalized (1:47,682 doses). Vaccines with pertussis component (DTwP/DTwP-Hib/DTwP-Hib-HeptatitisB) were the most reactogenic ( $73 \%$ of AEFV detailed reports: 25.2/10,000 doses). The frequency of Hypotonic-hyporesponsive episodes was 4.1/10,000 doses of vaccines with pertussis component (1:2452 doses). Parotid gland enlargement rate was 4.2/10,000 doses of measles-mumps-rubella vaccine (1:2376 doses). The rate of AEFV following yellow fever vaccine was 7.0/10,000 doses.

Discussion and conclusions: A limitation of AEFV systems is that they do not include rates in unvaccinated, and consequently events that are coincidental rather than caused by vaccination are included: AEFV are more likely to be notified when there are concerns about the specific vaccine. E-health systems are most useful at generating hypothesis for investigation in case control studies and for monitoring variations in the level of AEFV to indicate batch problems. This study highlights e-health vaccine system as an interesting tool for AEFV surveillance.

\section{Abstract \#: P 482}

\section{Burden of severe influenza disease in Norway 2008-2013}

Inger J. Bakken ${ }^{1}$, Siri H. Hauge ${ }^{2}$, Nina Gunnes ${ }^{1}$, Sara Ghaderi ${ }^{3}$, Birgitte F. de Blasio ${ }^{2}$, Lill Trogstad ${ }^{4}$, Ragnhild Tønnessen ${ }^{2}$, Per Magnus ${ }^{5}$, Olav Hungnes ${ }^{6}$, Siri E. Håberg ${ }^{5}$

${ }^{1}$ Department of genes and environment, Norwegian Institute of Public Health, Oslo, Norway; ${ }^{2}$ Department of Infectious Disease Epidemiology, Norwegian Institute of Public Health, Oslo, Norway; ${ }^{3}$ Department of Health Registries, Norwegian Institute of Public Health, Oslo, Norway; ${ }^{4}$ Department of Vaccines, Norwegian Institute 
of Public Health, Oslo, Norway; ${ }^{5}$ Department of Institute Management and Staff, Norwegian Institute of Public Health, Oslo, Norway; ${ }^{6}$ Department of Virology, Norwegian Institute of Public Health, Oslo, Norway; Corresponding author's e-mail: siri.helene.hauge@fhi.no

Background: In Norway, clinical influenza surveillance has been based on weekly reports from general practitioners' (GPs) offices and emergency clinics, covering approximately $15 \%$ of the population. Data on influenza hospitalizations and in hospital deaths in relation to influenza can be extracted from national registries, but has not been used for surveillance purpose due to a delay in reporting. We aimed to compare the burden and severity of the 2009-10 pandemic influenza to 2008-13 seasonal influenzas across different age groups by using data from all GPs and hospitals as reported to national health registries.

Methods: Information on influenza-like illness diagnosed in primary health care patients during 2008-13 was obtained from the Norwegian Directorate of Health. Reporting of the patient's personal identification number, the date of consultation, and diagnostic codes is mandatory for physicians in order to receive reimbursement. Information on hospitalized patients with a diagnosis of influenza was obtained from the Norwegian Patient Register (NPR), which holds individual-level information from all hospitals in Norway. The NPR was also our source of information on in-hospital deaths in patients with a diagnosis of influenza.

Results: Influenza hospitalizations followed a clear temporal pattern which coincided with the temporal pattern of influenza-like illness diagnosed in primary health care. However, in all seasons, there was a strong difference in the age-distribution between patients in primary health care and hospitalized patients. In terms of the number of hospitalizations and in-hospital deaths, the 2012-13 season was by far more severe than any other season during 2008-13, including the 2009-10 pandemic. There were approximately $50 \%$ more hospitalizations and three times as many in-hospital deaths in relation to influenza during the 2012-13 season compared with the 2009-10 pandemic. However, during the 2009-10 pandemic, younger age groups dominated among hospitalized patients, whereas more elderly patients were hospitalized during the 2012-13 season. The 2009-10 pandemic had the highest number of patients in primary health care compared with all the other seasons.

Conclusions: Data from national health registries can be used to gain insight in the burden of disease related to influenza infection. Data from hospital registries complement data from GPs and are necessary in order to investigate severe influenza infections.

\section{Abstract \#: P 483}

Tuberculosis cases and active screening among migrants, refugees and asylum seekers in Rome (2009-2014)

Ilaria Uccella $^{1}$, Marina Cammilli ${ }^{1}$, Laura Pecoraro ${ }^{1}$

${ }^{1}$ National Institute for Health, Migration and Poverty (INMPNIHMP), Rome, Italy; Corresponding author's e-mail: uccella@inmp.it

Background: Tuberculosis is the second cause of death from infectious diseases. Migrations affects tuberculosis epidemiology in developed countries. Around 3 million people are being 'missed' by health systems. Only $47 \%$ concluded latent infection treatment. The Italian Ministry of Health recommended TBC screening for people from high-prevalence countries, new arrivals and refugees. In 2013, 3.153 cases (58\% in migrants) were notified. Objective of the study was to evaluate active and latent $\mathrm{TBC}$ prevalence among migrants.
Methods: A retrospective monocentric study on TBC diagnosis and screening from 2009 to 2014 was conducted. All new migrants attending INMP/NIHMP outpatient department and patients with active tuberculosis symptoms were tested (Tuberculin Skin Test/ Quantiferon-TB gold). 19,

Results: Among 51.287 migrants, $22 \mathrm{TBC}$ cases were notified (0.4/ 1000): 17 new and 5 with TB history. Localization was pulmonary in 20 cases-sputum smear positivity in $40 \%$-extrapulmunary in 3 . $72 \%$ were males, median age 32 , origin: Africa (59.1\%), America (18.2\%) EU (18.2\%), Asia (4.5\%). Main countries: Peru (18.2\%), Romania, Nigeria, Eritrea (13.6\% each). $45.5 \%$ stayed in Italy $>4$ years and $27.3 \% 1$ year. TBC prevalence was $12 / 17.777$ among undocumented (0.6/1000) and 10/19.260 among documented (0.5/1.000). Among homeless and asylum seekers, 4/2.617 (0.15\%) and $6 / 5.163(0.1 \%)$ resulted affected by TBC. Out of 2.532 screened, $1.087(71.7 \%)$ were undocumented, $895(35.5 \%)$ asylum seekers, $262(10.4 \%)$ homeless and $44(1.7 \%)$ Roma. 659 (26\%) positive tests, $31(1.2 \%)$ missed results and $7(0.3 \%)$ refusals. Out of positive people, $84.7 \%$ males, median age $25,63.8 \%$ stayed in Italy 1 year. Origin: Africa (63\%), Asia (19.6\%), EU (11.8\%). Among 7.105 asylum seekers, $12.6 \%$ was tested, $29.2 \%$ positive, $89 \%$ males, median age 25 . Origin: Nigeria $(28.9 \%)$, Ivory Coast $(8.0 \%)$ Mali $(7.9 \%) .92 .5 \%$ stayed in Italy for 1 year. Among 3.222 homeless, $9 \%$ tested, $8.6 \%$ positive, $3.5 \%$ missed/delayed results, $1 \%$ refused. $81 \%$ males, median age 36, $46.6 \%$ stayed in Italy $>4$ years. Origin: Romania (22.7 \%), Afghanistan $(8.7 \%)$, Ivory Coast and Poland (5.8\% each).Out of 559 Roma people, $7.9 \%$ tested and $29.4 \%$ positive: nobody missed/refused/delayed results. $78 \%$ females, median age $41,56.8 \%$ stayed in Italy $>5$ years.

Conclusions: Active TBC prevalence among foreigners resulted low. No difference was observed between documented and undocumented. Most diagnoses were made among long-staying migrants, probably due to social determinants. Among high latent TBC prevalence groups, Roma people appear more compliant with screening. Different policies and rational screening for immigrant-associated tuberculosis must be evaluated to obtain higher treatment success rates.

\section{Abstract \#: P 484}

Epidemiological profile of malaria cases in a gold mining in Itaituba city The State of Pará, Brazil

Carlos F. C. de A. e Melo ${ }^{1}$, Alice C. M. das Neves ${ }^{2}$

${ }^{1}$ Pan American Health Organization. Brasília. Brazil; ${ }^{2}$ Postgraduate Programs in Public Health. University of Brasilia. Brasília. Brazil; Corresponding author's e-mail: acnmedeiros@gmail.com

Background: Malaria is a disease caused and perpetuating poverty and is associated with poor socioeconomic conditions. The city of Itaituba in Para state is the municipality with the highest number of malaria cases from gold mining in Brazil, keeping up in recent years, among the 10 municipalities with the highest number of cases. This study aimed to describe the epidemiology of malaria cases coming from the mining activity in the city of Itaituba, state of Pará, Brazil, in 2013.

Methods: Descriptive study was conducted with data from the Epidemiological Surveillance Information System and Malaria Cases Notification (SIVEP-Malaria) in 2013. Using the cases testing positive for malaria, not classified as a specific microscopic examination (LVC) and site of infection in the city of Itaituba/Pará, we estimated the number of malaria cases among Brazilian gold miners or "garimpeiros" and not gold miners, according parasitic species in the period 2003-2013. To calculate the risk of becoming ill from malaria 
calculated the annual parasite incidence (API), which expresses the number of positive tests per thousand inhabitants in a certain place and time.

Results: 24,390 positive cases for malaria in the state, and the city of Itaituba/Pará accounted for $42.6 \%(10,384)$ of cases in the state. Of the total municipal malaria cases, $6257(60.26 \%)$ were related to 'garimpeiros'. It is noteworthy that in the mines the proportion of cases is higher among men (90\%), with 15-59 years old (96\%) and less than 8 years of education $(78.6 \%)$. The average treatment time in 'garimpeiros' was 43 days, approximately 4 times more than the average time in not gold miners (13 days). There was a reduction of cases of plasmodium not P. falciparum in the years 2012 and 2013, however, among the 'garimpeiros' this reduction was lower. In the year 2013, the IPA Itaituba/Pará was 64, considered high risk, excluding matters relating to mining API reduces to 42 , in other words, medium risk.

Conclusion: Malaria is a disease with strong social determinants and mining conditions strengthen the maintenance of these determining factors and perpetuate the disease. The study concludes that it is necessary to malaria control strategies in areas of mining, noting the particular characteristics of the target population.

\section{Abstract \#: P 485}

HBV knowledge and vaccination uptake among patients attending GP clinics in Poland in the light of the anti-HBC total prevalence Maria Ganczak $^{1}$, Gabriela Dmytrzyk-Danilow ${ }^{2}$, Marcin Korzeń ${ }^{3}$

${ }^{1}$ Dept of Public Health, Pomeranian Medical University; ${ }^{2}$ Vaccination Unit, Primary Care Clinic, Zgorzelec, Poland; ${ }^{3}$ Dept of Methods of Artificial Intelligence and Applied Mathematics, Westpomeranian University of Technology, Szczecin, Poland; Corresponding author's e-mail: ganczak2@wp.pl

Background: Little is known of whether lack of knowledge of HBV transmission routes and adequate protective measures may create a major barrier regarding vaccination uptake.

Objectives: to assess knowledge about hepatitis B (HB) among vaccinated and unvaccinated patients from primary care clinics and to define factors associated with a high knowledge level, to estimate their $\mathrm{HBV}$ vaccination uptake and the prevalence of anti-HBc total in this population.

Methods: a cross-sectional sero-survey was conducted in March 2013 on adult patients, born before the universal neonatal vaccination era, from randomly selected primary care clinics from the Zgorzelec region, Poland, with the use of an anonymous questionnaire. Serum samples were assayed for anti-HBc total with enzyme immunoassay. Results: Response rate $99.3 \%$ (410/413): the median age 56 years (range 27-85), 66.1\% females. Previous HBV vaccination was reported by $55.4 \%$ participants (95\% CI: 50.5-60.1\%). The prevalence of anti-HBc total among unvaccinated patients was $13.6 \%(24 / 176$ : $95 \%$ CI: 9.3-19.5\%), among those vaccinated$6.0 \%$ (6/95: $95 \%$ CI: 2.9-13.1\%). Main sources of HB knowledge were media $(61.7 \%)$ and medical staff $(56.9 \%)$. The HB knowledge score ranged from 0 to 22 with the median 16.5 (76.7\% of the respondents had scores greater than $50 \%$ ): it was significantly higher in vaccinated patients than among unvaccinated $(p=0.002)$. Particular gaps were detected on knowledge on uprotected sexual intercourse, and MTCT (50.4 and $50.0 \%$ correct answers respectively): blood donation, mosquito bites and sharing a cup were all uncorrectly identified by $82.4,41.5$ and $39.0 \%$ respectively. Patient's low educational level was independently negatively associated with a "good" level of knowledge (OR 0.30). The willingness for further education on $\mathrm{HB}$ and $\mathrm{HBV}$ vaccination in the past were independently associated with good knowledge (OR 3.12 and OR 22.5 respectively). Conclusions: HBV remains a serious public health threat in Poland. Gaps in knowledge and myths regarding some modes of HBV infection among adult patients from primary care clinics call for educational actions which may result in a better protection against an infection. Altough information should reach the whole population, patiens representing low education level should be a group set apart for special attention. Individual knowledge on HB might serve as an effective tool in decision making regarding vaccination.

\section{Abstract \#: P 486}

\section{Direct effectiveness of meningococcal $C$ conjugate vaccine in children under 2 years of age}

Mônica Tilli Reis Pessoa Conde ${ }^{1}$, Rachel Maria Borelli Paradella Fernades $^{2}$, Eliana de Fátima Paulo ${ }^{2}$, Claudio Sacchi ${ }^{3}$, Ana Paula Lemos $^{3}$, Eliseu Alves Waldman ${ }^{1}$

${ }^{1}$ Faculdade de Saúde Pública da Universidade de São Paulo; ${ }^{2}$ Centro de Controle de Doenças/Secretaria da Saúde do Município de São Paulo; ${ }^{3}$ Instituto Adolfo Lutz; Corresponding author's e-mail: eawaldma@usp.br

Background: Meningococcal C conjugate vaccine (MCCV) was introduced in Brazil in 2010 for children under 2 years of age using a different strategy from that used in developed countries. An epidemic of meningococcal disease by meningococcus $\mathrm{C}$ is underway in the country at that time, therefore, was relevant to assess the direct effectiveness of this this vaccination scheme. This study aimed to assess the direct effectiveness of MCCV in birth cohorts vaccinated following the recommended immunization schedule of three doses in the first year of life plus a booster dose in the second year of life.

Methods: We conducted a population-based case-control study to assess vaccine effectiveness in children. We enrolled four controls for each case of MD matched by the area of residence of the cases. Cases and controls were selected among children born after January 2009 for whom vaccination with MCCV is recommended. All cases had MD confirmed by culture and/or real-time polymerase chain reaction (RT-PCR) and were selected among children admitted to hospitals in the city of Sao Paulo from January 2011 to December 2013 that were reported to the local MD passive surveillance. Controls were children living in the same area of the cases with no history of MD. All information for cases and controls was collected according to the day of admission of cases and vaccination information was checked against the child's vaccination record card. We estimated vaccine effectiveness using the formula 1 -odds ratio [OR] of vaccination. To estimate the unadjusted and adjusted odds ratios and $95 \%$ confidence interval (95\% CIs) we used conditional logistic regression. We investigated the association between MCCV vaccination and the dependent variable MD by estimating the OR after adjusting for age, gender, family income and number of people sharing a bedroom with the child.

Results: Our sample consisted of 35 cases and 140 controls. Six vaccine failures occurred among the cases. Effectiveness of at least one dose of MCCV ranged around $97.8 \%$ but not lower than $90.2 \%$. Effectiveness of one dose ranged around $98.6 \%$ but not lower than $86.2 \%$ and effectiveness of two doses or more ranged around $97.1 \%$ but not lower than $83.9 \%$, i.e., both dose schedules had similar vaccine effectiveness.

Conclusions: Assessing the direct effectiveness of MCCV, 2 years after its introduction, the study shown that the recommended vaccine schedule in Brazil was highly effective in the target population group, even with a single dose of MCCV. 


\section{Abstract \#: P 487}

Social network characteristics are associated with self-reported (symptoms of) infections in middle aged and elderly individualsthe Maastricht study

Stephanie Brinkhues ${ }^{1}$, Miranda T. Schram ${ }^{2}$, Christian J.P.A. Hoebe ${ }^{1}$, Coen D.A. Stehouwer ${ }^{2}$, Ronald M.A. Henry ${ }^{2}$, Simone J.S. Sep ${ }^{2}$, Annemarie Koster $^{3}$, Paul H.M. Savelkoul ${ }^{4}$, Nicole H.T.M. DukersMuijrers $^{1}$

${ }^{1}$ Department of Medical Microbiology, Maastricht University Medical Centre (MUMC+), Department of Sexual Health, Infectious Diseases and Environmental Health, Public Health Service (GGD) South Limburg, CAPHRI School for Public Health and Primary Care, Maastricht University, Netherlands; ${ }^{2}$ Department of Medicine, Maastricht University Medical Centre (MUMC+), CARIM Cardiovascular Research Institute Maastricht, Maastricht University, Netherlands; ${ }^{3}$ Department of Social Medicine, CAPHRI School for Public Health and Primary Care, Maastricht University, Netherlands; ${ }^{4}$ Department of Medical Microbiology, Maastricht University Medical Centre (MUMC+), CAPHRI School for Public Health and Primary Care, Maastricht University, Netherlands; Corresponding author's e-mail: stephanie.brinkhues@mumc.nl

Background: Elderly individuals are increasingly susceptible to infections due to an age-related decline in immune response. Most infections are spread through social relations/networks. However, social networks may also act as a buffer for infections by increasing immune function. This study aimed to describe the composition of social networks in a population aged 40-75 years and to assess associations between network characteristics and prevalence of selfreported respiratory and gastrointestinal infections. Thereby, we attempted to unravel whether networks act as a vehicle for transmission, a buffer against infections, or both.

Methods: In this population-based cross sectional cohort study $(\mathrm{N}=3004$, mean age 60.0 years, $48.8 \%$ female, $28.8 \%$ type 2 diabetes, $13.4 \%$ smokers), (symptoms of) infections within the past 2 months were assessed by self-administered questionnaires. Networks were assessed using a name generator/interpreter approach, yielding a maximum of 40 different network members per respondent. Network characteristics (e.g. density, diversity) were compared between age groups and sex using Chi square analyses. Univariable and multivariable logistic regression analyses were conducted to identify associations between network characteristics and infections (outcomes). Models were adjusted for diabetes status, age, sex, season, nationality, living situation, BMI, smoking status, alcohol use and educational level.

Preliminary

Results: Overall, participants reported a mean number of 10 network members, $26 \%$ daily or weekly contact with them, $43.1 \%$ had members living nearby, and $39.8 \%$ reported a high density in their network. Elderly (60 years and older) reported smaller networks, less frequent contacts and less diverse networks than younger individuals. Men reported smaller networks, less frequent contacts, more network members living nearby, lower density and less diversity. Cough, runny nose, sore throat (respiratory infections) and gastrointestinal infections were reported by $19.4,27.1,14.8$, and $12.5 \%$ of the participants, respectively. Sore throat and runny nose were more frequent in younger individuals and were significantly associated with larger networks and less frequent contacts. Cough was significantly associated with higher network density. Gastrointestinal infections were associated with younger age, female sex, larger networks, less frequent contacts, and having fewer network members living nearby. Conclusion: The preliminary results of this large observational study show that social networks can have transmission (network size, density) as well as buffering effects (contact frequency, nearness) on the prevalence of infections. Larger and denser networks increase the risk of exposure to infectious agents. Having more members living nearby and more frequent contacts may have a buffering effect on the ability to resist infection.

\section{Abstract \#: P 488}

Burden and mortality trends of hepatitis B and C, 1999-2013, São Paulo city, Brazil

Ana Paula S. Sato ${ }^{1}$, Inês K. Koizumi ${ }^{2}$, Norma S. O. Farias ${ }^{2}$, Maria Regina A. Cardoso ${ }^{1}$

${ }^{1}$ School of Public Health, University of São Paulo, São Paulo, Brazil; ${ }^{2}$ Center for Epidemiological Surveillance, São Paulo, Brazil; Corresponding author's e-mail: sah@usp.br

Background: Hepatitis B (HB) and hepatitis C (HC) infectious represent high burden of disease that affects millions of people each year, with disability and death. In the São Paulo city, a large Brazilian metropolis, mortality trends for $\mathrm{HB}$ and $\mathrm{HC}$ are little known.

Objective: To assess the burden and mortality trends of $\mathrm{HB}$ and $\mathrm{HC}$ from 1999 to 2013, in São Paulo city, Brazil.

Methods: This is an ecological time-series study using data from the National Mortality System. We studied multiple-cause mortality rates, according to gender, from $\mathrm{HB}$ and $\mathrm{HC}$ among residents in São Paulo city. The age-adjusted mortality rates were estimated using direct method and population from 2000 census. Prais-Winsten regression and annual percent change were performed for time series analysis. The Potential Years of Life Lost (PYLL) was obtained by the method of Romeder and McWhinnie, and its calculation considered all deaths from HB and HC occurred between 1 and 69 years old. The PYLL rate for 100.000 inhab was also estimated.

Results: HB mortality showed a decrease of $5.4 \%$ per year among men, but remained stationary in women. HC mortality showed an increase in both genders, but this trend was more evident among men (increase of $34 \%$ per year) than among women (increase of $19 \%$ per year). About $75 \%$ of deaths occurred in people under 70 years of age for both HBV (average age of death: 52.4 years) and HCV (average age of death: 54.6 years). The average PYLL for HB was 17.6 years and for $\mathrm{HC}$ was 15.4 years. The PYLL rate was higher in $\mathrm{HC}(31.5$ PYLL/100,000 inhab) than in HB (10.1 PYLL/100,000 inhab), especially in men.

Conclusion: This study showed the burden of mortality from viral hepatitis in São Paulo city, with a significant increase in HC mortality. Deaths from HC and HB disproportionately occurred in middle-aged persons. We highlight the importance of establishing policies for early diagnosis and treatment. In addition, the study provides a baseline for future studies given the introduction of new drugs for treatment of viral hepatitis.

\section{Abstract \#: P 489}

\section{Dietary glutamic acid intake and colorectal cancer risk: the Rotterdam study}

Gilson G. Viana Veloso ${ }^{1}$, Oscar H. Franco ${ }^{1}$, Rikje Ruiter ${ }^{2}$, Caterherina E. De Keyser ${ }^{1}$, Albert Hofman ${ }^{1}$, Bruno H. Stricker ${ }^{2}$, Jessica C. Kiefte-de Jong ${ }^{1,3}$

${ }^{1}$ Department of Epidemiology, Erasmus MC, Rotterdam, The Netherlands; ${ }^{2}$ Department of Internal Medicine, Groene Hart Hospital, Gouda: Department of Epidemiology, Erasmus MC, Rotterdam, The Netherlands; ${ }^{3}$ Leiden University College, The Hague, 
The Netherlands; Corresponding author's e-mail: j.c.kiefte@luc.leidenuniv.nl

Background: The role of glutamine in colorectal cancer (CRC) prevention is not well established. Animal studies have shown that glutamine supplementation may decrease colon carcinogenesis, but this has neither been studied in humans nor in population-based settings. The primary aim of this study was to assess whether dietary glutamic acid intake was associated with CRC risk in communitydwelling adults. A secondary aim was to assess whether this association was modified by body mass index (BMI).

Methods: This study was embedded in The Rotterdam Study, a prospective cohort that began in 1990. Our study population consisted of 5362 subjects aged 55 and over, and free of CRC at baseline. We calculated glutamic acid as a percentage of total protein intake using a validated food-frequency questionnaire at baseline. Incident cases of CRC were confirmed through medical reports. Statistical analyses were performed using Cox regression models after adjustment for confounders.

Results: During follow-up, 242 subjects developed CRC. We found that dietary glutamic acid intake was significantly associated with a lower risk of developing CRC (HR: 0.77: $95 \%$ CI: 0.61-0.98 per unit increase in glutamic acid intake). After stratification by BMI the association between dietary glutamic acid and CRC was only present in normal weight subjects (HR: 0.58: $95 \%$ CI: $0.40-0.85$ per unit increase in glutamic acid intake).

Conclusion: In this population-based prospective cohort study, the intake of dietary glutamic intake was associated with a lower risk of developing CRC in normal-weight individuals.

\section{Abstract \#: P 490}

Risk factors for anemia in children aged 6-59 months: case of Regional Hospital Ebolowa (south-cameroon)

Atangana Owona Armand ${ }^{1}$, Dr. Benjamin Alexandre Nkoum ${ }^{1}$, Dr. Joelle Laure Sobngwi ${ }^{1}$

${ }^{1}$ Catholic university of central africa/school of health sciences; Corresponding author's e-mail: atanganaowonaarmand@yahoo.ca

Background: The global prevalence of anemia in the general population is $24.8 \%$ and overall an estimated 1.62 billion the number of people suffering from anemia among which 293 million (47\%) of children under 5 years In Cameroon, three children 6-59 months of 5 $(60 \%)$ suffer from anemia: the southern region $(74 \%)$ has the highest prevalence.

Methods: To determine the risk factors for anemia in children aged 6-59 months in the Ebolowa Regional Hospital, we conducted a case-control study involving 66 children with anemia (cases) and 66 with no anemia (control), all hospitalized in the pediatric ward of the Regional Hospital Ebolowa. Sociodemographic data, nutritional children, and socioeconomic characteristics of mothers were collected. Food 24-h recall was used to assess the eating habits of children. Anemia (hemoglobin $<11 \mathrm{~g} / \mathrm{dl}$ ) was measured using a portable hemoglobin meter.

Results: the average hemoglobin levels were $7.3 \pm 2.1 \mathrm{~g} / \mathrm{dl}$ and $11.3 \pm 0.5 \mathrm{~g} / \mathrm{dl}$ in cases and controls respectively. Nutritionally, children who received mixed feeding during the first 6 months of life are 4 times more at risk of anemia (OR: $4,190 \% p=0.03$ ) than those with an exclusive breastfeeding: Furthermore, the non-consumption of cereals and tubers has a protective effect against anemia (OR: $0.390 \% p=0.07$ ). The children of mothers with lower monthly income 25,000 FCFA are 6 times more at risk of anemia (OR: 5,8 $90 \% p=0.02)$ than mothers with incomes above 50,000 FCFA: reside in urban areas decreases by $90 \%$ the risk of suffering from anemia (OR: $0.190 \% p=0.05$ ).

Conclusion: The results of this study support the hypothesis that the occurrence of anemia in children aged 6-59 months in Ebolowa Regional Hospital is due to a multifactorial etiology. Therefore, control programs against the community anemia should be directed to the promotion of exclusive breastfeeding, bio food fortification, disease prevention and improvement of maternal socioeconomic conditions.

\section{Abstract \#: P 491}

Association between dietary factors and plasma Fetuin-a concentrations in the general population

Katharina Nimptsch $^{1}$, Jürgen Janke ${ }^{1}$, Tobias Pischon ${ }^{1}$, Jakob Linseisen $^{2}$

${ }^{1}$ Molecular Epidemiology Research Group, Max-Delbrück Center for Molecular Medicine, Berlin, Germany; ${ }^{2}$ Institute of Epidemiology 2, Helmholtz Centre Munich, Neuherberg, Germany; Corresponding author's e-mail: katharina.nimptsch@mdc-berlin.de

Background: Fetuin-A, a protein synthesized and secreted by the liver, has been related to hepatic insulin resistance and subclinical inflammation and has been suggested as a novel marker for hepatic fat accumulation. There is growing evidence from prospective studies that high plasma fetuin-A levels are associated with higher risk of type 2 diabetes and cardiovascular diseases. However, little is known about dietary determinants of fetuin-A concentrations in the general population. Therefore, we aimed to investigate the cross-sectional association between dietary factors and fetuin-A concentrations in the Bavarian Food Consumption Survey II, in which dietary intake was assessed by three 24 -h dietary recalls.

Methods: We used data from 558 adults (aged 18-81 years) randomly chosen from the Bavarian population. Fetuin-A concentrations were measured in plasma using an enzyme-linked immunosorbent assay. The association between total energy intake, energy-providing nutrients, alcohol, or major food groups and fetuin-A concentrations was investigated using multivariable linear regression models.

Results: After multivariable adjustment for life style factors and body fatness, higher energy intake was associated with higher fetuin-A concentrations (per $500 \mathrm{kcal} /$ day $6.4 \mu \mathrm{g} / \mathrm{mL}, 95 \%$ CI 1.2, $11.5 \mu \mathrm{g} /$ $\mathrm{mL}$ in men, $2.2 \mu \mathrm{g} / \mathrm{mL}, 95 \% \mathrm{CI}-4.6,9.1 \mu \mathrm{g} / \mathrm{mL}$ in women). A $5 \%$ higher intake of energy from fat (in substitution with carbohydrates) was associated with $4.4 \mu \mathrm{g} / \mathrm{mL}(95 \% \mathrm{CI}-0.4,9.2 \mu \mathrm{g} / \mathrm{mL})$ higher fetuin-A in women, but not in men $(-1.3 \mu \mathrm{g} / \mathrm{mL}, 95 \% \mathrm{CI}-6.0$, $3.4 \mu \mathrm{g} / \mathrm{mL})$. Higher alcohol intake was associated with lower fetuin-A concentrations in men ( $p$ trend 0.03) and women ( $p$ trend 0.05): mean fetuin-A concentrations ( $95 \%$ confidence interval) for nondrinkers were $322 \mu \mathrm{g} / \mathrm{mL}(302,343 \mu \mathrm{g} / \mathrm{mL})$ in men and $321 \mu \mathrm{g} / \mathrm{mL}(308$, $335 \mu \mathrm{g} / \mathrm{mL}$ ) in women, and substantially lower in study participants who drank $\geq 30 \mathrm{~g}$ alcohol per day (men: $297 \mu \mathrm{g} / \mathrm{mL}, 95 \%$ CI 284 , $309 \mu \mathrm{g} / \mathrm{mL}$, women: $274 \mu \mathrm{g} / \mathrm{mL}, 95 \%$ CI $245,303 \mu \mathrm{g} / \mathrm{mL}$ ). When investigating major food groups, higher consumption of milk/dairy products was associated with lower circulating fetuin-A (per $150 \mathrm{~g} /$ day $-8.4 \mu \mathrm{g} / \mathrm{mL}, 95 \%$ CI $-14.0,-2.7 \mu \mathrm{g} / \mathrm{mL}$ in men, $-3.0 \mu \mathrm{g} / \mathrm{mL}, 95 \%-9.1,3.0 \mu \mathrm{g} / \mathrm{mL}$ in women). Dietary intakes of vegetables, meat or fish were not significantly associated with fetuinA concentrations in our study.

Conclusion: We observed that higher total energy intake tended to be associated with higher fetuin-A concentrations and that higher dietary intake of alcohol and milk/dairy products was associated with lower fetuin-A concentrations. Due to the preventive potential of our findings further exploration is warranted. 


\section{Abstract \#: P 492}

The influence of oral health on diet quality of older adults: a population-based study in Brazil

\author{
Ana Paula Gomes ${ }^{1}$, Ana Luiza Soares ${ }^{1}$, Helen Gonçalves ${ }^{1}$ \\ ${ }^{1}$ Postgraduate Program in Epidemiology, Federal University of \\ Pelotas, Pelotas, Brazil; Corresponding author's e-mail: \\ anapaulagomes.nutri@gmail.com
}

Background: Problems related to oral health as tooth loss, ill-fitting dentures and dry mouth, often seen in elderly, may hinder chewing, and are also associated to inadequate intake of some foods, such as meat, fruits and raw vegetables, which can result in low diet quality. This study aimed to evaluate the effect of oral health status on diet quality of older adults.

Methods: A cross-sectional, population-based study was carried out in Pelotas, Southern Brazil, with a sample of 1426 older adults. Diet quality was assessed using a score, which attributed points according to consumption frequency of some foods, such as rice and beans, whole grains, vegetables, fruits, meats, dairy products, sweets, soft drinks or juices, fried foods, preserved foods, processed meats or frozen foods and fast food. For healthy foods, higher consumption frequency had a higher score, while for unhealthy foods, score decreased with increase in consumption frequency. The total score ranged from 11 to 33 points and was divided in terciles, and the highest score tercile indicated good diet quality. Older adults oral health was assessed by the question: "Do you have any teeth problem or mouth problem that hinder to eat?". Those who responded affirmatively for this question were classified as having poor oral health. Multinomial logistic regression was used to evaluate the association between poor oral health and diet quality, and good diet quality category was the reference one.

Results: Poor oral health was associated to low diet quality and intermediate diet quality consumption, which were characterized by reduced consumption of rice and beans, whole grains, meats, vegetables, fruits and dairy products, and increased consumption of sweets, soft drinks, juices and fried foods. Men with poor oral health had 3.4 times greater chance to have a low quality diet and 3.7 times greater chance to have an intermediate quality diet, compared to those with good oral health. Women with poor oral health had 3.3 times greater chance to have a low diet quality and 2.2 times greater chance to have an intermediate diet quality, compared to those with good oral health.

Conclusion: More attention should be given to oral health of older adults, since poor oral health may be a barrier to healthy food consumption in this group.

\section{Abstract \#: P 493}

\section{Do Egyptian university students eat more or eat less when perceiving stress?}

\section{Gabriele Berg-Beckhoff ${ }^{1}$, Walid El Ansari ${ }^{2}$}

${ }^{1}$ Unit for Health Promotion Research, Institute of Public Health, University of Southern Denmark, 6700 Esbjerg, Denmark; ${ }^{2}$ Faculty of Applied Sciences, University of Gloucestershire, Gloucester GL2 9HW, United Kingdom; Corresponding author's e-mail: gbergbeckhoff@health.sdu.dk

Background: Most studies of stress and food choice found that individuals experiencing periods of stress over-ate food items they would customarily avoid, and that they ate these foods to feel better. This study examined perceived stress and food intake behavior of undergraduate university students in Egypt. We assessed students' levels of stress and correlated it with their consumption of a range of food groups.

Methods: The cross sectional study was conducted among undergraduates in Egypt $(\mathrm{N}=3.271$ : response rate: $90 \%)$. The questionnaire contained a short food frequency questionnaire (FFQ) and the four item short form Cohen's perceived stress scale. We assessed two composite food intake pattern scores (sweets, cakes and snacks score: and fruits and vegetables score) using FFQ. Spearman correlation coefficient examined the correlations between perceived stress on the one hand, and all food groups and the two food intake pattern scores. Multiple linear regression tested the associations of perceived stress with the two food intake pattern scores adjusted for age, sex, living situation, economic situation, moderate physical activity, BMI, and general stressor (overall burden).

Results: All the FFQ food groups were negatively associated with stress, suggesting that generally, perceived stress was associated with less food intake. These negative associations were also observed for the 2 composite food intake pattern scores, but it was more pronounced with some food groups (fruit, raw and cooked vegetables score) than with others (sweet, cookies and snacks score). Increased perceived stress was associated with decreased food intake, and the association was more pronounced in males than in females, suggesting that this sample of Egyptian students ate less across all the food groups we examined when they perceive stress.

Conclusion: Stress related under-eating was present in this sample of undergraduates. Recent studies suggest that stress could be associated with both, eating less and/or eating more depending on the study population, the stress or stressor amount/type, and the eating habit/s. Further research is necessary to differentiate between stress related over- and under-eater.

Acknowledgment: The authors thank the university and students who participated in this research and acknowledge Drs. S. Labeeb, S. Kotb and A. El-Houfy for participation in data collection.

\section{Abstract \#: P 494}

\section{Unprocessed and processed meat consumption and blood pressure: the INTERMAP study}

Linda M. Oude Griep ${ }^{1}$, Paraskevi Seferidi ${ }^{2}$, Jeremiah Stamler ${ }^{3}$, Queenie Chan ${ }^{1}$, Ioanna Tzoulaki ${ }^{1}$, Linda Van Horn ${ }^{3}$, Lyn M. Steffen ${ }^{4}$, Katsuyuki Miura ${ }^{5}$, Hirotsugu Ueshima ${ }^{5}$, Nagako Okuda ${ }^{6}$, Liancheng Zhao $^{7}$, Sabita S. Soedamah-Muthu ${ }^{2}$, Martha L. Daviglus ${ }^{8}$, Timothy MD Ebbels ${ }^{9}$, Elaine Holmes ${ }^{9}$, Paul Elliott ${ }^{1,10}$

${ }^{1}$ Department of Epidemiology and Biostatistics, School of Public Health, Imperial College London, London, United Kingdom; ${ }^{2}$ Division of Human Nutrition, Wageningen University, Wageningen, The Netherlands; ${ }^{3}$ Department of Preventive Medicine, Feinberg School of Medicine, Northwestern University, Chicago, Illinois, USA; ${ }^{4}$ School of Public Health, University of Minnesota,

Minneapolis, USA; ${ }^{5}$ Department of Health Science, Shiga University of Medical Science, Otsu, Japan; ${ }^{6}$ Section of Shokuiku, Department of Nutritional Education, National Institute of Health and Nutrition, Tokyo, Japan; ${ }^{7}$ Department of Epidemiology, Fu Wai Hospital and Cardiovascular Institute, Chinese Academy of Medical Sciences, Beijing, People's Republic of China; ${ }^{8}$ Institute for Minority Health Research, University of Illinois, Chicago, IL, USA; ${ }^{9}$ Department of Surgery and Cancer, Imperial College London, London, United Kingdom; ${ }^{10}$ MRC-HPA Centre for Environment and Health, Imperial College London, United Kingdom; Corresponding author's e-mail: 1.oude-griep@imperial.ac.uk 
Background: Evidence from prospective cohort studies suggests that relationships of red meat consumption with risk of cardiovascular diseases vary by processing due to added preservatives. Since evidence is limited, we investigated associations with BP of unprocessed and processed meat consumption, total and by type.

Methods: We used cross-sectional INTERMAP data of 4680 men and women aged 40-59 years from China, Japan, UK, and US. During four visits, eight BP measurements and four 24-h dietary recalls were collected. Meat was categorized by unprocessed or processed, type (red meat/poultry), and processing type. Processed red meats included fresh processed meat (ready-made, salted, and/or spiced, no curing), bacon, ham (cooked/cured), cold cuts and sausages, and canned meat products. Country-specific linear regression coefficients between meat intake and BP were estimated and pooled, weighted by inverse of their variance: extensively adjusted for lifestyle and dietary factors. The influence of correlated nutrients on the association was additionally investigated. To assess validity, P-trend and adjusted-R2 were calculated across quartiles of energy-adjusted meat intakes and urinary excretions of potential biomarkers (from literature) identified by ion exchange chromatography.

Results: Average daily total unprocessed/processed meat consumption (g/1000 kcal) was 38/21 in Western participants and 20/5 in Asian participants. Of total unprocessed meat intake, $52 \%$ was poultry in Western participants: $75 \%$ was red meat in Asian participants. In Western participants, processed red meat intake comprised mainly fresh processed meat (36\%), cold cuts and sausages (34\%), ham $(16 \%)$, and bacon $(7 \%)$ : processed poultry intake was negligible. Unprocessed meat intake by total, red meat, or poultry was significantly and directly associated with BP in Western and Asian participants, but did not persist with adjustment for BMI. In Western participants only, the direct association between processed red meat intake with SBP (per $25 \mathrm{~g} / 1000 \mathrm{kcal}:+0.94 \mathrm{mmHg}$ : $95 \%$ CI: 0.11 , 1.76) also attenuated with adjustment for BMI. Significant associations with SBP were observed for cold cuts and sausages (per $12.5 \mathrm{~g} /$ 1000 kcal: $+1.59 \mathrm{mmHg}: 95 \%$ CI: $0.75,2.43)$ and ham $(-0.95 \mathrm{mmHg}$ : $95 \%-1.84,0.06)$ and prevailed after adjustments for lifestyle factors, BMI, saturated fatty acids, and urinary sodium. Ham consumers tended towards a more health-conscious lifestyle that besides methodological issues as multiple testing may explain these findings.Strong associations were found for unprocessed red meat with urinary excretions of 3-methylhistidine, carnosine, and creatinine: and for (un)processed poultry with 1-methylhistidine.

Conclusion: Unprocessed and processed meat consumption was directly associated with BP, but findings were attenuated by BMI.

\section{Abstract \#: P 495}

No association of fruit and vegetable consumption with risk of cholecystectomy

Viktor Oskarsson $^{1}$, Caroline Nordenvall $^{2}$, Alicja Wolk ${ }^{1}$

${ }^{1}$ Institute of Environmental Medicine, Karolinska Institutet, Stockholm, Sweden; ${ }^{2}$ Department of Molecular Medicine and Surgery, Karolinska Institutet, Stockholm, Sweden; Corresponding author’s e-mail: viktor.oskarsson@ki.se

Background: Gallstone disease is common among adults in developed countries (Portincasa et al., Lancet, 2006). Although several constituents of fruit and vegetables (FVs) may reduce its occurrence (e.g., dietary fiber and vitamin C) (Gaby AR, Altern Med Rev, 2009), epidemiologic data on whether FV consumption is inversely associated with gallstone disease are sparse. In the only prospective study so far, which was performed among US women, it was observed that high-consumers of FVs had a reduced risk of cholecystectomy (as a proxy for symptomatic gallstone disease) (Tsai et al., Am J Med, 2006). Our objective was to examine the association between FV consumption and risk of cholecystectomy in two prospective cohorts of men and women.

Methods: We used data from 42,750 men (born 1918-1952) and 32,203 women (born 1914-1948), enrolled in the Cohort of Swedish Men and the Swedish Mammography Cohort, who at baseline were cholecystectomy-free and had completed a food-frequency questionnaire. Follow-up for cholecystectomy was conducted from 1998 to 2011 by linkage to the Swedish Patient Register. We used Cox models to obtain hazard ratios (HRs) according to sex-specific quartiles of FV consumption. $P$ values for trend were calculated by modeling the quartiles as a continuous variable. All multivariable models were adjusted for age (time-scale), sex, education, smoking, alcohol intake, physical activity, aspirin use, coffee consumption, and energy intake. In a separate model, we further adjusted for possible mediators (i.e., body mass index, diabetes, and hyperlipidemia).

Results: During 14 years of follow-up (1998-2011: 944,179 personyears), we identified 2129 cases of cholecystectomy (1002 in men and 1127 in women). In analyses adjusted for age and sex, there was an inverse association between FV consumption and risk of cholecystectomy $(P$ trend $=0.018)$ : but this association did not remain in analyses adjusted for other potential confounders $(P$ trend $=0.29)$. The multivariable-adjusted HR was 0.92 (95\% confidence interval [CI], 0.81-1.05) for the highest (median, 6.2 [men] and 7.8 [women] servings/day) compared with the lowest quartile of FV consumption [median, 1.5 (men) and 2.3 (women) servings/day]. The association was unchanged after adjustment for possible mediators (HR, 0.93: $95 \%$ CI, 0.82-1.06), and it did not differ by sex ( $P$ interaction $=0.79)$. Likewise, no association was observed for fruit $(P$ trend $=0.72)$ or vegetable consumption $(P$ trend $=0.58)$ when analyzed as separate items.

Conclusion: In this prospective study of men and women, there was no association between FV consumption and risk of cholecystectomy after adjustment for potential confounders.

\section{Abstract \#: P 496}

Different input variable quantification to obtain dietary patterns through principal component analysis

Ana Amélia Freitas Vilela ${ }^{1}$, Ilana Eshriqui ${ }^{1}$, Camila Benaim ${ }^{1}$, Maria Beatriz Trindade Castro ${ }^{1}$, Nadya Helena Alves do Santos ${ }^{1}$, Pauline M Emmett ${ }^{2}$, Marcelo Cunha ${ }^{3}$, Gilberto $\mathrm{Kac}^{1}$

${ }^{1}$ Nutritional Epidemiology Observatory, Department of Social and Applied Nutrition: Graduate Program in Nutrition, Institute of Nutrition Josué de Castro, Rio de Janeiro Federal University, Rio de Janeiro, Brazil; ${ }^{2}$ School of Social and Community Medicine,

University of Bristol, Bristol, UK; ${ }^{3}$ National School of Public Health, Oswaldo Cruz Foundation, Rio de Janeiro, Brazil; Corresponding author's e-mail: anaameliafv@gmail.com

Background: Principal Component Analysis (PCA) is a common approach used to derive dietary patterns (DP), likewise the input variables can be quantified in several different ways. Objective: To derive DP using different types of input variable and to compare the scores of these DP.

Methods: A prospective cohort study was conducted with 257 healthy pregnant women at baseline. A food frequency questionnaire was applied between the 5th and 13th gestational weeks to measure the dietary intake referring to the 6 months prior to pregnancy. PCA was used to identify DP according to the following input variables for each food group: daily frequency, daily portion size ( $\mathrm{g}$ or $\mathrm{ml} /$ day), percentage contribution to energy intake, energy-adjusted daily 
frequency and energy-adjusted portion size. Energy adjustment was made using the residuals method. The food groups and the number of extracted factors were the same for all methods aiming to allow comparisons. The best approach was selected according to the Kaiser-Meyer-Olkin test (KMO) and the communalities (h2). Pearson's correlation coefficients were estimated to compare the DP scores.

Results: Three DP were derived using 19 food groups. The DP characterized by Brazilian foods (rice, beans and meats) explained the highest percentage of variance for each input variable type, except daily portion size where it was the second pattern. The DP characterised by healthy foods (milk, vegetables, fruit and fish) explained the second highest percentage of variance using the three energyadjusted approaches. The KMO test was considerably higher for the input variables daily frequency, energy-adjusted daily frequency and daily portion size than for the other two variables. The $\mathrm{h} 2$ values were generally highest for the unadjusted frequency and portion size variables. The energy-adjusted variables showed lower factor loadings overall with excessive negative loadings and were more difficult to interpret. Higher correlations between the scores were observed between the DPs that were characterised by similar food groups. Similar DPs from the daily frequency and portion size variables were highly correlated $(0.869-0.916)$ but arose in a different order. The DPs from the energy-adjusted input variables correlated well with each other but less well with the two unadjusted input variables.

Conclusion: In this sample, the use of daily frequency as the input variable to obtain DP was adequate based on KMO and h2. There appeared to be no advantage in using daily portion size or energyadjusted input variables.

\section{Abstract \#: P 497}

\section{A posteriori and a priori dietary patterns at the age of 1 year and body composition at 6 years}

Trudy Voortman ${ }^{1}$, Elisabeth T.M. Leermakers ${ }^{1}$, Vincent W.V. Jaddoe $^{2}$, Albert Hofman ${ }^{1}$, Oscar H. Franco ${ }^{1}$, Edith H. van den Hooven $^{1}$, Jessica C. Kiefte-de Jong ${ }^{1}$

${ }^{1}$ Department of Epidemiology, Erasmus MC, University Medical Center, Rotterdam, the Netherlands; ${ }^{2}$ Departments of Epidemiology and Pediatrics, Erasmus MC, University Medical Center, Rotterdam, the Netherlands; Corresponding author's e-mail:

trudy.voortman@erasmusmc.nl

Background: and objectives: Dietary patterns have been linked to obesity in adults, however, not much is known about these associations in early childhood. We examined the associations between dietary patterns in toddlers and body composition at school-age in 2026 Dutch children participating in the Generation R study, a population-based cohort study.

Methods: Food intake at 1 year was assessed with a validated foodfrequency questionnaire. At the children's age of 6 years, we measured anthropometrics and body fat mass (with dual-energy-X-ray absorptiometry). We calculated age-and sex-specific SD scores for body mass index (BMI), fat mass index (FMI), and fat-free mass index (FFMI).

Dietary patterns: We used three different methods to derive dietary patterns. With principal component analysis (PCA) two a posteriori patterns were extracted that explained the maximal variance in food intake, which we called 'Health-conscious' (high intake of pasta and rice: vegetables: legumes: vegetable oils) and 'Western' (refined grains: savory snacks: confectionary: sugar-containing beverages). Reduced-rank regression (RRR) was used to identify two dietary patterns that explained the maximal variance in body composition: the first was positively associated with both FMI and FFMI, whereas the second was positively associated with FFMI, but not with FMI. A priori-defined diet quality was assessed using a diet score for preschool children based on dietary guidelines (high intake of vegetables, fruit, low-fat dairy, whole grains, fish, oils: low intake of sugarcontaining beverages, confectionary and snacks).

Results: In models adjusted for sociodemographic and lifestyle factors and total energy intake, children in the highest quartile of the PCA-derived 'Health-conscious' pattern had a 0.16 SD (95\% CI 0.04:0.28) higher FFMI: and those in the highest quartile of the diet score a $0.18 \mathrm{SD}(95 \% \mathrm{CI}$ 0.06:0.29) higher FFMI at 6 years than children in the respective lowest quartiles. Adherence to these patterns was not associated with FMI. Adherence to the PCA-derived 'Western' pattern was not associated with body composition at 6 years. The first RRR-derived pattern, positively associated with both FMI and FFMI, was characterized by high intake of meat, fish, sauces, and sugar-containing beverages. The second RRR-pattern, positively associated with FFMI only, was characterized by intake of whole grains, pasta and rice, and vegetable oils.

Conclusions: Our results indicate that dietary patterns characterized by high intake of vegetables, grains, and vegetable oils in early childhood are associated with a higher FFMI, but not FMI, at the age of 6 years. Dietary patterns in early childhood were not clearly associated with later FMI.

\section{Abstract \#: P 498}

Reproducibility and relative validity of the FFQ used in EPIC-NL to estimate the intake of specific saturated fatty acids

Jaike Praagman $^{1}{ }$, Anda P.J. Adolphs ${ }^{1}$, Caroline T.M. van Rossum ${ }^{2}$, Yvonne T. van der Schouw ${ }^{1}$, Joline J.W. Beulens ${ }^{1}$

${ }^{1}$ Julius Center for Health Sciences and Primary Care, University Medical Center, Utrecht, the Netherlands; ${ }^{2}$ National Institute for Public Health Environment (RIVM), Bilthoven, the Netherlands; Corresponding author's e-mail: j.praagman-2@umcutrecht.nl

Background: Previous studies suggest that the association between dietary saturated fatty acids (SFA) and cardiovascular disease (CVD) may differ depending on the carbon-chain length of these SFAs. To investigate this hypothesis in epidemiological research, a valid estimate of specific SFA intake is required. We investigated the reproducibility and relative validity of the food frequency questionnaire (FFQ), used in the Dutch European Investigation of Cancer and Nutrition cohort (EPIC-NL), for the assessment of intake of specific SFAs.

Methods: We used data from 121 Dutch men and women, aged 23-72 years, who filled out the FFQ three times at intervals of 6 months between 1991 and 1992. As a reference method we used data from twelve 24-h dietary recalls (24HDR) which they filled out monthly during the same time period. Intra-class correlation coefficients (ICC) were calculated to test the reproducibility of the FFQ. To test its relative validity Spearman rank correlation coefficients and weighted $\kappa$ coefficients were calculated, and Bland-Altman plots were constructed to test the absolute agreement between the FFQ and 24HDR.

Results: In general, the mean intakes measured by the FFQ were higher than intakes measured by the 24HDR. ICCs for the three FFQs were good for men and women, ranging from 0.58 to 0.72 , and from 0.66 to 0.83 respectively. In men, Spearman rank correlation coefficients between the FFQ and 24HDR were moderate-to-good (0.47-0.71). For women, Spearman rank correlations were moderateto-good for intakes of C10:0, C14:0, C16:0 and C18:0 (0.49-0.66), and moderate for the other SFAs $(0.30-0.43)$ of which intakes were very low. With the exception of C18:0 $(\kappa=0.27)$, weighted $\kappa$ 
coefficients ranged from 0.36 to 0.52 for men. For women, weighted $\kappa$ coefficients were fair-to-moderate, ranging from 0.21 to 0.25 for the low abundant SFAs C4:0, C6:0 and C17:0 and from 0.30 to 0.43 for the other SFAs. Bland-Altman plots showed good agreement between the FFQ and 24HDR, and showed proportional bias only for SFAs with very low intakes.

Conclusion: The FFQ, used in EPIC-NL, showed good reproducibility for the assessment of specific SFA intake. Furthermore, for the specific SFAs that are highly abundant in the Dutch diet this FFQ is an adequate tool to rank people according to intake. Relative validity for SFAs with very low intakes was less.

\section{Abstract \#: P 499 \\ The Dutch national dietary assessment reference database (NDARD)—an important tool in modern nutritional epidemiology}

Edith J.M. Feskens ${ }^{1}$, Anouk Geelen ${ }^{1}$, Marga Ocke ${ }^{2}$, Eva Corpeleijn ${ }^{3}$, Pieter van 't Veer' ${ }^{1}$ on behalf of the NDARD Users Group

${ }^{1}$ Div. Human Nutrition, Wageningen University, Wageningen, The Netherlands; ${ }^{2}$ National Institute of Public Health, Bilthoven, The Netherlands; ${ }^{3}$ Dept Epidemiology, UMCG, Groningen, The Netherlands; Corresponding author's e-mail: edith.feskens@wur.nl

Background: Food Frequency Questionnaires (FFQ) are commonly applied to collect dietary intake information in epidemiological studies. Ideally, an FFQ is generated using information on dietary intake of a large representative sample. The Dutch FFQ tool, to generate new FFQs, now uses data of the Dutch National Food Consumption Survey (VCP), but this lacks sufficient information on the individual variation of intake. Also, objective information as obtained from biochemical markers (biomarkers of intake) is needed to be able to evaluate the occurrence of under- and over-reporting and appropriate ranking of individuals based on their intake of interest. Aim of NDARD is to have a database to generate and validate FFQs for various research purposes, focusing not only on specific foods or nutrients (e.g. sugar-sweetened beverages or omega-3 fatty acids) but also on dietary patterns.

Methods: Between May 2011 and February 2013, 2048 men and women aged 20-70 year, from Wageningen and surrounding cities were recruited. Baseline measurements consisted of 2 methods of dietary assessment: FFQ and two to nine 24-h recalls, physical measurements, venepuncture, 24-h urine collection, and eating behaviour questionnaires. 24-h recalls were taken by telephone by trained dieticians, or self-administered using a newly developed webbased tool (Compl-eat). Physical examinations and FFQs were repeated after $1-2$ years.

Results: Mean energy intake of the men and women (mean age 52 year, mean BMI $26.2 \mathrm{~kg} / \mathrm{m} 2$ ) as assessed by FFQ was $2051 \mathrm{kcal} /$ day. Repeated 24-h recalls (3 or more) are available on 1595 subjects. Plasma fatty acids and carotenoids are available on 500 subjects: urinary nitrogen, potassium and sodium are available as well. These data will be used to develop regression calibration models with repeated recall outcomes and biomarkers as reference method to calibrate FFQ-results. This will serve as an important source for Dutch epidemiological studies, which will need only limited information on the validity of their FFQ in their population as they can calibrate their substudies to our large reference sample.

Conclusion: NDARD will serve as an important reference database for nutritional epidemiology in the Netherlands as the validity and repeatability of the FFQs can be established and calibration models can be used to assess diet-health relationships with less measurement error. Coming years the reference database will be enriched with data from recalls and/or FFQ and intake biomarkers from validation substudies of several Dutch cohort studies.

\section{Abstract \#: P 500}

Dietary diversity according to gender, socio-economic status and nutritional status among Moroccan rural adolescents

Rachid. Sbaibi ${ }^{1} /$ Youssef. Aboussaleh ${ }^{1} /$ Meriem. Sbai $^{1}$

${ }^{1}$ Ibn Tofail University; Corresponding author's e-mail: abou_85@yahoo.fr

Introduction: This study aims to compare the dietary diversity score (DDS) according to gender, socio-economic status and nutritional status among the rural middle school children (12-20 years) in the northwest of Morocco.

Materials and methods: The DDS represents the number of groups from 11 food groups consumed during the preceding 7 days by 300 middle school children ( 15.45 years \pm 1.64$)$ of the only middle school in the rural commune of Sidi El Kamel in northwestern Morocco. The comparison of means of DDS according to gender, socio-economic status and nutritional status is done by the MannWhitney test and the Kruskal-Wallis test at $5 \%$ error.

Discussion: $9 \%$ of children have a non-diversified diet (with DDS less than or equal to 7 of 11 food groups), against $91 \%$ who have a diversified diet with DDS strictly greater than 7 of 11 food groups, in average $(9.3 \pm 1.33)$ food groups consumed on the 11 groups during the preceding 7 days. The $t$ test Mann-Whitney confirms the equality of means of DDS between girls and boys $(u=9588.5$, $p=0.46>0.05)$. The DDS is only related to the function of the father. $\left(\chi^{2}=2.25, d f=5, P=0.033<0.05\right)$, where farmers and agricultural workers fathers have a direct and easy access to food crops. Also, the DDS is not related to nutritional status $(p>0.05)$.

Conclusions: The good dietary diversity among children in rural areas is linked to easy access to cereals, vegetables, dairy products via agricultural work of the father.

\section{Abstract \#: P 501}

Socio-economic differences in dietary patterns and their relation with cardiovascular disease and mortality in the EPIC-NL cohort

Mirjam C. Kneepkens ${ }^{1}$, Sander Biesbroek ${ }^{1}$, Saskia W. van der Berg ${ }^{1}$, Heidi P. Fransen ${ }^{2}$, Joline W. Beulens ${ }^{2}$, Petra H.M. Peeters ${ }^{2}$, Jolanda M.A. Boer ${ }^{1}$

${ }^{1}$ RIVM, Determinants of Chronic Diseases, Utrecht, The Netherlands; ${ }^{2}$ Julius Center for Health Sciences and Primary Care, University Medical Center Utrecht, Utrecht, The Netherlands; Corresponding author's e-mail: sander.biesbroek@rivm.nl

Background: Inequalities in health may be a result of different underlying dietary patterns associated with socio-economic status (SES). Dietary patterns associated with a reduced risk of cardiovascular disease (CVD) are more often seen in groups of higher SES. However, it is unclear whether different dietary patterns exist among groups with different SES or that similar dietary patterns are present, but with different percentages of adherence. Therefore, the aim of this study was to derive SES-specific dietary patterns and to investigate whether these SES-specific patterns, if any, result in different nutrient intakes, risk of CVD and risk of all-cause mortality.

Methods: We used data of the EPIC-NL cohort, consisting of 40,011 men and women aged 20-65 years at baseline, recruited between 1993 and 1997. Education, divided into 3 groups, was used as a 
measure of socio-economic status and was self-reported at baseline. Dietary intake was estimated using a validated 178-item food frequency questionnaire. Dietary patterns were derived using Principle Component Analysis (PCA) and K-means Cluster Analysis (KCA). Information on CVD and all-cause mortality was obtained by linkage to registries: follow-up data is available until January 1, 2008. Hazard ratios for cardiovascular disease and mortality were estimated using Cox Proportion Hazard analysis.

Results: Through PCA, three in general similar dietary patterns were obtained at all educational levels: a 'prudent', 'western' and 'traditional' pattern. With KCA, 'high-fibre' and 'low-fibre' patterns were found at all SES groups. The 'prudent' dietary pattern was associated with a reduced risk of CVD only in the high education level (adjusted HRQ4vsQ1: 0.71: $95 \%$ CI: 0.51, 0.98). None of the other PCAderived dietary patterns were associated with CVD or mortality. In the high-educated group, the low-fibre cluster was associated with an increased mortality risk compared to the high-fibre cluster (adjusted HR: 2.08: 1.09, 3.94). The clusters derived for the participants with low or middle educational level were not associated with CVD or mortality.

Conclusion: Similar underlying dietary patterns exist among groups with different educational levels. Therefore, there seems no need to identify these patterns separately for SES status in further research. However, significant associations with CVD and mortality were only observed in the high SES group. This is partly explained by differences in other baseline characteristics according to SES.

\section{Abstract \#: P 502}

\section{Socio-demographic variations in the prevalence of anaemia} in southern Kazakhstan

Karlygash Sadykova ${ }^{1}$, Gulnaz Nuskabaeva ${ }^{1}$, Mira Zhunissova ${ }^{1}$, Kamshat Madenbay ${ }^{\text {, Zhanar Shalkharova }}{ }^{2}$, Zhanat Shalkharova ${ }^{1}$, Andrej M. Grjibovski ${ }^{2,3}$

${ }^{1}$ International Kazakh - Turkish University, Turkestan, Kazakhstan; ${ }^{2}$ Kazakh Medical University of Continuing Education, Almaty, Kazakhstan; ${ }^{3}$ Northern State Medical University, Arkhangelsk, Russia; Corresponding author's e-mail:

karligash.sadykova@gmail.com

Background: While in most European countries the prevalence of anemia is low, it remains a significant public health problem in Central Asian part of the European WHO region with considerable variations between regions and little information outside large cities. The aim of the study was to determine the prevalence of anemia and its socio-demographic correlates with special emphasis to gender in an urban setting in Southern Kazakhstan, which is among the least privileged parts of Kazakhstan.

Methods: Altogether, 938 adults aged 18-74 years were enrolled at random in a cross-sectional study in a town of Turkestan (population 155.5 thousand), Southern Kazakhstan. All subjects completed an anonymous questionnaire and had blood tests taken. Anemia was defined as hemoglobin level below $130 \mathrm{~g} / \mathrm{L}$ for men and $120 \mathrm{~g} / \mathrm{L}$ for women. Ninety-five percent confidence intervals for the prevalence of anemia were calculated using Wilson's method. Categorical data were compared using Pearson's Chi squared tests and Fisher's exact tests. Results: Altogether 840 study participants had complete data on hemoglobin and basic socio-demographic variables. The overall prevalence of anemia was $30.6 \%$ (95\% CI: 27.1-34.3) among women and $15.6 \%$ (95\% CI: 11.4-20.9) among men, $p<0.001$. Among women the prevalence progressively decreased from $49.5 \%$ (95\% CI: 39.5-59.4) in those below 30 years to $4.1 \%$ (95\% CI: $1.1-13.7$ ) who were 60 years or older ( $p$ for trend $<0.001$ ). To the contrary, the corresponding data for men were $9.1 \%$ (95\% CI: 3.1-23.6) and $20.9 \%$ (95\% CI: 11.4-35.2), although the difference was not significant ( $p$ for trend 0.243 ). Women with higher education tended to have higher prevalence of anemia than those with secondary education or less (33.4 vs. $25.9 \%, p=0.050)$ while similar proportions were observed among men (14.8 vs. $16.4 \%, p=0.778)$. No difference in the prevalence of anemia was observed among married and unmarried women (29.0 vs. $36.2, p=0.106)$. No cases of anemia were registered among unmarried men while $16.8 \%$ of married men were anemic $(p=0.037)$.

Conclusions: We observed alarmingly high prevalence of anemia among women in the study setting with strong inverse association with age. Every second woman below 30 was anemic, which may have implications for reproductive and general health. Contrary to many other settings, anemia was positively associated with education among women and inversely associated with marital status among men. Generalization of our findings to other settings in Kazakhstan and Central Asia should be done with due caution. Lifestyle and cultural factors behind the observed associations will be discussed.

\section{Abstract \#: P 503}

Influence of socioeconomic and demographic factors on immunization coverage among children in the most populous state of India

$\underline{\text { Shambhavi Mishra }}^{1}$, Uttam Singh $^{1}$, C.M. Pandey ${ }^{1}$

${ }^{1}$ Department of Biostatistics \& Health Informatics, Sanjay Gandhi Postgraduate Institute of Medical Sciences, Raebareli road, Lucknow, Uttar Pradesh, India; Corresponding author's e-mail: chicksm19@gmail.com

Introduction: Uttar Pradesh, the most populous state of India according to 2011 Census had 199 million people. However, in the state immunization coverage is quite low. The Annual Health Survey (AHS) 2012-13 shows that only $53 \%$ children aged 12-23 months were fully immunized. Access to immunization with BCG coverage of $86 \%$ is somewhat satisfactory but due to overall dropout rate of $24 \%$, immunization coverage is low (AHS).

Objectives: To examine socio-economic and demographic factors influencing partial and non-immunization of children in Uttar Pradesh.

Data and Methods: Data for this study was taken from National Family Health Survey (NFHS- 3). The unit level information on 5157 children aged 12-59 months has been included in analysis. Covariates of low immunization coverage were examined using multinomial logistic regression model for partial and non-immunization including variables found significant in bi-variate analysis. Odds ratio and $95 \%$ confidence interval for each significant predictor was computed.

Results: Mother's education (OR: 3.75, CI: 2.29-6.15) and ANC visits (OR: 15.77, CI: 10.73-23.19) are the most dominant factor affecting partial and non-immunization among children respectively. Conclusions: District level health personnel should focus more on vulnerable groups identified in this study to motivate them to fully immunize their children.

\section{Abstract \#: P 504}

Epidemiological inconsistency of the vitamin D-breast cancer relationship: Are weight status and alcohol intake key modulators? 
Mélanie Deschasaux ${ }^{1}$, Jean-Claude Souberbielle ${ }^{2}$, Paule LatinoMartel $^{1}$, Angela Sutton ${ }^{3}$, Nathalie Charnaux ${ }^{3}$, Nathalie DruesnePecollo $^{1}$, Pilar Galan ${ }^{1}$, Serge Hercberg ${ }^{1,3}$, Sigrid Le Clerc ${ }^{4}$, Emmanuelle Kesse-Guyot ${ }^{1}$, Khaled Ezzedine ${ }^{5}$, Mathilde Touvier ${ }^{1}$

${ }^{1}$ Sorbonne Paris Cité Epidemiology and Statistics Research Center, Inserm U1153, Inra U1125, Cnam, Paris 13 University, Nutritional Epidemiology Research Team (EREN), Bobigny, France; ${ }^{2}$ Physiology Department, Necker Hospital, INSERM U845, Paris, France; ${ }^{3}$ Public Health Department, Avicenne Hospital, Bobigny, France; ${ }^{4}$ Conservatoire National des Arts et Métiers (CNAM), Genomics, Bioinformatics and Applications Team (EA4627), Paris, France; ${ }^{5}$ Dermatology department, Saint André Hospital, Bordeaux, France: Nutritional Epidemiology Research Team (EREN), Bobigny, France; Corresponding author's e-mail:

m.deschasaux@eren.smbh.univ-paris13.fr

Background: Vitamin D is a good candidate for breast cancer prevention thanks to anti-proliferative, pro-apoptotic and prodifferentiation properties along with reduction of estrogen signaling in breast tissues. However, epidemiological evidence is inconsistent, suggesting the existence of individual modulating factors. We investigated the prospective associations between plasma 25-hydroxyvitamin D (25OHD) concentration, polymorphisms of the vitamin D receptor (VDR) and vitamin D binding protein $(\mathrm{GC})$ genes, and breast cancer risk, along with two potential modulating factors: body mass index (BMI) and alcohol intake.

Methods: A nested case-control study was set up in the SU.VI.MAX cohort (233 cases/466 matched controls). Total plasma 25OHD concentration was assessed with Roche Cobas ${ }^{\circledR}$ electrochemoluminescent assay. Single nucleotide polymorphisms were determined with TaqMan assay (VDR BsmI and FokI, GC rs4588 and rs7041). Multivariate conditional logistic regression models were computed.

Results: While there was no association overall, 25OHD concentration $\geq 10 \mathrm{ng} / \mathrm{ml}$ was inversely associated with breast cancer risk for women with lower BMI (OR: $\geq 10$ vs $<10 \mathrm{ng} / \mathrm{ml}=0.38$ (95\% $\mathrm{CI}=0.20-0.74), \quad P=0.004)$ and with moderate-to-high alcohol intakes (OR: $\geq 10$ vs $<10 \mathrm{ng} / \mathrm{ml}=0.50(0.26-0.95), P=0.03)$ : whereas $25 \mathrm{OHD}$ concentrations $\geq 10$ or $\geq 20 \mathrm{ng} / \mathrm{ml}$ were positively associated with breast cancer risk for women with higher BMI (OR: $\geq 10$ vs $<10 \mathrm{ng} / \mathrm{ml}=2.22(1.12-4.40), P=0.02$ : OR: $\geq 20$ vs $<20 \mathrm{ng} / \mathrm{ml}=2.32(1.34-4.03), P=0.003)(P$ interaction $=0.002$ for BMI and 0.03 for alcohol). GC rs4588 TT genotype was positively associated with breast cancer risk (OR:TT vs. GG $+\mathrm{GT}=1.78$ (1.06-2.99), $P=0.03$ ), in particular for lean women. VDR BsmI BB genotype was inversely associated with breast cancer risk for moderate-to-high alcohol consumers (OR:BB vs. $\quad \mathrm{bb}+\mathrm{bB}=0.38$ (0.19-0.77), $P=0.007)$. VDR FokI and GC rs7041 polymorphisms were not associated with breast cancer risk.

Conclusion: This prospective study suggests that higher vitamin D status may be beneficial against breast cancer in some population subgroups (lean women, moderate-to-high alcohol consumers) but may be positively associated with breast cancer risk in other groups (e.g. women with higher BMI). Vitamin D-related gene polymorphisms were distinctly involved in breast carcinogenesis, being either inversely associated with breast cancer risk (VDR BsmI BB for moderate-to-high alcohol consumers), positively associated (GC rs4588 TT) or not associated (VDR FokI and GC rs7041). Our results provide interesting insights into the understanding of the role of vitamin $\mathrm{D}$ in the etiology of breast carcinogenesis. The observed modulations, consistent with previous epidemiological studies and supported by mechanistic plausibility, could partly explain the discrepancies existing between previous reported results and could be of importance for public health recommendations. Nonetheless, further research is needed to confirm these results.

\section{Abstract \#: P 505}

A prospective study of plasma $25 \mathrm{OHD}$ and PTH concentrations and prostate cancer risk

Mélanie Deschasaux ${ }^{1}$, Jean-Claude Souberbielle ${ }^{2}$, Paule LatinoMartel $^{1}$, Angela Sutton ${ }^{3}$, Nathalie Charnaux ${ }^{3}$, Nathalie DruesnePecollo ${ }^{1}$, Pilar Galan ${ }^{1}$, Serge Hercberg ${ }^{1,3}$, Sigrid Le Clerc ${ }^{4}$, Emmanuelle Kesse-Guyot ${ }^{1}$, Khaled Ezzedine ${ }^{5}$, Mathilde Touvier ${ }^{1}$

${ }^{1}$ Sorbonne Paris Cité Epidemiology and Statistics Research Center, Inserm U1153, Inra U1125, Cnam, Paris 13 University, Nutritional Epidemiology Research Team (EREN), Bobigny, France;

${ }^{2}$ Physiology Department, Necker Hospital, INSERM U845, Paris, France; ${ }^{3}$ Public Health Department, Avicenne Hospital, Bobigny, France; ${ }^{4}$ Conservatoire National des Arts et Métiers (CNAM), Genomics, Bioinformatics and Applications Team (EA4627), Paris, France; ${ }^{5}$ Dermatology department, Saint André Hospital, Bordeaux, France: Nutritional Epidemiology Research Team (EREN), Bobigny, France; Corresponding author's e-mail:

m.deschasaux@eren.smbh.univ-paris13.fr

Background: Mechanistic hypotheses suggest that vitamin D may be involved in prostate carcinogenesis through various effects on differentiation, apoptosis, and cell proliferation. Plasma parathyroid hormone (PTH) concentration, closely related to vitamin D metabolism may also play a role in prostate carcinogenesis. However, epidemiological evidence regarding the relationship between vitamin $\mathrm{D}$ status and prostate cancer risk is inconsistent and only one prospective study has investigated the association between PTH concentration and prostate cancer risk. Our objectives were thus to prospectively investigate the associations between vitamin D status, selected polymorphisms of vitamin D-related genes, plasma PTH concentration and prostate cancer risk.

Methods: 184 cases diagnosed within the SU.VI.MAX cohort were included in a nested case-control study and matched to 368 controls (13 years of follow-up). Baseline total plasma 25-hydroxyvitamin D (25OHD) concentration was assessed with Roche Cobas ${ }^{\circledR}$ electrochemoluminescent assay and plasma PTH was measured through Roche Cobas ${ }^{\circledR}$ electrochemoluminescent immunometric assay. Single nucleotide polymorphisms were determined with TaqMan assay (VDR BsmI, FokI and Cdx2, CYP24A1 rs4809958, GC rs4588 and rs7041, RXR rs7861779 and rs12004589, CaSR rs1801725 and rs4678174). Multivariate conditional logistic regression models were computed.

Results: Higher 25OHD concentration was inversely associated with the risk of prostate cancer in normal-weight men (OR per $1 \mathrm{ng} / \mathrm{ml}$ increment $=0.91(0.84,0.98), P$ trend $=0.02)$, but not in overweight men $(P=0.1)$. Higher PTH concentration was inversely associated with prostate cancer risk (OR per $1 \mathrm{ng} / \mathrm{L}=0.96(0.94,0.99)$, $P$ trend $=0.006:$ OR:Q4 vs Q1 $=0.44(0.24,0.79), P$ trend $=0.01)$. The studied SNPs were not associated with prostate cancer risk.

Conclusions: Our results suggest that vitamin D may be beneficial against prostate cancer in normal-weight men and that higher plasma PTH concentration may be associated with a decreased risk of prostate cancer, independently from 25OHD concentration. These associations, supported by biological plausibility, provide interesting insights into the understanding of the role of vitamin D and PTH in 
the etiology of prostate carcinogenesis and deserve further exploration.

\section{Abstract \#: P 506}

Urinary sodium and potassium excretion and risk of developing chronic kidney disease

Lyanne M. Kieneker ${ }^{1}$, Ron T. Gansevoort ${ }^{1}$, Rudolf A. de Boer ${ }^{2}$, Gerjan Navis $^{1}$, Stephan J.L. Bakker ${ }^{1}$, Michel M. Joosten ${ }^{1}$

${ }^{1}$ University of Groningen, University Medical Center Groningen, Department of Internal Medicine, Groningen, the Netherlands;

${ }^{2}$ University of Groningen, University Medical Center Groningen, Department of Cardiology, Groningen, the Netherlands;

Corresponding author's e-mail: 1.m.kieneker@umcg.nl

Background: The precise relationship between sodium and potassium intake and chronic kidney disease (CKD) risk in the general population remains uncertain. The aim of our study was to examine the associations of urinary sodium and potassium excretion $(\mathrm{UNaV}$ and UKV, respectively), as estimates of dietary uptake of these nutrients, with risk of developing CKD in the general population.

Methods: Data were used of the PREVEND study, an observational, general population-based cohort study in The Netherlands, in which participants (aged 28-75 years) were screened once every 3 years. $\mathrm{UNaV}$ and UKV were measured in two 24-h urine specimens at baseline (1997-1998) and midway during follow-up (2001-2003). Risk of CKD (defined as a creatinine + cystatin C-based estimated glomerular filtration rate [eGFR] $<60 \mathrm{ml} / \mathrm{min} / 1.73 \mathrm{~m} 2$ and/or albuminuria $>30 \mathrm{mg} / 24 \mathrm{~h}$ ) was prospectively studied in the 5392 subjects free of CKD at baseline.

Results: At baseline median UNaV and UKV were $135 \mathrm{mmol} / 24 \mathrm{~h}$ (interquartile range [IQR]: $106-169 \mathrm{mmol} / 24 \mathrm{~h}$ ) and $70 \mathrm{mmol} / 24 \mathrm{~h}$ (IQR: $57-85 \mathrm{mmol} / 24 \mathrm{~h}$ ), respectively. Median eGFR was 98 (IQR: $87-108) \mathrm{ml} / \mathrm{min} / 1.73 \mathrm{~m} 2$ and median urinary albumin excretion $8.1 \mathrm{mg} / 24 \mathrm{~h}$ (IQR: $6.0-12.1 \mathrm{mg} / 24 \mathrm{~h}$ ). During a median follow-up of 10.3 years (IQR: 6.2-11.4 years), 872 subjects developed CKD. After adjustment for age, sex, weight, height, smoking, alcohol consumption, family history of CKD, education, race, and 24-h urinary sodium, urea, and creatinine excretion, each $21 \mathrm{mmol} / 24 \mathrm{~h}$ (1 standard deviation) decrement in UKV was significantly associated with a $12 \%$ higher risk of developing CKD (hazard ratio, 1.12: $95 \%$ confidence interval, 1.03-1.21). No association between $\mathrm{UNaV}$ and risk of CKD was observed after multivariable adjustment (hazard ratio per $50 \mathrm{mmol} / 24 \mathrm{~h}$ decrement [1 standard deviation], 1.05: $95 \%$ confidence interval, 0.95-1.16). Sensitivity analyses studying CKD incidence defined by either impaired eGFR creatinine or increased albuminuria only, rendered essentially similar results.

Conclusion: Low dietary potassium uptake and not high sodium uptake was associated with an increased risk of developing CKD in the general population, suggesting that an increase in potassium intake may be beneficial to prevent incident CKD.

\section{Abstract \#: P 507}

Dietary habits associated with overweight and obesity among preschool children in Kenitra, northwest of Morocco

Meriem. Sbai ${ }^{1}$, Youssef. Aboussaleh ${ }^{1}$, Rachid. Sbaibi ${ }^{1}$

${ }^{1}$ Ibn Tofail University; Corresponding author's e-mail: abou_85@yahoo.fr
Objective: The aim of this study is to determine the dietary habits associated with obesity and nutritional status in preschool children in Morocco.

Design: Cross-sectional study of a representative sample of 5-6 yearold preschool childrenperformed from May to December 2012.

Setting: 9 primary schools located in Kenitra, the north-west part of Morocco.

Subjects: This study consisted of 247 preschool children, including 120 boys and 127 girls aged 60 to 84 months who were randomly recruited from Kenitra, Morocco.

Methods: Children's height, weight and body mass index (BMI) were measured using a standardized protocol. Indices of obesity (normal weight, overweight, obesity) were defined according to World health Organisation (WHO) standards published in 2007. Data were collected using an appropriate questionnaire completed by parents.

Results: The results showed that $14 \%$ of preschool children are overweight, against $6 \%$ having obesity. In the case of preschool children with normal weight, mean and z-score of weight were significantly higher in boys than in girls. Preschool children who do not eat olives or olive oil $(\mathrm{OR}=3.80,95 \% \mathrm{CI}=1.27,11.35)$ have a risk to developing an overweight or obesity 4 times more higher than children who consume at least once a day. Consuming unhealthy dietary habits is not associated with the development of overweight and obesity.

Conclusion: It appears that Moroccan preschool children are at risk of developing obesity which can persist into adolescence and adult causing cardiovascular disease, particularly among children not consuming healthy dietary habits especially olives and its oil on a daily basis. This study should be completed by the inclusion of additional questions about levels of physical activity and sedentary behaviour. Urgent interventions are strongly recommended to promote a healthy lifestyle among preschool children in Morocco.

\section{Abstract \#: P 508}

Food insecurity in relation to physical, cognitive, and emotional challenges among older adults: a multi country analysis

\section{Manisha Dubey ${ }^{1}$}

${ }^{1}$ International Institute for Populations Sciences, Mumbai, India; Corresponding author's e-mail: manikvdlw@gmail.com

Food security is considered an essential measure of population wellbeing. Even though, older adults are recognized as an age group at risk, comparatively little food security research has been conducted among aging populations.

The present study investigates links between physical and mental health conditions and food insecurity among adult participants 60 years and older $(n=20,863)$ in SAGE Wave 1 (2007-2010). SAGE is a longitudinal study with nationally representative samples of persons aged 50+ years in China, Ghana, India, Mexico, Russia and South Africa. These countries provide a broad representation from different geographic regions of the world, various stages in the demographic, economic and health transition, and include the two most populous countries of the world.

Among SAGE adults 60 years and older, $15.7 \%$ reported they ate less because of insufficient food, and $11.9 \%$ reported they experienced hunger in the past year, with both variables combined to reflect an overall prevalence of food insecurity at $15.8 \%$. Logistic regression results suggest that poorer physical mobility increases the likelihood of food insecurity among Indian and Mexican older adults by 27.2 and $32.2 \%$, respectively. Poorer cognitive function among South African adults predicted a $29.5 \%$ increase in food insecurity. In contrast, poorer cognitive function among Ghanaian adults 
predicted a $34.3 \%$ decrease in food insecurity. Depression increased the odds of older adult food insecurity in Ghana (489.6\%) and South Africa (258.6\%). Indian and Mexican adults' food security appears related to physical mobility, with Ghanaians' food security most challenged by depression. The finding that Ghanaian older adults faced reduced food insecurity risk with lower cognitive function is surprising given prior work showing that poorer cognitive function among older adults is associated with increased food insecurity. Findings suggest that older adults' vulnerability to food insecurity is intimately linked to country-specific cultural, social, and economic values and conditions.

\section{Abstract \#: P 509}

Patterns of biomechanical demands are associated with musculoskeletal pain in the beginning of professional life: a population-based study

$\underline{\text { Sara Lourenço }}^{1}$, Fábio Araújo ${ }^{1}$, Milton Severo ${ }^{1,2,3}$, Luís Cunha Miranda $^{4}$, Filomena Carnide, Raquel Lucas ${ }^{1,2}$

${ }^{1}$ EPIUnit - Institute of Public Health, University of Porto; ${ }^{2}$ Department of Clinical Epidemiology, Predictive Medicine and Public Health, University of Porto Medical School; ${ }^{3}$ Department of Medical Education and Simulation, University of Porto Medical School; ${ }^{4}$ Portuguese Institute of Rheumatology; ${ }^{5}$ Faculty of Human Kinetics, University of Lisbon; Corresponding author's e-mail: slourenco@med.up.pt

Background: The impact of occupational biomechanical demands on musculoskeletal pain in early stages of employment remains fairly unknown. Therefore, we aimed to assess whether short-term exposures to different patterns of work-related biomechanical factors have an effect on the presence and intensity of regional musculoskeletal pain since the beginning of professional life.

Methods: Cross-sectional data from participants aged 21 years were collected during the third wave of the EPITeen cohort $(n=1733$ : $37.5 \%$ were workers). Ten different work-related tasks were collected (sitting position, computer use, whole body vibrations, handling vibration tools, precision demands, repetitive tasks, overhead and kneeling work, bending or rotation movements and manual materials handling) and a latent class analysis was computed to identify patterns of occupational biomechanical demands. The presence and intensity of musculoskeletal pain with respect to four anatomical regions (neck/shoulder, upper limb, upper/lower back and lower limb) were evaluated through the Nordic Musculoskeletal Questionnaire.

Two methodological strategies were considered to estimate the associations between patterns of occupational biomechanical demands and regional musculoskeletal pain: a) whole sample, using as reference the non-workers: b) only workers, using as reference those with the lowest level of occupational biomechanical demands. Crude and adjusted odds ratios (OR) with $95 \%$ confidence intervals (95\% CI) were computed and estimates were adjusted for sex, body mass index, physical activity, smoking and education. Associations were additionally adjusted for occupational psychosocial context when only workers were considered.

Results: Four patterns of occupational biomechanical demands were identified: "low demands", "sitting demands", "repetitive \& asymmetric demands", and "high \& vibrational demands". Workers with occupations characterized by "repetitive \& asymmetric demands" or "high \& vibrational demands" presented respectively more than $50 \%$ (compared to non-workers) and $80 \%$ (compared to workers with "low demands" occupations) higher adjusted odds of reporting neck/ shoulder pain. These patterns were also significantly associated with the presence and severity of upper/lower back pain. Whether compared to the non-workers group or to the pattern of "low demands", occupations characterized by "sitting demands" were significantly related to the presence and intensity [adjusted OR (95\% CI): 1.62 [1.14-2.31) and 1.66 (1.03-2.66), respectively] of upper/lower back pain.

Conclusion: Patterns of highly physically demanding work-related tasks were associated with neck/shoulder pain and presence and severity of upper/lower back pain. This emphasizes the need to measure and monitor occupational biomechanical exposures since the beginning of professional life in order not only to prevent pain episodes but also to interrupt their evolution into chronicity.

\section{Abstract \#: P 511}

Co-benefit on mortality from pro-bicycle policy in Changwon, Korea: a health impact assessment

$\underline{\text { Sanghyuk Bae }}^{1}$, Yun-Chul Hong ${ }^{2}$

${ }^{1}$ Public Health Medical Service, Seoul National University Hospital, Seoul, Republic of Korea; ${ }^{2}$ Department of Preventive Medicine, College of Medicine, Seoul National University, Seoul, Republic of Korea; Corresponding author's e-mail: sanghyukbae@snu.ac.kr

Background: The city government of Changwon, Republic of Korea has implemented a pro-bicycle policy called "Bicycle City Changwon" since 2006. Shifting from motor vehicle to bicycle has direct and indirect health benefits. The present study was to assess the health benefits of shifting from motor vehicle to bicycle.

Method: We assessed the health benefit from reduced mortality due to increased physical activity and decreased air pollution, and the risk from increased traffic accidents. We conducted telephone survey to estimate the level of bicycle use among adults in the city. The Health Economic Assessment Tool (HEAT) was used to assess the health benefit from increased physical activity. We also conducted timeseries analysis to examine the time trend of traffic accident mortality and the concentration of PM10. The AirQ, a tool for health impact assessment for air pollution, was used to estimate the decreased mortality due to the reduction of air pollution.

Result: At the current level of bicycle use, 5.5 deaths per year were estimated to be prevented due to the increased physical activity. The trend of PM10 changed from increasing to decreasing after 2006. The estimated reduction of mortality due to the decreased PM10 was 21.9 cases over 4 years. The benefit exceeded the increase of mortality due to traffic accidents.

Conclusion: The present analyses shows that the implementation of pro-bicycle policy have brought significant health benefit due to the increased physical activities and reduced air pollution.

\section{Abstract \#: P 512}

Epidemiologic profile of male health workers in the state of Bahia, Brazil

Layla B. C. Wright ${ }^{1}$, Tania Maria de Araujo ${ }^{1}$, Maura Maria Guimarães de Almeida ${ }^{1}$, Fernanda Oliveira Souza ${ }^{1}$

${ }^{1}$ Núcleo de Epidemiologia, Universidade Estadual de Feira de Santana, Feira de Santana, Brazil; Corresponding author's e-mail: laylabc@hotmail.com

Introduction: health workers of the Unique Health System of Brazil are an important work force. But little is known of their working conditions and health, mainly the male category recently introduced 
in this context. It is understood that these professionals are the pillars of the health system and, therefore, characters of the development and improvement of this system, being a key arrangement to the work in health.

Methodology: it is a transversal study, of exploratory nature. We had the participation of male workers of the primary attention and of medium complexity of the health system. To calculate the size of the sample we used the outcome prevalence $(20.3 \%)$, confidence interval of $95 \%$ and $3 \%$ precision. Considering $20 \%$ of losses and refusals, the sample was estimated of 466 workers. The descriptive analysis was done using the absolute and relative frequencies of the interest variables. The research was approved by the Ethics Committee of the State University of Feira de Santana, under the protocol number 081/2009.

Results: Among the 672 male workers of primary and medium complexity health care who accepted participate in the study, $58.7 \%$ referred having a stable marriage relationship, $63.9 \%$ had children, $70.7 \%$ received up to three minimum wages, the average age was 40 years old, and $52.5 \%$ had completed high school. As for the main habits, $23.7 \%$ referred the use of tobacco and $58.7 \%$ informed ingestion of alcohol. As for the matters related to the work, $49.6 \%$ referred having a permanent working bond, $30 \mathrm{~h}$ of working hours was indicated by $71.7 \%$ of men, and in the last 12 months only $2.7 \%$ referred a working or journey accident. When asked about their quality of life, $68.8 \%$ considered it positive and as to their satisfaction at work, $72.6 \%$ were satisfied or very satisfied and $50.1 \%$ of them would candidate themselves again to the same job.

Conclusions: although the monthly wages are considered below the ideal, the workers consider their quality of life and satisfaction at work as positive. The evaluation of health conditions of the health workers has gained space in scientific publications. But the investigation of the aspects related to these health workers in primary attention and medium complexity health attention is still recent. This group's health needs a deeper investigation, mainly the men of this group, majorly female.

\section{Abstract \#: P 513}

\section{Vaccination against hepatitis be anti-HBs between health workers of achievement}

Fernanda de Oliveira Souza ${ }^{1}$, Tânia Maria de Araújo ${ }^{1}$, Paloma de Sousa Pinho Freitas ${ }^{1}$, Mariana Rabelo Gomes ${ }^{1}$, Layla Baptista Cavalcante Wright ${ }^{1}$

${ }^{1}$ Epidemiology Unit, Department of Health, Universidade Estadual de Feira de Santana, Bahia, Brazil; Corresponding author's e-mail: nandaolisouza@gmail.com

Background: The infection by the Hepatitis B Virus (HBV) can occur in any person, however there are groups substantially more exposed to the HBV, like the newborn from mothers that have the HBsAg, dyalisis patients and health-care professionals.

Methods: To verify the prevalence and the associated factors due to vaccination for Hepatitis B between primary health care professionals and mean complexity of the health sector, a cross-sectional study was conducted, with 506 professionals, whose data were collected in 2011 from the health sector workers in a city of the "Recôncavo Baiano". To investigate the immunization status for hepatitis B, were used questionnaire, sociodemographics and occupational characteristics. Uni and bivariate analysis were used for assessment of the associated factors from vaccination.

Results: The population studied were formed by women $(78.3 \%)$, permanent work contract $(63.8 \%)$. Most of them reported receiving three doses of vaccine against hepatitis B (59.9\%). The nurses, technicians and physicians were the most immunized $(91.8 \%)$. Regarding the associated factors with reported vaccination were: gender, age, education, personal protective equipment use and contact with biological material.

Conclusion: The health care professional's role as transmitting infectious diseases in clinical practice cannot be neglected, shall be taken into account that these are more exposed to communicable diseases, and they should be appropriately imunnized, in addition to having the confirmed immunity by the anti-HBs exam. The worker's health cannot be measured only by the absence of occupational diseases and accidents at work: but on the aspects that point to the workerl's insertion in the production process, from measures beyond those protection. Considerations: Points related to biosecurity in the academic curriculum may collaborate for the incorporation of more positive attitudes towards accession to vaccination and maintenance of vaccination status in view of the concern for occupational health.

\section{Abstract \#: P 514}

Factors associated to vaccination among community health agents Fernanda de Oliveira Souza ${ }^{1}$, Tânia Maria de Araújo ${ }^{1}$, Layla Baptista Cavalcante Wright ${ }^{1}$

${ }^{1}$ Epidemiology Unit, Department of Health, State University of Feira de Santana, Bahia, Brazil; Corresponding author's e-mail: nandaolisouza@gmail.com

Background: The Community Health Agent (CHA) represents a group of health workers and has become a political actor in the scenery of health assistance and its organization. The CHA is inserted in the family health program and should develop disease prevention activities and health promotion, with actions related to the vaccination of the community. Thus, it is interesting they have the complete vaccination calendar: hepatitis $B$, yellow fever, triple viral and diphtheria and tetanus.

Methodology: This is a transversal study of analytic nature. We had the participation of workers from the primary health and medium complexity health. To calculate the size of our sample we used the outcome prevalence $(21.8 \%$ ), a $95 \%$ reliable interval and precision of $3 \%$. Considering $20 \%$ by losses and refusals, the sample was estimated in 736 workers. The analytical description was done using the absolute and relative frequencies of the interest variables and the divaricated analyses, which confirmed the association between the variables of interest and vaccination. We also calculated the prevalence ratio: the $p$ value $(<0.05)$ was considered for statistic significance. The research was approved by the Ethical Committee in Research of the State University of Feira de Santana under the protocol number 081/2009.

Results: Among the 932 community agents, $86.5 \%$ were female, $86.0 \%$ were under 49 years old, $77.8 \%$ had children, $67.0 \%$ had partners and $90.1 \%$ declare themselves black or dark. As to the evaluation of the complete vaccination for the four vaccines, the prevalence among the CHA was $33.0 \%$. The complete vaccination was more prevalent in the women $(35.1 \%)$, in individuals under 49 years old $(34.1 \%)$, black or dark skin $(34.1 \%)$. Factors associated with vaccination: female gender, age over 50 years old and the skin color black or dark.

Conclusion: We considered very low the prevalence of global vaccination for the four vaccines, having in mind that they are offered free by the Health Ministry for all adult individuals (19-49 years old). Thus, it is necessary to propose sensibilization about vaccination of this group, having in mind the low prevalence of vaccination found. One must consider the social position that this worker occupies, since he lives in the community and he develops the role of multiplying 
health actions and that from his awareness they may become more effective.

\section{Abstract \#: P 515}

\section{Short-term mortality effects of ozone in patients with chronic obstructive pulmonary disease in the republic of Korea}

Jong-hun Kim ${ }^{1}$, Byoung-Hak Jeon ${ }^{1}$, Myung-jae Hwang ${ }^{1}$, Hae-kwan Cheong ${ }^{1}$

${ }^{1}$ Department of Social and Preventive Medicine, School of Medicine, Sungkyunkwan University; Corresponding author's e-mail: kimjh32@skku.edu

Background: Air pollutants have been associated with increased mortality according to several studies carried out. But few epidemiologic studies have assessed the effect of ambient ozone on mortality in Republic of Korea. We aimed to assess the short-term effects of ambient levels of ozone on daily COPD mortality.

Methods: Daily mortality of COPD data, maximum 8-h moving average concentration of ozone, ambient temperature, relative humidity and influenza like illness data were obtained from 2011. Season was divided two period as warm and cold by 10 degree Celsius of annual average temperature. The relationship between daily COPD mortality and maximum 8 -h moving average concentration of ozone was modelled using generalized linear regression analysis. Potential confounding factors due to ambient temperature, relative risk and influenza epidemics were adjusted.

Results: In the analyses stratified by season, both warm season and cold season showed most significant association in 2-lag day and in 1-lag day, respectively. The excess risks per $1 \mathrm{ppb}$ increase in concentration of ozone for daily COPD mortality increased $0.42 \%$ $(0.15-0.69)$ in warm season and $0.92 \%(0.12-1.73)$ in cold season. Conclusions: Our results showed that ambient maximum 8-h moving average concentration of ozone is associated with daily COPD mortality in both warm and cold seasons.

\section{Abstract \#: P 516}

Economic recession and incidence of sickness absence in Catalonia, Spain

Elena Zaballa ${ }^{1}$, Xavier Duran ${ }^{2}$, María Andreé López ${ }^{2}$, Albert Sanchez-Niubó $^{2}$, David Gimeno ${ }^{3}$, Fernando G. Benavides ${ }^{2}$

${ }^{1}$ Center for Research in Occupational Health (CiSAL), Universitat Pompeu Fabra (UPF), Barcelona, Spain/Hospital del Mar Medical Research Institute (IMIM); ${ }^{2}$ Center for Research in Occupational Health (CiSAL), Universitat Pompeu Fabra (UPF), Barcelona, Spain: CIBER in Epidemiology and Public Health (CIBERESP), Spain: Hospital del Mar Medical Research Institute (IMIM), Barcelona, Spain; ${ }^{3}$ Center for Research in Occupational Health (CiSAL), Universitat Pompeu Fabra (UPF), Barcelona, Spain: CIBER in Epidemiology and Public Health (CIBERESP), Spain: Hospital del Mar Medical Research Institute (IMIM), Barcelona, Spain: Department of Epidemiology, Human Genetics and Environmental Sciences, The University of Texas School of Public Health, San Antonio Campus, San Antonio, TX, USA.; Corresponding author's e-mail: elena.zaballa@upf.edu

Background: The global financial crisis began in 2007 led to a severe recession in Spain, with strong growth domestic product contractions in 2009 and 2102. The aim of this study was to compare incidence rates (IR) of sickness absence (SA) in 2009 and 2012 among salaried workers in Catalonia (Spain).

Methods: We examined salaried workers from Catalonia, 110,612 in 2009 and 105,854 in 2012, who were part of the Continuous Working Life Sample (CWLS). We obtained information on sex, age, company size, economic activity, occupation, contract of employment and diagnoses of SA episodes. First, we estimated sex-stratified incidence rates (IRs) of all-cause SA and cause-specific SA (i.e., due to musculoskeletal and mental disorders). Then, we compared the IRs, taking 2009 as the reference year, using rate ratios (RRs) and their corresponding $95 \%$ confidence intervals from Poisson regression models.

Results: IRs of all-cause SA decreased between 2009 and 2012 for both sexes: $20 \%$ in men (RR 0.80: $95 \%$ CI 0.78-0.82) and $19 \%$ in women (RR 0.81: $95 \%$ CI 0.79-0.83). These differences persisted after adjustment for age, company size, economic activity, occupation and contract of employment. For specific causes, the largest decrease between 2009 and 2012 was observed among women regarding SA episodes due to mental health problems (from 27.4 to 19.3 per 1000 person-years). Overall, sex-stratified patterns of decrease in the IRs of SA episodes due to musculoskeletal or mental disorders were similar to those of all-cause SA and with adjustment for covariates having minimal impact.

Conclusion: Our results show that, between 2009 and 2012, there was a significant decrease in the IRs of SA in both sexes and regardless of medical diagnoses and demographic and job-related covariates. Thus, our results suggest that the economic crisis contributed to the decline of sickness absence episodes. Future research should examine the role that other individual (e.g. perceived job insecurity) and contextual factors (e.g., a more strict labour legislation) might have had in the observed decrease.

\section{Abstract \#: P 517}

Prenatal Bisphenol a exposure may aggravate symptoms of autism spectrum disorder

Youn-Hee Lim ${ }^{1}$, Sanghyuk Bae ${ }^{1}$, Boong-Nyun Kim ${ }^{1}$, Choong Ho Shin $^{1}$, Young Ah Lee ${ }^{1}$, Yun-Chul Hong ${ }^{1}$

${ }^{1}$ Seoul National University College of Medicine; Corresponding author's e-mail: limyounhee@gmail.com

Objective: To estimate the impact of gestational and childhood Bisphenol A (BPA) exposures on symptoms of autism spectrum disorder (ASD) at 4 years of age and to determine whether child gender modified the associations.

Methods: We used a prospective birth cohort of 232 mothers and their 4-year-old children from Incheon and Seoul area in Korea. We characterized gestational and childhood BPA exposures by using BPA concentrations in maternal (during 2nd trimester of pregnancy) and child (4 years of age) urine samples, respectively. Symptoms of autism spectrum disorder were measured by using the Social Communication Questionnaire (SCQ) completed by parents or caregivers. The SCQ includes information on a child's body movements, use of language or gesture, and style of interaction. We used regression models adjusting for maternal age and education, a child's body mass index, age, gender, parity, and secondhand smoking, maternal BPA during 2nd trimester of pregnancy, and childhood BPA at 4 years old. Results: Mean gestational and childhood BPA concentrations were 2.1 and $7.2 \mu \mathrm{g} / \mathrm{L}$, respectively. With adjustment for confounders, each twofold increase in gestational BPA concentrations was associated with symptoms of ASD [relative risk (RR) 1.06, (95\% confidence intervals (CI), 1.01, 1.12], especially in style of interaction [RR 1.14 $(95 \% \mathrm{CI}, 1.02,1.26)]$ and use of language or gesture [RR $1.08(95 \%$ 
CI, 1.00, 1.18)]. Subgroup analysis according to sex showed that gestational BPA aggravated symptoms in use of language or gesture among girls $(P$ value 0.08$)$ and in style of interaction among boys $(P$ value 0.06$)$. We observed null associations between ASD and childhood BPA.

Conclusions: In this study, gestational BPA exposure affected child's symptoms of ASD, especially in use of language among girls and style of interaction among boys at 4 years of age.

\section{Abstract \#: P 518}

In-work poverty and health in Germany: Are trajectories of inwork poverty associated with between and within differences in self-rated health?

\section{Timo-Kolja Pförtner $^{1}$, Anika Nitzsche ${ }^{1}$}

${ }^{1}$ Institute of Medical Sociology, Health Services Research, and Rehabilitation Science (IMVR), Cologne, Germany; Corresponding author's e-mail: timo-kolja.pfoertner@uk-koeln.de

Introduction: This study investigated whether the presence of inwork poverty, the duration of in-work poverty and the elapsed time since last in-work poverty spell were associated with between and within differences in poor self-rated health (SRH).

Methods: We used logistic fixed- and random-effects hybrid model in a nationally representative German sample with 17 waves of data collection (1995-2012) to estimate between and within effects of different trajectories of in-work poverty on poor SRH. Three different trajectories of working poverty were studied: the current presence of in-work poverty, the duration of in work poverty and the elapsed time since last spell of in-work poverty. Interactions by age and sex were tested.

Results: In-work poverty was significantly associated with higher odds of poor SRH only between individuals. Between and within individuals, the odds of poor SRH were significantly higher with increasing duration of in-work poverty. Between individuals, recent spells of in-work poverty were significantly associated with higher odds of poor SRH, while within individuals odds of poor SRH were significantly higher the longer the last in-work poverty spell dated back. Differences in age and sex were observed but were not significant.

Conclusions: In-work poverty and different trajectories of in-work poverty along the life-course were significantly associated with poor SRH in Germany. In-work poverty is an emerging issue in public health and epidemiology and need urgent policy action.

\section{Abstract \#: P 519}

Development of a risk score for the prediction of COPD in the European community respiratory health survey (ECRHS) III cohort

Sofie Acke $^{1}$, Marcelius Atanga ${ }^{2}$, Hayat Bentouhami ${ }^{2}$, Marjoke Van $\overline{\text { Der Burg }}^{2}$, Vera Nelen ${ }^{2}$, Marc van Sprundel ${ }^{3}$, Joost Weyler ${ }^{2}$

${ }^{1}$ Mensura Occupational Health Services, Italiëlei 2, B-2000 Antwerp, Belgium; ${ }^{2}$ Epidemiology and Social Medicine, University of Antwerp, Universiteitsplein 1, B-2610 Antwerp, Belgium; ${ }^{3}$ Provincial Institute of Hygiene, Kronenburgstraat 45, B-2000 Antwerp, Belgium; Corresponding author's e-mail: Sofie.Acke@Mensura.be

Background: Chronic obstructive pulmonary disease (COPD) is a growing cause of morbidity and mortality worldwide, and accurate prediction of the individual development of this disease is needed to anticipate the future burden of COPD, target key risk factors, and plan for providing COPD-related health services.

Methods: 323 participants of the Antwerp part of the ECRHS III, completed in the year 2013 post-bronchodilator spirometry testing plus questionnaires about respiratory symptoms, health status and exposure to risk factors like smoking and occupation. A COPD prevalence estimate based on the Global Initiative for Chronic Obstructive Lung Disease 2011 diagnostic criteria was made. All potential predictive characteristics from the ECRHS II main questionnaire (with both non-clinical and clinical questions) and spirometric examination were explored and included in multiple logistic regression if $p \leq 0,20$ to develop a prediction model for COPD in ECRHS III, 12 years after part II.

Results: The prevalence of COPD (stage I to IV) was 11, $5 \%$ (SE 1, 7). All of these 37 subjects had persistent airflow limitation combined with respiratory symptoms and/or exposure to noxious agents but only in two of them, COPD had previously been diagnosed by a physician. Almost half of the subjects reported rhinitis and $25 \%$ ever had asthma. Hierarchical combination of the included predictors (smoking, occupation, traffic passing the house (cars/trucks), eating prepackaged food, snacks between meals, coughing, nasal allergies, asthma, pre-bronchodilation Tiffeneau $<70 \%$, etc.) resulted in one best discriminating prediction model after calibration, with the single spirometric predictor pre-bronchodilation Tiffeneau $<70 \%$. This model had an Area Under the Curve of 93, $1 \%$ (95\% CI 87, 4-98, $7 \%$ ). An individual risk score was derived, indicating a probability between $85,5 \%$ and $99,9 \%$ (depending on the degree of airflow limitation) to develop COPD for subjects with pre-bronchodilation Tiffeneau less than $70 \%$ in the ECRHS II.

Conclusions: A simple risk score based on only one spirometric characteristic to predict COPD 12 years later was developed. External validation and development of predicting models with other characteristics from the ECRHS II questionnaire is needed in the other participating ECRHS study centers through Europe. Although smoking cessation is becoming an increasingly urgent objective for an ageing worldwide population, a better understanding of prognostic factors that predict the individual probability to develop COPD is crucial to assist local public-health officials in elaborating the best possible primary prevention policies for their regions.

\section{Abstract \#: P 520}

Scarring and scaring effects of unemployment on heart disease during Europe's economic crisis: a prospective study from Germany

$\underline{\text { Jian } \mathrm{Li}^{1}}{ }^{1}$, Adrian Loerbroks ${ }^{1}$, Hans Bosma ${ }^{2}$, Peter Angerer ${ }^{1}$

${ }^{1}$ Institute of Occupational and Social Medicine, Center for Health and Society, Faculty of Medicine, University of Düsseldorf, Düsseldorf, Germany; ${ }^{2}$ Department of Social Medicine, Mastricht University, CAPHRI, Maastricht, The Netherlands; Corresponding author's e-mail: lijian1974@hotmail.com

Background: Much evidence has suggested that unemployment causes poor health, including heart disease. Studies among employed individuals indicate that their previous experience of unemployment (i.e., scarring effect) and anticipation of future unemployment (i.e., scaring effect) are associated with an increased risk of heart disease. Yet, potentially combined scarring and scaring effects of unemployment on heart disease have not been examined. We aimed to address this knowledge gap utilizing data gathered during Europe's recent economic crisis, which commenced in 2008.

Methods: We drew on prospective data from the German SocioEconomic Panel Study (2009-2011). A large working population 
$(n=7128)$ free from heart disease at baseline was included in the current analyses. Cumulative time of unemployment prior to 2009 was retrieved from the biography and life history. Anticipation of future unemployment was defined by respondents' ratings of the probability of losing their job within the next 2 years. Heart disease was measured by self-reports of a physician-diagnosis. Multivariate Poisson regression models were used to examine the effects with adjustment for covariates.

Results: During the prospective 2-year period, 114 heart disease events occurred (cumulative incidence $=1.6 \%$ ). The age- and sexadjusted models showed that the risk of heart disease was increased among those with long time of previous unemployment ( $\geq 2$ years) $(\mathrm{RR}=1.78,95 \% \mathrm{CI}=1.05-3.00)$, and also among those with high anticipation of future unemployment (probability $\geq 50 \%$ ) $(\mathrm{RR}=1.67,95 \% \mathrm{CI}=1.06-2.62)$ : among workers with both long time of previous unemployment and high anticipation of future unemployment, the risk was further elevated $(\mathrm{RR}=2.30,95 \%$ $\mathrm{CI}=1.48-3.58$ ). These associations were robust to additional adjustment for socioeconomic status, health behaviors, and health conditions at baseline, e.g., the combined scarring and scaring effects of unemployment on heart disease remained significant $(\mathrm{RR}=1.86$, $95 \%$ CI $=1.15-3.00)$.

Conclusions: During Europe's recent economic crisis, combined scarring and scaring effects of unemployment, for the first time, are observed to significantly increase the risk of incident heart disease in German workers.

\section{Abstract \#: P 521}

Trend in subjective well-being among Norwegian doctors from 2002 to 2012: a panel study

\section{Judith Rosta $^{1}$, Olaf G. Aasland ${ }^{2}$, Magne Nylenna ${ }^{3}$}

${ }^{1}$ Institute for Studies of the Medical Profession; ${ }^{2}$ Institute for Studies of the Medical Profession, Institute of Health and Society, University of Oslo; ${ }^{3}$ The Norwegian Knowledge Centre for the Health Services, Institute of Health and Society, University; Corresponding author's e-mail: judith.rosta@legeforeningen.no

Background: Doctors" well-being has gained increasing attention over the last decade. A high level of subjective well-being among doctors is important for the doctors themselves as well as for the quality of the health care systems. The aims of the study were to follow subjective well-being (SWB) among Norwegian doctors from 2002 to 2012, and to identify possible predictors of high levels of SWB in 2012.

Methods: The study population consisted of a representative sample of 1600-1800 doctors, selected from nationwide repeated postal surveys in 2002, 2004, 2006, 2008, 2010, and 2012. Response rates were between 65 and $73 \%$. SWB was measured with a single question on overall life satisfaction, scored from 1 (very dissatisfied) to 7 (very satisfied). Scores of 6 or 7 were considered high levels of SWB. General linear modelling, controlling for gender and age was used to describe the trend in SWB over time. Simultaneous effects on high levels of SWB were analyzed through a logistic regression model.

Results: Between 2002 and 2012, the mean-levels of SWB (5.3-5.4) were stable and the proportion of SWB above 5 increased from 52 to $57 \%$ among Norwegian doctors. High levels of SWB in 2012, controlled for gender, age, number of children and cohort effect, were significantly associated with reporting very good or good health (OR 3.7, $95 \%$ CI. 2.1-6.4), having high levels of job satisfaction (14.8, 8.6-25.6), living in very good quality of relationship $(1.9,1.1-3.4)$ vs. living without a partner (reference) and working as specialist in private practice $(2.4,1.2-4.8)$, full-time researcher $(2.7,1.5-5.0)$ or in administration (1.2, 0.6-2.4) vs. working in a hospital setting (reference).

Conclusions: No decline in SWB among Norwegian doctors was found over the study period. Job satisfaction, type of work, self-rated health and relationship status and quality showed effect on SWB.

\section{Abstract \#: P 522}

\section{Prenatal Bisphenol a exposure and child neuropsychological development}

Maribel Casas ${ }^{1}$, Joan Forns ${ }^{1,2}$, David Martínez ${ }^{1}$, Claudia Avella-García ${ }^{1,3}$, Damaskini Valvi ${ }^{1,4}$

Ana Ballesteros-Gómez ${ }^{5}$, Noelia Luque ${ }^{5}$, Soledad Rubio ${ }^{5}$, Jordi Julvez $^{1}$, Jordi Sunyer ${ }^{1}$, Martine Vrijheid ${ }^{1}$

${ }^{1}$ Centre for Research in Environmental Epidemiology (CREAL), Universitat Pompeu Fabra (UPF), CIBER Epidemiología y Salud Pública (CIBERESP), Barcelona, Spain; ${ }^{2}$ Department of Genes and Environment, Division of Epidemiology, Norwegian Institute of Public Health, Oslo, Norway; ${ }^{3}$ Unitat Docent de Medicina Preventiva i Salut Publica H. Mar-UPF-ASPB, IMIM (Hospital del Mar Medical Research Institute), Barcelona, Spain; ${ }^{4}$ Department of Environmental Health, Harvard T.H. Chan School of Public Health, Boston, Massachusetts, US; ${ }^{5}$ Department of Analytical Chemistry, University of Cordoba, Cordoba, Spain; Corresponding author's e-mail: mcasas@creal.cat

Background: There are concerns that bisphenol A (BPA) may be a neurodevelopmental toxicant, but evidence in humans for cognitive, psychomotor, and behavioral outcomes is very limited and inconsistent. Our aim was to examine the effects of prenatal BPA exposure on cognitive, psychomotor, and behavioral development in 438 children at 1,4 and 7 years of age.

Methods: BPA was measured in spot urine samples collected in trimester 1 and 3 of pregnancy from women participating in the INMASabadell birth cohort study. Cognitive and psychomotor development was assessed at 1 and 4 years using psychologist-based scales. Attention-deficit hyperactivity disorder (ADHD) symptoms and other behavioral problems were assessed at 4 years by teachers and at 7 years by parents using questionnaire-based rating scales.

Results: The average of the two creatinine-adjusted BPA concentrations was $2.5 \mu \mathrm{g} / \mathrm{g}$ creatinine. At 1 year of age, prenatal BPA exposure was associated with a reduction of psychomotor scores ( -4.28 points per $\log 10 \mu \mathrm{g} \mathrm{BPA} / \mathrm{g}$ creatinine, $95 \% \mathrm{CI}:-8.44$, $-0.41)$. At 4 years, BPA exposure was associated with an increased risk of ADHD-hyperactivity symptoms (incidence rate ratio $($ IRR $)=1.72: 1.08,2.73)$, particularly in boys ( $p$ for interaction $>0.1$ ). At 7 years, this association was not statistically significant but again stronger in boys ( $p$ for interaction $<0.1$ ). At 4 years, boys had an increased risk of ADHD-inattention symptoms whereas girls showed a reduced risk ( $p$ for interaction $<0.1$ ). BPA exposure was not associated with the cognitive scores at ages 1 and 4 years, or with other behavioral problems at 7 years.

Conclusion: Prenatal BPA exposure may affect children's behavioral development in a sex specific manner. There is a potential effect early in life on psychomotor development that disappears later in life.

\section{Abstract \#: P 523}

Vitamin B12 alleviate the adverse effects of PM2.5 on heart rate variability 
Youn-Hee Lim ${ }^{1}$, Yun-Chul Hong ${ }^{1}$

${ }^{1}$ Seoul National University College of Medicine; Corresponding author's e-mail: limyounhee@gmail.com

Background: Numerous epidemiologic studies have shown association between particulate matter less than 2.5 microns in aerodynamic diameter (PM2.5) and cardiovascular disease. However, only a few studies to determine individual level of a preventive strategy against the cardiovascular disease from air pollution were performed. We aimed to examine the possibility of alleviating effect of vitamin B intakes on the association between PM2.5 and heart rate variability and to further analyze which constituents are responsible.

Methods: We used a panel data of 329 elderly subjects in Seoul collected from 2008 to 2010 . Baseline data of food frequency questionnaires were used to compute dietary vitamin B intakes. Twenty three PM2.5 constituents and PM2.5 sources as well as PM2.5 total mass concentrations were examined. We measured the standard deviation of normal-to-normal intervals (SDNN), the square root of the mean squared differences of successive NN intervals (RMSSD), high frequency (HF), and low frequency (LF) for heart rate variability (HRV). We used mixed effect models with a compound symmetry covariance structure to assess the association of air pollution with HRV.

Results: Effects of total PM2.5 concentration on HRV was not significantly moderated by vitamin B intakes. However, PM2.5 constituents analysis showed that rMSSD and HF were reduced by 10.8 and $20.2 \%$, respectively, with an interquartile range (IQR) increase of organic carbon among those with low vitamin B12 intakes (below $10.6 \mu \mathrm{g} / \mathrm{day}$ ) whereas they displayed no associations among those with high vitamin B12 intakes. Reduction in rMSSD and HF associated with increases of Chromium was significantly different between those with low and high vitamin B12 intakes. Similar differences were observed in the relationship between Copper and HF. Among seven PM2.5 sources, an IQR increase of the four PM2.5 sources (gasoline and diesel exhaust, road dust, and burning of biomass) was related with reduction in rMSSD and HF by 11-12 and 20-21\%, respectively, among those with low vitamin B12 intakes, whereas no significant association was found among those with high vitamin B12 intakes.

Conclusion: The study may suggest that enriched intake of vitamin B12 may alleviate the effect on HRV by PM2.5, which was attributable to the particulate components, such as carbons and metals, or to the particulate sources, such as gasoline and diesel exhaust, road dust, and burning of biomass.

\section{Abstract \#: P 524}

Trends in sickness absence due to mental disorders among social workers in Finland and Sweden in 2005-2012

Otso Rantonen ${ }^{1}$, Paula Salo ${ }^{2}$, Tuula Oksanen ${ }^{1}$, Jaana Pentti ${ }^{1}$, Ellenor Mittendorfer-Rutz ${ }^{3}$, Jussi Vahtera ${ }^{4}$, Kristina Alexanderson ${ }^{5}$

${ }^{1}$ Finnish Institute of Occupational Health, Turku, Finland; ${ }^{2}$ Finnish Institute of Occupational Health, Turku, Finland: Department of Psychology, University of Turku; ${ }^{3}$ Department of Clinical Neuroscience, Division of Insurance Medicine, Karolinska Institute, Stockholm, Sweden; ${ }^{4}$ Finnish Institute of Occupational Health, Turku, Finland: Department of Public Health, University of Turku and Turku University Hospital, Turku, Finland; ${ }^{5}$ Department of Clinical Neuroscience, Division of Insurance Medicine, Karolinska Institute, Stockholm, Sweden; Corresponding author's e-mail: otso.rantonen@gmail.com
Objectives: To analyze trends in psychiatric work disability and subsequent returning to work in 2005-2012 among social workers in Finland and Sweden in comparison with pre-school teachers, special education teachers, and psychologists.

Methods: We used register data on long-term work disability (>14 days) due to mental disorders (ICD-10 codes F00-F99) from two prospective cohort studies (Finnish Public Sector study and All Sweden study). In the Finnish cohort the sample was 4849 employees working in Finland in 2005-2011 and in the Swedish sample 119,219 employees working in 2005-2012. In Finland this was 1155 social workers, 2419 pre-school teachers, 832 special education teachers and 443 psychologists and in Sweden the corresponding figures were $23,704,74,785,14,004,6726$. We used negative binomial regression models and estimated rate ratios (RR) for psychiatric work disability among social workers in reference to the other professions. In addition, we used Cox proportional hazard models to analyze the probability of returning to work after a work disability period due to mental disorders.

Results: Social workers had the highest rate of mean cumulative work disability days per person years due to mental disorders at the end of the study period both in Finland (7.2 days) and Sweden (10.5 days). Among social workers in Finland, risk of work disability due to mental disorders was 1.77 -fold (95\% CI 1.13-2.78) compared with pre-school teachers and 1.91-fold (95\% CI 1.08-3.41) compared with special education teachers, after adjustment for age and sex. There was no higher risk compared with psychologists. In Sweden, social workers were 1.43 (95\% CI 1.30-1.57) times more likely to have work disability due to mental disorders than pre-school teachers, 1.50 (95\% CI 1.31-1.72) than special education teachers, and 1.53 (95\% CI 1.29-1.81) than psychologists, when adjusted for age and sex. In the Finnish sample, there was no difference in the probability of returning to work in social workers compared with other occupations. In the Swedish sample, the probability of returning to work was 1.06 (95\% CI 1.03-1.09) times higher among pre-school teachers and 1.08 times (95\% CI 1.03-1.13) higher among special education teachers compared with social workers, after controlling for sex and age. There was no difference in the probability of returning to work between psychologists and social workers.

Conclusion: Our study showed that social workers are at a greater risk of work disability due to mental disorders compared with other professionals with similar educational level. The results were comparable both in Finland and Sweden.

\section{Abstract \#: P 525}

A cross sectional study of the association between heavy metals and uterine myomas in premenopausal Korean women

Shinhee $\mathrm{Ye}^{1}$, Eunhee $\mathrm{Ha}^{1}$, Hyewon Chung ${ }^{2}$, Kyungah Jeong, Yeon-ah Sung ${ }^{3}$, Hyejin Lee ${ }^{3}$, Changsun $\mathrm{Sim}^{4}$, Inbo $\mathrm{Oh}^{5}$

${ }^{1}$ Department of Preventive Medicine, College of Medicine, Ewha Womans University; ${ }^{2}$ Obstetrics and Gynecology, College of Medicine, Ewha Womans University; ${ }^{3}$ Internal Medicine, College of Medicine, Ewha Womans University; ${ }^{4}$ Department of Occupational and Environmental Medicine, Ulsan University Hospital, University of Ulsan College of Medicine; ${ }^{5}$ Environmental Health center, Ulsan University Hospital, University of Ulsan College of Medicine; Corresponding author's e-mail: shinheeye@ naver.com

Introduction: It has been hypothesized that exposure to exogenous estrogens may be associated with uterine myomas. We sought to investigate the association between heavy metals which have been shown to be hormonally active and these disorders using data from 
'Research on health effects of exposure to environmental risk factors in reproductive age women'.

Method: Study design is cross sectional. Women aged 30-49 years who had data on metals and the outcomes of interest, were premenopausal and non-pregnant were eligible $(n=307)$. Lead, cadmium, mercury were measured in whole blood. Diagnosis of uterine myomas was based upon pelvic ultrasonography. Logistic regression was used to examine the association between heavy metals and disease adjusting for use of age, birth control pills, age of menarche, parity, waist circumference.

Results: A significant association between blood cadmium concentration(GM: $0.98 \mu \mathrm{g} / \mathrm{L}, \mathrm{GSD} 1.41$ ) and uterine myomas was just observed. [higher than 90percentile $(1.567 \mu \mathrm{g} / \mathrm{L})$ versus lower than 90percentile: adjusted odds ratio $=3.245,95 \%$ confidence interval: 1.246-8.449].

Conclusions: Cadmium is known as metalloestrogen. Our results support the hypothesis that higher blood cadmium concentration is associated with the risk of uterine myomas. Because of this study design is cross sectional, the interpretation of results is limited. Further investigation in properly designed studies is needed.

\section{Abstract \#: P 527}

\section{Child injuries and risk factors}

$\underline{\text { Salma B. Galal }}^{1}$, Reda El Belbesi, Samia Hamad, Zeinab Al-Sayed

${ }^{1}$ EREN; Corresponding author's e-mail: salmagalal@yahoo.com

Background: Injury is a common cause for mortality in childhood. There are only few studies which look at the risk factors for injuries in children between 0 to 15 years of age.

\section{Aim:}

- To estimate the injuries incidence in children from 0 to 15 years of age in the household within last year

- To assess the risk factors contributing to injuries in children

Methods: A multistage random sample of households with a cluster survey technique (3) was conducted in a district in Cairo and a rural district. Only households with children $0-15$ years of age were included in this study. The sample was designed so as to take randomly 1200 questionnaires of clusters. Only one child was selected randomly from each eligible family with children from 0 to 15 years of age to avoid clustering effect of injuries in the same family. A proxy interview with mothers of children from 0 to 15 years of age was conducted with 1 year recall preceding the survey. Categories of injuries according to Manciaux and Romer (WHO) for laypersons were applied. SPSS 10 was used for the statistical analysis. Children with injuries were compared to those without.

Results: There is a significant difference between males (28\%) and females $(21 \%)$ in the occurrence of injuries. The mean age of mothers is significantly higher in injured children than in not injured. Distribution of serious injuries among age groups, gender and residence indicate that fractures were found more in 5-10 years and $10-15$ years old children, in males and rural areas. Burns were found more in 1-5 years old children, girls and urban area.

Distribution of mild injuries among age groups, gender and residence demonstrate that laceration was highest in age group 5-10 years, in females and rural area. Bruises are highest in 1-5 years old and in urban area. Abrasions were highest in 5-10 years and in urban area.

Conclusion: Around $30 \%$ of children $(0-15)$ had injuries within last year. There are rural/urban disparity, gender difference, and risk factors such as child labor and physical punishment.
Studies are needed to assess the time between injuries and reaching the health services.

\section{Abstract \#: P 528}

\section{Air temperature and mortality from hypertension in Astana, Kazakhstan}

Dinara Shalkarova ${ }^{1}$, Andrej M Grjibovski ${ }^{1,3}$, Nassikhat Nurgaliyeva $^{4,5}$, Telman Seisembekov ${ }^{4}$, Aliya Kosbayeva ${ }^{6}$, Bettina Menne ${ }^{6}$

${ }^{1}$ International Kazakh-Turkish University, Turkestan, Kazakhstan; ${ }^{2}$ Norwegian Institute of Public Health, Oslo, Norway; ${ }^{3}$ Northern State Medical University, Arkhangelsk, Russia; ${ }^{4}$ Medical University of Astana, Astana, Kazakhstan; ${ }^{5}$ WHO office in Kazakhstan, Astana, Kazakhstan; ${ }^{6} \mathrm{WHO}$ European Centre for Environment and Health, Bonn Office, Bonn, Germany; Corresponding author's e-mail: andrej.grjibovski@gmail.com

Background: Associations between temperature extremes and mortality from diseases of circulatory system have been observed in many cities in Europe. However, most of the studies have been performed in developed countries and in temperate climate while the evidence from developing settings with greater temperature extremes is still scarce. Astana-the capital of Kazakhstan-is the coldest capital in the European WHO region with one of the highest level of cardiovascular mortality provides unique opportunities for studying effects of climatic factors on diseases of circulatory system. The aim of this study was to explore associations between air temperature and daily mortality from hypertension in Astana, Kazakhstan.

Methods: Daily counts of deaths from hypertensive diseases (ICD-10 codes: I10-I15) among adults 18 years and older in Astana, Kazakhstan in 2000-2001 and 2006-2010, were obtained from the City Registry Office. The data on daily mean temperature, wind speed, humidity and barometric pressure were obtained from the National Hydrometeorological Service. The data were analyzed separately during warm (1 April-30 September) and cold (1 October-31 March) seasons and stratified by gender. Associations between mortality and apparent temperature (average for lags 0-3 in warm period and $0-15$ in cold periods) were studied using first order autoregressive Poisson regression models controlling for wind speed, barometric pressure and effects of month, year, weekends and holidays. Coefficients represent percent increase in the daily number of deaths by an increase in apparent temperature by $1{ }^{\circ} \mathrm{C}$. Apparent temperatures represent discomfort indices and are more appropriate for studies on the effects of weather since they combine the effect of temperature and humidity. Analyses were stratified by gender.

Results: An inverse association between the temperature and deaths from hypertensive diseases was found during warm period, but only among women: an increase in apparent temperature by $1^{\circ}$ was associated with a decrease in the daily number of deaths by $3.1 \%(95 \%$ CI: $0.2,6.1)$. No significant associations between apparent temperature and hypertension mortality during cold period were observed either for women $(\beta=2.0,95 \%$ CI: $-4.5,8.5)$ or for men $(\beta=-3.4 .0,95 \% \mathrm{CI}:-10.7,3.9)$.

Conclusions: Contrary to what has been reported from European settings, an inverse association was observed between mortality from hypertensive diseases and temperature during warm season. The association was limited to women. No association between cold temperatures and mortality from hypertension was observed. Factors behind these findings will be discussed. 


\section{Abstract \#: P 529}

\section{Atmospheric pressure and onset of episodes of Menière's disease:} a repeated measures study

$\underline{\text { Ralf Strobl }^{1}}$, Robert Gürkov ${ }^{2}$, Martin Müller ${ }^{1}$, Eva Grill ${ }^{1}$

${ }^{1}$ German Center for Vertigo and Balance Disorders, LudwigMaximilians-Universität München, Munich, Germany; ${ }^{2}$ Department of Otorhinolaryngology Head and Neck Surgery, LudwigMaximilians-Universität München, Munich, Germany;

Corresponding author's e-mail: ralf.strobl@ med.uni-muenchen.de

Background: Meniere disease (MD) is a peripheral inner ear disorder characterized by episodic vertigo, hearing loss and tinnitus. MD presents with attacks of up to several hours, often starting without a perceptible trigger, and belongs to the most debilitating known nonlethal diseases. With a prevalence of $200-500$ per 100,000 , it is a rather common inner ear disorder and causes a considerable socioeconomic burden due to its chronic and incurable nature. To date, the precise pathophysiologic mechanism of an MD attack is unresolved. Short-term changes in inner ear homeostasis are a putative mechanism that triggers MD attacks. Furthermore, changes in ambient air pressure are transmitted via the middle ear into the inner ear which subsequently may have a direct effect on the inner ear, i.e. changes in air pressure are potentially suitable to trigger an attack. The objective of this study was therefore to examine whether MD attacks are preceded by atmospheric pressure changes.

Methods: Patients of a tertiary outpatient dizziness clinic diagnosed with definite MD were asked to keep a daily vertigo diary to document MD attacks (2004-2009). Local air pressure, absolute temperature, and dew point temperature were obtained from the German Meteorological Service (DWD) on an hourly basis. Change in these meteorological parameters was conceptualized as the maximum difference in a $24 \mathrm{~h}$ time frame preceding each day. Effects were estimated using additive mixed models with a random participant effect. We included lagged meteorological parameters, age, sex, weekday and season in the model.

Results: A total of 56 persons, mean age 54 years (59\% female) were included. Mean follow-up time was 267 days. Persons experienced on average 10.3 episodes during the observation period (median 8). Age and change in air pressure 1 day before onset of a vertigo episode were significantly associated with vertigo onset risk $(\mathrm{OR}=0.978$ and $\mathrm{OR}=1.015)$. We could not show an effect of sex, weekday, season, air temperature, and dew point temperature.

Conclusion: Change in air pressure was significantly associated with onset of MD episodes, suggesting a potential triggering mechanism in the inner ear. MD patients may use air pressure changes as an early warning system for vertigo attacks.

\section{Abstract \#: P 531}

\section{Association between prenatal exposure to Bisphenol a and childhood blood pressure}

Sanghyuk Bae ${ }^{1}$, Youn-hee Lim², Young Ah Lee ${ }^{3}$, Choong Ho Shin ${ }^{3}$, Yun-Chul Hong ${ }^{2}$

${ }^{1}$ Public Health Medical Service, Seoul National University Hospital, Seoul, Korea; ${ }^{2}$ Environmental Health Center, College of Medicine, Seoul National University, Seoul, Korea; ${ }^{3}$ Department of Pediatrics, Seoul National University Children's Hospital, Seoul, Korea; Corresponding author's e-mail: sanghyukbae@snu.ac.kr

Background: Bisphenol A is considered as an endocrine disrupting chemical and its exposure is ubiquitous. Adverse health effects of bisphenol A exposure has been reported, including effects on cardiovascular system, and bisphenol $\mathrm{A}$ is suspected to cross placenta during pregnancy. The present study was aimed to evaluate the effect of prenatal exposure to bisphenol $\mathrm{A}$ on the blood pressure of the child at the age of 4 .

Methods: A cohort of 13,278 pregnant women was followed-up until the birth of the child from Aug. 2008 to Jul. 2011. Urinary bisphenol A concentration was measured at around 20 weeks of gestation. We followed up 440 randomly selected children at the age of 4 , and blood pressure was measured. We constructed linear regression model to examine the association between urinary bisphenol A concentration of mother at 20 weeks of pregnancy and blood pressure of the child, adjusting for potential confounders.

Result: Diastolic blood pressure of the child was significantly and positively associated with urinary concentration of bisphenol A measured during the pregnancy. For $1 \mu \mathrm{g} / \mathrm{L}$ increment of prenatal urinary bisphenol $\mathrm{A}$, the diastolic blood pressure was increased by $0.50 \mathrm{mmHg}(\mathrm{SE}=0.16, P$ value $=0.0022)$. However, the systolic blood pressure was not.

Conclusion: The present study suggests that exposure to bisphenol A during the pregnancy may affect the blood pressure of the child.

\section{Abstract \#: P 532}

Effect of air pollution on children's growth over the follow-up period of 24-Ãmonths: Mothers and ChildrenG's Environmental Health Study

Eunjeong Kim ${ }^{1}$, Eun-Hee $\mathrm{Ha}^{1}$, HyeSook Park ${ }^{1}$, Yun-Chul Hong ${ }^{1}$, Mina $\mathrm{Ha}^{1}$, Yangho Kim ${ }^{1}$, Byung-Mi Kim ${ }^{1}$

${ }^{1}$ Ewha Womans University, Department of Preventive Medicine, Ewha Medical Research Center, Ewha Womans University, Seoul, South Korea; Corresponding author's e-mail: lefty22@naver.com

Background: There have been some evidences that exposure to air pollution might be negatively associated with children's development. In this study, we investigated the association between pre/postnatal PM10 exposure and weight and length in children through 24 months of age.

Methods: This study is a part of MOCEH study which is an on-going, prospective birth cohort study in South Korea. Average exposure levels to PM10 of 1129 mother-child pairs during entire pregnancy and early childhood were estimated using inverse distance weighting (IDW) method. Birth outcomes such as birth weight, sex, and gestational age were obtained from medical records. Child weights and lengths were measured at each visits of children at 6, 12, and, 24 months of age and were transformed to weight for age z-scores and length for age $\mathrm{z}$-scores. Questionnaire was composed of residential factors, environmental exposure and general characteristics.

Results: In multiple linear regression model adjusting for mother's BMI, maternal age, maternal education, gestational age, and child sex, negative effect of PM10 exposure from 7 to 12 months was found on child to weight for age $\mathrm{z}$-scores at $12(\beta=-0.151 ; 95 \% \mathrm{CI}$ -0.238 to -0.063$)$ and 24 months $(\beta=-0.105 ; 95 \% \mathrm{CI}-0.200$ to $-0.010)$. The average concentration from 13 to $24-\tilde{A}$ months in children was also associated with weight for age $\mathrm{z}$-scores at 24 ( $\beta=-0.165 ; 95 \% \mathrm{CI}-0.310$ to -0.021$)$. In GEE analysis considering repeated measures at 6,12 , and 24 months, postnatal exposure to PM10 until 24 months of age was negatively associated with child weight $\mathrm{z}$ scores $(\beta=-0.057, \mathrm{CI}-0.104$ to -0.009 ) High exposure of PM10 from at birth to 6 months lowered length z-scores at 6 months $(\beta=-0.216 ; 95 \% \mathrm{CI}-0.311$ to -0.121$)$. No association was found between prenatal and postnatal exposure to PM10 and child length z-score at other time of measures. In GEE analysis, 
postnatal exposure to PM10 until 24-Ãmonths of age was negatively associated with child length $\mathrm{z}$ scores $(\beta=-0.094, \mathrm{CI}-0.146$ to -0.043 ) through 24 months of age.

Conclusion: Exposure to air pollution may affect the weight and length in early childhood. Further follow-up in the study will be needed to investigate whether the effect will be shown continuously or disappear at certain age as well as catch-up growth for children in low weight.* This study was supported by MOCEH (Mothers and Children's Environmental Health) project of the National Institute of Environmental Research, Republic of Korea.

\section{Abstract \#: P 533}

\section{Effort-reward-imbalance and sharps injuries in German} physicians: Is the association mediated by emotional exhaustion?

\author{
Natalie Riedel ${ }^{1}, \mathrm{Jian}_{\mathrm{Li}^{1}}{ }^{1}$, Adrian Loerbroks ${ }^{1}$, Peter Angerer ${ }^{1}$
}

${ }^{1}$ Heinrich-Heine-University of Düsseldorf, Medical Faculty, Institute of Occupational and Social Medicine; Corresponding author's e-mail: natalie.riedel@uni-duesseldorf.de

Objective: Sharps injuries are a major health concern for health care professionals around the world. Previous studies indicate that psychosocial work conditions such as long working hours, safety measures and work stress may account for sharps injuries. The potentially underlying mechanisms need further specification, though. In the hospital setting, empirical evidence exists for associations between work stress and burnout on the one hand and burnout and sharps injuries on the other hand. We therefore investigated whether work stress induced by an effort-reward imbalance was related to selfreported sharps injuries among physicians and whether this association was mediated by emotional exhaustion.

Methods: Our current analysis was based on the fourth wave (2014) of a German physician cohort. In that survey, 439 participants reported whether they had been hurt by a sharp object or cannula during the past year (sharps injuries, SI). Physicians' effort-rewardimbalance was measured by the ratio of perceived efforts and rewards. Emotional exhaustion (EE) was reflected by the Maslach Burnout Inventory scale. We adopted the four steps of mediation analyses recommended by Baron and Kenny: We examined the ageand gender-adjusted associations between (1) ERI and EE, (2) EE and SI, and (3) ERI and SI. (4) We added EE to the equation of (3) in order to observe whether the potential association between ERI and SI was statistically explained by EE. Finally, we evaluated the size of the indirect effect of EE in the association of ERI with SI using the bootstrap technique with 5000 replications.

Results: A total of 86 physicians (20\%) reported SI. Observations according to the four steps were as follows: (1) A higher ERI score was associated with a higher $\mathrm{EE}(\beta 0.50, p=0.000)$. (2) An increase in EE was linked to elevated odds of reporting SI [OR 1.56, $95 \%$-CI (1.24-1.97)]. (3) The association between ERI and SI appeared to be prominent [ERI OR 2.55, $95 \%$-CI (1.42-4.60)], but (4) was substantially attenuated by additional inclusion of EE in the model [ERI OR 1.67, $95 \%$-CI (0.85-3.24), EE OR $1.4295 \%$-CI (1.10-1.85)]. The indirect effect size of EE was estimated to be $50 \%$ [95\%-CI (11-94\%)].

Conclusions: In our sample, EE largely explained the association between ERI and SI. If confirmed elsewhere, this finding could justify expenses on work stress and burnout prevention programmes serving patients' safety, given that burnout is likely to cause medical errors in addition to sharps injuries.

\section{Abstract \#: P 534}

\section{Cause-specific work disability prior to the diagnosis of sleep} apnea

Paula Salo $^{1}$, Kristina Alexanderson ${ }^{1}$, Linnea Kjäldgård ${ }^{1}$, Jaana Pentti $^{1}$, Eleonor Mittendorfer-Rutz ${ }^{2}$, Mika Kivimäki ${ }^{1}$, Jussi Vahtera ${ }^{1}$

${ }^{1}$ Finnish Institute of Occupational Health, Turku, Finland; ${ }^{2}$ Insurance Medicine, Karolinska Institutet; Corresponding author's e-mail: paula.salo@ttl.fi

Background: Prior to sleep apnea diagnosis, increase in sickness absence have been observed. In men, lost work days due to any disease increased 1 year before sleep apnea diagnosis compared to controls, whereas in women the rates were higher already 5 years before the diagnosis. However, previous research used only inpatient data on diagnosed sleep apnea and all cause work disability.

Methods: We used data from the All Sweden study, a cohort of 9,704,655 individuals that were living in Sweden in years 2005-2010. Altogether 46,496 sleep apnea (primary or subsidiary diagnosis, ICD10 code G47.3) cases aged 20-64 years were identified from both inand outpatient registers in 2006-2010. Control group $(n=49,524)$ was formed by drawing a $1 \%$ random sample from the remaining cohort. Cause-specific work disability (ICD-10 code groups $\mathrm{C}$ and D, F, G, M, S and T) days in 2005-2010, obtained from a national register, for each individual were calculated as total net days of fulland part-time sickness absence or disability pension per each calendar year. Sex, age, educational level, marital status, area of residence, and country of birth were considered as covariates. The data were analyzed using negative binomial regression analyses with the generalized estimating equation.

Results: In work disability due to mental disorders, interaction of sleep apnea group and time was observed both for men $(p=.001)$ and women $(p=.02)$, after full adjustments. Among men, sleep apnea was associated with 1.27 -fold $(95 \%$ CI 1.12-1.44) greater risk of work disability due to mental disorders 5 years before the diagnosis and was at its highest (rate ratio [RR] 1.62, $95 \%$ CI 1.48-1.76) in the year of diagnosis. Among women, the RRs increased from 2.14 (95\% CI 1.91-2.41) 5 years before the diagnosis to $2.32(95 \%$ CI 2.13-2.52) in the year of diagnosis. Among men, sleep apnea was associated also with greater increase in work disability due to neurological diseases (IDC-10 code G) compared with controls. The RRs increased from 1.86 (95\% CI 1.33-2.59) 5 years before the diagnosis to 2.38 (95\% CI 1.87-3.05) in the year of diagnosis. In other disease categories analyzed, work disability rates were higher among those with sleep apnea compared with controls throughout the study period, and no interactions were observed.

Conclusion: Already 5 years before being diagnosed with sleep apnea, rates of work disability, particularly due to mental disorders, were higher in sleep apnea patients compared with controls.

\section{Abstract \#: P 535}

Fetal growth and maternal exposure to particulate air pollution more marked effects at lower exposure and modification by gestational duration

Ellen Winckelmans ${ }^{1}$, Bianca $\mathrm{Cox}^{1}$, Evelyne Martens ${ }^{2}$, Benoit Nemery $^{3}$, Tim S Nawrot ${ }^{1}$

${ }^{1}$ Hasselt University; ${ }^{2}$ Study Centre for Perinatal Epidemiology;

${ }^{3}$ University of Leuven; Corresponding author's e-mail:

ellen.winckelmans@uhasselt.be 
Background: While there is growing evidence that air pollution reduces fetal growth, results are inconclusive with respect to the gestational window of effect. We investigated maternal exposure to particulate matter (PM10) in the association with birth weight and fetus growth with a focus on shape of the association and gestational age.

Methods: The study population consisted of 648,711 singleton live births in Flanders (Belgium) between 1999 and 2009. PM10 exposure at maternal residence was averaged over various time windows. We used robust linear and logistic regression to estimate the effect of PM10 on birth weight and SGA. Segmented regression models were applied for non-linear associations.

Results: Among moderate preterm (32-36 weeks) and term ( $>36$ weeks) births, we found significant lower birth weight for all time windows. The estimated reduction in birth weight for a $10 \mu \mathrm{g} /$ $\mathrm{m} 3$ increase in average PM10 during pregnancy was $39.0 \mathrm{~g}$ [95\% confidence interval $(\mathrm{CI}): 26.4,51.5 \mathrm{~g}$ ] for moderate preterm births and $24.0 \mathrm{~g}$ (95 \% CI: 20.9, $27.2 \mathrm{~g}$ ) for term births. The corresponding odds ratios for SGA were 1.19 (95\% CI: 1.07, 1.32) and 1.09 (95\% CI: $1.06,1.12)$ respectively. Segmented regression models showed larger absolute estimates on fetal growth at lower PM10 concentrations.

Conclusion: Maternal PM10 exposure was significantly associated with a reduction in fetal growth among term and moderate preterm births, with a tendency of stronger effects for the latter and a flattening out of the slope at higher PM10 concentrations.

\section{Abstract \#: P 536}

\section{Sleep-related problems are associated with minor psychiatric} disorders among shift workers in southern Brazil

Maria Teresa Anselmo Olinto ${ }^{1}$, Anderson da Silva Garcez ${ }^{1}$, Jamile Block Araldi Macagnan ${ }^{1}$, Ruth Liane Henn ${ }^{1}$, Marcos Pascoal Pattussi $^{1}$

${ }^{1}$ University of Vale do Rio dos Sinos; Corresponding author's e-mail: mtolinto@gmail.com

Background: Psychiatric disorders have been shown an increasing prevalence in a worldwide population and this condition have been associated with increased mortality. In addition, the poor sleep quality has been demonstrated as an important cause for psychiatric disorders and shift work appears to impact the quantity and quality of sleep. Thus, the aim of this study was evaluate the association between sleep-related problems with minor psychiatric disorders in a sample of shift workers from a large plant located in southern Brazil.

Methods: A cross-sectional study was conducted with 1202 workers, 417 males and 785 females, between the ages of 18 and 50 years. Minor psychiatric disorders were assessed using the Self-Reporting Questionnaire (SRQ-20) and sleep-related problems were evaluated by the four following characteristics: sleep restriction $(5 \mathrm{~h}$ or less per day), difficulty in falling asleep, waking up during sleeping hours and sleep medication use. Multivariable analyses were calculated by Poisson regression and all analyses were stratified by gender.

Results: The prevalence of minor psychiatric disorders was $26.8 \%$ (95 \% CI: 24.3-29.3) among the total workers being more prevalent among females than males ( 30.2 vs. $20.4 \%: p<0.001)$. The presence of one sleep problem in the sample was $37.5 \%$ (95\% CI: 34.8-40.3) whereas $20.7 \%$ (95\% CI: 18.5-23.1) was classified as having two or more sleep problems. Sleep problems were associated with increased age, lower schooling and work on the night shift for both genders. Nightshift workers exhibited a significant association with sleep problems. According to the concomitant presence of sleeprelated problems the prevalence of minor psychiatric disorders showed a positive linear trend after adjusting for possible confounding factors (sociodemographic, behavioural, and occupational characteristics). The prevalence ratios for minor psychiatric disorders were 1.38 (95 \% CI: 0.86-2.20) and 3.25 (95\% CI: 2.07-5.11) in the males with one sleep problem and two or more sleep problems, respectively, compared with the reference category (absence of sleep problems). Similarly, among females, the prevalence ratios were 1.32 (95 \% CI: 0.99-1.77) and 2.28 (95\% CI: 1.74-3.00), respectively.

Conclusion: This study demonstrated a strong association between sleep-related problems and minor psychiatric disorders that can be influenced by working at night. Have two or more sleep problems increased the likelihood of minor psychiatric disorders in approximately threefold among males and twice among females. Furthermore, the prevalence of minor psychiatric disorders and sleeprelated problems were higher among females than males: however, the strengths of the association were higher in males.

\section{Abstract \#: P 537}

Patient's adherence to a walking program for non-specific low back pain

$\underline{\text { Ben van Koppen }}{ }^{1}$, Pim Zandwijk ${ }^{2}$

${ }^{1}$ PMC RijKo, Rotterdam, Netherlands; ${ }^{2}$ Gezondheidscentrum de Zorgmolen, Papendrecht, Netherlands; Corresponding author's e-mail: ben@pmcrijko.nl

Study Design. Cross sectional observational study.

Objective: To investigate adherence rate and influencing factors of patients referred to a physiotherapy clinic for non-specific low back pain to a one-off home activity advice.

Summary of background data: Research suggests that inadequate adherence to an activity advice during the intervention period might attenuate intervention effectiveness. Factors negatively influencing adherence, appeared to be of a behavioural nature related to person's beliefs and perceptions. Perceived barriers, low self-efficacy and unbeneficial Illness beliefs have been consistent predictors of nonadherence across studies.

Methods: Fifty one patients who reported non-specific low back pain received a one-off activity advice: daily 30 min-walk during the next week at a normal walking speed. Physical activity was observed with a validated tri-axial seismic accelerometer. Multiple patient related determinants were compared to the rate of adherence.

Results: Four patients ( $8 \%$ ) fully followed the walking advice. After conducting the Fisher's Exact test the group perceiving full realistic illness beliefs and the group scoring 200-299 on the self-efficacy scale showed significant best adherence to the activity advice. Nonadherence to the activity advice was identified in all other groups. Factors negatively influencing adherence behaviour were lack of time, weather conditions, and increasing low back pain.

Conclusion: Patient adherence in executing an activity advice for non-specific low back pain outside the clinical setting is poor and negatively influenced by specific factors.

Keywords: non-specific low back pain, adherence, activity advice, activity monitor, demographic information, clinical status, self-efficacy, illness beliefs, barriers, cut-off value.

\section{Abstract \#: P 538}

Trunk and pelvis motor control during motor and cognitive dualtasking in nonspecific chronic low back pain patients and healthy subjects 
Verbruggen Marlies ${ }^{1}$, Van Daele Ulrike ${ }^{2}$, Hallemans $\mathrm{Ann}^{2}$, Lagrin Sofie $^{1}$, Vaes Peter ${ }^{1}$

Corresponding author's e-mail: marlies.verbruggen@hotmail.com

Background: Poor trunk muscle coordination in response to postural adaptations of day-to-day activities is a reliable factor for chronic low back pain. Dual-tasking, or performing two or more activities at the same time, is a skill we often perform during these daily activities. Researchers have already investigated the effect of cognitive dualtasks on postural control of healthy subjects and low back pain patients. However, daily we perform many motor dual-tasks. According to the authors' knowledge, there has been no study that investigated both, cognitive and motor dual-tasks, in a low back pain population.

Purpose: The aim of this study was to investigate the influence of cognitive dual-tasks versus motor dual-tasks on motor control and muscle activity of pelvis and trunk in low back pain patients compared to healthy control subjects.

Methods: As basic task the participants performed a Waiters Bow, a standardised active trunk flexion movement test. This basic task was combined with two different cognitive tasks (= cognitive dual-tasks) and with a motor task (= motor dual-task). The Zebris, a 3-dimensional motion analysis system, measured the movements of pelvis and trunk. The ME 6000 registrated the activity of the M. Multifidus, M. Latissimus Dorsi, M. Obliquus internus and the M. Obliquus externus. Results: The control group $(n=16)$ moved significantly less with their thorax $(p<0.001)$, significantly more $(p=0.020)$ with their pelvis and showed a significant increase of M. Multifidus activity ( $p=0.020)$ during the motor dual-task when compared to the basic task or the cognitive dual-tasks. While the low back pain group $(\mathrm{n}=16)$, presented a similar but non significantly different trend.

Conclusions: The control group significantly changed their movement strategy when performing the motor dual-task compared to the basic or cognitive dual-tasks, in contrast with the low back pain group who did not change their movement strategy significantly.

Implications: The present study showed that chronic low back pain patients did not adapt their movement strategy during a motor dualtask in contrast with the healthy subjects who moved significantly different. Lack of neuromotor adaptation is typical in low back pain patients. It is important to keep in mind that a sudden low back pain onset is commonly reported during trunk flexion, like the Waiters Bow movement. Further research, about trunk flexion and dualtasking, should investigate the mechanism behind the deficiency of low back pain patients to adapt their movement strategy, given that this could lead to an appropriate treatment.

\section{Abstract \#: P 539}

Adherence to self-directed home exercises in patients with non-
specific low back pain: the relationship with readiness to change Laura M. Lundell, PT, MSc ${ }^{1}$, Dr. M.F. Pisters ${ }^{1}$, Prof. Dr. R.A. de $\mathrm{Bie}^{2}$

${ }^{1}$ Center for Physical Therapy Research and Innovation in Primary Care, Julius Health Care Centers, Utrecht, the Netherlands; ${ }^{2}$ Caphri School for Public Health and Primary Care, Maastricht University, Maastricht; Corresponding author's e-mail: lauralundell@live.nl

Background: Home-exercises are widely used in management of non-specific low back pain. Previous research on the effectiveness of home-exercise programs within musculoskeletal physiotherapy indicates that only one to two thirds of the patients are adherent to prescribed home-exercises during treatment. Adherence could be partly influenced by individual's readiness to change behavior and take an active role within treatment. The transtheoretical model has described the individual's readiness to change as a process of stages of change, unfolding over time (Precontemplation, Contemplation, Action, Maintenance). Several studies have hypothesized that the variation in the initial stage when entering physiotherapy might (partly) explain variation of adherence. The objective was to explore the relationship between readiness to change, as described in the Transtheoretical model, and adherence to self-directed home-exercises in patients with non-specific low back pain, treated with physiotherapy in primary care.

Methods: A cohort of patients was prospectively observed from the beginning of usual treatment in seven primary care practices in the Netherlands. Physiotherapists screened patients for eligibility and included patients with sub-acute, recurrent or chronic non-specific LBP. Readiness to change was measured with the Pain Stages of Change Questionnaire at baseline and in the second and sixth week. Adherence to the prescribed home exercises was measured in the second and sixth week with the Utrecht Home-Exercise Adherence Questionnaire. Association of readiness to change and adherence to home-exercises was explored using Relative Risk.

Results: In total 23 patients were followed during their treatment. At baseline, $8.7 \%(\mathrm{n}=2)$ of the patients were classified in the following stages: Contemplation, $73.9 \%(\mathrm{n}=17)$ in Action and $17.4 \%$ $(n=4)$ in Maintenance stage. Adherence to self-directed home-exercises (performance $>60 \%$ ) was $35.0 \%(n=7)$ in the second and sixth week. In comparison with higher stages, patients in Contemplation seemed to have 50-60\% higher chance for being nonadherent to the prescribed home-exercises.

Conclusion: The study shows that readiness to change was relatively high in the studied primary care population, implying that patients were prepared to take an active role in self-management and behavioral change. Although 'lower' readiness to change seems to be associated with higher chance for non-adherence, the number of adherent patients, even with high readiness to change, remains low. Self-directed home-exercises form an important part of the primary care physiotherapy for musculoskeletal disorders, and therefore new strategies are needed to improve adherence. Future research on the integration of Action and Coping Planning in self-management might offer new possibilities.

\section{Abstract \#: P 540}

Prognostic indicators of a back school program for nonspecific chronic low back pain patients: a retrospective cohort study

Lenie Denteneer ${ }^{1}$, Ulrike Van Daele ${ }^{1}$, Willem De Hertogh ${ }^{1}$, Steven Truijen $^{1}$, Gaetane Stassijns ${ }^{2}$

${ }^{1}$ University of Antwerp, Faculty of Rehabilitation Sciences and Physiotherapy, Belgium; ${ }^{2}$ University of Antwerp, Faculty of Medicine and Health Sciences, Belgium and the Antwerp University Hospital, Department of Physical Medicine and Rehabilitation; Corresponding author's e-mail: lenie.denteneer@uantwerpen.be

Background: Of all people who develop low back pain (LBP), $85 \%$ is named as being nonspecific. For these nonspecific LBP patients, exercise therapy is stated to be an effective intervention strategy. A common and effective type of therapy in the management of LBP are back school programs where exercise therapy (motor control and isometric training therapy) is often emphasized. Never before prognostic indicators for success or failure were derived for a back school program, which is the goal of this retrospective cohort study.

Methods: Out of 266 LBP patients, 49 chronic nonspecific LBP patients were retrospectively retained for further analysis based on predefined in- and exclusion criteria. As primary outcome the Oswestry Disability Questionnaire was used. All patients were 
evaluated and treated by physicians, psychologists, occupational therapists and physiotherapists. Thirty physiotherapy therapy sessions were preserved for isometric training therapy and three sessions to learn motor control floor exercises. Patients underwent therapy two times per week. Potential predictive variables were tested for their bivariate association with the reference standard using independent sample t tests and Chi square tests. Subsequently the possible predictors were then entered in a logistic regression analysis to determine the optimal predictive model.

Results: Nineteen patients were considered as therapy success, five patients as improved and twenty five as therapy failure. One possible predictor for success and three possible predictors for therapy failure were found. The strongest model for therapy failure was obtained with only one predictor namely the physical function subscale of the SF36 with a sensitivity of .79 and specificity of .68. As for therapy success we found one possible predictor namely the Zebris left rotation with a sensitivity of .41 and specificity of .72 .

Conclusion: The back school program mainly focusses on modifying body functions and contextual factors within the International Classification of Functioning, Disability and Health (ICF), activities and participation problems are not directly addressed. This study shows that targeting the ICF domains of body functions and contextual factors during therapy does not necessarily leads to an increased level of physical functioning, often one of the primary outcome measure of a back school program and accentuates the complexity and often scarcity in cohesion between the different ICF domains within a chronic LBP population. In future research we should use a RCT design and use a two or three arm design instead of the single arm design used here.

Keywords: Prognostic indicator, Nonspecific, Chronic, Low back pain.

\section{Abstract \#: P 541}

Structural and functional brain abnormalities in chronic low back pain: a systematic review

Jeroen Kregel $^{1,2,3}$, Mira Meeus ${ }^{1,3,4}$, Anneleen Malfliet ${ }^{1,2,3}$, Mieke Dolphens $^{1}$, Lieven Danneels ${ }^{1}$, Jo Nijs ${ }^{2,3}$, Barbara Cagnie ${ }^{1}$

${ }^{1}$ Ghent University, Department of Rehabilitation Sciences and Physiotherapy, Ghent, Belgium; ${ }^{2}$ Vrije Universiteit Brussel, Departments of Human Physiology and Physiotherapy, Brussels,

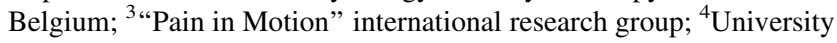
of Antwerp, Department of Rehabilitation Sciences and Physiotherapy, Antwerp, Belgium; Corresponding author's e-mail: jeroen.kregel@ugent.be

Background: Due to increasing evidence of maladaptive neuroplastic changes in CLBP and other chronic pain disorders, analyzing brain properties may be of great value. The purpose of this systematic review is to analyze the available literature on structural and functional brain abnormalities in chronic low back pain (CLBP) based on several brain magnetic resonance imaging (MRI) techniques.

Methods: PubMed and Web of Science were systematically screened for relevant literature using different combinations of keywords regarding structural and functional brain imaging techniques in patients with CLBP. Reference lists of included articles were handsearched for additional literature. Eligible articles were assessed on risk of bias and reviewed by two independent researchers.

Results: The search query returned 27 articles meeting the inclusion criteria. Ten studies evaluated structural gray matter changes. There is conflicting evidence in global gray matter changes, with both increases and decreases shown in different studies. Gray matter changes were demonstrated in specific brain regions. Structural white matter changes were reported in five studies. There is conflicting evidence in total white matter volume due to both increases and unchanged white matter. Several regional differences were identified in which white matter changes were shown. Functional organization during rest was evaluated in ten studies. CLBP patients showed increased activation in specific regions, together with a disrupted default mode network. A total of six studies evaluated brain activity in response to a nociceptive stimulus. Findings suggest that patients demonstrated increased activity in pain related regions, and decreased activity in analgesic regions.

Conclusions: Overall, there is moderate evidence for regional changes in gray and white matter, together with an altered functional connectivity during rest and increased activity in pain related areas following painful stimulation, evidencing an upregulated pain matrix. More longitudinal research is needed to clarify the temporal relationship regarding pain and neuroplastic changes and integration of different brain imaging techniques is warranted.

\section{Abstract \#: P 542}

\section{Development of clinical prediction rules in chronic nonspecific low back pain patients: study protocol for a randomized controlled trial}

Lenie Denteneer ${ }^{1}$, Gaetane Stassijns ${ }^{2,3}$, Willem De Hertogh ${ }^{1}$, Steven Truijen $^{1}$, Nienke Jansen ${ }^{3}$, Ulrike Van Daele ${ }^{1}$

${ }^{1}$ University of Antwerp, Faculty of Rehabilitation Sciences and Physiotherapy, Belgium; ${ }^{2}$ University of Antwerp, Faculty of Medicine and Health Sciences; ${ }^{3}$ Antwerp University Hospital, Physical Medicine and Rehabilitation, Belgium; ${ }^{3}$ Free University of Brussels, Faculty of Medicine and Pharmacy, Belgium;

Corresponding author's e-mail: lenie.denteneer@uantwerpen.be

Background: There is a consensus that exercise therapy should be used as a therapeutic approach in chronic low back pain (CLBP) but little consensus has been reached about the preferential type of therapy. Due to the heterogeneity of the population no clear effect of specific therapy interventions are found. Probably a specific subgroup of the investigated population will benefit from the intervention and another subgroup will not benefit, looking at the total investigated population no significant effects can be found. Therefore there is a need for the development of clinical prediction rules (CPRs). Objectives for this trial are first, the derivation of CPRs to predict treatment response to three forms of exercise therapy for patients with nonspecific CLBP. Secondly, we aim to validate a CPR for the three forms of exercise therapy for patients with nonspecific CLBP.

Methods/Design: The study design is a randomized controlled trial. Patients with nonspecific CLBP of more than 3 months duration are recruited at the Antwerp University Hospital (Belgium) and Apra Rehabilitation Hospital. After examination, patients are randomly assigned to one of three intervention groups: motor control therapy, general active exercise therapy and isometric training therapy. All patients will undergo 18 treatment sessions during 9 weeks. Measurements will be taken at baseline, 9, 6 months and at 1 year. The primary outcome used is the Modified Oswestry Disability Questionnaire score. For each type of exercise therapy a CPR will be derived and validated. For validation, the CPR will be applied to divide each treatment group into two subgroups (matched and unmatched therapy)using the baseline measurements. We predict a better therapeutic effect for matched therapy.

Conclusion: A randomized controlled trial has not previously been performed for the development of a CPR for exercise therapy in CLBP patients. Only one CPR was described in a single-arm design for motor control therapy in sub-acute non-radicular LBP patients. In 
this study, a sufficiently large sample will be included in both the derivation and validation phase.

Keywords: Clinical prediction rule, Nonspecific, Chronic, Low back pain.

\section{Abstract \#: P 543 \\ Is there evidence for central sensitization in nonspecific, nontraumatic neck pain? \\ Malfliet Anneleen ${ }^{1}$, Kregel Jeroen ${ }^{2}$, Cagnie Barbara ${ }^{2}$, Kuipers Mandy $^{1}$, Dolphens Mieke ${ }^{2}$, Roussel Nathalie ${ }^{3}$, Meeus Mira ${ }^{2}$, Danneels Lieven ${ }^{2}$, Bramer Wichor ${ }^{4}$, Nijs Jo ${ }^{1}$ \\ ${ }^{1}$ Vrije Universiteit Brussel, Belgium; ${ }^{2}$ Universiteit Gent, Belgium; ${ }^{3}$ Universiteit Antwerpen, Belgium; ${ }^{4}$ University Medical Center Rotterdam, the Netherlands; Corresponding author's e-mail: anneleen.malfliet@vub.ac.be}

Introduction: Chronic neck pain (NP) is a common, but poorly understood problem. Often no underlying structural pathology can be found and radiological imaging findings are more related to age than to a patients' symptoms. Besides this, chronic idiopathic NP is also very disabling with almost $50 \%$ of all these patients showing disability at long-term follow-up. Central sensitization (CS) is defined as "amplification of neural signaling within the central nervous system that elicits pain hypersensitivity" or "increased responsiveness of nociceptive neurons in the central nervous system to their normal or subthreshold afferent input". There is evidence for involvement of CS in many chronic pain conditions. Within the area of chronic idiopathic $\mathrm{NP}$, there is consistent evidence for the presence and clinical importance of CS in patients with traumatic NP (i.e. whiplash-associated-disorders). However, the majority of chronic idiopathic NP patients are unrelated to a trauma, and hence are termed chronic idiopathic non-traumatic NP. When comparing whiplash with idiopathic non-traumatic NP, indications for different underlying mechanisms are found. The goal is to review the existing scientific literature on the role of CS in patients with chronic idiopathic, non-traumatic NP.

Methods: A systematic search of relevant literature was performed via several scientific, electronic databases. Titles and abstracts were checked to identify relevant articles. Eligible articles had to meet following inclusion criteria: (1) subjects had to be human adults (>18 years) diagnosed with idiopathic, non-traumatic chronic NP (present $>3$ months): (2) papers had to report outcomes related to CS, and (3) had to be full-text reports or original research.

Results: Six articles were found eligible after screening title, abstract and-when necessary-full text for in- and exclusion criteria. All selected studies were case-control studies. Overall, results regarding the presence of CS were divergent. While the majority of patients with chronic traumatic NP are characterized by CS, this is not the case for patients with chronic idiopathic NP, with available evidence suggesting CS not being a major characteristic. Individual cases might have CS pain, but further research should reveal their typical features. Conclusion: Literature about CS in chronic idiopathic, non-traumatic $\mathrm{NP}$ patients is rare and provides an inconclusive message. CS is not a characteristic feature of chronic idiopathic, non-traumatic NP, but can be present in some individuals of the population. In the future a subgroup with CS might be defined, but based on current knowledge it is not possible to characterize this subgroup.

\section{Abstract \#: P 546}

Aqua-cycling, a supporting power; preliminary results

Deborah Thijssen ${ }^{1}$, Darcy Ummels ${ }^{1}$, Stefanie Rewald ${ }^{2}$, Rob A. de Bie ${ }^{2}$, Ilse Mesters ${ }^{2}$, A.F. Lenssen ${ }^{3}$, Pieter J. Emans ${ }^{4}$, Emmylou Beekman ${ }^{1,5}$

${ }^{1}$ Bachelor Physiotherapy, Zuyd University of Applied Sciences, Heerlen, The Netherlands; ${ }^{2}$ Department of Epidemiology, CAPHRI School for Public Health and Primary Care, Maastricht University, The Netherlands; ${ }^{3}$ Department of Physiotherapy, Maastricht University Medical Centre, Maastricht, The Netherlands; ${ }^{4}$ Department of Orthopaedic Surgery, Maastricht University Medical Centre, Maastricht, The Netherlands; ${ }^{5}$ Physical therapy/multidisciplinary centre, ParaMedisch Centrum Sittard Zuid, Sittard, The Netherlands; Corresponding author's e-mail: 1100262thijssen@zuyd.nl

Objective: The purpose of this study was to investigate the effects of a 12 week aqua-cycling program on functional capacity in patients with knee osteoarthritis, in comparison with regular care, on short and long term.

Method: The design of this study was a randomised controlled trial in which the intervention group received a 12 week aqua-cycling program and the control group received care as usual. There were measurements at baseline (T0), post-intervention (T1, short term) and a follow-up 3 months post-intervention (T2, long term). Primary outcome was distance walked during the Six Minute Walking Test (6MWT). Secondary outcomes were knee pain measured with the Numeric Pain Rating Scale (NPRS), perceived exertion measured with the Borg Ratings of Perceived Exertion (BORG-RPE) and selfreported level of physical activity with the Short Questionnaire to Assess Health (SQUASH).

Results: There were 42 patients randomised into the intervention group and 35 into the control group. The intervention group walked significantly more meters than the control group during the $6 \mathrm{MWT}$ on short term (34 m, $p=0.000)$. Within the intervention group there was a significant improvement on the 6MWT on long term $(42.5$ meters, $p=0.031)$. On long term there was no significant difference between the groups $(p=0.086)$.

The NPRS did not change significantly on short and long term in both groups. On short term the BORG-RPE increased significantly in the intervention group. The control group showed higher self-reported physical activity level on short and long term.

Discussion: After 12 weeks of aqua-cycling participants walked significantly more meters compared to baseline and compared to the control group. However, these preliminary results must be interpreted with caution. The higher BORG-RPE scores and lower physical activity levels in the intervention group might suggest that the aquacycling training was exhausting. The study had several limitations: $6 \mathrm{MWT}$ was done in a square of 44 meters, instead of the regular protocol of a 20 or $50 \mathrm{~m}$-cours. Further, the control group was allowed to get physiotherapy, so there was no uniform and controlled usual care program to compare to. Due to the low number of patients the reliability of this study is slightly decreased. However, the power of this study was $91.7 \%$, which means the conclusion from this study is representative.

Conclusion: This study shows that aqua-cycling may improve functional capacity in patients with knee osteoarthritis on a short term. 


\section{Abstract \#: P 547}

\section{Het effect op te leveren krachten bij het gebruik van een} Strykerm $1{ }^{\circledR}$ brancard, met en zonder gebruik van de move-in

Mitch M. van Dijk ${ }^{1}$, Daniel C. Nijman ${ }^{1}$, Jasper M. van Steenhoven ${ }^{1}$

${ }^{1}$ Gezondheid, Gedrag en Maatschappij (GGM), instituut

paramedische studies, Nijmegen, Nederland; Corresponding author's e-mail: danielnijman2006@hotmail.com

Inleiding: Ambulance personeel in Nederland heeft een hoger ziekteverzuim in vergelijking tot het landelijk gemiddelde. Waarschijnlijk ligt fysieke overbelasting hieraan ten grondslag. Het in- en uitrijden van een brancard in de ambulance is een onderdeel van deze fysieke belasting. In dit onderzoek wordt gekeken naar de til-, duw- en trekkrachten bij het in- en uitrijden van een Stryker $\mathrm{M} 1{ }^{\circledR}$ brancard, zowel met als zonder gebruik van het Move-In systeem.

Methode: Dit onderzoek is experimenteel (within-subject). Ambulancepersoneel heeft de brancard 12 keer in- en uit de ambulance gereden met en zonder gebruik van het Move-In systeem. Hierbij verschilden de gewichten tussen de 0,75 en 150 kilogram. De krachten die zij hierbij hebben geleverd zijn geregistreerd door middel van krachtsensoren. Het praktijkonderzoek is uitgevoerd in de loods van PENKO B.V.

Resultaten: Dit onderzoek toont aan dat tilkrachten met gebruik van het Move-In systeem het meest gereduceerd worden. In vergelijking met de Stryker M1 ${ }^{\circledR}$ neemt de tilkracht bij het inrijden van de brancard met gemiddeld $49 \%$ af. Bij het uitrijden van de brancard wordt de tilkracht tot $75 \%$ gereduceerd. Alle resultaten van de praktijkmeting zijn op de trekkracht bij 0 en 150 kilogram na significant gebleken.

Conclusie: Dit onderzoek laat zien dat er bij het gebruik van het Move-In systeem, zowel bij het in- als uitrijden van de brancard in de ambulance, bij 0,75 en 150 kilogram significant minder tilkrachten geleverd zijn. Bij het inrijden reduceert de Move-In de tilkrachten met ongeveer $50 \%$. Wanneer er gekeken wordt naar het uitrijden, laten de resultaten zien dat de Move-In naar mate het gewicht op de brancard toeneemt in verhouding meer tilkrachten reduceert, tot wel $75 \%$.

Aangezien er bij het in- en uitrijden van een brancard in de ambulance voornamelijk getild moet worden, is het aan te bevelen om de tilkrachten tot minimaal de norm $(23 \mathrm{~kg})$ te verminderen. Hiervoor is de Move-In een mogelijke optie. De manier waarop het personeel de brancard in de ambulance rijdt heeft effect op de te leveren kracht. Daarom is het aan te bevelen om voorafgaand het gebruik van de Move-In een training te volgen. Dit om optimaal gebruik te kunnen maken van het systeem.

\section{Abstract \#: P 548}

\section{Physical training in sarcoidosis}

Marcellis R.G.J. ${ }^{1}$, Van der Veeke M.A.F. ${ }^{2}$, Mesters I. ${ }^{3}$, Drent M. ${ }^{4}$, De Bie R.A. ${ }^{3}$, De Vries G.J. ${ }^{5}$, Lenssen A.F. ${ }^{1}$

${ }^{1}$ Department of Physical Therapy, Maastricht University Medical Centre (MUMC), Maastricht, The Netherlands; ${ }^{2}$ Physiotherapy practice FysioMedica, Geleen, The Netherlands \& Department of Epidemiology, Faculty of Health, Medicine and Life Sciences (FHML), Maastricht University, Maastricht, The Netherlands; ${ }^{3}$ Department of Epidemiology, Faculty of Health, Medicine and Life Sciences (FHML), Maastricht University, Maastricht, The Netherlands; ${ }^{4}$ ild care expertise team, Hospital Gelderse Vallei, Ede, The Netherlands \& Department of Toxicology, FHML, Maastricht University, Maastricht, The Netherlands; ${ }^{5}$ Department of Respiratory
Medicine, ORBIS MC, Sittard-Geleen, The Netherlands; Corresponding author's e-mail: rik.marcellis@mumc.nl

Background: Sarcoidosis patients frequently experience fatigue, exercise intolerance and muscle weakness, resulting in reduced quality of life (QOL). Physical symptoms may be partially reversible by following a structured physical training program. Scientific studies on the benefits of physical training in sarcoidosis have been scarce, so the aim of this pilot study with a single-group pre-post measurement design was to examine the impact of a 13-week physical training program on fatigue, physical functions and QOL in fatigued sarcoidosis patients and/or patients with exercise intolerance.

Methods: Sarcoidosis patients with fatigue complaints and/or exercise intolerance, referred for physical therapy by their pulmonologist, were included. Eighteen sarcoidosis patients participated in a 13-week physical training program for $1 \mathrm{~h}$, thrice a week, including peripheral muscle and endurance training. The Fatigue Assessment Scale (FAS), World Health Organization Quality of Life-BREF assessment instrument (WHOQOL-BREF), Medical Research Council (MRC) dyspnea scale, visual analogue scale (VAS), six-minute walk test (6MWT), submaximal bicycle test and muscle strength of the quadriceps and elbow flexors were assessed at baseline and after the program.

Results: FAS scores had decreased (mean difference -2.7 points, $95 \% \mathrm{CI}-4.4$ to -1.1 ) after completion of the training program, along with improvements in WHOQOL-BREF psychological health domain (mean difference 0.9 points, $95 \%$ CI $0.2-1.7$ ) and MRC dyspnea score (mean difference -0.4 points, $95 \% \mathrm{CI}-0.8$ to -0.1 ). 6MWD improved by $34.6 \mathrm{~m}$ (95\% CI 20.3-49.0) and mean heart rate on the bicycle test improved (mean difference 8.4 beats $/ \mathrm{min}, 95 \% \mathrm{CI}$ -13.8 to -3.0 ), as did quadriceps strength (mean difference $10.7 \mathrm{~kg}$, $95 \%$ CI 5.5-15.9). No changes were observed in VAS score or elbow flexor muscle strength. The mean number of training sessions attended was $29.0 \pm 6.8$ of the potential number of 39 . No adverse events were recorded. After the training program $72 \%$ of the patients continued a training program comparable to that used in this study.

Conclusion: Physical training appeared to be useful and safe for sarcoidosis patients to decrease fatigue and improve psychological health and physical functions (dyspnea, 6MWD, bicycle heart rate, quadriceps strength). Patients compliance was high and most patients continued a physical training program. Future studies are warranted to assess the benefits of physical training in sarcoidosis.

\section{Abstract \#: P 549}

\section{The effect of constraint-induced movement therapy in stroke} patients

\section{Pieter Vromans $^{1}$ \\ ${ }^{1}$ Student Thim van der Laan; Corresponding author's e-mail: pietervromans@msn.com}

Background: A stroke is literally an accident in the blood vessels of the brain. In about eighty percent of the cases a stroke is caused by a cerebral infarction and twenty percent by a cerebral hemorrhage. Symptoms are all-round, depend on range and localization of the lesion. The average neurological functional recovery takes about 5-17 weeks.

The incidence in 2013, at an age range from 15 to 64 years, is 1 on 1000 people a year. In the age range from 65 years and older this is $10-11$ on 1000 people a year. The guideline describes CIMT as an intervention with the highest proof of evidence. On the other hand it also describes that there are a lot of variations in performance and intensity. 
Objective: What is the effect of CIMT for adults at the subacute and postacute phase after a stroke on the recovery of the arm- and hand function?

Design: To answer the main question there has been searched on Pubmed. Terms to find relevant studies are: Stroke AND (Forced Use OR CIMT OR Constraint induced movement therapy) AND (Arm OR Upper limb).

Results: Five studies have been taken. Four of five with a good methodological quality. Five studies found significant improvements inside the intervention- and control group. Two studies found significant improvements between both groups.

Conclusion: The effect of CIMT is plausible for adult stroke patients at the subacute and postacute phase focused on the recovery of the arm and hand function.

\section{Abstract \#: P 550 \\ Oefentherapie abductoren en exorotatoren van de heupmusculatuur bij patellofemoraal pijnsyndroom \\ $\underline{\text { Jesper C.P.J.W. Gabriels }}^{1}$ \\ ${ }^{1}$ Hogeschool voor fysiotherapie Thim van der Laan, Nieuwegein, Netherlands; Corresponding author's e-mail: \\ jesper.gabriels@gmail.com}

Background: Patellofemoral pain syndrome is a pathology that indicates anterior knee pain caused by dysfunction of the patellofemoral joint, this causes that the patella doesn't track properly through the trochlea of the femur. Most common symptoms that the patient may experience include: pain around the peripattelar or retropatellar side of the kneecap, crepitus, pain during or after long time sitting, function problems, swelling and a feeling of pseudo giving away is possible to experience. Recent literature more often describes the involvement of an imbalance in the hipmuscles in relation to patellofemoral pain syndrome. Exercise of the hip abductor and lateral rotator muscles restored the imbalance of the hipmuscles and could contribute to the knee function in patients with patellofemoral pain syndrome.

Objective: What is the effect of exercise of hip abductor and lateral rotator muscles on knee function in patients with patellofemoral pain syndrome?

Design: To answer the main question there has been searched in Pubmed and PEDro for randomized controlled trials of 5 years or younger with the following terms: patellofemoral pain syndrome, exercise, hip, knee function. After in- and exclusioncriteria four randomized controlled trials of good methodological quality are included.

Results: Function of the knee is significantly improved in all four included studies in the interventiongroups that followed exercise therapy of the hip abductors en lateral rotators.

Conclusion: Exercise of hip abductor and lateral rotator muscles during 4 weeks show significant improvements on knee function in patients with patellofemoral pain syndrome.

\section{Abstract \#: P 551}

Comparative analysis of headtilt and forward head position during laptop use between females with postural induced headache and healthy

Sarah Mingels ${ }^{1}$, Ludo van Etten ${ }^{2}$, Wim Dankaerts ${ }^{3}$, Marita Granitzer ${ }^{4}$, Herbert Thijs ${ }^{5}$
${ }^{1}$ Department of Rehabilitation Sciences and Physiotherapy, Faculty of Medicine and Life Sciences, Hasselt University, Diepenbeek, Belgium; ${ }^{2}$ Centre of Expertise in Life Sciences, Zuyd University, Heerlen, The Netherlands; ${ }^{3}$ Musculoskeletal Research Unit, Department of Rehabilitation Sciences, Faculty of Kinesiology and Rehabilitation Sciences, University of Leuven, Leuven, Belgium; ${ }^{4}$ REVAL Rehabilitation Research Centre, Biomedical Research Institute, Faculty of Medicine and Life Sciences, Hasselt University, Diepenbeek, Belgium; ${ }^{5}$ I-BioStat, Interuniversity Institute for Biostatistics and statistical Bioinformatics, Hasselt University, Diepenbeek, Belgium; Corresponding author's e-mail: sarah.mingels@uhasselt.be

Objectives: To compare head tilt, forward head posture, the ratio between forward head posture and maximum (manually induced) head protraction between a postural induced headache group and healthy controls. To explore the evolution trends of head tilt and forward head posture during a laptop task and comparing this evolution between the two groups. To evaluate the correlation between the evolution of head tilt and forward head posture.

Methods: Angles for maximum head protraction and retraction, head tilt and forward head posture of 12 female students with postural induced headache and 12 female healthy controls were calculated from digital pictures within a cross-sectional design (while neutral sitting and performing a laptop task).

Results: Significant differences $(p<0.05)$ were found in maximum head protraction between the headache group $\left(23.7^{\circ} \pm 7.9^{\circ}\right)$ and the healthy controls $\left(30.5^{\circ} \pm 6.0^{\circ}\right)$. The ratio of forward head posture during habitual sitting to the maximum head protraction differed significantly $(p<0.05)$ between the headache group $(1.4 \pm 0.4)$ and the healthy controls $(1.1 \pm 0.2)$. Within the headache group biphasic head tilt and forward head posture profiles were observed during the laptop task. These profiles differed significantly between groups and seemed to be negatively correlated within groups $(-0.927)$. Head tilt and forward head posture variances were larger in the headache group.

Conclusion: During sitting the headache group showed a larger maximum head protraction and a less close to the end range forward head posture. During the laptop task forward head posture and head tilt behaved biphasically with a more static forward head posture pattern and a more dynamic head tilt pattern in the headache group.

\section{Abstract \#: P 552}

Afname van elleboogklachten na een zomerprogramma voor handbalkeepers: een case-series onderzoek

$\underline{\text { Linda van Maanen-Coppens }}{ }^{1}$, Maarten Barendrecht ${ }^{2}$

${ }^{1}$ Master sportfysiotherapeut bij TotalFysio, Eindhoven; ${ }^{2}$ Avans + , Breda; Corresponding author's e-mail: lindacoppens@live.nl

Inleiding: Van alle handbalkeepers krijgt $75 \%$ gedurende hun loopbaan elleboogklachten maar een afdoende oplossing voor de aanpak van deze blessure, die ontstaat als gevolg van veelvuldige hyperextensie en valgusbelasting tijdens het blokken van de bal, ontbreekt. Veel handbalkeepers blijven langdurig doorspelen met klachten, waardoor herstel uitblijft. Gesteld wordt dat elleboogklachten positief beïnvloed worden met een gefaseerd programma gedurende de zomerstop.

Methode: Dit onderzoek was een prospectieve case serie. Handbalkeepers volgden een gefaseerd zomerprogramma onder fysiotherapeutische begeleiding, gericht op het versterken van pronatoren/flexoren van de onderarm en een geleidelijke opbouw in belasting op de elleboog. Gedurende het onderzoek waren er zes meetmomenten: bij aanvang (t0), na zes weken trainen (t1), na 
afronding van het zomerprogramma ( $\mathrm{t} 2)$, en een aantal follow-up metingen gedurende het seizoen 2013-2014 na één (t3), drie (t4) en zes maanden (t5). Tijdens alle meetmomenten werden pijn (Visual Analoge Scale en Numeric Pain Rating Scale), handknijpkracht (Jamar dynamometer) en belemmeringen in ADL (Nederlandse Disability of the Arm, Shoulder and Hand questionnaire) in kaart gebracht.

Resultaten: Negen handbalkeepers (vijf mannen/vier vrouwen: gemiddelde leeftijd 23,2 jaar) die in het seizoen 2012-2013 allen minimaal één of meer dagen uitvielen als gevolg van elleboogklachten, namen deel aan het zomerprogramma en werden gevolgd tijdens de follow-up periode. De keepers keerden na afloop van het zomerprogramma terug op minimaal hun oude competitieniveau, en lieten na afloop van het programma ( $\mathrm{t} 2)$ individueel klinisch relevante verbeteringen zien in hun pijnklachten (VAS $>70 \%$ afname, NPRS $>66 \%$ afname) en algemeen functioneren (DASH $>95 \%$ afname). Deze verbeteringen bleven gedurende de follow-up (t5) behouden. Op groepsniveau was tijdens 5 sprake van een significante verbetering ten opzichte van t0 op de VAS $(p=0.001)$, NPRS $(p=0.000)$ en DASH $(p=0.000)$, en in de handknijpkracht $(\mathrm{kg})$ van de aangedane arm $(p=0.009)$. Bij geen enkele keeper was gedurende het seizoen 2013-2014 sprake van uitval als gevolg van elleboogklachten.

Conclusie, discussie en aanbevelingen: Een specifiek zomerprogramma lijkt zinvol te zijn voor handbalkeepers met elleboogklachten, om terug te keren op het oude niveau en uitval gedurende het seizoen te voorkomen. Verder onderzoek op grotere schaal en met controlegroep is echter geïndiceerd om de resultaten uit deze case series te kunnen bevestigen.

\section{Abstract \#: P 553}

Psychometric properties of lower extremity handheld dynamometry strength measurement in children with cerebral palsy: a systematic review

Angelique N. Mulder-Brouwer ${ }^{1}$, Eugene A.A. Rameckers ${ }^{2}$, Caroline H. Bastiaenen ${ }^{3}$

${ }^{1}$ University for Professionals in Pediatric Physical Therapy, AVANSplus, Breda, the Netherlands + Medicort, Centre for Physiotherapy, Utrecht, the Netherlands; ${ }^{2}$ University for Professionals in Pediatric Physical Therapy, AVANSplus, Breda, the Netherlands + Department of Rehabilitation Medicine, CAPHRI, Maastricht University, the Netherlands + Centre of Expertise, Adelante Valkenburg, the Netherlands; ${ }^{3}$ Department of Epidemiology, Musculoskeletal Research Division CAPHRI, Maastricht University, Maastricht, the Netherlands; Corresponding author's e-mail: amulder@medicort.nl

Background: Quantifying muscle strength is essential for early identification of muscle groups that need targeting in treatment and for the adaptation and evaluation of training programs for children with Cerebral Palsy (CP). For this purpose it is important to use measurement instruments with sound clinimetric properties. To reinforce a physiotherapists or researchers choice of an appropriate measure and protocol we decided to perform a systematic review on reliability and validity of isometric muscle testing using HHD protocols of the muscles of the lower extremities in children with $\mathrm{CP}$, including a comparison of relevant protocol content. The purpose of this study is to review the current level of evidence on reliability and validity of handheld dynamometry (HHD) protocols to quantify maximal isometric strength of the muscles of the lower extremities of children with cerebral palsy $(\mathrm{CP})$.
Method: A systematic search of MEDLINE, CINAHL, Cochrane and EMBASE databases up to December 2013 was performed. Studies that reported clinimetric properties of handheld dynamometry protocols to measure isometric strength of muscle groups of the lower limbs for children with $\mathrm{CP}$ aged 3-18 years were identified and examined. The COSMIN checklist with 4-point rating scale was used by two independent raters to evaluate the methodological quality of the included studies. Best-evidence synthesis was performed using COSMIN outcomes and the quality of clinimetric properties.

Results: Seven eligible studies were identified. Validity was not covered in any of the included studies. Best evidence synthesis revealed "unknown" to "moderate" evidence. ICC values were "positive" for most muscle groups for intrarater reliability and showed mixed results for interrater reliablity. Because of low sample sizes (10-25) in all studies, the final levels of evidence are rated as "unknown".

Conclusions: Research on clinimetric properties of HHD to measure lower extremity strength in children with $\mathrm{CP}$ is rare. Best evidence synthesis revealed moderate evidence for reliability. An important limiting factor is the low sample size. When sample size is excluded from the methodological quality rating, they score better and suggest that the reliability data reported have some value for clinical practice and research, but should be considered with great care. Establishment of valid and reliable protocols for HHD for children with $\mathrm{CP}$ of various age ranges and various Gross Motor Function Classification System levels (GMFCS) levels is required to ensure clinical utility of the measurement instrument.

\section{Abstract \#: P 555}

Effects of cognitive stress and relaxation on central pain modulation in patients with chronic whiplash-associated disorders, fibromyalgia and healthy controls

Iris Coppieters, $\mathrm{MSc}^{1,2}$, Barbara Cagnie, $\mathrm{PhD}^{1}$, Jessica Van Oosterwijck, $\mathrm{PhD}^{1,2}$, Robby De Pauw, MSc ${ }^{1}$, Mira Meeus, $\mathrm{PhD}^{1,2,3}$, Jo Nijs, $\mathrm{PhD}^{2,4}$

${ }^{1}$ Department of Rehabilitation Sciences and Physiotherapy, Faculty of Medicine and Health Sciences, Ghent University, Belgium; ${ }^{2}$ Pain in Motion international research group, www.paininmotion.be;

${ }^{3}$ Department of Rehabilitation Sciences and Physiotherapy, Faculty of Medicine and Health Sciences, University of Antwerp, Belgium;

${ }^{4}$ Department of Physical Medicine and Physiotherapy, University Hospital Brussels, Belgium; Corresponding author's e-mail: iris.coppieters@ugent.be

Background: A growing body of research has demonstrated that impaired central pain modulation or central sensitization (CS) is a crucial mechanism for the development of persistent pain in patients with chronic whiplash-associated disorders (WAD) and fibromyalgia (FM). However, there is limited research concerning the influence of cognitive stress and relaxation on central pain modulation in chronic WAD and FM patients. The present study aims at investigating the effects of cognitive tasks and relaxation on central pain modulation in chronic WAD and FM patients compared to healthy individuals.

Methods: A randomized controlled trial with crossover design was conducted. Fifty-seven subjects, 16 chronic WAD patients, 17 FM patients and 22 pain-free healthy volunteers, were enrolled and subjected to various pain measurements. Pressure pain thresholds, temporal summation of pain (TS) and conditioned pain modulation (CPM) were evaluated. Subsequently, participants were randomly allocated to either a group that performed a battery of cognitive tests or a group that received progressive relaxation therapy. Afterwards, all pain measurements were repeated. One-week later participants groups were switched. 
Results: A significant interaction effect was found for TS in the different study groups after relaxation $(p=0.008)$ and after the cognitive tests $(p=0.003)$. TS decreased after the relaxation therapy and after the cognitive tests in chronic WAD patients and healthy controls. In contrast, TS increased after both interventions in FM patients. CPM efficacy decreased after both interventions in all study groups. Subsequently, a significant time effect was found for CPM after relaxation $(p=0.002)$ and after the cognitive tests $(p=0.001)$.

Conclusion: Lower TS values point towards reduced bottom-up sensitization, possibly due to a smaller amount of incoming nociceptive signals from the periphery as a result of the relaxation or distraction by focusing on cognitive processing. In the FM group, TS worsened after both interventions. Therefore, it can be assumed that a single relaxation session as well as cognitive stress may have negative acute effects on pain modulation in patients with FM, while cognitive stress and relaxation reduced bottom-up sensitization in both chronic WAD patients and controls. However, both cognitive stress and relaxation induced more dysfunctional descending pain inhibition in all study groups. These results should be taken into consideration when developing therapy strategies for patients with chronic WAD and FM.

\section{Abstract \#: P 556}

\section{Adverse events after cervical spinal manipulative therapy: consensus based classification and definitions}

Rik Kranenburg $^{1}$, Sandra E. Lakke ${ }^{1}$, Renske Peters ${ }^{2}$, Maarten Schmitt ${ }^{2}$, Cees P. van der Schans ${ }^{3}$

${ }^{1}$ Research and Innovation Group in Health Care and Nursing, Hanze University Groningen, University of Applied Sciences, Groningen, the Netherlands; ${ }^{2}$ SOMT, Institute for Master Education in Manual Therapy, Amersfoort, the Netherlands; ${ }^{3}$ Research and Innovation Group in Health Care and Nursing, Hanze University Groningen, University of Applied Sciences, Groningen, the Netherlands: Department of Rehabilitation, University Medical Center Groningen, Groningen, the Netherlands; Corresponding author's e-mail: h.a.kranenburg@pl.hanze.nl

Background: Cervical spinal manipulations (CSM) are frequently employed techniques to alleviate neck pain and headache. Minor and major complications following CSM have been described, though clear consensus on definition and the classification of the complications had not yet been achieved. As a result, incidence rates may be underestimated.

Objectives: The aim of this study was to develop a consensus-based classification of adverse events following cervical spinal manipulations which has good feasibility in clinical practice and research.

Design: A three round Delphi-study.

Method: Medical specialists, manual therapists, and patients $(\mathrm{n}=30)$ participated in an online survey. In Round 1, participants were invited to select a classification system of adverse events. Potential complications were inventoried and detailed in accordance with the ICF and the ICD-10. In Round 2, panel members categorized the potential complications in their selected classification. During the third round, it was inquired of the participants whether they concurred with the answer of the majority of participants.

Results: Thirty four complications were defined. Consensus was achieved for 29 complications for all durations [hours, days, weeks]. For the remaining five complications, consensus was reached for two of the three durations [hours, days, weeks].

Conclusions: A consensus-based classification system of adverse events after cervical spinal manipulation was developed which comprises patients' and clinicians' perspectives and has only a small number of categories. The classification system includes a precise description of potential adverse events and is based on international accepted classifications (ICD-10 and ICF). This classification system may be useful for utilization in both clinical practice and research.

\section{Abstract \#: P 557}

Patellofemoral pain syndrome: specify the diagnostics of PFPS by a flowchart, based on scientific evidence

$\underline{\text { Rick van de Ven }}{ }^{1}$, Benjamin Jansen ${ }^{1}$, Marten Berends ${ }^{1}$

${ }^{1}$ Fysiotherapie, HAN Hogeschool van Arnhem en Nijmegen, Nijmegen, Nederland.; Corresponding author's e-mail: rickvdven@hotmail.com

Objective: Patellofemoral Pain Syndrome (PFPS) is difficult to diagnose due to the wide range of complaints patients may present themselves with. Because of the lack of clear guidelines, physiotherapists often work based on clinical expertise. The purpose of this research is to develop a flowchart based on scientific evidence, in which causes and symptoms of PFPS and treatable factors are shown. The purpose of this flowchart is to specify the diagnostics of PFPS. Method: In this systematic review 80 articles have been included, which were all judged on quality and applicability in a primary physiotherapy practice. These articles were conducted from various databases and fellow physiotherapists. From these articles, only significant factors which are possible to test in a primary physiotherapy practice were included in the flowchart.

Results: There is consensus that PFPS is associated with an increased level of activities, pain during certain activities (prolonged sitting with bended knees, squatting, kneeling, stair climbing and cycling), enlarged hip adduction, general hypermobility, decreased abduction-, extension-, and exorotation-force of the hip, decreased mobility and strength of the $\mathrm{mm}$. Hamstrings and $\mathrm{m}$. Quadriceps, including specifically the $\mathrm{m}$. Vastus Medialis Obliquus (VMO), neurological factors, psychological factors and a lateralisation of the patella. There is no consensus about the mobility of the $\mathrm{m}$. Gastrocnemicus and the hip, the length of the Iliotibial Band (ITB), the amount of pronation of the foot and the angle of the $\mathrm{m}$. Quadriceps (Q-angle). There is no association between PFPS and leg length difference, genu varum/valgum, pes planus/cavus, mobility of the knee and timing of the onset between VMO and the m. Vastus Lateralis (VL).

Discussion/conclusion: The factors that are associated with PFPS and that are applicable in a primary physiotherapy practice are included in the flowchart. Therefore, the strength of the VMO, neurological factors and the lateralisation of the patella are not included. From the factors in which there is no consensus, the enlarged Q-angle and the limited mobility of the $\mathrm{m}$. Gastrocnemius and ITB are included. The added value of this flowchart is that it is a simple and quick screening tool for the diagnosis of PFPS in a primary physiotherapy practice. More research needs to be done on ways to measure the stance of the patella, Q-angle, and the strength and volume of the VMO in a primary physiotherapy practice and the association between PFPS and pronation of the foot and the mobility of the hip.

\section{Abstract \#: P 558}

Translation, validation and reliability of the Dutch late-life function and disability instrument computer adaptive test

$\underline{\text { Remco M. Arensman }}^{1}$, Martijn F. Pisters ${ }^{1}$, Janneke M. de Man ${ }^{1}$, Marieke J. Schuurmans $^{1}$, Alan M. Jette ${ }^{2}$, Rob A. de Bie ${ }^{3}$ 
${ }^{1}$ Department of Rehabilitation, Nursing Science and Sport, Physical Therapy Science, University Medical Center Utrecht, Utrecht, The Netherlands; ${ }^{2}$ Boston University, Health \& Disabilities Research Institute, Boston, United States of America; ${ }^{3}$ Maastricht University, Caphri, Maastricht, The Netherlands; Corresponding author's e-mail: rmarensman@live.com

Background: Adequate and user-friendly instruments to assess physical function and disability in the elderly population are vital for estimating and predicting healthcare needs in clinical practice. Computerized adaptive testing (CAT) has multiple advantages over conventional instruments (e.g. individualized measurement process, increased measurement precision, and decreased respondent burden). The Late-Life Function and Disability Instrument Computer Adapted Test (LLFDICAT) appears promising to assess physical functioning and disability in gerontology research and clinical practice. The aim of this study was to translate the LLFDICAT to the Dutch language and to investigate the validity and reliability in a sample of Dutchspeaking community dwelling older persons.

Methods: The item bank of the LLFDICAT was translated using a forward-backward procedure. A sample of 54 older adults was recruited and completed the LLFDICAT, WHO-Disability Assessment Schedule 2.0 (WHODAS 2.0), PF-10 and 10-meter walk test (10MWT). The LLFDICAT was retested in 2-10 days. Pearson's $r$ was calculated to assess the concurrent validity of the LLFDICAT and the ICC 2,1, group-level reliability and participant-level reliability were calculated to assess reliability.

Results: A correlation of 0.74 was found between the LLFDICAT Function scale and the PF-10. Additionally, the LLFDICAT Disability scale showed a correlation of -0.57 with the WHODAS 2.0 and -0.53 with the 10MWT respectively.

The LLFDICAT Function scale showed an ICC 2,1 of 0.84, with a group-level reliability score of 0.85 . The LLFDICAT Disability scale showed an ICC of 0.76 and a group-level reliability of 0.81 .

Conclusions: The Dutch LLFDICAT shows strong validity and high reliability when used to assess physical function and disability in community dwelling elderly.

The LLFDICAT is a short, valid and reliable instrument for use in both research and clinical practice.

\section{Abstract \#: P 559}

\section{Mediterranean diet adherence and cognitive decline in middle- aged men and women: the Doetinchem Cohort Study}

Lisa G. Hendriks ${ }^{1,2}$, Astrid C.J. Nooyens ${ }^{1}$, Jolanda M.A. Boer ${ }^{1}$, Martin P.J. van Boxtel ${ }^{3}$, W.M. Monique Verschuren ${ }^{1}$

${ }^{1}$ RIVM; ${ }^{2}$ WUR; ${ }^{3}$ Maastricht University; Corresponding author's e-mail: astrid.nooyens@ rivm.nl

Background: Cognitive function declines with age, which can eventually lead to dementia. The underlying mechanism of dementia is not exactly known and treatment options are limited. Therefore, primary prevention is important and should preferably start at middle age or earlier. The Mediterranean diet, characterized by a limited intake of animal derived and processed foods and a high intake of plant foods, has been associated with reduced cognitive decline and risk of dementia, in older persons. The aim of this study was to assess whether adherence to a Mediterranean diet was associated with less cognitive decline in a middle-aged Dutch population.

Methods: In 2971 men and women from the Doetinchem Cohort Study, on average 55 years old at baseline (1995-2007), cognitive function (global cognitive function and the domains memory, information processing speed, and cognitive flexibility) was assessed at least twice, with a 5-year time interval. Diet was assessed twice with a
178 item validated food-frequency questionnaire and the modified Mediterranean Diet Score (mMDS) was calculated at a 10-point scale and categorized in three groups (low: 0-3, intermediate 4-5, and high 6-9 adherence). Multivariate linear regression analyses were used to assess the association of the mMDS and change in cognitive function. Because of effect modification by age, longitudinal regression analyses with repeated measurements was used to study the effect of mMDS on the association between age and cognitive function. The following confounders were included in the statistical analyses: age, sex, level of education, marital status, BMI, physical activity, smoking, cholesterol ratio, hypertension, diabetes, mental health, vitality, energy intake, Apo 84 genotype and baseline cognition.

Results: Although not yet statistically significant at 5-years of followup $(P=0.07)$, a higher mMDS was associated with less decline in global cognitive function. After 10-year follow-up a higher mMDS was significantly associated with less decline in both global cognitive function $(P<0.01)$ and memory $(P<0.01)$. Similar results were observed for the longitudinal analyses. Global cognitive function and memory declined less with age in the highest mMDS group as compared to the intermediate mMDS group, respectively $P<0.05$ and $P<0.06$. Differences between the other mMDS groups (lowest vs. intermediate and lowest vs. highest) were not statistically significant.

Conclusions: In our middle-aged population, better adherence to a Mediterranean diet was associated with less decline in global cognitive function and memory. These findings are in line with results in older populations.

\section{Abstract \#: P 560}

Alcohol drinking and abdominal adiposity for longitudinal study of adult health participants-ELSA-Brazil

Gabrielle Guidoni Torres ${ }^{1}$, Jorge Gustavo Velasquez Melendez ${ }^{2}$, Maria Carmen Viana ${ }^{1}$, Bruce Duncan ${ }^{3}$, Maria del Carmen Bisi Molina $^{1}$

${ }^{1} \mathrm{UFES} ;{ }^{2} \mathrm{UFMG} ;{ }^{3} \mathrm{UFRGS}$; Corresponding author's e-mail: mdmolina@uol.com.br

Introduction: Abdominal adiposity is a risk factor for several noncommunicable diseases, such as hypertension, diabetes, dyslipidemia and coronary heart disease, and this risk exists independent of the presence of overall obesity. Is not well defined yet the association between alcohol consumption and the development of abdominal adiposity.

Objective: To evaluate the association between the consumption of alcoholic beverages and abdominal obesity.

Methods: Cross-sectional study conducted from the database of the Longitudinal Study of Adult Health-ELSA-Brazil. Were measured weight, height and waist circumference (WC) and calculated the body mass index (BMI). Alcohol consumption among participants was measured from a structured questionnaire with closed questions based on the questionnaire of the National Center for Health Statistics. The questions were about the type of beverage and the daily intake, weekly and yearly. Considered is a $14 \mathrm{~g}$ dose of alcohol being equivalent to $120-150 \mathrm{~mL}$ of $350 \mathrm{~mL}$ of wine and beer. For spirits was considered a dose of whiskey, rum, vodka, among others. The Mann-Whitney test and Spearman correlation was used to test the association between doses of alcoholic beverages and WC.

Results: Of the 15,071 participants, 7242 ingest alcoholic beverages (wine, beer or spirits). Among those who consume alcoholic beverages $17.4 \%$ are smokers, about $59 \%$ are male, and $40 \%$ are between 45 and 54 years and over $60 \%$ are overweight. The amount of alcohol ingested at doses was $3.3 \pm 3.5$ for wine $(n=3744)$, 
$6.8 \pm 9.0$ for beer $(\mathrm{n}=5707), 3.9 \pm 4.6$ for distillates $(\mathrm{n}=1773)$ and $1.2 \pm 1.4$ for all beverages $(n=7242)$. In the assessment of average intakes of each type of alcoholic beverage and fitness WC, by sex, we observed a significant difference for adequate or inadequate WC for all drinks in males $(1.3 \pm 1.5,1.6 \pm 1.7)$, while for women there is a difference when assessed all beverages $(0.61 \pm 0.64$, $0.72 \pm 0.76)$ and beer separately $(3.4 \pm 3.9,4.5 \pm 5,2)$. The correlations between the $\mathrm{CC}$ and the intake of weekly doses were low and not significant.

Conclusion: Research shows a positive association between alcohol consumption in general and some types of drinks and abdominal obesity marker. The importance of this information is given in the development of measures to monitor the progress of chronic diseases.

\section{Abstract \#: P 561}

\section{Changes in biological parameters in a population of pregnant women}

Amina Boufars ${ }^{1}$, Hakim Belghiti ${ }^{1}$, Guerinech Hassania ${ }^{1}$, Elarbi Bouaiti $^{1}$, Rachid Razine ${ }^{2}$, Mustapha Mrabet $^{1}$

${ }^{1}$ Health service and community medicine; ${ }^{2}$ Department of public health; Corresponding author's e-mail: boufarsamina@gmail.com

Background: During pregnancy many biological parameters can have fluctuations in the upward or downward, reflecting the physiological adaptations but sometimes pathological metabolism in pregnant women. The aim of our study is to analyze the changes in biological parameters of Moroccan pregnant woman.

Methods: this is a cohort study of a sample of pregnant women followed in 2009 at the nutrition unit of the military hospital of instruction Mohammed V in Rabat. The recruitment was carried out by simple random sampling with the list of pregnant women who consult to the same unit. Were included in our study women at 12 weeks gestation, with no associated pathology and having a single pregnancy. For each woman a standardized questionnaire was filled in and $5 \mathrm{ml}$ of blood was taken. Various biological parameters were defined in the three consultations (12SA, between 22 and 24 weeks and between 34 and 36 weeks).the variables studied were fasting glucose, insulin, lipid profile, hemoglobin and CRP.The objectives of the study were clearly explained to women while respecting confidentiality and anonymity.

Results: Have been included in our study 50 pregnant women whose average age was $27 \pm 6$ years. The analysis showed a significant increase in total cholesterol between the three consultations with respectively $(1.58,2.14$ and $2.30 \mathrm{~g} / \mathrm{l})$ and triglyceride $(0.91,1.53$ and $1.82 \mathrm{~g} / \mathrm{l})$ in contrast to hemoglobin which has fallen of $11.86-11.13 \mathrm{~g} / \mathrm{dl}$. Conclusion: our study shows that biological parameters change in pregnancy where the interests of the proper monitoring and the adoption of a balanced diet.

\section{Abstract \#: P 562}

\section{Child weight and its association with food habits in Portuguese children}

Aristides M. Machado-Rodrigues $^{1}$, Rômulo Fernandes ${ }^{2}$, Augusta Gama ${ }^{3}$, Helena Nogueira ${ }^{3}$, Victor Rosado ${ }^{3}$, Isabel Mourão ${ }^{4}$, Cristina Padez ${ }^{3}$

${ }^{1}$ Research Centre for Anthropology and Health, University of Coimbra, Coimbra: ESE-IPV, Viseu, Portugal; ${ }^{2}$ Faculty of Science and Technology, Paulista State University - UNESP, Brazil;
${ }^{3}$ Research Centre for Anthropology and Health, Un iversity of Coimbra, Coimbra, Portugal; ${ }^{4}$ University of Tras-os-Montes and Alto Douro, Vila Real, Portugal; Corresponding author's e-mail: rodriguesari@hotmail.com

Background: The prevalence of childhood obesity has reached epidemic proportions in Portugal, with greater numbers of people becoming affected earlier in their lives. The literature has identified several sociodemographic and behavioural risk factors which play an important role on overweight/obesity. Among others, some dietary habits are frequently assumed as a main concern (i.e. consumption of sweetened beverages and candy: skipping breakfast: and so on). In context of the preceding trends, the purpose of the present study was to examine associations between different food habits and the risk of overweight/ obesity in children.

Methods: The sample of the present study included 2258 male children aged 7-9 years randomly selected. Height and weight were measured. Body Mass Index (BMI) was calculated subsequently and participants were classified as normal weight or overweight/obese (Cole et al., 2000). Eating behaviours (i.e. have breakfast: consumption of soup and vegetables, and soft drinks and chocolate) were assessed by questionnaire as well as sedentary behaviours (i.e. TV viewing). Logistic regressions were used, with adjustments for age, sex, time spent sedentary, and parental education.

Results: After controlling for the afore-mentioned confounders, children who have breakfast in a daily basis were 2.8 times less likely to be classified as overweight/obese than their peers who skipping breakfast regularly. Furthermore, and contrary to our expectation, there was no significant main effect neither of the soft beverages and chocolate consumption nor soup and vegetables in the weight status of children. Conclusion: The present cross-sectional study showed an inverse association between breakfast consumption and child weight. Future research should extend similar design in males and females to assess multiple meals that include a wider range of foods and beverages to confirm or not some of the afore-mentioned findings. However, action should be taken to improve breakfast eating, as well as other healthy habits in order to prevent the epidemic proportions of obesity among Portuguese children.

\section{Abstract \#: P 563}

Do demographic and lifestyle determinants of an unhealthy dietary pattern differ by socio-economic status?

Heidi P. Fransen $^{1,2}$, Anne M. May ${ }^{1}$, Joline W.J. Beulens ${ }^{1}$, Ellen A. Struijk $^{1,2}$, Jolanda M.A. Boer ${ }^{2}$, G. Ardine de Wit ${ }^{1,2}$, N. Charlotte Onland-Moret ${ }^{1}$, Yvonne T. van der Schouw ${ }^{1}$, H. Bas Bueno-deMesquita $^{3,4,5,6}$, Jeljer Hoekstra ${ }^{2}$, Petra H.M. Peeters ${ }^{1,5}$

${ }^{1}$ Julius Center for Health Sciences and Primary Care, University Medical Center Utrecht, Utrecht, the Netherlands; ${ }^{2}$ National Institute for Public Health and the Environment (RIVM), Bilthoven, the Netherlands; ${ }^{3}$ National Institute for Public Health and the Environment (RIVM), Bilthoven, the Netherlands; ${ }^{4}$ Department of Gastroenterology and Hepatology, University Medical Center Utrecht, Utrecht, the Netherlands; ${ }^{5}$ School of Public Health, Imperial College London, London, United Kingdom;

${ }^{6}$ Dt. of Social \& Preventive Medicine, Faculty of Medicine, University of Malaya, Kuala; Corresponding author's e-mail: h.p.fransen@umcutrecht.nl

Background: Diet is an important modifiable lifestyle factor associated with healthy aging. Dietary patterns have been associated with other socio-demographic and lifestyle characteristics such as educational level, smoking status and level of physical activity. These determinants may interact and we aimed to investigate whether 
determinants of an unhealthy dietary pattern differed by socio-economic status.

Methods: We conducted a cross-sectional analysis in the EPIC-NL cohort. At study entry (1993-1997) 39,393 men and women, aged 20-70 years, filled out a lifestyle questionnaire and a validated food frequency questionnaire. We used individual educational level as proxy for socio-economic status (SES). Lifestyle characteristics studied were sex, Body Mass Index (BMI), smoking status, and physical activity level. We defined an unhealthy dietary pattern as low adherence to a Mediterranean-style diet (a score of 0-3 out of 9).

Results: Of our study population, $30 \%$ reported an unhealthy dietary pattern at study entry. In the low SES group higher odds for an unhealthy dietary pattern were found for females and current smokers. Odds ratios for females compared to males in the low, intermediate and high SES group were 1.29 [95 \% CI: 1.20: 1.39], 0.97 [95 \% CI: 0.88: 1.08]) and 0.95 [95\% CI: 0.84: 1.06 ], respectively. Odds ratios for current smokers compared to never smokers in the low, intermediate and high SES group were 1.19 [95 \% CI: 1.11: 1.27], 1.02 [95\% CI: 0.91: 1.14]) and 0.92 [95\% CI: 0.81: 1.06], respectively. Similar results were found when using a social status score at neighborhood level as proxy for SES. BMI and physical activity level were associated with an unhealthy dietary pattern in all SES groups. Higher odds for an unhealthy dietary pattern were found for participants with a high BMI (OR for obese vs normal weight 1.38 [95 \% CI: 1.29: 1.47]) and participants with an inactive lifestyle (OR for inactive vs active participants 1.58 [95\% CI: 1.46: 1.71]).

Conclusion: Determinants of an unhealthy dietary pattern differ by socio-economic status. BMI and physical activity level were associated with an unhealthy dietary pattern in all SES groups, while sex and smoking status were only significantly associated with unhealthy dietary patterns in participants with a low SES.

\section{Abstract \#: P 564}

\section{Measuring food insecurity in a urban sample of northern Portuguese adults}

Brenda Frias $^{1}$, Cláudia Campos ${ }^{1}$, Henrique Barros ${ }^{1}$

${ }^{1}$ Institute of Public Health, University of Porto, Portugal; Corresponding author's e-mail: brendafriasbs@gmail.com

Background: In Portugal as in many developed countries the limited or uncertain to nutritionally adequate and safe food access caused by the economic crisis affects the household food security. This study aims to estimate the prevalence of food insecurity in an urban sample of Portuguese adults, as well as the relationship with sociodemographic determinants. Furthermore, we aim to evaluate the psychometric characteristics of the scale.

Methods: This study was conducted in a sample from EpiPorto cohort composed of Portuguese adults in the metropolitan region of Porto, 309 individuals responsible for feeding in their homes were selected on a convenience sample. The short version of the Food Security Scale was administered to eligible participants with the question "Did you feel changes in their ability to buy essential foods after the crisis (2011) due to economic difficulties?". The evaluation of the psychometric characteristics was assigned according to the exploratory factor analysis (EFA) and confirmatory factor analysis (CFA), the general distribution of food insecurity was also calculated for the sample. A logistic regression analysis was performed to determine factors associated with food insecurity.

Results: The scale used showed satisfactory psychometric characteristics, with good accuracy (Cronbach's alpha was 0.824). The study showed that about $21 . \%$ of households were food insecure. Our findings also indicated, $18.7 \%$ reported concern that food ran out before they had money to buy more and $19.7 \%$ reported that they could not make balanced meals. Food insecurity showed a moderate association with income and education, that is, individuals with lower socioeconomic level has more risk of food insecurity, therefore will have a negative impact on the well-being of families.

Conclusion: The understanding of the broad range of food insecurity is the starting point for an identifying at-risk households and targeting interventions to reduce their risk of further worsening is increasingly recognized as critical to preventing the physical and economic consequences.

\section{Abstract \#: P 565}

The effects of a healthy diet program toward water drinking of pupils in the elementary school in Taiwan

Fu-Hui Lee ${ }^{1}$, Tzu-I Chiu ${ }^{1}$, Li-Feng Lin ${ }^{1}$

${ }^{1}$ Central Taiwan University of Science and Technology;

Corresponding author's e-mail: fhlee@ctust.edu.tw

Background: In recent years, there has been a growing importance of issue concerned with Sugar-sweetened beverages and students' weight gain in Taiwan. The main purpose of this study was to explore the effectiveness of a healthy diet education program about drinking more water compared with one control group.

Methods: A quasi-experimental research design was conducted. The research target contained 132 students as the experimental group and 125 students as the control group from the fifth and sixth grade students of four urban elementary schools in central Taiwan. Students of the experimental group received a healthy diet teaching courses including water drinking, sugar-sweetened beverages or unsweetened drinks and choose healthy drinks in three units and the control group did not receive these instructions during the experimental period. Both groups were pre-tested before the intervention and post-tested 2 month later and the obtained data was statistically analyzed by using paired $t$ test and t-test. In order to evaluate the effectiveness of the program, a questionnaire including the Scale of Water Drinking Knowledge (Kuder-Richardson reliability $=0.59$ ), Attitude (Cronbach's $\alpha=0.77$ ), and Behavior (Cronbach's $\alpha=0.75$ ) was designed. Results: The results on the experimental group showed that the knowledge, attitude and behavior scores of the posttest significantly increased than the pretest. For the healthy drinking knowledge, attitude and behavior on the posttest, the experimental group exhibited significantly higher than the control group, with no significant difference between the two groups on the pretest.

Conclusion: The results indicated that the water drinking education program had positive effects on students' knowledge, attitude and behavior in elementary school. Base on the findings of the study, several recommendations for body weigh control program and health promoting school were proposed.

\section{Abstract \#: P 567}

Iron supplementation in schools and colleges by educational institutes is essential to combat higher proportion of anaemia among adolescents

$\underline{\text { Dr. Padmavathi Dyavarishetty }^{1}}$, Dr. Shobha Kowali ${ }^{1}$

${ }^{1}$ K.J. Somaiya Medical College \& Research Center, Mumbai; Corresponding author's e-mail: shettypadma@hotmail.com 
Background: Anaemia is a common problem in adolescent girls in India. Poor nutritional status is a vicious cycle of adolescent anaemia leading to anaemia in adulthood in turn giving birth to low birth weight babies who grow up to be malnourished and anaemic child and adolescent. Nutritional Intervention Programmes in India focuses on provision of weekly iron folic acid supplementation to adolescent girls. To what extent this programme has been able to make an impact is not known.

Methods: It is an observational, cross-sectional study of adolescents in 7th, 8th, 9th standard in an urban school. The data was analysed using averages, proportions and statistical tests like Chi square.

Results: 202 adolescent's haemoglobin levels were assessed. Of these $62 \%$ were girls and $38 \%$ were males. Mean age of the adolescents was 12.6 years. $25 \%$ of the adolescents had haemoglobin levels less than 12 gms $\% .53 \%$ were mildly anaemic and $20 \%$ had moderate anaemia. Only $1.5 \%$ had severe anaemia. Mean $\mathrm{Hb}$ was $10.88 \mathrm{gms} \%$ with a standard deviation of 1.5 . The difference observed between the haemoglobin status between boys and girls was statistically significant.

Conclusion: The higher proportion of adolescents with anaemia is a cause of concern. Weekly iron folic acid supplementation provided by the government does not seem to have made the desired impact as yet. There is a need therefore to further strengthen the weekly iron folic acid supplementation by inter-sectoral collaboration between education and health departments. If all the educational institutes make a policy decision to implement weekly iron folic acid supplementation, then a large number of children who are in the educational institute could benefit making it a cost-effective and a feasible modality.

\section{Abstract \#: P 568}

Binge drinking: a problematic of public health among students in higher education

M.P. Tavolacci ${ }^{1,2}$, Laure Richard ${ }^{3}$, Pierre Déchelotte ${ }^{2,3}$, Joël Ladner ${ }^{2,4}$

${ }^{1}$ Rouen University Hospital, Clinical Investigation Center 1404, 1 Rue de Germont, Rouen Cedex 76031, France; ${ }^{2}$ Rouen University Hospital, Inserm U1073, Rouen, France; ${ }^{3}$ Rouen University Hospital, Department of Nutrition, Rouen, France; ${ }^{4}$ Rouen University Hospital, Department of Epidemiology and Public Health, Rouen, France; Corresponding author's e-mail: marie-pierre.tavolacci@chu-rouen.fr

Background: Binge drinking (BD) and behavioural risk factors among students in higher education are unknown. The aim was to identify the prevalence and the factors associated with the frequent BD in university student.

Methods: French Students in higher education completed an anonymous online questionnaire on the website specifically dedicated to students: www.tasanteenunclic.org. The questionnaire included age, gender, housing, curricula, smoking, cannabis consumption, alcohol abuse problems (ADOSPA test), tendency to cyber-addiction (Orman test), risk of eating disorders (SCOFF test), depression (Beck test) and perceived stress (Cohen score). Frequent BD was determined as a consumption of five or most alcoholic drinks on one occasion at least twice a month. A risk profile of consumers was conducted according to the consumption of alcohol and frequency of BD.

Results: A total of 3569 students were included with a mean age of $20.5(\mathrm{SD}=2.2)$ and a sex ratio $(\mathrm{M}: \mathrm{F})$ of 0.58 . Smoking concerned $22.3 \%$ of students, $39.8 \%$ were occasionally consumers of cannabis, $25.6 \%$ had a risk of internet addiction and $20.5 \%$ risk of eating disorders. The prevalence of frequent BD was about $13.8 \%(24.7 \%$ in men and $7.6 \%$ in female: $p<10-4$ ) and $26.1 \%$ had an alcohol abuse problems. Non-drinkers was for $13.0 \%$ of students, $18.0 \%$ was no-risk consumers, $50.0 \%$ was episodic risk consumers and $19.0 \%$ was chronic risks consumers. After logistic regression, the frequent $\mathrm{BD}$ was significantly associated with the male sex $\mathrm{aOR}=4.65,95 \% \mathrm{CI}$ [3.40-6.37], living in rental aOR $=1.71,95 \%$ CI [1.21-2.42], business school aOR $=4.72,95 \%$ CI [2.76-8.08], smoking aOR $=5.57,95 \% \mathrm{CI}[3.83-8.08]$ and consuming cannabis $\mathrm{aOR}=12.76,95 \%$ CI [9.09-17.93]. Students granting, not regularly practicing sport and living in couple had a lower risk of frequent BD, respectively aOR $=0.67,95 \% \mathrm{CI}[0.48-0.93]$, aOR $=0.52,95 \%$ CI [0.38-0.71] and aOR $=0.42,95 \%$ CI [0.26-0.68].

Conclusion: Frequent BD concerns one on six students and especially male students. Alcohol consumption is at risk (episodic or chronic) for near $70 \%$ of students. We also identify a target population for prevention and interventions: male students, living on campus or in rented accommodation and with other addictive behavior. These interventions should be not only for freshmen but also throughout the academic years of study.

\section{Abstract \#: P 569}

Food practices of childcare staff and the association with dietary intake of Dutch children at childcare

$\underline{\text { Jessica S. Gubbels }^{1}}{ }^{\text {, Sanne M.P.L. Gerards }}{ }^{1}$, Stef P.J. Kremers ${ }^{1}$

${ }^{1}$ Dep. of Health Promotion, NUTRIM School of Nutrition and Translational Research in Metabolism, Maastricht University, the Netherlands; Corresponding author's e-mail:

jessica.gubbels@maastrichtuniversity.nl

Background: The study explored the associations between various childcare staff food practices and children's dietary intake at childcare centres.

Methods: Energy balance-related dietary intake (fruit, vegetables, sweet and savoury snacks, water and sweet drinks) of a total of 398 1to 3 -year-old children at childcare was registered on 2 weekdays within 1 week, using observations by dieticians and childcare staff. Childcare staff practices of 24 staff members were assessed using questionnaires administered by dieticians. Data were analysed using multilevel regression analyses.

Results: Children consumed much fruit and sweet snacks at childcare, and they mainly drank sweet drinks. When staff explained to the children what they were doing during food preparation, children ate significantly more fruit. Children ate significantly less sweet snacks when they were allowed to help with the preparation of meals. When staff stimulated children to continue eating, they ate more vegetables and when staff gave children food without asking them whether they wanted any, the children ate less sweet snacks. If staff ate together with the children, children ate significantly more sweet snacks.

Conclusion: The study showed the importance of childcare staff food practices for children's food intake at childcare. Although more research is needed to examine the conditions under which these practices can have a positive impact on children's dietary intake, especially child involvement in meal preparation at childcare seems to hold promising results.

\section{Abstract \#: P 570}

Association between healthy and unhealthy dietary habits with weight status in pre-school children

Aristides M. Machado-Rodrigues ${ }^{1}$, ${\text { Augusta } \mathrm{Gama}^{2} \text {, Isabel Mourão }}^{3}$,

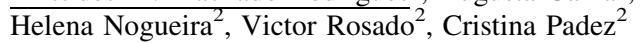


${ }^{1}$ Research Centre for Anthropology and Health, University of Coimbra, Coimbra: ESE-IPV, Viseu, Portugal; ${ }^{2}$ Research Centre for Anthropology and Health, University of Coimbra, Coimbra, Portugal; ${ }^{3}$ Research Centre in Health and Human Development, University of Tras-os-Montes, Vila Real, Portugal; Corresponding author's e-mail: rodriguesari@hotmail.com

Background: In the Southern countries of Europe, the childhood obesity is an important public health issue with a multifactorial nature. A likely source of excess energy intake, and thus a potential contributor to the increased prevalence of overweight and obesity, is the consumption of sweetened beverages, such as regular soft drinks, and sweet snacks and candy. Although several risk factors for consumption of the above-mentioned nutritional items have been identified among school-aged children and adolescents, limited research has been conducted among pre-school children. Therefore, the present study aimed to examine the relationships between healthy and unhealthy dietary intake and overweight risk in a sample of female pre-school children.

Methods: The sample included 4349 pre-school children (2210 males) aged 3-5 years randomly selected. Height and weight were measured. Body Mass Index (BMI) was calculated subsequently and participants were classified as normal weight or overweight/obese (Cole et al. 2000). Eating behaviours were assessed by questionnaire as well as sedentary behaviours (i.e. TV viewing). Logistic regressions were used, with adjustments for age, sex, time spent sedentary, and parental education.

Results: After controlling for the above-mentioned confounders, children who consumed soft drinks in a daily basis were 1.52 times more likely to be classified as overweight/obese than their peers without regular consumption of those beverages. The final regression model also revealed that children who consumed chocolate regularly were $72 \%$ more likely to be classified as overweight/obese children. Conclusion: The present cross-sectional study revealed that regular sweet beverages and chocolate intake were independently associated with an increased prevalence of overweight in pre-school children. These results may help health professionals to advocate for policies that reduce sweet food, particularly soft drink consumption among children. Furthermore, interventions are probably needed to improve healthy eating habits, particularly fruit and vegetable intake in order to prevent the high prevalence of childhood obesity in the Southern countries of Europe.

\section{Abstract \#: P 571 \\ Validity of the measure of the estimated consumption of sodium and potassium in the ELSA-Brasil}

Taísa Sabrina Silva Pereira ${ }^{1}$, Jorge Gustavo Velasquez Melendez ${ }^{2}$, Isabella Bensenor ${ }^{3}$, Nagela Valadão Cade ${ }^{1}$, José Geraldo Mill ${ }^{1}$, Maria del Carmen Bisi Molina ${ }^{1}$

${ }^{1} \mathrm{UFES} ;{ }^{2} \mathrm{UFMG} ;{ }^{3} \mathrm{USP}$; Corresponding author's e-mail:

mdmolina@uol.com.br

Introduction: The high sodium intake is associated with elevations in blood pressure, and high consumption of potassium is associated with decreased blood pressure and reduction of cardiovascular events. Thus, the measurement of these electrolytes is useful for clinical and epidemiological approach to cardiovascular diseases in general. The Food Frequency Questionnaire (FFQ) has been used to evaluate the energy consumption, macro and micronutrients, however, may have limitations when it comes to specific nutrients estimates as sodium and potassium.
Objective: To validate the measurement of sodium and potassium intake estimated by FFQ in Study of Adult Health Longitudinal (ELSA-Brasil).

Methods: Cross-sectional study conducted from the database online ELSA-Brasil. Urinary collection $12 \mathrm{~h}$ night was adopted to estimate creatinine clearance and excretion of electrolytes. A semi-quantitative food frequency questionnaire with 114 food items was applied. The NDSR was used for evaluation of the FFQ food items. To assess the agreement between the methods was used the Bland-Altman and intraclass correlation coefficient (ICC). Medium and consumption quintiles were compared.

Results: 12,593 participants analyzed data obtained through the FFQ and the urinary excretion of $12 \mathrm{~h}$ night. Significant differences were found between the estimated mean values in grams of sodium in the urine $(4.3 \pm 2.1)$, crude FFQ $(4.5 \pm 1.7)$ and FFQ adjusted for energy $(4.3 \pm 0.7)(p<0.001)$ between the average potassium intake (urine: $2.4 \pm 1$ : FFQ crude: $4.8 \pm 1.8$ and FFQ adjusted: $4.6 \pm 1$ : $p<0.001$ ) and estimated salt (urine: $10.9 \pm 5.4$ : FFQ crude: $11.4 \pm 4.2$ and FFQ adjusted: $10.8 \pm 1.7: p<0.001)$. The ICC between the FFQ and urinary excretion was 0.31 for crude sodium and 0.11 for adjusted and 0.27 for potassium crude and 0.34 adjusted potassium. The exact agreement (same quintile) was $23.7 \%$ for sodium and potassium crude, $22.6 \%$ for sodium adjusted and $23.4 \%$ for potassium. According to the Bland-Altman analysis measures are not consistent and show significant bias.

Conclusion: There was no agreement between the methods. The choice of a reference method that accurately reproduces the usual dietary intake of sodium and potassium is still a major challenge given the need for indicators to be used.

\section{Abstract \#: P 572}

\section{Nutritional status of students from a rural area in sao tome and principe-Africa}

Lilian Ramos Sampaio ${ }^{1}$, Raquel Mara Teixeira ${ }^{2}$, Andrêa Jacqueline Fortes Ferreira $^{1}$, Ligia Amparo dos Santos ${ }^{1}$, Albaneide Peixinho ${ }^{3}$, Nelson Vinicius Melo Andrade ${ }^{4}$, Emanuelle Silva ${ }^{1}$, Iracema dos Santos Veloso ${ }^{1}$

${ }^{1}$ Federal University of Bahia (UFBA) - Salvador, Brazil; ${ }^{2}$ Brazilian Cooperation Agency (ABC)-Brasilia, Brazil; ${ }^{3}$ National Fund for Education. Development (FNDE)-Brasilia, Brazil; ${ }^{4}$ University of Porto - Porto, Portugal; Corresponding author's e-mail: raquelmt100@gmail.com

Background: Undernutrition in children and adolescents continues to be a serious global public health problem in many countries, especially in Sub-Saharan Africa, where meeting food needs is a great challenge. This study aimed to assess the nutritional status of children in the Democratic Republic of Sao Tome and Principe.

Methods: This is a cross-sectional study, involving 1759 children and adolescents aged from 5 to 14 years-old, attending primary school in a rural area of Sao Tome and Principe. The health situation, socioeconomic and demographic profile of students and their parents or guardians were investigated. The prevalence of stunting (height-forage $<-2.0 \mathrm{z}$-scores), thinness (Body Mass Index (BMI)/Age $<-2.0$ $\mathrm{Z}$-scores and BMI/Age $<-3.0 \mathrm{z}$-cores), overweight/obesity (BMI/ Age $>2.0 \mathrm{z}$-scores and $>3.0 \mathrm{z}$-cores) and body composition measurement (Arm Muscle Area (AMA)/Age and $\sum$ Triceps skinfolds (TCS) + Sub Scapular (SBCS)) were calculated.

Results: The prevalence of stunting among schoolchildren was $62.5 \%$. The adolescents were the most affected, with a prevalence of $25.0 \%$ in males and $21.7 \%$ in females. Among children, males had 
the highest prevalence of stunting, $12.7 \%$. Male students were the most affected by thinness, highlighting the high prevalence among adolescents $(25.0 \%)$. Overweight has been observed in $7.6 \%$ of the students and obesity has been slightly observed in children $(0.7 \%)$. Female children and adolescents had 32.3 and $47.4 \%$ of deficits of adipose tissue, respectively, while males had a greater impairment of muscle mass, with percentages of 46.5 and $36.8 \%$, among children and adolescents, respectively.

Conclusion: The nutritional status of school children in this country is a major concern, considering the significant percentage of impairment of weight, height and lean mass reserves, as well as the cases of overweight and obesity among children assessed. Those results highlight the need to adopt strategies that can improve the nutritional status of children and adolescents in this region, given the high prevalence of malnutrition and its severe consequences.

\section{Abstract \#: P 573 \\ Reasons for emergency re-admission to hospital in children and young people in England: a cohort study using hospital administrative data \\ Linda P.M.M. Wijlaars $^{1}$, Pia Hardelid ${ }^{1}$, Jenny Woodman ${ }^{1}$, Janice Allister ${ }^{2}$, Ronny Cheung ${ }^{3}$, Ruth Gilbert ${ }^{1}$ \\ ${ }^{1}$ Population, Policy and Practice, University College London, London, UK; ${ }^{2}$ Clinical Innovation and Research, Royal College of General Practitioners, London, UK; ${ }^{3}$ Department of General Paediatrics, Imperial Healthcare NHS Trust, London, UK; Corresponding author's e-mail: linda.wijlaars@ucl.ac.uk}

Background: Rates of emergency admissions for children and young people have been increasing over the last 10 years. To incentivise prevention of repeat admissions, payments for emergency readmissions within 30 days of discharge are restricted in the National Health Service (NHS) in England. Yet evidence from the US shows that adult patients are readmitted with different problems, suggesting that readmissions might not be entirely preventable. We determined the proportion of children and young people (CYP) in England who are readmitted for the same problem.

Methods: We performed a retrospective cohort study using national administrative hospital data. We included children and young people (0-24 year olds) who were discharged after an emergency admission to the NHS in England in financial year 2009/10. We used the primary diagnosis codes (International Classification of Disease version 10, ICD10) to classify six categories indicating reason for admission (infection, chronic condition, injury, perinatal- or pregnancy-related, sign or symptom, or other). We grouped readmissions as $\leq 30$ days, or between 30 days and 2 years after the index discharge.

We used multivariable logistic regression to determine factors available at the index admission (GP registration, sex, ethnicity, deprivation quintile, age, primary diagnosis group, and underlying chronic conditions recorded during the year before index admission discharge) that were predictive of readmission within 30 days.

Results: Overall, $8 \%$ of CYP were readmitted within 30-days. Only half of the 30 day readmissions and $40 \%$ of the readmissions between 30 days and 2 years had the same primary diagnosis group as the original admission. These proportions were consistent across age, sex and diagnostic groups, except for young women (15-24 years) with pregnancy-related problems and infants who were more likely to be readmitted for the same primary diagnostic group. A sensitivity analysis grouping primary diagnoses into 22 ICD10 chapters produced similar results.

CYP with underlying chronic conditions were readmitted within 30 days twice as often (OR: 1.93, $95 \%$ CI: 1.89-1.99) compared to
CYP without chronic conditions. Ethnicity and deprivation had relatively small effects on readmission rates.

Conclusion: Financial penalties for readmission are expected to incentivise more effective care of the original problem, thereby avoiding readmission. Our findings, that half of children come back with different problems, do not support this presumption. Strategies to improve care in hospital, during transfer and/or in primary care need to address the breadth of health problems faced by children discharged from hospital, not just specific conditions.

\section{Abstract \#: P 574}

Mortality in children aged 0-9 years: a nationwide cohort study from three Nordic countries

$\underline{\text { Yongfu Yu }}{ }^{1}$, Jørn Olsen ${ }^{1}$, Naiqing Zhao ${ }^{2}$, Jiong $\mathrm{Li}^{1}$

${ }^{1}$ Section for Epidemiology, Department of Public Health, Aarhus University, Aarhus, Denmark; ${ }^{2}$ Department of Biostatistics, School of Public Health, Fudan University, Shanghai, China; Corresponding author's e-mail: yoyu@ph.au.dk

Background: Mortality in children under 5 years has already been widely studied, whereas little is known about mortality in late childhood especially in 5-9 years of age. We aim to characterize mortality in children aged $0-9$ years using unique data from national registers in three Nordic countries.

Methods: The population-based register study used nationwide data in Denmark from 1973 to $2008(\mathrm{n}=2,433,758)$ and Sweden from 1973 to $2006(\mathrm{n}=3,400,212)$, and a random sample of $89.3 \%$ of children born in Finland from 1987 to 2007 ( $n=1,272,083$ ). The children entered the cohort after birth and follow-up ended at the following events: death, emigration, the day before the 10th birthday or end of follow-up (31 December 2009 in Denmark, 31 December 2008 in Sweden and 31 December 2010 in Finland), whichever came first. Cumulative mortality and mortality rate stratified by sex, age groups, type of death and calendar periods with 5-year interval were estimated.

Results: A total of 7,105,962 children contributed with person-time to the study and 48,299 deaths were identified. Mortality rate decreased with age before 5 years and afterwards was relatively stable between 5 and 9 years. Mortality rate in males was higher than in females in each age group and difference was more pronounced in children aged 5-9 years (rate ratio, 1.39, $95 \%$ CI: 1.31-1.49). Neoplasms $(\mathrm{N}=2473,5.12 \%)$ were the most dominant cause of death in children aged 0-9 years, followed by disease of nervous system $(\mathrm{N}=1816,3.76 \%)$ and transport accidents $(\mathrm{N}=1381,2.86 \%)$. Across the study period from 1981-1985 to 2001-2005, all-cause mortality reduced by from 33.99 to $61.93 \%$ in different age groups and mortality difference between sexes decreased with time. Mortality in infancy $(<1$ year), particularly in neonates, is higher than that of all other age-groups in 2006-2010. Mortality due to transport accidents increased with age and was higher in males aged 5-9 years. Conclusions: Mortality in children aged $0-9$ years in three Nordic countries have fallen dramatically and mortality difference between sexes have decreased during the past years. Infants are still the focus of next action and need continued efforts because of their high mortality. Children aged 5-9 years should receive more attention to reduce the gap between sexes and diminish avoidable death despite their stable and low mortality. Although mortality rates in the three Nordic countries are among the lowest in the world, there is still scope for improvement and special attention should focus on preventable factors related to death. 


\section{Abstract \#: P 575}

Relationship between nurse practitioners' knowledge and implementing health literacy strategies in patient education

Homamodin Javadzade $^{1}$, Mahnoush Reisi ${ }^{1}$, Firouzeh Mostafavi ${ }^{1}$, $\overline{\text { Gholamreza Sharifirad }}^{2}$

${ }^{1}$ Department Health Education and Health Promotion, Faculty of Health, Isfahan University of Medical Sciences, Isfahan, Iran; ${ }^{2}$ Department of Public Health, Faculty of Health, Qom University of Medical Sciences, Qom, Iran; Corresponding author's e-mail: homam_j@hotmail.com

Introduction: Communication is one of the basic and most important skills for health care providers. Using health literacy strategies in patient education as a communication tactics by nurse practitioners make the information understandable for patients and also the effectiveness of the education will increase dramatically. The aim of this study was to assess nurse practitioners knowledge and practice of health literacy strategies in patient education and the correlation between this two factors.

Methods: This cross-sectional descriptive study was done in May and June 2014 on 148 nurse practitioners of Al-Zahra educational hospital, Isfahan, Iran. Data collected via researcher made standardized questionnaire consist of three parts respectively: Demographics, Knowledge about health literacy and its strategies, and health literacy strategies practice. Data analyzed by SPSS v.17 using descriptiveanalytic statistics.

Results: The mean score of knowledge was dramatically low $5 / 89 \pm 2 / 1$ (from maximum available score of 14) and the mean score of nurses in health literacy strategies practice was 21/74 $\pm 3 / 47$. There was a positive relationship between total knowledge of respondents and their practice and also there was statistically significant correlation between basic facts about health literacy and standards for designing health materials subscales and practice.

Conclusion: According to the findings nurse practitioners have limited knowledge about health literacy strategies and doesn't have desirable use of health literacy strategies in practice. Considering that nurses who had better knowledge about health literacy had also better practice in using health literacy strategies in patient education and the key role of health literacy in high quality patient centered health care, it seems retrain courses for nurses with aim of empowering them in this field is necessary.

\section{Abstract \#: P 576}

Prevention of falls in hospitalized patients: validation of the modified johns Hopkins fall risk assessment

Lena Johannsen ${ }^{1,2}$, Andreas Hanel ${ }^{2,3}$, Maria Eberlein-Gonska ${ }^{2,3}$, Jochen Schmitt ${ }^{2,4}$, Thomas Petzold ${ }^{1,2}$

${ }^{1}$ Center for Evidence-Based Healthcare, Department of Quality and Medical Risk Management; ${ }^{2}$ University Hospital Carl Gustav Carus Dresden, Dresden, Germany; ${ }^{3}$ Department of Quality and Medical Risk Management; ${ }^{4}$ Center for Evidence-Based Healthcare; Corresponding author's e-mail: thomas.petzold@uniklinikumdresden.de

Background: Falls constitute a critical health issue for all individuals. The WHO defines falls as "inadvertently coming to rest on the ground, floor or other lower level, excluding intentional change in position to rest in furniture, wall or other objectical risk for inpatients so that preventive measures are necessary. We aimed to investigate
(1) predictors of falls in inpatients and (2) the performance of the modified Johns Hopkins fall risk assessment (FRA).

Methods: We conducted a prospective cohort study based on data from routine documentation from the University Hospital Dresden, Germany. The database included all inpatient cases admitted to and discharged in 2012 and $2013(\mathrm{n}=112,523)$. Documentation of fall risk by means of an in-house developed FRA and incident falls by trained staff.

Results: A total of $1.3 \%$ of patients experience a fall event $(\mathrm{n}=1473)$. The overall fall rate was $2.4 / 1000$ treatment-days. Significant predictors for an inpatient fall event during a hospital stay included age (OR 1.04, $95 \%$-CI 1.041 to 1.047 per year), duration of stay (OR 1.03, $95 \%$-CI 1.034 to 1.4 per day), treatment on ICU (OR $2.8,95 \%$-CI 2.5 to 3.2 ). Content validity of the FRA was very good with a Cohen's Kappa of 0.918. With a sensitivity of $69.3 \%$, specificity of $73.4 \%$, and Youden-Index of 0.427 criterion validity of the FRA was also adequate. The area under the curve (AUC) value was 0.75 and rated as acceptable.

Conclusion: This validation study based on data of a very large prospective study indicates that the modified Johns Hopkins is an appropriate instrument to determine the risk of falls in hospitalized patients. Further modifications to increase the sensitivity of the risk assessment are currently being considered. The use of evidence-based risk assessments is an important step for the establishment of a living safety culture in hospital care.

\section{Abstract \#: P 577}

The health behavior difference in complementary and alternative medicine between mainlanders, Taiwanese and Hakka elders in Taiwan

$\underline{\text { Tzui Chiu }}^{1}$, Li-Feng Lin ${ }^{1}$, Fu-Hui Lee ${ }^{2}$

${ }^{1}$ Nursing Department, Central Taiwan University of Science and Technology, Taichung, Taiwan; ${ }^{2}$ Department of Eldercare, Central Taiwan University of Science and Technology, Taichung, Taiwan; Corresponding author's e-mail: tichiu101929@ctust.edu.tw

Background: The use of complementary and alternative medicine $(\mathrm{CAM})$ in Asia has risen and has attracted research and interest in the general public.

Aim: This study is concerned with the health behavior difference in CAM between Mainlanders Taiwanese and Hakka elders in Taiwan. Method and Participants: A total of 96 participants filled in an questionnaire comprising various sections that deal with their health behavior and experiences with orthodox and complementary treatment.

Results: (1) Mainlanders elders places more value on maintaining and having a higher level of health consciousness than Taiwanese and Hakka elders. (2) A higher level of income have a more positive relationship with one's level of health consciousness and maintenance of health. (3) Taiwanese elders were in greater favour of using Traditional Chinese Therapies as compared to Mainlanders and Hakka elders. (4) Mainlanders elders were more willing than Taiwanese and Hakka elders to use energy therapies. (5) There were no significant effects of sex on any of the measures.

\section{Abstract \#: P 578}

Prevalence and correlates of chronic cholecystitis in a Kazakhstani setting: a cross-sectional study 
Kanat Sakiyev ${ }^{1}$, Aigul Amanbekova ${ }^{1}$, Lyazat Ibrayeva ${ }^{1}$, Lazat Batyrbekova ${ }^{2}$, Kanat Sadykov ${ }^{3}$, Saule Atshabarova ${ }^{1}$, Otegen Gazizov ${ }^{2}$, Andrej M Grjibovski ${ }^{4,5}$

${ }^{1}$ National Centre of Industrial Hygiene and Occupational Diseases, Karaganda, Kazakhstan; ${ }^{2}$ Karaganda State Medical University, Karaganda, Kazakhtsan; ${ }^{3}$ Karaganda State University named after E.A. Buketov, Karaganda, Kazakhstan; ${ }^{4}$ Northern State Medical University, Arkhangelsk, Russia; ${ }^{5}$ Norwegian Institute of Public Health, Oslo, Norway; Corresponding author's e-mail: andrej.grjibovski@gmail.com

Background: Chronic cholecystitis is a lower intensity inflammation of the gallbladder that lasts a long time, may transform into gallbladder cancer and is treated surgically. It is often associated with advanced age, obesity and female gender. Its considerable prevalence and costs of surgical treatment make this condition a substantial public health problem in developed countries. Less is known about the prevalence of chronic cholecystitis in developing countries, although the obesity epidemic and population aging have recently become important public health issues in these countries. The aim of this study is to assess the prevalence of chronic cholecystitis and its correlates in a rural setting of Kazakhstan.

Methods: A cross-sectional study. A random sample of 896 adults aged 18+ living in Aiteke-Bi area (Kyzylorda region, Kazakhstan) was taken from a total population of 38 thousand. Of them, 809 had data on all studied variables. Chronic cholecystitis was diagnosed using anamnestic information and ultrasound test. The prevalence was calculated with $95 \%$ confidence intervals using Wilson's method. Associations between chronic cholecystitis, age-groups, gender, and BMI categories were tested using Pearson's Chi squared tests.

Results: The prevalence of chronic cholecystitis was $27.8 \%(95 \%$ CI: $24.2-31.7)$ among women and $23.6 \%$ among men $(p=0.172)$. In women, the prevalence of chronic cholecystitis progressively increased with age from $21.2 \%$ (95\% CI: 16.1-27.5) in 18-39 year olds to $46.3 \%$ (95\% CI: 36.6-56.3) among 60 years and older ( $p$ for linear trend $<0.001)$. The increase in men was less pronounced and did not reach statistical significance $(p$ for linear trend $=0.301)$. The corresponding proportions were $21.2 \%$ (95\% CI: 15.1-28.9) and $30.3 \%$ (95\% CI: $21.1-41.3$ ). Altogether, $11.5 \%$ of the sample was underweight while $30.7 \%$ were overweight and $28.3 \%$ were obese. The prevalence of chronic cholecystitis was similar across BMI categories in both men $(p=0.528)$ and women $(p=0.151)$.

Conclusions: Every fourth resident of Aiteke-Bi was diagnosed with chronic cholecystitis. Contrary to most other studies, we did not observe differences in the prevalence of the condition across genders or BMI categories. Association with age was significant only for women. Generalization of our results to other settings in Kazakhstan or Central Asia should be done with due caution. The reasons behind our findings and factors which are particularly relevant for development of chronic cholecystitis in Kazakhstan will be discussed.

\section{Abstract \#: P 579}

\section{Knowledge about contraceptive devices and attitude towards family size among post-graduating girls}

\section{Sonam Bedi $^{1}$, C.M. Pandey ${ }^{1}$, R.N. Mishra ${ }^{2}$, Uttam Singh ${ }^{1}$}

${ }^{1}$ Department of Biostatistics \& Health Informatics, Sanjay Gandhi Postgraduate Institute of Medical Sciences, Lucknow, India; ${ }^{2}$ Division of Biostatistics, Department of Community Medicine, Institute of Medical Sciences, Banaras Hindu University, Varanasi, India; Corresponding author's e-mail: sonam.bedi09@gmail.com
Background: Dramatic Indian population increase needs immediate check for its control. The focus should be to the rural part especially north India. The present study was carried on post graduating girls: To assess the opinion of girls about the population size and the present growth rate of the country, to assess the opinion of girls about their future family size along with the desired sex composition, to assess the present knowledge of girls about various contraceptive methods and their sources.

Methods: This was a cross sectional study carried during December to January, 2010 among 100 girls of non co-education Degree Colleges of Varanasi City. The sample size of 100 was randomly chosen from colleges representing girls from all corners of the country: local and medium class society and affluent community of the city. Unpaired $\mathrm{t}$ test was applied.

Results: More than 2/3rd girls were below the age of 25 years with preponderance of Hindus girls. Nearly half of girls were of general caste and more than $80 \%$ from nuclear families. More than $80 \%$ fathers of girls were either graduate or of above qualification with $50 \%$ involved in service: while nearly $50 \%$ mothers were graduates, mostly being housewives. Out of $90 \%$ responses, $95.6 \%$ stated overpopulation leads to financial problem causing crises of food, basic amenities. Out of $83 \%$ responses, $74.7 \%$ girls viewed accelerating awareness of family planning methods may lead to population control. About 3/4th of the girls were in favor of 2 children out of which $4 / 5$ th preferred one is to one male-female ratio. SC/ST had desired significantly larger family size than general and OBC. The most commonly available and practiced spacing methods e.g. condom (male), oral pills, $\mathrm{Cu}-\mathrm{T}$ was known to more than 3/4th of the girls: highest condom male $(98.0 \%)$ followed by oral pills $(87.0 \%)$ and $\mathrm{Cu}-\mathrm{T}$ (75.0). The major sources for commonly practiced spacing contraceptives were TV/Transistor.

Conclusion: The education of the girls is the important component to have better realization of problem of over population, its control strategy and to follow the small family norm. Educating girls means making aware of more and more contraceptive devices. More frequent advertisement through TV, news papers and magazines should be made, especially on traditional methods of contraception.

\section{Abstract \#: P 580}

Family physicians' roles in cancer care: a quantitative and qualitative study from turkey

Raziye Ozdemir $^{1}$, Merve Karacali ${ }^{2}$, Ayse Ozkan ${ }^{2}$, Hasan Yildirim ${ }^{1}$

${ }^{1}$ Department of Occupational Health and Safety, Karabuk University School of Health, Karabuk, Turkey; ${ }^{2}$ Eflani Community Health Center, Karabuk, Turkey; Corresponding author's e-mail: ozdemirr75@hotmail.com

Background: This study aimed to explore the role of Family Physicians (FPs) in cancer care, including prevention, screening, diagnosis, and palliative care of cancer survivors.

Methods: The study consisting of two methodological stages was conducted in Karabuk province of Turkey in 2014.

Stage I: A descriptive study was established in this stage. The data were collected from $56(87.5 \%)$ out of 64 FPs in primary care by means of a questionnaire and analyzed using SPSS employing descriptive statistics.

Stage II: A qualitative exploratory study was performed. Data were collected using in-depth interviews with 15 FPs. Interviews were conducted and recorded on tape by two researchers. Interview 
transcripts were analyzed using content analysis and the main themes were identified.

Ethics committee approval was obtained from the Bulent Ecevit University Research Ethics Committee.

Findings: Stage I: The half $(50.0 \%)$ of the FPs reported that they routinely provided cancer prevention information focused on especially smoking cessation $(83.9 \%)$. The two-third of FPs promoted screening for cervical, breast and colorectal cancers which are covered by the national cancer screening program. The one-third $(32.1 \%)$ FPs did not know their number of cancer patients. Only $16.1 \%$ of FPs reported that they made regular home visits for cancer patients.

Stage II: Four themes were emerged:

1. Perceived responsibilities of FPs on cancer care: All FPs remarked that their tasks have included prevention and early diagnosis of cancer but not included follow-up and palliative care of patients.

2. Demand-oriented care: FPs reported that cancer prevention and screening services limited to people registered with FPs and consulting Family Health Unit (FHU) for any reason, and they remarked that they have not made any effort to reach to the people who are not using FHU services.

3. The small number of team members: FPs works with single family health worker in FHU. The majority of FPs stated that it is impossible for them to deal with early detection and management of cancer with only one family health worker along with their own daily routines.

4. Lack of coordination within health system: All FPs expressed that coordination could only be ensured when patients informed their FPs about the services they received from other sources or specialists. Three FPs stated that it was impossible to ensure coordination without referral system.

Conclusion: The study showed that services provided by FPs in primary care have important problems in terms of content, accessibility and coordination of cancer care.

\section{Abstract \#: P 581}

Mortality from diseases, conditions and injuries where alcohol is a necessary cause in Brazil, 2009-2011

Leila Posenato Garcia ${ }^{1}$, Lúcia Rolim Santana de Freitas ${ }^{2}$, Vilma Pinheiro Gawryszewski ${ }^{3}$, Elisabeth Carmen Duarte ${ }^{2}$

${ }^{1}$ Institute for Applied Economic Research; ${ }^{2}$ University of Brasilia;

${ }^{3}$ Pan American Health Organization; Corresponding author's e-mail: leilapgarcia@gmail.com

Background: Alcohol use is an important risk factor for premature death. Brazil is one of the countries in the Americas with higher mortality rates where alcohol was a necessary cause, which means deaths that wouldn't have occurred in the absence of alcohol consumption. The aim of this study is to describe the mortality from diseases, conditions and injuries where alcohol was a necessary cause in Brazil and its regions in the period 2010-2012.

Methods: This is a descriptive study using data from the National Mortality Information System. Deaths from diseases, conditions and injuries where alcohol was a necessary cause were defined from the International Statistical Classification of Diseases and Related Health Problems, 10th revision (ICD-10) codes, and combined into four groups: liver diseases (K70.0-K70.4 and K70.9): neuropsychiatric disorders (F10.0-F10.9, G32.1 and G62.1): poisoning/intoxication (X45.0-X45.9, X65.0-X65.9, Y15.0-Y15.9, Y90.0-Y90.9 and Y91.0-Y91.9): and other diseases (G31.2, G62.1, G72.1, I42.6,
K29.2, K85.2, K86.0, O35.4, P04.3, Q86.0, R78.0). Triennial average crude and age-standardized mortality rates were calculated per 100,000 inhabitants for Brazil and its regions, and according to sex, age, and group of causes.

Results: In 2010-2012, a total of 55.380 deaths where alcohol was a necessary cause were registered in Brazil. The overall crude and standardized mortality rates were, respectively, 9.6 and 9.7 per 100,000 inhabitants. Standardized mortality rates per 100,000 inhabitants were higher in the Northeastern (11.7), Center-Western (11.0) and Southern (10.1) regions, compared to the Southeastern (8.9) and Northern (5.4) regions. Men comprised $88.5 \%$ of deaths and had a mortality rate around 8 times higher than women. The crude mortality rates according to sex were 17.4 per 100,000 men and 2.1 per 100,000 women. Higher mortality rates were found among people aged 50-59 years (28.5 per 100,000 inhabitants) and 60-69 years (27.2 per 100,000 inhabitants). People aged 50-69 years comprised $56 \%$ of deaths. Most deaths were due to liver diseases (55\%) and neuropsychiatric disorders (39\%).

Conclusion: Alcohol is an important necessary cause of death in Brazil, mainly through liver diseases and neuropsychiatric disorders. The risk of death varied among the country's regions and was substantially higher among men and people aged 50-69 years.

\section{Abstract \#: P 582}

The health status of the elderly population of Iran in 2012

Farid Najafi $^{1}$, Parisa Taheri ${ }^{2}$

${ }^{1}$ School of Public Health, Kermanshah University of Medical Sciences, Kermanshah, Iran; ${ }^{2}$ Shahid Beheshti University of Medical Sciences, Tehran, Iran; Corresponding author's e-mail:

fnajafi@kums.ac.ir

With advancing age comes dramatic increase in the incidence of chronic diseases, disabilities and mental problems. This study was conducted to epidemiologically describe the health status of the elderly population of Iran. People aged $\geq 60$ were selected. MiniNutritional Assessment, Activity of Daily Living, Geriatric Depression Scale questionnaires were administered. Physical diseases and risk factors were also investigated. For the purpose of this study, 1350 elderly individuals were randomly selected. The mean age of sample was $69.1 \pm 7.3$ and $65.9 \%$ were illiterate. From total, $727(53.8 \%)$ had vision problems. The most common problem was cataracts with a prevalence of $305(23.6 \%)$ patients who were either suffering from cataracts or had undergone a surgery for it. From 634 individuals who used dentures, $446(70.3 \%)$ wore the same first denture for over 10 years, without being examined. $524(38.8 \%)$ of our seniors spent their free time alone at home. $13.2 \%$ of women and $12.6 \%$ of men were dependent on others for at least one daily activity. In terms of nutrition, $70(5.5 \%)$ of the elderly (46 women and 24 men) were severely malnourished with 554 (41.3\%) (243 men and 311 women) at risk of malnutrition. The health status of the elderly in Iran was similar to other countries in the world. However there continues to be room for improvement. Taking into account the rapidly aging population of Iran, health providers and policy makers are required to take steps at a national level and direct more resources into supporting the elderly population.

\section{Abstract \#: P 583}

Prevalence of anemia among adults in a rural setting adjacent to the disappearing Aral Sea, Kazakhstan: a cross-sectional study 
Lyazat Ibrayeva $^{1}$, D.T. Amirkhanova ${ }^{2}$, F.U. Nidilbayeva ${ }^{2}$, A.A. Serikbayeva ${ }^{2}$, G.G. Ospanova ${ }^{2}$, A.M. Aitkulov ${ }^{3}$, M.A. Mukasheva ${ }^{3}$, Andrej Grjibovski ${ }^{4,5}$

${ }^{1}$ National Centre of Industrial Hygiene and Occupational Diseases, Karaganda, Kazakhstan; ${ }^{2}$ Karaganda State Medical University, Karaganda, Kazakhtsan; ${ }^{3}$ Karaganda State University named after EA Buketov, Karaganda, Kazakhstan; ${ }^{4}$ Northern State Medical University, Arkhangelsk, Russia; ${ }^{5}$ Norwegian Institute of Public Health, Oslo, Norway; Corresponding author's e-mail: andrej.grjibovski@gmail.com

Background: Anemia is the most common disorder of the blood affecting nearly a quarter of people globally. Anemia increases costs of medical care and lowers a personl's productivity through a decreased ability to work. Moreover, anemia is a well-known risk factor for adverse pregnancy outcomes. While in most European countries the prevalence of anemia among both men and women is low, it remains a significant public health problem in Central Asia. However, most of the studies in these countries have been performed in relatively wealthy urban areas. The aim of this study was to assess the prevalence of anemia among adults living in Aiteke-Bi, one of the least privileged rural areas situated close to the disappearing Aral Sea, Kazakhstan.

Methods: A cross-sectional study. A random sample of 896 adults aged 18+ living in Aiteke-Bi area (Kyzylorda region, Kazakhstan) was taken from a total population of 38 thousand. Hemoglobin level was estimated among 790 individuals (men and non-pregnant women). Anemia was defined as hemoglobin level $<12.0 \mathrm{~g} / \mathrm{dL}$ for non-pregnant women and $<13.0 \mathrm{~g} / \mathrm{dL}$ for men. The prevalence was calculated with $95 \%$ confidence intervals using Wilson's method. Variations in the prevalence of anemia by age and gender were tested using Pearson's Chi squared tests.

Results: The overall prevalence of anemia was greater among women (73.7 \%, $95 \%$ CI: 68.2-78.5) than among men $(58.2 \%, 95 \% \mathrm{CI}:$ 53.8-62.4), $p<0.001$. The prevalence of anemia decreased with age in both genders ( $p$ for both linear trends $<0.001$ ). While among women the prevalence of anemia was $69.7,53.1$ and $49.5 \%$ in age groups $18-39$, 40-59, and 60+ years, respectively, the corresponding proportions for men were 98.2, 63.3 and 49.2\%. Gender differences reached the level of statistical significance for the youngest age-group $(p<0.001)$, but not for middle $(p=0.074)$ and the oldest $(p=0.976)$ groups.

Conclusions: Almost three thirds of women and more than a half of men in this rural Kazakhstani setting have anemia, which exceeds the prevalence of the condition in most settings of the European WHO region. Contrary to most other settings, the prevalence of anemia was higher among men than among women in Aiteke-Bi. Extremely high prevalence of anemia among young adults may have serious consequences for reproductive health of the local population. Factors behind the observed findings as well as preventive strategies at the regional level will be discussed.

\section{Abstract \#: P 584}

Predicting nurse practitioners intention and use of health literacy strategies in patient education from theory of planned behavior perspective

Homamodin Javadzade ${ }^{1}$, Mahnoush Reisi ${ }^{1}$, Firoozeh Mostafavi ${ }^{1}$, Gholamreza Sharifirad $^{2}$

${ }^{1}$ Department Health Education and Health Promotion, Faculty of Health, Isfahan University of Medical Sciences, Isfahan, Iran; ${ }^{2}$ Department of Public Health, Faculty of Health, Qom University of Medical Sciences, Qom, Iran; Corresponding author's e-mail: homam_j@hotmail.com
Background: Health literacy is one of the most important priorities for improving health care quality through enhancing patient-provider communication. Implementing health literacy strategies enables nurses to provide information and instructions for patients in a manner that is more commensurate and understandable. The purpose of this study was to investigate the factors affecting nurses' intention to implement health literacy strategies in patient education based on theory of planned behavior.

Methods: A cross-sectional study was done on 148 nurse practitioners of AL-Zahra educational hospital in Isfahan, Iran, using descriptive-analectic method. Data collected via standardized questionnaire based on theory of planned behavior constructs and analyzed by SPSS v.17 using ANOVA, Independent T-test, Pearson correlation and linear regression.

Results: There were statistically significant correlation between using health literacy strategies and marriage status, attending in retraining courses, employment type, job history, and job status. Perceived behavioral control was the most powerful predictor of intention $(\beta=0 / 417)$ and use health literacy strategies in patient education and behavior of nurses $(\beta=0 / 319)$.

Conclusion: According to the findings of this study, perceived behavioral control is a powerful determinant of nurses' intention and behavior of using health literacy strategies in patient education. Hence we recommend nurse educators to pay special attention to the constructs of this theory mainly perceived behavioral control in retrain courses about patient education and health literacy strategies.

\section{Abstract \#: P 585}

Association between gestational weight gain and prenatal care visits in Rio de Janeiro, Brazil

Débora Souza Gigante ${ }^{1}$, Ana Amélia Freitas Vilela ${ }^{1}$, Amanda C. Cunha Figueiredo ${ }^{1}$, Patricia de Carvalho Padilha ${ }^{1}$, Amanda Rodrigues Amorim Adegboye $^{2}$, Maria Beatriz Trindade de Castro ${ }^{1}$

${ }^{1}$ Federal University of Rio de Janeiro; ${ }^{2}$ Copenhagen University and University of Westminster; Corresponding author's e-mail: mbtcastro@gmail.com

Background: Inadequate gestational weight gain (GWG) is significantly associated with negative pregnancy outcomes. In developing countries the reduced number of prenatal visits leads to increased adverse outcomes for the mother and infant. Yet, the association between adequate prenatal-care and GWG has not been investigated in Brazil.

Objective: To investigate the association of adequacy of prenatalcare and GWG.

Methods: The cross-sectional study has included 322 low-income adult Brazilian women. Pre-pregnancy nutritional status was defined according to body mass index (BMI) categories: underweight, normal-weight, overweight and obese. To describe inadequate Gestational Weight Gain (GWG), it was defined as weight gain below or above the Institute of Medicine (IOM) Recommendations based on pre-pregnancy BMI. Inadequate number of prenatal visits was defined as $\leq 6$ and inadequate initiation of prenatal-care as 1 st visit at $>13$ th gestational week. Prenatal care index (PNC) combining the two dimensions of adequacy (initiation of prenatal care and number of prenatal received visits) was developed. PNC was classified as adequacy of both dimensions was attended: or inadequacy of at least one dimension or both dimensions. GWG was used as continuous variable in linear regression models, adjusted for confounders were performed to estimate the associations between adequacy of number of prenatal visits, initiation of prenatal care and PNC, and GWG. 
Results: On average the prenatal-care started at $12.9( \pm 7.1)$ gestational weeks and $43.1 \%$ of women $(n=144)$ had $\leq 6$ visits. Near of $60.0 \%(\mathrm{n}=181)$ of women presented inadequate gestational weigh gain: $90(29.3 \%)$ below IOM recommendations and $91(29.6 \%)$ above IOM recommendations. After adjustment for civil status, maternal age, pre-pregnancy nutritional status and schooling, it was observed a positive association of number of prenatal care visits ( $\beta=0.17230: p=0.0067$ : IC $95 \%$ : 0.04807-0.29653) and GWG: and a negative association of index of PNC $(\beta=-0.09727$ : $p=0.0890$ : IC 95\%: $-0.20947: 0.01492)$ : and gestational age ( $\beta=-0.13522: p=0.0218$ : IC $95 \%$ : $-0.25066-0.01978)$.

Conclusion: This study highlights the importance of adequacy of contact with health care services during pregnancy and provision of nutritional education during prenatal visits to promote adequate GWG and ultimately maternal-infant health outcomes.

\section{Abstract \#: P 586}

\section{Ambient temperature as trigger of preterm delivery in a temperate climate}

Bianca Cox ${ }^{1}$, Ana M. Vicedo-Cabrera ${ }^{2}$, Antonio Gasparrini ${ }^{3}$, Evelyne Martens $^{4}$, Jaco Vangronsveld ${ }^{1}$, Bertil Forsberg ${ }^{2}$, Tim S. Nawrot ${ }^{1}$

${ }^{1}$ Centre for Environmental Sciences, Hasselt University, Diepenbeek, Belgium; ${ }^{2}$ Occupational and Environmental Medicine, Department of Public Health and Clinical Medicine, Umeå University, Umeå, Sweden; ${ }^{3}$ Department of Medical Statistics, London School of Hygiene and Tropical Medicine (LSHTM), London, United Kingdom; ${ }^{4}$ Study Centre for Perinatal Epidemiology, Brussels, Belgium; Corresponding author's e-mail: bianca.cox@uhasselt.be

Background: Preterm birth is the leading cause of perinatal morbidity and mortality in developed countries. Studies have reported peaks of preterm birth in summer as well as in winter, but findings from studies on the short-term effects of meteorological variables are inconclusive, especially for colder climates. Therefore, we investigated the triggering effect of temperature on the risk of preterm birth during the warm and cold season.

Methods: We used a quasi-Poisson model on 446,110 singleton natural deliveries in Flanders (Belgium) between 1998 and 2011, taking into account the daily pregnancies at risk and their gestational age distribution. Delayed and non-linear temperature effects were modelled by a distributed lag non-linear model (DLNM).

Results: Relative to the median value of $12.8{ }^{\circ} \mathrm{C}$, the risk of preterm birth in the warm season increased by $14.1 \%$ (95\% Confidence Interval (CI): 4.7: 24.2) when minimum temperature up to 3 days before delivery (lag $0-3$ ) exceeded the 95 th percentile $\left(17.9^{\circ} \mathrm{C}\right)$. The corresponding estimate for maximum temperature was not significant $(2.6 \%, 95 \% \mathrm{CI}:-8.7: 15.4)$. In the cold season, we observed a significant effect of low minimum temperature on the risk of preterm birth 2 days before birth (lag 2), but cumulative cold effects were not significant.

Conclusion: Even in a temperate climate, low as well as high temperatures might trigger preterm delivery, suggesting that pregnant women should be protected from temperature extremes.

\section{Abstract \#: P 587}

Oral health status among oldest-old elders and associated factors

Clarice Santana Milagres ${ }^{1}$, Luísa Helena do Nascimento Tôrres ${ }^{1}$, $\underline{\text { Maria da Luz Rosario de Sousa }}{ }^{1}$
${ }^{1}$ Piracicaba Dental School - University of Campinas; Corresponding author's e-mail: luzsousa@fop.unicamp.br

Background: The rising in life expectance highlights the need for knowing better the oral health status of the older population especially the oldest ones in developing countries as Brazil. The aim is to verify the association between oral health status of the oldest-old elders in relation to the youngest ones in six Brazilian municipalities.

Methods: It was used data from a multicenter and cross-sectional study conducted by the "FIBRA Study, which studies frailty in the Brazilian elderly. The total sample consisted of 2343 individuals without cognitive deficit of both genders aged $\geq 65$ years-old in the period between 2008 and 2009 of which $11.7 \%$ were $\geq 80$ years-old. The elderly was recruited at home and submitted to a single data collection session. The outcome was age dichotomized into 65-79 and 80 years or more. Associations between the outcome and sociodemographic, behavioral, general health and oral health data was assessed using Poisson Regression adjusted for confounding variables.

Results: Complete data was available for 2126 elders. The mean age was $72.28( \pm 5.48)$ and $65.6 \%$ were females and $50.6 \%$ married. The oral health variables associated with being older were self-report of not having any natural teeth ( $\mathrm{PR}=1.46,95 \% \mathrm{CI} 1.14-1.87)$ and difficulty or pain to chew hard food ( $P R=1.34,95 \%$ CI 1.05-1.70). Other variables that were associated with being 80 years or more were lower schooling ( $\mathrm{PR}=1.47,95 \%$ CI 1.15-1.88), being female $(\mathrm{PR}=0.48,95 \%$ CI $0.37-0.62)$, being single/divorced/widow $(\mathrm{PR}=2.27,95 \% \mathrm{CI} 1.77-2.92)$, not being white $(\mathrm{PR}=0.73,95 \%$ CI 0.57-0.95), not working ( $\mathrm{PR}=1.55,95 \%$ CI 1.06-2.28), smoking cigarettes $(\mathrm{PR}=0.56,95 \% \quad \mathrm{CI}$ 0.33-0.97), and having comorbidity ( $\mathrm{PR}=0.76,95 \%$ CI $0.59-0.98)$.

Conclusions: The results suggest an association between worst oral health status and being an oldest-old subject. Also being older seems to be associated with worst general health and sociodemographic condition.

\section{Abstract \#: P 588}

Impact of grip strength on mortality in the old age: results from the Kora-age study

$\underline{\text { Barbara Strasser }}^{1}$, Marjan Arvandi ${ }^{2}$, Christa Meisinger ${ }^{3}$, Raffaella Matteucci Gothe $^{2}$, Uwe Siebert ${ }^{2}$, Karl-Heinz Ladwig ${ }^{3}$, Eva Grill ${ }^{4,5}$, Alexander Horsch ${ }^{6}$, Michael Laxy ${ }^{7}$, Annette Peters ${ }^{3}$,

Barbara Thorand ${ }^{3}$

${ }^{1}$ Institute for Nutritional Sciences and Physiology, UMIT, Hall in Tirol, Austria; ${ }^{2}$ Department of Public Health and Health Technology Assessment, UMIT, Hall in Tirol, Austria; ${ }^{3}$ Institute of Epidemiology II, German Research Center for Environmental Health, Neuherberg, Germany; ${ }^{4}$ Institute for Medical Information Processing, Biometrics and Epidemiology (IBE); ${ }^{5}$ German Center for Vertigo and Balance Disorders, Ludwig-Maximillians-University Munich, Munich, Germany; ${ }^{6}$ Institute of Medical Statistics and Epidemiology, TUM, Munich, Germany; ${ }^{7}$ Institute of Health Economics and Health Care Management, German Research Center for Environmental Health, Neuherberg, Germany; Corresponding author's e-mail: barbara.strasser@umit.at

Background: Impaired muscular strength is strongly related to activity limitations and physical disability in middle-aged and aged individuals. However, studies evaluating the relation between muscular strength and mortality in the old are scarce. Objective of this study was to examine the association between handgrip strength and mortality in a prospective population-based cohort study. 
Methods: Data originate from the KORA-Age study in Augsburg. A total of 1079 persons $75.9 \pm 6.5$ (mean $\pm \mathrm{SD}$ ) years old (537 men, 542 women) were enrolled in 2009 and followed-up for mortality 3 years later. Handgrip strength was measured using the JAMAR Dynamometer (Saehan Corp). Three trials, with brief pauses, were allowed for each hand alternately. Participants were encouraged to exert their maximal grip. The best result was chosen for analyses. A Cox proportional hazard model was used to determine adjusted hazard ratios for handgrip strength with $95 \%$ confidence intervals (95\% CI). Plausible confounders were pre-selected based on literature and expert opinion. Further variable selection was based on statistical significance and change-in-estimate criteria.

Results: Maximum grip strength was $24 \pm 5 \mathrm{~kg}$ in women and $39 \pm 8 \mathrm{~kg}$ in men aged 65-72 years (first tertile) and $17 \pm 5 \mathrm{~kg}$ and $28 \pm 8 \mathrm{~kg}$ in women and men aged over 80 years (third tertile), respectively. Age-adjusted mortality rates per 1000 person years across incremental sex-specific thirds of muscular strength $(95 \% \mathrm{CI})$ were 57 (39-81), 14 (7-27), and 1 (0-19) for women and 77 (59-106), 24 (13-41) and 14 (7-30) for men. After adjusting for significant confounders (age, sex, diet score, physical activity, number of drugs and chronic diseases), hazard ratios (95\% CI) across incremental thirds of muscular strength for mortality were 3.33 $(1.53-7.22)$ for the first tertile and $1.42(0.61-3.28)$ for the second tertile as compared to the third tertile (reference).

Conclusions: Muscular strength is inversely and independently associated with mortality in old age. Older adults should be encouraged to perform muscle-strengthening exercises in order to reduce their mortality risk.

Acknowledgement: The KORA research platform (KORA, Cooperative Health Research in the Region of Augsburg) was initiated and financed by the Helmholtz Zentrum München-German Research Center for Environmental Health, which is funded by the German Federal Ministry of Education and Research and by the State of Bavaria. Furthermore, KORA research was supported within the Munich Center of Health Sciences (MC Health), Ludwig-Maximilians-University, as part of LMUinnovativ. This research was partially supported by the Tyrolean Science Fund (Project No. 0404/1398, 2014-15).

\section{Abstract \#: P 589}

\section{Unemployment, mental disorders and suicide: a systematic review} $\underline{\text { Marcos Antonio dos Santos }}{ }^{1}$

${ }^{1}$ Doctoral Program in Occupational Safety and Health; Corresponding author's e-mail: marcosmdrj@gmail.com

Introduction: Suicide is a major problem in public health and is related to many risk factors as unemployment and changing in a labour market. Unemployment, understood as compromising situation of access to income, goods and services, causes profound impact on health's conditions. For Castelhano (2005), the greatest fear of those who have jobs today is losing it. The global inequality in employment and labour, the growing unemployment, the intensified labour precariousness, of slavery or forced labour and other hazardous forms of employment are associated with serious accidents and injuries to multiple diseases that affect workers, their families and their communities, with unemployment being a serious threat to mental health. Objective: This article aims to demonstrate the current escalating impacts of work on mental health workers from the study of the relationship between unemployment and suicide as the direct effects of this new morphology of labour.

Method: The search was conducted using the following databases: PubMed, Medline, Web of Science, Scopus, Library of knowledge online (b-on), Social Care Online, EBSCO, PsycINFO, SciELO.
These databases were chosen to ensure that search strategy in the literature examined thoroughly covered the areas of medicine, sociology and psychology, beyond the textbooks and official documents of the above areas. The terms used in the search were: "unemployment", "mental health", "suicide", "mental disorder". Using the PRISMA methodology, the 628 articles were considered if the search terms were included and were published in the period 2005-2014: after a review of the title and summary, 53 eligible studies-review articles, editorials and documents in English, French, German, Spanish, Portuguese and Italian-were included, but 19 articles were used in this review after duplicates and documents dealing with mental health in retirees, children and adolescents were excluded.

Conclusion: Findings suggest that long-term unemployment is associated with greater incidence of suicide. Changes in the workplace, such as the reduction of employment, expansion of unemployment, have led to an increase in unemployment, which, in turn, has led the worker to the disease process, especially mentally. Community programs mental and psychotherapeutic strategies evidence-based health can help reduce and prevent suicide in the working world, a global imperative.

\section{Abstract \#: P 590}

\section{Lifestyle and working memory in children}

Mónica López-Vicente $^{1}$

${ }^{1}$ Child Health, CREAL, Barcelona, Spain; Corresponding author's e-mail: mlopez8@creal.cat

Background: Sleep duration and frequency of exercise and sedentary behaviors, as video games and television use, in children are a matter of concern for public health. These lifestyle habits have an important role on neurodevelopment. We aimed to study how the current lifestyle patterns are associated with working memory (WM) in healthy children.

Methods: 2897 children between 7 and 10 years old from 39 schools participated in this study. WM was repeatedly measured using n-back task in 4 visits during a period of 1 year. Sleep duration, exercise, video games use, and television viewing were reported by parents. We performed unadjusted and adjusted linear mixed-effects models for the 4 outcomes (2- and 3-back d' with numbers and words) including school and subject as random effects.

Results: Exercising less than once per week and not using video games at weekends were negatively associated with n-back scores. Playing video games more than $2 \mathrm{~h}$ per weekend day was associated with better n-back performance. Sleep duration and watching TV were not associated with the WM measure in the adjusted models. Conclusions: Both exercise and video games use were positively related to WM in a large sample of healthy children. Future longitudinal research is needed in order to confirm these important findings.

\section{Abstract \#: P 591}

Probing know do gaps for inadequate hand washing before vaccination by ANMs during routine immunization in indian Kosi riverine belt

$\underline{\text { Dr. Rishbah Kumar Rana }}^{1,2,3}$, Dr. Manu Batra ${ }^{4}$, Dr. C. Roy ${ }^{2,3,4,5}$

${ }^{1}$ IEA; ${ }^{2}$ IAPSM; ${ }^{3}$ IMA; ${ }^{4}$ IPHA; ${ }^{5}$ APCRI; Corresponding author's e-mail: bakwasandsony@gmail.com 
Background: "Clean care is safe care". Hand hygiene is the primary measure to reduce infections. A simple action, perhaps, but the lack of compliance among health-care providers is problematic worldwide. Auxillary Nursing Midwives are an integral component in the community setting in India. In Kosi riverine area, their involvement to increase Routine Immunization coverage is integral to its success. This study intended to determine the Know-Do Gaps for Inadequate Hand washing during Routine Immunization Vaccine Administration. Methods:

(a) Objectives: To determine the know do gaps for inadequate practice of proper hand washing techniques among ANMs during Routine Immunization Vaccine Administration in the District of Begusarai In the Kosi Riverine Area.

(b) Design and Setting: A prospective study conducted across the district with ANMs being observed during RI session sites before Vaccine Administration, and were asked questions regarding Handwashing.

(c) Study Method and Material: A questionnaire was designed in the Department of Community Medicine Darbhanga Medical College.

Results: Poor Knowledge about the importance of Hand washing before starting the session site, Before administration of vaccine, Poor emphasis on Hand Washing during training session, Lack of Hand washing logistics at most session sites, and No monitoring regarding Handwashing, were some of the important factors causing the Know Do Gaps.

Conclusion: From Training sessions to session site the emphasis will have to be on Effective Implementation of Hand washing. Systems Approach is the need of Hour.

\section{Abstract \#: P 593}

\section{Surgical risks associated with winter sport tourism}

Stéphane Sanchez ${ }^{1}$, Cécile Payet ${ }^{1}$, Jean Christophe Lifante ${ }^{2}$, Stéphanie Polazzi $^{1}$, François Chollet ${ }^{1}$, Antoine Duclos ${ }^{1}$

${ }^{1}$ Pôle IMER, Hospices Civils de Lyon; ${ }^{2}$ Digestive Surgical Department, Lyon Sud, Hospices Civils de Lyon; Corresponding author's e-mail: stephane.sanchez@chu-lyon.fr

Background: Mass tourism during winter in mountain areas may cause significant clustering of body injuries leading to increasing emergency admissions at hospital. We aimed at assessing if surgical safety and efficiency was maintained in this particular context.

Methods: We selected all emergency admissions of open surgery performed in French hospitals between 2010 and 2012. After identifying mountain areas with increasing volume of surgical stays during winter, we considered seasonal variations in surgical outcomes using a difference-in-differences study design. We computed multilevel regressions to evaluate whether significant increase in emergency cases had an effect on surgical mortality, complications and length of stay. Clustering effect of patients within hospitals was integrated in analysis and surgical outcomes were adjusted for both patient and hospital characteristics.

Results: A total of 381 hospitals had 559,052 inpatient stays related to emergency open surgery over 3 years. Compared to other geographical areas, a significant peak of activity was noted during winter in mountainous hospitals (Alps, Pyrenees, Vosges), ranging 6-77\% volume increase. Peak was mainly explained by tourists' influx $(+124.5 \%, 4351 / 3496)$ and increased need for orthopaedic procedures $(+36.8 \%, 4731 / 12,873)$. After controlling for potential confounders, patients did not experience increased risk for postoperative death (ratio of OR $1.01,95 \%$ CI $0.89-1.14, p=0.891$ ), thromboembolism $(0.95,0.77-1.17, p=0.621)$ or sepsis $(0.98$, $0.85-1.12, p=0.748)$. Length of stay was unaltered $(1.00,0.99-1.02$, $p=0.716$ )

Conclusion: Surgical outcomes are not compromised during winter in French mountain areas despite a substantial influx of major emergencies.

\section{Abstract \#: P 594}

Risk factors associated with asthma among rural women of India Ankita Chourasia $^{1}$, C.M. Pandey ${ }^{1}$, Ashish Awasthi ${ }^{1}$, Uttam Singh ${ }^{1}$

${ }^{1}$ Department of Biostatistics \& Health Informatics, Sanjay Gandhi Postgraduate Institute of Medical Sciences, Raebareli road, Lucknow, Uttar Pradesh, India; Corresponding author's e-mail: ankitasgpgi@gmail.com

Background: Asthma is a complex condition resulting from an interaction between genetic vulnerability and exposure to environmental factors. Globally, Prevalence of asthma is increasing. Women in rural India are more exposed to household and environmental factors causing asthma. It is necessary to determine the risk factors associated with asthma among rural women of India. The objective of this study is to examine the risk factors associated with asthma among rural women of India.

Methods: The data for this study has been drawn from National Family Health Survey 3 (2005-2006). 64,049 women aged 15-49 are included. Outcome variable is taken as women suffering from asthma. Women's age, education, body mass index, smoking practices including cigarettes, cigar/beedi, and pipe, and alcohol, type of house, type of cooking fuel, cooking done under chimney, and wealth index are considered as independent variables. Prevalence of asthma is computed for all variables. The variables found significant in bivariate analysis are included in multivariate analysis. Population-attributable risk is also computed for all variables.

Results: Overall prevalence of asthma is 17 per thousand women. As compared to underweight women, overweight women are at two times more risk of having asthma. Women with exposure to smoking cigarettes/beedi and drinking alcohol are at higher risk of having asthma as compared to those who are non-exposed. Women who do not cook food under chimney are at 1.3 times more risk of having asthma. Women with lower educational level are at 1.2 times more risk of having asthma as compared to women with higher education. Not cooking under chimney, no education, and higher age group (35-49) has higher population-attributable risk percent. The corresponding PAR is PAR not cooking under chimney $=19 \%$, PAR no education $=15 \%$, PAR age $35-49=35 \%$.

Conclusion: Fostering education and healthy habits might help in declining morbidity associated with asthma. Cooking under chimney reduces the risk of asthma.

\section{Abstract \#: P 595}

\section{Prevalence of fetal alcohol spectrum disorder (FASD): a meta-} analysis

Sylvia Roozen $^{1}$, Gjalt-Jorn Y. Peters ${ }^{2}$, Gerjo Kok $^{3}$, David Townend ${ }^{4}$, Jan Nijhuis', Leopold Curfs ${ }^{6}$

${ }^{1}$ Governor Kremers Centre (GKC), Maastricht University Medical Centre+ (MUMC+) - Department of Work and Social Psychology, Maastricht University; ${ }^{2}$ Faculty of Psychology and Education Science, Open University of the Netherlands; ${ }^{3}$ GKC, MUMC+ - 
Department of Work and Social Psychology, Maastricht University; ${ }^{4}$ GKC, MUMC+ - Department of Health, Ethics \& Society, Maastricht University; ${ }^{5}$ GKC, MUMC + - Department of Obstetrics \& Gynaecology, MUMC +; ${ }^{6} \mathrm{GKC}$, MUMC + - Department of Genetics, MUMC+; Corresponding author's e-mail:

sylvia.roozen@maastrichtuniversity.nl

Background: Alcohol use during pregnancy is one of the leading preventable causes of intellectual or developmental disability. FASD is the non-diagnostic umbrella term used to characterize the full range of damage caused by prenatal alcohol exposure, varying from mild to severe, and encompassing a broad array of physical defects and cognitive, behavioral, emotional, and adaptive functioning deficits. This situation clearly warrants intervention. Development of a focused, effective and evidence based intervention requires insight in the burden of FASD and the underlying dynamics. The current literature synthesis aims to provide the required evidence base by compiling the literature on FASD, specifically addressing what we know of the prevalence of FASD, which behaviors contribute to FASD, and which preventive measures have been recommended so far.

Methods: A search was conducted in multiple electronic bibliographic databases up to November 2014. A query was generated and the resulting hits were exported and screened by two independent screeners. The constructed query yielded 395 hits. Three different screening rounds were conducted based on: titles, titles and abstract, full text articles. In addition, two review studies were cross checked for possible missing articles and all authors were asked to confirm our interpretation of their results. A number of 49 records $(11 \%)$ remained useful for further extraction and meta-analysis. The Freeman-Tukey double arcsine transformation was used for analyzing proportional data. Then, multiple meta-analyses were conducted with the transformed proportions. These were then back-transformed to prevalence rates to facilitate interpretation of the outcomes and confidence intervals $(\mathrm{CI})$ bounds were calculated.

Results: On the basis of population based studies, the overall FASD pooled prevalence estimates ranged from 1.31 to 17.49 per 1000 . FASD was calculated to be 15.4 per 1000 (95\% CI: 1.02-45.56). The individual diagnostic differences and challenges will be presented.

Conclusion: The results show that alcohol use during pregnancy is an important health problem. The present study within our project is a first step for improving health promotion related to FASD.

\section{Abstract \#: P 596}

\section{Psychic disorders minor in the causal chain of periodontitis}

Samilly S. Miranda ${ }^{1}$, Julita M. F. Coelho ${ }^{1}$, Isaac S. Gomes-Filho ${ }^{1}$, Silas Carvalho $^{2}$, Johelle de S. Passos ${ }^{1}$

${ }^{1}$ Health, UEFS, Feira de Santana, Brazil; ${ }^{2}$ Health, FAT, Feira de Santana, Brazil; Corresponding author's e-mail: samillymiranda@gmail.com

Background: Few studies have investigated the association between periodontitis and minor psychiatric disorders that has prompted interest in the subject. It is known that the lack of hygiene, psychomotor damage, diminished salivary flow due to the use of drugs added to the difficulty of access to dental services have been allocated as potential links to such association. Aim: To estimate the association between Minor Psychic Disorders and periodontitis.

Methods: A case-control study with secondary data from a previous study with a subsample of 414 adults in an urban area was delineated. A group of cases of periodontitis was compared to a control group without this condition. Sociodemographic and lifestyle habits were obtained from questionnaires, the diagnosis of chronic periodontitis was made from complete periodontal examination. Laboratory tests from the collection of blood samples were requested. Individuals with minor psychiatric disorders were identified from the application of the Self-Reporting Questionnaire (SRQ-20) in the case and control groups. The analysis procedures included descriptive analysis and stratified using the Chi square test and t test, and logistic regression analysis. This study design was submitted to and approved by the Research Ethics Committee of the State University of Feira de Santana, Bahia (Protocol no. 025/2004).

Results: The prevalence of minor psychiatric disorders was higher among subjects with periodontitis compared to those without periodontitis (73.1 vs $24.2 \%$ ) and the crude OR was 8.50: $95 \% \mathrm{CI}$ : 5.13-14.10: $p<0.001$. After adjustment for Drinking and Diabetes and the presence of acute myocardial infarction was obtained OR 2.16: $95 \%$ CI: 1.27 to 3.65: $p=0.004$.

Conclusion: Compared to higher frequency of minor psychiatric disorders in patients with periodontitis is possible to identify a causal link between these clinical conditions. We emphasize high rates of periodontitis and tooth loss in the sample, which raises reflections about difficulties in accessing health services to control these adverse conditions.

\section{Abstract \#: P 597}

\section{Determinants of dental healthcare utilization in chronic disease patients in Hong Kong}

$\underline{\text { Michelle J.W. Chang }}{ }^{1}$, Janice M. Johnston ${ }^{1}$

${ }^{1}$ School of Public Health, the University of Hong Kong, Hong Kong; Corresponding author's e-mail: cjwlele@gmail.com

Introduction: Many studies report a bi-directional association in the clinical pathology between chronic disease (e.g., diabetes mellitus, coronary heart disease) and dental disease (e.g., periodontal disease). However, people who experience increased oral disease burden because of chronic disease may not be found to use increased dental healthcare utilization. This study aims to identify barriers of dental healthcare seeking behavior among chronic disease patients and propose new approaches to general health management in Hong Kong.

Methods: Self-reported past 12-month dental healthcare service utilization and chronic health conditions were extracted from the Thematic Household Survey (THS) in 2011. Andersen's Health Behavioral Model will be used as conceptual model for the study which includes predisposing (age, sex, education attainment, household income, and smoking status), enabling (Medical benefit, insurance coverage), and need factors (presence of doctor-diagnosed chronic disease). Dental healthcare service utilization was defined as individual past 12 months teeth examined or treated experience based on self-reported ever use any type of dental care services. Firstly, logistic regression models were fitted to age-adjusted ORs of dental visits by each of the above factors separately. Secondly, a full model which contained all predisposing, enabling, and need factors was used for identifying factors associated with dental health service utilization.

Results: 24,699 residents over 18 years old were recruited for the THS survey. $19.8 \%$ of the respondents reported chronic health conditions as diagnosed by Western medicine practitioners. $22.7 \%$ diabetes mellitus, $24.1 \%$ hypertension and $23.8 \%$ heart disease (including both coronary heart disease and other heart disease) selfdiagnosed respondents reported had at least once teeth examined or treated experience during the past 12 months compared with $27.5 \%$ for those without chronic health conditions. The prevalence of dental healthcare utilization was significantly lower among the residents 
with diabetes mellitus and hypertension compared with those without chronic disease. However, following adjustment for all covariates showed, hypertension and heart disease were significantly associated with higher dental care utilization.

Conclusion: Not simply the same results compared with elsewhere, higher dental care utilization among residents with selected chronic health conditions was found after adjusted for different predisposing, enabling and need factors. Also chronic disease patients with different predisposing, enabling and need factors have different health seeking behaviors for dental health care service. Further research will investigate why these factors would influence residents' dental health care seeking behaviors in Hong Kong.

\section{Abstract \#: P 598}

Early sexual initiation and alcohol, tobacco and illicit drugs use among Brazilian adolescents

$\underline{\text { Ana Luiza SoaresTiago }^{1}}{ }^{1}$ Neuenfeld Munhoz ${ }^{1}$, Helen Gonçalves ${ }^{1}$

${ }^{1}$ Postgraduate Program in Epidemiology, Federal University of Pelotas, Pelotas, Brazil; Corresponding author's e-mail:

nutri.analuiza@hotmail.com

Background: In Brazil, sexual initiation mean age is 14 years old among boys and 15 years among girls. Early sexual intercourse is related to higher health risks during and after adolescence, such as sexually transmitted diseases and pregnancy. Some studies have shown that adolescents who use alcohol, tobacco and illicit drugs are also those who have started sexual activity earlier. The aim of this study was to estimate the prevalence of early sexual intercourse, and to assess its relationship with alcohol, tobacco and illicit drugs use in Brazilian adolescents.

Methods: Data from National Adolescent School-based Health Survey (PeNSE, 2012) was used. This cross-sectional study evaluated a representative sample of all 9th grade students in Brazil. Early sexual intercourse was defined as first sexual intercourse up to age 14 . Alcohol and tobacco use were considered the use of any quantity of alcohol or tobacco in the 30 days before the interview. Illicit drug use was considered the use any illicit drug ever. The most commonly used drugs were assessed, such as marijuana, cocaine, crack, solvent-based glue, general ether-based inhalants, ecstasy and oxy. Statistical analyses, stratified by sex, were carried out in Stata 12.1, considering study designs and sample weight. Poisson regression was used in crude and adjusted analysis, and the confounding variables considered were age, mother education, skin colour and school administration (public or private). PeNSE was approved by the Research Ethics Committee of the Ministry of Health.

Results: From the 109,104 scholars who answered the interview, $15.7 \%$ had early sexual initiation, and the prevalence was higher among boys $(23.9 \%)$ than among girls $(8.1 \%)$. Alcohol use was referred by $26.1 \%$ of the adolescents, tobacco use by $17.2 \%$, and illicit drug use was reported by $7 \%$ of them, with no difference between genders. The risk of alcohol, tobacco and illicit drugs use was 2.0, 2.6 and 3.1 times, respectively, higher among those who had early sexual initiation, and the risk of substance use was even higher among girls than among boys. The association remained statistically significant after adjustment for confounders.

Conclusions: Sexual initiation before 15 years old is associated to alcohol, tobacco and illicit drugs use in adolescence. Health strategies should take into account that risk behaviours tend to cluster together and health policies should consider this when planning interventions.

\section{Abstract \#: P 599}

Adverse childhood and adolescence experiences in a birth cohort in southern Brazil

Ana Luiza G. Soares ${ }^{1}$, Alicia Matijasevich ${ }^{1}$, Ana Menezes ${ }^{1}$, Fernando Wehrmeister $^{1}$, Maria Cecilia Assunção ${ }^{1}$, Helen Gonçalves ${ }^{1}$

${ }^{1}$ Postgraduate Program in Epidemiology, Federal University of Pelotas, Pelotas, Brazil; Corresponding author's e-mail: analuiza.nutri@gmail.com

Background: Adverse childhood or adolescence experiences are a lifecourse social determinant of health. Exposure to trauma early in life may have not only a psychological effect, influencing mental disorders, but also an impact on physical health, increasing the risk of cardiovascular diseases and obesity. The aim of this study was to estimate the prevalence of domestic violence, physical abuse and sexual abuse, and to assess the sociodemographic factors related to it in a Brazilian birth cohort.

Methods: All children born in 1993 in the city of Pelotas $(\mathrm{N}=5265)$, Southern Brazil, were interviewed and followed until adolescence. At 15 -years-old follow-up ( $85.7 \%$ follow-up rate) the occurrence of domestic violence, physical abuse and sexual abuse until adolescence was evaluated using a self-reported and confidential questionnaire. Domestic violence was assessed by asking: "Has there ever been fights with physical assault in your household among adults or has an adult ever assaulted a child or adolescent?". Physical abuse was evaluated by the question: "Has an adult of your family or someone who was looking after you hit you in a way that left you hurt or bruised?". Sexual abuse was assessed by asking: "Has anyone ever tried to do sexual things to you against your wish, threatening or hurting you?". Sociodemographic information included gender, mother education and family asset index. Descriptive and bivariate analyses were carried out in Stata 12.1, and Poisson regression with robust variance adjustment was used. The study was approved by the Research Ethics Committee of the Medical School of the Federal University of Pelotas.

Results: Until 15 years old, $10.4 \%$ of the adolescents referred domestic violence, $7.1 \%$ reported physical abuse and $1.5 \%$ reported sexual abuse. About $15 \%$ of the interviewees have had at least on type of adverse experience during childhood or adolescence. The prevalence of physical abuse, domestic violence and sexual abuse was 1.4, 1.5 and 3.4 times higher among girls than among boys, respectively. The three outcomes were inversely related to family asset index, but only sexual abuse was associated to low mother education. Conclusions: Domestic violence, physical abuse and sexual abuse are more likely to happen among girls and poorer adolescents in Brazil. Health strategies should prioritize these groups in the prevention of childhood and adolescence adverse experiences.

\section{Abstract \#: P 600}

Self-harm and suicide attempts during a dramatic national economic transition: a population-based study in Iceland

Hildur Guðný Ásgeirsdóttir ${ }^{1}$, Tinna Laufey Ásgeirsdóttir ${ }^{2}$, Pórdís Katrín Porsteinsdóttir $^{3,4}$, Brynjólfur Mogensen ${ }^{3,5}$, Ullakarin Nyberg ${ }^{6}$, Sigrún Helga Lund ${ }^{1}$, Unnur Valdimarsdóttir ${ }^{1,7}$, Arna Hauksdóttir ${ }^{1}$

${ }^{1}$ Center of Public Health Sciences, Faculty of Medicine, University of Iceland, Reykjavik, Iceland; ${ }^{2}$ Faculty of Economics, University of Iceland, Reykjavik, Iceland; ${ }^{3}$ Research Institute in Emergency Care, National University Hospital in Reykjavík; ${ }^{4}$ Faculty of Nursing, University of Iceland, Reykjavik, Iceland; ${ }^{5}$ Faculty of Medicine, 
University of Iceland, Reykjavik, Iceland; ${ }^{6}$ St. Göran Norra Stockholms Psykiatri, Stockholm, Sweden; ${ }^{7}$ Department of Epidemiology, Harvard TH Chan School of Public Health, Boston, Massachusetts, USA; Corresponding author's e-mail: hga@hi.is

Background: Macroeconomic downturns have repeatedly been associated with increased suicide rates.

Aims: We aimed to study potential change in self-harm and suicide attempts in Reykjavik, the capital of Iceland, during a period of major national economic transition, from 2003 to 2012.

Methods: We retrieved data from electronic hospital records of the National University Hospital in Reykjavik (population size: 204.725), containing all ICD-10 diagnoses of intentional self-harm and suicide attempts (ICD-10 diagnostic codes: X60-85, X40-49, Y10-34, T36-50, Z91.5). Information on date of the attendance and on the individual's age, gender and postcode was obtained from these resources and population size from Statistics Iceland. We used Poisson regression models to compare attendance rates during the economic recession (end 2008 through 2012) to attendance rates precrisis (2003-2008).

Results: During the study period, a total of 4537 attendances were recorded due to self-harm or suicide attempts: $1874 / 100.000$ pre and $1309 / 100.000$ post economic crisis ( $p$-value 0.135). Attendance rates were relatively stable among women during the study period (difference in slope $p$-value 0.9336) while attendance among men seemed to reach a high point in 2007, i.e. in the economic boom. The annual increase was 1.83 per 100.000 men pre-crisis and decreased postcrisis, by 3.06 per 100.000 male inhabitants per year ( $p$-value 0.00679). Age-specific analysis revealed an increase in attendance among women $25-35$ years of age post-crisis, as compared to precrisis (RR 1.28: CI 1.08-1.51) and among men older than 45 years of age (RR 1.23: 1.04-1.47). No other age groups showed significant differences in attendance pre-and post-crisis.

Conclusion: These data suggest limited overall impact of the 2008 Icelandic economic collapse on attendance rates due to self-harm and suicide attempts except in certain age groups. In fact, the economic boom was associated with a high-point in self-harm and suicide attempts among Icelandic men.

\section{Abstract \#: P 601}

\section{Individual and community-level predictors of suicidal ideation and attempt in South Korea, 2009: a multi-level analysis}

\section{$\underline{\text { Hyungryul Lim }}^{1}$, Ho-Jang Kwon ${ }^{1}$, Mina $\mathrm{Ha}^{1}$}

${ }^{1}$ Department of Preventive Medicine, College of Medicine, Dankook University; Corresponding author's e-mail: matrixlhr@gmail.com

Background: Since 2007, South Korea has consistently ranked first place in the suicide rate among OECD countries and it has been great challenges to prevent suicides in the country. According to OECD statistics, the suicide rate in South Korea is 29.10 per 100000 persons in 2012. We conducted this study to examine potential associated factors with suicidal ideation and attempt in both individual and community-levels in whole South Korea.

Methods: We conducted a cross-sectional study using the 2009 Community Health Survey which has representative adult respondents' health data and KOSIS (Korean Statistical Information Service) data for community level information (fist level: metropolitan city/province, and second level: city/town levels) regarding contextual or regional welfare and economic situation. We considered urbanization, monthly household income, basic living security recipient, subjective health status, subjective stress level, experience of depression mood, education level and marital and living status as well as age and gender as individual-level factors and elderly welfare leisure facilities, social welfare facilities, welfare budget share, GRDP per capita, employment rate and CPI growth as community-level factors. We performed the survey analysis considering the complex sampling frame and the mixed effects logistic regression for multilevel modeling by using $\mathrm{R}$ ver. 3.0.3.

Results: Of 230715 respondents in 2009 Community Health Survey, we analyzed 222715 respondents for our modeling excluding those with incomplete response. The prevalence of suicidal ideation was $10.5 \%$ and suicidal attempt was $0.6 \%$. In final fully adjusted multilevel model, in terms of suicidal ideation, most risk factors are from individual-level factors, such as older age, female, lower household income, lower education level, living alone without spouse, and national basic living security recipient. There was one exception, more elderly welfare leisure facilities at provincial level showed a preventive effect against suicidal ideation $(\mathrm{OR}=0.98,95 \% \mathrm{CI}$ 0.97-1.00). In terms of suicidal attempt, younger age, female, lower household income, lower education level, living alone without spouse, and national basic living security recipient were individuallevel risk factor. There was no significant risk factor from community-level factors.

Conclusion: In South Korea, elderly over 60 years old showed the highest prevalence of suicide. Even if a suicidal ideation is not directly connected to committing suicide, we could not help but try to prevent old people thinking commit suicide as considering senior welfare at provincial level.

\section{Abstract \#: P 602}

The gap between training for and the practice of prescribing drugs by nurses in primary health care in Brazil

Claudia Santos Martiniano ${ }^{1,2}$, Emanuela de Castro Marcolino ${ }^{3,4}$, Marize Barros Araújo de Barros ${ }^{1,5}$, Ardigleusa Alves Coelho ${ }^{1,2}$, Ricardo Alexandre Arcêncio ${ }^{6,7}$, Inês Santos Estevinho Fronteira ${ }^{8}$, Severina Alice da Costa Uchôa ${ }^{1}$

${ }^{1}$ Programa de Pós-Graduação em Ciências da Saúde. Universidade Federal do Rio Grande do Norte; ${ }^{2}$ Rua Juvêncio Arruda, s/n. Campus Universitário, Bodocongó. CEP: 58.429-600. Campina Grande Paraíba. Brazil; ${ }^{3}$ Mestranda em Saúde Pública. Universidade Estadual da Paraíba; ${ }^{4}$ Endereço: Rosendo Pereira de Lucena, 92, São José, Campina Grande, Paraíba. Brazil; ${ }^{5}$ Rua Industrial João Motta, n 1541, B1 D, apto 102. Condomínio Liberdade. Capim Macio, Natal-RN, Brazil; ${ }^{6}$ Escola de Enfermagem de Ribeirão Preto. Universidade de São Paulo; ${ }^{7}$ Av. Bandeirantes, 3900 Campus Universitário. 14049900 - Ribeirão Preto, SP - Brazil; ${ }^{8}$ Rua da Junqueira, 100.1349-008 Lisboa. Portugal; ${ }^{9}$ Av. Senador Salgado Filho, S/N. Lagoa Nova ICEP: 59078-970| Natal/RN. Brazil; Corresponding author's e-mail: cmartiniano@ibest.com.br

Background: In many countries, the inclusion of nurses as prescribers is considered to be an advanced practice. In Brazil, such prescriptions are legally regulated and restricted to Primary Health Care protocols. The presence of prescribing nurses has provoked a debate among medical and nursing corporations. However, there are few studies examining the qualifications, protocols and in-service training that are aimed at prescribing nurses in Primary Health Care. Objective: We sought to investigate possible gaps between the education, qualifications, self-assessments and practice of the prescribing of medicine in Primary Health Care from the nursés perspective.

Design: This investigation is a case study with a qualitative approach. Setting: This research occurred in a Brazilian municipality with $84.04 \%$ Family Health Strategy coverage and 400,002 inhabitants in the northeast of Brazil. 
Participants: The participants were an intentional sample of 37 nurses in primary health care who were linked to the Family Health Strategy.

Methods: The study was conducted between August and November 2011 with four focus groups and a script validated by Delphi technique and a pilot study. This study addressed the qualifications for the prescription of medication, the sufficiency of the Ministry of Health protocols and self-assessments of the ability to prescribe. Bardin's qualitative analysis was applied.

Results: Of the 37 participants, $97.3 \%$ were female, $54 \%$ had graduated less than 10 years ago, $27 \%$ had graduated between 10 and 20 years ago, $16.2 \%$ had graduated more than 20 years ago, and $83.8 \%$ had graduated with a specialisation in Public Health. All nurses reported having received insufficient training in the discipline of pharmacology to qualify them for prescriptive practice. The nurses emphasised the need for post-graduation training, the importance of clinical experience, and the lack of discussions and training. Only a small number of nurses self-assessed themselves as competent in prescribing drugs, and the others revealed fears of causing adverse reactions to medication.

Conclusions: There are gaps in the education, training, and daily demands of the prescription of medication by nurses in primary health care. It is suggested that prescription practices should be incorporated into undergraduate studies and continuing education in health services.

\section{Abstract \#: P 603}

Severe obese children benefit to the same extent as overweight and obese children from a long-term ambulatory interdisciplinary lifestyle intervention

J.M. Rijks ${ }^{1}$, J. Plat ${ }^{2}$, R.M. Mensink ${ }^{2}$, E. Dorenbos ${ }^{1}$, W.A. Buurman ${ }^{3}$, A.C.E. Vreugdenhil ${ }^{1}$

${ }^{1}$ Department of Paediatrics \& School of Nutrition and Translational Research in Metabolism (NUTRIM), Maastricht University Medical Centre; ${ }^{2}$ Department of Human Biology \& School of Nutrition and Translational Research in Metabolism (NUTRIM), Maastricht University; ${ }^{3}$ Department of Neuropsychology, Maastricht University Medical Centre; Corresponding author's e-mail: j.rijks@mumc.nl

Objectives: The prevalence of childhood overweight and obesity has increased rapidly worldwide. This is a major health problem since children with overweight and obesity are predisposed to significant health problems. Particularly the most severe obese children are at risk whereas lifestyle interventions often struggle to be effective in this group. Here we studied whether severe obese children can benefit equally from a long-term ambulatory interdisciplinary life style program as overweight and obese children.

Methods: 172 children and adolescents with overweight, obesity, severe obesity or morbid obesity according the International Obesity Task Force (IOTF) criteria ( $42 \%$ boys: $58 \%$ girls) were included in this non-randomized longitudinal intervention. They participated in the personalized life style intervention program of $\mathrm{COACH}$ (Centre for Overweight Adolescent and Children's Healthcare) where they received ambulatory, personalized guidance on a monthly basis by an interdisciplinary team.

Results: After 12 and 24 months de BMI z-score decreased significant with $-0.12 \pm 0.3$ and $-0.21 \pm 0.3$ respectively. Also cardiovascular risk parameters like waist circumference z-score, diastolic blood pressure, $\mathrm{HbA1c}$, total cholesterol, LDL-cholesterol and paediatric non-alcoholic fatty liver index-score were significantly improved after 1 year of lifestyle intervention. Most important, regarding long term weight loss and health benefits the intervention program was equally effective in morbid obese and severe obese children as compared with overweight and obese children.
Conclusion: Even severe obese children benefit from a long-term, ambulatory, personalized lifestyle intervention carried out by an interdisciplinary team in hospital setting and demonstrate significant weight loss and improvement of cardiovascular risk markers to the same extent as overweight and obese children.

\section{Abstract \#: P 604}

An investigation into some risk factors for oral submucous fibrosis and oral leukoplakia

$\underline{\text { Shambhavi Mishra }}^{1}$, Sumit kumar ${ }^{2}$, C.M. Pandey ${ }^{1}$, Divya Mehrotra ${ }^{2}$

${ }^{1}$ Department of Biostatistics \& Health Informatics, Sanjay Gandhi Postgraduate Institute of Medical Sciences, Lucknow, Uttar Pradesh. India; ${ }^{2}$ Department of Oral \& Maxillofacial Surgery, KG Medical University, Lucknow, Uttar Pradesh, India; Corresponding author's e-mail: chicksm19@gmail.com

Introduction: Oral submucous fibrosis (SMF) is a well-recognised, potentially malignant condition of the oral cavity that is defined as a chronic condition that can affect any part of the oral mucosa. Likewise, oral leukoplakia is one of the oral lesions with high premalignant potential. Oral SMF and oral leukoplakia are the most common types of oral precancer. WHO has defined a precancer to hold an increased risk of cancer transformation. It is reported that $25 \%$ of all cancer related deaths in India are caused due to oral cancer.

Objectives: To explore various epidemiological, social and dietary risk factors for oral submucous fibrosis and oral leukoplakia.

Data and Methods: A population based case-control study was conducted in rural and urban areas of Lucknow, the capital of most populous state, Uttar Pradesh, India. The data was collected on 3437 individuals aged 15-60 years. Out of which 448 were suffering from Oral submucous fibrosis, 128 from oral leukoplakia and 2688 were not suffering from any of these two diseases and are considered as controls for the study. Data was collected over a period of 2.5 years from April 2006 to October 2009. First univariate analysis was performed to identify factors that cause Oral submucous fibrosis and leukoplakia and later factors found significant were included in the multivariate logistic regression model. Odds ratio with $95 \%$ CI was calculated.

Results: Oral Submucous Fibrosis: Non-tobacco Areca Nut locally known as 'Sada Pan Masala' emerged as the most dominant factor affecting oral SMF. Users of sada pan masala were sixteen times more likely to develop oral SMF as compared to those not consuming. This was followed by tobacco Areca Nut (known as 'tobacco pan masala') OR 9.95 (CI: 7.67-12.90), khaini/mainpuri/surti, supari and tobacco pan. Also those eating non vegetarian food were 1.4 times more likely of developing oral SMF as compared to vegetarians.

Oral Leukoplakia: Tobacco and Non-tobacco Areca Nut (Tobacco pan masala and sada pan masala) are the two most dominant factors affecting oral leukoplakia. Those consuming sada pan masala and tobacco pan masala are seven times more likely to develop leukoplakia as compared to those not consuming these products. This was followed by khaini/mainpurti/surti OR: 3.53 (CI: 1.75-7.11) and tobacco pan.

\section{Abstract \#: P 605}

\section{Levels and sources of physical activity in german adolescents}

Maia Smith, MS ${ }^{1}$, Dietrich Berdel, MD $^{2}$, Joachim Heinrich ${ }^{1}$, Holger Schulz 
${ }^{1}$ Epidemiologie 1, Helmholtz Zentrum München, Munich, Germany; ${ }^{2}$ Department of Pediatrics, Marien-Hospital Wesel, Wesel, Germany; ${ }^{3}$ Comprehensive Pneumology Center Munich (CPC-M), Member of the German Center for Lung Research, Munich, Germany;

Corresponding author's e-mail: maia.smith@helmholtz-muenchen.de

Introduction: Physical activity (PA) is a well-known protective factor for numerous health outcomes. However, activity levels of adolescents are known to be too low. We combined accelerometers with a diary to gain insight into the relative importance of sources of objectively measured activities during school and leisure time, including school physical education (PE), transportation to school and leisure sport.

Methods: Accelerometric and diary data from 1394 adolescents ( $45 \%$ male, mean age $15.6+0.5$ years) was combined to evaluate daily levels and patterns of sedentary, light, moderate and vigorous activity, as well as moderate-to-vigorous (MVPA) in both 1 and 10-min bouts. Total activity was broken down into school (including PE) and leisure (including sport and transportation to school).

Results: About $2 / 3$ of adolescents' time is spent sedentary, $1 / 3$ in light activity, and about $5 \%$ in moderate to vigorous PA (MVPA, about $40 \mathrm{~min} /$ day on average). Boys and girls achieve $60 \mathrm{~min}$ of objectively measured MVPA on 27 and $18 \%$ of days. Roughly $20 \%$ of total MVPA takes place in continuous bouts of at least $10 \mathrm{~min}$. During school time outside PE, adolescents spent about $75 \%$ of time sedentary and $22 \%$ of time in light activity, respectively, while during leisure time, they were less sedentary ( $64 \%$ of time) and more engaged in light activity ( $31 \%$ ). School outside PE provided $17 \%$ of MVPA in 1-min bouts, transportation $7 \%$ and PE only $5 \%$. Leisure sport contributed $18 \%$ of MVPA in 1-min bouts, but $30 \%$ of 10 -min bouts. Most MVPA, particularly in short bouts, was accumulated outside of either transportation or dedicated sporting activity during leisure time.

Conclusion: German adolescents are typical of Europeans in showing low levels of activity, with significant contributions from leisure sport and transportation but relatively little from PE. Adolescents are most sedentary during school, and they are most active during episodes of leisure sport. However, frequency and duration of an activity has a greater effect on its contribution to total MVPA than does engagement level, so fostering activity during common leisure time appears to be an option to increase activity in adolescents.

\section{Abstract \#: P 606}

Prevalence and assessment of iodine deficiency disorder among the rural population in the coastal area of Villupuram (TN), South India

Senthilvel Vasudevan ${ }^{1}$, Jayanthi Sureshbabu ${ }^{2}$, Sumathi Senthilvel ${ }^{3}$

${ }^{1}$ King Saud Bin Abdulaziz University for Health Sciences;

${ }^{2}$ Pondicherry Institute of Medical Sciences; ${ }^{3}$ Formerly Lecturer in Kasturba Gandhi College of Nursing; Corresponding author's e-mail: senthilvel99@gmail.com

Background: Iodine is an essential micronutrient needed for normal human growth and development. It is required in minute quantities ranging from 100 to 150 micrograms every day. In this study, our objectives are to estimate the prevalence of goiter in the rural population, to assess the knowledge, attitude and practice regarding iodized salt among the study population and to estimate the level of iodine in salt samples at household and trader levels.

Methods: Study area: Marakkanam block of Villupuram district, Tamil Nadu, India is the field practice area of the PIMS Rural Health center, Anaichenkuppam. Study period: 1st-26th February 2012. Study design: Community-based, cross sectional study and it was a secondary data analysis. Study population: All individuals residing in the villages under the field practice area. Sample size: A total of 1233 households belonging to 8 villages and 2830 individuals were interviewed and examined for goiter with oral consent. Sampling methodology: Villages were randomly 8 were selected from the 16 villages' data collected by house-to-house survey and they were included in this present study.

Tool: pre-designed and pre-tested questionnaire. Statistical Analysis and software: mean, S.D. and proportion, Chi square test and for analysis done by SPSS 16.0 version.

Results: Among 1233 surveyed households, Hinduism was the major religion followed accounting for $85 \%$. The major sources of drinking water were bore well $(46 \%)$, piped water from the panchayat water tank $(42.6 \%)$ and hand pumps $(9.6 \%)$. Overall prevalence in goiter was $7.89 \%(\sim 8 \%)$. The prevalence increased from $2.8 \%$ in upper class to $12.4 \%$ in the lower class and it was found to be statistically significant $(p<0.001)$.

Conclusion: From this study, we concluded that only less no. of the respondents were aware that iodine deficiency caused some disease or health problem. We will eliminate IDD status of the rural community by involving Govt. and non-Govt. organizations by conducting awareness programs and IEC activities.

\section{Abstract \#: P 607}

Brazilian health system and screening programs in vulnerable population: population-based health surveys in São Paulo-Brazil Camila Nascimento Monteiro ${ }^{1}$, Sheila Rizzato Stopa ${ }^{2}$, Chester Luiz Galvão Cesar ${ }^{2}$, Marilisa Berti de Azevedo Barros ${ }^{3}$, Reinaldo José Gianini $^{1}$, Moisés Goldbaum ${ }^{1}$

${ }^{1}$ Department of Preventive Medicine, Faculty of Medicine, University of São Paulo, São Paulo, Brazil; ${ }^{2}$ Faculty of Public Health, University of São Paulo, São Paulo, Brazil; ${ }^{3}$ Faculty of Medicine, University of Campinas, Campinas, Brazil; Corresponding author's e-mail: c.nascimentomonteiro@gmail.com

Background: Screening programs among adults with diabetes and hypertension are interventions in way to promote health and living conditions in this population. Brazilian Unified Health System includes preventive screenings (for breast, prostate and cervical cancer) to identify and control possible risks, to reduce disease mortality or its possible damage, especially in vulnerable populations, such as hypertensive and diabetics.

Methods: Data from two cross-sectional population-based Health Surveys (ISA-Capital 2003 and ISA-Capital 2008), carried out by home interviews. Target population was from both sexes, 20 years old and greater, who reported suffering from diabetes mellitus and/or hypertension, which realized preventive screenings. Data analysis was carried out by comparison of prevalences at two different periods of time and their $95 \%$ confidence intervals. The difference between these two periods was considered statistically significant when there was no overlap between the confidence intervals.

Results: There was a statistically significant increase in health services utilization for preventive screenings between 2003 and 2008. In 2003, mammography proportion for diabetic and hypertensive population was 70 and $80 \%$ in $2008(p=0.022)$. In 2003, for prostate exam in hypertensive population, the proportion was 51 and $69 \%$ in $2008(p=0.013)$. However, regarding to Unified Health System coverage to perform all preventive screenings, no significant difference in the period was found. Concerning to cervical cancer, there was no significant difference in the period.

Conclusions: The increase in preventive screenings may be explained by many awareness campaigns in the period. Also, the coverage 
enlargement of Brazil's Family Health Programme may have contributed as well. Although, there was no increase in Unified Health System coverage for screening costs, which elucidates a challenge to the universality of the health system.

\section{Abstract \#: P 608}

Trends in hospitalizations for pregnancy, childbirth and the puerperium in São Paulo Brazil

Camila Nascimento Monteiro ${ }^{1}$, Bárbara Laisa Alves Moura ${ }^{1}$, Márcia Furquim de Almeida ${ }^{\mathrm{I}}$

${ }^{1}$ University of São Paulo; Corresponding author's e-mail: c.nascimentomonteiro@gmail.com

Background: Pregnancy, childbirth and the puerperium (PCP) hospitalizations represent high magnitude in public health system (Unified Health System-SUS) in São Paulo city (SP). Objective: To analyse trends in hospitalization for conditions of Chapter XV PCD of International Classification of Diseases System (ICD10).

Methods: Evaluation of trends in hospitalization of Chapter XVICD10. Data were gathered from the Hospital Information System (SIH -SUS) and the Brazilian Institute of Geography and Statistics (IBGE). Admissions for PCP of women aged 15-49 years in the period of 2003-2012. Historical series and descriptive analysis was performed. Exponential regression was employed to evaluate time trend.

Results: PCP hospitalizations represented an average $61.2 \%$ of total admissions (SD: 4.0) and there was a decrease of its hospitalization rate of $5.3 \%$ over the period. Delivery showed the highest rate of hospitalization, followed by maternal disorders, abortion, hypertensive disorders in pregnancy, respectively of: 20.2 (SD 0.9): 8.8 (SD 0.4), 4.2 (SD 0.5), 1.3 (SD 0.3)/1000 women. PCP showed an average of 3 days of hospitalization (SD 0.3). There was an annual reduction trend of hospitalization rate of PCP conditions: more than $5 \%$ for hypertensive disorders in pregnancy $\left(\mathrm{y}=1.71 \mathrm{e}-0.054 \mathrm{x} \mathrm{R}^{2} 0.60\right)$ and placenta previa $\left(y=0.25 \mathrm{e}-0.055 \mathrm{x} \mathrm{R}^{2} 0.60\right)$. Abortion complications also declined $\left(y=5.08 \mathrm{e}-0.034 \mathrm{x} \mathrm{R}^{2} 0.81\right)$. Only obstructed delivery labour showed an increase of hospitalization rate $(\mathrm{y}=0.88$ $\mathrm{e}-0.0125 \mathrm{x} \mathrm{R}^{2}$ 0.35).

Conclusions: The reduction of PCP hospitalization rate may be the result of fertility the decline in Sao Paulo City. Abortion is an illegal act in Brazil, although there was a reduction trend of its complications, this was still the third cause of hospitalization of these conditions. The maternal disorders hospitalizations reduction suggests an improvement of prenatal care in this period.

\section{Abstract \#: P 609}

\section{Self-rated health in Brazilian population: national health survey, Brazil}

$\underline{\text { Sheila Rizzato Stopa }}{ }^{1}$, Camila Nascimento Monteiro ${ }^{2}$

${ }^{1}$ Department of Epidemiology, School of Public Health - University of São Paulo. São Paulo-Brazil; ${ }^{2}$ Faculty of Medicine - University of São Paulo. São Paulo-Brazil; Corresponding author's e-mail: sheilarizzato@usp.br

Background: self-rated health has been widely used as a predictor of individual and collective welfare, also for morbidity, depression, inactivity and mortality reflecting an integrated perception of the individual, which includes a biopsychosocial dimension. The aim was to describe self-rated health in Brazilian adults (aged 18 years or older).

Methods: data from the National Health Survey-Brazil 2013, crosssectional population-based study. The proportion of self-rated health was calculated and presented by sex, age (18-29, 30-59, 60-64, 65-74, 75 years or older), schooling (no instruction level or incomplete middle school, complete middle school and incomplete high school, complete high school and incomplete undergraduate, complete undergraduate), ethnicity (white, black, brown) and macro regions (North, Northeast, West Central, Southeast, South), with a $95 \%$ confidence interval, along with the absolute values.

Results: $66.1 \%$ (CI $95 \%$ : 65.4-66.8) self-rated their health as good or very good, being higher at the Southeast region of the Brazil $71.5 \%$ (CI 95\%: 70.1-72.8) and lower at the Northeast $56.7 \%$ (CI 95 \%: 55.6-57.8). Regarding to sex, $70.3 \%$ (CI $95 \%$ : 69.3-71.2) of men considered their health good or very good, compared to $62.4 \%$ (CI $95 \%$ : 61.5-63.4) of women. Concerning age groups, these proportions varied from $81.6 \%$ (CI $95 \%$ : 80.5-82.7) for $18-29$ years and $39.7 \%$ (CI 95\%: 36.6-42.8) for people aged 75 years or older. About the schooling level, it was observed that $49.2 \%$ (CI $95 \%$ : 48.0-50.4) of people without any instruction or with incomplete primary school self-rated their health good or very good, while those with complete under graduation self-rated $84.1 \%$ (CI $95 \%$ : 82.7-85.5). Still, according to race/color skin, self-rated health as good or very good was bigger in white people $70.3 \%$ (CI 95\%: 69.4-71.3) than in black $62.0 \%$ (CI 95\%: 59.7-64.3).

Conclusions: Commitment to social welfare and health programs are necessary to improve health for those at the lowest socioeconomic segments, such as sex and schooling, in way to decrease inequalities in health.

\section{Abstract \#: P 610}

Sedentary activities in preschool children: analysis of the week and weekend screen time

$\underline{\text { Aparício, Graça }^{1}, \text { Cunha, Madalena }}{ }^{1}$, Duarte, João ${ }^{1}$

${ }^{1}$ Polytechnic Institute of Viseu, Portugal/Health School - CI\&DETS; Corresponding author's e-mail: gaparicio5@hotmail.com

Background: Watching television and other audiovisual screens may be useful but when in excess have been related to a wide range of problems in children, like sleep patterns, nutritional status, behavioral problems and/or with kids socialization. Aims: to analyze the time that preschool children spend every day watching TV or playing video games during the week and weekend.

Methods: Cross-sectional and observational study comprised of 792 preschool children, average age $=4.39$ years old $(\mathrm{SD}=0.91)$ and their parents, living in Centre of Portugal. Children's screen time classification was based on AAP recommendations.

Results: globally the average daily time watching TV during week (70.5 $\mathrm{min})$ is less than in the weekend $(127.6 \mathrm{~min})$ and much higher than the average time playing video games, during the week (42.28 $\mathrm{min})$, as in weekend $(84.8 \mathrm{~min})$. Between sexes there were significant differences only at weekend $(\mathrm{t}=2.009, p=0.044)$.

Considering the screen time average (TV and video games), it reaches a minimum of $1 \mathrm{~h}$ and maximum about $8 \mathrm{~h}$, with higher values in boys, with significance at weekend $(\mathrm{t}=1944$ : $p=0.050)$. Analyzing the weekly screen time versus the children's age, there were significant differences between 3 and 4 years and 3-5 years $(p<0.000)$, while in weekend we found that as ages increases, children spend more screen time. Considering the AAP cut-off, it was found that both during the week as in weekend most children watch TV about one 
hour a day, mainly the children with 3 years and between 1 and $2 \mathrm{~h}$ those with 5 years. The period not recommended appears in $8.5 \%$ of girls, in $11.1 \%$ in those with 4-year during the week and $34.5 \%$ of boys in weekend, with significance $(p<0.000)$. Considering the total screen time, children stay about $1 \mathrm{~h}$ front the screen during the week, and $3.4 \%$ of 4 years stay for more than two hours, while during the weekend the time rises to $15.3 \%$ children who stay more than $2 \mathrm{~h}$ front the screen, especially boys (17.1\%) and $17.4 \%$ those with 5 years, with significance to the age $\left(\chi^{2}=22.31, p=0.001\right)$.

Conclusion: although the results are less worrisome than others reported in some countries, a significant number of children spend screen time above the recommended especially at the weekend. As a public health issue, is important to promote prevention activities, because preventive care interventions in early childhood have potential for improving health outcomes.

\section{Abstract \#: P 611}

The role of school social environment on dental caries experience in 8-12 years old Brazilian children: a multilevel analysis

Maria R. Fernandez ${ }^{1}$, Marcos B. Correa ${ }^{1}$, Marília L. Goettems ${ }^{1}$, Thiago M. Ardenghi ${ }^{2}$, Flavio F. Demarco ${ }^{3}$

${ }^{1}$ Post-Graduate Program in Dentistry, Federal University of Pelotas, Pelotas, Brazil; ${ }^{2}$ Post-Graduate Program in Dentistry Science, Federal University of Santa Maria, Santa Maria, Brazil; ${ }^{3}$ Post-Graduate Programs in Dentistry and Epidemiology, Federal University of Pelotas; Corresponding author's e-mail: ffdemarco@gmail.com

Background: Although children spend most of their time involved in activities related to school, few studies have focused on the association between school social environment and oral health. This crosssectional study assessed individual and school-related social environment correlates of dental caries from Brazilian schoolchildren aged 8-12 years.

Methods: A sample of children from 20 private and public schools $(\mathrm{n}=1.211)$ was selected. Socioeconomic data were collected from parents and data regarding children characteristics were collected from children using a questionnaire. Dental examinations were performed to assess the presence of dental plaque: dental caries prevalence $(\mathrm{DMFT} \geq 1)$ and dental caries experience (mean $\mathrm{dmft}+\mathrm{DMFT}$ ). The social school environment was assessed by a questionnaire based on the National Survey of Students' Health (PeNSE) administered to schools coordinators. Data analyses were carried out in Stata 11.0 (StataCorp, College Station, TX, USA). Descriptive statistics were used to characterize the sample by outcomes and independent variables. Multilevel Poisson Regression was used to assess the association between school social environment and dental caries prevalence and experience.

Results: Dental caries prevalence was $32.4 \%$ (95 \% CI 29.7-35.2) and the mean dmft + DMFT was 1.84 ( \pm SD 2.2).. Multilevel models showed that $(\mathrm{dmft}+\mathrm{DMFT})$ and $(\mathrm{DMFT} \geq 1)$ were associated with lower maternal schooling and higher levels of dental plaque. For contextual variables, schools with sports activities out of the time were associated with lower $(\mathrm{dmft}+\mathrm{DMFT})$ and $(\mathrm{DMFT} \geq 1)$, while the occurrence of violence and theft episodes were positively associated with dental caries.

Conclusions: School social environment have an effect on prevalence and experience of dental caries in children. The results suggest that strategies focused on promotion of healthier environments should be stimulated to reduce inequalities in dental caries.

\section{Abstract \#: P 612}

\section{Preconception care: women's experiences and health behavior}

M. Poels ${ }^{1}$, MSc., Dr. H.F. van Stel $^{2}$, Dr. M.P.H. Koster ${ }^{3}$

${ }^{1} \mathrm{PhD}$ student, Department of Obstetrics and Gynaecology, UMC Utrecht, the Netherlands; ${ }^{2}$ Assistant Professor, Department of Health Technology Assessment, Julius Center UMC Utrecht, the Netherlands; ${ }^{3}$ Assistant Professor, Department of Obstetrics and Gynaeceology, UMC Utrecht, the Netherlands; Corresponding author's e-mail: m.poels@umcutrecht.nl

Background: Preconception care (PCC) includes all information and care around conception and pregnancy and has the potential to identify and reduce risk factors that can adversely affect pregnancy outcomes. The attention for PCC has grown substantially over the past decades. However, not many couples are aware of the possibility of PCC and the use of PCC remains low. The aim of this study was to assess attitudes, experiences and health behavior changes of women regarding PCC.

Methods: Participants were recruited from the one midwifery practice in the municipality Zeist, the Netherlands. A specifically developed questionnaire was sent to 455 women who delivered a live born infant in 2013.

Results: A total of 283 women (62.2\%) filled out the questionnaire. Women were mostly Dutch $(\mathrm{n}=215: 79.9 \%)$ or Moroccan $(\mathrm{n}=22$ : $8.2 \%)$. The majority had a high educational level $(\mathrm{n}=153: 60.5 \%)$. A total of 160 women $(59.3 \%)$ searched for PCC-related information before they conceived, mostly on the internet. Their main reason to gather information was to be prepared for future pregnancy $(\mathrm{n}=139$ : $86.9 \%)$. Only 68 respondents $(24.5 \%)$ consulted a healthcare provider regarding their wish to conceive. The topic most discussed was fertility $(n=46: 67.6 \%)$. Women stated that the primary reason not to attend a PCC consult was that they feel they already have sufficient knowledge.

During consults a personal advice was given in $42(61.8 \%)$ of the cases. At time of conception 39 of all respondents $(15.5 \%)$ were overweight (BMI 25-30) and $16(6.3 \%)$ were obese (BMI $>30)$. Of the women who were smokers $21(37.5 \%)$ quit smoking preconceptionally. Women who had a PCC consult were more than 12 times more likely to quit smoking. Of the women who were used to drinking alcohol, 40 (26.1\%) quit drinking before they conceived. Women who actively searched for PCC-related information were twice as likely to quit drinking. Folic acid was used by 160 respondents $(59 \%)$ during the preconceptional phase.

Conclusion: This study showed that most women search for information on PCC by themselves rather than to consult a healthcare provider. However, only a minority of women changed their health behavior preconceptionally. Women tend to primarily associate PCC with fertility issues, whilst major health benefits can be obtained by addressing lifestyle in the preconceptional phase. Therefore, more research is needed regarding barriers and facilitators for the delivery of PCC.

\section{Abstract \#: P 613}

Patterns of internet queries on skin cancer prevention along Brazilian summers: the "summer effect" exists?

Vasconcellos-Silva, P.R. ${ }^{1}$, Griep, R.H. ${ }^{1}$, Souza, M.C. ${ }^{2}$

${ }^{1}$ Oswaldo Cruz Foundation; ${ }^{2}$ National Cancer Institute - Brazil;

Corresponding author's e-mail: bioeticaunirio@yahoo.com.br 
Background: Patterns of internet queries, linked to "windows" of collective interests, have been increasingly researched in the field of health promotion. The present project describes patterns of queries related to information on skin protection and early detection of skin cancer along the summer in Brazil—what we call the "summer effect".

Methods: National Cancer Institute website, the most used source of informational resources on cancer in Brazil, was used as observation point. A log analyzer (a software which tracks websites accesses) was used along 48 months to track the accesses to prevention webpages. During 48 months the pages on skin UV protection and skin selfexamination (pages of interest) were followed.

Results: Although the average number of hits to the website has grown significantly along the period, the results of analysis of variance showed no significant differences concerning summer averages in comparison with the rest of the year $(p=0.7491)$.

Conclusion: The absence of the "summer effect" is discussed in terms of the dissonance between perception of risk (exposure to summer UV) and the lack of interest on information about prevention. The common overexposure did not raise further interest to searches on prevention and early detection of skin cancer. We discuss the "rational choice" theory and the relevance of individual knowledge about risk prevention in health promotion.

\section{Abstract \#: P 614}

Frequency of comorbidities and their association with clinical disability and relapse in multiple sclerosis

Prudence Tettey ${ }^{1}$, Dylan Siejka ${ }^{2}$, Steve Simpson ${ }^{1}$, Bruce Taylor ${ }^{1}$, Leigh Blizzard $^{1}$, Anne-Louise Ponsonby ${ }^{3}$, Terence Dwyer ${ }^{3}$, Ingrid van der $\mathrm{Mei}^{1}$

${ }^{1}$ Menzies Institute for Medical Research, Australia; ${ }^{2}$ School of Medicine, University of Tasmania, Australia; ${ }^{3}$ Murdoch Children's Research Institute, University of Melbourne, Australia;

Corresponding author's e-mail: prudence.tettey@utas.edu.au

Background: MS patients may be at an increased risk of comorbidities due to the debilitating and chronic nature of the disease.

Objectives: To investigate the frequency of comorbidities and their associations with clinical disability and relapse in MS.

Methods: A prospective cohort of 198 MS patients, followed 2002-2005, and queried about specific doctor-diagnosed comorbidities. Prevalences of comorbidities in the MS cohort were compared to the 2007 general population in Australia. Multilevel mixed-effects linear regression was used to assess the difference in disability between those who reported comorbidities and those who did not. The association with hazard of relapse was assessed using survival analysis.

Results: The age-standardised prevalences of hypertension, dyslipidaemia, asthma, psoriasis, eczema and anaemia were significantly higher in the MS cohort compared to the general Australian population. The level of disability (MSSS) in those who reported overweight/obesity ( $\beta$ : 0.76 (95\% CI: $0.04,1.48), p=0.037$ ), or dyslipidaemia ( $\beta$ : 1.05 (95\% CI: $0.07,2.02), p=0.036$ ) was significantly higher compared to those who did not report these comorbidities, even after adjustment for potential confounders. For relapse analyses, rheumatoid arthritis and anaemia were associated with more than three-fold (HR: 3.70 (95\% CI: 1.80, 7.58), $p<0.001$ ) and two-fold (HR: 2.04 (95\% CI: 1.11, 3.74), $p=0.022$ ) increased risk of subsequent relapse respectively.

Conclusions: The prevalences of some comorbidities were higher in MS patients and associated with greater disability and relapse risk.
Studies of comorbidity treatment and prevention in patients with MS have the potential to improve our understanding of the prognosis and outcomes of MS.

\section{Abstract \#: P 617}

Generation of knowledge-, attitude- and practice-scores as predictors for vaccination in the elderly

Carolina J. Klett-Tammen ${ }^{1}$, Gérard Krause ${ }^{1}$, Linda Seefeld ${ }^{2}$, Jördis J. Ott ${ }^{1}$

${ }^{1}$ Dept. of Epidemiology, Helmholtz Centre for Infection Research, Braunschweig, Germany; ${ }^{2}$ Dept. of Basic Medical Issues: Preventive and Medical Activities in Health Education, Federal Centre for Health Education, Köln, Germany; Corresponding author's e-mail: Carolina.Klett-Tammen@helmholtz-hzi.de

Background: Many cases of hospitalization and death among elderly are caused by vaccine-preventable diseases. Given the relatively low vaccination coverage in these age-groups for recommended vaccinations, information on determinants of influenza, pneumococci and tetanus vaccine uptake is crucial. Until now, only few studies have generated scores applying statistical methods to provide concise public health relevant information. The aim of this analysis is to identify areas of knowledge, attitude and practice (KAP) which are of relevance for prioritizing public health actions regarding vaccination in the elderly.

Methods: A KAP-survey on infection prevention, including vaccination was conducted among a representative sample of the German non-institutionalized population aged 60-85 years. Data from 1.223 questionnaires were analyzed. In addition to the analysis of sociodemographic factors impacting on vaccine uptake, factor analyses were applied to generate a knowledge-, an attitude- and a practicescore. Each score was dichotomized and logistic regression was used to assess the extent to which each score predicts uptake of the influenza-, pneumococcal- and tetanus-vaccination, respectively. Further analysis was carried out to evaluate influences of the knowledge- and the attitude-score on the practice score and thereby assessed how vaccination-related knowledge and attitude result in concrete vaccination-related behavior.

Results: For each of the three vaccines analyzed, the practice-score was most predictive indicating that other vaccination-related behavior such as possession of a vaccination record and utilization of vaccination counseling is a good predictor for the uptake of specific vaccinations. A better knowledge was associated with the uptake of the influenza $(\mathrm{OR}=1.3)$ and the pneumococcal vaccination $(\mathrm{OR}=1.2)$, while only for the influenza vaccination a more positive attitude increased vaccine utilization $(\mathrm{OR}=1.1)$. A better knowledge on vaccination raised the chance to have a higher practice-score $(\mathrm{OR}=4.8)$ : a more positive attitude implied a chance twice as high to perform more vaccination-related practices.

Conclusion: The high predictability of the practice-score for three vaccinations considered implies that actual behavior has a greater impact on vaccine uptake than knowledge and attitude. Not knowledge on a specific vaccine but rather an elevated general awareness of vaccine recommendations might be the main determinant for using a specific vaccine. Public health agencies may therefore focus rather on communicating general knowledge and providing directions on where to access this information. Including physicians in further research studies and providing hints for target-group specific vaccination counseling can lead to evidence-based and appropriate utilization of vaccination in groups of potential risk such as the elderly. 


\section{Abstract \#: P 619}

Race and sex differences in the role of occupational cognitive requirements on late-life cognitive aging

Lindsay R. Pool ${ }^{1}$, Jennifer Weuve ${ }^{2}$, Robert S. Wilson ${ }^{3}$, Ute Bültmann ${ }^{4}$, Denis A. Evans ${ }^{2}$, Carlos F. Mendes de Leon ${ }^{1}$

${ }^{1}$ Center for Social Epidemiology and Population Health, Department of Epidemiology, University of Michigan School of Public Health, Ann Arbor, MI, USA; ${ }^{2}$ Rush Institute for Healthy Aging, Department of Internal Medicine, Rush University Medical Center, Chicago, IL, USA; ${ }^{3}$ Rush Alzheimer's Disease Center, Department of Behavioral Science, Rush University Medical Center, Chicago, IL, USA;

${ }^{4}$ Department of Health Sciences, Community and Occupational Medicine, University Medical Center Groningen, The Netherlands; Corresponding author's e-mail: lpool@umich.edu

Background: There is increased attention on the degree to which cognitive activity throughout adulthood affects late-life cognitive aging, particularly through occupationally-related cognitive stimulation. In this analysis, we focus on occupational cognitive requirements as marker of adulthood cognitive activity, with particular attention to how race and sex may lead to systematic differences in occupational characteristics.

Methods: Occupational cognitive requirements were measured using main lifetime occupation information for 7637 participants aged $>65$ years of the Chicago Health and Aging Project (CHAP). This information was linked with standardized data on worker attributes and job characteristics from the Occupational Information Network $\left(\mathrm{O}^{*} \mathrm{NET}\right)$. Ratings of cognitive processes required in 10 work-related tasks were used to create a summary measure of occupational cognitive requirements (range 0-7). Linear mixed models were used to estimate the association between the occupational cognitive requirements score and a global measure of cognitive function (z-score). For race (black/white) and sex (female/male), two-way interactions with the occupational cognitive requirements score and three-way interactions with the occupational cognitive requirements score and time were fit to estimate any differential association with cognitive aging. Results: In the sample, $62.5 \%$ were female and $64 \%$ were black. The average occupational cognitive requirements score was 2.2 (SD 0.9). In adjusted analyses, the occupational cognitive requirements score showed a curvilinear association with late-life cognition at baseline, including a strong linear association $(\beta=0.17$ : $p<0.0001)$ and a weaker negative quadratic association $(\beta=-0.03$ : $p<0.0001)$. Higher occupational cognitive requirements score was associated with slower decline in global cognitive function over time $(\beta=0.006: p=0.004)$. Black race was associated with lower cognition at baseline $(\beta=-0.39$ : $p<0.0001)$, but this association was attenuated by 0.04 for each increase in level occupational cognitive requirements $(p=0.0193)$. Black race was associated with slower decline in cognitive function for lower levels of occupational cognitive requirements, but was associated with faster decline for higher levels of occupational requirements. Female sex was associated with higher cognitive function at baseline $(\beta=0.095$ : $p=0.0160)$, but was not significantly associated with differential decline in cognitive function over time nor did these effects differ by levels of occupational cognitive requirements.

Conclusion: Findings suggest that occupational cognitive requirements are associated with better cognition and a slower rate of cognitive decline in older age, but black race may modify the association between occupational cognitive requirements and cognitive aging. Therefore, adulthood cognitive activity may contribute to cognitive reserve in late life, with differing effects based on social context.

\section{Abstract \#: P 620}

Epidemiologic profile of children attended the reception with risk classification of an emergency hospital

Aline Santana Macedo ${ }^{1}$, Bianka Sousa Martins Silva ${ }^{2}$, Carolina Vinhas Andrade Pacheco Silva ${ }^{1}$, Izabel Laís de Oliveira ${ }^{1}$, Taiana Silva Ramos ${ }^{1}$

${ }^{1}$ Universidade Estadual de Feira de Santana; ${ }^{2}$ Faculdade Anísio Teixeira; Corresponding author's e-mail: biankabio@bol.com.br

Whereas health services should be organized hierarchically in three levels of attention aimed at comprehensiveness of care, this study aims to evaluate the profile of children treated at Home with Risk Rating of a hospital emergency unit. This is a descriptive study with a quantitative approach. Data collection was conducted through secondary data in a computerized system of the service itself. We conducted a descriptive analysis and it was observed that the users rated as somewhat urgent and non-urgent calls correspond to $64 \%$ in this emergency. The pain and other nonspecific symptoms followed by respiratory disorders represent 45 and $13 \%$ of complaints, respectively, and $17 \%$ of calls were from surrounding municipalities. The study indicates that this service receives considerable contingent resolvable with complaints in primary care. It is considered that the fragility of primary care involves issues of political, technical, and management of these services, which reinforce common sense, which privileges the hospital and plays the biomedical model of care centered on complaints conduct, low impact indicators of population health.

\section{Abstract \#: P 621}

Accumulation of adverse childhood events and overweight in childhood-a systematic review

Leonie K. Elsenburg ${ }^{1,2}$, Kim J. E. van Wijk ${ }^{2,3}$, Ronald P. Stolk ${ }^{1}$, Aart C. Liefbroer ${ }^{2,3,4}$, Nynke Smidt ${ }^{1}$

${ }^{1}$ Department of Epidemiology, University of Groningen, University Medical Center Groningen, Groningen, the Netherlands; ${ }^{2}$ Netherlands Interdisciplinary Demographic Institute (NIDI-KNAW), the Hague, the Netherlands; ${ }^{3}$ University of Groningen (RUG), University Medical Center Groningen, Groningen, the Netherlands; ${ }^{4}$ Faculty of Social Sciences, VU University Amsterdam, the Netherlands; Corresponding author's e-mail: elsenburg@nidi.nl

Background: Overweight and obese children have an increased risk of overweight and obesity in adulthood and this is associated with increased morbidity and mortality later in life. Adverse events during childhood, such as child maltreatment, diseases or death of a family member and parental divorce, can threaten the individual's mental and physical well-being. However, evidence for a relationship between adverse childhood events and overweight in children is still inconclusive and a comprehensive systematic review investigating the relation between adverse childhood events and overweight in childhood is currently lacking. Therefore, we conducted a systematic review in which we studied whether accumulation of adverse childhood events is related to overweight and obesity in childhood.

Methods: PubMed, EMBASE, PsycINFO and CINAHL were searched. Search terms consisted of medical subject headings (MeSH) and keywords related to (1) life events, adversities and stress, (2) (childhood) overweight/obesity, (3) children, adolescents, teens and youth. Included studies consisted of cross-sectional, longitudinal and casecontrol studies that investigated the relation between accumulated 
adverse childhood events and overweight in children $\leq 18$ years, presented the data in a quantitative way and were published as a full-text English paper in a peer-reviewed journal. Selection, data-extraction and quality assessment of the included papers were done by two reviewers independently. References of all included studies were also screened for eligibility. A narrative synthesis of the relation between accumulation of adverse events and overweight in childhood will be performed. If possible, subgroup analyses will be conducted for: type of study design (cross-sectional, longitudinal or case-control), overweight measure (e.g. body mass index, waist-to-hip ratio, waist circumference), age of participants, measure of cumulative adversity, and follow-up period.

Results: The search identified 3948 hits, of which 35 papers were identified as eligible. Currently, we are conducting the data-extraction and quality assessment of all eligible papers. The results of this systematic review will be presented at the conference.

\section{Abstract \#: P 622}

\section{Interaction of unexpected pregnancy and young motherhood} predict infant abuse: a prospective study in Japan

\section{Takeo Fujiwara $^{1}$}

${ }^{1}$ Department of Social Medicine, National Research Institute for Child Health and Development, Tokyo, Japan; Corresponding author's e-mail: fujiwara-tk@ncchd.go.jp

Background: Unexpected pregnancy and young motherhood can be a risk factor for infant abuse: however, few studies investigate whether interaction of unexpected pregnancy and younger motherhood increases the risk of infant abuse. The aim of this study is to investigate whether unexpected pregnancy and younger motherhood predicts infant abuse.

Method: Questionnaire inquiring on infant abuse, defined as shaking and smothering at least once during past month, was disseminated at 4-month-old health checkup at 11 municipalities in Aichi prefecture, Japan (valid response $\mathrm{N}=6358$ ). Then, the data collected at registration of pregnancy, including maternal age and feeling on her pregnancy were linked with the questionnaire response (linked $\mathrm{N}=6056$ ). Logistic regression was employed to see the association between 4 categorization (young motherhood (defined as less than 25 years old) or not and unexpected pregnancy or not) and infant abuse, adjusted for covariates (parity, gestational week at registration, whether returning to maternal house of origin to give birth, and depressive symptom).

Result: At 4-month-old health checkup, 193 (3.2\%) mothers reported infant abuse. In crude model, younger motherhood with unexpected pregnancy 2.87 (95\% confidence interval (CI): 1.61-5.11) times more likely abuse their infants than older mother with expected pregnancy. Moreover, younger motherhood with expected pregnancy showed no higher risk on infant abuse than older mother with expected pregnancy. After adjusted for covariates, young motherhood with unexpected pregnancy remained to show significant association with infant abuse (odds ratio: 2.28, $95 \%$ CI: $1.26-4.12$ ).

Conclusion: Unexpected pregnancy among younger motherhood predicted infant abuse. The current finding should be used to identify high-risk mothers on infant abuse.

\section{Abstract \#: P 623}

\section{Chronic kidney disease in free-living Brazilian adults. ELSA-Brasil}

Sandhi M Barreto ${ }^{1}$, Roberto M. Ladeira ${ }^{1}$, Bruce B. Duncan ${ }^{2}$, Maria Ines Schmidt $^{2}$, Antonio Lopes ${ }^{3}$, Isabela Bensenor ${ }^{4}$, Dora Chor ${ }^{5}$, Rosane Griep ${ }^{5}$, Pedro G. Vidigal ${ }^{1}$, Antonio P. Ribeiro ${ }^{1}$, Jose G. Mill ${ }^{6}$
${ }^{1}$ Universidade Federal de Minas Gerais; ${ }^{2}$ Universidade Federal do Rio Grande do Sul; ${ }^{3}$ Universidade Federal da Bahia; ${ }^{4}$ Universidade de São Paulo; ${ }^{5}$ Fundação Oswaldo Cruz; ${ }^{6}$ Universidade Federal do Espírito Santo; Corresponding author's e-mail: sandhi.barreto@gmail.com

Background: Chronic Kidney Disease (CKD) is one of the major public health problems around the world. However, data on CKD prevalence are sparse in Latin American countries.

Methods: Analysis of data from the baseline (2008-2010) examination of the Brazilian Longitudinal Study of Adult Health (ELSABrasil) consisting of 14,636 public sector employees from six Brazilian capitals aged 35-74 years. CKD defined as the presence of albuminuria as increased $(>30 \mathrm{mg} / \mathrm{g})$ urinary albumin-creatinine ratio (ACR) or decreased estimated glomerular filtration rate (GFR) $<60 \mathrm{~mL} / \mathrm{min} / 1.73 \mathrm{~m}^{2}$. GFR was estimated by the CKD-EPI equation without correction for skin color. Multiple logistic regression was used to investigate the association of education and race/skin color with CKD after considering the effect of age, sex, smoking, diabetic and hypertension status, history of cardiovascular disease, obesity, total to HDL cholesterol ratio and uric acid.

Results: According to KDIGO criteria, the overall prevalence of CKD was $9.0 \%$, being significantly higher in men, older people and in black participants. ACR, in isolated form, detected $50 \%$ more CKD in the 65-74 year-olds compared to the 35-44 year-olds. On the other hand, isolated eGFR detected over 20 times more CKD. After adjustments, being black (OR, 1.23: $95 \%$ CI, 1.03-1.47) or brown (OR, 1.19: $95 \%$ CI, 1.02-1.38) or Indigenous (OR, 1.90: $95 \%$ CI, 1.18-3.06) and having lower education level (OR, 1.21: $95 \% \mathrm{CI}$, 1.01-1.44) remained statistically associated with greater chances of CKD.

Conclusions: We found a high prevalence of CKD in this large cohort of free living Brazilians, especially among black and indigenous as well as lower educated individuals. There was marked discrepancies in the increases of eGFR and ACR by age. The health related factors considered in this analysis did not explain the race/skin color and educational disparities found in CKD prevalence and need further investigation.

\section{Abstract \#: P 624}

Regional deprivation and computed tomography use in pediatric patients in Germany

Steffen Dreger ${ }^{1}$, Lucian Krille ${ }^{2}$, Werner Maier $^{3}$, Roman Pokora ${ }^{4}$, $\overline{\text { Maria Blettner }}^{4}$, Hajo Zeeb ${ }^{1}$

${ }^{1}$ Leibniz Institute for Prevention Research and Epidemiology - BIPS; ${ }^{2}$ Institute of Medical Biostatistics, Epidemiology and Informatics, University Medical Center Mainz: International Agency for Research on Cancer; ${ }^{3}$ Helmholtz Zentrum München, German Research Center for Environmental Health; ${ }^{4}$ Institute of Medical Biostatistics, Epidemiology and Informatics, University Medical Center Mainz; Corresponding author's e-mail: dreger@bips.uni-bremen.de

Background: Conflicting findings were observed in recent studies assessing the association between patients' socio-economic status (SES) and the received number of computed tomography examinations. The aim was to investigate SES and variation in CT examination practice for pediatric patients in Germany.

Methods: As part of large epidemiological study, this sub-analysis used data from Radiology Information Systems for children aged 0-14.5 years without cancer who had at least one CT examination between 2001 and 2010 which were extracted in 20 hospitals across Germany. The small-area German Index of Multiple Deprivation 
(GIMD) was used to assess deprivation. The GIMD scores were classified into least, medium and most deprived areas based on the frequency distribution of all German municipalities. The respective GIMD category was assigned to each patient by linking their residential postal code at time of last known CT to the municipal level. A multinomial logistic regression model was used to assess the association of GIMD with patients' CT examination numbers $(1,2-3,4-5$, $6+$ CTs).

Results: A total of 37,810 pediatric patients received 59,572 CT scans during the study period. 27,287 (72\%) children received only one CT examination, while $\mathrm{n}=885(2.3 \%)$ received six or more. Patients living in most deprived areas had higher odds of having more than one CT examination compared to patients from least deprived areas, which was similar for higher CT examination numbers: '2-3 CT' odds ratio $(\mathrm{OR})=1.45$, $95 \%$ CI: 1.40-1.50: '4-5 CT' $\mathrm{OR}=1.48,95 \% \mathrm{CI}: 1.38-1.59$ : ' $6+\mathrm{CT}$ ' $\mathrm{OR}=1.54,95 \% \mathrm{CI}$ : 1.41-1.69. Males had a higher chance of receiving more CT examinations than females ('4-5 CT': OR $=1.13,95 \% \mathrm{CI}$ : $1.07-1.19$ ). Additional stratified analysis of cancer patients who received either diagnostic or treatment-related CT examinations showed diverging results.

Conclusion: The observed results in our study are in line with research results from the UK and the Netherlands. Limitations of the ecological approach and the lack of differentiation of CT details have to be acknowledged. More research is therefore needed to identify other potential reasons for CT examinations, e.g. specific indications and other contextual measures such as the rate of traffic accidents which may explain the differences in CT examinations.

\section{Abstract \#: P 625}

\section{Associations of life course socioeconomic position and job stress with carotid intima-media thickness. The Brazilian longitudinal study of adult health (ELSA-Brasil)}

Lidyane V. Camelo ${ }^{1}$, Luana Giatti ${ }^{2}$, Dóra Chor $^{3}$, Rosane Härter Griep $^{4}$, Isabela M. Benseñor ${ }^{5}$, Itamar S. Santos ${ }^{6}$, Ichiro Kawachi ${ }^{7}$, Sandhi Maria Barreto ${ }^{1}$

${ }^{1}$ Postgraduate Program in Public Health, Faculty of Medicine, Universidade Federal de Minas Gerais ${ }^{2}$ School of Nutrition, Universidade Federal de Ouro Preto; ${ }^{3}$ Escola Nacional de Saúde Pública, Fundação Oswaldo Cruz; ${ }^{4}$ Laboratory of Health and Environment Education, Fundação Oswaldo Cruz; ${ }^{5}$ Center for Clinical and Epidemiologic Research, Hospital Universitário, Universidade de São Paulo; ${ }^{6}$ Internal Medicine Department, School of Medicine, Universidade de São Paulo; ${ }^{7}$ Department of Social and Behavioral Sciences, Harvard School of Public Health; Corresponding author's e-mail: lidyanecamelo@gmail.com

Background: Our aims were to examine whether life course socioeconomic position (SEP) is associated with carotid intima-media thickness (IMT), and we investigated whether this association is partially mediated by job stress.

Methods: We use data from the baseline (2008-2010) of 8830 current workers from the Brazilian Longitudinal Study of Adult Health (ELSA-Brasil), a multicenter prospective cohort study of civil servants. Subclinical atherosclerosis was assessed by carotid intimamedia thickness (IMT) and it was defined as the mean of the right and left carotids mean values (mean-mean). Maternal education, social class of first occupation, and occupational social class were used to measure childhood, youth and adulthood SEP, respectively. Job stress was assessed by the Brazilian version of the Swedish Demand-Control-Support Questionnaire. We evaluated the job strain following the Karasek's model and the job control was also analyzed separately, categorized according to tertiles. Directed acyclic graph, and linear regression models were used.

Results: In the minimally adjusted model, maternal education was not associated with IMT in men, but low versus high maternal education was associated with higher IMT in the order of $0.022 \mathrm{~mm}(p<0.01)$ in women. Compared with participants with high social class of first occupation, men and women with low social class of first occupation presented $0.031 \mathrm{~mm}(p<0.01)$ and $0.015 \mathrm{~mm}(p<0.05)$ of increase in IMT, respectively. Low versus high social class of current occupation was associated with greater IMT in the order of $0.037 \mathrm{~mm}$ $(p<0.001)$ in men and $0.039 \mathrm{~mm}(p<0.001)$ in women. The simultaneous adjustment for all SEP indicators showed that childhood SEP did not remain statistically associated to IMT in women, and youth SEP persisted borderline associated with IMT only among men $(p=0.04)$. However, adulthood SEP continued statistically associated with IMT in a dose-response manner in men and women. Job stress did not explain the association between SEP and IMT, since job strain and low job control were not associated with IMT independently of SEP.

Conclusion: Exposure to social disadvantage in adulthood was consistently associated with higher IMT in both genders, and the same was observed with youth SEP among men. However, childhood SEP was associated with higher IMT only among women and this association disappeared after adjustment for youth and adulthood SEP. Both measures of job stress failed to explain the higher IMT among participants with social disadvantage over the life span, suggesting that strategies to address socioeconomic inequalities in CVD should target additional steps besides reducing job stress.

\section{Abstract \#: P 626}

Inclusion of immigrants and ethnic minorities in European birth cohort studies-a review

Angelique Grosser $^{1}$, Tanja Vrijkotte ${ }^{2}$, Ina Hinz ${ }^{1}$, Oliver Razum ${ }^{1}$, Jacob Spallek $^{1}$

${ }^{1}$ Department of Epidemiology and International Public Health, School of Public Health, Bielefeld University; ${ }^{2}$ Department of Social Medicine, Academic Medical Center, Amsterdam; Corresponding author's e-mail: angelique.grosser@uni-bielefeld.de

Background: Europe hosts increasing numbers of immigrants and members of ethnic minority groups. They may experience health disadvantages relative to the majority populations of host countries. Birth cohort studies can help to disentangle factors producing health inequalities over the life course. This review assesses how birth cohorts in European countries i) consider migration history and ethnicity in their study design and ii) use this information in data analysis.

Methods: Birth cohort studies were identified via searches of MEDLINE and of three web-based registries of mother and child cohorts (e.g. www.birthcohorts.net.). Two reviewers systematically searched all birth cohort studies to identify those fulfilling a priori defined inclusion criteria. Where applicable, data on the inclusion of migrants and ethnic minorities in study design and data analysis was extracted.

Results: Eighty-eight European birth cohorts were included, totalling more than 380,000 children in 21 different European countries. Four birth cohort studies $(4.5 \%)$ explicitly aimed to investigate the development of health inequalities in populations with ethnic minorities or immigrants. Six birth cohorts $(7 \%)$ provided study materials in languages other than the national language, and 49 cohorts $(56 \%)$ collected some information on migration history or ethnic background of study participants. As for data analysis, 30 birth 
cohorts (34\%) stated the proportion of migrants among study participants (ranging from 2 up to $60 \%$ ), half of which $(16,18 \%$ ) could also provide this information stratified for specific migrant or ethnic groups. Ten birth cohort studies $(11 \%)$ have used information on migration history or ethnicity for comparative analyses or plan to do so in future.

Conclusion: The majority of birth cohorts assessed participants? Migration history or ethnic background in some detail: however, information on the proportion of immigrant or ethnic group members, and how this information is used for comparative analyses, is still scarce. Performing birth cohort studies explicitly aiming to investigate health inequalities in populations with ethnic minorities or immigrants remain rare though desirable. Birth cohort studies should routinely include and possibly oversample migrant or ethnic groups, actively enable their participation and conduct comparative analyses.

\section{Abstract \#: P 627}

Use of healthcare services by immigrant in Italy: first results from Italian National Institute of Statistics (ISTAT) National Survey

Alessio Petrelli ${ }^{1}$, Antonella Sferrazza ${ }^{1}$, Lidia Gargiulo ${ }^{2}$, Laura Iannucci $^{2}$, Gianfranco Costanzo ${ }^{1}$, Concetta Mirisola ${ }^{1}$

${ }^{1}$ National Institute for Health, Migration and Poverty (INMP); ${ }^{2}$ ISTAT; Corresponding author's e-mail: petrelli@inmp.it

Background: The better health status of immigrants compared with the native population is well known. Nevertheless, poor information is available about presence and strength of barriers to access healthcare services.

Methods: The study was conducted on data from the ISTAT survey on "Health status and use of health care services" $(49,811$ families and 119,073 individuals representative of the resident Italian population). For the purposes of this study, the questionnaire collected information on: perceived health status: chronic morbidity, socioeconomic conditions, use of health care services.

A synthetic indicator of the use of health care services was used (IHS). It aggregates information on hospital admissions, surgery, specialist examinations, outpatient diagnostics, medicines, rehabilitation and primary health care provided in the last 12 months. The indicator is based on a continuous scale and is organized into 4 hierarchic levels (no access, and three categories based on distribution tertiles).

Ordinal multivariate logistic models were created by using IHS as dependent variable and citizenship (foreigners/Italians) as determinant. Models were progressively adjusted for age (6 classes), gender, perceived health status (very good/good, fair/bad/very bad), geographical area (North, Centre, South and islands of Italy), educational level (high, medium, low).

Results: Out of the total sample, $7.8 \%$ are foreigners (of which $3 \%$ are from developed countries): $48 \%$ are males, median age is 44.5 (SD: 23.3) for Italians and 31.2 for foreigners (SD: 17.6). $36.5 \%$ of the sample has a high educational level (37\% among Italians and $32 \%$ among foreigners).

$71 \%$ declare they have excellent or very good health $(84 \%$ among foreigners).

Out of total foreigners, $55 \%$ have not accessed health care services in the last 12 months (37\% among Italians), whereas $9 \%$ result to be great consumers ( $21 \%$ among Italians).

After covariates adjustment, the results of the logistic model show that the probability of high use of services by foreigners is 0.63 (IC $95 \%$ : 0.60-0.66). No statistically significant differences are observed for geographical area.
Conclusion: After taken into account the perceived health status, foreigners use healthcare services $37 \%$ less than Italians. In order to assess the presence of possible barriers to access health care, the causes of such a difference must be identified.

\section{Abstract \#: P 628}

Risk factors for violence against women: a multicenter casecontrol study in emergency services in Brazil

Leila Posenato Garcia $^{1}$, Elisabeth Carmem Duarte ${ }^{2}$, Lúcia Rolim Santana de Freitas ${ }^{2}$, Gabriela Drummond Marques da Silva ${ }^{2}$

${ }^{1}$ Institute for Applied Economic Research; ${ }^{2}$ University of Brasilia; Corresponding author's e-mail: leila.garcia@ipea.gov.br

Background: Violence against women is an important public health problem in Brazil. This study aimed to investigate risk factors for violence against women among victims treated in emergency services in Brazil.

Methods: This is a multicenter case-control study conducted with data from the Brazilian Surveillance System for Violence and Accidents (VIVA). This study included data collected in 69 sentinel emergency services, located in 25 capitals from all the country's regions, in 2011. Women with 18 years-of-age or more that were treated in the study centers were included in the sample. Cases were defined as female victims of violence perpetrated by a partner, expartner, family member, friend or acquaintance. Controls were defined as women who suffered other types of injuries, including traffic accidents, falls and burns. Crude and adjusted odds ratios (OR), and $95 \%$ confidence intervals (95\% CI) were estimated through nonconditional logistic regression.

Results: The analysis included overall 10.743 women, 623 cases and 10,120 controls. In the adjusted analysis, risk factors for violence against women were: younger age (18-29 years OR 1.00: 30-44 years OR 0.79 IC $95 \%$ 0.64-0.97: 45-59 OR 0.41 IC $95 \%$ 0.31-0.54: 60 years and over OR 0.13 IC $95 \% 0.08-0.21$ ), lower education (OR 1.96, IC $95 \%$ 1.62-2.37), not having a paid job (OR 1.34 , IC $95 \% 1.12-1.61$ ), alcohol consumption by the victim in the $6 \mathrm{~h}$ previous to the episode that prompted care seeking (OR 4.49, IC $95 \% 3.59-5.59)$, having previously sought treatment for the same reason in other service (OR 1.28, IC $95 \%$ 1.05-1.57), occurrence during weekends compared to weekdays (OR 1.33, IC $95 \%$ 1.10-1.59), and occurrence from 6 p.m. to 12 p.m. (OR 2.02, IC $95 \%$ 1.57-2.61) or 12 p.m. to 6 a.m. (OR 2.82, IC $95 \% 2.06$ a 3.88), compared to 6 a.m. to 12 a.m.

Conclusion: This study reinforces that violence against women is a multifactorial problem and provides important information for policies regarding the care of the victims. The results indicate that the services for the victims should be available during nights and weekends, not only because those are the periods of higher demand, but also because having previously sought treatment for the same reason in other service was an associated factor, what could indicate the unavailability of inability of services to provide care for the victims. Other risk factors included alcohol consumption by the victim, younger age and lower socioeconomic status, which should be considered by healthcare workers in the approach of suspected cases of violence against women.

\section{Abstract \#: P 630}

Inequalities in coronary heart disease risk by education and area deprivation among 1.2 million UK women: prospective study 
Sarah Floud $^{1}$, Angela Balkwill ${ }^{1}$, Kath Moser $^{1}$, Valerie Beral ${ }^{1}$, Benjamin J. Cairns ${ }^{1}$

${ }^{1}$ Nuffield Dept of Population Health, University of Oxford, Oxford, Oxfordshire; Corresponding author's e-mail:

sarah.floud@ceu.ox.ac.uk

Background: Educational inequalities in coronary heart disease (CHD) risk have been reported for women. Inequalities in relation to area deprivation have also been reported, even after accounting for individual socio-economic status. However, this evidence has mainly come from US and Scandinavian studies, with limited evidence from the UK. We have investigated the association between education and area deprivation and risk of CHD in UK women.

Methods: After excluding women with heart disease, stroke or cancer at recruitment, a total of 1,202,983 middle-aged women from the prospective Million Women Study were followed for CHD hospital admissions and deaths. Cox regression models were used to calculate relative risks (RRs) and $95 \%$ confidence intervals (CI) for first CHD events (hospital admission or death) and for CHD mortality, according to education and area deprivation. The highest achieved educational qualification was reported at recruitment. Area deprivation at recruitment was quantified by quintiles of the Townsend score. We also assessed the proportion of the association that could be explained by smoking, body mass index, physical activity and alcohol intake.

Results: Thirteen percent of women had tertiary educational qualifications, $26 \%$ had secondary qualifications, $17 \%$ had technical qualifications, $41 \%$ had no qualifications but completed compulsory schooling, and $2 \%$ did not complete compulsory schooling. During 12 years of follow-up, 72,070 women had a first CHD event and, overall, 6242 died from CHD. Lower levels of education were associated with higher risks of CHD $(P$ heterogeneity $<0.0001$ for first events and for mortality). For example, women with no qualifications who had completed compulsory schooling had almost twice the risk of a first CHD event as women with tertiary qualifications (RR adjusted only for age and region $=1.92,95 \%$ CI 1.86-1.97). Risks of CHD also increased as the level of deprivation increased ( $P$ heterogeneity $<0.0001$ for first events and for mortality). An effect of education on CHD risk was seen within every level of deprivation and an effect of deprivation was seen within every level of education. The risk estimates were substantially reduced with additional adjustment for smoking, body mass index, physical activity and alcohol intake, with these four factors accounting for most (70-85\%) of the associations seen when adjusting for age and region of residence only.

Conclusion: Education and area deprivation appear to act independently of each other in their association with CHD risk: however a large proportion of these associations are explained by some modifiable risk factors.

\section{Abstract \#: P 633}

Factors associated with the occurrence of gender violence

Bianka Sousa Martins Silva ${ }^{1}$, Maura Maria Magalhães ${ }^{1}$, Tânia Maria Araújo

${ }^{1}$ Universidade Estadual de Feira de Santana; Corresponding author's e-mail: biankabio@bol.com.br

Objective: To assess factors related to the occurrence of gender violence in a population of northeastern Bahia in 2007.

Methods: This cross-sectional study was conducted in Feira de Santana, Bahia a probabilistic sample of clusters derived from census tracts being used. Participants 4170 individuals, of both sexes, aged
15 and more. Data were collected in semi-structured questionnaire with home visits and home record. To verify the factors associated with violence, we used a hierarchical logistic regression analysis.

Results: The prevalence of physical and/or emotional violence was $18.63 \%$. Women showed a higher frequency $(19.7 \%)$ than men $(16.5 \%)$ with 1.2 times higher prevalence of victimization. Adjusted by hierarchical logistic regression analysis showed a positive association between women suffer physical and/or emotional violence with age (RP $=1.23$ : IC 95\%: 1.01: 1.50), household type $(\mathrm{RP}=1.28:$ IC $95 \%: 1.10: 1.54)$, type of building $(\mathrm{RP}=1.66:$ IC $95 \%: 1.14: 2.41)$, smoking (RP $=1.36:$ IC $95 \%: 1.10: 1.70)$ and violence in childhood ( $\mathrm{RP}=2.13$ : IC $95 \%: 1.79: 2.53)$.

Conclusions: The data revealed that young women aged between 15 and 49 years, nonwhite, smokers, alcoholics, living in rented houses or transferred with a history of violence against children are the worst victims of physical and/or emotional. Thus, the study reiterates the need to deepen the understanding of social determinants and interaction with the phenomenon of violence.

\section{Abstract \#: P 634}

Factors associated with the occurrence of leishmaniasis in children of a specialized hospital fair Santana

Simara Rubens Pereira ${ }^{1}$, Maria Margarete Brito Martins ${ }^{1}$, $\underline{\text { Bianka Sousa Martins Silva }}^{1}$

${ }^{1}$ Faculdade Anísio Teixeira; Corresponding author's e-mail: biankabio@bol.com.br

Objective: To describe the demographic profile of children with visceral leishmaniasis (VL) of a state hospital in Feira de Santana, Bahia in the period 2008-2011.

Methods: This was a retrospective study in a specialized hospital in Feira de Santana, BA. The study subjects were children younger than 12 years diagnosed with LV totaling 12 individuals of both sexes. Data were collected from medical records through proper form containing socioeconomic, family and demographic information. A descriptive analysis was performed by calculating the absolute and relative frequencies.

Results: About $58.3 \%$ of children were from families with lower socioeconomic status and less than a minimum wage: $58 \%$ were from rural areas and $50 \%$ lived in places with no infrastructure: $33 \%$ had piped water and $17 \%$ sewage. The largest number of cases was in children aged $0-5$ years being the most prevalent female $(83 \%)$. The research showed that $83 \%$ of families had no knowledge of the disease.

Conclusion: The implementation of actions for interventions and health education, along with health team approach to conditioning and determining the factors that lead the individual to acquire the disease is necessary.

\section{Abstract \#: P 635}

Educational, occupational, and income inequalities in a longitudinal measurement of successful aging

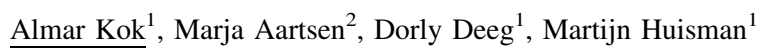

${ }^{1}$ VU University Medical Center Amsterdam, Netherlands; ${ }^{2} \mathrm{VU}$ University Amsterdam, Netherlands; Corresponding author's e-mail: a.kok1@vumc.nl

Background: The consequences of low socioeconomic position for health and functioning in old age are a-specific, operating through 
many pathways towards multiple health outcomes. Since the concept of successful aging typically includes a combination of physical, cognitive, and psychosocial health, it may represent a key outcome in relation to which socioeconomic disparities in the quality of aging can be studied.

Methods: A Successful Aging Index was calculated which included nine domains of physical, cognitive, emotional and social functioning. Based on 16-year longitudinal data from 2095 participants aged 55-85 in 1992 in the Longitudinal Aging Study Amsterdam, Latent Class Growth Analysis distinguished subgroups of older adults with similar trajectories for each of the nine domains. The Successful Aging Index expressed the number of domains (range 0-9) for which each respondent showed a favorable ('successful') trajectory. Regression analyses were used to examine the associations between education, occupational skill level, and income and the Successful Aging Index. Additionally, associations between the three indicators of socioeconomic position and each separate domain of functioning were examined.

Results: Higher education, occupational skill level, and income were independently associated with higher numbers of successful trajectories. Education was more strongly related to successful aging than income and occupational skill level. Analyses of the separate domains of successful aging showed that individuals with lower socioeconomic position were especially at risk for less favorable trajectories in functional limitations, subjective health, cognitive functioning, and emotional support provided to others. Limited socioeconomic inequalities were found in satisfaction with life, instrumental support given and depressive symptoms. No inequalities were found in social activity and social loneliness.

Conclusion: Lower socioeconomic position in society adversely affects the longitudinal and multidimensional process of aging. However, while inequalities were relatively large in physical and cognitive functioning, older adults with lower SEP were as likely as individuals with higher SEP to show high functioning and stability in most psychosocial aspects of aging. While summary measures of aging thus provide a comprehensive picture of inequalities across relevant domains of functioning, they may entail a danger of glossing over crucial inequalities in specific aspects of functioning that bear importance for public health.

\section{Abstract \#: P 636}

Does rural/urban location moderate associations between weight status and children's dietary habits?

Aristides M. Machado-Rodrigues ${ }^{1}$, Rômulo Fernandes ${ }^{2}$, Augusta Gama $^{3}$, Isabel Mourão ${ }^{4}$, Helena Nogueira ${ }^{3}$, Cristina Padez ${ }^{3}$

${ }^{1}$ Research Centre for Anthropology and Health, University of Coimbra, Coimbra: ESE-IPV, Viseu, Portugal; ${ }^{2}$ Faculty of Science and Technology, Paulista State University - UNESP, Brazil; ${ }^{3}$ Research Centre for Anthropology and Health, University of Coimbra, Portugal; ${ }^{4}$ University of Tras-os-Montes and Alto Douro, Vila Real, Portugal; Corresponding author's e-mail: rodriguesari@hotmail.com

Background: The literature has commonly pointed out the area of residence as a factor that influences lifestyle, especially opportunities for healthy eating habits, physical activity and in turn public health policy. On the other hand, the burden of obesity is not borne equally and some disparities in prevalence should be clearly documented, particularly for low-income, minority and rural communities. Furthermore, understanding the causes of these disparities is crucial to the development and implementation of effective interventions to address obesity and its related conditions. The main purpose of this study was to examine whether rural/urban location moderate associations between weight status and dietary habits among female children.

Methods: The sample comprised 2055 randomly selected female children aged 7-9 years. Height and weight were measured. Body Mass Index (BMI) was calculated subsequently and participants were classified as normal weight or overweight/obese (Cole et al., 2000). Eating behaviours (i.e. have breakfast: consumption of soup and vegetables, and soft drinks and chocolate) were assessed by questionnaire as well as sedentary behaviours (i.e. TV viewing). Logistic regressions were used, with adjustments for age, time spent sedentary, and geographic location (i.e. rural vs. urban).

Results: The crude model revealed an inverse significant association between obesity and healthy eating habits (i.e. eating soup and milk in a daily basis). However, the final model, after controlling for rural/ urban location, just showed eating soup in a daily bases as significant predictor of child weight: children who consumed soup regularly had $62 \%$ less likely to be classified as overweight/obese children than their peers who skipping soup daily.

Conclusion: The present cross-sectional study revealed interesting relationships between some healthy eating habits and child weight, especially after controlling for biological and geographic confounders. However, future research should extend similar design in males to assess multiple meals that include a wider range of foods and beverages to confirm or not some of the afore-mentioned results.

\section{Abstract \#: P 637}

Predisposing factors related to methadone maintenance treatment intention among Iranian addicts

Mehdi Mirzaei Alavijeh $^{1}$, Farzad Jalilian ${ }^{2}$, Behzad Karami Matin ${ }^{2}$

${ }^{1}$ Department of Health Education \& Promotion, Faculty of Medical Sciences, Tarbiat Modares University, Tehran, Iran; ${ }^{2}$ Department of Public Health, School of Health, Kermanshah University of Medical Sciences, Kermanshah, Iran; Corresponding author's e-mail: m.mirzaeialavijeh@modares.ac.ir

Background: Methadone maintenance treatment (MMT) is a general treatment suite that involves the long-term prescribing of methadone as an alternative to the opioid on which the consumer was dependent: studies indicated that MMT is a cost-effective treatment for opioid dependence. The purpose of this study was to determine the predisposing factors related to MMT intention among sample of Iranian addicts.

Methods: This cross-sectional study was conducted in Kermanshah, Iran in 2013. A total of 762 male opiate addicts, who were referred to addiction treatment centers for receiving MMT, were randomly selected to participate voluntarily in the study. Participants filled out a writing questionnaire including the attitude toward MMT, perceived behavioral control not to use the drug, and MMT intention. Data were analyzed by the SPSS software (ver. 21.0) using the bivariate correlations, and linear regression at $95 \%$ significant level.

Results: Bivariate correlations showed MMT intention was significantly related to attitude $(r=0.455)$, and perceived behavioral control $(r=0.475)$. Linear regression analysis indicated attitude, and perceived behavioral control, accounted for $25 \%$ of the variation in the outcome measure of the MMT intention.

Conclusion: Based on our result, it seems that designing and implementation of educational programs to increase negative belief about drug and increasing perceived behavioral control not to use the drug may be usefulness of the results in order to prevent of relapse among addicts. 


\section{Abstract \#: P 638}

High perinatal mortality rate among immigrants in Belgium. A population-based cohort study from 1998 to 2010

\section{J. Racape ${ }^{1}$, S. Alexander ${ }^{1}$, M. De Spiegelaere ${ }^{1}$}

${ }^{1}$ School of Public Health - Université Libre de Bruxelles, Brussels, Belgium; Corresponding author's e-mail: jracape@ulb.ac.be

Background: Reproductive health among migrants is a major topic in most industrialized countries. Several epidemiological studies have described the pregnancy outcome of immigrant women in receiving countries with conflicting results. The objectives of this work were to describe and measure inequalities in pregnancy outcomes and perinatal mortality according to the nationality and socio-economic status of mothers in Belgium during 1998-2010.

Methods: This study is a population-based cohort study using the data from linked birth and death certificates from the Belgian civil registration system. Data are related to all babies born during 1998-2010 and whose mother was living in Belgium. Perinatal mortality, prematurity and low birth weight were estimated based on socio-demographic characteristics and nationality of the mother. We used maternal nationality at mother's own birth and at delivery of her child, as stated by the parents on the birth certificate. We grouped the nationalities in 7 categories: Belgian, EU27, Eastern Europe including Russia, Maghreb, Turkey, Sub-Sahara Africa and others. We used logistic regression to estimate the odds ratios (ORs) for the association between perinatal health and nationality.

Results: A total of 1,512,829 singleton births were recorded between 1998 and 2010 in Belgium. Women of Maghreb, sub-Saharan Africa, Turkey and Eastern Europe experience a significant excess in perinatal mortality compared to Belgian. For sub-Saharan Africa women, this excess reflects a high rate of preterm deliveries $(p<0.0001$ compared to Belgian), low birth weight $(p<0.0001$ compared to Belgian) and a low socio-economic level. Paradoxically, despite a lower risk of prematurity and low birth weight, Maghrebian mothers experience an excess of perinatal mortality even after adjustment for parity, mother's age and education and number of incomes in the household $(p<0.0001)$. Eastern European mothers have a significant lower perinatal mortality compared to Belgian after adjustment $(p<0.0001)$. This excess of perinatal mortality doesn't persist for naturalized mothers. Even after adjustment, Maghrebian naturalized mothers present a lower perinatal mortality than the native Belgian $(p=0.02)$.

Conclusion: In Belgium, patterns of inequalities in perinatal mortality and pregnancy outcomes vary according to nationality. Some nationality groups present an increased perinatal mortality regardless of socioeconomic status. The access to and use of healthcare could contribute to these differences. Analysis to understand how migration trajectories and socioeconomic status interact are currently underway.

\section{Abstract \#: P 639}

Social inequalities in self-rated health in central Kazakhstan: a population-based cross-sectional study

Lyudmila Turgunova $^{1}$, Yelena Laryushina ${ }^{1}$, Andrej M Grjibovski ${ }^{2,3}$, Dana Amirkhanova $^{1}$, Tatyana Bayesheva ${ }^{1}$, Assel Alina ${ }^{1}$

${ }^{1}$ Karaganda State Medical University, Karaganda, Kazakhstan; ${ }^{2}$ Norwegian Institute of Public Health, Oslo, Norway; ${ }^{3}$ Northern State Medical University, Arkhangelsk, Russia; Corresponding author's e-mail: andrej.grjibovski@gmail.com
Background: Self-rated health (SRH) has been widely used to assess health inequalities in both developed and developing countries. However, very few studies have been performed in the Asian part of the European WHO region. Those few published studies have reported large health inequalities in major cities warranting further research in less privileged areas. The aim of this study was to assess gender-, ethnic, and social inequalities in SRH in Central Kazakhstan.

Methods: The sample consisted of 3286 individuals aged 18-65 years randomly selected from two urban and two rural settings in Karaganda region (Central Kazakhstan) outside the regional centre. SRH was classified as poor, satisfactory, good, very good and excellent. Associations between SRH and age, gender, ethnic background, education, self-reported income, marital status, presence of chronic conditions and rural versus urban residence were assessed using multivariable logistic regression. Crude and adjusted odds ratios (OR) for "good or better" versus "worse than good" SRH were calculated with $95 \%$ confidence intervals (CI). Tests for linear trends were used for ordinal variables by including them as continuous in the model.

Results: Altogether, poor, satisfactory, good, very good and excellent health was reported by $6.5,49.5,36.7,4.2$ and $3.0 \%$ of the responders, respectively. Men were less likely to report "worse than good" health $(\mathrm{OR}=0.66,95 \% \mathrm{CI}: 0.55-0.79)$ then women. Clear gradients in SRH were observed by age, education and income in adjusted analysis (all tests for trends $p<0.001)$. The oldest age-group $(60+)$ had nearly three times as high odds as the youngest (18-29 years) group $(\mathrm{OR}=2.87,95 \% \mathrm{CI}: 2.11-3.90)$. Individuals with secondary $(\mathrm{OR}=1.37,95 \% \mathrm{CI}: 1.13-1.67)$ or vocational $(\mathrm{OR}=1.41,95 \%$ CI: 1.17-1.72) education had grated odds for "worse than good" SRH. The least economically privileged group had 1.72 (95\% CI: 1.29-2.28) greater odds for "worse than good" SRH compared to their most privileged counterparts. Chronic conditions increased the odds of worse than good SRH (OR $=2.46,95 \%$ CI: 2.11-2.86). No difference in SRH was found by marital status, ethnic background or place of residence.

Conclusions: Substantial levels of inequalities in SRH by age, gender, education and income, but not by ethnicity, place of residence or marital status were found in Central Kazakhstan. The results can be generalized to other regions of Kazakhstan with similar socio-economic conditions. Further research is warranted to identify the factors behind the observed associations in Kazakhstan.

\section{Abstract \#: P 640}

Which socioeconomic indicators are useful for social epidemiology studies-an ethical-theoretical approach

\section{Nina-Alexandra Götz ${ }^{1}$}

${ }^{1}$ University Osabrück/Hannover Medical School; Corresponding author's e-mail: nina.goetz@uni-osnabrueck.de

Most of the social epidemiology studies focus on traditional social indicators like income, education level and so on, as an approach to define deprivation resp. wealth in a population. The lack of alternative indicators for empirical studies makes it necessary to use these output-orientated indicators. Even as it is evident that e.g. income is not a reliable indicator to appropriate the well-being of a person or a following statement of the individual real opportunities is possible. According to justice-theoretical conceptions (like the Capability Approach by Amartya Sen) it is necessary to evaluate what persons are able to be or do and so to define there capabilities to do certain things in life. So, the question is if there is an urgency to discuss other alternative indicators for social epidemiology studies. 
As there is little known about the issue of capability operationalization it was used in a first step of the study a qualitative design with interviews of nine elderly persons (aged $55+$ ). Focus of this study are the elderly population, because of the hypothesis that especially in older age, other factors as income and so on are important for the elderly and brings quality in their life. This is an outstanding fact regarded to the official aim of social structure analyses by identifying deprivation individuals and measures what is helpful for quality of life. In the second part of the study is now used a Delphi-Method to define opportunity-based social indicators by experts of the fields of psychology, gerontology and sociology.

As the Delphi-Method is in progress there is up to now not a result of a finished social indicator set. But the field study will end in April and the result will be an indicator set of required capabilities with regarding to the theoretical-concept by Amartya Sen's Capability Approach.

The results of the qualitative interviews suggest the need for other dimensions to define as the used 'traditional' indicators in social epidemiology studies. Using these set of opportunity-based indicators could prospectively help to examine social epidemiology causeand-effect chains in an innovative way.

\section{Abstract \#: P 641}

Do social network aspects buffer the impact of neighborhood disadvantage on health related quality of life? The lifelines cohort study

Bart Klijs ${ }^{1}$, Carlos F. Mendes de Leon ${ }^{2}$, Eva U.B. Kibele ${ }^{3}$, Ronald P. Stolk ${ }^{1}$, Nynke Smidt ${ }^{1}$

${ }^{1}$ University of Groningen, University Medical Center Groningen, the Netherlands; ${ }^{2}$ University of Michigan School of Public Health, United States; ${ }^{3}$ University of Groningen; Corresponding author's e-mail: b.klijs01@umcg.nl

Background: A large social network and fulfillment of social needs are associated with better quality of life. It is unknown whether these aspects of the social network also buffer the potential impact of living in a disadvantaged neighborhood on quality of life. Our aim is to investigate to what extent socioeconomic deprivation, social isolation and community recourses in the neighborhood are associated with quality of life. Furthermore, we investigate to what extent more personal contacts and fulfilment of social needs buffer the potential impact of neighborhood disadvantage on quality of life.

Methods: A sub sample of the baseline data of the LifeLines Cohort Study $(n=67.830)$ was used and linked with data from Statistics Netherlands (i.e. demographic composition, socioeconomic characteristics and facilities in the participants' neighborhoods). Quality of life was assessed using the RAND-36 (physical and mental component scores). Using principal component analysis, composite indices of socioeconomic deprivation, social isolation and community resources in the neighborhood were constructed. Mixed effect linear regression models were fitted to assess associations of the indices with quality of life, adjusting for age, sex, marital status, education, income, and address density. Interactions of social network aspects*neighborhood index were added to the models to assess whether the associations differed by number of personal contacts $(<5$, $5-9,10-14, \geq 15$ per 2 weeks) and social need fulfillment $(<24$, $24-26, \geq 27$ points on the SPF-IL scale).

Results: Socioeconomic deprivation and social isolation are associated $(p<0.05)$ with lower physical (betas -0.30 and -0.29$)$ and mental quality of life (betas -0.20 and -0.37 ). Fewer community recourses is only associated with lower physical quality of life (beta $-0.24)$. Individuals with more social contacts or higher social need fulfillment have a better mental, but not a better physical quality of life. The associations of higher socioeconomic deprivation and social isolation with lower mental quality of life are smaller or absent among individuals with a large number of personal contacts or high social need fulfillment (all $p<0.001$ ).

Conclusion: Neighborhood disadvantage is associated with lower mental quality of life, but this association is less strong or absent for those with a large number of personal contacts or a high level of social need fulfillment. This suggests that interventions to increase the number of personal contacts or improve social support can contribute to a better mental quality of life of those living in disadvantaged neighborhoods.

\section{Abstract \#: P 642}

Social relationships and cognitive decline: a systematic review of longitudinal cohort studies

Jisca S. Kuiper ${ }^{1}$, Marij Zuidersma ${ }^{2}$, Richard C. Oude Voshaar ${ }^{2}$, Sytse U. Zuidema ${ }^{3}$, Ronald P. Stolk ${ }^{1}$, Nynke Smidt ${ }^{1}$

${ }^{1}$ Department of Epidemiology, University of Groningen, University Medical Center Groningen, Groningen, The Netherlands;

${ }^{2}$ Department of Psychiatry, University of Groningen, University Medical Center Groningen, Groningen, The Netherlands;

${ }^{3}$ Department of General Practice, University of Groningen, University Medical Center Groningen, Groningen, The Netherlands;

Corresponding author's e-mail: j.s.kuiper@umcg.nl

Background: Cognitive decline is considered as a normal process of ageing. However, in some cases the cognitive decline may proceed faster than normally is expected and results in limited daily functioning and a lower quality of life. Persons with cognitive impairment are at risk to develop mild cognitive impairment (MCI) and dementia. Poor social relationships are a risk factor for cognitive decline. However, conclusions from the existing literature are contradictory. Therefore, we conducted a systematic review of longitudinal cohort studies, to investigate the relation between various social relationship factors and cognitive decline in the general population.

Methods: We systematically searched in MEDLINE, Embase, and PsycINFO to identify longitudinal cohort studies investigating the association between social relationships and cognitive decline in the general population. The study selection, data extraction and the assessments of the methodological quality of included studies was performed by two reviewers independently. For each study, the odds ratio (OR) was calculated to present the association between the social relationship factor and cognitive decline. In case relative risk, mean, or (un)standardized regression coefficient was used, this was converted to ORs. Results were pooled in a meta-analysis using random effects models and summarized in a forest plot. Subgroup analyses were conducted for 1) the structural (i.e. social network size, frequency of social contact) and functional (i.e. social support, loneliness) social relationship factors, 2) duration of follow-up (short, intermediate, and long term) and 3) baseline risk for cognitive decline.

Results: Our initial search yielded 8.527 articles. After applying exclusion criteria, 44 studies (73.939 participants) were eligible for inclusion. The follow-up duration varied from 1.5 to 12.4 years and the mean age of participants ranged from 47 to 84 years old. More than half of the studies (55\%) found a statistically significant association between poor social relationship factors and cognitive decline. The final results of the meta-analysis and subgroup analyses will be presented at the conference.

Conclusion: This review will provide information on the association between social relationships and cognitive decline. 